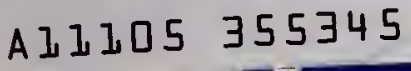

PUBLICATIONS
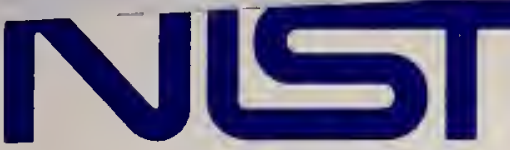

United States Department of Commerce

Technology Administration

National Institute of Standards and Technology

NIST Special Publication 918

PROCEEDINGS OF THE 1997

INTERNATIONAL CONFERENCE ON

INTELLIGENT SYSTEMS AND SEMIOTICS:

A Learning Perspective

A. M. Meystel, Editor

\section{ISAS '97 \\ World Model}

QC

100

.057

NO. 918

1997

Sponsored by:

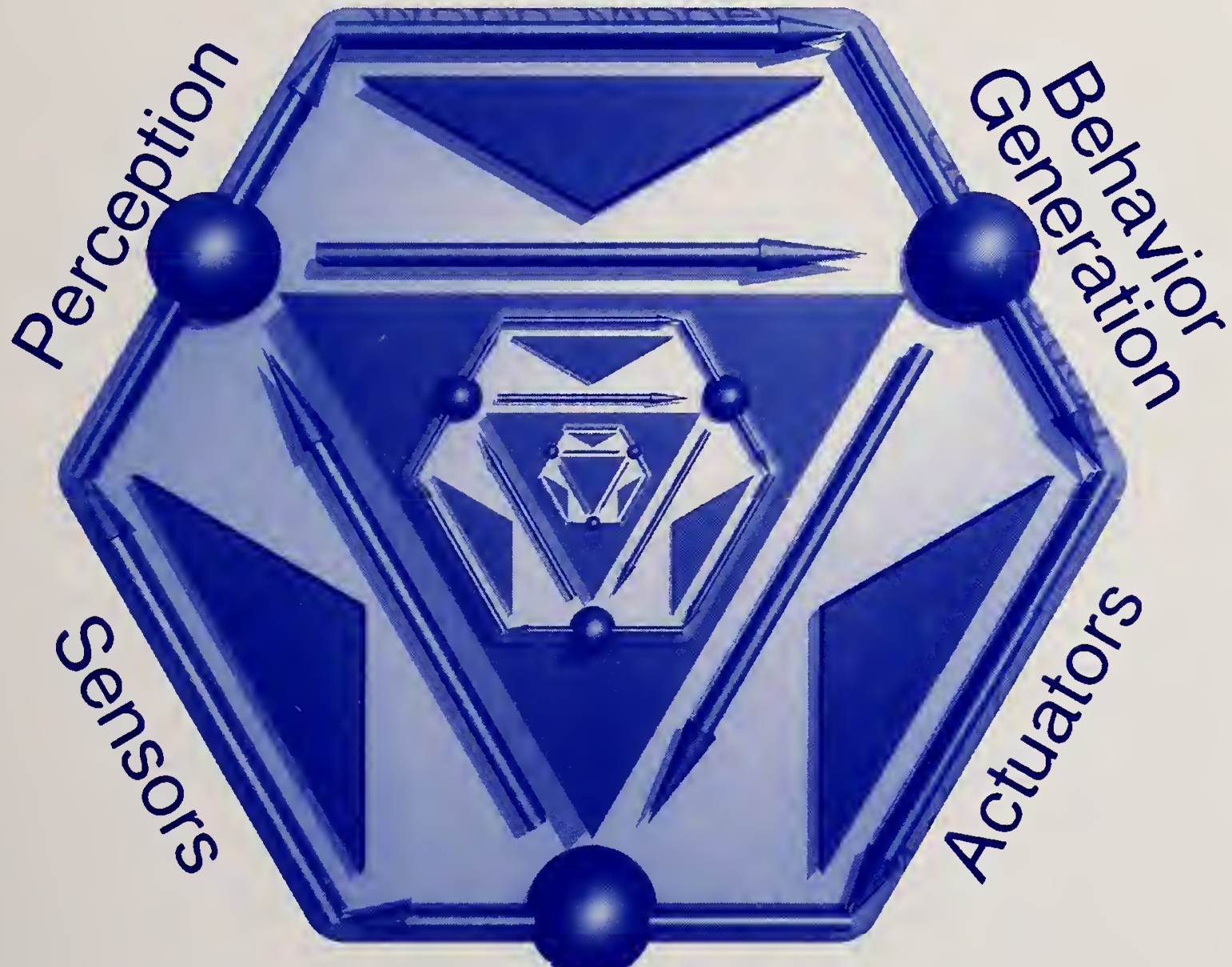

World

National Institute of Standards and Technology (NIST)

Institute of Electrical and Electronic Engineers (IEEE)

National Science Foundation (NSF)

Army Research Office (ARO) 



\section{NIST Special Publication 918}

\section{PROCEEDINGS OF THE 1997 \\ INTERNATIONAL CONFERENCE ON \\ INTELLIGENT SYSTEMS AND SEMIOTICS: \\ $A$ Learning Perspective}

ISAS ' 97

Gaithersburg, MD

September 22-25, 1997

A.M. Meystel, Editor

Manufacturing Engineering Laboratory

Intelligent Systems Division

National Institute of Standards and Technology

Gaithersburg, Maryland 20899-0001

September 1997

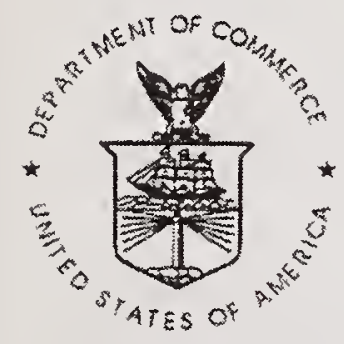

\section{SPONSORS}

National Institute of Standards and Technology

The Institute of Electrical and Electronics

Engineers, Inc.

National Science Foundation

Army Research Office

U.S. Department of Commerce

William M. Daley, Secretary

Technology Administration

Gary R. Bachula, Acting Under Secretary for Technology

National Institute of Standards and Technology

Robert E. Hebner, Acting Director 
ISBN: 1-88684-02-3

National Institute of Standards and Technology

Special Publication 918

Natl. 1nst. Stand. Technol.

Spec. Publ. 918

587 pages (Sept. 1997)

CODEN: NSPUE2
U.S. Government Printing Office

Washington: 1997

For sale by the Superintendent of Documents

U.S. Government Printing Office

Washington, DC 20402 


\title{
SEMIOTICS: THE TOOLBOX OF INTELLIGENCE
}

\author{
Editor's Preface
}

\section{Intelligent Systems}

Intelligence is magic.

We do not call things that we easily understand intelligence. Then, it is considered just an algorithm and as any algorithm, it seems to be mundane and mechanical.

Yet, magic accomplishes the goals in a presumably non-algorithmic way.

(I mean, magic not sorcery because although they both rely on incantations, i.e. use some kind of linguistic tools, sorcery employs evil forces, while our focus will be on useful applications like robotics, intelligent manufacturing, language translation, automated discovery, unmanned energy systems, large health care programs, analysis of music and poetry, well...battlefield too.)

This is why Sukhan Lee, one of the organizers of the Symposium on Computational Intelligence (Monterey, July 9-11, 1997), was surprised by the discovery that the conference could actually be called "IEEE International Symposium on Computational Magic." The panel, at which it was discussed, noticed that the similarity between magic and intelligence is in the depth and clarityof our knowledge of them.

This reminds us of the commonality between intelligence and quantum mechanics. Many people are looking forward to uncovering the mystery of intelligence by the means of quantum mechanics. This commonality is in the similarity among them in both the depth and the clarity of our knowledge of them.

We cannot avoid using the term intelligence. We can even see some benefits in attributing the term, intelligent system, to many not clearly defined real world systems: the ones that demonstrate some magic that is typical for intelligence. We are dreaming of intelligent robots that are capable of getting to the core of an unexpected situation. Say, a particular situation is not presented in the list of behavior rules. Yet, the robot can figure out how to deal with it. That is intelligence!

When the famous Deep Blue browses the list of precomputed possibilities with breathtaking speed, we are disappointed. We would prefer a slower thinker to come up with a winning solution with no browsing at all-possibly by magic. 
Michael Botvinnik, the Chess Champion of the Post-WWII era, envisioned a multiresolutional system of planning in future chess-playing computers. Multi-level decision making was his interpretation for his own mechanisms of winning.

Intelligent System is a metaphor for the system which functions but yet cannot be fully understood. To understand it, we have to use another intelligent system -ourselves-which we cannot fully understand either. The metaphor of intelligent system allows for prediction and control of the systems of interest, although the properties and the mechanisms of these systems are not totally clear to us. We are compelled to use this metaphor and this system, although its algorithm cannot be clear to us now (and maybe will never be clear at all.)

We are interested in scientific and practical benefits in using this metaphor and its theories. The metaphor of intelligent system maybe is applicable in all domains and at all levels of granularity, or resolution. We have to show this in the future. If we succeed, then the embedded incompleteness of our theory will be forgiven by the scientific community, (especially because in the case of intelligent systems this incompleteness is forever!)

Can we say something substantial about intelligent systems today?

We can say that intelligent systems are generalizing machines. Their way of surviving is to establish a trade-off between unbearable multiplicity of needs and desires and an unbearable amount of information processing, which is required for this. They generalize by the virtue of grouping (clustering), focusing attention, and combinatorial searching.

In the meantime, the procedure of generalization is always associated with a loss of information. This is why the bottom-up process of re-generalization should be repeated constantly. Sooner or later, the entities of the upper level would change inadvertently. When new information is incorporated, the procedure of regrouping, refocusing, and re-searching will be performed. Thus, the previously created multiresolutional system of representation will be changed as a partial effect of such a learning process.

(See? Would it be sufficient to look for the explanation for intelligence only within the quantum physics? Quantum mechanics itself is a product of the multiresolutional system of intelligence.)

\section{Semiotics}

Semiotics is the only system of thought that dares to consider itself as an object of its own analysis. Like intelligence, semiotics is a self-referential and self-reflective system. All components of intelligence including generalization with its focusing attention, search and clustering are parts of semiotics. Actually, what we called learning process, in semiotics is called semiosis. 
Semioticians are concerned with the issue "where is our niche?" This is an important issue, but you have to specify what level of resolution you are talking about. Indeed, you may look for a niche in one room versus another, in one country or state versus another, or even in one galaxy versus another.

When I talk about this search for a niche, limagine crowds of scientists intruding the Wild West of Intelligence looking for a place to prosper, or the crowd of scientists like gold miners trying to stake a place in the Klondike of Intelligence. Remember that everything happened literally on our eyes. Indeed, Ampere's "Kybernetes" and Maxwell's "On Governors" appeared in in the middle of 19th Century. At about the same time, C. Peirce wrote his papers on semiotics. Wiener's "Cybernetics" was published a century later. We did not even have enough time to properly digest these works.

The invariance of results in different scientific domains is not in looking for answers to the question "What?" but rather in looking for answers to the question "How?"

Semiotics is the one which is looking for "How?" for all domains together because our intelligence is common for all domains.

The well-known saying is that mathematics is the language of Science.

Yes, but it uses Semiotics as its Toolbox, the Metalanguage.

Semiotics supplies its Metalanguage to all disciplines of Science that are therefore dialects of Semiotics.

Semiotics is mathematics that aspires for symbol grounding.

Semiotics is mathematics that does not delegate symbol-grounding to engineers.

Semiotics is mathematics that remembers that all axiomatic theories are just alternatives of World simulation or for World simulation. It teaches engineers how to perform symbol grounding after a simulation is done.

Let us ask the question, "What is Life, a playground, or a place with situated meaning?" Semiotics does not follow the temptation to answer "a little bit of both." Semiotics is looking for, interested in, and striving toward a situated meaning of Life.

For semiotics, the existentialist issue "I think, therefore I am" does not exist. It is merely a philosophical question. For semiotics, the insight "I think, therefore laxiomatize" has only a partial importance. This is a mathematical issue. For semiotics, the issue is, "I think, therefore, I must understand how do I do it." 
Yes, mathematics is a dialect of semiotics. But, semiotics always attacks the problem before mathematics does. Mathematics can be applied only after we understand the system well or can pretend to understand it well.

\section{Conference ISAS'97}

Jourdain, the protagonist of Moliere's play "Le Bourgeois Gentilhomme, " was surprised when he found out that he speaks prose. Most of the professionals in science and other intellectual areas have no idea that they speak semiotics.

We have a mission: to demonstrate the power of semiotic tools to them, to promulgate understanding, to diminish confusion, to enhance clarity.

Semiotics is the invariance of all disciplines represented at our conference. Participants of the conference and readers of this volume will easily recognize the relevance of each discipline both to intelligent systems and to semiotics.

Some of these quests will be easy; some will require an effort. The theory of games is ultimately semiotic. It is self-referential. Its implicit component is reflection, and it realizes that known theory does not guarantee to win the game. When you think about it, the theory of automata can be considered an attempt to decompose Peircean triangle into its components.

I hope that the toolbox of semiotics will be useful in your particular domain of science, in its particulardialect. I hope that it will enhance your power of analysis and interpretation, and allow you to express the meaning we always search for.

I wish you happy semiotic analysis of multiple complex intelligent systems you will encounter att this Conference.

\section{A. Meystel}

July 26, 1997 


\section{Conference Committee}

Honorary Conference Chairs - Thomas A. Sebeok, Indiana University and Lotfi Zadeh, University of California at Berkeley

General Chair - James Albus, NIST

Program Chair - Alex Meystel, NIST/Drexel University

Educational Activities Chair - Kevin Passino, Ohio State University

Local Arrangements Chair - Richard Quintero, NIST

Publication Chair - David Coombs, NIST

Publicity Chair - Elena Messina, NIST

Vice-Chair, Publications - Patricia Flanagan, NIST

\section{International Program Committee}

K. Baheti, NSF

Z. Bien, KAIST, Korea

K. Bellman, DARPA

P. Borne, Ecole Centrale de Lille, France

B. Bouchon-Meunier, U. P Et M Curie, France

J. L. R. Chandler, WESS

A. Chikrii, Institute of Cybernetics, Ukraine

M. Coombs, NMSU, USA

B. Dean, Sandia National Laboratories, USA

E. D. Dickmanns, U. der Bundeswehr, Germany

V. Finn, VINITI, Russia

D. Fogel, Natural Selection, USA

K. Furuta, Tokyo Inst. of Technology, Japan

J. Goguen, U. of California at San Diego, USA

J. Gray, U. of Salford, UK

W. Gruver, SFU, Canada

M. Gupta, U. of Saskatchevan, Canada

M. Herman, NIST, USA

C. Joslyn, Los Alamos Nat'l Lab

M. Jubert, NIST, USA

S. Kawaji, Kumamoto U., Japan

O. Kaynak, Bogazici U., Turkey

Y. Klimontovich, Moscow State University, Russia

L. Kohout, Florida State University, USA

M. Kokar, Northeastern University, USA

V. Kreinovich, U. of Texas at El Paso

S. Lee, U. of So. California, USA

V. Lefebvre, U. of California at Irvine, USA

D. A. Linkens, U. of Sheffield, UK

R. Lumia, U. of New Mexico, USA

R. Mann, Oak Ridge National Laboratory, USA

J. Maulbetsch, EPRI, USA

G. T. McKee, U. of Reading, UK

L. Miller, NGIS, USA

B. Mirkin, DIMACS, Rutgers U., USA

A. Mironov, Moscow State University, Russia

M. Modarres, U. of Maryland, USA

I. Muchnik, DIMACS, Rutgers U., USA 
C. Nehaniv, U. of Aizu, Japan

V. Novak, U. of Ostrava, Czech Republic

U. Ozguner, Ohio State University, USA

L. Perlovsky, Nichols Research Corporation, USA

D. Pospelov, Comp. Ctr. Academy of Science, Russia

K. Pourrezaei, Drexel U., USA

T. Reader, WSMR, USA

B. Rieger, O. of Trier, Germany

G. Ritter, U. of Florida, USA

P. Schenker, JPL, USA

K. Schlussel, NGIS, USA

D. Skatrud, ARO, USA

A. Stopp, Daimler-Benz, Germany

M. Sulcoski, NGIS, USA

H. Szu, U. of SW Louisiana, USA

S. G. Tzafestas, NTUA, Greece

J. Umiker-Sebeok, Indiana U., USA

B. Turksen, University of Toronto, Canada

J. C. A. van der Lubbe, Delft U. of Technology, The Netherlands

F.-Y. Wang, U. of Arizona, USA

P. Wang, Duke U., USA

J. M. Weiss, EPRI, USA

P. Werbos- National Science Foundation, USA

F. Zhao, Ohio State U. 


\section{ISAS'97 CONFERENCE PROCEEDINGS}

\section{TABLE OF CONTENTS}

Semiotics: The Toolbox Of Intelligence: Editor's Preface

\section{PLENARY LECTURES}

Why Now Semiotics.

James S. Albus, National Institute of Standards and Technology

Top-Down Engineering

Lawrence J. Fogel, Natural Selection, Inc.

Ordinal Optimization and Soft Computing Yu-Chi Ho, Harvard University

Introduction to Quantum Theory of Open Systems 14 Yu.L. Klimontovich, Moscow State University

Information Granulation and its Centrality in Human and Machine Intelligence 26 Lotfi A. Zadeh, University of California at Berkeley

\section{WORLD REPRESENTATION}

The Role of Semiotic Descriptors in Relational Representation of Fuzzy Granular Structures

Ladislav J. Kohout and Eunjin Kim, Florida State University

An Integrated Approach to Fuzzy Learning and Reasoning in Hierarchical Knowledge Structures

Jan C.A. van der Lubbe, Delft University of Technology and Emile Egbers, Zappwerk

Conceptual Categories as Knowledge Structures

Christopher Landauer, The Aerospace Corporation

Knowledge Representation Language in Semiotic Systems

V.N. Vagin and E.Yu. Golovina, Moscow Power Engineering Institute 
"Intelligence and Semiotics in Pattern Recognition and Neural Networks"

David Casasent, Carnegie Mellon University

Morphological Perceptrons

Gerhard X. Ritter and Peter Sussner, University of Florida

Traps and Tricks in Standard Benchmark Problems for Neurocontrol

Ludmila M. Dolmatova, Scientific Cybernetics, Inc.

Paul J. Werbos, National Science Foundation

Neural Network Based Planner/Learner for Control Systems

J. Albus, National Institute of Standards and Technology

A. Lacaze, National Institute of Standards and Technology, University of Maryland

A. Meystel, National Institute of Standards and Technology, Drexel University

N. DeClaris, University of Maryland

A Hybrid Hierarchical Neural-AI Model of Mammal-Like Intelligence. 82

Paul J. Werbos, National Science Foundation

\section{LOGICAL CALCULI FOR SEMIOSIS}

Plausible Reasoning and Its Logics

Oleg M. Anshakov, Victor K. Finn and Dmitry P. Skvortsov

VINITI - All Russian Institute of Scientific and Technical Information

Application of JSM-Theory of Plausible Reasoning to Social Data Analysis and Social

Actions Prediction

V.K. Finn and M.A. Mikheyenkova

VINITI - All Russian Institute for Scientific and Technical Information

Algorithmic Complexity of Generating Hypotheses and Forecasts in Version Spaces

Formed by Concept Lattices

S.O. Kuznetsov, Intelligent Systems Laboratory

To the Scaleable Technology of Automated Document Understanding based on Quasi-Axiomatic Theories

M.I. Zabezhailo, Intelligent Systems Laboratory

NonMonotonic Reasoning in the Modal Quantificational Logic Z

Frank M. Brown, University of Kansas

\section{LEARNING}

Inductive Theory of Semiotic Systems

Lev Goldfarb, University of New Brunswick 
Unified Learning/Planning Automaton: Generating and Using Multigranular

Knowledge Hierarchies

Alex Meystel, National Institute of Standards and Technology, Drexel University

Alberto Lacaze, National Institute of Standards and Technology, University of Maryland

Structural Learning for Classification of High Dimensional Data

Paul M. Baggenstoss, Naval Undersea Warfare Center

Learning from Large Dimensional, Small Data by a Potential Function Method with

Nonlinear Transformation: A Cancer Diagnostic Study

Mark Modell, MediSpectra, Inc., Victor Kamensky, Albert Einstein College of Medicine,

Casimir Kulikowski and Ilya Muchnik, Rutgers University and

Louis Burke and Jonathan Niloff, Harvard Medical School

Incremental Learning of Inheritance.

Jose Neves, Evgueni Smirnov and Cesar Anlide, University of Minho

\section{MULTIRESOLUTIONAL SEMIOSIS}

Multi-Resolution Data Processing: It is Necessary, It is Possible, It is Fundamental .... 145 S. A. Starks, V. Kreinovich, University of Texas at El Paso

A. Meystel, National Institute of Standards and Technology, Drexel University

Multiresolutional Planning with Minimum Complexity

J. Albus, National Institute of Standards and Technology

A. Lacaze, National Institute of Standards and Technology, University of Maryland

A. Meystel, National Institute of Standards and Technology, Drexel University

Multi-Resolution Data Processing is Optimal: Case Study of Detecting Surface Mounted

Devices

A.E. Brito, O.M. Kosheleva, and S.D. Cabrera, University of Texas at El Paso

An Artificial Life View on Intelligent Enterprises - Fuzzy Evolution Multi-Agent

Systems.

Valery Tarassov, Bauman Moscow State Technical University

\section{ORDINAL OPTIMIZATION}

Ordinal Optimization for a Class of Combinatorially Hard Problems

Christos G. Cassandras, Boston University, Liyi Dai, Washington University and

Christos G. Panayiotou, University of Massachusetts at Amherst

Three Techniques for Ordinal Optimization: Short Simulation Runs, Crude Analytical

Models, and Imprecise Simulation Models

Jeffery E. Wieselthier, Naval Research Laboratory, Craig M. Barnhart, $T R W$, and

Anthony Ephremides, University of Maryland 
Selection Schemes in Ordinal Optimization and Their Computational Advantages

T.W. Edward Lau, Harvard University

Intelligent Computing Budget Allocation for Discrete Event Simulation and

Optimization

Chun-Hung Chen, Karen Donohue of Pennsylvania

Liyi Dai, Washington University and Enver Yucesan, INSEAD

Comparison of Performance Orders of Different Design for Discrete-Event Dynamic Systems

Liyi Dai, Washington University, and Chun-Hung Chen, University of Pennsylvania

\section{ARCHITECTURES}

A Case-Based Integrated Learning Architecture and Its Applications

Gennady Agre, Bulgarian Academy of Sciences

Computational Embodiment: Software Architecture.

Christopher Landauer, The Aerospace Corporation, and

Kirstie L. Bellman, Defense Advanced Research Projects Agency

Emergence of Complex Organized Natural Systems, A Hierarchically - Structured Conceptualization and Notation of Organization.

Jerry L.R. Chandler, Krasnow Institute for Advanced Studies

A Holonic Approach to Intelligent Control of a Flexible Transfer System for

Manufacturing

Mihaela Ulieru, Simon Fraser University

Learning Coordination Patterns from Emergent Behavior in a Multi-Agent-Based

Manufacturing System.

F. Maturana, S. Balasubramanian, and D.H. Norrie, University of Calgary

\section{EVOLUTION}

Speculation on the Evolution of Symbols 233

David B. Fogel, Natural Selection, Inc.

The Evolution of Intelligence from the Semiotic Perspective

John R. Alexander, Jr., Towson State University

On Hardware Evolvability and Levels of Granularity Adrian Stoica, Jet Propulsion Laboratory

Evolution of Learning: The Merging of Semiotic and Evolutionary-System Theories . 248 Wolfgang Hofkirchner, Vienna University of Technology 
The Effects of Biological Aging on Genetic Algorithms

Tim Schnelmer and Frank M. Brown, University of Kansas

\section{AUTONOMY}

Adaptive Autonomy: The Key to Dynamic, Responsive Formation of Sensible Agent Organizations

K. Suzanne Barber, The University of Texas at Austin

Finding and Using Multiple Models for Autonomous Learning

I.N.P. Davidson, CSIRO

Agent Based Task Viewing in Telerobotic Environments

Gerard T. McKee, Bernard G. Brooks, University of Reading

Paul S. Schenker, Jet Propulsion Laboratory

A Unified Approach for Fast Recognition, Learning and Interpretation of the

Environment by an Autonomous Mobile Robot

Andreas Stopp and Lars Küttner, Daimler-Benz AG

Learned Emergence of Functional Symbol Systems in Adaptive Autonomous Agents,

Guided by Skinner's Analysis of Verbal Behavior

William R. Hutchison, Behavior Systems LLC

\section{LIKELIHOOD, MIXTURES, AND SYMBOLS}

Towards Quantum Field Theory of Symbol

Leonid I. Perlovsky, Nichols Research Corporation

Semiotic Compounds - Mixtures of Components Invariant Under Specified

Transformations

Roy L. Streit, Naval Undersea Warfare Center

Information, Likelihood, and Einstein

Leonid I. Perlovsky, Nichols Research Corporation

A Symbolic Representation of Misconceptions

Benjoe A. Juliano, Coastal Carolina University

\section{SEMIOTICS OF TEXTS AND IMAGES}

Mathematical Aspects of Cyberaesthetics

Leonid I. Perlovsky, Nichols Research Corporation 
Semiotics and Modeling Computer Classification of Text with Genetic Algorithm:

Analysis and First Results

Vincent Rialle, Sofiane Oussedik, Université Joseph Fourier and

Jean-Guy Meunier, Georges Nault, Université du Québec à Montréal

Dynamic Word Meaning Representations and the Notion of Granularity. Text

Understanding as meaning constitution by SCIPS

Burghard B. Rieger, University of Trier

Cyberasthetics in Light of Zoosemiotics

Victor Dmitriev, Oklahoma State University

Leonid I. Perlovsky, Nichols Research Corporation

The Hole in the System

Floyd, Merrell, Purdue University

\section{MULTIDISCIPLINARY APPLICATIONS OF SEMIOTICS}

Control Mechanisms for a Nonlinear Model of International Relations

Áron Péntek, Jim Kadtke, University of California at San Diego

Suzanne Lenhart, University of Tennessee and

Vladimir Protopopescu, Oak Ridge National Laboratory

Galen in Medical Semiotics

Thomas A. Sebeok, Indiana University

Introduction in Physics of Open Systems: Main Principles. Diagnostics on the "S-Theorem" Criterion. Norm of Chaos. Degradation and Self-Organization.

Phase Transitions 364

Yu. L. Klimontovich, Moscow State University

Non-Equilibrium Themodynamics Explains Semiotic Shapes: Applications to Astronomy and to Non-Destructive Testing of Aerospace Systems.

Roberto Osegueda, Carlos Ferregut, Mary J. George, Jose M. Gutierrez, and

Vladik Kreinovich, University of Texas at El Paso

Towards Computers of Generation Omega - Non-Equilibrium Thermodynamics, Granularity, and Acausal Processes: A Brief Survey

M. Koshelev and V. Kreinovich, University of Texas at El Paso

\section{SEMIOTICS OF OPEN SYSTEMS}

'Seeing' the Battlefield through Virtual and Constructive Simulation

Ken Hunt, Mounted Maneuver Battlespace Lab 
TVA's Kingston Unit 9 Distributed Control System (DCS) Retrofit Benefit

Documentation.

Dennis Linkins, TVA Technology Advancement

David Lewis, TVA Kingston Fossil Plant, Rob Frank, EPRI U\&C Plant and

Joe Weiss, EPRI

Evaluation Measures for the Collective Intelligence of Closed Social Structures

Szuba Tadeusz, Kuwait University

The Semiotics of Autonomous Investigations of Natural Systems

Paul S. Prueitt, Highland Technologies, Inc.

\section{SOFT MODELING}

Soft Modeling for Intelligent and Complex Systems.

Paul P. Wang, Duke University

Computational Embodiment: Biological Considerations

Kirstie L. Bellman, Defense Advanced Research Projects Agency

Christopher Landauer, The Aerospace Corporation

The Neurodynamics of Intentionality in Animal Brains Provides the Basis of Intelligent Behavior

Walter J. Freeman, University of California at Berkeley

Hypermedia Integration of Information Resources for Plant Operations

Lefteri H. Tsoukalas, Purdue University, and

Robert E. Uhrig, University of Tennessee at Knoxsville

\section{GAMES AND REFLEXION}

Game Problem on Soft Landing for Moving Objects Arkadii A. Chikrii, Cybernetics Institute

Game Problems of Control with Delay of Information

Greta Ts. Chikrii, Cybernetics Institute

Reflexive Game Theory.

Vladimir A. Lefebvre, University of California at Irvine 


\section{COMPUTATIONAL APPROACHES IN SEMIOTICS}

Supporting End-User Programming with Explanatory Discourse.

Clarisse Sieckenius de Souza, PUC-Rio

An Approach to Computational Semiotics

Ricardo Gudwin and Fernando Gomide, DCA-FEEC-UNICAMP

Sequential Algorithm for the Semantic Representation of Actions in Terms of Moving

Parts

Hiroyuki Nishina, Saitama University

Solving the Forward Kinematics Problem for the Stewart Platform by Focusing Attention and Searching.....
A. Lacaze, National Institute of Standards and Technology, University of Maryland
C. Tasoluk, Drexel University
A. Meystel, National Institute of Standards and Technology, Drexel University

\section{BRAIN, MENTALITY, AND MEANING}

A Neurobiological Interpretation of Semiotics: Meaning, Representation, and Causality

Walter J. Freeman, University of California at Berkeley

Intelligence vs. Mentality: Important but Independent Concepts

James H. Fetzer, University of Minnesota

Functional Organization of Cortico - Basal Ganglia - Thalamocortical Loops

K.V. Baev, Barrow Neurological Institute

Learning How to Know: Semiotics and Multiscale Cybernetics 505

A. Meystel, National Institute of Standards and Technology, Drexel University

\section{SEMIOTICS IN INTELLIGENT CONTROL}

Semiotics: a Key to Intelligent Control

Tariq Samad, Honeywell Technology Center

Intelligent vs. Reflex Control for Complex Dynamical Systems Michel Cotsaftis, LTME/ECE

Introduction to Integrated Learning/Planning Paradigm

A. Meystel, National Institute of Standards and Technology, Drexel University

A. Lacaze, National Institute of Standards and Technology, University of Maryland 


\section{ISAS ‘96 PREVIOUSLY UNPUBLISHED PAPERS}

A Roadmap to the Future for Intelligent Systems and Semiotics. James S. Albus, National Institute of Standards and Technology

Computational Semiotics and Fuzzy Linguistics: On Meaning Constitution and Soft

Categories

Burghard B. Rieger, University of Trier

Linguistic Geometry: Model Evaluation

Boris Stilman, University of Colorado at Denver

\section{ISAS'96" CONFERENCE REVIEW}

ISAS'96: Conference Review

Prepared by A. Meystel, K. Bellman, D. Filev, J. Goguen, C. Hewitt

C. Joslyn, L. Kohout, C. Landauer, I. Muchnik, L. Perlovsky,

V. Stefanuk, and Y. Yufuk

\section{LATE SUBMISSIONS}





\section{PLENARY LECTURES}





\title{
Why Now Semiotics? \\ From Real-Time Control \\ to Signs and Symbols
}

\author{
James S. Albus \\ Chief, Intelligent Systems Division \\ Manufacturing Engineering Laboratory \\ National Institute of Standards and Technology \\ albus@cme.nist.gov
}

Why is NIST hosting a conference on Semiotics? NIST's primary interest is in intelligent control systems for manufacturing and in standards and measures for open architecture controllers. What has that to do with the science (dare I say the philosophy) of signs and symbols? What has semiotics to say to manufacturing? You can read for a long time in the semiotics literature before you encounter a reference to the problem of data communications between a CAD database and a numerically controlled machine tool. So where is the connection? What does the engineering of intelligent real-time control systems have in common with the science of signs and symbols?

\section{Control}

To answer this last question, let's take a look at the field of control. The history of real-time control begins with the invention of the fly-ball governor. The problem was how to regulate the flow of steam to control the speed of a steam engine under variable load conditions. The solution was feedback. If the engine was running too slowly, the fly-ball arms fell down, opening a valve and allowing more steam to flow. If the engine was running too fast, the fly-ball arms flew out, closing the valve and slowing the flow of steam.

The development of feedback control theory reached a peak during World War II with the development of radar controlled anti-aircraft guns. Norbert Wiener was the intellectual leader of this effort. He founded the field of cybernetics. Feedback control theory matured in the aerospace industry during the Cold War race for supremacy in the skies and outer space. Today, feedback control theory provides the mathematical foundations for servo controllers used in millions of applications.

Feedforward control is simply a recognition that if you know enough about the system you are controlling, you can design a pattern of inputs to the controller that will produce the desired output from the system.
One example of feedforward control is the control program for a machine tool. The machine tool and the metal cutting processes are sufficiently well characterized so that low level servo controllers can guarantee that the tool will go to the points along the paths designated in the program. A typical machine tool program may include thousands of steps which proceed from start to finish with essentially no feedback from the machine.

A more interesting example of feedforward control is the use of plant models (and inverse plant models) for process control. If you want to get a plant to produce a certain output, first develop a plant model (transition matrix between inputs, states, and outputs). Then, invert the model. When you apply the desired output to the inverse plant model, it will produce the proper control sequence that if input to the plant will cause it to generate the desired output. In other words, an inverse plant model put in series with a modeled plant produces a transfer function of unity. Thus, the desired output applied to the series combination of the inverse model and the plant itself will produce the desired output from the plant. It is amazing that this works. How well it works depends on the fidelity of the inverse plant model. Bernie Widrow just published a book on how to use neural nets to develop inverse plant models even for non-minimum phase plants.

In the field of artificial intelligence (AI) and robotics, a great deal of research has been done on planning. A plan expressed as a series of commands is essentially a feedforward control sequence. Thus, planning is a form of feedforward control. A planner uses a plant model to predict the results of hypothesized actions. The ability to predict is a key (perhaps THE key) to intelligent behavior. The development of prediction theory was first developed by Kalman for use in designing recursive filters. A Kalman filter predicts one sample period into the future. An AI planner requires a model that can predict the future out to a 
planning horizon which may be many seconds, minutes, hours, or days into the future.

Recently, controller developers began to combine feedback and feedforward control techniques to achieve the advantages of both. AI system designers have begun to combine deliberative planners (feedforward) with reactive (feedback) controllers. Control theory is beginning to focus on prediction -- to anticipate the requirements of the future in addition to compensating for errors from the past.

Both feedback and feedforward control systems employ concepts such as signals, states, state variables, events, and event tokens. Knowledge of plants, processes, entities, attributes, and plant behavior are stored in a variety of numerical and symbolic formulae, rules, state-transition matrices, and state graphs consisting of characters and words, images, frames, graphs, and pointers.

Control systems must also deal with real-time issues such as response time, bandwidth, sampling rate, stability, observability, and controllability. Control theory has developed an arsenal of mathematical tools for optimizing designs and for incorporating adaptive and learning algorithms into the control system. Most recently, attention has been focused on fuzzy systems, neural nets, and genetic algorithms.

\section{Semiotics}

Semiotics is the science of signs and symbols. Fundamental to signs and symbols is the notion of representation. The dictionary definition of the word "represent" means "to be a symbol for or an image of something in the world." Representation requires data structures that represent (and presumably) correspond to entities and situations in the world.

Symbol grounding refers to the problem of establishing and maintaining correspondence between symbols in the data structure and things in the world. In an intelligent system, signal grounding is achieved by sensory processing and world modeling functions that establish and maintain correspondence between the internal world model and external reality.

It was Immanual Kant who first made clear the distinction between a representation that exists in the mind and objective reality that exists in the world. We need to keep this distinction clear in the design of intelligent semiotic systems. Entities and situations in the world are sensed producing observed signals and images that are segmented into observed entities. Observed entities and images are filtered to form estimated entities and images. Dynamic models can then produce predicted entities and images. Predicted entities and images plus desired goals can produce plans and actions in the world.

Semiotics has a lot to do with language, particularly regarding semantics, pragmatics, causality, and plausibility.

Semantics defines the meaning of what is encoded in grammatically correct strings of symbols and words. One of the most basic forms of meaning is contained in the logical calculus of deduction, inference, and probability. Semantics can be formalized in relational graphs consisting of networks of nodes and edges where the nodes are words and the edges are relationships.

Relational graphs can also be used to describe pragmatics (what things are used for) and causality (what things cause other things). Rules, equations, formulae, and algorithms can be used to define how things work and what effect some things have on other things. For example, $\mathrm{F}=\mathrm{ma}$ is a formula that describes the relationship between force applied to a mass and the acceleration that will be experienced by that mass. The formula $\mathrm{F}=\mathrm{ma}$ can be used to compute how much force is needed to produce a desired acceleration or how much acceleration to expect when a particular force is applied.

Situations can be represented by words and narrative text. However, situations can also be represented by images, drawing, maps, and pictures. For reasons that I do not fully understand, semiotics seems much less concerned with images and maps than with words and symbols. Apparently, a strong preference exists among researchers in semiotics, as well as in artificial intelligence, and linguistics to represent the world in symbolic form -- i.e., in frames, relational graphs, and text, rather than in pictures and maps.

But words are often inadequate to fully describe the richness and dynamic vibrancy of real world situations. Especially if we take into account the totality of the sensory experience -- vision, hearing, smell, taste, touch, and gut feelings -- words become a cumbersome and sparse medium for representation. 


\section{Images as Representation}

In this presentation, I want to argue the case that image representations are much more important than anyone would guess from reading either the semiotic or the control theory literature. I would argue that images are not just sensory input to be dispensed with as soon as it is possible to derive a symbolic representation. Images are repositories of information about spatial and temporal detail that cannot be represented as efficiently in any other format.

It is clear that the use of images for representation of knowledge and computing developed very early in the evolution of the brain. Visual competence existed in the brains of sea creatures and insects almost a billion years ago. Fish, crustaceans, and insects have eyes and neural computing structures for processing images. Birds and mammals all represent the visual world and process images for purposes of locomotion and manipulation. Vision is crucial to hunting for food and evading danger in almost every species. There is good neurophysiological evidence from birds and primates that neurons on the surface of the superior colliculus are anatomically arranged so as to form a visual map on which targets of attention are displayed. Signals from this genetically designed map representation are then transformed directly into commands to muscles to move the eyes.

In marked contrast, language comes very late in the evolutionary history of the brain. Birds, mammals, and even insects use a variety of signs and symbols to signal their presence and their sexual interests. Many animals use symbolic gestures or sounds to communicate intentions such as aggression, submissiveness, friendliness, or fear, or to warn others of danger. Nevertheless, in all species, language competence is primitive when compared to visual processing capabilities. Among humans, the level of language competence that most occupies researchers probably did not exist in verbal or sign language form more than 100,000 ago. Written forms of language first appeared only about 10,000 years ago.

It is said that a picture is worth a thousand words. Clearly, the capabilities of the brain for representing and processing visual information far exceeds those for language representation and processing. From this fact alone, I would think that more emphasis should be placed on the use of images as a mechanism for thinking about and planning for the future. We should pay more attention to computing with images, rather than computing on images to extract symbolic data and then discarding the images.

Images are derived directly from sensors. The sensor egosphere is a very natural coordinate frame for representing knowledge about the world -- especially knowledge about where things are and how they are moving. We often communicate information through pointing to things. We show each other how to do things. We compute where to place our feet based on where supporting surfaces appear in our visual world. We visually track targets we intend to pursue and look directly at things we intend to manipulate. We flee from parts of the egosphere that contain threatening images.

Images that are overlaid with symbolic information about objects are called maps. Maps can be used to represent situations and to make plans. For example, military maps of the battlefield typically show the elevation and slope of the terrain, the type of ground cover (trees, grass, buildings, roads), the type of terrain (lake, mountain, forest, open fields), and the disposition of friendly and enemy forces. Soldiers frequently used maps and physical models of the terrain to plan battles. Travelers rely on maps for planning and executing trips. Navigators for ships and airplanes use maps to figure out where they are, and to plan what direction to go, and when to turn.

I intend to pursue this issue of visual (or iconic) versus symbolic (or language based) representations throughout the body of my talk. I want to suggest that the study of signals, images, and symbols, and the processes that transform one into the other form the basis for integration of semiotics with real-time control.

\section{Representation to Action}

I will argue that intelligent systems can improve its performance by using both iconic and symbolic representations and computational methods. Iconic and symbolic formats can and should be tightly linked so that information computed in one domain can be transformed into the other and vice versa. For example, combining iconic representations with symbolic information produces maps that can be used to plan and control behavior. Regions on maps can be specified as goals. Task objects can be specified symbolically by name and identified visually in images. Goals can also be defined by means of visual representations similar to 
"key frames" used in cartoon movies. Situations can be specified on maps showing the desired configuration of objects relative to each other and the terrain. Attention can be specified in terms of where on the egosphere to look or where to point high resolution sensor arrays.

I also want to describe a reference model architecture that incorporates both symbolic and iconic representations and outline an engineering methodology for the design and development of intelligent control systems. I will briefly describe some application examples developed using this methodology.

But, in the end, I return to the question "Why Now Semiotics?" Why should real-time control theorists care about the science of signs and symbols? And why should semioticists study issues related to real-time control?

The answer, I believe, can be found in those areas where the two fields intersect and address common problems. These areas include the acquisition, representation, and use of knowledge about the world. How can signals from sensors be transformed into images, maps, and symbols that represent states, attributes, entities, events, values, and situations in the world? How can knowledge be used to focus attention, plan action, and reason about the best course of action? How does an intelligent system generate and express goals and intentions? How does it know how to behave in order to achieve goals in an uncertain and often hostile environment? The answers to these questions will be found sooner if we combine what is known and is being discovered in the two fields of semiotics and real-time intelligent control.

I believe semiotics can help control engineers better understand how to represent images, maps, entities, actions, rules, situations, and relationships. I think recursive filtering theory and image processing research can help semioticists better establish correspondence between the real world and representations of the world. Given these better representations, intelligent systems can better plan and execute tasks amid uncertainty. Given a reference model architecture, researchers can better understand how to partition the control system so that various kinds of information can be learned. Learning can only take place within a prior structure where incremental improvements in performance can be rewarded and failures can be incrementally corrected. Given an engineering methodology, engineers can better understand how to approach the design of intelligent systems.

\section{Semiosis}

I would argue that the combination of real-time control and semiotics will enable us to build more intelligent systems for practical applications. Semiotics gives us a better understanding of semantics, pragmatics, causality, logic, inference, probability, and plausibility. Intelligent real-time control theory gives us a better understanding about how sense and process sensory data into knowledge about the world, and how to use that knowledge to plan and control behavior. Combined, these two diverse fields provide insight as to how better to acquire, represent, and use knowledge and how to transform knowledge into actions that produce real wealth for the benefit of society.

This then is the answer to "Why Now Semiotics?" Now is the time to integrate semiotics with real-time control to produce intelligent systems for practical applications of great importance to the future of human kind. 


\title{
Top-Down Engineering
}

\author{
Lawrence J. Fogel, Ph.D. \\ Natural Selection, Inc. \\ 3333 N. Torrey Pines Ct., Suite 200 \\ La Jolla, CA 92037
}

\begin{abstract}
Rather than design by arranging (and rearranging) components, why not use fast-time mutation and selection to evolve the required design? Simply generate a random set of alternative designs. Suggestions/hints can be added at any time, but are not required, for the process learns without instruction. Increasingly appropriate designs are found through successive generations. This evolutionary computation has been used to detect unknown signals in noise (discovering new templates for later pattern recognition), to design optimal neural networks (even when the response surface is discontinuous), for forecasting nonstationary time series given an arbitrary payoff function, for determining the relationship among variables (modeling an unknown transducer/plant), for the control of that plant within the limits of its controllability, and for gaming if that plant has its own purpose. There are, indeed, a wide range of other applications.
\end{abstract}

Of course, all this presumes the problem at hand is well defined, that is, that a meaningful fitness/payoff function has been specified. This requires more than simply measures of effectiveness and related measures of performance. The problem becomes well defined only if these measures are weighted in relative importance and combined, taking into account their degree of criticality. The Valuated State Space Approach provides a convenient way to translate subjective judgment with respect to intent into a concise specification of the purpose to be achieved. Top-down engineering can be realized by coupling the Valuated State Space Approach with evolutionary programming.

\section{KEYWORDS:}

top-down engineering, evolutionary programming. stochastic optimization, evolutionary computation

\section{INTRODUCTION}

"en॰gi•neer'ing, $\boldsymbol{n}$. the planning, designing, construction, or management of machinery," [1] begins with the recognition of a need. The available resources/components/building blocks are arranged within the imposed constraints. This construct is evaluated. The weak points/bottlenecks are identified. Adjustments are made to improve the design. This process continues until further improvements are no longer cost effective. The entire procedure is bottom-up.
But a much better design may exist ... one that cannot be found by continually improving on the original concept. Searching the streets of Los Angeles for the quickest route may take you away from a freeway entrance that might provide much faster transit. Continually improving the gaslamp will never make it into an electric light. Why not design top-down, that is, use fast-time computation to directly search the domain of candidate solutions/designs until one of sufficient worth has been found?

At first glance this might seem unreasonable, for the domain of possible solutions is so immense. Exhaustive search is clearly impossible. It becomes feasible only if an extremely efficient search technique is available. Why not simulate the way nature evolves increasingly appropriate creatures/designs over millennia? Each organism (design) faces the challenge posed by its environment and is thus scored. Those that are sufficiently fit produce offspring that are similarly tested. This optimization process is extremely efficient and can be used to address welldefined engineering problems, that is, to find a good enough solution fast enough for it to be useful.

\section{DISCUSSION}

Real-world problems are, indeed, extremely complex. The constraints are nonlinear and the objective function may change as the situation develops. To solve these, conventional engineering relies on simplifying assumptions. For example, the actual problem is often separated into components that can be more easily addressed. But locally optimal components do not integrate to yield a globally optimal solution unless the components are independent ... and they rarely are. We call upon linear programming even when the constraints are known to be nonlinear. Steepest descent is used even when the response surface may have multiple modes, be discontinuous, noisy or, in the limit, have no gradient. Spectral analysis and Markov processes are used to predict time series, even when the actual environment is known to be nonstationary. Such simplification of ten yields the right answer, to the wrong problem!

Another way to simplify is to use expert systems. Hcre, the design is based on using generally useful rules (heuristics) derived from experience. But remembered rules may be in error. The sample size documenting the worth of individual heuristics is likely to be insufficient. This is especially true when dealing with a dynamic environment where the objective function is changing while the problem is being solved. And rules that are good "on the average," may not be good enough. In practice, we need an particularly appropriate way to solve the problem at 
hand. Incidentally, when dealing with a nonstationary environment, the rules that are best at any particular time are likely to be far less than best at other times. To make matters worse, obeying any set of rules is certainly not the best way to operate in an environment that includes intelligently interactive competitors.

Traditional engineering is, indeed, bottom-up. The design process becomes increasingly complicated and cumbersome. Computers are used to solve complex sets of differential equations . . . rather than to directly address the problem at hand. But we can now generate alternative solutions at negligible cost, and thus simulate the fundamental process of evolution in order to design top-down. Alternative solutions are scored as separate entireties. No direct attempt is made to improve any of these individual designs. Those found inadequate are simply discarded with no penalty. The solutions that are found to be worthy are varied to yield offspring, thereby generating a new population (generation) that is similarly scored. As in nature, the selection process is phenotypic. Alternative designs/organisms are scored only in terms of their expressed behavior, not their components or structure. The mutation/selection process iterates until a sufficiently worthy solution has been found (or the available computational resources have been expended).

This fast-time neo-Darwinian evolution has a significant history. There have been various approaches: evolution strategies, evolutionary programming, genetic algorithms and related efforts in genetic programming and classifier systems. The first two of these are very similar, the primary distinction being whether or not the parents are part of the next generation. Genetic algorithms are quite different. They often use binary string representations, even when this is not an intuitive representation for the problem at hand. In contrast, in evolutionary programming, the representation follows the designer's intuition. Any suitable choice can be made. Genetic algorithms strongly rely on crossing over to generate new offspring, cutting and splicing existing solutions in the hopes that such recombination will be useful. In evolutionary programming, no emphasis is placed on any particular operator: again the mode of variation can reflect the representation chosen as well as the known interactivities between components of the solutions. It may be wise to recombine ideas from different solutions (for example, mix ice cream and root beer), or it may not (for example, combining the first half of Hamlet with the second half of Macbeth . . . it is still Shakespear, but it doesn't make sense). Recent mathematical proofs have indicated that crossover provides no general advantage to solving problems [2], and so, in order to maximize the practical use of evolutionary algorithms, the search operators must be tailored to the task at hand. This requires expertise.

Finally, with regard to the selection mechanisms, the traditional genetic algorithm relies on proportional selection, with the expected number of copies of each parent being made in proportion to its relative fitness. In contrast, in evolutionary programming, selection simply eliminates unfit solutions and can also be made probabilistic such that there is a tunable likelihood of accepting solutions with lower fitness. This ability to adjust the selection pressure offers yet another versatility to evolutionary programming. Note that it naturally lends itself to parallel processing and can be operated in a hierarchic manner, that is, with a higher level of evolution adapting the variables of the lower level. This may involve adjusting the population size, number of generations before each decision, the selection pressure, the mutation noise distribution, and so forth.

Indeed, evolutionary programming [3], [4], [5] is a most general optimization technique. The only "rules" are iterative mutation and selection. In its standard form, the basic evolutionary program uses the four main components of all evolutionary computation algorithms: initialization, variation, evaluation (scoring), and selection [6], [7]. At the basis of this, as well as other evolutionary computation algorithms, is the presumption that, at least in a statistical sense, learning is encoded phylogenically versus ontologically in each member of the population. 'Learning' is a byproduct of the evolutionary process as successful individuals are retained through stochastic trial and error. Variation (such as mutation) provides the means for moving solutions around on the search space, preventing entrapment in local minima. The evaluation function directly measures fitness, or equivalently the behavioral error, of each member in the population with regard to the environment. Finally, the selection process probabilistically culls suboptimal solutions from the population, providing an efficient method for searching the topography.

More specifically, evolutionary programming starts with a population of randomly chosen trial solutions (designs/plans of action, that is, alternative ways of allocating the available resources over time). These may include suggestions, but they are not required. Each of these trial solutions is evaluated with regard to the specified fitness function. Only some are retained to serve as parents. Each parent is altered through application of a mutation process. Mutations on the degrees of freedom are chosen with respect to a probability distribution, typically uniform. The number of mutations per offspring is also chosen with respect to a probability distribution or it may be fixed $a$ priori. These offspring solutions are then evaluated over the existing environment in the same manner as their parents. After the fitness or behavioral error is assessed for all offspring, the selection process is performed in one of several ways, depending on current knowledge of the response surface. In most applications, the size of the population remains constant, but there is no restriction in the general case. The process is halted when either the solution reaches a predetermined quality, a specified number of iterations has been achieved, or some other criteria (such as sufficient convergence) stops the algorithm.

Evolutionary programming differs philosophically from other evolutionary computational techniques such as genetic algorithms in a crucial manner. It is purely top-down rather than 
bottom-up optimization. It is important to note that (according to neo-Darwinism) selection operates only on the phenotypic expressions of a genotype; the underlying coding of the phenotype is only affected indirectly. The realization that a sum of optimal parts rarely leads to an optimal overall solution is key to this philosophical difference. Genetic algorithms rely on the identification, combination, and survival of "good" building blocks (schemata) iteratively combining these to form larger "better" building blocks. In a genetic algorithm, the coding structure (genotype) is of primary importance as it contains the set of optimal building blocks discovered through successive iterations. The building block hypothesis is an implicit assumption that the fitness is a separable function of the parts of the genome. This successively iterated local optimization process is fundamentally different from evolutionary programming which is an entirely global approach to optimization. Solutions/designs (or, by analogy, organisms) in an evolutionary programming algorithm are judged solely on their fitness with respect to the given environment. No attempt is made to partition credit to individual components of the solutions. In evolutionary programming (and in evolution strategies), the variation operator allows for simultaneous modification of all variables at the same time. Fitness, described in terms of the behavior of each population member, is evaluated directly, and is the sole basis for survival of an individual in the population. A crossover operation designed to recombine building blocks is not utilized in the general forms of evolutionary programming.

Evolutionary programming can be used to detect unknown signals in noise (discovering templates for pattern recognition), to design optimal neural networks (even when the response surface is discontinuous), for forecasting nonstationary time series given an arbitrary payoff function, for discovering the relationship among variables (modeling an unknown transducer/plant), for the control of that plant within the limits of its controllability, and for gaming if that plant has its own purpose. It has been demonstrated for improving drug design (Agouron Pharmaceuticals, Inc.), for adaptive control of freeway ramp signals (CalTrans), for scheduling the production of clothing (Levi Strauss \& Company), for the distribution of fuel (Chevron), for aiding the detection of breast cancer (U.S. Army Medical Research and Materiel Command), and for various other military programs that concern improved training and real-time mission planning.

But all too often engineering problems as posed are ill-defined. Are the stated requirements hard or soft constraints? If soft, how soft? What else should be measured? Are all these measures equally important? Are some, or all of them, critical (that is, failure in this regard nullifies the value of any other achievement)? Are there degrees of criticality? We often simply minimize the mean squared error rather than refer to the real payoff matrix wherein equally correct outcomes are not of equal worth and equal, but opposite, errors have different costs. Clearly, measures of effectiveness (MOEs) and the associated measures of performance (MOPs) do not tell the whole story.
Indeed, effective engineering begins with a clear understanding of what must be achieved by when. But what if that outcome is not realized? Surely some value is found in lesser degrees of achievement. There are even times when our primary concern is to avoid a particularly undesirable situation. In other words, to be meaningful, we must identify each of the significantly different futures, all the way from utopia to catastrophe, for only then can we measure the overall worth of any given situation.

Unfortunately, it is difficult to envision these significantly different futures, no less their relative worth. The Valuated State Space Approach provides a convenient way to overcome this difficulty. This method allows the responsible individual to portray the significantly different futures in terms of preferentially independent ${ }^{1}$ aspects of concern. These are the dimensions of a hyperspace, each being attributed some relative importance and made measurable by designating mutually exclusive class intervals that indicate those differences that make a difference in degree of achievement. The class intervals exhaustively span the range of each parameter from the most to the least desirable degree, each of these being attributed some value. When parameters are not directly measurable, they are expressed in terms of subparameters that are measurable or extended to still lower levels in the hierarchy that include directly measurable subparameters. Thus, the purpose to be achieved takes the form of a hierarchic Valuated State Space together with a normalizing function that can be used to combine contributions on the various parameters and subparameters in a manner that reflects their degree of criticality.

In many situations some overall worth is realized if there is some achievement in any regard. It is then appropriate to use the weighted arithmetic mean. In other situations, all of the parameters may be critical. Under these conditions, the normalizing function takes the form of a weighted geometric mean, that is, the overall worth is the product of the relative degrees of achievement, each raised to the power of the normalized importance of that parameter. If all the achievement values are high, the weighted arithmetic mean can be used as an optimistic estimate of the overall worth, for the difference between these means becomes negligible at successively higher values of achievement in every regard. [6]. Alternative "what ifs" can then be scored in view of their corresponding futures. The best is selected and there is a list of the remaining deficiencies, by priority. The worth of partially resolving any of these remaining problems can easily be computed.

But organizations operate in a cooperative or competitive environment. Our best move may not be best if it significantly injures a friendly organization and/or greatly benefits an adversary. The Valuated State Space Approach can also be used to express the presumed purposes of the other players so that

\footnotetext{
${ }^{1}$ wherein any change in the degree of achievement on a parameter does not alter the relative importance of the parameters.
} 
their best moves and ours can be estimated in light of their mutual attitudes and the state of the game.

Engineering decisions are improved when the purpose is made clear and concise, for means have value only in terms of ends. evolutionary programming can then be used to discover increasingly appropriate designs and/or modes of behavior in view of the presumed dynamics/constraints.

This capability has recently been demonstrated in gaming. Effective training requires an interactive adversary. Having qualified individuals simulate the opposing force is dangerous, for it presumes an understanding of their culture and intent. Such simulations are non-repeatable and cannot be calibrated. It is equally dangerous to train against any rule-based enemy (expert system), for the real enemy is intelligently interactive, learns, may demonstrate initiative, and is likely to behave in a generally unpredictable manner. by generating non-rule-based intelligently interactive adversaries in tank platoon combat simulation for the sake of improved training. Non-rule-based intelligently interactive adversaries were generated for simulating tank platoon combat. Three series of experiments were conducted using the Modular Semi Automated Forces (ModSAF) simulation program that embodies the terrain, platforms, and low-level deconfliction [8]. These tank warfare experiments were performed using a Sparc 20 Work Station in real-time. In the first series, opposing tank platoons were situated arbitrarily, each given different missions in the form of a Valuated State Space and normalizing function. These include the weighted probabilities of kill and survival, as well as the time and distance remaining to reach a specified goal-point. In one experiment, both platoons had unlimited visibility and equal firing range of 1200 meters. The primary concern of both platoons was survival. Realistic physical constraints were imposed, for example, the tanks could not move faster than 25 meters per second. Tactical decisions were made every 20 seconds after 30 generations of mutation and selection, the best evolved behavior being implemented. In this manner, there was learning across successive decision points.

The platoons moved toward their goal-points and, in this case, toward one another. They stopped just before reaching the firing range. They behaved erratically until finding themselves in synchrony. Then took a path toward its goal that remains outside the firing range. In essence, both platoons discovered "evasion" ... . without understanding this concept. In another experiment a platoon trades-off the worth of continuing toward its goal-point as opposed to pursuing the remaining enemy tanks. Throughout these experiments, the exhibited behavior seems rational.

In the second series of experiments, one platoon was provided information concerning the mission of the other platoon. This resulted in improved performance. In the third series of experiments, the same platoon was given misinformation about the enemy's mission, this resulting in a measurable cost. Obviously, the decision points can be made a function of the combat tempo. The less time between the decisions, the greater the population and the larger the number of generations before each decision, the greater the level of simulated intelligence. Note that the level of intelligence and, indeed, the mission can be changed at any time during the engagement. This combat simulation can be extended to higher levels of command wherein diverse platforms are interactively controlled in a coordinated manner. In essence, here is a simulation of coevolution.

This non-rule-based simulation can also be used to provide effective decision support, for evaluating alternative weapon systems capabilities and the tactics/doctrine required for these systems. In fact, it may be used on-line to generate particularly worthwhile tactics/doctrine in light of the developing situation. This simulation can also be used as a basis for measuring the impact of soft intelligence factors on the outcome of combat. To what extent does levels of training, loyalty, fatigue, cohesiveness, and so forth modify force effectiveness?

\section{CONCLUSION}

Mathematics has long been the primary tool of engineering. Linear approximations yield first cut solutions. Far more complex representations are required to treat actual real-world problems. This formalism requires significant computation. We allocate extensive CPU time to solve differential equations that portray time-dependent interactive dynamic systems.

But computers can be used more directly. There is no need for the intervening mathematics. The machine itself becomes a fasttime model of natural evolution. Rather than yield a single best design, it provides an evolved population of solutions, each of measured worth. The engineer can review these, taking into account concerns that were not directly stated in the payoff function: this design scored well but may have undesireable side effects or unintentional consequences, that design may prove too costly, and so forth. The ultimate design is selected from those that have survived extensive competition. If conventional designs are introduced as suggestions and are of true worth they will be so recognized by having survived the selection process. evolutionary computation is not in competition with other methods of optimization, it simply provides a way of improving on their results. In essence, it is a simulation of the scientific method and, therefore, an embodiment of creativity.

Of course, we must still recognize the need for a design and express that need in concise terms for, to be effective, any optimization technique must reference an explicit scoring function. But ordinarily ends are stated in terms of means. The contractor sees a new building in terms of brick and mortar rather than the efficiency of layout, comfort, cost of maintenance, longevity of service and, of course, ability to withstand environmental extremes. This no longer need be the case. We now have a convenient formalism for representing purpose/intent/mission. We can now solve complex problems 
using top-down engineering by coupling the Valuated State Space Approach with evolutionary programming.

But there is more to do. We need to determine the fundamental capabilities and limitations of evolutionary computation. What is the simplest way to generate useful heredity through reproduction in a finite noisy environment? What form of evolutionary computation should be used to treat which class of problems? What are the trade-offs between population size, number of generations, type of variation, and so forth? Are there heuristics for selecting a most suitable representation for any given problem? Are there ways to estimate the convergence rate under different conditions? Under what conditions is it better to evolve weighted rules rather than simply direct combinations of the available resources?

It might also prove worthwhile to examine the manner in which evolutionary computation relates to natural evolution. Is it worthwhile to replicate the specific mutation/selection process that takes place in nature? How should genetic variation be combined with phenotypic selection? Is it worthwhile to replicate natural evolution across the hierarchy from genes through organisms (their organization and species)? What theory of natural evolution (Lamarckian, Darwinian, and so forth) is most appropriate for addressing certain kinds of problems? Would it be advantageous to combine aspects of these different theories?

How can evolutionary computation be made still more efficient and effective? Is it worthwhile to use asynchronous evolution to allow some of the better parents to reproduce across generations and/or to artificially alter the response surface (the adaptive landscape) to facilitate finding the global optimum? How can evolutionary computation benefit from self-adaptation (internally and/or through the use of meta-levels)? What are the limits of evolving self-referential, self-modeling, self-aware, conscious automata that, in an artificial social setting, may even exhibit conscience?

How should evolutionary computation be realized under different conditions/constraints? What is the most appropriate language and coding procedure and why, given the circumstance? When and how should distributed/parallel processing be used for evolutionary computation? When is it advantageous to hardwire evolutionary computation into a chip? These and other questions are worthy of serious consideration.

In the meantime, there are diverse applications worthy of topdown engineering.

\section{REFERENCES}

[1] Websters New Twentieth Century Dictionary, 1983, Simon and Schuster, New York, pp. 602.

[2] D.H. Wolpert and W.G. Macready (1997) "No free lunch theorems for optimization," IEEE Trans. evolutionary Computation, Vol. 1:1.

[3] Fogel, L.J., 1964, On The Organization of Intellect, Ph.D. dissertation, University of California, Los Angeles.

[4] Fogel, L.J., Owens, A.J., and Walsh, M.J., 1966, Artificial Intelligence through Simulated Evolution, New York, NY: John Wiley.

[5] Fogel, D.B., 1995, Evolutionary Computation. Toward a New Philosophy of Machine Intelligence, Piscataway, NJ, IEEE Press.

[6] Fogel, David B., Bäck, Thomas, and Michalewicz, Zbigniew, 1997, Handbook of Evolutionary Computation, Oxford University Press and Institute of Physics Publishing.

[7] Fogel, David B., 1997, An Introduction to Evolutionary Computation (video presentation), Piscataway, NJ., IEEE Press.

[8] Fogel, L.J, Porto, V.W., and Owen, M., 1996, An Intelligently Interactive Non-Rule-Based Computer Generated Force, Proceedings of the Sixth Conference on Computer Generated Forces and Behavioral Representation, Orlando, FL: Institute for Simulation and Training, STRICOM-DMSO, pp. 265-270. 


\section{Ordinal Optimization and Soft Computing ${ }^{\dagger}$ Yu-Chi Ho \\ Division of Engineering and Applied Sciences \\ Harvard University \\ Cambridge, MA 02138}

\section{EXTENDED ABSTRACT}

The world is full of large and complex engineering problems that we do not know how to solve effectively or efficiently. Examples range from the design and operation of communication networks and manufacturing plants, to traffic control on land, sea, and in the air, to military C3I and logistic management. Such discrete event systems permeate the modern human-made civilization. Typically they do not possess simple analytical structures compared to the differential equation based physical systems; stochastic effects abound in such systems; and finally their designs involve search in a huge parameter/configuration space. While simulation is a general purpose performance analysis tool of choice, fundamental theoretical limitations on computation rule it out as an optimization tool for such problems. In this talk we advocate a strategic re-direction of this general optimization problem and show that effective in-road can be made in practice. We will discuss the results that have been achieved so far and the problems remain. Our thesis also advocates the inclusion of this optimization tool as one elements of the computing intelligence arsenal. A live demonstration of the tool will be conducted during the talk to illustrate its generality and properties. A separate session immediately following this talk also addressed more recent developments.

Additional details can be found in the author's web-site:

http://hrl.harvard.edu/people/faculty/ho/CRCD

and the following references.

\section{REFERENCES}

[1] Ho, Y.-C., R. Sreenivas, and P. Vakili, "Ordinal Optimization of Discrete Event Dynamic Systems,", J. of DEDS, vol. 2(2), pp. 61-88, 1992.

[2] Patsis, N., C.-H. Chen, and M. Larson, "Parallel Simulation of DEDS," IEEE Trans. on Control Technology, 1996.

[3] Deng, M., Y.-C. Ho, and J.-Q. Hu, "Effect of Correlated Estimation Error in Ordinal Optimization," Proceedings of the Winter Simulation Conference, pp. 466-475, December 1992.
[4] Ho, Y.-C., "A New Paradigm for Stochastic Optimization and Parallel Simulation," Proc. of 1993 DES Workshop in IMA/U. Minn. Lecture Notes Series, Springer-Verlag, 1994. Presented at the IMA workshop 5/23/93 in Minneapolis and the ORSA/TIMS/INRIA Conference on Applied Probability in Paris 6/18/93.

[5] Ho, Y.-C., "Heuristics, Rules of Thumb, and the 80/20 Proposition," IEEE Trans. on Automatic Control, vol. 39(5), pp. 1025-1027, May 1997.

[6] Ho, Y.-C., and M. Larson, "Ordinal Optimization and Rare Event Probability Simulation," J. DEDS, vol. 5, \#2-3, 1995.

[7] Wieseltheir, J. E., C. M. Barnhart, and A. Ephremides, "Ordinal Optimization of Admission control in Wireless Multihop Voice/Data Network via Standard Clock Simulation," J. DEDS, Vol.5, \#2-3, 1995.

[8] Ganz, A., and X. Wang, "Efficient algorithm for virtual topology design in multihop lightwave networks," IEEE/ACM Transactions on Networking, vol. 2, June 1994.

[9] Xie, X.-L., "Dynamics and convergence rate of ordinal comparison of stochastic discrete event systems," IEEE Transactions on Automatic Control, to appear, 1997.

[10] Xie, X.-L., "An Ordinal Optimization Approach to a Token Partition Problem for Stochastic Timed Event Graphs," Proceedings of the 1994 Winter Simulation Conference, Orlando, Florida, pp. 581-588, December 1994.

[11] Dai, L.-Y., "Convergence Properties of Ordinal Comparison in the Simulation of Discrete Event Dynamic Systems," JOTA, Vol. 91(2), pp. 363-388, 1996.

[12] Lau, T.-W. E., and Y.-C. Ho, "Universal Alignment Probabilities and Subset Selection for Ordinal Optimization," JOTA, Vol. 39(3), pp. 455-490, June 1997.

[13] Deng, M., and Y.-C. Ho, "Sampling-Selection Method For Stochastic Optimization Problem," submitted to AUTOMATICA, 1995.

[14] Lee, L.-H, T.-W. E. Lau, and Y.-C. Ho, "Explanation of Goal Softening in Ordinal Optimization," IEEE Trans. on Automatic Control, to appear, 1997.

[15] Ho, Y.-C., "An Explanation Of Ordinal Optimization," Proceedings of Joint Conference on Information Sciences, Raleigh, NC, March 1997.

\footnotetext{
${ }^{\dagger}$ The work in this paper is partially supported by NSF grants EID-9212122, EEC9402384, Army contracts DAAL03-92-G0115, DAAH04-95-0148 and Air Force contract F49620-95-1-0131.
} 
[16] Tang, Z. B., and Y.-C. Ho, "Modification of Adaptive Partitioned Random Search," Proc. of 1995 Conference on Decision and Control, New Orleans, December 1995.

[17] Chen, C. H., and V. Kumar, "Motion Planning of Walking Robots in Environments with Uncertainty," Proc. of IEEE Conference on Robotics and Automation, 1996.

[18] Yang, M. S., L.-H. Lee, and Y.-C. Ho, "On Stochastic Optimization and Its Applications to Manufacturing," Proc. of AMS Conference on Stochastic Problems in Manufacturing, to appear in Lecture Notes in Applied Mathematics, 1997.

[19] Dai, L.-Y., and C.-H. Chen, "Rates of convergence of ordinal comparison for dependent discrete event dynamic systems," JOTA, Vol. 94(1), July 1997.

[20] Ho, Y.-C., "On the Numerical Solution of Stochastic Optimization Problems," IEEE Trans. on Automatic Control, Vol. 42(5), 1997. 


\title{
Introduction to Quantum Theory of Open Systems
}

\author{
Yu.L.Klimontovich \\ Physics Faculty \\ Moscow State University \\ 117234 Moscow Russia \\ E-mail: ylklim@hklim.phys.msu.su
}

\section{ABSTRACT}

In this Lecture we are going to pave the way for the quantum theory of open systems. The question of completeness of description of physical phenomena on the basis of quantum mechanics and other eternal questions of the quantum theory are considered. Applications of the S-theorem and the conservation law of Information and Entropy are demonstrated. In this domain there are the intersections between the Physics of Open Systems and Semiotics.

\section{INTRODUCTION}

As and in the classical kinetic theory, in the quantum one the simplest model is the one-component rarefied gas of structureless particles (the Boltzmann gas). This model it is possible to expand in two main direction, and consider two more complicated models.

The first one is the rarefied electron-ion plasma, which is a three-component system comprised of gases of electron and ions, and electromagnetic field.

The second is the system of atoms and field, which in the simplest case consists of two components. The gas component differs from Boltzmann gas in that the structure of atoms or molecules is taken into account. The second component is again the electromagnetic field.

A natural generalization of these two particular models is the so-called plasma-molecular system, which of at least four components (KlimontovichWilhelmsson-Yakimenko-Zagorodnii 1989). An example of such system is provided by partially ionized plasma. Foundation of the kinetic of systems of this kind have been laid in (Klimontovich 1980 (1983); 1982 (1986) Klimontovich-Kremp-Kraeft 1981, 1987).

A consistent description of plasma-molecular systems is only possible within the framework of quantum theory; hence the need of bridging the gap between the classical theory of open systems to the appropriate quantum theory.

This is best done with the concrete examples of simple but real systems, such as the system of noninter- acting hydrogen atoms or free electrons in electromagnetic field. We shall use these examples to illustrate the transition from the reversible microscopic operator equations to irreversible quantum kinetic equations. Such transition may be interpreted as replacement of system of particles and field oscillators by a continuous medium.

In particular, this implies that Schroedinger equation of quantum mechanics for deterministic (not operator) wave functions describes the evolution of continuous medium, but ignores the dissipative terms. In this sense, there is an analogy between Schroedinger equation in quantum mechanics and Euler equation in hydrodynamics (Klimontovich 1993, 1995).

It would be natural to begin our study of quantum system of atoms and field with the microscopic equations, which give complete quantum electrodynamic description of atoms and electromagnetic field.

Like in the case of Boltzmann gas, we again are faced with important questions. What are the smallest scales on which the initial equations of quantum electrodynamics lose their reversibility? What is the cause of irreversibility?

Similar question arise at different levels of description. We might ask, for instance, what are the smallest scales which allow going over from operator equations of quantum electrodynamics to the corresponding Schroedinger equation for the deterministic distribution function of, let us say, the atom of hydrogen.

The fact is that quantum mechanical Schroedinger equation is more coarse than the corresponding equation of quantum electrodynamics, since it only involves the mean field rather than the coordinates of individual field oscillators.

The problem consists therefore in finding the starting point of transition towards irreversible equations. In this connection we should mention simulating influence of the work of Ilya Prigogine (Prigogine 1980; Prigogine - Stengers 1984), who for many years has been studying the possible generalization of the second law of thermodynamics to the microscopic level. The main role is assigned to the dynamic instability 
of motion of microscopic objects. Dynamic instability in quantum theory is manifested in significant change of wave functions when the relevant initial conditions are varied even slightly.

Recall that in case of Boltzmann gas the procedure of smoothing over physically infinitesimal volume (the "point" of continuous medium), which is necessary for going over from the system of particles to the approximation of continuous medium, reflects the existence of dynamic instability of motion. The smoothing over the physically infinitesimal scales in the construction of irreversible equations of statistical theory is based on the existence of dynamic instability of motion of particles of system. In this respect the dynamic instability of motion plays a constructive role in the statistical theory.

Since the theory of dynamic instability in quantum theory is still in its early stage, it will be expedient to start with the evaluation of the smallest scales on which smoothing is possible. This is necessary for defining the limit of applicability of the initial microscopic equations of quantum electrodynamics.

The question of completeness of description of physical phenomena on the basis of quantum mechanics was the subject of the famous debate between Einstein and Bohr at the 5th Solvay Conference in 1927 (see Mehra 1975). Later this problem was discussed in the paper entitled Can quantum-mechanical description of physical reality be considered complete? (Einstein - Podolski - Rosen 1935, and also in the papers by de Broglie (1953) and Bohm (1952). The issue of "deficiency of quantum-mechanical description" is closely related to the problem of the so-called hidden parameters in quantum mechanics.

Most physicists argued that quantum mechanical description is complete, and that the problem of hidden parameters does not exists. This view was based for the most part on the book by John Neuman (1932), in which he proved that hidden parameters are incompatible with the foundations of quantum theory. It was not mentioned, however, that Schroedinger equation of quantum mechanics is itself approximate.

Further theoretical and experimental studies were simulated by the results of Bell (Bell 1965, 1966: Belinski, Klyshko 1993), who formulated the condition of existence of hidden parameters as Bell's inequality. This was seen as a new possibility of experimental verification: if quantum mechanical description is complete, then Bell's inequality does not hold.

In a recent review (Belinskii - Klyshko 1993) we read:

"The problem formulated many years ago by Einstein, Podolski and Rosen, by Bohm and Bell, still excite the new generation of physicists. To a large extent this is due to the fact that the contradiction between the prediction of quantum theory and the theory of hidden parameters can be settled in a convincing way (in favor of quantum theory, of course) by experimentum crucis, unlike most other quantum paradoxes. The theory of hidden parameters is closely related to the ensemble-statistical interpretation of quantum theory, and this is the reason why such experiments (real or imaginary) add serious evidence to the eternal debate between the advocates of statistical (Einstein) and orthodox (Bohr's or Copenhagen) interpretations, and their numerous modifications."

And in the next paragraph:

"It is yet possible that in the future this debate will be settled (perhaps in favor of a third way), and historian of science will be see it as a vivid example of fallacies which plagued even the brightest minds of the past"

So the authors do note rule out possibility of a third way.

Let us take advantage of this option. Our treatment will be largely based on the ideas and methods described in detail in (Klimontovich 1993, 1995, 1997). We would like to point the recent paper (Kadomtsev, 1994) in which the principle problems of quantum theory are discussed.

\section{Microscopic and Macroscopic SChroedinger Equations}

Imagine a quantum system which consists of an ideal gas of "particles" (hydrogen atoms, as example) and fluctuation electromagnetic field.

Microscopic description of quantum processes in such system starts with the reversible dynamic equations for the particles and field. There two possible approaches. On is based on the Schroedinger equation for the wave function of complete set of variables of particles and field. The other relies on the equation for quantum (operator) wave function of particles and electromagnetic field's oscillators. In both cases the initial dynamic equations give, in principle, an exhaustive quantum mechanical description of time evolution of the system under consideration. Such level of description corresponds to a pure ensemble.

For the sake of simplicity, we shall describe interaction with the electromagnetic field in the dipole approximation. So we have a closed set of equations for operator wave function $\Psi(r, R, t)$ of electron in hydrogen atom, and field operator $E^{\wedge}(R, t)$. Instead the $\Psi(r, R, t)$ operator it is useful to use the quantum operator $\hat{f}(r, p, t)$ which is corresponding to Wigner quantum distribution function $f(r, p, t)$ in the sixdimensional phase space.

However, owing to the nonlinearity of operator equations, averaging over the Gibbs ensemble results in a very sophisticated system of meshing equations in 
the moments of different order. Accordingly, we once again have to deal with the problem of closure, so as to obtain a closed system of approximate equations.

In quantum mechanics the equation for operator wave function $\Psi(r, R, t)$ is replaced by Schroedinger equation for the deterministic wave function $\psi(r, R, t)$ of the electron in a hydrogen atom $(\Psi(r, R, t) \rightarrow$ $\psi(r, R, t))$

In quantum mechanics the equation for operator wave function is replaced by Schroedinger equation for deterministic wave function of the electron:

$$
\begin{gathered}
i \hbar \frac{\partial \psi}{\partial t}=\frac{\hbar^{2}}{2 m} \frac{\partial^{2} \psi}{\partial R^{2}}+U(r) \psi-d E(R, t) \psi \\
\int|\psi|^{2} \frac{d r d R}{V^{2}}=1
\end{gathered}
$$

This equation approximative but still reversible. From (1) we can go over to the equation for deterministic "quantum distribution function" in Wigner's form

$$
\begin{gathered}
f(r, p, R . t= \\
\frac{1}{(2 \pi)^{3}} \int \psi\left(r+\frac{1}{2} \hbar \tau, R, t\right) \psi^{*}\left(r-\frac{1}{2} \hbar \tau, R, t\right) \\
\exp (-i \tau p) \frac{(2 \pi \hbar)^{3}}{V} d \tau \\
\int f(r, p, R, t) \frac{d r d p d R}{(2 \pi \hbar)^{3} V}=\int \mid \psi\left(r, R,\left.t\right|^{2} \frac{d r d R}{v^{2}}=1\right.
\end{gathered}
$$

Let us also write the corresponding kinetic equation:

$$
\begin{gathered}
\frac{\partial f}{\partial t}+v \frac{\partial f}{\partial r}+E(R, t) \frac{\partial f}{\partial p}+ \\
\frac{i}{\hbar(2 \pi \hbar)^{3}} \int\left[U\left(r+\frac{1}{2} \hbar \tau\right)\right]-\left[U\left(r-\frac{1}{2} \hbar \tau\right)\right] . \\
\exp \left[i \tau\left(p^{\prime}-p\right)\right] d \tau d p=0
\end{gathered}
$$

which in the classical limit becomes

$$
\frac{\partial f}{\partial t}+v \frac{\partial f}{\partial r}+E(R, t) \frac{\partial f}{\partial p}-\frac{\partial U}{\partial r} \frac{\partial f}{\partial p}=0 .
$$

We see that Schroedinger equation (1) for deterministic wave function corresponds in the classical limit to the kinetic equation for the one-point distribution function $f(r, p, R . t)$. Like Schroedinger equation, this equation is reversible since it does not take into account the dissipation due to the interaction of electron with fluctuation electromagnetic field.

Now, to facilitate the transition to the irreversible equations< let us refresh some points from Boltzmann's kinetic theory.

The kinetic Boltzmann equation differs from (5) in that it includes the "collision integral" which take care of the dissipation due to redistribution of the particle's velocities because of collisions between them. By $\tau$ and $l$ we denote the relaxation parameters - the free path time and length. We also introduce the characteristic parameters of the problem $T$ and $L$.

As we know from gas kinetic theory, important are two extreme cases, corresponding to the approximation of gas dynamics and of free molecular flow. The gasdynamic approximation is used when $\tau \ll$ $T, \quad l \ll L$. Then the kinetic Boltzmann equation may be replaced by the simpler equation for the gasdynamic functions $\rho(R, t), \mathbf{u}(R, t), T(R, t)$.

In the opposite extreme case, when $\tau \gg T, l \gg$ $L$, the dissipative term (the "collision integral") in the zero approximation may be dropped. This brings us to the reversible kinetic equation which formally coincide with (5).

It is possible to exploit this analogy?

Recall first of all that the nature of description is changed dramatically when we go over from the Liouville equation, which carries all information about the motion of particles of system, to the Boltzmann equation. To wit, ¿from the system of particles whose motion is described by the reversible Hamilton equations, we go to a continuous medium in six-dimensional space of coordinates and momenta. Naturally, the transition to the approximation of continuous medium is associated with restriction from the side of small scales which define the size of the "point". Since the information about the motion of particles within the "point" is lost, the equation of continuous medium must be dissipative and therefore irreversible.

This transition is necessitated by dynamic instability of motion of microscopic elements of the system. It is the dynamic instability of motion combined with uncontrollable small exertions form the surrounding world, that makes the transition to irreversible equation inevitable.

In this connection it is worthwhile to recall that dissipation is usually seen as damping of motion, scattering of energy, loss of information. At nonequilibrium phase transitions, however, which may result in the appearance of new structure, the dissipation plays a constructive role.

This explains why the construction of dissipative equations is of crucial importance for the statistical theory of open systems. The first step in this direction 
consists in the revision of the concept of continuous medium. This requires given a concrete definition of physically infinitesimal scales.

It is known how to do this for Boltzmann gas and for a plasma (Klimontovich 1983, 1985, 1987). How then do we apply this knowledge to the quantum system of atoms and electromagnetic field.

Let us return to the classical kinetic equation (5). For the Boltzman gas, for example it can be used for describing free molecular flows. The approximation of continuous medium still holds, however, since this equation does not carry information about the motion of individual particles. This restrict the admissible values of $T$ and $L$ from below: they must be much greater than the 6relevant physically infinitesimal scales:

$$
\tau \gg T \gg \tau_{p h}, \quad l \gg L \gg l_{p h}
$$

And yet, equation (5) is reversible, because it corresponds to the zero approximation in small parameter $L / l$.

What is the situation in quantum theory?

\section{Continuous medium APPROXimation in QUANTUM THEORY}

To take care of the dissipation, we must include into consideration the interaction of atoms with fluctuating electromagnetic field. This will result in dissipative kinetic equation for the quantum distribution function (or density matrix) and the field.

The appropriate "collision integrals" are determined by the small-scale fluctuations whose characteristic scales are much smaller than the characteristic scales of the kinetic equations. In this way, the problem of the structure of "continuous medium" is brought up explicitly in the derivation of kinetic equations.

This problem is closely associated with the definition of kinetic equations.

In the pure ensemble, the operator density matrix (for example, in Wigner's representation (Klimontovich,1958, 1995; Klimontovich, Silin, 1960; Brittin and Chapell, 1961; Hillery, O'Connell, Scully, Wigner 1994) is expressed via product of operator wave functions $\Psi(r, R, t)$. Upon transition to the continuous medium, when the kinetic equations become irreversible, the pure ensemble is replaced by "mixed" ensemble. Then there is no representation in which the density matrix can be expressed exact via the product of wave functions!

It might seem that the above formulas defy this statement. Indeed, the quantum distribution function (2)is defined as product of wave function which satisfy Schroedinger equation (1). It is as if we returned to the "pure ensemble" again.
Naturally, there is a fundamental difference between these two definition of pure ensemble. The first exactly renders the statistical properties of quantum mechanical description, whereas the second definition of pure ensemble corresponds to approximation of continuous medium. The approximation amounts to neglecting the dissipation altogether.

For the system of $N$ particles this approximation corresponds to Hartree equation in quantum mechanics, or to self-consistent Vlasov approximation in plasma theory, or, at last, to Euler approximation to hydrodynamics which disregards the dissipation due to viscous friction and heat conduction.

In order to define the structure of "continuous medium" in quantum theory, we must first of all introduce the characteristic scales of the system in question. The characteristic length and frequency for hydrogen atom are:

$$
r_{0}=\frac{\hbar^{2}}{n e^{2}}, \quad \omega_{0}=\frac{m e^{4}}{2 \hbar^{3}}, \quad v_{0}=\frac{e^{2}}{\hbar} .
$$

The Bohr radius $r_{0}$ is the characteristic scales of the ground state distribution $|\Psi(r)|^{2}$, the frequency $\omega_{0}$ determines the energy of ground state.

Additional parameters arise when we go over to the dissipative kinetic equations. We divide them into two classes.

The first class includes those parameters which characterize the process of relaxation towards equilibrium. This time is defined by Einstein's coefficient for spontaneous transitions:

$\tau_{n m}=\frac{1}{A_{m}^{n}} . \quad A_{m}^{n}=\frac{4\left|d_{n m}\right|^{2} \omega_{n m}^{3}}{3 m c^{3}} \sim \gamma\left(\omega_{n m}\right)=\frac{2 e^{2} \omega_{n m}^{2}}{3 m c^{3}}$.

Einstein's coefficient is proportional to the coefficient of radiation friction at the transition frequency. The following estimate then holds good:

$$
A_{m}^{n} \sim \mu^{3} \omega_{n m} \ll \omega_{n m}, \quad \text { where } \mu=\frac{e^{2}}{\hbar c}
$$

is the "constant of fine structure".

At the zero temperature the atoms are in the ground state, and the density of electron's position in the atom is isotropic. The most probable distance ¿from the nucleus is characterized by Bohr's radius $r_{0}$.

Now let us try to answer the following question: does the distribution of electron's position in the ground state display a finer structure? What are the smallest times which the isotropic distribution takes to become established in the ground state? 
It is obvious that the structure of "continuous medium" is defined by the scales smaller then those of (8). In classical and quantum electrodynamics there are two scales which are smaller than Bohr's radius $r_{0}$ :

$$
r_{e}=\frac{e^{2}}{m c^{2}}, \quad \lambda_{C}=\frac{\hbar}{m c} \sim \mu r_{0} \ll r_{0}, \text { where } \mu=\frac{e^{2}}{\hbar c} .
$$

The first is the "classical electron radius". The main contribution to the effective cross section of scattering of photons by free electrons (the Tomson's scattering) is proportional to $r_{0}^{2}$. The second parameter is Compton's length, which defines the shift of wavelength in case of scattering of $x$-rays by free electrons. Which of these characterizes the beginning of irreversibility, and thus defines the finest structure of "continuous medium" the size of "point" in quantum mechanics?

Remark that the study of the structure of "continuous medium" in quantum mechanics is stimulated, in particular, by those difficulties which is associated with the attempts to calculate quantum fluctuations, like fluctuations of velocity of free electron moving in a fluctuation electromagnetic field (Klimontovich 1990(1991), 1993,1995). The solution of this problem gives us possibility to define the smallest relaxation time over which the irreversibility sets up. This smallest relaxation time is defined by the classical electron radius $r_{0}$ :

$$
\tau_{r e l} \sim \tau_{e}=\frac{r_{0}}{c} \equiv \frac{1}{\Gamma} ; \quad \Gamma=\frac{3 m c^{3}}{2 e^{2}} \sim \frac{c}{r_{e}} .
$$

Now we shall show that the scales $r_{e}, \tau_{e}$ define the fine structure of the atom's ground state.

\section{ESTABLishment of GROUND STATE IN ATOM-OSCILLATORS}

Consider a gas of noninteracting atoms in equilibrium electromagnetic field. Following to Tomson model, we regard the atom as a sphere of radius $r_{0}$ which carries a uniformly distributed positive charge. The electron vibrates with respect to the center of the sphere. The frequency of oscillations is found from the expression for the elastic force

$$
e \mathrm{E}=-\frac{e^{2}}{r^{3}} \mathbf{r}=-m \omega_{0}^{2} \mathbf{r} ; \quad \omega_{0}=\sqrt{\frac{e^{2}}{m r^{3}}} .
$$

For the sake of simplicity we consider onedimensional oscillations of the atom-oscillators. In the equilibrium state the mean kinetic and potential energies are defined by expressions:

$$
m\left\langle v^{2}\right\rangle=m \omega_{0}^{2}\left\langle x^{2}\right\rangle=k T_{\omega_{0}}=\hbar \frac{1}{2} \hbar \omega_{0} \operatorname{coth} \frac{\hbar \omega_{0}}{2 k T}
$$

For atom-oscillators at room temperature the $\operatorname{coth} \hbar \omega_{0} / 2 k T$ is close to one, and the amplitudes $v_{0}, x_{0}$ are therefore defined by the zero-point energy $\hbar \omega_{0} / 2$. So we have the following expressions for the frequency and amplitude of atom-oscillators which are in equilibrium with electromagnetic field:

$$
\omega_{0}=\sqrt{\frac{e^{2}}{m x_{0}^{3}}} ; \quad m v_{0}^{2}=m \omega_{0}^{2} x_{0}^{2}=\frac{1}{2} \hbar \omega_{0} .
$$

We find from them the expressions the amplitude, frequency and velocity of atom-oscillator (Klimontovich 1995)

$$
x_{o}=\frac{\hbar^{2}}{m e^{2}}, \quad \omega_{0}=\frac{m e^{4}}{\hbar^{3}}, v_{0}=\frac{e^{2}}{\hbar},
$$

which are known in quantum mechanics as Bohr's parameters. Now these parameters are not consequence of Schroedinger equation for atom-oscillators, but they represent the condition of equilibrium between atom-oscillators and electromagnetic field.

To describe the distribution in the space $(x, v)$ for a gas of noninteracting atom-oscillators in equilibrium electromagnetic field, we can use the generalized Fokker-Planck equation (17.3.13) in (Klimontovich 1995) for the distribution function $f(x, v, t)$ :

$$
\begin{gathered}
\frac{\partial f}{\partial t}+v \frac{\partial f}{\partial x}-\quad \omega_{0}^{2} x \frac{\partial f}{\partial v}=\frac{\partial}{\partial v}\left[D_{(v)} \frac{\partial f}{\partial v}\right]+\frac{\partial}{\partial v}[\gamma v f]+ \\
\frac{\partial}{\partial x}\left[D_{(x)} \frac{\partial f}{\partial x}+\frac{\omega_{0}^{2} x}{\gamma} f\right] .
\end{gathered}
$$

Here the diffusion and friction coefficients in velocity space are given be expressions:

$$
D_{(v)}=\gamma \frac{k T_{\omega_{0}}}{m} ; \quad \gamma=\frac{2 e^{2} \omega_{0}^{2}}{3 m c^{3}} \equiv \frac{\omega_{0}^{2}}{\Gamma}
$$

The spatial diffusion and friction coefficients are:

$$
D_{(x)}=\frac{k T_{\omega_{0}}}{m \gamma} \equiv \Gamma x_{0}^{2} ; \quad \frac{\omega_{0}^{2}}{\gamma}=\Gamma .
$$

The equilibrium solution of this equation is defined by Planck's formula: 


$$
\begin{gathered}
f(x, v)=C \exp \left(-\frac{m v^{2} / 2+m \omega_{0}^{2} x^{2} / 2}{k T_{\omega_{0}}}\right), k T_{\omega_{0}}= \\
\frac{1}{2} \hbar \omega_{0} \operatorname{coth} \frac{\hbar \omega_{0}}{2 k T}
\end{gathered}
$$

Now we can show the that relaxation times $\tau_{(v)}, \tau_{(x)}$ differ very much.

Indeed, the corresponding diffusion times are defined by expressions

$$
\tau_{(v)}=\frac{v_{0}^{2}}{D_{(v)}}=\frac{1}{\gamma} ; \quad \tau_{(x)}=\frac{x_{o}^{2}}{D_{(x)}}=\frac{1}{\Gamma} \ll \tau_{(v)} .
$$

The "strength" of inequality « can be expressed in terms of "fine structure constant" $\mu=e^{2} / \hbar c$ :

$$
\tau_{(x)} \sim \mu^{6} \tau_{(v)} \quad \text { and } \tau_{(x)} \sim \mu^{3} \frac{1}{\omega_{0}} ; \quad \tau_{(v)} \sim \frac{1}{\mu^{3} \omega_{0}} .
$$

We see that the spatial diffusion is much more "faster" than the diffusion in velocity space. Thus, are two stage ("fast" and 'slow") of the evolution to equilibrium distribution (19).

The "fast" process of evolution characterizes by the time of spatial diffusion $\tau_{(x)}=x_{o}^{2} / D_{(x)}=1 / \Gamma$. We see that the quantum mechanical ground state is established during very short time interval $\tau_{(x)} \sim r_{e} / c$ $\sim \mu^{3}\left(1 / \omega_{0}\right)$ which much less of the period of Bohr oscillations.

The diffusion time for "slow" process is defined by the time spontaneous emission $\tau_{(v)} \sim 1 / \gamma\left(\omega_{0}\right)$.

Thus, in within the atomic limits the spatial diffusion is the fastest process, As a result, Boltzmann distribution with respect to $x$ is established on this scale within the time $\tau_{(x)} \sim r_{e} / c$ and can be used as the smoothing function for carrying out the transition to the smoother function $f(x, v, t)$ (Klimontovich 1995).

Marking off the function which satisfies (16)with tilde, we may define the operation of smoothing as

$$
\begin{array}{r}
f(x, v, t)=\int \hat{f}\left(x-x^{\prime}, x, t\right) F\left(x^{\prime}\right) d x^{\prime} \\
F(x)=\frac{1}{\sqrt{2 \pi x_{0}^{2}}} \exp \left(-\frac{x^{2}}{2 x_{0}^{2}}\right) .
\end{array}
$$

We see that the scale $x_{0}$ ("Bohr's radius") defines the size of "point" of quantum continuous medium for system is considered, and scale $\tau_{e}$ defines the corresponding physically infinitesimal time.
After smoothing, the second dissipative term in (E.15) drops out, and we arrive at the standard Fokker-Planck equation commonly used for describing the Brownian motion of harmonic oscillator:

$$
\frac{\partial f}{\partial t}+v \frac{\partial f}{\partial x}-\omega_{0}^{2} x \frac{\partial f}{\partial v}=\frac{\partial}{\partial v}\left[D_{(v)} \frac{\partial f}{\partial v}\right]+\frac{\partial}{\partial v}[\dot{\gamma} v f] .
$$

For quantum atom-oscillators the diffusion and friction coefficients are defined by the expressions (17).

We see that in this equation the scale on which the spatial diffusion takes place within the volume of a atom (in the volume of a ground state) is a "hidden parameter" (or "hidden scales"!

¿From the last relation (21) follows inequality $\tau_{(v)} \gg 1 / \omega_{0}$ allows us to further simplify equation (23) by averaging over the period of oscillations. As a result we have the following equation:

$$
\begin{aligned}
\frac{\partial f}{\partial t} & =\frac{1}{2}\left[D_{(v)}\left(\frac{\partial^{2} f}{\partial v^{2}}+\frac{1}{\omega_{0}^{2}} \frac{\partial^{2} f}{\partial x^{2}}\right)\right. \\
& \left.+\frac{\partial}{\partial v}(\gamma v f)+\frac{\partial}{\partial x}(\gamma x f)\right]
\end{aligned}
$$

This equation describes only the slowest process of relaxation over the time of the order $1 / \gamma \gg 1 / \omega_{0}$.

We see that it is possible to describe Brownian motion of quantum atom-oscillators with a varying degree of finesse. The most detailed information is contained in the Fokker-Planck equation (16), where the negligibly small ("hidden") scales are less than $\tau_{e}, r_{e}$. The least detailed information is contained in the Fokker-Planck equation (24)

Other example of Ground State Structure it is possible to find in the last chapter of the book (Klimontovich 1995).

\section{ABOUt SOME ETERnAl QUestions of QUANTUM MECHANICS}

We saw that the concept of "pure ensemble" is clear defined only in the case of complete quantum mechanical description system of atoms and electromagnetic field.

In quantum mechanics, however, the term "pure ensemble" is used also in those cases when the description is based on the Schroedinger equation (1)for the deterministic wave function $\psi(r, t)$ of only of the variables of particles. This approach corresponds to approximation of continuous medium and when the dissipation is not taking into account. On this ground it is impossible to describe transitions between stationary levels accompanied by emission of radiation.

Since the description based on the Schroedinger equation is not complete, there exist "hidden parameters" ("hidden scales") which are revealed the use of 
a more realistic approach to problem under consideration.

We have illustrated this possibility with a concrete example of the system of atom-oscillators and field. By introducing the scales of "continuous medium" we were able to take into account the small-scale fluctuation in quantum Fokker-Planck equation.

The calculation of small-scale fluctuations is also of interest by itself: these fluctuations, for instance, are definitive for the effective cross section of a scattering of photons by free electrons (Klimontovich 1993,1995)

It is important that the effective cross section in the quantum domain (visible and $x$-ray ranges) does not depend on Planck's constant, and can therefore be found from classical calculations. The relevant scales $\tau_{e}, r_{e}$ are so small that Heisenberg's uncertainty relation does not hold.

In this way, there are two "exits" from quantum theory. One of these corresponds to the domain of large scales and slow a spatial variations. The other is associated withe the transition to the scales which are much smaller than any of the quantum scales of the system in question, which brings us into the realm of "hidden parameters" ("hidden scales").

The second question of this section which was the subject of the famous debate between Einstein and Bohr is "Is Quantum Mechanical Description Complete?" - the title of the famous paper (Einstein,Podolski, Rosen 1935).In the light of arguments developed above, the answer to this question is negative. The fact is that quantum mechanical description based on the reversible Schroedinger equation can always supplemented by the inclusion of fluctuations. Then, owing to the existence of fluctuation dissipation relations the dissipation is inevitable. Consequently, any kind of quantum mechanical description is in practice incomplete, and the concept of "pure ensemble" is just an abstraction.

The above arguments score in favor of Einstein's standpoint. In this connection it would be interesting to note that the problem of incompleteness of quantum mechanical description had been actually solved by Einstein long before the emergence of quantum mechanics as such.

As early as 1916 Einstein formulated the concept of spontaneous emission for the case of two-level atom interacting with the equilibrium electromagnetic field. This term emphasize the inevitability of energy loss by radiation (Einstein 1916). The dissipation thus being inseparable from real processes. The Schroedinger equations for hydrogen atom and other mechanical systems can only be regarded as a useful idealization.

At this point we would like to quote Ilya Progogine, who said (Prigogine 1980, p.70):
"...Or, on the contrary, The should we argue that nobody has ever seen an atom that would not decay when brought into an exited level? The physical "reality then corresponds to systems with continuous spectra< whereas standard quantum nechanics appears only as a useful idealization, as a simplified limiting case".

Prigigine does not belong to the founding fathers of quantum mechanics. His statement, however, closely echoes the words of Louis de Broglie.

De Broglie expressed doubt in the completeness of quantum mechanical description even at the heyday of the quantum theory. His scepticism was not, however, shared by his contemporaries, and so he also abandoned this attitude, becoming one of the most brilliant advocates of the "Copenhagen" formulation of quantum mechanics. He recalled this period in the following words (de Broglie 1952):

"Soine people, remembering that I abandoned my first attempts and used the interpretation of Bohr and Heisenberg in all niy works for twenty-fifes years thereafter, will accuse ine of being inconsistent when they see that $I$ ain again doubtful and ask myself whether my initial orientation had been right after all. Should I feel like joking, I can reply in Voltaire's words, "Only foolish people never change their minds"

"The answer, however, call be more prudent.

"The progress of science is continually harassed by the tiranic influence of certain concepts which in the course of time have become dogmas. Because of this, the principles which have been recognized as final must be subject to most thorough revision".

At that time these words were the voice of one crying in the wilderness. This can be illustrated with a quotation from the article published in the influential American newspaper;

"The principle of uncertainty has eventually made all contemporary physicists (with the exception of Dr.Einstein) recognize that there is no causality or determinism in nature. Dr.Einstein in majestic solitude has held out against all these concepts of quantum theory"

(The New York Times, 30 March 1952).

Yes another pertinent passage is taken from Dirac's paper published shortly before his death (Dirac 1978):

"I think it might turn out that ultimately Einstein will prove to be right, because the present form of quantum mechanics should not be considered as the final form. There are great difficulties (which I shall mention later $\}$ in connection with the present quantum mechanics. It is the best that one can do up till now.

But, one should not suppose that it will survive indefinitely into future. And I think that it is quite likely that at some future time we may get an improve 
quantum mechanics in which there will be a return to determinism and which will, therefore, justify the Einstein point of view.

But such return to determinism could only be made at the expense of giving up some other basic idea which we now assume without question. We would have to pay for it some way which we yet cannot guess at, if we are to reintroduce determinism."

We do not wish to comment on these statements:the clarity and boldness of the classics can only be admired. Their words encourage further studies in the quantum statistical theory of open systems, which will be continued in the next part of this Lecture. We tried to define the author's position with regard to the fundamental problems of quantum theory. I shall try go on by this way.

\section{Statistical presentation of Heisenberg UNCERTAINTY PRINCIPLE}

\section{VI.A. The oscillatory form of Heisenberg relation}

As well known from the text books on quantum mechanics, the Heisenberg uncertainty principle follows from a inequality

$$
\int\left|\frac{x}{L} \psi+L \frac{d \psi}{d x}\right|^{2} \frac{d x}{L} \geq 0 ; \quad \int|\psi|^{2} \frac{d x}{L}=1 .
$$

Here $L$ is any length parameter.

Let us $f(x, p, t)$ is a quantum distribution function - Wigner function (2) then the last inequality we can present in the following form:

$$
\int\left(\frac{x^{2}}{L^{2}}+\frac{L^{2} p^{2}}{\hbar^{2}}\right) f(x, p, t) \frac{d x d p}{2 \pi \hbar} \geq 1 .
$$

The left side of this inequality we can present as mean value of energy for harmonic oscillator with the proper frequency is defined relation

$$
\omega_{0}=\frac{\hbar}{m L^{2}} ; \quad \frac{\hbar^{2}}{2 m L^{2}}=\frac{1}{2} \hbar \omega_{0} .
$$

Thus

$$
\int\left(\frac{m \omega_{0}^{2} x^{2}}{2}+\frac{p^{2}}{2 m}\right) f(x, p, t) \frac{d x d p}{2 \pi \hbar} \geq \frac{1}{2} \hbar \omega_{0} .
$$

This means that the mean value of a harmonic oscillators can not be less than the corresponding zero energy

$$
\frac{m \omega_{0}^{2}\left\langle x^{2}\right\rangle}{2}+\frac{\left\langle p^{2}\right\rangle}{2 m} \geq \frac{1}{2} \hbar \omega_{0} .
$$

The inequality are presented here it is possible to write in the followings forms:

$$
L^{4}-\frac{\hbar^{2}}{\left\langle p^{2}\right\rangle} L^{2}+\hbar^{2} \frac{\left\langle x^{2}\right\rangle}{\left\langle p^{2}\right\rangle} \geq 0
$$

and

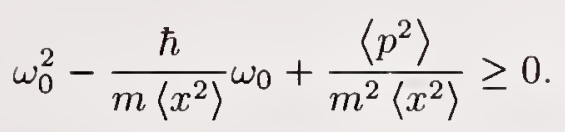

¿From this inequality the Heisenberg uncertainty relation follows

$$
\left\langle x^{2}\right\rangle\left\langle p^{2}\right\rangle \geq \frac{\hbar^{2}}{4}
$$

In a general case parameters $L$ and $\omega_{0}$ have arbitrary values. For the sign " =" these parameters are not there are some restrictions on its

$$
L^{2}=\frac{\hbar}{m \omega_{0}}=2\left\langle x^{2}\right\rangle=\frac{\hbar^{2}}{2\left\langle p^{2}\right\rangle}
$$

or in other form

$$
\frac{\left\langle p^{2}\right\rangle}{m}=m \omega_{0}^{2}\left\langle x^{2}\right\rangle=\frac{\hbar^{2}}{2 m L^{2}}=\frac{1}{2} \hbar \omega_{0} .
$$

\section{VI.B. The sign "=". Distribution functions}

For the sigh " =" the equation (25) has the following solution

$$
|\psi(x)|^{2}=\frac{1}{\sqrt{2 \pi\left\langle x^{2}\right\rangle}} \exp \left(-\frac{x^{2}}{2\left\langle x^{2}\right\rangle}\right) .
$$

The corresponding solution of the equation (26)

it is possible to present as the Wigner distribution for the harmonic oscillator with the proper frequency $\omega_{0}$

$$
f(x, p)=\frac{\hbar}{\sqrt{\left\langle x^{2}\right\rangle\left\langle p^{2}\right\rangle}} \exp \left(-\frac{x^{2}}{2\left\langle x^{2}\right\rangle}-\frac{p^{2}}{2\left\langle p^{2}\right\rangle}\right) .
$$

The dispersions $\left\langle x^{2}\right\rangle,\left\langle p^{2}\right\rangle$ are defined by relations (34).

\section{S-THEOREM FOR QUANTUM SYSTEMS. RELATIVE ORDERING OF STATES "=", "> "}

\section{VII.A. S-theorem}

Of all macroscopic functions, only entropy $S$ possesses a combination of properties that allow it to be used as a measure of uncertainty in the statistical description of processes in macroscopic systems.

Entropy being the sole function with properties of a measure of chaos, there is but one option. It is necessary to redefine entropy so that the average energy remains constant in the course of evolution. 
But evolution in time it is equally possible to consider the evolution of stationary states in open systems at slowly changing of control (governing) parameters. It is for this type of evolution, that criterion was introduced (Klimontovich 1983, 1984, 1988,1990 (1981), 1995). This criterion was for the first time formulated for specific cases (Klimontovich 1983, 1984) and called "S-theorem". Later, its general formulation was suggested, to make possible to direct comparison between the relative degree of order from experimental date (Klimontovich 1988).

Here we shall consider the evolution of quantum states corresponding, accordingly, to the sigh "=" and "> " in Heisenberg relation.

In general case, the degree of order of the distinguished state differs, which account for one of them being more chaotic than the other. Let us term it "physical chaos". As a rule, this state is nonequilibrium and more ordered than the equilibrium state.

Let us, by assumption, the quantum state corresponding to the sign "=" is most chaotic. For this state the quantum distribution function $f_{0}(x, p)$ is determined by the expression (36). The corresponding entropy

$$
\begin{gathered}
S_{0}[x, p]=-\int f_{0}(x, p) \ln f_{0}(x, p) \frac{d x d p}{2 \pi \hbar}= \\
-\int f_{0}(x) \ln f_{0}(x) \frac{d x}{L}- \\
-\int f_{0}(p) \ln f_{0}(p) \frac{L d p}{2 \pi \hbar} \equiv S_{0}[x]+S_{0}[p] .
\end{gathered}
$$

The mean energy for this state is defined by the zero energy

$$
\langle E\rangle=\frac{m \omega_{0}^{2}\left\langle x^{2}\right\rangle}{2}+\frac{\left\langle p^{2}\right\rangle}{2 m}=\frac{1}{2} \hbar \omega_{0} .
$$

¿From the inequality (29) the mean more then this. But according the S-theorem to determine the relative degree of order it is necessary to compare the states at equal values of the mean energy To satisfy this condition it is necessary to replace the distribution function by the renormalized one

$$
f_{0}(x, p) \rightarrow \tilde{f}_{0}(x, p) .
$$

The renormalized distribution function is also Gaussian but with renormalized values $\left\langle\tilde{x}^{2}\right\rangle,\left\langle\tilde{p}^{2}\right\rangle$ for dispersions

$$
\tilde{f}_{0}(x, p)=\frac{\hbar}{\sqrt{\left\langle\tilde{x}^{2}\right\rangle\left\langle\tilde{p}^{2}\right\rangle}} \exp \left(-\frac{x^{2}}{2\left\langle\tilde{x}^{2}\right\rangle}-\frac{p^{2}}{2\left\langle\tilde{p}^{2}\right\rangle}\right) \geq 0 .
$$

To change the mean value of energy we introduce some the non zero temperature $T$

$$
\frac{\left\langle\tilde{p}^{2}\right\rangle}{m}=m \omega_{0}^{2}\left\langle\tilde{x}^{2}\right\rangle=k_{B} T_{\omega_{0}}=\frac{1}{2} \hbar \omega_{0} \operatorname{coth} \frac{\hbar \omega_{0}}{2 k_{B} T} \geq \frac{1}{2} \hbar \omega_{0} .
$$

Let us the quantum distribution function $f(x, p, t)$ characterizes any nonstationary state with the sign "> " in the Heisenderg uncertainty relation. The quantum distribution function $f(x, p . t)$ may have and negative values, but the corresponding distribution functions separately for coordinates and momenta in any cases are positive

$$
\begin{gathered}
\int f(x, p, t) \frac{L d p}{2 \pi \hbar}=f(p, t) \geq 0 \\
\int f(x, p, t) \frac{d x}{L}=f(x, t) \geq 0
\end{gathered}
$$

The corresponding entropy

$$
\begin{gathered}
S[x, p]=-\int f_{0}(x, p) \ln f_{0}(x, p) \frac{d x d p}{2 \pi \hbar}= \\
-\int f(x) \ln f(x) \frac{d x}{L}- \\
-\int f(p) \ln f(p) \frac{L d p}{2 \pi \hbar} \equiv S[x]+S[p] .
\end{gathered}
$$

To find the necessary value of temperature $T$ we must solve the equation

$$
\begin{aligned}
& \int\left(\frac{m \omega_{0}^{2} x^{2}}{2}+\frac{p^{2}}{2 m}\right) \tilde{f}_{0}(x, p) \frac{d x d p}{2 \pi \hbar}= \\
& \int\left(\frac{m \omega_{0}^{2} x^{2}}{2}+\frac{p^{2}}{2 m}\right) f(x, p) \frac{d x d p}{2 \pi \hbar} \equiv \\
& \int \frac{m \omega_{0}^{2} x^{2}}{2} f(x) \frac{d x}{L}+\int \frac{p^{2}}{2 m} f(p) \frac{L d p}{2 \pi \hbar} .
\end{aligned}
$$

The solution of this equation

$$
T(t) \geq 0
$$

therefore the choice of the state with the sign "=" in the Heisenberg uncertainty relation as the more chaotic state is correct. In the (44) variable $t$ for nonequilibrium states plays the role of parameter.

Using the expression (40) for the renormalized distribution function $\tilde{f}_{0}(x, p)$ and the constancy condition (44) for the average energy, the expression for 
the entropy difference of states with signs $"=", ">"$ can be present as inequality

$$
\begin{gathered}
\tilde{S}[x, p]-S[x, p] \equiv \tilde{S}[x]-S[x]+\tilde{S} p]-S[p]= \\
\int f(x, t) \ln \frac{f(x, t)}{\tilde{f}(x)} \frac{d x}{L}+\int f(p, t) \ln \frac{f(p, t)}{\tilde{f}(p)} \frac{L d p}{2 \pi \hbar} \geq 0 .
\end{gathered}
$$

Thus, the state with the sign " $="$ in the Heisenberg relation is the most chaotic. The last expression serves as the quantitative measure for relative degree of order any quantum state - stationary or nonstationary and most chaotic state which corresponds to sign " =" in the Heisenderg uncertainty relation.

It is necessary remember that the oscillatory model was exploited above only in the special case concern of real physical oscillator. The model was considered in much more general. The parameter $L$ in the previous formulas is some general length parameter. If $L$ is the size of the system then the relation $L$ and $\omega_{0}$ allows to use "as example" the oscillatory model for description of a free particle motion Kadotsev 1994; Klimontovich 1995,1997 (in press)).

\section{INFORMATION}

There are to different statistical definition of Shannon information. The first one coincides with definition (by form) with Boltzmann definition of entropy. If $f(X)$ is any dimensionless distribution function of dimensionless variable $X$, then the Shannon information (entropy or " $S$ - information") is defined by expression (Haken 1988; Kadomtsev, 1994)

$$
I[X]=S[X]=-\int f(X) \ln f(X) d X .
$$

or for discrete variables

$$
I[n]=S[n]=-\sum_{n} f_{n} \ln f_{n}
$$

Although in many cases the calculation of $S-$ information is certainly useful, it does not reflect the existence of self-organization in open systems (Klimontovich 1990 (1991), 1995).

To define the changing of information in processes in open systems it is better to use more general definition of information (Stratonovich 1975; Klimontovich $1982(1986))$

$$
I[X \mid Y]=S[X]-S[X \mid Y] .
$$

Here is $S[X]$ is ordinary Boltzmann - Shannon entropy

$$
S[X]=-\int f(X) \ln f(X) d X
$$

and $S[X \mid Y]$ the conditional entropy. It is connected with the conditional distribution function $S[X \mid Y]$ $(f(X, Y)=S[X \mid Y] f(Y))$ by relation

$$
S[X \mid Y]=-\int f(X, Y) \ln f(X \mid Y) d X d Y .
$$

The expression for the information it is possible to present in more symmetrical form

$$
I[X \mid Y]=\int \ln \frac{f(X, Y)}{f(X) f(Y)} f(X, Y) d X d Y \geq 0 .
$$

We see that is defined by such way the information is positive. The equality corresponds to the case when the quantities $X, Y$ are statistically independent.

\section{Conservation law of Entropy and INFORMATION}

Let us the distribution function is defined completely defined be the first moment

$$
f(Y)=\delta(Y-a)
$$

$a$ is any characteristic parameter. In open systems it can play the role of control (governing) parameter. Introduce this function in the expressions (51), (50). After integrating over $Y$ we will obtained the following expression for the information

$$
\begin{gathered}
I[X \mid a]=S[X]-S[X \mid a] \equiv S[X]+ \\
\int f(X \mid a) \ln f(X \mid a) d X .
\end{gathered}
$$

If the nonconditional entropy $S[X]$ does not depend on values of the control parameter $a$,then, using the last equality, it is possible obtain the corresponding relationship between the information and the entropy for any two values of control parameter

$$
I\left[X \mid a_{1}\right]-I\left[X \mid a_{2}\right]=S\left[X \mid a_{2}\right]-S\left[X \mid a_{1}\right] .
$$

¿From it follows the conservation law for the sum information and entropy

$$
I[X \mid a]+S[X \mid a]=\text { const } .
$$

We see that for the cases, when the nonconditional entropy $S[X]$ does not depends on the value of the control parameter. 
In the paper (Kadomtsev 1994) represents the conservation law as sum of the nonconditional information and entropy

$$
I[X]+S[X]=\text { const }
$$

but such presentation of the conservation law contradicts to the definition (47) of the nonconditional information and entropy.

For the example we can use conservation law (56) for quantum systems. Let us $S[X] \rightarrow S_{0}[x, p]$ is the nonconditional entropy for the state with the sigh " =" in the Heisenberg uncertainty relation. It corresponds to the ground state of the system at the temperature $T=0$. Let us also $\tilde{S}[x, p]$ is the renormalized entropy for the state with the sigh "=" in the Heisenberg uncertainty relation, but for temperature $T>0$, and, at last, $S[x, p, t]=S[x, t]+S[p, t]$ is the entropy of any nonequilibrium of stationary states. The corresponding notation will be used and for conditional information. Then, in accordance with inequality (45) ([in accordance with $S$ - theorem), we have the relation

$$
I[z, p, t]-\tilde{I}[x, p]=\tilde{S}[x, p]-S[x, p, t] \geq 0 .
$$

Thus, at the transition from the more chaotic state to the more ordered state the amount if the information increase.

As and in the classical theory the $S$-theorem it is possible now to use for the diagnostic of quantum systems and, thus, to solve one from the most important problems of Semiotics (Meystel, 1995; Coombs,and Sulcoski (Eds.) 1966).

\section{References}

[1] Belinski A.V.,Klyshko D.N. 1993: The Interference of Light and the Bell's Theorem (in Russian) Uspechi Fiz.Nauk, 163,1

[2] Bell J.S.1965:On the Einstein-Podolski-Rosen. Physics, 1,165 .

[3] Bell J.S.1965: On the Problem of Hidden Variable in Quantum Mechanics. Rev.Mod.Phys.38,447.

[4] Brittin W.E., Chapell W.R. 1961. The Wigner Distribution Function and Second Quantization in Phase Space. Rev of Mod. Physics 34620.

[5] Bohm D. On the possible Interpretation of Quantum Mechanics on the Basis of Concept of "Hidden Parameters", Phys.Rev.85,166,180.

[6] Coombs M., Sulcoski M. (Eds.) 1996. Control Mechanisms for Complex Systems. Las Cruces, New Mexico.

[7] De Broglie L.1953: La Physik Quantique Resterat-Elle Indeterministe? Paris.

[8] Dirac P.A.M. Directions in Physics. John Wiley and Sons New York, 1978.

[9] Einstein A. 1916: Strahlung Emission und Absorption nach der Quantentheorie. Verhandl.Dtsch.Phys.Ges.18,318.
[10] Einstein A.,Podolski B.,Rosen N.1935: Can Quantum-Mechanical Description of Physical Reality Be Considered Complete? Phys.Rev.47,777.

[11] Haken H. Information and Selforganization. Springer Heqdelberg, Berlin, New York, 1988.

[12] Hillery M., O'Connell R.F., Scilly M.O., Wigner E.P. 1994. Distribution functions in physics: Fundamentals. Physics Reports 106122167.

[13] Kadomtsev B.B. 1994: Dynamics and Information. Uspechi Fiz. Nauk 164,449

[14] Klimontovich Yu.L. 1958. On the Method of "Second Quantization" in Phase Space. Soviet Physics JETF 6 (33) 752.

[15] Klimontovich Yu.L. and Silin V.P. 1960 (1962). On the Spectra of Systems of Interacting Particles and he Collective Losses on Passage of Particles Through Matter. Uspechi Fiz. Nauk 70 247; Fortschr.Physik, 10389.

[16] Klimontovich Yu.L. 1982 (1986) Statistical Physics. Moscow "Nauka, 1982; Harwood Academic Publishers New York.

[17] Klimontovich Yu.L. 1980 (1983) The Kinetic Theory of Electromagnetic Processes. Nauka Moscow; Springer Berlin, Heidelberg, New York.

[18] Klimontovich Yu.L. 1983 Entropy Decrease in the Processes of Self-Organization, (in Russian). Pis'ma v ZhTF 91089.

[19] Klimontovich Yu.L. 1984. Entropy and Entropy Production in the Laminar and Turbulent Flows, (in Russian).Pis'ma v ZhTF 1080.

[20] Klimontovich Yu.L.1990,1991 Turbulent Motion and Structure of Chaos. Nauka, Moscow; Kluwer Academic Publishers Dordrecht.

[21] Klimontovich Yu.L. 1993: To the Statistical Ground of Schroedinger Equation. (in Russian) TMF, 97,3.1995:

[22] Klimontovich Yu.L.1995:Statistical Theory of Open Systems. Nauka, Moscow; Kluwer Academic Publishers, Dordrecht, 1995.

[23] Klimontovich Yu.L.1996: Relative ordering criteria in open systems. Uspechi Fiz. Nauk 1661231.

[24] Klimontovich Yu.L. 1997:To Kinetic Theory of Collisionless Plasma. Uspechi Fiz. Nauk, 167, 23.

[25] Klimontovich Yu.L.,Kremp D.1981:Quantum Kinetic Equation with Bound States. Physica A, 109,512 .

[26] Klimontovich Yu.L.,Kremp D.,Kraeft W.1987: Kinetic Theory for Chemically Reacting Gases and Partially Ionized Plasmas. Adv.Chem.Phys.58,175.

[27] Klimontovich Yu.L., Wilhelmsson H., Yakimenko I.P., Zagorodnii A.G. 1989: Statistical Theory of Plasma Molecular Systems. Phys.Rev.175, 264.

[28] Mehra J.1875: The Solvay Conferences on 
Physics. D.Reidel, Dordrecht, Boston.

[29] Meystel A.1995 Semiotic Modeling and Situation Analisis. AfRem, Inc.

[30] Neumann J. Mathematische Grundlagen der Quantummrchanik. Springer, Berlin.

[31] Prigogine I.1980:From Being to Becoming. Freeman, San Francisco.

[32] Prigogine I., Stengers I. 1984: Order out of Chaos. Heinemann, London.

[33] Stratonovich R.L.1975. Theory of Information, (in Russian). Moscow "Sov. Radio". 


\title{
Information Granulation and its Centrality in Human and Machine Intelligence
}

\author{
Lotfi A. Zadeh ${ }^{*}$
}

Abstract

\begin{abstract}
In a general setting, granulation involves a partitioning of a real or mental object into granules, with a granule being a clump of points (objects) drawn together by undistinguishability, similarity, proximity or functionality.

Granulation is crisp or fuzzy depending on whether the granules are crisp or fuzzy. In the case of age, for example, the time-intervals $\{1,2,3, \ldots$ $, 130\}$ are crisp, whereas the fuzzy time-intervals \{very young, young, middle-aged, old, very old\} are not.

Granulation -- and especially information granulation (IG) -- are ubiquitous in human actions and cognition. We employ granulation when we speak, write, eat, walk and analyze an image; we employ fuzzy information when we partition a human body into body parts: head, neck, chest, arms, legs, etc.; and more generally, we apply granulation when we partition a complex problem into simpler subproblems.

Modes of information granulation in which the granules are crisp (crisp IG) play important roles in a wide variety of methods, approaches and techniques. Among them are: interval analysis, quantization, rough set theory, diakoptics, divide and conquer, Dempster-Shafer theory, machine learning from examples, chunking, qualitative process theory, decision trees, semantic networks, analog-to-digital conversion, constraint programming, cluster analysis and many others.

Important though it is, crisp IG has a major blind spot. More specifically, it fails to reflect the fact that in much -- perhaps most -- of human reasoning and concept formation the granules are fuzzy rather than crisp. For example, the fuzzy granules of a human head are the nose, ears, forehead, hair, cheeks, etc. Each of the fuzzy granules is associated with a set of fuzzy attributes, e.g., in the case of the fuzzy granule hair, the fuzzy attributes are color, length, texture, etc. In turn,
\end{abstract}

each of the fuzzy attributes is associated with a set of fuzzy values. Specifically, in the case of the fuzzy attribute length(hair), the fuzzy values are long, short, not very long, etc. The fuzziness of granules, their attributes and their values is characteristic of the ways in which human concepts are formed, organized and manipulated.

In human cognition, fuzziness of granules is a direct consequence of fuzziness of the concepts of indistinguishability, similarity and functionality. Furthermore, it is entailed by the finite capacity of the human mind to store information and resolve detail. In this perspective, fuzzy information granulation (fuzzy IG) may be viewed as a form of loose data compression.

Fuzzy information granulation underlies the remarkable human ability to make rational decisions in an environment of imprecision, uncertainty and partial truth. And yet, despite its intrinsic importance, fuzzy information granulation has received scant attention except in the context of fuzzy logic, in which fuzzy IG underlies the basic concepts of linguistic variable, fuzzy rule-set and fuzzy graph. In fact, the effectiveness and successes of fuzzy logic in dealing with real-world problems rest in large measure on the use of the machinery of fuzzy information granulation. This machinery is unique to fuzzy logic.

Recently fuzzy information granulation has come to play a central role in the methodology of computing with words (CW). More specifically, in a natural language words play the role of labels of fuzzy granules. In computing with words, a proposition is viewed as an implicit fuzzy constraint on an implicit variable. The meaning of a proposition is the constraint which it represents.

In $\mathrm{CW}$, the initial data set (IDS) is assumed to consist of a collection of propositions expressed in a natural language. The result of computation -- referred to as the terminal data set 
(TDS) -- is likewise a collection of propositions expressed in a natural language. To infer TDS from IDS the rules of inference in fuzzy logic are used for constraint propagation from premises to conclusions.

There are two main rationales for computing with words. First, computing with words is a necessity when the available information is not precise enough to justify the use of numbers. And second, computing with words is advantageous when there is a tolerance for imprecision, uncertainty and partial truth that can be exploited to achieve tractability, robustness, low solution cost and better rapport with reality. In coming years, computing with words is likely to evolve into an important methodology in its own right with wide-ranging applications on both basic and applied levels.

Inspired by the ways in which humans granulate human concepts, we can proceed to granulate conceptual structures in various fields of science. In a sense, this is what motivates computing with words. An intriguing possibility is to granulate the conceptual structure of mathematics. This would lead to what may be called granular mathematics. Eventually, granular mathematics may evolve into a distinct branch of mathematics having close links to the real world.
A subset of granular mathematics and a superset of computing with words is granular computing.

In the final analysis, fuzzy information granulation is central to human reasoning and concept formation. It is this aspect of fuzzy IG that underlies its essential role in the conception and design of intelligent systems. What is conclusive is that there are many, many tasks which humans can perform with ease and that no machine could perform without the use of fuzzy information granulation, This conclusion has a thought-provoking implication for AI: Without the methodology of fuzzy IG in its armamentarium, AI cannot achieve its goals.

*Professor in the Graduate School and Director, Berkeley Initiative in Soft Computing (BISC), Computer Science Division and the Electronics Research Laboratory, Department of EECS, University of California, Berkeley, CA 94720-1776;

Telephone: 510-642-4959; Fax: 510-642-1712;

E-mail:zadeh@cs.berkeley.edu.

Research supported in part by NASA Grant NCC 2 275, ONR Grant N00014-96-1-0556, LLNL Grant 442427-26449, ARO Grant DAAH 04-961-0341 and the BISC Program of UC Berkeley. 

II WORLD REPRESENTATION 



\title{
The Role of Semiotic Descriptors in Relational Representation of Fuzzy Granular Structures
}

\author{
Ladislav J. Kohout and Eunjin Kim \\ Department of Computer Science \\ Florida State University \\ Tallahassee, Florida 32306-4019, USA \\ kohout@cs.fsu.edu, ejkim@cs.fsu.edu
}

\begin{abstract}
This paper describes the role of semiotic descriptors in representation of knowledge by relational structures.

Fuzzy relations can manipulate semantic information that is carried by linguistic labels. The conditions imposed on logic operations applied to fuzzy relational systems can be strictly mathematically defined. But the logic semantic is not sufficient to deal satisfactorily with the meaning of linguistic labels that carry the conceptual meaning of applications. It has to be supplemented by the notion of semiotic descriptors. These can be expressed as algebraic restrictions over the basic fuzzy relational system [5].

Semiotic Fuzzy Knowledge Representation Structure consists of the pair of structures, namely $\langle F R S, S D\rangle$. FRS is a Fuzzy Relational Structure consisting of a family of fuzzy relations, and $S D$ is collection of semiotic descriptors [4].

After describing the general semiotic model, we shall also show a specific application - use of Semiotic Fuzzy Knowledge Representation approach in study of cost and affordability in engineering design. Specific examples to be presented are taken form the domain of aeronautic industry. BK-products of relations [7] and fast fuzzy relational algorithms [1] are the technical tools by which we extract meaning form the answers to specific questions presented to engineers in our case study.
\end{abstract}

\section{Introduction}

This paper describes the role of semiotic descriptors in representation of knowledge by relational structures. Fuzzy relations can manipulate semantic information that is carried by linguistic labels. The conditions imposed on logic operations applied to fuzzy relational systems are strictly mathematically defined. But the logic semantic is not sufficient to deal satisfactorily with the meaning of linguistic labels that carry the conceptual meaning of applications. It has to be supplemented by some semiotic notions that can be expressed as algebraic restrictions over the basic fuzzy relational system.

Semiotic Fuzzy Knowledge Representation Structure consists of the pair of structures, namely $<$ FRS, SD $>$. FRS is Fuzzy Relational Structure consisting of a family of fuzzy relations, and SD is collection of semiotic descriptors $[4],[5]$.

Semiotic descriptors are obtained by exploratory knowledge elicitation [6]. We use repertory grids to elicit the meaning as used by human experts [3] Once the relevant semiotic descriptors are identified, relationships between them can be captured by repertory grids using bi-polar constructs, each construct consisting of a pair of semiotic descriptors.

After the repertory grids are applied to a particular problem, and the semantic relationship captured by the grids, the grids are transformed into fuzzy relations which relate the semiotic descriptors.

Relational methods of analysis are then applied in order to discover meaningful conceptual structures implicit in these fuzzy relations. This is done by forming fuzzy relational products and further processing thus obtained composed relations by algorithms computing closures and interiors of fuzzy relations.

Particularly useful structural relationships are equivalences, similarities and preorders between individual semiotic descriptors captured by the bi-polar repertory grids. These structural properties relating the meaning of concepts intrinsically contained in the data captured by repertory grids must be made explicit by appropriate relational computations. The computational algorithms for this purpose are based on BK-Products of fuzzy relations and Fast Fuzzy Relational Algorithms [8] computational tools are used to identify relational structures and properties intrinsically contained in data.

After describing the general semiotic model, we shall also show a specific application - use of Semiotic Fuzzy Knowledge Representation approach in study of cost and affordability in engineering design. Specific examples to be presented are taken form the domain of aeronautic in- 
dustry.

A specific example on which we demonstrate our methods will be knowledge elicited from human experts characterising an ingot process of the Lower Pressure Turbine (LPT) Cover Plate. The data of this example is evaluated by means of fuzzy relational subproducts with respect to the structural relationships of cost factors. Crucial to this approach is the use of semiotic descriptors that allow for linguistic interpretation of relational computations. These descriptors potentially denote the technological and fiscal entities and concepts that are relevant to the problem of affordability.

\section{Semiotic Descriptors, Granularity and Meaning}

\subsection{Semiosis and Interpretation}

The main goal of semiosis is interpretation of signs and symbols - recognition of their meaning. The meaning interpretation unit is a very complicated one. The meaning of something can be different depending on the scale which is used for representation. This makes the notion of granularity a very important one. Levels of granularity also referred to as levels of resolution are closely related to generalization.

Interpretation and meaning depend on context. A process considered with all its details cannot be properly understood unless the details irrelevant within a particular context or perspective are brushed away. But this interpretation will often be incomplete. With incompleteness at work, there may be more than one interpretation. Indeed, a whole family of interpretations may be possible, some of which may be conflicting. Fuzzy sets, relations and logic can play an important role here: they allow us, through the theory of potentiality (or virtual plurality) to deal with the whole family of virtual outcomes, and also to measure the degree of conflict of individual members of some possiblistic family of outcomes produced by the meta-process of interpretation.

\subsection{Object Emergence}

The phenomenon of object emergence is linked with formation of crisp and fuzzy classes (generalized groupings). Indeed, here we deal with the logic theory of fuzzy relations, which can be used to expose the inadequacy of currently used logical structure of crisp (i.e. non-fuzzy objects). Logically, generalization is a process in which, by means of grouping together relevant properties (intensional specifications) of objects, we create new objects - structures (given as such by extension) by new intensional specification. So OOP objects can be viewed as special cases of the pragmatics of groupings, the semantics of which is given by many-valued logic based relations with special meta-properties.

\subsection{Triadic Distinction of Semiotics: Syntax, Semantics and Pragmatics}

One of the classic notions of semiotics is the triadic distinction: syntax - semantics - pragmatics coined by Morris in late 1930s. That is the place where the duality Semiotics - Mathematics comes in. In logic methods of proofs, only the "form", the syntactic composition plays the role. In the so called logic theory of models, the primary goal is to interpret syntax in semantic meta-structures. But what we need here is the fully fledged duality: syntax - semantics, and the pragmatics of emergence of either of these. Here the fuzzy logic can play an important role: we have the duality of linguistic descriptors and fuzzy structures to which these descriptors apply. We also have the duality symbolic vs. numerical that are both addressed by what Zadeh calls "the fuzzy logic in wider sense". "Numerical" in our relational setting will be extended to the many-valued logic valuation of relational structure, and "symbolic" will be represented by semiotic descriptors, which are special instances of linguistic labels.

Now, we have to provide the link of Semiotic Fuzzy Knowledge Representation Structure (SFKRS) with the semiotic triangle. Semiotic Fuzzy Knowledge Representation Structure consists of the pair of structures, namely $<$ FRS, SD $>$. FRS is Fuzzy Relational Structure consisting of a family of fuzzy relations, and SD is collection of semiotic descriptors [4],[5]..

The semiotic triangle has three vertexes, namely name, meaning and represented object. The vertex "name" of the triangle maps into a semiotic descriptor SD, while the vertex "meaning" is represented by a Fuzzy Relational Structure (FRS). The vertex "object" maps into the object of the real world characterisation of which is captured by the pair <FRS, SD > of SF KRS. See Figure 1 for more further details.

\section{$3 \quad$ Semiotic Relational Model}

\subsection{Representing Activites in the Domain of Manufactur- ing in the Aeronautic Industry}

In this second part of our paper, we look at a specific relational model from the domain of manufacturing in the aeronautic industry. First we give an overview of the global relational structure in terms of semiotic descriptors SD. This is followed by a section that shows how the Semiotic Fuzzy Knowledge Representation Structure SFKRS (which consists of a pair of structures, namely $<$ FRS, SD $>)^{1}$ is further completed by eliciting the fuzzy member-

\footnotetext{
${ }^{1}$ We have noted previously that FRS is a Fuzzy Relational Structure consisting of a family of fuzzy relations, and SD is collection of
} 


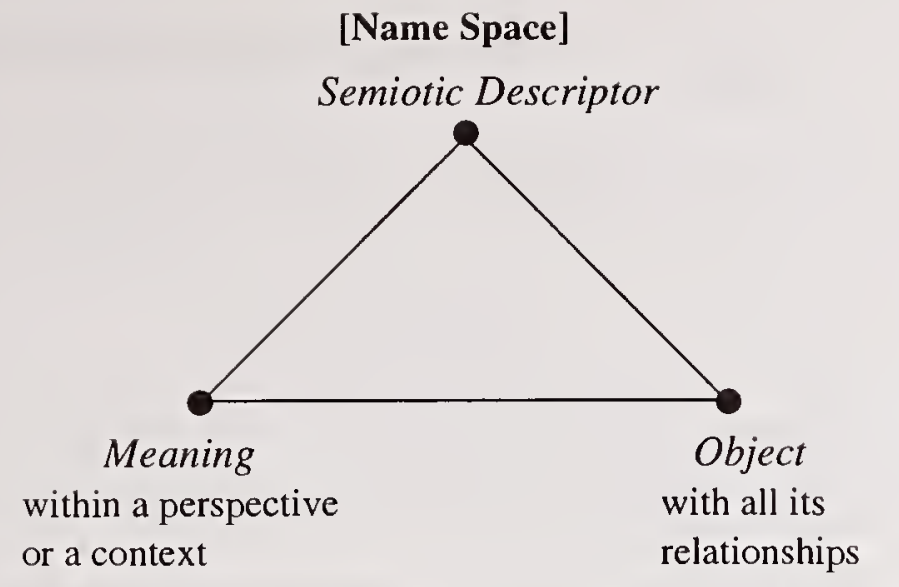

[Conceptual Characterization]

[Physical Reality]

\section{Figure 1. Semiotic Triangle}

ship values of FRS by repertory grids. Repertory grids are presented to human experts, in this case engineers who fill the grids. The grids are the turned into fuzzy relations which are further processed by relational computations by which the meaning within a context is extracted.

We have noted previously that FRS is Fuzzy Relational Structure consisting of a family of fuzzy relations, and SD is collection of semiotic descriptors.

\subsection{Conceptual Categories of Semiotic Descriptors}

The relational model presented in this case study is intended for the capturing and representing the features relevant to the affordability analysis and prediction. It cosists of the following conceptual categories of relations :

- Objects,

- attributes,

- values,

- agents,

- perspectives,

- contexts,

- views.

Each conceptual category listed above represents a specific level of granularity. Each level of granularity may posses conceptual refinements that are interlinked with other granular structures. Each conceptual category has specific meaning that can be understood and interpreted by a domain expert.

Objects are, for example, either parts of a manufactures product, manufactured products or whole technologies, depending on the resolution level.

semiotic descriptors.
Attributes are characterised by linguistic descriptors. Examples of these are: low_raw_material_cost, smallprocessing_windows, high_temperature, good_lubricity, low_variance_in_raw_material_costs, etc. But attributes can also be characterised by measurable physical or fiscal parameters, such as:

temperature, lubricity, cost_reducing_potential, potential investment, cost, etc.

Interactions (special kinds of relations), for example: REL_1,5: low_variance_in_raw_material_costs $\longrightarrow$ low_cracking_probability

REL_2,3:

good_processing_control

low_raw_material_cost

REL_3,7: low_raw_material_cost common_standard_material_alloy.

Values. These are either linguistic variables or numerical variables determining the degree to which an object possesses an attribute.

Agents. In the context of this project, agents are the observers (e.g. engineers or accountants) assessing the degree to which an attribute is possessed by an object. For example, in [R4], [R8], [R10] describing the evaluation of an LPT cover plate the observers were engineers evaluating to what degree various attributes can be assigned to the LPT plate. Perspectives. An object or a family of objects can be evaluated within different perspectives. For example, an LPT cover plate can be evaluated from the perspective of an engineer, or from the perspective of a business analyst performing value analysis of the part, or from the perspective of an accountant. Each perspective may employ attributes that are different from the attributes of a different perspective for the same object. Some attributes may, however, be shared by different perspectives.

Contexts. Each object or family of objects can appear in several different contexts. For example an LPT cover plate may appear e.g.in context of ingot process, forging process, extrusion process, or other processes.

Views. Even in one particular perspective or context, different experts may assess the objects and situations in which objects appear differently. These differences of views of different experts can be captured by repertory grids and compared by relational methods using algorithms provided by TRYSIS.

\section{A Case Study: Low Pressure Turbine (LPT) Cover Plate}

\subsection{Semantics of the Relationships of the Relational Model of LPT Cover Plate}

\subsubsection{Semiotic Descriptors of Relational Products}

In representing knowledge structures, not only quantitative but also qualitative notions are involved. Productrelations formed by the relational products represent new 
entities composed from the original data. Each relations possesses meaning carried by its linguistic label. This label, which is in fact an instance of semiotic descriptors carries a concrete interpretation within a particular knowledge domain. This interpretation also determines the meaning of the composed relation computed by the relational product.

Our model relation $M$ to be used in the sequel $M \in \mathcal{R}(Y \times$ $O \times P$ ), relates the following three lists (sets) of entities:

- the set $Y$ of process attributes;

- the set $O$ of observers, assessors or measuring sensors; with the set $G$ of process identifiers;

- the set of Parts or Components.

Applying the usual selection and projection operators the ternary relation is decomposed into a family of 2-ary relations in $\mathcal{R}(Y \times O)$, indexed by the set $P$. The processes $P_{1}$ and $P_{2}$ are extrusion and forging, respectively. The relation $R 1$ and $R 2$ are from the set of process attributes $Y$ to the set $O$ ) is composed with its transpose $R^{T}$ by means of triangle subproduct and the local preorder closure computed by the TRYSIS system. The result of this computation is a relation from the process attributes $Y$ to $Y$. This relation shows the dependencies of process attributes represented as a preorder relation.

A sample result displaying dependences and equivalences of process attributes is shown in Figure 2. These figures show the Hasse Diagram (HD) structures displaying the preorders of process attributes computed by the fuzzy relational triangle subproduct over processes. The object processed in both processes and evaluated by the engineers is LPT cover plate made of gamma-titanium.

A number of evaluative schemes can be formulated, showing inter-process dependences, inter-observer dependences, etc. Because the purpose of this paper is to show basic techniques of relational analysis as applied to manufacturing processes, not to provide a detailed analysis of engineering of LPT cover plate, further details of these computations are not presented in this paper.

Three scenarios for using a repertory grid on LPT parts have been used [3]. Here it will suffice to present just one scenario, namely

\section{SCENARIO A:}

1 object (LPT cover plate) is rated by group of respondents (engineers) where each of the respondents does assess the object independently in a selected process. The aim is to find the dependences between process characteristics and the inter respondent consistency.

There may be several situations or processes in which the object may appear. In our example these are extrusion and forging.
4.2 Context Dependency of the Meaning of Semiotic Descriptors

In each context defined by a specific industrial process (e.g. forging or extrusion) there is a set of semiotic descriptors that is relevant to the knowledge representation structure that has been created for a specific purpose. Thus in our project [2], we study the question of affordability that is related to the cost of production. This determines which semiotic descriptors will appear in the SFKRS relevant to each industrial process.

For example, looking at process of forging and extrusion for a particular part, i.e. LPT cover plate, we have large/small process window bipolar semiotic descriptors for extrusion and large/small process window bipolar semiotic descriptors for forging (see the list of SD in Table 1). Out of these, Large/small process window are identical and appear in both processes.

This however does not mean that a specific list of semiotic descriptors has the same meaning in two different contexts. A distinct difference in the meaning which a particular list of semiotic descriptors can acquire in different contexts is clearly shown in Table 1.

Fig. 2 shows that in the context of extrusion, semiotic descriptors large process window $(\mathrm{C} 2)$ and long die life $(C 9)$ are captured within the equivalence class of FRS, hence they are equivalent in their meaning. In the context of forging on the other hand, it is different. Large process window $(C 2)$ is equivalent with air furnace atmosphere(C5) in its meaning. The equivalence of large process window $(\mathrm{C} 2)$ and long die life $(C 9)$, however, does not hold in the context forging despite of the fact that it holds for the process of extrusion.

Let us look at these results now in semiotic terms. As can be seen from Table 1, the list of semiotic descriptors used for evaluation of the process of forging is the same as the list of the semiotic descriptors used for the process of extrusion. So in terms of Fig. 1, we use the same name space for both processes. The Hasse diagram structure of Fig. 2 for computed for extrusion is different from the structure that was obtained for forging. In terms of Fig. 1, The Hasse diagram structures represent the third vertex (3) of the semiotic triangle, i.e. relational conceptual characterisation. This characterisation has been elicited experimentally from a group of human experts-engineers by repertory grids. The fuzzy relation oftained from these repertory grids was then tested for the relational property of preorder that can be graphically presented as a Hasse diagram.

We can also consider the SFKRS with the negative side of bi-polar features of the semiotic descriptors. As shown in Figures 3 and 4 the SFKRS can also be formed with the negative semiotic descriptors. SFKRS depicted in Figure 3 shows the property of contrapositive symmetry ${ }^{2}$ while

\footnotetext{
${ }^{2}$ A logic proposition is contrapositive if $a \rightarrow b=\neg b \rightarrow \neg a$.
} 
SFKRS of Figure 4 does not show it.

The implication operators used to compute the Hasse diagrams displayed in Figure 2 of this paper are indicated directly in the figures ${ }^{3}$

\section{Conclusion}

In this paper, we have shown by an experimental study, that the semiotic triangle which is usually applied indiscriminately, is in fact context dependent. We have demonstrated that

- In one context, two different fragments of SFKRS elicited by questioning engineers by the repertory grid techniques may be equivalent in meaning. In another context their equivalence may not hold.

- In two different contexts defined by two distinct industrial processes the meaning of a set of the identical semiotic descriptors may be different in their meaning.

Our paper also shows that the use of basic semiotic notions such as a semiotic triangle, if properly applied may add another dimension to enginnering. It is widespread opinion, in particular in the US that semiotic is useful only for literary studies. We hope that we have managed to persuade our readers that semiotics enriched by fuzzy relational techniques has important applications in technology.

ACKNOWLEDGEMENT: This work has been partially supported by the NSF grant DMI 9525991 entitled Decision-Making with Incomplete Information in an Integrated Product and Process Development Enterprise: A Management Decision Tool for Cost Modeling and Affordability Applications.

\section{References}

[1] W. Bandler and L.J. Kohout. Special properties, closures and interiors of crisp an d fuzzy relations. Fuzzy Sets and Systems, 26(3):317-332, June 1988.

[2] B. Dubrosky, L.J. Kohout, R.M. Walker, E. Kim, and H.P. Wang. Use of fuzzy relations for advanced technology cost modeling and affordability decisions. In 35 th AIAA Aerospace Sciences Meeting and Exhibit (Reno, Nevada, January 6-9,1997), pages 1-12, Reno, NV, January 1997.

[3] E.. Kim, L.J. Kohout, B. Dubrosky, and W. Bandler. Use of fuzzy relations for affordability decisions in high technology. In R.A. Adey, et al. Applications of Artificial Intelligence in Engineering XI. Computational Mechanics Publications, Southampton, UK - Bilerica, MA, USA, 1996.

\footnotetext{
${ }^{3}$ These are defined by formulas Łukasiewicz implication operator $a \stackrel{5}{\rightarrow} b=\min (1,1-a+b)$; Gaines-Goguen implication operator $a \stackrel{4}{\rightarrow} b=\min (1, b / a)$ and $S^{*}$ (Heyting-Gödel) implication operator $a \stackrel{3}{\rightarrow} b=1$ if $a \leq b, b$ otherwise.
}

[4] L.J. Kohout. A Perspective on Intelligent Systems: A Framework for Analysis and Design. Chapman and Hall \& Van Nostrand, London \& New York, 1990. A Scientific Monograph, 255 pages.

[5] L.J. Kohout. Activity Structures: A methodology for design of multi-environment and multi-context knowledge-based systems. In [6].

[6] L.J. Kohout, J. Anderson, and W. et al. Bandler. Knowledge-Based Systems for Multiple Environments. Ashgate Publ., Aldershot, U.K., 1992.

[7] L.J. Kohout and W. Bandler. Fuzzy relational products in knowledge engineering. In V. Novák et al., eds, Fuzzy Approach to Reasoning and Decision Making, pages 51-66. Kluwer, Dordrecht, 1992.

[8] L.J. Kohout and W. Bandler. Use of fuzzy relations in knowledge representation, acquisition and processing. In L.A. Zadeh and J. Kacprzyk, editors, Fuzzy Logic for the Management of Uncertainty, pages 415-435. John Wiley, New York, 1992.

\section{FRS of Extrusion}

$(\mathrm{S}, \mathrm{H}, \mathrm{harsh})=(\mathrm{S},(\mathrm{H}, \mathrm{HU}, \mathrm{M})$, mean $)$

$=\left(\mathrm{S}^{*},(\mathrm{H}, \mathrm{HU}), \mathrm{harsh}\right)=\left(\mathrm{S}^{*},(\mathrm{H}, \mathrm{HU})\right.$, mean $)$

$=(\mathrm{G} 43,(\mathrm{H}, \mathrm{HU})$, harsh $)=(\mathrm{G} 43, \mathrm{H}$, mean $)$

$=\left(G 43^{\prime},(\mathrm{H}, \mathrm{HU})\right.$, harsh $)=\left(G 43^{\prime},(\mathrm{H}, \mathrm{HU})\right.$, mean $)$

$=(\mathrm{L},(\mathrm{H}, \mathrm{HU})$, harsh $)=(\mathrm{E},(\mathrm{H}, \mathrm{HU})$, mean $)$

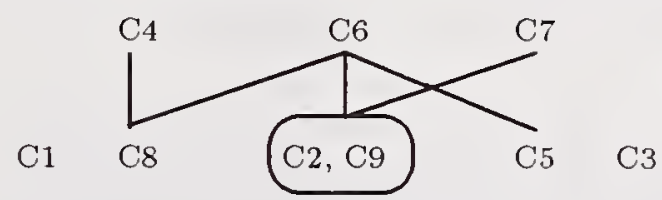

\section{FRS of Forging}

$(\mathrm{S}, \mathrm{H}$, harsh $)=(\mathrm{S}, \mathrm{H}$, mean $)$

$=\left(S^{*},(H, H U, M)\right.$, harsh $)=\left(S^{*},(H, H U, M)\right.$, mean $)$

$=(\mathrm{G} 43,(\mathrm{H}, \mathrm{HU})$, harsh $)=(\mathrm{G} 43,(\mathrm{H}, \mathrm{HU})$, mean $)$

$=\left(G 43^{\prime},(\mathrm{H}, \mathrm{HU})\right.$, harsh $)=\left(G 43^{\prime},(\mathrm{H}, \mathrm{HU})\right.$, mean $)$

$=(\mathrm{E},(\mathrm{H}, \mathrm{HU})$, harsh $)=(\mathrm{L},(\mathrm{H}, \mathrm{HU})$, mean $)$

$=(\mathrm{KDL}, \mathrm{HU}$, harsh $)$

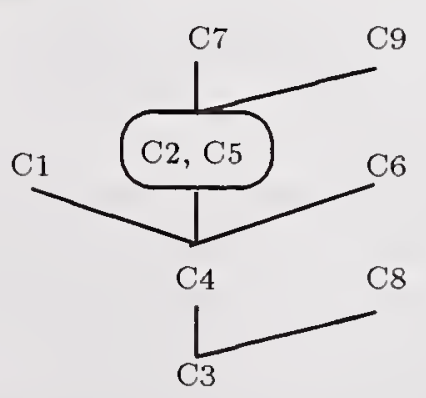

Figure 2. SFKRS $(<$ FRS,SD $>$ ) with the positive bipolar fetures in the context of Extrusion and Forging 


\begin{tabular}{|c|l||c|l|}
\hline \multicolumn{2}{|c||}{ Positive Semiotic Descriptors } & \multicolumn{2}{c|}{ Negative Semiotic Descriptors } \\
\hline Symbol & \multicolumn{1}{|c|}{ Meaning } & Symbol & \multicolumn{1}{|c|}{ Meaning } \\
\hline C1 & Capable Analytical Modeling & $\overline{C 1}$ & Limited Analytical Modeling \\
C2 & Large Process Window & $\overline{C 2}$ & Small Process Window \\
C3 & Low Temperature & $\overline{C 3}$ & High Temperature \\
C4 & Good Lubricity & $\overline{C 4}$ & Low(or Difficult) Lubricity \\
C5 & Air Furnace Atmosphere & $\overline{C 5}$ & Vacuum Furnace Atmosphere \\
C6 & Good Process Control & $\overline{C 6}$ & Limited Process Control \\
C7 & Available Tooling & $\overline{C 7}$ & New Tooling \\
C8 & Flat Die Shape & $\overline{C 8}$ & Shaped Die Shape \\
C9 & Long Die Life & $\overline{C 9}$ & Short Die Life \\
\hline
\end{tabular}

Table 1. SD both in the context of Extrusion and Forging

\section{FRS in Extrusion}

$(\mathrm{S}, \mathrm{H}, \mathrm{harsh})=(\mathrm{S},(\mathrm{H}, \mathrm{HU}, \mathrm{M})$, mean $)$

$=\left(\mathrm{S}^{*},(\mathrm{H}, \mathrm{HU})\right.$, harsh $)=\left(\mathrm{S}^{*},(\mathrm{H}, \mathrm{HU})\right.$, mean $)$

$=(\mathrm{G} 43,(\mathrm{H}, \mathrm{HU})$, harsh $)=(\mathrm{G} 43, \mathrm{H}$, mean $)$

$=\left(G 43^{\prime},(\mathrm{H}, \mathrm{HU})\right.$, harsh $)=\left(G 43^{\prime},(\mathrm{H}, \mathrm{HU})\right.$, mean $)$

$=(\mathrm{E},(\mathrm{H}, \mathrm{HU}), \mathrm{harsh})=(\mathrm{E},(\mathrm{H}, \mathrm{HU})$, mean $)$

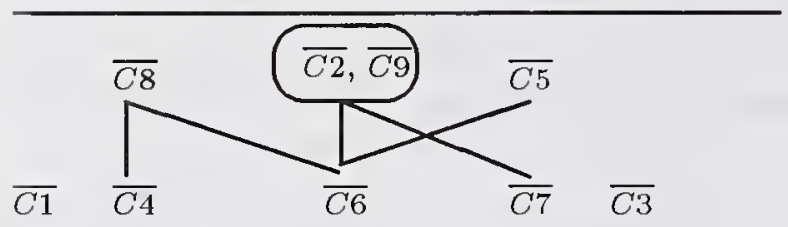

FRS in Forging

$(\mathrm{S}, \mathrm{H}, \mathrm{harsh})=(\mathrm{S}, \mathrm{H}$, mean $)$

$=\left(\mathrm{S}^{*},(\mathrm{H}, \mathrm{HU}, \mathrm{M})\right.$, harsh $)=\left(\mathrm{S}^{*},(\mathrm{H}, \mathrm{HU}, \mathrm{M})\right.$, mean $)$

$=(\mathrm{G} 43,(\mathrm{H}, \mathrm{HU})$, harsh $)=(\mathrm{G} 43,(\mathrm{H}, \mathrm{HU}, \mathrm{M})$, mean $)$

$=\left(G 43^{\prime},(\mathrm{H}, \mathrm{HU})\right.$, harsh $)=\left(G 43^{\prime},(\mathrm{H}, \mathrm{HU})\right.$, mean $)$

$=(\mathrm{L},(\mathrm{H}, \mathrm{HU})$, harsh $)=(\mathrm{L},(\mathrm{H}, \mathrm{HU})$, mean $)$

$=(\mathrm{KDL}, \mathrm{HU}, \mathrm{harsh})$

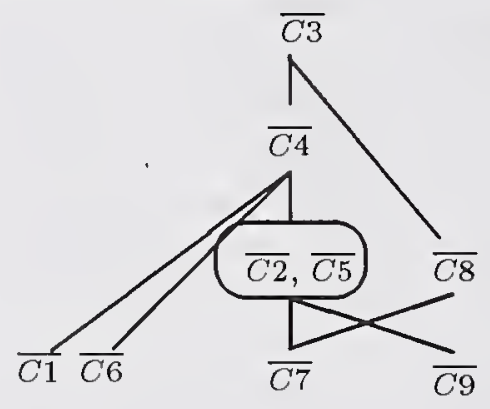

Figure 3. SFKRS $(<$ FRS,SD $>)$ with the negative bipolar fetures in the context of Extrusion and Forging - holding the property of ContraPositive Symmetry

Extrusion

(G43, M, mean)
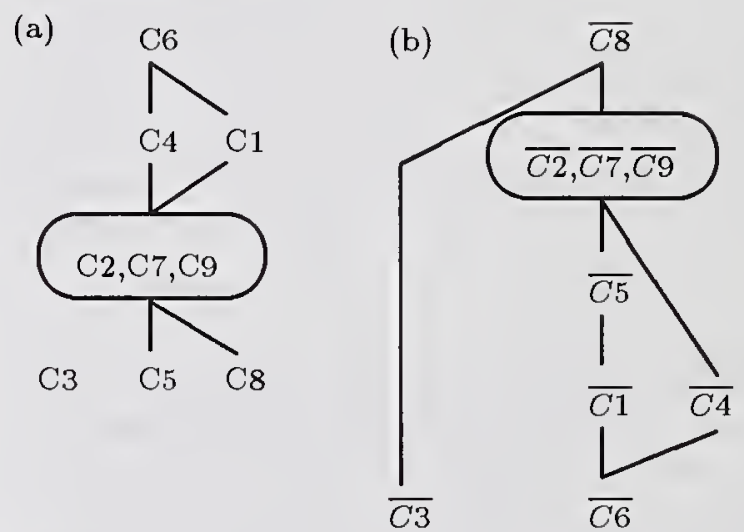

Forging

(G43, M, (harsh,mean))

(c)

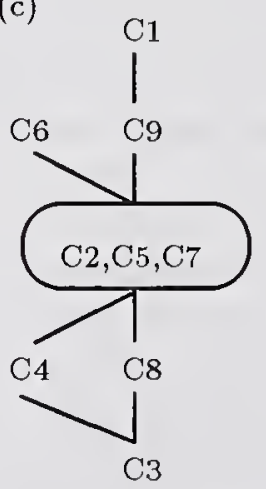

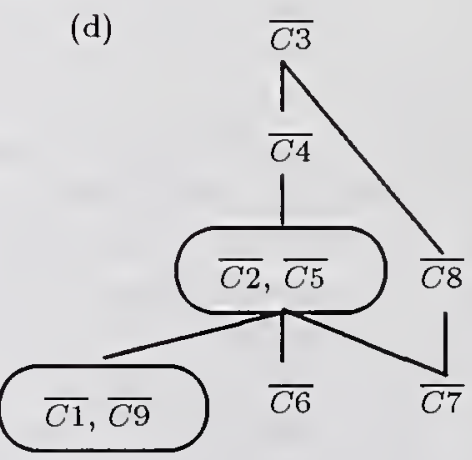

Figure 4. SFKRS $(<$ FRS,SD $>)$ with both positive and negative bipolar fetures in the context of Extrusion and Forging - withouth holding the property of ContraPositive Symmetry 


\title{
AN INTEGRATED APPROACH TO FUZZY LEARNING AND REASONING IN HIERARCHICAL KNOWLEDGE STRUCTURES
}

\author{
Jan C.A. van der Lubbe ${ }^{1} \&$ Emile Egbers ${ }^{2}$ \\ 1 Delft University of Technology, Dept. Electrical Engineering, Information Theory Group, P.O. \\ Box 5031, 2600 GA Delft, the Netherlands, E-mail: vdlubbe@it.et.tudelft.nl \\ ${ }^{2}$ Zappwerk, P.O. Box 3363, 2601 DJ Delft, the Netherlands, E-mail e.egbers@ zappwerk.nl
}

\begin{abstract}
In this paper an integrated system is developed which can perform the three inference methods introduced by Peirce (1931-35) (abduction, deduction and induction) and which is also suitable for case-based reasoning. The hierarchical representation of knowledge guarantees an optimal strategy for inference.
\end{abstract}

\section{Introduction}

In Backer (1995) and Van der Lubbe \& Backer (1995) an expert system approach for fuzzy data analysis was presented. By means of learning from samples (i.e. data sets) implicit functional relations are transformed into explicit knowledge rules, including the uncertainty associated with them. On the basis of the knowledge rules which are ordered hierarchically inference is made for new samples. As such the system is capable for both inductive learning and deductive reasoning. In addition to this also case-based reasoning can be performed using the exceptional samples which cannot be generalized adequately to general knowledge rules.

In the present paper the system is extended to more general applications. Furthermore, the induction part is enhanced by adapting the method proposed by Ho et al. (1988). Specific attention is paid to the inference mechanism, where a membership function should be assigned to the inferred conclusion(s). Situations whereby more than one knowledge rule will fire are considered. In order to obtain a system which can deal with the three semiotic inference types distinguished by the American philosopher Charles Sanders Peirce (1931-35) the problem of abduction in hierarchical knowledge trees is studied. By means of so-called pseudo-abduction the problem of abduction can be solved in a deductive manner.

\section{2: The reliable training set and inductive learning}

Let us assume that we have a set $\mathrm{W}$ of training samples from the entire instance space supplied by the expert. A member of the training set $W$ is denoted by w. It corresponds to a specific sample. It can be represented by an n-dimensional attribute vector $\left\{a_{1}(w), \ldots, a_{1}(w), \ldots, a_{n}(w)\right\}$, expressing features of that sample. The attributes can be quantitative or qualitative. It is assumed that all qualitative attributes do possess the same domain of possible modalities $\left\{v_{1}, \ldots, v_{p}\right\}$, like very low, low, medium, high etc. For each attribute $a_{1}(w)$, the corresponding modality is denoted by $v_{i}(w)$, whereby $v_{i}(w) \in$ $\left(v_{1}, \ldots, v_{p}\right)$. And thus

$$
w:\left\{a_{1}(w)=v_{1}(w), \ldots, a_{n}(w)=v_{n}(w)\right\} .
$$

To each sample corresponds a decision or judgement with respect to some phenomenon. The domain of modalities of a decision is denoted by $D$ $=\left\{d_{1}, \ldots, d_{j}, \ldots d_{m}\right)$. The learning problem now is to find the set $\{R\}$ of rules that predict the correct decision or domain modality for each training sample if its attribute values given by the expert is taken into account. Rules have the form:

$$
\begin{aligned}
& \operatorname{IF}\left\{a_{1}(w)=v_{1}(w), \ldots, a_{n}(w)=v_{n}(w)\right\} \\
& \text { THEN }\left\{d=d_{j}, f_{j}(w)\right\}
\end{aligned}
$$

This can be read as follows. If a sample has attribute values according to the conditional part of the rule, then $\mathrm{dj}$ is a correct decision with membership function $f_{j}(w)$. Example: IF $\{a 1=$ high, $\mathrm{a} 3=$ low $\}$, THEN $\left\{\mathrm{d}_{2}=\right.$ very low, 0.8$\}$. Remark that not all attributes are per se part of the conditional part of the rule.

Clearly, from the expert point of view, the set $D$ $=\left\{d_{1}, \ldots, d_{j}, \ldots, d_{m}\right\}$ can be associated with a partitioning $P$ of training set $W$ in $m$ classes 
$\left\{\mathrm{P}_{1}, \ldots, \mathrm{P}_{\mathrm{j}}, \ldots, \mathrm{P}_{\mathrm{m}}\right\} ;$ the concept-driven partitioning. The rule generation problem is to find for each class $P_{j}$ a representative set of rules $\left\{R_{j}\right\}=\left\{R_{j 1}, \ldots, R_{j h}, \ldots\right\}$ that predicts $d_{j}$ for all the samples of $P_{j}$ as good as possible. There are two requirements on the representative set of rules: the requirements of covering and discrimination. The first says that for each sample there should be a rule that can recognize this sample. Thus, there should be no samples that are not recognized. The discrimination property implies that a rule which is aimed at a specific decision is supposed not to recognize samples related to other decisions. Key concept is coverage; the coverage of a rule $R_{j h}$ is the number of samples of $P_{j}$ which is recognized by this rule. The coverage is denoted by $P_{j}\left(R_{j h}\right)$. The membership function $\mathrm{f}_{\mathrm{j}}(\mathrm{w})$ of a representative rule $\mathrm{R}_{\mathrm{jh}}$ is considered to be equal to the relative coverage $Q\left(R_{j h}\right)$ of that rule, expressed by

$$
\underline{Q}\left(R_{j h}\right)=P_{j}\left(R_{j h}\right) /\left|P_{j}\right| \text {. }
$$

I.e. the number of samples covered by the rule relative to the total number $\left|\mathrm{P}_{\mathrm{j}}\right|$ of training samples in the partition.

The quality of representative rule $\mathrm{R}_{\mathrm{jh}}$ with respect to decision $\mathrm{d}_{\mathrm{j}}$ is defined as:

$$
\mathrm{Q}\left(\mathrm{R}_{\mathrm{jh}}\right)=\mathrm{P}_{\mathrm{j}}\left(\mathrm{R}_{\mathrm{jh}}\right) / \sum_{\mathrm{j}} \cdot \mathrm{P}_{\mathrm{j}} \cdot\left(\mathrm{R}_{\mathrm{jh}}\right)
$$

which takes into account the number of samples recognized by rule $R_{j h}$, which are not an element of $P_{j}$.

For the generation of representative rules the method of Ho et al. (1988) can be used. One starts with the determination of the representative rule for say sample $w_{j} \in P_{j}$. Therefore, one begins with an 'empty' rule and ties an attribute that covers the maximal number of samples of $\mathrm{P}_{\mathrm{j}}$. This is repeated by adding other attributes until it is discriminative and coverage cannot be improved. By doing so for all samples of $\mathrm{P}_{\mathrm{j}}$, a number of rules will be the result. The first rule that belongs to the representative set is the rule with the largest coverage. The second rule is the rule that has the largest coverage with respect to the samples of $P_{j}$ which were not covered by the first rule. Etcetera. Rules are added one after eachother, until all samples of $\mathrm{P}_{\mathrm{j}}$ are covered.

Experiments were performed by modifying the algorithm of Ho et al. (1988) in order to improve its efficiency. First of all equally general and equally parsimonious rules are all added to the rule set. I.e. rules that cover the same training samples and tie the same number of attributes in their conditional part are thought to be equally acceptable to represent a concept or decision as no reasonable criterion exists to decide which one is the best.

Furthermore, on rules tying more than one attribute in their conditional parts an extra parsimonious check is carried out. This is necessary by the way the attributes are tied to the rules by the original algorithm. In this only the coverage of each attribute is considered, under the expectation that this will give the best chance on achieving a general, discriminative rule in the fastest way. By leaving out the tied attributes one by one from the conditional part of the rule and checking for disrimination, redundant attributes can be detected and excluded from the rule resulting in a more parsimonious (and therefore more general) rule. Sometimes, as in the case of equal rules as outlined above, this will even cause rules to become redundant.

Nevertheless, in general the result will be still poor, since the quality of the samples will differ. In order to rule out these effects, a data-driven partitioning is performed, which is based on the application of some numerical fuzzy clustering technique leading to maximum intra-cluster similarity and minimum inter-cluster similarity. The result of the data-driven partitioning is a hard partitioning $P$ ' into clusters with for each sample the membership value with which it belongs to each cluster. By comparing the concept-driven partitioning and the data-driven partitioning poor samples or exceptions can be found.

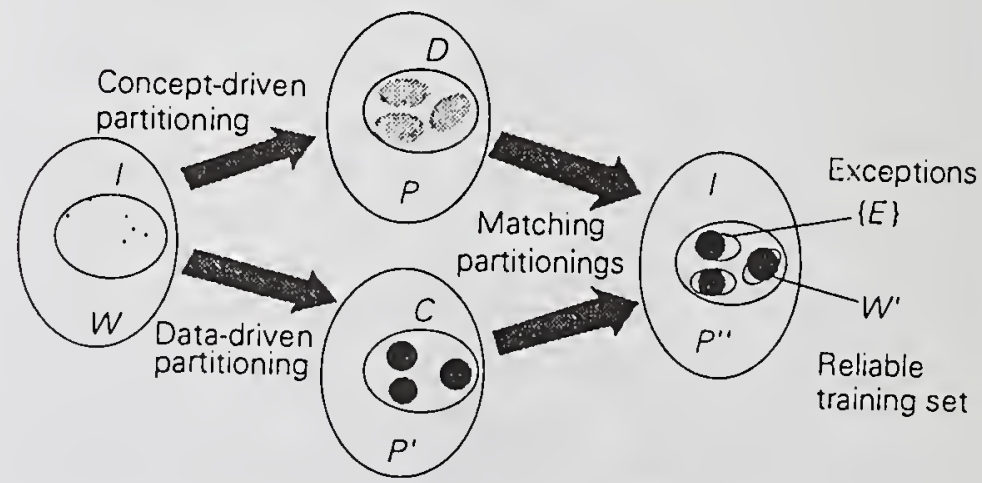

Figure 1. Reliable training set and exceptions. 
This can be simply done by ruling out those training samples that are not included in the intersection of $P$ and $P^{\prime}$ and those samples that have no membership value to any of the clusters above a certain threshold. These samples are considered as exceptions. The corresponding set is denoted by $E$. The remaining samples of $W$ form together the reliable training set $W^{\prime}=W / E$. The corresponding partitioning is $\mathrm{P}^{\prime \prime}$. See figure 1.

The reliable training set $\mathrm{W}^{\prime}$ with its partitioning $\mathrm{P}^{\prime \prime}$ is the proper training set on the basis of which knowledge rules are determined. This is performed according to the modified method of $\mathrm{Ho}$ et al. (1988), mentioned above. Once the rules are found the system can be used for new samples for which the decision should be inferred.

\section{3: Incremental inductive learning}

If the rules are determined and new training samples become available then it depends on the situation whether the rule base should be modified or not. Assume a new training data set/sample w', for which a decision should be found. This sample is characterized by its attribute values, as follows: $w^{\prime}:\left\{a_{1}\left(w^{\prime}\right)=v_{1}\left(w^{\prime}\right), \ldots, a_{n}\left(w^{\prime}\right)=v_{n}\left(w^{\prime}\right)\right\}$.

The corresponding decision according to the expert's judgement is given by

$$
J:\left\{d=d_{k}, f_{k}\left(w^{\prime}\right)\right\} \text {. }
$$

However, the system predicts the concept $d=d_{k}$. instead of $d_{k}$ with its corresponding membership function:

$$
S:\left\{d=d_{k^{\prime}}, f_{k} \cdot\left(w^{\prime}\right)\right\} \text {. }
$$

The following situations are distinguished. Compare Figure 2.

a) If $d_{k} \neq d_{k^{\prime}}$, then sample $w^{\prime}$ should be considered as an exception, since system and expert come to different decisions.

b) If $d_{k}=d_{k^{\prime}}$ and $D(S, J)=\left|f_{k}\left(w^{\prime}\right)-f_{k^{\prime}} \cdot\left(w^{\prime}\right)\right| \leq 0.5$, then the difference between system and expert is small, and thus this new sample is covered by the existing knowledge base and only the membership function of the rule should be modified.

c) Modifications of the rules itselves are only needed if $d_{k}=d_{k^{\prime}}$ and $D(S, J)=\left|f_{k}\left(w^{\prime}\right)-f_{k^{\prime}} \cdot\left(w^{\prime}\right)\right|>$ 0.5 . The sample $w^{\prime}$ is then added to group $P_{k}$ and $a$ new representative set of rules is generated.

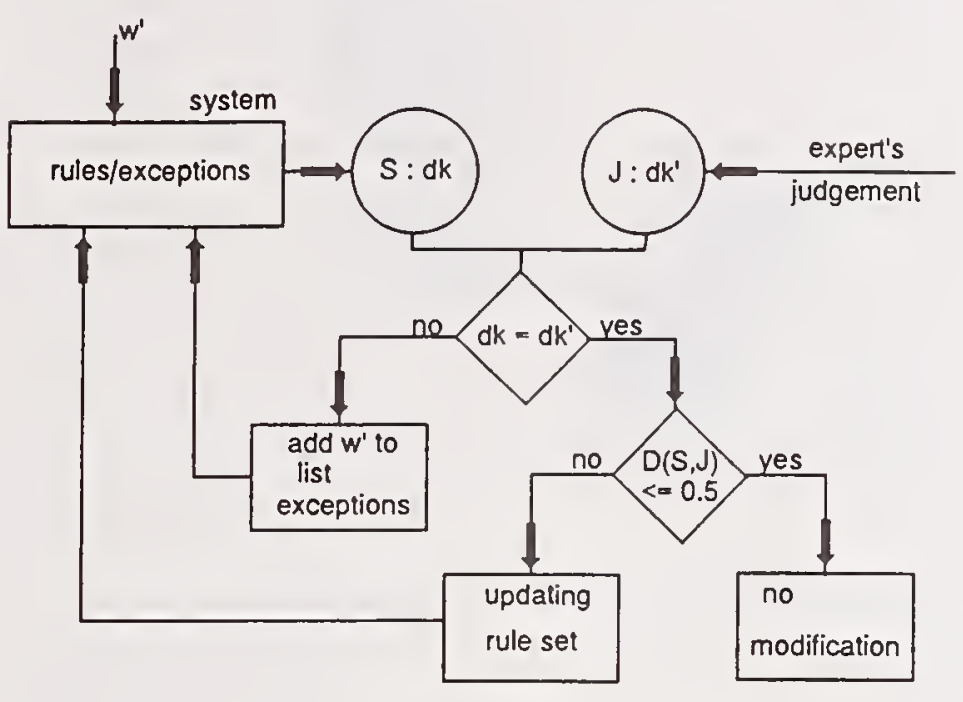

Figure 2. Rule updating and identifying exceptions.

\section{4: Hierarchical knowledge organization and deductive reasoning}

Now hierarchical knowledge organization in the form of rule trees is considered. We assume that we have been able to construct a nesting of partitionings, $\mathrm{P}^{0}, \mathrm{P}^{1}, \mathrm{P}^{2}, \ldots$. The null partition $\mathrm{P}^{0}$ contains all training samples. At level 1 we have a partitioning $\mathrm{P}^{d}=\left\{\ldots, \mathrm{P}_{j}^{1}, \ldots\right\}$, where $\mathrm{P}_{j}^{\mathrm{d}}$ is one of the subsets into which the sample space is divided. The rule set which covers partitioning $\mathrm{P}^{\prime}$ is denoted by $\left\{R^{\prime}\right\}=\left\{\ldots, R_{j}^{1}, \ldots\right\}$, whereby $R_{j}^{1}$ is the set of rules related to subset $P_{j}^{\prime}$ :

$$
\left\{R_{j}^{1}\right\}=\left\{R_{j 1}^{1}, R_{j 2}^{1}, \ldots\right\}
$$

At each level 1 it holds that:

$-\forall \mathrm{i} \neq \mathrm{j}: \mathrm{P}_{\mathrm{i}}^{\mathrm{d}} \cap \mathrm{P}_{\mathrm{j}}^{\mathrm{l}}=\varnothing$.

$-\Sigma_{\mathrm{j}}\left|\mathrm{P}_{\mathrm{j}}^{\mathrm{j}}\right|=$ total number of samples.

- If $\mathrm{P}_{j}^{\mathrm{l}}$ is split up into $\mathrm{P}^{\mathrm{l+1}}{ }_{\mathrm{i}}$ and $\mathrm{P}^{\mathrm{l}+1}{ }_{\mathrm{i}}$, then: $\mathrm{P}^{\mathrm{l+1}}{ }_{\mathrm{i}} \subseteq \mathrm{P}_{\mathrm{j}}^{\mathrm{l}}$. and $\left|\mathrm{P}^{l+1}{ }_{i}\right| \leq\left|\mathrm{P}_{j}^{l}\right|$, and similarly for $\mathrm{P}^{\mathrm{l}+1}{ }^{\prime}$.

It can be shown, considering the rules for the various partitions, that for increasing 1 the rules become more specific. Thus at the top of the tree are the most general (and thus less discriminative) rules, whereas at the bottom of the tree most specific rules are. At the lowest level (1 large) we have the rules corresponding to the individual samples. However, the tree structure has also another interesting property. 


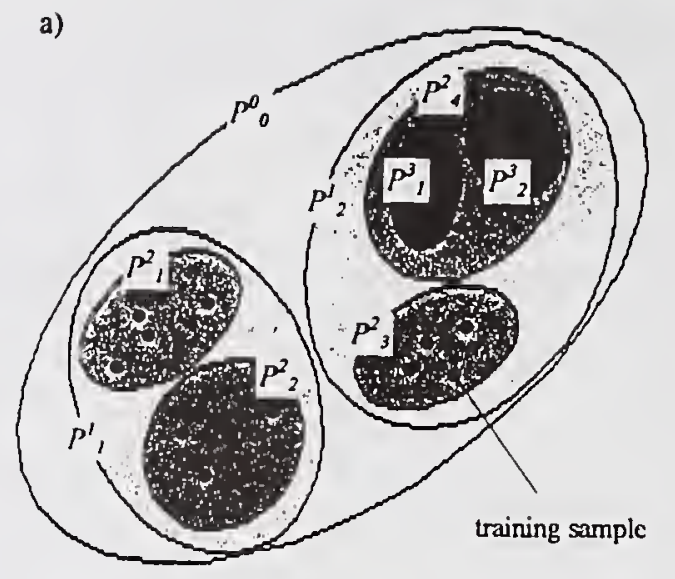

b)

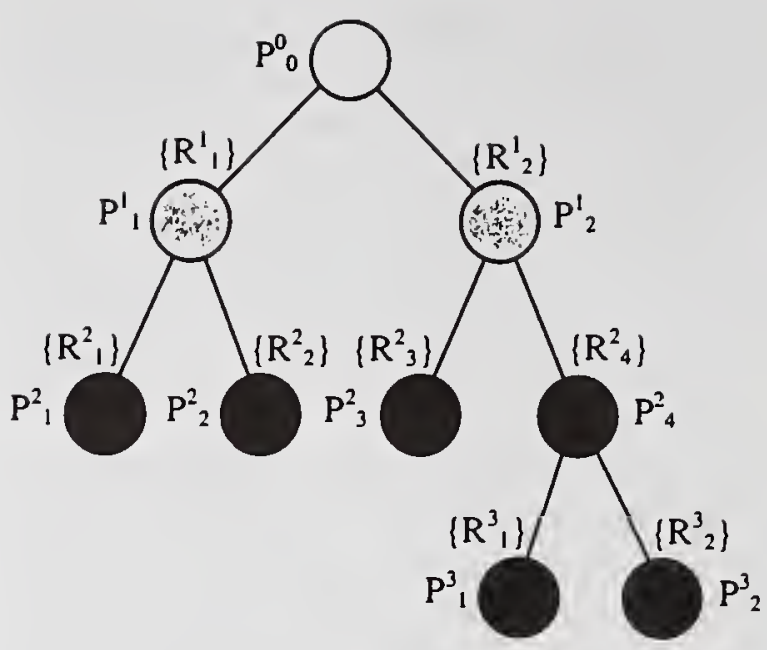

Figure 3. Partitioning and knowledge tree.

If the hierarchical knowledge tree is used for inference e.g. on sample $w^{\prime}$ :

$\left\{a_{1}\left(w^{\prime}\right)=v_{1}\left(w^{\prime}\right), \ldots, a_{n}\left(w^{\prime}\right)=v_{n}\left(w^{\prime}\right)\right\}$ (whereby now the decision is not known a priori), then in general one has the following situation. Consider rule $R_{j h}^{1}$ at level 1 and related to subset $P_{j}^{1}$ :

$$
\begin{aligned}
R_{j h}^{\prime}: & \operatorname{IF}\left\{a_{1}(w)=v_{1}(w), \ldots, a_{n}(w)=v_{n}(w)\right\} \\
& \operatorname{THEN}\left\{d=d_{j}^{\prime}, f_{j}^{\prime}(w)\right\}
\end{aligned}
$$

If this rule is applicable, i.e. the attributes (with their modalities) of sample w' correspond to those of the conditional part of the rule, the inferred decision will be

$$
\left\{d=d_{j}^{1}, f_{j}^{\prime}\left(w^{\prime}\right)\right\},
$$

whereby $f_{j}^{\prime}\left(w^{\prime}\right)$ the relative coverage $\underline{Q}\left(R_{j h}^{\prime}\right)$ $\left(=f_{j}^{1}(w)\right)$ of the rule.

Since every partition in the tree is in general represented by a rule set rather than by one single rule, $w$ ' may be covered by more than one rule in the rule set and therefore determination of the membership of the inferred decision is not trivial anymore. Each applicable rule contributes to the evidence for a new sample $w$ ' to belong to the partition. I.e. the plausibility for a sample to belong to the partition increases if more rules are firing at the same time.

Then simply adding all the relative coverages of the applicable rules together is not correct, as it does not take into account possible overlap of the coverages of the rule. Yet, as we have seen before a rule will only be added to the rule set:

1. If a rule covers at least (worst case) one currently uncovered training sample, or

2. If rules are identical. I.e. they cover the same training samples and tie an equal number of attributes (equally parsimonious).

For that very reason, the effective relative coverage is a more appropriate membership function.

Suppose a ruleset $\left\{R_{j}^{\prime}\right\}$ for partition $P_{j}^{\prime}$ at level $l$, containing $n+m$ rules of which $n$ rules $\left\{R_{j 1}^{1}, \ldots, R_{j n}^{1}\right\}$ are applicable to a new sample $w^{\prime}$ and the other $m$ rules $\left\{R_{j,+1}^{1}, \ldots, R_{j, n+m}^{1}\right\}$ are non-applicable, and which are ordered for decreasing coverage $P_{j}^{l}\left(R_{j h}^{l}\right)$. The effective (relative) coverage $Q\left(R_{j}^{1}\right)$ then can be calculated by the following two-step algorithm:

1. $\quad Q_{1}\left(R_{j}^{l}\right)=P_{j}^{l}\left(R_{j 1}^{\prime}\right)$; for $\mathrm{i}=2$ to $\mathrm{n}$ : if $P_{j}^{1}\left(R_{j i}^{1}\right)<P_{j}^{l}\left(R_{j,-1}^{1}\right)$ or $\operatorname{pars}\left(R_{j i}^{1}\right) \neq$ $\operatorname{pars}\left(R_{j, i-1}^{\prime}\right) \quad$ (i.e. the \# of tied attributes of rule $R_{j i}^{1}$ and $R_{j, j-1}^{1}$ are unequal),

$$
\text { then } Q_{j}\left(R_{j}^{\prime}\right)=Q_{j}\left(R_{j}^{\prime}\right)+1 \text {. }
$$
$\begin{array}{ll}2 . . \quad & \mathrm{Q}_{2}\left(\mathrm{R}_{\mathrm{j}}^{\mathrm{l}}\right)=\left|\mathrm{P}_{\mathrm{j}}^{\mathrm{l}}\right|-\left\{\mathrm{P}_{\mathrm{j}}^{\mathrm{l}}\left(\mathrm{R}_{\mathrm{j}, \mathrm{n}+1}^{1}\right) . .+\right. \\ & \left.. \mathrm{P}_{\mathrm{j}}^{\mathrm{j}}\left(\mathrm{R}_{\mathrm{j}, \mathrm{n}+\mathrm{m}}^{1}\right)\right\} .\end{array}$

The first step determines a worst case $Q_{1}\left(R_{j}^{1}\right)$ according to all applicable rules. The second step takes into account the maximum number of trainingsamples that the non-applicable rules may 
cover. Hence, the latter will result in a second worst case $Q_{2}\left(R_{j}^{1}\right)$.

Now the resulting worst case $Q\left(R_{j}^{1}\right)$ can be found as: $\underline{Q}\left(R_{j}^{1}\right)=\max \left[Q_{1}\left(R_{j}^{1}\right), \underline{Q}_{2}\left(R_{j}^{1}\right)\right] /\left|P_{j}^{\prime}\right|$. As a worst case $Q\left(R_{j}^{1}\right)$ is calculated, the membership can be thought of as a pessimistic lower bound.

It can be shown that for increasing 1 the range of uncertainty with respect to the inferred decision will decrease. This implies that in practice making inferences it is preferable when rules at the lower levels of the knowledge tree can fire, since these ones give the smallest range of uncertainty. However, starting at the lowest level leads to a computational complexity compatible with that of usual case-based reasoning. Starting at the top of the tree is more appropriate. One goes down to lower levels as long as there are rules that fire. If at a level where no rule fires than one stops. Although the top-down approach is better than the bottom-up one, a more adequate approach is searched for.

Considering the hierachical partitioning tree, not all partitionings will be equally obvious. There should be levels of which the partitioning corresponds more to the internal test set structure than other levels. It may be expected that the rules of the rule sets related to these levels have a higher chance of firing than rules of other levels, since these rules set are related to a more natural underlying partitioning. Having found such levels this can be used as starting point for making efficient inferences in the hierarchical knowledge tree.

In fact the problem is to find the most probable partitioning as well as the 'true' number of clusters. Based on the idea of core zones as discussed in Bezdek et al. (1988), Jennes et al. (1994) introduced the core zone index (CZI) for estimating this true number of clusters. CZI is computed for each level in the partitioning tree. That level with the largest CZI is called the core zone index level (see also Van der Lubbe, 1995). It is the starting point for the inference process in the hierarchical knowledge tree. Let the core zone index level be indexed by 1 , then in search for the most specific rule the successive levels $1+1,1+2$ etcetera are considered until the level where no rule can fire. The last but one level is the level which generates the ultimate decision(s). If more than one rule fires at that level, i.e. in the same node, the membership function with respect to the ultimate conclusion is according to the two-step algorithm mentioned above. If the lower levels do not succeed, then the higher levels 1-1, 1-2 etcetera are considered. If only at a high level there are rules can fire with respect to inference on a sample w', the conclusion should be that the knowledge in the tree is not applicable to the inference for that specific sample w', for the core zone index level is the most plausible level. The fact that only rules at higher levels succeed, implies that a decision about a sample w' can only be made if the optimal partitioning is neglected. Then sample $w^{\prime}$ should be considered as an exception.

In practice starting at the core zone index level will lead to a faster solution than starting at the bottom (or top).

\section{Abductive reasoning}

Whereas the methods described above are dealing with induction and deduction, it is of interest to study the possibilities for abductive inference, since in practice abduction is the most important of the three Peircean inference forms. In literature many methods for abduction are given. Compare Van der Lubbe (1996). These methods are not applicable here, taking into account the structure of the system developped until now. Considering abduction as the inverse of deduction, methods for abductive inference should be compatible with the methods followed for deduction. That means that also in the case of abduction a similar hierarchical knowledge structure should be chosen as the basis. Clearly, the hierarchical knowledge tree used in the foregoing sections can not be applied for abduction, since the conditional part of the rule is related to the attributes and the concluding part to the decision or conclusion. In the case of abduction we are just reasoning from decisions to attributes.

Let us assume that we have $\mathrm{k}$ decision domains $\left\{D_{1}, \ldots, D_{k}\right\}$ instead of just one decision domain. In the case of deductive reasoning we now have $k$ knowledge trees. For abductive reasoning there are $\mathrm{n}$ knowledge trees, if there are $\mathrm{n}$ attributes. The $\mathrm{n}$ abductive knowledge trees are generated in the same way as the deductive knowledge trees are. I.e. by means of the modified method of $\mathrm{Ho}$ et al. (1988), taking into account possible exceptions. Compare Figure 4.

The final abductive result is achieved by deductive inference in the $\mathrm{n}$ abductive knowledge 


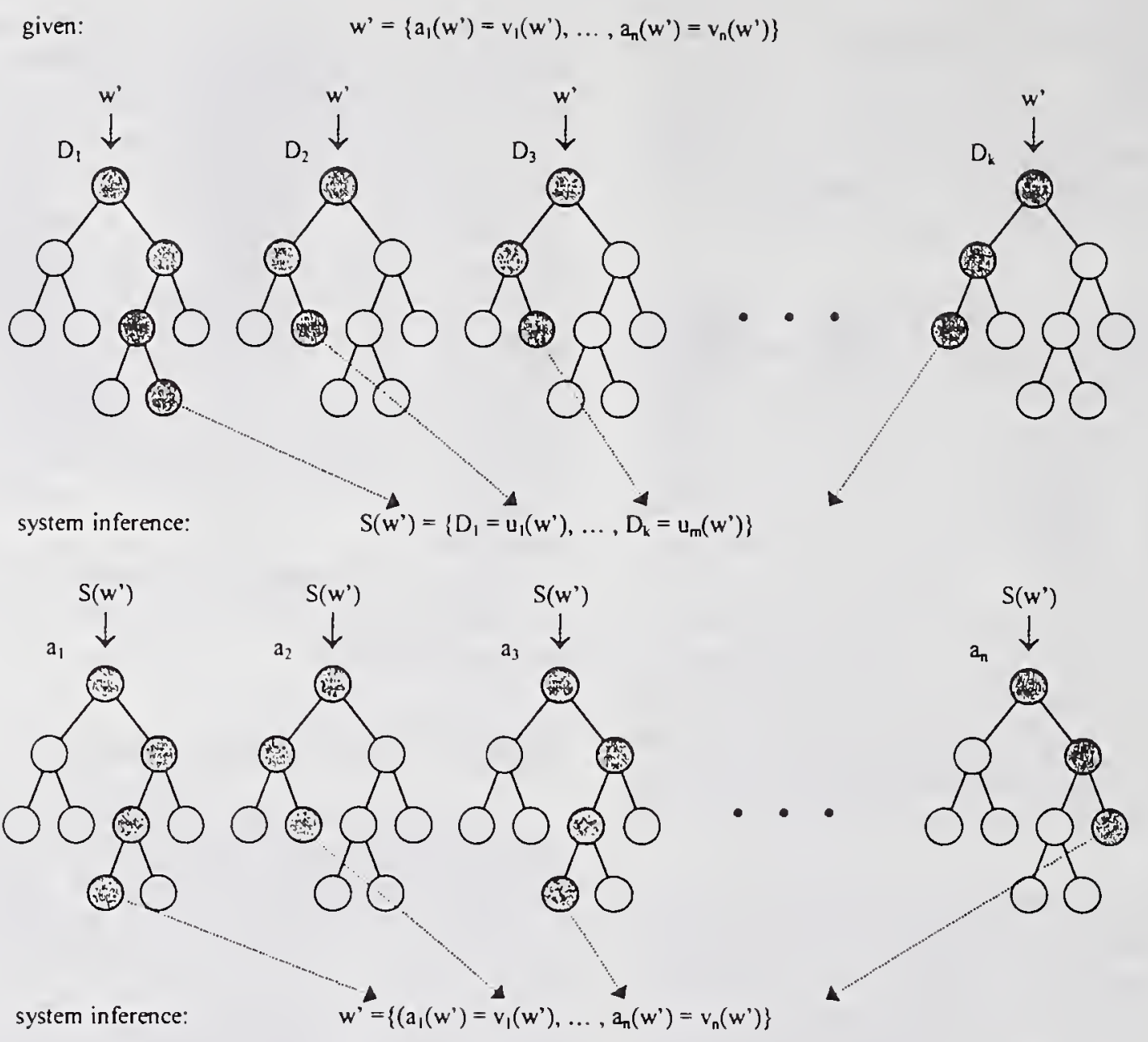

Figure 4. Deductive and abductive knowledge trees.

trees. Therefore, it can be considered as a form of pseudo-abduction. For each of the attributes an indication is given of its possible modalities on the basis of the given decisions or conclusions.

Experiments were performed by first reasoning on the basis of the deductive knowledge trees from attributes to decisions - followed by pseudoabductive reasoning on the basis of the abductive knowledge trees: from decisions to attributes. In all cases the original attributes with their modalities were refound. This underlines the usefullness of pseudo-abduction.

Considering the overall system we may conclude that instead of comparing the unknown sample with all other samples of the training set, as is the case in usual case-based reasoning, the hierarchical knowledge organization enables a more efficient inference; not in the least by determination of the core zone index level. By means of the hierarchical structure there can be inferred to the most probable decision step-by-step. If during the process inference stops half-way by not further firing of rules at the lower levels, more general decisions

will result than at the bottom of the tree. If the knowledge tree is not applicable at all to the sample to be judged, then it is considered as an exception. Then, the only thing one can do is using case-based reasoning and comparing it with earlier exceptions in the hope that then some inference becomes possible.

\section{Conclusion}

It has been shown that on the basis of a training set an adequate hierarchical knowledge tree can be generated by matching concept- and data-driven partitionings of the sample space and by searching for representative rules by means of induction. These hierarchical knowledge trees are appropriate 
for incremental learning and for handling exceptions. Further, it enables inference for new samples, by means of deductive as well as abductive reasoning, in an efficient way. This efficiency is due to the fact that in general not all samples of the training set should be considered. The core zone index and the determination of the core zone index level guarantee the optimal start level. Usual case-based reasoning is only applied to exceptions.

\section{References}

- Backer, E. (1995): Computer-assisted reasoning in cluster analysis, Prentice Hall, New York.

- Bezdek, J.C. and Er-Woon Chiou (1988): A new approach to feature extraction for visual displays, In: Computer Vision, Graphics, and Image Processing, Vol. 41, pp. 186-209.

- Davies, D.L. and D.W. Bouldin (1979): A cluster separation measure, IEEE Trans. Pattern Anal. Mach. Intell., PAMI-1. pp. 224-227.

- Dubes, R.C. (1987): How many clusters are best? - An experiment, In: Pattern Recognition, Vol. 20, no 6, pp. 645-663.

- Egbers, E. (1996): Pseudo-abduction in hierarchical knowledge structures (in Dutch), M.Sc. Thesis, Delft University of Technology.

- Ho, T.B., Diday, E. and M. Gettler-Summa (1988): Generating rules for expert systems from observations, In: Pattern Recognition Letters, Vol. 7, pp. 265-271.

- Jennes, C., Jeedella, J.S.Y. and E.G.P. Bovenkamp (1994): Finding the true number of clusters using a core-zone-based index, Delft University of Technology, unpublished report.

- Peirce, Ch.S. (1931-35): Collected papers of Ch.S. Peirce, C. Hartshorne and P. Weiss (eds.), Cambridge (Mass.)

- Van der Lubbe, J.C.A. and E. Backer (1995): Hierarchical classification inference for fuzzy data analysis, In: Proc. ISUMA-NAFIPS '95, College Park (Maryland), September 17-20, 1995, pp. 402407.

- Van der Lubbe, J.C.A. (1996): A semiotical approach to abduction in artificial intelligence, Delft University of Technology, Technical Report TUD-EE-IT 96101.

- Van der Lubbe, J.C.A. (1996): A Peircean approach to artficial intelligence, In: Proc. Intelligent Systems: A Semiotic Perspective, Vol. II, Gaithersburg (Maryland), October 20-23, 1996, pp. 7-13. 


\title{
Conceptual Categories as Knowledge Structures
}

\author{
Christopher Landauer \\ National Systems Group, The Aerospace Corporation \\ The Hallmark Building, Suite 187, 13873 Park Center Rd., Herndon, Virginia 20171 \\ Phone: (703) 318-1666, FAX: (703) 318-5409 \\ E-mail: cal@aero.org
}

\begin{abstract}
This paper introduces an approach to knowledge representation as an active process. More than merely recording knowledge in some extractable form, we recognize that representation must include the processes that perform the activities of insertion as well as extraction. Our intention is to develop a representational method that is powerful enough and flexible enough that systems built using it can represent, reason about, and change their own structure and behavior.
\end{abstract}

The approach is based on recent work on knowledge representation for our "wrapping" integration infrastructures, and on some comparative semiotics: studies of mathematical and linguistic reasoning.

The basic notion is a generalization of set theory in four directions: our collective objects have (1) indefinite boundaries and (2) indefinite elements; (3) the context is allowed to leak into the interpretation of the objects; and (4) there is a notion of multiplicity of structures that correspond respectively to considering the same object or class from different points of view, in different contexts. This notion allows us to model the modeling decisions explicitly, and to keep track of the modeling simplifications so we can relate them to each other and to the processes that create, change and use them.

\section{Introduction}

The main goal of this work is to invent a more interesting and flexible mechanism for representing the knowledge and information needed by, in, and for constructed complex systems in general, and computing systems based on wrappings in particular. We have written elsewhere [5] about the barrier imposed by any well-founded system, and our problem is to try to build something that does not have this same barrier (i.e., indefinitely refinable knowledge structures) from something that is inherently grounded in computer hardware (i.e., computer software). The genesis of this work was thinking about the differences between mathematical and linguistic reasoning [7].

In this section, we describe some of the background for our attitude, and introduce some of the concepts and terminology we use.

\subsection{Background}

In our research, we have considered two different mapping activities: modeling some phenomenon in the real world (using simulation and modeling techniques we have developed [8]), and implementing some abstract idea (using software engineering and software system development techniques that we have developed [5]). In both cases, there is a representation issue, and there are simplifications needed to make the representation. One of our goals is to allow explicit treatment of the modeling process and the representation issues.

We also want to get away from the use of sets as the only model for categories of knowledge, since they artificially limit the kinds of categories that can be considered [4]. Sets have both definite boundaries and specific elementary units. There are many models of "uncertainty" that generalize the first constraint: probability distributions, fuzzy sets, belief functions, rough sets, and many others. As near as we can tell, there is no appropriate model that generalizes the second constraint. This lack is mainly due to the nature of mathematics as our most concrete form of reasoning: the elementary units must be defined before we can start mathematical reasoning. This constraint is not present in linguistic reasoning [7].

In addition, we want to generalize sets in two other directions: we want to allow context to leak into the de- 
scriptions [1] [10], and we want to allow individuals to have different structures in different viewpoints. In many cases, the indefinite boundaries of a category are made more precise (though still somewhat indefinite) by specifying the context under which the category is being considered, thereby allowing the context to become part of the specification of the category. For this reason, we make context a part of viewpoint. We also note that the different viewpoints can give the category different structure, one of which can even be to take the category as an individual.

\subsection{A First Look}

In this subsection, we introduce the concepts that define our approach. Do not expect formal or precise definitions here; they begin in the next section. Terms in italics in this section are technical terms in our approach that are defined in the next sections.

The most important source idea is the "thing" ness of all concepts: anything we can describe with language can be "reified", that is, viewed as a "thing", and referred to as a unit or viewed in a number of different, not wholly compatible, ways. That is, each viewpoint leads to a possibly different structure for the individual (and certainly to a different interpretation of the significance of the structure). These "thing"s can be grouped naturally into categories. We think of categories as including our notion of concepts or classes. We call these "thing"s individuals, and think of the categories as collections of them. It is this property that leads us to consider all concepts as individuals, at least under certain viewpoints, and to recognize that different viewpoints lead to different structures for the individuals. We can view a category as an individual under certain viewpoints, and we can view an individual as an assemblage under certain viewpoints. We can also view an individual under a certain viewpoint to be a member of a category under some viewpoint.

There are two ways to study grouping of individuals into categories. We can begin with some individuals and try to identify appropriate categories, or we can begin with categories and try to examine individuals in them. Both approaches are used here.

Categories also describe relationships among other categories. We want to generalize the notion that categories contain other categories, which are separated by their distinctions into different roles. A relationship has roles that may be filled by other categories, so we can talk about roles without using the categories that might fill them. In this sense, they are similar to frames with variable slots for other objects.

A category can be divided partially by distinctions into several other categories, and the distinctions become new roles for the division (partially means not necessarily either disjoint or exhaustive, i.e., not only into subsets). The "other" categories are called constituents. Distinctions are important only relative to a certain viewpoint, so division of a category is defined only in terms of a viewpoint. Different viewpoints may divide the category in different ways. The divisions include our notions of knowledge structures (including abstract datatypes such as "set of X" and other structures).

Constituent categories for different divisions of a category generally have different viewpoints from each other. When we write that a category "contains" another, it means that there is a viewpoint according to which the second category is one of the constituents of the first one.

If we extend this decomposition further to many levels, we get a kind of hierarchy of categories, related by various divisions according to various viewpoints. This hierarchy becomes a kind of "ontology" for the application domains involved, and it allows us to make much more flexible use of the usual sorts of ontologies defined in knowledge representation languages.

\subsection{Systems and Environments}

A knowledge representation scheme makes no sense outside a context of use. The context we consider for this paper is autonomous systems [2] [3] [9]. An autonomous system must somehow classify its surroundings in ways that allow appropriate responses, or have some way to recognize features of its surroundings that instigate appropriate responses. An autonomous system also needs to learn from its experience in the world, adjusting its internal "knowledge" structures to improve its knowledge of the world, and therefore improve the effectiveness of its behavior.

This kind of system has internal structures that include interpreter definitions in addition to the usual data and explicit knowledge base structures, because the architecture of our systems (with their active integration activities coordinated by wrapping processes) means that the interpreters are also defined by explicit data structures (e.g., 
wrex programs). The system also has four processes for knowledge construction and use: "recording" experiences (as traces) in processible structures; assessing it according to different criteria; extracting knowledge (as models) in processible structures; and using it for different purposes.

\section{Organization Rules: Structures}

We can organize knowledge either "top-down", from collectives to instances, or "bottom-up", from instances to collectives. Our top-down approach to modeling knowledge depends on one notion of organizational entity and one notion of organizational relation. The "categories" refer to classes of real world phenomena, and the "divisions" represent them. Our bottom-up approach depends on these notions as if we were collecting "individuals" into classes.

\subsection{Category}

Categories are descriptions of all phenomena. The fundamental organizational entity is the category, which represents anything that can be labeled by a symbol. In fact, we want our notion of category to be co-extensive with the notion of "concept" and the notion of something that we can describe and reason about. That makes it the same notion as the one we have considered before [7] for "symbols": we can make up and use names for categories long before we know everything (or anything!) that is in the categories (e.g., black holes, phlogiston, public-key cryptosystems). This is the connection between our notion and natural language processes: we are attempting to model the effectiveness of language in referring to concepts, even though we expect there to be many more categories than words or phrases [6].

\subsection{Division}

The fundamental organizational relation is division

$$
\begin{aligned}
& \text { of a category } \\
& \text { into roles for other categories } \\
& \text { according to a viewpoint. }
\end{aligned}
$$

This division is our replacement notion for the usual notion of set or structure. Our notion of division corresponds to structural decomposition of categories, but it is much more general; it allows all structuring methods to coexist. We describe what a viewpoint is a little later on.

Divisions make some things about a category more definite. We make divisions because the more information we have about a category, the more precisely we can use it. We make multiple divisions because we think of categories as being different in different contexts, or when we consider them from different viewpoints.

One kind of division is to specify that the category plays a particular role in some other division. This features allows us to build up as well as down with the categories.

Another kind of division shows the structure of the category when considered from one viewpoint, with decomposition corresponding to subcategories of the category, and constraints corresponding to distinguishing properties of the subcategories.

We can also describe the structure of the individuals in a category, at least according to a particular viewpoint, by first defining a division that corresponds to considering the category as a collection of individuals and thereby defining a category of those individuals. Then we can either use a division of that category for the individual structure, using architectures and roles for other categories, with "boxes and lines" diagrams or hierarchies for structure, or we can imagine an abstract space containing (representations of) the individuals as elements of the space, or as a subregion in which they must lie, or as a mapping from some other structure into the space.

We use a viewpoint to emphasize certain distinctions among entities in a category. Distinctions can be differences in content, structure, available information, or intended use. They are often explicit, but sometimes unknown at first.

Distinctions define roles in the division of a category. For example, a partition of a set is a division that has roles for the various subsets, which may or may not be explicitly distinguished. The different components of a structured entity are roles in the division that defines that decomposition. The different steps of a structured process are roles in the division of that process into parts. Some other common ways to write about divisions are that a category "contains" entities, or that a category "describes a relationship" among entities. 


\subsection{Individuals}

Individuals are particular objects or agents or actions or events or relationships, usually associated with one or more categories. The individuals are the "things" we referred to in our introduction. An individual is intended to represent a real-world entity, the same way a category does. In many cases, what we would normally consider to be an individual is not uniquely specified, so we must make do with a class of possibilities. In this case, we are using the individual as a "typical" member of a category (which derives from a particular viewpoint on the individual).

We talk about individuals as members of a category, even though membership requires a viewpoint, and therefore is really only for divisions. That means that an individual must be a member of a division, not a category. Every individual is therefore an instance of one or more divisions, and we consider the corresponding categories as "types" of the individual (according to the viewpoint that leads to the division).

Sometimes we are given an individual (including the current situation), and we try to form or understand the categories it may lie in. We may have to infer one or more viewpoints, and consider different structures for the individual.

As we get deeper in the category hierarchy, the categories become more and more concrete, and we eventually get to sets and data structures, which in our scheme are divisions of categories.

Divisions also apply to individuals, but we usually try to put the individual into a category first, so that the divisions apply to the category instead.

\subsection{Viewpoint}

We use viewpoints to distinguish different structures for our categories, either for the individuals in the category or for the category itself. The viewpoint defines some of the assumptions and modeling decisions that are reflected in the presented division.

A viewpoint is a focus within a context. A focus is a presumed scope and level of detail, which defines the scope of quantifiers, and the level of detail required to express the viewpoint. A different focus means a different viewpoint, which means a different division. Different viewpoints of a category often differ only in their focus. The scope de- fines the extent of the conceptual units, both structures and processes, and the level of detail defines their conceptual "size". It is part of our ongoing research to develop notations that express or define scope and level of detail. There is something "compatible" about the "size" of the conceptual units in a focus that makes it seem "natural".

It is important in this approach that the same category may have many different divisions, each with a different viewpoint, and that we can therefore easily imagine adding new divisions as other viewpoints become interesting.

A viewpoint implies a choice of descriptive language for the corresponding division, and a corresponding language interpreter. The viewpoint is outside the division, but refers to it.

One of the difficult outstanding research problems is to determine how and when viewpoints are related to each other.

\subsection{Context}

We have long maintained that context provides all the interpreters for symbols, and that semantics is_ the interpretation of syntax. For this paper, it means that context provides all the interpreters for categories, and that semantics is the interpretation of divisions in categories.

A context does not _do_ anything. It is a passive provider of and reference to information and processes.

An interpreter is a mapping from elements to actions or objects, and from constructs to patterns of action or structures. So just as focus is a collection of categories that are used as units, context is a collection of categories that are used as relationships (mappings) from some categories to others.

There is a notion of specificity in context. There is a relation of "more concrete" among divisions, and interpretation is intended to map towards more concrete categories. The simplest implementation of context is a collection of mappings from attributes to values, but others are possible.

A context is a state of knowledge, which is a collection of assumptions, specializations, relationships, and current situation.

A context contains or refers to the domain-specific prior information structures and interpreters. The environment is the situation-specific current dynamic activity to which 
the system must respond.

It is never the case that we know all of the context of a division, so it is essential that we be able to augment the context at any time. When we write of a category "in" a context, we actually mean the division of the category according to the viewpoint given by the context and the focus of the category.

\section{Process Rules: Behaviors}

Our knowledge representation defines more than just what the concepts are and how they are related. It also defines how things change and what can happen.

\subsection{Activity and Action}

Everything that happens is an activity. Every activity has an effect, which we may or may not be able to detect. All activities take time, which may or may not be representable as instantaneous, according to the time scale in use. An activity description includes all intermediate steps or stages (we do not presume discrete steps). An activity is a kind of category. An action is a kind of activity. An activity can be made up of actions.

An action description is an indivisible activity. It is a kind of division that has the form of a collection of constraints among prior and posterior variables (i.e., roles for individuals), and their times of occurrence. There is no intermediate "step" in an action; the values of the affected variables can only be queried before the action or after the action. During the action, the values are presumed to be the same as at the starts of the action, but they are also considered to be "in transit", so they cannot be relied on to hold fixed for any positive amount of time. An activity description allows querying of actions at all intermediate times. For example, a discrete sequence of steps implements an activity as a set of actions, whereas a continuous motion is an activity for which we know all the intermediate places as a function of time.

A division is a model in our usual sense, with component roles for other models and interactions styles which distinguish different classes of divisions. Actions on a division are converted into actions on the roles, the interactions or both. This conversion is part of the definition of a division. Different kinds of divisions correspond to different collections of conversion processes.
Every action is a change of something. Action classes have pre- and post-conditions, and descriptions of effects.

Every action has an effect, possibly an actor (which can be an agency instead of an individual), and an actee, which is usually one or more roles for categories (or individuals).

The difference between an event and an action is that events are instantaneous; we don't use them. Actions can take some amount of time.

\subsection{Situations}

Situations are particular parts of the environment (as if they were a component or aspect of the environment, except that we don't necessarily know anything else about the environment). A situation describes part of the environment at a particular time, and in that sense is like a context individual; the difference between the situations and other parts of context is that situations are instances of the environment in time.

There is some interesting new mathematics associated with situations [1], and much work on situated planning [10] and situated behavior [9], and we believe that our approach allows us to describe the situations, so that they may be made explicit in the computations.

Computationally, the application of an action to a situation has three parts: the situation provides individuals, the action roles match up to those individuals, and the action effects change those individuals.

For these purposes, a situation can be thought of as a time and a collection of individuals. It is not a set. It is a partly determined category. Such a category is determined by coincidence in time and space rather than coincidence in defining properties.

\subsection{Current Behaviors}

We distinguish the rules of behavior, which are about classes of situations and the appropriate corresponding activities, from the actual behavior, which is about the current situation and the current activities as time progresses. Classes of possible behaviors can be defined by stringing together the rules according to classes of possible situations, but they must be specialized to represent the actual (or presumed) situation.

What actually happens in a system (or, more properly, within a system _and_ between it and its environment) is 
a temporal pattern of instances of activities. We call them "activities" instead of "events" because the latter term is used more often for instantaneous events, which we allow only as a conceptual shorthand.

While the system is operating, the relevant part of the "current" situation at any given time, or even the currently available knowledge about the current situation at that time, is almost never enough to identify it uniquely. There is therefore always a class of possible situations. The class is organized not so much by its internal structure as it is by its component's coexistence in time.

\section{Conclusions}

The notions we have discussed here provide a different approach to representing knowledge in a complex system. We believe that they allow incorporation of most other methods of representation, and that something along these lines is necessary to allow the representation to take the current context and environment into account.

We have provided a number of ideas for what a new representational approach would have to provide. Specifically, we have stressed how "representing" must be changed from static notions of the organization and definitions (data dictionary style) to one that must include the active processes for interpreting the knowledge constructs. In this processing plus the metaknowledge required for our software architecture approach [5] [9], we will begin to have the necessary capabilities to handle and explicit contextual information which we consider essential.

The current research project is constructing a software system that implements this approach, and in which we can perform various studies of effective knowledge collection, reorganization, and extraction.

\section{References}

[1] Jon Barwise, The Situation in Logic, CSLI Lecture Notes No. 17, Center for the Study of Language and Information, Stanford U. (1989)

[2] Kirstie L. Bellman, "When Intelligence is in Control", pp. 10-12 in Intelligent Systems: A Semiotic Perspective, Proceedings of the 1996 International Multidisciplinary Conference, Volume I: Theoretical Semiotics, Workshop on Biologically Inspired Complex Systems, 20-23 October 1996, Gaithersburg, Maryland (1996)
[3] Kirstie L. Bellman, Christopher Landauer, "A Note on Improving the Capabilities of Software Agents", (poster summary) pp. 512-513 in Proceedings A A'9\%: The First International Conference on Autonomous Agents, 5-8 February 1997, Marina Del Rey (1997)

[4] George Lakoff, Women, Fire, and Dangerous Things, U. Chicago Press (1987)

[5] Christopher Landauer, Kirstie L. Bellman, "Knowledge-Based Integration Infrastructure for Complex Systems", International Journal of Intelligent Control and Systems, Volume 1, No. 1, pp. 133-153 (1996)

[6] Christopher Landauer, Kirstie L. Bellman, "Semiotics of Constructed Complex Systems", pp. 35-40 in Intelligent Systems: A Semiotic Perspective, Proceedings of the 1996 International Multidisciplinary Conference, Volume I: Theoretical Semiotics, Workshop on Intelligence in Constructed Complex Systems, 20-23 October 1996, Gaithersburg, Maryland (1996)

[7] Christopher Landauer, Kirstie L. Bellman, "Mathematics and Linguistics", pp. 153-158 in Intelligent Systems: A Semiotic Perspective, Proceedings of the 1996 International Multidisciplinary Conference, Volume I: Theoretical Semiotics, Workshop on New Mathematical Foundations for Computer Science, 20-23 October 1996, Gaithersburg, Maryland (1996)

[8] Christopher Landauer, Kirstie L. Bellman, "ModelBased Simulation Design with Wrappings", pp. 169174 in Proceedings of OOS'97: Object Oriented Simulation Conference, WMC'97: 1997 SCS Western Multi-Conference, 12-15 January, Phoenix, SCS International (1997)

[9] Christopher Landauer, Kirstie L. Bellman, "Computational Embodiment: Constructing Autonomous Software Systems", pp. 42-54 in Judith A. Lombardi (ed.), Continuing the Conversation: Dialogues in Cybernetics, Volume I, Proceedings of 1997 ASC Conference, 8-12 March 1997, U. Illinois (1997)

[10] Lucy A. Suchman, Plans and Situated Actions: The problem of human-machine communication, Cambridge U. Press (1987) 


\title{
KNOWLEDGE REPRESENTATION LANGUAGE IN SEMIOTIC SYSTEMS
}

\author{
Vagin V.N., E. Yu. Golovina \\ Moscow Power Engineering Institute, Applied Mathematics Department. \\ Krasnokazarmennaja, 14, 111250 , Moscow, Russia. \\ Electronic mail: vagin@apmsun.mpei.ac.ru \\ gol@apmsun.mpei.ac.ru
}

\begin{abstract}
A semiotic system is a multi-layer one, consisting of multilayer networks from signs-frames. One of the main components of any semiotic system is a knowledge-based repository (semiotic knowledge base). The difference of a semiotic knowledge base from an ordinary one is in a structure of basic units for knowledge representation (sign structure) and in operation set over these basic units.

Multi-layer logic (MLL), developed by $\mathrm{S}$. Ohsuga and $\mathrm{H}$. Yamauchi, is proposed as knowledge representation language in semiotic knowledge bases. MLL is appropriate apparatus for discription of knowledge structuring and aggregation.

We have been developed the extension of MLL syntax, which allows to increase efficiency of the deductive inference. The Scolemization algorithm and the unification algorithm for extented MLL syntax is developed. The system KM (Knowledge Model), based on deductive inference in MLL, as a modelling tool of complex-structured problem domains in semiotic knowledge bases is developed. The conceptual language for modelling a complex-structured problem domain is researched. The translator of queries with conceptual language into MLL is designed.
\end{abstract}

KEYWORDS: semiotic system, knowledge base, knowledgc representation language, problem domain, multi-layer logic.

\section{INTRODUCTION}

The widespread using of computer technology in different spheres of human activity has made necessary to develop semiotic systems.

A semiotic system is a multi-layer one, consisting of multi-layer networks from signs-frames. The sign used in semiotic systems has three entities: a name, a concept and representation which are interpreted as syntax, semantics and pragmatics. A sign structure is matched with a frame structure that also has similar entities: name, protoframe and frame example. One of the main components of any semiotic system is a knowledge-based repository (semiotic knowledge base). The difference of a semiotic knowledge base from an ordinary one is in a structure of basic units for knowledge representation (sign structure) and in operation set over these basic units.

The central problem of semiotic system development is problem domain modelling. To model the complex- structured problem domains, it is necessary to choice the knowledge representation language, which is available for the description of such domains.

\section{A FORMAL MODEL OF A COMPLEX- STRUCTURED PROBLEM DOMAIN IN SEMIOTIC SYSTEMS}

As a formal model of a complex-structured problem domain we have been proposed a hybrid model that combines the "program engineering" paradigm $[1,2,3,4]$ and the "knowledge engineering" one $[5,6]$, i.e. an object-oriented approach $[7,8]$ and procedures, and a model for representing knowledge about a problem domain, should be used in order to represent a formal description of a problem domain in semiotic systems $[9,10,11,12]$.

We have been proposed that a logical model based on multi-layer logic (briefly MLL) $[13,14]$, should be used as a formalism for representing knowledge about a complexstrucrured problem domain. MLL is an integration of the logical approach and of an approach based on semantic networks. It may be considered as an object-oriented firstorder predicate calculus that describes knowledge structuring and aggregation.

By a slash we will mean a kind of delimiter used in prefix of a formula. Thus, the simple slash $(\mathrm{Q} \times \mathrm{X})$ is used to denote that $x$ is an element of the set $X(x \in X)$, yhe simple "thick" slash $(Q x / X)$ denotes that $x$ is defined on a set whose elements are the components of the object $X$ $(X \nabla X)$, while the double slash $(Q x / / X)$ denotes that $x$ is defined on a set whose elements are part of the object $X$ $(\mathrm{X} \cdot \mathrm{x})$.

We are developed the extension of MLL syntax, which allows to increase efficiency of the deductive inference. The extented MLL syntax is represented.

Alphabet :

(1) constants: à,b,c, .., X,Y,Z (constant sets), ..

(2) variables: $x, y, z, \ldots$

(3) function symbols: $f, g, h, \ldots$

(4) predicate symbols : $P, Q, R, \ldots$

(5) quantifier: $\forall, \exists$

(6) logic connectives: $\neg, \&, V, \rightarrow$

(7) auxilliary symbols: $\#,{ }^{*}, I, l, I /,\{\},,($,) 


\section{Terms:}

(1). Any constant and variable are terms.

(2). If $\mathrm{f}$ is a $\mathrm{n}$-ary function symbol and $t_{1}, t_{2}, \ldots, t_{n}$ are terms, then $f\left(t_{1}, t_{2}, \ldots, t_{n}\right)$ is a term.

(3). All terms are obtained by applying the rules (1) and (2).

The rules of designing well-formed formules (WFF) for :extented MLL syntax is represented.

$\mathrm{F} 1$. If $\mathrm{P}$ is a $\mathrm{n}$-ary predicate symbol and $t_{1}, t_{2}, \ldots, t_{n}$ are terms, then $P\left(t_{1}, t_{2}, \ldots, t_{n}\right)$ is WFF.

$F 2$. If $F$ and $G$ are WFF, then $\neg F, F \& G, F \vee G, F \rightarrow G$ are WFF.

$\mathrm{F}$. If $\mathrm{F}$ is a WFF and $\mathrm{x}$ is an object variable, then

(1). $(\forall x / y) F$ and $(\exists x / y) F$ are WFF, where $y$ is a constant or variable (here $/$ is an ordinary slash).

(2). $(\forall x / y) F$ and $(\exists x / y) F$ are WFF, where $y$ is a constant or variable (here / is a fat slash).

(3). $(\forall x / / y) F$ and $(\exists x / / y) F$ are WFF, where $y$ is a constant or variable(here // is a double slash).

(4). $(\forall(x / Z) / / y)$ Fand $(\exists(x / Z) / / y) F$ are WFF, where $y$ is a constant or variable, $Z$ is a constant set.

F4. There are no other rules of designing WFF.

An inference algorithm for extented MLL syntax has been developed.

At the present time the system KM (Knowledge Model) based on deductive inference in MLL is designed.

\section{THE MODELLING SYSTEM KM OF A COMPLEX-STRUCTURED DOMAIN}

The system KM (Knowledge Model) as a modelling tool of a complex-structured problem domains is developed. The system KM allows :

- to construct the ISA-hierarchy of object classes;

- to define the object classes attributes and the relations between object classes;

- to construct the Part-of -hierarchy of object classes;

- to define the class representatives;

- to construct the representatives Part-of -hierarchy;

- to browse the class objects and the hierarchies;

- to describe the logic formulas;

- to receive answer to queries.

The architecture of the KM system is represented in Fig.ure 1.

The main components of the system $\mathrm{KM}$ are:

- subsystem of the problem domain model controlling;

- subsystem of modelling;

- subsystem of browser;

- knowledge base;

- subsystem of the deductive inference.

The subsystem of deductive inference, based on inference in MLL, is served for:

- logical verification of information represented in a knowledge base;
- obtaining attribute values and extensions of relations what allows to "compress" the extensional component of the knowledge base;

- receiving the new knowledge from knowledge, represented in the knowledge base;

- obtaining answers to queries.

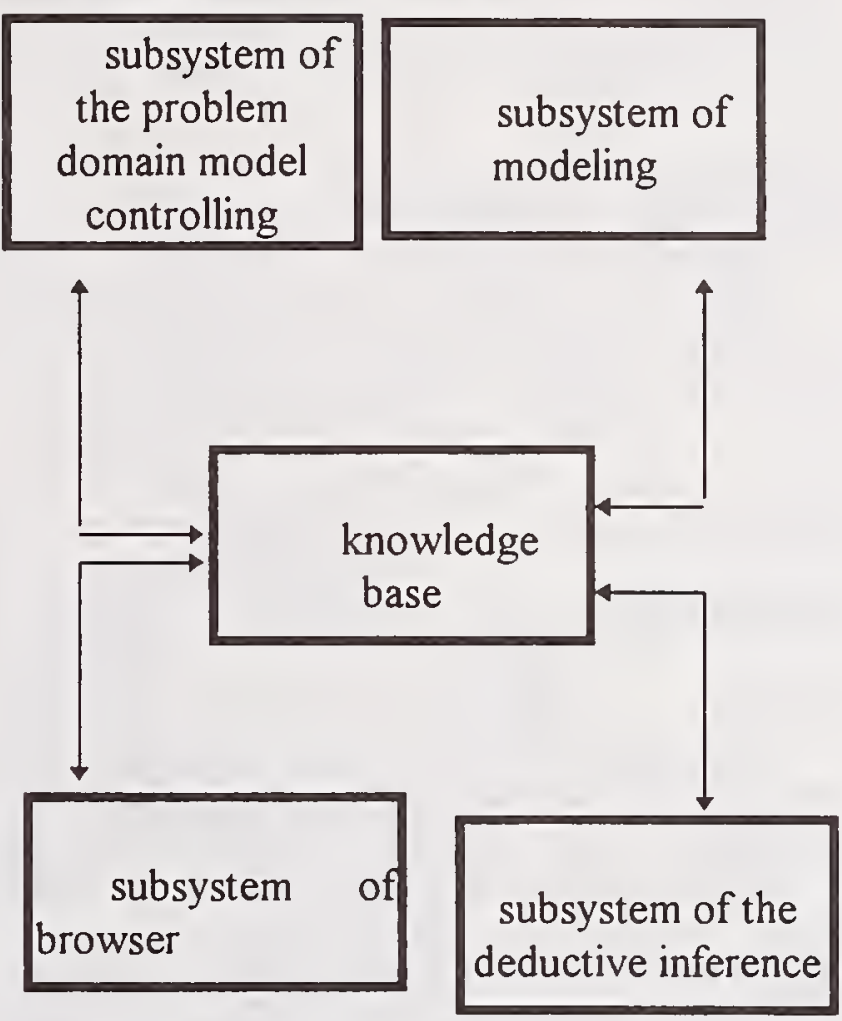

Figure 1. The architecture of the KM system Example 1.

A program component $\mathrm{x}$, including in the control support system (CSS) \#P, provides landing an airplane $y$, assigned to the airport \#A, if there are:

- $\mathrm{PC} t$, in which $\mathrm{x}$ operates;

- a radiolocation station (RLS) s, connected with PC $\mathrm{t}$;

- information flow \#Il, containing message flow $\mathrm{tl}$, accepted by RLS $s$ and containing message class $\mathrm{rl}$, describing an airplane $\mathrm{y}$ and processing by a program component $\mathrm{x}$;

- information flow \#I2, produced by a program component $\mathrm{x}$ and accepted by an airplane $\mathrm{y}$, containing message flow $t 2$, transfering RLS $s$, in which there is a message class $r 2$, containing information about airplane landing $y$.

The structures of the problem domain is represented in Figure 2.

Formalization in MLL with extented syntax:

( $\exists(x /$ program_component $) / / \# \mathrm{P})(\forall(y /$ airplane $) / / \# \mathrm{O})$

$(\exists(\mathrm{s} / \mathrm{RLS}) / / \# \bar{A})(\exists(\mathrm{t} / \mathrm{PC}) / / \# \mathrm{~A})(\exists(\mathrm{t}$ l/message_flow $) / / \# \mathrm{Il})$

$(\exists(\mathrm{rl} / \mathrm{message}$ class $) / / \mathrm{t} \mathrm{l})(\exists(\mathrm{t} 2 /$ message_flow $) / / \# \mathrm{I} 2)$

( $\exists(\mathrm{r} 2 / \mathrm{message}$ class $) / / \mathrm{t} 2)$

Operate $(x, t)$ \& Connect( $(s, t)$ \& Accept_RLS(s,tl) \& Describe $(r \mathrm{r}, \mathrm{y})$ \&Process $(\mathrm{x}, \mathrm{rl})$ \& Transfer_RLS(s,t2) $\&$ Produce $(x, r 2) \quad \& \quad$ Accept_airplane $(y, r 2) \rightarrow$ Landing $(\mathrm{x}, \mathrm{y})$ 


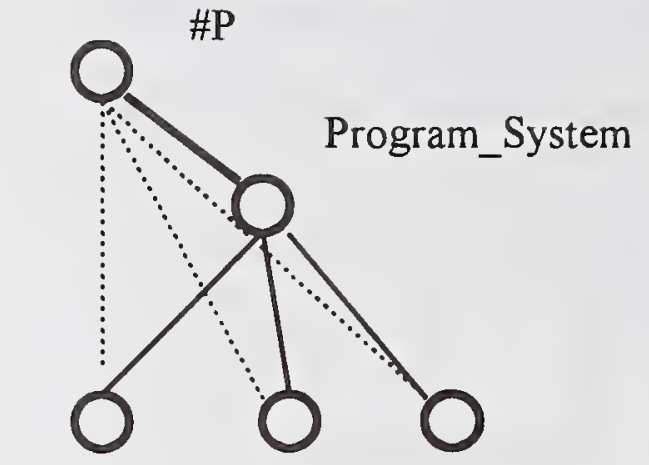

P1 P2

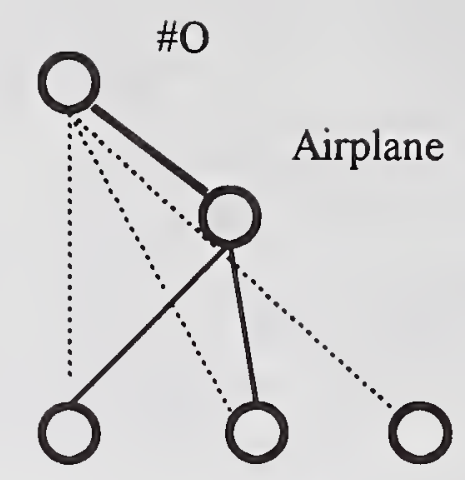

AN24 TU154 $\amalg$ 76

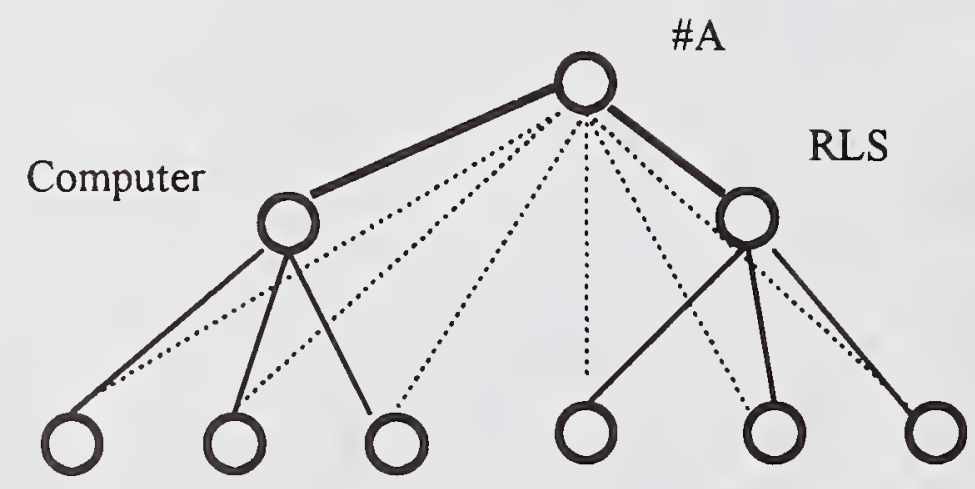

SUN 1000 SUN 10000 Pentium 133 RLS1 RLS2 RLS3

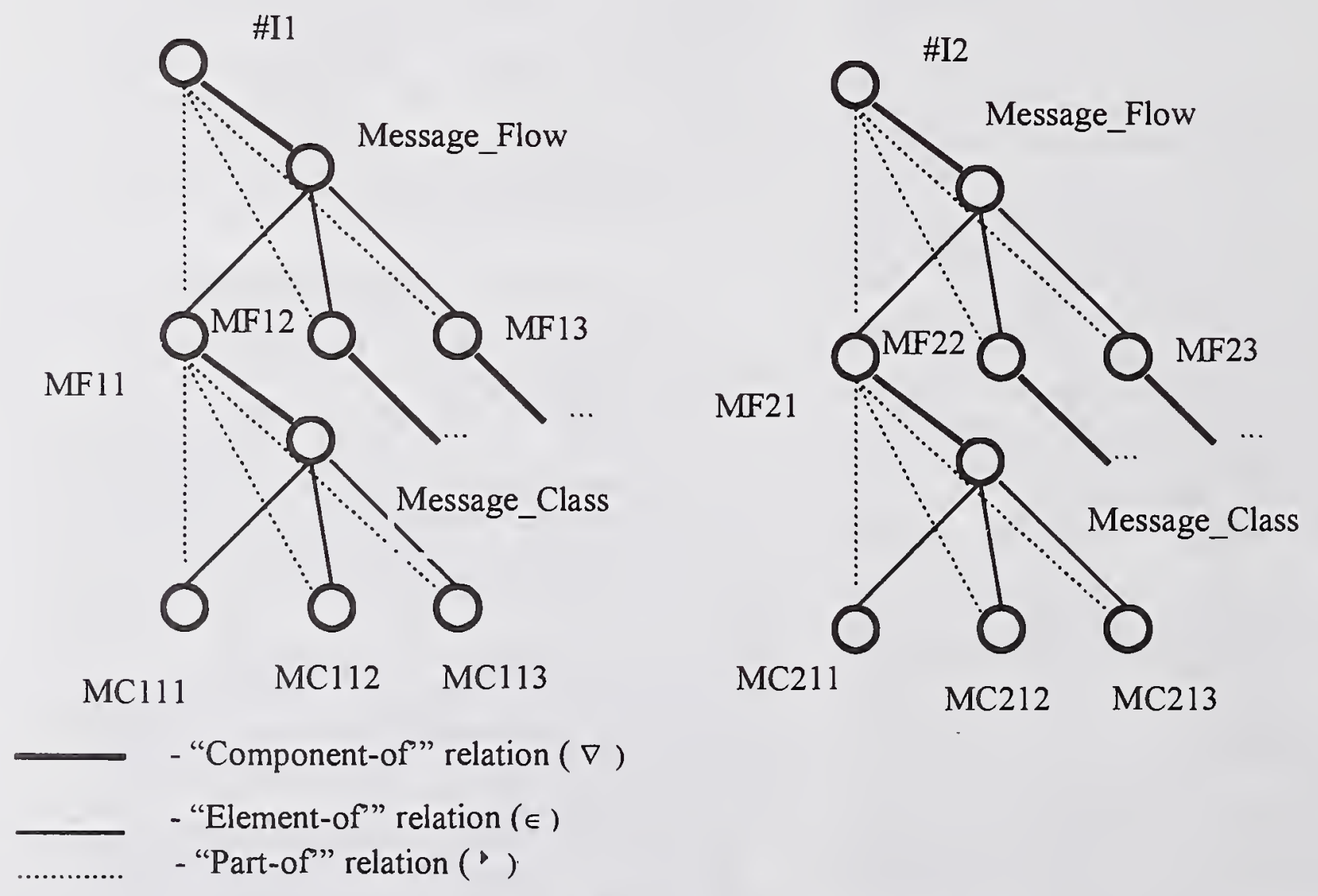

Figure 2. The structures of the problem domain 
The conceptual language for modelling a complexstructured problem domain is developed. The translator of queries with conceptual language to MLL is designed.

The examples of queries .

Example 2.

Let us define the program components, which are part of the developed system $P$, and the airplanes, which are part of the controlling object $\mathrm{O}$, between which the specific relation "provide fly_up_to_landing" is set up.

Formalization in MLL:

( $\exists(\mathrm{x} /$ Program_component $) / / \# P)(\exists(y /$ airplane $) / / \# O)$

provide_fly_up_to_landing $(x, y) \rightarrow$.

\section{Example 3.}

Let us define the program components, which are part of the developed system $P$, and the computers, which are part of the airport $\mathrm{A}$, between which function relation is set.

\section{Formalization in MLL: \\ $(\exists(\mathrm{x} /$ Program_component $) / / \# \mathrm{P})(\exists(\mathrm{t} /$ computer $) / / \# A)$ function $(x, t) \rightarrow$.}

The system KM supports "a free connection" [15] of KB and DB under Paradox DBMS. Such implementation of the system KM provides using all opportunities Paradox DBMS such as distributed processing, high performance, complex verification, supporting of integrity and safety of data, failure and recovery of DB, supporting of very large $\mathrm{DB}$. The system KM is running under Windows'95. The development language of the system $\mathrm{KM}$ is Borland $\mathrm{C}++$.

\section{CONCLUSIONS}

We have considered a new approach to desinging semiotic systems. The knowledge representation language in semiotic systems is proposed. The system KM as a tool of modelling a complex-structured problem domain is developed.

This work was supported by the Russian Fund of Fundamental Researches (project code 96-01-00125).

\section{REFERENCES}

[1] Modern Software Engineering. Foundation and Current Perspectives.- Edited by Peter A.Ng., Raymond T. Yeh. VAN NOSTRAND REINHOLD, New York,-1990.- c.591.

[2] J.E. Cooling. Software Design for Real-time Systems. CHAPMAN AND HALL (University and Professional Division).- 1991 , p. 505
[3] Harel D. and ets. Statemate: a Working Environment for the Development of Complex Reactive Systems.-IEEE Transactions on Software Engineering, Vol. 16, No. 4, April 1990, pp.403-413

[4] Robert V. Rubin, James Walker II, Eric J. Golin Early Experience with the Visual Programmer's Workbench.IEEE Transactions on Software Engineering, vol.16, No. 10,1990

[5] Andrew J.Symohds Creating a Software-Engineering Knowledge Base.- Software Development Computer-Aided Software engineerig (CASE).Edited by Chirofsky E.J.IEEE Computer Society press technology series, 1989

[6] Marc Eisenstadt, J.Domingue, T.Rajan, E.Motta Visual Knowledge Engineering,- IEEE Transactions on Software Engineering, Vol.16, No. 10, October 1990

[7] Sally Shlaer and Stephen J. Mellor "Object Lifecycles: Modeling the World in States",Prentice-Hall, Englewood Cliffs, N.J., 1992

[8] E. Gibson Objects - Born and Bred.- BYTE, October, 1990

[9] M. Eisenstadt, M. Brayshaw A Knowledge Engineering Toolkit.- BYTE, vol.15, No.10,12,1990

[10] Yokoyamat An object-oriented and constraint-based knowledge representation system for design object modeling.- Tokyo, Japan, 1COT Research Center, 1989

[11] V.N. Vagin, E.Yu. Golovina, N.O.Salapina. Intelligent CASE for Decision Making Systems. Proceedings of JCKBSE'96. Second Joint Conference on Knowledge-Based Software Engeneering. Sozopol, Bulgaria. September 2122, 1996,-pp. 122-127.

[12] Vagin V.N., Viktorova N.P., Golovina E.Yu. Multilayer Logic as a Knowledge Representation Model in the CASE System.- Journal of Computer and Systems Sciencis International.-Vol.33, No.3, 1995, pp.72-83.

[13] Ohsuga S., Yamauchi H. Multi-layer logic - a predicate logic including data structure as knowledge representation language.- New generation computing, Vol.3,-NO.4,-1985 -c.451-485.

[14] Ohsuga S. Toward inlelligent CAD systems.Computer Aided Desing, Vol.21,-NO.5,- 1989.-c.315-337.

[15] H.Yamauchi, S. Ohsuga Loose coupling of KAUS with existing RDBMSs.- Data \& Knowledge Engineering, 5, 1990,-pp.227-251. 

III

NN - MODELS 



\title{
"Intelligence and Semiotics in Pattern Recognition and Neural Networks"
}

\author{
David Casasent \\ Carnegie Mellon University \\ Department of Electrical and Computer Engineering \\ Pittsburgh, PA 15213
}

\section{ABSTRACT}

Pattern recognition is a vital part of many intelligent systems. Various uses of intelligence in several new algorithms are described for a range of image processing problems: detection, algorithm fusion, feature extraction, object representation, optimal features for both representation and discrimination, object classification and pose estimation. The techniques employed include biologically motivated Gabor wavelet filters, new neural nets, and nonlinear image processing. Test results on IR, SAR, robotic and active vision data are presented.

\section{DETECTION}

This is the first step in general image processing or scene analysis, locate all objects of interest in the scene independent of object distortions and object class. These regions of interest (ROIs) are then further processed to reduce false alarms and determine the class and pose of the contents of each ROI. There is no one magic detection filter or algorithm, similarly humans do not use just one technique for detection. The best results we have seen are obtained by applying several different detection algorithms to a scene and then fusing the output results to obtain one detection plane with peaks at all ROI locations.

\section{ALGORTHMS}

The best detection algorithms we have found are the morphological wavelet transform (this applies morphological and Gabor wavelet filters to the scene and fuses their outputs) [1] and a Gabor basis function filter [3]. The morphological processing removes spatially-varying background information while the Gabor processing locates clutter regions of the scene where target detection confidence will be low; the Gabor basis function filters detection objects. All results are distortioninvariant.

\section{ALGORITHM FUSION}

To combine the results from different detection filters, the best algorithm fusion technique we found [1] was to use fuzzy logic concepts to produce nonlinear membership mapping functions to apply to each detection algorithm's analog output. These map the outputs for the different algorithms to the same range. We use different mapping functions for different ranges of object contrast. We then use pointwise minimum fusion (a fuzzy logic AND) to fuse the different algorithm outputs.

These techniques thus use a variety of different image processing techniques (morphology, distortion-invariant filters, wavelets, fuzzy logic, fusion).

\section{IR AND SAR RESULTS}

For $\mathbb{R}$ data $\left(360^{\circ}\right.$ aspect distortions of 8 different object classes in severe clutter, with poor object contrast) we achieved excellent results (Table 1) for detection $\left(\mathrm{P}_{\mathrm{D}}\right)$ and false alarms per scene $\left(\mathrm{P}_{\mathrm{FA}}\right)$. Algorithm fusion reduces $\mathrm{P}_{\mathrm{FA}}$ by up to factor 10. Subsequent processor stages further reduce $P_{\mathrm{FA}}$ to below 0.1 per $\mathrm{km}^{2}$ with $\mathrm{P}_{C}=95 \%$ correct recognition (Section 2 ).

Table 1: Algorithm fusion detection results (TRIM-2)

\begin{tabular}{|c|c|}
\hline $\mathrm{P}_{\mathrm{D}}(\%)$ & $\mathrm{P}_{\mathrm{FA}}$ \\
\hline 90.6 & 3.1 \\
$80 \%$ & 0.9 \\
\hline
\end{tabular}

For SAR data [2], we designed a new set of distortioninvariant filters (about 3 per class). These are applied to an input scene and the WTA output if above a threshold indicates an ROI, the filter with the largest output above a threshold denotes the class of the ROI data. This technique requires many fewer templates than in model based methods. The filters are shiftinvariant and hence easily implemented in DSP hardware. We tested these filters on two different real SAR target databases and a synthetic database of SAR target models. For clutter, we used half of the man-made and natural clutter false alarms over $76 \mathrm{~km}^{2}$ that passed the first two stages of the Lincoln Labs SAR processor. For four class data with $360^{\circ}$ of distortion, we obtained $\mathrm{P}_{\mathrm{D}}=99.8 \%, \mathrm{P}_{\mathrm{C}}=96.6 \%$ and a superb $\mathrm{P}_{\mathrm{FA}}=0.026 /$ $\mathrm{km}^{2}$. We tested the filters at $\mathrm{SNR}=0 \mathrm{~dB}$ and found a negligible decrease in $\mathrm{P}_{\mathrm{C}}$ of $0.5 \%$. We tested the filters for $25 \%$ object obscuration and achieved $\mathrm{P}_{\mathrm{C}}=80 \%$. Thus, these intelligent filters are robust.

\section{NEW INTELLIGENT FILTER/FUSION DESIGN}

We now advance a new neural net (NN) data driven concept to use intelligence to automate the design of several different Gabor wavelet detection filters (selection of their parameters) AND the simultaneous design of the combination coefficients to be used to fuse different filter outputs [3]. This represents an automated data-driven intelligent image processing system. We discuss this approach using Gabor wavelet filter functions

$$
g_{n}=e^{\pi\left(x^{2} / a^{2}+y^{2} / b^{2}\right)} e^{-j 2 \pi r \omega \cos (\theta-\phi)}
$$

When such a filter is applied to a scene, its analog output denotes the amount of a given spatial frequency $\omega$ at a given orientation $\phi$ present in each local scene region defined by a and b. We consider Gabor wavelet (GW) functions with modulation 
in 1-D only, we select $\omega=1 / 2 d$ (where $d=a$ or $b$ for horizontal and vertical features respectively). We use filters formed from the real and the imaginary parts of the Gabor function (GF); real Gabor filters are proven blob detectors and imaginary Gabor filters are proven edge detectors.

We use $6 \mathrm{GFs}$, two real (these detect object height and width) and four imaginary (these detect the left, right, top and bottom object edges). We developed a separate algorithm to determine the optimal parameters for each GF separately. These 6 GF outputs or GF filters are the $P_{1}$ inputs we consider to the neural net processor of Figure 1. The $P_{1}$ to $P_{2}$ weights combine these different GF functions into $\mathrm{N}_{2}$ different macro GFs at $\mathrm{P}_{2}$. The $\mathrm{P}_{2}$ outputs represent different detection correlation planes for different macro GF filters. These outputs are then combined $\left(\mathrm{P}_{2}\right.$ to $P_{3}$ weights) into $N_{3}$ fused output correlation planes at $P_{3}$, where a WTA determines if each local input scene region is an ROI or not (and the confidence of each estimate). The $P_{1}$ to $P_{2}$ weights are complex-valued and the $\mathrm{P}_{2}$ nonlinearity used is a simple square law. These are radically new neural net concepts. We have shown that this produces general quadratic nonlinear decision surfaces of higher rank than other methods. The $P_{2}$ inputs represent the $\mathrm{N}_{2}$ new macro $\mathrm{GW}$ filters (combinations of the basic 1-D GF input $P_{1}$ functions), the $P_{2}$ to $P_{3}$ weights represent the algorithm fusion of these $\mathrm{N}_{2}$ detection filters. Both the filters and their output fusion are automatically selected and are data driven. This thus represents a very intelligent and general distortion-invariant filter synthesis and algorithm fusion concept. Initial results show better $\mathrm{P}_{\mathrm{C}}$ and $\mathrm{P}_{\mathrm{FA}}$ on distorted multi-class objects than any other Gabor filter method.

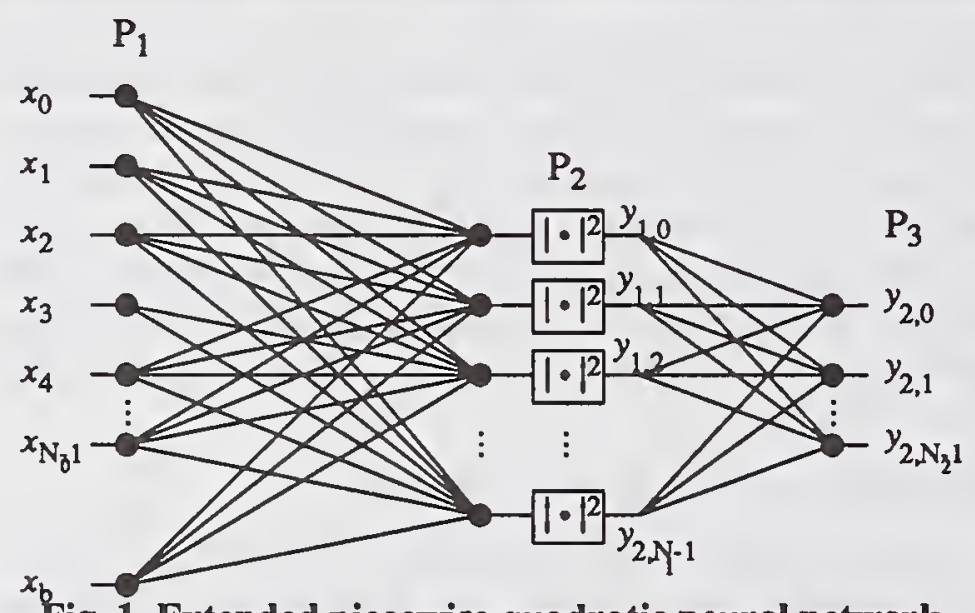

Fig. 1. Extended piecewise quadratic neural network (E-PQNN) architecture.

\section{DISTORTED OBJECT CLASSIFICATION}

\section{FEATURE SPACE TRAJECTORY (FST) PROCESSOR}

Our distortion-invariant filters represent one method to drastically compress a model base of different aspect views etc. (distortions) of an object. When object class and pose are necessary, the FST representation is very novel and useful $[4,5]$. In this case, different aspect views of an object are represented by different points in feature space. Points corresponding to adjacent aspect views are connected by straight lines. This produces an FST (piecewise linear trajectory). Different FSTs are produced for different objects. This is an attractive semiotic representation for all distorted views of an object. To classify an unknown input, we plot it as a point in feature space. The closest FST provides the class estimate; the closest line segment on that FST provides a pose estimate for the input. If an input point is too far from an FST, it is rejected as clutter. This representation overcomes many standard classifier problems (training set size, generalization etc.) and requires very low online calculations.

IR and SAR Results. For our 8-class distorted IR database, we used 11 aspect views of each object as a training set and produced an FST for each object using shift-invariant wedge magnitude Fourier features [5] (each FST has only 11 vertices). We correctly classified $95 \%=\mathrm{P}_{\mathrm{C}}$ of all test set aspect view inputs. Of more importance, we input the 200 clutter region false alarms (from our detection stage) to the FST processor. We were able to reject all but one clutter input and reduce $P_{F A}$ below $0.1 / \mathrm{km}^{2}$.

We also applied the FST to SAR data. For 2 classes of objects in real SAR (with every other aspect view used for training), we achieved $\mathrm{P}_{\mathrm{C}}=100 \%$. With $0 \mathrm{~dB}$ SNR, we achieved negligible loss $\left(\mathrm{P}_{\mathrm{C}}=99.77 \%\right.$ ). In both cases, (for 100 of the 200 clutter chips that passed the first two stages of the Lincoln Labs processor), we achieved perfect $P_{F A}=0$ false alarm rejection. For a 4-class synthetic SAR object database, we achieved $\mathrm{P}_{\mathrm{C}}=98.33 \%$ and $\mathrm{P}_{\mathrm{FA}}=0.026 / \mathrm{km}^{2}$. Thus, the FST is a very attractive representation and allows for a unique processor using semiotic concepts. It requires only $6.5 \mathrm{kbytes}$ of storage per object and only 6.5 kops per class.

Object Representation. We have developed a technique to reduce the number of vertices (aspect views) necessary to represent an object and to determine which aspect views to use. We have used this to analyze the representation error in our 8class $\mathbb{R}$ data (use of only 11 vertices (aspect views) per FST produces a worst-case average representation error of only $3 \%$ ). We used it to reduce the number of training set images in our SAR data from 180 to 80 aspect views per class for a 2 class real SAR database (we achieved $P_{C}=96 \%$ with $P_{F A}=0$ still).

Feature Spaces. Wedge or ring Fourier features are attractive as they are shift-invariant. Another attractive semiotic description of distorted object views are the dominant eigenfeatures (KL); these perform better for SAR data. For several object classes, we use FK eigenfeatures (they provide better discrimination). We note that calculating FK features from $\mathrm{KL}$ features rather than imagery is preferable (outliers are automatically removed), that use of more $\mathrm{KL}$ features is not best (outlier data is included), and that we have developed eigen techniques to determine if a training and test set are compatible and to estimate $P_{C}$ to be expected. We find these new eigenspaces an new eigenanalysis methods to be new intelligent semiotic data analysis tools. 


\section{ACTIVE VISION USES}

We have used a time sequence of views of an object over several frames as input to the FST [5] and shown that by matching an input sequence to FST sections that individual errors in separate frames (as much as 50\%) could be overcome $\left(\mathrm{P}_{\mathrm{C}} \approx 100 \%\right)$. This new semiotic representation and processor thus also handles time sequences of data.

We recently [5] also considered various active vision applications of the FST in robotics. In active vision, a sensor (or robot) acquires a view of an object and then moves to another location to better learn the object (or to grasp it, inspect it, etc.). In such robotics case, the object or a model-based CAD description of it is available and hence an infinite number of distorted object aspect views are possible. The FST representation and our adaptive algorithm are essential here to select the number of aspect views needed and which aspect views. We have shown that our algorithm yields better representation than use of aspect views evenly-spaced in aspect angle. We have also developed new analysis measures to determine which aspect view produces a pose estimate with the best accuracy and which aspect view provides a class estimate with the best confidence.

We use the FST to estimate the class and poise of the object from a given view; we then use this to determine where to drive the sensor or robot to look next to obtain: a better aspect pose estimate, a better class estimation, a better view for inspection or to grasp the object, etc.

\section{HIGHER-ORDER FEATURES}

Higher-order features can provide more information regarding input data (higher-order correlations). Nonlinear PCA has been applied to such cases, but all techniques are iterative and have various problems (most notably the rank of the decision surfaces produced). We have various new intelligent semiotic ideas on how to extract preferable nonlinear features (that provide both representation and discrimination) and how to calculate such discriminant functions in closed form. Standard KL features provide only representative, standard FK (or Fisher etc.) features provide only discrimination. To select features that are good for both representation and discrimination we define a representative measure $E_{R}$ (using the covariance matrix $C$ of the data matrix) and a discrimination measure $E_{D}$ using the matrix

$$
\mathrm{R}_{12}=\sum \sum\left(\underline{x}_{1 \mathrm{p}}-\underline{x}_{2 q}\right)\left(\underline{x}_{1 p}-\underline{x}_{2 q}\right)^{T} \text {, }
$$

where $x_{1}$ and $x_{2}$ are class 1 and 2 samples. For both good representation and discrimination, we select the dominant eigenvectors of $\underline{C}_{1}+\underline{C}_{2}+\underline{B}_{12}$ (for the two-class case) as our features. To produce nonlinear features, we describe each data vector in a higher-dimensional space as $\mathrm{x}_{\mathrm{H}}$ (with higher order products included) and we use higher-order $\mathrm{C}$ and $\mathrm{R}$ matrices. We solve for nonlinear higher-order features in the higher-order $\mathrm{H}$ space (the solution has a closed form); the solutions are nonlinear in the original space.
Initial tests show that this approach yields decision surfaces of higher rank (than nonlinear PCA can), that higher-order correlations exist in real data, and that their use improves $\mathrm{P}_{\mathrm{C}}$. This is a very attractive, new (intelligent and automated) semiotic method to nonlinearly represent and process data.

\section{REFERENCES}

[1] D. Casasent and A. Ye, "Detection filters and algorithm fusion for ATR", IEEE Trans. on Image Processing, Vol. 6, no. 1, pp. 114-125, January 1997.

[2] D. Casasent and S. Ashizawa, "SAR Detection, Recognition and Clutter Rejection with new MINACE filters", Optical Engineering, accepted, October 1997.

[3] D. Casasent and D. Weber, "Quadratic Gabor correlation filters for object detection", Proc. SPIE, Vol. 3078, April 1997. [4] D. Casasent and R. Shenoy, "Feature space trajectory for distorted-object classification and pose estimation in SAR", Optical Engineering, accepted, October 1997.

[5] D. Casasent, L. Neiberg and M. Sipe, "FST distorted object representation for classification and pose estimation", Optical Engineering, submitted May 1997. 


\title{
MORPHOLOGICAL PERCEPTRONS
}

\author{
Gerhard X. Ritter and Peter Sussner \\ University of Florida \\ Center for Computer Vision and Visualization \\ Room E301, CSE Building \\ Gainesville, Florida 32611
}

\section{ABSTRACT}

The theory of artificial neural networks has been successfully applied to a wide variety of pattern recognition problems. In this theory, the first step in computing the next state of a neuron or in performing the next layer neural network computation involves the linear operation of multiplying neural values by their synaptic strengths and adding the results. Application of a nonlinear activation function usually follows the linear operation in order to provide for nonlinearity of the network. In this paper we discuss a novel class of artificial neural networks, based on lattice algebra, in which the operations of multiplication and addition are replaced by addition and maximum (or minimum), respectively. By taking the maximum (or minimum) of sums instead of the sum of products, computation is nonlinear before the application of a nonlinear activation function. As a consequence, the properties of these networks, which are also known as morphological neural networks, are drastically different than those of traditional neural network models. The main emphasis of the results presented here is on morphological perceptrons. Within the constraints of this short paper, we define the basic computational model of a morphological neuron and examine some differences between morphological perceptrons and traditional perceptron models.

Keywords: Artificial neural networks, perceptrons, morphological neural networks, morphological perceptrons.

\section{INTRODUCTION}

In recent years a novel theory of neural computing based on lattice theory has emerged. Network models based on this theory have, in several cases, proven to be superior to traditional models. Some references of the underlying theory and applications of these networks can be found in $[5,2,1,10,3,11,8]$. In this paper we restrict our attention to morphological perceptrons.

Artificial neural network models are specified by the network topology, node characteristics, and training or learning rules. The basic equation governing the theory of computation in the standard neural network model is:

$$
x_{j}(t+1)=f\left(\sum_{i=1}^{n} x_{i}(t) \cdot w_{i j}-\theta_{j}\right),
$$

where $x_{j}(t)$ denotes the value of the $j$ th neuron at time $t, n$ represents the number of neurons in the network, $w_{i j}$ the synaptic connectivity value between the $i$ th neuron and the $j$ th neuron, $\theta_{j}$ a threshold, and $f$ the next-state function which usually introduces a nonlinearity into the network. Although not all current network models can be precisely described by this equation, they nevertheless can be viewed as variations of this equation. In contrast, the basic equation governing neural computation based on lattice algebra is vastly different from the weighted sum of linear algebra used in most neural computation. The next-state value of a neuron is obtained from an additively weighted maximum (or minimum) of inputs from neighboring neurons connected to the neuron and the resultant value is then passed through a hard-limiter. More precisely, computation of the next state value of a particular neuron $N_{j}$ assumes the form

$$
x_{j}(t+1)=f\left(p_{j} \bigvee_{i=1}^{n} r_{i j}\left(x_{i}(t)+w_{i j}\right)\right),
$$

where $r_{i j}= \pm 1$ denotes the excitatory or inhibitory influence of the $i$ th neuron on the $j$ th neuron, and $p_{j}= \pm 1$ denotes the post-synaptic response of the $j$ th neuron to the total received input. The values +1 and -1 denote excitatory and inhibitory influences, respectively. In particular, if $r_{i j}=1$, then the $i$ th neuron has an excitatory influence on the $j$ th neuron, and if $r_{i j}=-1$, then the $i$ th neuron tries to inhibit the firing of the $j$ th neuron. Similarly, if $p_{j}=1$, then the post-synaptic response of the $j$ th neuron is excitatory and if $p_{j}=-1$, then the post-synaptic response of the $j$ th neuron is inhibitory. The function $f$ is a hard-limiter defined as $f(x)=1$ if $x>0$ and $f(x)=0$ if $x \leq 0$.

Neurons that compute their next state using Equation 2 are also called morphological neurons and neural networks based on lattice theory computation are known as morphological neural networks [8]. Figure 1 illustrates the computational framework of neurons in a general morphological 
neural net. An obvious advantage of this framework is that it more aligned with the biological model and does not involve multiplication, thus providing for faster computation.

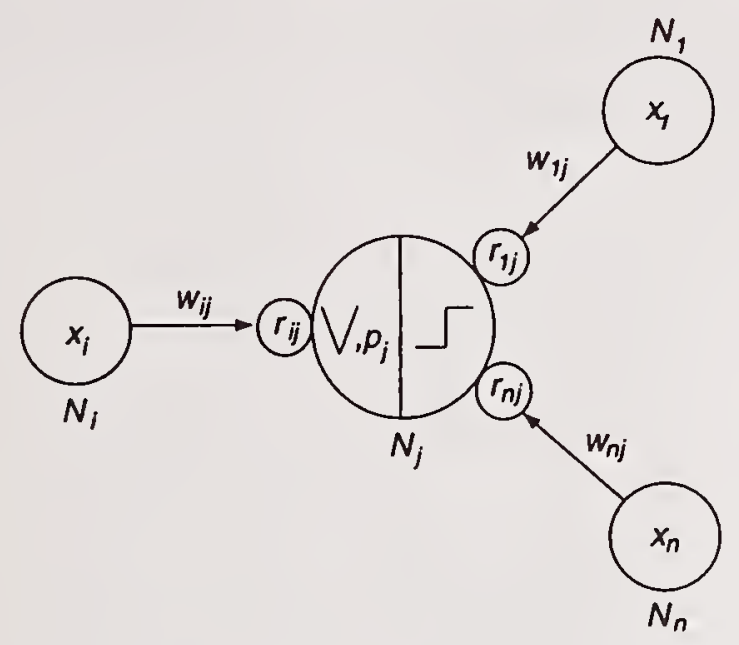

Figure 1. Computational model of a morphological neuron.

The basic neural computations as expressed by Equations 1 and 2 are based on two fundamentally different algebraic systems. Observe that the computation represented by Equation 1 is based on the operations of the algebraic structure $(\mathbf{R},+, x)$, where $\mathbf{R}$ denotes the set of real numbers. In particular, the computation $\sum x_{i} w_{i}$ is a linear operation involving multiplication and addition. In contrast, the basic computation occurring in a morphological neuron, as expressed by Equation 2, is based on thesemi-ring structure $\left(\mathbf{R}_{-\infty}, \vee,+\right)$ (or $\left(\mathbf{R}_{\infty}, \wedge,+\right)$ if we replace the operation of maximum by minimum). Here $\mathbf{R}_{-\infty}$ represents the extended real number systems $\mathbb{R}_{-\infty}=\mathbb{R} \cup\{-\infty\}$. The basic arithmetic and logic operations in the extended real number systems are as follows. The symbol + denotes the usual addition with the additional stipulation that $a+(-\infty)=(-\infty)+a=-\infty \forall a \in \mathbb{R}_{-\infty}$, while the symbol $\vee$ denotes the binary operation of maximum with the additional stipulation that $a \vee(-\infty)=(-\infty) \vee a=a$ $\forall a \in \mathbf{R}_{-\infty}$. Note that the symbol $-\infty$ acts like a zero element in the system $\left(\mathbf{R}_{-\infty}, \vee,+\right)$ if one views $\vee$ as addition and + as multiplication. Also, the role of the multiplicative identity in the structure $(\mathbb{R},+, x)$ is played by the number 1 ; i.e., $1 \cdot a=a \cdot 1=a \quad \forall a \in \mathbb{R}$. In the structure $\left(\mathbf{R}_{-\infty}, V,+\right)$ his role is played by the number 0 since $0+a=a+0=a \quad \forall a \in \mathbf{R}_{-\infty}$.

Several advantages of morphological neural networks over traditional neural networks models have been established in the area of associative memories. In [9] it was shown that morphological analogues of the Hopfield autoassociative memory have infinite storage capacity, provide perfect output for perfect input patterns, and are more robust in the presence of noise. It has also been proven that morphological bidirectional associative memories easily outperform traditional bidirectional associative memories in terms of storage capacity, bidirectional recall, as well as recall under noisy conditions [6]. In this paper we discuss some of the advantages of morphological perceptrons

\section{MORPHOLOGICAL PERCEPTRONS}

Due to page restrictions we shall concentrate our discussion mostly on single-layer morphological perceptrons (SLMPs) and indicate how these can be used to construct powerful multilayer perceptrons.

Some of the most successful associative memories have been multilayer perceptrons. These are feedforward nets that can be trained using such well-known algorithms as back propagation. Some of the problems associated with these nets are the selection of the number of hidden layers, the choice of the number of neurons within a hidden layer, very lengthy training procedures, and convergence problems associated with training algorithms. In contrast, morphological perceptrons have very simple learning algorithms that converge in two passes and these algorithms actually specify the number of neuron in a hidden layer necessary for a given classification problem [7].

The basic building blocks of morphological perceptrons are single-layer morphological perceptrons. A singlelayer perceptron consists of an input layer of $n$ neurons and an output layer consisting of a single neuron that computes a weighted sum of the input neuron values, subtracts a threshold, and passes the result through a binary-valued non-linearity. Each of the two possible outputs corresponds to a different classification response. Typically, a pattern vector $\mathbf{x}=\left(x_{1}, x_{2}, \ldots, x_{n}\right)$ is presented to the input neurons, with each input neuron assuming the value (or state) of the corresponding vector coordinate value. The pattern $\mathrm{x}$ is said to belong to class $C_{0}$ or class $C_{1}$ depending as to whether the output neuron's value is 0 or 1 , respectively. As shown in Figure 2, the topology of single-layer morphological perceptrons mimics that of the standard single-layer perceptron. However, computation at the output neuron is based on the computational model given by Equation 2. In particular, the result of the output neuron is given by

$$
f\left(p \bigvee_{i=1}^{n} r_{i}\left(x_{i}+w_{i}\right)\right)
$$

where $x_{i}$ denotes the value of the $i$ th input neuron, $w_{i}$ the synaptic weight between the $i$ th input neuron and the output neuron, $r_{i}$ indicates whether the input of the $i$ th input 
neuron to the output neuron is excitatory or inhibitory, and $p$ denotes the post-synaptic response of the output neuron.

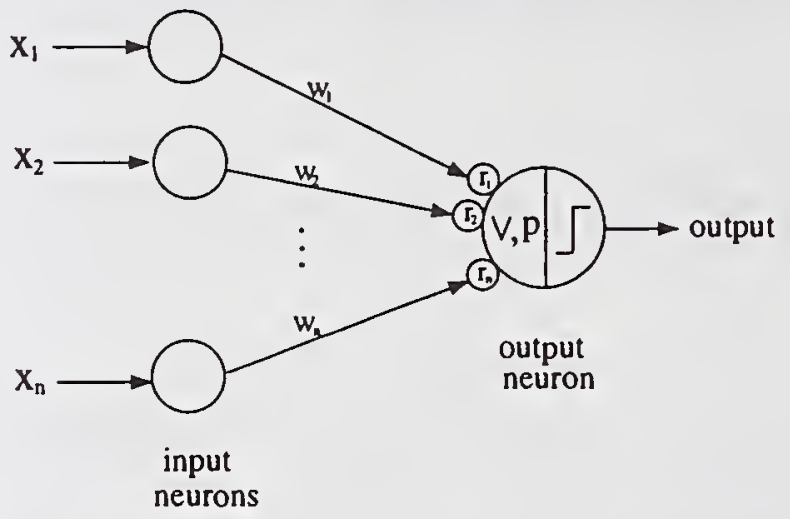

Figure 2. Single-layer morphological perceptron.

It is not difficult to ascertain that a single-layer morphological perceptron can provide solutions to problems that cannot be solved by traditional single-layer perceptrons. We provide a simple example of this.

Example 1. Suppose we want a single-layer morphological perceptron to classify pattern vectors $(2,0),(0,2)$, $(2,2)$ and close neighbors to belong to class $C_{1}$, and pattern vectors $(1,0),(0,1),(1,1)$ and close neighboring points to belong to class $C_{0}$. Simple computation will verify that the net shown in Figure 2 with $n=2, w_{1}=w_{2}=-1.5$, $r_{1}=r_{2}=1$, and $p=1$ will correctly solve this problem. For example, using the inputs $x=(0,1)$ and $x=(2,2)$ we obtain

$$
\begin{gathered}
f[(0-1.5) \vee(1-1.5)]=f(-.5)=0 \\
\text { and } \\
f[(2-1.5) \vee(2-1.5)]=f(.5)=1
\end{gathered}
$$

respectively. Therefore, $(0,1) \in C_{0}$ and $(2,2) \in C_{1}$.

An interesting observation is that the singlelayer perceptron of this example provides a solution for a problem that cannot be solved by the traditional single-layer perceptron model since the two classes of patterns are not linearly separable. This observation follows from the fact that the equation $\pm\left[r_{1}\left(x_{1}+w_{1}\right) \vee r_{2}\left(x_{2}+w_{2}\right) \vee \cdots \vee r_{n}\left(x_{n}+w_{n}\right)\right]=0$ represents an $(n-1)$-dimensional surface, called the perceptron's decision boundary, which divides Euclidean $n$-space into two regions. The regions separated by the decision boundary correspond the output neuron's on-off regions as any input from one region will always result in an on state (value 1) of the neuron while any input from the other region will result in an off state (value 0 ) of the neuron. For instance, the decision boundary generated by the perceptron of Example 1 is the infinite step function shown in Figure 3. Any point in the quarter-plane bounded by the lines $x=-w_{1}$ and $y=-w_{2}$ will result in a 0 -state value if used as input to the perceptron. Therefore, this perceptron could solve the two-class problem illustrated in Figure 3, where patterns of one class belong to the region marked "class 0 " and patterns of the other class belonging to the region marked "class $1 . "$

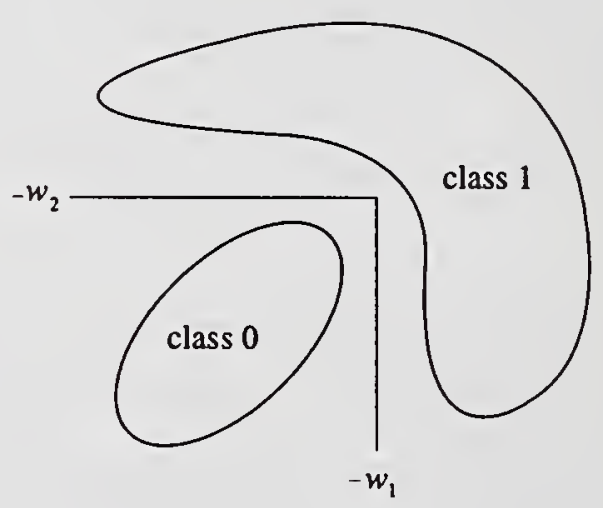

Figure 3. Decision boundary for the single-layer morphological perceptron of Example 1.

Single-layer morphological perceptrons are the basic building blocks of multilayer morphological perceptrons. Thus, in order to construct multilayer morphological perceptrons, it is necessary to have a thorough understanding of SLMPs. The output neurons of SLMPs form the hidden neurons multilayer morphological perceptrons. The decisions made by these hidden neurons depend on their synaptic responses to inputs as well as the synaptic weights of the input axons. These responses and weights determine the decision boundaries for the neuron's on-off regions. For a two-input nodes $(n=2)$ SLMP, the simplest decision boundary is obtained by setting one of the weights, $w_{1}$ or $w_{2}$, equal to $-\infty$. If $w_{1}=-\infty$, then the decision boundary is the horizontal line $y=-w_{2}$, and if $w_{2}=-\infty$, then the decision boundary will be the vertical line $x=-w_{1}$. Figure 4 illustrates these two possibilities for $r_{1}=r_{2}=1$ and $p=1$. Changing the value of $p$ to $p=-1$ interchanges the two classes $C_{0}$ and $C_{1}$ since the on region becomes the off region and vice versa. If $r_{1}=1$ and $w_{2}=-\infty$, then changing $r_{1}$ to $r_{1}=-1$ will also interchange the neuron's on-off regions. However, it is important to note that if $w_{i}=-\infty$, then we must have $r_{i}=1$ since $-\left(x_{i}-\infty\right)=\infty-x_{i}=\infty$, which is outside the computational framework of the algebra $\left(\mathbb{R}_{-\infty}, \vee,+\right)$. 

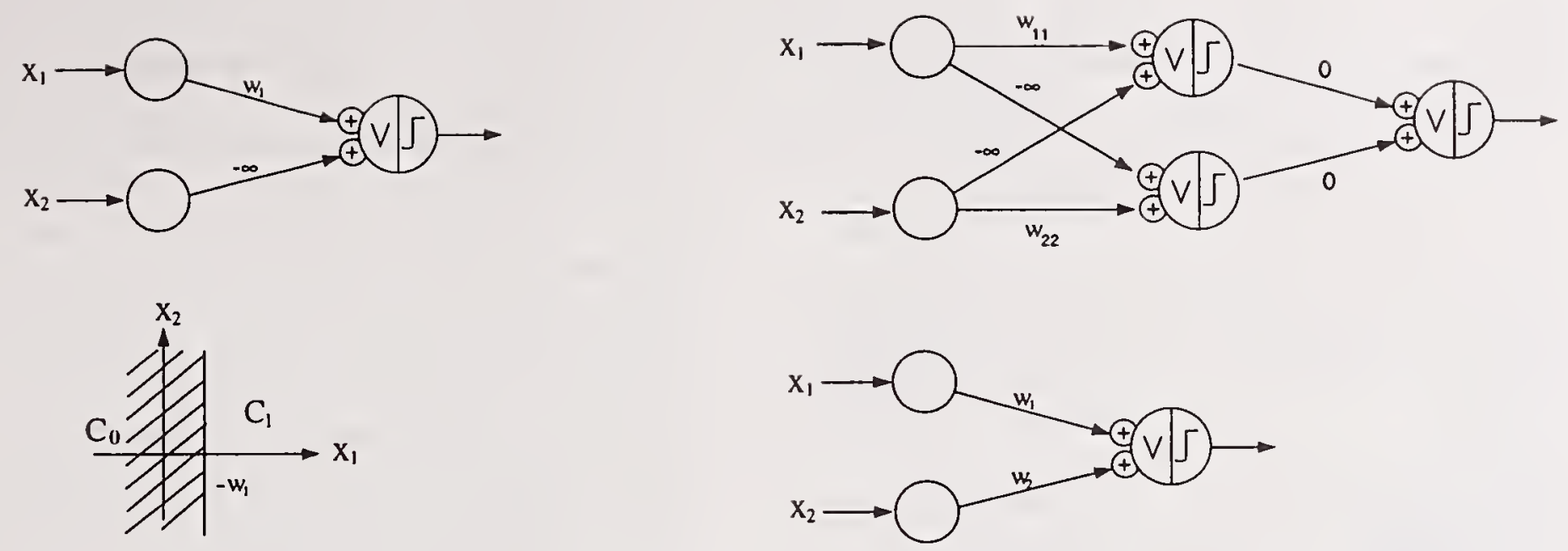

Figure 5. Two equivalent morphological

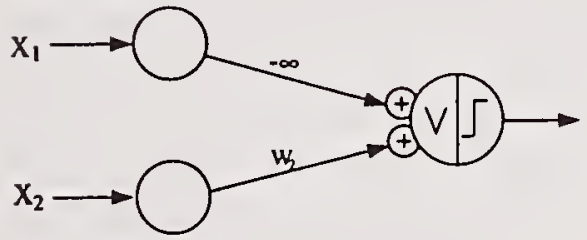

perceptrons. Here $w_{1}=w_{11}$ and $w_{2}=w_{22}$.
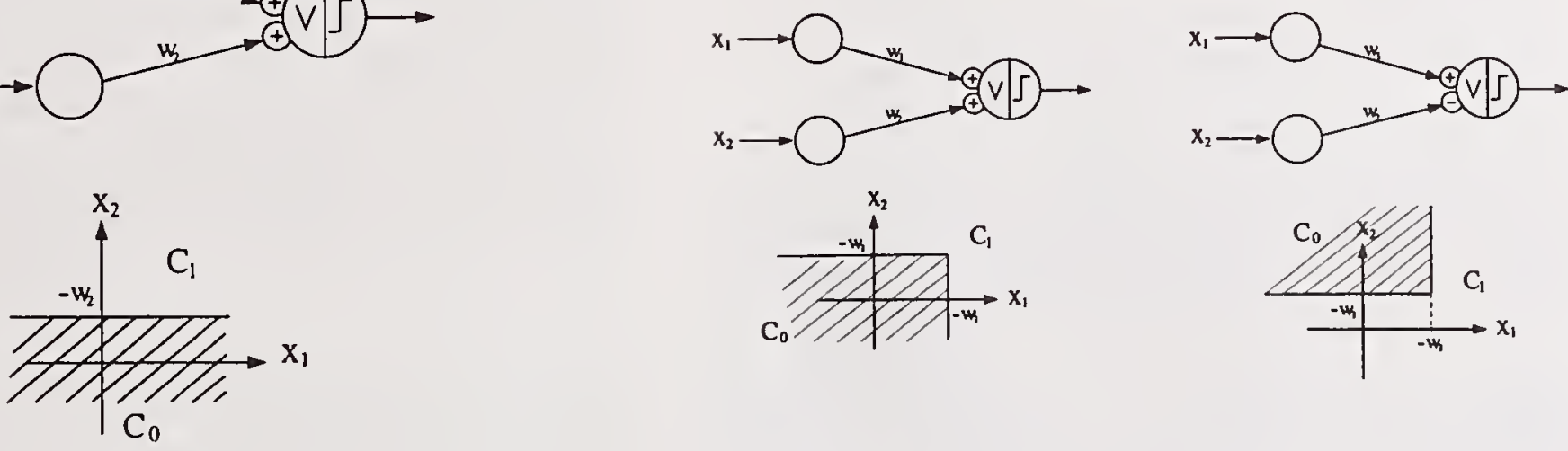

Figure 4. Decision boundaries for two-input, single-layer morphological perceptron having one synaptic weight equal to $-\infty$.

The two SLMPs shown in Figure 4 can be combined to form a two-input multilayer morphological perceptron with one hidden layer as shown in Figure 5. The output neuron of this multilayer perceptron computes the maximum of its two hidden layer input neurons. Hence, a pattern point will belong to class $C_{0}$ if and only if it was classified by both single-layer perceptrons as belonging to class $C_{0}$. It is easy to see that this multilayer perceptron is equivalent to the single-layer perceptron shown on the bottom of Figure 5 if $w_{1}=w_{11}$ and $w_{2}=w_{22}$; a pattern vector $\mathbf{x}=\left(x_{1}, x_{2}\right)$ is classified by both perceptrons as belonging to class $C_{0}$ if and only if $x_{1} \leq-w_{1}$ and $x_{2} \leq-w_{2}$.

The preceding discussion shows that a two-input SLMP can have one of three distinct types of basic decision boundaries; a horizontal or vertical line, or an infinite step function formed by a vertical and horizontal half-line. Figure 6 shows the four possible types of infinite step functions for two-input SLMPs.
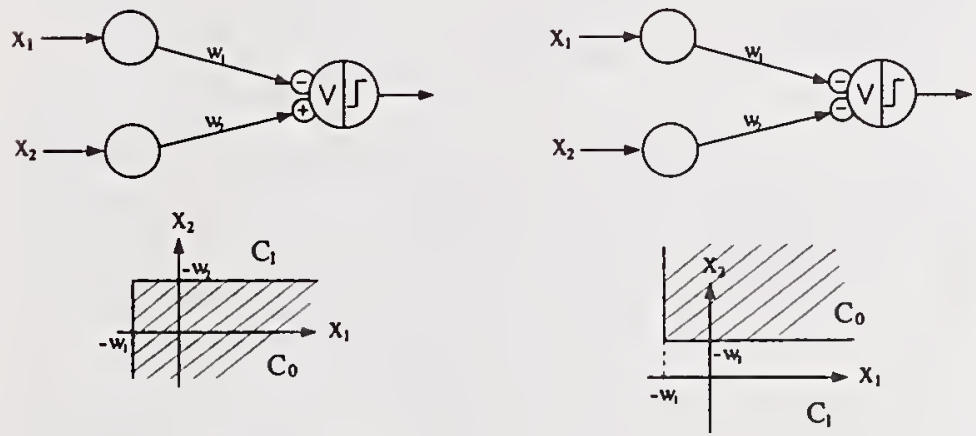

Figure 6. Decision boundaries for two-input, single-layer morphological perceptrons with real-valued synaptic weights.

The two algebras $\left(\mathbb{R}_{-\infty}, \vee,+\right)$ and $\left(\mathbb{R}_{\infty}, \wedge,+\right)$ are dual algebras. The simple isomorphism $h: \mathbb{R}_{-\infty} \rightarrow \mathbb{R}_{\infty}$ defined by $h(r)=-r \forall r \in \mathbb{R}_{-\infty}$ defines this duality. Thus, it does not matter which of these two algebras we use for the underlying mathematical foundation of morphological neural networks. This duality can be used for interchanging classes. For example, by negating neural inputs (i.e., interchanging $r_{i}$ with $-r_{i}$ ) and interchanging the operation of maximum with minimum interchanges the classes $C_{0} \leftrightarrow C_{1}$. This interchange of classes is illustrated in Figure 7. Of course, as mentioned earlier, in order to interchange the on-off regions we could have simply used an inhibitory post-synaptic response for the output neuron of 
the perceptron on the left side of Figure 7. This follows from the fact that

$$
\begin{aligned}
& f\left[-\left(-\left(x_{1}+w_{1}\right) \vee\left(x_{2}+w_{2}\right)\right)\right] \\
& =f\left[\left(x_{1}+w_{1}\right) \wedge-\left(x_{2}+w_{2}\right)\right] .
\end{aligned}
$$
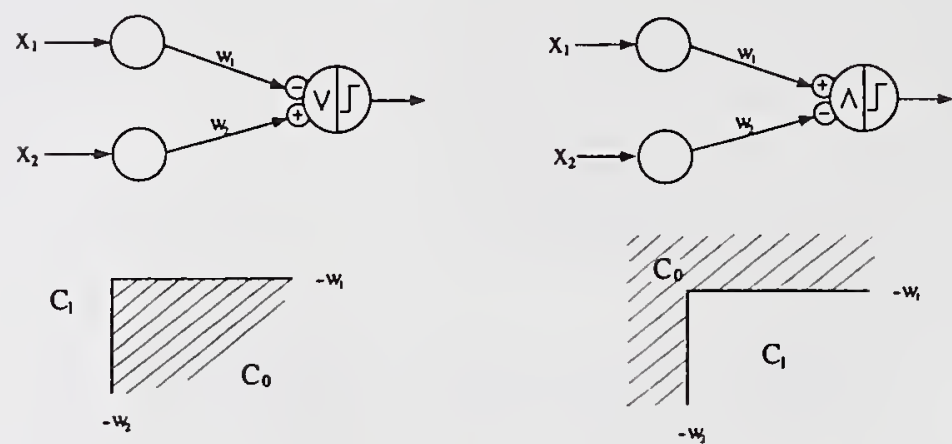

Figure 3. Two SLMPs with identical decision boundaries but reversed on-off regions.

In 3-dimensional space lines are replaced by planes and half-lines by half-planes. For example, setting $w_{1}=$ $w_{2}=-\infty$ and $w_{3} \in \mathbf{R}$ yields the plane $z=-w_{3}$ as the decision boundary for the corresponding three-input, single-layer morphological perceptron. Hence, by choosing $w_{i} \in \mathbf{R}$ for some $i=1,2$, or 3 , and setting the remaining two weights equal to $-\infty$, three basic types of planar decision boundary can be obtained. In analogy to the decision boundaries shown in Figure 4, each of these 2-dimensional decision boundaries will be a plane normal to one of the coordinate axes and parallel to the remaining two axes. Using two such decision surfaces (i.e., setting only one of the weights equal to $-\infty$ ) a more complex decision boundary can be formed. This decision boundary corresponds to a 2-dimensional step function. For instance, if $w_{1}=-\infty$ and $w_{2}, w_{3} \in \mathbb{R}$, the decision boundary divides 3-space into two regions, namely the region $\left\{(x, y, z): y \leq-w_{2}, z \leq-w_{3}, x \in \mathbb{R}\right\}$ and its complement if all pre- and post-synaptic influences are positive. Since any two of the decision planes will intersect in a line, 12 such basic 2-dimensional step functions can be constructed, yielding 24 possible on-off regions.

An even more complex decision boundary is obtained when all three weights are real-valued. In this case, the decision boundary corresponds to the infinitely extended boundary of a three-sided rectangular box. These analogies carry over into higher dimensional spaces.

Two-layer morphological perceptrons can solve any class problem in which the classes are compact sets. This is the case in basically all realistic pattern classification problems, where the patterns of a given class belong to some bounded set. For instance, in the plane, any compact set can be approximated arbitrary closely by rectangles. Thus, decision surfaces can be obtained via horizontal and vertical lines to form any set approximating rectangles. This concept extrapolates to higher dimensional compact sets. For example, the two-input, 2-layer perceptron shown in Figure 8 will classify all patterns falling within the set bounded by the lines $x_{1}=-w_{11}, x_{1}=-w_{12}, x_{1}=-w_{13}$, $x_{2}=-w_{21}, x_{2}=-w_{22}$, and $x_{2}=-w_{23}$ shown in the bottom half of Figure 8 as belonging to class $C_{0}$. It should be clear that any planar configuration can be boxed in by such sequences of step functions. The number of neurons in the hidden layer depends, of course, on the number of step functions required to approximate a region. Another interesting observation is that the class $C_{0}$ in this example is not convex, showing that a single hidden layer is sufficient for both convex as well as non-convex regions. This is in contrast to standard perceptrons that require two hidden layers for non-convex class decisions [4]. Note also that if we eliminate the third neuron $N_{3}$ in the hidden layer, then the new net would classify all points in the infinite, non-convex and non-compact region $R=\left\{\left(x_{1}, x_{2}\right): x_{2} \geq-w_{23}\right.$ if $-w_{11} \leq x_{1} \leq-w_{12}$ or $-w_{21} \leq x_{2} \leq-w_{22}$ if $\left.x_{1} \geq-w_{12}\right\}$ as belonging to class $C_{0}$. Thus, multilayer morphological perceptrons can also classify patterns belonging to unbounded regions.

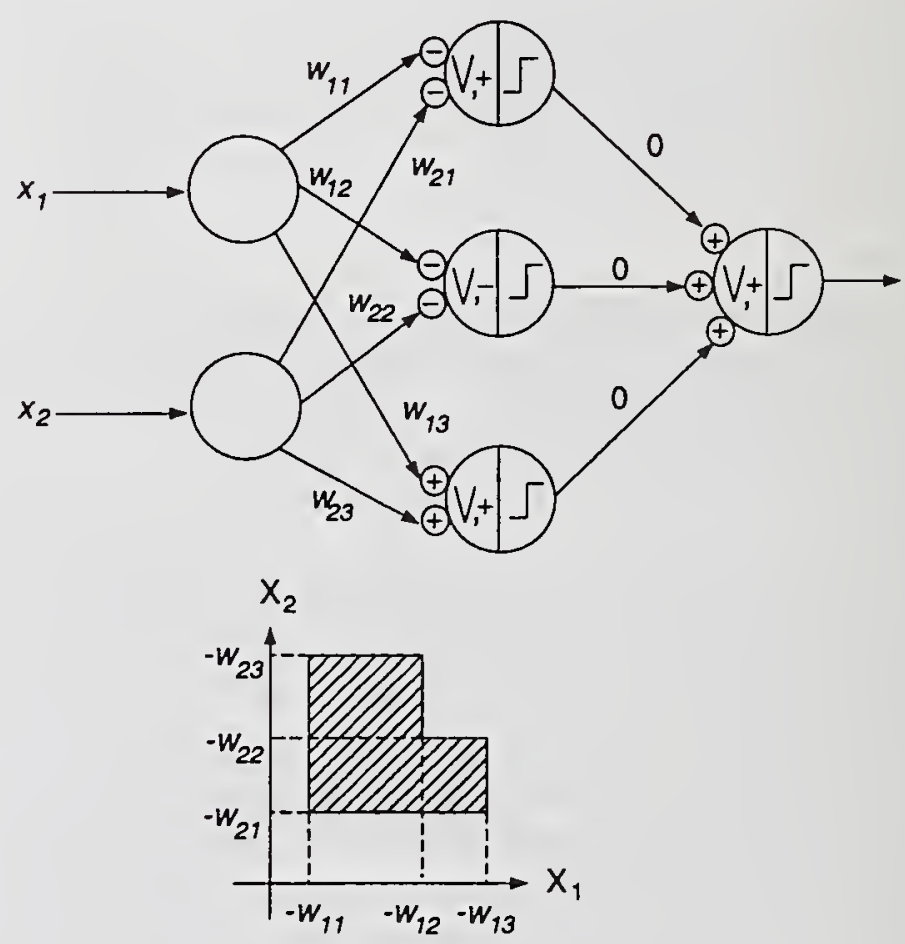

Figure 7. Non-convex decision boundary of a 2-layer morphological perceptron.

Using the different decision surfaces that are provided by the single-layer morphological perceptron discussed earlier, it will be an interesting and instructive exercise for the reader to construct 2-layer morphological perceptrons whose decision boundaries approximate the boundaries of different geometric regions in the plane. 


\section{CONCLUSION}

This paper introduces neural network computing based on lattice algebra, which provides a new paradigm for artificial neural networks. The resulting morphological networks are radically different in behavior than traditional artificial neural network models. One advantage of using morphological neural networks over traditional artificial neural networks is that the algebra $\left(\mathbb{R}_{-\infty}, \vee,+\right)$ does not involve the operation of multiplication and thus provides for greatly reduced computational overhead. Additionally, due to the global maximum operation, the usual convergence problems that exist in most of the traditional approaches do not occur in the lattice algebraic approach. For example, in [7] we showed that training algorithms for morphological perceptrons converge in at most two steps. Furthermore, these training algorithms also add all necessary neurons in the hidden layer. Our main emphasis this paper was on morphological perceptrons. We laid the foundations of single-layer morphological perceptrons and indicated how these can be used to construct multilayer morphological perceptrons

\section{REFERENCES}

[1] J.L. Davidson. Simulated annealing and morphological neural networks. In Image Algebra and Morphological Image Processing III, volume 1769 of Proceedings of SPIE, pages 119-127, San Diego, CA, July 1992.

[2] J.L. Davidson and F. Hummer. Morphology neural networks: An introduction with applications. IEEE Systems Signal Processing, 12(2):177-210, 1993.

[3] P.D. Gader, Y. Won, and M.A. Khabou. Image algebra network for pattern recognition. In Image Algebra and
Morphological Image Processing V, volume 2300 of Proceedings of SPIE, pages 157-168, July 1994.

[4] R.P. Lippmann. An introduction to computing with neural nets. IEEE Transactions on Acoustics, Speech, and Signal Processing, ASSP-4:4-22, 1987.

[5] G.X. Ritter and J. L. Davidson. Recursion and feedback in image algebra. In SPIE's 19th AIPR Workshop on Image Understanding in the 90's, Proceedings of SPIE, McLean, Va., October 1990.

[6] G.X. Ritter, J.L. Diaz de Leon, and P. Sussner. Morphological bidirectional associative memories. Preprint available from authors. Submitted to Neural Networks.

[7] G.X. Ritter and P. Sussner. An introduction to morphological perceptrons. Preprint available from authors. Submitted to IEEE Transactions on Neural Networks.

[8] G.X. Ritter and P. Sussner. An introduction to morphological neural networks. In Proceedings of the 13th International Conference on Pattern Recognition, pages 709-717, Vienna, Austria, 1996.

[9] G.X. Ritter, P. Sussner, and J.L. Diaz de Leon. Morphological associative memories. Preprint available from authors. To appear in IEEE Transactions on Neural Networks.

[10] C.P. Suarez-Araujo. Novel neural network models for computing homothetic invariances: An image algebra notation. Journal of Mathematical Imaging and Vision, 7(1):69-83, 1997.

[11] Y. Won and P.D. Gader. Morphological shared weight neural network for pattern classification and automatic target detection. volume 4 of Proceedings of the 1995 IEEE International Conference on Neural Networks, pages 2134-2138, Perth, Australia, November 1995. 


\section{Traps and Tricks in Standard Benchmark Problems for Neurocontrol}

\author{
Ludmila M. Dolmatova \\ Scientific Cybernetics, Inc. \\ Arlington, VA 22201 \\ dolm(juno.com
}

\author{
Paul J. Werbos \\ National Science Foundation, Room 675 \\ Arlington, VA 22230 \\ pwerbos@nsf.gov
}

\begin{abstract}
In the last few years neurocontrol has made enormous progress, in terms of new engineering applications, new mathematical designs and ideas, and new links to the brain. The success of real life applications depends on a vast variety of factors, from general design ideas down to programming details. Sometimes it is not easy to find the real reason for success or failure in a particular application, because the usual approach to neural modeling is somewhere between art and science. So, it's time to demystify the process of design, from idea to implementation to interpretation of results. There exists a set of benchmark problems described in [1] which became a de facto standard for exploring the possibilities of new modeling and control methods. However, most of these problems (even though they were intentionally designed for instructive purposes) can be implemented them in different ways, which complicates the comparison of results. The goal of this paper is to demonstrate some "underwater rocks" in the application of neurocontrol methods using standard benchmark problems as a commonly available touchstone.
\end{abstract}

KEYWORDS: neurocontrol, benchmark problems; model evaluation.

\section{INTRODUCTION}

Adaptive Critic Designs (ACD) [2,3], one of the most powerful tools for solving multidimensional optimization problems, are gaining growing popularity in research community [4-8, etc]. It has been demonstrated that ACD methods allow one to solve real-life problems with large numbers of state variables with better performance than traditional methods allow.

According to the definition of a "brain-like" system, as suggested in $[9,10]$, it should contain at least three major general-purpose adaptive components: (1) an Action or Motor system, capable of outputting effective control signals to the plant or environment; (2) an "Emotional" or "Evaluation" system or "Critic," used to assess the long-term costs and benefits of near-term alternative outcomes; (3) an "Expectations" or "System Identification" component, which serves as a model or emulator of the external environment or of the plant to be controlled. Therefore, 3 separate but interacting subsystems are considered: Critic, Model, and Action.

The implementation of these subsystems is always a matter of serious choice. In the most general case, each of them may be implemented as some kind of function approximator. Of course, neural networks can be regarded as universal approximators $[11,12]$; thus, in the majority of applications the Model is chosen to be neural network with straight feedforward or recurrent architecture. However, in industrial processes some kind of analytical model is usually available. So in this paper we use analytical models to illustrate the basic concepts of design. It is clear that such models in real applications might be incomplete, contradictory or ambiguous (sometimes they are such even in test cases), but end users nıost often prefer to have first principles or empirical models used directly in the development of an overall control system, which is quite reasonable according to the concept of utilization of all available information.

The organization of the paper is as follows: Section 2 briefly reviews the logic of dual heuristic programming (DHP) used here as the example of ACD design, and backpropagation through time. These methods are applied in Section 3 which describes ACD for 2 traditional benchmark problems: pole balancing on a moving cart, and aircraft autolander. Section 4 summarizes the results of computer experiments with these benchmark problems, and suggests directions for further development. Finally, the conclusions are drawn.

\footnotetext{
- The views herein are those of the author, not those of NSF.
} 


\section{DHP AND BACKPROPAGATION THROUGH TIME AS GENERAL METHODS.}

A complete description of backpropagation through time (BTT) can be found in [13], and that for various $A C D$ including Dual Heuristic Programming (DHP) in [3]. It is worth mentioning, that the description in this source (Handbook of Intelligent Control) is complete, but concise, as is determined by the nature of a Handbook. Therefore, almost every subsequent publication has added something from the author's understanding of those methods. We review here the main ideas of BTT and DHP just for the convenience of readers.

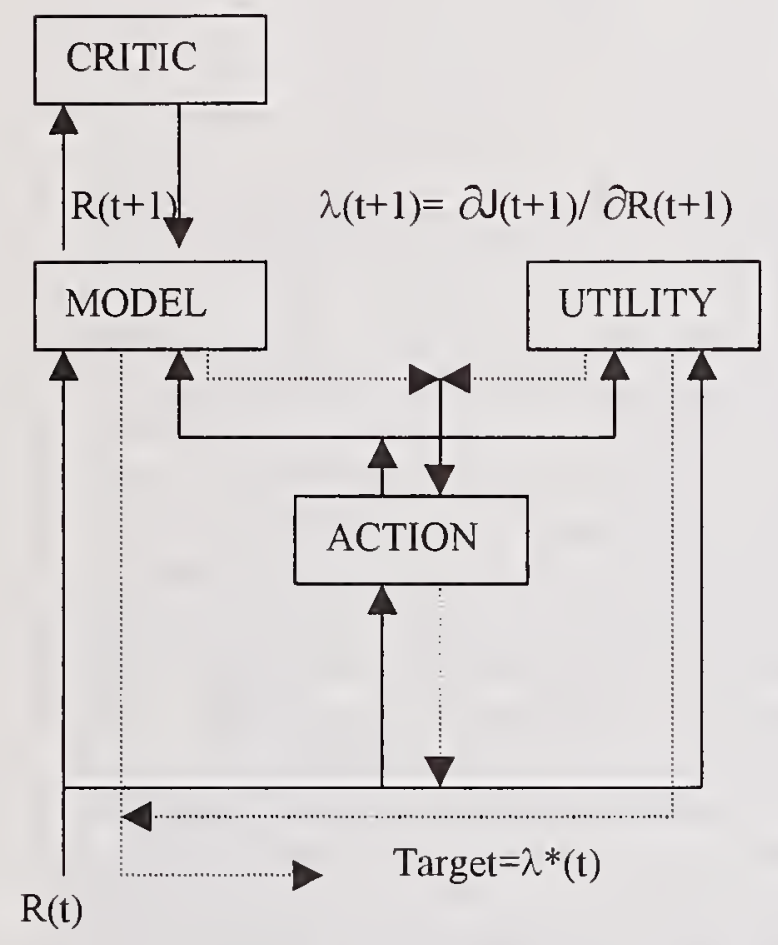

Fig. 1. An outline of DHP

DHP is a procedure for adapting that network which approximates the derivatives of the secondary, or strategic utility function $\mathrm{J}$ (Critic network). The primary utility function $U$ is supplied by the user. The process of network adaptation is governed by dual subroutines which backpropagate derivatives from the Model and the Action as shown in Fig. 1 reproduced from [3] (the forward flow of information is drawn in solid lines; the backward flow is in dotted lines).

The Backpropagation of utility, from the other side, is an exact and straightforward method for adaptation of the Action weights based on explicit calculation of the utility function $\mathrm{N}$ steps forward in time. Usually it is used for off-line learning, but in the test example considered here (aircraft autolander) it can be implemented as online method, because the control interval is only 10 times larger than the sampling interval, thus if the system is able to calculate the utility function 10 steps forward reasonably quickly, it can adjust weights in Action network between two consecutive control moments.

\section{COMPUTER EXPERIMENTS WITH BENCHMARK PROBLEMS.}

In both cases presented in Section 3, the model of dynamical system is described as a set of equations, not as a neural network or other approximator. If the model is exact, it allows us to avoid prediction errors, and also to get exact derivatives through the model, which are necessary to adapt the Critic and Action networks.

\subsection{Inverted pendulum on a moving cart: balancing forever.}

The pole-balancing problem is one of the simplest test problems in classical control theory [14], reinforcement learning [15], and artificial intelligence [16]. The specific goals of researchers have been different: from training the pole-and cart system so as to keep balance with only a fail signal [15], to the demonstration of the optimal control strategy [17]. Here we consider the intermediate task, where learning of the Critic Network on-line evaluation of selected utility function, so that the resulting controller (trained Action network) is able to balance the pole without evaluation of the situation, using just the vector of state variables.

For our numerical experiments we have used the equations from [15], and their finite difference approximation with time step equal to 0.02 .

$$
\begin{aligned}
& \ddot{\theta}(t)=\frac{g \sin \theta(t)+\cos \theta(t) \frac{-f(t)-m l \dot{\theta}^{2}(t)+\mu_{c} \operatorname{sgn}(\dot{x}(t))}{m_{c}+m}-\frac{\mu_{p} \dot{\theta}(t)}{m l}}{l\left(\frac{4}{3}-\frac{m \cos ^{2} \theta(t)}{m_{c}+m}\right)} \\
& \ddot{x}(t)=\frac{f(t)+m l\left(\dot{\theta}^{2}(t) \sin \theta(t)-\ddot{\theta}(t) \cos \theta(t)\right)-\mu_{c} \operatorname{sgn}(\dot{x}(t))}{m_{c}+m}
\end{aligned}
$$

$x(t+1)=x(t)+\delta \ddot{x}(t)$

$\dot{x}(t+1)=x(t)+\delta \dot{x}(t)$

$\theta(t+1)=\theta(t)+\delta \dot{\theta}(t)$

$\dot{\theta}(t+1)=\theta(t)+\delta \ddot{\theta}(t)$

The control interval is equal to the sampling interval, i.e. force can be applied each $0.02 \mathrm{sec}$, and during this interval it is constant. A description of model parameters and their default values is given in Table 1 . 


\begin{tabular}{|c|c|c|}
\hline Parameter & Value & Comments \\
\hline$g$ & $9.81 \mathrm{~m} / \mathrm{s} / \mathrm{s}$ & $\begin{array}{l}\text { acceleration due to } \\
\text { gravity }\end{array}$ \\
\hline$m_{c}$ & $1.0 \mathrm{~kg}$ & mass of cart \\
\hline$m$ & $0.1 \mathrm{~kg}$ & mass of pole \\
\hline$l$ & $0.5 \mathrm{~m}$ & half-pole length \\
\hline$\mu_{c}$ & 0.0005 & $\begin{array}{l}\text { coefficient of } \\
\text { friction of cart on } \\
\text { track }\end{array}$ \\
\hline$\mu_{p}$ & 0.000002 & $\begin{array}{l}\text { coefficient of } \\
\text { friction of pole on } \\
\text { cart }\end{array}$ \\
\hline$f(t)$ & $\begin{array}{l}-10 \mathrm{~N} \\
10 \mathrm{~N}\end{array}$ & $\begin{array}{l}\text { force, applied to } \\
\text { cart's center of mass } \\
\text { at time t }\end{array}$ \\
\hline$\delta$ & $0.02 \mathrm{sec}$ & sampling interval \\
\hline
\end{tabular}

Table 1. Description and default values of parameters for pole balancing problem.

A principal difference from the previous work consists in the choice of utility function U:

$U=-f(t)(10 \sin \theta+\sin \dot{\theta}+x / 25)-0.5 \theta^{2}-0.5 \dot{\theta}^{2}-0.5 x^{2}-0.5 \dot{x}^{2}$

In contrast with learning by trials and errors [15], we provide the possibility for the system to evaluate its state from the viewpoint of some external observer. In this approach the system should not fail at all if the utility function is defined correctly, if the system is evaluating its state frequently to have time to respond, and if the range of control signal is wide enough. In fact, under such conditions a dynamical system guided by DHP should demonstrate one-trial learning -and it does.

With the pole starting in an upright position of pole, after applying arbitrary force (about $2 \mathrm{~N}$ ) the system actually converges to the neighborhood of a stable state (zero angle from vertical, fixed coordinate) after 3000 time intervals, i.e. in 60 seconds. That's what it costs to learn the balancing. This process is shown at Fig.2, where it can be seen how the contradictory requirements of stabilizing with respect to the angle and to the coordinate are satisfied. The oscillations of utility function certainly correspond to oscillations of force (control action) when the system changes priorities: after stabilizing the angle it comes to stabilizing the coordinate of cart.

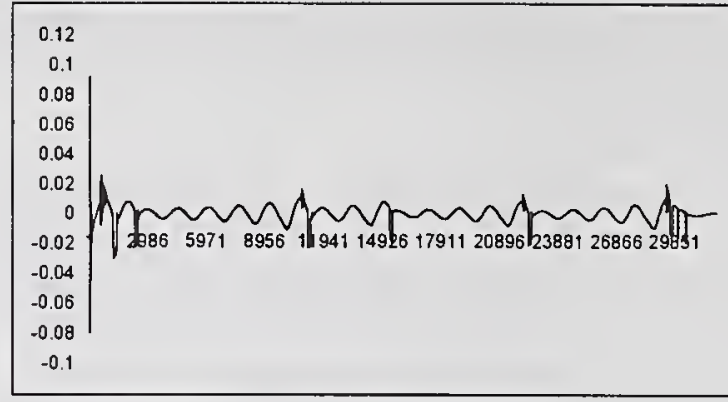

(a)

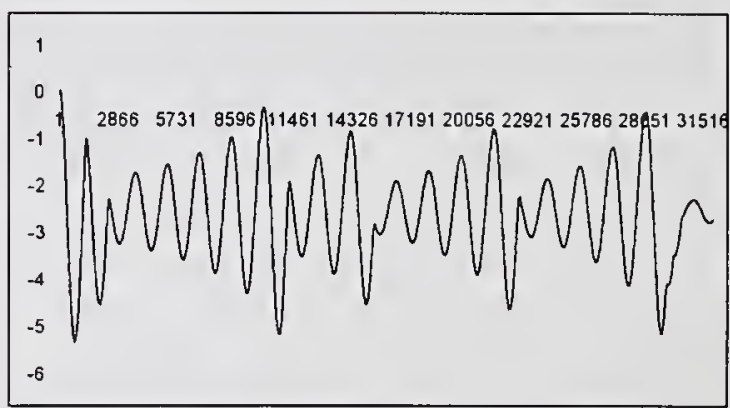

(b)

Fig. 2. Pole angle (a) and cart coordinate (b) during training phase. Time interval is equal to $0.02 \mathrm{sec}$.

In this picture one can see a periodical increase of angle and coordinate amplitude with time. Sounds strange, but this is exactly what was desired. Not to maintain the upright pole position, because it is unrealistic for inherently unstable system, but to maintain proper frequency of oscillations, or combination of few frequencies, because the system is optimizing several parameters simultaneously -- angle, angular velocity, coordinate of cart, cart velocity.

In training mode the controller was balancing the pole at least 45 minutes without any growth of oscillations. So, the initial goal - to keep balancing forever having the possibility to evaluate system state was reached in one trial.

\section{Comments on choosing $U$ function.}

A standard caveat in ACD description is that the utility function $U$ is essentially problem dependent, therefore the correct definition of $U$ is crucial for the success of the application. There is also a source of possible danger, however. Consider, for example, our expression for the $U$ function in the inverted pendulum problem (pole balancing). It seems relatively simple and natural; much more complicated expressions may be derived when one attempts to incorporate human experience [4]. However, this is false simplicity: in fact, the major term in the utility function.

$-v(10 \sin \theta+\sin \dot{\theta})$ 
implicitly refers to a desired control law. Even without learning, it would be enough to choose the external force as

$$
f=5 \sin \theta+\dot{\theta}
$$

to push the system into the equilibrium state [18].

Though it's correct only when there are no constraints on $\mathrm{x}$ coordinate (as in the cited reference), this example clearly demonstrates that the utility function should reflect general optimization requirements like energy minimization, being in the neighborhood of desired point, and so on (as does the utility function implemented here). As can be seen from data analysis, at the beginning of training the derivative of the utility function with respect to control actually determines the direction of control changes, then it becomes constant, and if the dynamical system still followed such instructions it would move away from the neighborhood of equilibrium point. Starting from approximately 50 $\mathrm{sec}$ of system time the influence of derivatives through the model is more noticeable, and after that moment those derivatives (and, in fact, other components of utility function) determine the changes of control action.

\section{Generalizing abilities.}

When the system learned pole balancing starting from the upright position, the problem was a bit complicated. With the same arbitrary starting control action it had to learn convergence to the stable mode starting from a small angle. Using Action and Critic networks trained for zero-starting balancing, it was possible to train them further, gradually increasing the starting angle to maintain one-trial learning. At 0.6 rad training was stopped. The Action network obtained at this step was later used as the control network for test examples. In fact, trained Action network was able to stabilize cart-and-pole system with starting angle up to 0.68 rad, which it was not trained for.

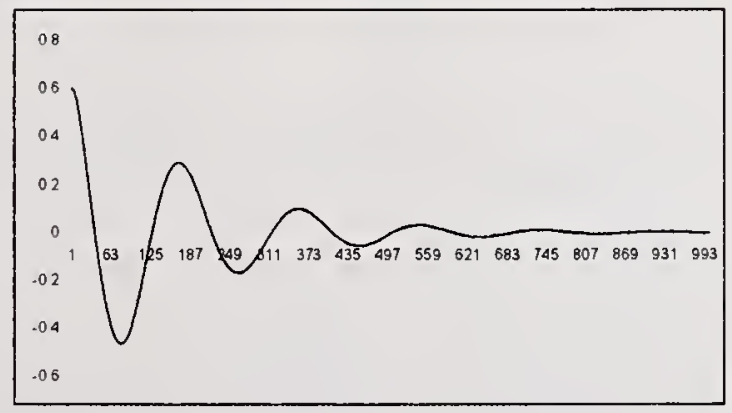

(a)

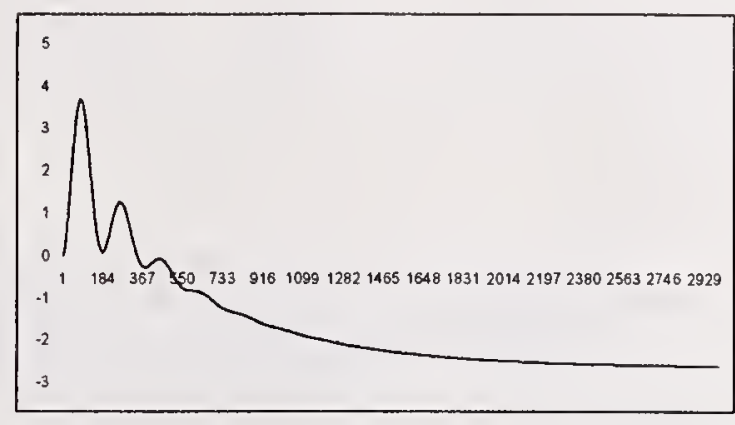

(b)

Fig. 3. Stabilizing the system staring from the angle $0.6 \mathrm{rad}$. Time interval is equal to $0.02 \mathrm{sec}$

The results of a test for starting angle $0.6 \mathrm{rad}$ are shown in Fig. 3. After $20 \mathrm{sec}$ of system time the pole angle reached 0 (actually, small oscillations around the zero point were maintained). Stabilizing of the cart coordinate took about $1 \mathrm{~min}$ of system time.

The next question was: is the controller truly adaptive, i.e. what if we change the parameters of pole and cart (mass, length of pole) or external parameters (friction, gravity)? Yes, it was really able to re-learn; moreover, this adaptation to new conditions occurred instantly (Fig.4).

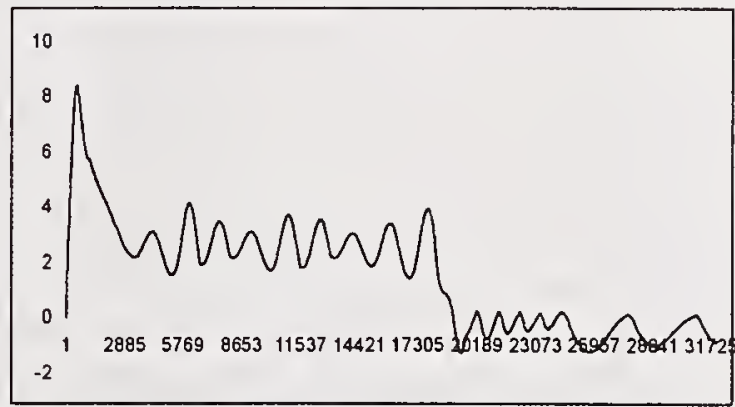

Fig. 4. Cart coordinate for training of the system with variable parameters. Time interval is equal to $0.02 \mathrm{sec}$

Learning was started from the standard parameter set defined in Table 1. After $2 \mathrm{~min}$ of system time the mass of the pole was changed to $0.3 \mathrm{~kg}$, and $2 \mathrm{~min}$ later it was changed to $0.05 \mathrm{~kg}$ without interrupting of learning. As can be seen at the chart for cart coordinate, such changes (point 6000 and 12000) were hardly noticeable. Again after $2 \mathrm{~min}$ of system time the length of the pole was changed to $3 \mathrm{~m}$. and the mass of the pole was set back to standard mass $0.1 \mathrm{~kg}$. This time it was noticed (point 18000), and it coincides with human experience -- the easiness of balancing depends more on pole length, than on its mass. At the point 24000 (next 2 minutes of system time) all parameters were reset to standard one; however gravity was decreased 10 times. The general structure of learning process remained the same, 
but the frequency of oscillations decreased, which was entirely expected according to physical laws.

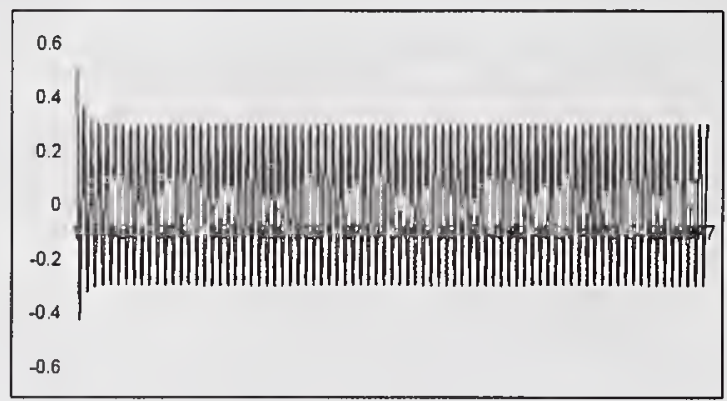

(a)

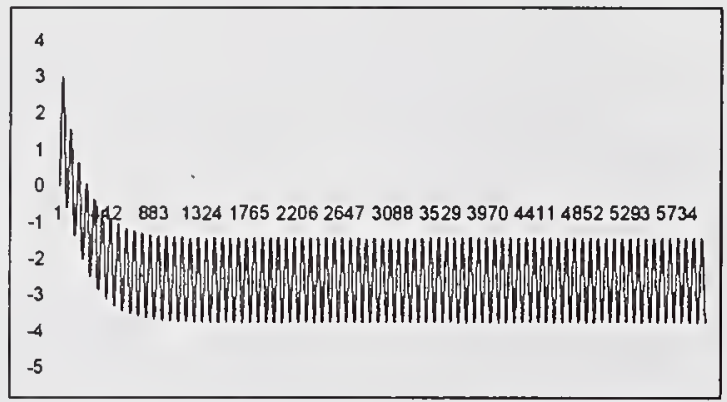

(b)

Fig. 5. Action strategy for larger control interval. Time interval is equal to $0.05 \mathrm{sec}$.

The last computer experiment that can be done with our controller is to put it into really hard conditions. It was trained for a control interval 0.02 sec; let us now assume that it is possible to apply control actions only every $0.05 \mathrm{sec}$. It can be seen from Fig. 5 that even with such a restriction, the Action network provides reasonable control without retraining, though the control strategy is quite different: instead of small oscillation around zero angle the system maintains a stable mode with large amplitude of oscillations.

Comparing our results with those of Balakrishnan [17] we can conclude that the resulting controller shows behavior close to the optimal control. It can be seen that choice of different utility function does not influence the nature of the stand-alone controller. The utility function is important only during the training stage; the resulting Action network should be close to optimal control law, whichever was the learning method. Thus, the most general type of utility function, derived, for example, from energy conserving conditions (minimal intensity of control) or desired set point description (minimal square of all state variables) should be preferred over the fancy "problem-dependent" functions.

\section{Shaping: an easy way to train the controller.}

Now we come to the exploitation of scaleinvariance of the model. Since the system should stabilize both pole angle and cart coordinate, the simplest utility function is

$U=-0.5 \theta^{2}-0.5\left(x / x_{\max }\right)^{2}$

where coefficients are chosen so as to simplify the derivatives of utility function.

However, it is very difficult to train the Action and Critic networks with such a general utility function. With high learning rate the force oscillates, but with low learning rate it is to slow to react properly. Note that now the system should learn to react only to target variables, not to their derivatives.

One technique, that is very efficient in such situations is called "shaping" in [13]. In shaping, one first adapts a simpler controller to a simplified version of the problem, one then uses the weights of the resulting controller as the initial values of the weights of the controller to solve the more complex problem, and so on up to the required level of complexity.

It is clear which is the critical parameter that determines the complexity of pole-and-cart control: gravity. So, the training was started from $\mathrm{g}=0.0981 \mathrm{~m} / \mathrm{sec}^{2} ;$ then, after convergence (about 60 $\mathrm{sec}$ of system time) this parameter was set to $\mathrm{g}=0.981 \mathrm{~m} / \mathrm{sec}^{2}$. This time training took a bit longer, but still after 5 trials the system learned to keep its balance starting from zero position and random (about $2 \mathrm{~N}$ ) initial force, as is shown in Fig.6.

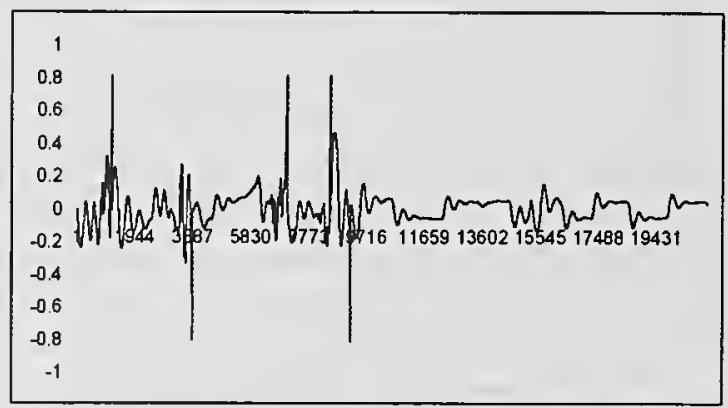

(a)

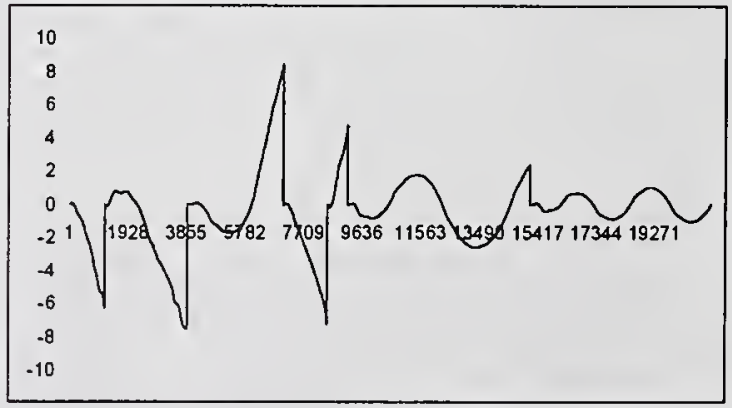

(b)

Fig.6. Training phase for $g=0.981 \mathrm{~m} / \mathrm{sec}^{2}$, general type of utility function: angle (a), $\mathrm{x}$-coordinate (b). Time interval equal 0.01 . 
After that the system was able to stabilize the pole with starting angle $0.5 \mathrm{rad}$ without re-learning, with the same high learning rates of Action and Critic networks. Before, with a problem-dependent utility function an increase of starting angle from 0.1 to $0.6 \mathrm{rad}$ required a decrease of learning rate from 0.7 to 0.1 .

The essential point in the training process, regardless of the utility function, (and mostly regardless of the concrete example) was that there be a difference between "convergence for training with possibility of weights correction" and "convergence for stand-alone action". In the first meaning, the system Action+Critic converged after at most 12000 iterations (120 sec of system time, see Fig.6). However, Action alone has been converged, in the second meaning, only 30000 iterations later. Moreover, the controller for $\mathrm{g}=0.981 \mathrm{~m} / \mathrm{sec}^{2}$ was able to control pole-and cart system up to the value of $g=4.905 \mathrm{~m} / \mathrm{sec}^{2}$.

Then the same shaping procedure was repeated for the standard value of gravity. After few trials it learned new external conditions, and at the end again Action network was able to control pole-and-cart system autonomously. For the last case the utility function was slightly changed to gave more importance to the changes in $x-$ coordinate.

None of the ACD pole balancer descriptions cited here (except, possibly [8], which implementation we had not possibility to evaluate in details yet) was able to learn a stable stand-alone controller working with simple general-type utility function under the conditions of full gravity. Like Balakrishnan [17] we were able to receive optimal control strategy under reduced gravity ( $\mathrm{g}=0.981$ ), however, then we scaled it up to real-life conditions. Shaping, as learning tactic was extremely useful and time-efficient for this purpose.

\subsection{AIRCRAFT AUTOLANDER: THE SIMPLEST DESIGN IS ENOUGH.}

The Autolander problem described in [1] concerns the simulation of aircraft landing. This linearized model of commercial aircraft and its environment includes such variables as longitudial velocity, vertical angular velocity, pitch rate and angle, altitude, horizontal position, autothrottle state, and wind disturbance state. Pitch angle command is used as control action. The equations, parameters, and constraints are those from [1], problem A.3.

The process of landing is guided by an airport-based lnstrumental Landing System (ILS), which provides desired values of height and vertical velocity of aircraft, and in this example controller must track the prespecified trajectory. In fact, it must learn the proper approach angle relative to the runway threshold, which should be maintained during the first phase of landing. During the second phase, starting at 45 feet height, the aircraft follows the exponential law, decreasing both altitude and vertical velocity, and elevating the nose.

As was said before, almost every model allows some undesirable freedom at the implementation stage, which can be treated in different ways. First, we re-defined the intermediate variable $a_{w}=U_{0} / h(t)$, where $h(t)$ is the height of aircraft at the moment $t$, to be $a_{w}=U_{0} / 10$ for $h<10$ to avoid division by zero. The height $\mathrm{h}=10$ was rather arbitrary. Another modification was not so small, but it seemed to be reasonable in the modeling context: during the flare phase in the original model ILS provided the desired values for height and vertical velocity starting from actual height of aircraft ( 45 feet) at the beginning of the flare regardless of what the desired height was just before this moment.

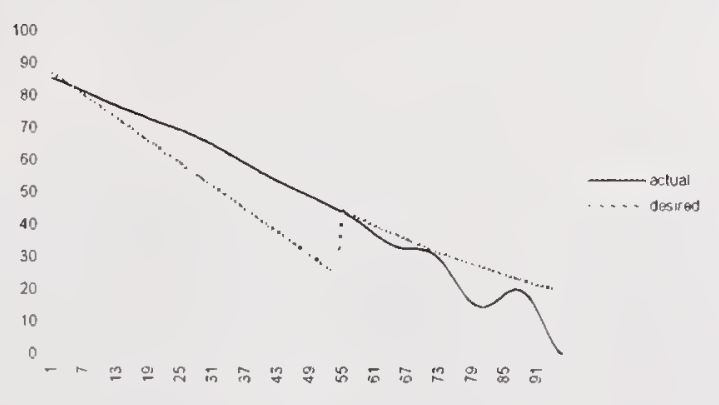

(a)

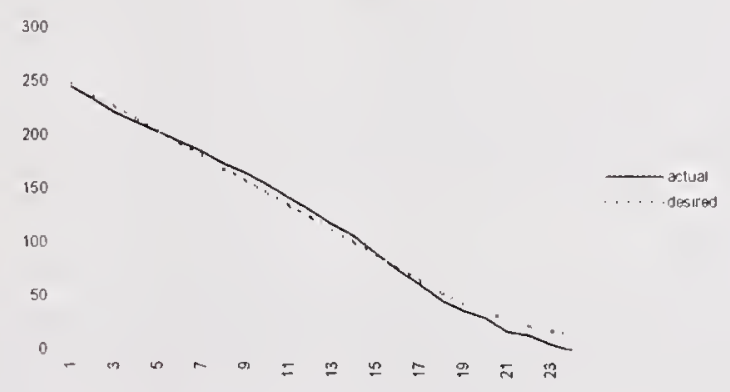

(b)

Fig. 7. Broken desired trajectory for the original model (a), and continuos one for modified model (b).

As a result, the desired trajectory had a break at $\mathrm{h}=45 \mathrm{ft}$, and the process of learning during the flare phase was biased. The difference between original (a) and implemented here (b) desired trajectory is shown in the Fig. 7. 
The last modification was related to starting conditions: the desired interception point calculated from ILS data was at the end of the runway, so instead of using real coordinate $\mathrm{x}$ to calculate the desired (ILS) height we used $x=x+500$.

Under these conditions the utility function can be written as

$U=-a_{1} 0.5\left(h-h_{c}\right)^{2}-a_{2} 0.5\left(\dot{h}-\dot{h}_{c}\right)^{2}$

where $h(\dot{h})$ is actual height (vertical speed) of the aircraft, and $h_{c}\left(\dot{h}_{c}\right)$ is the height (vertical speed) value supplied by ILS. Coefficients $a_{1}$ and $a_{2}$ varied during the training phase: first, $a_{1}$ was much greater than $a_{2}$, reflecting the priority of landing in the target zone of runway; then $\mathrm{a}_{2}$ was increased to provide fine tuning of vertical speed.

The state vector comprised all aircraft and environment variables, thus allowing us to avoid including the information from previous time intervals as additional input to Action and Critic networks. Besides, using an analytical model we can calculate exact derivatives with respect to control and input variables, and the accuracy of simulation and evaluation of state essentially increases, thus decreasing the number of iterations necessary for convergence.

\section{How it was really going}

Training of the system was done with backpropagation of utility [13] with calculation of utility function 10 steps forward. Taking into account that the sampling interval was $0.01 \mathrm{sec}$, and control interval $0.1 \mathrm{sec}$, it was almost real-time learning. We include here some comments to illustrate the concrete organization of this process. First, input vector normalization of at least some components of the input vector was necessary (division of height $(\mathrm{h})$ and horizontal coordinate $(\mathrm{x})$ by maximal values of these parameters, known in advance).

Next, for this kind of modeling it is important to simulate the noise vector outside the Model itself, because when we use dual subroutine DualModel for calculating the derivatives with respect to control and state vectors at the backward step, we need the same values of noise parameters as were used during the forward step, so it should be either the same input vector for Model and DualModel or an external vectors available for both. Another important point is already commonplace: don't use the standard $\mathrm{C}$ random number generator, which has extremely bad statistical properties.

\section{Wind shear, gusts, and generalization abilities}

Initially the system was trained in the absence of wind gusts, with wind shear only. Learning was fast, within 10 attempts the difference between actual and desired trajectory decreased significantly, and the Action network was able to land the aircraft starting from default conditions subject to constraints on touchdown region, vertical speed, horizontal speed and pitch value.

Then wind gusts were added, and learning was continued. Still the Action network was able to land the aircraft if control signals were generated using the information about utility values 10 steps forward. What really took time here, was the training of the Action network to generate control signals responding only to the state vector, without dynamic weights correction. After a few hundred iterations it was able to land the aircraft in wind conditions in the majority of cases.

As can be seen from Fig. 8(a), representing test results for 200 landings with random noise, most of the landings ended in the zone called "shortened runway" in [5] (below $600 \mathrm{ft}$. The distribution of vertical velocity is within a narrow range, though it doesn't satisfy the constraints of original model.

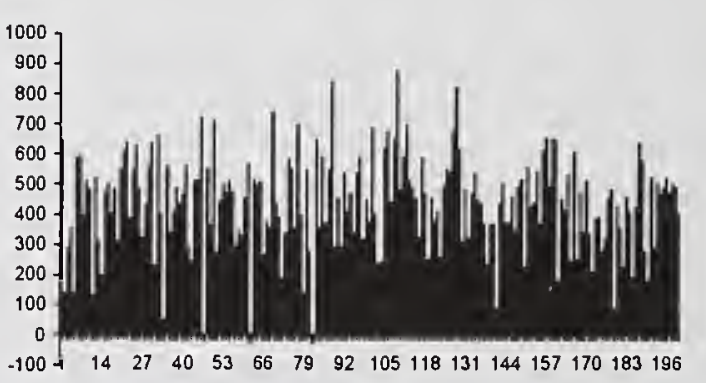

(a)

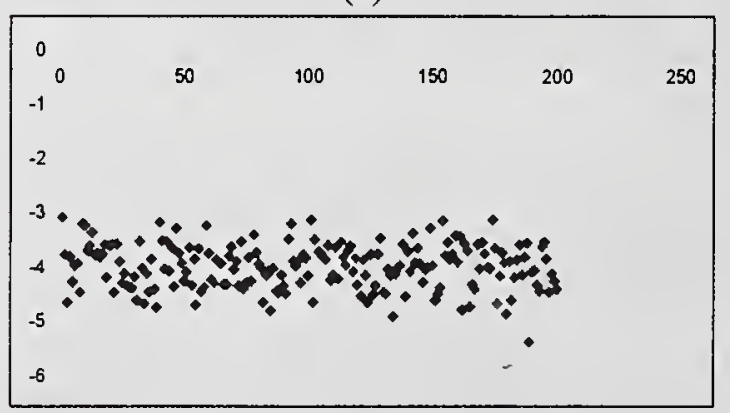

(b)

Fig. 8. Test results for landings with random wind gusts: $x$-coordinate (a), and vertical speed (b).

We can conclude that with a full and exact analytical model of aircraft it is possible to achieve a high rate of success even without guidance from an Instrumental Landing System. What's more important here, this rate has been achieved with 
simplest system design possible - a feedforward Action network and backpropagation of utility as learning method. This tells us that the autolander problem in this formulation hardly can be considered as suitable example for demonstrating the advantages of more sophisticated designs. DHP, for example, demonstrates approximately the same success rate, though with different distribution of $x$-coordinate and vertical speed; however DHP training took much more time for this case.

For examples of successful application of HDP, BU, and DHP to this problem one may see $[5,7]$. A brief description of successful application of GDHP can be found in [6]. A lot of model details are discussed in [19] where the problem was originally posed.

\section{FIRST OBSERVATIONS AND FURTHER DEVELOPMENT}

Though the examples described here are simply test problems, they allow us to make some informal observations that may be helpful for reallife applications. First, these problems are not complex enough to demonstrate the advantages of more sophisticated designs over simpler ones, like BTT . Next, they allow multiple final formalization that leads to different quality of models thus reducing the compatibility of results. The last technical remark: usage of de facto standard test problems should be more formal than we can see now. None of the papers cited here deals with exactly the same problem that any other paper deals with. There are always small differences -either the dimensions and components of input vector, or different values of coefficients, or slightly modified model. This complicates real comparison and reproducibility of results.

The same problems were recognized long ago in supervised learning, and the benchmark set PROBEN1 appeared [20]. In summary, there is still a need for the creation of more challenging and more fully specified benchmark problems and benchmarking rules for neurocontrol, which (ideally) should be disseminated over the Web and addressed in "challenge" sessions in major conferences.

It is important to distinguish between the complexity of ACD methods and the complexity of the problem in hand. The application of these methods can be straightforward, but the problem may be underdetermined or contradictory. The final formalization of a model may be done in different ways; the details of its implementation are also essential. Even the simplest examples described in this paper demonstrate these possibilities. Fortunately, ACD designs are powerful enough, and sometimes they can handle ill-defined problems, but this is not always the case. It is necessary, for instance, to provide proper normalization, and the same range of calculated and desired values of derivatives of utility function before final judgement about modeling quality. The optimal choice of parameters, like learning rate or network architectures is not an ACD-specific problem. It's just an inner problem of implementation modules that constitute the whole system, and relationships between parameters of different modules like those mentioned in [8] are most often the phantoms of more serious problems inside the modules.

If we consider the "ladder of designs", defined first in [2] then in greater detail in [21], an example clearly demonstrating the advantages of higher-level designs over lower-level ones should be something like this:

-- self-complete, not allowing to extend it using commonsense or physical knowledge;

-- formalized up to the end, including input-output parameters, integration scheme, list of adjusted parameters, normalization procedures;

-- showing visible increase of some quality measure while climbing on the ladder of designs;

Besides, it should be physically interesting. To develop such an example will be our next task.

Another direction of further research is to clarify some stability issues. As could be seen from more detailed analysis of the inverted pendulum example, if the system is inherently unstable the increase in calculated Critic value leads to greater increase in target Critic value, and the system quickly moves to or behind the accepted range of parameters. This can be avoided by programming tricks, like dynamic renormalization, but it would be preferable and more reliable is to solve such divergence problems at conceptual level.

\section{CONCLUSIONS}

When the problem at hand is solved, at least to the degree acceptable to potential users, it seems so easy that even the developer forgets the tricks he applied and the traps he avoided. Yes, his abilities as a developer have increased, and at the subconscious level he remembers the way to do things right, but here the question of expertise transfer arises. If something has been done, it would be useful to formalize a method of problem solving, even if it is not the way the problem was actually solved. First, it makes it possible to understand even one's own results more deeply. Next, such "step-by-step" instructions allow 
newcomers to the field to get their first results easier, thus creating positive motivation, which in turn stimulates their productivity. Also, it provides guidelines for future research. And finally, the most ambitious though long-term claim: such formalization of human experience leads to the possibility of automating some routine procedures and tactical decisions now reinvented again and again.

\section{REFERENCES}

[1] Anderson, C. W., and Miller, W. T.

Challenging control problems. In W. T. Miller, R.

S. Sutton, and P. J. Werbos (Eds.) Neural Networks for Control, The MIT Press, 1990, pp. 475-510.

[2] Werbos, P.J. A menu of designs for reinforcement learning over time. In W. T. Miller, R. S. Sutton, and P. J. Werbos (Eds.) Neural Networks for Control, The MIT Press, 1990, pp. 67-95.

[3] Werbos, P.J. Approximate dynamic programming for real-time control and neural modeling. In D. A. White, D. A. Sofge (Eds.) Handbook of Intelligent Control, Van Nostrand Reinhold, 1992, pp. 493-525.

[4] Santiago, R. A., Werbos P. J New progress toward truly brain-like intelligent control. In Proceedings of World Conference on Neural Networks, 1994, Vol. I, pp. 27-33.

[5] Prokhorov, D. V., Santiago, R.A., and Wunsch, D. C. (1995) Adaptive critic designs: a case study for neurocontrol. Neural Networks, Vol.8, No.9, pp. 1367-1372.

[6] Prokhorov, D. V (1995) A globalized dual heuristic programming and its application to neurocontrol. Proceedings of the World Congress on Neural Networks, Washington, D.C. July 17-21, Vol. II, pp. 41-43.

[7] Saini, G., and Balakrishnan, S. N. Adaptive critic based neurocontroller for autolanding of aircrafts with varying glideslopes. Proceedings of the International Conference on Neural Networks, Houston, June 9-12, 1997, pp. 2288-2293.

[8] Lendaris, G. G., and Paintz, C. Training strategies for critic and action neural networks in dual heuristic programming method. Proceedings of the International Conference on Neural Networks, Houston, June 9-12, 1997, pp. 712-717. [9] Werbos, P. J. Applications of advances in nonlinear sensitivity analysis, in R.Drenick \& F.Kozin (Eds), System Modeling and Optimization: Proc. IFIP Conf. (1981), Springer 1992.

[10] Werbos, P.J. Building and understanding adaptive systems: a statistical/numerical approach to factory automation and brain research, IEEE Trans. SMC, March/April 1987.
[11] Kolmogorov, A. N. On the representation of continuous functions of many variables by superposition of continuous functions of one variable and addition, Doklady Akademii Nauk SSR, V. 114, p. 953-956, 1957. English translation: Mathematical Society Translation, V. 28, p. 55-59, 1963

[12] Kurkova, V. Kolmogorov's theorem and multilayer neural networks. Neural Networks, V. 5, p. 501-506, 1992

[13] Werbos, P.J. Neurocontrol and supervised learning. In D. A. White, D. A. Sofge (Eds.) Handbook of Intelligent Control, Van Nostrand Reinhold, 1992, pp. 65-89.

[14] Friedland, Control System Design, McGraw Hill, 1985, Ch.4.

[15] Barto, A. G., Sutton, R. S., and Anderson, C. W. Neuronlike adaptive elements that can solve difficult learning control problems, IEEE transactions on Systems, Man, Cybernetics, Vol. 13, N. 5, 1983, pp. 834-846.

[16] Michie, D., and Chambers, R.A. BOXES: an experiment in adaptive control. In E. Dale and D. Michie (Eds.) Machine Intelligence, 2, Ediburgh, Scotland: Oliver and Boyd, pp. 137-152.

[17] Balakrishnan, S. N., and Biega, V. A dual neural network architecture for linear and nonlinear control of inverted pendulum on a cart. Proceedings of the IEEE International Conference on Control Applications, Deaborn, MI, Sept. 15-18, 1996, pp. 614-619.

[18] Ritter, H., Martinetz, T., and Schulten, K. Neural Computation and Self-Organizing Maps, Addison-Wesley Publ. Co., 1992, pp. 133-138. [19] Jorgensen, C. C., and Schley, C. A neural network baseline problem for control of aircraft flare and touchdown. In W. T. Miller, R. S. Sutton, and P. J. Werbos (Eds.) Neural Networks for Control, The MIT Press, 1990, pp. 403-425. [20] Pretchel, L. Proben 1 - a set of neural network benchmark problems and benchmarking rules, 1994. (ftp.ira.uka.de/pub/neuran/proben1.tar.gz) [21] Werbos, P.J. Learning in the brain: an engineering interpretation. In K. Pribram (Ed.) Learning as Self-Organization, Erlbaum, 1996. 


\title{
Neural Network Based Planner/Learner for Control Systems
}

\author{
J. Albus $\star$ N. DeClariso A. Lacaze $\star \circ$ A. Meystel $\star \bullet$ \\ $\star$ National Institute for Standard and Technology, \\ - University of Maryland, • Drexel University \\ lacaze@eng . und . edu
}

\section{ABSTRACT}

A neural network based planning/learning system that prunes its decision tree by generalizing upon results of previous experiences is proposed. The results for the case of and inverted pendulum controller are demonstrated [1].

\section{INTRODUCTION}

Neural Networks (NN) for intelligent control as a part of well known structures with "Adaptive Critic" are recommended $[2,3]$. However, the advantages of search are not fully used because the NN elements do not have any capability for searching.

Real-time Control System (RCS) architecture of intelligent systems [4] determines the functionality of Behavior Generation. The latter uses planning as a combination of job assignment and scheduling $[4,5]$ which determines the need in a subset selection (focusing attention) and alternatives exploration (searching). Complexity of doing this can be substantially reduced by forming a multi-resolutional system [6].

In this paper, we will follow the conceptual paradigm of multi-resolutional planning/learning outlined in $[7,8]$. We will build upon the learning algorithms used in Baby-Robot, $[9,10]$ but will implement them with the planning algorithm.

Planning/learning as a joint process relies upon simulation of multiple alternatives of the process, Construction of decision trees is necessary in many areas where reactive strategies are insufficient to achieve the desired performance (for example, manufacturing, obstacle avoidance, control systems, medical interventions, etc). Most of these planning problems are solved in one of the following ways:

- Search techniques. These include Dijkstra, A*, beam search and most of the AI techniques like (genetic algorithms and expert systems). These solutions are considered to be too slow for real time operations and are in most cases nonlearning search techniques.
- Dispatching techniques. Frequently the designers decide that planning is altogether too expensive. Instead, they supply several general rules of thumb that are supposed to reactively improve performance. Unfortunately, these rules of thumb are very general (FIFO, LIFO, bidding) and usually under-perform most search algorithms. The dispatching techniques are equivalent to "greed" algorithms and generally, cannot ensure the optimality of solutions.

The proposed NN-based Planner/learner has performance advantages over the dispatching algorithms with adaptation. It plans (instead of reacting), and it learns rules (or rules of thumb) that allow it to search only a reduced set of the decisions in the decision tree.

The proposed NN Planner/learner learns rules of actions for different lengths of strings of experiences, and it learns to simulate the results of this actions. Then, the NN-planner creates a look ahead search where the different action rules are explored, and the simulator predicts new states. This tree is explored before any control is applied.

Before giving the details of the NN planner/learner, we present its building blocks (NNs) and how are they trained to learn both the action rules and the simulation rules.

\section{The Neural Network Algorithm}

In order to develop the NN based planner/learner. Standard NNs were applied. The training algorithm uses the Levenberg-Marquardt variation of Newton's method to modify the NNs weights. The algorithm approximates the Hessian matrix:

$$
\nabla^{2} F(\mathbf{x}) \tilde{=} 2 \mathbf{J}^{T}(\mathbf{x}) \mathbf{J}(\mathbf{x})
$$

where $\mathbf{J}(\mathbf{x})$ is the Jacobian matrix. And Newton method can be written as:

$$
\mathbf{x}_{k+1}=\mathbf{x}_{k}-\mathbf{A}_{k}^{-1} \mathbf{g}_{k}
$$




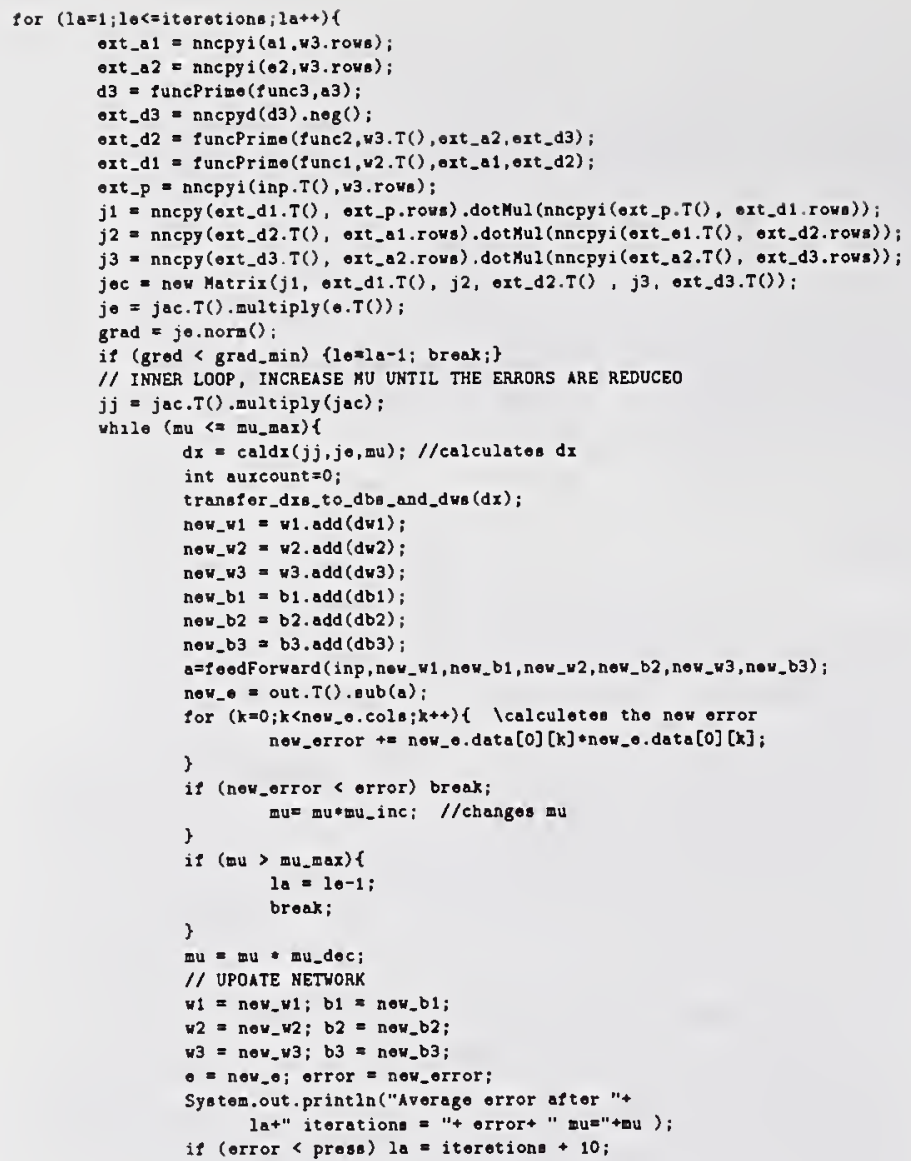

Figure 1: Levemberg-Marquardt JAVA implementation

where $\left.\mathbf{A}_{k} \equiv \nabla^{2} F(\mathbf{x})\right|_{\mathbf{x}=\mathbf{x}_{k}}$ and $\left.\mathbf{g}_{k} \equiv \nabla F(\mathbf{x})\right|_{\mathbf{x}=\mathbf{x}_{k}}$. Thus, by plugging in we get,

$$
\mathbf{x}_{k+1}=\mathbf{x}_{k}-\left[\mathbf{J}^{T}\left(\mathbf{x}_{k}\right) \mathbf{J}\left(\mathbf{x}_{k}\right)\right]^{-1} \mathbf{J}^{T}\left(\mathbf{x}_{k}\right) \mathbf{v}\left(\mathbf{x}_{k}\right)
$$

the problem with the previous equation is that $\mathbf{J}^{T}\left(\mathbf{x}_{k}\right) \mathbf{J}\left(\mathbf{x}_{k}\right)$ may not be invertible. Thus, LevenbergMarquardt algorithm warrants invertibility,

$$
\mathbf{x}_{k+1}=\mathbf{x}_{k}-\left[\mathbf{J}^{T}\left(\mathbf{x}_{k}\right) \mathbf{J}\left(\mathbf{x}_{k}\right)+\mu_{k} \mathbf{I}\right]^{-1} \mathbf{J}^{T}\left(\mathbf{x}_{k}\right) \mathbf{v}\left(\mathbf{x}_{k}\right)
$$

by looking at the previous equation, we see that if $\mu_{k}$ is large, then the algorithm approaches the steepest descent algorithm. If it is 0 , it becomes the GaussNewton algorithm. In our implementation, different $\mu$ are tested, and the best one is selected at each epoch. The core of the Java implementation shows how this was implemented is shown in Figure 1.

The complete algorithm was successfully tested using the standard problems (XOR, etc.). As far as transfer functions the $\log$ sigmoid $a=\frac{1}{1+e^{-n}}$ and the hyperbolic tangent sigmoid $a=\frac{e^{n}-e^{-n}}{e^{n}+e^{-n}}$ were implemented. The hyperbolic tangent was used in the examples for this paper.

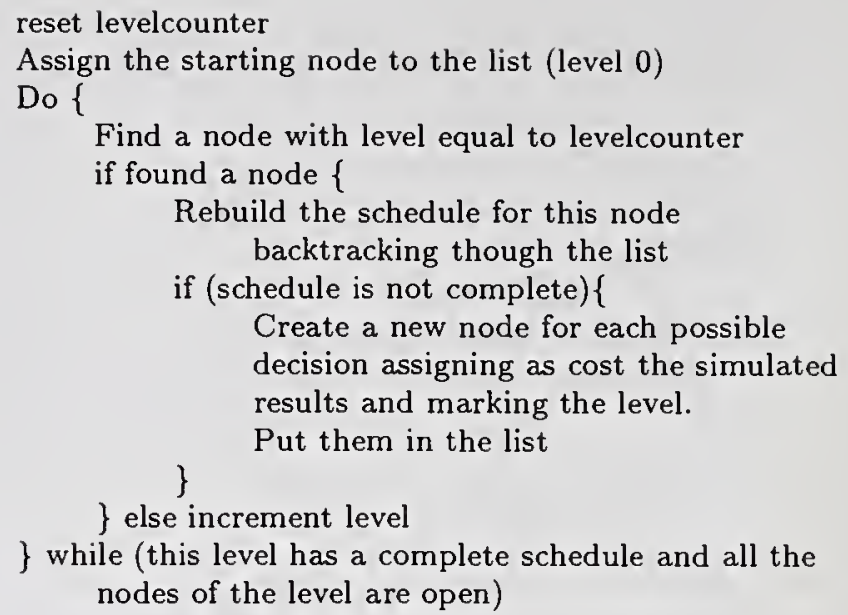

Figure 2: Pseudocode for scheduling using dynamic programming principle

\section{The Planning Algorithm}

This paper will go over the two most known planning algorithms. Most of the other algorithms are derived from these two, and they normally lose generality by doing so.

\section{III.A. Dynamic Programming}

The fundamentals of dynamic programming were introduced in the 1950's by Bellman $[11,12]$ and thoroughly explained in $[13,14]$. Dynamic Programming interprets any optimization as a multi-stage decision process. The results of dynamic programming can become known locally only after the total search for the best trajectory is completed.

To calculate the optimal criterion value for any subset of size $k$, we first have to know the optimal value for each subset $k-1$. In this case, $k$ represents the level in the tree of search. Figure 2 shows the pseudocode dynamic programming process.

Dynamic programming originally was applied in cases where the branches of the tree cost the same. The space was intentionally discretized such that moving to successors would generate exactly one unit of cost. In these cases, dynamic programming opens exactly the same amounts of nodes as Dijkstra Algorithm (next). In cases when the cost of the edges of the graph are not the same, Dynamic programming open significantly more nodes than Dijkstra. No matter which form is chosen, dynamic programming requires completion of the search to the goal .

\section{III.B. Dijkstra Search}

The average computational requirements are considerably less than those of the dynamic programming 


\section{AN ExAmple}

Assign the starting node to the open list

Do \{

Find the node with the smallest cost in the open list and move it to the closed list

Rebuild the schedule for this node backtracking through closed list

if (schedule is not complete)

Create a new node for each possible decision assigning as cost the simulated results.

\} Put them in the open list

\} while (schedule is not complete)

Figure 3: Pseudocode for scheduling using Dijkstra algorithm

[15]. A pseudocode for Dijkstra algorithm applied to scheduling is presented in Figure 3 . The Dijkstra algorithm, in the same manner as dynamic programming warrants that the solution is optimal. The difference between dynamic programming and Dijkstra algorithm is that dynamic programming iterates in the number of arcs in the graph while Dijkstra iterates on the length (or cost) of the arcs. An interesting comparison between Dijkstra, dynamic programming and Floyd-Warshall algorithm is presented in [15].

\section{III.C. Using Neural Networks to Prune the Dijkstra Tree}

We can use NNs within the Dijkstra Planner in two possible ways:

1. As simulators. NNs can be trained to predict what will be the next state given the current state and the decision. Of course there are some cases where the plant complexity itself will make this mapping hard to train. In these cases (which may be most) the system will have to be divided in different hierarchical levels to handle its complexity. This issue is outside of the scope of this paper. For the examples given, one level is sufficient, and this mapping can be learned with sufficient accuracy to make the planning plausible.

2. As control rules. If information of the previous working of a controller for the plant or results of previous search with the same goal, this information can be used to train NNs to suggest patterns of decisions that where successful in the past. We propose to use abduction: search the tree of future decisions by exploring first paths that were successful in the past. Of course, this technique will not give better results than the full search, but there are many cases where full search is not an option.
For this example we have chosen the inverted pole. The dynamical equations are as follows:

$$
\begin{aligned}
x_{1}^{\prime}= & x_{2} \\
x_{2}^{\prime}= & \frac{\left(\frac{-\mu_{p} \cdot x_{2}}{m \cdot l}+g \cdot \sin \left(x_{1}\right)+\frac{\cos \left(x_{1}\right)}{M+m}\right)}{l \cdot\left(\frac{I}{(m * l * l)}+1-\left(\cos \left(x_{1}\right)\right)^{2} \cdot \frac{m}{M+m}\right)} * \\
& \frac{\left(U-\mu_{r} * \operatorname{sign}\left(x_{4}\right)-m \cdot l \cdot x_{2}^{2} * \sin \left(x_{1}\right)\right)}{l \cdot\left(\frac{I}{(m * l * l)}+1-\left(\cos \left(x_{1}\right)\right)^{2} \cdot \frac{m}{M+m}\right)} \\
x_{3}^{\prime}= & x_{4} \\
x_{4}^{\prime}= & \frac{U-\mu_{r} \cdot \operatorname{sign}\left(x_{4}\right)}{M+m}+ \\
& \frac{m \cdot l \cdot\left(x_{2}^{\prime} \cdot \cos \left(x_{1}\right)-x_{2}^{2} * \sin \left(x_{1}\right)\right)}{M+m}
\end{aligned}
$$

where

$$
\begin{array}{rc}
\text { mass of pendulum } & m=1 \mathrm{Kg} \\
\text { pivot of center of mass } & l=0.5 \mathrm{~m} \\
\text { inertia } & I=\frac{\mathrm{m} . \mathrm{l}^{2}}{3} \\
\text { pivot friction } & \mu_{p}=2 * 10^{-6} \frac{\mathrm{kgm}}{\mathrm{s}} \\
\text { mass of cart } & M=1 \mathrm{~kg} \\
\text { rolling friction } & \mu_{r}=5 * 10^{-4} \frac{\mathrm{kgm}}{\mathrm{s}^{2}} \\
\text { gravity } & g=9.81 \frac{\mathrm{m}}{\mathrm{s}^{2}}
\end{array}
$$

In order to simulate this equations, Runge-Kutta was implemented in JAVA (see Figure 4).

The JAVA applet is shown in Figure 5

\section{LeARning the Control Rules}

\section{V.A. The Standard Way}

The first step was to test the NN algorithm in the pole simulation using the standard technique of learning the input-output mapping. The following experiment was performed:

1. A controller was built using the following parameters:

$$
\begin{gathered}
U=(\text { random } \\
+400 * 2-1) * \text { error } \\
+\left(x_{3}-\text { goalpos }\right) \\
+400 * x_{4} \\
-1089.172 * x_{1} \\
-401.27 * x_{2}
\end{gathered}
$$

To make it a little bit more realistic, a maximum force of $U$ was not allowed to get out of the interval $[-300,300]$. An error is introduced as function of error. The position of the goal goalpos is randomly placed at random intervals.

2. A string of 1000 states and actions were recorded to simulate the normal working of the plant with some error. 


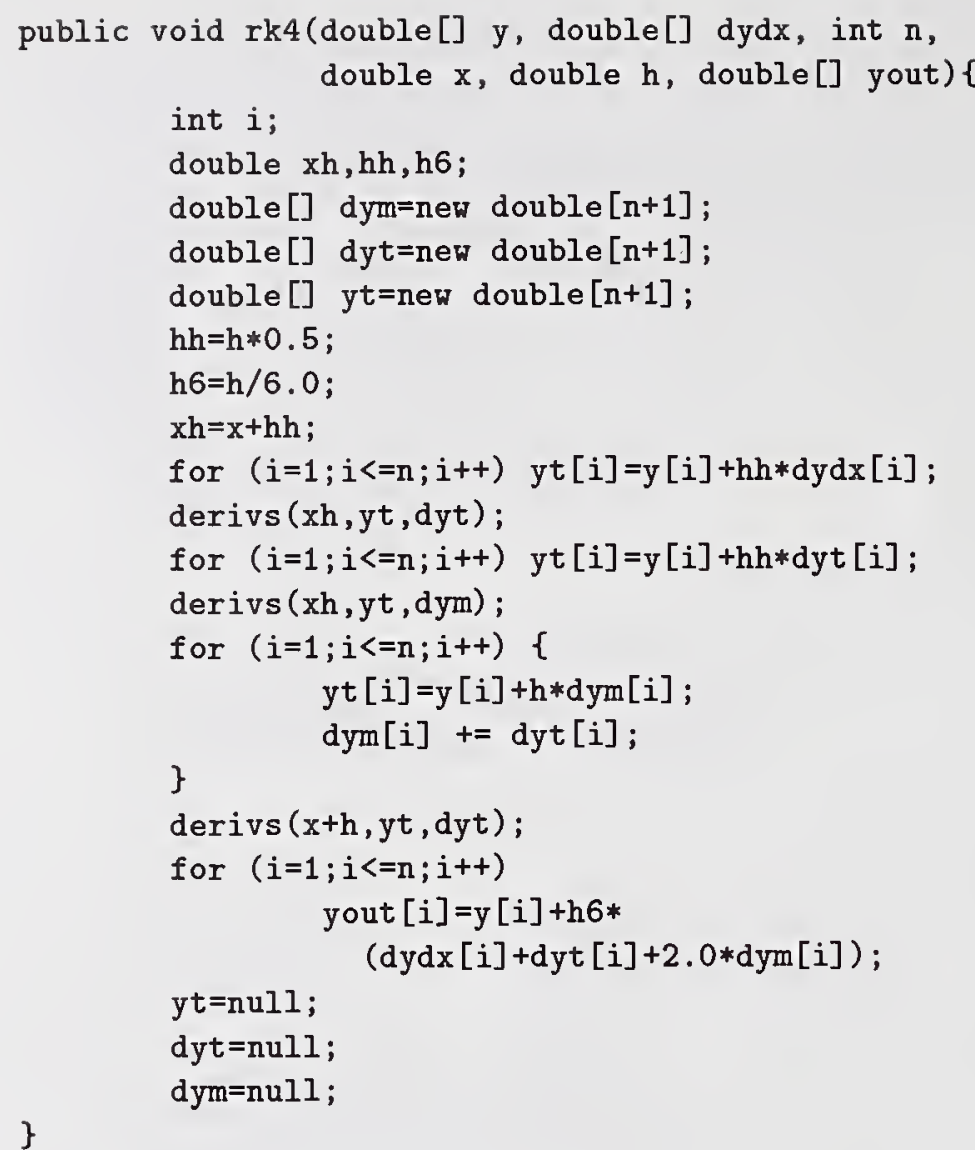

Figure 4: Runge-Kutta Java implementation

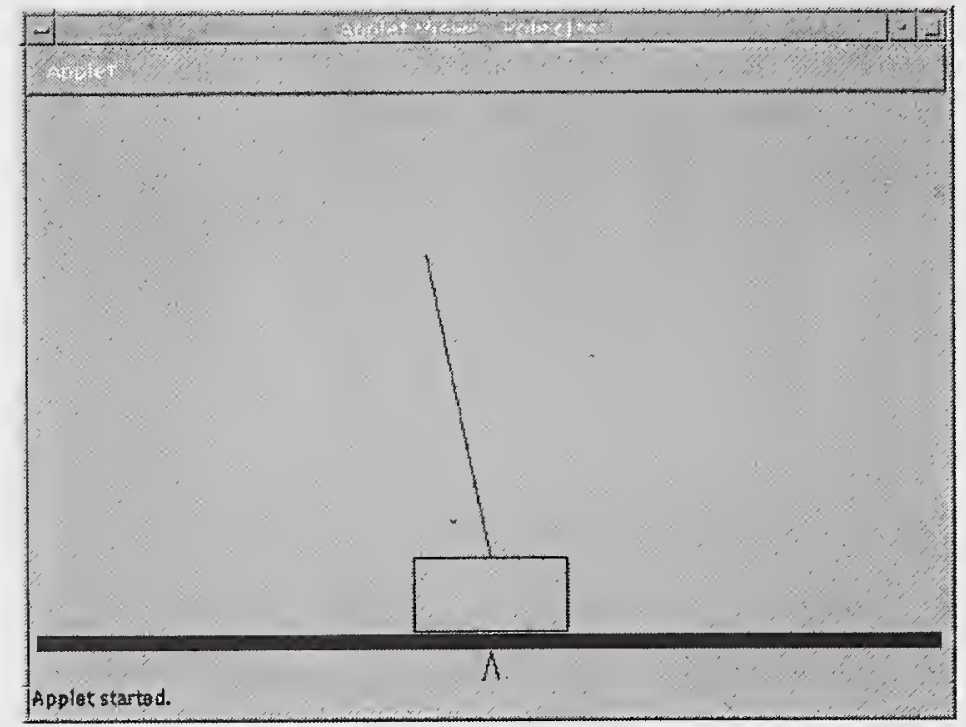

Figure 5: Pole.class applet of the inverted pole

A NN was trained by using the chosen control algorithm to learn the $U$ given the states. The structure of the net was a 6-6-1 net using the tangential logarithmic activation function. Figure 6 shows the error function of the network while training.

Figure 7 shows the error of the plant operating with the trained NN and the error of the plant operating with the original controller. This figure was created by accumulating the $e r=a b s\left(x_{1}-\right.$ goalpos $)+a b s\left(x_{3}\right)$

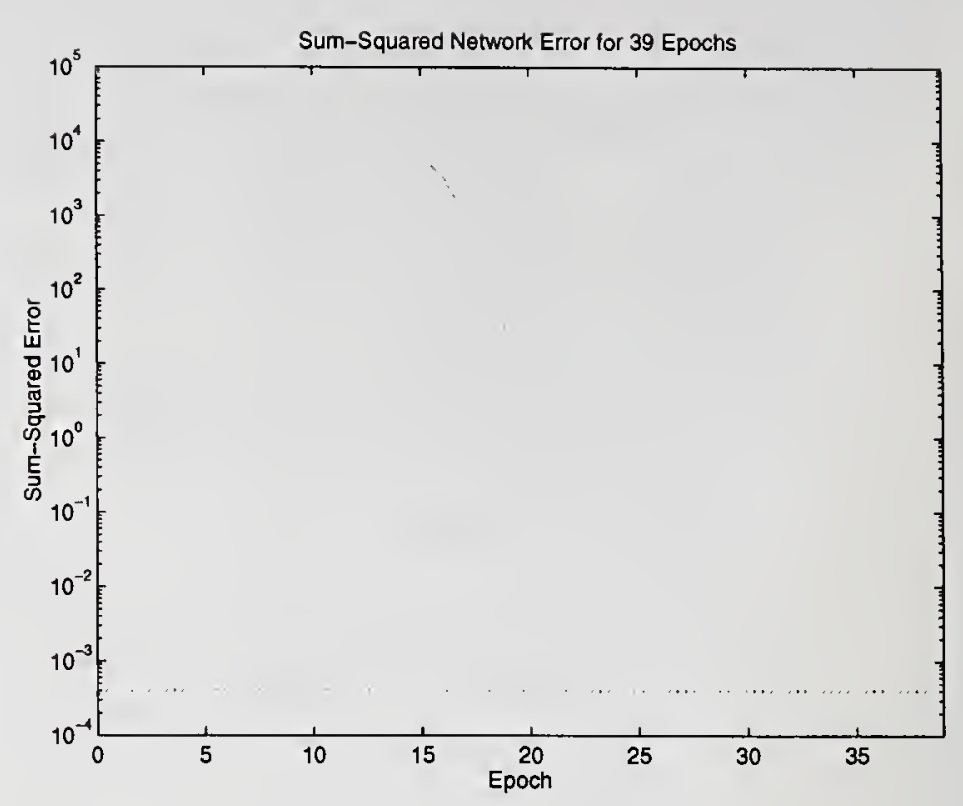

Figure 6: Learning error of the input-output function

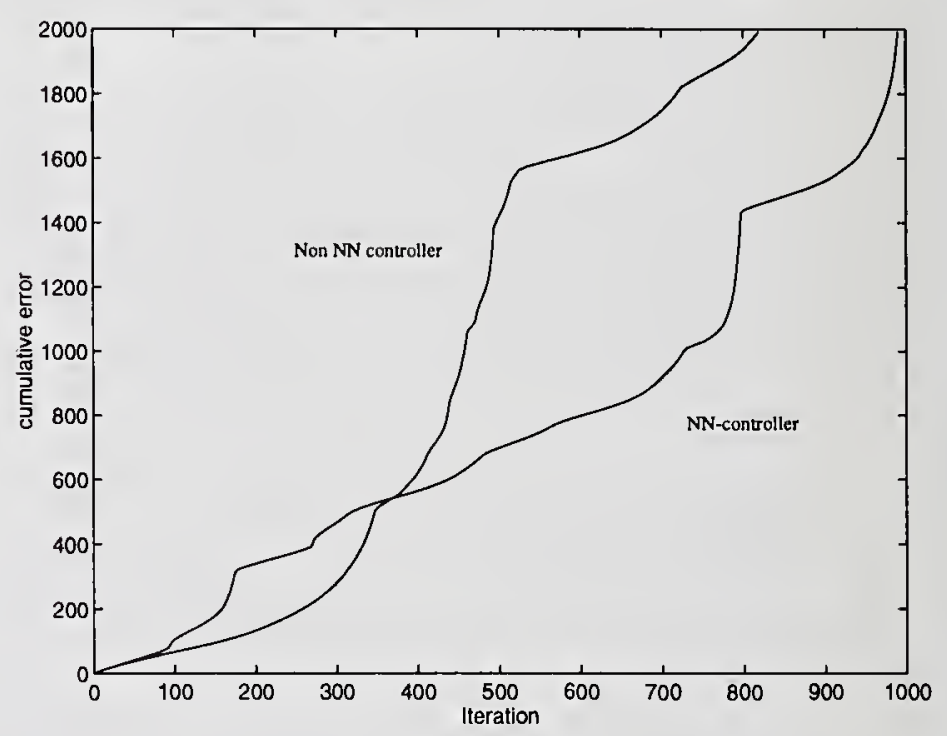

Figure 7: Similar cumulative error values for the NN controller and the non-NN controller, for multiple goals and compound cost function

during the normal operation of the plant. The goal is randomly changed, causing of the waves seen in the figure. The error (error is not er) was not modified. The results show similar error functions for the NN and the original controller. Figures 8 and 9 show also similar results using different cost functions: $e r=$ $a b s\left(x_{1}-\right.$ goalpos $)$ and $e r=a b s\left(x_{3}\right)$ respectively. For the last two examples, the goal was kept fixed, which is why the function is not wavy.

Please note that these results where achieved without the planner and are only intended to show that the NN is learning the control rule.

\section{V.B. Learning for Planning}

One of the biggest problems in learning is finding the correct measure of goodness that allows the al- 


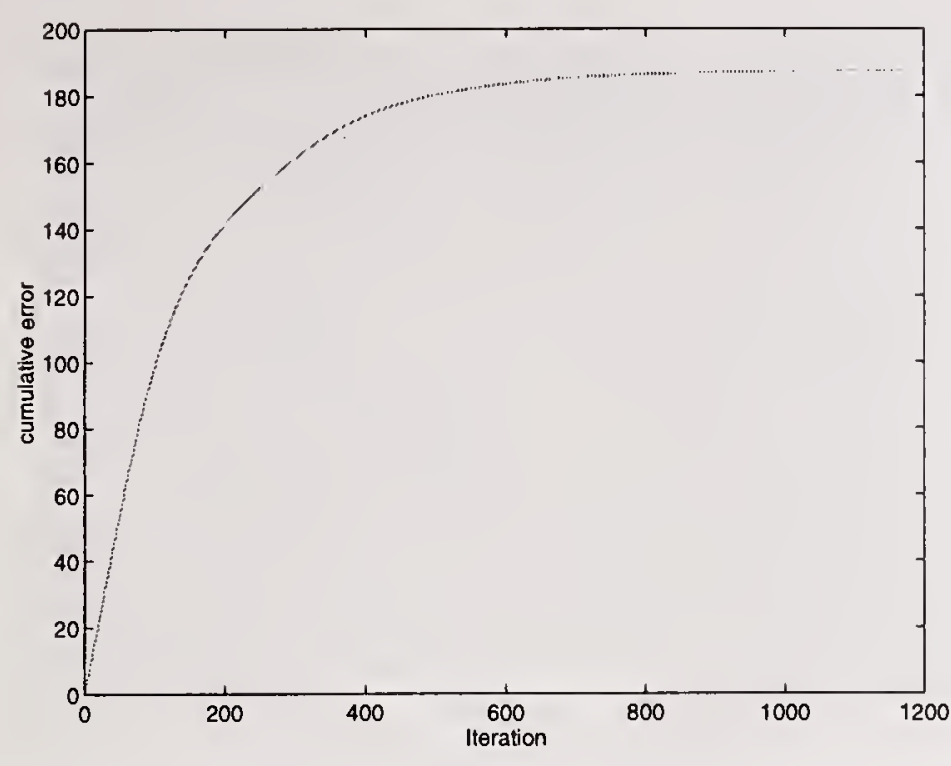

Figure 8: Similar cumulative error values for the NN controller (lighter curve) and the non-NN controller, for one goal and position cost function.

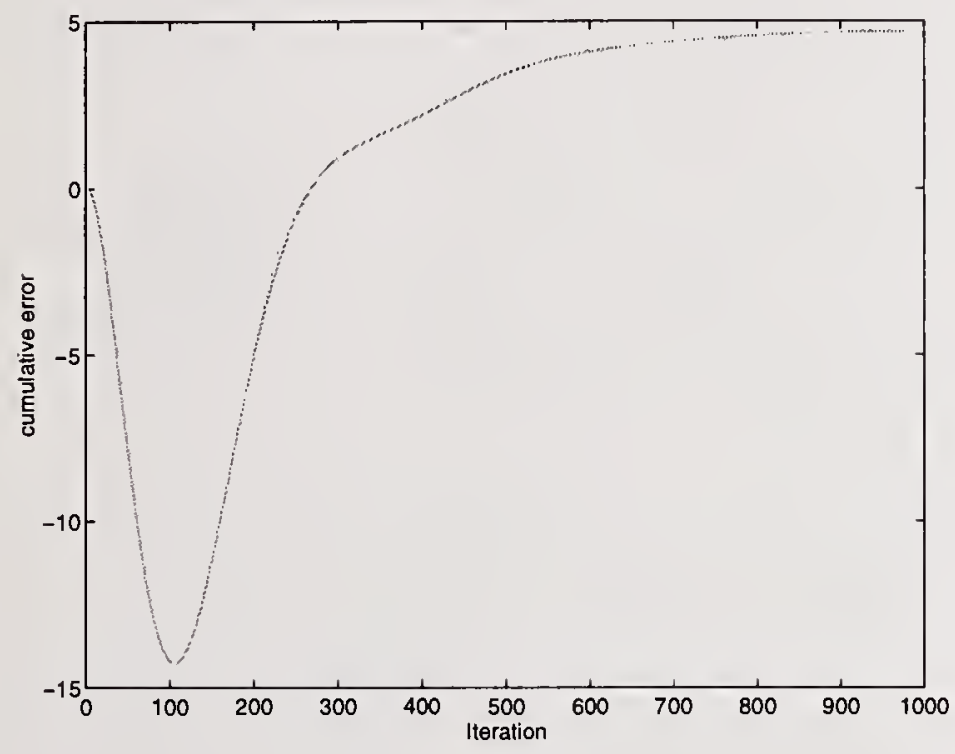

Figure 9: Similar cumulative error values for the NN controller (lighter curve) and the non-NN controller, for one goal and angular cost function

gorithm to separate the "good" examples from the "bad" examples. This measure cannot be introduced unless many circumstances of functioning are taken into account. For example, if the cart is to the left of the goal and the pole is angled to the left, one would imagine that a force to the left could bring the cart and the balanced pole to the left. If the force is high, in the short term, the impression will be that the cart has made good progress. But unfortunately this may change as soon as the goal is achieved. The forces of inertia will "over-regulate" the pole. On the other hand, we can have long term measures of goodness that are worthless in the short term.

Our planning system uses not a single one of these

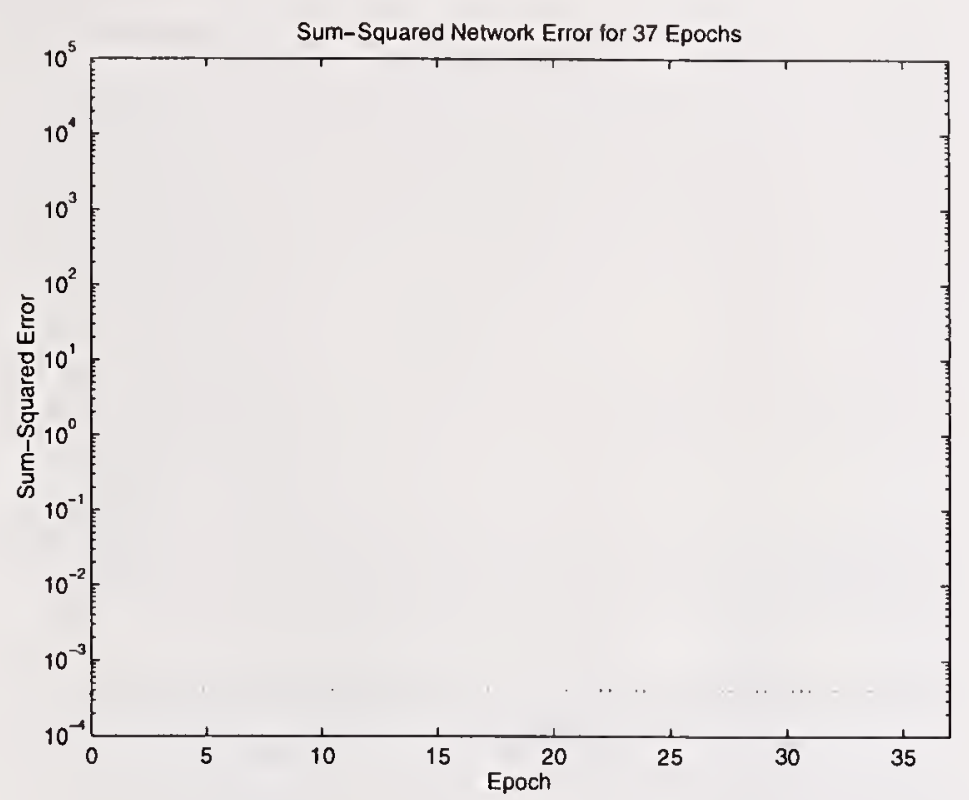

Figure 10: Learning error of the state-action to new state function

heuristics but all of them in a planning system. In this case, we can create control rules by training NNs on experiences that are good at different length experiences (strings of state-action-state...). For example, we can take all the $S A S$ (state action state) triplets that are above a certain measure of goodness. Goodness is calculated as the difference to the distance to the goal before and after the string of actions. The same can be repeated for $S A S A S$ quintuples and with longer strings. The NNs trained with shorter strings will have many experiences, since there is higher number of shorter strings than longer ones. The cost measure will give correct results only in the short term, which may not be good. On the other hand NNs trained with longer strings will have less strings, but the cost measure is trustworthy in a longer term. In this case we are interested in minimizing the cumulative error.

The idea is to have these networks store behavior that was successful in the past at different string lengths. The planner will start exploring the decision space along this successful paths by creating tentative combinations of strings with different lengths. In most plants behavior that was successful in the past has a higher probability of future success. From the errors that were introduced in the old plant controller we want to learn behaviors that where successful not because of the controller but by chance.

The NN for simulation was trained with the state and action as input and the next state as output. The Levemberg-Marquardt algorithm was used and the structure of the net is 5-5-4 net. The evolution of the training error can be seen in Figure 10, and the output is compared to the Runge-Kutta value in Figure 11. 

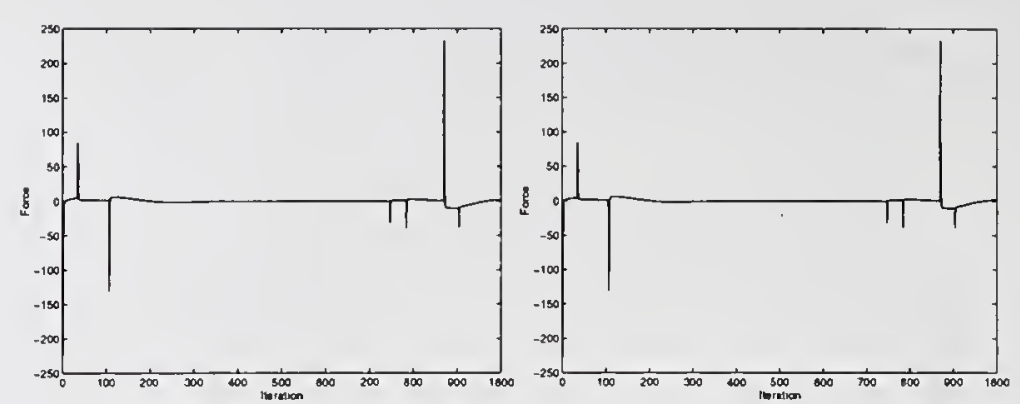

Figure 11: Runge Kutta simulation output (top) and NN output

The complete simulator can be trained using only one NN in very small problems. Where it becomes computationally costly to train to increase the number of nodes to allow the network to properly model the system, the problem can be divided into smaller pieces. There could be a set of networks covering all or some of the space that we want to simulate. This interesting topic is outside of the scope of the paper.

\section{THE NN PLANNER}

The neural network planner learns two kinds of rules from previous experiences: action rules, and simulation rules. Action rules have the form

$$
S \rightarrow A, g
$$

where $S$ is a situation vector, $A$ is a action vector, $g$ is goodness estimate, and $\rightarrow$ implies mapping. These rules will be used by the planner to select the actions that where good performers in the past.

Simulation rules are the second kind of rules that are learned by this algorithm. They have the form

$$
S_{i}, A_{i} \rightarrow A_{i+1}
$$

where $S_{i}$ is a situation vector, $A_{i}$ is a action vector, and $S_{i+1}$ is an approximation of the state vector where the system is likely to be after action $A_{i}$ is applied at $S_{i}$

The NN planner uses all the networks shown before. The steps performed by the planner are as follows:

1. The system runs using the previous controller introducing noise in its output to the plant. The state $(S)$ used by the old controller and the action $(A)$ applied by the controller (with the noise) will be stored in a database.

2. Experiences $\left(S_{i} A_{i} S_{i+1}\right)$ (Figure 12) are ordered based on the goodness. Where goodness is the difference of the distances to the goal before and after $A$.

3. The "best" experiences are selected.

4. A neural network with $S_{i}$ as input and $A_{i}$ as output is trained. We will call these NNs control rules.

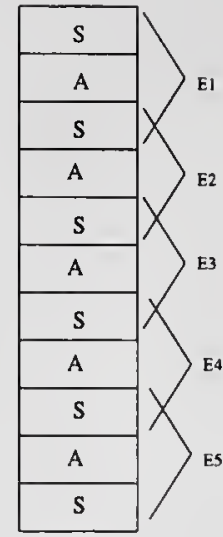

All experiences

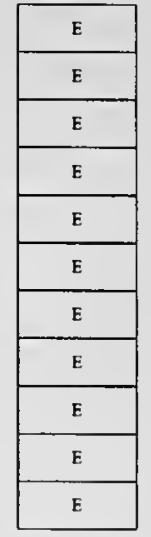

Good experiences

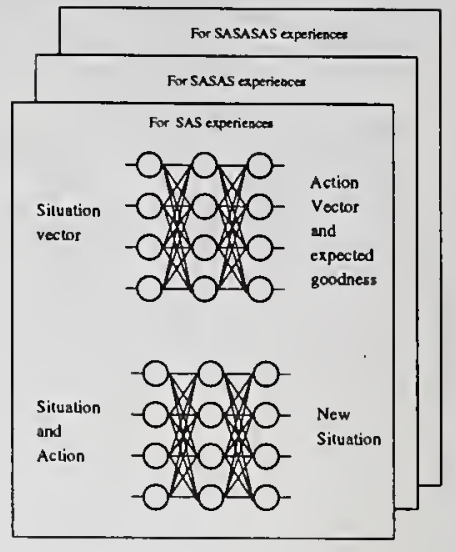

Figure 12: Proposed Approach

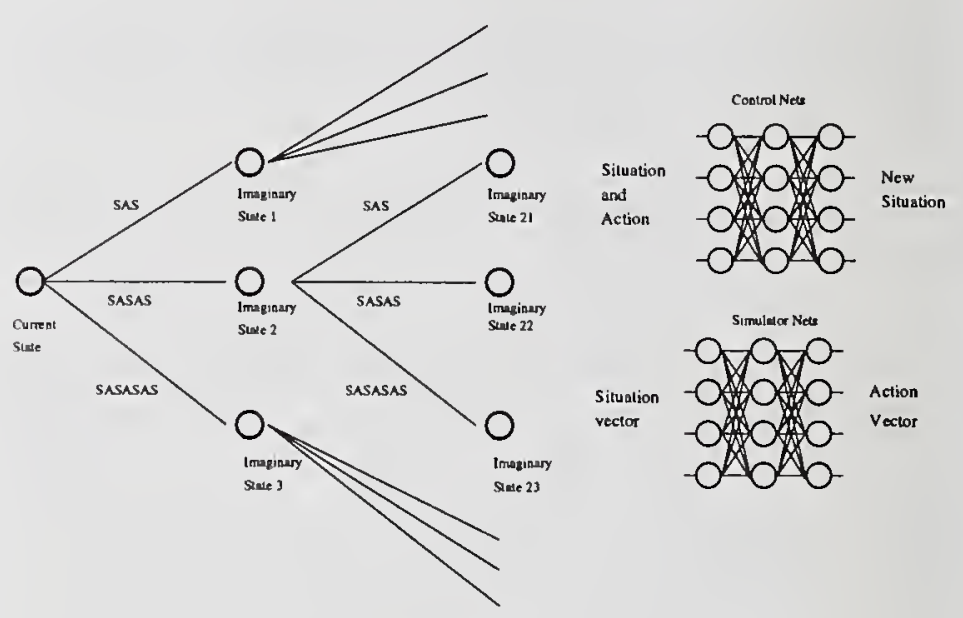

Figure 13: Decision tree

5. A neural network with $S_{i}$ and $A_{i}$ as input and $S_{i+1}$ as output trained. We call these NNs simulation elements.

6. The previous 4 steps are repeated for $S_{i} A_{i} S_{i+1} A_{i+1} S_{i+2}$, $S_{i} A_{i} S_{i+1} A_{i+1} S_{i+2} A_{i+2} S_{i+3}$, etc.

7. A lookahead search tree is generated using the current state as the starting point and the control rules for $S_{i} A_{i} S_{i+1}, S_{i} A_{i} S_{i+1} A_{i+1} S_{i+2}$, etc where $S_{i}$ match the current situation. Once these actions are applied, we use the simulation elements to simulate the next imaginary state (because we have not applied the action yet). We repeat this procedure again until we achieve the desired imaginary state or we run out of time. This lookahead planner is illustrated in Figure 13. Please note that if enough time is allocated, paths with actions that are not recommended by the control rules can also be explored.

8. The first action in the best path through this decision tree is chosen as the action to execute. 


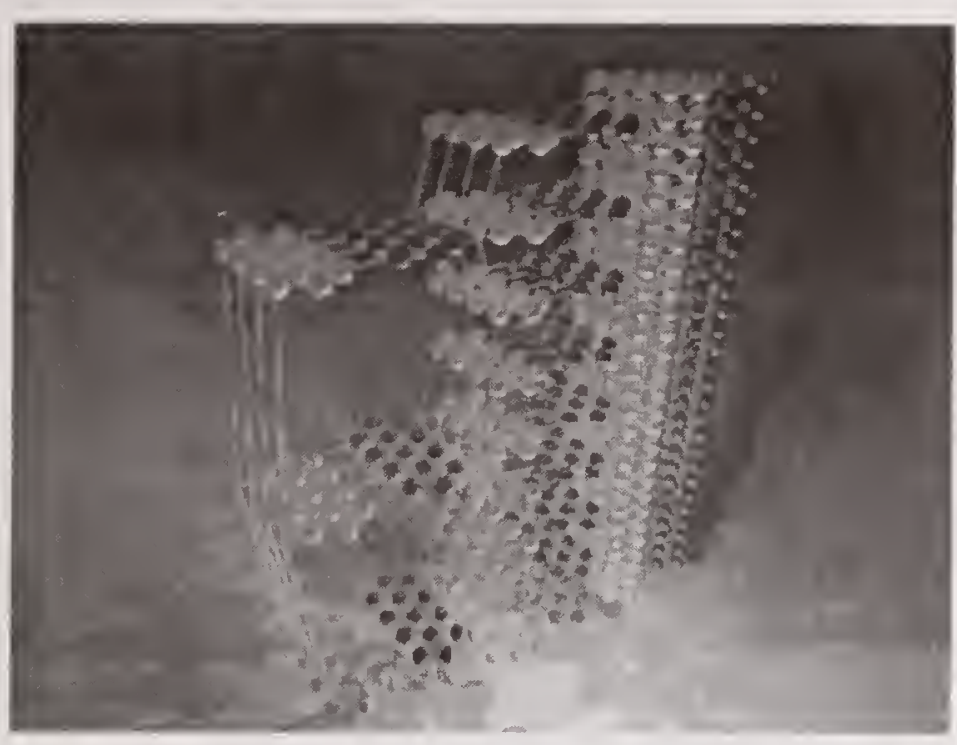

Figure 14: NN planner

Figure 14 shows a $3 \mathrm{D}$ visualization of the process of developing a decision plan. As shown, only three control rules exist in this example. The opening of this decision tree follows the Dijkstra algorithm.

\section{CONClusions AND Future Work}

1. A planning/learning subsystem is constructed to use in control systems

2. The planner first explores the previously successful paths but it continues with the search if sufficient time is given. This planner will find optimal trajectories.

3. The system incorporates both learning and searching which makes it different and advantageous in comparison with the known systems

4. In the future, we are going to implement and compare other NN engines: CMAC, conjugate gradient, etc.

5. The approach seems to outline a constructive model of brain-like intelligence.

\section{REFERENCES}

[1] A. Lacaze. A neural network planner that prunes its own tree. Term Paper, Knowledge Engineering, Electrical Engineering Department, University of Maryland, May 1997.

[2] A. G. Barto. Neuronlike adaptive elements that can solve difficult learning control problems. IEEE Transactions on System, Man and Cybernetics, 13:834-846, 1983.

[3] D. A. White and D. A. Sofge, editors. Handbook of Intelligent Control. VNR, New York, 1992.

[4] J. Albus. Outline for a theory of intelligence. IEEE Transactions on Systems, Man, and $\mathrm{Cy}$ bernetics, 21:473-509, 1991.

[5] J. Albus and A. Meystel. A reference model architecture for design and implementation of in- telligent control in large and complex systems. International journal of Intelligent control and Systems, 1:15-30, 1996.

[6] J. Albus, A. Meystel, and A. Lacaze. Multiresolutional planning with minimum complexity, in this volume.

[7] A. Meystel and A. Lacaze. Unified learning/planning automaton: generating and using multigranural knowledge hierarchies, in this volume.

[8] A. Meystel and A. Lacaze. Introduction to integrated/learning planning, in this volume.

[9] J. Albus, A. Lacaze, and M. Meystel. Evolution of knowledge structures during the process of learning. In Semiotics Workshop of the 10th IEEE International Symposium on Intelligent Control, Monterey ,CA, August 1995.

[10] J. Albus, A. Lacaze, and M. Meystel. Multiresolutional intelligent controller for baby robot. In Proceedings of the 10th IEEE International Symposium on Intelligent Control, Monterey ,CA, August 1995.

[11] R. Bellman. Dynamic Programming. Princeton Univesity Press, 1957.

[12] R. Bellman and S. E. Dreyfus. Applied Dynamic Programming. Princeton University Press, 1962.

[13] D. Bertsekas. Dynamic Programming. Prentice Hall, 1987.

[14] R. Parker and R. Rardin. Discrete optimization. Academic Press, 1988.

[15] D. Bertsekas and R. Gallager. Data Networks. Prentice Hall, 1992. 


\title{
A HYBRID HIERARCHICAL NEURAL-AI MODEL OF MAMMAL-LIKE INTELLIGENCE
}

\author{
Paul J. Werbos \\ National Science Foundation, Room $675^{*}$ \\ Arlington, Va. 22230 \\ pwerbos@nsf.gov
}

\begin{abstract}
This paper briefly sumarizes and cites new work which tries to bridge the gap between advanced neural network designs and some of the known capabilities of mammalian intelligence. The new design draws heavily on concepts of hierarchy and temporal chunking from AI, and on relational representation of objects and space.
\end{abstract}

\section{Overview}

By 1995, several groups of researchers had finally implemented neural network designs which met certain basic criteria for what a brainlike intelligence should look like[1]. These designs were intended to be able to learn "any task" involving goal-directed behavior over time, without prior knowledge of the task. In technical terms, such designs have been called "modelbased adaptive critics" or model-based approximate dynamic programming (ADP). They have sometimes been classified as a type of reinforcement learning system.

The engineering benefits of such designs have become increasingly clear over the past few years. By now ten groups have successfully implemented such designs [2]. The strengths and weaknesses relative to conventional designs, in terms of stability, etc., are becoming better understood [3]. In formal terms, these designs provide a capability for optimization over time in uncertain noisy nonlinear environments; that, in turn, permits systems to learn how to track a narrow desired trajectory or performance envelope, which is especially useful when trying to operate highly dynamical nonlinear plants.

In 1970 or so, when I first developed the theory behind these designs, I hoped that they would be enough to describe at least those aspects of higher-order intelligence which exist in the mammalian brain. This is not the same thing as explaining consciousness in the minds of humans. In order to understand consciusness in the minds of humans, one must also address issues such as symbolic reasoning, quantum computaing and the soul, etc.; although I do have ideas about those subjects [4], they are beyond the scope of this paper. In my view, a scientific understanding of the mammal-brain level of intelligence is a prerequisite to a truly deep understanding of those more advanced levels of intelliegnce[2,4]. Indeed, the new design to be mentioned here does begin to provide some ideas about the deep structure underlying verbs, nouns, and adverbs, at the very least.

Nevertheless, by 1996 [1], it was apparent that these advanced designs still had two major deficiencies -- major gaps between the kinds of learning capability they provide, and the kind which exists in the mammalian brain.

First of all, in order to learn many difficult planning problems, such as generalized spatial navigation, it would be necessary to "fill in the boxes" in these designs with a certain type of slow but powerful neural network, the "SRN." $[5,6]$. To explain how the brain also achieves

\footnotetext{
- The views herein are those of the author, not those of NSF, but the paper was written on NSF time and may therefore be freely copied subject to proper citation.
} 
high-bandwidth control of muscle movements, one then needs to assume a "two brains in one" master-slave design described in [1].

Second, and more seriously, in order to learn how to "see" far into the future, the underlying learning rules have to be changed, to allow some sort of "temporal chunking." The usual critic adaptation rules train a network at time $t$ based on information about time $t+1$. One can do far better by allowing the use of $t+T$, at times, where $\mathrm{T}$ is a larger time interval. But how can one do this? My concerns about this wedre magnified by the growing evidence from neuroscience that the basal ganglia are a crucial part of higher-order intelligence, and that they perform something very similar to task-based planning in $\mathrm{AI}[1]$.

A careful analysis of the mathematics of temporal chunking in ADP forced me into the development of an hierarchical task-based design, made up of "decision blocks." An international patent disclosure was filed on this design on June 4, 1997, through Scientific Cybernetics, courtesy of Sam Leven. The main specification section of that disclosure is being published in [7]. Of course, that paper mainly deals with global implementation details. (The disclosure also required 300 pages of additional papers -- mainly papers previously published -in order to describe some of the subsystems.) In order to complement that discussion, another paper is being published very soon [2] which summarizes the preceding history and the intellectual strategy here in great detail. It expands on the implications for neuroscience, stressing the empirical data on the mammalian brain.

I am very grateful to Alex Meystel for drawing my attention to [8], soon after the publication of [2]. This paper made me appreciate more seriously a third limitation of the earlier model-based ADP systems -- their treatment of space. In a formal sense, this problem can be handled without changing the higher-level architecture of the model [2,7]. It is a subsystem issue, at least for the mammalian level of intelligence. (But mammals do not have to coordinate 100 quasi-independent robotic pieces of themselves spread out over an acre!) Nevertheless, the required change in neural network subsystems is very large, and has major implications for neuroscience [2] and engineering applications[7]. It is particularly relevant to tasks like predicting the behavior of molecules or controlling complex networks like heat exchangers, precoolers, fuel processors, utility grids, etc.

\section{A Few Details}

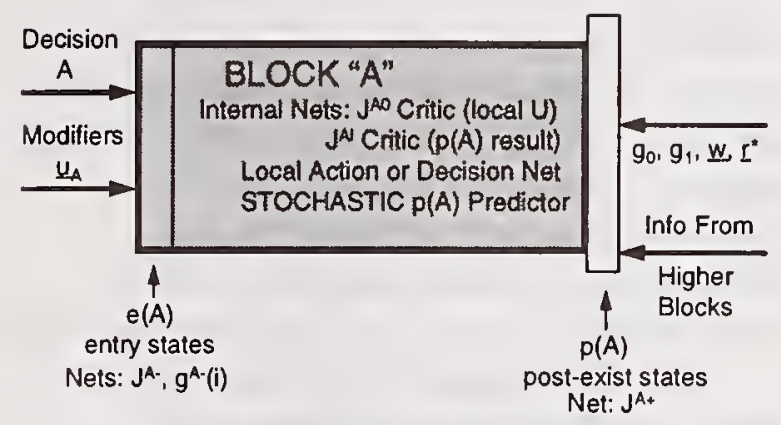

Figure 1. Structure of a Decision Block

Figure 1 is a reduced version of a figure which appears in [2]. It summarizes the structure of a "decision block." In this theory, the upper level of the mammalian brain (excluding the higher motivational systems) is essentially made up of a library of such decision blocks. The blocks are formally distinct, but in practice (as in the brain) they may share hidden nodes, and must share a common estimated state vector $\underline{\mathbf{R}}$.

The task of each decision block is to decide which lower-level decision blocks to activate. This is a crisp, discrete decision. (In [1], I speculated that the activation might be fuzzy instead of crisp; however, because of the problem of local minima, and the need for rational learning, this did not turn out to be promising.) For the lowest level block, the decisions are really just actions $\underline{\mathbf{u}}$ sent to the lower brain. Decision blocaks may be thought of as "active verbs." The arrangement is strictly sequential in nature, with only one decision block activated at any time on any level of the hierarchy.

When a decision block is activated, three kinds of additional information are also needed, to guide the actions taken nside the block: "adverb" modifiers, a fuzzy goal object $\mathbf{g}$, and compressed information about the goals inherited from higher levels of the hierarchy. In [7], mechanisms for adapting all of these structures or networks are provided.

The fuzzy goal object mainly consists of an image of a desired state $\mathbf{r}^{*}$ plus a vector of weights (indicating which components of that image vector are most important). In effect, this object provides a kind of explicit quadratic representation of a value function. The need for 
this kind of representation became clear only after considerable analysis of the problem of communication between decision blocks.

Within each decision block, neural networks must be adapted which are very similar to those in the usual model-based adaptive critics -- local critic networks, a local Model or prediction network, and a local Decion or action network. In this case, however, it is especially critical that the latter two networks be stochastic networks, rather than the usual sorts of deterministic neural network[2,7]. Two additional networks are needed in order to optimize the interface with other decsiion blocks.

A key aspect of using stochastic networks is that one can replicate such phenomena as "imagination" or "dreaming" or novelty-seeking behavior which is crucial to effective higher-order problem-solving and mammalian behavior $[4,9]$.

\section{References}

1. P.Werbos, Learning in the Brain: An Engineering Interpretation, in K.Pribram and J.King, eds, Learning as Self-Organization, Erlbaum, 1996.

2. P.Werbos, Values, goals and utility in an engineering-basxed theory of mammalisn intelligence, in K.Pribram, ed, Values (tentative title), Erlbaum 1997.

3. P.Werbos, Neurocontrollers, in J.Webster, ed., Encyclopedia of Electronics and Electrical Engineering, Wiley, forthcoming.

4. P.Werbos, Optimization: A

Foundation for understanding consciousness. In D.Levine \& W. Elsberry (eds) Optimality in Biological and Artificial Networks?, Erlbaum, 1996.

5. P.Werbos \& X.Z.Pang, Generalized maze navigation: SRN critics solve what feedforward or Hebbian nets cannot.Proc. Conf. Systems, Man and Cybernetics (SMC) (Beijing), IEEE, 1996. (An earlier version appeared in WCNN96.)

6. X.Z.Pang \& P.Werbos, Neural network design for $\mathrm{J}$ function approximation in dynamic programming, Math. Modelling and Scientific Computing (a Principia Scientia journal), special issue on neural nets planned for circa December 1996. See also www.glue.umd.edu/ pangxz.

7.P.Werbos, A Brain-Like Design To Learn Optimal Decision Strategies in Complex Environments, in M.Karny, K.Warwick and V.Kurkova, eds, Dealing with Complexity: A Neural Networks Approach. Springer, London, 1997.

8. J.Albus, Outline of Intelligence, IEEE Trans. Systems, Man and Cybernetics, Vol.21, No.2, 1991.

9. . D.S.Levine and S.J.Leven, eds, Motivation, Emotion, and Goal Directio $n$ in Neural Networks, Erlbaum, 1992. 
IV

LOGICAL CALCULI FOR SEMIOSIS 



\title{
Plausible Reasoning and Its Logics
}

\author{
Oleg M. Anshakov, Victor K. Finn, Dmitry P. Skvortsov \\ VINITI - All-Russian Institute of Scientific and Technical Information \\ Usievitcha St. 20a, Moscow, Russia, 125219 \\ Phone: +7 095 152-5447, Fax: +7 095 152-5447 \\ email: lis@viniti.msk.su
}

\begin{abstract}
The paper deals with some formal models of plausible reasoning. The so-called JSMmethod of automatic hypothesis generation is one of these models. Some important notions of JSMreasoning and its logical basis are informally introduced in this paper.
\end{abstract}

\section{INTRODUCTION}

Reasoning can be considered as synthesis of cognitive procedures. These procedures include valuating of elements of our knowledge such as facts, hypotheses, and arguments. General scheme of plausible reasoning is represented by the notion of Quasi-Axiomatic Theory (QAT). [Finn 1991], [Finn 1995].

Let $\Sigma$ be the set of axioms that are known to provide an incomplete characterization of the application domain, let $\Sigma^{\prime}$ be an open set of elementary (protocol) propositions representing facts (we simply call these propositions facts), where $\Sigma^{\prime}=\cup_{\mathrm{n}_{\mathrm{E}}} \Sigma_{\mathrm{n}}$, and $\Sigma_{\mathrm{n}}$ is the set of facts corresponding to the $n$-th state of the QAT. Let $\Re$ be the set of inference rules such that $\Re=R^{\prime} \cup \Re_{0}$, where $\Re^{\prime}$ is the set of plausible inference rules (PIR) and $\Re_{0}$ is the set of reliable (deductive) inference rules. Then $\Gamma^{\prime}=<\Sigma, \Sigma^{\prime}, \Re>, \Gamma=<\Sigma, \Re>$ and $\Gamma_{\mathrm{n}}=<\Sigma, \Sigma_{\mathrm{n}}, \Re>$ will be called QAT, QAT carcass, and n-th state of QAT, respectively.

Computer analogs of these concepts are obvious: $\Sigma_{\mathrm{n}}$ is the state of the data base (the fact base) and $\Gamma$ is the knowledge base containing declarative knowledge $(\Sigma)$ and procedural knowledge $(\mathfrak{R})$. The QAT itself is the limiting (ideal) notion that all the states of the carcass $\Gamma$ are attainable in the limit, e.g., we have $\Sigma_{1} \subseteq \Sigma_{2} \subseteq \ldots \subseteq \Sigma_{\mathrm{n}} \subseteq \ldots$

Particular realization of QAT is JSM-method of automatic hypothesis generation. The paper discuss this apparatus and its logical foundation.

Applications of JSM-method are considered in [Zabezhailo et al. 1995], [Mikheyenkova 1995]

\section{INFINITE-VALUED LOGIC WITH FINITE NUMBER OF TRUTH VALUE TYPES}

We use a logic with the following types of truth values: empirically true (denoted by +1 ), empirically false (denoted by -1), empirically contradictory (denoted by 0 ), uncertain (denoted by $\tau$ ), logically true (denoted by t), logically false (denoted by f), other (they can be added in some special versions of JSM-method). By $E$ denote the set of all types of truth values. Thus, $E=\{+1$, $-1,0, \tau, \mathbf{t}, \mathbf{f}\}$. In some special cases, $E$ can be extended. An informal interpretation of the truth value types is as follows:

- the truth value of predicate $P$ is a value of type +1 (empirically true) iff it is known that $P$ (possibly) holds;

- the truth value of predicate $P$ is a value of type -1 (empirically false) iff it is known that $P$ (possibly) does not hold;

- the truth value of predicate $P$ is a value of type 0 (empirically contradictory) iff there are arguments both for $P$ and against it;

- the truth value of predicate $P$ is a value of type $\tau$ (uncertain) iff there are neither arguments for $P$ nor against it.

The types $+1,-1,0$, and $\tau$ are called internal. The types $\mathbf{t}$ and $\mathbf{f}$ are called external. We denote by $V_{\varepsilon}$ the set of all truth values of type $\varepsilon$. By $V$ we denote the set of all truth values.

We say that $\varepsilon$ is a non-split type if $V_{\varepsilon}$ is a one-element set. We say that $\varepsilon$ is a (denumerable) split type if $V_{\varepsilon}$ is a denumerable set. The types $+1,-1$, and 0 are split types. The types $\mathbf{t}, \mathbf{f}$, and $\tau$ are non-split types.

By $E^{\text {int }}$ denote the set of all internal types of truth values. $E^{\text {int }}=\{+1,-1,0, \tau\}$. By $E^{\text {ext }}$ denote the set which consists of two external types of truth values. $E^{\text {ext }}=\{\mathbf{t}, \mathbf{f}\}$. By $E_{\omega}$ denote the set of all (denumerably) split types of truth values. $E_{\omega}=\{+1,-1$, $0\}$. By $E_{0}$ denote the set of all non-split types of truth values. $E_{0}=\{\tau, \mathbf{t}, \mathbf{f}\}$. If it is necessary, we can extend the set $E$ by adding a finite set of non-split internal types of truth values. We denote this set by $E_{0}^{\text {int }}$.

For any non-split type $\varepsilon$ there exists a unique truth value in $V_{\varepsilon}$ denoted by $\langle\varepsilon, 0\rangle$ (or by $\varepsilon$ ). If $\varepsilon$ is a split type, then $V_{\varepsilon}=\left\{\langle\varepsilon, k\rangle k \in \omega^{\prime}\right\}$, where $\omega^{\prime}=\{0,1, \ldots, \omega\}=\omega U\{\omega\}$, i.e., $\omega^{\prime}$ is a successor 
of the first limit ordinal $\omega$. In other words, $\omega^{\prime}$ is obtained from the set of all natural numbers by adding the item $\omega$, which is greater than any natural number.

Suppose we have a procedure of hypothesis generation such that, using it for an arbitrary predicate $P$, we can look for the truth value type of this predicate. Assume that the truth value of the predicate $P$ is $\langle\varepsilon, k\rangle$, where $\varepsilon$ is a split type of truth values. Then $k$ is the number of the step, where the truth value type of this predicate was found. We can use $k$ as a measure of plausibility. The more $k$ the less plausible is a hypothesis. If $k=0$, then the truth value type of the predicate is known before the beginning of the above procedure.

In the standard JSM-method, the truth value $\langle 0,0\rangle$ is not considered. In other words, in the standard JSMmethod the empirically contradiction cannot be a fact, but it can be a hypothesis.

By definition, put $V_{(\tau, n)}=\{\tau\} \bigcup \underset{\varepsilon \in E_{\omega}}{\bigcup}\{\langle\varepsilon, k\rangle \mid k>n\}$. If $\varepsilon \in E_{\omega}$ (i.e., $\varepsilon$ is a split type), then by definition we put $V_{(\varepsilon, n)}=\{\langle\varepsilon, k\rangle \mid k \leq n\}$. Intuitively, the fact that the truth value of the predicate $P$ belongs to $V_{(\tau, n)}$ means that we did not find the truth value type of this predicate within $n$ steps. The fact that the truth value of the predicate $P$ belongs to $V_{(\varepsilon, n)}$, where $\varepsilon$ is a split type, means that we found truth value type of this predicate within $n$ steps.

We will use shorter notation for $V_{(\varepsilon, n)}$ and $V_{(\tau, n)}$, namely: $(\varepsilon, n)$ and $(\tau, n)$ respectively. Note that $(\tau, n)=(\tau, n+1) \mathrm{U}\{\langle+1, n\rangle,\langle-1, n\rangle,\langle 0, n\rangle\}=(\tau, n+1) \mathrm{U} \bigcup_{\varepsilon \in E_{\omega}}\langle\varepsilon, n\rangle$

In our logic we use the following connectives:

$$
\begin{gathered}
\mathbf{J}_{\langle\varepsilon, n\rangle}(\alpha)= \begin{cases}\mathbf{t} & \text { if } \alpha=\langle\varepsilon, n\rangle, \\
\mathbf{f} & \text { otherwise, }\end{cases} \\
\mathbf{J}_{\langle\tau, n)}(\alpha)= \begin{cases}\mathbf{t} & \text { if } \alpha \in(\tau, n), \\
\mathbf{f} & \text { otherwise, }\end{cases} \\
\mathbf{J}_{(\varepsilon, n)}(\alpha)= \begin{cases}\mathbf{t} & \text { if } \alpha \in(\varepsilon, n), \\
\mathbf{f} & \text { otherwise, }\end{cases}
\end{gathered}
$$

where $\varepsilon$ is a split type $(\varepsilon \in\{+1,-1,0\})$; $\neg, \&, \vee, \rightarrow, \leftrightarrow, \forall, \exists$; the latter are the classical logical connectives and quantifiers over $\{\mathbf{t}, \mathbf{f}\}$.

We can describe the syntax of this infinite-valued logic and define terms and formulas in a general way. Both infinite-valued and two-valued (external) predicates can be considered in this logic. We can take the following connectives as basic symbols: $\neg, \&, \vee, \rightarrow, \leftrightarrow, \forall, \exists$ (the classical two-valued connectives and the quantifiers; it is sufficient to take only
$\mathbf{J}_{\varepsilon}(\varepsilon \in E), \mathbf{J}_{\langle\varepsilon, n\rangle},\left(\varepsilon \in E_{\omega}\right)$ where $\mathrm{J}_{\varepsilon}$ and $\mathrm{J}_{\langle\varepsilon, n\rangle}$ can be defined as follows:

$$
\begin{aligned}
\mathbf{J}_{\varepsilon}(\alpha)= \begin{cases}\mathbf{t} & \text { if } \alpha \in V_{\varepsilon}, \\
\mathbf{f} & \text { otherwise, }\end{cases} \\
\mathbf{J}_{\langle\varepsilon, n\rangle}(\alpha)= \begin{cases}\mathbf{t} & \text { if } \alpha=\langle\varepsilon, n\rangle, \\
\mathbf{f} & \text { otherwise. }\end{cases}
\end{aligned}
$$

Then by definition, we put

$$
\begin{aligned}
& \mathbf{J}_{(\varepsilon, n)}(P)=\bigvee_{k \leq n} \mathbf{J}_{\langle\varepsilon, k\rangle}(P), \quad \text { where } \quad \varepsilon \in E_{\omega}, \quad \text { and } \\
& \mathbf{J}_{(\tau, n)}(P)=\mathbf{J}_{\tau}(P) \vee \bigvee_{\varepsilon \in E_{\omega}}\left(\mathbf{J}_{\varepsilon}(P) \& \neg \mathbf{J}_{\langle\varepsilon, n)}(P)\right)
\end{aligned}
$$

The above logic is an example of the $\mathbf{J}$-definable $\mathbf{J}$ compact logics considered in [Anshakov, Finn, Skvortsov 1989]. In this paper it is proved that every Jdefinable J-compact logic is strongly axiomatizible, i.e., for any $\mathbf{J}$-definable $\mathbf{J}$-compact $\operatorname{logic} \boldsymbol{L}$ one can construct a calculus $I$ such that the strong completeness theorem holds: $\left.\Gamma\right|_{I} A$ iff $\Gamma \mathrm{I}_{L} A$, where $\Gamma \vdash_{I} A$ means that the formula $A$ is deducible from the set of formulas $\Gamma$ in the calculus $I, \Gamma \mid=_{L} A$ means that the formula $A$ is a semantic consequent of the set of formulas $\Gamma$ in the $\operatorname{logic} L$.

\section{SIMPLIFIED FORMULATION OF ISM- RULES}

We consider items of three types. The items of the first type are objects of arbitrary nature. The items of the second type are fragments (parts) of these objects. Assume that we can find the common part (fragment) of any set of objects. The fragments may be objects, but they are not neccesarily objects. The items of the third type are sets of properties of objects. We can imagine the objects as sets, the fragments as subsets, the common part of several objects as their intersection.

Suppose $X$ is an object, $x$ is a fragment (part) of an object, $A$ is a set of some properties of the objects. Consider two many-valued binary predicates denoted by $\Rightarrow_{1}$ and $\Rightarrow_{2}: X \Rightarrow_{1} A$ means that the object $X$ (possibly) has all the properties from $A ; \quad x \Rightarrow_{2} A$ means that the fragment $x$ is a (possible) cause of any property from $A$.. In the simplest case $A=\{a\}$. In this case we write $X \Rightarrow_{1} a$ and $x \Rightarrow_{2} a$ instead of $X \Rightarrow_{1}\{a\}$ and $x \Rightarrow_{2}\{a\}$ respectively.

For the sake of simplicity, assume that our objects are represented by sets; their fragments are subsets of objects; the common part of several objects is their settheoretic intersection. Moreover, suppose that all sets of properties of the objects are one-element. 
We write $\mathbf{J}_{(\tau, n)}\left(x \Rightarrow_{2} a\right)$ if we do not yet know whether the fragment $x$ is a (possible) cause of the property $a$, after $n$ steps of Hypothesis Generation Procedure. We write $\mathbf{J}_{\langle+1, n\rangle}\left(x \Rightarrow_{2} a\right)$ if we discovered that $x$ is a (possible) cause of the property $a$ just at step $n$. Similarly, we write $\mathbf{J}_{\langle-1, n\rangle}\left(x \Rightarrow_{2} a\right)$ if at step $n$ we found arguments for $x$ to be a cause of absence of $a$, and we write $\mathrm{J}_{\langle 0, n\rangle}\left(x \Rightarrow_{2} a\right)$ if at step $n$ we found arguments for $x$ to be a (possible) cause of both presence and absence of $a$

The Fist-type JSM-rules are formulated as follows:

$$
\begin{gathered}
("+" \text {-rule }) \frac{\mathbf{J}_{(\tau, n)}\left(x \Rightarrow_{2} a\right), \mathbf{M}_{n}^{+}(x, a), \neg \mathbf{M}_{n}^{-}(x, a)}{\mathbf{J}_{\langle+1, n+1\rangle}\left(x \Rightarrow_{2} a\right)} \\
\frac{\left("-{ }^{\prime \prime}-\text { rule }\right)}{\mathbf{J}_{(\tau, n)}\left(x \Rightarrow_{2} a\right), \mathbf{M}_{n}^{-}(x, a), \neg \mathbf{M}_{n}^{+}(x, a)} \\
\mathbf{J}_{\langle-1, n+1)}\left(x \Rightarrow_{2} a\right) \\
\left(" 0^{\prime \prime} \text {-rule }\right) \frac{\mathbf{J}_{(\tau, n)}\left(x \Rightarrow_{2} a\right), \mathbf{M}_{n}^{+}(x, a), \mathbf{M}_{n}^{-}(x, a)}{\mathbf{J}_{\langle 0, n+1\rangle}\left(x \Rightarrow_{2} a\right)} \\
\frac{\mathbf{J}_{(\tau, n)}\left(x \Rightarrow_{2} a\right), \neg \mathbf{M}_{n}^{+}(x, a), \neg \mathbf{M}_{n}^{-}(x, a)}{\mathbf{J}_{(\tau, n+1)}\left(x \Rightarrow_{2} a\right)}
\end{gathered}
$$

We write $\mathbf{J}_{(+1, n)}\left(X \Rightarrow_{1} a\right)$ if we discovered that the object $X$ (possibly) had the property $a$ within $n$ steps of Hypothesis Generation Procedure. Similarly we write $\mathbf{J}_{(-1, n)}\left(X \Rightarrow_{1} a\right)$ if we discovered that $X$ do not had the property $a$ within $n$ steps.

Suppose $S_{n}^{+}(a)=\left\{X \mid \mathbf{J}_{(+1, n)}\left(X \Rightarrow_{1} a\right)\right\}$, $S_{n}^{-}(a)=\left\{X \mid \mathbf{J}_{(-1, n)}\left(X \Rightarrow_{1} a\right)\right\} \cdot \mathbf{M}_{n}^{+}(y, a)$ means that there exists $X \subseteq S_{n}^{+}(a)$ such that the following conditions hold:

(1) $X \subseteq S_{n}^{+}(a)$;

(2) $y=\prod_{X \in X} X$;

(3) $y \neq \varnothing$.

$\mathbf{M}_{n}^{-}(y, a)$ means that there exists $X \subseteq S_{n}^{-}(a)$ such that the following conditions hold:

(1)

$$
|x| \geq 2 \text {; }
$$

$$
\begin{aligned}
& y=\underset{X \in X}{I} X ; \\
& y \neq \varnothing ;
\end{aligned}
$$

Suppose $\quad \mathbf{J}_{(+1, n)}\left(X_{i} \Rightarrow_{1} a\right), \quad \mathbf{J}_{(-1, n)}\left(Z_{i} \Rightarrow_{1} a\right)$, $(i=1,2,3) ; \quad \mathbf{J}_{(+1, n)}\left(Y_{1} \Rightarrow_{1} a\right), \quad \mathbf{J}_{(-1, n)}\left(Y_{2} \Rightarrow_{1} a\right)$; (see Fig. 3-1). Using first-type JSM-rules, we obtain,
$\mathbf{J}_{\langle+1, n+1\rangle}\left(x \Rightarrow_{2} a\right), \mathbf{J}_{\langle 0, n+1\rangle}\left(y \Rightarrow_{2} a\right), \mathbf{J}_{\langle-1, n+1\rangle}\left(z \Rightarrow_{2} a\right)$.

We write $\mathbf{J}_{(\tau, n)}\left(X \Rightarrow_{1} a\right)$ if we do not yet know whether the object $X$ has the property a after $n$ steps of Hypothesis Generation Procedure. We write $\mathbf{J}_{\langle+1, n\rangle}\left(X \Rightarrow_{1} a\right)$ if we discovered that $X$ has the property $a$ just at step $n$. Similarly, we can describe the conditions $\mathrm{J}_{\langle-1, n\rangle}\left(X \Rightarrow_{1} a\right)$ and $\mathrm{J}_{\langle 0, n\rangle}\left(X \Rightarrow_{1} a\right)$.

The Fist-type JSM-rules are formulated as follows:

$$
\begin{aligned}
& ("+" \text {-rule }) \frac{\mathbf{J}_{(\tau, n)}\left(X \Rightarrow_{1} a\right), \Pi_{n}^{+}(X, a)}{\mathbf{J}_{\langle+1, n+1\rangle}\left(X \Rightarrow_{1} a\right)} \\
& \left("{ }^{\prime \prime-} \text {-rule }\right) \frac{\mathbf{J}_{(\tau, n)}\left(X \Rightarrow_{1} a\right), \Pi_{n}^{-}(X, a)}{\mathbf{J}_{\langle-1, n+1)}\left(X \Rightarrow_{1} a\right)} \\
& \left(" 0^{\prime \prime} \text {-rule }\right) \frac{\mathbf{J}_{(\tau, n)}\left(X \Rightarrow_{1} a\right), \Pi_{n}^{0}(X, a)}{\mathbf{J}_{\langle 0, n+1\rangle}\left(X \Rightarrow_{1} a\right)} \\
& \left(" \tau^{\prime \prime} \text {-rule }\right) \\
& \frac{\mathbf{J}_{(\tau, n)}\left(X \Rightarrow_{1} a\right),-\neg \Pi_{n}^{+}(X, a), \neg \Pi_{n}^{-}(X, a), \neg \Pi_{n}^{0}(X, a)}{\mathbf{J}_{\langle+1, n+1)}\left(X \Rightarrow_{1} a\right)}
\end{aligned}
$$

$\Pi_{n}^{+}(X, a)$ holds iff the following conditions are satisfied:

$$
\begin{aligned}
& \mathbf{J}_{(+1, n)}\left(y \Rightarrow_{2} a\right) \text { for some } y \subset X ; \\
& \mathbf{J}_{(-1, n)}\left(z \Rightarrow_{2} a\right) \text { for not any } z \subset X .
\end{aligned}
$$

$\Pi_{n}^{-}(X, a)$ and $\Pi_{n}^{+}(X, a)$ are defined dually. $\Pi_{n}^{0}(X, a)$ holds iff the following conditions are satisfied:

(1) $\quad \mathbf{J}_{(+1, n)}\left(y \Rightarrow_{2} a\right)$ for some $y \subset X$;

(2) $\mathbf{J}_{(-1, n)}\left(z \Rightarrow_{2} a\right)$ for some $z \subset X$.

For example, let $\mathbf{J}_{(+1, n)}\left(x \Rightarrow_{2} a\right), \quad \mathbf{J}_{(-1, n)}\left(y \Rightarrow_{2} a\right)$ (see Fig. 3-2). Then using the second-type JSM-rules, we obtain $\mathbf{J}_{\langle+1, n+1\rangle}\left(X \Rightarrow_{2} a\right), \quad \mathbf{J}_{\langle 0, n+1\rangle}\left(Y \Rightarrow_{2} a\right)$, $\mathbf{J}_{\langle-1, n+1\rangle}\left(Z \Rightarrow_{2} a\right) \quad \mathbf{J}_{(\tau, n+1)}\left(W \Rightarrow_{2} a\right)$. 


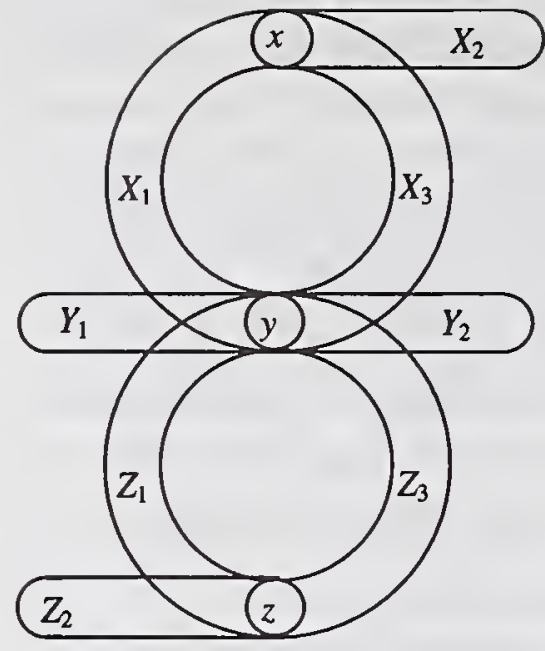

Figure 3-1: First-Type Rules

\section{JSM-THEORY}

The JSM-theory $\mathrm{T}(\mathrm{M})$ imitating system $\mathrm{M}$ of rules of the JSM-inference is constructed on the base of the Jlogic $\mathrm{V}$ with finite number of types of truth values. This theory is constructed in some many-sorted language L containing individual variables of three sorts: for objects, subobjects and properties (or sets of properties).

The JSM-theory consists of three families of axioms.

Procedural axioms imitate hypothesis generation procedures of the JSM-method. Namely, the plausible rules of the first and second type are rewritten in the form of implications (so-called axioms of step-by-step inference):

$(\varepsilon, \mathrm{r}, \mathrm{n})$

$\mathrm{J}_{(\tau, n)}\left(X \Rightarrow_{1} A\right) \& \Pi_{1, n}^{\varepsilon}(X, A) \rightarrow \mathbf{J}_{\langle\varepsilon, n+1\rangle}\left(X \Rightarrow_{1} A\right)$,

$(\omega, \mathrm{r}, \mathrm{n})$

$\mathbf{J}_{(\tau, n)}\left(X \Rightarrow_{1} A\right) \& \underset{\varepsilon \in E_{\omega}}{\&} \neg \Pi_{1, n}^{\varepsilon}(X, A) \rightarrow \mathbf{J}_{(\tau, n+1)}\left(X \Rightarrow_{1} A\right)$.

where $\mathrm{r}=1,2$ and $\varepsilon \in E_{\omega}$.

Similarly, the axioms imitating the first-type rules can be formulated.

Then the axioms of stabilization are added:

$$
\begin{aligned}
& \forall X \forall A\left(\mathbf{J}_{(\tau, n)}\left(X \Rightarrow_{1} A\right) \rightarrow \mathbf{J}_{(\tau, n+1)}\left(X \Rightarrow_{1} A\right)\right) \rightarrow \\
& \rightarrow \forall X \forall A\left(\mathbf{J}_{(\tau, n)}\left(X \Rightarrow_{1} A\right) \rightarrow \mathbf{J}_{\tau}\left(X \Rightarrow_{1} A\right)\right)
\end{aligned}
$$

Similarly, the axiom of stabilization with respect to the predicate $\Rightarrow_{2}$ can be formulated. (namely, if on the step $n$ no one new value $\langle\varepsilon, n+1\rangle$ is attributed then all remained subdefined up to their moment acquires the ultimate value $\omega)$.

Remark. For each axiom of step-bystep inference, the converse holds., i.e. the correspondence equivalencies are provable in the

Figure 3-2: Second-Type Rules

JSM-logic. These equivalencies correspond to the constructivity of generation of truth values in the JSMinference.

The generating condition are expressible by the formulas of the language $\mathrm{L}$. These formulas involve the notions of similarity and difference of the objects considered. More precisely, the operations (e.g. settheoretical intersection or the corresponding operation on graphs etc.).

Algebraic axioms $\mathrm{T}_{\mathrm{a}}$ describe the properties of these operations, e.g. semilattices or Boolean conditions on $\&$ (namely, axioms of atomic Boolean algebras)

Some means of description of finite families of objects (and subobjects) are necessary too. Namely, one can use quantifiers on sequences of an arbitrary finite length (i.e. finite functions on natural numbers) or quantifiers over finite sets of objects (and the corresponding axioms of weak monadic second order logic are included in $\mathrm{T}_{\mathrm{a}}$ ).

Hence, algebraic part $T_{a}$ describes the structure of domains (of our three sorts) and corresponds to the structure of data in database with incomplete information involved.

Finally, the JSM-theory can conclude some declarative axioms $\mathrm{T}_{\mathrm{d}}$ corresponding to procedures of verification and falsification of results of the JSM-reasoning, in particular:

(i) axioms of formal correctness representing the interrelation between the predicates (e.g. axioms of monotonicity and additivity),

(ii) axioms of control of JSM-inference (axioms of causal completeness, consistency etc.)

(iii) descriptive axioms characterizing peculiarities of a subject domain (e.g., some principles of sociology or another field to which the JSM-method is applied).

\section{REFERENCES}

Anshakov, O.M., Finn, V.K., Skvortsov, D.P. 1989. On axiomatization of many-valued logics associated with 
formalization of plausible reasoning. Studia Logica, vol. XLVIII, N 4, pp. 423-447.

Finn, V.K. 1991. Plausible inferences and plausible reasoning. Jour. Soviet Math., Plenum Publ. Cor., Vol. 56, N 1, pp. 2201 - 2248.

Finn, V.K. 1995. JSM-reasoning for control problem in open ( \pm )-worlds. Proceedings of the 1995 ISIC Workshop. 10th IEEE International Symposium on Intelligent Control, Monterey, CA, USA. pp. 75 - 79.

Josephson, J.R., Josephson, S.G. 1994. Abductive Inference: Computation, Philosophy, Technology. Cambridge University Press.

Mikheyenkova, M.A. 1995. Applications of JSMmethod to problem of sociology. Proceedings of the 1995 ISIC Workshop. 10th IEEE International Symposium on Intelligent Control, Monterey, CA, USA. pp. 429 - 433.

Zabezhailo, M.I. et al. 1995. Reasoning Models for Decision Making: Application of the JSM-method in Intelligent Control System. Proceedings of the 1995 ISIC Workshop. 10th IEEE International Symposium on Intelligent Control, Monterey, CA, USA. pp. 99 108. 


\title{
Application of JSM-Theory of Plausible Reasoning to Social Data Analysis and Social Actions Prediction
}

\author{
V.K.Finn and M.A.Mikheyenkova \\ All-Russian Institute for Scientific and Technical Information (VINITI) \\ Usievitcha 20, Moscow, Russia, 125219 \\ Phone/Fax: +(7-095)-152 5447 \\ e-mail: lis@viniti.msk.su
}

\begin{abstract}
The present report continues the series of papers, devoted to attempts to describe formal frames of theory of reasoning for sociological investigation and to apply it to real sociological problems. Logicalcombinatorial JSM-method of automatic hypotheses generation is chosen to be the basis of our approach. The method realizes special class of plausible reasoning in open subject domains with large amount of empirical material and ill-formalized knowledge.
\end{abstract}

KEYWORDS: plausible reasoning, quasiaxiomatic theory, modelling in the humanities

\section{INTRODUCTION}

The present report continues series of papers, devoted to attempts to describe formal frames of theory of reasoning for sociological investigation and to apply it to real sociological problems [13]. Logical-combinatorial JSM-method of automatic hypotheses generation is chosen to be the basis of our approach. The method realizes special class of plausible reasoning in open subject domains with large amount of empirical material and ill-formalized knowledge [4, 5].

Informally the method can be considered as a reasoning scheme "similarity - cause - analogy": the reasons of events (phenomena) are searched by the analysis of their structural similarity and then these reasons are employed to forecast new events (phenomena) by structural analogy. This scheme corresponds to the principle "similarity of objects involves similarity of their properties". Here similarity (or it's n-ary generalization) is considered to be reflexive and symmetric relation.

\section{APPLICATION OF JSM- METHOD IN SOCIOLOGY}

Thus, sociology as the humanity investigating social phenomena' similarity, the humanity with ill-formalized knowledge generated on the basis of huge empirical data, can be supposed to be a field for JSM-method application. However, certain condition must be fulfilled for success of
JSM-method's application (see, for example [2, $6]$ ), in particular, empirical data must really contain causal relations.

The possibility of JSM-application in sociology is based on consideration of social systems as systems with multi-factor ( \pm )-influences. ${ }^{1}$ Consequently, we speak about studying the relation "subject (individual) $\Rightarrow$ behaviour" in supposition that the individuality of the person is responsible for his behaviour. Comparison of individuals' behaviour, similarity and difference revealing is necessary to use proposed tools for analysis of the involved relation.

For this analysis we need structural description of the subject (individual) by the system of differential indications. Part of them is to describe social characteristics (in accordance with idea of E.Fromm about social character). Another part defines the characteristics of individual irrelatively to social interactions. And the last part represents features of person's biography, essential for the problem involved. By another words, proposed approach gives the possibility of individual behaviour prediction, when combinatorial analysis is inaccessible (because of substantial amount of information) for human's brain.

Let's underline, that the relation "subject (individual) $\Rightarrow$ behaviour" is the micro-level representation of the macro-level (macrosociology) relation "situation $\Rightarrow$ behaviour". The latter relation to be investigated "situation" concept must be specified and formalized.

General scheme of JSM-reasoning (see $[4,5]$ ) in application to problem involved is interpreted as followes.

(1). Creation of statements about reasons of objects' properties (by observing of facts (examples) from the initial data base with incomplete information - DBII) application of plausible inference rules of first kind (PIR-I). Determinants of behaviour are defined on this stage.

\footnotetext{
${ }^{1}$ We are aware that more objective picture of social reality must include a description of irregular disturbances [7], treated by statistical methods.
} 
(2). Prediction of unknown properties of objects in DBII (the first-step hypotheses about reasons are used in this prediction), inference by analogy - application of plausible inference rules of second kind (PIR-II). Forecast of individual behaviour is carried out on this stage. Forecast control is realized experimentally (in corresponding period of time).

(3). Explanation of facts and results and inference control on the base of sufficient-basis-forconclusion criterion (CSBC). We generate falsificators for DBII automatically by CSBC use.

\section{JSM-METHOD WITH SCALES}

Let $\mathbf{U}^{(1)}=\left\{d_{1}, \ldots, d_{r_{1}}\right\}, \mathbf{U}^{(2)}=\left\{a_{1}, \ldots, a_{r_{2}}\right\}$ be two sets, on which we define two Boolean algebras, $B_{1}=\left\{2^{U^{(1)}},-, \cap, \cup\right\}$ - algebra of objects, $B_{2}=\left\{2^{U^{(2)}},-, \cap, \cup\right\}$ - algebra of properties. Consider 2 partially defined relations: $\Rightarrow_{1}{ }^{*}$ and $\Rightarrow_{2}{ }^{*}$. 2-place predicate symbol $\Rightarrow_{1}$ corresponds to $\Rightarrow_{1}{ }^{*}, 2$-place predicate symbol $\Rightarrow_{2}-$ to $\Rightarrow_{2}{ }^{*} . \mathrm{X} \Rightarrow_{1} \mathrm{Y}$ means, that the object $\mathrm{X}$ possesses the set of properties $\mathrm{Y}$; the predicate describes data base with incomplete information (DBII). $\mathrm{V} \Rightarrow{ }_{2} \mathrm{~W}$ means, that the subobject $\mathrm{V}$ causes the set of properties $\mathrm{W}$; the predicate describes automatically generated fragments of knowledge base (KB). Inner truth values (for real facts and semi-facts representation) have the form $\bar{v}=\langle v, n\rangle, n$ - number of step of PIR using. Traditionally four types of inner truth values $v$ in JSM-logic are used: + 1 - factual true, -1 - factual false, 0 - empirical contradiction and $\tau$ uncertainty. Types of external truth values (for facts' with valuation and PIRs' representation): $t$ - "logical true", $f$ - "logical false". Let $J_{v} \Phi$ be the operator, $J_{v} \Phi=$ true, if $v(\Phi)=v, J_{v} \Phi=$ false, if $\mathrm{v}(\Phi) \neq \mathrm{v}$, where $\mathrm{v}[\Phi]$ - valuation.

We suppose the knowledge in empirical theories (that deal with the open world, progressively replenished with new facts) to be represented in the form of quasiaxiomatic theory (QAT) $[4,5] \mathfrak{I}$ $=\left\langle\Sigma, \Sigma^{\prime}, R\right\rangle$, where $\Sigma$ is an open axiom set, describing a subject domain (SD) incompletely; $\Sigma^{\prime}$ is an open set of empirical elementary statements about subjects from SD $(\Sigma$ corresponds to knowledge base KB, $\Sigma^{\prime}$ corresponds to data base DB). $R$ is an inference rules set, $R=R_{\mathrm{d}} \cup R_{\mathrm{p}}$, where $R_{\mathrm{d}}$ is a set of deductive rules, $R_{\mathrm{p}}$ is a set of plausible inference rules (PIR).
In QAT for JSM $\Sigma^{\prime}$ - set of empirical facts $J_{\langle v, 0\}}\left(X \Rightarrow{ }_{1} Y\right)(v \in\{1,-1, \tau\})$ of the considered SD. $J_{\langle v, 0\}}(X \Rightarrow, Y)$ means, that the statement "object $X$ possesses set of properties $Y$ " has the valuation $v$ (true, false or uncertainty) in the initial state (on the 0-th step of reasoning). Plausible inference rules $R_{\mathrm{p}}$ in the JSM-QAT are PIR-I and PIR-II.

Let's turn to our problem.

Let $U^{(1)}=\left\{u_{1}, \ldots, u_{r}\right\}$ be a set of differential indications, describing individual personalities' qualities, several social features and biographical data (sociological data are collected by methods of formalized interview and psychological tests in our investigation). $\mathrm{X}_{i} \quad(i=1, \ldots, k)$ - objects (persons, subjects of sociological investigation, $X_{i} \in 2^{U^{(1)}} \cdot U^{(2)}=\left\{b_{1}, \ldots, b_{s}\right\}$ - a set of behaviouristic readinesses, $Y_{i}(i=1, \ldots, k)$ - a set of $i$-subject's behaviouristic readinesses, $Y_{i} \in$ $2^{\mathrm{U}^{(2)}}$.

The relation $\Rightarrow 1^{*}$ is formed on the basis of expert analysis (which is fulfilled by sociologists and psychologists) of questions complexes. Since answers of "possibly, yes" and "possibly, no" types are very often in sociopsychological practice (side by side with "yes" $(+1)$ and "no" (1) - answers), we introduce two new types of truth values $-+1 / 2$ and $-1 / 2$ - to characterize the degree of properties presence or absence. Logical connectives $\&$ and $\vee$ are defined in a standard manner - see, for example, [4].

Three strategies of empirical data investigation with different degrees of properties' presence or absence (different scales) are possible.

(a). The difference between degrees is supposed to be unimportant, only direction ( + or -) is essential. Then types of truth values $+1 / 2$ and +1 (as far as $-1 / 2$ and -1) merge, $\forall \mathrm{X} \forall \mathrm{Y}\left(J_{(1,9)}\left(\mathrm{X} \Rightarrow_{1} \mathrm{Y}\right) \leftrightarrow J_{\left(\frac{1}{2}, g\right.}\left(\mathrm{X} \Rightarrow_{1} \mathrm{Y}\right)\right)$, and we return to traditional four types of truth values (see above).

(b). Mechanisms of different degrees of properties presence or absence exposing are different.

Then

$\forall \mathrm{X} \forall \mathrm{Y} \neg\left(J_{\langle 1,0\}}\left(\mathrm{X} \Rightarrow_{1} \mathrm{Y}\right) \leftrightarrow J_{\left\langle\frac{1}{2}, 0\right\}}\left(\mathrm{X} \Rightarrow_{1} \mathrm{Y}\right)\right)$,

special predicates $\tilde{\mathbf{M}}_{a, \mathrm{n}}^{+\frac{1}{2}}(\mathrm{~V}, \mathrm{~W}, \mathrm{k})$ for treating facts $J_{\frac{1}{2},{ }_{0}}\left(\mathrm{X} \Rightarrow_{1} \mathrm{Y}\right)$ (n - number of calculation step) are introduced (analogous to $\tilde{\mathbf{M}}_{a, \mathrm{n}}^{+}(\mathrm{V}, \mathrm{W}, \mathrm{k})$ for treating facts $J_{\langle 1,0}\left(X \Rightarrow_{1} Y\right)$; for $-1 / 2$ and -1 - symmetrically) and then usual scheme of JSM-reasoning is applied.

(c). $\pm 1 / 2$ is considered to be "weak" (expressed insufficiently) \pm 1 correspondingly (see also 
[1]), $\forall X \forall Y\left(J_{\{1,0\}}\left(X \Rightarrow_{1} Y\right) \rightarrow J_{\left(\frac{1}{2}, 9\right.}\left(X \Rightarrow_{1} Y\right)\right)$. In this case we take into account examples with both types of truth values $(+1 / 2$ and +1$)$ to discover the reasons of relation's $\Rightarrow 1^{*}$ exposing with the truth value $+1 / 2$. This process is formally described by the predicate $\tilde{\mathbf{M}}_{a, \mathrm{n}}^{+\frac{1}{2}}(\mathrm{~V}, \mathrm{~W}, \mathrm{k}$ ) (which differs from section (b) predicate). This predicate reveals local similarity on $(+1 / 2)$ - and (+1)-examples $J_{\left(\frac{1}{2}, \mathrm{n}\right)}\left(\mathrm{Z}_{i} \Rightarrow_{1} \mathrm{U}_{i}\right)$ and $\mathrm{J}_{(1, \mathrm{n})} \quad\left(\mathrm{Z}_{j} \quad \Rightarrow_{1} \quad \mathrm{U}_{j}\right)$ correspondingly, $i=1, \ldots, p, j=p+1, \ldots, k$, where $k$ is variable $(1 \leq p \leq k, k \geq 2)$. (For $1 / 2$ - symmetrically.)

Analogously, the predicate $\tilde{\Pi}_{n}^{+\frac{1}{2}}(\mathrm{~V}, \mathrm{~W}, \mathrm{k})$ for 2-nd kind rules PIR-II (rules for prediction) includes the subformula, expressing the statement, that $\mathrm{V}$ contains positive $(+1 / 2)$ - and $(+1)$ reasons $\mathrm{X}_{1}, \ldots, \mathrm{X}_{p}$ and $\mathrm{X}_{p+1}, \ldots, \mathrm{X}_{k}$ of properties $\mathrm{Y}_{1}, \ldots, \mathrm{Y}_{k}$ correspondingly, $W$ being covered by these sets of properties. (For - $1 / 2$ - symmetrically.)

PIR-I gets the form.

$$
\begin{aligned}
& J_{(\mathrm{t}, \mathrm{n})}\left(\mathrm{V} \Rightarrow_{2} \mathrm{~W}\right) \text {, } \\
& \mathbf{M}_{a, \mathrm{n}}^{+}(\mathrm{V}, \mathrm{W}) \& \neg \mathbf{M}_{a, \mathrm{n}}^{-}(\mathrm{V}, \mathrm{W}) \& \neg \mathbf{M}_{a, \mathrm{n}}^{-\frac{1}{2}}(\mathrm{~V}, \mathrm{~W}) \\
& \left(\mathrm{I}^{(+)}\right) \\
& J_{\langle 1, \mathrm{n}+1}\left(\mathrm{V} \Rightarrow_{2} \mathrm{~W}\right) \\
& J_{(\mathrm{t}, \mathrm{n})}\left(\mathrm{V} \Rightarrow_{2} \mathrm{~W}\right) \text {, } \\
& \neg \mathbf{M}_{a, \mathrm{n}}^{+}(\mathrm{V}, \mathrm{W}) \& \neg \mathbf{M}_{a, \mathrm{n}}^{-}(\mathrm{V}, \mathrm{W}) \& \\
& \mathrm{M}_{a, \mathrm{n}}^{+\frac{1}{2}}(\mathrm{~V}, \mathrm{~W}) \& \neg \mathrm{M}_{a, \mathrm{n}}^{-\frac{1}{2}}(\mathrm{~V}, \mathrm{~W}) \\
& \left(\mathrm{I}^{\left(+\frac{1}{2}\right)}\right) \\
& J_{\left(\frac{1}{2}, \mathrm{n}+1\right)}\left(\mathrm{V} \Rightarrow{ }_{2} \mathrm{~W}\right)
\end{aligned}
$$

$\left(\mathrm{V} \Rightarrow{ }_{2} \mathrm{~W}\right)$ gets truth value $+1 / 2$, only if it does not simultaneously gets truth value +1 . (For $-1 / 2$ and -1 - symmetrically.)

$$
\begin{aligned}
& J_{(\mathrm{r}, \mathrm{n})}\left(\mathrm{V} \Rightarrow_{2} \mathrm{~W}\right) \text {, } \\
& \mathbf{M}_{a, \mathrm{n}}^{+}(\mathrm{V}, \mathrm{W}) \& \mathbf{M}_{a, \mathrm{n}}^{-}(\mathrm{V}, \mathrm{W}) \mathrm{V} \\
& \mathbf{M}_{a, \mathrm{n}}^{+}(\mathrm{V}, \mathrm{W}) \& \mathbf{M}_{a, \mathrm{n}}^{-\frac{1}{2}}(\mathrm{~V}, \mathrm{~W}) \vee \\
& \mathbf{M}_{a, \mathrm{n}}^{-}(\mathrm{V}, \mathrm{W}) \& \mathrm{M}_{a, \mathrm{n}}^{+\frac{1}{2}}(\mathrm{~V}, \mathrm{~W}) \vee \\
& \mathbf{M}_{a, \mathrm{n}}^{+\frac{1}{2}}(\mathrm{~V}, \mathrm{~W}) \& \neg \mathrm{M}_{a, \mathrm{n}}^{-\frac{1}{2}}(\mathrm{~V}, \mathrm{~W}) \\
& J_{(0, \mathrm{n}+1)}\left(\mathrm{V} \Rightarrow{ }_{2} \mathrm{~W}\right) \\
& J_{(t, n)}\left(V \Rightarrow{ }_{2} \mathrm{~W}\right) \text {, } \\
& \neg \mathbf{M}_{a, \mathrm{n}}^{+}(\mathrm{V}, \mathrm{W}) \&-\mathbf{M}_{a, \mathrm{n}}^{-}(\mathrm{V}, \mathrm{W}) \& \\
& \neg M_{a, \mathrm{n}}^{+\frac{1}{2}}(\mathrm{~V}, \mathrm{~W}) \& \neg \mathrm{M}_{a, \mathrm{n}}^{-\frac{1}{2}}(\mathrm{~V}, \mathrm{~W})
\end{aligned}
$$$$
\left(\mathrm{I}^{(0)}\right)
$$

$$
J_{(\tau, \mathrm{n}+1)}\left(\mathrm{V} \Rightarrow{ }_{2} \mathrm{~W}\right)
$$

In comparison with $[4,5]$ representation of rules for PIR-II is changed only for $\pm 1 / 2$.

$$
\left(\mathrm{II}^{\left(+\frac{1}{2}\right)}\right) \frac{J_{(\xi, \mathrm{n})}\left(\mathrm{V} \Rightarrow_{1} \mathrm{~W}\right), \Pi^{+\frac{1}{2}}{ }_{\mathrm{n}}(\mathrm{V}, \mathrm{W}) \&-\Pi^{+}(\mathrm{V}, \mathrm{W})}{J_{\left\langle\frac{1}{2}, \mathrm{n}+1\right\}}\left(\mathrm{V} \Rightarrow_{1} \mathrm{~W}\right)}
$$

$\left(\mathrm{V} \Rightarrow{ }_{1} \mathrm{~W}\right)$ gets truth value $+1 / 2$, only if it does not simultaneously gets truth value +1 . (For $-1 / 2$ and -1 - symmetrically.)

The former consideration involves two degrees of properties presence or absence $- \pm 1 / 2$ and \pm 1 . This scheme can be generalized to the case of $n$ degrees.

\section{CONTEXTUAL METHOD}

But the simple (binary) model of causal relation "cause - effect" appears to be quite insufficient for many empirical fields. More profound contextual - analysis of causal relation is provided by the so-called "generalized" JSMmethod. We shall try to develop an idea of contextual analysis in application to the problem involved.

Let us introduce new sorts of variables.

Variables of the $5^{\text {th }}$ sort - for set of attendant (promoting or deterrent) factors, $U^{(5)}=\left\{c_{1}, \ldots\right.$, $c_{p}$, constants and variables of the $5^{\text {th }}$ sort, $Z \in 2^{U^{(5)}}$. Variables of the $4^{\text {th }}$ sort for sets $Z, Z_{1}$, $(\zeta)^{\prime}$, $Z_{\mathrm{m}}$...(and corresponding constants), $Z \in 2^{2^{\mathrm{U}^{\left(j^{\prime}\right)}} \text {. }}$. Alongside with Boolean algebras $B_{i}(i=1,2)$ (see above) Boolean algebras $\mathbb{B}_{4}=\left\{2^{U^{(5)}},-\cap, \cup\right\}$ algebra of attendant factors - and $B_{3}=\left\{2^{2^{U^{(5)}}}\right.$, $-, \cap, \cup\}$ - algebra of external circumstances are considered.

Let's consider ternary predicate $\mathrm{F}(\mathrm{X} \mathrm{Z}, \mathrm{Y})$ "objects $\mathrm{X}$ under the circumstances $\mathrm{Z}$ possesses the set of properties $Y$ " - instead of binary predicate $\mathrm{X} \Rightarrow_{1} \mathrm{Y}$ - "object $\mathrm{X}$ possesses (does not possess) the set of properties $Y$ ". Binary predicate $\mathrm{V} \Rightarrow_{2} \mathrm{~W}$ - "the subobject $\mathrm{V}$ causes the set of properties $W$ " is substituted by 2 ternary predicates: $T_{p r}(V, Z, W)$ - "the subobject $V$ under the circumstances (promoting factors) from the set $Z$ causes (does not cause) the set of properties $W "$ - and $T_{d}(V, Z, W)$ - "the subobject $V$, if circumstances (deterrent factors) from the set $Z$ are absent, causes (does not cause) the set of properties W".

There is not enough place here to present the complete description of formula for predicates $\mathbf{M}_{a p r, \mathrm{n}}^{+}(\mathrm{V}, \mathrm{Z}, \mathrm{W})$ and $\mathbf{M}_{a d, \mathrm{n}}^{+}(\mathrm{V}, \mathrm{Z}, \mathrm{W})$. We can 
only say, that first one describes a set of examples (this set being a base for plausible inference), expresses the exaustability condition - demand to consider all appropriate examples from initial DB. Empirical regularity, that describes predicted causal relation in $\mathbf{M}_{a p r, \mathrm{n}}^{+}(\mathrm{V}, \mathrm{Z}, \mathrm{W})$, is expressed by subformula

$\forall \mathrm{X} \forall \mathrm{Z} \forall \mathrm{Y}\left(\left(\left(J_{(1, \mathrm{n})} \mathrm{F}(\mathrm{X}, \mathrm{Z}, \mathrm{Y}) \& \forall \mathrm{Y}^{\prime} \forall \mathrm{Z}^{\prime}\left(J_{(1, \mathrm{n})} \mathrm{F}(\mathrm{X}, \mathrm{Z}\right.\right.\right.\right.$ $\left.\left.\left.{ }^{\prime}, \mathrm{Y}^{\prime}\right) \rightarrow \mathrm{Z}^{\prime} \subseteq Z \& \mathrm{Y}^{\prime} \subseteq \mathrm{Y}\right) \& \mathrm{~V} \subset \mathrm{X}\right) \&\left(\mathrm{~V}_{\mathrm{l}}{ }^{\mathrm{pr}} \subseteq \mathrm{Z} \vee \ldots \vee \mathrm{V}_{\mathrm{r}}^{\mathrm{pr}}\right.$ $\subseteq \mathrm{Z})) \rightarrow \mathrm{W} \neq \varnothing \& \mathrm{~W} \subseteq \mathrm{Y}$ )

("V causes set of properties $W$ under the condition that elements $\mathrm{V}_{1}{ }^{\mathrm{pr}}, \ldots, \mathrm{V}_{\mathrm{r}}^{\mathrm{pr}}$ from the set of attendant (promoting) factors $Z$ are present"). Other fragments of the predicate $\mathbf{M}_{a p r, \mathrm{n}}^{+}(\mathrm{V}, \mathrm{Z}, \mathrm{W})$ describe local similarity of the situations, where promoting factors $\mathrm{Z}$ act, exaustability condition - taking into account all these situations, - condition of $\mathrm{V}_{\mathrm{i}}^{\mathrm{pr}}$ minimality ( $i$ $=1, \ldots, r)$ and condition, that their set is unique.

The predicate $\mathbf{M}_{a d, \mathrm{n}}^{+}(\mathrm{V}, \mathrm{Z}, \mathrm{W})$ is formulated analogously to generalized predicate of agreement $\mathbf{M}_{a g, \mathrm{n}}^{+}(\mathrm{V}, \mathrm{X}, \mathrm{W})$ from [8]. The only difference is in sorts of elements from $Z$ and $X$. The elements of the set of obstacles $X$ from $\mathbf{M}_{a g, \mathrm{n}}^{+}(\mathrm{V}, \mathrm{X}, \mathrm{W})$ are subobjects (from $2^{\mathbf{U}^{(1)}}$ ) whereas the elements of the set of attendant factors $\mathrm{Z}$ from $\mathbf{M}_{a d, \mathrm{n}}^{+}(\mathrm{V}, \mathrm{Z}, \mathrm{W})$ represent external circumstances (from $2^{\mathbf{U}^{(5)}}$ ), under which causal relation exists. Empirical regularity, that describes predicted causal relation in $\mathbf{M}_{a d, \mathrm{n}}^{+}(\mathrm{V}, \mathrm{Z}, \mathrm{W})$, is expressed by subformula $\forall \mathrm{X} \forall \mathrm{Z} \forall \mathrm{Y}\left(\left(\left(J_{(1, \mathrm{n})} \mathrm{F}(\mathrm{X}, \mathrm{Z}, \mathrm{Y}) \& \forall \mathrm{Y}^{\prime} \forall \mathrm{Z}^{\prime}\left(J_{(1, \mathrm{n})} \mathrm{F}(\mathrm{X}, \mathrm{Z}\right.\right.\right.\right.$ $\left.\left.\left.{ }^{\prime}, Y^{\prime}\right) \rightarrow Z^{\prime} \subseteq Z \& Y^{\prime} \subseteq Y\right) \& V \subset X\right) \& \neg\left(V_{1}{ }^{\mathrm{pr}} \subseteq Z \vee \ldots \vee V\right.$ $\left.\left.\left.{ }_{\mathrm{r}}^{\mathrm{pr}} \subseteq \mathrm{Z}\right)\right) \rightarrow \mathrm{W} \neq \varnothing \& \mathrm{~W} \subseteq \mathrm{Y}\right)$

("V causes set of properties $\mathrm{W}$ under the condition that elements $\mathrm{V}_{1}{ }^{\mathrm{pr}}, \ldots, \mathrm{V}_{\mathrm{r}}^{\mathrm{pr}}$ from the set of attendant (deterrent) factors $Z$ are absent").

It is obvious, that context consideration is essential in investigation of individual (collective) behaviour. The proposed approach to context consideration does not, of cause, rule out the necessity to formalize "situation" concept and to investigate "situation $\Rightarrow$ behaviour" relation, the latter problems being the problems of macrosociology.

\section{PRESENT STATUS}

We have just begun to study the particular case of behaviour - predisposition to one or another social identification and solidary actions. We collaborate with the group of researchers from Institute of sociology, Russian Academy of Sciences - E.N.Danilova, S.G.Klimova, O.N.Dudchenko - headed by Director of Institute professor V.A.Yadov. First results of JSManalysis of solidary behaviour have been received. They are in accordance with theoretical models of sociological investigation. The results give the possibility to represent the totality of social actions' determinants reflecting different types of consciousness.

\section{ACKNOWLEDGEMENTS}

Most part of computer work has been carried out by S.S.Moscovsky with participation of N.I.Melnicov. The authors are grateful to D.W.Vinogradov for fruitful discussions.

\section{REFERENCES}

[1] Finn, V.K., Mikheyenkova, M.A. "On the application of JSM-method of automatic hypotheses generation in sociological investigations", Artificial Intelligence News, special issue, pp.91-98, 1993.

[2] Mikheyenkova, M.A. "Application of JSMreasoning to problems of sociology", in Proceedings of the 1995 ISIC Workshop, 1995, pp.429-433.

[3] Mikheyenkova, M.A., Danilova, E.N., Finn, V.K., Ivashko, V.G., Yadov, V.A. "Application of JSM-method of automatic hypotheses generation to some problems of sociology", in Proceedings of Workshop on Applied Semiotics, European Conference on Artificial Intelligence96, 1996, p.22-25

[4] Finn, V.K. "Plausible Inferences and Plausible Reasoning", Journal of Soviet Mathematics, Plenum Publ.Cor., vol.56, N 1, 1991.

[5] Finn, V.K. "Plausible Inferences in Intellectual Systems of JSM-type", Itogi Nauki i Tekhniki, ser. Informatica., V.15, pp.54-101, 1991 (in Russian).

[6] Finn, V.K. "JSM-reasoning for control in open ( \pm )-worlds", in Proceedings of the 1995 ISIC Workshop, 1995, pp.75-79.

[7] Finn, V.K. "On intelligent systems of automated support of scientific research", Nauchno-tekhnicheskaya informatsiya, ser.2, N 5-6, p.1-2, 1996 (in Russian).

[8] Finn, V.K. "On generalized JSM-method of automatic hypotheses generation", Semiotics and Informatics, Issue 29, pp.93-123, 1989 (in Russian). 


\title{
Algorithmic Complexity of Generating Hypotheses and Forecasts in Version Spaces Formed by Concept Lattices
}

\author{
S.O. Kuznetsov \\ Intelligent Systems Laboratory, \\ Moscow, RUSSIA \\ serge@viniti.msk.su
}

\begin{abstract}
A survey of results concerning algorithmic complexity of learning in the version space formed by Galois (concept) lattices is given. The results are related to some work in machine learning and abductive reasoning.
\end{abstract}

KEYWORDS: concept lattices, machine learning, classification, abductive reasoning

\section{INTRODUCTION}

Recent studies [12], [3] show that formal concept analysis based on Galois (or concept) lattices is a good means for learning of concepts. However, this approach faces much computational difficulties, since the number of concepts can be exponential in the number of examples. Therefore, the problem of computational complexity is crucial here. Algorithm reported in [3] is quadratic in the number of concepts generated. In this paper, we present some results concerning the complexity of generating the set of all hypotheses in the version space formed by Galois lattices. We describe an algorithm which is linear in the number of hypotheses (and, therefore, in the number of concepts), and can work both in batch and incremental modes. Unlike the model of concept clustering method from [3], the JSM-method from [4, 5] uses both positive and negative examples. This allows us to relate the model presented to a generalization of version spaces [8] known as disjunctive version space approach [10], [9]. We discuss some extensions of the approach to more general data structures (e.g., to graphs).

\section{MAIN DEFINITIONS: HYPOTHESES AND FORE- CASTS (CLASSIFICATIONS)}

The model presented here was first proposed in [4] as JSMmethod of automatic hypothesis generation and described in more detail in [5].

Let $E^{+}$be a set of positive examples and $E$ be a set of negative examples of a class (defined by a property $W$, in the sequel, we do not mention the name of the property when possible), and $U$ be a set of attributes, $I^{+}$and $I$ be relations defined on $E^{+} \times U$ and $E \times U$, respectively. The triples $\left(E^{+}, U, I^{+}\right)$and $(E$ $, U, I)$ are called positive and negative contexts, respectively.
Definition 1. Let $A \subseteq U, B \subseteq E^{+}$. The Galois connections on the set $E^{+} \times U$ are given in the following way:

$s(A)=\left\{e \in E^{+} \mid e I^{+} a\right.$ for all $\left.a \in A\right\}$,

$t(B)=\left\{a \in U \mid a I^{+} e\right.$ for all $\left.e \in B\right\}$.

As in [11], instead of $s(A)$ and $t(B)$ we write $A^{\prime}$ and $B^{\prime}$. We will use the notation $A$ "' and $B$ "' as abbreviations for $t(s(A))$ and $s(t(B))$.

A pair $(A, B)$ such that $A^{\prime}=B, B=A^{\prime}$ is called a (positive) concept, $A$ is called the intent, and $B$ is called the extent of the concept [11]. The set of all concepts forms a lattice (for properly defined sup and inf operations) called Galois lattice [3] or concept lattice [11].

Definition 2. Let $\left(E^{+}, U, I^{+}\right)$and $(E, U, \Gamma)$ be positive and negative contexts. A positive concept $\left(H, H^{\prime}\right), H \subseteq U$ is a positive hypothesis generated for these contexts if $H \subseteq e$ for no negative example $e \in E$.

Analogs of this definition are used in various paradigms of machine learning. It says, that a hypothesis (concept, description, etc.) should cover some positive examples and should not cover any negative example.

A positive hypothesis $\left(H, H^{\prime}\right)$ is called minimal by iuclusion if there is no positive hypothesis

$\left(H_{l} H_{l}^{\prime}\right)$ such that $H_{l} \subseteq H$ (minimality of a hypothesis means that it is most general among the set of all hypotheses).

Galois connections for negative examples and (minimal) negative hypotheses are defined similarly.

The following definition gives the rule of forecast (classification).

Definition 3. Let $\left(E^{+}, U, I^{+}\right)$and $(E, U, I)$ be positive and negative contexts, and $Q$ be an object that belongs neither to $E^{+}$nor to $E$ (i.e., we do not know whether it has or does not have the property $W$ ). A positive hypothesis $\left(H_{+}, H_{+}{ }^{\prime}\right)$ is called a hypothesis in favor of $Q$ if $H^{+} \subseteq Q$. A negative hypothesis $\left(\mathrm{H}_{\text {, }} \mathrm{H}\right.$. ') is called a hypothesis against $Q$ if $H . \subseteq Q$ is called a positive forecast if there is a positive hypothesis in favor of it and there is no negative hypothesis against it. Negative forecasts are defined dually. 
The relation "be in favor of" corresponds to the relation "cover" (when the talk is about descriptions covering examples). We avoid using this word here, since "covering" has the opposite order-theoretic connotation.

\section{COMPLEXITY RESULTS}

\subsection{Theoretical Limitations}

It is easily shown that the number of hypotheses can be exponential. Moreover, this number is hard to compute and estimate (which could have been useful for resources allocation). The following result is a testimony to this statement.

Theorem 1. The problem of determining the number of all positive (negative) hypotheses is \#P-complete.

Theorem 2. The problem of determining the number of all positive (negative) hypotheses minimal by inclusion is \#Pcomplete.

Theorem 3. The following problem is NP-complete: INSTANCE Positive context $\left(E^{+}, U, I^{+}\right)$, negative context $(E-, U, I)$, query $Q$.

QUESTION Does there exist a positive hypothesis in favor of $Q ?$

The theorem is proved by considering a combinatorial interpretation of the problem: a problem related to a quadripartite graphs [6].

The following particular cases of the problem from Theorem 4 where polynomial-time algorithms are possible were indicated in [6]:

- The set of negative examples is empty,

- The number of attributes from $U$ that correspond to the query $Q$ (i.e., $|Q|$ ) is bounded from above by a constant. This case is very important for applications, for example, in computer drug design, where the size of a chemical compound is limited, but the number of compounds involved as positive and negative examples is large. The algorithm used in this case is linear w.r.t. the number of positive hypotheses in favor the query.

In the following table, we present results from [6] about the complexity of decision problems concerning hypotheses with restrictions on the sizes of their intents and extents.

\begin{tabular}{|l|l|l|}
\hline & $\leq$ & $\geq$ \\
\hline$|H|$ & $\mathrm{NP}$ & $\mathrm{P}$ \\
\hline$\left|H^{\prime}\right|$ & $\mathrm{P}$ & $\mathrm{NP}$ \\
\hline$|H|+\left|H^{\prime}\right|$ & $\mathrm{NP}$ & $\mathrm{NP}$ \\
\hline
\end{tabular}

As above, $|H|$ and $\left|H^{\prime}\right|$ denote the sizes of intents and the extents of hypotheses, respectively; $P$ denotes that there exists a polynomial algorithm for solving the problem, NP denotes NP-completeness of the problem. For instance, the upper left element of the table means that the problem "does there exist a hypothesis such that $|H| \leq k$ ?" ( $k$ is a parameter) can be solved by a polynomial time algorithm. The element in the bottom line and right column is indicative of the fact that the problem "does there exist a hypothesis such that $|H|+\left|H^{\prime}\right| \geq k$ ?" is NPcomplete.

\subsection{Close-by-One (CbO) Algorithm for Computing $\mathrm{Hy}$ - potheses and Its Complexity}

We assume that all objects from $E^{+}$are numbered, and so a set $X \subseteq E^{+}$can be represented by a respectively ordered tuple. The numbering of objects from $E^{+}$induces lexicographic ordering of sets from the powerset of $E^{+}$. For the sake of convenience, we can represent the process of computing hypotheses as a top-down one, which generates some tree whose vertices correspond to hypotheses. During this process, the examples from $E^{+}$can be labeled or remain unlabeled in each vertex independently. The following algorithm is based upon the depth-first strategy, though other strategies are possible as well. $Y$ denotes the extent of a current hypothesis.

\section{Close-by-One (CbO) Algorithm}

Step 0. There is only one root vertex where all examples are unlabeled, $Y:=\varnothing$.

Step 1. The current vertex corresponds to the concept with the extent $Y$. The first unlabeled element of $E^{+}$, say $X_{i}$, is taken, $\left(Y \cup\left(X_{i}\right)\right)^{\prime}$ and $\left(Y \cup\left\{X_{i}\right\}\right)^{\prime}$ ' are computed. A new vertex that corresponds to $\left(Y \cup\left(X_{i}\right)\right)^{\prime \prime}$ 'is generated and connected by an edge to the vertex associated with $Y$.

Step 2. Check conditions (a) and (b):

(a) $\left(Y \cup\left\{X_{i}\right\}\right)$ "'contains objects with numbers less than those of the objects from $Y$ or the number of $X_{i}$, (i.e., the concept with the extent ( $\left.Y \cup\left\{X_{i}\right\}\right)^{\prime}$ 'has already been generated).

(b) $\left(Y \cup\left(X_{i}\right\}\right)^{\prime}$ is contained as a subset in a negative example from $E$

If either of these cases holds, all elements of $E^{+}$are labeled at the vertex $\left(Y \cup\left\{X_{i}\right\}\right)$ '"(thus, the branch will not be extended). If neither of conditions (a) and (b) holds, we label additionally the element $X_{i}$ at the vertex $Y$ and all elements of $\left(Y \cup\left\{X_{i}\right\}\right)^{\prime}$ 'at the vertex $\left(Y \cup\left\{X_{i}\right\}\right)$ '.

Step 3. If all elements of $E^{+}$are labeled at $\left(Y \cup\left(X_{i}\right\}\right)$ ', we go to Step 4. Otherwise, $Y:=\left(Y \cup\left\{X_{i}\right\}\right)^{\prime \prime}$, we return to Step 1 .

Step 4. We backtrack the tree upwards to the nearest vertex with unlabeled elements of $E^{+}$. If such a vertex exists and corresponds, say, to the object $Z$, then $Y:=Z$ and we have to go to step 1 . If there is no such vertex, this means that all concepts have been generated and the algorithm halts.

Theorem 4. Let $\left(E^{+}, U, I^{+}\right)$and $(E, U, I)$ be positive and negative contexts, respectively. Then the set of all positive hypotheses can be generated in time $O\left(\left|E^{+}\right||E||U| K\right)$ and space 
$O\left(\left(\left|E^{+}\right|+|U|+|E|\right) K\right)$. by the algorithm $\mathrm{CbO}$, where $K$ is the number of resulting hypotheses. The same estimate holds for negative examples.

Thus, the resources required are linear w.r.t. to the number of hypotheses generated, which is better than results reported in [3], where an algorithm is proposed that has quadratic complexity w.r.t. to the number of hypotheses.

Algorithm $\mathrm{CbO}$ can work in purely incremental way without recomputing old hypotheses: new examples are given sequential numbers greater than those of the existing examples and the generation of hypotheses continues. The principle of canonical ordering of hypotheses used in the algorithm enables the parallelization of the algorithm: all hypotheses are generated with the use of local operations and tests, which does not require the interaction of processors, the use of a supervisor, and shared memory.

The $\mathrm{CbO}$ algorithm can be applied to more general type of data than contexts from Definition 1, e.g., in the case where examples are represented by sets of graphs. In this case, we do not use Galois connections, but define operation $G^{\prime}$ on a set of graphs $G$ as the set of all maximal by inclusion common subgraphs of the graphs from $G$. Though the problem of computing such operation is NP-hard, the algorithm remains linear with respect to the number of hypotheses generated. More generally, the algorithm can be used for arbitrary data that allows the definition of an idempotent, commutative and associative operation $\Pi$ (i.e., of inf type) on pairs of objects. The expression. $\left(Y \cup\left(X_{i}\right\}\right)^{\prime}$ in the formulation of the $\mathrm{CbO}$ algorithm is replaced by $Y_{l} \Pi \ldots \Pi Y_{k} \Pi X_{i}$, where $\left\{Y_{1} \ldots, Y_{k}\right\}=Y$, and expression $\left(Y \cup\left(X_{i}\right\}\right)$ " is replaced by the operation of taking the set of all objects $S$ that contain $Z$ (i.e., $S \Pi Z=Z$ ) In the case of contexts the operation $\Pi$ corresponds to the settheoretic intersection $\cap$. The time complexity of the algorithm in the general case is $O\left(\left|E^{+}\right| K\right) \alpha+O\left(\left|E^{+}\right|^{2}|E| K\right) \beta$, where $m$ is the number of positive examples, $K$ is the number of resulting hypotheses, $\alpha$ is the complexity of computing operation $\Pi, \beta$ is the complexity of testing the order relation corresponding to the operation $\Pi$.

\section{RELATION TO OTHER WORK AND DISCUSSION}

As mentioned above, our algorithm constructs Galois or concept lattices as their version spaces. Machine learning method reported in [3] uses the same version spaces, but the algorithms used therein are less efficient and do not take into account negative examples (concept is a particular case of a hypothesis when $E=\varnothing$ ). The version space used in this work is similar to the disjunction version space proposed in $[9,10]$ as the extension of Mitchell's version spaces [8]: examples must be covered by a disjunction of concepts, not by a single concept as in the work by Mitchell. Hypotheses defined in this paper correspond to the set $S$ of the lower boundary of the disjunctive version space. The work [10] reports on the quadratic algorithm for the generation of $S$, which is outperformed by our algorithm. Definition 3 presents a way of plausible reasoning similar to abduction [2]. Moreover, this definition uses a method for generating hypotheses (Definition 2), whereas in abduction models they appear like deus ex machina. Computational results concerning forecasts are similar both in pessimistic (related to \#P-completeness) and optimistic (linear complexity in the number of hypotheses) aspects to those obtained in [2]. A more close look on the connection of JSM-method with the formal concept analysis along the lines of [11] is presented in [7].

The CbO Algorithm was used in an applied system for computer drug design [1]. By means of this algorithm the system detects structural causes of biological acitivity and classifies unstudied compounds w.r.t. biological activity under study. Chemical compounds can be presented both in descriptor form (as sets of descriptors) and as molecular graphs. Though the latter case is more preferable from the point of view of applications (better forecasts are obtained), computing all hypotheses on average Pentium PC computer is feasible only for small samples (20-30 examples), as it was in the case of octane series [1]. The use of $\mathrm{CbO}$ algorithm drastically reduced the computation time ( $60 \%$ on average) as compared with the time needed for previous algorithms used in the system, which, like those in [3] and [10] had quadratic time complexity in the number of hypotheses.

\section{REFERENCES}

[1] Blinova, V.G. On Results of Applying JSM-method of Hypothesis Generation to the Problem "Structure of Chemical Compound - Biological Activity," Automat. Document. Math. Linguist., No. 5, 1995.

[2] Bylander, T., Allemang, D., Tanner, M. C., and Josephson, J. R., "The Computational Complexity of Abduction," Artif. Intell., 49, No. 1, 25-60, 1991.

[3] Carpineto, C. and Romano, G. "A Lattice Conceptual Clustering System and Its Application to Browsing Retrieval," Mach. Learn., 24, 95-122, 1996.

[4] Finn, V. K. Data bases with incomplete information and a new method of automatic hypothesis generation, in: "Dialog and Factographic Information Systems" [in Russian], Moscow, (1981), pp.153-156.

[5] Finn, V. K. "Plausible Inference and Plausible Reasoning", J. Sov. Math., 56, No.1, 1991.

[6] Kuznetsov, S. O. "Algorithmic Complexity of the Generation of Hypotheses and Classification Based on the Search for Set Intersection", Doklady Akad. Nauk, 335, No. 3, 300-303, 1994 (Physics-Doklady), 39, No. 3, 142-145, 1994.

[7] Kuznetsov, S. O. "Mathematical Aspects of Concept

Analysis," J. Math. Sci., ser. Sovremennaya Matematika i ee Prilozheniya, 18, 27-71, 1995.

[8] Mitchell, T. "Generalization as Search," Artificial Intelligence, 18, 203-226, 1982. 
[9] Nikolaev, N. I.and Smirnov, E. N. "Analytical Learning Guided by Empirical Technology: An Approach to Integration," in: N. Lavrac and S. Wrobel, Eds., Proc. European Conference on Machine Learning, Lecture Notes on Artificial Intelligence, 912, Springer Verlag, 327-330, 1995.

[10] Smirnov, E. N. "Space Fragmenting - A Method for Disjunctive Concept Acquisition," in: B. Du Boulay and V. Sgurev, Eds., Artificial Intelligence V: Methodology, Systems, Applications, Elsevier, 97-104, 1992.

[11] Wille, R. "Restructuring lattice theory: an approach based on hierarchies of concepts," in: I. Rival, Ed., Ordered Sets, Dordrecht-Boston (1982), pp.445-470.

[12] Wille, R."Concept Lattices and Conceptual Knowledge Systems," Comput. Math. Appl., 23, No. 6-9, 493-515, 1992. 


\title{
To the Scaleable Technology of Automated Document Understanding based on Quasi-Axiomatic Theories.
}

\author{
M.I. Zabezhailo \\ Intelligent Systems Laboratory, \\ Moscow, RUSSIA \\ lis@viniti.msk.su
}

\begin{abstract}
A new approach to full-text processing is presented. The discussed text-processing technique is based on the logical formalism of quasiaxiomatic theories (QAT). A core element of the described approach is in use of original machine learning technique - so-called JSMmethod of automated hypotheses formation. The described QATbased technique is used as a tool for analysis of similarities in contents of full-text documents. Finally, possible applications of the presented tools as a practical instrument for automated message understanding are discussed.
\end{abstract}

KEYWORDS: quasi-axiomatic theories, plausible reasoning, machine learning, automated document understan ding.

\section{INTRODUCTION}

To design a semiotic model of open system in many cases means to provide an ability to process data of different types describing open (unclosed, partially described, etc.) subject area. In more details it means to support the following two characteristic features:

- to provide the ability (for the system controller and for the model under design) to learn by experience (e.g., by examples $^{1}$ );

- to provide the ability to process (by "unified" data OLAP2 and decision making instruments applied in a "unified" decision making environment) data of different types (e.g., symbolic and numeric, structured and unstructured, textual and graphical, etc.).

Our approach to design the presented type mechanisms of modeling is based [1] on the so called Quasi Axiomatic Theories (QAT). Every QAT may be characterized (for more details see, for example [1] and [4]) by a tuple

where

$$
\Gamma=\left\langle\Sigma, \Sigma^{\natural}, \mathbf{R}\right\rangle
$$

- $\Sigma$ is an axiom set (describing a "current state" of the subject area under investigation and the controlled system.

\footnotetext{
${ }^{1}$ I.e. to base current decision making process on the experience of both "good" (i.e. "positive") and "bad" (i.e. "negative") previously made decisions.

2 OLAP - OnLine Analytical Processing.
}

Roughly speaking it's a set of "empirical dependencies" invariant lows ${ }^{3}$ - describing a situation under analyșis);

- $\Sigma^{6}$ is a set of empirical statements (i.e., elementary "facts") characterizing empirical knowledge (e.g., examples of previously made decision, etc.);

- $\mathbf{R}$ is a set of reasoning rules consisting of two parts

where

$$
\mathbf{R}=\mathbf{R}_{\mathbf{r}} \cup \mathbf{R}_{\mathbf{p}}
$$

- $\mathbf{R}_{\mathbf{r}}$ is a set of reliable inference rules (e.g., rules of deductive inference);

- $\mathbf{R}_{\mathbf{p}}$ is a set of plausible reasoning rules (e.g., formalized variants of reasoning by analogy rules, "common sense" reasoning rules, rules of abductive reasoning, etc.).

From our viewpoint the challenge is to provide the designed semiotic model of the analyzed open system by the following two features:

- to be able to learn (by use of reasoning rules from

$\mathbf{R}=\mathbf{R}_{\mathbf{r}} \cup \mathbf{R}_{\mathbf{p}}$ ) new empirical dependencies from "examples" (i.e. from elements of $\Sigma^{6}$ ) and

- to be able to use this new dependencies (i.e., procedural extension of $\Sigma$ processed from $\Sigma^{\mathbf{6}}$ by $\mathbf{R}$ ) in "understanding" and "processing" of new situations (i.e. control activities in new situations) for open system under analysis.

\section{AN APPROACH.}

We start from the methodological hypothesis characterizing a formal model of natural language as a set of local dependencies (i.e. grammatical rules, axioms) and a set of exclusions (i.e. facts, that can not be described by the mentioned axioms). Our aim is to learn by examples of facts and to extend (where it's possible) some known rules on new cases (new facts). We use the JSM-method (see [1]) as the tool of machine learning and reasoning formalization realized by means of QAT. This technology may be introduced as a methodological platform for machine intelligence systems that implement a synthesis of cognitive procedures. The technology is based on the original and sophisticated mathematical formalism of inductive learning. The described mathematical formalism provides an effective co-operation of the following reasoning techniques:

3 Providing only partial (i.e. - uncomplete) description of the situation under analysis. 
- constructive formalization of abductive reasoning (in the sense of C.S.Peirce);

- constructive formalization of inductive reasoning (in the sense of J.S.Mill);

- automated deduction;

- formalized reasoning by analogy.

The presented tools are oriented on the processing of large volumes of information in full-text data bases. The discussed tools provide sufficiently "flexible" and effective search of documents that are "relevant" to fixed themes. Using this type tools it's possible to implement NL-text-processing based on "understanding" documents by computer (this "understanding" is provided by original instruments that model NL-text semantics by special syntactic structures).

The key characteristic of our text-processing tools is in automatic (i.e. implemented by computer) "understanding" of a target set of full-text documents. This "understanding" is provided in the process of co-analysis of the target texts and automatically extracted from the semantic structures (so-called concepts). The discussed concepts are designed in the process of machine learning by analyzed texts. We use QuasiAxiomatic Theories (QAT) as an original methodological platform for automated text understanding: the techniques used for machine learning is formalized by means of QAT.

Basic elements of our approach are the following:

- automatic extraction and analysis of the "semantic similarity" classes for processed documents;

- an ability to use, in the process of machine learning, both positive and negative text-precedents;

- a flexible structure of logical inference rules for document classification (i.e. for similarity classes generation);

- an ability to combine logical and statistical methods of data analysis.

The most important features that differs our approach from other solutions used in the discussed problem area may be characterized by the following:

- we are able to provide effective machine learning from small samples of texts-precedents;

- we are able to process automated text "understanding" based on both positive (i.e. examples of texts that are relevant to the analyzed theme) and negative information (i.e. examples of texts that are not relevant to the analyzed theme);

- we are able to design non-monotonic (i.e. dynamically restructured in accordance with the extension of text corpora under analysis) structure of the "semantic similarity" classes for processed documents.

\section{APPLICATIONS}

We suppose that QAT-based techniques may be used as a background for a qualitative break and effective spurt in the area of automated text understanding. JSM-style document processing tools can provide NL-document processing which realize an automated document clustering (e.g., "natural kind"4 clustering of theme vectors extracted from documents by linguistic analysis tools of the ORACLE ConText type [2]). In opposite to the approach based on the analysis of statistical correlations $^{5}$ (and realized, for example, in PATHFINDER [3]) the JSM-style document processing and "understanding" technique can provide an exhaustive deterministic analysis and "context-dependent" computational document understanding

We suppose that the most important applications of our theoretical approach and software tools might be designed in the following problems:

- the improvement of effective and rapid information searches on the INTERNET;

- automated message understanding;

- automated classification of full-text documents;

- improvement of the text-processing effectiveness for standard document management systems;

- improvement (based on the use of machine learning technique) of effectiveness for information search tools in standard text based information retrieval systems;

- an analysis of "semantic analogy" for sets of full-text documents (e.g., an analysis of how closely connected, in the sense of their content, two sets of texts are);

- automatic diagnostics for the existence of "many aspects" (multi-dimensional semantics) in the content of the text corpora under analysis;

- automatic diagnostics of a "new content" occurrence caused by an extension of analyzed text corpora by new elements (i.e. by new texts);

and some other fields, characterizing applications of intelligent information systems (see for example [5]).

\section{REFERENCES}

[1] Finn V. K. "Plausible inferences and plausible reasoning". - Journ. Soviet Mathematics. Plenum Publ. Corp., vol. 56, N 1, 1991.

[2] "CONTEXT: Introduction to Oracle ConText". - ORACLE White Paper.- ORACLE Corp., September, 1993.- Pp.1-17.

${ }^{4}$ I.e. clustering based on machine learning by examples of documents that relevant to the processed query (i.e., by so called positive examples) and by examples of documents that are not relevant to the processed query (i.e., by so called negative examples).

5 More correctly: it is based on the analysis of "distances" between "lexical units" - e.g., words, themes, ... - and on the analysis of there "coexistence" in analyzed documents. 
[3] "PATHFINDER (Version 7.0)".- User's Guide. - Presearch Inc., Fairfax, VA. - 1994.

[4] Zabezhailo M.I. et al. "Reasoning Models for Decision Making: Applications of JSM-Method for Intelligent Control Systems". - Architectures for Semiotic Modeling and Situa
August 1995, Monterey, CA. - AdRem, Inc., 1995. - Pp. 99108.

[5] Zabezhailo M.I., Finn V.K. "Intelligent information systems". - International Forum on Information and Documentation (FID, The Hague, Netherlands).- 1996. - Vol.21. - N2. Pp.21-31 tion Analysis in Large Complex Systems.- Proc. of the Workshop of 10th (1995) IEEE Symp. on Intelligent Control (Eds.: J.Albus, A.Meystel, D.Pospelov, T.Reader). - 27 -29 


\title{
NonMonotonic Reasoning in the Modal Quantificational Logic Z
}

\author{
Frank M. Brown \\ Artificial Intelligence Laboratory \\ University of Kansas \\ Lawrence, Kansas, 66045 \\ brown@eecs.ukans.edu
}

\begin{abstract}
Nonmonotonic reasoning can be recursively axiomatized as a formal axiomatic theory which is monotonic. This paper describes how this is done beginning with an axiomatization of logical truth with the modal quantificational logic $\mathrm{Z}$ which builds on previous works of Leibniz, Carnap, Prior, and especially Bressan. It explains how to form default statements in terms of modal possibility with respect to a theory, how to reduce possibility with respect to a theory to logical possibility, how to determine when something is logically possible, how to form reflective equations where the default is formed using the theory containing the default itself, how to solve various types of reflective equations in quantificational logic to obtain their "fixed points", and how to deal with multiple solutions by taking their commonalty. In a broader sense this work supports Lewis's original claim that modal logic is a missing part of classical logic in that it illustrates how classical logic extended with modal logic accounts for nonmonotonic reasoning.
\end{abstract}

\section{Introduction}

NonMonotonic Reasoning is a form of deductive reasoning that allows general laws to be asserted and general conclusions to be drawn from them without contradiction in the presence of numerous exceptions. For example, in a nonmonotonic system one may assert the general law that birds fly even though we also know that penguins are birds which do not fly. In a nonmonotonic system the assumption that there are such penguins will not be contradictory. This is achieved by representing general laws as defaults rather than merely as universally quantified implications. Thus instead of writing "birds fly" as for all $x$ if $x$ is a bird then $x$ flies:

$$
\forall \mathrm{x}((\text { bird } \mathrm{x}) \rightarrow(\text { fly } \mathrm{x}))
$$

it is written as for all $\mathrm{x}$ if $\mathrm{x}$ is a bird and if it is logically possible with respect to a theory $\Gamma$ for $x$ to fly then $x$ flies:

$$
(\forall x((\operatorname{bird} x) \wedge(<>(\Gamma \wedge(\text { fly } x))) \rightarrow(\text { fly } x))))
$$

The underlined phrase speaks of logical possibility thereby implying a reduction of nonmonotonic reasoning to modal logic There are of course many different modal logics, but there is one that is particularly relevant, namely the modal quantificational logic Z [Brown90] whose possibility operator is analogous to the semantical concept of satisfiability in classical first order logic. For all sentences $\Gamma$ of first order logic,

(Satisfiable (kwote $\Gamma)$ ) if and only if $(<\Gamma)$ where (kwote $\Gamma$ ) is the name of $\Gamma$ in the metatheory. The remainder of this paper describes nonmonotonic reasoning in terms of the $\mathrm{Z}$ modal logic. $\mathrm{Z}$ is axiomatized in Section 2. Proof techniques for determining what is logically possible are given in Section 3. Section 4 introduces reflective equations and describes an approach to solving them. Section 5 describes the propositional quantifier disjunction idiom, which is used to represent the disjunction of solutions to a reflective equation. Some conclusions are drawn in section 6.

\section{Axiomatization of $\mathbf{Z}$ Modal Logic}

The modal quantificational logic $\mathrm{Z}$ is an eight tuple: $(\rightarrow, \# \mathrm{f}, \forall,=,[]$, vars, predicates, functions) where $\rightarrow$, \#f, $\forall,=$, and [] are logical symbols, vars is a set of variable symbols, predicates is a finite set of predicate symbols each of which has an implicit arity specifying the number of terms to be associated with that predicate, and functions is a finite set of function symbols each of which has an implicit arity specifying the number of terms to be associated with that function. The set of logical symbols, the set of variables, and the set of predicate and function symbols are pairwise disjoint. The set of terms is the smallest set which includes the variables and is closed under the process of forming new terms from other terms using the functions symbols of the language. The set of sentences is the smallest set which includes \#f, the variables, and each of the predicates followed by an appropriate number of terms, and is closed under the process of forming new sentences from other sentences using the logical symbols of the language, provided that no variable in any subexpression of a sentence has free occurrences both as a sentence and as a term. Variables which occur only in term positions are called object variables. Variables which occur only in sentence positions are called propositional variables. Roman letters possibly indexed with digits are used as variables of $Z$; for example: $p, q, r, w, v, x_{1} \ldots x_{n}$, $\mathrm{y}_{1} \ldots \mathrm{y}_{\mathrm{n}}$. Greek letters are used as syntactic metavariables. $\gamma$ ranges over the variables, $\pi, \pi_{1} \ldots \pi_{\mathrm{n}}$ range over the predicate symbols, $\infty, \infty_{1} \ldots \infty_{n}$ range 
over function symbols, $\delta, \delta_{1} \ldots \delta_{\mathrm{n}}$ range over terms, and $\alpha, \alpha_{1} \ldots \alpha_{n}, \beta, \beta_{1} \ldots \beta_{n}, \Gamma$, and $\Gamma_{1} \ldots \Gamma_{n}$ range over sentences. Thus, the terms are of the forms $\gamma$ and $(\infty$ $\delta 1 \ldots \delta n)$, and the sentences are of the forms $(\alpha \rightarrow \beta)$, $\#$ f, $(\forall \gamma \alpha),([] \alpha),\left(\pi \delta_{1} \ldots \delta_{\mathrm{n}}\right),\left(\delta_{1}=\delta_{2}\right)$, and $\gamma . \quad A$ nullary predicate $\pi$ or function $\infty$ is written as a sentence or term without parentheses, i.e., $\pi$ instead of $(\pi)$ and $\infty$ instead of $(\infty)$.

The primitive symbols of $Z$ are shown in figure 1 .

\begin{tabular}{|l|l|}
\hline Symbol & Meaning \\
\hline$\alpha \rightarrow \beta$ & if $\alpha$ then $\beta$. \\
\hline$\# \mathrm{f}$ & falsity \\
\hline$\forall \gamma \alpha$ & for all $\gamma, \alpha$. \\
\hline$\delta 1=\delta 2$ & $\delta 1$ is (contingently) equal to $\delta 2$. \\
\hline$\square \alpha$ & $\alpha$ is logically necessary \\
\hline \multicolumn{2}{|l|}{ Figure 1: Primitive Symbols of $Z$} \\
\hline
\end{tabular}

The defined symbols of $Z$ are listed in figure 2 below with their intuitive interpretations .

\begin{tabular}{|c|c|c|}
\hline Symbol & Definition & Meaning \\
\hline$\neg \alpha$ & $\alpha \rightarrow \# \mathrm{f}$ & not $\alpha$ \\
\hline$\# \mathrm{t}$ & $\neg \# \mathrm{f}$ & truth \\
\hline$\alpha \vee \beta$ & $(\neg \alpha) \rightarrow \beta$ & $\alpha$ or $\beta$ \\
\hline$\alpha \wedge \beta$ & $\neg(\alpha \rightarrow \neg \beta)$ & $\alpha$ and $\beta$ \\
\hline$\alpha \leftrightarrow \beta$ & $(\alpha \rightarrow \beta) \wedge(\beta \rightarrow \alpha)$ & $\begin{array}{l}\alpha \text { if and only } \\
\text { if } \beta\end{array}$ \\
\hline$\exists \gamma \alpha$ & $\neg \forall \gamma \neg \alpha$ & some $p$ is $\alpha$ \\
\hline$<>\alpha$ & $\neg[] \neg \alpha$ & $\begin{array}{l}\alpha \text { is logically } \\
\text { possible }\end{array}$ \\
\hline$\alpha \equiv \beta$ & {[]$(\alpha \leftrightarrow \beta)$} & $\begin{array}{l}\alpha \text { and } \beta \text { are } \\
\text { synonymous }\end{array}$ \\
\hline$[\beta] \alpha$ & $([](\beta \rightarrow \alpha))$ & $\beta$ entails $\alpha$ \\
\hline$<\beta>\alpha$ & $(<>(\beta \wedge \alpha))$ & $\begin{array}{l}\alpha \text { is possible } \\
\text { in } \beta\end{array}$ \\
\hline$\delta 1[=] \delta 2$ & $\left([]\left(\delta_{1}=\delta_{2}\right)\right)$ & $\begin{array}{l}\delta_{1} \text { necessarily } \\
\text { equals } \delta_{2}\end{array}$ \\
\hline (world $\alpha$ ) & $\begin{array}{l}(<>\alpha) \wedge \\
(\forall \gamma(([\alpha] \gamma) \vee([\alpha] \neg \gamma))) \\
\text { where } \gamma \text { is not in } \alpha\end{array}$ & $\alpha$ is a world \\
\hline$(\operatorname{gen} \alpha)$ & $\begin{array}{l}\left(\vee\left(\exists \gamma_{11 \ldots} \ldots \gamma_{\mathrm{m} 1}\right.\right. \\
\left.\left.\left(\alpha \equiv\left(\pi_{1} \gamma_{11} \ldots \gamma_{\mathrm{m} 1}\right)\right)\right)\right) \\
\ldots\left(\exists \gamma_{1 \mathrm{n}} \ldots \gamma_{\mathrm{mn}}\right. \\
\left.\left.\left.\left(\alpha \equiv\left(\pi_{\mathrm{n}} \gamma_{1 \mathrm{n}} \ldots \gamma_{\mathrm{mn}}\right)\right)\right)\right)\right) \\
\text { where all the } \\
\text { predicates are } \pi_{1} \ldots \pi_{\mathrm{n}} \\
\text { and the arity of } \pi_{\mathrm{i}} \text { is } \\
\mathrm{m}_{\mathrm{i}} \text { and for each i and } \\
\mathrm{j}, \gamma_{\mathrm{ij}} \text { is a distinct } \\
\text { variable not in } \alpha\end{array}$ & $\begin{array}{l}\alpha \text { is a } \\
\text { generator }\end{array}$ \\
\hline
\end{tabular}

$\mathrm{Z}$ is effectively axiomatized with a recursively enumerable set of theorems as the set of axioms is itself recursively enumerable and its inference rules are recursive. The classical (i.e., non-modal) axioms and inference rules of $Z$ include those of quantificational logic [Mendelson] given in figure 3 . The laws MR1, MR2, MA1, MA2, MA3, MA4, MA5, MA6, and MA7 are a standard set of axioms and inference rules for first order quantificational logic except for the following two points: First, because $\gamma$ in laws MR2 and MA4 may be a propositional variable these two laws constitute an additional fragment of $2 \mathrm{nd}$ order logic beyond first order logic. Propositional quantification in modal logics has been investigated by [Fine 70]. A conceptual interpretation of quantification [Garson $84]$ is at the heart of this logic, although this will not be noticed until one deals with necessity, because all the normal laws of first order logic hold otherwise.

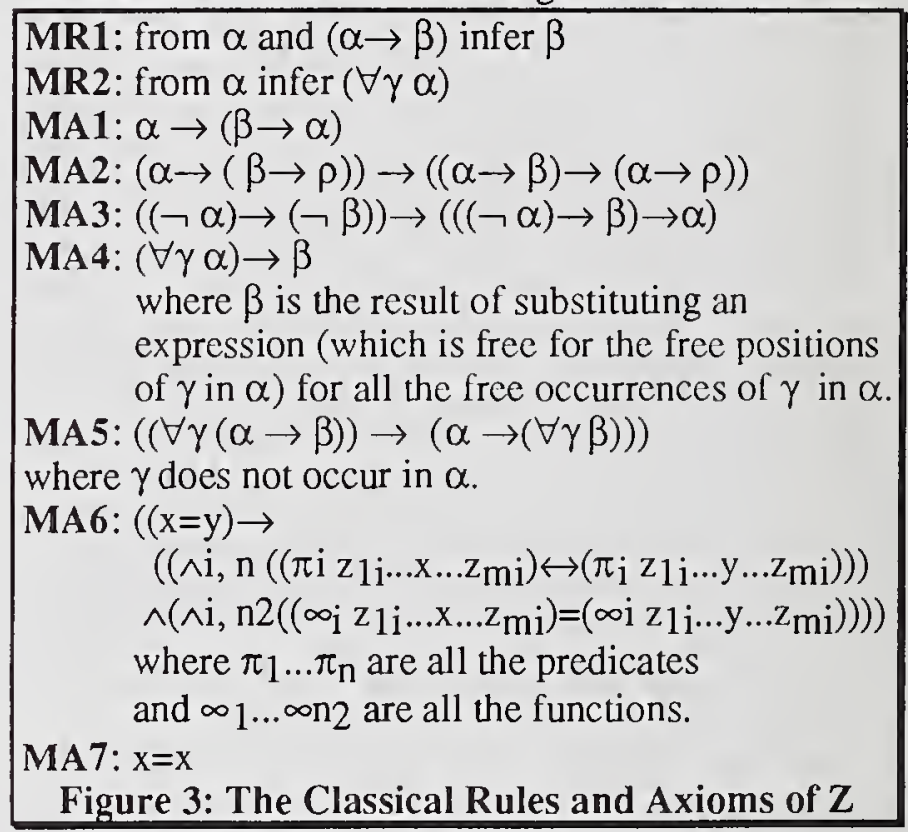

The modal inference rule and axioms of $\mathrm{Z}$ which are about logical necessity (i.e., []) are given in figure 4 . The laws R0, A1, A2 and A3 constitute an S5 modal logic[Hughes\&Cresswell 68] which, with the nonmodal laws, is similar to [Carnap46,Carnap56] and a first order logic version of [Bressan 72]. A4 says that a proposition is logically necessary if it is entailed by every world proposition. This law was implied in [Leibniz] and has been used by a number of authors including [Prior\&Fine 77, Fine 70]. Laws $\mathrm{A} 5, \mathrm{~A} 6$, and $\mathrm{A} 7$ axiomatize the predicates. $\mathrm{A} 5$ is the key axiom which says that any exhaustive conjunction of negated or unnegated distinct generators is a world, provided that there is a sentence $\alpha$ expressible in the formal language of $Z$ which holds when $p$ is an unnegated generator of that conjunction. Like the A5 axiom in [Brown87] it handles quantifiers over arbitrary domains. The A5 axiom is far stronger than the trivial modality axioms such as $\exists \mathrm{pq}((\neg[\mathrm{p}] \mathrm{q}) \wedge(\neg[\mathrm{p}] \neg \mathrm{q}))$ assumed in [Lewis 36] and $\exists \mathrm{p}((<>\mathrm{p}) \wedge(<>\mathrm{p}))$ assumed in [Bressan 72]. It also extends certain axiom schemas used in propositional logic S5c [Hendry\&Pokriefka 85] and 
propositional logic S13 [Cocchiarella]. Laws A6 and A7 axiomatize the intensionality of predicate arguments and are compatible with Bressan's [Bressan 72] view that predicates map tuples of concepts into propositions. Laws $\mathrm{A} 8$ and $\mathrm{A} 9$ axiomatize the functions which may be viewed as mappings of tuples of concepts into concepts.

R0: from $\alpha$ infer $([] \alpha)$

A1: ([]p) $\rightarrow p$

A2: $([p] q) \rightarrow(([] p) \rightarrow([q))$

A3: ([]p) $\vee([] \neg[] p))$

A4: $(\forall \mathrm{w}((\mathrm{WORLD} w) \rightarrow([\mathrm{w}] \mathrm{p}))) \rightarrow[\mathrm{l}] \mathrm{p}$

A5: WORLD $(\forall \mathrm{p}((\mathrm{GEN} \mathrm{p}) \rightarrow(\mathrm{p} \leftrightarrow[\mathrm{[}] \alpha)))$

for every expression $\alpha$.

A6: $\neg\left(\equiv\left(\pi_{1} \mathrm{x}_{1} \ldots \mathrm{x}_{n}\right)\left(\pi_{2} \mathrm{y}_{1} \ldots \mathrm{y}_{\mathrm{m}}\right)\right)$

where $\pi_{1}$ and $\pi_{2}$ are different predicate symbols.

A7: $\left(\left(\pi \mathrm{x}_{1} \ldots \mathrm{x}_{n}\right) \equiv\left(\pi \mathrm{y} 1 \ldots \mathrm{y}_{n}\right)\right)$

$\leftrightarrow\left(\left(\mathrm{x}_{1}[=] \mathrm{y}_{1}\right) \wedge \ldots . .\left(\mathrm{x}_{\mathrm{n}}[=] \mathrm{y}_{\mathrm{n}}\right)\right)$

A8: $\neg\left(\left(\infty_{1} \mathrm{x}_{1} \ldots \mathrm{x}_{\mathrm{n}}\right)[=]\left(\infty_{2} \mathrm{y}_{1} \ldots \mathrm{y}_{\mathrm{m}}\right)\right)$

where $\infty_{1}$ and $\infty_{2}$ are diffcrent function symbols.

A9: $\left(\left(\infty \mathrm{x}_{1} \ldots \mathrm{x}_{n}\right)[=]\left(\infty \mathrm{y}_{1} \ldots \mathrm{y}_{n}\right)\right)$ $\leftrightarrow\left(\left(\mathrm{x}_{1}[=] \mathrm{y}_{1}\right) \wedge . . \wedge\left(\mathrm{x}_{\mathrm{n}}[=] \mathrm{y}_{\mathrm{n}}\right)\right)$

Figure 4: The Modal Rule and Axioms of $Z$

Example: A particular $\mathrm{Z}$ language contains the binary predicate: loves and the unary predicate: happy, the unary function father-of, and the 0 -ary function John. The definitions and axioms depending on the this language are as follows. First, the generators are defined to be those propositions beginning with the loves and happy properties:

$($ gen $\alpha)=\operatorname{df}(\exists x \exists y(\alpha=($ loves $x y))) \vee(\exists x(\alpha=($ happy $x)))$ The equality axiom MA6 becomes:

MA6: $\quad \mathrm{x}=\mathrm{y} \rightarrow((($ loves $\mathrm{x} \mathrm{z}) \leftrightarrow($ loves y $\mathrm{z}))$

$\wedge(($ loves z x $) \leftrightarrow($ loves z y ))

$\wedge(($ happy $\mathrm{x}) \leftrightarrow$ (happy $\mathrm{y}))$

$\wedge(($ father-of $x)=($ father-of $y)))$

Using the above definition of generators, the axiom scheme A5 becomes:

A5: $\quad($ world $(\forall p((\exists x \exists y(p \equiv($ loves $x y)))$

$\vee(\exists x(p \equiv($ happy $\mathrm{x}))))$

$\rightarrow(\mathrm{p} \leftrightarrow[] \alpha)))$

for every expression $\alpha$.

Finally, axiom schemas A6,A7, A8, and A9 become:

A6: $\quad \neg(($ loves $\times 1 \times 2) \equiv($ happy $\mathrm{y} 1))$

A7a: $\quad(($ loves $\times 1 \times 2) \equiv($ loves y1 y2))

$$
\leftrightarrow((\mathrm{x} 1[=] \mathrm{y} 1) \wedge(\mathrm{x} 2[=] \mathrm{y} 2))
$$

A7b $\quad(($ happy $\mathrm{x} 1) \equiv($ happy $\mathrm{y} 1)) \leftrightarrow \mathrm{x} 1[=] \mathrm{y} 1)$

A8: $\quad \neg(($ father-of $x 1)[=]$ john $)$

A9: $\quad(($ father-of $x 1)[=]($ father-of $y 1)) \leftrightarrow(x 1[=] y 1)$

\section{What is Logically Possible?}

Axiom scheme A5, which has a recursively enumerable number of instances, expresses what is logically possible to the extent that it can be so expressed. In the previous example, instantiating $\alpha$ in $\mathrm{A} 5$ to ( $\mathrm{p} \equiv$ (happy John) produces: $(\operatorname{world}(\forall p((\exists x \exists y(p \equiv($ loves $x y)))$ $\vee(\exists \mathrm{x}(\mathrm{p} \equiv($ happy $\mathrm{x}))))$ $\rightarrow(p \leftrightarrow[](p \equiv($ happy John $)))))$

By S5 modal logic this becomes:

(world

$((\forall x \forall y(($ loves $x \mathrm{y}) \leftrightarrow(($ loves $\mathrm{x} y) \equiv($ happy John $))))$ $\wedge(\forall \mathrm{x}(($ happy $\mathrm{x}) \leftrightarrow(($ happy $\mathrm{x}) \equiv($ happy John $))))))$

By Axioms A6 and A7 this becomes:

(world $((\forall x(($ loves $x \mathrm{y}) \leftrightarrow \# f))$ $\wedge(\forall x(($ happy $x) \leftrightarrow(x[=] J o h n)))))$

Since, by definition, worlds are logically possible it follows that:

$<>((\forall \mathrm{x}(($ loves $\mathrm{x} \mathrm{y}) \leftrightarrow \# \mathrm{f}))$

$\wedge(\forall x(($ happy $x) \leftrightarrow(x[=] J o h n))))$

Since $(<>(\alpha \wedge \beta))$ implies $(<>\beta)$ in $S 5$ it follows that: $<>\forall \mathrm{x}(($ happy $\mathrm{x}) \leftrightarrow(\mathrm{x}[=] \mathrm{John})$

The main problem with using axiom scheme A.5 to prove that something is logically possible lies in finding the appropriate substitution for the parameter $\alpha$. The theorem ZPl given below is the basis of a heuristic for finding such instances. Intuitively, we know that the conjunction of instances of a predicate and the conjunction of instances of the negation of a predicate are logically possible whenever the two do not coincide on any instance. For example: $((p) a) \wedge(p$ b) $\wedge(\neg(p \quad c)))$ is logically possible but $((p a) \wedge(p$ b) $\wedge(\neg(\mathrm{p}$ a $)))$ is not. Thus if a sentence can be written in the form: $(\Gamma \wedge(\forall x(\alpha \rightarrow(\mathrm{p} \mathrm{x}))) \wedge(\forall \mathrm{x}(\beta \rightarrow(\neg(\mathrm{p} \mathrm{x})))))$ where $p$ does not occur in $\alpha, \beta$, and $\Gamma$, then it is logically possible if and only if $\{x: \alpha\} \cap\{x: \beta\}$ is empty, which is to say that $\exists x(\alpha \wedge \beta)$ does not follow from $\Gamma$ or to say that $\Gamma$ and $\neg \exists x(\alpha \wedge \beta)$ is logically possible. In this manner determining whether a sentence with $n$ predicates is logically possible can sometimes be reduced to determining whether a sentence with $n-1$ predicates is logically possible without having to guess any instances of the A5.

Theorem ZP1: The possibility of a disjoint predicate definition: If $\Gamma, \alpha$, and $\beta$ are sentences of $Z$ containing no unmodalized occurrence of the predicate $\pi,=$, or a propositional variable, $\pi$ is of arity $\mathrm{n}$, and $\mathrm{x}=\mathrm{x} 1 \ldots \mathrm{xn}$ is a tuple of $\mathrm{n}$ variables then:

$$
\begin{aligned}
& (<>(\Gamma \wedge(\forall x(\alpha \rightarrow(\pi x))) \wedge(\forall x(\beta \rightarrow \neg(\pi x))))) \\
& \leftrightarrow(<>(\Gamma \wedge(\neg \exists x(\alpha \wedge \beta)))) \\
& \text { Proof }
\end{aligned}
$$

Assume: $\pi$ is not in $\Gamma, \alpha$, nor $\beta$ then

$(<>(\Gamma \wedge(\forall x(\alpha \rightarrow(\pi x))) \wedge(\forall x(\beta \rightarrow \neg(\pi x)))))$

$\leftrightarrow(<>(\Gamma \wedge(\neg \exists x(\alpha \wedge \beta))))$

divides into two cases:

Case 1:

$(<>(\Gamma \wedge(\forall x(\alpha \rightarrow(\pi x))) \wedge(\forall x(\beta \rightarrow \neg(\pi x)))))$

$\rightarrow(<>(\Gamma \wedge(\neg \exists x(\alpha \wedge \beta))))$

resolving on the implications with $\pi$ and $\neg \pi$ gives:

$(<>(\Gamma \wedge(\forall x(\alpha \rightarrow(\pi x))) \wedge(\forall x(\beta \rightarrow \neg(\pi x))))$ $\wedge(\forall x(\neg \alpha \vee \neg \beta))) \rightarrow(<>(\Gamma \wedge(\neg \exists x(\alpha \wedge \beta))))$

which may be rewritten as: 
$(<>(\Gamma \wedge(\forall x(\alpha \rightarrow(\pi x))) \wedge(\forall x(\beta \rightarrow \neg(\pi x))))$ $\wedge(\neg(\exists x(\alpha \wedge \beta)))) \rightarrow(<>(\Gamma \wedge(\neg \exists x(\alpha \wedge \beta))))$

which holds by the laws of S5 modal logic.

Case 2:

$(<>(\Gamma \wedge(\neg \exists x(\alpha \wedge \beta))))$

$\rightarrow(<>(\Gamma \wedge(\forall x(\alpha \rightarrow(\pi x))) \wedge(\forall x(\beta \rightarrow \neg(\pi x)))))$

Using axiom $A 4$ twice we get:

$(\exists w(($ world $w) \wedge([w](\Gamma \wedge(\neg \exists x(\alpha \wedge \beta))))))$

$\rightarrow(\exists \mathrm{w}(($ world $\mathrm{w})$ $\wedge([w](\Gamma \wedge(\forall x(\alpha \rightarrow(\pi x))) \wedge(\forall x(\beta \rightarrow \neg(\pi x)))))))$

Let $(h w)$ represent the world in which $(\pi x)$ holds if and only if $\alpha$ holds in $w$ and every other generator holds if and only if it holds in w:

$$
(\mathrm{h} w):=\forall \mathrm{g}(\text { Gen } \mathrm{g}) \rightarrow(\mathrm{g} \leftrightarrow((\exists \mathrm{x}((\mathrm{g} \equiv(\pi \mathrm{x})) \wedge([\mathrm{w}] \alpha)))
$$$$
\vee((\neg(\exists \mathrm{x}(\mathrm{g} \equiv(\pi \mathrm{x})))) \wedge([\mathrm{w}] \mathrm{g}))))
$$

By A5 (h w) is a world. From this assignment it follows that: $([(h w)](\forall x((\pi x) \leftrightarrow([w] \alpha))))$. The $w$ in the conclusion is instantiated to (h w) giving:

$(($ world $w) \wedge([w](\Gamma \wedge(\neg \exists x(\alpha \wedge \beta)))))$

$\rightarrow([(\mathrm{h} w)](\Gamma \wedge(\forall \mathrm{x}(\alpha \rightarrow(\pi \mathrm{x}))) \wedge(\forall \mathrm{x}(\beta \rightarrow \neg(\pi \mathrm{x})))))$

Since $([(h w)](\forall x((\pi x) \leftrightarrow([w] \alpha)))),(\pi x)$ may be

replaced by $([w] \alpha)$ giving:

$(($ world $w) \wedge([w](\Gamma \wedge(\neg \exists x(\alpha \wedge \beta)))))$

$\rightarrow([(h w)](\Gamma \wedge(\forall x(\alpha \rightarrow([w] \alpha))) \wedge(\forall x(\beta \rightarrow \neg([w] \alpha)))))$

Pushing $\neg$ through the the world $w$ entailment gives:

$(($ world $w) \wedge([w](\Gamma \wedge(\neg \exists x(\alpha \wedge \beta)))))$

$\rightarrow([(h \mathrm{w})](\Gamma \wedge(\forall \mathrm{x}(\alpha \rightarrow([w] \alpha))) \wedge(\forall \mathrm{x}(\beta \rightarrow([\mathrm{w}] \neg \alpha)))))$

The hypothesis may be rewritten giving:

$(($ world $w) \wedge([w] \Gamma \wedge(\forall x(\beta \rightarrow \neg \alpha))))$

$\rightarrow([(\mathrm{h} w)](\Gamma \wedge(\forall \mathrm{x}(\alpha \rightarrow([\mathrm{w}] \alpha))) \wedge(\forall \mathrm{x}(\beta \rightarrow([\mathrm{w}] \neg \alpha)))))$

Reducing the scope of $[w]$ in the hypothesis gives

$(($ world $w) \wedge([w] \Gamma) \wedge(\forall x(([w] \beta) \rightarrow([w] \neg \alpha))))$

$\rightarrow([(\mathrm{h} w)](\Gamma \wedge(\forall \mathrm{x}(\alpha \rightarrow([\mathrm{w}] \alpha))) \wedge(\forall \mathrm{x}(\beta \rightarrow([\mathrm{w}] \neg \alpha)))))$

Since $[w] \neg \alpha$ is implied by $[w] \beta$ if it is entailed by

$[w] \beta$, it suffices to prove:

$(($ world $w) \wedge([w] \Gamma) \wedge(\forall x(([w] \beta) \rightarrow([w] \neg \alpha))))$

$\rightarrow([(h w)](\Gamma \wedge(\forall x(\alpha \rightarrow([w] \alpha))) \wedge(\forall x(\beta \rightarrow([w] \beta)))))$

Reducing (hw) to lower scope gives:

$(($ world $w) \wedge([w] \Gamma) \wedge(\forall x(([w] \beta) \rightarrow([w] \neg \alpha))))$

$\rightarrow(([(\mathrm{h} w)] \Gamma) \wedge(\forall \mathrm{x}(([(\mathrm{h} w)] \alpha) \rightarrow([\mathrm{w}] \alpha)))$

$$
\wedge(\forall x(([(h w)] \beta) \rightarrow([w] \beta))))
$$

Since $\pi,=$, nor any propositional variable occur unmodalized in $\Gamma, \alpha$, or $\beta,(h w)$ entails these sentences if and only if $w$ does, thus (h $w$ ) in the conclusion may be replaced by $w$ giving:

$(($ world $w) \wedge([w] \Gamma) \wedge(\forall x(([w] \beta) \rightarrow([w] \neg \alpha))))$

$\rightarrow(([w] \Gamma) \wedge(\forall x(([w] \alpha) \rightarrow([w] \alpha)))$

$$
\wedge(\forall x(([w] \beta) \rightarrow([w] \beta))))
$$

which is a tautology. QED.

ZP1 is applicable to any theory (such as propositional logic, monadic predicate logic, and the case of a finite theory) which can be put into a prenix conjunctive normal form such that no disjunct contains more than one unmodalized occurrence of $\pi$, since by the laws of classical logic such theories are equivalent to a disjunction of expressions of the form: $(\Gamma \wedge(\forall x(\alpha \rightarrow(\pi$ $x))) \wedge(\forall x(\beta \rightarrow \neg(\pi x))))$.
Example: The database $\mathrm{K}$ contains the following facts: Tweety and Chilly-Willy are birds, but (being a penguin) Chilly-Willy does not fly. By default birds fly. That is, for all $x$, if it is possible (with respect to the theory that Tweety and Chilly-Willy are birds, and Chilly-Willy does not fly) for $x$ to fly, then $x$ does fly:

$\mathrm{K} \equiv(($ bird cw $) \wedge($ bird $\mathrm{tw}) \wedge(\neg($ fly $\mathrm{cw}))$ $\wedge(\forall x)(($ bird $x)$ $\wedge(<($ bird cw $) \wedge($ bird $t w) \wedge(\neg($ fly $c w))>($ fly $x))$ $\rightarrow($ fly $\mathrm{x})))$ )

The possibility may be rewritten by the laws of $\mathrm{Z}$ so as to divide the positive and negative occurrences of the predicate fly as follows:

$$
\begin{aligned}
<>((\text { bird cw }) \wedge(\text { bird tw }) & \wedge \forall \mathrm{z}((\mathrm{z}[=] \mathrm{x}) \rightarrow(\text { fly z })) \\
& \wedge \forall \mathrm{z}((\mathrm{z}[=] \mathrm{cw}) \rightarrow(\neg(\text { fly z }))))
\end{aligned}
$$

Using ZP1 with $\Gamma:=($ bird $c w) \wedge($ bird tw $), x:=z, \pi:=$ fly, $\alpha:=(z[=] x)$, and $\beta:=(z[=] c w)$ gives the equivalent possibility:

$$
\begin{aligned}
& <>((\text { bird cw }) \wedge(\text { bird } \mathrm{tw}) \\
& \wedge(\neg(\exists \mathrm{z}((\mathrm{z}[=] \mathrm{x}) \wedge(\mathrm{z}[=] \mathrm{cw})))))
\end{aligned}
$$

Since $[=]$ is transitive, symmetric and reflexive the $\exists z$ quantifier may be eliminated giving:

$$
<>((\text { bird } c w) \wedge(\text { bird } t w) \wedge \neg(\mathrm{cw}[=] \mathrm{x}))
$$

The same process is now applied to the Bird predicate. First it is rewritten as:

$<>(\neg(\mathrm{cw}[=] \mathrm{x}))$ $\wedge \forall \mathrm{z}(((\mathrm{z}[=] \mathrm{cw}) \vee(\mathrm{z}[=] \mathrm{tw})) \rightarrow($ bird $\mathrm{z}))$ $\wedge \forall \mathrm{z}(\# \mathrm{f} \rightarrow(\neg($ bird $\mathrm{cw}))))$

Using ZP1 with $\Gamma:=\neg(\mathrm{cw}[=] \mathrm{x}), \mathrm{x}:=\mathrm{z}, \pi:=$ bird, $\alpha:=(\mathrm{z}[=] \mathrm{cw}) \vee(\mathrm{z}[=] \mathrm{tw})$, and $\beta:=\# \mathrm{f}$ gives the equivalent possibility:

$<>(\neg(\mathrm{cw}[=] \mathrm{x})) \wedge(\neg(\exists \mathrm{z}(((\mathrm{z}[=] \mathrm{cw}) \vee(\mathrm{z}[=] \mathrm{tw})) \wedge \# \mathrm{f})))$

which by classical logic is just: $\langle>(\neg(\mathrm{cw}[=] \mathrm{x}))$ which by $\mathrm{Z}$ is just: $(\neg(\mathrm{cw}[=] \mathrm{x}))$. Therefore, the original expression then becomes:

$\mathrm{K} \equiv(($ bird cw $) \wedge($ bird tw $) \wedge(\neg($ fly cw $))$ $\wedge(\forall \mathrm{x}((($ bird $\mathrm{x}) \wedge(\neg(\mathrm{cw}[=] \mathrm{x})) \rightarrow($ fly $\mathrm{x}))))$

which is equivalent in $Z$ to:

$\mathrm{K} \equiv($ (bird tw)^(bird cw)

$$
\wedge(\forall x((\text { bird } x) \rightarrow((\text { fly } x) \leftrightarrow \neg(x[=] c w)))))
$$

The sentence: $\forall x(($ bird $x) \rightarrow(($ fly $x) \leftrightarrow \neg(x[=] c w)))$ which contains a necessary equality entails the nonmodal sentence: $\forall \mathrm{x}(($ bird $\mathrm{x}) \rightarrow(($ fly $\mathrm{x}) \leftrightarrow \neg(\mathrm{x}=\mathrm{cw})))$.

ZP1 implies that contingent definitions added to a theory are conservative extensions:

Theorem ZP2: Definability of a predicate: If $\Gamma, \alpha$ are sentences of $Z$ containing no unmodalized occurrence of a predicate $\pi,=$, or propositional variable then: $(<>(\Gamma \wedge(\forall x((\pi \mathrm{x}) \leftrightarrow \alpha)))) \leftrightarrow(<>\Gamma)$

proof: Let $\beta$ be $\neg \alpha$ in ZP1 and simplify. QED.

\section{Reflective Reasoning in $\mathrm{Z}$}

One problem with computing defaults with respect to a theory $\Gamma$ is that the default itself is not part of the theory $\Gamma$. When $\Gamma$ essentially contains a disjunction 
this often causes the resulting theory to be inconsistent. For example, if $\Gamma$ were the statement that either Tweety does not fly or Chilly-Willy does not fly then the proposition that Chilly-Willy does not fly is possible with $\Gamma$ and likewise the proposition that Tweety does not fly is also possible with $\Gamma$. Thus both propositions would be true by default. Unfortunately, their conjunction contradicts $\Gamma$ if it were also asserted. Thus, the following is \#f:

$(($ bird cw $) \wedge($ bird tw $) \wedge((\neg($ fly cw $)) \vee(\neg$ fly tw $)))$ $\wedge(\forall \mathrm{x}(($ bird $\mathrm{x}) \wedge(<($ bird cw $) \wedge($ bird tw $)$

$\rightarrow($ fly $\mathrm{x}))))$

$$
\wedge((\neg(\text { fly cw })) \vee(\neg(\text { fly tw })))>(\text { fly } x))
$$

For this reason, the expression is reformulated so that the default itself becomes a proposition within the possibility expression. This is done by writing a reflective (i.e. fixed point) equation in $\mathrm{K}$ where the variable $\mathrm{K}$ is made synonymous to the theory and the default and where $K$ replaces $\Gamma$ in the default itself: $\mathrm{K} \equiv(($ bird tw $) \wedge($ bird cw $) \wedge((\neg($ fly cw $)) \vee(\neg($ fly tw $)))$

$\wedge \forall \mathrm{x}((($ bird $\mathrm{x}) \wedge(<\mathrm{K}>($ fly $\mathrm{x}))) \rightarrow($ fly $\mathrm{x})))$

A reflective equation has the form $K \equiv(\Phi \mathrm{K})$ where $\mathrm{K}$ is a propositional constant (i.e. a globally scoped universally quantified variable such as a Skolem function). The goal is to transform the initial equation into a disjunction, $\left(\left(K \equiv \beta_{1}\right) \vee \ldots \vee\left(K \equiv \beta_{n}\right)\right)$ with each $\beta_{i}$ free of $K$. If $K \equiv \beta_{i}$ implies the original equation then $\beta_{\mathrm{i}}$ is a solution to the original equation. We call the process of finding solutions to a reflective equation reflective reasoning.

Example: A Reflective Equation: Tweety and ChillyWilly are birds. At least one of them does not fly. By default Birds Fly.

$(\mathrm{K} \equiv(($ bird tw $) \wedge($ bird cw $) \wedge(\neg$ fly tw $) \vee(\neg$ fly cw $))$ $\wedge(\forall \mathrm{x}($ bird $\mathrm{x}) \wedge(<\mathrm{K}>($ fly $\mathrm{x})) \rightarrow($ fly $\mathrm{x})))))$

where bird and fly are lary predicates. To solve this equation we divide the default into three instances:

$(\mathrm{K} \equiv(($ bird tw $) \wedge($ bird $\mathrm{cw}) \wedge(\neg$ fly tw $) \vee(\neg$ fly $\mathrm{cw}))$

$\wedge(<K>($ fly tw $)) \rightarrow($ fly tw $)))))$

$\wedge(<K>($ fly cw $)) \rightarrow($ fly cw $)))))$

$\wedge(\forall x($ bird $x) \wedge(\neg((x[=] \mathrm{tw}) \vee(x[=] c w)))$

$$
\wedge(<\mathrm{K}>(\text { fly } \mathrm{x})) \rightarrow(\neg \text { fly } \mathrm{x})))))
$$

Splitting into 4 cases we then get:

$((<\mathrm{K}>($ fly tw $)) \wedge(<\mathrm{K}>$ (fly cw $))$

$\wedge(\mathrm{K} \equiv(($ bird tw $) \wedge($ bird $\mathrm{cw}) \wedge((\neg$ fly tw $) \vee($ fly $\mathrm{cw})$ $\wedge$ (fly tw $) \wedge($ fly cw $)$

$\wedge(\forall \mathrm{x}(($ bird $\mathrm{x}) \wedge(\neg((\mathrm{x}[=] \mathrm{tw}) \vee(\mathrm{x}[=] \mathrm{cw})))$ $\wedge(<K>($ fly $x))) \rightarrow($ fly $x)))$

$\vee((<\mathrm{K}>($ fly tw $)) \wedge(\neg<\mathrm{K}>$ (fly cw $)))$

$\wedge(\mathrm{K} \equiv(($ bird tw $) \wedge($ bird cw $) \wedge((\neg$ fly tw $) \vee(\neg$ fly cw $))$ $\wedge$ (fly tw)

$\wedge(\forall \mathrm{x}(\operatorname{bird} \mathrm{x}) \wedge(\neg((\mathrm{x}[=] \mathrm{tw}) \vee(\mathrm{x}[=] \mathrm{cw})))$

$\wedge(<K>($ fly $x)) \rightarrow($ fly $x)))))$

$\vee((\neg<\mathrm{K}>($ fly tw $)) \wedge(<\mathrm{K}>$ (fly cw) $)$

$\wedge(\mathrm{K} \equiv(($ bird tw $) \wedge($ bird cw $) \wedge((\neg$ fly tw $) \vee(\neg$ fly $\mathrm{cw})$ $\wedge$ (fly cw)

$\wedge(\forall x($ bird $x) \wedge(\neg((x[=] t w) \vee(x[=] c w)))$

$$
\begin{gathered}
\wedge(<\mathrm{K}>(\text { fly } \mathrm{x})) \rightarrow(\text { fly } \mathrm{x}))))) \\
\vee((\neg<\mathrm{K}>(\text { fly tw })) \wedge(\neg<\mathrm{K}>\text { (fly cw }))) \\
\wedge(\mathrm{K} \equiv((\text { bird tw }) \wedge(\text { bird cw }) \wedge((\neg \text { fly tw }) \vee(\neg \text { fly cw })) \\
\wedge(\forall \mathrm{x}(\text { bird } \mathrm{x}) \wedge(\neg((\mathrm{x}[=] \mathrm{tw}) \vee(\mathrm{x}[=] \mathrm{cw}))) \\
\wedge(<\mathrm{K}>(\text { fly } \mathrm{x})) \rightarrow(\text { fly } \mathrm{x}))))))
\end{gathered}
$$

which simplifies to:

$$
\begin{aligned}
& ((<\mathrm{K}>\text { (fly tw })) \wedge(<\mathrm{K}>\text { (fly cw })) \wedge(\mathrm{K} \equiv \# \mathrm{f})) \quad \text { case } 1 \\
& \vee((<\mathrm{K}>(\text { fly tw })) \wedge(\neg<\mathrm{K}>\text { (fly cw }))) \quad \underline{\text { case2 }} \\
& \wedge(\mathrm{K} \equiv((\text { bird tw }) \wedge(\text { bird cw }) \wedge(\neg \text { fly } \mathrm{cw}) \wedge(\text { fly tw }) \\
& \wedge(\forall x(\text { bird } x) \wedge(\neg((x[=] \text { tw }) \vee(x[=] c w))) \\
& \wedge(<\mathrm{K}>(\text { fly } \mathrm{x})) \rightarrow(\text { fly } \mathrm{x}))))) \\
& \vee((\neg<\mathrm{K}>\text { (fly tw })) \wedge(<\mathrm{K}>(\text { fly } \mathrm{cw})) \quad \text { case } 3 \\
& \wedge(\mathrm{K} \equiv((\text { bird tw }) \wedge(\text { bird cw }) \wedge(\neg \text { fly tw }) \wedge \text { (fly cw }) \\
& \wedge(\forall x(\text { bird } x) \wedge(\neg((x[=] t w) \vee(x[=] c w))) \\
& \wedge(<K>(\text { fly } x)) \rightarrow(\text { (fly } x))))) \\
& \vee((\neg<\mathrm{K}>(\text { fly tw })) \wedge(\neg<\mathrm{K}>\text { (fly cw }))) \quad \text { case } 4 \\
& \wedge(\mathrm{K} \equiv((\text { bird tw }) \wedge(\text { bird cw }) \wedge((\neg \text { fly } t w) \vee(\neg \text { fly } c w)) \\
& \wedge(\forall \mathrm{x}(\text { bird } \mathrm{x}) \wedge(\neg((\mathrm{x}[=] \mathrm{tw}) \vee(\mathrm{x}[=] \mathrm{cw}))) \\
& \wedge(<\mathrm{K}>(\text { fly } \mathrm{x})) \rightarrow(\text { fly } \mathrm{x}))))))
\end{aligned}
$$

In case 1 , since $\mathrm{K}$ is \#f, which cannot be possible, that case is eliminated. In case 4, the negated possibilities say that $[\mathrm{K}](\neg$ fly $\mathrm{tw}) \wedge[\mathrm{K}](\neg$ fly $\mathrm{cw})$, but since the equation implies that:

$[(($ bird tw $) \wedge($ bird cw $) \wedge((\neg$ fly tw $) \vee(\neg$ fly cw $))$

$\wedge(\forall x($ bird $x) \wedge(\neg((x[=] t w) \vee(x[=] c w)))$

$$
\wedge((\mathrm{K}>(\text { fly } x)) \rightarrow(\text { fly } x))))))] \mathrm{K}
$$

it could only hold if:

$[(($ bird tw $) \wedge($ bird cw $) \wedge((\neg$ fly tw $) \vee(\neg$ fly $c w))$

$$
\wedge(\forall x((\text { bird } x) \wedge(\neg((x[=] t w) \vee(x[=] c w)))
$$

$((\neg$ fly tw $) \wedge(\neg$ fly cw $))$

$$
\wedge(<\mathrm{K}>(\text { fly } \mathrm{x})) \rightarrow(\text { fly } \mathrm{x}))))]
$$

but the negation of this is

$\diamond(($ bird tw $) \wedge($ bird cw $) \wedge((\neg$ fly tw $) \vee(\neg$ fly cw $))$

$$
\wedge(\forall x((\text { bird } x) \wedge(\neg((x[=] \operatorname{tw}) \vee(x[=] c w)))
$$

$\wedge(($ fly tw $) \vee($ fly $c w))$

$$
\wedge(<\mathrm{K}>(\text { fly } \mathrm{x})) \rightarrow(\text { fly } \mathrm{x}))))))
$$

which is implied by:

$$
\begin{aligned}
& <((\text { bird tw }) \wedge(\text { bird cw }) \wedge((\neg \text { fly tw }) \vee(\neg \text { fly cw })) \\
& \wedge(\forall x((\text { bird } x) \wedge(\neg((x[=] \text { tw }) \vee(x[=] \mathrm{cw}))) \rightarrow(\text { fly } x)))) \\
& \wedge((\text { fly tw }) \vee(\text { fly cw }))
\end{aligned}
$$

which is true by ZP1. So this case is also false and may be eliminated. For the remaining two cases the inequalities are repeated inside the possibilities:

$$
\begin{gathered}
(((<\mathrm{K}>(\text { fly tw })) \wedge(\neg<\mathrm{K}>(\text { fly cw }))) \\
\wedge(\mathrm{K} \equiv((\text { bird tw }) \wedge(\text { bird cw }) \wedge(\neg \text { fly cw }) \wedge(\text { fly tw }) \\
\wedge(\forall \mathrm{x}((\text { bird } \mathrm{x}) \wedge \neg((\mathrm{x}[=] \mathrm{tw}) \vee(\mathrm{x}[=] \mathrm{cw})) \\
\wedge(<\mathrm{K}>((\neg((\mathrm{x}[=] \mathrm{tw}) \vee(\mathrm{x}[=] \mathrm{cw}))) \rightarrow(\text { fly } \mathrm{x}))) \\
\rightarrow(\text { fly } \mathrm{x}))))) \\
\vee((\neg<\mathrm{K}>(\text { fly tw })) \wedge(<\mathrm{K}>(\text { fly cw })) \\
\wedge(\mathrm{K} \equiv((\text { bird tw }) \wedge(\text { bird cw }) \wedge(\neg \text { fly tw }) \wedge(\text { fly cw }) \\
\wedge(\forall \mathrm{x}(\text { bird } \mathrm{x}) \wedge(\neg((\mathrm{x}[=] \mathrm{tw}) \vee(\mathrm{x}[=] \mathrm{cw}))) \\
\wedge(<\mathrm{K}>((\neg((\mathrm{x}[=] \mathrm{tw}) \vee(\mathrm{x}[=] \mathrm{cw}))) \rightarrow(\text { fly } \mathrm{x}))) \\
\rightarrow(\text { fly } \mathrm{x})))))
\end{gathered}
$$

Since the parts inside the $<>$ of the following two sentences entail $\mathrm{K}$ for cases 2 and 3 respectively, and since they are \#t by ZP1:

$<($ bird $t w) \wedge($ bird $c w) \wedge(\neg$ fly $c w) \wedge($ fly $t w) \quad \underline{\text { for } 2}$ $\wedge(\forall x(($ bird $x) \wedge(\neg((x[=] \operatorname{tw}) \vee(x[=] c w))) \rightarrow($ fly $x)))>$ 
$\wedge((\neg((x[=] t w) \vee(x[=] c w))) \rightarrow($ fly $x)))$

$<($ bird tw $) \wedge($ bird cw $) \wedge(\neg$ fly tw $) \wedge($ fly cw $) \quad$ for 3

$\wedge(\forall x(($ bird $x) \wedge(\neg((x[=] t w) \vee(x[=] c w))) \rightarrow($ fly $x)))>$

$\wedge((\neg((x[=] \mathrm{tw}) \vee(\mathrm{x}[=] \mathrm{cw}))) \rightarrow($ fly $\mathrm{x})))$

the above cases 2 and 3 simplify to just:

$(((<\mathrm{K}>($ fly tw $)) \wedge(\neg<\mathrm{K}>($ fly cw $)))$

$\wedge(\mathrm{K} \equiv(($ bird tw $) \wedge($ bird $c w) \wedge(\neg$ fly cw $) \wedge($ fly tw $)$ $\wedge(\forall \mathrm{x}(($ bird $\mathrm{x}) \wedge \neg((\mathrm{x}[=] \mathrm{tw}) \vee(\mathrm{x}[=] \mathrm{cw})) \wedge \# \mathrm{t} \wedge$ $\rightarrow($ fly $x)))))$

$\vee((\neg<\mathrm{K}>($ fly tw $)) \wedge(<\mathrm{K}>$ (fly cw $))$

$\wedge(\mathrm{K} \equiv(($ bird tw $) \wedge($ bird cw $) \wedge(\neg$ fly tw $) \wedge($ fly cw $)$ $\wedge(\forall x($ bird $x) \wedge(\neg((x[=] t w) \vee(x[=] c w))) \wedge \#(\wedge$ $\rightarrow($ fly $x))))$ )

Substituting the solution for $\mathrm{K}$ in each case into the remaining possibility statements and using $Z P 1$ gives:

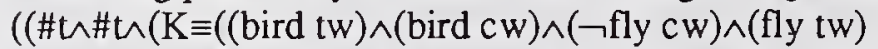
$\wedge(\forall x(($ bird $x) \wedge \neg((x[=] \mathrm{tw}) \vee(x[=] c w)) \wedge \rightarrow($ fly $x)))))$

$\checkmark(\# \downarrow \wedge \# \wedge \wedge(\mathrm{K} \equiv(($ bird tw $) \wedge($ bird cw $) \wedge(\neg$ fly tw $) \wedge($ fly cw $)$ $\wedge(\forall x($ bird $x) \wedge(\neg((x[=] \operatorname{tw}) \vee(x[=] \mathrm{cw}))) \wedge \rightarrow($ fly $x)))))$

By the laws of classical logic this simplifies to:

$((\mathrm{K} \equiv(($ bird $t w) \wedge($ bird $c w)$ $\wedge(\forall x(($ bird $x) \rightarrow(($ fly $x) \leftrightarrow \neg(x[=] c w))))$

$\vee(\mathrm{K} \equiv(($ bird tw $) \wedge($ bird $c w)$ $\wedge(\forall x(($ bird $x) \rightarrow(($ fly $x) \leftrightarrow \neg(x[=] t w)))))$

which results in a disjunction of two solutions; one where $c w$ is the only bird which does not fly and one where tw is the only bird which does not fly.

\section{Propositional Quantifier Idiom}

When a reflective equation has more than one solution there arises the question as to which solution is to be used. One way of avoiding this question is to adopt the conservative approach of only accepting what is common to all the solutions. This is achieved with the idiom: $K \equiv \exists k(k \wedge(k \equiv \alpha))$ which may be read as the database $\mathrm{K}$ is the (possibly uncountably infinite) disjunction of all the solutions $\mathrm{k}$ to the reflective equation $k=\alpha$. When there are a finite number of solutions $\beta_{1} \ldots \beta_{n}$ to such an equation: $\left(k \equiv \beta_{1}\right) \vee \ldots \vee\left(k \equiv \beta_{n}\right)$, the expression $\exists k(k \wedge(k=\alpha))$ is equivalent to $\exists k\left(k \wedge\left(\left(k \equiv \beta_{1}\right) \vee \ldots \vee\left(k \equiv \beta_{n}\right)\right)\right)$ which by the distribution properties of $\wedge$ and $\vee$ and by the fact that $\exists$ associates through $\vee$ gives the equivalent expression: $\quad\left(\exists k\left(k \wedge\left(k=\beta_{1}\right)\right)\right) \vee \ldots \vee\left(\exists k\left(k \wedge\left(k=\beta_{n}\right)\right)\right)$. Since $\exists k\left(k \wedge\left(k \equiv \beta_{i}\right)\right)$ is logically equivalent to just $\beta_{i}$ $\mathrm{K}$ will be equivalent to the disjunction of solutions: $\beta_{1} \vee \ldots \vee \beta_{n}$. For example, the disjunction of solutions from section 4 gives:

$\mathrm{K} \equiv(($ bird $\mathrm{tw}) \wedge($ bird $\mathrm{cw}) \wedge(\neg$ fly $\mathrm{cw}) \leftrightarrow($ fly $\mathrm{tw})$ $\wedge(\forall x((($ bird $x) \wedge \neg((x[=] \mathrm{tw}) \vee(x[=] \mathrm{cw}))) \rightarrow($ fly $x)))$

\section{Conclusion}

Nonmonotonic reasoning captures a deep and important aspect of human reasoning. Essentially it allows us to express laws and to reason without cluttering up our laws with numerous exceptions. The Modal Quantificational Logic Z provides an effective (i.e. recursively enumerable) method for automatically deducing nonmonotonic consequences for many interesting problems. $\mathrm{Z}$ improves upon previous methods of defining nonmonotonic reasoning via complicated metatheories by allowing for quantification through modal scopes and by eliminating those theories' inherent ineffective processes for computing consistency. In a broader sense, $Z$ supports Lewis's [Lewis 36] original claim that modal logic is a missing part of classical logic in that this modal logic also accounts for nonmonotonic reasoning.

\section{Acknowledgments}

This research was supported by the National Science Foundation Grant No. MIP9526532. I thank Guy Jacobs for his help.

\section{References}

Bressan,Aldo, A General Interpreted Modal Calculas, Yale University Press, 1972.

Brown87a,F.M. "The Modal Logic Z", The Frame Problem in AI: Proceedings of the 1987 AAAI Workshop, Morgan Kaufmann, LosAltos,CA 1987.

Brown91,Frank M., "The Modal Quantificational Logic Z Applied to the Frame Problem", International Journal of Expert Systems Research and Applicatiions, Special Issue: The Frame Problem. Part A. eds. Keneth Ford and Pattrick Hayes, vol. 3 number 3, pp169-206 JAI Press 1990. Reprinted in Reasoning Agents in a Dynamic World: The Frame problem, editors: Kenneth M. Ford, Patrick J. Hayes, JAI Press 1991. Carnap46, Rudolf, "Modalities and Quantification" Journal of Symbolic Logic, vol.11, number 2, 1946.

Carnap56, Rudolf, Meaning and Necessity: A Study in the Semantics of Modal Logic, The University of Chicago Press, 1956.

Cocchiarella,N.B., "Philosophical Perspectives on Quantification in Tense and Modal Logic", Handbook of Philosophical Logic, D. Reidel,1984

Fine, K., "Propositional Quantifiers in Modal Logic" Theoria 36, 1970.

Garson,J.W. "Quantification in Modal Logic", Handbook of Philosophical Logic, Volume II, Extensions of Classical Logic, 1984.

Herbert E. Hendry and M. L. Pokriefka, "Carnapian Extensions of S5", Journal of Phil. Logic 14, 1985.

Hughes,G.E. and Creswell,M.J., An Introduction to Modal Logic, Methuen \& Co. Ltd., London, 1968.

Leibniz,G. "Necessary and Contingent Truths" 1686, Leibniz Philosophical Writings, Dent\&Sons. 1973.

Lewis,C.I. Strict Implication, Journal of Symbolic Logic, vol I. 1936.

Mendelson,E. Introduction to Mathematical Logic, Van Norstrand, Reinhold Co., New York, 1964.

Prior,A.N. \& Fine,K., Worlds, Times, and Selves, Gerald Duckworth, 1977. 
V

LEARNING 



\title{
INDUCTIVE THEORY OF SEMIOTIC SYSTEMS
}

\author{
Lev Goldfarb
}

\author{
Faculty of Computer Science \\ University of New Brunswick \\ Fredericton, N.B., E3B 5A3, Canada \\ goldfarb@unb.ca
}

\begin{abstract}
Induction, which has been called the "scandal of philosophy", has become the scandal of psychology and artificial intelligence as well.
\end{abstract}

J. H. Holland et al., Induction (1986)

\begin{abstract}
- ln light of the ongoing discussion about the name of the emerging field, l propose and partly motivate the name Inductive Semiotic Systems as an appropriate one. The three postulates given in section 5 capture the essence of the justification for the proposed name. They can be further reduced to the following. Since all objects, in the universe have emergent compositional structure, the term "object structure" (and therefore the term "meaning") cannot be properly understood, defined, and captured outside the inductive learning process. Such a process, based on the appropriate formal mathematical structure, try to capture the object-class structure by constructing the "inductive sign" that represents in the given context both the object and the corresponding class of objects. Moreover, I conjecture that all other representations and semiotic processes have evolved around these, inductive, representations. The main link with the classical sciences is outlined: the working hypothesis (which we currently pursue) that the proper sensors for all intelligent systems are symbolic, or inductive, measurement devices, a far-reaching generalization of the classical, or numeric, measurement devices. In this connection, l briefly review the inductive nature of the cognitive processes responsible for the emergence of numbers.
\end{abstract}

Keywords: intelligent systems, symbolic representations, numbers, inductive learning, inductive class representation, evolving transformation system, symbolic (inductive) measurement devices.

\section{INTRODUCTION}

This paper was motivated by a number of developments all originating from my work on the foundation of pattern recognition began 20 years ago and intended to

This work is partially supported by the NSERC research grant number OGP0002686. unify the two basic but incompatible approaches: vector space, or the classical statistical, approach, and formal grammar, or the syntactic, approach. The work gradually culminated in a fundamentally new mathematical model, Evolving Transformation System (ETS), that attempts to capture formally the nature of "symbolic" representations $[1,2,3,4,5]$. The model eventually led to the outline of the inductive theory of vision [6], which, in turn, prompted the re-examination and generalization of the classical, or numeric, measurement processes [7]. What, basically, motivated a serious re-examination of the classical measurement processes is not, as one might expect, the well-documented problematic state of the quantum measurement problem, but the realization that ETS embodies a fundamental generalization of the classical mathematical concept of (measurement) space $[8,9]$ such that the former cannot be reduced to the latter. In other words, it became clear that most processes in a symbolic representation space are not reducible to those in a numeric space, the fact whose importance cannot be overestimated. In particular, ETS suggested that the currently accepted understanding of biological information processing (inevitably dictated by our current state of measurement and instrumentation) as proceeding from numeric to symbolic representations is not correct: all stage of biological information processing, including the initial stage of transduction, can properly be understood using the appropriate symbolic representations only [10].

Thus, with the understanding of fundamental irreducibility of the symbolic, or semiotic, processes to the numeric ones, the question of the structure of intelligent semiotic systems could be addressed in a more appro- 
priate and satisfactory manner, i.e. the basic formal language should be that of a formally specified "symbolic" mathematical model, or ETS. Since the latter was developed to capture the nature of inductive learning processes, it is quite natural to call the emerging theory the inductive theory of semiotic systems. Moreover, the name is also (and mainly) justified on the basis of the fact that, according to the ETS, the modification of "geometry" of the symbolic representation space, and, as it turns out, of the very representation itself, is actually accomplished during inductive learning processes, which thus emerges as the central intelligent processes. It appears also that the concept of emergent representation cannot be adequately understanding outside inductive learning processes.

The exposition in the paper is as informal as possible in order to be accessible to a wider range of researchers, and therefore no technical details of the ETS model, which led to all the considerations, are given.

\section{THE CENTRAL ROLE OF INDUCTIVE LEARNING PROCESSES}

The pre-eminent role of inductive processes in epistemology and science has been recognized almost from the very beginning and increasingly so as science matured. Thus, passing Bacon, Hume, Mill and confining ourselves to the latest period, this century, we find, for example, B. Russell devoting to induction a chapter in his book "Problems of Philosophy"(1912). In "Outline of Philosophy" (1927) he states:

Induction raises perhaps the most difficult problem in the whole theory of knowledge. Every scientific law is established by its means, and yet it is difficult to see why we should believe it to be a valid logical process. ... When mankind took to science, they tried to formulate logical principles justifying this kind of inference. I will only say that they seem to me very unsuccessful. I am convinced that induction must have validity of some kind in some degree, but the problem of showing how or why it can be valid remains unsolved.

Another leading philosopher, A.N. Whitehead, in spite of the basic philosophical differences with his important collaborator B. Russell, in "Science and the Modern World" (1925) also agrees with the latter on the important role of induction: "The theory of induction is the despair of philosophy - and yet all our activities are based upon it".

Before proceeding further, a "fresh" model-independent definition of inductive learning process proposed by me might be useful.

Definition: Given a small finite set $C^{+}$of positive objects that are randomly chosen from a (possibly infinite) class $C$-class to be learned-and a small finite set $C^{-}$of negative objects, i.e. not from $C$, the inductive learning process has to construct an inductive class representation (ICR) of $C$ such that

- ICR can generate an approximation $C_{a p p}$ of $C$, and

- if $C_{a p p}^{*}$ is a noisy perturbation of $C_{a p p}$ and $C^{*}$ is a noisy perturbation of $C$, then the ratio of cardinalities

is close to 1 .

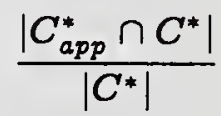

Note both the generative (and, therefore, mainly discrete) nature of the ICR, as well as a fuzzy boundary of the "generated" class (and, therefore, the presence of continuous element in the ICR, which is not obvious from the definition). These two features, combined in a natural way, make the above definition unique among the other definitions of inductive learning processes. Furthermore, both of them are supported by psychological experiments ([11], pp. 33-38; [12], pp. 97-102). The uniqueness of the definition is further clarified by our recent experiments, which strongly suggests that the well known "inductive learning" models, including connectionist models, are, in fact, not inductive learning models at all.

What does make the inductive learning process a prime candidate for the central cognitive process? The answer, I believe, is strongly suggested by the ubiquitous fact that any object/event representation is guided to a considerable extent, by the finite number of the agent's encounters, direct and indirect, with that object/event. In fact, it is not difficult to see how the construction of practically all cherished concepts in cognitive psychology, including frames and mental models, can be accomplished based on the concept of ICR. Moreover, H. Plotkin in [13] makes a book-length argument that only inductive mechanisms can allow each biological species to convert the "Humean uncertainty ... into a pragmatic issue of dividing the world into band width of frequencies of change and fluctuation, and [to employ these] ... mechanisms that are able to match the rates of perturbation of the world with organic structures ... to alter their own states at equivalent rates" (see also [14]).

\section{INDUCTIVE ORIGIN OF NUMBERS}

Remarkably, it appears that the decisive and irreplaceable role of the inductive axiom in the well known 
Peano axiomatization of natural numbers is a reflection of the inductive cognitive origin of numbers.

Following [15], sect. 1.2, I first summarize the 4 stages in the emergence of numbers. The presence of the first stage (which has evolved in many animals many millions of years ago) is indicated by the ability to reasonably accurately compare the sizes of some temporal and spatial sets of events or objects (see, for example $[16,17,18,19])$.

The second stage is manifested in the primitive cultures in the form of the choice of very few selected reference, or standard, sets of objects mainly for the purpose of explicit storage of various set sizes (see, for example $[16,17,18])$.

The third stage is achieved when a single reference set of object is selected, and the fourth stage is characterized by the emergence of the abstract concept of number, independent of any particular set of reference objects. One should note that the last stage is quite recent (4000-2000 B.C.) and it is reflected in many of our measures of length: the height of a horse is measured in "hands" and length generally in "feet" (from foot) and earlier also in "ells" (from elbow, 1 ell $=45$ inches). Also, the word "digit" means not only the numbers $1,2,3, \ldots$ but a finger or a toe as well. Similar phenomenon is observed in practically all languages $[16,17,18]$.

One point to observe in the progression of the four stages-and this has actually became perfectly clear only at the end of the last century - is that a number is, in fact, a sign representing the (infinite) class of all finite sets whose sizes are all equal to this number. This, plus the other point, which will be discussed next, lead quite naturally, as we shall see, to the hypothesis proposed in the title of this section that the concept of number has been acquired by means of the evolutionary inductive learning processes, when the evolving agent gradually acquires the concept of number via embedded in the agent (and therefore also evolving) cognitive inductive learning processes interacting with the environment.

Let us next look at this process from a little more formal perspective. The definition of inductive learning process given in section 2 is model-independent, i.e. no concrete mathematical model is specified to account, first, for the generativity and, second, for the measure of proximity of elements in the set. Thus, to continue, we shall need to choose some concrete mathematical model satisfying the two general and absolutely essential conditions of the definition. Since at present no model except ETS satisfies the requirements of the definitions, the latter is quite naturally my choice. Hence, in what follows I simply adopt the ETS as the model of inductive learning (see, for example, my paper in the last year proceedings of this conference [5] or [2, 3, 4])

In a few words, ETS can be described as a formal mathematical explication of the idea of the "symbolic" object space as opposed to the idea of "numeric" object space. The latter is captured, for instance, by concept of Euclidean vector space. It turns out that there is a profound difference in the nature of the (metric) geometry for the two classes of spaces, numeric object spaces forming a very special, "degenerate", subclass of the class of symbolic object spaces. (The distance between two symbolic objects-or, more precisely , object representations-is defined as the "shortest" sequence of weighted symbolic transformations connecting the two objects, e.g. deletions, insertions and substitutions.) The important new idea here is that the distance function is defined by the fixed set of weighted symbolic operations, each of which can transform one object into the other. In other words, the new idea is that the concept of distance is introduced via the fixed set of symbolic operations, which in a numeric case, e.g vector space, becomes "trivial". Moreover, the representation itself is not fully determined by the "structure" of the object itself but also by the latter set of operations, specifying the emergent multi-resolutional structure that can only be discovered during various inductive learning processes. For example, in case of strings, to indicate such an emergent structure, the usual string representation aacdbaacdcabba is not sufficient, and more adequate form of representation might be, for example, a.acd.ba.acd.ca.b.ba reflecting the corresponding symbolic operations that are constructed during the learning stage from the basic singleletter operations with the help of several fixed composition forming operators [3].

In ETS, the inductive class representation (ICR), constructed during the learning process, consists of a small selected subset of the positive training set plus the learned set of weighted operations (that can act on the selected training set to generate the approximation $C_{a p p}$ of the class $C$ to be learned (see Definition in section 2). The very simple nature of numeric object representation space manifests itself in the simplicity of the ICR for the set $\mathbf{N}$ of natural numbers: $1 \&$ the successor operation. The latter can be seen, for example, from the Peano axiomatization of $\mathbf{N}$, which is now universally accepted as the standard one. This symbolic, or compositional, simplicity becomes perhaps more apparent when we view natural numbers as the set of strings over a single letter alphabet $\{a\}$ :

$$
\mathbf{N}=\{a, a a, a a a, a a a a, \ldots\} .
$$

Thus, looking at $\mathbf{N}$ as the result of application of the corresponding ICR (e.g. string aaa plus the deletioninsertion of $a$ ), it is not difficult at all to connect this 
ICR with the actual learning process as reflected in the above four stages: the positive training set may consist of several strings of $a$ 's, for example, a, aaa, aaaaaa, and the negative training set is simply empty. Since the final set of operation consists of just one singleletter deletion-insertion operation, no multiresolutional structure enters the "picture". In this manner a very natural connection is established between the emergence of natural numbers and the central intelligent processes - inductive learning processes.

\section{GENERALIZED MEASUREMENT PROCESS AS INDUCTIVE LEARNING PROCESS}

In the last section we have connected the structure of natural numbers - and therefore of most of our presentday mathematics - to the simplest form of inductive class representation (ICR), consisting of a single object plus a single operation. It is not difficult to see now the gradual development of the classical scientific paradigm as application of this (Peano) ICR to the representation of various important "numeric" features of reality: mass, distance, time, speed, etc. etc.. I want to emphasize the fact that in spite of apparent multitude of "variables" introduced in basic sciences, the structure of the representation space for all these variables-e.g. Euclidean vector space-is essentially determined (via the real numbers) by the structure of the ICR of natural numbers.

Given the above, one can propose to view more general, i.e. "symbolic, or semiotic", measurement processes simply as various concrete implementations of general inductive learning processes. (We were lead to these general measurement processes [7] after the investigation of the foundations of computational vision processes [6].)

To better understand the meaning of "symbolic measurement process", let us first look from the new perspective at a concrete example of the classical, or numeric, measurement process-length measurement. It goes without saying that the emergence of numbers has preceded the idea of the ruler. Hence, the idea of length measurement should be looked at as a quite natural application of the above view of natural numbers (via Peano ICR) to the concept of length, i.e. the above $\boldsymbol{a}$ becomes (linear) unit of measurement.

We have recently proposed [7] to generalize the classical idea of length measurement to the idea of plane shape "measurement". The basic idea is to expand the "measurement alphabet" from $A=\{a\}$ to, for example, $A^{\prime}=\{a, b, c\}$, where the nonlinear units $b$ and $c$ are the oriented concave and convex corners, as in the example in [1]. In other words, if the idea of the clas- sical linear ruler is to assemble successively identical copies of a single linear unit of measurement, the basic idea of the above "plane ruler" is to allow one to assemble dynamically several, including nonlinear, units of measurement and to apply the inductive learning process to discover the corresponding symbolic operations that specify the multiresolutional structure of the shape representation as a member of a certain class of shapes. For a more detailed exposition of this example see the companion paper [7], where, in particular, it is illustrated how the noise in the symbolic representation can also be eliminated during the inductive learning process by discovering the corresponding symbolic operation of weight zero.

\section{INDUCTIVE SEMIOTIC SYSTEMS}

In the tradition of these conferences, I propose to address both the "computational semiotics" ([20], p. 155) and the semiotics understood by Metz as "the formalization of the natural sciences" ([21], p. 30) in one framework-Inductive Semiotic Systems (ISS). The corresponding new science can then be defined as the science concerned with the study and modeling of autonomous (including biological) systems that can symbolically encode a functionally necessary (but possibly unbounded) class of events/objects in the environment after an exposure to a very small number of events from the class.

As was partly outlined in the last section, the questions related to "the problem of how signals from sensors can be reliably transformed into symbolic data structures that are suitable for logical reasoning" [22] are also addressed by the proposed framework but in a manner much more radical than implied by that statement: the sensors themselves must embody inductive (symbolic) measurement devices. Such intelligent devicescontrary to the classical sensors based on the very simple form of the ICR-would be modeled in accordance with the new mathematical structure, ETS, that allows the measurement device to capture in a much less restrictive, symbolic, form the structure of the corresponding class of events in the environment. The outputs of such measurement devices, as in the case of biological systems, would be the current multiresolutional symbolic representations of events, or objects. Hence no transduction of the numeric input into symbolic representation is necessary. Moreover, as became gradually clear to us, such a transduction appears to be meaningless, since, as was mentioned above, the class of numeric spaces forms a degenerate subclass of the class of symbolic spaces, so that in this case the very rational for symbolic representation collapses.

Semiotics is often defined as a field "involved in 
analyses of trilateral unity: object-sign-meaning" ([23], p. 45). The latter view goes back to C. Peirce, who introduced semiotics as "the doctrine of the essential nature and fundamental varieties of possible semiosis", where "by the semiotics I [he] mean[s] an action, an influence, which is, or involves, a cooperation of three subjects, such as a sign, its object and its interpretant, this tri-relative influence not being in anyway resolvable into actions between pairs" ([21], p. 15).

In accordance with the above (ISS) view of semiotics, one can introduce a somewhat more precise perspective on the "analysis of the trilateral unity", as the the study and modeling of the following triad: combinative, or compositional, object structure, the corresponding sign (i.e. object representation) structure, and the mathematical structure that captures the former. And it is the inductive learning processes, through the discovery of the object-class structure, that relate the members of the triad. This interpretation, as well as the following postulates, are adopted from the similar view of the computational vision given in [6]. The following three postulates may be viewed as a more detailed description of the relation between the members of the triad.

Postulate 1. All objects in the universe have emergent compositional structure. Moreover, the term "object structure", and therefore the term "meaning", cannot be properly understood and defined outside the inductive learning process.

Postulate 2. The inductive learning process is an evolving process that tries to capture the emergent compositional object-class structure mentioned in Postulate 1. The mathematical structure on which the inductive learning model is based should have the intrinsic capability to capture the evolving compositional object structure.

(As discussed in section 3, the appropriate mathematical structure is fundamentally different from the classical mathematical structures.)

Postulate 3. All basic representations, i.e. signs, are constructed on the basis of the "inductive signs", which, in turn, are constructed by the inductive learning processes (see Postulate 2). Thus, the inductive learning processes form the core around which all other semiotic processes have evolved.

\section{CONCLUSION}

I have proposed to put the inductive object-class representation constructed during the inductive learning process at the basis of the semiotic "analysis of the trilateral unity [of] object-sign-meaning" [23]. There is considerable evidence (mainly not discussed in this paper) to suggest that the inductive learning processes are the central intelligent processes responsible for the construction of the current basic object representations ("signs"). The emergent symbolic operations constructed during learning and inducing on the symbolic space the "optimal (metric) geometry" clarify the meaning of "meaning" as well as the meaning of the multiresolutional representation.

As discussed in section 3 , the emergence of numbers appears to be a result of application of the perceptual inductive learning processes to the representation of the set size only, the latter being a very restricted, numeric, form of inductive object-class representation. Moreover, the application of the general inductive learning processes to a less trivial, symbolic, form of object-class representation suggests, in particular, the future, qualitatively quite different, line of development of mathematics, as was also suggested, for example, in ([24], p. 36). Such "symbolic" rather than "numeric" mathematics would be about the entities that are compositionally structured in a manner less "trivial", or more general, than the "numeric" entities. In connection with this, it is interesting to note the considerable (non-technical) similarity of Fodor and Pylyshyn's important argument [25] in favor of symbolic representations as opposed to numeric ones on the basis of thoughts having non-trivial compositional structure and directed against the connectionist cognitive models.

The real possibility of development and the nature of symbolic, or inductive, measurement devices are also briefly discussed, in order to suggest the essential scientific link between the classical sciences and that of the inductive semiotic systems.

Thus, the present paper can be considered as an attempt to fundamentally address the great recurring "scandal" mentioned in the epigraph.

\section{REFERENCES}

[1] L. Goldfarb, On the foundations of intelligent processes I: An evolving model for pattern learning, Pattern Recognition 23, 595-616, 1990.

[2] L. Goldfarb, What is distance and why do we need the metric model for pattern learning, Pattern Recognition 25, 431-438, 1992.

[3] L. Goldfarb and S Nigam, The unified learning paradigm: A foundation for AI, in Artificial Intelligence and Neural Networks: Steps toward Principled Integration, eds. V. Honavar and L.Uhr, Academic Press, Boston, 1994.

[4] L. Goldfarb, What is inductive learning? Construction of inductive class representation, Proc. 
of Workshop on What Is Inductive Learning, ed.

L. Goldfarb, 9-21, 1996.

[5] L. Goldfarb, Inductive class representation and its central role in pattern recognition, Proc. 1996 Conf. on Intelligent Systems: A Semiotic Perspective, eds. J. Albus, A. Meystel, R. Quintero, Vol. $1,53-58$.

[6] L. Goldfarb, S. Deshpande and V.C. Bhavsar, Inductive theory of vision, Technical Report TR96108, Faculty of Computer Science, U.N.B., 1996.

[7] L. Goldfarb and S. Deshpande, What is the symbolic measurement process?, to appear in Proc. IEEE SMC'97 Conf. Computational Cybernetics and Simulation, Oct. 1997.

[8] L. Goldfarb, On some mathematical properties of the ETS model, Technical Report TR93-079, Faculty of Computer Science, U.N.B., 1993.

[9] L. Goldfarb, J. Abela, V.C. Bhavsar and V.N. Kamat, Can a vector space based learning models discover inductive generalization in a symbolic environment?, Pattern Recognition Letters 16:7, 719-726, 1995.

[10] L. Goldfarb and K. Cormier, On the central role of symbolic representations in biological processes: Symbols as operations executed by molecules, synapses and neuronal systems, Technical Report TR97-114, Faculty of Computer Science, U.N.B., 1996.

[11] L.W. Barsalou, Cognitive Psychology: An Overview for Cognitive Scientists, Lawrence Erlbaum Associates, Hillsdale, New Jersey, 1992.

[12] A.R. Damasio, Descartes' Error: Emotion, Reason, and the the Human Brain, G.P. Putnam's Sons, New York, 1994.

[13] H. Plotkin, Darwin Machines and the Nature of Knowledge: Concerning Adaptations, Instinct and the Evolution of Intelligence, Penguin, 1994.

[14] H. Plotkin, Induction processes and evolution, to appear in Pattern Recognition, Special Issue on What Is Inductive Learning?, ed. L. Goldfarb, 1998.

[15] G.I. Ruzavin, On the Nature of Mathematical Knowledge: Essays on the Methodology of Mathematics, Mysl' Publ., Moscow, 1968 (in Russian).

[16] J. McLeish, The Story of Numbers: How Mathematics Has Shaped Civilization, Ballantine, New York, 1994.
[17] J.N. Crossley, The Emergence of Number, World Scientific, Singapore, 1987.

[18] T. Dantzig, Number: The Language of Science, 4th ed., The Free Press, New York, 1954.

[19] R. Kuntzig, A head for numbers, Discover 18:7, 108-115, 1997.

[20] C. Landauer and K.L. Bellman, Mathematics and linguistics, Proc. 1996 Conf. on Intelligent Systems: A Semiotic Perspective, eds. J. Albus, A. Meystel, R. Quintero, Vol. 1, 153-158.

[21] U. Eco, A Theory of Semiotics, Indiana University Press, Bloomington, Indiana, 1979.

[22] J. Albus, Retina, decision making, and semiotic control, Preface in [23].

[23] A. Meystel, Semiotic Modeling and Situation Analysis: An Introduction, AdRem Inc., Bala Cynwyd, Pennsylvania, 1995.

[24] C. Landauer and K.L. Bellman, Semiotics of constructed complex systems, Proc. 1996 Conf. on Intelligent Systems: A Semiotic Perspective, eds. J. Albus, A. Meystel, R. Quintero, Vol. 1, 35-40.

[25] J.A. Fodor and Z.W. Pylyshyn, Connectionism and cognitive architecture: A critical analysis, in S. Pinker and J. Mehler, eds., Connections and symbols, MIT Press, Cambridge, Massachusetts, 1988. 


\title{
Unified Learning/Planning Automaton: Generating and Using Multigranular Knowledge Hierarchies
}

\author{
Alex Meystel $^{* 0}$ and Alberto Lacaze ${ }^{*}$ \\ * National Institute of Standards and Technology \\ Drexel University \\ ${ }^{¥}$ University of Maryland
}

\begin{abstract}
This paper introduces an approach for analysis of systems employing unity of learning and planning processes. An architecture of an automaton with joint learning and planning (LPA) is presented which determines a class of learning algorithms. An algorithm of learning that employs grouping, focusing attention and combinatorial search is described. LPA searches for an appropriate set of rules, and then, for a preferable motion trajectory. It creates top down planning/control processes which are based on multigranular hierarchies of knowledge and lead to the temporal evolution of the system.

Key words: complexity, control, evolution in biology, experiences, focusing attention, generalization, granularity, grouping, intelligence, multigranular knowledge hierarchies, multiresolutional architecture, planning.
\end{abstract}

\section{Introduction}

In 1986 the concept of "baby-robot" was introduced in [1]. This concept emphasizes processes of unsupervised learning and is associated with the joint evolution of behavior and knowledge incorporated within a system. It seems plausible that learning from multiple experiences is inseparable from the architecture of applying the acquired knowledge for shaping the desirable behavior.

We introduce a formal system-a learning automaton which has the faculties required to obtain and use knowledge: sensors, subsystems for storing and organizing information, subsystems for generating commands, actuators for changing the world, but initially it has no world model and no rules to achieve a goal. This knowledge should be learned and our goal is to understand how.

The concepts of experience as a part of behavior, as well as desirability of behavior and its components-experiences, will be introduced and explored (Section 3). Behavior is shaped by the actions which emerge as a result of planning the goal-states, actions that lead to goal-states, and strings of them. The process of finding the string of desirable states and actions leading to them is called planning. The latter would be impossible unless the ability to judge the degree of desirability of states and actions would not be acquired in advance. Generating and applying proper commands to execute the planned trajectory and compensate for errors is called execution. Both planning and execution are a part of control. The process of acquiring the relevant information and processing it so that a proper behavior could be generated is called learning. Planning/control and learning are complementary procedures of intelligent computation.

The process of planning starts with focusing attention which selects the initial representation of the world or the map with its boundaries. The space is discretized into tessellata which determine the space resolution, or granularity. Combinatorial search is performed as a procedure of choosing one string (the minimum cost string) out of the multiplicity of all possible strings formed out of the space tessellata at this particular level of resolution.

Grouping the tessellata in a variety of feasible strings allows selection of one of them. This determines an envelope of focusing attention around the vicinity of the minimum cost string. All information within the envelope is being transformed to the higher level of resolution and grouping of behavior units starts again. Focusing attention presumes proper distribution of nodes in the state space so that no unnecessary search be performed. Combinatorial search forms the alternatives. All of these three procedures together can be considered as a process of generalization. Generalization is a generation of the representation at the lower level of resolution.

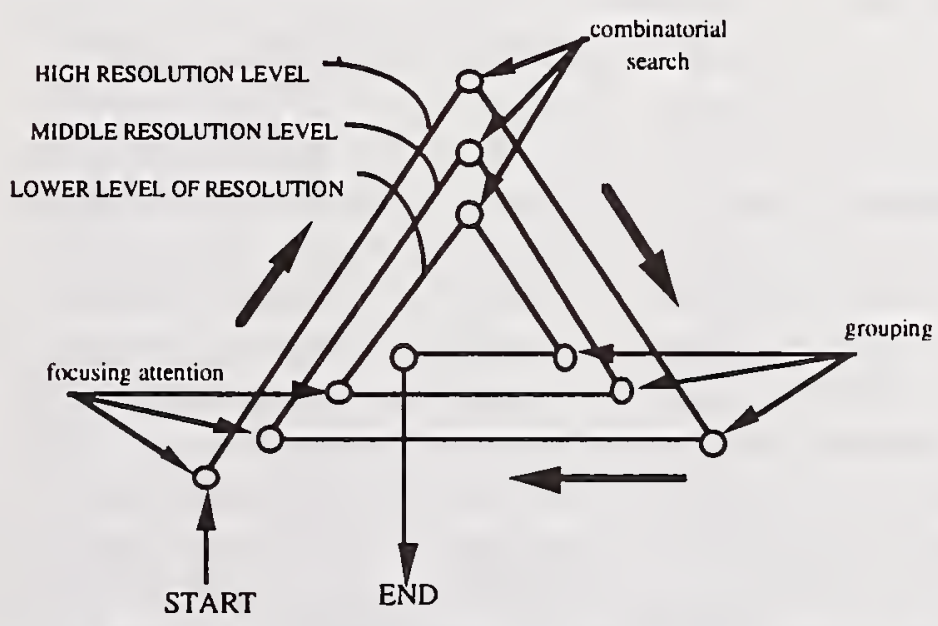

Figure 1. Multiresolutional Consecutive Knowledge Acquisition by the virtue of Generalization

An example of joint functioning of these three operations is shown in Figure 1. The operations work jointly as a triplet, which can be considered an elementary 
unit of intelligence [2]. The process is shown in Figure 1 for learning. It presumes generalization of high resolution units of information into lower resolution clusters. This process can proceed in the opposite direction, from a lower resolution to a higher resolution, i. e. counterclockwise. Then it will demonstrate planning and presume instantiation via decomposition of low resolution units into higher resolution. Both generalization and instantiation are the key processes of intelligence.

All of these phenomena presume that the system under consideration can "behave" as a result of its perceiving the states, planning the decisions and acting by using the actuators. The overall system can be represented using the concept of elementary loop of functioning [2-4].

This paper demonstrates that learning leads to emergence of a multiresolutional representation and a hierarchy of planning/control.

\section{Algorithms of Unsupervised Learning with Combinatorial Enhancement and Generalization}

2.1 Learning Automata. An automaton is a state machine which generates outputs by using its transition function and state-output function tabulated in advance for all possible states. Conventional automata are capable of demonstrating "reactive behaviors" according to the prescriptions stored in their transition and output functions $[21,22]$. In this paper, a class of learning automata is outlined which are state machines whose transition and stateoutput functions are updated and modified based upon prior experiences which are stored in the memory and transformed into sets of rules. The transition and output functions of these automata have open lists of rules. New rules can be added to these lists.

This concept is similar to the one described in [5]. In this paper, we will equip learning automaton with a new capability: to synthesize their output by combining together previously stored rules in search of the most appropriate behavior. In addition to reactive responses, our learning automata demonstrate the skill of deliberation, or planning.

This new type of automata will be called Learning and Planning Automata (LPA). LPA are presumed to have a learning system $L$ which allows for enriching both the transition and the output functions and a planning system, as a part of the mechanism of behavior generation $B G$.

As the system of rules develops it becomes a hierarchical one. This is equivalent to formation of the hierarchy of automata as a result of the evolution of a single learning automaton and affects the corresponding input and output vocabularies. Operators of $L$ are equipped by minimum initial set of tools including the ability to form strings, to construct hypotheses, and to infer tautologies.

Learning system $L$ can be defined as a system of acquisition of experiences, transformation of these experiences into rules of action, derivation of new concepts, and organization of these concepts into enhanced knowledge base (entity-relational network similar for the long term knowledge called ontology, base of hypotheses, concept base, etc.) This improves decision-making for achieving the goal.

In order to support processes of learning, each learning automaton is equipped by the set of actuators that follow commands at the output of learning automaton. Changes in the world are measured by the sensors. A set of sensors is the only source of information for the learning system about the state of the World (state.) The automaton is also equipped by subsystems of Sensory Processing and World Model which allow for interpreting the input from sensors.

The Learning System can judge upon truthfulness of this information only by the results of actions (behavior) which are undertaken to achieve the goal. The subsystem of Behavior Generation (BG) contains transition function and output function $[2,3,6]$. Other devices of BG are described in Section 5. This approach is presented earlier in $[7,8]$.

The state of the world is understood as a set of $n$ sensors' outputs which arrive at a particular moment of time. Action is a set of $\mathrm{m}$ action outputs which are generated by the system between two consecutive states. States can be represented as sets (lists), or as vectors within a particular system of coordinates. Actions are understood as causes of changes that are sensed after the actions are applied.

Goal is a state which must be achieved as a result of the behavior. The goal is often presumed to be given to a system. From [2-6] we know that goals can emerge also as a result of planning. From $[3,4]$ we know that the process of learning generates subgoals.

It is presumed that any valued experience is associated with a certain measure of "goodness". It can be interpreted as a reward for the pursuit of the goal $G$. The value of reward will be used for the subsequent process of hypotheses generation and selection. Ultimately, it determines the rules necessary for "survival." A rule can be obtained as a result of transforming the cause-effect relations discovered within repetitive experiences.

After the rules are confirmed, some of their parts which emerged as a result of generalization, may not belong to the initial vocabularies. These parts are to be treated as new concepts. Concept is a label for the entity which can be obtained recursively as a cluster or group of higher resolution concepts. All new concepts are obtained from two available classes of rules (see Figure 2.) states and actions are concepts and so are clusters of states and actions, as well as their components.

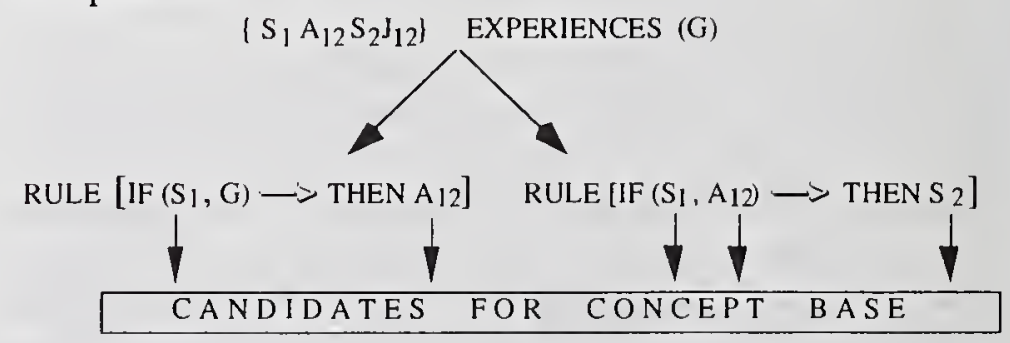

Figure 2. Experiences $\longrightarrow$ Rules $\longrightarrow$ Concepts

(S-state, A-action, J-reward, G-goal)

The new concept obtained from the generalized experiences as a result of their transformation into rules are 
novel words which are not present in the lists of previously defined input and output vocabularies. These novel words are words of the new vocabularies: at the lower level of resolution. They describe the phenomena related to groups of units of experiences.

\subsection{The Algorithm of Inductive}

Generalization. The following sequence of activities can be explicated from the definition of learning:

1. Experiences are collected and stored in memory.

2. Experiences are compared, resemblances are determined, and clusters are formed by the virtue of resemblance. Clusters of experiences which already have cause-effect relationships, are transformed into rules, and control rules and events rules are separated.

3. Search for the meaningful cause-effect relationships can be done not only among the stored set of experiences but among the synthesized experiences too.

4. The clusters of states and actions that are parts of the newly created rules are stored as concepts of lower resolution; then the growth of the concept base begins. New concepts emerge as a result of clustering: the new clusters are labeled and receive a status of a new word.

5. Each of the clusters of similar enhanced experiences is considered to be a candidate for becoming a rule hypothesis.

6. The hypotheses are stored. Subsequently, the new experiences are stored in parallel in two new vocabularies: the original and the one formed by the newly created concepts.

7. The process of consecutive operations

$\{$ collection of experience $\longrightarrow$ hypothesis formation$\rightarrow$ generation of rules $\rightarrow$ concepts emergence $\}$

is repeated each time as a new experience arrives.

8. Hypotheses are validated by statistics of their use and then enter the base of hypotheses. As vocabularies grow, they allow to represent and control functioning of the system by using their new words. The sequence of steps 1-6 can be repeated which forms a lower level of representation.

The algorithm (Figure 3) describes a recursive process which leads to a multiresolutional (MR) system of world representation and an MR systems of rules of actions, both acquired as a result of learning. Inductive generalization claims that multiple occurrences of similar experiences testify for existence of a particular rule, if most of these occurrences have the same (or similar) explanation of causes [14]. If the number of occurrences is not statistically persuasive, then we can talk about the case of hypotheses generation by means of abductive generalization. In both cases, it is important to account for the list of attributes/variables and for the relations among them [15].

Learning employs the algorithm of generalization which presumes a multiple iteration of the triplet from Figure 1: focusing attention on the subset of experiences and searching among them and their combinations until a grouping can be performed, i.e. a cluster of similar units could be substituted by a single generalized hypothesis [10]. If the subsequent experiences confirm the hypothesis, it becomes stronger. Automata with generalization have not been previously discussed in the literature on learning.

Since the algorithm of generalization as applied recursively to its own results, each two adjacent levels of resolution use generalization applied to the initial information which can be considered experiences and to generalized information which is called hypotheses. Thus, we can distinguish two kinds of generalization pertaining to the level to which they are applied: generalization from experiences before any hypothesis is available and generalization from hypotheses.

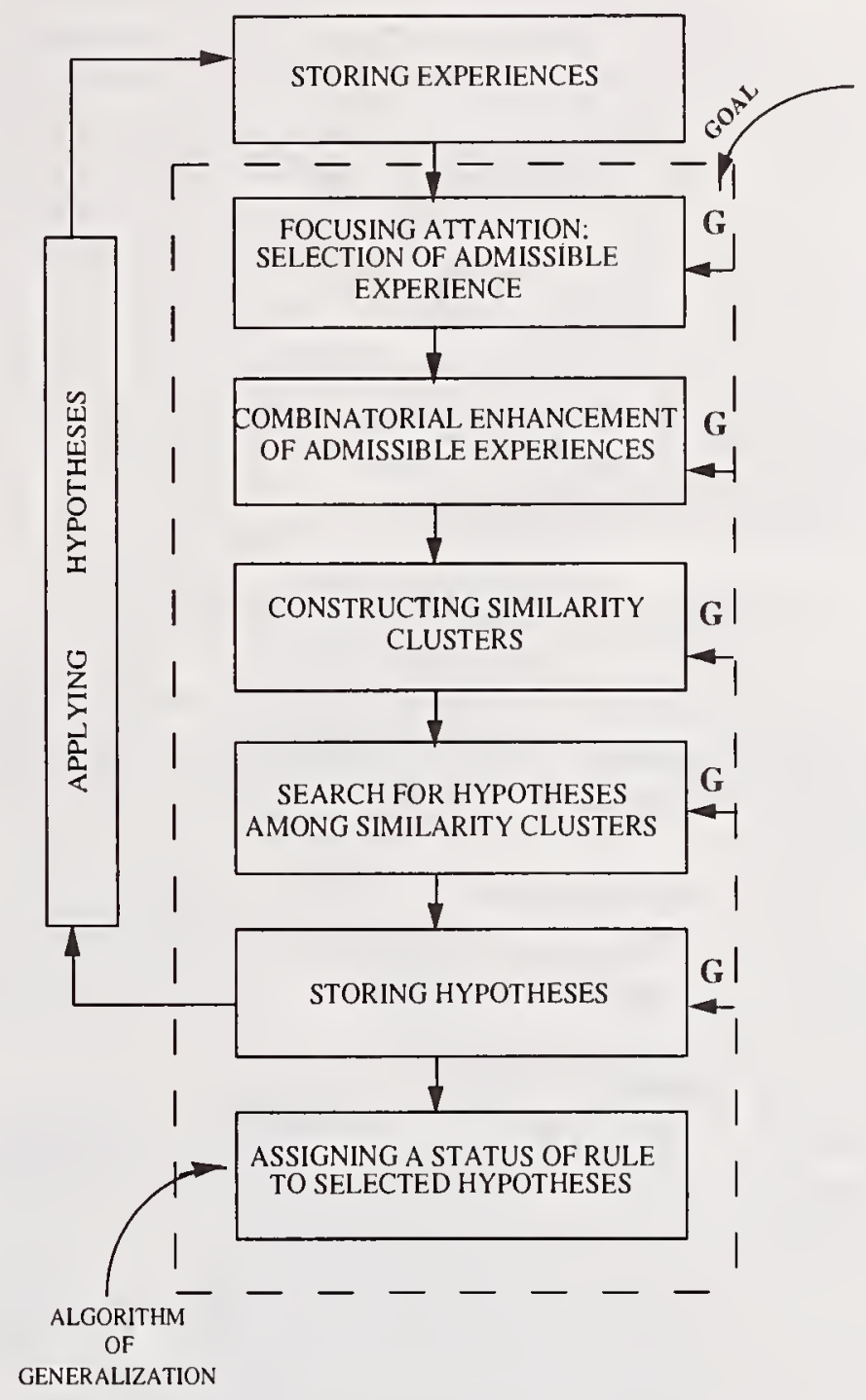

Figure 3. The Generalization Algorithm

Learning with generalization allows for using experiences in a very efficient way: it is a tool of reducing complexity. In order to receive innovations, some combinatorics should be introduced for generating words beyond the existing experiences. Similar mechanism has been proven to be very useful for design purposes [10].

\subsection{Combinatorial Enhancement: Searching} for Hidden Implications. Each experience can testify only about some part of the overall state. The available sensor information not necessarily can be a good basis for generating a hypothesis. Consider an example with autonomous mobile robot which must learn how to act in a particular environment. In a particular state, a single actuator command cannot be a proper response. Frequently, a combined command (steering + propulsion) must be assigned. Just value of the sensor reading of angle of "heading", $\angle \alpha$, or value of "angle to the goal", $\angle \sigma$, cannot 
be an antecedent in a rule what to do. However, their difference $(\angle \alpha-\angle \sigma)$ is a perfect variable of control.

The enhancing creates a new set containing the initial set and all possible combinations of its elements.

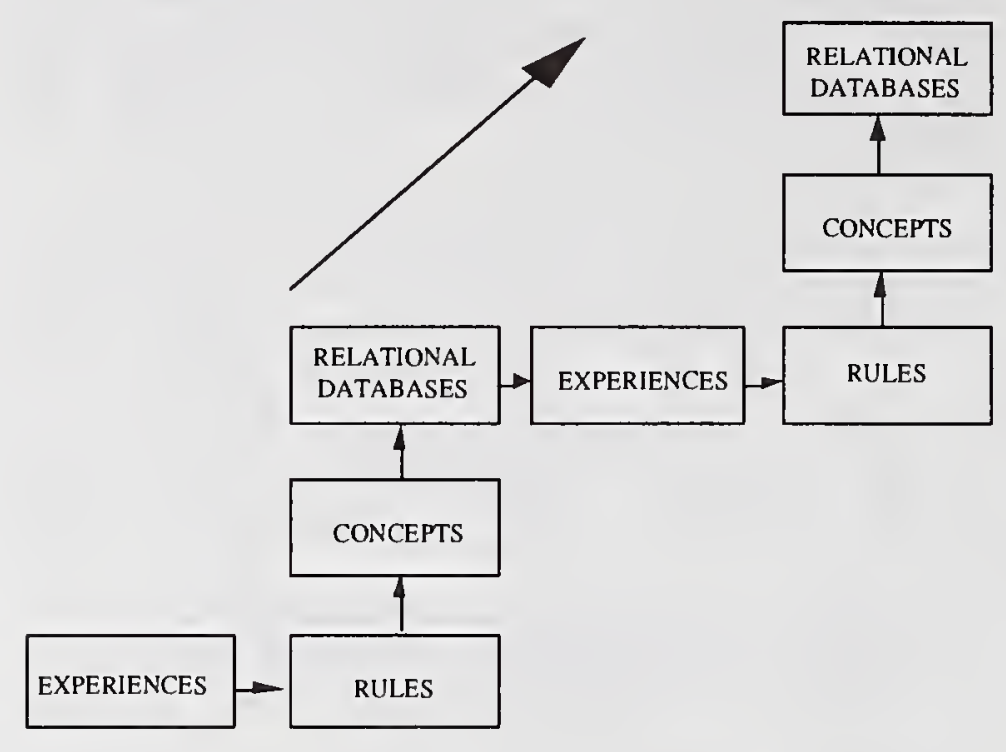

Figure 4. Evolution of Acquired Knowledge

The enhanced states is a set formed by a state, all possible combination of its components, and all possible combinations between components of the action $\mathrm{A}$ and the state $S$.

Such two persuasive examples of learning as the evolution of living creatures and the evolution of knowledge would be substantially impaired if no combinatorial enhancement would be possible.

The following factors are considered critical for stimulating evolution of living creatures: reproduction, mutation, competition, selection [9]. These factors are applied to knowledge in a similar way.

The algorithm of generalization creates new hypotheses, rules, and concepts which become new words in vocabularies. The same algorithm is applied to its own results. Now the previously generalized experiences are generalized again, and the hypotheses and rules of "lower resolution" are obtained. Evolution of acquired knowledge is illustrated in Figure 4. This diagram shows that the joint system of knowledge representation and behavior generation converges to a multiresolutional one.

2.4 Searching for Valid Hypotheses among Clusters. The reason behind the creation of clusters is our belief that each of these clusters is a candidate to become a hypothesis of a rule of a different behavior [7, 8].

The hypotheses are then stored in the database of hypotheses as a tree where each hypothesis is related to its "parent" by the goal. If no hypothesis was found for a certain state, then the state in the hypothesis with closest state to ours becomes a subgoal. This is one more source for the emerging hierarchies of acquired knowledge.

2.5 Combinatorics of refinement. When hypotheses are sought for within the level of higher resolution, the objects and experiences are supposed to be "enhanced" combinatorially in the way similar to one used at a level. This enhancement is used for the problems of design. It is a part of the process of "task decomposition $[3,6]$."

\section{Behavior Generation as a Multiresolutional Search for a Motion Trajectory in the State Space}

The second process characteristic for the learning automaton is Behavior Generation (BG) (see [4,16-20].) This process is understood as a sequence of top-down planning activities which end with receiving a set of control commands. The purpose of learning is to enable the subsequent process of planning. Conventional automata are only capable of reactive decisions; they are not capable of "look-ahead" decision making processes which are typical for deliberative planning.

Behavior generation consists of two components: goal refinement and trajectory generation. The goal is given from the upper level at a particular resolution. In order to plan the trajectory, this representation of goal should be refined ("job assignment".) Then, "each member of the team" can plan its trajectory ("scheduling.") Unlike learning, which develops bottom-up and works via generalization (or coarsening), the process of planning develops top-down and works via instantiation (or refinement.) The process of planning is determined as choosing the desirable behavior by anticipating admissible alternatives among possible behaviors and selecting the best of them.

From Section 2, we conclude that all experiences acquired and hypotheses generated contain some knowledge of some reactive rules. For example, "if it is necessary to get to $S_{2}$ from $S_{1}$, apply $A_{12}$." This rule reacts by evoking $A_{12}$ to the need of getting into $S_{2}$ from $S_{1}$ at $i$ level of resolution.

Trajectory is a string of adjacent admissible elementary subdomains (or tessellata, tiles of the discretized space).

Any well posed problem of planning should start with assigning the initial point and the final point of the trajectory which should be determined at the higher resolution within the tessellatum of the resolution under consideration. The feasible trajectory is determined at the lower level of resolution.

PT is called a feasible trajectory if it has an initial and a final points, and all tiles of the string are contained in the feasible trajectory at the lower level of resolution. Thus, a feasible trajectory for the level $i$ is always represented as a string of tiles for the $i+1$ level of resolution and is a subspace of the $\mathrm{i}$ level of resolution. Clearly, the needs in and the algorithms of finding a feasible trajectory are tools of focusing attention. We determine the subspace in which the wpp of planning should be resolved, and the optimum trajectory should be found. Indeed, the feasible trajectory determined at the i+1 level of resolution becomes an "envelope," a bound domain of space at the i level of resolution. Search in the state space or $S^{3}$-search, (see $[8,14$ 20]) is done by synthesizing the feasible trajectories for a particular level of resolution and then building the alternatives of possible motion trajectories for the lower 
resolution level within the envelope cost space.

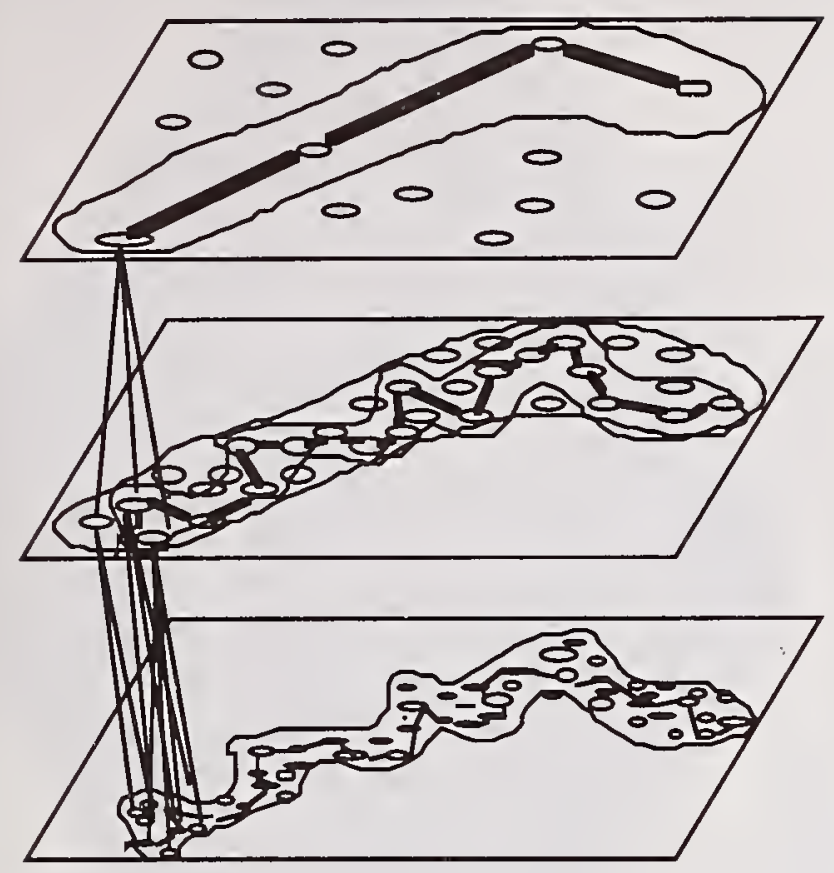

Figure 5. Illustration to the $\mathrm{MS}^{3}$-algorithm.

Some particular volume of the state spaces designated for a subsequent search for a solution, Operation of contraction puts constraints on this volume and should be properly justified. We need to reduce the probability that contraction eliminates some or all of the opportunities to find the optimum path trajectory. The following heuristic strategy of contraction is chosen. After the search at the lowest resolution level is performed, the optimum trajectory is surrounded by an envelope. It is a convex hull which has a width $w$ determined by the context of the problem. Then, the random points generation at the next level of resolution is performed only within this envelope of search. The problem of consistency of representation under the contraction heuristic has to be addressed in the future.

The Behavior Generation part of LPA functioning is illustrated in Figures 5 and 6.

\section{Conclusions: The Issues of Further Research}

The results presented in this paper has been confirmed by the experimental results [1,7,8]. "Baby-Robot" was able to learn how to reach the arbitrary situated goal only after implementing the algorithm of generalization with combinatorial enhancement. Before this algorithm was implemented, Baby-Robot was able to learn how to reach the particular located goal. If the location of the goal was changed, the successful learning process for the previous goal could not help to find a new one. Generalization n with combinatorial enhancement has enabled the robot to make the discovery, and initiate the process of hierarchical learning. Other positive results are recorded in $[19,20]$.

1. In this paper, we introduce and analyze an algorithm of MR unsupervised learning with inductive generalization and a search for hidden implications. This algorithm is applied recursively to its own results at the output. Thus, LPA develops an evolving MR system of representation. It enables the system of behavior generation (BG) also to evolve. LPA provides for an evolution of the automata equipped with such systems. LPA becomes an MR automaton, and its levels of resolution can change as the evolution of knowledge and behavior proceeds. This evolution can be illustrated by Figure 7 .

2. From Figure 8 , one can see the behavior evolves. This producing different plans and motion trajectories as shown as a horizontally developing tree. At the same time, its knowledge evolves as shown in the vertical hierarchical structures.

This process seems to be even more important to analyze of the evolution of living creatures. We believe that LPA allows for analysis of the processes of evolution of its systems as species. It is possible to equip the automaton by the system of reproduction. It would be possible to analyze how the process of knowledge evolution is affected by different mechanisms of reproduction.

3. This line of research takes advantage of the uniqueness of LPA among other known systems of automata with learning. The mechanism of unsupervised learning allows for the ultimate freedom in the way the learning process organizes the acquired knowledge. It is possible to anticipate that as the knowledge base evolves, the knowledge becomes utterly diversified. Rules concerning the external world will emerge, and the rules concerning processes of inner knowledge organization and procedures of processing will follow.

4. LPA can be used to analyze all stages of learning including the "early learning" stage. Certainly, some initial knowledge ("bootstrap knowledge") is presumed. This bootstrap organization of knowledge can strongly affect the subsequent processes of knowledge evolution. On the other hand, the learning system is presumed to be free of a building up of all subsequent knowledge organization. How it will organize the knowledge acquired and why? - this is the research issue for LPA.

5. The processes of knowledge acquisition are affected by the knowledge stored. They start creating some bias in the subsequent knowledge acquisition since the results of automaton functioning will be induced by the knowledge previously stored. So, if the results of functioning were "good" or led to a "better" behavior, the system might assume that its goodness is due to the knowledge used. It might happen that the experiences the system acquires are limited by its predisposition.

6. Since the system has been developed to demonstrate some particular behavior, the evaluation of this behavior should be the ultimate measure for the process and results of knowledge organization as well as the processes and results of the ways knowledge is acquired from the external world and knowledge used for behavior generation. This determines an interesting interconnection between further developing the hierarchy of functions for evaluation of goodness which precipitate at different levels of resolution. The 
interconnection between learning, behavior, and emerging "system of values" in LPA is an important research issue.

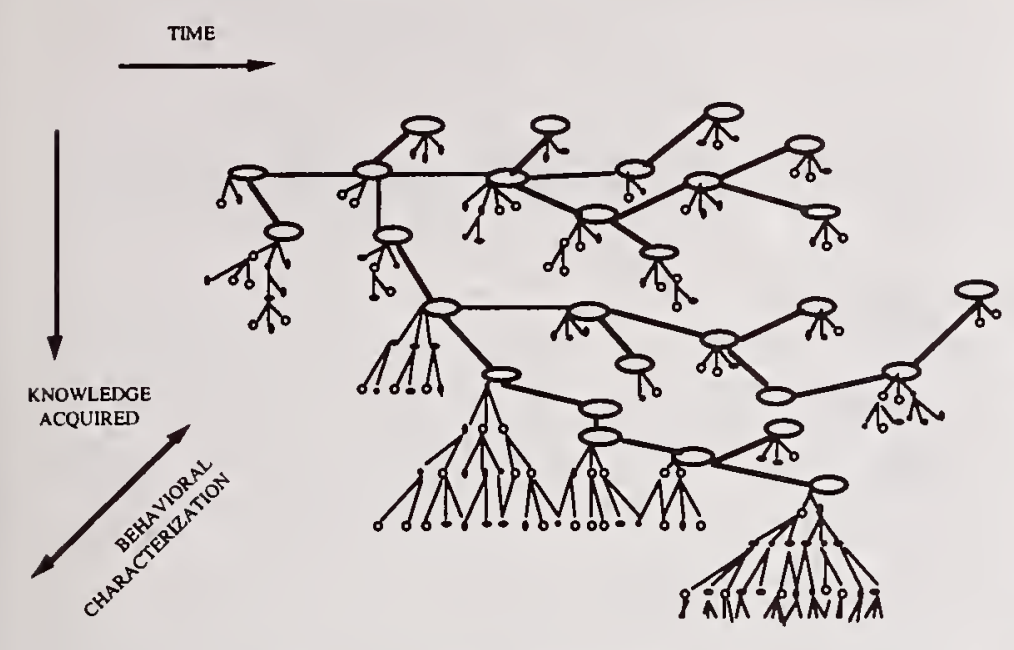

Figure 7.Evolution of Knowledge and Behavior of LPA

7. All these three processes: acquisition, organization and use affect the overall system functioning. Therefore, other systems of learning can modify and alter the system of LPA. At the present time the following mechanisms of knowledge acquisition are known from the literature:

a). Learning by transfer. In this case all knowledge which subsequently is required for behavior generation is transferred from another source where it was stored and organized in advance based upon existing design decisions and experiences of functioning. This method of knowledge acquisition presumes that the structure of the system of interest and its functioning in required circumstances are previously known.

b). Learning by examples. In this case, we presume a "Teacher" which has substantial knowledge about my cases of possible functioning, stores knowledge of previous experiences and spells out a set of possible scenarios in which the functioning of the system is expected. Undoubtedly, a set of tests can be developed in which a behavior of system is entertained and after each case of behavior the system receives the teacher's evaluation whether it was good, and how good it was.

The difference between these systems and LPA can be easily seen.

8. Learning and Behavior Generation produce structural and behavioral hierarchies. It is possible to state that using LPA reduces computational complexity by increasing the structural complexity.

\section{References}

1. Meystel A. Baby-robot: On the analysis of cognitive controllers for robotics, Proc. IEEE Int'l Conf. on Man \& Cybernetics, Tucson, AZ, Nov. 11-15, 1985, pp. 327-222

2. Meystel A., Intelligent Systems: A Semiotic Perspective, International Journal of Intelligent Control and Systems, Vol. I, No.1, 1996, pp. 31-57

3. Albus J., Meystel A., A Reference Model Architecture for Design and Implementation of Semiotic Control in Large and Complex Systems, Proc. of the 1995 IEEE ISIC Workshop, Architectures for Semiotic Modeling and Analysis in Large Complex Systems, Monterey, CA 1995, pp. 33-45
4. Meystel A., Nested Hierarchical Control, in Eds. P. Antsaklis, K. Passino, An Introduction to Intelligent and Autonomous Control, Kluwer Academic, Boston, MA 1992

5. Rajaraman K.,Sastry P.S., Finite Time Analysis of the Pursuit Algorithm for Learning Automata, IEEE Transactions on Systems, Man \& Cybernetics, Part B: Cybernetics, Vol.26, No.4, August 1996, pp. 590-598.

6. Albus J., Outline for a Theory of Intelligence, IEEE Transactions on Systems, Man and Cybernetics, ol. 21, No. 3, May/June 1991, pp. 473-509

7. Albus J., Lacaze A., Meystel A., Autonomous Learning Via Nested Clustering, Proc. of the 34th IEEE Conference on Decision and Control, New Orleans LA, 1995, pp. 3034-3039

8. Albus J., Lacaze A., Meystel A., Theory and Experimental Analysis of Cognitive Processes in Early Learning, Proc. of the 1995 IEEE International Conference on Systems, Man and Cybernetics, vol. 4, Vancouver, BC, Canada, 1995, pp. 44044409

9. Fogel D.B., Evolutionary Computation: Toward a New Philosophy of Machine Intelligence, IEEE Press, NY, 1995

10. Meystel A.,Thomas M. Computer aided conceptual design in robotics, Proc. IEEE Int'l Conf. in Robotics, Atlanta, GA, March 13-151984, pp. 220-229

11. Mirkin B., Mathematical Classification and Clustering, Kluwer Academic, Dordrecht, 1996

12. Mirkin B., The Method of Principal Clusters, Automation and Remote Control, 1988, pp. 1379-1388

13. Mirkin B., Method of Fuzzy Additive Types for Analysis of Multidimensional Data, Automation and Remote Control, 1990, pp. 683-821

14. Michalski R.S, A Theory and Methodology of Inductive Learning, Artificial Intelligence, Vol. 20, No.2, Elsevier, Amsterdam

15. Goldfarb L.,Abela J., Bhavsar V.C., Kamat V.H. Can a Vector Space Based Learning Model Discover Inductive Class Generalization in a Symbol Environment, Pattern Recognition Letters, V. 16, 1995, pp.719-726

16. Meystel A., Planning in a hierarchical nested controller for autonomous robots, Proc. IEEE 25th Conf. on Decision and Control, Athens, Greece, 1986

17. Meystel A., Planning in a Hierarchical Nested Control System, in Eds. W. Wolfe, N. Marquina, Mobile Robots, Proc. of SPIE, Vol. 727, Cambridge, MA 1986, p.p.42-76

18. Saridis G., An Integrated Theory of Intelligent Machines by Expressing the Control Performance as Entropy, Control: Theory and Advanced Technology, Vol. 1, No. 2, p.p. $125-$ 138, MITA-Press, Tokyo, Japan, 1985

19. Meystel A., Uzzaman S., Landa G., Wahi S., Navathe R., Cleveland B., State Space Search For An Optimal Trajectory Proc. of the IEEE Symposium on Intelligent Control, Vol. II, Philadelphia, PA, 1990

20. Grevera G., Meystel A., Searching for a path through Pasadena, Proc. of the IEEE Symposium on Intelligent Control, Arlington, VA, 1989

21. K. Naim, A. S. Poznyak, Learning Automata: Theory and Applications, Pergamon, 1994

22. S. Lakshmivarahan, Learning Algorithms Theory and Applications, Springer-Verlag, New York, 1981 


\title{
Structural Learning for Classification of High Dimensional Data
}

\author{
Dr. Paul M. Baggenstoss \\ Naval Undersea Warfare Center \\ Newport RI, 02841 \\ 401-841-7505 x 38240 (TEL) \\ 401-841-7453 (FAX) \\ p.m.baggenstoss@ieee.org (EMAIL) \\ EDICS 6.1.6 / $3.5 / 6.17$
}

July 1,1997

\section{Abstract}

This paper is concerned with probability density estimation in high-dimensional settings. A breakthrough in classifier performance has been achieved by assuming that the multidimensional feature vector is composed of statistically independent "groups". The method, which we call independence grouping, determines a mapping of features into separate lowdimensional feature groups, which are regarded as independent. The optimal size of the groups and the best mapping is determined either by large numbers of random trials, or by pairwise test for mutual independence of features. The bias associated with the independence assumption is traded off with large improvements in PDF estimation from the dimensionality reduction.

\section{Introduction}

In many classification problems of current interest, no concise statistical model is available the competing hypotheses. Example: a microphone is placed at a busy street intersection, the noises received at the microphone are to be classified. There are two choices for such difficult problems:

1. Employ a single general model with a large number of unknown parameters (such as Neural Net or Kernel-based PDF estimator).

2. Employ several low-dimensional models, each specific to a hypothesis.

Either way, a large number of parameters (features) must be estimated and used jointly for classification. In such problems, the curse of dimensionality strikes causing the amount of data required to train the classifier to rise exponentially, far outstripping the ability to collect, store or process.

Many researchers hypothesize that many natural phenomana are inherently low-dimensional, but in some unknown subspace or manifold of the high-dimensional feature space. Learning this lowdimensional structure we call structural learing.

In this paper, we expose a novel method of structural learning that has achieved a breakthrough in classifier performance. The method, which we call independence grouping, determines a mapping of features into separate low-dimensional feature groups, which are regarded as independent. The best mapping is determined either empirically by large numbers of trials, or by direct determination of the degree of independence. The bias associated with the independence assumption is offset with large improvements from the dimensionality reduction.

Because of practical considerations (computer and time resources) many of the results presented in this paper were obtained from different data sets. However, the results are presented in the order in which they were obtained along with the thinking that led to them. The author hopes that this style this will result in a very readable presentation.

\section{Independence Grouping}

Let there be $N$ features organized into a vector $\mathbf{x}=$ $\left\{x_{1}, x_{2}, \ldots, x_{N}\right\}$. The full-dimensional (FD) classifier for $M$ hypotheses,

$$
\max _{j=1, \ldots, M} \hat{p}\left(\mathbf{x} \mid H_{j}\right) p\left(H_{j}\right)
$$

makes use of the FD PDF estimate $\hat{p}\left(\mathbf{x} \mid H_{j}\right)$ which is derived from some training data. We use $K$ to 
denote sample size and superscripts to denote sample number:, $\left\{\mathbf{x}^{1}, \ldots, \mathbf{x}^{K}\right\}$.

Let the $N$ features be divided into $G$ groups, each group having $K_{j}$ features, thus $N=\sum_{j=1}^{G} K_{j}$. The feature mapping are denoted by $\beta_{i} \in[1, G], i=$ $1, \ldots N$ which maps each feature $x_{i}$ into a group.

Note that that $G=1, \beta_{i}=1$ for $i=1, \ldots, N$ is the mapping for the FD PDF.

For each mapping $\boldsymbol{\beta}=\left\{\beta_{1}, \ldots, \beta_{N}\right\}$, the independence-grouped (IG) PDF for $\mathbf{x}$ is constructed as a product of group-PDF's.

$$
\begin{aligned}
\hat{p}\left(\mathbf{x} \mid \boldsymbol{\beta}, H_{j}\right)=\hat{p}\left(\left\{x_{i}: \beta_{i}=1\right\} \mid H_{j}\right) \\
\cdot \hat{p}\left(\left\{x_{i}: \beta_{i}=2\right\} \mid H_{j}\right) \\
\cdots \hat{p}\left(\left\{x_{i}: \beta_{i}=G\right\} \mid H_{j}\right)
\end{aligned}
$$

The above expression carries with it an implication that the groups are statistically independent of one another. Note that (1) must be trained on a training data set for each $j$. Training requires a double maximization/optimization: optimize jointly over $\boldsymbol{\beta}$ and the parameters of the PDF estimator for the data set avaliable. When training the lower-dimensional PDF's, less data is required. Or, with a fixed training data size, they will be more accurate than the FD counterpart. But, is the increase in accuracy enough to counter the bias associated with the assumption of independence? In the cases we have studied, the implicit assumption of independence among groups simply is not true - there always seems to be statistical dependence between any two features. We now argue that the goodness this assumption can be directly tested.

Note that (1) is a PDF in its own right on the same feature space and may be directly compared with the FD classifier using likelihood value. The apropriateness of the independence assumption will be reflected in the total log-likelihood of the PDF estimate obtained under the assumption. There is no need for guesswork (note that we assume here that the total log-likelihood "score" of the PDF estimate must be obtained from a statistically independent set of data as was used for training or else "overtraining" occurs).

We claim that the high dimensional PDF, when optimized to fit a limited set of training data may be making far worse compromises in the process of training, although these compromises are hidden from view in the vast reaches of $\mathcal{R}^{N}$. Should (1) provide better performance than the FD counterpart, then there is no sane reason to not accept it. After all, recall that the FD classifier is contained in the set of all groupings.

\section{PDF Estimation}

In this paper, estimates of PDF's for FD or IG PDF's are derived from training data using a standard multivariate PDF estimation approach called heteroscedastic Gaussian mixture (GM) modeling. A widely accepted technique for estimating the parameters of the GM model is the EM algorithm [1],[2]. The EM algorithm suffers from numerical problems when there is insufficient data leading some researchers to avoid it [3] or constrain the covariances of the kernels to be identical [4], or of uniform size with variable rotation [5]. Adding to the covariance estimates based on a Bayesian prior density argument is the preferred method of dealing with the problem [6], [7]. We have obtained excellent results using Bayesian priors to represent an implied assumption of measurement noise. Measurement noise may represent quantization noise or subjectively determined error variances.

\section{Optimum Group Size}

We have not mentioned how $G$ or $k_{j}$ are determined. We start by selecting $G$ and $k_{j}$ empirically. We will shortly see that for a given problem, algorithm performance has a broad peak at a certain group-size, say $k^{*}$.

In the following experiment, a data set of time series "snapshots" from $M=6$ signal types was available. The data was divided into 5 sets containing different amounts of additive noise to artificially produce a variation of SNR in $3 \mathrm{~dB}$ increments. A total of $N=44$ features were extracted from each time series. An average of about 150 samples was available from each class at each SNR for training and testing.

The group sizes were fixed and $\boldsymbol{\beta}$ was common across hypotheses. For each $\boldsymbol{\beta}$, PDF's were estimated on the groups from training data, the combined PDF (1) was formed, and classification performance (probability of correct classification, $P_{c c}$ ) was measured using a separate testing data set. This was repeated for a range of group sizes. When we speak of trials, we refer to independent random data splits into training and testing halves. The $P_{c c}$ was averaged across trials. $P_{c c}$ was measured by counting the number of correct decisions as a fraction of the total number of data samples used in testing.

The 44 features were grouped into sets of $2,3,4,5,6,7,9,11,17$, and 21 features. The last group contained the remainder number of features when 44 was perfectly divisible. A total of 16 statistical trials were run at each SNR and for each group size. In each trial, not only was the data randomly split, but the features were randomly assigned to groups. 
It was expected that using very small groups would induce poor performance due to the (incorrect) assumption of independence between groups. For larger groups, the dimensionality issues would dominate. The net result would be an optimal dimension. The experiment was repeated for various levels of additive Gaussian noise (SNR reductions) in $3 \mathrm{~dB}$ steps. This expectation is very clearly supported in Figure 1 . The group size (dimension $P$ ) is plotted on the abscissa.

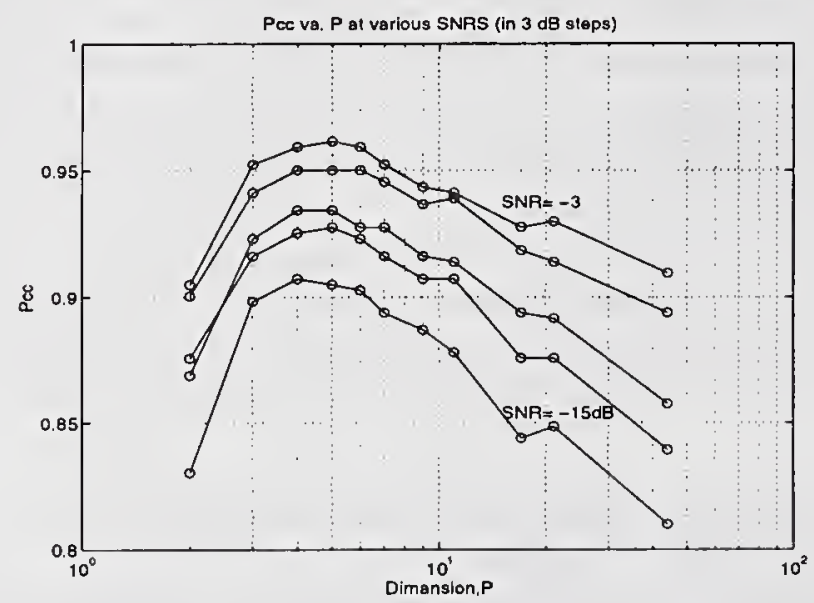

Figure 1: Classification performance as a function of feature group size, average of 16 random trials.

The importance of Figure 1 cannot be overstated. Conventional thinking in classification has been to increase $N$ using the FD classifier until a collapse in peformance is noted due to dimensionality issues. Here, we keep $N$ fixed but are able to adjust the dimension nevertheless by adjusting group size. The performance peak at $P \sim 5$ shown in Figure 1 cannot be found by existing methods of model order selection which do not utilize all the features at once.

\section{$5 \quad$ Best Grouping}

The limitations of the previous experiment are that no attempt was made to find an optimal gouping, which should be different for each class hypothesis. However, it still clearly indicates the existence of a broad maximum for group size around $k^{*}=5$.

The following experiment is a variation of the previous $M=6$ experiment. A total of $N=64$ features were used in the random grouping scheme. Random group sizes in the range of 3 to 6 in length were used. As before, the groupings were held constant across hypotheses. For each grouping, $P_{c c}$ was averaged across SNR. A total of 1800 groupings were tested on the CRAY T3D at NUWC in Newport, RI. The best grouping of the 1800 was selected. Multiple trials were then run using this "best grouping" to more accurately measure its performance.

When measuring $P_{c c}$, it was necessary to add a constant to each log-PDF (equivalent to adjusting prior probabilities of the hypotheses in a Bayesian framework). The 6 constants were adjusted to minimize the total number of errors. This optimization was repeated for each trial. The grouping with the best average performance was then used in 16 trials at each SNR step. In Figure 2, the resulting performance of the best grouping is plotted in which RG stands for random grouping. This graph shows the $P_{c c}$ per-

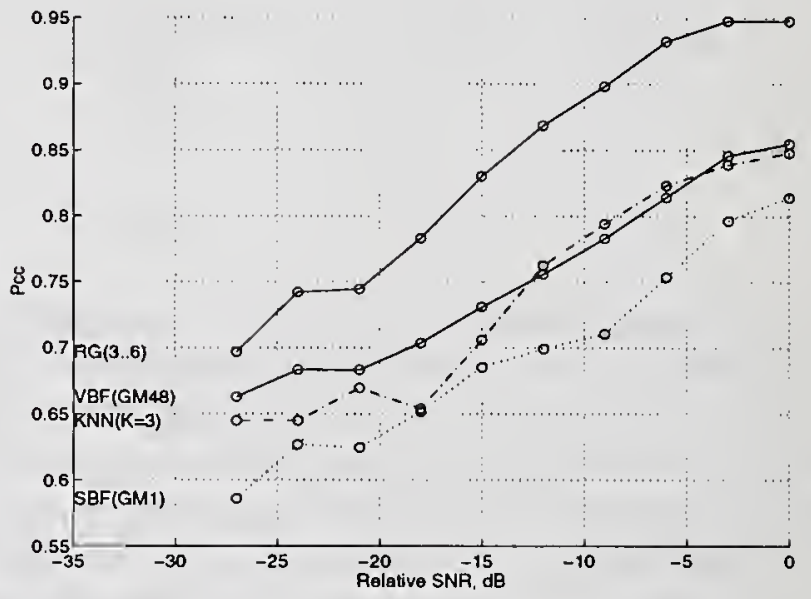

Figure 2: Classification performance vs. SNR of the best grouping out of 1800 trials for IG PDF (denoted $\mathrm{RG}$ ) vs. three other classifiers (see text).

formance as a function of SNR for the best random grouping as compared with three other classifiers:

1. GM48: Full-dimensional classifier for 44 features using 48 mixture components.

2. $\mathrm{KNN}(k=3)$ : K-nearest neighbor classifier $(k=$ $3)$.

3. GM1: Full-dimensional classifier for 44 features using 1 mixture components, essentially a quadratic Bayesian classifier.

For each data point on the graph represents an average of 16 trials, each using a different random data split of the same overall data set. The IG classifier clarly outperforms the other classifiers.

\subsection{Measure of Fit (MOF)}

Up to now, we have optimized the feature grouping by maximizing estimated $P_{c c}$. This has two disadvantages:

1. It requires a lot of computation each time $P_{c c}$ is estimated. This limits the number of combinations that can be tried. 
2. It constrains each class to have the same grouping (it would not be practical otherwise).

In an attempt to avoid these problems, alternative ways were sought to optimize the groupings.

Consider an algorithm for training a Gaussian Mixture estimate of $p\left(\mathbf{x} \mid H_{j}\right)$. Such an algorithm operates my maximizing the average or total $\log$-likelihood of the data $\sum_{k} \log p\left(\mathbf{x}^{k}\right)$. But, as mentioned earlier, (1) is a PDF on $\mathcal{R}^{N}$ in its own right. There is no reason not to jointly optimize the groupings at the same time as the parameters of the Gaussian Mixture based on total likelihood. This incorporates grouping selection (structure learning) directly in the PDF estimation of a given class. Thus, grouping selection is separated from classification performance. More grouping trials could be tested per class for a given amount of computer resources. In addition, differences in the data structure of different classes could be taken into account.

In Figure 3, we show 32 histograms. Each histogram is created from 37 random groupings for a given random data split. It may be seen that the distribution of the average likelihood depends greatly on the data split. It is theorized that some data splits contain data points that are likely to be "alone", without neighbors and not likely to be "covered" by PDF mixture kernels, which tends to bias the likelihood value down. Thus, the determination of the "best grouping" overall had to factor in the bias of each random data split.

The best grouping overall for each class hypothesis was selected. An improvement in classification performance was noted, however, it was not significant. The author believes that this is due to the high-dimensional space in which to search for the best $\boldsymbol{\beta}$. In the following, we break through this barrier.

\subsection{Independence Measure}

If the implied assumption of independence groupings is the statistical independence between groups, then it makes sense to obtain a measure of feature interdependence. Unfortunately, for practical reasons, we must limit ourselves to pair-wise tests. We are not interested is simple correlation coefficients since this is not descriptive enough. Uncorrelated random variables can be statistically dependent. The scheme we use is based on a hypothesis test for independence vs. dependence. More specifically, the independence hypothesis says that

$$
p\left(x_{i}, x_{j}\right)=p\left(x_{i}\right) p\left(x_{j}\right)
$$

The procedure is then to estimate the three PDF's $\hat{p}\left(x_{i}, x_{j}\right), \hat{p}\left(x_{i}\right), \hat{p}\left(x_{j}\right)$ from available data, then form

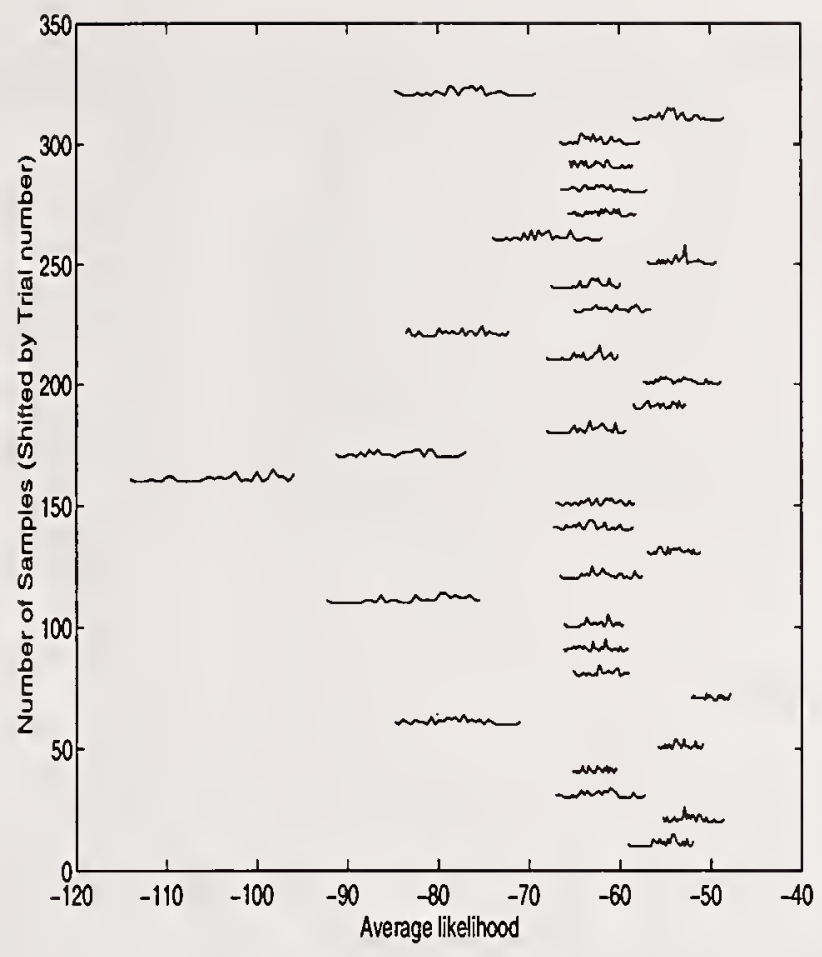

Figure 3: Histograms of random Groupings for 32 different random splits

the empirical independence measure

$$
Q(i, j)=\frac{1}{K} \sum_{k=1}^{K} \log \left(\frac{\hat{p}\left(x_{i}^{k}\right) \hat{p}\left(x_{j}^{k}\right)}{\hat{p}\left(x_{i}^{k}, x_{j}^{k}\right)}\right)
$$

where $K$ is the number of available training samples and the superscript indicates the sample index.

This measure was computed for all feature pairs in a 70-feature example. The results are provided in Figure 4 . In the graphic, feature dependence measure is scaled and quantized to a number between 0 and 15 , represented as a hexadecimal character. Anything below 3 is shown as ".". These types of graphics are then used to manually or automatically determine the best feature groupings.

The following procedure was found to be satisfactory: .

1. Determine what the largest group size that can be accepted.

2. Any two features with a dependence measure greater than a threshold $\tau$ are placed in the same group.

3. Begin with $\tau$ equal to some large value.

4. Lower $\tau$ until the largest group size has been reached.

5. Continue to lower $\tau$, grouping together features, but do not add to groups that have exceeded the maximum group size. 


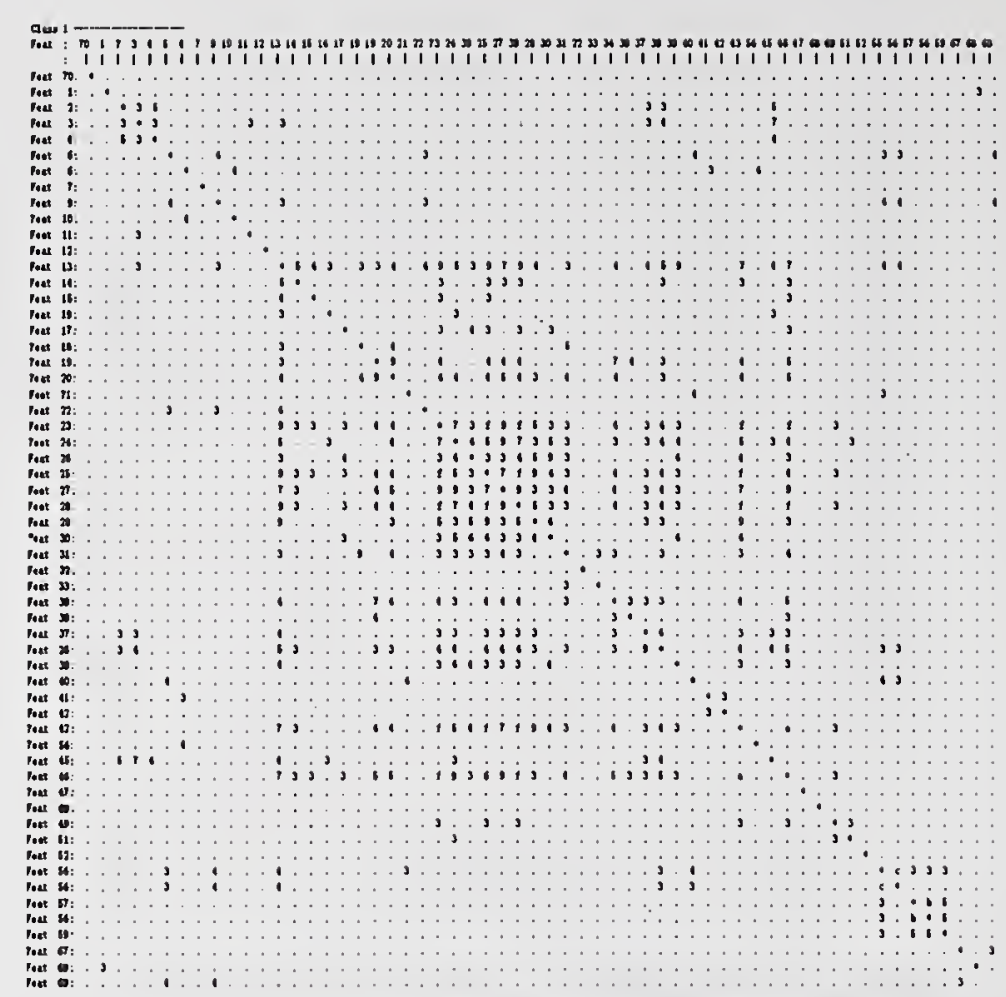

Figure 4: Graphic of feature interdependence

This procedure can be automated. The graphic in Figure 4 produced the grouping in Table 1 . The reader may notice that the largest group size is 11. Many of the features in the largest group were highly dependent and could not be justifiably separated. Furthermore, highly dependent (or correlated) features could be grouped together without greatly increasing the ultimate voulme of the space. Thus, groups with highly dependent features could be larger.

\subsection{Results of Groupings based on Independence-Measure}

Consider the second (from top) histogram in Figure 3 (trial number 31 of 32). We expand this histogram in Figure 5. In addition, a single point is added at the far right. This point is the likelihood value of the PDF using the grouping determined from pair-wise independence measures and tested with exactly the same data split as the rest. The fact that this point is far outside the population of the randomly selected groupings graphically illustrates how significant the independence measure method is with respect to random grouping selection.

\begin{tabular}{|c|c|}
\hline Group & Features \\
\hline 1 & 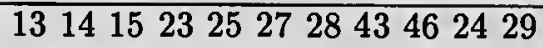 \\
\hline 2 & 58402122 \\
\hline 3 & 1920341831 \\
\hline 4 & 5556575859 \\
\hline 5 & 676869 \\
\hline 6 & 4453 \\
\hline 7 & 61044 \\
\hline 8 & 263017 \\
\hline 9 & 3738 \\
\hline 10 & 4142 \\
\hline 11 & 7 \\
\hline 12 & 11 \\
\hline 13 & 12 \\
\hline 14 & 16 \\
\hline 15 & 32 \\
\hline 16 & 33 \\
\hline 17 & 36 \\
\hline 18 & 39 \\
\hline 19 & 47 \\
\hline 20 & 48 \\
\hline 21 & 49 \\
\hline 22 & 51 \\
\hline 23 & 52 \\
\hline
\end{tabular}

Table 1: Feature grouping corresponding to Figure 4

\subsection{Genetic Algorithm for Grouping Optimization}

Neither random search nor search based on pair-wise independence measure are expected to locate the best grouping. Since the search is on a discrete space, gradient-based methods cannot be employed. However, a genetic algorithm is well suited for the task. In the preliminary implementation, the following approach was employed. A starting point was selected based on random search or independence measure. Then, a mutatiuon was performed by applying one of three possible changes:

- Swap 2 features.

- Move 1 feature.

- Merge 2 groups.

All operations used a uniformly distributed random variable to select the group or feature. If a feature was moved outside the range of existing groups, a new group was created. Approximatelty 1000 single mutations were performed and the MOF was determined. The 8 mutations which produced the largest change in MOF with respect to the original parent for the same random split were selected. These 8 children were then used as parents (asexually) for the next generation. The MOF was always calculated on testing data, i.e. unseen by the PDF training algorithm. A graphic 


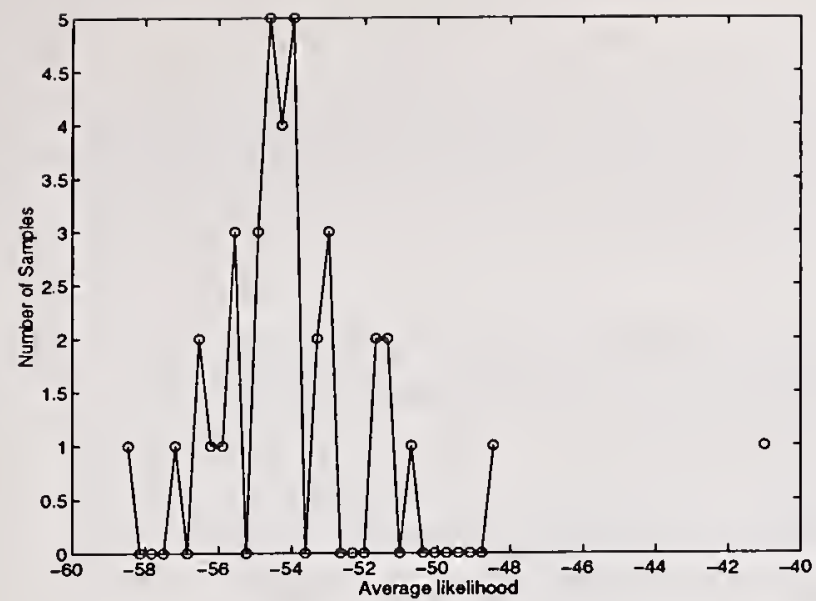

Figure 5: Histogram of 37 random Groupings using same data split

showing a histogram of the MOF of each generation is shown in Figure 6. Beginning from the bottom, the

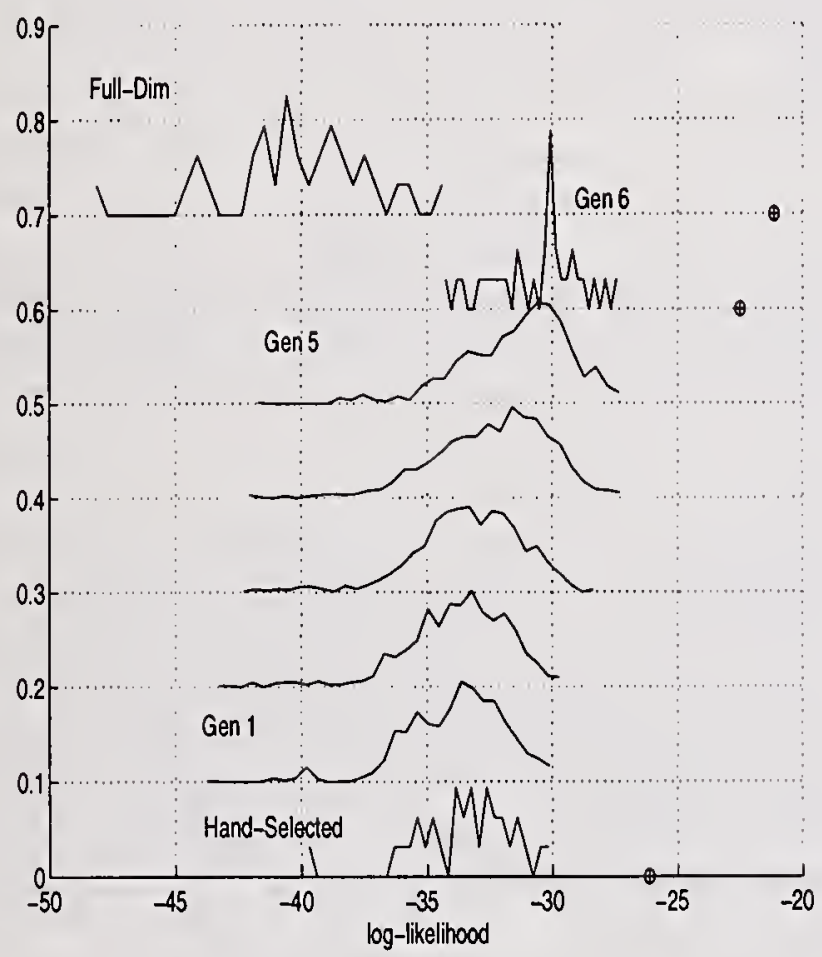

Figure 6: Five generations of Genetic Algorithm

MOF histogram for the parent "hand-seleted" grouping determined from pair-wise independence is shown along with a single data point on the right indicating the MOF for the training data. Loosely speaking, this represents an upper limit of achievable MOF for testing data. Next, the MOF histograms of generations 1 through 5 are shown. The smoothness of these histograms reflects a large number of groupings included in contrast to the single parent grouping. Note a shift to the right with each generation. The best grouping of generation 5 was seleted. The next histogram, dennoted "Gen 6" is its MOF histogram. Note that again we include the "uppper limit" point, the average MOF for training data. The final histogram of the FD PDF (at top) provides a contrast that clearly illustrates the characteristic of IG PDF's. Note that the average likelihood for the training data (single points at right) is higher for FD vs. IG. This is expected since it reflects the fact that the IG PDF is more constrained than the FD PDF and cannot fit the training data as well. However, when testing data is used, the more robust IG PDF's far outperform the FD PDF.

\section{Conclusions and Future Work}

An entirely new dimension has been added to the PDF training phase of classifiers (or any PDF-based algorithm). Structural learning finds the best structure upon which to train the PDF. While PDF estimation must be performed by matching the PDF parameters to best fit the given training data, structural learning must be performed by fitting to the testing data, unseen by the PDF estimator. This encourages the combined structure-PDF estimate to be robust while at the same time optimizing the measure of fit.

\section{References}

[1] R. L. Streit, "A neural network for optimum Neyman-Pearson classification," IEEE Trans. Neural Nets, vol. 5, no. 5, pp. 764-783, 1994.

[2] L. I. Perlovsky, "A model-based neural network for transient signal processing," Neural Networks, vol. 7 , no. 3, pp. 565-572, 1994.

[3] J.N.Hwang, S.R.Lay, and A. Lippman, "Nonparametric multivariate density estimation: A comparative study," IEEE Trans SP, vol. 42, pp. 2795-2810, October 1994.

[4] R. L. Streit and T. E. Luginbuhl, "Maximum likelihood training of probabilistic neural netsworks," IEEE Trans. on Neural Networks, vol. 5, pp. 764$783,1994$.

[5] R. L. Streit and T. E. Luginbuhl, "Estimation of Gaussian mixtures with rotationally invariant covariance matrices," Unknown, 1996.

[6] D. M. Titterington, Statistical Analysis of Finite Mixture Distributions. Wiley, 1985.

[7] G. J. McLachlan, Mixture Models. Dekker, 1988. 


\section{LEARNING FROM LARGE DIMENSIONAL, SMALL SAMPLE DATA BY A POTENTIAL FUNCTION METHOD WITH NONLINEAR TRANSFORMATION: A CANCER DIAGNOSTIC STUDY}

\author{
Mark Modell \\ MediSpectra, Inc., Cambridge, MA \\ Victor Kamensky \\ Albert Einstein College of Medicine, \\ Department of Epidemiology \\ and Social Medicine, Bronx, N.Y.
}

\section{Introduction}

Machine learning methods are especially well suited to medical diagnostic applications. One of the application is the screening and diagnosis of cancerous and pre-cancerous conditions. In the present paper we discuss novel techniques of machine learning applied to the analysis of data from a new optical biopsy device where the dimensionality of each original observation of the data is very large $(60,000$ items) and where the number of observations is very small (approximately one hundred).

We have analyzed data collected by the ColpoProbe device (MediSpectra, Inc., Cambridge, MA) at the colposcopy clinic of the Beth Israel Hospital, Boston [L.Burke et al, 1996]. It is widely accepted that Pap Smear testing has been successful in reducing cervical cancer in the U.S. by more than $70 \%$. It is, however, also recognized that the methods are far from ideal and not cost effective. Each year, 50 million women in the U.S. receive a Pap Smear test and of those, approximately $6 \%$ are returned with a finding of ASCUS (Atypical Squamous Cells of Undetermined Significance). This diagnosis represents minor Pap Smear abnormalities that are not sufficiently clear-cut to permit a more specific diagnosis. Because of obvious prognostic concerns, this population requires thorough evaluation to determine whether benign or malignant processes are more likely.

The triage of patients with ASCUS results from Pap smears is a growing problem. Currently, patients are either referred for repeat Pap smears or directly to colposcopy. There have been several studies in the literature on the use of Pap smears for triaging non-negative Pap smears. These show that the sensitivity of the Pap smear when used as a follow-up is typically between 40 and $62 \%$ (Slawson, D.C., et. al., 1994; Slawson, D.C., et. al., 1993;
Casimir Kulikowski and Ilya Muchnik

Rutgers University, Piscataway, NJ

Louis Burke and Jonathan Niloff

Department of Obstetrics, Gynecology

Reproductive Biology, and Pathology

Harvard Medical School

and Beth Israel Hospital, Boston, MA

Xiao-Wei Sun, et. al., 1995). This results in a significant number of patients with ASCUS Pap smears not being referred to colposcopy. Additionally, the relatively high false positive rates of the repeated Pap smear implies that many women would be inappropriately referred to colposcopy [MF. Mitchell, 1994].

MediSpectra has developed the ColpoProbe device, an optical biopsy instrument, as a triage tool for following an ASCUS Pap Smear result in order to select those women most likely to require colposcopy. Increasing the sensitivity of the procedure will result in more patients receiving the required treatment, while increasing the specificity will result in fewer unneeded colposcopies. In order to quantify the accuracy of the ColpoProbe device, MediSpectra has completed a feasibility study of the instrument. We have analyzed the data collected by the ColpoProbe to assess its performance. The present paper evaluates the diagnostic power of the method using a novel machine learning strategy to efficiently process the large amount of data produced. It also emphasizes methodological questions surrounding the application of these machine learning techniques.

\section{Data description}

A single observation from the original data set comprises 300 spectrograms of fluorescence emission. These emissions were less or equal 200 nsc. of each. Each emission spectrum is divided into $185 \mathrm{spec}-$ tral components. Usually the fluorescence emission spectra have high noise. Therefore, it is necessary to aggregate the observations to increase reliability in the processing. We used the following aggregation before analysis:

1. The average spectrum, i.e. mean spectrogram, was computed from the $\mathbf{3 0 0}$ individual observa- 
tions;

2. The average spectrum was filtered to decrease the noise;

3. The filtered average spectrum was aggregated in the following way: the original 185 components were separated in a small number of groups. Each group was represented by the mean and the standard deviation of original spectral components in the group.

These steps of "pre-analysis" yield an efficient data representation. Thus, after the preparation each spectrum was represented by 51 variables. $\mathrm{Fi}$ nally, we got the set of the vectors in 51-dimensional space. We will call them below also records or cases. The set consisted of 81 cases classified by histophathologist into five biopsy result categories, denoted as CC, Metapl, NED, SILHI and SILLO with the distribution indicated in the table 1 below:

Table 1: BIOPSY

\begin{tabular}{||c|c|c|c|c|c||}
\hline CC & Metapl & NED & SILHI & SILLO & Total \\
\hline 7 & 26 & 19 & 21 & 8 & 81 \\
\hline
\end{tabular}

In this paper we present results of discrimination of these 81 records when they were combined into two groups of biopsy categories namely, "precancerous" (denoted as SIL and including SILHI and SILLO categories) and "non-precancerous" (nonSIL, consisting of the other three categories of CC, Metapl and NED). Thus there are 29 SIL and 52 nonSIL records.

\section{A New Machine Learning Algorithm based on the Potential Function Method and on a Nonlinear Transformation for Extracting Discriminating Features}

\subsection{Overview of Method}

Most machine learning methods build objectclassification functions in a space of features. Input objects are represented as vectors of features and can be interpreted geometrically as points in the feature space. Thus, any surface which splits the space into regions associated with the different classes can serve as an object classification function.
The main problem with machine learning is to derive or learn a classification surface from a limited number of cases of known classification. In other words, the goal of learning is to generalize the knowledge from a limited set of samples. In geometrical terms, the goal is to construct a discriminating surface using the limited set of labeled points in the feature space so that it will be able to identify any new set of input vectors (points) of unknown classification. This surface is called a discriminant surface, and the mathematical function which describes it in the feature space is called a recognition function, or a recognizer [Debroye et al., 1996].

The potential function method is an universal machine learning method $[\mathrm{O}$. Bashkirov, E.Braverman and I. Muchnik, 1964, Aizerman et al., 1974, E. Braverman and Muchnik, 1983]. Recently it took on renewed importance because the support vector machine approach [V. Vapnik, 1995] has demonstrated great efficiency in applying integral kernels as a system of functions for building discriminants. Learning procedures based on this method allow practitioners to incorporate and test a wide variety of alternative sub-methods that can be adapted or customized to specific problems. The principal idea is to identify a similarity function between a vector and a class represented by a collection of vectors of known classification (known as the training set). Each class will be identified by its particular similarity function. This function is constructed as a linear combination of integral kernels (the potential functions), each of which is associated with one vector from the training set.

A discriminant is defined as the maximum of the similarity functions. Thus, for each vector, the values of the similarity functions are calculated and the largest of these determines the class associated with the vector. To learn the class identification is to determine the coefficients of the similarity functions. As in all machine learning methods, the potential function method has free parameters that must be learned from the training data. Also, in addition, it allows to choose an integral kernel types to correspond to the implicit structure of similarity or dissimilarity in the training data. The disadvantage of this additional freedom is the fact that the search for the best kernel and its parameters can be quite expensive and time-consuming.

Over 30 years ago, a two-stage recognizer design was proposed to transform original variables into "informative" in a particular sense [Sebestyen, 1962]. The simple rationale here was that the fit 
between learning procedure and training samples is expected to be better in the new space than in that of the original one. Mostly, the mentioned transformation was linear[W. Weiss and C.A. Kulikowski, 1991; S. Watanabe, 1969]. Further development of this approach for recogner design using a non-linear transformation model was originally suggested by Fukunaga [K. Fukunaga, 1992]. However, this method does not lend itself to wide practice because, for example, it does not explore combining potential function method at all.

In this paper, we describe and test a new nonlinear procedure for transforming a space of original variables into one that is more discriminating . The result of applying this technique is a set of new features with approximately the equal degrees of information content. The potential function method can work better with these features since they can be then combined directly without the need for separate weights. The potential functions, then, correspond to simple distance functions.

The new nonlinear transformation is based on three simple steps:

1. Extend the set of original variables by adding to them non-linear transformations such as their products, ratios, etc.

2. Build Boolean variables based on applying cutpoints derived from the training data to yield the best predictive performance for the given classifications based on a statistical similarity criterion. Generally, the idea of using Boolean space for a recognizer was very popular in the 60's [Bongard, 1968] and are implemented now when the machine learning area is integrated with AI-approach [P. Hammer et all., 1995]. Our realization of the idea is based on a particular systematical approach using of the Exact Fisher Test.

3. keep a part of generated Boolean variables which are not correlated.

The result of the first two steps of the procedure is a set of Boolean variables instead of the original numerical ones. A third step suppresses redundant information from these derived boolean variables. It keeps as a final set only those informative features which are correlated no higher than some chosen threshold. Because the procedure produces a
Boolean space instead of a numerical one, it is natural to use the potential function method with kernels based on a Hamming distance function. Next we concentrate on the specific implementation of the described combined algorithm for our problem. One can easily change the algorithm by varying the described procedure.

Note, in order to maximize the use of a data set it is common to derive a feature space transformation such as the above based on the entire data set (i.e. training and testing sets combined). However, we determine the weights of the kernels for the potential function algorithm only from the training data set alone.

\subsection{Principal Steps of the Learning Proce- dure}

Below we give a formal description of the main procedures for the presented method.

Transformation from numerical data into boolean data. Three procedures are described here which require the following notation and definitions. Let us denote by $X=\left\|x_{i j}\right\|, i=$ $1,2, \ldots, N ; j=1,2, \ldots, n$ any given matrix of $\mathrm{N}$ rows $\left\{x_{i}\right.$. $\}$ which presents objects characterized by $\mathrm{n}$ numerical variables.

The first procedure extends $X$ to $Y=\left\langle X, X^{\prime}\right\rangle$ where new variables from $X^{\prime}$ are, as mentioned above, the products and ratios for all possible pairs of $\mathrm{n}$ variables from $\mathrm{X}$. Every pair of original variables $\left[x_{k}, x_{k^{\prime}}\right]$ determines three new numerical variables, - $\left(x_{k} * x_{k^{\prime}}, x_{k} / x_{k^{\prime}}, x_{k^{\prime}} / x_{k}\right)$. The total number variables in $Y$ becomes $(n *(n-1)+n(n+1) / 2)$.

The second procedure analyzes all possible cutpoints on every $y$-variable ( $y$ is a column of the matrix $Y$; a cut-point is a value of y which splits all given in the matrix $Y$ y-values into 2 groups: which are smaller then the cut-point and which are higher it). This analysis permits us to evaluate how well the associated boolean variable correlates with the output-classification, which is also is a boolean variable. We will denote the output variable as $C$ : if for an i-th record the corresponded value $C_{i}=1$ it means the $\mathrm{i}$-th record belongs into the class $\mathrm{A}$ and if $C_{i}=0$ it belongs into the class $\mathrm{B}$.

One determines one boolean variable $z_{s k}$ by conditions

$z_{s k}=1$ if $y_{k} \geq y_{s k}^{*}$ and $z_{s k}=0$ if $y_{k}<y_{s k}^{*}$ where $y_{s k}^{*}$ is the $s$-th cut-point on the corresponding numerical variable $y_{k}$. One evaluates the variable 
$z_{s k}$ by calculating the $2 * 2$ table

$$
\begin{array}{ll}
a & b \\
c & d
\end{array}
$$

where $a=\# i\left(z_{i s k}=1 \& C_{i}=1\right), b=\# i\left(z_{i s}(k)=\right.$ $\left.1 \& C_{i}=0\right), c=\# i\left(z_{i s}(k)=0 \& C_{i}=1\right), d=$ $\# i\left(z_{i s}(k)=0 \& C_{i}=0\right)$.

In the above equalities were used the following notation:

a) a number of observation in a given data which matches conditions definded by the content

The table allows to use a statistical measure to estimate the probability $P$ of the event $" z_{i s}(k)=$ $1 \& y_{i} .=A$ and $z_{i s}(k)=0 \& y_{i} .=B "$. After the evaluation one chooses a threshold $P^{*}$ for the probability $P$ : if $P \geq P^{*}$ (or, $1-P \geq P^{*}$ ) then $z_{s}(k$ ) is lep as a candidate significant boolean variable. Mai' different statistics can be used for this purpose, of which the simplest is to directly use the ratio $\rho=(a+d) /(a+b+c+d)$ : if for example $P^{*}=.7$ then $z_{s}$ is significant if $\rho \geq .7$ or if $(1-\rho)=\geq .7$. In our concrete research we used so called the Exact Fisher Test [Fleiss,1982; Hays,1978; Woolson,1987]. The quantities $a, b, c$, and $d$ are the numbers of cases in the $2 X 2$ confusion matrix where the biopsy classes nonSIL and SIL denote the true classification and $V B O O L i=0$ denotes a negative screening result from using the $i$-th Boolean variable related to a significant variable, while $V B O O L i=1$ would correspond to a positive result for the same variable.

\begin{tabular}{|llll|}
\hline \multicolumn{4}{c|}{ BIOPSY } \\
VBOOLi & nonSIL & SIL & Total \\
\hline 0 & $\mathrm{a}$ & $\mathrm{b}$ & $\mathrm{a}+\mathrm{b}$ \\
1 & $\mathrm{c}$ & $\mathrm{d}$ & $\mathrm{c}+\mathrm{d}$ \\
Total & $\mathrm{a}+\mathrm{c}$ & $\mathrm{b}+\mathrm{d}$ & $\mathrm{a}+\mathrm{b}+\mathrm{c}+\mathrm{d}$ \\
\hline
\end{tabular}

For this table the calculation of Fisher's exact (2tailed) test was carried out. The probability of measuring $\mathrm{a}, \mathrm{b} \mathrm{c}$ and $\mathrm{d}$ in the table under the assumption that the percentages $1-\mathrm{s}$ and $\mathrm{s}$ for the two classes (nonSIL and SIL ) are the same is then given by the following formula:

$$
\text { Fisher }_{t}=\frac{(a+b) !(c+d) !(a+c) !(b+d) !}{(a+b+c+d) ! a ! b ! c ! d !}
$$

Variables are evaluated and selected by using the values of

$$
F_{\min }=\min \text { Fisher }_{t} .
$$

This procedure screens all $n$ yk-th for all the corresponded cut-points $s(k)$ and collects a complete set of the candidates for significant boolean variables. We denote the set as $S$ and let the module of $S$ be $M$. This completes the second procedure.

Significant variable extraction. The final step is to extract a set $S^{\prime}$ of highly significant boolean variables which we define as the smallest subset of $S$ which has a high enough level of correlation with the variables from the complementary subset $(S-$ $S^{\prime}$ ). One can apply different heuristic procedures to obtain this type of the core set. We used one of the simplest:

1) building a matrix of correlations on the defined set of all $M$ candidates for the significant variables;

2) for every candidate calculate the sum of the moduli of coefficients of its correlations with all other variables;

$3)$ to extract a set $S^{\prime}$ with cardinality corresponding to a prior chosen value $M^{\prime}$ with the largest values of the sums.

Potential function procedure. We chose to use the potential function method in its nonparametric form modeled by a parametric family of integral kernels [Aizerman et al. 1976]:

$$
K(u, v)=1 /\left(a+b r^{q}(u, v)\right),
$$

where $u$ and $v$ are vectors with components which are extracted significant boolean variables (vectors in the $M^{\prime}$-dimensional space); $r(u, v)$ is a distance function defined on an arbitrary pair of vectors and $\{a, b, q\}$ are three scalar free parameters. Because $K$ is a monotonically decreasing function of the distance function we can interpret it as a similarity measure. The method introduces a similarity function $K(u, G)$ with values that are average similarities between a vector $\mathrm{u}$ and a class of vectors $G$. The function is the mean of the above described elementary similarity measure $K(u, v)$ :

$$
K(u, G)=\frac{1}{|G|} \sum_{v \in G} c(v) K(u, v),
$$

where $|\cdot|$ is the notation for a modulus, and $c(v)$ is a function of weights defined on all elements of $G$. Let us assume that the training set for class $A$ contains $N_{1}$ vectors and the training set for class contains $\mathrm{N}_{2}$ vectors then the discriminant rule is given by the function: 


$$
F(u)=K(u, A)-K(u, B) .
$$

The rule is simple: if $F(u) \geq 0$ then $u$ belongs in class $A$ and vice versa. The function of weights $c(v)$ is defined on the training set by a standard algorithm of the potential function method: the training set is observed iteratively, - an observed element $\mathrm{u}$ is recognized by the above rule based on current values of the function $c(v)$, and if it is recognized incorrectly the corresponding weight $c(u)$ is increased to $[c(u)+1]$; if it is recognized correctly no weights change. The procedure is stopped when a cycle of observations of all elements in the training set don't yield changes in the value of the function $c(v)$.

\section{Procedures for performance evaluation}

Two types of tests have been used for the performance evaluation. The first is a computationally inexpensive single test procedure, while the second is a form of cross-validation:

A) Single Test Procedure:

1. Randomly divide each class (nonSIL and SIL) into approximately equal subsets

2. Perform the procedure described in section 3.5 twice. First let SUBSET 1 be the learning sample, and SUBSET 2 be the testing sample. Then reverse this, using SUBSET 2 as the learning sample, and SUBSET 1 as the testing sample.

Recognition results for both applications of the method are then combined into the following standard performance table (or confusion matrix):

\begin{tabular}{|c|cc|}
\hline DISCR. DECISION & \multicolumn{2}{|c|}{ CLASS BY BIOPSY } \\
\hline & nonSIL $(\mathrm{N})$ & SIL $(\mathrm{P})$ \\
$\operatorname{nonSIL}(\mathrm{N})$ & $T_{N}$ & $F_{P}$ \\
$\operatorname{SIL}(\mathrm{P})$ & $F_{N}$ & $T_{P}$ \\
\hline
\end{tabular}

where: $T_{P}$ - True Positives:the number of SIL cases correctly recognized as SIL;

$F_{P}$ - False Positives:the number of SIL cases incorrectly recognized as nonSIL;

$T_{N}$ - True Negatives: the number of nonSIL correctly recognized as nonSIL

$F_{N}$ - False Negatives: the number of nonSIL incorrectly recognized as SIL
Their percentages with respect to the correct categorization (based on biopsy results) can then be used to evaluate the performance of the discriminant method in terms of true positive and true negative frequencies $\left(T_{P_{\%}}, T_{N_{\%}}\right.$ respectively), and their percentages with respect to the classifier decision provides the predictive power of a positive result vs. that of a negative result ( $P_{P_{\%}}$ and $P_{N_{\%}}$, respectively).

B) Multiple test procedure:

The test described in A) above is performed 100 times - for 100 random splits of the data set into two parts. Summary statistics for frequencies of true positives, false positives, true negatives and false negatives will provide the appropriate performance results for the discriminant method. This is a type of cross-validation.

\section{Results of ColpoProbe Data Analysis}

All the results described below were obtained by applying the potential function method on significant boolean variables derived from the entire set of 81 observations. While from an application perspective statistically it would have been more appropriate to have derived these Boolean variables from the learning set data alone, the present analysis suits the purpose of providing an evaluation of the potential function learning method. The application of the cut-point procedure produces 20 significant boolean variables generate from the set of 81 observations.

For the selected variables we performed 100 tests of the discriminant function using 100 random splits, yielding the performance Table 2 (in the first, fourth and seventh line of the table are numbers of observations were tested, - because every original observation was tested 100 times the total number of tests was 8,100; respectively, second and fifth line of the table present results in rows; finally, the third and sixth line present for columns of the table).

Statistics for percents is the table 3 .

The best performance is given by a split with the results shown in the table 4 . 
Table 2: 100 Tests result

\begin{tabular}{|l|lll|}
\hline Decision & \multicolumn{3}{|c|}{ BIOPSY } \\
& nonSIL & SIL & Total \\
\hline nonSIL & 4766 & 507 & 5273 \\
& 90.38 & 9.62 & \\
& 91.65 & 17.48 & \\
\hline SIL & 434 & 2393 & 2827 \\
& 15.35 & 84.65 & \\
& 8.35 & 82.52 & \\
\hline Total & 5200 & 2900 & 8100 \\
\hline
\end{tabular}

Table 3: Average results

\begin{tabular}{|c|ccccc|}
\hline Variable & $\mathrm{N}$ & Min & Max & Mean & Std Dev \\
\hline$T_{P_{\%}}$ & 100 & 65.5 & 93.1 & 82.5 & 6.7 \\
$P_{P_{\%}}$ & 100 & 70.4 & 92.9 & 84.8 & 4.9 \\
$T_{N_{\%}}$ & 100 & 82.7 & 96.2 & 91.7 & 3.0 \\
$P_{N_{\%}}$ & 100 & 81.5 & 96.1 & 90.5 & 3.4 \\
\hline
\end{tabular}

There are nine splits that yield the minimum number of two false negatives $\left(F_{N}\right.$, where SIL is mistaken for nonSIL) like in the above best performance table.

From examining the statistics of these results we can see that, for a preliminary data set with a relatively small amount of data of large dimensionality, the performance provides excellent results with tight bounds for controlling (expensive) false positives, while keeping the number of false negatives within reasonable bounds. Its negative predictive power is remarkably high $(96 \%$ with a standard error of $3 \%$ ) for a first analysis of the data.

Further analyses and refinements of the preprocessing and the method are being carried out with the goal of tightening the bounds and further improving the false negative performance of the test and hence its positive predictive power.

Table 4: The best result

\begin{tabular}{|l|cc|}
\hline & \multicolumn{2}{|c|}{ DECISION BIOPSY } \\
& nonSIL & SIL \\
\hline nonSIL & 49 & 2 \\
SIL & 3 & 27 \\
\hline
\end{tabular}

\section{REFERENCES}

1. L. Burke, J. Niloff, M. Kobelin, G. Abu-Jawdeh, A. Zelenchuk and M. Modell(1996)

Use of Autofluorescence of Cells to Evaluate Cervical Neoplasia Journal of Gynecologic Techniques, Vol. 2, No.4:187-190.

\section{M. F. Mitchell(1994)}

The accuracy of colposcopy Clinical Consultations in Obstetrics and Gynecology, No.6:70-73.

\section{Slawson, D.C., et al.(1994)}

Should all women with cervical atypia be referred for colposcopy: a HARNET study J. of Family Practice, 38:4, 387-392.

\section{Slawson, D.C., et al.(1993)}

Follow-up Papillomavirus Smear for Cervical Atypia: Are we missing Significant Disease? J. of Family Practice, 36:289-293.

5. Xiao-Wei Sun, et. al.(1995)

Evaluation of the Hybrid Capture Human Papillomavirus Deoxyrbonuckeic acid detection test Am. J. Obstetrics and Gynecology, 173:1432-1437.

6. Devroye, Gyorfi and Lugosi(1996)

A Probabilistic Theory of Pattern Recognition. Springer, p. 654

7. Bashkirov, O.A., Braverman,E.M. and Muchnik,I.B. (1964),

Potential Function Algorithms for Pattern Recognition Learning Machines, Automation and Remote Control,25, n 5, 629-631.

8. M. Aizerman, E. Braverman and L. Rozonoer(1970)

Potential Function Method in Machine Learning Theory Nauka, Moscow, 384 p.(in Russian)

9. Braverman, E.M. and Muchnik, I.B.(1983) 
Structural Methods for Analysis of Empirical Data, Moscow: Nauka, 464p.(in Russian)

10. V. Vapnik(1995)

The nature of statistical learning theory Springer, London, 267p.

11. W. Weiss and C.A. Kulikowski(1991)

Computer Syttems that learn: Classification and Prediction Methods from Statistics, Neural Net, Machine Learning, and Expert Systems, Morgan Kaufmann, Palo Alto, 233p.

12. Sebastyen(1962)

Decision Making Processes in Pattern Recognition Macmillan, New York, 1962, 162p.

13. S. Watanabe(1969)

Knowing and Guessing Wiley, New York,

14. M. Bongard(1968)

Problem of recognition

15. P.L. Hammer, E. Boros, V. Kamensky, A. Kogan and Ilya Muchnik(1995)

Logical analysis of data(LAD): an implementation in SAS(R) language, Proceedings of the Eighth Annual NorthEast SAS Users Group Conference, Washington, DC,PP 683-688.

16. K. Fukunaga(1992)

Introduction to Statistical Pattern Recognition Academic Press, London, $591 \mathrm{p}$.

17. Fleiss Joseph L.(1982),

Statistical Methods for Rates and Proportions, Second Edition,John Wiley and Sons, New York. p.25-26.

18. Hays, William L.(1988)

Statistics. Fourth Edition, Holt, Rinehart and Winston, New York. p.783-787.
19. Woolson, Robert F.(1987),

Statistical Methods for the Analysis of Biomedical Data, John Wiley and Sons,New York. p.215-221. 


\title{
Incremental Learning of Inheritance
}

\author{
Jose Neves, Evgueni Smirnov and Cesar Analide \\ Department of Informatics \\ The University of Minho \\ Largo do Paço \\ 4719 BRAGA CODEX-PORTUGAL
}

e-mail: (jneves, smirnov, analide\}@di-ia.uminho.pt

\begin{abstract}
This paper proposes a cycled system for learning of the hierarchical models of inheritance of Multiagent Systems (MAS), using a logical framework. The system acquires the appropriate models from the descriptions of the most informative models with respect to the learning process. The determination of the most informative models is accomplished by analysing hypothetical space of the models and evaluating the problem solving capabilities of the MAS, based on these models. The system presents two advantages: its components are symbolic, and it requires significantly small number of the "training" models. This allows learning of "understandable" symbolic descriptions of the hierarchical models of inheritance in reasonable time.
\end{abstract}

Keywords: Machine Learning, Multiagent Systems, Logic Programming.

\section{Introduction}

Learning in MAS is on the intersection between the multiagent paradigm and the machine learning one [3, 8]. It is motivated by the insight that it is not possible to determine a-priori the complete knowledge that must exist within each component of a distributed system, in order to allow for a satisfactory performance of such a system. It is therefore broadly agreed in both the Distributed Artificial Intelligence and $\mathrm{Ma}$ chine Learning communities, that there is need to provide these systems with the ability to learn, i.e. to selfimprove their future behaviour.

Learning in MAS consists of two types of learning. First, single-agent learning; i.e. learning that is done by a single agent as a result of its interaction with the environment or a set of other agents. This type of learning presupposes the application of the traditional machine learning techniques. Second, distributed learning, that is defined as learning what is possible, only because several agents are presented. This type of learning comprises learning in teams, learning to act in teams, learning to coordinate agents, learning of the organisational structure of MAS, etc. The main techniques used in distributed learning are borrowed from Reinforcement Learning and Neural Networks $[6,8]$, which ensure perceptiveness, correctness and reactiveness of the MAS, but at the same time retain the main shortcomings of the non-symbolic approaches to Artificial Intelligence, namely:

- incomprehensibility of the knowledge and behaviour of the systems;
- high time complexity as a result of exploring a sufficient number of environmental states.

The research considered in this paper aims at overcoming these problems in the learning organisational structure of MAS, endorsed via the disjunctive logic programming paradigm facing inheritance [1] That is why the learning organisational structure is reduced to the task of learning of the hierarchical models of inheritance. Solving this task is proposed to be done in a closed cycle:

WHILE there is no acceptable description of the models of inheritance $\mathbf{D O}$

Determine the satisfactory performance of the MAS with respect to their current models of inheritance; Consider the descriptions of the models, as their examples with positive or negative classification in respect to the performance;

Learn the general descriptions of the "good" models with the new examples;

Determine the most informative (examples) models with respect to learning;

Change the model of the systems with the most informative ones.

\section{END}

The steps 1-2 and 4-5 are completely symbolic sub-processes, that require explicit descriptions of the inheritance models. That is why the learning step obeys this requirement, and the communication within the cycle is accomplished on a symbolic level. This means that the intermediate results of the learning process are symbolic descriptions of the models of inheritance. Their complete acquisition is accomplished when the determination of the most informative models is not possible, or when the designers of the MAS agree on some of the acquired descriptions of the "good" models, as satisfactory. Therefore, the process of learning is completely autonomous, and requires only the most informative models, in contrast with existing algorithms [8]. The final results are the symbolic descriptions of the models of inheritance, that guarantee a satisfactory performance and understandable behaviour of the corresponding MAS.

This paper sketches the logical knowledge representation scheme in section 2 . Section 3 considers a system for learning of the models of inheritance, based on the proposed cycle. Section 4 analyses the system and proposes future directions for research. 
2 Knowledge Representation with Inheritance

This work considers knowledge representation by means of a hierarchical model, with inheritance, that endorse the treatment of incomplete information, allowing for commonsense reasoning.

The system structure is supported by two kinds of entities: agents, that represent specific entities such as objects or their individual classes or instances; and links, which are used as a way of formalising relationships among those entities (Figure 1). One link may represent a hierarchical relation between agents, or the association of a set of data to an agent (the agent's theory).

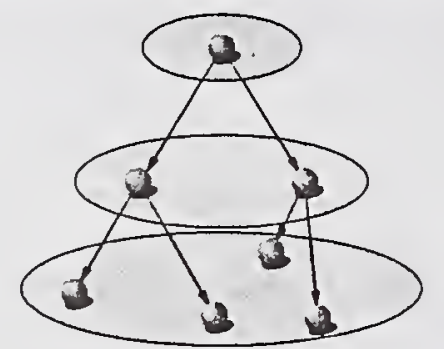

Figure 1. A Hierarchical Model of Inheritance

In terms of a logic program, this kind of relation between two agents can be described by a predicate of the type ISA links, that are found in most of the works about hierarchical structures, namely:

isa: Agent, Class, Cancelled

where the first argument labels the agent on a hierarchical structure, linked to the class given by the second parameter. The last argument presents the set of information that must be cancelled in the inheritance process.

One may say that Tweety is a bird, where the knowledge about whether it may or may not fly, is not inherited, but represented in terms of the production: isa(tweety, bird, [fly])

About the agents knowledge, a predicate is defined, being the first argument the identification of the agent, and the second its theory:

agent: Agent, Theory

The fact that birds can fly, that have wings, but do not have wheels, can be declared as:

agent(bird, [fly, wings, - wheels])

Note that the classical negation, ' $\neg$ ', is being used in a way that permits one to denote what kind of knowledge must be interpreted as known to be false, distinguishing from other situations where one does not have information about the truth of knowledge.

In this system the agent's data is represented at the level that it appears on the hierarchy. For those agents which are subclasses of other entities, its knowledge may be inherited from the hierarchy, where the proof mechanism to provide the system with this kind of flavour may be stated as follows:

- a question may be proved at the agent's level; or
- the solution to a question may be found at a level on the hierarchy that is meta to the agent to which the question was initially for.

This type of reasoning will be performed by a proof predicate, defined as:

\section{proof: Agent, Question}

where Agent identifies the agent questioned and Question represents the question itself.

The first bullet above can be declared, as:

$$
\begin{aligned}
& \text { proof(Agent, Question) } \leftarrow \\
& \text { agent(Agent, Theory), } \\
& \text { process(Question, Theory) }
\end{aligned}
$$

where " $\leftarrow$ " names "if" and the proof for a question stated for a specific agent is found if it is possible to process that question in the agent's theory.

The second bullet, states that the solution to one's question must be searched up to the hierarchy, through those branches with permission to inherit knowledge:

$$
\begin{aligned}
& \text { proof(Agent, Question) } \leftarrow \\
& \text { isa(Agent, Class, Cancel), } \\
& \text { not exists(Question, Cancel), } \\
& \text { proof(Class, Question) }
\end{aligned}
$$

\section{Learning Hierarchical Models of Inheritance}

Learning hierarchical models of inheritance, based on the proposed knowledge representation scheme, are needed, because it is not possible to determine a-priori the models, such that a satisfactory performance of the systems is guaranteed. This presupposes that the hierarchical models of the systems have to be refined (learned), using the feedback from their decision making processes. The definition of the learning task, and proposition of a system for its solving, are the subjects of this section.

\subsection{The Definition of the Task}

A system based on the knowledge representation scheme from section 2, can be considered as a MAS. The system consists of a set of agents and classes, whose relationships are determined by a given hierarchical model of inheritance. The task for learning of the models is a task of acquiring new models, that ensures the system effectiveness with respect to a given criterion of performance.

The Task of Learning the Hierarchical Models of Inheritance

Given:

- A multiagent system:

- A set of agents and agent's classes;

- A hierarchical model of inheritance.

- A criterion of performance of the system;

- A set of the tasks of the system.

Find

- The hierarchical models of inheritance of the system that maximise its performance criterion.

The task of learning the models of inheritance is a task of learning the relationships among the agents and their classes. It is motivated by the fact that the 
The correspondence between the ISA predicates and the attributes in DNF language

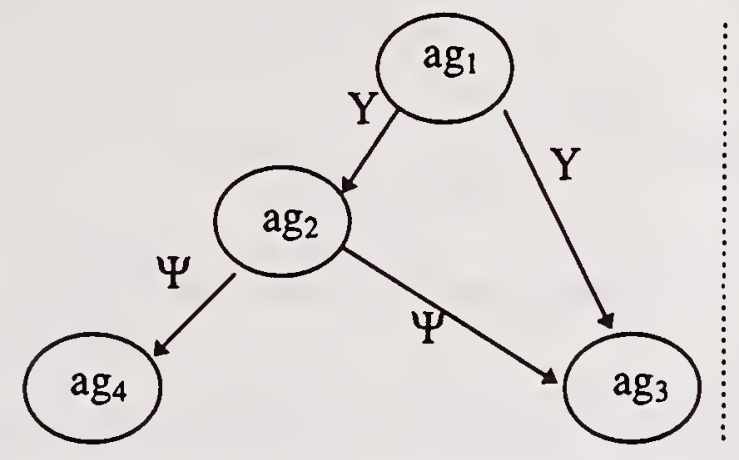

$$
\begin{aligned}
& \text { isa }\left(a g_{2}, a g_{1}[Y]\right) \leftrightarrow A_{l}=0 \\
& \text { isa }\left(a_{2}, a_{1},[]\right) \leftrightarrow A_{1}=1 \\
& \text { isa(ag, } \left.a_{3},[Y]\right) \leftrightarrow A_{2}=0 \\
& \text { isa }\left(a_{g_{3}}, a_{1},[]\right) \leftrightarrow A_{2}=1 \\
& \text { isa(ag } \left.{ }_{3} a_{2},[\Psi]\right) \leftrightarrow A_{3}=0 \\
& \text { isa }\left(a g_{3}, a g_{2},[]\right) \leftrightarrow A_{3}=1 \\
& \text { isa }\left(a g_{4}, a g_{2},[\Psi]\right) \leftrightarrow A_{4}=0 \\
& \text { isa }\left(a g_{4}, a g_{2},[]\right) \leftrightarrow A_{4}=1
\end{aligned}
$$

Figure 2. An Example of a Multiagent Logic Programming System, its Hierarchical Model of In. heritance, and Corresponding Description Languages

model of inheritance is formed by such relationships, and therefore is described by a conjunction of the corresponding ISA predicates. As different hierarchical models can have "positive or negative classification" with respect to the performance criterion of the system, their "ISA" descriptions can be considered as training examples of these models. Forming the examples is a gradual process of transition, of a system's model to another, by means of an association of the models with the classification determined by the criterion of performance. This means that the task of learning models of inheritance is an incremental one.

The result of the learning process has to be a set of the most important models with respect to the high quality performance of the system. The set is defined as a set of models, such that does not exist any other set with smaller number of models, which lead to the satisfactory performance of the system.

\subsection{The Choice of the Description Language of the Hierarchical Models of Inheritance}

The description of models of inheritance has to be made within languages that ensure solvability of the incremental learning task in real time. Therefore the DNF attributive languages are chosen, because the corresponding learning algorithms are very simple, and lead to suitable average complexity for both batch and incremental learning.

Encoding the descriptions of models of inheritance in DNF attributive languages is possible by the following scheme: every ISA link corresponds to an attribute, and every cancelled list corresponds to an integer (Figure 2). The integer number represents a vector with $\mathrm{N}$ binary digits, where $\mathrm{N}$ is the number of the possible predicates, whose extensions can be inherited. Therefore a position in the vector corresponds to a predicate, and the value $1(0)$ of the digit means, that the extension of the predicate can(not) be inherited. The integer belongs to the closed interval $\left[0,2^{\mathrm{N}}\right]$ which boundaries mean forbidden and full inheritance.

This scheme of representation aims at decreasing the description complexity of the learning task, and at the same time preserving the exactness of the model descriptions. In this way the task can be solved in reasonable time by the incremental decision tree learning.

\subsection{Incremental Decision Tree Learning}

The requirements: $i$ ) the task of learning the models of inheritance is incremental; $i i$ ) the learning has to identify the important models; and iii) the representative scheme of the models is attributive, determine ITI algorithm as the most suitable one [7]. ITI is an algorithm for incremental concept learning by induction on decision trees, that handles both numeric and symbolic values of attributes of the examples in DNF attributive languages. Algorithm understanding presupposes the consideration of the notion of the decision tree and the method of learning.

\subsubsection{Decision Trees}

A decision tree with respect to a set of pre-classified examples, that are presented in a conjunctive attributive language, is a tree whose nodes correspond to different attributes of the language, and the branches (arcs) correspond to different values of the attributes, so that every example from the set can be coded by simple traversing from the root of the tree to the corresponding example's leaf (Figure 3). The use of the decision tree in induction is due to the natural fact, that the process of learning consists of finding the nodes within the tree, corresponding to examples with the same classification. The descriptions of the paths from the root of the tree to these nodes are represented in the conjunctive attributive language. Each of these descriptions is discriminant; i.e. it contains only those attributes which values discriminate examples from the corresponding node with respect to other examples. Therefore the disjunction of the conjunctive descriptions with the same classification form the disjunctive discriminant description (in DNF attributive languages) of a concept, that is associated with the classification. This is a natural result in decision tree learning, that can be re-interpreted as acquiring the maximal descriptions of the concepts in the partially ordered DNF attributive languages.

The main problem in decision tree learning is the fact that the number of possible decision trees is equal 


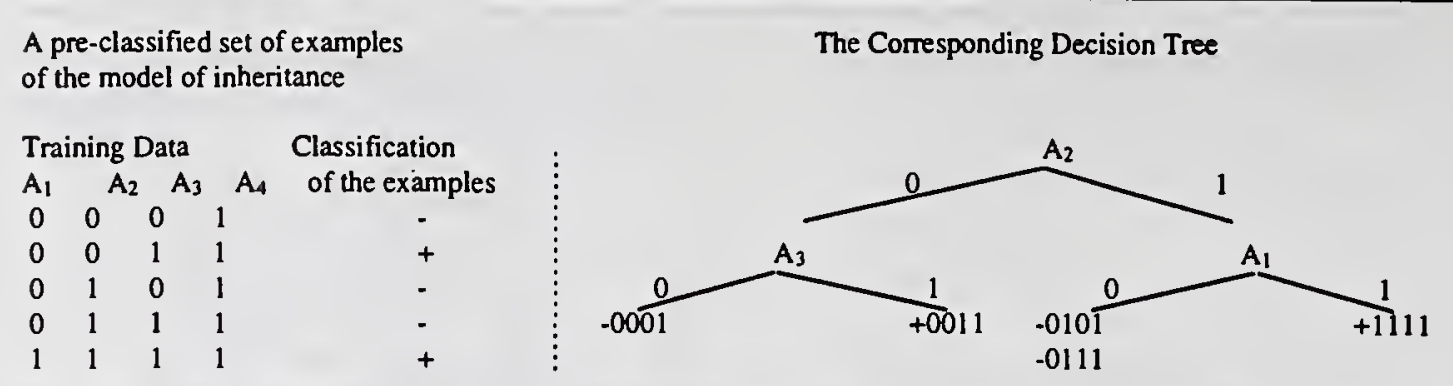

The resulted description of the "good" hierarchical models of inheritance derived by traversing the paths in the tree:

$\left(\left(A_{2}=0 \wedge A_{3}=1\right) \vee\left(A_{1}=1 \wedge A_{2}=1\right)\right) \leftrightarrow$

$\leftrightarrow\left(\left(i s a\left(a_{3}, a g_{1},[Y]\right) \wedge i s a\left(a g_{3}, a_{2},[]\right)\right) \vee\left(i s a\left(a g_{2}, a g_{1},[]\right) \wedge i s a\left(a_{3}, a g_{1},[]\right)\right)\right)$

Figure 3. A Decision Tree from the Training Examples of the Inheritance Model

to the possible combinations of the attributes in the languages. That is why the problem of finding the most minimal decision tree is very important, and at the same time unsolvable in the general case. In spite of this negative fact there exists a general decision tree algorithm [4], that builds decision trees using a topdown, divide-and-conquer approach: select an attribute; divide the example set into subsets, characterised by the possible values of the attribute; and follows the same procedure recursively with each subset until no subset contains examples with different classification. The algorithm is able to construct near-optimal decision trees when the choice of the attribute on every step is made by the use of the following information theoretic method: suppose that the training example set contains p positive and n negative examples; at the same time any attribute $A$ divides the training set $E$ into the subsets El...Er, according to its $r$ distinct values; each subset $\mathrm{Ei}$ has pi positive and ni negative examples; so the information gain from the test of the attribute $\mathrm{A}$ is:

$\operatorname{Gain}(A)=I\left(\frac{p}{p+n}, \frac{n}{p+n}\right)-\sum_{i=1}^{r} \frac{p i+n i}{p+n} I\left(\frac{p i}{p i+n i}, \frac{n i}{p i+n i}\right)$

where $I$ is the informative formula.

In this way on every step the algorithm calculates the information gain of the attributes, and chooses the one that has the best gain. This guarantees the building of near-optimal decision trees in batch manner, whose validity has been shown in [4].

\subsubsection{Incremental Algorithm}

ITI algorithm builds incrementally Decision Trees (DT) in contrast with the general decision tree algorithm (Figure 4). It uses the following scheme: the initial tree is the empty tree (NIL). When an example is to be incorporated into a tree, if the tree is NIL then the tree is replaced by a leaf that indicates the class of the leaf, and the example is attached to the leaf. Whenever an example is to be incorporated, the branches of the tree are followed according to the values in the example until a leaf is reached. If the example has the same classification as the leaf, the example is simply added to the set of examples, saved at the node. If the example has a different classification from the leaf, the algorithm attempts to turn the leaf into a decision node by the choice of the proper attribute. Immediately after an example incorporation, the tree is balanced according to the gain's expression.

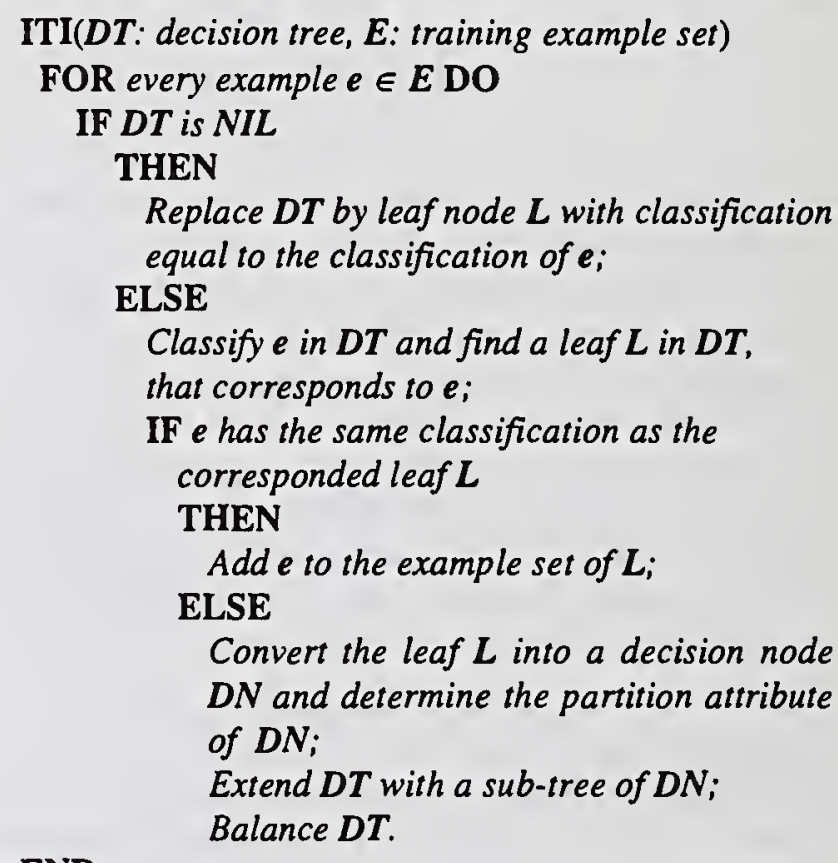

END.

\section{Figure 4. ITI's Algorithm}

\subsubsection{The Most Informative Learning}

The incremental learning can be speed up, if it is carried out by the most informative training examples, which are defined as examples that correspond to a half of the descriptions in the hypothesised space of the concepts to be learned. In this way, regardless of their classification, the learning is dramatically speed up, because a half of the currently plausible concept descriptions is rejected.

Applying this idea to decision tree learning presupposes the determination of the DNF hypothetical spaces of the concepts, presented in decision trees (Figure 5). These hypothetical spaces are determined by two boundaries in the partial ordered structure of DNF attributive languages [2]. The first, upper boundaries, are disjunctive discriminant descriptions 
The structure of DNF hypothetical space

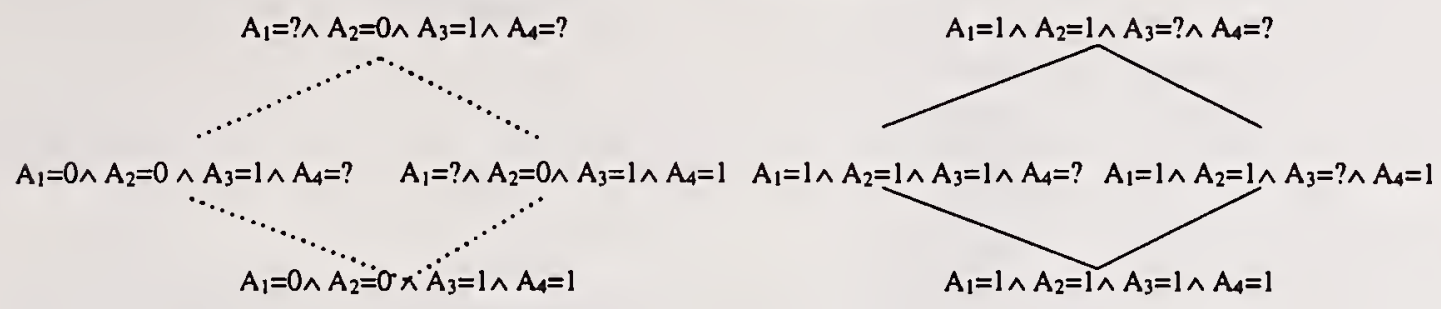

$\begin{array}{ll}\text { The upper boundary: } & \text { The lower boundary: } \\ \left(A_{2}=0 \wedge A_{3}=I\right) \vee\left(A_{I}=I \wedge A_{2}=I\right) & \left(A_{1}=0 \wedge A_{2}=0 \wedge A_{3}=I \wedge A_{4}=I\right) \vee\left(A_{1}=I \wedge A_{2}=I \wedge A_{3}=I \wedge A_{4}=I\right)\end{array}$

Both conjunctive hypothetical spaces in DNF space are shown with ..... and _ lines.

The upper and lower boundaries of the first conjunctive hypothelical space are,

respectively: $\left(A_{2}=0 \wedge A_{3}=1\right)$ and $\left(A_{1}=0 \wedge A_{2}=0 \wedge A_{3}=1 \wedge A_{4}=1\right)$

The upper and lower boundaries of the second conjunclive hypolhetical space are,

respectively: $\left(A_{1}=I \wedge A_{2}=I\right)$ and $\left(A_{1}=I \wedge A_{2}=I \wedge A_{3}=I \wedge A_{4}=I\right)$

\section{Figure 5. The Hypothetical Spaces of the "Good" Models of Inheritance}

of the concepts, that according to the properties of decision trees are the maximal descriptions of the concepts in the DNF attributive languages. The second, lower boundaries, are disjunctions of examples of the concepts, which ensure their minimality in these languages [5].

The most informative examples with respect to the first conjunctive hypothetical space:

$\left(A_{1}=I \wedge A_{2}=0 \wedge A_{3}=1 \wedge A_{4}=1\right)$ and

$\left(A_{1}=0 \wedge A_{2}=0 \wedge A_{3}=1 \wedge A_{4}=0\right)$;

These examples are near misses examples with respect to $\left(A_{1}=0 \wedge A_{2}=0 \wedge A_{3}=\ln A_{4}=1\right)$ and correspond to $\left(A_{2}=0 \wedge A_{3}=1\right)$.

Figure 6. Determining the Most Informative Examples with respect to the First Conjunctive Hypothetical Space (presented in Figure 5).

The DNF hypothetical spaces can be considered as unions of conjunctive hypothetical sub-spaces (Figure 5 ). These sub-spaces are determined by the conjunctive discriminant descriptions of the concepts (upper boundaries) and corresponding concepts examples (lower boundaries), which are mutually disjointed with respect to examples, once their upper boundaries are discriminant. Therefore it is not possible to determine the most informative examples for DNF hypothetical spaces. However that is possible for the conjunctive hypothetical sub-spaces, once they are convex and definite (for the chosen coding scheme), and there exists a simple near-optimal heuristic procedure for determining the most informative examples with respect to them. It determines the most informative examples, as near-misses examples; i.e. the examples whose descriptions are different with respect to the descriptions from the lower boundaries only with the value of one attribute (Figure 6) [2]. The simplicity of the procedure makes possible the development of ora- cle for support of most informative incremental learning of decision trees (Figure 7). The oracle gets, as an input a decision tree and acquires the most informative training examples with respect to the tree and the concepts to be learned. In order to do that, the oracle determines conjunctive hypothetical spaces of the concepts by acquiring their boundaries from the decision tree. The lower boundaries serve to determine the most informative examples by the use of the heuristic procedure, and the upper boundaries for sifting out those of them, that do not correspond to the spaces.

Oracle $\left(D T\right.$ : decision tree; $\left\{E_{C}\right\}$ : set from sets of the most informative examples of the concepts)

Determine the upper $(U)$ and lower $(L)$ boundary sets of the conjunctive hypothetical spaces of the concepts $C$ encoded in DT;

FOR every concept $C$ DO

FOR every boundary set of concept $C$ DO

Determine the set of near-misses examples $E^{\prime} C$ with respect to $L$;

Remove from $E^{\prime}$ c those examples that do not correspond to $U$;

END

$E_{C}=E_{c} \cup E_{C}^{\prime}$

Figure 7. The Oracle for Determining The Most Informative Examples

\subsection{A System for Learning of the Hierarchical Model of Inheritance}

The system for learning of the hierarchical models of inheritance consists of a set of embedded (six) subsystems, that form a closed cycle (Figure 8). The first one is a MAS, which models of inheritance have to be determined. This system tries to solve a particular task from a given task set, as its initial model of inheritance is preliminarily determined. The results of the 
problem solving process are analysed by the second sub-system, that identifies their quality by a given criterion of performance of the MAS. The logic description of the current hierarchical model is translated to DNF attributive language by the third subsystem, that uses the coding scheme, proposed in the sub-section 3.2. The resulted attributive description is considered, as a training example with positive or negative classification, depending on the quality of the current solution. The fourth sub-system ITI algorithm updates the decision tree of the concept corresponding to the models of inheritance with the new example. The new decision tree is used by the fifth sub-system of oracle, that determines an ordered set of the most new informative examples. This set of examples is translated by the sixth sub-system from DNF attributive language to the initial logic language. The first example of the set represents the description of the new most suitable model of inheritance with respect to the learning process. That is why the MAS changes its model of inheritance according to the new description and continues to solve its tasks. This closed process of learning of the hierarchical models of inheritance goes on till the moment when the oracle is not able to generate new examples, or the designers of the MAS determine some of the models, as satisfactory. In both cases the final result is a symbolic description of the "good" inheritance models of the MAS.

\section{Conclusion}

This article proposes a system for learning the hierarchical models of inheritance of MAS, that are represented under the logical knowledge representation scheme, presented in section 2. The main advantages of the system are the following:

- The system acquires the symbolic descriptions of the most important symbolic models of inheritance, that ensures satisfactory and understandable performance of the corresponding MAS.

- The system learns the models of inheritance in reasonable time, because the most informative examples of the models are only used, that leads to rejection of a half of the hypothetical spaces of models in the average case. This property contrasts the proposed system with the existing system for refinement of the agent relationships, that use arbitrary examples $[6,8]$.

- The system is completely autonomous; i.e. it does not require external help.

- The system can be applied to different MAS with inheritance models.

The main shortcomings of the system pointed the directions for future research:

- Impossibility to learn, when the criterion of performance of the MAS is not well-defined, that leads to the presence of noise in the training descriptions of the models;

- Impossibility to learn, when the agent's theories are changed. In this case some of the models of inheritance change their performance classification

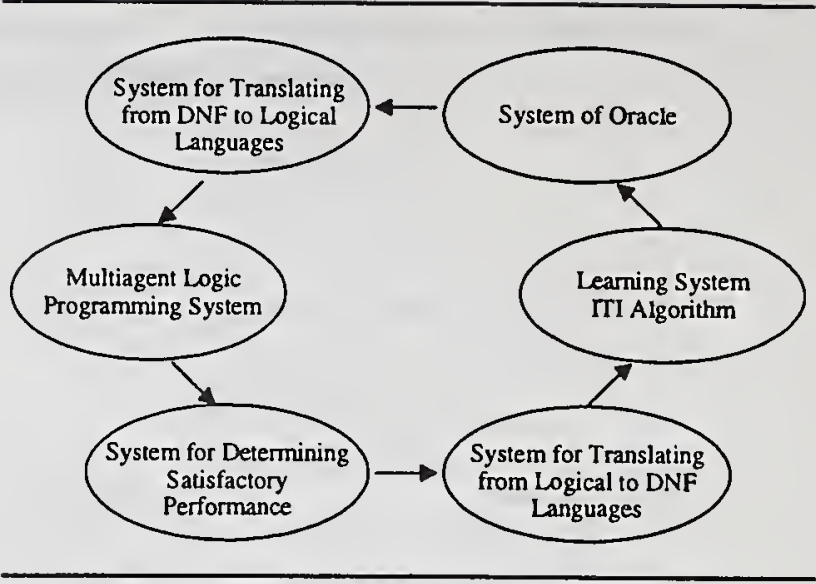

Figure 8. The System for Learning of the Models of Inheritance

and the learned "good" models became invalid.

Both shortcomings correspond to learning in the presence of noise and concept drift. They can be easily avoided for the learning sub-system, but not for the oracle one. That is why the main efforts for improvements have to be made in the oracle based decision tree learning, that is a completely new area of research in machine learning, ensuring the extension of the capabilities of the system.

\section{References}

[1] Analide, C., and Neves, J., Hierarchical Structures with Inheritance, Technical Report, Department of Informatics, University of Minho, April 1997.

[2] Mitchell, T., Generalisation as Search, in: Dietterich, T. and Shavlik, J., (eds.) "Readings in Machine Learning”, Morgan Kaufmann, 96-107, 1991.

[3] Kaiser, M., Dillman, R., and Rogalla, O., Communication as the Basis for Learning, in: Proceedings on Workshop on Multiagent Learning, ECAI, 1996.

[4] Quinlan, R., C4.5: Programs for Machine Learning, Morgan Kaufmann, 1993.

[5] Smirnov, E.N. and Neves, J., Example Order Independent Learning of Disjunctive Concepts, AIDRI97 Conference, Switzerland, 1997, (in print).

[6] Stone, P., and Veloso, M., Multiagent Systems: A Survey from a Machine Learning Perspective, (in print).

[7] Utgoff, P., An Improved Algorithm for Incremental Induction of Decision Trees, in Proc. on International Conference on Machine Learning, 1994.

[8] Weiss, G. and Sen, S., Adaptation and Learning in Multiagent Systems, Springer-Verlag, 1996. 
VI

MULTIRESOLUTIONAL METHODS 



\title{
Multi-Resolution Data Processing: It is Necessary, It is Possible, It is Fundamental
}

\author{
S. A. Starks ${ }^{1}$, V. Kreinovich ${ }^{1}$, and A. Meystel ${ }^{2}$ \\ ${ }^{1}$ NASA Pan-American Center for \\ Environmental and Earth Studies (PACES) \\ University of Texas at El Paso, El Paso, TX 79968 \\ emails \{sstarks,vladik\}@utep.edu \\ ${ }^{2}$ National Institute of Standards and Technology \\ Gaithersburg, MD 20899
}

\section{ABSTRACT}

Experience shows that many data processing problems are difficult to solve, and some of these problems have even been proven to be computationally intractable. Human experts successfully solve many such problems by using a hierarchical, multi-resolution approach. These multiresolution methods are, in several cases, provably optimal. However, due to the computational intractability of the problem itself, the multi-resolution approach can only work if the systems that we are analyzing are themselves hierarchical. We show that, first, due to (inevitable) measurement inaccuracies, an arbitrary input data is consistent with the hierarchical model, and second, that in many cases, the actual physical world is indeed fundamentally hierarchical.

Since traditional statistical methods have been designed primarily for non-hierarchical models, their direct application to multi-resolution data processing can lead to biased estimates. On a simple example, we show how these methods can be corrected to avoid this bias. Surprisingly, the analysis of this problem leads to new unexpected symmetries.

KEYWORDS: multi-resolution data processing, granularity, computational intractability, wavelets, fractals, satellite image, semiotics, environmental studies, Schroedinger's paradox

\section{DATA PROCESSING IS DIFFICULT}

Data processing is difficult: an empirical fact. From the engineering viewpoint, the main problem with data acquisition and processing is to manage to acquire the data. To launch a successful space mission to other planets is indeed an extraordinary engineering achievement. However, even for such missions, the pure volume of acquired data is so huge that processing all this data becomes a very difficult task.

Computers become faster and faster, new algorithms are designed, and therefore, data processing becomes faster and faster; however, at the same time, this same progress leads to better data acquisition devices that drastically increase the amount of raw data, and to new ideas of what additional information we can extract from the old data. A typical NASA-related example: the University of Texas at El Paso, together with Jet Propulsion Lab, is currently analyzing the data from Mariner missions to find relativistic effects on the spaceships' trajectories.

The complexity of data processing is a difficult problem for many different application areas, but is especially difficult for areas in which it is relatively easy to get new data. Environmental and earth studies are one of such areas: lots of relatively easily accessible data come from satellites, and processing this data becomes more and more difficult.

This problem is going to become even more acute in the nearest future: Indeed, currently, environmental-related satellites use a few frequencies (no more than 10). From the resulting measurement results, we only get a small sample of the spectrum, and from this small portion, it is often difficult to tell one type of terrain from another (or even from a cloud formation). To get a better understanding of Earth features, NASA is currently planning to launch a series of new multi-spectral satellites that, for each point, will measure up to several hundred intensities instead of the usual few. This increase in data flow is definitely advantageous, but it makes data processing even more more complicated.

Data processing is difficult: theoretical results. Every once in a while, new algorithms appear that drastically decrease the computation time of different data processing problems. This continuous progress in algorithm design may lead to an (over)optimistic viewpoint that sooner or 
later, ideal algorithms will emerge that will perform all the data processing tasks in real time (i.e., the current data will be processed by the time when new data will arrive).

Alas, this optimism is unfounded: numerous theoretical results show that in the general case, data processing problems are computationally intractable (or, to use the precise term from the theory of computing, NP-hard). This is true for general data processing (see [8] and references therein), for the problems of reconstructing the past $[1,2]$, for problems of quantum mechanics [10] and space-time geometry [7], etc.

Crudely speaking, these results mean that for any algorithm that solves a data processing problem, there exist possible data on which this algorithm takes exponentially long time, i.e., time that grows as $2^{n}$, where $n$ is the length of the input (measured, e.g., in bits). Already for reasonably small $n$ (e.g, for $n \approx 300$ ), the required time exceeds the lifetime of the Universe. In short, such problems are indeed intractable.

The problem. The problem is: how to process real-life data?

\section{MULTI-RESOLUTION DATA PRO- CESSING IS NECESSARY}

How do we humans solve this problem? Enter the idea of multi-resolution data processing. There are many data processing problems in which human experts are much better than computers, image processing one of them. To be more precise:

- If we have a small amount of data, e.g., a blurry noisy image, a human eye will only see the noise, while a computer can perfectly well filter this noise out and get, e.g., all these nice pictures that we see coming from the Hubble telescope.

- On the other hand, if we have a huge amount of data, e.g., if we need to identify a face on a photo or a geological pattern on satellite image, then a trained human eye does it in no time, while supercomputers often take forever or even sometimes fail.

How do we humans do it? Definitely not because our brain is faster than a computer: its main processing elements (neurons) have a processing time of 10-100 milliseconds. The main reason why we can do this job is because we do not store and we do not process the pixel-by-pixel image as a computer would normally do. Instead, we store and remember image in a very compressed form, usually, as a small collection of standard images described, usually, in semiotic form, i.e., by words and symbols (mental or real). Moreover, this description is usually hierarchical, multiscale: first, we remember and describe the "big picture" (main features), then we go into more details (i.e., into a somewhat smaller scale), etc.

Since we humans use this idea, and use it successfully, it is desirable to make computer programs use this idea as well.

Wavelets: a mathematical representation of the idea of multi-resolution data processing. One of the most practically successful formalizations of the idea of multi-resolution data processing is the wavelet technique (see, e.g., [13]). Crudely speaking, a wavelet transform decomposes the original image into "sub-images" that correspond to the large-scale details, medium-scale details, etc. So, we can store and process these sub-images instead of the entire original image.

Multi-resolution methods are indeed optimal. Many people have heard about wavelets, and it is reasonably well known that in many application areas (e.g., in data compression) wavelet-based methods are often indeed better than more traditional techniques. What is less known is that wavelet-based methods are not simply biologically motivated and empirically good: there are mathematical results that show that in many problems, waveletbased methods are indeed optimal. We will mention two such results:

- The first result is closer related to human way of data processing: it tells that among all possible neural networks, neural networks that use wavelet-type activation functions have (asymptotically) the best approximation property [9].

- The second result is directly related to data processing, namely, to image processing: it shows that in a certain class of problems (like the problem of automatically detecting whether a surface mounted device is correctly mounted on a chip) wavelets are indeed the best data compression method [6]. This second result is not just an asymptotic optimality result: it has actually led to successfully wavelet-based image processing results $[3,5]$.

Multi-resolution methods may be the best of the possible ones, but how come they are good? The very fact that multi-resolution methods are the best (i.e., the fastest) of all possible methods of solving data processing problems does not invalidate the above-cited pessimistic result that this problem is computationally intractable. In other words, theoretically, one can describe possible combinations of data for which these methods will not work.

However, both our own experience (as human experts we use multi-resolution methods) and the experience of data 
processing algorithms that use multi-resolution techniques show that these methods are practically very feasible, in other words, that these horror worst-cases practically do not happen in real life. The question is: why? Is nature designed in such a way that these methods always work, or simply we were lucky so far and bad cases will still appear in the future?

Our answer is optimistic: yes, nature is designed in this manner.

\section{MULTI-RESOLUTION DATA PRO- CESSING IS POSSIBLE AND IS FUNDA- MENTAL}

Two explanations. We will give two explanations why nature is designed in this way:

- Our first explanation will say, crudely speaking, that even when nature can form arbitrarily complicated images and data strings, the inevitable presence of noise and measurement errors makes every observation compatible with a hierarchical model (i.e., with a model that can be handled by multi-resolution techniques)

- Our second explanation is that not only the approximate image of nature is hierarchical, but the nature itself is granular and hierarchical.

First explanation: Tsirelson's theorem. [15] Tsirelson noticed that in many cases, when we reconstruct the signal from the noisy data, and we assume that the resulting signal belongs to a certain class, the reconstructed signal is often an extreme point from this class. For example, when we assume that the reconstructed signal is monotonic, the reconstructed function is often (piece-wise) constant; if we additional assume that the signal is smooth (one time differentiable, from the class $C^{1}$ ), the result is usually one time differentiable but rarely twice differentiable, etc.

Tsirelson provides an elegant geometric explanation to this fact: namely, when we reconstruct a signal from a mixture of a signal and a Gaussian noise, then the maximum likelihoodestimation (a traditional statistical techniques) means that we look for a signal that belongs to the priori class, and that is the closest (in the $L^{2}$-metric) to the observed "signal+noise". In particular, if the signal is determined by finitely many (say, $d$ ) parameters, we must look for a signal $\vec{s}=\left(s_{1}, \ldots, s_{d}\right)$ from the a priori set $A \subseteq R^{d}$ that is the closest (in the usual Euclidean sense) to the observed values $\vec{o}=\left(o_{1}, \ldots, o_{d}\right)=\left(s_{1}+n_{1}, \ldots, s_{d}+n_{d}\right)$, where $n_{i}$ denotes the (unknown) values of the noise.

Since the noise is Gaussian, we can usually apply the central limit theorem and conclude that the average value of $\left(n_{i}\right)^{2}$ is close to $\sigma^{2}$, where $\sigma$ is the standard deviation of the noise. In other words, we can conclude that $\left(n_{1}\right)^{2}+\ldots+\left(n_{d}\right)^{2} \approx d \sigma^{2}$. In geometric terms, this means that the distance $\sqrt{\sum\left(o_{i}-s_{i}\right)^{2}}=\sqrt{\sum n_{i}^{2}}$ between $\vec{s}$ and $\vec{o}$ is $\approx \sigma \sqrt{d}$. Let us denote this distance $\sigma \sqrt{d}$ by $\varepsilon$.

Let us (for simplicity) consider the case when $d=2$, and when $A$ is a convex polygon. Then, we can divide all points $p$ from the exterior of $A$ that are $\varepsilon$-close to $A$ into several zones depending on what part of $A$ is the closest to $p$ : one of the sides, or one of the edges. Geometrically, the set of all points for which the closest point $a \in A$ belongs to the side $e$ is bounded by the straight lines orthogonal (perpendicular) to $e$. The total length of this set is is therefore equal to the length of this particular side; hence, the total length of all the points that are the closest to all the sides is equal to the perimeter of the polygon. This total length thus does not depend on $\varepsilon$ at all. However, the set of all the points at the distance $\varepsilon$ from $A$ grows with the increase in $\varepsilon$; its length grows approximately as the growth of a circle, i.e., as const. $\varepsilon$. When $\varepsilon$ increases, the (constant) perimeter is a vanishing part of the total length. Hence, for large $\varepsilon$, the fraction of the points that are the closest to one of the sides tends to 0 , while the fraction of the points $p$ for which the closest is one of the edges goes to 1 .

Similar arguments can be repeated for any dimension. For the same noise level $\sigma$, when $d$ increases, the distance $\varepsilon=$ $\sigma \sqrt{d}$ also increases, and therefore, for large $d$, for "almost all" observed points $\vec{o}$, the reconstructed signal is one of the extreme points of the a priori set $A$.

Much less probable is that the reconstructed signal belongs to the 1-dimensional face of the set $A$, even much less probable that $s$ belongs to a 2-D face, etc.

The main methodological consequence of this result is that even when the actual state space is continuous, when we determine the state from measurements result, we inevitably obtain (most often) one of the discretely many states. On the large-scale level, we get one of the few clusters. When we add new measurements and thus, get to the next level, each original cluster sub-divides into new clusters, etc., so that we get a hierarchical structure.

\section{Comment: Schroedinger's paradox and other} methodological applications of Tsirelson's result. In quantum mechanics, this result explains why pure states (extremal points) are much more frequent that mixed ones; in history, it explains why there are finitely many types of social organization; in logic, it explains why in spite of the clearly fuzzy character of most human reasoning, binary logic describes most of this reasoning pretty well, etc. In particular, it explains the famous "cat" paradox proposed by $E$. Schroedinger, one of the founding fathers of quantum mechanics,

In classical physics, it is assumed that for each state of a physical system, every property is either true or false. For example, a particle is either located in a certain interval 
of space coordinates $[x-\Delta, x+\Delta]$, or it is not located inside this interval. In quantum mechanics, in addition to the states in which a particle is located within this interval, and to the states in which the particle is definitely outside it, there are states in which some measurements of the coordinate will lead to results within the interval, and some to the results outside this interval. In such states, we cannot say that a statement "the particle is located in the given interval" is true or that this statement is false; at best, we can determine the probability of the "yes" answer. (To describe such unusual "truth value", quantum logic has been introduced.)

States with unusual "truth values" are not an exception, but rather a general rule in quantum mechanics: e.g., for every two states $\psi$ and $\psi^{\prime}$ with certain values $\lambda \neq \lambda^{\prime}$ of a measured quantity, there exists a state called their $s u$ perposition in which the value of this quantity is no longer certain. (In the standard formalism of quantum mechanics, where states are described by vectors in a Hilbert space, superposition is simply linear combination.)

Such superposition state is easy to generate. Schroedinger has shown that this superposition principle seemingly contradicts our intuition: indeed, suppose that we have a cat in a box, and a light-controlled rifle is aimed at the cat in such a way that a left-polarized photon would trigger the rifle and kill the cat, while the right-polarized photon would keep the cat alive. If we send a photon with a circular polarization (that is, according to quantum mechanics, a superposition of left- and right-polarized states), we would get (due to the linear character of the equations of quantum mechanics), the superposition of the states resulting from using left- and right-polarized photons. In other words, we will get a superposition of a dead and alive cat states. This is, however, something that no one has ever observed: for macroscopic objects (cats included), an object is either dead or alive. Tsirelson's result explains why such non-extremal states are indeed difficult to observe.

Second explanation: Fractal (hierarchical) structure of the Universe [11]. At first glance, the Universe as a whole seems to be uniform: in whatever direction we look, there are, on a large scale, approximately the same amount of galaxies. However, as early as the 19th century, Olbers showed (in his famous paradox) that this impression is false: If indeed the matter was homogeneously distributed, then the total brightness of all the stars located at distances between $R$ and $R+\Delta R$ would be proportional to the volume $R^{2} \cdot \Delta R$ of the corresponding spherical segment. Since the brightness dims with distance as $R^{-2}$, the resulting Earth-observed brightness would be the same irrespective of $R$, and the total brightness caused by all the stars would be infinite or at least very large. As a result, argued Olbers, it would be as bright at night as it is at daytime. The only way to avoid this paradox and to re- tain the observable homogeneity with the observable night darkness is to take into consideration that the Universe is hierarchical: stars form galaxies, galaxies form galaxy clusters, etc. The larger scale we go it, the less space is taken by matter, and the more by vacuum. The resulting fractal description of matter distribution is indeed consistent.

Olbers paradox was the first but not the only occurrence of meaningless infinity in seemingly meaningful physical equations. Such infinities consistently emerge in field theory, both classical and quantum. An interesting mathematical fact that is that if we consider field theories in space-time of arbitrary dimension $d$, then infinities only occur for (small) integer $d$, in particular, for the physically meaningful $d=4$, but they do not occur for $d=4-\varepsilon$ for a small $\varepsilon>0$. Currently, this idea is used as a formal trick, to compute the physical quantities by using fractal dimensions, but it is reasonable to conclude, from this result, that the actual dimension of space-time is fractal, i.e., that space-time indeed has a fractal structure [4]. Since spacetime is also homogeneous, this conclusion means, crudely speaking, that not all points from the 4-D continuum describe events from the actual space-time, but that these events actually form a hierarchical structure.

\section{MULTI-RESOLUTION DATA PRO- CESSING: PROBLEMS AND CHAL- LENGES}

Traditional statistical methods are based on nonhierarchical data. Traditional statistical methods treat the entire data processing as a single process, going from input (initial data) to the output (classification or values of different quantities). To be more precise, there exist multi-step methods, but these are methods that simplify the computations at the expense of the artificially added hierarchical structure, and not by using the actual hierarchical structure.

Traditional statistical methods and multi-resolution data processing: a problem. Since traditional statistical methods are oriented towards one-step data processing, when we have a multi-resolution, multi-stage processing, we apply the traditional statistical methods to each stage separately, as if at each stage, we start with the raw data, and return the final results of data processing.

In reality, after, e.g., the first step of data processing, we do not have raw data any more, we have pre-processed data; due to this pre-processing, the error probability distribution for pre-processed data is different from a typical error probabilities for raw data, and therefore, strictly speaking, traditional methods are no longer applicable.

This problem is very urgent for processing environmental data, especially for processing earth-based environmental 
data (that usually supplements the data coming from satellite imaging). This data, usually, does not come directly from measurements: the raw measurement results are processed and generalized; then measurements corresponding to a certain small area are processed together, etc.; quite a few levels of data processing pass before we even get the data.

2-D example. How does this multi-stage processing affect the results? Let us give a simple illustrative example. We have already mentioned (when describing Tsirelson's result) that, from the geometric viewpoint, standard data processing techniques correspond to finding the point $\vec{s}$ from the a priori set $A$ that is the closest to the observation point $\vec{o}$ (the closest in the sense of either the standard Euclidean distance or of its multi-dimensional analogue). Let us start, for simplicity, with a $2-\mathrm{D}$ case $(d=2)$, and let us consider an a priori ellipse

$$
A=\left\{\left(x_{1}, x_{2}\right) \mid \frac{x_{1}^{2}}{A^{2}}+x_{2}^{2}=R^{2}\right\}
$$

To get a point $\vec{s} \in A$ is the ultimate goal of data processing. At each intermediate step, we achieve this goal only partly, i.e., we get a intermediate point from some larger set; at each stage, this set gets smaller and smaller until finally, we get a point from the desired set $A$. It is natural to assume that these intermediate sets are also ellipses that are similar to the desired set $A$, with the only difference that they correspond to larger values $R$; from one step of data processing to the next, the value $R$ gets smaller and smaller until we reach the desired value.

In other words, we start with a point $\vec{o}$ that comes from observations. On the first step of data processing, we find a point $\vec{s}^{(1)}$ from the ellipse $A^{(1)}$ (corresponding to the value $\left.R^{(1)}\right)$ that is the closest to $\overrightarrow{0}$. One the second step, we find the point $\vec{s}^{(2)}$ from the ellipse $A^{(2)}$ (corresponding to the value $\left.R^{(2)}<R^{(1)}\right)$ that is the closest to $\vec{s}^{(1)}$, etc. After $N$ processing steps, we get a point $\vec{s}^{(N)}$ from the desired ellipse $A$. This point is the closest to the previous point $\vec{s}(N-1)$ but, as one can easily see geometrically, this point is not necessarily the closest to the original observation $\vec{o}$ from all the points from $A$. In other words, the result of multi-stage processing algorithm is different from the desired point $\vec{s}$. How different is it? And how can we compensate for this difference?

To simulate the effect of a large number $N$ of stages on the result of data processing, let us consider the limit of infinitely many stages. In this limit, instead of finitely many different ellipses $A^{(1)}, \ldots, A^{(N)}$ that correspond to decreasing values $R^{(1)}>\ldots>R^{(N)}=R$, we get a continuous family of ellipses that correspond to decreasing value of the parameter $R$. Similarly, instead of finitely many intermediate results $\vec{s}^{(1)}, \ldots, \vec{s}^{(N)}$ of data processing, we get a continuous family of points. Geometrically, this continuous family of points forms a curve $x_{2}=f\left(x_{1}\right)$.

To describe this continuous process, let us describe how the "next" point on the curve (that describes different stages of data processing) is related to the "previous" one, i.e., to be more precise, let us describe a differential equation for this curve. Each point $\left(x_{1}, x_{2}\right)$ on this curve belongs to a certain ellipse (with the value $R=\sqrt{x_{1}^{2} / A^{2}+x_{2}^{2}}$ ). To get the "next" point on this curve, we consider a slightly smaller ellipse, with the parameter $R-\Delta R$, and take the point on that smaller ellipse that is the closest to the given one. The straight line that connects the original point with the "next" one is, in geometric terms, a tangent to the curve. It is well known from geometry that the straight line segment from any point to its closest point on any surface or curve (in particularly, on an ellipse) is orthogonal to this surface or curve (i.e., to the tangent to this surface or curve). Thus, at any point, the tangent to the desired curve is orthogonal to the tangent to the ellipse that this curve currently passes through.

The tangent to the ellipse $x_{1}^{2} / A^{2}+x_{2}^{2}=$ const can be obtained if we differentiate both sides of the ellipse's equation: $\left(2 x_{1} / A^{2}\right) d x_{1}+2 x_{2} d x_{2}=0$. Dividing both sides by 2 , we get $\left(x_{1} / A^{2}\right) d x_{1}+x_{2} \cdot d x_{2}=0$. The orthogonal line to this tangent is, therefore, described by the equation $d x_{1} /\left(x_{1} / A^{2}\right)=A^{2} d x_{1} / x_{1}=d x_{2} / x_{2}$. This differential equation can be easily integrated, leading to $c+a \cdot \ln \left(x_{1}\right)=\ln \left(x_{2}\right)$, where $a=A^{2}$ and $c$ is an arbitrary constant, i.e., to $x_{2}=C \cdot x_{1}^{a}$ (where we denoted $C=\exp (c))$.

We can use this equation to correct the effects of multistage data processing: namely, if we know the values $\left(x_{1}, x_{2}\right)$ that correspond to several different stages of data processing, then we can:

- use the least squares method to find the parameters $a$ and $c$ from the equation $\ln \left(x_{2}\right)=c+a \cdot \ln \left(x_{1}\right)$;

- use the resulting formula $x_{2}=\exp (c) \cdot x_{1}^{a}$ to reconstruct the original values $x_{1}$ and $x_{2}$; and then,

- use least square method again to find the point $\vec{s}$ on the ellipse $A$ that is the closest to the original observations $\left(x_{1}, x_{2}\right)$.

Multi-dimensional case. In a 2-D case, we get reasonably simple formulas. It turns out that in a more realistic multi-D case, the resulting formulas are only slightly more complicated. Indeed, if instead of ellipses, we consider ellipsoids

$$
A=\left\{\left(x_{1}, \ldots, x_{n}\right) \mid \frac{x_{1}^{2}}{A_{1}^{2}}+\ldots+\frac{x_{n}^{2}}{A_{n}^{2}}=R^{2}\right\},
$$

then a similar orthogonality condition means that a tangent to the curve (that represents the consequent intermediate results of multi-stage data processing) is orthogonal 
to the surface of the ellipsoid. This condition leads to a system of equations

$$
\frac{d x_{1}}{x_{1} / A_{1}^{2}}=\frac{d x_{2}}{x_{2} / A_{2}^{2}}=\ldots=\frac{d x_{n}}{x_{n} / A_{n}^{2}},
$$

from which we conclude that $\ln \left(x_{i}\right)=a_{i} \cdot \ln \left(x_{1}\right)+c_{i}$, i.e., that $x_{i}=C_{i} \cdot x_{1}^{a_{i}}$.

Surprising emergence of symmetries. An interesting side effect of our analysis is that the resulting curve has an unexpected symmetry: namely, if we change a unit in which we measure $x_{1}$ to a unit that is $\lambda>0$ times smaller (i.e., if we replace $x_{1}$ by $\tilde{x}_{i}=\lambda \cdot x_{i}$ ), then we get exactly the same formulas for the relationship between $x_{i}$ if we appropriately change the units for all other variables $x_{i}$. Moreover, the relationship $x_{i}=C_{i} \cdot x_{1}^{a_{i}}$ is the only possible relationship with this property.

This particular symmetry is very important (for numerous examples of using this and more complicated symmetries in computer science and data processing, see, e.g., [14]). The very fact that this important symmetry comes as a consequence of the hierarchical structure of data processing makes us believe that, maybe, symmetry in general, with all its important applications in physics and in other areas, can be explained based on the granular hierarchical structure of the Universe.

Acknowledgments. This work was supported in part by NSF under grant No. EEC-9322370 and by NASA under cooperative agreement NCCW-0089.

\section{REFERENCES}

[1] Alefeld, G., Koshelev, M., and Mayer, G. "Fixed Future and Uncertain Past: Theorems Explain Why It Is Often More Difficult To Reconstruct the Past 'Than to Predict the Future", in Proceedings of the NASA University Research Centers Conference, Albuquerque, New Mexico, February 16-19, 1997, pp. 23-27.

[2] Alefeld, G., Koshelev, M., and Mayer, G. "Why is it computationally harder to reconstruct the past than to predict the future?", International Journal of Theoretical Physics, Vol. 36, pp. 1709-1715, 1997.

[3] Carrillo, G., Cabrera, S.D., and Portillo, A.A. "Inspection of Surface-Mount-Device images using wavelet processing", in Proceedings of SPIE Applied Imagery Pattern Recognition, Washington D.C., October 1996.

[4] Finkelstein, A.M., and Kreinovich, V. "The singularities in quantum cosmology," Astrophysics and Space Science, pp. 73-76, 1987.
[5] Gallegos, J.M., Villalobos, J.R., Carrillo, G., and Cabrera, S.D., "Reduced-dimension and wavelet processing of SMD images for real-time inspection", In Proceedings of the IEEE Southwest Symposium on Images Analysis and Interpretation, San Antonio, April 1996.

[6] Kosheleva, O., and Brito, A.E. "Interval + Image = Wavelet", submitted to Reliable Computing.

[7] Kreinovich, V. "Spacetime isomorphism problem is intractable (NP-hard)," International Journal of Theoretical Physics, Vol. 30, pp. 1249-1257, 1991.

[8] Kreinovich, V., Lakeyev, A., Rohn, J., and Kahl, P. Computational complexity and feasibility of data processing and interval computations, Boston: Kluwer, 1997 (to appear).

[9] Kreinovich, V., Sirisaengtaksin, O., Cabrera, S.D. "Wavelet neural networks are optimal approximators for functions of one variable," in Proceedings of the IEEE International Conference on Neural Networks, Orlando, FL, July 1994, Vol. 1, pp. 299-303.

[10] Kreinovich, V., Vazquez, A., and Kosheleva, O. "Prediction problem in quantum mechanics is intractable (NPhard)," International Journal of Theoretical Physics, 1991, Vol. 30, pp. 113-122, 1991.

[11] Mandelbrot, B.B. The fractal geometry of Nature, San Francisco: Freeman, 1982.

[12] Meystel, A. "Evolution of knowledge in large complex systems", in Control Mechanisms for Complex Systems: Issues of Measurement and Semiotic Analysis, Proceedings of the Workshop, Las Cruces, NM, 1996, pp. 231-254.

[13] Meyer, Y. Wavelets algorithms and applications, Philadelphia: SIAM, 1993.

[14] Nguyen, H.T., and Kreinovich, V. Applications of continuous mathematics to computer science, Dordrecht: Kluwer, 1997 (to appear).

[15] Tsirel'son, B.S. "A geometrical approach to maximum likelihood estimation for infinite-dimensional Gaussian location. I", Theory Probab. Appl., Vol. 27, pp. 411-418, 1982. 


\title{
Multi-resolutional Planning with Minimum Complexity
}

\author{
J. Albus A. Lacaze $\star$ A. Meystel $\star 0$ \\ * National Institute of Standards and Technology \\ - Drexel University \\ - University of Maryland
}

\section{AbSTract}

This paper introduces a method for evaluating the planning complexity. Finding the optimal branching allows for evaluation of the optimum number of levels in a hierarchy of planning.

\section{Problem Definition}

A classical planning problem can be characterized by a number of tasks to be performed $(t)$, a number of resources that can perform those tasks $(r)$ and the value of a cost function to be minimized $(\Phi)$. This description is general enough that tasks and resources can be of different levels of coarseness, and thus make the solution more general.

The duty of the planner is to assign the tasks to the resources and schedule the times at which these tasks should be performed so that they minimize the cost function $[1,2,3]$.

Earlier, it was demonstrated that planning a minimum-cost trajectory in a graph organizing the process of planning in a hierarchical fashion and the optimum number of levels was obtained [4]. In this paper, we find the optimum number of levels levels for a multi-resolutional hierarchy of planning.

"Assignment" is a task-resource couple. Plan is defined as a time tagged concatenation of assignments. "Decision" is all the assignments in a plan with the same time tag. A plan is a list of decisions. "First decision" has earliest time tag "second decision" has the second.

Planners will create possible plans, investigate them and choose the least expensive. The complexity of the planner depends on the maximum amount of combinatorially possible plans that can be created. It the worst case, optimal planners will study all plans.

In this paper, the assumption of full allocation will be taken. No task will be left unprocessed if there is a free resource. This is an assumption that reduces the amount of plans calculated by the planner.

\section{One Level Planning}

Theorem II.1 If the number of tasks $t$ is smaller than the number of resources $r$ then, the worst case number of decisions that the planner investigates is $D(t, r)=\frac{r !}{(r-t) !}$

Proof. The number of combinations of resources in tasks is $C_{t}^{r}=\left(\begin{array}{l}r \\ t\end{array}\right)=\frac{r !}{t !(r-t) !}$. In order to find all the possible assignments, we have to permutate each of those combinations:

$$
\begin{aligned}
D(t, r) & =C_{t}^{r} \cdot P_{t} \\
& =\frac{r !}{t !(r-t) !} \cdot t ! \\
& =\frac{r !}{(r-t) !}
\end{aligned}
$$

Since $t<r$ the planner only needs to make one decision to complete the plan, it is not necessary to assign each resource with more than one task.

In most cases, $t>r$, thus plans will have more then one decision.

Theorem II.2 If the number of tasks $t$ is larger than the number of resources $r$ then, there are $D(t, r)=$ $\frac{t !}{(t-r) !}$ possible first decisions among all possible plans.

Proof. The number of combinations of tasks in resources is $C_{r}^{t}=\left(\begin{array}{l}t \\ r\end{array}\right)=\frac{t !}{r !(t-r) !}$. In order to find all the possible assignments, we have to permutate each of those combinations:

$$
\begin{aligned}
D(t, r) & =C_{r}^{t} \cdot P_{r} \\
& =\frac{t !}{r !(t-r) !} \cdot r ! \\
& =\frac{t !}{(t-r) !}
\end{aligned}
$$

Theorem II.3 If the number of tasks $t$ is equal to the number of resources $r$, then the worst case number of decisions that the planner investigates is

$$
D(t, r)=r !=t !
$$

Proof. In this case, we have a simple permutation. Note that both Equation 1 and 2 reduce to Equation 3 when $t=r$. 
Theorem II.4 The worst case number of plans that the planner must investigate in a one level system where $t>r$ is

$$
P(t, r)=\frac{r !}{(r-m) !} \cdot \prod_{i=1}^{n} \frac{[t-(i-1) r] !}{[t-i \cdot r] !}
$$

where $m=t \bmod r$ and $n=\operatorname{int}\left(\frac{t}{r}\right)$

Proof. Since plans are strings of decisions, the total number of plans the planner must investigate in the worst case can be found by multiplying the number of different first decisions times the number of different second decisions, and so forth.

$$
P(t, r)=\prod_{i=1} D_{i}
$$

where $D_{i}$ are the number of $i$ th decisions. For the number of first decisions, Equation 2 shows that $D_{1}=\frac{t !}{(t-r) !}$ assignments are created. For the second decisions, the number of resources is the same, but $r$ tasks have already been processed by the first decision. Thus, if $t-r>=r, D_{2}=\frac{(t-r) !}{(t-2 \cdot r) !}$; or if $t-r<r$ by using Equation $1, D_{2}=\frac{r !}{(r-t \bmod r) !}$. The same thing happens for the third decision $D_{3}=\frac{(t-2 \cdot r) !}{(t-3 \cdot r) !}$ if $t-2 \cdot r>=r$, or $D_{3}=\frac{r !}{(r-t \bmod r) !}$ if $t-2 \cdot r<r$. We need to multiply Equation $2 \frac{t}{r}$ times adjusting for the previously processed tasks, and then multiply the number of possible last decisions created by the leftover $(t \bmod r)$ tasks using Equation 1

$$
P(t, r)=\underbrace{\frac{r !}{(r-m) !}}_{\text {last decisions }} \cdot \underbrace{\prod_{i=1}^{n} \frac{[t-(i-1) r] !}{[t-i \cdot r] !}}_{\text {first } n \text { decisions }}
$$

where $m=t \bmod r$ and $n=\operatorname{int}\left(\frac{t}{r}\right)$. Please note that if $t<r$, then $n=0$, the part labeled as "first decisions" disappears, and $m=t$. Therefore, Equation 6 becomes Equation 1. If $t=r$ then $m=0$, the part labeled "last decisions" disappears, and $n=1$. Therefore, Equation 6 becomes Equation 3.

\section{Using the Scheduler to Reduce the Number of Investigated Plans}

In Section II, the planner worked in two distinct stages. First, the tasks are assigned to the resources until all tasks are finished. This creates all the possible string of assignments. Then, the scheduler takes these strings and assigns time tags to the taskresource couples. This creates plans that can be evaluated against the cost function. In [5] a planning method where the assignment of the tasks is woven with the scheduler is presented. In [5], this planner is called a tightly coupled JA-SC planner, where JA-SC means "Job Assigner-SCheduler." The scheduler is invoked after each decision. So, this planning procedure knows (or estimates) which resource will finish earlier in all the decisions. [5] assumes of "full allocation," where no unprocessed task (that is within the assigned ordering constraints) and free resources will be left unassigned.

Theorem III.1 The worst case number of plans that a tightly coupled $J A-S C$ planner must investigate in one level where $t>r$ is

$$
P(t, r)=t !
$$

assuming that no two resources are available exactly at the same time except in the first decisions.

Proof. For this case, Equation 5 still applies. For the set of first decisions, the tightly coupled JA-SC planner behaves the same. Equation 2 shows that $D_{1}=\frac{t !}{(t-r) !}$ assignments are created. For the set of second decisions, however, the scheduler that investigates each of the assignments in the first decisions, can estimate which resource finishes the assigned task first. At that time, only one resource is available and $t-r$ tasks to process; therefore, $D_{2}=t-r$. Similarly, $D_{3}=t-r-1$. In turn,

$$
\begin{aligned}
P(t, r) & =\frac{t !}{(t-r) !} \cdot(t-r) \cdot(t-r-1) \cdot \ldots \cdot 1 \\
& =\frac{t !}{(t-r) !} \cdot(t-r) ! \\
& =t !
\end{aligned}
$$

The ratio between the plans explored by the loosely and tightly coupled JA-SC planners can be expressed as follows:

$$
\begin{aligned}
\frac{P(t, r)_{L}}{P(t, r)_{T}} & =\frac{\frac{r !}{(r-m) !} \cdot \prod_{i=1}^{n} \frac{(t-(i-1) r) !}{(t-i \cdot r) !}}{t !} \\
& =\frac{r !}{(r-m) !(t-r) !} \cdot \prod_{i=2}^{n} \frac{[t-(i-1) r] !}{(t-i \cdot r] !}
\end{aligned}
$$

Figure 1 shows this ratio plotted for different number of tasks and resources. As expected, the ratio is one when $t<=r$ but, it quickly increases with increasing number of tasks and resources.

\section{EFFECTS OF ORdering CONSTRAINTS}

Very seldom is a planning problem set up where there are no constraints in the task order execution. In Sections II and III, constraints were not considered when calculating the amount of plans created and their complexity. There are two main changes caused by the insertion of constraints to the complexity of the planner:

1. The most obvious change is that the number of tasks and resources available to execute at any point of time is reduced. This reduces the total 


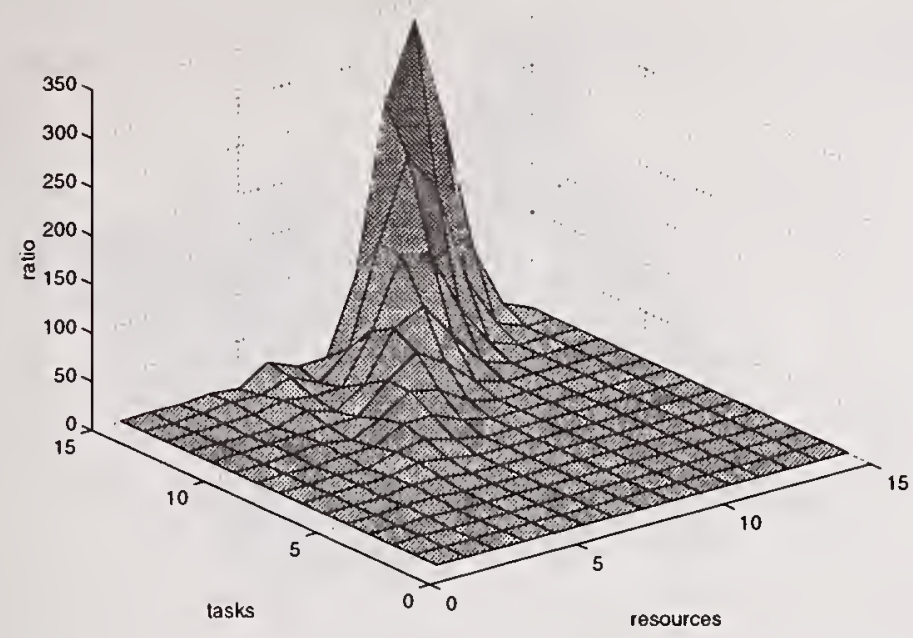

Figure 1: Ratio between the number of plans of the loosely coupled to the tightly coupled JA-SC planners

amount of plans. Unfortunately, because there various kinds of constraints it is not a simple job to determine the effect that many ordering constraints can make into the total number of plans.

2. The other change causes Equation 7 to overstate the number of decisions created. Suppose there are a set of tasks waiting to be processed, but cannot be executed because of an ordering constraint. Once the constraint is satisfied, the planner will have to deal with all the new tasks that are now available for processing and one or more than one available resources. If we have more than one resource available and more than one task ready to execute, then the conditions of Theorem III.1 are not satisfied. In those cases, the worst case number of plans lays between Equation 4 and Equation 7. Note that the tightly coupled JA-SC planner cannot be more complex than the loosely coupled, since the loosely coupled is creating all the possible alternatives.

Theorem IV.1 Every ordering constraint imposed in a planning problem can only decrease the number of plans that need to be investigated.

Proof. Equation 4 shows the total number of plans; all possible alternatives are counted. Among those possible alternatives, some follow the newly imposed constraints and some do not. But, there are no newly created plans, because there are no more possible plans. By imposing the constraint, we should only count the plans that follow the constraint, which is smaller (or equal) than the original number of plans.

Theorem IV.2 if $t>r$ then by introducing an ordering constraint in the form: $t_{i}$ must be processed after $t_{j}$ is finished, where $t_{i}$ and $t_{j}$ are two tasks, then the number of plans is reduced by at least $\frac{t}{t-r}$.
Proof. By expanding Equation 4 we find that

$$
P(t, r)=\frac{t !}{(t-r) !} \cdot \frac{(t-r) !}{(t-2 \cdot r) !} \cdot \frac{(t-2 \cdot r) !}{(t-3 \cdot r) !} \cdot \ldots
$$

but since we know that in the first decision, task $t_{i}$ cannot be processed since $t_{j}$ has not been processed, then the amount of decisions made in the first decisions is $\frac{(t-1) !}{(t-r-1) !}$. The second decisions becomes more complex since there are some alternatives in the set of first decisions that have processed $t_{j}$ and some have not. The total number of alternatives in the set of second decisions is smaller than $\frac{(t-r) !}{(t-2 \cdot r) !}$ since this equation contains the number of all alternatives of the second decisions. Then we find the ratio using only the first term,

$$
\begin{aligned}
& \frac{P(t, r)}{P(t, r)_{1} \text { con } s t}=\frac{\frac{t !}{(t-r) !}}{(t-1) !} \\
&=\frac{t}{(t-r-1) !} \\
&(t-r)
\end{aligned}
$$

\section{COMPLEXITY}

If we assume the following, the complexity of the planner can be represented as a polynomial:

- the complexity planner is directly proportional to the amount of times that the simulator is invoked. This simulator has a complexity of $\Theta_{S}$ necessary to find the next state for each explored decision

- the complexity of the planner is directly proportional to the complexity of the combinatorics that are necessary to create each assignment. In this case since the nodes in the hierarchy are composed of decisions and we assume a loosely coupled JA-SC case, the complexity of a decision $\Theta_{D}=r \Theta_{A}$ where $\Theta_{A}$ is the complexity of creating an assignment.

For example, if we have one resource and one task, the complexity of the planner is $\Theta_{P}=\Theta_{S}+\Theta_{D}$ For this paper, we assume that $\Theta_{S}+\Theta_{D}$ is a constant.

Theorem V.1 If the complexity of the planning algorithm $\Theta_{P}(t, r)$ is directly proportional to the complexity of the each investigated decision $\Theta_{D}$ and its simulation $\Theta_{S}$ then assuming loosely coupled JA-SC planner:

$$
\begin{aligned}
\Theta_{P}(t, r) & =\left(\Theta_{D}+\Theta_{S}\right) \cdot\left[\sum_{j=1}^{n}\left[\prod_{i=1}^{j}\left[\frac{[t-(i-1) r] !}{[t-i \cdot r] !}\right]\right]+\right. \\
& \left.\frac{r !}{(r-m) !} \cdot \prod_{i=1}^{n} \frac{[t-(i-1) r] !}{[t-i \cdot r] !}\right]
\end{aligned}
$$

where $m=t \bmod r$ and $n=\operatorname{int}\left(\frac{t}{r}\right)$ 
Proof. since the complexity of the planner depends on the amount of assignments explored,

$$
\Theta_{P}(t, r)=\left(\Theta_{D}+\Theta_{S}\right) \cdot \sum_{j=1}^{n} \text { Different } j^{t h} \text { decisions }
$$

We use Equation 4 to calculate the number of decisions at each decision column. Since in the first $n-1$ decision columns, there are more unprocessed tasks than resources available the part labeled, "last decisions," in Equation 6 cancels out. This "last decisions" part will only count for the $n^{\underline{t h}}$ decision column. That is,

$$
\begin{aligned}
\Theta_{P}(t, r)= & \left(\Theta_{D}+\Theta_{S}\right) \cdot\left[\frac{t !}{(t-r) !}+\right. \\
& \prod_{i=1}^{2} \frac{[t-(i-1) r] !}{[t-i \cdot r] !}+ \\
& \prod_{i=1}^{3} \frac{[t-(i-1) r] !}{[t-i \cdot r] !}+ \\
& \prod_{i=1}^{n} \frac{[t-(i-1) r] !}{[t-i \cdot r] !}+ \\
& \left.\frac{r !}{(r-m) !} \cdot \prod_{i=1}^{n} \frac{[t-(i-1) r] !}{[t-i \cdot r] !}\right]
\end{aligned}
$$

which reduces to Equation 11

\section{Hierarchical Planning}

The benefits of hierarchical control systems are obvious. Although there is no consistent theory of hierarchical system design or optimization, general recommendations are often based upon heuristics or intuition. Hierarchical control systems are based on the idea that tasks and resources can be clustered into lower resolution tasks and resources. NIST-RCS is an example of how these control structures can be organized.

In order to go from a one level planner to a system with multiple levels, lets start by assuming the following:

- The tasks cluster in $a$ clusters. These clusters are lower resolution tasks.

- The resources cluster in $b$ resource clusters. We will call these cluster lower resolution resources.

- The number of tasks in each lower resolution task is approximately equal .

- The number of resources in each lower resolution resource is approximately equal.
Theorem VI.1 In a two level control hierarchy, the worst case planning complexity created by both levels is

$$
\begin{aligned}
& \Theta_{2 \text { lev }}(r, t)=\Theta(a, b)+ \\
& b \cdot \Theta\left(\operatorname{ceil}\left(\frac{t}{a}\right), \operatorname{ceil}\left(\frac{r}{b}\right)\right)
\end{aligned}
$$

Proof. The total planning complexity should be equal to the complexity created by the low resolution level plus the complexity created in the high resolution level. The complexity created in the high resolution level are $\Theta(a, b)$ since we have $a$ low resolution tasks and $b$ low resolution resources. When the planner of this lower resolution level is finished and there is one low resolution plan that is chosen based upon the cost function, this plan goes to the higher resolution level. This level has $b$ control nodes, each one with its own planner. The number of low resolution tasks sent to the higher resolution level is $a$, so, the number of high resolution tasks that have to be planned in each low resolution resource is $\frac{t}{a}$, and they should be processed by $\frac{r}{b}$ high resolution resources. The ceil function is used to round up. This compensates for the branching factor which may not exactly divide the number of tasks or the number of resources.

Theorem VI.2 In a l level control hierarchy, the worst case planning complexity created in all levels is

$$
\begin{aligned}
& \Theta_{l \text { lev }}(r, t)=\sum_{i=0}^{l-2} b^{i} \cdot \Theta(a, b)+ \\
& b^{l-1} \cdot \Theta\left(\operatorname{ceil}\left(\frac{t}{a^{l-1}}\right), \text { ceil }\left(\frac{r}{b^{l-1}}\right)\right)
\end{aligned}
$$

Proof. The lowest level in the hierarchy will always create $\Theta(a, b)$ complexity regarding the amount of levels that we have. If $l>2$ then the second level of the hierarchy will have $b \cdot \Theta(a, b)$. If $l>3$ then the third level will have $b^{2} \cdot \Theta(a, b)$ complexity. In other words, $\sum_{i=0}^{l-2} b^{i} \cdot \Theta(a, b)$ will account for all the complexity created at each level of the $l$ levels control hierarchy except the highest level of resolution. This last level will have $b^{l-1} \cdot \Theta\left(\operatorname{ceil}\left(\frac{t}{a^{l-1}}\right)\right.$, ceil $\left.\left(\frac{r}{b^{l-1}}\right)\right)$ complexity, since there are ceil $\left(\frac{t}{a^{l-1}}\right)$ highest resolution tasks in each cluster, and ceil $\left(\frac{r}{b^{l-1}}\right)$ resources in each cluster. Thus Equation 14.

\section{ViI. Optimizing the Control Hierarchy}

Important questions include the following: Given $t$ and $r$, what is the best clustering criterion? What is the optimal number of levels that should be used to minimize complexity? The answers come from numerically inspecting the complexity equations. 


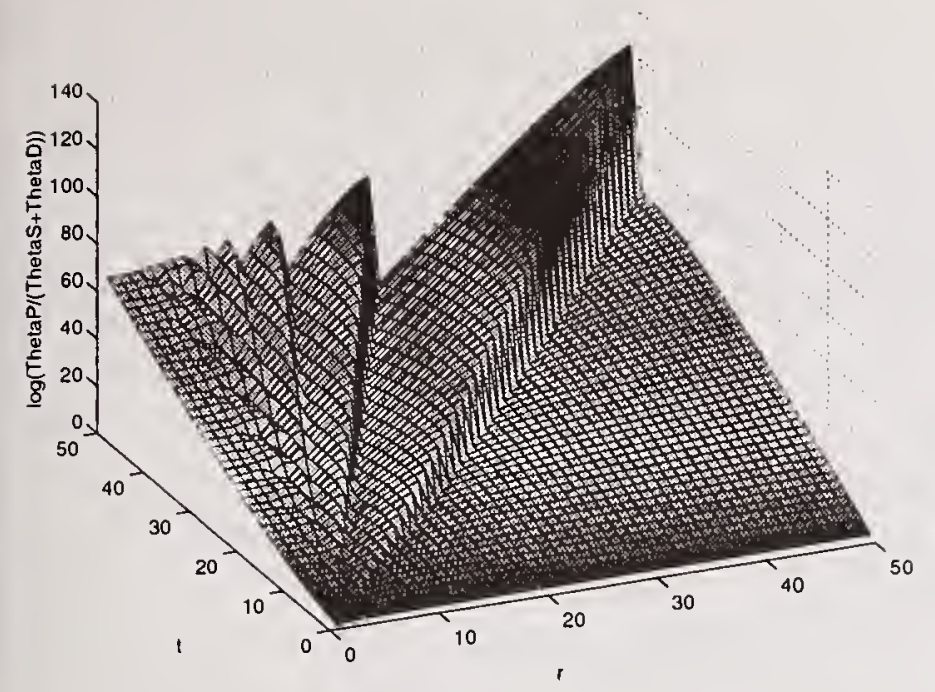

Figure 2: $\log \left(\frac{\Theta_{p}}{\Theta_{s}+\Theta_{D}}\right)$ for a one level control system as a function of the $r$ and $t$

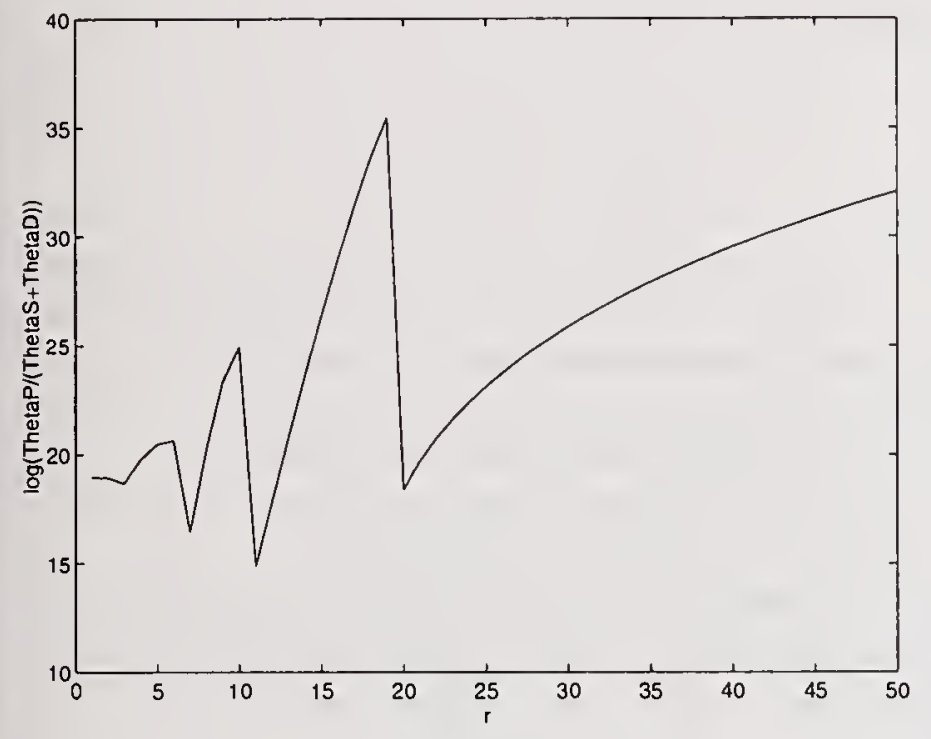

Figure 3: $\log \left(\frac{\Theta_{p}}{\Theta_{S}+\Theta_{D}}\right)$ for a one level control system for 20 tasks as a function of $r$

The following experiment was conducted. The complexity of a one level system was calculated (Figure 2) as a function of the number of tasks and resources. The graph was calculated by finding the logarithm of Equation 11 by assigning $l=2$, assuming $\Theta_{S}+\Theta_{D}$ constant and equal to one unit of complexity. The folds that this figure presents (See Figure 3) can be explained by the fact that the number of assignments can decrease when the number of resources increases. An obvious example of this is $\Theta_{P}(3,2)=18>\Theta_{P}(3,3)=6$ (using Equation 11), that can be seen in the big fold at $r=t$, the other folds occur when $r$ is a multiple of $t$.

Equation 14 was used to find the optimal $a$, and $b$ for each possible combination of $t$ and $r$ within the shown range. These optimal values where found by using brute force search over the $a$ and $b$ spaces. Figure 4 and Figure 5 show these optimum values af-

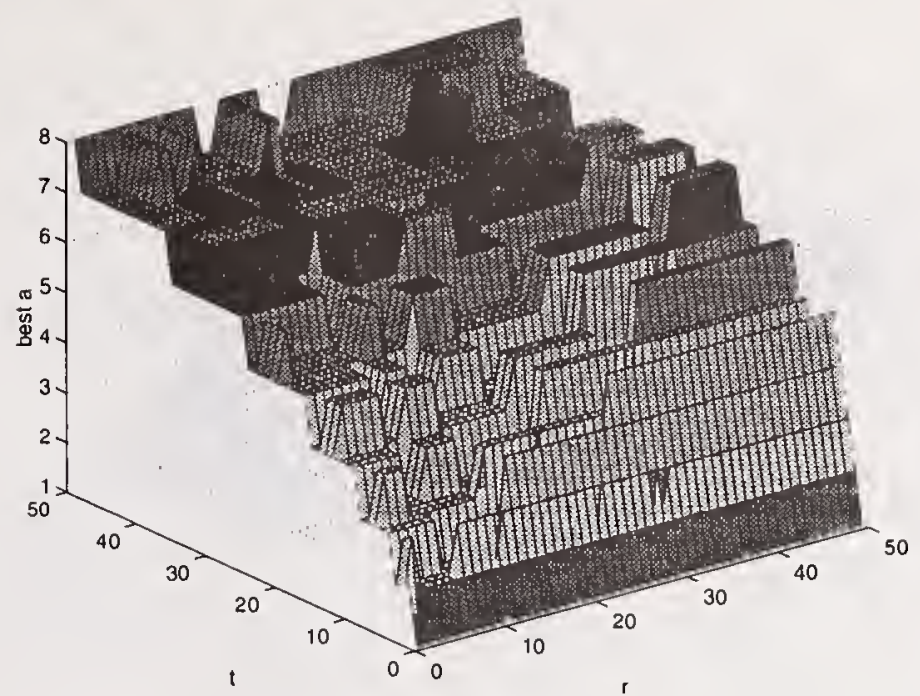

Figure 4: Results of searching for the best $a$ as a function of the $r, t$ and $b$

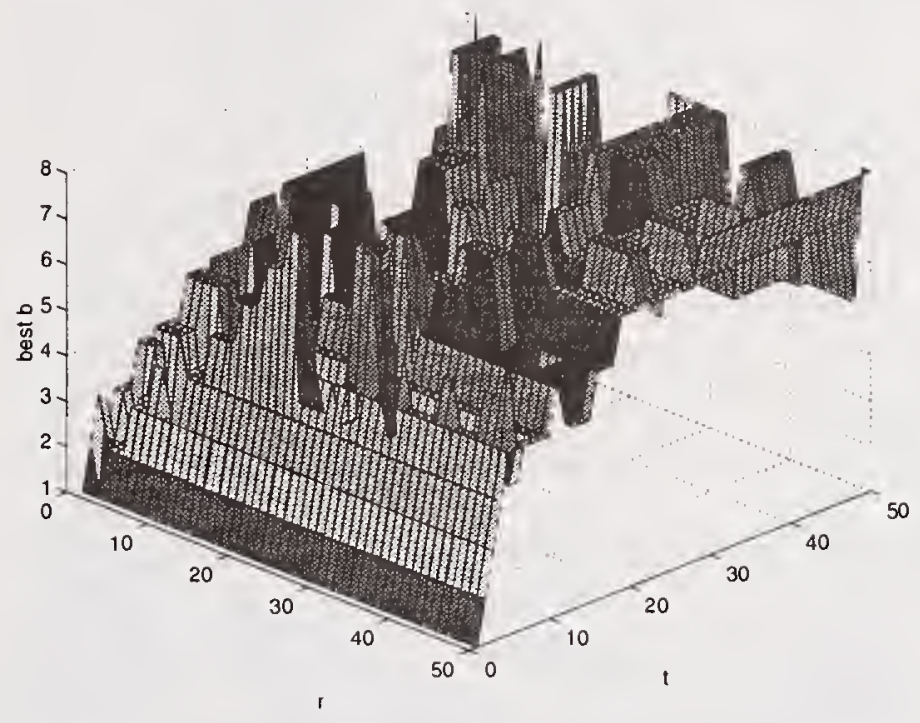

Figure 5: Results of searching for the best $b$ as a function of the $r, t$ and $a$

ter the search procedure is finished. The results are non-monotonically increasing as $r$ (in the case of $a$ in Figure 4 ) and $t$ (in the case of $b$ in Figure 5) are increasing.

Then, using these optimal $a$ and $b$, the complexity of the 2 level system was found for the same range of $t$ and $r$. The results are shown in Figure 6. Compare the difference in complexity with the one level system shown in Figure 2 This same procedure can be repeated for more levels.

Although it is interesting to find what is the optimal clustering criterion for a given amount of tasks and resources, it has more applications to find the optimal number of levels for a certain number of tasks and resources. This is done in the following way:

1. Given the number of tasks $t$ and the number of resources $r$, the optimal $a$ and $b$ are numerically found by searching using Equation 14 for 


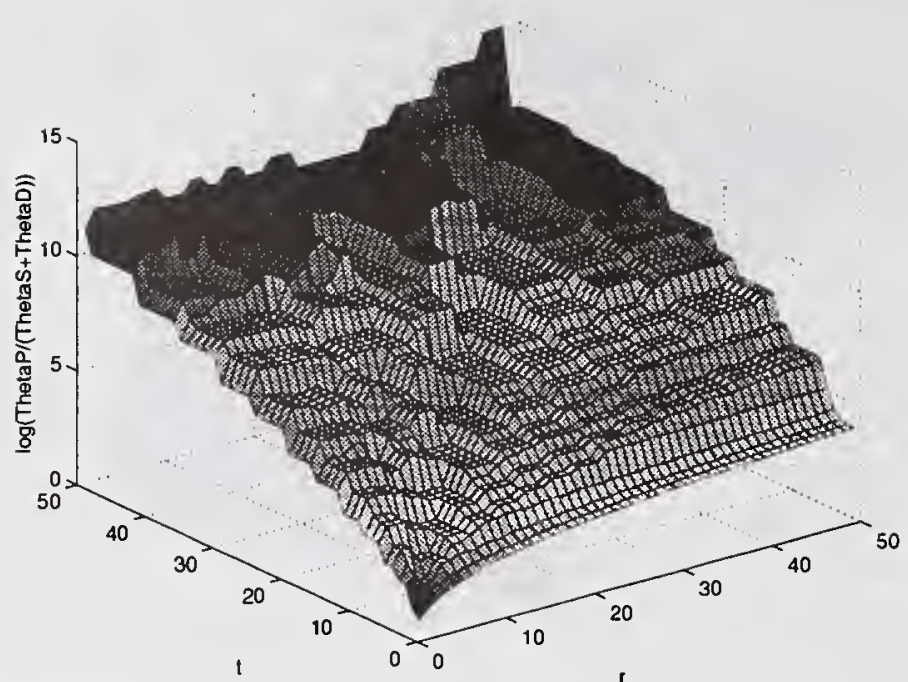

Figure 6: $\log \left(\frac{\Theta_{P}}{\Theta_{S}+\Theta_{D}}\right)$ for a two level control system as a function of the $r$ and $t$ and using optimal $a$ and b

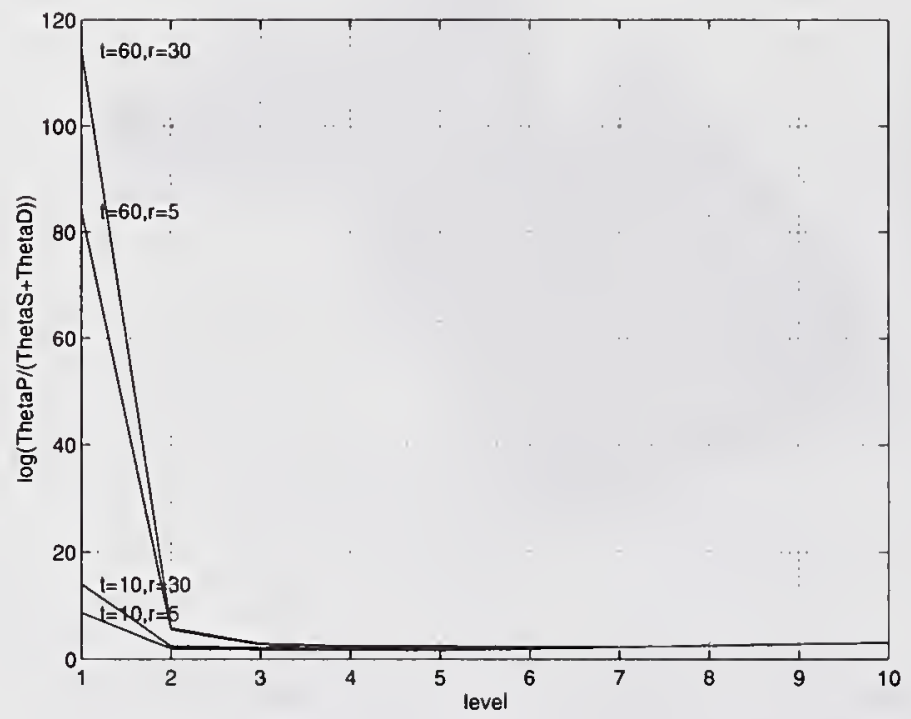

Figure 7: $\log \left(\frac{\Theta_{P}}{\Theta_{S}+\Theta_{D}}\right)$ as a function of the number of levels (1-10) for some fixed combinations of $r$ and $t$

\section{$2,3,4, \ldots, 10$ levels.}

2. The minimum complexity versus level function is numerically found.

Figure 7 show 4 examples: $\{t=50, r=30\}$, $\{t=50, r=5\},\{t=10, r=30\},\{t=10, r=5\}$. Figure 8 shows the same figure excluding the one level data. If both $t$ and $r$ increase, the general rule is that the number of levels necessary to minimize their complexity will also increase. Different levels create significantly different complexity and that having this curve determines the optimum amount of levels necessary to minimize complexity. Some overhead is created by adding levels of resolution, this overhead is partly accounted for in Equation 14, where extra terms may be necessary.

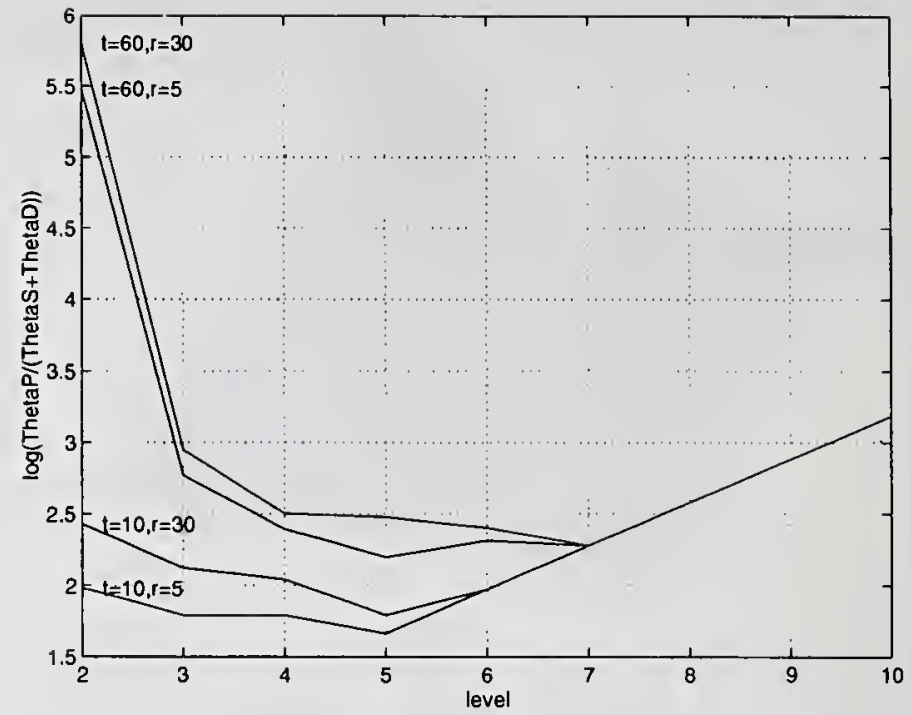

Figure 8: $\log \left(\frac{\Theta_{P}}{\Theta_{S}+\Theta_{D}}\right)$ as a function of the number of levels (2-10) for some fixed combinations of $r$ and

\section{CONCLUSIONS}

- The process of planning was analyzed and mathematical expressions were obtained for both the single level and multi-resolutional cases.

- The complexity of loosely and tightly JA-SC planners was analyzed and compared.

- Some of the effects of ordering constraints in the planners complexity was studied.

- The optimal number of levels which minimizes the complexity of a hierarchical control system is found.

\section{REFERENCES}

[1] J. Albus and A. Meystel. A reference model architecture for design and implementation of semiotic control in large and complex systems. In Semiotic Workshop of the 10th IEEE Intenational Symposium on Intelligent Control, pages 33-45, Monterrey, CA, 1995.

[2] J. Albus. An intelligent system architecture for manufacturing (ISAM). Technical report, NIST, 1996.

[3] J. Albus and A. Meystel. Behavior generation. Technical report, NIST, 1995.

[4] Y. Maximov and A. Meystel. Optimum design of multiresolutional hierarchical control systems. In Proceedings of IEEE Int'l Symposium on Intelligent Control, pages 514-520, Glasgow, U.K., 1992.

[5] J. Albus, A Lacaze, and A. Meystel. Planning in the hierarchy of NIST-RCS for manufacturing. In Intelligents Systems: A Semiotics Perspective, 1995. 


\title{
Multi-Resolution Data Processing is Optimal: Case Study of Detecting Surface Mounted Devices
}

\author{
A.E. Brito, O.M. Kosheleva, and S.D. Cabrera \\ Department of Electrical and Computer Engineering \\ University of Texas at El Paso \\ El Paso, TX 79968, USA \\ email \{alexb,olga,cabrera\}@ece.utep.edu
}

\section{ABSTRACT}

In computer and electronic manufacturing, it is very important to be able to automatically check whether the surface mounted devices (SMD) are correctly placed on the printed circuit boards. The inspection of these boards has to be done on a shop floor, where statistical characteristics of the noise vary so much that, in essence, we only have interval estimates for this noise.

We show that under this interval uncertainty, the optimal image processing technique consists of using Haar wavelets. Wavelets indeed lead to much better results than previously used Fourier transform techniques.

On a more fundamental level, our result is a step towards solving an important problem related to wavelets and multi-resolution data processing: these methods often empirically work much better than other methods, but there are very few theoretical explanations of this efficiency. Our results shows that, probably, such a theoretical explanation can be obtained if we take (interval) uncertainty into consideration.

\section{INTRODUCTION TO THE PROBLEM}

\subsection{Case Study: Inspection of Surface Mounted Devices}

Modern electronics manufacturing requires fast and efficient production. As a result, the assembly of printed circuit boards (PCB) with surface mounted devices (SMD) is usually done by robots. This manufacturing and assembly process is usually at the edge of the current manufacturing abilities, with a reasonable amount of PCB produced with defects. Therefore, it is extremely important to inspect and test the devices in order to weed out the defective ones.

Most SMD devices are so small that it is very difficult and very time-consuming for a human inspector to check whether the device is mounted at all, and whether it is mounted correctly. This problem is further complicated by the fact that many PCB have hundreds of SMD. Therefore, we need an automatic inspection. For that, we take a photo of the board, and we process the resulting image; see, e.g., $[4,5,12]$ and references therein.

\subsection{It is Necessary to Compress the Image}

The image that we need to process is a photo. The camera produces an array of electronic signals $\widetilde{f}(x)$ that describe the brightnesses $f(x)$ at different pixels $x$. The values $\tilde{f}(x)$ corresponding to different pixels $x$ are fed into the computer.

In the manufacturing environment, we need to process lots of images, and for each image, we need to process all these values fast, ideally, on a reasonably cheap PC-type computer. If we were to apply complicated processing techniques to all the pixel values, this would require lots of computer processing time: a good image consists of about 1 million pixels, and even the most crude images that we have been processing still consist of $53 \times 27 \approx 1,400$ pixels. It is therefore desirable to compress this data to a few numbers, and then base our decisions on the compressed data only.

\subsection{How Can We Compress the Image?}

How is data compressed in general? For example, how do physicists represent a dependency $y=f(x)$ between the two quantities?

Usually, this dependency is smooth (and even analytical), and therefore, the corresponding function $f(x)$ can be represented (at least for small $x$ ) as a sum of its Taylor series:

$$
f(x)=f(0)+f^{\prime}(0) \cdot x+\frac{1}{2} \cdot f^{\prime \prime}(0) \cdot x^{2}+\ldots
$$

Since measurements are usually imprecise, we do not need all these terms to represent the measurement results; it is sufficient to take only a few first terms in this expansion: 
- To describe the most crude measurements, it may be possible to keep only the first (constant) term and take $f(x) \approx c_{1}$ for some constant $c_{1}$.

- To describe better measurements, we may need linear terms as well. In other words, we take

$$
f(x) \approx c_{1}+c_{2} \cdot x
$$

and use the coefficients $c_{1}$ and $c_{2}$ to make decisions about the analyzed dependence.

- To get an even better approximation, we may want to retain quadratic terms as well, and take

$$
f(x) \approx c_{1}+c_{2} \cdot x+c_{3} \cdot x^{2}
$$

etc.

In this case, we start with a basis consisting of the functions $e_{1}(x)=1, e_{2}(x)=x, e_{3}(x)=x^{2}$, etc., a basis in which every function (at least every function that is smooth enough) can be represented as an infinite series

$$
f(x)=c_{1} \cdot e_{1}(x)+c_{2} \cdot e_{2}(x)+\ldots+c_{k} \cdot e_{k}(x)+\ldots,
$$

and then we take several first coefficients $c_{1}, \ldots, c_{N}$ as the desired compressed representation of the function $f(x)$. In most physical cases, the monomials $1, x, x^{2}$, etc., form a physically reasonable basis; in other cases, sines or other functions may be a better first approximation that linear or quadratic ones. But in general, the idea of using the first few coefficients of the expansion seems to be a reasonable data compression method. This approach is used in imaging as well. In this paper, therefore, we will consider data compression methods based on this idea.

\subsection{It is Best to Use Orthonormal Bases}

Some of the known bases (e.g., sines and cosines) are orthonormal in the sense that

$$
\begin{gathered}
\int e_{i}(x) \cdot e_{j}(x) d x=0 \text { when } i \neq j \\
\int e_{i}^{2}(x) d x=1 \text { for all } i
\end{gathered}
$$

In principle, a physical basis need not be orthonormal; e.g., the monomials do not have this property. However, it is always possible to transform each basis $\left\{e_{1}(x), e_{2}(x), \ldots\right\}$ into a new orthonormal basis $\left\{\bar{e}_{1}(x), \bar{e}_{2}(x), \ldots\right\}$ by using the known orthonormalization procedure:

$$
\begin{gathered}
\bar{e}_{1}(x)=g_{11} \cdot e_{1}(x) \\
\bar{e}_{2}(x)=g_{21} \cdot e_{1}(x)+g_{22} \cdot e_{2}(x)
\end{gathered}
$$

etc., for appropriate coefficients $g_{i j} ;$ e.g., $g_{11}=$ $1 / \sqrt{\int e_{1}^{2}(y) d y}$.

When we move to an orthonormal base, we do not lose anything, and we do not change the actual compression: indeed, for all $N$, the class of all functions of the type $c_{1} \cdot e_{1}(x)+\ldots+c_{N} \cdot e_{N}(x)$ is exactly the same as the class of all functions of the type $c_{1} \cdot \bar{e}_{1}(x)+\ldots+c_{N} \cdot \bar{e}_{N}(x)$. On the other hand, we do gain in computation time when we turn to orthonormal bases (and computational time is what we try to minimize in the first place): Indeed, for orthonormal bases, the computation of the coefficients becomes much computationally simpler than for the general bases:

$$
c_{i}=\int f(y) \cdot \bar{e}_{i}(y) d y .
$$

In view of this important advantage of orthonormal bases (and also in view of the absence of any disadvantages), it makes perfect sense to use such bases. In the following text, we will therefore assume that the basis $e_{i}(x)$ is orthonormal, i.e., that the conditions (2)-(3) are satisfied.

\subsection{Taking Measurement Errors into Considera- tion}

Brightness values are measured with a non-negligible inaccuracy. In general, in image processing, we use statistical methods of processing data that are based on the assumption that we know the probabilities of different imaging errors. It is often possible to collect these probabilities: we measure the frequencies of different errors and we see that as the number of experiments grow, these frequencies tend to a certain limit which is the desired probability. However, in our case, we are dealing with images taken on the shop floor. The situation on the shop floor changes so frequently and so unpredictably that there are no stable frequencies of errors. Therefore, we do not know the probabilities of different value of noise; the only information that we have about the noise is the upper bound $\Delta$ on its value: For every point $x$, the difference $\Delta f(x)=f(x)-\tilde{f}(x)$ between the actual (unknown) brightness $f(x)$ and its measured value $\tilde{f}(x)$ is bounded by $\Delta$ :

$$
|\Delta f(x)| \leq \Delta .
$$

In other words, after measuring brightness, we only know, for each point $x$, the interval $[\widetilde{f}(x)-\Delta, \widetilde{f}(x)+\Delta]$ of possible values of brightness $f(x)$ at this point $x$.

This measurement inaccuracy leads to inaccuracy in the coefficients $c_{i}$, i.e., to the difference $\Delta c_{i}=c_{i}-\tilde{c}_{i}$ between the ideal values (4) of these coefficients (the values that we would have gotten if we had the ideal image) and the actually computed values

$$
\tilde{c}_{i}=\int \tilde{f}(y) \cdot e_{i}(y) d y .
$$


If this inaccuracy is huge, then the resulting values of the coefficients $c_{i}$ are very unreliable and cannot be used to make any conclusions about the actual image. So, we must make this difference as small as possible.

How can we express this idea numerically? If we reconstruct the image from the compressed data, we get the following formula:

$$
\tilde{f}_{\text {rec }}(x)=\tilde{m}_{1}(x)+\ldots+\tilde{m}_{N}(x),
$$

where we denoted

$$
\tilde{m}_{i}(x)=\tilde{c}_{i} \cdot e_{i}(x) .
$$

If we used the precise image instead, we would have gotten a similar (but more accurate) representation

$$
f_{\text {rec }}(x)=m_{1}(x)+\ldots+m_{N}(x),
$$

where we denoted

$$
m_{i}(x)=c_{i} \cdot e_{i}(x) .
$$

It makes sense, therefore, to estimate the relative quality of choosing a function $e_{i}(x)$ as the largest possible value of the difference

$$
\Delta m_{i}(x)=m_{i}(x)-\widetilde{m}_{i}(x) .
$$

So, we arrive at the following definitions.

\section{MATHEMATICAL FORMULATION OF THE PROBLEM}

Let $X$ be a space with a measure $\mu$ (e.g., a line $R$ or a plane $R^{2}$ equipped with a standard (Lebesgue) measure $\mu$ ). By an orthonormal basis, we mean a sequence of squarely integrable functions $e_{i}: X \rightarrow R\left(e_{i} \in L^{2}(X)\right)$ that satisfy the properties (2)-(3).

Definition 1. By a compression scheme, we mean a pair $\left(\left\{e_{1}(x), e_{2}(x), \ldots\right\}, N\right)$, in which:

- $\left\{e_{1}(x), e_{2}(x), \ldots\right\}$ is an orthonormal basis, and

- $N$ is an integer.

Definition 2. Let $\left(\left\{e_{i}(x)\right\}, N\right)$ be a compression scheme, and let $\Delta>0$ be a positive real number. This number $\Delta$ will be called measurement inaccuracy. By the reconstruction inaccuracy $q_{i}$ of $i$-th term of this basis $e_{i}(x)$, we mean the largest possible value $q_{i}$ of the difference $\left|\Delta m_{i}(x)\right|$ :

$$
q_{i}=\max _{x, f(x), \Delta f(x)}\left|\Delta m_{i}(x)\right|,
$$

where maximum is taken over:
- all points $x \in X$,

- all functions $f \in L^{2}$,

- all function $\Delta f \in L^{2}$ for which $|\Delta f(x)| \leq \Delta$ for all $x \in X$,

and $\Delta m_{i}(x)$ is determined by the formulas (4) - (8) with $\tilde{f}(x)=f(x)-\Delta f(x)$.

Our goal is to find, for each $N$, the basis for which the reconstruction accuracy is the smallest possible. We want to minimize $N$ different numbers $q_{1}, \ldots, q_{N}$. In general, if we minimize one objective function, it is difficult to expect that any other objective function will be simultaneously minimized. However, in our case, we are lucky: for the cases of $X=R$ and $X=R^{2}$ that correspond to imaging, there is a basis for which all the reconstruction inaccuracies $q_{i}$ take the smallest possible value.

Definition 4. By a $1 D$ Haar basis, we mean the basis that consists of the following functions:

- the function $\phi(x)$ that is equal to 1 for $0 \leq x<1$ and to 0 otherwise;

- the function $w(x)=\phi(2 \cdot x)-\phi(2 \cdot x-1)$ (that is equal to 1 for $0 \leq x<1 / 2$, to -1 for $1 / 2 \leq x<1$, and to 0 for all other $x$ );

- functions $w_{j k}(x)=2^{-j / 2} \cdot w\left(2^{j} \cdot x-k\right)$, where $j$ and $k$ are arbitrary integers.

Comment. In general, if we have a function $w(x)$ that tends to 0 as $|t| \rightarrow \infty$, and for which the functions $w\left(2^{j} \cdot x-\right.$ $k$ ) are orthogonal to each other, so that after normalization, we get an orthonormal basis $c_{j k} \cdot w\left(2^{j} \cdot x-k\right)$, then this basis is called an (orthonormal) wavelet basis; see, e.g., $[2,7,11,14]$.

Definition 5. By a 2D Haar basis, we mean the basis consisting of the functions $f_{i j}\left(x_{1}, x_{2}\right)=e_{i}\left(x_{1}\right) \cdot e_{j}\left(x_{2}\right)$, where $e_{i}$ and $e_{j}$ are functions from the $1 D$ Haar basis.

THEOREM. For every $i$, the imaging inaccuracy of the Haar wavelet basis is the smallest possible.

\section{Comments.}

- The proof of this theorem is given in the following section.

- For SMD, wavelets, in particular, Haar wavelets, indeed lead to much better results than more traditional Fourier transform techniques $[1,5,6,8,9]$ 
- On a more fundamental level, our result is a step towards solving the following important problem related to wavelets and multi-resolution data processing:

- these methods often empirically work much better than other methods, but

- there are very few theoretical explanations of this efficiency.

Our result shows that, at least on some cases, such a theoretical explanation can be obtained if we take interval uncertainty into consideration.

- A related problem is: Which wavelet is the best? This problem is raised, e.g., in [11].

- In $[3,10]$, this problem is analyzed under the assumption that the measurement errors are random, Gaussian, and independent. In this case, the best approximation corresponds to minimizing the sum of the squares of these errors. Special wavelets are presented that minimize this sum.

- In our paper, we consider a similar problem, but under the assumption that the measurement errors belong to the corresponding intervals. The optimization of the corresponding worst-case error leads to Haar wavelets.

\section{PROOF}

1. Let us first show that for an arbitrary basis $\left\{e_{1}(x), e_{2}(x), \ldots,\right\}$, we have

$$
q_{i}=\Delta \cdot \max _{x}\left|e_{i}(x)\right| \cdot \int\left|e_{i}(y)\right| d y .
$$

Indeed, for each function $f(x)$, we have

$$
\begin{gathered}
\Delta m_{i}(x)=m_{i}(x)-\widetilde{m}_{i}(x)=c_{i} \cdot e_{i}(x)-\widetilde{c}_{i} \cdot e_{i}(x)= \\
e_{i}(x) \cdot \int f(y) \cdot e_{i}(y) d y-e_{i}(x) \cdot \int \tilde{f}(y) \cdot e_{i}(y) d y= \\
e_{i}(x) \cdot \int e_{i}(y) \cdot \Delta f(y) d y .
\end{gathered}
$$

Hence, $\left|\Delta m_{i}(x)\right|=|I| \cdot\left|e_{i}(x)\right|$, where we denoted $I=$ $\int e_{i}(y) \cdot \Delta f(y) d y$. Since the function $\left|\Delta m_{i}(x)\right|$ is proportional to $\left|e_{i}(x)\right|$, the maximum of $\left|\Delta m_{i}(x)\right|$ is proportional to the maximum of $\left|e_{i}(x)\right|$, i.e.,

$$
\max _{x}\left|\Delta m_{i}(x)\right|=|I| \cdot \max _{x}\left|e_{i}(x)\right|
$$

For a given basis $e_{i}(x)$, the maximum of the left-hand side is, therefore, attained when the value $|I|$ is the largest possible.

In the definition of $q_{i}$, the maximum is taken over all function $\Delta f \in L^{2}$ for which $|\Delta f(x)| \leq \Delta$ for all $x \in X$. For each such $\Delta f(x)$, we have $\left|e_{i}(y) \cdot \Delta f(y)\right| \leq \Delta \cdot\left|e_{i}(y)\right|$, and therefore,

$$
\begin{gathered}
|I|=\left|\int e_{i}(y) \cdot \Delta f(y) d y\right| \leq \int\left|e_{i}(y) \cdot \Delta f(y)\right| d y \leq \\
\int \Delta \cdot\left|e_{i}(y)\right| d y=\Delta \cdot \int\left|e_{i}(y)\right| d y .
\end{gathered}
$$

On the other hand, for arbitrary $B$, we can take $\Delta f(y)=$ $\Delta \cdot \operatorname{sign}\left(e_{i}(y)\right)$ for $|y| \leq B$ and $\Delta f(y)=0$ otherwise. For this choice of $\Delta f(y)$, we have

$$
I=\int e_{i}(y) \cdot \Delta f(y) d y=\Delta \cdot \int_{|y| \leq B}\left|e_{i}(y)\right| d y .
$$

The larger $B$, the closer this value to $\Delta \cdot \int\left|e_{i}(y)\right| d y$. Therefore, the largest possible value of $I$ is indeed $\Delta \cdot \int\left|e_{i}(y)\right| d y$, and therefore, the largest possible value $q_{i}$ of $\left|\Delta m_{i}(x)\right|$ is indeed equal to

$$
\Delta \cdot \max _{x}\left|e_{i}(x)\right| \cdot \int\left|e_{i}(y)\right| d y .
$$

The formula is proven.

2. Let us now show that for an arbitrary basis, we have $q_{i} \geq 1$.

Indeed, clearly, for all $y$,

$$
\max _{x}\left|e_{i}(x)\right| \geq\left|e_{i}(y)\right| .
$$

Therefore,

$$
\begin{gathered}
q_{i}=\max _{x}\left|e_{i}(x)\right| \cdot \int\left|e_{i}(y)\right| d y= \\
=\int \max _{x}\left|e_{i}(x)\right| \cdot\left|e_{i}(y)\right| d y \geq \int\left|e_{i}(y)\right| \cdot\left|e_{i}(y)\right| d y .
\end{gathered}
$$

Since $\left\{e_{i}(x)\right\}$ is an orthonormal basis, the right-hand side is equal to 1 and therefore, $q_{i} \geq 1$.

3. To complete the proof, we must show that for Haar basis, $q_{i}=1$.

Indeed, for each of the functions from this basis, there exists a non-zero real number $C$ such that for every $x$, either $e_{i}(x)=0$, or $\left|e_{i}(x)\right|=C$. Therefore,

$$
q_{i}=\max _{x}\left|e_{i}(x)\right| \cdot \int\left|e_{i}(y)\right| d y=
$$




$$
C \cdot \int\left|e_{i}(y)\right| d y=\int C \cdot\left|e_{i}(y)\right| d y .
$$

We know that for every $y$, either $\dot{e}_{i}(y)=0$, or $\left|e_{i}(y)\right|=C$. Therefore:

- When $e_{i}(y)=0$, we have $C \cdot\left|e_{i}(y)\right|=0=e_{i}^{2}(y)$.

- When $\left|e_{i}(y)\right|=C$, then $C \cdot\left|e_{i}(y)\right|=e_{i}^{2}(y)$.

In both cases, $C \cdot\left|e_{i}(y)\right|=\left|e_{i}(y)\right|^{2}$ and therefore,

$$
q_{i}=\int C \cdot\left|e_{i}(y)\right| d y=\int\left|e_{i}(y)\right|^{2} d y
$$

Since $\left\{e_{i}(y)\right\}$ is an orthonormal basis, we have $q_{i}=1$. The statement is proven, and so is the theorem.

Acknowledgments. The authors are thankful to Vladik Kreinovich and Mikhail Zakharevich for valuable discussions.

\section{REFERENCES}

[1] Carrillo, G. Cabrera, S.D., and Portillo, A.A. "Inspection of Surface-Mount-Device images using wavelet processing", in Proceedings of SPIE Applied Imagery Pattern Recognition, Washington D.C., October 1996.

[2] Chui, C. An Introduction to Wavelets, Academic Press, 1992.

[3] Coifman, R.R., Matviyenko, G., and Meyer, Y. "Modulated Malvar-Wilson bases", Applied and Computational Harmonic Analysis, Vol. 4, pp. 58-61, 1997.

[4] Computer vision: theory and industrial applications, Berlin-N.Y.: Springer-Verlag, 1992.

[5] Gallegos, J.M., Villalobos, J.R., Carrillo, G., and Cabrera, S.D. "Sequential algorithms for the inspection of Surface Mounted Devices", in Proceedings of the 13th SPIE Conference on Intelligent Robots and Computer Vision, Boston, MA, SPIE, 1994, Vol. 2354, pp. 215-226.

[6] Gallegos, J.M., Villalobos, J.R., Carrillo, G., and Cabrera, S.D. "Reduced-dimension and wavelet processing of SMD images for real-time inspection", in Proceedings of the IEEE Southwest Symposium on Images Analysis and Interpretation, San Antonio, April 1996.

[7] Mallat, S. "A theory for multiresolution signal decomposition: the wavelet representation", IEEE Transactions on Pattern Analysis and Machine Intelligence, Vol. 14, pp. 710-732, 1992.
[8] Mallat, S., and Hwang, W.L. "Singularity detection and processing with wavelets", IEEE Transactions on Information Theory, Vol. 38, pp. 617-643, 1992.

[9] Mallat, S., and Hwang, W.L. "Characterization of signals from multiscale edges", IEEE Transactions on Pattern Analysis and Machine Intelligence, Vol. 14, pp. 710$732,1992$.

[10] Matviyenko, G. "Optimized local trigonometric bases", Applied and Computational Harmonic Analysis, Vol. 3, pp. 301-323, 1996.

[11] Meyer, Y. Wavelets algorithms and applications, Philadelphia: SIAM, 1993.

[12] Pau, L.F. Computer vision for electronics manufacturing, N.Y.: Plenum, 1990.

[13] Strang, G., and Nguyen, T. Wavelets and Filter Banks, Wellesley, MA: Wellesley-Cambridge Press, 1996.

[14] Vetterli, M., and Kovacevic, J. Wavelets and subband coding, Englewood Cliffs, New Jersey: Prentice Hall, 1995. 


\title{
AN ARTIFICIAL LIFE VIEW ON INTELLIGENT ENTERPRISES - FUZZY EVOLUTIONARY MULTI-AGENT SYSTEMS
}

\author{
Valery TARASSOV* \\ Bauman Moscow State Technical University, \\ 2nd Baumanskaya st., 5; Moscow 107005, Russia \\ Tel. +(7095)-921.73.66 \\ Fax: +(7095)-263.61.70 \\ E-mail: tar@srv-m.mpei.ac.ru \\ * Presently a visiting professor of \\ University of Valenciennes and Hainaut-Cambresis, \\ Le Mont Houy BP311, \\ 59304 Valenciennes Cedex, France \\ Fax: +(333)-27.14.12.88 \\ E-mail: tarasov@pulsar.univ-valenciennes.fr
}

\begin{abstract}
The aim of this paper is to propose a new methodological framework for large-scaled intelligent systems based on bioreengineering concepts, and more generally, on artificial life principles. Two viewpoints on artificial life content are presented: a narrow one where artificial life models are considered as a special case of multi-agent systems, and general approach concerning the specification of basic principles and mechanisms of living. These approaches, underlie the suggested model of fuzzy evolutionary multi-agent system seen here as a kernel of intelligent enterprise. After specifying main properties of intelligent and virtual enterprises, principal architectures of basic fuzzy multi-agent system and generic fuzzy evolutionary multi-agent system are built and studied in the context of enterprise integration and reengineering problems.
\end{abstract}

Keywords: Artificial Intelligence, Multi-Agent Systems, Distributed Intelligence, Bioreengineering, Artificial Life, Evolutionary Semiotics, Intelligent Enterprise, Fuzzy Evolutionary Multi-Agent System

\section{INTRODUCTION}

Classical AI models are penetrated by individual rationalism, because they reduce natural intelligence mainly to rational individualized problem solving with the use of some heuristics. Such a conventional view seems to be very restrictive: it does not make into account the emergence and evolution of intelligence in communication, cooperation and coordination processes. A brilliant criticism of classical AI paradigm may be found in [1], where Winograd and Flores have suggested a new approach to model the intelligence phenomena based on Searle's speech acts theory. This approach studying the interactions between two agents - Customer and Performer has been successfully applied to represent the coordination processes in organizations by means of workflow model. Another important keystone which has considerably contributed to the change of classical AI paradigm was the book [2] by Minsky where a social AI paradigm has been introduced in detail. Following this social standpoint, the modeling (and specifically, semiotic modeling) of abstract agents interacting in some organization represents the main content of AI studies [3].

Such a social paradigm underlies two main tendencies in the modern AI - integration and decentralization. Following Minsky, for the sake of versatility, one can exploit and manage the advantages of several types of representations at the same time. Specifically, the problem is to find out how to build an efficient bridge between top-down and bottom-up design [4]. One may speak about various levels of integration. First of all, since eighties, one has began to couple expert systems with conventional informational technologies that implies the arrival of hybrid expert systems or integrated intelligent systems. 
The development of integrated intelligent systems has necessitate the elaboration of some empirical and theoretical integration and communication techniques, in particular, the blackboard architecture that is also of primary concern in distributed AI systems. Such integrated systems are required to support various knowledge models and various reasoning types, to realize both knowledge and image processing.

Furthermore, one proceeds the way of integrating some intelligence attributes (or, some specific working definitions of intelligence) with the aim of obtaining non-linear, synergetic effects [5] by compensating the shortcomings and enhancing the advantages of component models in coupled models. Some typical examples are: integrated neuro-logical and specifically neuro-fuzzy models [6-8] underlying Zadeh's soft computing [9] and often using logic-based neurons and distributed fuzzy system modeling [10], neuro-optical models by Kuznetsov [11], computational intelligence models [12], integrated concurrent engineering models [13], Petri net models of fuzzy neural networks [14], etc.

The most well-known instances of hybrid AI strategies are computational intelligence and soft computing. So in the framework of soft computing three AI trends - logical, neuronal and evolutionary - or, in other words, three intelligence aspects - uncertainty/ fuzziness processing, learning and adaptation - are amalgamated by representing fuzzy production rules in a trainable neural network which is optimized with the use of genetic algorithms. These three components also form a kernel of a more general area called computational intelligence; besides, it involves chaotic systems, inductive learning, probabilistic reasoning and some other techniques.

Integrated concurrent engineering models tending to model design process (and more generally, product's life cycle) require building hybrid (fuzzy modal and fuzzy nonmonotonic) logics. At last, neuro-optical models suggest a coupling of connectionism with wave (oscillation) theory.

Second, more fundamental tendency, is closely related to the first one - AI distribution and/or decentralization. Here distributed intelligent systems may have a unique centralized control module (or agent), and the control in decentralized systems is reduced to local interactions between agents [15]. Sometimes, this difference is taken as the main classification criterion for dividing multi-agent systems (MAS) into: a) distributed AI systems composed from a few intelligent (and often heterogeneous) agents (where one of them may play the subordinator role); b) artificial life (in the narrow sense of term) or purely collective intelligence $[16,17]$ systems, where group intelligent behavior emerges from local communications between many not necessarily intelligent agents.

\section{SYSTEMIC APPROACH VERSUS LOGICAL APPROACH IN AI}

The classical intelligent systems using symbolic (or, more specifically, logical) approaches in AI based on cognitive psychology satisfy the following main postulates [18, 19].

1. Centralization postulate

2. Internal representation postulate (AI epistemology);

3. Knowledge-centered postulate;

4. Disembodied AI postulate;

5. Closed world assumption (CWA)

6. Independence postulate (cognitive processes may be considered independently from evolution);

7. Concertation postulate (between cognition and language in $\mathrm{AI})$

8. Homogeneity postulate (interacting models have the same, homogeneous nature).

Nowadays, due to the integration and decentralization tendencies mentioned above, these earlier unchanging postulates must be revised. What postulates are to be revised depends on the kind of integrated models and on the integration basis. For example, the advocates of artificial neural networks related to neurophysiological studies emphasize a crucial role of knowledge distribution, massive parallelism and learning, as well as the emergence of symbolic representations in the system of artificial neurons. The development of evolutionary modeling in AI means making over the independence postulate. Such AI (or close to AI) trends as moboticism [20] and virtual reality [21] represent completely opposite viewpoints to compare with logical approach. So the moboticism introducing «beings» as reactive agents without representations focuses on the importance of coordinated local reactions for intelligent behavior and denies the ideas of knowledge-centrism, disembodiment, rational kinematics, centralization and independence. In addition, virtual reality systems suppose direct experience acquisition and introduce artificial sensory-motor coordinations. A rather close but less radical position concerning the withdrawal from the postulates above is proper to the adepts of such approaches as «societies of minds» by Minsky [2], decentralized [15] and distributed AI [22, 23], etc.

In our opinion, the essence of the paradigm change in modern AI may be caught by recalling the title of the well-known monograph «From Being to Becoming» by Prigogine [24] (here it is interpreted as a call to modeling the genesis of intelligence for AI needs) and the words by Bobrow [25] «to throw away the isolation assumption». It is necessary to introduce the interaction dimension enabling to move from individual intelligen! systems to intelligent organizations and societies of intelligent agents. 
So, a convergence of symbolic, neuronal and evolutionary approaches takes place in modern AI. Here various integration schemes lead to the generation of hybrid (or synergetic) AI. Below we shall discuss artificial life issues in the context of intelligent organizations.

\section{ARTIFICIAL LIFE}

Artificial life (AL) is an interdisciplinary field that focuses on the functional analysis, modeling and simulation of living systems. The sources of AL raise to von Neumann's theory of self-reproducing automata [26] and complexity theory introduced by Kolmogorov [27] and Chaitin [28]. Its first practical results are related to simulated evolution concept [29] and genetic algorithms [30]. The official AL birth date is 1987 when Langton organized at Los-Alamos (USA) an interdisciplinary workshop on the synthesis and simulation of living systems [31] (recent results in the field of $\mathrm{AL}$ are collected in [32]).

Actually there are two different viewpoints of AL. In a narrow sense, AL is seen as a branch of multi-agent systems, where intelligent behavior emerges from local interactions between agents, and it does not exist any centralized control agent [16, 17]. More precisely, this viewpoint on AL content may be expressed by the following 5 statements:

- the MAS consists of a «population» of rather simple and relatively independent agents;

- each agent himself specifies his own reactions to events of his local environment and his interactions with other agents;

- there is no special agent who subordinates other agents;

- there is no rules for specifying global agents behavior;

- all behavior, properties and structure on the superior (collective) level emerges from local interactions between agents.

In a more general context, $\mathrm{AL}$ is a pluridisciplinary branch that integrates some trends of biology and cybernetics, artificial intelligence and robotics, physics and mathematics, chemistry and synergetics (Figure 1). In this sense, AL embraces and extends AI studies. Like artificial intelligence tending to comprehend and to model various phenomena of human psyche and specifically of natural intelligence (or at least to solve problems or to perform actions related to intelligent behavior), $\mathrm{AL}$ deals with general principles and mechanisms of life. Artificial life aims at abstracting crucial properties of living systems (for example, autoreproduction, autoconservation and autoregulation) and putting them into practice via computer-based simulation and development of various applied systems. This definition is based on functionally-structural approach to life phenomena: the essence of life is mainly related to specific modes of organizing units, functions and processes and cannot be completely reduced to the properties of material substratum (carbon chains and DNA structures). So according to Kolmogorov, basic AL metaphor can be formulated in the following way: if an artificial organization is in some sense equivalent to a living organism and it has analogical output functions to compare with living system, then such an organization may be refered as "living". In other words, AL considers mainly "living processes" beyond the usual scope of organic chemistry and concentrates on their computer analysis and simulation (virtual life processes mediated by computer).

In this paper we shall use both narrow and general $\mathrm{AL}$ concepts. On the one hand, in our model of fuzzy evolutionary multi-agent system (FEMAS) underlying the concept of intelligent organizations, the analysis of local interactions between agents and coordination links specifying decentralized system management will be of primary concern. On the other hand, general life principles, such as autonomy, homeostathis and autopoiesis principles, and specifically basic evolutionary theory principles including evolution, adaptation for survival, mutation and natural selection, will be used to drive the evolution of FEMAS model.

\section{INTELLIGENT ENTERPRISES}

Generally the concept of intelligent enterprise includes both the organizational structure aspects and the way of its development and functioning.

On the one hand (from a structural viewpoint), enterprise intelligence is attributed to the progressing reversibility of its functionally-dynamic structures, or the increasing number of symmetrical horizontal relations that implies the higher level of coordination and collaboration.

On the other hand, following the intelligence definition given by Piajet [33], enterprise intelligence as a systemic attribute may be defined from a behavioral viewpoint as a flexible and at the same time stable equilibrium of enterprise behavior. If the forms of enterprise's influence on its environment become more complex and variable, leading to progressive compositions and associations, then its behavior may be refered as more intelligent. Generally speaking, intelligence 


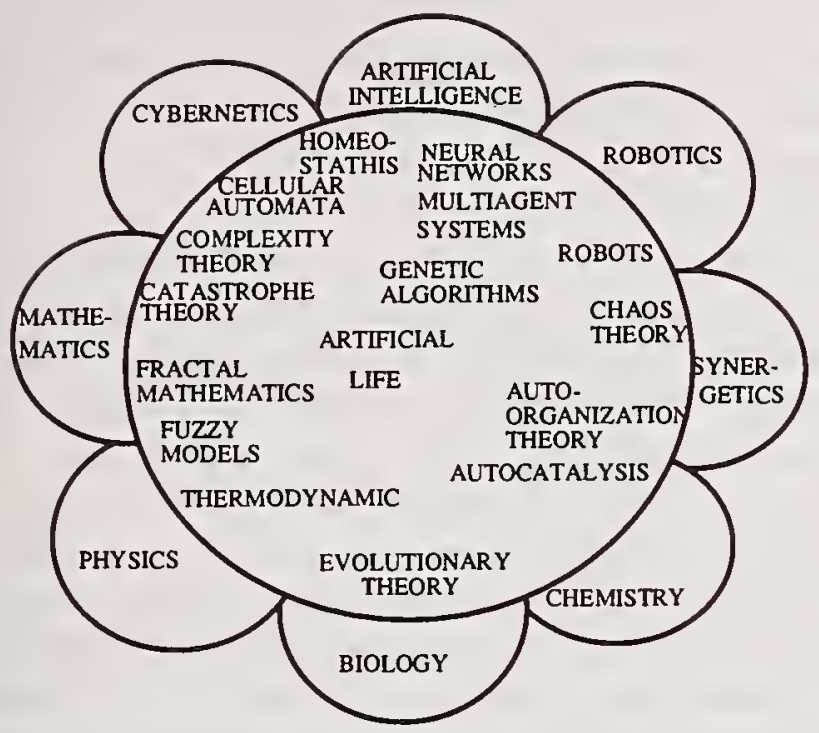

Figure 1. Branches and models of artificial life

includes the capacities of learning, understanding, acquiring and using competences acquisition to adapt to new situations. Intelligent enterprise is characterized not only by a capacity of using in an efficient manner the knowledge and competences of its personnel. Moreover, it is conceived as a collective learning system which can permanently apprehend and to transform itself for attaining its goals. So the enterprise intelligence underlies the ability to continuously develop the capacity to co-evolve and to build its own future and associates the competitivity level with the capacity to learn and to adapt faster than competitors.

Definition 1. Intelligent enterprise (IE) is a kind of posttaylorian enterprise that emerges as a result of both interenterprise and intra-enterprise communication processes, enabling enterprise semiosis, and is driven by principles of maximal adaptation to its environment, optimal autoreengineering for autoconservation, and activity autoregulation via permanent knowledge mining and accumulation, needs anticipation and strategic planning, shared motivation and collective learning.

So the genesis of the enterprise intelligence in communication processes means that the enterprises and their divisions act as semiotic entities. To differ from conventional formal systems, semiotic systems formally introduced by Pospelov [34] and thoroughly studied by Meystel [35], are open and take into account semantic and pragmatic informations aspects of knowledge; moreover, they support multi-resolution, nonmonotonic and common-sense reasoning. Thus, semiotic systems admit a variable sign interpretation and open the possibilities to change knowledge and its processing mode (inference rules, axioms and even language).
So enterprise semiosis is the process of enterprise knowledge generation, accumulation and transformation through communications with specifying its syntax, semantics and pragmatics. Here the syntax is associated with the enterprise knowledge structure, semantics refers to the sense (a relation "knowledge - source") and pragmatics corresponds to some knowledge use (a relation "knowledge - consumer").

The intrinsic features of intelligent enterprises are the capacities to deal with uncertain and fuzzy informations. The main "product-based" or "service-based" sources of imprecision and fuzziness in enterprise environment are: (a) multiple interpretation in "client - supplier" communications; in particular, product specification or service request formulation in terms of natural language that leads to semantical ambiguity and multivalued interpretation; (b) incomplete and fragmentary character of available data on new products and services; (c) lack or complexity of analytical equations describing future product parameters and, as a consequence, rather vague knowledge about their relationships (d) principal limitations to the precision in specifying both quantitative and (in particular) qualitative parameters.

Below we shall introduce fuzzy evolutionary multi-agent systems as a formal tool of representing intelligent enterprises.

\section{FUZZY EVOLUTIONARY MULTI-AGENT SYSTEMS}

Evolutionary modeling of multi-agents systems in the context of intelligent and virtual enterprises represents an important step from traditional mechanistic vision toward a new living paradigm in enterprise engineering and reengineering. On the one hand, this living paradigm is inspired by modern artificial life approaches and specifically by neodarwinian approach reposed on the principles of survival, reproduction and mutation. On the other hand, the introduction of evolutionary mechanisms into MAS provides a natural framework for studying various situations of human and artificial agents interactions within the enterprise with taking into account mental and social aspects. A comparative analysis of agents goals, resources and competences enables to specify a concrete intermediate type of interaction from pure competition to pure cooperation, or from pure conflict to pure collaboration.

In this session the concept of fuzzy evolutionary multi-agent system is introduced and formalized, and a generic FEMAS architecture is proposed. We consider a special case of intelligent organization, where agents roles are specified through virtual enterprise metaphor [37, 38]. 
Definition 2. Virtual enterprise (VE) is a sort of metaenterprise merging in an electronic way the objectives, the resources and the competences of different enterprises in order to develop complex innovative projects and to obtain new world-class products.

So the birth of VE as an innovative intelligent organization is connected with selecting and sharing common goals, unique competences, compatible resources, including the most advanced technologies, from different enterprises situated in different places around some complex project that cannot be achieved by isolated VE components. The composition of VE also fulfills a compensatory function: it gives the opportunity to diminish disadvantages and to enhance advantages of heterogeneous components. In particular, it permits bridging the gap between big enterprises (powerful but not very reactive) and small enterprises (rather weak but ordinarily having prompt reaction and better adaptation facilities to changes).

So the development of VE environments enables to model multi-sites and multi-trade enterprise aspects, including the transactions between producer, customer, supplier and subcontractor. It is aimed at attaining better enterprise flexibility, reactivity or agility to compare with its components and integrates various informations, applications and processes concerning a given project.

Definition 3. A basic fuzzy multi-agent system (BFMAS) is a pair

$$
\text { BFMAS = }(\mathbf{X}, \mathbf{R}) \text {, }
$$

where $X=\{1, \ldots n\}$ is a set of heterogeneous intelligent agents, $\quad R$ is a family of basic types of (generally) fuzzy relations between agents.

According to virtual enterprise metaphor, BFMAS is seen as a FEMAS cell, and the set $X$ consists of 5 elements or contains 5 roles: $X=\{1, \ldots, 5\}$, where: 1 - Customer agent; 2 Coordinator agent, 3 - Performer agent, 4 - Subordinator agent, 5 - Observer (Meta-Coordinator) agent. Let us discuss in a more detailed way the content of roles of these intelligent agents.

The Customer is defined as the agent which initiates and determines the tasks to accomplish or the works to do for satisfying its need. Customers evaluate performed work and declare if it meets their satisfactions. So the customer may be any physical or civil person outside or inside the enterprise. The customer function based mainly on gnoseological considerations (specifying the content of the work) may be seen in some sense as enterprise's service function and expressed in terms of conversation systems by the questions of type "What to produce?" or "For what can the product be used? . From informational viewpoint it determines the semantics of information exchange during virtual enterprise agents transactions.

The Coordinator (or the Manager) is defined as the agent that creates, supports and coordinates a network of requests and commitments necessary to perform some work. The Coordinator distributes the works and selects available resources to ensure a good performance. Hence his (or its in case of electronic coordinator) principal function may be refered as resource function. He inspects also work performance and evaluates the quality. So the coordinator may be seen as a mediator between the customer and the performer. The Coordinator function based mainly on ontological considerations may be considered as enterprise's operatory function and corresponds to the questions of type "Who, When and Where must do the work?" Moreover it is related to structural aspect of information.

The Performer is defined as the agent (or agents team) personally responsible for the work being done and for declaring its completion. It needs to be emphasized that mainly praxeological considerations related to methods and techniques of accomplishing given work underlie performer's activity. The Performer function is in fact enterprise's basic technical function containing its know-how; it corresponds to the question "How to do the work?" and specifies the pragmatics of information exchange in agents' transactions

The Subordinator (or the Supervisor) is defined as the agent carrying up the integrating meta-function of organizing and supervising the virtual enterprise functions mentioned above and consequently governing the enterprise processes viewed here in the scope of the transactions "customer - coordinator performer". His principal functions are the enterprise functioning supervision, inspection and evaluation (including the agents efficiency evaluation). He (it) has the power to intervene at critical situations and to redistribute enterprise's resources in a timely manner. For instance, he (it) can replace the coordinator and hence organize a new business process. The Subordinator functions concern the specification of virtual enterprise policies including customers choice, leading management personnel selection, functional duties definition, conflict resolution. To summarize, it means activating enterprise functions and neutralizing enterprise dysfunctions.

So the Subordinator meta-function (to compare with the functions performed by other agents) is based on axiological consideration related to the field of motivation, principal objectives and strategies development. It needs anticipating future tendencies and investigating possible ways of 
development. Specifically it includes the questions like "Why one will begin to manufacture or to sell a new product (to render a new service) and how much of it will be produced (sold)?" From the semiotics viewpoint it gives the necessary meta-information and ensures the semiosis of virtual enterprise cell.

In a general case the family of fuzzy relations $\mathrm{R}$ contains three types of relations and may be represented by a partition

$$
\mathbf{R}=\mathbf{R}_{1} \cup \mathbf{R}_{2} \cup \mathbf{R}_{3}
$$

where $R_{1}=\{1 \rightarrow 2 ; 2 \rightarrow 1 ; 2 \rightarrow 3 ; 3 \rightarrow 2 ; 1 \rightarrow 3 ; 3 \rightarrow 1\}$ is a set of fuzzy horizontal relations;

$R_{2}=\{4 \rightarrow 2 ; 4 \rightarrow 1 ; 4 \rightarrow 3\}$ is a set of fuzzy vertical top-down relations;

$R_{3}=\{2 \rightarrow 4 ; 1 \rightarrow 4 ; 3 \rightarrow 4\}$ is a set of fuzzy vertical bottom-up relations.

To depend from the context, the set $R_{1}$ may contain coordination, collaboration or cooperation relations, and the set $\mathrm{R}_{2}$ - subordination, competition or conflict relations. The set $\mathrm{R}_{3}$ mainly contains informing relations.

These fuzzy relations can be specified by gradual transitions on continuous "gray» scales. They depend on the following principal factors: a) goal compatibility; b) presence of commitments and mutual responsibility; c) level of competences; d) level of individual resources.

So the agents above together with the appropriate relations compose the basic multi-agent structure (or cell) for virtual organization called SuPerCoC (derived from Subordinator Performer - Coordinator - Customer). This cell is invariant in respect to organization type and activities, as well as environment influences.

In this paper one reduces the enterprise cell representation to a two-level BFMS represented by a tetrahedron whose basis is composed by the agents (1), (2), (3) together with their links (the PerCoC-basis) and the summit corresponds to the supervising agent (4) (Figure 2). This representation is inspired by a structural-functional unit introduced in [39].

Here the exchange of roles is possible. Moreover each agent can play concurrently several roles (the cases of coinciding (1) and (2), (1) and (4), (1), (2) and (4)), which reduce the

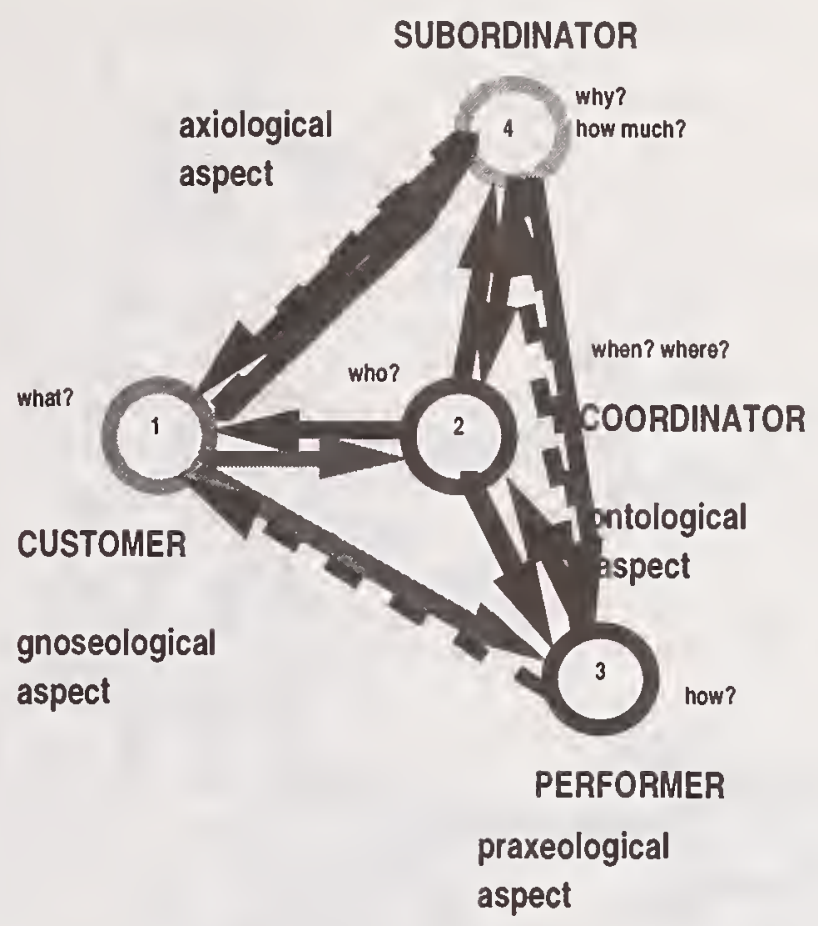

Figure 2. Representation of basic fuzzy multi-agent system

tetrahedron SuPerCoC to the triangle $\mathrm{PerCoC}$ or the segment PerC.

Definition 4. A generic fuzzy evolutionary multi-agent system is given by a sextuple

where:

$$
\text { FEMAS }=\left(\mathbf{X}, \mathbf{R}, \mathbf{A}, \mathbf{A}_{c}, \mathbf{S}, \mathbf{E}\right),
$$

$\mathrm{X}=\{1, \ldots, \mathrm{n}\}$ is an heterogeneous set of agents; $\mathrm{R}$ is a family of basic fuzzy relations between agents; $A$ is a set of agents actions; $A_{c} \subset A$ is a finite set of agents communication acts called a communication protocol; $S$ is a set of fuzzy states of FEMAS; $E$ is a set of fuzzy evolutionary strategies in multiagent system. The sets $A, A_{c}, S$ and $E$ may include fuzzy parameters.

A typical communication protocol for VE agents can be written in the following form $\mathrm{P}=$ \{request, offer, accept, decline, transfer, cancel, etc. \}. Here some linguistic modifiers and quantifiers like very, rather, often, rarely, possibly, necessarily etc., which may act on the verbs above, as well as inexact time constraints imply the need in fuzzy models given by possibility distributions.

The state space includes such states as initial, normal, critical, degraded, etc. Each evolutionary strategy implies some transformation in FEMAS such as vertical or horizontal growth, bifurcation, self-reproduction, etc.

A basic FEMAS architecture underlying the representation of intelligent organizations is given in Figure 3. The processes 


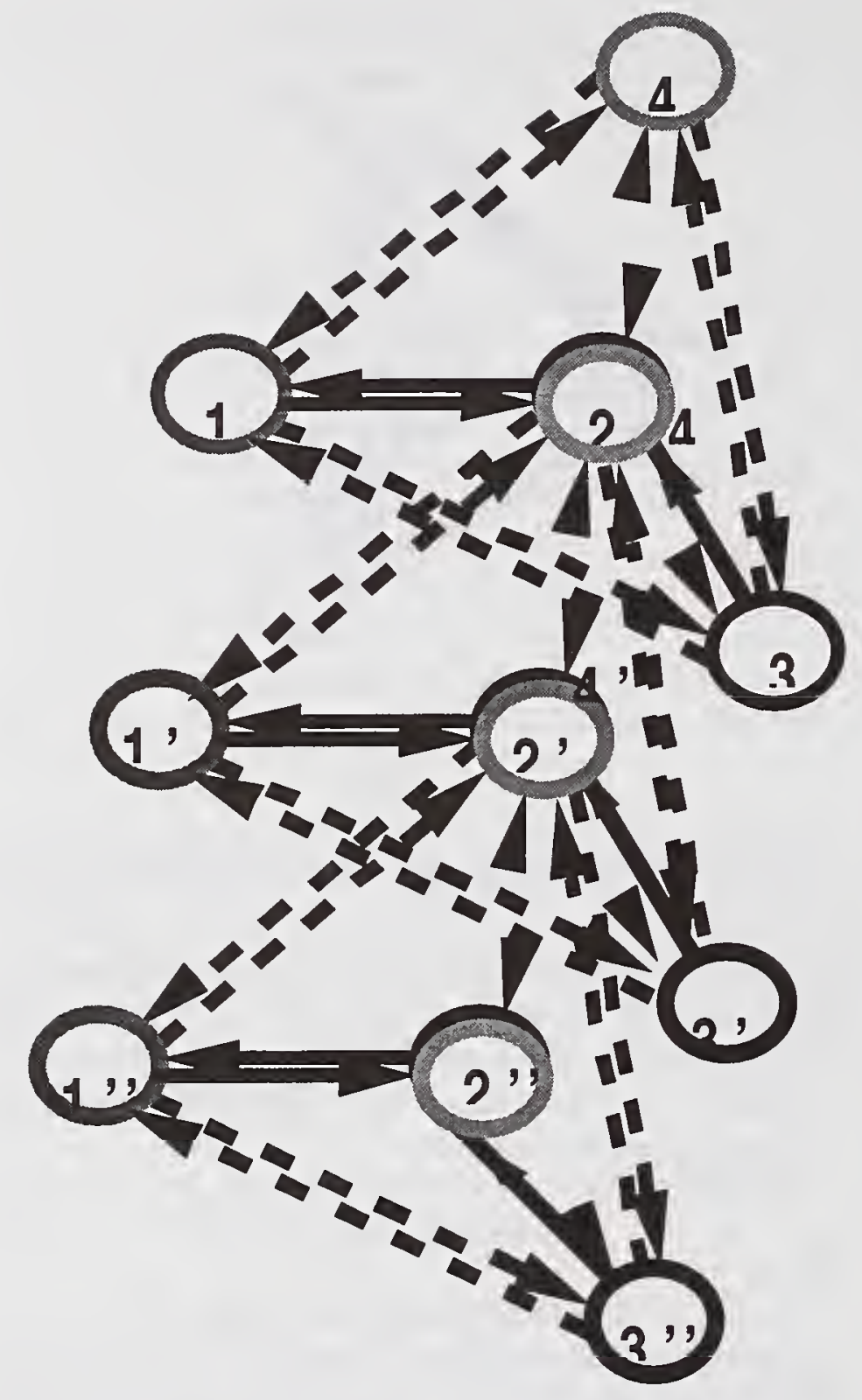

Figure 3. Basic FEMAS architecture

of intelligent organization emergence, development and degradation may be modeled on the basis of FSU. So the results of using different evolutionary strategies in enterprise management may be specified by defining some transformations in FSU, such as its vertical or horizontal growth, self-reproduction or bifurcation, etc. For example, the adoption of empowerment strategy may be seen as Subordinator's (4) power delegation to Coordinator (2) who becomes the Subordinator on the inferior level (4') and hence initiates the next coordination process $\left(1^{\prime} \leftrightarrow 2^{\prime} \leftrightarrow 3\right)$, etc. (Figure 3). This top-down scheme may be attributed to the enterprise growth via $\left(2 \rightarrow 4^{\prime}\right)$-type transformations. On the other hand, the inverse bottom-up scheme is possible, where the next organization level is generated after exhausting the resources of preceeding level. Here the Ex-Subordinator (4)

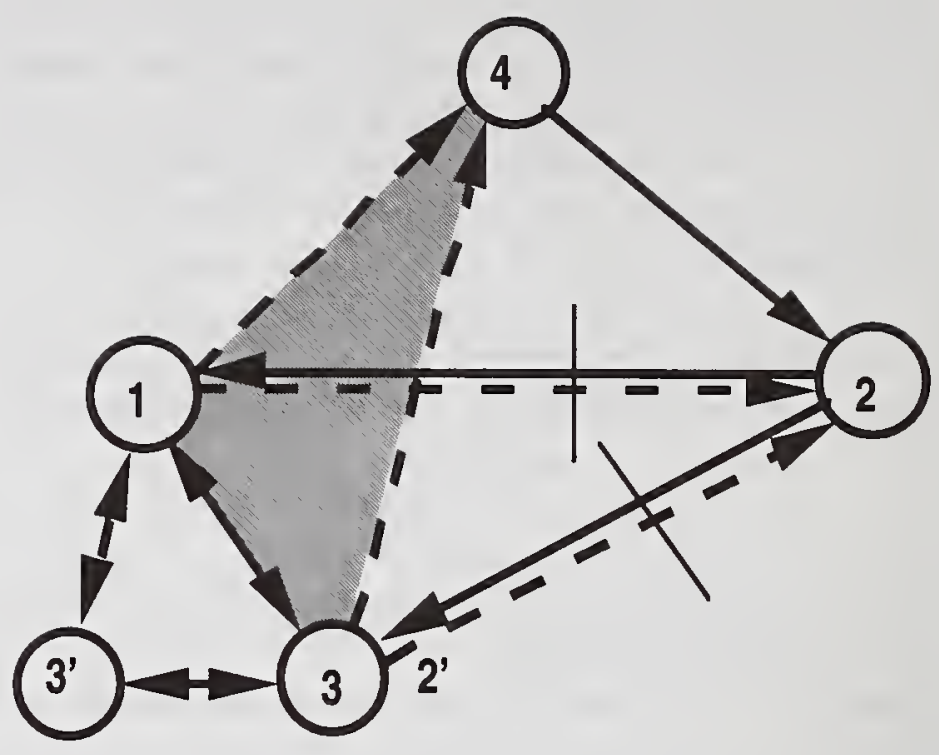

Figure 4. Representation of autonomous team evolution: a case of Coordinator's degraded functions

is affected by new subordination and coordination links, and becomes the Coordinator (2') on the new level. This.

$(4 \rightarrow 2)$ model corresponds for instance to generating enterprise associations and consortiums. Moreover both normal and degraded modes of virtual enterprise management processes may be modeled on the basis of FSU. Normal management process is associated with coordination loop $(1 \leftrightarrow 2 \leftrightarrow 3)$ and only episodic activation of $(4 \rightarrow 2)$ and $(2 \rightarrow 4)$ relations (Principle of Exceptional Subordinator's Intervention). Besides, the direct "customer-performer" $1 \rightarrow 3$ and $3 \rightarrow 1$ are not activated too. Here a complete routing may usually have the form $(1 \rightarrow 2 \rightarrow 4 \rightarrow 2 \rightarrow 3 \rightarrow 2 \rightarrow 4 \rightarrow 2 \rightarrow 1)$.

Degraded mode may result, for example, from the conflicts between Coordinator and Customers, or Coordinator and Performer. In this case informing links $1 \rightarrow 4$ or $3 \rightarrow 4$ may be used, and as a rule the Subordinator intervention. expressed by $(4 \rightarrow 1)$ and $(4 \rightarrow 3)$ links is necessary. For instance, in case of conflict between the Customer and the Coordinator, the Subordinator can temporarily perform the Coordinator functions and hence activate the loop (4314). Moreover he can replace the Coordinator 2 by some other agent 2'. For instance, the Subordinator may entrust the Coordinator functions 2 to Ex-Performer 3 who finds a new Performer that corresponds to horizontal evolution of enterprise FSU via 3(2' transformations). These situations are depicted in Figure 4.

\section{CONCLUSION}


A special language for intelligent enterprise evolutionary modeling is being constructed on the basis of the introduced formal representation. It will contribute to the implementation of agent-oriented approach to enterprise reengineering related to the development and use of new integrated software tools combining the capacities of groupware and liveware.

\section{REFERENCES}

[1] Winograd, T., Flores, F. Understanding Computers and Cognition: a New Foundation For Design. Norwood: Ablex, 1986.

[2] Minsky, M. The Society of Mind. New York: Simon and Shuster, 1986.

[3] Russell, S., Norvig, P. Artificial Intelligence: a Modern Approach. Englewood Cliffs, NJ: Prentice Hall, I995.

[4] Minsky, M. "Logical vs. Analogical or Symbolic vs. Connectionnist or Neat vs. Scruffy", in Artificial Intelligence at MIT: Expanding Frontiers, P.H.Winston and S.A.Shellard (Eds.). Cambridge Massachusetts: The MIT Press, 1990, pp.219-243.

[5] Fanabashi, M., Maeda, A., Morooka, Y., Mori, K. "Fuzzy and Neural Hybrid Expert Systems: Synergetic AI", IEEE Expert, August 1995, pp.3240.

[6] Keller, J.M., Yager, R.R., Tahani, H. "Neural Network 1mplementation of Fuzzy Logic", Fuzzy Sets and Systems, Vol. 45, n 1, pp.1-12, 1992.

[7] Kosko, B. Neural Networks and Fuzzy Systems. Englewood Cliffs: Prentice Hall, 1992.

[8] Buckley, J.J, Hayashi Y. "Neural Nets for Fuzzy Systems", Fuzzy Sets and Systems, 1995, vol.71, pp.265-276, I995.

[9] Zadeh, L.A. "Fuzzy Logic, Neural Network -and Soft Computing", Communications of the ACM, vol.37, $\mathrm{n}^{\circ} \cdot 3, \mathrm{pp} .77-84,1994$.

[10] Pedrycz, W., Lam, C.W., Rocha, A. "Distributed Fuzzy System Modelling", IEEE Transactions on Systems, Man and Cybernetics, vol.25, $n^{\circ}$ 5, pp. 769-780, 1995,

[11] Kuznetsov, O.F. "Non-Classical Paradigms in AI", Control Theory and Systems, $n^{\circ}$ 5, pp.3-23, 1995 (in Russian - translated into English by InterPeriodics publishers)

[12] Zimmermann, H.-J. "Recent Developments in Fuzzy Logic and Intelligent Technologies", in Proceedings of the 1st International Workshop "Fuzzy Logic and Intelligent Technologies in Nuclear Sciences" (FLINS'94, Mol, Belgium, September 1994), Ruan Da et al., ed., 1994, p p. 3-7.

[13] Pospelov, D.A., Averkin, A.N., Tarassov, V.B. "Soft Computing, Concurrent Engineering, What Else?", in Proceedings of IFSA'95 World Congress (Sao Paulu, Brazil, July, I995), I995, p p. 361-363.

[14] Ahson, S. "Petri Net Models of Fuzzy Neural Networks", IEEE Transactions on Systems, Man and Cybernetics, vol.25, $\mathrm{n}^{\circ}$ 6, pp.926-933, 1995.

[15] Demazeau Y., Muller J.-P. (Eds.). Decentralized Artificial Intelligence. Amsterdam: Elsevier North-Holland, 1990.

[16] Ferber, J. Les Systemes Multi-Agents. Vers une Intelligence Collective. Paris: InterEditions, 1995.

[17] Heudin, J.-C. La Vie Artificielle. Paris: Hermes, 1994

[18] Kirsh, D. "Foundations of AI: the Big Issues", Artificial Intelligence, vol.47, n¹/3, pp. 3-30, I 991 .

[19] Tarassov, V.B. "From Artificial Intelligence to Artificial Life: New Branches in the Sciences of Artificial", Artificial Intelligence News, $n^{\circ} 4$, pp.93-117, 1995 (in Russian).

[20] Brooks, R. "Intelligence Without Representation", Artificial Intelligence, vol.47, pp.139-I59, 1991.
[21] Theasby, P.J. "The Virtues of Virtual Reality", GEC Review, vol.7, n³, pp.131-145, 1992.

[22] Bond, A., Gasser, L. (Eds.). Readings in Distributed Artificial Intelligence. New York: Morgan Kaufman, 1988.

[23] Avouris, E., Gasser L. (Eds.). Distributed Artificial Intelligence: Theory and Praxis. Amsterdam: Kluver Academic Publishers, 1992.

[24] Prigogine, 1. From Being to Becoming. San Francisco: Freeman, 1980.

[25] Bobrow, D. "Dimension of Interaction: AAAI-90 Presidential Address", Al Magazine, vol.12, n³, pp.64-80, 1990.

[26] von Neumann, J. Theory of Self-Reproducing Automata. Urbana: University of Illinois Press, 1966.

[27] Kolmogorov, A.N. "Automata and Life", in What Is Possible and Impossible in Cybernetics. Moscow: USSR Academy of Sciences Press, 1964 (in Russian).

[28] Chaitin, G.J., "Toward a Mathematical Definition of Life", in Maximum Entropy Formalism", R.D.Levine and M.Tribus (Ed.), Cambridge Ma: MIT Press, 1979, pp. 477-498.

[29] Fogel, L.J., Owens A.J., Walsh M.J. Artificial Intelligence Through Simulated Evolution. New York: John Wiley and Sons, 1966.

[30] Holland, J.H. Adaptation in Natural and Artificial Systems. Ann Arbor: The University of Michigan Press, 1975.

[3I] Langton C. (Ed.). Artificial Life. New York: Addison-Wesley, 1988.

[32] Langton, C., Shimohara, T. (Eds.). Artificial Life V. Cambridge MA: MIT PRESS, 1996.

[33] Piaget, J. Biologie et Connaissance: Essai sur les Relations entre les Regulations Organiques et les Processus Cognitifs . Paris: Gallimard, 1967.

[34] Pospelov, D.A. Situative Control. Moscow: Nauka, 1986 (in Russian). [35] Meystel, A. Semiotic Modeling and Situatiion Analysis. Bala Cynwyd PA: AdRem Inc., 1995.

[36] Tarassov, V.B. "Artificial Meta-Intelligence: a Key to Enterprise Reengineering", in Proceedings of the Second Joint Conference on Knowledge Based Software Engineering (JCKBSE'96, Sozopol, Bulgaria, September 1996). Sofia: BAAI, pp. 15-24.

[37] Davidow, W., Malone, M. The Virtual Corporation: Structuring and Revitalizing the Corporation for the 21st Century. New York: Harper Business, 1992.

[38] Goldman, S.L., Nagel, R.N., Preiss K. Agile Competitors and Virtual Organizations: Strategies for Enriching the Customer. New York: Van Nostrand Reinhold, 1995.

[39] Volkov, A.M., Mikadze, Y.V., Solntseva, G.N. Activity: Structure and Regulation. Moscow: Moscow State University Press, 1987 (in Russian). 

VII ORDINAL OPTIMIZATION 


\title{
ORDINAL OPTIMIZATION FOR A CLASS OF COMBINATORIALLY HARD PROBLEMS
}

\author{
Christos G. Cassandras \\ Dept. of Manufacturing Engineering \\ Boston University \\ Boston, MA 02215 \\ cgc@enga.bu.edu \\ Liyi Dai \\ Dept. of Systems Science and Mathematics \\ Washington University \\ St. Louis, MO 63130 \\ liyi@zach.wustl.edu \\ Christos G. Panayiotou \\ Dept. of Electrical and Computer Engineering \\ University of Massachusetts \\ Amherst, MA 01003 \\ panayiot@ecs.umass.edu
}

\begin{abstract}
We consider a class of discrete resource allocation problems which are hard due to the combinatorial explosion of the feasible allocation search space. In addition, if no closed-form expressions are available for the cost function of interest in a stochastic environment, one needs to estimate the cost function through direct on-line observation or through simulation. We will present an optimization scheme driven by ordinal comparisons of cost estimates and show that it converges in probability to the global optimum. An important feature of this scheme is that it exploits the fast convergence properties of ordinal comparisons, as well as eliminating the need for "step size" parameters whose selection is always difficult in optimization algorithms. An application to a stochastic discrete resource allocation problem is included.
\end{abstract}

\section{EXTENDED SUMMARY}

Discrete optimization problems often arise in the context of resource allocation. In the basic model we consider here, there are $K$ identical resources to be allocated over $N$ user classes so as to optimize some system performance measure (objective function). Let the resources be sequentially indexed so that the "state" or "allocation" is represented by the $K$-dimensional vector $s=\left[s_{1}, \ldots, s_{K}\right]$ where $s_{j} \in\{1, \ldots, N\}$ is

This work was supported in part by the National Science Foundation under grants EEC-95-27422 and ECS-9624279, by AFOSR under contract F49620-95-1-0131, and by the Air Force Rome Laboratory under contract F30602-95-C-0242 the user class index assigned to resource $j$. Let $S$ be the finite set of feasible resource allocations

$$
S=\left\{\left[s_{1}, \ldots, s_{K}\right]: s_{j} \in\{1, \ldots, N\}\right\}
$$

where "feasible" means that the allocation may have to be chosen to satisfy some basic requirements such as stability or fairness. Let $L_{i}(\mathbf{s})$ be the class $i$ cost associated with the allocation vector $\mathbf{s}$. The class of resource allocation problems we consider is formulated as:

$$
\min _{s \in S} \sum_{i=1}^{N} \beta_{i} L_{i}(\mathbf{s})
$$

where $\beta_{i}$ is a weight associated with user class $i$. (RA) is a special case of a nonlinear integer programming problem and is in general NP-hard. However, in some cases, depending upon the form of the objective function (e.g., separability, convexity), efficient algorithms based on finite-stage dynamic programming or generalized Lagrange relaxation methods are known (see [1] for a comprehensive discussion on aspects of deterministic resource allocation algorithms). Alternatively, if no a priori information is known about the structure of the problem, then some form of a search algorithm is employed (e.g., Simulated Annealing, Genetic Algorithms).

In general, the system we consider operates in a stochastic environment; for example, users may request resources at random time instants or hold a particular resource for a random period of time. In this case, $L_{i}(\mathbf{s})$ in (RA) becomes a random variable and it is usually replaced 
by $E\left[L_{i}(\mathrm{~s})\right]$. Moreover, we wish to concentrate on complex systems for which no closed-form expressions for $\left.L_{i}(\mathbf{s})\right]$ or $E\left[L_{i}(\mathbf{s})\right]$. are available. Thus, $E\left[L_{i}(\mathbf{s})\right]$. must be estimated through Monte Carlo simulation or by direct measurements made on the actual system. Problem (RA) then becomes a stochastic optimization problem over a discrete state space.

While the area of stochastic optimization over continuous decision spaces is rich and usually involves gradient-based techniques as in several well-known stochastic approximation algorithms [2]-[3], the literature in the area of discrete stochastic optimization is relatively limited. Most known approaches are based on some form of random search with the added difficulty of having to estimate the cost function at every step. Such algorithms have been recently proposed by Yan and Mukai [4] and Gong et al [5]. Another recent contribution to this area involves the ordinal optimization approach presented in [6]. Among other features, this approach is intended to exploit the fact that ordinal estimates are particularly robust with respect to estimation noise compared to cardinal-estimates; that is, estimating the correct order of two costs based on noisy measurements is much easier than estimating the actual values of these costs. The implication is that convergence of such algorithms is substantially faster. These recent contributions are intended to tackle stochastic optimization problems of arbitrary complexity, which is one reason that part of the ordinal optimization approach in [6] includes a feature referred to as "goal softening". On the other hand, exploiting the structure of some resource allocation problems can yield simpler optimization schemes which need not sacrifice full optimality. This is the approach we take in tackling problems of the form (RA)

In this work, we first consider the deterministic version of problem (RA) and provide a necessary and sufficient condition for global optimality, based on which we develop an optimization algorithm. We analyze the properties of this algorithm and show that it yields a globally optimal allocation in a finite number of steps. We point out that, unlike resource allocation algorithms presented in [1], an important feature of the proposed algorithm is that every allocation in the optimization process remains feasible, so that our scheme can be used on line to adjust allocations as operating conditions (e.g., system parameters) change over time. Next, we address the stochastic version of the resource allocation problem. By appropriately modifying the deterministic algorithm, we obtain a stochastic optimization scheme. We analyze its properties treating it as a Markov process and prove that it converges in probability to a globally optimal allocation under mild conditions.

Details are provided in a full paper [7]. Here, we limit ourselves to pointing out two features of the resource allocation scheme we analyze which are worth noting because of their practical implications. All iterative reallocation steps are driven by ordinal comparisons, which, as mentioned earlier, are particularly robust with respect to noise in the estimation process. Consequently, (i) As in other ordinal optimization schemes, convergence is fast because short estimation intervals are adequate to guide allocations towards the optimal, and (ii) There is no need for "step size" or "scaling" parameters which arise in algorithms driven by cardinal estimates of derivatives or finite differences; instead, based on the result of comparisons of various quantities, allocations are updated by reassigning one resource with respect to the current allocation. This avoids the difficult practical problem of selecting appropriate values for these parameters, which are often crucial to the convergence properties of the algorithm.

\section{REFERENCES}

[1] Ibaraki, T., and Katoh, N., Resource Allocation Problems: Algorithmic Approaches, MIT Press, 1988.

[2] Kiefer, J., and Wolfowitz, J., "Stochastic Estimation of the Maximum of a Regression Function", Annals of Mathematical Statistics, 23, pp. 462-466, 1952.

[3] Robbins, H., and Monro, S., "A Stochastic Approximation Method", Annals of Mathematical Statistics, 22, pp. 400-407, 1951.

[4] Yan, D., and Mukai, H., "Stochastic Discrete Optimization", SIAM Journal on Control and Optimization, 30, 1992.

[5] Gong, W.B., Ho, Y.C., and Zhai, W., "Stochastic Comparison Algorithm for Discrete Optimization with Estimation", Proc. of 31st IEEE Conf. on Decisiona nd Control, Dec. 1992.

[6] Ho Y.C., Sreenivas, R.S., and Vakili, P., "Ordinal Optimization in DEDS", J. of Discrete Event Dynamic Systems: Theory and Applications, Vol. 2, pp. 61-88, 1992.

[7] C.G. Cassandras. L. Dai, and C.G. Panayiotou, "Ordinal Optimization for Deterministic and Stochastic Discrete Resource Allocation," to appear in Proc. of 35th Conference on Decision and Control, Dec. 1997. 


\section{Three Techniques for Ordinal Optimization: Short Simulation Runs, Crude Analytical Models, and Imprecise Simulation Models}

\author{
Jeffrey E. Wieselthier \\ Information Technology Division \\ Naval Research Laboratory \\ Washington, DC 20375 \\ wieselthier@itd.nrl.navy.mil
}

\author{
Craig M. Barnhart* \\ TRW Data Technologies Division \\ 16201 Centretech Parkway \\ Aurora, CO 80011 \\ Craig.Barnhart@trw.com
}

\author{
Anthony Ephremides \\ Electrical Engineering Dept. and \\ Institute of Systems Research \\ University of Maryland \\ College Park, MD 20742 \\ tony @ eng.umd.edu
}

\begin{abstract}
In the study of discrete-event dynamic systems (DEDS), accurate analytical models are generally either unavailable or too complex to evaluate numerically. Thus, the determination of optimal control policies often entails the simulation of many alternative systems in parallel. To reduce the computational burden, we investigate the use of ordinal optimization. This approach focuses on determining control policies that perform relatively well (although not necessarily optimally), without necessarily obtaining accurate performance measures.

In this paper we study three techniques for the ordinal optimization of DEDS, namely the use of short simulation runs, crude analytical models, and imprecise simulation models.
\end{abstract}

KEYWORDS: optimization, ordinal optimization, simulation, standard clock

\section{INTRODUCTION}

The motivation underlying ordinal optimization [1] is that finding the optimal solution (or control policy) is often too costly or time consuming, although a suboptimal solution (that may be found quite easily) may provide sufficiently good performance.

In this paper we study three techniques for the ordinal optimization of DEDS. First, we demonstrate that remarkably accurate performance rankings can be obtained on the basis of short simulation runs. In particular, for our example of admission control in communication networks, we demonstrate that the high degree of correlation introduced by the Standard Clock parallel simulation technique provides considerably better performance (i.e., faster convergence to accurate rankings) than the use of Common Random Numbers. Our second approach is the use of crude analytical models that capture the crucial aspects of system behavior. Again, remarkably accurate policy rankings are achieved (in this case without using simulation at all), even though such crude models provide poor estimates of actual performance; if accurate performance measures are needed, it is sufficient to simulate performance for only the best few policies determined in this manner. Our third approach is the use of imprecise simulation models that, like the crude analytical models, incorporate the salient features of the communication network model.

\footnotetext{
* C. M. Barnhart's contributions to this paper were made while he was at the Naval Research Laboratory.

This work was supported by the Office of Naval Research.
}

\section{THE STANDARD CLOCK TECHNIQUE}

The SC simulation technique $[2,3,4]$, which permits the simultaneous evaluation of system performance under a large number of control policies, is a primary component of our ordinal optimization techniques. Although the basic SC simulation technique is limited to exponential interevent times, the technique has been extended to some more general examples as well [5], [6], [7].

In SC simulation, each event is determined by two random numbers, one to specify the timing of the next event, and the other to specify its type. It is possible that an event determined in this manner turns out to be infeasible (e.g., a departure from an empty system). The interevent time of such a "fictitious" event is used to update the system time as if the event were "real" and did in fact occur, but no state change occurs (the fictitious event is discarded).

The improved efficiency of the SC method is achieved by using the resulting sequence of (interevent time, event type) pairs, known as the clock sequence, to simultaneously generate sample paths for a number of structurally similar, but parametrically different, systems. This reduction in the number of events that has to be generated has a dramatic effect on the overall simulation time because the generation of events is considerably more time consuming than the consequent updating of system state [7]. Furthermore, the use of a common event sequence introduces a high level of correlation among the parallel simulations, greatly accelerating the determination of good control policies by means of ordinal-optimization techniques, as we demonstrate in this paper.

\section{THE ADMISSION-CONTROL PROBLEM IN CIRCUIT-SWITCHED NETWORKS}

We have studied the problem of admission-control in circuit-switched, multihop, wireless networks. In our examples, a circuit is established over a predetermined path between the originator of a call and its destination node for the duration of the call. Contention-free channel access is implemented by means of frequency-division multiple access (FDMA); thus a necessary and sufficient condition for a call to be established is that a transceiver is available at every node in its circuit. The model is described in greater detail in [8] and [9].

Figure 1 shows an example of a ten-node wireless network that supports circuit-switched voice. The multihop paths corresponding to each of $J=5$ circuits are distinguished by different shadings. The dashed lines connect nodes that are within communication range of each other, but that do not support any of the five circuits. 


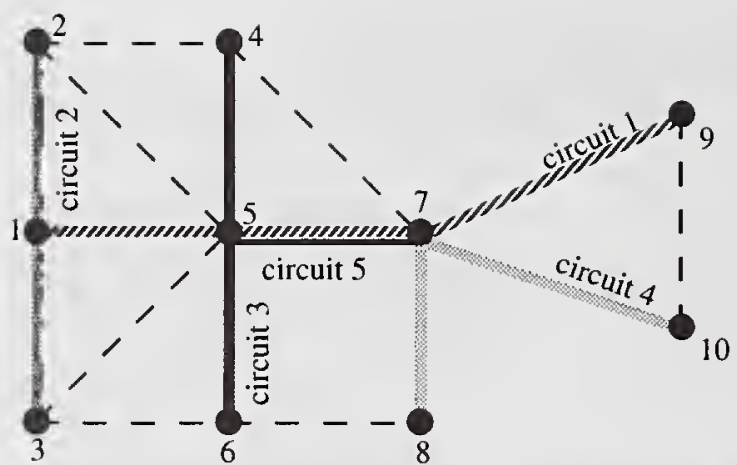

Fig. 1 - An example circuit-switched voice network.

Calls for circuit $j$ are generated according to a Poisson process with rate $\lambda_{j}$, and their durations are exponentially distributed with parameter $\mu_{j}$. A vector description of circuit $j$ in terms of the nodes it traverses is given by $c_{j}=\left\{c_{j}, c_{2}, \ldots\right.$, $\left.c_{j N}\right\}$, where

$$
c_{j i}=\left\{\begin{array}{l}
1, \text { if circuit } j \text { traverses node } i \\
0, \text { otherwise }
\end{array},\right.
$$

and $N$ is the number of nodes in the network. The state of the system with $J$ source-destination pairs, and hence $J$ circuits, is described by the vector $x=\left\{x_{1}, x_{2}, \ldots, x_{j}\right\}$, where $x_{j}$ is the number of calls currently active on circuit $j$, each of which is referred to as a call of type $j$. A central controller makes the decisions on whether or not to accept calls based on perfect knowledge of the number of calls of each type that are currently in progress (i.e., the system state $\boldsymbol{x}$ ), and hence the set of resources that are available for new calls. The transceivers needed to establish a circuit are acquired simultaneously when the call arrives, and are released simultaneously when the call is completed. Calls are blocked when one or more nodes along the path do not have a transceiver available, or when a decision is made not to accept a call despite the availability of transceivers. Blocked calls are lost from the system. These assumptions, coupled with the class of coordinate-convex admission-control policies discussed below, lead to a mathematically tractable description of system performance.

The number of transceivers at node $i$ is denoted by $T_{i}$. No more than $T_{i}$ calls can simultaneously use the resources at node $i$, i.e.,

$$
\sum_{j=1}^{J} x_{j} c_{j i} \leq T_{i}, \quad i=1, \cdots, N .
$$

These equations, which are termed the "system constraints," limit the state space $\Omega_{\mathrm{o}}$ in which $\boldsymbol{x}$ is allowed to take values. We refer to a system that is solely constrained by these system constraints as an "uncontrolled" system.

Network performance can often be improved by administering an admission-control policy that blocks some calls even though resources are available [9]. We define the admission-control policy, denoted by $\Omega$, in terms of a set of circuit "thresholds" and a set of "linear-combination constraints." Thresholds restrict the number of calls that will be admitted to the individual circuits, and can be expressed as

$$
x_{j} \leq X_{j}=\text { threshold on circuit } j, \quad j=1, \ldots, J .
$$

The linear-combination constraints are defined as

$$
\sum_{j \in S_{I}} x_{j} \leq Y_{I}=\begin{aligned}
& \text { threshold on total number of call types } \\
& \text { that are members of set } S_{I}
\end{aligned}
$$

for suitable subsets $S_{l}$ of the set of all call types. In particular, for the example of Fig. 1, three linear-combination constraints are imposed by node $5\left(x_{1}+x_{3} \leq Y_{1}, x_{1}+x_{5} \leq Y_{3}\right.$, and $x_{3}+x_{5} \leq$ $\left.Y_{4}\right)$ and two are imposed by node $7\left(x_{1}+x_{4} \leq Y_{2}\right.$ and $x_{4}+x_{5} \leq$ $\left.Y_{5}\right)$. Thus, the control policy is given by $\Omega=\left\{X_{1}, \ldots, X_{J}, Y_{1}\right.$, ... $\}$. Now the problem is the determination of the optimal admission-control policy $\Omega^{*}$, i.e., the values of the $X_{j}$ and the $Y$, that yield the optimal network performance.

The use of this form of control policy assures us that the state space is "coordinate convex" [10]. A coordinate-convex policy is specified in terms of the set of admissible states; completed calls are not blocked from leaving, on-going calls do not get rerouted, and new calls are admitted with probability 1 if the state to be entered is in the admissible region.

Under our assumption of Poisson arrival statistics, the use of coordinate-convex policies results in the so-called productform characterization of the system $[11,12]$. To determine the optimal policy, performance must be evaluated for many candidate policies. In view of the computationally intensive nature of the evaluation of product-form solutions, it is desirable to develop efficient simulation approaches for this problem.

\section{SC SIMULATION OF CIRCUIT- SWITCHED NETWORKS}

We have used SC techniques to evaluate the performance of the circuit-switched network of Fig. 1 under a number of different admission-control policies. The discrete parameters of interest in this case are the circuit thresholds $X_{j}$ and the linear-combination constraints $Y_{l}$. The events that must be generated are arrivals and departures. An arrival to circuit $j$ is denoted by $a_{j}$, and occurs at a rate of $\lambda_{j}$. The departure rate for each active call on circuit $j$ is $\mu_{j}$; thus, if there are $x_{j}$ active calls on circuit $j$, the departure rate for calls of type $j$ is $x_{j} \mu_{j}$. This situation is translated to events in the simulation as follows. We define $X_{j \max }$ to be the maximum value $X_{j}$ can have over the entire set of policies. Thus

$$
X_{j \max }=\min _{i \ni c_{j i}=1}\left(T_{i}\right) .
$$

Because there may be up to $X_{\text {jmax }}$ active calls on circuit $j$, we must consider $X_{\text {jmax }}$ different departure events $d_{j n}(n=1, \ldots$, $X_{\text {jmax }}$ ) for circuit $j$, namely

$$
\begin{aligned}
d_{j n} \Rightarrow & \text { feasible departure from circuit } j \text { if } x_{j} \geq n, \\
& \text { otherwise fictitious event. }
\end{aligned}
$$

Following the usual technique used in uniformization [13], the maximal rate of this system is

$$
\Lambda=\sum_{j=1}^{J}\left(\lambda_{j}+X_{j \max } \mu_{j}\right) .
$$

The upper part of Fig. 2 shows the "ratio yardstick" for the network of Fig. 1, where the maximum threshold on each circuit is three. To determine the event type, a random number, uniformly distributed over $[0,1]$, is generated. The event type (e.g., $a_{j}$ or $d_{j n}$ ) is determined by the interval into which the random number falls. ${ }^{1}$ This ratio yardstick corresponds to operation of the network at the maximal rate, and it can generate all events that are needed for the simulation of systems with control policies in which the circuit

\footnotetext{
${ }^{1}$ The computational effort involved in determining the event type by means of the alias method [14] is independent of the number of event types, thus making this method suitable for complex systems.
} 
thresholds do not exceed 3. In our example $\lambda_{j}=\mu_{i}=1$, which implies that all events are equally likely; therefore, all the intervals have the same width. All arrival events in this system are real, and the corresponding calls are accepted as long as their acceptance does not violate any of the system constraints or control constraints. However, departure events are fictitious if an insufficient number of calls of that type are currently active, as discussed above. For example, referring again to the upper part of Fig. 2, an event of type $d_{13}$ will be fictitious (and hence ignored, although time will be updated) if there are less than three calls of type 1 currently active.

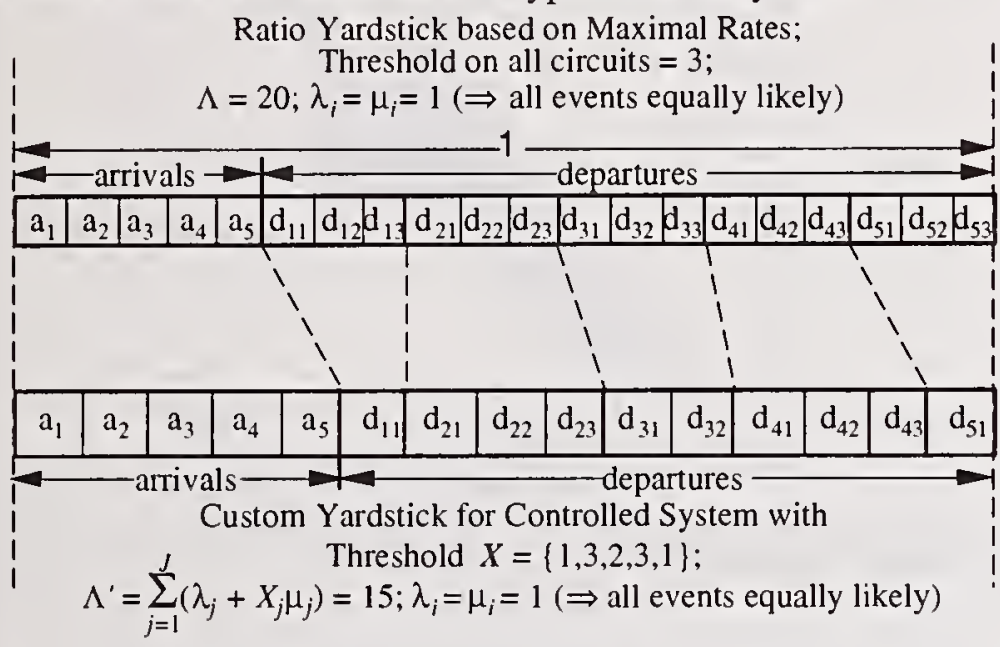

Fig. 2 - Ratio yardsticks for the circuit-switched network.

To reduce the number of fictitious events (and thus further improve the efficiency of the simulations) we have considered the use of "custom" ratio yardsticks, which are constructed to conform with the particular parameters of each sample path. The lower part of Fig. 2 shows a custom ratio yardstick for an example in which the circuit thresholds have been lowered to $\{1,3,2,3,1\}$. A number of events have been eliminated because they will always be fictitious, thus resulting in a reduction in the event rate from $\Lambda=20$ to $\Lambda^{\prime}=$ 15; e.g., with the threshold on circuit 1 reduced to 1 , the departure events $d_{12}$ and $d_{13}$ will always be fictitious. Clearly, by eliminating them from the ratio yardstick we can increase the percentage of real events. The remaining events are again equally likely, although there are only 15 of them instead of 20.

Despite the potential advantages of custom ratio yardsticks, we have found in our SC simulations that the added burden of maintaining and accessing separate custom yardsticks for every sample path outweighs the reduction in the number of fictitious events provided by their use [15]. Furthermore, the use of custom ratio yardsticks reduces the level of correlation among the sample paths, thus resulting in poorer ordinal rankings, as is shown in Section 5.2.4.

\section{ORDINAL OPTIMIZATION USING SHORT SIMULATION RUNS}

We have performed SC simulations of the network shown in Fig. 1, with the goal of determining the optimal policy, by using the model discussed in Sections 3 and 4. Eight transceivers are assumed present at each node in the network. Recall from Section 3 that a policy for this network can be written as $\Omega=\left\{X_{1}, \ldots, X_{5}, Y_{1}, \ldots, Y_{5}\right\}$. For $\lambda \mu=4.0,{ }^{2}$ we

${ }^{2}$ Blocking probability does not depend on the individual values of $\lambda$, and $\mu_{j}$, but only on the ratios $\rho_{j}=\lambda / \mu_{j}$. have simultaneously evaluated the 120 different control policies $\left\{X_{1}, 6,6,6, X_{5}, 8,8, Y_{3}, 8,8\right\}$, where $X_{1}, X_{5}=0, \ldots 6$; $Y_{3}=0, \ldots, 8 ; Y_{3} \leq X_{1}+X_{5} ; X_{1} \leq Y_{3} ;$ and $X_{5} \leq Y_{3}$.

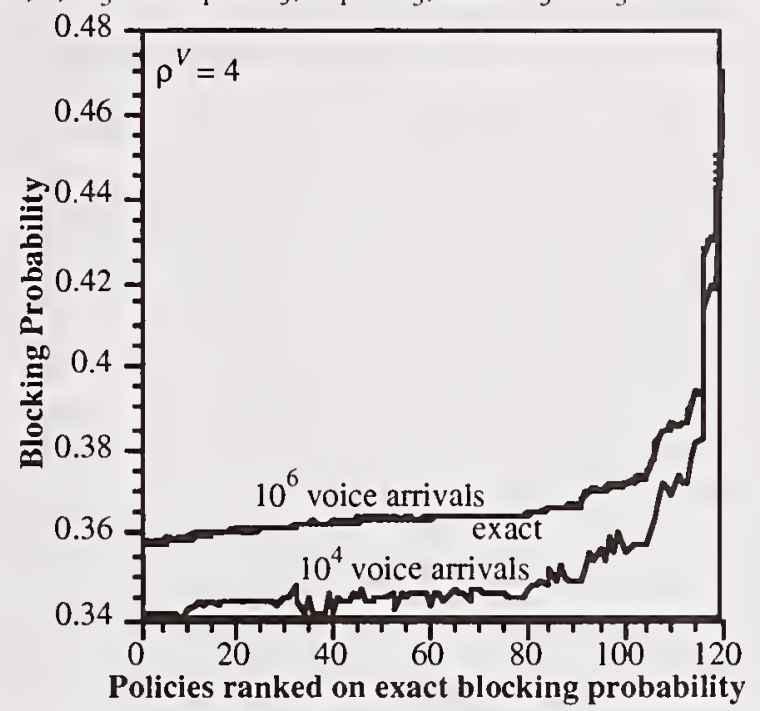

Fig. 3 - Probability of blocking across the range of policies.

\subsection{Evaluation of Voice-Call Blocking Probability}

In Fig. 3 we show the exact and simulated voice-call blocking probability associated with the 120 control policies. The exact results are determined numerically from the product-form solution, and simulated results are based on runs of $10^{4}$ and $10^{6}$ voice-arrival events. The horizontal axis is simply the ordering from the best (minimum blocking probability) policy to the worst, based on the exact model; thus blocking probability is a monotonically nondecreasing function of the horizontal axis. It is apparent from the closeness of the curves in Fig. 3 that the results of the longer simulation are extremely accurate; the simulation error is never more than $0.2 \%$. It is also apparent that the shorter simulation is not long enough to predict blocking probability accurately.

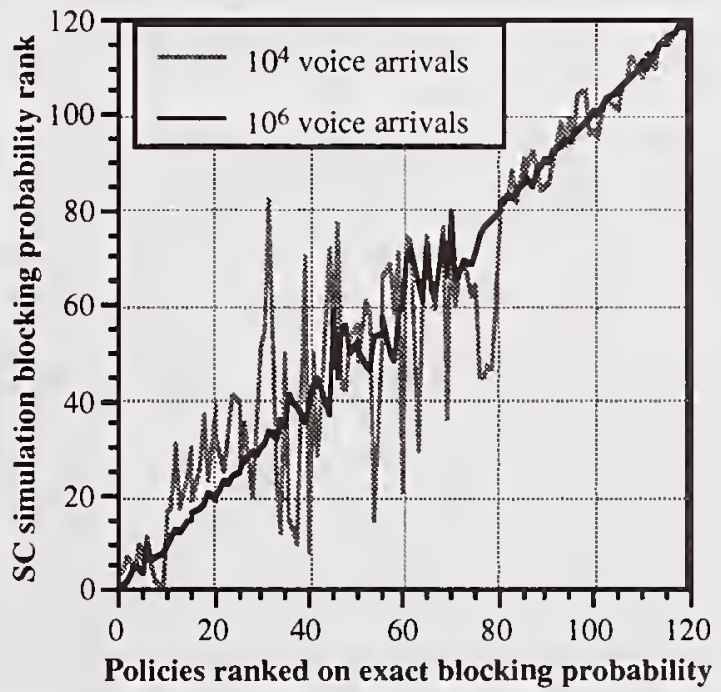

Fig. 4 - Blocking probability found in SC simulations compared to the exact value, $\rho=4$.

\subsection{Ordinal Ranking of Policies in Terms of Voice-Call} Blocking Probability

In Fig. 4 we compare ordinal rankings of the voice-call blocking probability obtained from two SC simulations to the exact rankings. The two SC simulations differed only in their durations, which were based on $10^{4}$ and $10^{6}$ voice-call arrivals. The ordinal ranking of the simulation results shows 
remarkable agreement with the exact ordinal ranking (ideally the curve would be a straight line with unit slope). For long simulations this is not surprising; however, it is remarkable that the short simulation placed eight of the top ten policies in the top ten positions. This agreement is achieved despite the insensitivity of blocking probability to the policy used. It is apparent from Fig. 3 that there is little sensitivity to the policy that is used when blocking probability is the only performance measure of interest. For example, as the policies are examined from the best to the $80^{\text {th }}$ out of 120 , the blocking probability increases by only a small amount, i.e., from 0.358 to 0.365 .

\subsection{The Impact of Common Event Sequences on Rankings}

The SC method uses a common event sequence for all control policies; thus it permits the direct comparison of the performance of these policies under the same input conditions. The benefits of the use of common event sequences for comparing policies, as compared to the use of independent brute force (BF) simulations, has been noted in $[14,16,17$, $18]$. It was shown in $[19,20]$ that the use of different randomnumber generator seeds can produce differences in performance in our problem that are considerably greater than those resulting from the use of different admission-control policies, especially for short simulation runs.

The failure of the independent BF simulations to provide good rankings for short simulation runs clearly demonstrates the benefits obtained by using a common event sequence. In our example, the use of independent event streams appears to require a simulation duration of about two orders of magnitude greater than that required for SC simulation to obtain comparable performance. Thus, in addition to the benefit of simulation speedup (in terms of the time required to simulate a specified number of events), the SC technique's use of a common event sequence introduces correlation into experiments so that accurate rankings are obtained early in the simulation. Therefore, highly accurate ordinal rankings can be obtained with much shorter simulations than would be required with independent $\mathrm{BF}$ simulation runs.

\subsection{The Use of Common Random Numbers, but Different Event Sequences}

In Section 4 we noted that the use of custom alias tables (custom ratio yardsticks) may, in some cases, permit higher efficiency in SC simulations because of the consequent reduction in the number of fictitious events. However, when custom alias tables are used, different events may be passed to different sample paths, even though the same random-number sequence is used to drive all of the parallel experiments. We now address the quality of the ordinal rankings that are obtained when common random numbers (CRN) are used in conjunction with custom alias tables to simulate the same example considered above for SC simulation with a common event stream and for independent BF simulations. In particular, we address the conjecture that the use of a common underlying random-number sequence can introduce beneficial correlation among the sample paths, even though the event sequences are different. It was recently demonstrated that the use of CRN maximizes the rate of convergence for ordinal comparison in systems that satisfy "positive quadrant dependence" 3 [21]; however, this condition is not satisfied in our problem.

Figure 5 shows the blocking probability and ordinal

\footnotetext{
${ }^{3}$ Two random variables $X \in R$ and $Y \in R$ are said to be positively quadrant dependent if $\operatorname{Prob}[X \geq x, Y \geq y] \geq \operatorname{Prob}[X \geq x] \operatorname{Prob}[Y \geq y]$.
}

rankings for simulations of length $10^{6}$ events for the present case of CRN simulations; results for six sample paths are shown. A comparison with curves for the case of independent experiments $[19,20]$ shows that little, if any, benefit is achieved by using the CRN method. The rankings appear to be comparable to those shown in Fig. 5 for the case of a simulation of duration $10^{4}$ events.

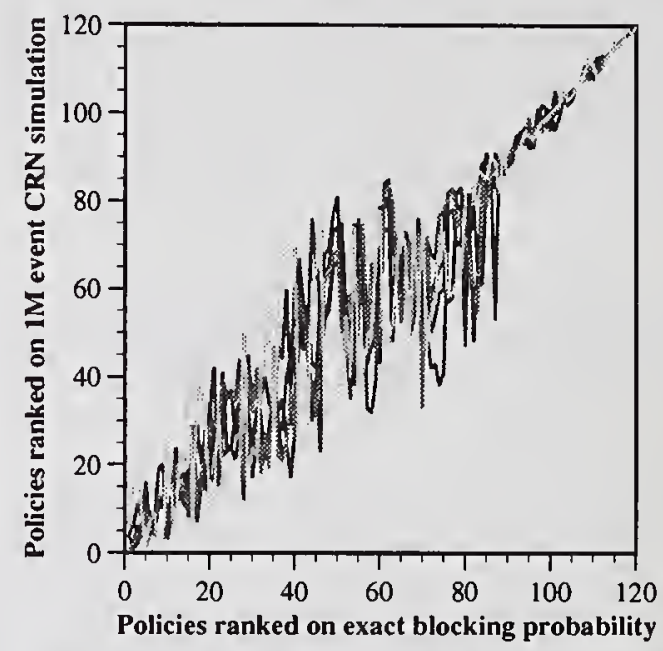

Fig. 5 - Ordinal rankings for CRN simulations with custom alias tables; six random-number generator seeds; simulation duration $=10^{6}$ events.

\subsection{A Performance Measure for Ordinal Rankings: The Spearman Rank Correlation Coefficient}

To facilitate the comparison of sets of rankings, it is helpful to use a quantitative measure of the quality of a set of rankings. To provide the basis for such a metric, let $\Xi_{i}$ be the performance measure value (in this case blocking probability) associated with policy $i(1 \leq i \leq N)$, based on a simulation run; and let $\Psi_{i}$ be the exact performance measure value associated with policy $i$ (where the index $i$ is arbitrary, i.e., not based on performance measure values). Thus the pair $\left(\Xi_{i}, \Psi_{i}\right)$ represents the simulated and exact performance values associated with policy $i$. Now define $R_{i}=\operatorname{rank} \Xi_{i}$ (where $R_{i}=$ 1 means that policy $i$ is the policy with the best value of $\Xi_{i}$ ) and $S_{i}=\operatorname{rank} \Psi_{i}$. One method of comparing these sets of rankings is the use of Spearman's rank correlation coefficient [22]

$$
r_{s}=1-\frac{6 \sum_{i}\left(R_{i}-S_{i}\right)}{N\left(N^{2}-1\right)},
$$

which provides a scalar measure of the "association" between two sets of rankings. If all of the simulated rankings are correct (i.e., $\left.R_{i}=S_{i}, 1 \leq i \leq N\right), r_{s}=1$.

Figure 6 shows the Spearman rank curves for the three approaches to simulation we have used, namely the commonevent, independent-event (BF), and CRN examples. Six sample paths are shown for each case to demonstrate the degree of variation among of the results. (The Spearman rankings for the CRN examples show much greater variation among the different random seeds than those for the commonevent and independent-BF examples.) The use of custom alias tables in SC simulations is detrimental, apparently because the differences introduced into the experiments reduces the correlation among them (as compared to the case of commonevent SC simulation), thus resulting in poor ordinal rankings of policies. To obtain comparable ordinal rankings, it may be necessary to run simulations that are several orders of magnitude greater in length than those required for common- 
event SC simulations. In that case, however, all of the efficiency gained through the reduction of fictitious events would be eliminated.

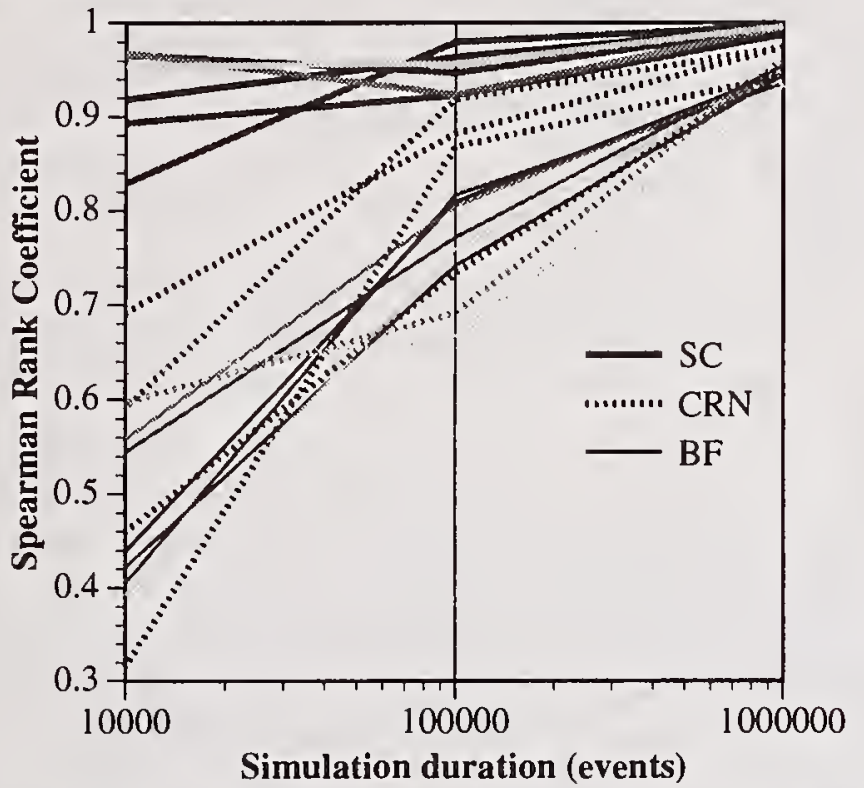

Fig. 6 - Quality of ordinal rankings of blocking probability, based on Spearman's rank coefficient, for SC, BF, and CRN simulations of various lengths.

\section{ORDINAL OPTIMIZATION USING CRUDE ANALYTICAL MODELS AND IMPRECISE SIMULATION MODELS}

In this section, we demonstrate the use of crude analytical models and imprecise simulation models for ordinal optimization. The particular problem we address is the determination of the voice-call admission-control policy that minimizes the delay of data packets in integrated voice/data networks, subject to a constraint on the blocking probability of voice calls. Data traffic, which consists of fixed-length packets that arrive according to exponential interarrival times, is added to the basic model of Fig. 1. The integrated network model is described fully in $[7,20]$, where it is demonstrated how fixed-length packets can be incorporated into the SC methodology.

The development of accurate data-packet delay models is a difficult problem. Data-packet delay depends not only on the statistics of the data-packet process itself, but also on the time-varying properties of the circuit-switched voice-call process. In particular, a data packet can be successfully transmitted only if a transceiver is available at the designated receiving node when the transmitting node transmits. The availability of a transceiver for reception depends on the current state of the voice-call process at that node because data traffic is permitted to use only those transceivers that are not currently supporting an active voice call. Nevertheless, we make the oversimplification of modeling the data-packet process at node $i$ as an M/D/1 queueing system, for which the delay (under admission-control policy $\Omega$ ) is

$$
\tilde{D}_{i}(\Omega)=\frac{\rho_{i}^{d}}{2 C_{i}\left(1-\rho_{i}^{d}\right)}
$$

where $C_{i}$ is the "residual capacity" of node $i$ (the expected number of transceivers available for data, i.e., not being used for voice traffic), $\rho_{i}^{d}$ is the expected normalized load at node $i$, and $\mu$ is set equal to 1 without loss of generality (i.e., implicitly, we assume that delay is measured in slots, where a slot is the time required by a transmitter to transmit one fixedlength data packet (all transceivers transmit at the same rate)). In the subsequent discussions, the delay metric we have used is the average nodal delay taken over all nodes.

This delay model is deficient in several ways. In addition to the fact that it ignores the need to pair transceivers at the transmitting and receiving nodes, it also ignores other critical aspects of network operation. Most significant is the implicit assumption that the number of data packets that are serviced per unit time at node $i$ is constant at $C_{i}$ (the fact that $C_{i}$ is not, in general, integer-valued is a minor point here), whereas the number of servers available in the real system varies, based on the voice state. An accurate estimate of delay would have to take into account not only the expected voice state (which determines residual capacity) as we do in our model, but also the fraction of time spent in each voice state as well as the statistics of the duration of each state visit. Thus, not surprisingly, the delay estimate based on this model is not accurate. However, we demonstrate shortly that this estimate does provide a remarkably accurate indication of the relative performance of a large number of different policies (and thus of their rankings), as demonstrated by SC simulations.

In Fig. 7 we compare the ordinal rankings obtained from four of our SC simulations of the integrated network to the ordinal rankings that were obtained by using the simple analytical $\mathrm{M} / \mathrm{D} / 1$ model for delay. We again consider the network of Fig. 2 with eight transceivers at each node, and examine the same 120 policies as in the voice-call blocking probability example; however, we plot our results only for the best 87 policies because the estimated delay based on the analytical $\mathrm{M} / \mathrm{D} / 1$ model is infinite for the remaining policies (the system is in saturation because the offered load is greater than the residual capacity at one or more nodes). Two of the simulations used the "receivers-assumed" model [7, 20], which neglects the need to verify that a transceiver is actually available at the intended receiving node. The other two used the more-accurate "receivers-verified" model, under which a data packet is not transmitted unless a transceiver is, in fact, available at the intended receiving node. In all cases, the duration of the simulation was $10^{6}$ voice arrivals.

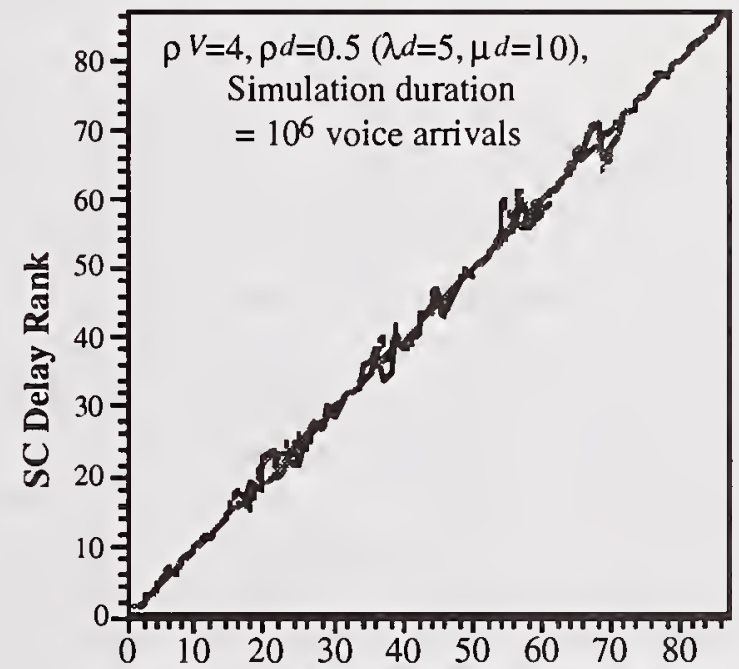

Policies Ranked on Analytical Delay Estimate

Fig. 7 - A comparison of the ranking of the policies by $\mathrm{SC}$ simulation and by the analytical delay estimate.

For all four runs shown, $\rho_{j}^{V}=\rho^{V}=4(j=1,2, \ldots, 5)$ and the loading on each of ten data queues is $\rho^{d}=0.5$. For each model (i.e., receivers-assumed and receivers-verified) we used two different expected voice-call durations $\left(\lambda^{V}=4.0, \mu^{V}=1.0\right)$ and $\left(\lambda^{V}=0.4, \mu^{V}=0.1\right)$. The data service rate $\mu^{d}$ was 10.0 in all the simulations, thus, the expected voice-call duration is 10 
times the data-packet length when $\mu^{V}=1.0$, and 100 times the data-packet length when $\mu^{V}=0.1$. Therefore, the total offered voice and data loads are the same in these simulations, but the parameter sets differ in the voice rates $\lambda^{V}$ and $\mu^{V}$. This is of interest because our analytical delay estimate, which is based on the product-form solution of the voice process, only considers the offered voice load (i.e., $\rho_{j}^{v}$ ). It does not account for the actual values of $\lambda^{V}$ and $\mu^{V}$. By keeping the offered load the same, and varying the voice rate (i.e., vary $\lambda^{V}$ and $\mu^{V}$ subject to $\lambda^{V} / \mu^{V}=4$ ), we can evaluate the impact of this simplification in the analytical delay estimate.

The $y$ axis represents the rank assigned to the policy ( $x$ axis) as a result of SC simulations. Again, a straight line with unit slope would indicate perfect agreement. The figure shows that the agreement for all four simulations, although imperfect, is impressively good; the four curves are virtually indistinguishable, and therefore they are not labeled. The deviation in ranking among the top 54 policies is never more than three. Furthermore it is interesting that, despite the significant differences between the simulation models (data receivers assumed vs. data receivers verified) and the voice rates $\left(\lambda^{V}=4\right.$ vs. $\left.\lambda^{V}=0.4\right)$, which produce significantly different values of delay (the difference can be several orders of magnitude $[7,20])$, the rankings are very similar among the different simulation runs. This is true even though, for the case of the receivers-verified model, all but the top four policies represent operation in the saturation region. Thus, we see that both the crude analytical model (i.e., the $M / D / 1$ model) and the imprecise simulation model (the receiversassumed model) produce highly accurate ordinal rankings, even though they provide poor estimates of actual performance.

\section{CONCLUSIONS}

A variety of approaches are available for ordinal optimization. Most of our work has been based on the use of the Standard Clock approach, which provides an efficient means to simulate structurally similar, but parametrically different, systems in parallel. With this technique, the ability to rapidly determine good ordinal rankings is a consequence of the use of a common event sequence to drive all of the parallel simulations. This common event sequence introduces a high level of correlation among the parallel experiments. In this paper we compared SC techniques to the use of the method of Common Random Numbers (CRN), which is implemented by using custom ratio yardsticks (and hence custom alias tables). We have observed that the use of the CRN approach provides little improvement over the use of independent brute force simulations, apparently because there is little correlation among the experiments.

We also demonstrated that crude analytical models and imprecise simulation models, which are incapable of providing acceptable estimates of system performance, nevertheless can be useful tools for ordinal optimization.

\section{REFERENCES}

[1] Y.-C. Ho, R. S. Sreenivas, and P. Vakili, "Ordinal Optimization of DEDS," Journal of Discrete Event Dynamic Systems, 2 pp. 61-88, 1992.

[2] P. Vakili, "Using a Standard Clock Technique for Efficient Simulation," Operations Research Letters, 10 pp. 445-452, 1991

[3] Y.-C. Ho, S. Li, and P. Vakili, "On the Efficient Generation of Discrete Event Sample Paths under Different Parameter Values," Mathematics and Computation in Simulation, 30 pp. 347-370, 1988.
[4] C. G. Cassandras, J.-I. Lee, and Y.-C. Ho, "Efficient Parametric Analysis of Performance Measures for Communication Networks," IEEE J. SAC, 8-No. 9 pp. 1709-1722, December 1990.

[5] Y.-C. Ho, C. G. Cassandras, and M. Makhlouf, "Parallel Simulation of Real Time Systems via the Standard Clock Approach," Mathematics and Computers in Simulation, 35 pp. 33-41, 1993.

[6] C.-H. Chen and Y.-C. Ho, "Extension of the Standard Clock Method for Discrete Event Simulation," submitted to IEEE Transactions on Control Systems Technology, 1993.

[7] J. E. Wieselthier, C. M. Barnhart, and A. Ephremides, "Standard Clock Simulation and Ordinal Optimization Applied to Admission Control in Integrated Communication Networks," Journal of Discrete Event Dynamic Systems: Theory and Applications, 5 pp. 243-279, April/July 1995.

[8] C. M. Barnhart, J. E. Wieselthier, and A. Ephremides, "An Approach to Voice Admission Control in Multihop Wireless Networks," Proceedings of IEEE INFOCOM'93, San Francisco, CA, pp. 246-255, March 1993.

[9] C. M. Barnhart, J. E. Wieselthier, and A. Ephremides, "Admission-Control Policies for Multihop Wireless Networks," Wireless Networks, 1-4 pp. 373-387, December 1995.

[10] J. M. Aein, "A Multi-User-Class, Blocked-Calls-Cleared, Demand Access Model," IEEE Transactions on Communications, COM-26-No. 3 pp. 378-385, March 1978.

[11] S. Jordan and P. Varaiya, "Control of Multiple Service, Multiple Resource Communication Networks," IEEE Transactions on Communications, 42-11 pp. 2979-2988, November 1994.

[12] S. Jordan and P. Varaiya, "Throughput in Multiple Service, Multiple Resource Communication Networks," IEEE Transactions on Communications, 39-No. 8 pp. 1216-1222, August 1991.

[13] H. C. Tijms, Stochastic Modelling and Analysis: A Computational Approach, Chichester: John Wiley \& Sons, 1986.

[14 P. Bratley, B. L. Fox, and L. E. Schrage, A Guide to Simulation, New York: Springer Verlag, 1987.

[15] J. E. Wieselthier, C. M. Barnhart, and A. Ephremides, "Efficient Simulation of DEDS by Means of Standard Clock Techniques: Queueing and Integrated Radio Network Examples," NRL/MR/5521-93-7392, Naval Research Laboratory, September 1993.

[16] P. Glasserman and P. Vakili, "Comparing Markov Chains Simulated in Parallel," Probability in the Engineering and Informational Sciences, 8-3, 1994.

[17] P. Glasserman and D. D. Yao, "Some Guidelines and Guarantees for Common Random Numbers," Management Science, 38-6 pp. 884-908, June 1992.

[18] P. Heidelberger and D. I. Iglehart, "Comparing Stochastic Systems Using Regenerative Simulation with Common Random Numbers," Adv. Appl. Prob., 11 pp. 804-819, 1979.

[19] J. E. Wieselthier, C. M. Barnhart, and A. Ephremides, "Ordinal Optimization of Discrete-Event Dynamic Systems: A Comparison of Standard Clock and Common-Random-Number Methods," Proceedings of the 1997 Conference on Information Sciences and Systems (CISS), Johns Hopkins University, Baltimore, MD, March 1997.

[20] J. E. Wieselthier, C. M. Barnhart, and A. Ephremides, "Ordinal Optimization of Admission Control in Wireless Multihop Integrated Networks via Standard Clock Simulation," NRL Report NRL/FR/5521-95-9781, Naval Research Laboratory, August 1995.

[21] L. Dai and C.-H. Chen, "The Effect of Correlation on Ordinal Comparison of Discrete Event Dynamic Systems," Proceedings of the 35th Conference on Decision and Control, Kobe, Japan, pp. 3316-3321, December 1996.

[22] S. Kotz and N. L. Johnson, ed., Encyclopedia of Statistical Sciences, New York: Wiley, pp. 584-587, 1982. 


\title{
Selection Schemes in Ordinal Optimization and Their Computational Advantages ${ }^{\ddagger}$
}

\author{
T. W. Edward Lau ${ }^{\dagger}$ \\ Division of Engineering and Applied Sciences \\ Harvard University \\ Cambridge, MA 02138
}

\begin{abstract}
In this talk, we explain the advantages of selection-based search schemes from a theoretical perspective. The role of selection in tackling many difficult engineering problems has been empirically demonstrated with noticeable successes. This is also confirmed in the recent AI researches. We show that one can achieve impressive gain in computational efficiency when certain strategic definition of optimization goal is adopted. In this light, we propose a generalized optimization paradigm called ordinal optimizarion. which pertains to performance analysis of many computationally hard problems in a pragmatic sense.
\end{abstract}

KEYWORDS: stochastic optimization. sampling and selection, goal soflening, learning algorithms

\section{INTRODUC.TION}

The following optimization objective is often sought in various design problems:

$$
\min _{\epsilon \Theta} J(\theta)=\mathbf{E}[L(\theta)]
$$

where $\Theta$ is the design space in which $\theta$ is a design parameter, and $J(\cdot)$ is an expected value of performance measurements $L(\theta)$. While mathematically succinct, equation (1) is difficult to solve in many real-world design scenarios, e.g. intelligent systems, owing to the following challenges:

A. Search - the design space $\Theta$ is hugc and may have little structure to be exploited. Typical examples are problems in combinatorial optimization, which involves discrete or even symbolic variables. On top of the exponential growth in the size of search space, local properties such as gradient and convexity information may not be easily constructed.
B. Estimation - the functional $J(\cdot)$ has to be calculated via monte-carlo simulation or through crude approximating models. Lengthy and repeated computer experiments for averaging out $L(\theta)$ 's are needed in order to obtain quality answers. Theoretical limit on how fast statistical estimates can converge also makes simulation by itself an arguably insufficient means to solve statement (1) in view of limited computing budget.

Despite the above challenges, problems such as (1) are still faced by designers who are eager to obtain quality answers in a time-efficient and computationally affordable manner. Ordinal optimization, proposed by Ho et al. [1] (see also Ho [15]), aims at getting around the above mentioned challenges. In fact, ordinal optimization is a complementary technique which enhances the capabilities offered by existing search methods and estimation algorithms. We shall elaborate ordinal optimization in Section 2.

Sampling and search methods have recently gained attention in AI researches. Successes were reported in chess toumament, planning and scheduling applications [2], in control problems [3], and in the networking area [4]. Intelligent search schemes combined with the advent of faster and parallel computing technology provide a new avenue of problem solving possibilities. A pcrtinent idea is to iteratively learn about good solution "properties" by means of sampling the search space. These "properties" can be, for example, usefil solution representation, promising regions of the search space, or better hcuristics, etc. Genetic algorithms and reinforcement learning are techniques that embrace these ideas of iterative sampling and learning.

Meanwhilc, due to the imprecision or randomness involved in estimating design performances (e.g., in montecarlo experiments), it becomes necessary to devise selection methods so that learning can be effectively achieved through

\footnotetext{
${ }^{\ddagger}$ This work is partially supported by NSF grants EID-9212122, EEC 9402384, Army contracts DAAL03-92-G0115, DAAH04-950148 and Air Force contract F49620-95-1-0131.

${ }^{\dagger}$ Post-doctoral fellow, Division of Enginecring and Applied Sciences, Harvard University. Email: twel@arcadia.harvard.edu.
} 
evahuating the design samples. This motivates our main topic in this paper - selection schemes in ordinal optimization, which will be discussed in Section 3. Subsequently, we will connect the selection schemes to some ideas of iterative learning in Section 4.

\section{GENERALIZED SELECTION AND GOALS}

Consider a collection of $N$ design samples drawn from the design space. There is a true order of these $N$ designs with respect to the true performances. If this order were known, then we could easily pick out the best, the second best, etc., and the selection problem is essentially solved. However, the observed order of these $N$ designs is inevitably perturbed from the true order due to noisy evaluation of design performances. In particular, suppose the designer insists on getting one design which is the true best. Then lengthy evaluation is needed so that the resulting order of the $N$ designs would tum out the true best design showing up as the observed best. If the noise factor is large, then it is possible that the true best is displaced to the last position of the observed order of the $N$ designs. Therefore, we bring forth two major ideas in ordinal optimization:

- Ordinal comparison - it is much easier to decide whether " $A>B$ ?" than " $A-B=$ ?" In other words, one can estimate the observed performance order better than the magnitude of performance values. In fact, this has been formally established by Dai [5] and Xie [6], and extended by Dai and Chen [14]. Their results show that the probability of answering the former question correctly occurs exponentially fast along with the simulation horizon.

- Goal softening - instead of picking a single design to match the very best in a population, which is an unlikely event, one may soften the goal to settle on the top- $n \%$ of the population by selecting a subset of designs. Lee et al. [7] has studied this viewpoint using an order statistics formulation, and show that it also has exponential advantage in terms of correct selection.

These two tenets are manifested by a quantity called alignment probability, defined as

$$
P_{t}(g, s, k)=P(|\mathrm{G} \cap \mathbf{S}| \geq k, t)
$$

where $\mathbf{G}$ is the set of top-g true best designs (called good enough designs), and $\mathbf{S}$ is the set of top-s observed best designs, $k$ is called the alignment level and $t$ is the index for simulation horizon. Notice that in conventional optimization one often asks for $g=s=1$. The alignment probability of such a case is often too small to be of any interest. On the other hand, Dai [5] has shown that $1-P_{\mathcal{A}}(1,1,1)$, decays exponentially as the simulation proceeds, i.e., for some $\alpha>0$ (which depends on the $N$ design performances), we have

$$
1-P_{A}(1,1,1) \leq \exp (-\alpha t)
$$

Xie [6] has generalized the result for other values of $g, s$ and $k$. Meanwhile, in Lee et al. [7], it is shown that $1-P_{f}(g, s, 1)$ decreases exponentially as the sizes $g$ and $s$ are relaxed, i.e.,

$$
1-P_{f}(g, s, 1) \leq \exp (-\mathrm{fcn}(g, s, t)) .
$$

The implication of these results is that if one is willing to relax the goal and select a subset of design for further analysis or learning, then it is possible to

i) execute shorter simulation to tolerate large confidence intervals of performance estimates; and/or

ii) run crude or surrogate model that is computationally cheaper and more amenable to analysis.

These advantages translate into impressive computational saving and speed-up, and have been confirmed by various empirical studies (see [4] and [8] to [12]).

\section{EXPECTED ALIGNMENT LEVELS}

In this section, we look closer into the effects of goal softening. Given that the designer is willing to accept a good enough subset $\mathbf{G}$ and to select a subset $\mathbf{S}$ from short simulation or crude evaluation, a useful quantity to assist decision is the expected alignment level $\mathrm{E}_{t}[k]$ given by

$$
\mathbf{E}_{f}[k]=\sum_{k} k P(|\mathbf{G} \cap \mathbf{S}|=k ; t)
$$

It is not difficult to see that the expected alignment level is an increasing function of $g$ and $s$. In the absence of true performances, one has to estimate $\mathbf{E}_{t}[k]$ based on noisy observed performances. However, we shall demonstrate that this can be approximately obtained if the designer is able to estimate the type of underlying true performance profile and noise magnitude. The true performance profile will be referred to as the ordered performance curve (OPC). It is a nondecreasing curve of true performances plotted against the integral values of the true performance order. In general there are intinitely many possible form of OPCs but several types of $\mathrm{OPC}$ can be categorized, which are shown in Figure 1 below. See Lau and Ho [13] for detailed discussion regarding ordered performance curves. 


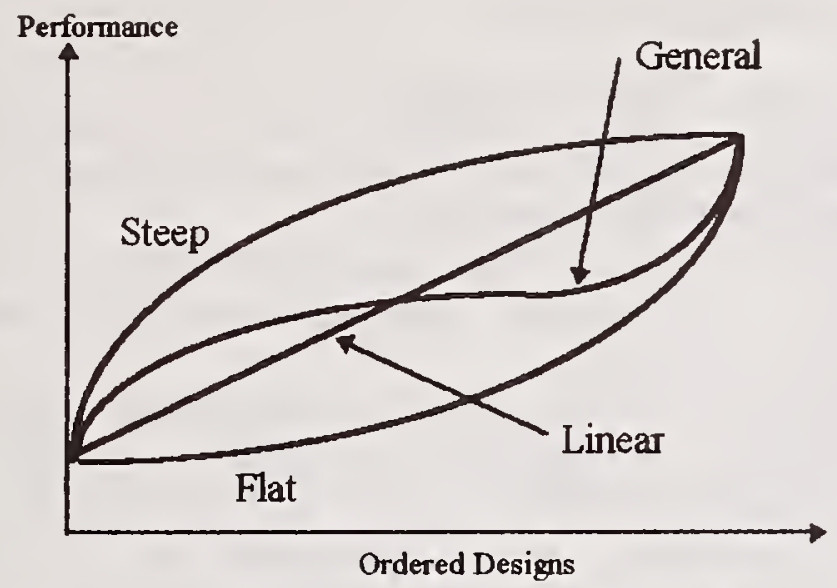

Figure 1. Different types of OPCs

For illustrative purpose, let us consider the case that all true performances are equally spaced apart, which is equivalent to a linear OPC of the form

$$
J\left(\theta_{i l}\right)=c \rho\left(\theta_{i j}\right)+c_{0}
$$

where $\theta_{i j}$ is the $i$ th true best design, and $\rho(\cdot)$ gives the true rank of a design. Notice that $\rho\left(\theta_{1,}\right)=i$. Clearly this relationship would not be available, for otherwise there would be no problem for selection in the first place. Consider that the observed performance values $J^{\prime}(\cdot)$ are generated by the additive noise model

$$
J^{\prime}\left(\theta_{[i]}\right)=J\left(\theta_{i[]}\right)+\omega\left(\theta_{i]}\right)
$$

where $\omega\left(\theta_{i j}\right)$ is the noise disturbance which we assume to be independent across designs. Typical picture of observed performances is shown in Figure 2.

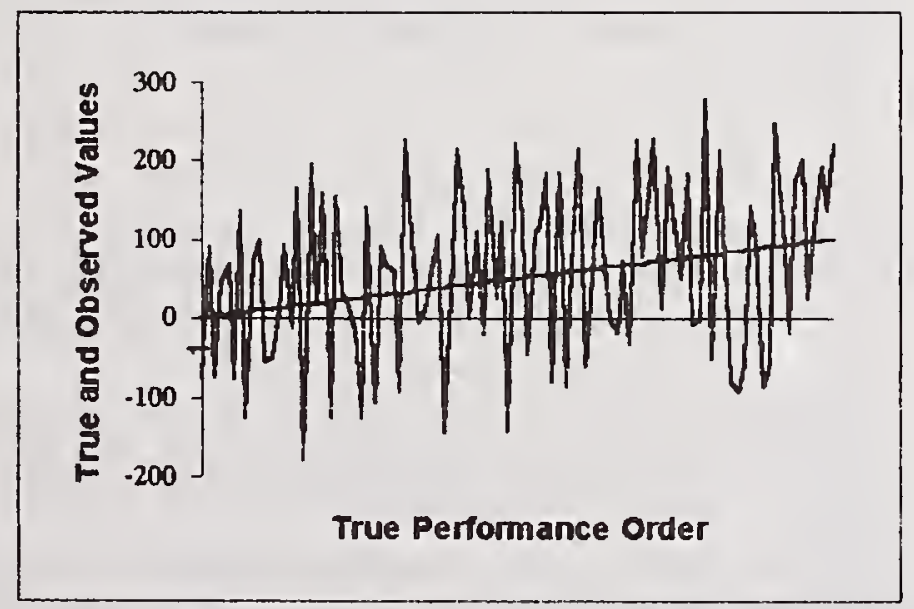

Figure 2. Design order is perturbed by noisy observations

Suppose the designer is able to give a rough guess that the underlying true performances are approximately uniformly separated (by means of short simulation, for example). Then, the expected alignment levels can be shown as in Figure 3a, 3b and $3 \mathrm{c}$ for signal-to-noise ratios 1:5, 1:2 and 1:1 respectively. ${ }^{1}$ These diagrams are generated based on a total of 1,000 design samples with linear OPC and independent uniform noise density $[-W, W]$.

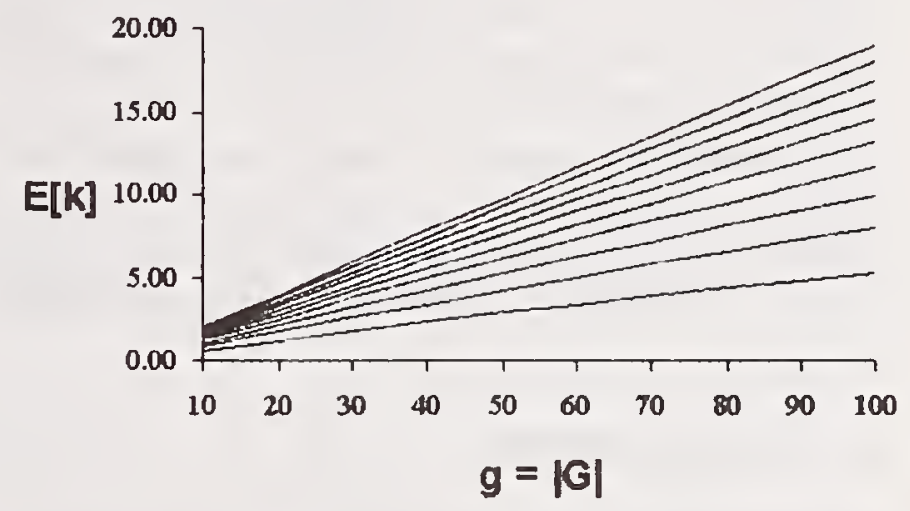

Figure 3a. $\mathrm{E}[k]$ vs. $g$ for $s=10$ to 100 with linear OPC and signal-to-noise ratio 1:5.

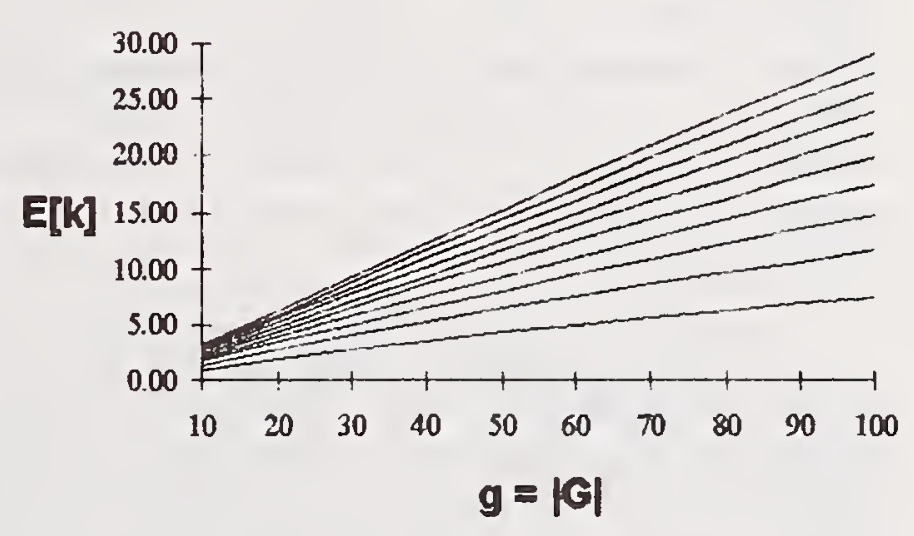

Figure $3 \mathrm{~b}$. $\mathbf{E}[k]$ vs. $g$ for $s=10$ to 100 with linear OPC and signal-to-noise ratio 1:2.

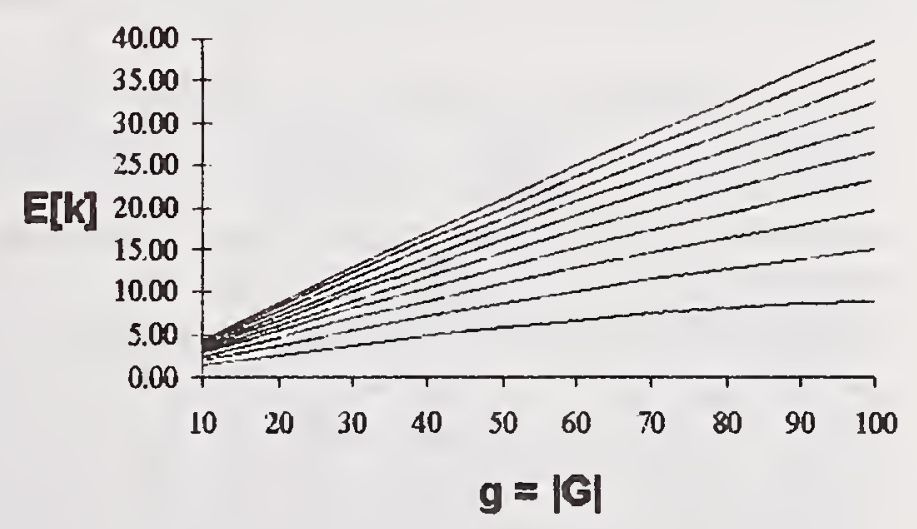

Figure 3c. E. $[k]$ vs. $g$ for $s=10$ to 100 with linear OPC and signal-to-noise ratio 1:1.

\footnotetext{
${ }^{1}$ Signal-to-noise ratio is defined as the ratio between the range of true performances to the range of noise values.
} 
Let us interpret these diagrams. In the presence of very large noise, such as $1: 5$, if one is willing to relax the goal to $g=50$ designs, then one can expect about 5.5 designs in a selection of $s=50$ desions. If more simulation is performed and signal-to-noise ratio is reduced to $1: 2$, then on average about 10.5 of the top-50 true best designs are found in the top50 observed best designs. Notice that should the designer insist on getting $g=s=1$, then much longer simulation is needed in order to get the selected best to be indeed the true best. The computational advantagc hore is several foldcd.

\section{DISCUSSIONS}

We have seen in the previous section that softening the goal of selection provides practical savings in the simulation budget. The selection schcmc is based on obscrvcd pcrformanccs and thus the name Horse Race selection rule is coined. Other selection rules are also possible and generally produce different alignment results. The study of various selection rules is an on-going rescarch topic. Besides thc above illustrative results, we also have extensive calculations based on a variety of types of OPC and noise ranges, also reported in Lau and Ho [13].

From an algorithmic point of view, with a selected subset that guarantees certain alignment of good enough designs, a designer can run longer simulation on this subset to lcarn about good "propertics" of solutions, as mentioncd in Section 1. The subsequent steps would be to develop adaptive search schemes that utilize as much as possible the knowledge learned from this selected subset. This sampling-selectionleaming approach laid out by ordinal optimization carrics important implications in the design of intelligent systems.

Furthermore. ordinal optimization advocates a new paradigm to pcrform stochastic optimization for computationally difficult problems; namely, ordinal comparison and goal softening. It serves as a complementary methodology to existing approaches such as genetic algorithms, reinforcement learning, other artificial intclligcnce techniques, and nonetheless conventional procedures discussed in many optimization literature. We believe that this should warrant further research attention. In the theoretical development of ordinal optimization, we have current rcsults that tackle noise estimation, design-dependent noises and constrained optimization problems.

\section{REFERENCES}

[1] Ho, Y.-C., R. Sreenivas, and P. Vakili, "Ordinal Optimization of Discrete Event Dynamic Systems,", J. DEDS, vol. 2(2), pp. 61-88, 1992.

[2] Ginsbcrg, M. L., "Do Computcrs Nced Common Scnse?" Proc. of Knowledge Representation and Reasoning Conference, 1996.

[3] Deng, M., and Y.-C. Ho, "Sampling-Selection Method For Stochastic Optimization Problcm," submittcd to AUTOMATICA, 1995.

[4] Wieseltheir, J. E., C. M. Bamhart, and A. Ephremides, "Ordinal Optimization of Admission control in Wireless Multihop Voicc/Data Nctwork via Standard Clock Simulation," J. DEDS, Vol.5, \#̈2-3, 1995.

[5] Dai, L.-Y., "Convergence Properties of Ordinal Comparison in the Simulation of Discrete Event Dynamic Systems," JOTA, Vol. 91(2), pp. 363-388, 1996.

[6] Xie, X., "Dynamics and convergence rate of ordinal comparison of stochastic discrete event sy'stems," IEEE Trans. on Automatic Control, vol. 42(4), pp. 586-590, 1997.

[7] Lec, L.-H, T.-W. E. Lau, and Y.-C. Ho, "Explanation of Goal Softening in Ordinal Uptimization," IEEE Trans. on Automatic Control, to appear, 1997.

[8] Patsis, N., C.-H. Chen, and M. Larson, "Parallel Simulation of DEDS," IEEE Trans. on Control Technology, 1996.

[9] Ho, Y.-C., and M. Larson, "Ordinal Optimization and Rare Event Probability Simulation," J. DEDS, vol. 5, \#2-3, 1995.

[10] Xie, X.-L., "An Ordinal Optimization Approach to a Tokcn Partition Problcm for Stochastic Timed Event Graphs," Proc. of the 1994 Winter Simulation Conference, Orlando, Florida, pp. 581-588, December 1994.

[11] Yang, M. S., L.-H. Lee, and Y.-C. Ho, "On Stochastic Optimization and Its Applications to Manufacturing," to appear in Lectures in Applied Mathematucs, pp. 317-331, AMS, 1997.

[12] Tang, Z. B., and Y.-C. Ho, "Modification of Adaptive Partitioncd Random Scarch," Proc. of 1995 Conference on Decision and Control, New Orleans, December 1995.

[13] Lau, T.-W. E., and Y.-C. Ho, "Universal Alignment Probabilities and Subset Selection for Ordinal Optimization," JOTA, Vol. 39(3), pp. 455-490, Junc 1997.

[14] Dai, L.-Y., and C.-H. Chen, "Rates of convergence of ordinal comparison for dependent discrete event dynamic systems," JOTA, Vol. 94(1), July 1997.

[15] Ho, Y.-C., "On the Numcrical Solution of Stochastic Optimization Problems," IEEE Trans. on Automatic Control, Vol. 42(5), 1997. 


\title{
Intelligent Computing Budget Allocation for
}

\section{Discrete Event Simulation and Optimization}

\author{
Chun-Hung Chen \\ Department of Systems Engineering, University of Pennsylvania, Philadelphia, PA 19104-6315 \\ Karen Donohue \\ Department of Operations and Information Management, The Wharton School, \\ University of Pennsylvania, Philadelphia, PA 19104-6315 \\ Liyi Dai \\ Department of Systems Science and Mathematics, Washington University, St. Louis, MO 63130 \\ Enver Yucesan \\ Technology Management Area, INSEAD, 77305 Fontainebleau Cedex, France
}

\begin{abstract}
Simulation plays a vital role in analyzing many discrete-event systems. Usually, using simulation to solve such problems can be both expensive and time consuming. We present an effective approach to intelligently allocate computing budget for discrete-event simulation. This approach can intelligently determine the best simulation lengths for all simulation experiments and significantly reduce the total computation cost to obtain the same confidence level. Numerical testing results are included. Also we compare our approach with traditional two-stage procedures. Numerical results show that our approach is much faster than the traditional two-stage procedures.
\end{abstract}

KEYWORDS: Discrete-event simulation, optimization, ranking and selection.

\section{WHY INTELLIGENT COMPUTING BUDGET ALLOCATION?}

It is often necessary to apply extensive simulation to design or efficiently manage large real-life systems such as communication networks, traffic systems, and automated

* This work was supported in part by the National Science Foundation under grant No. DMI-9322830 and grant No. ECS962479. manufacturing plants. Unfortunately, simulation can be both expensive and time consuming. Suppose we want to compare $k$ different discrete-event systems (designs or alternatives), we do $N$ simulation replications for all $k$ designs. (Without loss of generality, we consider terminating simulation in this paper. Our approach is also applicable to steady-state simulation although we need to have $N$ independent samples rather than $N$ independent simulation replications.) Therefore, we need $k N$ simulation replications. The simulation results become more accurate when $N$ increases. If the accuracy requirement is high ( $N$ is not small), and if the total number of designs in a design problem is not small ( $k$ is large), then $k N$ can be very large, which may easily make total simulation cost extremely high and preclude the feasibility of the simulation approach. How to effectively reduce computation cost to obtain a good decision is crucial to apply simulation.

Instead of equally simulating all $k$ designs, a more efficient way is to have different simulation replication numbers for different designs. The numbers of replications are determined using preliminary simulation information. A central issue is how to effectively utilize the available information and how to intelligently determine the best simulation replication numbers for al designs. Our goal is to significantly reduce simulation cost while obtaining the desired simulation quality. In fact, this question is equivalent to optimally decide which designs will receive computing budget for continuing simulation or to find an optimal way to reach an optimal design.

Dudewicz and Dalal (1975) develop a two-stage procedure for selecting the best design or a design which is very close to 
the best system. In the first stage, all systems are simulated with $n_{0}$ replications. Based on the results obtained from the first stage, we estimate how many more simulation replications for each design should be conducted in the second stage in order to reach the desired confidence level. Rinott (1978) presents another way to estimate the number of required simulation replications in the second stage. Many researchers have extended this idea to more general ranking and selection problems in conjunction with new developments. Among of them are Chiu (1974), Gupta and Panchapakesan (1979), Charnes (1991), Matejcik and Nelson (1993), Bechhofer, Santner, and Goldsman (1995), Futschik and Pflug (1996), and Hsu (1996).

With further development from Chen (1995), we will present an effective approach to intelligent allocate computing resource for discrete-event simulation. Also, we will compare our approach with the traditional two-stage procedures by conducting a numerical experiment. Numerical results show that our approach is more than ten times faster than the twostage procedures. We formulate the problem of intelligent computing resource allocation as a "optimal computing budget allocation" problem in the next Section. In Section 3, we present a simple sequential approach. We will show the numerical results in Section 4. Section 5 compares our method with the two-stage procedure. Section 6 concludes this paper.

\section{OPTIMAL COMPUTING BUDGET ALLOCATION}

Suppose that our goal is to select a design associated with the smallest mean performance measure among $k$ designs with unknown variances that are not necessarily equal. Further assume that the computing budget is limited and the number of designs is not small.

Denote

$k$ : the total number of designs,

$X_{i j}$ : the $j$ th i.i.d. sample of the performance measure from Design $i$,

$N_{i}$ : the number of simulation replications for Design $i$,

$\bar{\mu}_{i}$ : the sample mean for Design $i ; \bar{\mu}_{i}=\frac{1}{N_{i}} \sum_{j=1}^{N_{i}} X_{i j}$,

$\mu_{i}:$ the mean performance measure; $\mu_{i}=E\left(X_{i j}\right)$.

For steady-state simulation, Batch Means method (Schmeiser 1982) can be used if the simulation samples from any design are not independent. Again, we consider terminating simulation in this paper. Independence assumption is not a problem for practical applications.
When $N_{i}$ 's are large, $\bar{\mu}_{i}$ can be a good approximation for $\mu_{i}$, since, according to the law of large numbers, $p\left\{\bar{\mu}_{i} \rightarrow \mu_{i}\right\}$ $\rightarrow 1$, as $N_{i} \rightarrow \infty$. Given the fact that we can only do a finite number of simulation replications, $\bar{\mu}_{i}$ is simply an approximation to $\mu_{i}$. Using the approximation results to select the best design (without loss of generality, we consider minimization problems in this paper; thus the "best" design means the design with the smallest $\mu_{i}$ ), we have to ask what the probability of correct selection is. Correct selection can be defined by that a design with the smallest sample performance measure is actually the best design (the smallest population performance). In the remaining part of this paper, let "CS" denote the event "correct selection."

Chen (1996) provides an effective way to quantify confidence level when the number of systems is large. Furthermore, the sensitivity information of the confidence level with respect to simulation replications can be easily obtained when the approach in Chen (1996) is applied, which will provide the basis to determine how to allocate computing in this paper. From Chen (1996) we have

$$
\begin{aligned}
& P\{\mathrm{CS}\}=P\left\{\begin{array}{l}
\text { a system with the smallest sample mean } \\
\text { performance is actually the best system }\}
\end{array}\right. \\
& \geq \prod_{\substack{i=1 \\
i \neq b}}^{k} P\left\{\hat{\mu}_{b}<\hat{\mu}_{i}\right\}=A P C S
\end{aligned}
$$

where index $b$ is the design having the smallest sample mean performance, i.e.,

$$
b=\arg \min _{i}\left\{\bar{\mu}_{i}\right\}
$$

and $\hat{\mu}_{i}$ is the posterior distribution which consists of information from both prior distribution and the samples $\left\{X_{i j}\right.$, $\left.j=1,2, \ldots, N_{i}\right\}$. Under the assumption of normality

$$
\hat{\mu}_{i} \sim \mathrm{N}\left(\frac{1}{N_{i}} \sum_{j=1}^{N_{i}} X_{i j}, \frac{\sigma_{i}^{2}}{N_{i}}\right), \quad \text { for } i=1,2, . ., k
$$

We refer to this lower bound of the correct selection probability as the Approximate Probability of Correct Selection $(A P C S)$. While $P\{C S\}$ is very difficult to obtain, $A P C S$ can be computed very easily. We will use $A P C S$ to approximate $P\{C S\}$. Numerical testing in Chen (1996) shows that it can provide reasonably good approximation.

As motivated in Section 1, we intend to minimize the total computation cost while obtaining a desired confidence level. If simulations are performed on a sequential computer, the computation cost can be approximated by $N_{1}+N_{2}+\cdots+$ $N_{k}$. Ideally we want to 


$$
\begin{aligned}
& \min _{N_{1}, \cdots, N_{k}}\left\{N_{1}+N_{2}+\cdots+N_{k}\right\} \\
& \text { s.t. } A P C S \geq P^{*} .
\end{aligned}
$$

where $P^{*}$ is a user-defined confidence level requirement.

\section{A SEQUENTIAL APPROACH}

We now present a sequential approach to intelligently determine the number of simulation replications. Instead of finding the best $N_{1}, N_{2}, \ldots, N_{k}$ at the beginning of simulation, we sequentially select some PROMISING designs and simulate these selected PROMISING designs. The PROMISING designs are those by simulating which we anticipate the improvement of $A P C S$ is maximized.

Before doing the simulation, there is neither knowledge about $A P C S$ nor an idea about how to allocate budget, therefore all designs are simulated with $n_{0}$ replications, and the posterior distribution for design $i$ is

$$
\mathrm{N}\left(\frac{1}{n_{0}} \sum_{j=1}^{n_{0}} X_{i j}, \frac{\sigma_{i}^{2}}{n_{0}}\right)
$$

We use this statistical information to decide on the further allocation. In other words, after running $n_{0}$ replications for each design, we have the basic idea about each design and can decide which designs are worthy of being allocated more computing budget. Let $\Delta_{I}$ be the additional computing budget allocated to system $i$ in each step $\left(\Delta_{I}\right.$ is a non-negative integer). In order to find the PROMISING designs and effectively allocate computing budget for further simulation, it is necessary to know how APCS would be affected if additional simulation budget $\Delta_{I}$ is added to system $i$. Under the Bayesian model, it is convenient to use the statistical information at $n_{0}$ to estimate $A P C S$ at $n_{0}+\Delta_{I}$ by using an approximated posterior distribution

$$
\mathrm{N}\left(\frac{1}{n_{0}} \sum_{j=1}^{n_{0}} X_{i j}, \frac{\sigma_{i}^{2}}{n_{0}+\Delta_{i}}\right) \quad \text { for design } i
$$

We refer this approximation to EAPCS (estimated approximate probability of correct selection). We assume that $\Delta_{I}$ is not large and $n_{0}$ is close to $n_{0}+\Delta_{I}$, otherwise EAPCS is not a good estimator for APCS.

Similarly, when simulation proceeds until $\left(N_{1}, N_{2}, \ldots, N_{i-}\right.$ ${ }_{1}, N_{i}, N_{i+1}, . ., N_{k}$ ), we can also use the available information to estimate how APCS will change if design $i$ is given additional budget $\Delta_{I}$, i.e., $\operatorname{EAPCS}\left(N_{1}, N_{2}, \ldots, N_{i-1}, N_{i}+\Delta_{l}, N_{i+1}, \ldots, N_{k}\right)$, by using an approximated posterior distribution

$$
\mathrm{N}\left(\frac{1}{N_{i}} \sum_{j=1}^{N_{i}} X_{i j}, \frac{\sigma_{i}^{2}}{N_{i}+\Delta_{i}}\right) \quad \text { for design } i
$$

Let $\Delta \equiv \sum_{i=1}^{k} \Delta_{i}$. Thus $\Delta$ is one-time incremental computing budget in our sequential algorithm. In Section 5 we have more details about how to choose $n_{0}$ and $\Delta$. We hope that $A P C S$ becomes larger as simulation proceeds, we sequentially add computing budget by $\triangle$ each time until that $A P C S$ achieves a satisfactory level $P^{*}$. In order to reduce to the total computation cost, this budget $\Delta$ should be optimally allocated so that the EAPCS is maximized. Thus, at step $l, l=1,2$, ...., we have

$$
\begin{gathered}
\max _{\Delta_{1}^{l}, \ldots, \Delta_{k}^{l}} \operatorname{EAPCS}\left(N_{1}^{l}+\Delta_{1}^{l}, N_{2}^{l}+\Delta_{2}^{l}, \cdots, N_{k}^{l}+\Delta_{k}^{l}\right), \\
\text { s.t. } \sum_{i=1}^{k} \Delta_{i}^{l}=\Delta \text { and } \Delta_{i}^{l} \geq 0 \text { for all } i .
\end{gathered}
$$

In summary, we have the following algorithm:

\section{A Sequential Algorithm for Optimal Computing Budget Allocation (OCBA)}

Step 0. Perform $n_{0}$ simulation replications for all designs,

$l \leftarrow 0$,

$N_{1}^{l}=N_{2}^{l}=\cdots=N_{k}^{l}=n_{0}$.

Step 1. If $\operatorname{APCS}\left(N_{1}^{l}, N_{2}^{l}, \cdots, N_{k}^{l}\right) \geq P^{*}$, stop, otherwise, go to Step 2.

Step 2. Solve (3),

Step 3. Perform additional $\Delta_{i}^{l}$ simulation replications for design $i, i=1, \ldots, k$.

$$
\begin{aligned}
& N_{i}^{l+1}=N_{i}^{l}+\Delta_{i}^{l}, \text { for } i=1, \ldots, k . \\
& l \leftarrow l+1 .
\end{aligned}
$$

To solve (3), we assume the variables are continuous and apply steepest-descent method (Luenberger 1984 and Nash and Sofer 1996) to approximately solve (3). Then the results for the numbers of simulation replications are rounded off to integers. The gradient of EAPCS with respect to $N_{i}$ is estimated by the following formula: 


$$
\begin{aligned}
& \frac{\partial}{\partial N_{i}} \operatorname{EAPCS} \approx \\
& \frac{\operatorname{EAPCS}\left(N_{1}, N_{2}, \cdots, N_{i}+\tau, N_{i+1}, \ldots, N_{k}\right)-\operatorname{APCS}\left(N_{1}, N_{2}, \cdots, N_{i}, N_{i+1}, \ldots, N_{k}\right)}{\tau}
\end{aligned}
$$

where $\tau$ is a small number. To avoid spending much time in iteratively finding the solution of (3), we only do a very limited numbers of iterations when applying steepest-descent method. In fact, we can also use other optimization techniques to solve (3), please refer to Chen et al. (1997) for more details. Chen et al. 1997 also gives guidelines for selection of $n_{0}$ and $\Delta$.

\section{NUMERICAL TESTING}

This section present the numerical testing results using our OCBA algorithm. We test a simple $G / G / 1 / \infty$ queue $(k=10)$. There is one server with uniformly distributed service times and interarrival times. In this single-node example, all designs have the same arrival time uniform $[0.1,1.9]$, and service time in system $i$ is uniform $[0.1,1.8-0.05 i], i=1,2, \ldots, 10$. We want to find a design with minimum average system time for customers served in the first 10 time units (terminating simulation). Obviously, higher service rate results in shorter system time in this simple example, therefore, design 1 is the true best design. In the numerical experiment, we compare their computation costs and the actual convergence probabilities $P\{\mathrm{CS}\}$ for using OCBA and without using OCBA approaches.

We set $\Delta=12$ and $n_{0}=10$ in this example. To avoid spending too much time in solving (3), we only do two iterations in the gradient method. 10,000 independent experiments are performed so that the average computation cost and $P\{C S\}$ can be estimated. Different confidence level requirements are also tested. Table 1 contains the numerical results using OCBA algorithm and Table 2 contains the results without using OCBA.

\begin{tabular}{ccc}
\hline $\mathrm{P}^{*}$ & $\begin{array}{c}\text { Total \# of } \\
\text { Replications }\end{array}$ & $P\{\mathrm{CS}\}$ \\
\hline $60 \%$ & 196.4 & $72.2 \%$ \\
$80 \%$ & 344.3 & $86.6 \%$ \\
$90 \%$ & 523.6 & $96.3 \%$ \\
$95 \%$ & 735.4 & $98.1 \%$ \\
\hline
\end{tabular}

Table 1. Average total number of simulation replications and $P\{C S\}$ for OCBA application $\left(n_{0}=10\right)$.

\begin{tabular}{ccc}
\hline $\mathrm{P}^{*}$ & $\begin{array}{c}\text { Total \# of } \\
\text { Replications }\end{array}$ & $P\{\mathrm{CS}\}$ \\
\hline $60 \%$ & 541.8 & $82.9 \%$ \\
$80 \%$ & 863.6 & $90.7 \%$ \\
$90 \%$ & 1474.6 & $96.9 \%$ \\
$95 \%$ & 2175.5 & $99.0 \%$ \\
\hline
\end{tabular}

Table 2. Average total number of simulation replications and $P\{\mathrm{CS}\}$ without applying OCBA algorithm $\left(n_{0}=10\right)$.

Comparing Tables 1 and 2, we observe that our OCBA can achieve the desired $P\{C S\}$ with much lower computation cost. Figure 1 shows how the OCBA algorithm allocates computing budget to different designs.

\section{COMPARISON WITH OTHER METHODS}

In this section we will compare our approach with twostage procedures given by Dudewicz and Dalal (1975) and Rinott (1977) using the testing example in Section 4. Unlike our Bayesian approach, these two-stage procedures are developed based on classic statistical model. Also the idea of indifference-zone is required to apply such two-stage procedures.

Two confidence level $P^{*}=90 \%$ and $95 \%$ have been tested. When applying Rinott's and Dudewicz's procedures, we set the indifference zone $d^{*}=0.059\left(\mu_{(2)}-\mu_{(1)}=0.059\right)$. In both cases 10,000 independent experiments are run to evaluate the computational efficiency and to estimate the actual convergence probabilities. The computation cost and $P\{\mathrm{CS}\}$ are given in Table 3. From Table 3, significant speedup is observed for our method over both two-stage procedures, while the actual convergence probabilities for all approaches are no less than the desired levels. The speedup factors are higher than 14 in all cases.

\begin{tabular}{cccc}
\hline Methods & $\begin{array}{c}\text { Total \# of } \\
\text { Replications }\end{array}$ & $P\{\mathrm{CS}\}$ & $\begin{array}{c}\text { Speedup } \\
\text { factor using } \\
\text { OCBA }\end{array}$ \\
\hline OCBA & 523.6 & $96.2 \%$ & \\
Dudewicz's Proc. & 8059.9 & $98.3 \%$ & 15.3 \\
Rinott's Proc. & 9479.3 & $98.6 \%$ & 18.1 \\
\hline
\end{tabular}

Table 2. Comparison of our OCBA approach and traditional two-stage procedures. $P^{*}=90 \%$. 


\begin{tabular}{cccc} 
Methods & $\begin{array}{c}\text { Total \# of } \\
\text { Replications }\end{array}$ & $P\{C S\}$ & $\begin{array}{c}\text { Speedup } \\
\text { factor using } \\
\text { OCBA }\end{array}$ \\
\hline OCBA & 735.4 & $98.1 \%$ & \\
Dudewicz's Proc. & 11023.2 & $99.4 \%$ & 14.6 \\
Rinott's Proc. & 12464.1 & $99.5 \%$ & 16.5 \\
\hline
\end{tabular}

Table 3. Comparison of our OCBA approach and traditional two-stage procedures. $P^{*}=95 \%$.

\section{Concluding Remarks}

In this paper we present an optimal computing budget allocation technique that can intelligently determine the best simulation replication numbers for all designs. We also compare our approach with traditional two-stage procedures by conducting a numerical experiment. Numerical testing shows that our approach is more than ten times faster than two-stage procedures. For more details regarding this techniques readers are recommended to see Chen et al. (1997).

\section{References}

1. Bechhofer R. E., Santner, T. J., and Goldsman, D. M. 1995. Design and Analysis of Experiments for Statistical Selection, Screening, and Multiple Comparisons. John Wiley \& Sons, Inc.

2. Charnes, J. M. 1991 "Multivariate Simulation Output Analysis," Proceeding of the 1991 Winter Simulation Confidence, 187-193.

3. Chen, C. H. 1995. "An Effective Approach to Smartly Allocate Computing Budget for Discrete Event Simulation," Proceedings of the 34th IEEE Conference on Decision and Control, 2598-2605.

4. Chen, C. H. 1996. "A Lower Bound for the Correct Subset-Selection Probability and Its Application to Discrete Event System Simulations," IEEE Transactions on Automatic Control., Vol. 41, No. 8, 1227-1231.

5. Chen, C. H., Chen, H. C., and Dai, L. 1996 "A Gradient Approach for Smartly Allocating Computing Budget for
Discrete Event Simulation," Proceedings of the 1996 Winter Simulation Conference, 398-405.

6. Chen, C. H., Dai, L., Chen, H. C., and Yucesan, E. 1997. "Efficient Computation of Optimal Budget Allocation For Discrete Event Simulation," submitted to Operations Research.

7. Chiu, W. K. 1974. "Selecting the Populations with Largest Means From Normal populations with Unknown Variances," Australian Journal of Statistics, 16, 144-147.

8. Dudewicz, E. J. and Dalal, S. R. 1975. "Allocation of Observations in Ranking and Selection with Unequal Variances," Sankhya, B37:28-78.

9. Futschik, A. and Pflug, G. C. 1996. "Asymptotically Optimal Allocation of Simulation Experiments in Discrete Stochastic Optimization," Working Papers, International Institute for Applied Systems Analysis, Laxenburg, Austria.

10. Gibbons, J. D., Olkin, I., and Sobel, M. 1977. Selecting and Ordering Populations: A New Statistical Methodology, John Wiley \& Sons, Inc.

11. Goldsman, G., B. L. Nelson, and B. Schmeiser. 1991. "Methods for Selecting the Best System," Proceedings of the 1991 Winter Simulation Conference, 177-186.

12. Goldsman, G., and B. L. Nelson. 1994. "Ranking, Selection, and Multiple Comparison in Computer Simulation," Proceedings of the 1994 Winter Simulation Conference, 192-199.

13. Gupta, S. S. and S. Panchapakesan. 1979. Multiple Decision Procedures: Theory and Methodology of Selecting and Ranking Populations, John Wiley.

14. Luenberger, D. G., 1984. Linear and Nonlinear Programming, Addison-Wesley.

15. Matejcik, F. J., Nelson, B.L. 1993. "Simulations Ranking, Selection and Multiple Comparisons for Simulation," Proceeding of the 1993 Winter Simulation Confidence, 386-391.

16. Rinott, X. 1978. "On two-stage Selection Procedures and Related Probability Inequalities," Communications in Statistics A7, 799-811.

17. Schmeiser, B. 1982, "Batch Size Effects in the Analysis of Simulation Output," Operations Research, Vol. 30, No. 3, , 556-567. 


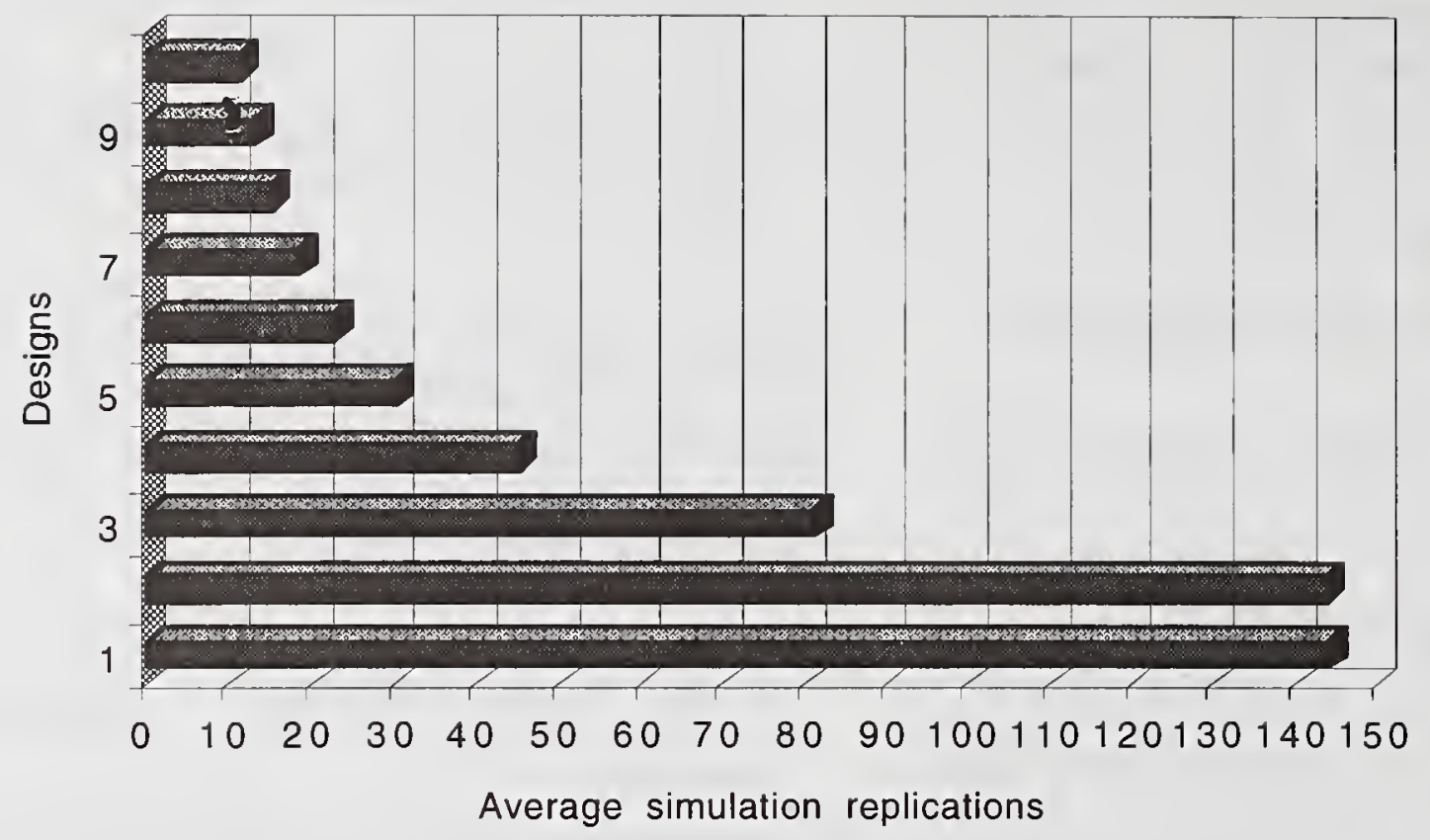

Figure 1. Computing budget allocation determined by OCBA. $P^{*}=90 \%$ and $n_{0}=10$. (design 1 is the best design). 


\section{Comparison of Performance Orders of Different Designs for Discrete-Event Dynamic Systems*}

\author{
Liyi Dai \\ Dept. of Systems Science and Math. \\ Washington University \\ St. Louis, MO 63130
}

\author{
Chun-Hung Chen \\ Dept. of Systems Engineering \\ University of Pennsylvania \\ Philadelphia, PA 19104-6315
}

\section{Abstract}

In this paper, properties of ordinal comparison for discreteevent dynamic systems are investigated by employing the large deviation principle which allows one to have an expression for the rate of convergence of ordinal comparison. With this expression, conditions are obtained under which the rate of convergence of ordinal comparison is exponential. Such expression also enables one to obtain bounds on the rate of convergence and design sample path generation schemes that maximizes the convergence rate of ordinal comparison.

\section{Introduction}

Many design problems in Discrete-Event Dynamic Systems (DEDS) require choosing one design from a large, discrete design space, such as the problem of resource allocation [5]. Such problems are of combinatorial nature and known to be NP hard (difficult to solve [17]). The design of DEDS is further complicated by the fact that not only the design spaces are large and discrete but also the performance measures on which designing is based do not have closed-form solutions. Except for special cases, discrete-event simulation or sample path observation is usually used in performance estimation and evaluation of designs in DEDS. Such performance estimation is time-consuming and costly. Typically, a performance estimator converges slowly at rate of $O(1 / \sqrt{t})$ in simulation or observation time $t[9]$.

On the other hand, in reality, we often face a decision making problem of choosing one relatively better design from all possible alternatives. As long as we can single out the "good" and "satisficing" designs, the exact values of their performance measures are of secondary importance. Such goal relaxation can bring great saving of effort. In the context of recently proposed ordinal optimization methods, ordinal comparison of different designs by their relative ranks is very efficient, as observed in many experiments. Ordinal comparison is able to discern quickly the good designs using noisy information $[1,6,7,10,13,14,18]$. By ordinal comparison, we mean comparing the relative

*This work was supported in part by the National Science Foundation under grant No. ECS-9624279 and by the University of Pennsylvania Research Foundation. goodness (rank) of different designs without knowing the exact values of corresponding performance measures. Recent research has revealed a more interesting phenomenon: Ordinal comparison is beneficial when used in conjunction with conventional methods such as stochastic approximation and simulated annealing as reported in [3, 11, 22].

Consider the problem of finding the best or a good design among $N$ possibilities. Let $\Theta=\left\{\theta_{1}, \theta_{2}, \ldots, \theta_{N}\right\}$ denote the set of all designs and $J(\theta) \in R$ denote the performance measure of a particular design $\theta \in \Theta$. In general DEDS, the exact form of $J(\theta)$ is not available. We can only use noisy estimate of $J(\theta)$ in our decision making. Let us consider the dynamics of the following experiment. We simultaneously simulate $N$ parallel DEDS with designs $\theta_{i}$, $i=1,2, \ldots, N$. This can be done, for example, using the standard clock [21] or the augmented system analysis [4]. As the simulation continuous, we collect data and output an estimate of $J(\theta)$, denoted by $L(\theta, t)$, for every $\theta \in \Theta$. Convergence of $L(\theta, t)$ to $J(\theta)$ is generally slow at the rate of at most $O(1 / \sqrt{t})$ according to the law of large numbers (assuming the validity of convergence). However, it has been observed that the observed order of performance measures $L\left(\theta_{i}, t\right), i=1,2, \ldots, N$, can quickly converge to an order very close to the true order of performance measures $J\left(\theta_{i}\right), i=1,2, \ldots, N$, despite possible presence of large noises $[1,6,7,10,13,14,18]$.

This paper is concerned with several fundamental properties of ordinal comparison in DEDS. By applying the large deviation principle one is able to obtain an expression for the rate of convergence of ordinal comparison. With this expression, conditions are obtained under which the rate of convergence of ordinal comparison is exponentially fast. Such expression also enables one to obtain bounds on the rate of convergence and design sample path generation schemes that maximizes the convergence rate of ordinal comparison.

\section{Problem Statement}

The DEDS under investigation is described by a rightcontinuous stochastic process $\{X(\theta, \xi, t) \in \mathcal{X}, t \geq 0\}$ parameterized by $\theta \in \Theta$ and defined on a common probability space. Here, $\mathcal{X} \subset R$ is the state space, $\theta$ is used simply to indicate a design, and $\xi$ represents all the randomness 
involved. For each design $\theta \in \Theta$, let $L(\theta, t) \in R$ be an estimate of a performance measure $J(\theta) \in R$ based on a particular sample trajectory of $\{X(\theta, \xi, t)\}$ over $[0, t]$. In DEDS, $J(\theta)$ is often a steady state performance and $L(\theta, t)$ is a sample performance over $[0, t]$. Note that $L(\theta, t)$ converges slowly with rate at most $O(1 / \sqrt{t})$. Long and timeconsuming simulation has to be performed in order to have a good estimate of the (steady state) performance measure $J(\theta)$.

Now consider the problem of comparing performance measures corresponding to a set $\Theta$ of $N, 1 \leq N<\infty$, designs. Design $\theta_{i}$ is said to be better than design $\theta_{j}$ if $J\left(\theta_{i}\right)>J\left(\theta_{j}\right)$. Without loss of generality, we assume that the $N$ designs are indexed in such a way that $\infty>J\left(\theta_{1}\right)>J\left(\theta_{2}\right)>J\left(\theta_{3}\right)>\ldots>J\left(\theta_{N}\right)>-\infty$. Particularly, we are interested in finding a design that is one of the $M, 1 \leq M \leq N$, true best designs in $\Theta$. For convenience, let us define $\Theta_{g}=\left\{\theta_{i}, i=1,2, \ldots, M\right\}$ and $\Theta_{b}=\left\{\theta_{i}, i=M+1, M+2, \ldots, N\right\}$ the sets of "good" and "bad" designs, respectively. For the problem of finding one of the $M$ best designs based on the simulation over $[0, t]$, we choose the design with the largest sample performance, i.e.,

$$
\theta_{t}^{*}=\arg \max _{\theta \in \Theta} L(\theta, t) .
$$

Experimental results have shown that (2.1) can $q 1$ : $k$ kly find the true desired design $[1,6,13,18]$. Intuitively, implies that relative order of performance measures converges very fast.

In order to characterize the convergence of ordinal comparison, we define the following indicator process

$$
I(t)= \begin{cases}1 & \text { if } \max _{\sigma \in \Theta_{g}} L(\sigma, t) \geq \max _{\theta \in \Theta_{b}} L(\theta, t) \\ 0 & \text { otherwise. }\end{cases}
$$

Then $I(t)$ is equal to 1 if the observed best design is among the true good designs and equal to 0 otherwise. Since $\max _{\sigma \in \Theta_{g}} L(\sigma, t)$ is the maximum of the observed true "good" designs and $\max _{\theta \in \Theta_{b}} L(\theta, t)$ is the maximum of the observed true "bad" designs, $I(t)$ is a function of $t$ indicating when the observed best design determined by (2.1) is one of the $M$ true best designs.

Our main goal is to examine the behavior of

$$
\operatorname{Prob}[I(t)=1] \text { and } \operatorname{Prob}[I(t)=0]
$$

as a function of time $t$. If $\lim _{t \rightarrow \infty} L(\theta, t)=J(\theta)$, a.s., then $([6])$

$$
\lim _{t \rightarrow \infty} \operatorname{Prob}[I(t)=1]=1, \quad \lim _{t \rightarrow \infty} \operatorname{Prob}[I(t)=0]=0 .
$$

The convergence rate of $I(t)$ will be taken as the rate at which $\operatorname{Prob}[I(t)=0]$ goes to zero (or the rate at which $\operatorname{Prob}[I(t)=1]$ goes to 1$)$.

\section{The Large Deviation Principle}

The concept of large deviation has been proven useful in the study of the convergence of ordinal comparison. The following definition is adopted from [8].

Definition 3.1 Let $\left\{X_{\epsilon}\right\}$ be a family of random variables defined on a state space $\mathcal{X}$. We say that $\left\{X_{\epsilon}\right\}$ satisfies the large deviation principle with a rate function $\phi$ if, for every closed set $A \subset \mathcal{X}, \lim \sup _{\epsilon \rightarrow 0} \epsilon \log \operatorname{Prob}\left[X_{\epsilon} \subset\right.$ $A] \leq-\inf _{\lambda \in A} \phi(\lambda)$, and for every open set $B \subset \mathcal{X}$, $\liminf _{\epsilon \rightarrow 0} \epsilon \log \operatorname{Prob}\left[X_{\epsilon} \subset B\right] \geq-\inf _{\lambda \in B} \phi(\lambda)$, where the mapping $\phi: \mathcal{X} \rightarrow[0, \infty]$ is lower semicontinuous, i.e., $\liminf _{\lambda_{n} \rightarrow \lambda} \phi\left(\lambda_{n}\right) \geq \phi(\lambda)$ for all $\lambda \in \mathcal{X}$.

Now define the logarithmic moment generating function $\Lambda_{\epsilon}(s)=\log E\left[e^{s X_{\epsilon}}\right]$ and suppose that the limit $\Lambda(s)=$ $\lim _{\epsilon \rightarrow 0} \epsilon \Lambda_{\epsilon}(s / \epsilon)$ exists pointwise. The following theorem from [8] gives an expression for the rate function $\phi$.

Theorem 3.2 Assume that $\left\{X_{\epsilon}\right\}$ satisfies the large deviation principle with a rate function $\phi$. If $\phi$ is convex and, for all $c \in R^{+}$, the set $\{\lambda: \phi(\lambda) \leq c\}$ is a compact subset of $\mathcal{X}$, then $\phi$ is the Fenchel-Legendre transform of $\Lambda(s)$, namely,

$$
\phi(\lambda)=\sup _{s}\{\lambda s-\Lambda(s)\} .
$$

If, in addition,

$$
\inf _{\lambda \in A^{\circ}} \phi(\lambda)=\inf _{\lambda \in \bar{A}} \phi(\lambda)
$$

for $A \subset \mathcal{X}$ where $A^{\circ}$ and $\bar{A}$ are the interior and closure of $A$, respectively, then we know from Definition 3.1 and Theorem 3.2 that

$$
\lim _{\epsilon \rightarrow 0} \epsilon \log \operatorname{Prob}\left[X_{\epsilon} \subset A\right]=-\inf _{\lambda \in \bar{A}} \phi(\lambda) .
$$

Choose $A=(0, \infty)$. Then,

$$
\inf _{\lambda \in[0, \infty)} \phi(\lambda)=\inf _{\lambda \in[0, \infty)} \sup _{s \geq 0}\{\lambda s-\Lambda(s)\}=-\inf _{s \in[0, \infty)} \Lambda(s) .
$$

Therefore,

$$
\lim _{\epsilon \rightarrow 0} \epsilon \log \operatorname{Prob}\left[X_{\epsilon} \subset(0, \infty)\right]=\inf _{s \in[0, \infty)} \Lambda(s) .
$$

It gives an expression for the convergence rate of $\operatorname{Prob}\left[X_{\epsilon} \subset(0, \infty)\right]$ as $\epsilon$ goes to zero.

\section{Rate of Convergence}

The large deviation principle can be used to investigate the convergence rate of ordinal comparison.

Lemma $4.1([16,19])$ Let $f_{\epsilon}(a)$ be a sequence of functions on $\mathcal{A}$. Suppose $\lim _{\epsilon \rightarrow 0} f_{\epsilon}(a)=f(a)$ for every $a \in \mathcal{A}$. If $\left\{f_{\epsilon}(a)\right\}$ are convex, differentiable at $a_{0} \in \mathcal{A}$, and $f(a)$ is differentiable at $a_{0}$, then

$$
\lim _{\epsilon \rightarrow 0} \frac{d f_{\epsilon}\left(a_{0}\right)}{d a}=\frac{d f\left(a_{0}\right)}{d a} .
$$


let

Theorem 4.2 For any pair of designs $\sigma \in \Theta_{g}, \theta \in \Theta_{b}$,

$$
X_{t}(\sigma, \theta)=L(\theta, t)-L(\sigma, t)
$$

and

$$
\Lambda(s, \sigma, \theta)=\lim _{t \rightarrow \infty} \frac{1}{t} \log E\left[e^{s t X_{t}(\sigma, \theta)}\right] .
$$

Assume that

(i) $X_{t}(\sigma, \theta)$ satisfies the large deviation principle and the rate expression (3.5) holds,

(ii) $\log E\left[e^{s t X_{t}(\sigma, \theta)}\right]$ and $\Lambda(s, \sigma, \theta)$ are differentiable at $s=0$

(iii) and for every design $\theta \in \Theta$,

$$
\lim _{t \rightarrow \infty} E[L(\theta, t)]=J(\theta)
$$

Then there exist positive constants $\alpha>0, \beta>0$ such that

$$
\operatorname{Prob}[I(t)=0] \leq \beta e^{-\alpha t}
$$

in other words, the convergence of ordinal comparison is exponentially fast.

Proof. According to the definition of the indicator process,

$$
\begin{gathered}
\operatorname{Prob}[I(t)=0]=\operatorname{Prob}\left[\max _{\sigma \in \Theta_{g}} L(\sigma, t)<\max _{\theta \in \Theta_{b}} L(\theta, t)\right] \\
\leq \min _{\sigma \in \Theta_{g}} \operatorname{Prob}\left[L(\sigma, t)<\max _{\theta \in \Theta_{b}} L(\theta, t)\right] \\
=\min _{\sigma \in \Theta_{g}} \operatorname{Prob}\left[\bigcup_{\theta \in \Theta_{b}}\{L(\sigma, t)<L(\theta, t)\}\right] \\
\leq \min _{\sigma \in \Theta_{g}} \sum_{\theta \in \Theta_{b}} \operatorname{Prob}[L(\sigma, t)<L(\theta, t)] .
\end{gathered}
$$

Since $X_{t}(\sigma, \theta)$ satisfies the large deviation principle, we know from (3.5) that

$$
\begin{gathered}
\lim _{t \rightarrow \infty} \frac{1}{t} \log \operatorname{Prob}[L(\sigma, t)<L(\theta, t)] \\
=\lim _{t \rightarrow \infty} \frac{1}{t} \log \operatorname{Prob}\left[X_{t}(\sigma, \theta) \subset(0, \infty)\right] \\
=\inf _{s \in[0, \infty)} \Lambda(s, \sigma, \theta) .
\end{gathered}
$$

Since $\log E\left[e^{s t X_{t}(\sigma, \theta)}\right]$ is convex in a neighborhood of $s=0$ and is differentiable at $s=0$, we know from Lemma 4.1 that

$$
\begin{aligned}
\left.\frac{d \Lambda(s, \sigma, \theta)}{d s}\right|_{s=0} & =\left.\lim _{t \rightarrow \infty} \frac{1}{t} \frac{d}{d \theta} \log E\left[e^{s t X_{t}(\sigma, \theta)}\right]\right|_{s=0} \\
& =\lim _{t \rightarrow \infty} E\left[X_{t}(\sigma, \theta)\right] .
\end{aligned}
$$

The assumption (iii) implies that

$$
\lim _{t \rightarrow \infty} E\left[X_{t}(\sigma, \theta)\right]=J(\theta)-J(\sigma)<0 .
$$

Therefore, $\Lambda(s, \sigma, \theta)<0$ for small $s$, which implies that there exists an $\alpha>0$ such that

$$
\inf _{s \in[0, \infty)} \Lambda(s, \sigma, \theta) \leq-\alpha .
$$

Combining the previous inequality with (4.7) yields,

$$
\lim _{t \rightarrow \infty} \frac{1}{t} \log \operatorname{Prob}[L(\sigma, t)<L(\theta, t)] \leq-\alpha .
$$

The proof of the theorem follows from this inequality and (4.6).

Theorem 4.2 assures the exponential convergence of ordinal comparison under some conditions in terms of the large deviation principle. Other conditions that also guarantee such exponential convergence are given in $[6,10]$. In particular cases, the requirement for the rate expression (3.5) can be relaxed. Some important cases are discussed in detail in [6].

\section{Bounds on Convergence Rate}

In this section, we find bounds for the rate of convergence of ordinal comparison. Based on these bounds, we propose simulation schemes that maximize the rate of convergence of the indicator process. The results also shed useful insight for sample path reconstruction in performance analysis.

Let

$$
Y_{t}=\max _{\theta \in \Theta_{\mathrm{b}}} L(\theta, t)-\max _{\sigma \in \Theta_{g}} L(\sigma, t) .
$$

If $Y_{t}$ satisfies the large deviation principle and if the rate expression (3.5) holds, then the convergence rate $r$ of $\operatorname{Prob}[I(t)=0]$ is

$$
r=-\inf _{s \in[0, \infty) t \rightarrow \infty} \lim _{t \rightarrow \infty} \frac{1}{t} \log E\left[e^{s t\left(\max _{\theta \in \Theta_{b}} L(\theta, t)-\max _{\sigma \in \Theta_{g}} L(\sigma, t)\right)}\right] .
$$

Therefore, maximizing the rate is equivalent to maximizing (5.8). It is sufficient to minimize

$$
\Lambda_{t}(s) \stackrel{\text { def }}{=} E\left[e^{s t\left(\max _{\theta \in \Theta_{b}} L(\theta, t)-\max _{\sigma \in \Theta_{g}} L(\sigma, t)\right)}\right]
$$

for all $s \geq 0, t \geq 0$, and consequently

$$
r=-\inf _{s \in[0, \infty)} \lim _{t \rightarrow \infty} \frac{1}{t} \log \Lambda_{t}(s) .
$$

A function $g\left(X_{1}, X_{2}\right): R^{2} \rightarrow R$ is said to be superadditive if, for any $X_{1} \leq X_{1}^{\prime}, X_{2} \leq X_{2}^{\prime}$,

$$
g\left(X_{1}^{\prime}, X_{2}\right)+g\left(X_{1}, X_{2}^{\prime}\right) \leq g\left(X_{1}, X_{2}\right)+g\left(X_{1}^{\prime}, X_{2}^{\prime}\right)
$$

If $g\left(X_{1}, X_{2}\right)$ is twice differentiable, it is superadditive if and only if $\partial^{2} g\left(X_{1}, X_{2}\right) / \partial X_{1} \partial X_{2} \geq 0$. The following result is due to $[2,20]$. 
Lemma 5.1 Let $X_{1}, X_{2}$ be random variables with marginal distributions $F_{i}\left(x_{i}\right), i=1,2$, and joint distribution $H\left(x_{1}, x_{2}\right)$. Assume that $g\left(X_{1}, X_{2}\right)$ is rightcontinuous and superadditive and $E\left[g\left(X_{1}, X_{2}\right)\right]$ is finite for all $H\left(x_{1}, x_{2}\right)$. Then

$$
\begin{gathered}
\sup _{\{H\}} E\left[g\left(X_{1}, X_{2}\right)\right]=E\left[g\left(F_{1}^{-1}(u), F_{2}^{-1}(u)\right)\right] \\
\inf _{\{H\}} E\left[g\left(X_{1}, X_{2}\right)\right]=E\left[g\left(F_{1}^{-1}(u), F_{2}^{-1}(1-u)\right)\right]
\end{gathered}
$$

where the random variable $u$ is uniform over $[0,1]$ and the inverse $F^{-1}(u)$ is defined as

$$
F^{-1}(u)=\inf \{x \in R \mid F(x) \geq u\} .
$$

Lemma 5.1 says that $E\left[g\left(X_{1}, X_{2}\right)\right]$ is maximized by sampling $X_{1}$ and $X_{2}$ using the same random number $u$ according to

$$
X_{i}=F_{i}^{-1}(u), \quad i=1,2 .
$$

This scheme of generating samples of random variables is known as the scheme of common random numbers (CRN). It is perhaps the most popular scheme for variance reduction in simulation.

Let fix $t$ and denote by $G_{\theta}(w)=\operatorname{Prob}[L(\theta, t) \leq w]$ the c.d.f of $L(\theta, t)$ for each $\theta \in \Theta$. Define

$$
\begin{gathered}
\bar{G}_{g}(w) \stackrel{\text { def }}{=} \min _{\theta \in \Theta_{g}}\left\{G_{\theta}(w)\right\}, \underline{G}_{g}(w) \stackrel{\text { def }}{=} \max \left\{0,1-\sum_{\theta \in \Theta_{g}}\left[1-G_{\theta}(w)\right]\right\} \\
\bar{G}_{b}(w) \stackrel{\text { def }}{=} \min _{\theta \in \Theta_{b}}\left\{G_{\theta}(w)\right\}, \underline{G}_{b}(w) \stackrel{\text { def }}{=} \max \left\{0,1-\sum_{\theta \in \Theta_{b}}\left[1-G_{\theta}(w)\right]\right\}
\end{gathered}
$$

Then the following theorem gives lower and upper bounds for the convergence rate $r$ of ordinal comparison.

Theorem 5.2 Suppose that $Y_{t}$ satisfies the large deviation principle and that the rate expression (3.5) holds. Then

$$
-\inf _{s \in[0, \infty) t \rightarrow \infty} \lim _{t \rightarrow \infty} \frac{1}{t} \log \underline{\Lambda}_{t}(s) \leq r \leq-\inf _{s \in[0, \infty)} \lim _{t \rightarrow \infty} \frac{1}{t} \log \bar{\Lambda}_{t}(s)
$$

where

$$
\begin{array}{r}
\bar{\Lambda}_{t}(s)=E\left[e^{s t\left(\bar{G}_{b}^{-1}(u)-\underline{G}_{g}^{-1}(u)\right)}\right], \\
\underline{\Lambda}_{t}(s)=E\left[e^{s t\left(\underline{G}_{b}^{-1}(u)-\bar{G}_{g}^{-1}(1-u)\right)}\right] .
\end{array}
$$

\section{Proof. Let}

$$
W_{1}=\max _{\theta \in \Theta_{b}} L(\theta, t), \quad W_{2}=\max _{\sigma \in \Theta_{g}} L(\sigma, t) .
$$

For any $t \geq 0, s \geq 0$, consider the function

$$
-e^{s t\left(W_{1}-W_{2}\right)} \text {. }
$$

It is continuous and superadditive in $\left(W_{1}, W_{2}\right)$. Therefore, Lemma 5.1 applies and, for all $t \geq 0$, all $s \geq 0$, and all $\theta \in \Theta_{b}$

$$
\sup _{\left\{H_{w}\right\}} E\left[-e^{s t\left(W_{1}-W_{2}\right)}\right]=E\left[-e^{s t\left(F_{W_{1}}^{-1}(u)-F_{W_{2}}^{-1}(u)\right)}\right],
$$

$$
\inf _{\left\{H_{w}\right\}} E\left[-e^{s t\left(W_{1}-W_{2}\right)}\right]=E\left[-e^{s t\left(F_{W_{1}}^{-1}(u)-F_{W_{2}}^{-1}(1-u)\right)}\right]
$$

where $\left\{H_{w}\right\}$ is the set of all correlations among $\left(W_{1}, W_{2}\right)$ and $F_{W_{1}}$ and $F_{W_{2}}$ are the distribution functions of $W_{1}$ and $W_{2}$, respectively.

However, from the definitions of $W_{1}$ and $W_{2}$ and according to basic probability properties, we know that

$$
\begin{gathered}
\operatorname{Prob}\left[W_{1} \leq w\right]=\operatorname{Prob}\left[\max _{\theta \in \Theta_{b}} L(\theta, t) \leq w\right] \\
\leq \min _{\theta \in \Theta_{b}} \operatorname{Prob}[L(\theta, t) \leq w]=\min _{\theta \in \Theta_{b}} G_{b}(w)=\bar{G}_{b}(w) .
\end{gathered}
$$

On the other hand,

$$
\begin{gathered}
\operatorname{Prob}\left[W_{1}>w\right]=\operatorname{Prob}\left[\max _{\theta \in \Theta_{b}} L(\theta, t)>w\right] \\
\leq \sum_{\theta \in \Theta_{b}} \operatorname{Prob}[L(\theta, t)>w]=\sum_{\theta \in \Theta_{b}}\left[1-G_{\theta}(w)\right]
\end{gathered}
$$

which implies

$$
\operatorname{Prob}\left[W_{1} \leq w\right] \geq \max \left\{0,1-\sum_{\theta \in \Theta_{b}}\left[1-G_{\theta}(w)\right]\right\}=\underline{G}_{b}(w) .
$$

Therefore,

$$
\underline{G}_{b}(w) \leq F_{W_{1}}(w) \leq \bar{G}_{b}(w) .
$$

Similarly,

$$
\underline{G}_{g}(w) \leq F_{W_{2}}(w) \leq \bar{G}_{g}(w) .
$$

With those bounds, it is easy to verify that

$$
\begin{gathered}
-e^{s t\left(F_{W_{1}}^{-1}(u)-F_{W_{2}}^{-1}(u)\right)} \leq-e^{s t\left(\bar{G}_{b}^{-1}(u)-\underline{G}_{g}^{-1}(u)\right)}, \\
-e^{s t\left(F_{W_{1}}^{-1}(u)-F_{W_{2}}^{-1}(1-u)\right)} \geq-e^{s t\left(\bar{G}_{b}^{-1}(u)-\underline{G}_{g}^{-1}(1-u)\right)}
\end{gathered}
$$

for any $s t \geq 0$. Combining this with (5.15) and (5.16), we know that

$E\left[e^{s t\left(\bar{G}_{b}^{-1}(u)-\underline{G}_{g}^{-1}(u)\right)}\right] \leq E\left[e^{s t Y_{t}}\right] \leq E\left[e^{s t\left(\underline{G}_{b}^{-1}(u)-\bar{G}_{g}^{-1}(1-u)\right)}\right]$.

or equivalently,

$$
\underline{\Lambda}_{t}(s) \geq \Lambda_{t}(s) \geq \bar{\Lambda}_{t}(s) .
$$

The combination of the preceding inequality with (5.9) yields (5.13). The proof is thus complete.

Theorem 5.2 makes it possible to design simulation experiments so that the rate of convergence of ordinal comparison is maximized.

Corollary 5.3 Assume $M=1$. Suppose that $Y_{t}$ satisfies the large deviation principle and that the rate expression (3.5) holds. Then the rate of convergence of the indicator process is maximized by sampling $L(\theta, t)$ using the scheme of CRN.

Proof. For $M=1, \Theta_{g}=\left\{\theta_{1}\right\}$ contains only one design $\theta_{1}$. In this case, $\bar{G}_{g}(w)=\underline{G}_{g}(w)=G_{\theta_{1}}(w)$. Then Theorem 5.2 shows that the rate of convergence of ordinal 
comparison is given by (5.9) in which $\Lambda_{t}(s)$ is bound from below by

$$
\Lambda_{t}(s) \geq \bar{\Lambda}_{t}(s)=E\left[e^{s t\left(\bar{G}_{b}^{-1}(u)-G_{\theta_{1}}^{-1}(u)\right)}\right],
$$

that is

$$
\inf _{H_{L}} \Lambda_{t}(s)=E\left[e^{s t\left(\bar{G}_{b}^{-1}(u)-G_{\theta_{1}}^{-1}(u)\right)}\right]
$$

where $\left\{H_{L}\right\}$ is the set of all correlations among $\{L(\theta, t)\}$. Since the lower bound can be achieved by sampling all $L(\theta, t)$ using the scheme of CRN. Therefore, (5.8), or the convergence rate of ordinal comparison, is maximized by the scheme of CRN.

As far as ordinal comparison is concerned, it is important to be able to design simulation experiments so the upper bound in (5.13) is achieved. Let us consider this problem for different values of $M$.

(i) $M=1$. In this case, Corollary 5.3 shows that the upper bound in (5.13) can be achieved by the scheme of CRN.

(ii) $M=2$. We know from the proof of Theorem 5.2 that the upper bound is achieved when $W_{1}$ and $W_{2}$ are sampled using the scheme of CRN when the distribution of $W_{1}=\max _{\theta \in \Theta_{b}} L(\theta, t)$ is

$$
F_{W_{1}}(w)=\tilde{G}_{b}(w),
$$

and the distribution of $W_{2}=\max _{\sigma \in \Theta_{g}} L(\sigma, t)$ is

$$
F_{W_{2}}(w)=\underline{G}_{g}(w) .
$$

The distribution (5.20) is achieved by setting

$$
L(\theta, t)=G_{\theta}^{-1}(u), \quad \theta \in \Theta_{b} .
$$

The distribution (5.21) is achieved by setting

$$
L\left(\theta_{1}, t\right)=G_{\theta_{1}}^{-1}(u), \quad L\left(\theta_{2}, t\right)=G_{\theta_{2}}^{-1}(1-u) .
$$

It is easy to verify that such sample scheme yields the upper bound in (5.13). However, it should be pointed out that such scheme is not practical because the set $\Theta_{g}$ and $\Theta_{b}$ are unknown a priori. Nevertheless, this fact shows that the scheme of CRN does not give the maximum rate for ordinal comparison.

(iii) $M \geq 3$. In this case, it can be shown using counterexamples that $\underline{G}_{g}(w)$ is not a distribution but can be asymptotically achieved by a sequence of random variables [20]. As a result, it is impossible to find sampling schemes that achieve the bounds in (5.13) for $M \geq 3$. This means that the scheme of CRN does not give the maximum rate for ordinal comparison for $M \geq 3$.
To sum up, it is difficult. if not impossible, in practical simulation to achieve the maximum rate for $M \geq 2$. For $M=1$, or for the problem of selecting the best from possible $N$ designs, Corollary 5.3 shows that the maximum rate of convergence in (5.13) can be achieved by sampling using the scheme of CRN. This assures the positive role of CRN when $L(\theta, t)$ can be sampled from a single random number $u$. Of course, for practical problems $L(\theta, t)$ has more complicated form. In simulations or sample path constructions of DEDS, $L(\theta, t)$ is usually a function a set of random numbers, i.e.,

$$
L(\theta, t)=L(u(1, \theta), u(2, \theta), \ldots, u(n, \theta))
$$

where $n$ is an integer determined by the system structure and $t$. It is possible to write $L(\theta, t)$ as a non-decreasing function of $u(i, \theta)$ for a large class of DEDS (we shall not describe the exact class of systems because of the limited space. See [7] for further details). The following discussion is restricted to a case that is easily realizable in simulation and that has explicit solution. We assume that $\left\{\left(u\left(n, \theta_{1}\right), u\left(n, \theta_{2}\right), \ldots, u\left(n, \theta_{N}\right)\right)\right\}$ is a sequence of independent random vectors. We would like to find the correlation among $u\left(n, \theta_{1}\right), u\left(n, \theta_{2}\right), \ldots, u\left(u, \theta_{N}\right)$, for every fixed $n$, that maximizes the rate of ordinal comparison. This scheme can be easily realized and most useful.

Assume again $M=1$. For any $\theta \in \Theta_{b}$, let

$$
X_{t}(\theta)=L(\theta, t)-L\left(\theta_{1}, t\right)
$$

Then it has been proved that the scheme of CRN maximizes the rate of convergence of ordinal comparison as summarized by the following theorem. The details can be found in [7].

Theorem 5.4 Assume that $L(\theta, t)$ is right-continuous, non-decreasing in $u(n, \theta)$ for every $t$ and every $n \geq 1$. If, for every $\theta \in \Theta_{b}, X_{t}(\theta)$ satisfies the large deviation principle and if the rate expression (3.5) holds, then the rate of convergence for the indicator process is maximized by choosing

$$
u(n, \theta)=v(n),
$$

where $\{v(n)\}$ is a sequence of i.i.d. random numbers uniform on $[0,1]$, in other words, the rate is maximized by using the scheme of CRN.

Additional results regarding the effect of correlation on the convergence of ordinal comparison can be found in $[7$, $10,15]$.

The following example, modified from an example of [12], illustrates the difference in the rates of convergence for independent simulations and simulations using CRN.

Example 5.4 Consider an M/G/1 FCFS queue with arrival rate $\lambda$. The service times have the Weibull distribution $G(x)=1-e^{-(\gamma x)^{\alpha}}, x \geq 0$ for constants $\gamma, \alpha>0$. Let the design parameter be $\theta=(\lambda, \gamma, \alpha)$. The performance measure is the mean queue length in steady state. We consider 5 designs with the following parameters: 


\begin{tabular}{|c|c|c|c|c|c|}
\hline$\theta$ & $\theta_{1}$ & $\theta_{2}$ & $\theta_{3}$ & $\theta_{4}$ & $\theta_{5}$ \\
\hline$\lambda$ & 1.0 & 0.5 & 0.5 & 1.0 & 0.7 \\
\hline$\alpha$ & 1.3 & 2.0 & 1.0 & 1.0 & 2.5 \\
\hline$\gamma$ & 2.0 & 0.75 & 1.0 & 1.6 & 1.0 \\
\hline$J(\theta)$ & 0.78 & 1.12 & 1.0 & 1.64 & 1.21 \\
\hline
\end{tabular}

The performance is taken as the negative of the mean queue length. The true best design is $\theta_{1}$. The simulation performances for independent simulations and for simulation using CRN are shown respectively in Figures 1 and 2. Note that the displayed time is the real queue time, not the simulation time. The simulation was completed within seconds.

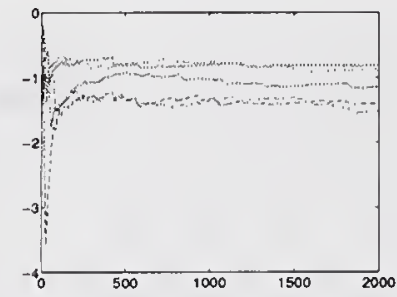

(a) Sample performances

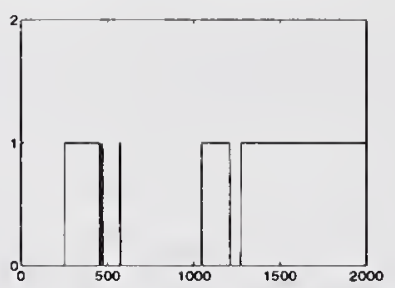

(b) Indicator process
Figure 1. Independent simulations

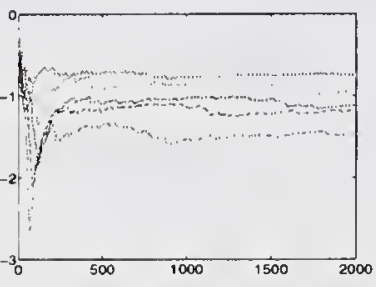

(a) Sample performances

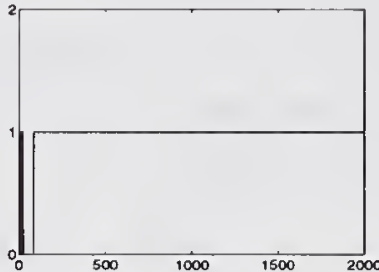

(b) Indicator process
Figure 2. Simulations using CRN

Figures 1 and 2 show that correlation could significantly speed up the convergence of ordinal comparison.

\section{References}

[1] C.M. Barnhart, J.E. Wieselthier, and A. Ephremides, "Ordinal Optimization by Means of Standard Clock Simulation and Crude Analytical Models," in Proc. 33rd Conf. Decision Control, 1994, pp. 2645-2647.

[2] S. Cambanis, G. Simons, and W. Stout, "Inequalities for $E k(X, Y)$ When the Marginals are Fixed," Zeitschrift für Wahrscheinlichkeitstheorie und verwandte Gebiete, Vol. 36, pp. 285-294, 1976.

[3] C.G. Cassandras and G. Bao, "A Stochastic Comparison Algorithm for Continuous Optimization with Estimations," in Proc. 33rd Conf. Decision and Control, 1994, pp. 676-677.

[4] C.G. Cassandras and S. Strickland, "Observable Augmented Systems for Sensitivity Analysis of Markov and Semi-Markov Processes," IEEE Trans. Automatic Control, Vol. 34, pp. 1026-1037, 1989.

[5] C.G. Cassandras, L. Dai, and C.G. Panayiotou, "Ordinal Optimization for Deterministic and Stochastic Discrete Resource Allocation," manuscript, 1997.
[6] L. Dai, "Convergence Properties of Ordinal Comparison in the Simulation of Discrete Event Dynamic Systems," J. Opti. Theory Appli., Vol. 91, pp. 363-388, 1996.

[7] L. Dai and C.-H. Chen, "Rates of Convergence of Ordinal Comparison for Dependent Discrete Event Dynamic Systems," J. Opti. Theory Appli., Vol. 94, 1997.

[8] A. Dembo and O. Zeitount, Large Deviations Techniques, Boston: Jones and Bartlett, 1993.

[9] V. Fabian, "Stochastic Approximation," Optimizing Methods in Statistics, Edited by J. S. Rustagi, New York: Academic Press, 1971.

[10] P. Glasserman and P. Vakili, "Comparing Markov Chains Simulated in Parallel," Prob. Engr. Info. Sci., Vol. 8, pp. 309-326, 1994.

[11] W.B. Gong, Y.C. Ho, and W. Zhai, "Stochastic Comparison Algorithm for Discrete Optimization with Estimations," manuscript, 1996.

[12] P. Heidelberger and" D.L. Iglehart, "Comparing Stochastic Systems Using Regenerative Simulation with Common Random Numbers," Adv. Appl. Prob. Vol.11, pp. 804-819, 1979.

[13] Y.C. Ho, "Heuristics, Rule of Thumb, and the 80/20 Proposition," IEEE Trans. Automatic Control, Vol. 39, pp. 1025-1027, 1994.

[14] Y.C. Ho and M. Deng, "The Problem of Large Search Space in Stochastic Optimization," in Proc. 33rd Conf. Decision and Control, 1994, pp. 1470-1475.

[15] Y.C. Ho, M. Deng, and J. Hu, "Effect of Correlated Estimation Error in Ordinal Optimization," in Proc. 1992 Winter Simulation Conf., 1992, pp. 466-475.

[16] J.Q. Hu, "Strong Consistency of Infinitesimal Perturbation Analysis Estimates," Ph.D. Thesis, Division of Applied Sciences, Harvard University, 1990.

[17] T. Ibaraki and N. Katoh, Resource Allocation Problems: Algorithmic Approaches, Cambridge: The MIT Press, 1988.

[18] N. Patsis, C.-H.Chen, and M. Larson, "Parallel Simulations of DEDS," IEEE Trans. on Control Technology, 1997, to appear.

[19] R.T. Rockafellar, Convex Analysis, Princeton: Princeton University, 1972.

[20] A.H.T. Tchen, "Inequalities for Distributions with Given Marginals," Annals of Prob., Vol. 8, pp. 814827, 1980.

[21] P. Vakili, L. Mollamustaflaglu, and Y.C. Ho, "Massively Parallel Simulation of a Class of Discrete Event Systems," in Proc. IEEE Frontiers MPC '92 Symposium, Washington, D.C., 1992.

[22] D. Yan and H. Mukai, "Optimization Algorithm with Probabilistic Estimation," J. Opti. Theory Appli., Vol. 79, pp. 345-371, 1993. 


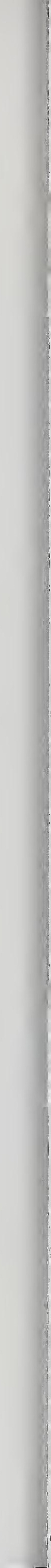




\title{
A Case-Based Integrated Learning Architecture And Its Applications*
}

\author{
Gennady Agre \\ Institute of Information Technologies - Bulgarian Academy of Sciences \\ Acad. G. Bonchev St. Block 29A, 1113 Sofia, Bulgaria \\ E-mail: agre@iinf.acad.bg
}

\section{ABSTRACT}

The paper presents an approach to building an Integrated Learning Architecture in which Case-Based Reasoning is used both as a corrective of solutions inferred by other problemsolving paradigms and as a method for accumulating and refining knowledge of a knowledge based system. The proposed architecture is intended to solve the classification task. The applications of the approach for case-based maintenance of rulebased systems and for case-based refinement of neural networks are briefly described.

KEYWORDS: Machine Learning, Integrated Learning Architecture, Case-Based Reasoning, Knowledge Refinement, Neural Networks.

\section{INTRODUCTION}

An important requirement to the modern knowledge based systems (KBS) is the ability to operate in the real (open) environment [1]. In order to do this they should be provided by the mechanisms allowing them to correct automatically partially incomplete and incorrect expert knowledge used. This means that the modern KBS should be able to learn.

From the other side the modern technology of KBS development is radically changed. The emphasis now is on analyzing, identifying and adapting the already developed KBS components (databases, acquired domain knowledge, KBS shell and models) rather then on the development of KBS "on scratch".

A promising approach to solve both problems is the development of Integrated Learning Architectures (ILA) which are able to use several different problem-solving paradigms or/and machine learning methods $[15,1,2]$.

The present paper describes an approach to building such ILA in which case-based reasoning (CBR) [10] is used both as a corrective of solutions inferred by other problem-

*This research was partially supported by National Science Foundation, Grant I-606/96 solving paradigms and as a method for accumulating and refining system knowledge. The proposed ILA is intended to solve the classification task which is assumed to be described by one of its existing generic model (e.g. $[5,14])$.

\section{INTEGRATED PROBLEM- SOLVING AND LEARNING}

\subsection{The System Architecture}

The case-based ILA structure (see Fig. 1). consists of three main parts: an original KBS, a Case Base Formation Module (CBF) and a case-based reasoning module (CBReasoner).

\subsubsection{The Case Base Formation Module}

The CBF module uses domain knowledge, training examples and the KBS problem-solving model for creating a memory of cases. A case is represented as a single information structure containing the case name, a list of case features (attribute - value pairs) and the case solution. The module should decide i) what training examples are worth to be transformed and stored into the memory as useful past cases, ii) how the memory should be indexed to retrieved only relevant to the current situation cases and iii) how to construct failure predictors for recognizing potentially "dangerous" situations in which the KBS solutions should be corrected. The module should also be able choose an appropriate similarity metrics which measures the degree of likeness between the problem at hand and the retrieved past cases. The CBF module runs off-line and initializes the CB-Reasoner.

\subsubsection{The Case-Based Reasoner}

As an input to the reasoner the output case and its solution formed after solving the problem by KBS are used. The CB-reasoner architecture is an instance of the general architecture of a case-based planer adapted for solving the classification task $[9,2]$. 


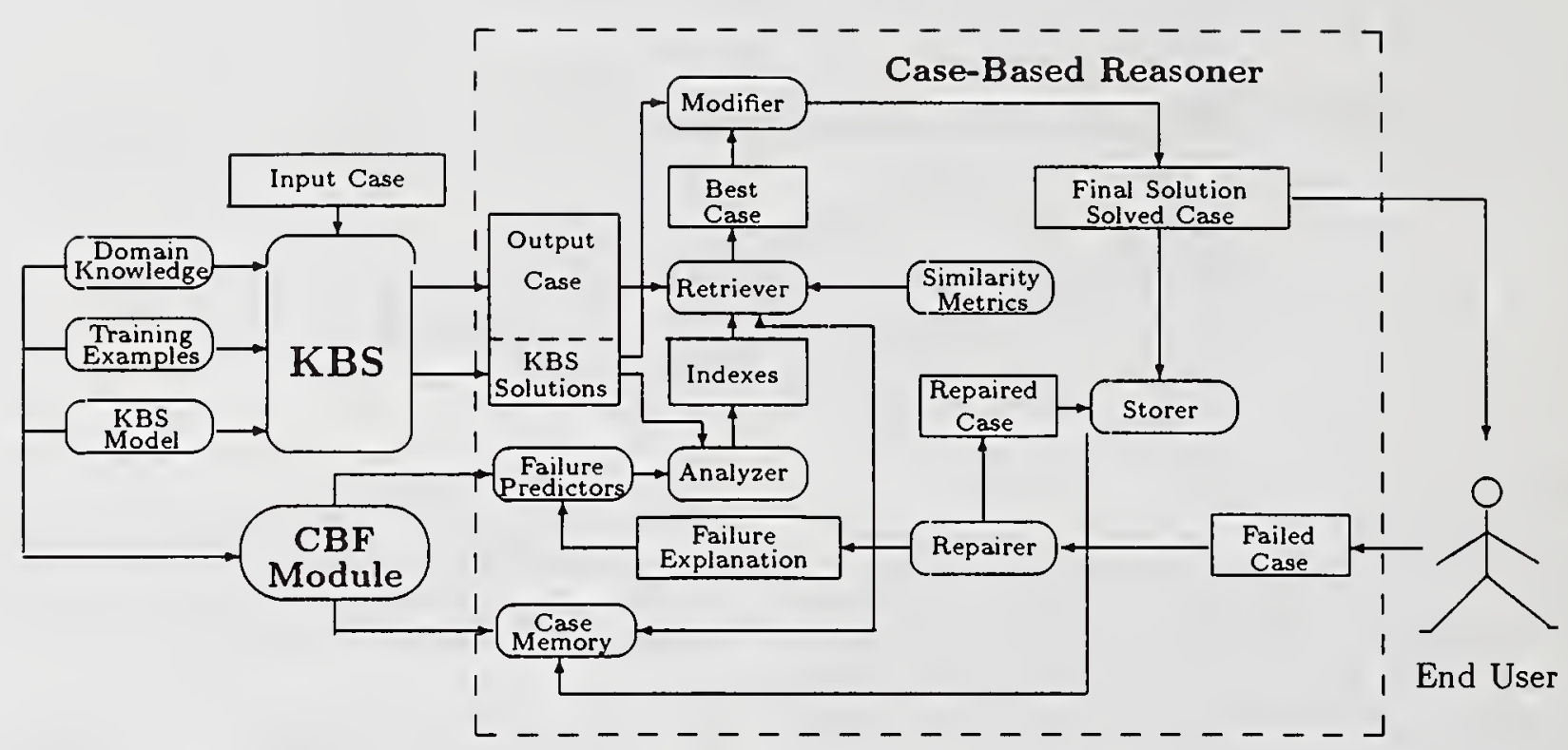

Figure 1. The Case-Based Integrated Learning Architecture

The KBS solutions are initially processed by the Analyzer which should decide whether the solutions must be corrected and if so which set of indexes should be used to determine relevant past cases. The indexes and the current problem description along with the predefined similarity metrics are used by the Retriever to find the most similar ("best") case which solution could be applied to the current problem. The decision about what solution should be preferred - provided by KBS or by the CB-reasoner - is the responsibility of the Modifier. The final solution is given to the end user and the Storer forms and "remembers" the current case into the memory.

After receiving a "feedback" from the user who approves or rejects the final solution, the Repairer starts its operation. In case of approval, the Repairer sends the confirmation to the Storer which then decides whether it is worthwhile to continue remembering this correctly solved case or it may be forgotten. Otherwise, the Repairer tries to "explain" the failed case. In such situations the Repairer creates failure predictors which then used by the Analyzer to avoid repetition of similar failures in the future.

\subsection{Failure Prediction}

The process of searching a case-based solution starts only when the current situation is recognized as "dangerous", i.e. if there is a sufficiently convincing evidence that the KBS solution is wrong. This prediction process is based on comparing the KBS solution to the set of failure predictors formed by the CBF module and extended by the Repairer. The roles of such predictors are played by the KBS solutions of the training examples included in the case base (if such examples are available) and/or by the system solutions recognized as wrong by the user. In other words, the fact that KBS has inferred once an erroneous solution is considered as a sufficiently convincing evidence for suspecting the system every time when it infers the same solution of another problem. Conversely, if a KBS solution of a new problem is different from the known failure predictors, there are no reasons to "suspect" the system and its solution is accepted without any doubt.

\subsection{Case Retrieval and Conflict Reconciling}

The success of integrated approaches combining several problem-solving paradigms crucially depends on the scheme for reconciling the conflicts between them. Most of the proposed methods (see e.g. [8] for integration of CBR with rule-based reasoning) use a threshold scheme in which the threshold values are selected ad hoc. We avoid this deficiency by forming a proper set of cases to be retrieved. In this set we include not only the cases denying the proposed KBS solution but also the cases confirming it. In such a way the retrieval set reflects the ILA problem solving experience and restricts the search space only to the relevant (from the ILA point of view) cases.

\section{CASE-BASED MAINTENAN- CE OF RULE-BASED SYSTEM}

The described above architecture was applied to the task of improving a rule-base system (RBS) problem-solving behavior in the course of its operation [3] - an important part of the adaptive maintenance task [6]. In this application 
the KBS training examples are assumed to be unknown ${ }^{1}$.

\subsection{Linowledge Components}

Domain Knowledge is non-probabilistic rules represented as flat structures associating conjunctions of the problem features with problem solutions. A feature is a nominal attribute-value pair. KBS Model treats the system problem-solving behavior as a hypothesis driven process.

It is assumed that KBS is able to perform both backward and forward chaining since forward chaining is normally used for generation of a list of differential hypotheses (possible solutions) and backward chaining - for testing them. A hypothesis is considered to be confirmed if there is a satisfied rule having the solution as its conclusion and to be rejected if all rules leading to it have failed. The system stops its operation either if a confirmed hypothesis has been found or all generated hypotheses have been tested and rejected.

\subsection{Case Base Formation}

To avoid the problem with the absence of training examples the CBF module treats the rules as generalized "typical" cases describing the domain concepts. Each rule is interpreted as a case represented by a list of features extracted from the rule conditions. The rule conclusion defines the case classification. The typicality of a case is defined as its family resemblance and measured by the ratio of its intra-concept similarity to its inter-concept similarity. The intra-concept similarity of a case is its average similarity to other members of the same concept and the inter-concept similarity - its average similarity to members of all others concepts. The similarity $s(X, Y)$ between two cases with known classifications is defined as the inverse of the distance between them [18]: $s(X, Y)=1-\delta(X, Y)$, where $\delta(X, Y)$ is computed as the normalized Euclidean distance between the corresponding cases:

$$
\delta(X, Y)=\sqrt{\frac{1}{k} * \sum_{j=1}^{k} w_{j} *\left(x_{j}-y_{i}\right)^{2}}
$$

$x_{j}\left(y_{j}\right)$ is the value of $j$-th attribute of case $X(Y), k=$ $\left|A^{x} \cup A^{y}\right|, A^{z}(z=x, y)$ - the set of attributes of the corresponding case. Weight $w_{j}$ denotes the importance of $j$-th attribute of the case and is calculated as the ratio of the number of all case containing this attribute to the whole number of all cases (rules) in the rule base. $x_{j}-y_{j}=1$ if $x_{j} \neq y_{j}$ and $x_{j}-y_{j}=0$ otherwise. For missing values $\left(x_{j}-y_{j}\right)^{2}=\frac{1}{L_{j}} *\left(1-\frac{1}{L_{j}}\right)$, where $L_{j}$ is the number of possible values of $j$-th attribute.

\footnotetext{
${ }^{1}$ See [2] for more detailed description of the task.
}

\subsection{Indexing Scheme}

A case is indexed by all possible roles it may play in the process of rule-based problem solving. Each solved case is indexed as true negative (TN) by each hypothesis rejected during problem solving and as true positive (TP) by the solution found along with the name of the rule inferred this solution.

TP- and TN-indexes are used for indexing cases which have been successfully solved by the RBR system, i.e. when the user has confirmed their solutions. A case is indexed as false positive (FP) by the rule if it satisfies the rule but the real case solution differs from the inferred one. A case is indexed as false negative with respect to (w.r.t.) a given concept which is the real solution of the case if it has been tested and rejected by the concept rules.

A failed case is indexed as untested w.r.t. a given concept - its real solution - if the solution has not been tested by the concept rules.

\subsection{Case Matching}

The distance between a stored case $X$ and a new problem $Y$ is defined as: $\Delta(X, Y)=W_{X} * \delta(X, Y)$, where $\delta(X, Y)$ is the distance measure described in the previous section and $W_{X}$ is the weight of $X$. The weight of a stored case is simply reciprocal of its typicality. The matching procedure is organized as a nearest neighbor algorithm in which a new case is classified according to the class of the best matched case - the case with the minimum value of distance $\Delta$. In the case of ties the most typical case (i.e. with the minimum value of weight $W$ ) is preferred.

When measuring the similarity between an example with unknown classification and a case belonging to a known category, it is naturally to consider only such features of this example which are relevant to the case class. For each category the corresponding set of relevant attributes is defined as the union of all attributes which the rules corresponding to this category refer to. In such a way we avoid the influence of any redundant (for a particular category) features.

\subsection{Storing New Cases}

All cases erroneously solved by the system are stored. Such cases are indexed as false positive or true negative w.r.t. the faulty solution and false negative, true positive or untested (depending on the results of the failure analysis made by the CB-reasoner) w.r.t. the real solution of the problem. The solved case is also indexed as true negative w.r.t. all hypotheses tested and rejected by the system during problem solving session.

A case successfully solved by the system is stored only if its solution has been found as a result of reconciling a conflict between the paradigms and an identical case has not been stored. Such cases confirm the correctness of applying 
the concrete rule or using the concrete case to obtain the problem solution. For each newly stored case the value of weight $W$ reflecting the case typicality is calculated.

\subsection{Selection of Cases to be Retrieved}

The set of cases to be retrieved is determined by comparing the main characteristics of the current situation (the rule which has inferred the problem solution and the set of rejected hypotheses) with indexes connecting stored cases rules. The retrieval set is formed not only by the exceptional cases rejecting the particular rule but also by the cases confirming it. Since the set of such true positive cases may be empty the case representing the rule itself is also retrieved.

In situations when no KBS solution has been found all generalized cases associated with the rejected hypotheses are retrieved along with all solved cases uncovered by the corresponding rules. In both situations the final solution is determined by the best matched case.

\subsection{Empirical Evaluation}

The approach was implemented in the experimental ILA system CoRCase (Correcting Rules by Cases) and tested on two medical domains - prognosis of breast cancer recurrence (BC) and location of primary tumor (PT) - well known in ML community benchmark data bases ${ }^{2}$ (see Table 1).

\begin{tabular}{|l|l|l|l|l|}
\hline Domain & Examples & Classes & Attrs & Vals/Attr \\
\hline BC & 286 & 2 & 9 & 5.8 \\
PT & 339 & 22 & 17 & 2.2 \\
\hline
\end{tabular}

Table 1. Main characteristics the databases used in empirical evaluation.

For the evaluation of the results the random sub-sampling strategy was used [17]. Each database was randomly split into two non-overlapping subsets, one for training (70\% of examples) and one for testing ( $30 \%$ of examples). The experiments were repeated ten times for different splits and the results were averaged.

The training sets were used to induce corresponding sets of rules. Since we wanted to prove applicability of the approach to the expert rules with unknown principles of building, two algorithms of different types were used to simulate the rules. The first one was an ID3-like algorithm inducing discriminating rules and the second - an AQ-type one producing covering rules. The CBF module transformed each rule set into generalized cases which ware then tested on the corresponding set of testing examples.

To evaluate the contribution of CBR and RBR to the final classification accuracy of the system four different problem-solving algorithms were tested. The first one

\footnotetext{
${ }^{2}$ The data was prepared by M. Zwitter and M. Soklic from the University Medical Center, Institute of Oncology, Ljubljana, Slovenia.
}

was pure RBR. In the second algorithm the solution was searched by matching a problem at hand against the generalized cases produced after the rule transformation. In this algorithm (named TC-search) no testing cases had been stored. The third algorithm was an incremental extension of the second one. In this algorithm each solved case had been stored and then used for searching solution of the next problem. The algorithm may be seen as a naive CBR with exhaustive search in the case space. And the last algorithm was an implementation of the method for integrating RBR and CBR described in this section. The average accuracy and standard deviations obtained are presented in Table $2^{3}$.

The results of the experiments show that the best accuracy is achieved by the proposed scheme for combination of rules and cases. It is particularly interesting that on the PT database both the TC-search and the naive CBR methods used separately have worse performance than RBR. It once again proves the effectiveness of the used indexing scheme used which allows to retrieve for matching comparatively relevant cases.

\begin{tabular}{|l|l|l|l|l|}
\hline Domain & $R B R(A Q)$ & TC-search & $C B R$ & CoRCase \\
\hline BC & $62.0 \pm 0.6$ & $70.2 \pm 2.3$ & $70.6 \pm 1.4$ & $71.3 \pm 3.0$ \\
PT & $34.7 \pm 4.2$ & $33.5 \pm 1.8$ & $33.1 \pm 2.0$ & $37.0 \pm 1.6$ \\
\hline
\end{tabular}

Table 2. Classification accuracy $(\%)$ of the tested problem-solving methods.

\section{CASE-BASED REFINEMENT OF NEURAL NETWORKS}

The second application of the proposed approach for building ILA was to the problem of case-based refinement of neural networks $(\mathrm{NN})^{4}$. Our main goal was to study the potential of CBR for further improvement of a trained NN. That is why in that application only a part of the CBreasoner architecture was used (Analyzer, Retriever and Modifier).

\subsection{Knowledge Components}

Training examples used for training $\mathrm{NN}$ is a main source of knowledge. The same training set along with information how the examples were solved by NN after completion of its training phase were used as an implicit problem-solving model of the network.

\subsection{Indexing Scheme}

A case is represented by a list of (normalized) attributevalue pairs and its real solution (classification). Each case

\footnotetext{
${ }^{3}$ See [3] for more detailed description and evaluation of the experimental results.

${ }^{4}$ Initial results are described in [4]
} 
is indexed both by its real and NN solutions. If an example has been correctly classified, it is considered as a "typical" case confirming the NN solution, otherwise - as an "exceptional" one.

\subsection{Case Base Formation}

In our previous research [4] the process of case base formation was implemented as a two steps procedure trying to identify that part of "noisy" (from NN point of view) training examples which might be used by a CBR method. However, the analysis of the initial experiments with the system has shown the low effectiveness of this procedure so now all training examples unsolved by NN are stored as useful cases.

The case matching process is implemented as a Nearest Neighbor algorithm which uses the weighted Euclidean distance $^{5}$ :

$$
\Delta(X, Y)=\sqrt{\sum_{i=1}^{n} w_{i}\left(\frac{x_{i}-y_{i}}{\max x_{i}-\min _{i}}\right)^{2}}
$$

where $n$ is the number of attributes describing a case, $x_{i}$ and $y_{i}$ stand for the values of $i$-th attribute for cases $X$ and $Y$, and $\max _{i}$ and $\min _{i}$ - for the maximal and minimal values of $i$-th attribute.

The attribute weights $w_{i}$ are calculated by the CBF procedure using Relief $F$ algorithm [11].

\subsection{Failure Prediction and Case Retrieval}

The process of prediction is based on comparing the NN solution to the set of failure predictors formed after construction of the case base. The roles of such predictors are played by the erroneous NN solutions of the training examples included into the case base.

In all "suspected" situations the final system solution is found by means of CBR method that matches the current situation against the past cases which are not only exceptional but also typical w.r.t. the NN solution.

The formation of the case retrieval set is organized as a two steps procedure. During the first step the exceptional case which is the most similar to the current problem is found. Then $k$ nearest to this case neighbors confirming NN classification are retrieved. The final solution is searched among these $k+1$ cases.

\subsection{Empirical Evaluation}

The approach was implemented in an experimental ILA system CorNCase2 (Correction Network by Cases - version 2). The system was tested on four well-known ML benchmark databases described by numerical attributes -

\footnotetext{
${ }^{5}$ Only numerical attributes are used.
}

glass (GL), diabetes (DB), breast cancer $(B C)^{6}$ and iris (IR)[13] (see Table 3).

\begin{tabular}{|c|c|c|c|c|}
\hline Domain & Examples & Atts. & Classes & Missing (\%) \\
\hline BC & 699 & 10 & 2 & 2.2 \\
DB & 145 & 5 & 3 & 0.0 \\
GL & 214 & 9 & 6 & 0.0 \\
IR & 150 & 4 & 3 & 0.0 \\
\hline
\end{tabular}

Table 3. Main characteristics of the databases used in the empirical evaluation:

Each database was randomly split into two nonoverlapping subsets, one for training ( $70 \%$ of examples) and one for testing ( $30 \%$ of examples). The experiments were repeated ten times for different splits and the results were averaged.

The TB-RBF system [12] was selected as an instance of NN. TB-RBF uses domain knowledge in the form of decision trees (or rules) to define the topology of a radial basis function network. Each class is mapped to an output unit, each attribute - to an input unit and hidden units correspond to the branches of the tree. To evaluate the CorNCase 2 behavior we also tested weighted (ReliefF-1NN) and unweighted variants of the Nearest Neighbor (1NN) algorithm working on the TB-RBF training set. The average accuracy and standard deviations obtained are presented in Table 4.

\begin{tabular}{|c|c|c|c|c|}
\hline Domain & TBRBF & INN & ReliefF-1NN & CorNCase2 \\
\hline BC & $95.5 \pm 1.2$ & $95.6 \pm 1.1$ & $95.8 \pm 1.2$ & $96.3 \pm 1.2$ \\
DB & $91.9 \pm 4.1$ & $93.9 \pm 4.4$ & $94.4 \pm 3.3$ & $92.3 \pm 4.0$ \\
GL & $70.2 \pm 4.6$ & $69.5 \pm 6.5$ & $70.6 \pm 6.2$ & $75.0 \pm 6.8$ \\
IR & $97.1 \pm 2.3$ & $95.3 \pm 1.9$ & $96.4 \pm 2.1$ & $97.3 \pm 1.7$ \\
\hline
\end{tabular}

Table 4. Classification accuracy (\%) on the test data bases used in the experiments.

It can be seen that CorNCase 2 outperforms TB-RBF in all databases. Moreover, in 3 of them (BC, GL and LD) the proposed integrated method outperforms both problemsolving paradigms used in the integration. These results prove the effectiveness of the proposed scheme for indexing and retrieving cases.

It should be mentioned that the value of parameter $k$ (the number of typical nearest neighbors for each exceptional case) used in the experiments was set to 30 (for DB, GL and IR the same classification accuracy was achieved when $k=15$ ). That means that it is not necessary to store the whole training set - we need only those cases which are members of such lists of neighbors. As a result CoRNCase 2 uses in testing phase about $80 \%$ of training examples for DB, $72 \%$ - for GL, $60 \%$ - for IR and only $36 \%$ - for BC. This shows that the selection of the proper value for $k$ (e.g. by applying some cross-validation techniques) may lead to significant data compression.

\footnotetext{
${ }^{6}$ Missing values were replaced with the most frequently occurring values for the respected attributes.
} 


\section{CONCLUSION}

In this paper we propose an approach to building an integrated learning architecture in which case-base reasoning is used both as a corrective of solutions inferred by other problem-solving paradigms and as a method for accumulating and refining domain knowledge. The architecture is intended to solve the classification task. The applications of the approach for case-based maintenance of rule-based systems and for case-based refinement of neural networks are briefly described. The different aspects of the architecture have been tested on several well-known machine learning databases. The experimental results prove that the approach is an encouraging step towards creating the integrated learning architectures

\section{ACKNOWLEDGMENTS}

The author thanks Dr. Irena Koprinska for providing the results of the experiments with TB-RBF system.

\section{References}

[1] Aamodt, A. Knowledge-intensive, integrated approach to problem solving and sustained learning. Ph.D. Dissertation, University of Trondheim, 1991.

[2] G. Agre. Improvement of KBS Behavior by Using Problem-Solving Experience, Proceedings of the 6th International Conference AIMSA'94, World Scientific Publ., pp. 257-266, 1994.

[3] G. Agre. KBS Maintenance as Learning Two-Tiered Domain Representation, In: Lecture Notes in Artificial Intelligence 1010, Springer, pp. 109-120, 1995.

[4] G. Agre and I. Koprinska. Case-Based Refinement of Knowledge-Based Neural Networks. In: Proceedings of the International Conference "Intelligent Systems: A Semiotic Perspective", October 20-23, Gaithersburg, MD, USA, 1996, Vol. 2, pp. 221-226.

[5] W. Clancey. Heuristic classification. Artificial Intelligence, 27(3), pp. 289-350, 1985.

[6] F. Coenen and T. Bench-Capon. Maintenance of Inowledge-Bases Systems: Theory, Techniques and Tools, Academic Press, 1993.

[7] S. Cost, S. Salzberg. A Weighted Nearest Neighbor Algorithm for Learning with Symbolic Features. $M a$ chine Learning 10, pp. 56-78, 1993.

[8] Golding A.R., Rosenbloom, P.S.: Improving RuleBased Systems Through Case-Based Reasoning. Proceedings of the National Conference on Artificial Intelligence, Anaheim, MIT Press, pp. 22-27, 1991.
[9] K. Hammond. Case-Based Planning: Viewing Planning as a Memory Task, Academic Press, 1989.

[10] J. Kolodner. Case-Based Reasoning. Morgan Kaufmann Publ., 1993.

[11] I. Kononenko, M. Robnik-Sikonia. ReliefF for Estimation and Discretization of Attributes in Classification, Regression and ILP Problems. In: A.M Ramsay (Ed.) Artificial Intelligence: Methodology, Systems, Applications, IOS Press, 1996, pp. 31-40.

[12] M. Kubat and I. Ivanova (Koprinska). Initialization of RBF Networks with Decision Trees. In: Proceedings of the 5th Belgian-Duch Conference on ML (BENELEARN'95), pp. 61-70, 1995.

[13] P. Murphy. UCI Repository of Machine Learning Databases, @ics.uci.edu, Irvine, CA: University of California, Department of Information and Computer Sciences, 1995.

[14] Patil, R.S. Artificial Intelligence Techniques for Diagnostic Reasoning in Medicine. In: H.E. Shobe (Ed.). Exploring Artificial Intelligence: Survey Talks from the National Conferences on Artificial Intelligence, Morgan Kaufmann, San Mateo, CA, pp. 347-379, 1988.

[15] E. Plaza, A. Aamodt, A. Ram, W. van de Velde, M. van Someren. Integrated Learning Architectures. Pavel B. Brazdil (Ed.) Proceedings of Machine Learning: ECML-93, Springer-Verlag, pp. 429-441, 1993.

[16] J. Quinlan. C4.5. Programs for Machine Learning, Morgan Kaufmann Publ., 1992.

[17] S. Weiss and C. Kulikowski. Computer Systems That Learn, Morgan Kaufmann, 1990.

[18] J. Zhang. Selecting Typical Instances in InstanceBased Learning. In: Proceedings of the Ninth International Workshop on Machine Learning (ML'92), Morgan Kaufmann, San Mateo, California, pp. 470-479, 1992. 


\title{
Computational Embodiment: Software Architecture
}

\author{
Christopher Landauer \\ National Systems Group, The Aerospace Corporation \\ The Hallmark Building, Suite 187, 13873 Park Center Rd., Herndon, Virginia 20171 \\ Phone: (703) 318-1666, FAX: (703) 318-5409, E-mail: cal@aero.org \\ Kirstie L. Bellman \\ Information Technology Office, Defense Advanced Research Projects Agency \\ 3701 N. Fairfax Dr., Arlington, Virginia 22203-1714 \\ Phone: (703) 696-2219, FAX: (703) 696-0564, E-mail: kbellman@arpa.mil
}

\begin{abstract}
Computational Embodiment is the computer implementation of principles that we believe will lead to more autonomous and self-generating behaviors that will allow software systems to exist in and interact with complex environments. We restrict our attention here to symbolic environments (MUDs), as an initial step towards understanding and constructing "interaction spaces" in which humans and computer programs can interact on an equal footing.
\end{abstract}

Our approach to constructing autonomous software systems is based on theoretical work on the structures that underly language and movement in biological systems and on the structure of constructed complex systems mediated or integrated by software.

This paper describes how our "Wrapping" approach to software integration, which is a computationally reflective dynamic integration infrastructure, can implement autonomous systems. The wrapping approach supports autonomy by providing at least primitive versions of the functions that are necessary, and by providing an infrastructure that makes changing those functions easy.

\section{Introduction}

In this paper and its companion [4], we describe an approach to constructing autonomous agents that is based on theoretical work on the organization of structures that underly language and movement and on the structure of constructed complex systems mediated or integrated by software.

We are constructing autonomous software agents in symbolic environments called Multi-User Domains (MUDs)
[18] [19] [11], using a style of "computational embodiment", which is part of our research program on interaction spaces [15].

In our view, a system is "autonomous" if it can be said to have "purposeful behavior", e.g., act independently based on internally generated intentions [5]. There are really only two classes of (difficult) requirements for effective autonomy: robustness and timeliness. Robustness means graceful degradation in increasingly hostile environments, as well as an ability to exploit unexpected advantages in the environment, which to us implies a requirement for adaptability. Timeliness means that situations are recognized "well enough" and "soon enough", and that "good enough" actions are taken "soon enough". There is not necessarily any optimization here.

In this paper, we describe our notion of wrappings as dynamic infrastructure (Section 2). Then we turn to a description of the architecture we use (Section 3), and show how wrappings are used to implement the architecture, so we can study some fundamental theory of computational embodiment from the companion paper [4]. Finally, we discuss our prospects for the future (Section 4).

\section{Wrapping Background}

Our research in complex systems has shown the importance of infrastructure, that is, explicit components and activities of the system whose function is to help organize the rest [16]. No matter what kinds of computational models are used, the system will need infrastructure which supports complex interactions. Our "wrapping" approach to intelligent integration infrastructure for constructed complex systems provides a natural means for incorporating adaptation and other processes as computational resources. It is based on automatic processing of ex- 
plicit qualitative information about all of the system components and their interconnection architecture. Its advantages are (1) a simplifying uniformity of description using the meta-knowledge organized into Wrapping Knowledge Bases, and (2) a corresponding simplifying uniformity of processing that meta-knowledge using algorithms called Problem Managers, which are active integration processes that use the meta-knowledge to organize the system's computational resources in response to problems posed to it by users (who can be either computing systems or humans). In particular, since the entire process is recursive [13] [14], wrappings provide a general way to allow specialized methods to participate.

We believe that effective use of any sufficiently complex system requires the system itself to provide many different kinds of assistance to its users, whether they are humans or other computer systems. The system must provide what we have called the Intelligent User Support (IUS) functions:

- Selection (which resources can be applied to a particular problem),

- Assembly (how to let them work together),

- Integration (when and why they should work together),

- Adaptation (how to adjust them to work on certain kinds of problems), and

- Explanation (why certain resources were or will be used or not used).

\subsection{Wrapping Overview}

The wrapping theory has four basic features:

1. ALL parts of a system architecture are resources, including programs, data, user interfaces, and everything else;

2. ALL activities in the system are problem study, (i.e., apply a resource to a posed problem), including user interactions, information requests and announcements within the system, and service or processing requests, etc.;

3. Wrapping Knowledge Bases (WKBs) contain wrappings, which are explicit machine-processable descriptions of all of the resources and how they can be applied to problems to support the five IUS functions above;
4. Problem Managers (PMs), including the Study Managers (SMs) and the Coordination Manager (CM), are algorithms that process those wrappings to collect and select resources to apply to problems. They are also resources, so they are also wrapped.

The wrapping information and processes form expert interfaces to all of the different ways to use resources in a heterogeneous system that are known to the system.

\subsection{Wrapping Information}

The main information entities are Wrapping Knowledge Bases (WKBs). The WKBs contain explicit, machinereadable descriptions of each computational or information processing element (called a resource) in the system: not just how to use it, but also whether and when and why and in what kinds of combinations it should or can be used. The entries in the WKB are called "wrappings". Wrappings have a variable syntax, according to our "variables all the way down" principle, but there are some common features in the semantics [12], which we describe briefly next.

Each wrapping is a list of "problem interpretation" entries, each of which describes one way in which this resource can be used to deal with a problem. There may be several problem interpretation entries for the same problem if the resource has many different ways of dealing with it. Each of these problem interpretation entries has lists of conditions that must hold for the resource to be considered or applied. These sets of conditions are important at different times; one at resource consideration or planning time, and the other at resource application time. These conditions range from data type and interaction protocol statements (for "how" to apply the resource) to qualitative knowledge about the context under which it is appropriate to apply the resource. These act as pre-conditions for the application of the resource. The post-condition is the "product" list of context component assignments, which describe what information or services this resource makes available when it is applied.

\subsection{Wrapping Processes}

The wrapping processes are active coordination processes that use the wrappings for the Intelligent User Support functions [12] [13]. They also provide overview via perspective and navigation tools, context maintenance functions, monitors, and other explicit infrastructure activities. Since they are also wrapped, the entire system is Computationally Reflective [17] [10] [13]. It is this ability 
of the system to analyze its own behavior that provides some of the power and flexibility of resource use.

\subsubsection{Coordination Manager}

The alternation between problem definition and problem study, and the determination of an appropriate context of study, are organized by the Coordination Manager (CM), which is one of the resources that coordinates the wrapping processes. The basic problem study sequence is managed by a kind of resource called the Study Manager (SM), which organizes problem study into a sequence of basic steps that we believe represent a fundamental part of problem solving.

The CM runs a sequence of steps that manages the overall system behavior:

Find context : get a containing context from the user

loop:

Pose problem : determine the current problem

Study problem : use an SM to address it

Present result : to user

Each step is a problem posed to the system by the CM, which then uses the default SM to manage the system's response to the problem.

The main purpose of the CM is cycling through the other three problems, which are posed by the CM in the context found by the first step. This way of providing context and tasking for the SM is familiar from many interactive programming environments: the "Find context" part is usually left implicit, and the rest is exactly analogous to LISP's "read-eval-print" loop, though with rather different processing at each step, mediated by one of the SMs. In this sense, the CM steps represent the basic heartbeat of the system.

\subsubsection{Study Manager}

The Study Manager is the central algorithm of our problem study strategy. It is the default resource for the problem "Study problem". Its purpose is to organize the resources that process the wrappings, and to cause and monitor the behaviors the wrappings describe. There may be other resources that are intended for this same problem, but the SM is the one that is chosen if no other resource applies. This overlap of function illustrates a general principle of resource coordination we have used throughout: instead of trying to find one general method for all cases (which we do not believe is possible anyway), we combine general methods for certain processes with powerful specialized methods that apply in certain contexts.

The SM process begins with a problem poser, a problem defined by its name and associated data, and the context in which the problem was originally posed.

We have divided the "Study problem" process into three main steps: "Interpret problem", which means to find a resource to apply to the problem; "Apply resource", which means to apply the resource to the problem in the current context; and "Assess results", which means to evaluate the result of applying the resource, and possibly posing new problems. We further subdivide problem interpretation into five steps:

\section{Interpret problem :}

Match resources : get list of candidates

Resolve resources : reduce list, make bindings

Select resource : choose one resource to apply

Adapt resource : finish the bindings

Advise poser : describe bindings chosen

Apply resource : go do it

Assess results : evaluate

\subsubsection{SM Recursion}

Up to this point in the description, the SM is just a (very) simple type of planning algorithm. Several additional design features make it a framework for something more. First, all of the wrapping processes are themselves wrapped. Second, the processing is completely recursive. All of the steps listed above for the SM are also posed problems. Third, there are other SMs that have slightly more interesting algorithms (such as looping through all the candidate resources to find one that succeeds, as described in the next section). These three things mean that every new planning idea that applies to a particular problem domain (which information would be part of the context) can be written as a resource that is selectable according to context; it also means that every new mechanism we find for adaptation or every specialization we have for application can be implemented as a separate resource and selected at an appropriate time. It is this recursion that leads to the power of wrapping, allowing basic problem study algorithms to be dynamically selected and applied according to the problem at hand and the context of its consideration.

This recursion has profound implications in other applications of wrapping [14], but for its application to software development, the fact that the SM can be selected is a key. 
Since the SM steps above that interact with the Wrapping Knowledge Bases (WKB) are themselves posed problems, we can use completely different syntax and largely different semantics for different parts of the W KB in different contexts, and select the appropriate processing algorithms according to context. In particular, whether one writes about one WKB or several is a matter of taste and viewpoint. Finally, the SM is only one of a family of processes called Problem Managers (PMs), each of which is wrapped and selectable according to context, and each of which can pose problems and organize their study in different ways.

\section{Implementation Issues}

In this section, we first describe the MUD environment with which the agent must interact, and then the architecture of the experimental system we intend to use for our autonomous agent, based on wrapping. Multi-User Domains (MUDs) are an interesting new kind of groupware that incorporates people into the program. MUDs have become enormously popular as games and as educational support tools over the last few years because they get the human interactions right in some fundamental sense, and because they engage our sense of "place" [18] [19] [11] [15]. MUD clients and servers are easy to obtain and run (most servers and clients are free), but they usually only provide text worlds; there is little interaction with existing tools that are outside the MUD; though some have construction languages that allow complex programming, it is the usual kind of programming; and it is not very easy to access large volumes of information.

\subsection{MUD Architecture}

We start with a description of MUD architecture [15], because that characterizes the environment in which the agent needs to act. We also characterize the MUD environment via the important styles of interaction, so that the agent can distribute incoming interaction items appropriately.

The MUD environment is defined by its architecture:

- Connectivity Management,

- Virtual World, and

- Computational Infrastructure.

The Connectivity Management Layer is responsible for the multi-user capabilities of a MUD. This layer is responsible for the information transmission in both directions between users (i.e., humans or computer programs that use the MUD), and characters (i.e., objects within the MUD that have externally provided behavior). It is common across many styles of MUD, and will not be discussed further in this paper.

Our autonomous agent interacts mainly with the Virtual World, using a small separate layer for interaction with the Connection Management part of the MUD. The Virtual World consists of locations (called "rooms") and interconnections (called "exits"), each of which has a description. We say that an exit is "linked" to a room if the room is its destination (all links are one-way). The only kinds of behavior that are allowed are issuing commands to the server and following links.

For our discussions of MUD architecture, we use one of the simplest MUD servers, called TinyMUD [2] [18] [19], since it has all the essential technical features we want. It is not too complex to extract and describe those features easily, and because it illustrates one of the most important aspects of MUDs: it is the writers and artists that define the culture (not the technical substrate), and it is the culture that makes a MUD viable or not.

The important aspects of the Virtual World are:

- places and connections between them,

- movements,

- objects and their locations,

- actions, and

- relationships among aspects

Everything in the system is a model, which we use to represent objects, relationships, and actions. Everything has attributes, one of which is location. Connections are maps from one place to another. Movements are actions that attempt to use connections or connectionless maps (e.g., teleport). Actions can change any attributes. This is one classification hierarchy of everything, which is derived from the ones common to many MUD servers and other Virtual environments. It will be mapped into a hierarchy appropriate for understanding our experimental environment.

\subsection{Architecture of Experimental System}

The basic architecture of a wrapping system has three layers [12] [13]: the bottom two layers are the Message Distribution Layer, which can be implemented with explicit messages or in other ways, and the Wrapping Core Layer, which contains the default Coordination Manager and Study Manager, and the default resources for CM and 
SM steps. Above that are the wrapping resources (other SMs and PMs), utilities (optimizers and such), and applications. These can be thought of as a single Resource Layer.

It follows that our architecture problem becomes a matter of identifying appropriate resources and their wrappings. We need to describe the problems, resources, and wrappings for the autonomous agent. The architecture is adaptive, so that we can insert new computational resources into connections when they become available. It is also different for different experiments, and there is a meta-architecture that helps build new architectures.

\subsection{Agent Architecture}

Our computational version of the language and movement theory [3] is that there are layers of symbol systems that separately normalize external signals into interesting information spaces, so that the useful processing can take place in those spaces (this separation is the analogue of the behavior of different senses).

The agent runs a set of steps that manages its overall behavior:

Login : to remote server

concurrent loop:

Look around : determine current local environment

Talk : to characters, local or remote

Move arcund : to other places

Do commands : choose some available commands

All of these processes are concurrent, making suggestions about what to do next, but the agent usually doesn't move around or do commands and talk locally at the same time, (these processes interfere with each other because of focus of attention).

Some of the major low-level communication resources in the agent have the following tasks (these are problems):

- login to remote server,

- convert incoming character strings into interaction items,

- convert outgoing items to characters, and

- distribute interaction items according to context and content.
The agent interacts with the Virtual World with other tasks:

- parse room descriptions and other information,

- identify connections, commands, and conversational streams,

- generate movement and other actions, and

- generate conversational interaction items.

We have constructed a number of local interaction grammars, using CYKPL (my variant of V. Pratt's combination of the widely used Cocke-Younger-Kasami and Early parsers [7] [12]), but we can use any of the common table or chart parsers [9], or any other mechanism we choose [1], and with that mechanism we can use whatever new user language we choose (of course, some language choices are better suited to certain kinds of parsing, and vice versa).

\section{Prospects}

Autonomous systems must be complex systems, with an ever-increasing repertoire of possible behaviors and processes for selecting them, fallback choices to account for incorrect situation estimation, and quick partial solutions to reduce decision time. All activity is situated, strongly dependent on context, and there need to be different decision processes in different situations. This flexibility requires an architecture in which many parts of the system are infrastructure, organizing other parts of the system to identify and address problems, and monitoring their behavior [8].

The wrapping approach provides several simplifying uniformities for the infrastructure: Wrapping Knowledge Bases uniformly represent the computational resources (uniformity of description), wrapping processes uniformly treat system activity as problem study (uniformity of processes), and treating the wrapping processes as resources also provides a computationally reflective system, which can reason about its own behavior. The ability to reflect on one's own behavior (and modify it) is one of the main keys to effective autonomy.

Our study of autonomy is part of a larger research program on Interaction spaces [15], which are computer-mediated virtual places in which computer programs and humans meet. Since wrappings allow many special case processes to be combined with general case methods, and MUDs allow multiple programs to be connected together with multiple humans as users, all in the same environment, we have taken the wrapping architecture and applied it to the MUD servers to define these interaction spaces. 
Interaction spaces are a new way for computer programs to be integrated, by combining the interactions among the different kinds of users of the space, be they human or computer programs. The idea is that explicit attention to using co-experience as an integration mechanism can lead to very different styles of interaction among computational agents and humans, and therefore possibly much more interesting program structures and behaviors. This paper is about the integration infrastructures and architectures that support adaptive and reflective behaviors. We believe that using them, we can build agents that are much more useful partners in an increasingly complex information environment.

\section{References}

[1] Alfred V. Aho, Jeffrey D. Ullman, The Theory of Parsing, Translation, and Compiling, Volume I: Parsing, Prentice-Hall (1973)

[2] David Applegate, James Aspnes, Timothy Freeman, and Bennet Yee, TinyMUD, source code available via anonymous ftp from host "ftp.math.okstate.edu", in file "/pub/muds/servers/ tinymud-1.5.4.tar.Z", 130Kb, last changed 13 December 1991 (availability last checked 7 July 1996)

[3] Kirstie L. Bellman and Lou Goldberg, "Common Origin of Linguistic and Movement Abilities", American Journal of Physiology, Volume 246, pp. R915-R921 (1984)

[4] Kirstie L. Bellman, Christopher Landauer, "Computational Embodiment: Biological Considerations", (to appear) in Proceedings ISAS'97: Intelligent Systems and Semiotics 1997, 23-25 September 1997, Gaithersburg, Md. (1997)

[5] Kirstie L. Bellman and Donald O. Walter, "Biological Processing", American Journal of Physiology, Volume 246, pp. R860-R867 (1984)

[6] Paul K. Davis, Richard Hillestad (eds.), Proceedings of DARPA Variable-Resolution Modeling Conference, 5-6 May 1992, Herndon, Virginia, Conference Proceedings CF-103-DARPA, RAND Corp. (March 1993)

[7] Susan L. Graham, Michael A. Harrison, Walter L. Ruzzo, "An Improved Context-Free Recognizer", ACM Transactions on Programming Languages and Systems, Volume 2, No. 3, pp. 415-462 (July 1980)

[8] Barbara Hayes-Roth, Karl Pfleger, Philippe Lalanda, Philippe Morignot, Marka Balabanovic, "A Domain-Specific Software Architecture for Adaptive Intelligent Systems", IEEE Transactions on Software Engineering, Vol. SE-21, No. 4, pp. 288-301 (April 1995)

[9] Martin Kay, "Algorithm schemata and data structures in syntactic processing", Report CSL-80-12,
Xerox PARC (1980); reprinted in Barbara Grosz, Karen Sparck-Jones, Bonnie Lynn Webber (eds.), Readings in Natural Language Processing, Morgan Kauffman (1986)

[10] Gregor Kiczales, Jim des Rivieres, Daniel G. Bobrow, The Art of the Meta-Object Protocol, MIT Press (1991)

[11] Christopher Landauer, The MUDpage, file accessible via the World-Wide Web at URL "http: //tinylondon.ucsd.edu/ cal/MUDpage.html", first version (December 1994), updated (23 January 1995) (availability last checked 7 July 1996)

[12] Christopher Landauer, Kirstie L. Bellman, "Integrated Simulation Environments" (invited paper), pp. 409-431 in [6]; shortened version in Christopher Landauer, Kirstie Bellman, "Integrated Simulation Environments", Proceedings of the Artificial Intelligence in Logistics Meeting, 8-10 March 1993, Williamsburg, Va., American Defense Preparedness Association (1993)

[13] Christopher Landauer, Kirstie L. Bellman, "The Role of Self-Referential Logics in a Software Architecture Using Wrappings", Proceedings of ISS '93: the 3rd Irvine Software Symposium, 30 April 1993, U. C. Irvine, California (1993)

[14] Christopher Landauer, Kirstie L. Bellman, "The Organization and Active Processing of MetaKnowledge for Large-Scale Dynamic Integration", pp. 149-160 in Proceedings 10th IEEE International Symposium on Intelligent Control, Workshop on Architectures for Semiotic Modeling and Situation Analysis in Large Complex Systems, 27-30 August 1995, Monterey (August 1995)

[15] Christopher Landauer, Kirstie L. Bellman, "Integration Systems and Interaction Spaces", pp. 161-178 in Proceedings of the First International Workshop on Frontiers of Combining Systems, 26-29 March 1995, Munich (March 1996)

[16] Christopher Landauer, Kirstie L. Bellman, "Constructed Complex Systems: Issues, Architectures and Wrappings", pp. 233-238 in Proceedings EMCSR 96: Thirteenth European Meeting on Cybernetics and Systems Research, Symposium on Complex Systems Analysis and Design, 9-12 April 1996, Vienna (April 1996)

[17] Pattie Maes, D. Nardi (eds.), Meta-Level Architectures and Reflection, Proceedings of the Workshop on Meta-Level Architectures and Reflection, 27-30 October 1986, Alghero, Italy, North-Holland (1988)

[18] Mike O'Brien, "Playing in the MUD", Ask Mr. Protocol Column, SUN Expert, Volume 3, No. 5, pp. 19-20, 23, 25-27 (May 1992)

[19] R. Riner and J. Clodius, "Simulating Future Histories", Anthropology and Education Quarterly (Fall 1994) 


\title{
Emergence of Complex Organized Natural Systems. A Hierarchically - Structured Conceptualization and Notation of Organization.
}

\author{
Jerry LR Chandler \\ Krasnow Institute for Advanced Studies, \\ George Mason University, 837 Canal Drive, McLean, VA 22102 \\ and \\ Washington Evolutionary Systems Society. \\ JLRChand @ erols.com
}

\section{ABSTRACT}

Our natural world is dynamically composed in real time. Despite frequent perturbations and structural irregularities, substantial zones of stability are observable. The historical emergence of such structurally stable zones from simpler components is supported by lines of evidence from many disciplines $(2,3,12,10,16)$. In order to construct a common semiotic lineage among the diverse symbol systems used by various disciplines, a theory of organization was constructed. The foundations of the theory are developed from observations on the nonlinear dynamics of organisms within ecoments ecoments being defined as the immediate surroundings of systems. Each system, (sub-system, sub-sub-system ...) is assigned four primitives attributes (closure, conformation, concatenation and cyclicity). In principle, each of these four terms is enumerable for a local system. Degrees of organization (symbolized as $\mathrm{O}^{\circ}$ ) are composed from lesser organized systems to higher organized systems in terms of the enumeration of the four primitives. The emergent organizations are enumerated: $1^{\circ}, 2^{\circ}, 3^{\circ}, \ldots$. The patterns of organization at any particular level, $\mathrm{O}^{\circ}$, are composed from patterns at other levels. Thus, no particular science or philosophy is assigned a privileged role in the unfolding of the dynamics.

Mathematically, the systems are composed under the scientific representations of categories as developed in Ehresmann and Vanbremeersch, [10] and Chandler, [4,5,7]. Categorical transitions can be viewed as homologous to Thom's four archetypal singularities $[17,18]$. This notation parallels natural history and allows the facile accounting of the molecular biological mechanisms within a living system. Implications of this theory of natural organization for the design of artificial hierarchical systems are apparent.

Keywords: Category theory, complexity, hierarchy, emergence, theoretical biology, biochemistry, semiotics, modeling, information theory, nonlinear dynamics, structuralism, organizational theory, conceptual bindings, cell, genetics, health and disease

\section{SEMIOTICS OF REPRESENTATIONS}

How are dynamics of the organic components organized to form organisms? The semiotic character of the problem is exemplified by noting that the terms organic, organism, and organization are all derived from the same root! The common semantic origin of these terms suggests the existence of a common perceptual pattern underlies the phenomena of complex pattern generators. If such a perceptual pattern exists, then it should be possible to construct a network of symbols and languages such that the representation of physical, chemical, biochemical, cellular, individual and population processes are meaningfully interrelated. For example, for material objects, a criteria of meaning could be a common understanding of the scale of an object, it parts, its forms of birth and death, and its capacities to unite with or separate from other objects (analogous to Thom's archetypal singularities (17)). Meaningful transdisciplinary communication depends on a mutual understanding among the participants, for example, between the business manager and the mathematician. To understand the meaning of a semantic term for a material object implies some knowledge of the relationships among the parts, the whole and the embedding system surrounding the object itself (6). This is a major task for a biological notation because of the unique sequences of macromolecules which generate the unique dynamics of each individual organism. Since the role of individual DNA base changes (mutations) in the genesis of specific disease states is frequently observed, a useful notation must represent both the structural form of a complex system as well as its dynamic unfolding. Causal pathways are intrinsic to complex behaviors. A semiotics of complexity should include a crisp representation of causality in order to construct to a useful notation. Analogously, the meaning of the mathematical models should provide a basis for predictions. If the scientific models of complex systems are not relatable to practice, the primary goal will not be achieved.

\section{Structures for Scientific Semiotics}

If science is to become a unified body of knowledge, a congruency must be extensible from simple to complex systems. Can the search for congruence among what we observe, what we symbolize and what we describe be viewed as an alternative expression of a desire for scientific predictions? Predictability for a complex system requires a congruence among observations, languages and symbols, as geometrically illustrated in Figure 1. Can this figure also be viewed as a genitive symbol which is independent of the scale, history, or behavior of any particular system 
and for organizing transdisciplinary communication? Are these questions complementary to one another?

\section{Observations}

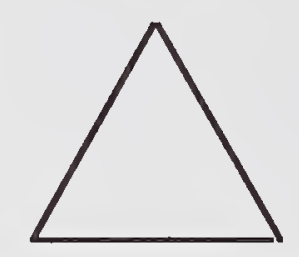

\section{Symbols Languages}

Figure 1. Inchoate symbol for a common scientific semiotics.

Traditionally, scientists have sought to isolate individual objects or classes of objects for study. Disassembly of natural systems into component parts and analysis of the individual units was a successful strategy for the study of simple natural systems. Such work tends to accent the independence of the components. Indeed, physical theories often further accent the notion of independence or autonomy, both in the conceptualization and the mathematical representation. The examples of quantum mechanics and thermodynamics illustrate the critical importance of the concept of independence to scientific thought. However, emergence of a new degree of organization depends on interdependence - a special cooperation among the components such that a vertical genesis process occurs in order to construct a hierarchy. For example, the organic bases within a DNA sequence of a genetic system are not independent, but are tightly linked to each other to form a double helix. This assessment of interdependence among objects was crucial to a logical construction of complexity (the $\mathrm{C}^{*}$ hypothesis) in the WESScomm I, II, and III papers, $(4,5,7)$ where the observed logical interdependence among the classes explicitly motivated the symbolic representation. The description of complexity was built by organized systems of constraints. Thus, conceptual interdependence formed the basis for the organization of the $C^{*}$ hypothesis.

A motivation for logical interdependence comes from the necessary and sufficient conditions for life (5). More explicitly stated, causality in biological systems requires an interdependence among three degrees of organization the genetic system, the organism and the ecosystem as illustrated by the following relations:

A genome is a necessary but not a sufficient condition for the existence of an organism.

An ecosystem is a necessary but not a sufficient condition for the existence of an organism.

The sufficiency of a specific ecosystem to sustain life of a specific organism depends on the genome.
The sufficiency of a specific genome to sustain life depends on the specific ecosystem.

Abstractly stated, internal and external causes fit together dynamically to create necessary and sufficient conditions for the emergence and sustenance of life's' patterns.

A primary objective this notation is express the structure of complexity in symbolic terms such that mathematical relationships among the patterns can be explored. Which mathematical structures are sufficiently rich to support a representation of interdependence, of hierarchical degrees of organization and of nonlinear dynamics? Ehresmann [11] noted an abstract freedom in creating mathematical structures and that the "theory of categories seems to be the most unifying trend in present day mathematics;... ." Ehresmann and Vanbremeersch (10), Thom (17), Rosen (15), and Baas (1) have suggested the use of category theory in science. The structural simplicity of category theory (objects, morphisms, and compositions) lends itself to logical applications. It has found wide application in the design of computer algorithms (object oriented programming) (19). Ehresmann and Vanbremeersch, in a long series of papers starting in 1987, have pioneered the use of category theory to describe mental processes by constructing categorical "Memory Evolutive Systems." A hierarchical perspective on complexity (the $C^{*}$ hypothesis) and the Memory Evolutive Systems are closely related theories (8). We have published a model of a cell based on category theory and pointed out the critical role of time scales in decision making processes (17). Enhanced graph theory, which is somewhat related to category theory, is widely used in the computations of chemical structures. It appears that augmented category and graph theories, along with singularity theory will play a substantial role in the construction of emergent dymamics of complex systems.

A robust, constructive, scientific notation is needed to support the mathematical explorations of organizations of interdependent objects.

\section{Proposal for a robust notation for organized systems}

Historically, a symbol is used to denote, among other possibilities, a category or object or concept or belief or a class of categories, objects, concepts or beliefs.

Let $\mathrm{O}^{\circ}$ be any degree of organization of an object.

Here, a symbol of the form $\mathrm{O}^{\circ}$ is used to denote a general class of organization. The natural question now arises: How can I create a procedure for assigning scientific meaning to the natural sequence of symbols? Since science has a number of organizational principles, a selection is necessary to construct an ordering relationship. The objective of the procedure is to construct a notation such that a one to one correspondence between the languages of scientific observations and the symbolic 
representation of the degree of organization creates a conceptual basis for the enumeration of complexity. Therefore, I choose to select the meaning of the symbols $\mathrm{O}^{\circ} 1, \mathrm{O}^{\circ} 2, \mathrm{O}^{\circ} 3, \ldots$ such that a construction of one to one correspondences between natural numbers and material objects is feasible. I have selected the following specific semantic ordering for material objects of a cell:

$$
\begin{aligned}
& \mathrm{O}^{\circ 1} \text { subatomic particles } \\
& \mathrm{O}^{\circ 2} \text { atoms } \\
& \mathrm{O}^{\circ 3} \text { molecules } \\
& \mathrm{O}^{\circ 4} \text { biomacromolecules } \\
& \mathrm{O}^{\circ 5} \text { cells } \\
& \mathrm{O}^{\circ 6} \text { ecoment } \\
& \mathrm{O}^{\circ 7} \text { environment }
\end{aligned}
$$

This ordering relationship was selected for living systems; it may also be sufficient for general chemical and physical usage, since it uses the atomic table as a guide. The meaning of these symbols is given.

$\mathrm{O}^{\circ 1}$, subatomic particles, consist of three material objects: protons (+), electrons (-) and neutrons.

$\mathrm{O}^{\circ 2}$, atoms, consists of somewhat over one hundred unique objects, composed from the three subatomic particles. The composition of atoms from particles can be enumerated systematically in terms of the natural numbers, preserving the one to one correspondence between particles and particles bound into atoms.

$\mathrm{O}^{\circ 3}$, molecules, consists of a very large number of different material objects composed from atoms or ions. The binding operations which form neutral molecules from atoms preserve one to one correspondences between atoms and atoms bound into molecules. These binding operations concomitantly form the patterns of chemical structures. The patterns formed in molecules are created from the organization of the quantum numbers.

$\mathrm{O}^{\circ 4}$, biomacromolecules, consists of many material objects (not an infinite number), composed from specific classes of molecules. The binding operations preserve one to one correspondences between subgraphs the molecules and the bound subgraphs within the macromolecules.

$\mathrm{O}^{\circ 5}$, cells, consists of living objects which can be represented as have a boundary sustained by a genetic system. The genetic system is composed of components of a consisting of $\mathrm{O}^{\circ 1}$, subatomic particles, $\mathrm{O}^{\circ 2}$ atoms, $\mathrm{O}^{\circ 3}$ molecules, and $\mathrm{O}^{\circ 4}$ biomacromolecules. The ordering relationships among the components of a cell are not completely specified by internal relationships. Nonetheless, orderings relate all the essential components of a cell.

$0^{\circ 6}$, ecoment, consists of the surrounds of the cell. In natural systems, the surround may include $\mathrm{O}^{\circ 1}$, subatomic particles, $\mathrm{O}^{\circ 2}$ atoms, $\mathrm{O}^{\circ 3}$ molecules, $\mathrm{O}^{\circ 4}$ biomacromolecules, $\mathrm{O}^{\circ} 5$ other cells and potentially more highly organized systems. The ordering relationships among the components of an ecoment are not readily specified for natural systems. However, the minimal essential components of an ecoment are known for many cells and higher organisms - they are named "essential nutrients."

$\mathrm{O}^{\circ 7}$, environment, is the embedding system for the surrounds of organisms.

As suggested by the ordering relationships, the term ecoment is introduced to describe the immediate surrounding of the living organism. It is this immediate surroundings which provides the nutrients (the necessary and sufficient conditions) for sustaining life. The term ecoment implies a specific subset of the environment which is experienced by the organism.

A sequence of degrees of organization can be designed for other systems. These designs may require either a smaller number or a larger number of degrees of organization. For example, less complex systems composed from mechanical and / or electrical components would not require as many degrees of organization as a living organism.

As noted above, the conceptual basis of this proposal for a scientific semiotics is the recognition that three degrees of organization are essential to predicting the complete behavior of any system and these three $\mathrm{O}^{\circ}$ must form an ordering relationship $(6,7)$. Philosophically, these three degrees of organization can be expressed in common language as the parts, the whole and the surroundings of the whole. Co-extensive with this language and these symbols are the scaling factors -- the parts are smaller than whole, the whole is smaller than it's ecoment.

\section{Language of description of a degree of organization}

The next step in designing a scientific semiotics for accounting for complexity is to list a common set of concepts which can be applied to a specific system. Four terms can be used to describe the behavior of any degree of organization (7). These four terms are interdependent with each other and must be defined sequentially.

Closure: a domain of discourse, a category, a system, an object, a unity. 
Conformation: the components of the closure of a system, the internal patterns of the system, the relationship among the parts of the system, a three dimensional depiction of the internal description of a system, a specific geometric and algebraic description of components of the system.

Concatenation: binding parts together, linking changes in the conformation, changes in the internal patterns of a system, the specific linkages between parts of the whole, dynamic processes of the system linking patterns to patterns.

Cyclicity: a pattern of concatenations which sustains the system, the potential cyclic walks or pathways over the conformations of the system, the habitual behaviors of the closure.

These four concepts serve as the basis for a linguistic description of simple and complex material systems. In principle, each concept can be applied to the enumeration of each degree of organization. When the material state of a system is known, then these concepts provide a basis for specifying specific objects and may allow an accounting of the complexity. An example illustrating the application of these symbols and concepts to fermentation of wine was reported (Chandler, in press). The description of the fermentation of sugars from natural juices illustrates the interrelationship between scientific languages and scientific symbols at degrees $\mathrm{O}^{\circ 1}, \ldots, \mathrm{O}^{\circ 6}$. Indeed, the organization of entire internal process of fermentation can be symbolized in terms of causal pathways among the labeled asymmetric psuedographs and dynamic processes symbolizing the role of collaborative configurations in generating singularities and accelerating the flow of energy. Since fermentation has been cultivated by human cultures since ancient times, these examples can also be extended to higher degrees of organization to describe the role of fermentation in the history of science and law. For example, cultural controls (laws) governing the purity and wholesomeness of beer were among the earliest food safety rules.

\section{Complex Causal Pathways}

Examination of the differential equations representing fermentation illustrate that the cause - effect relationships in complex systems can be deterministic, highly organized, and extremely selective. Of all the possible singularities which could occur among the $\mathrm{O}^{\circ 1}, \mathrm{O}^{\circ 2}, \mathrm{O}^{\circ 3}, \mathrm{O}^{\circ 4}, \mathrm{O}^{\circ 5}$ and $\mathrm{O}^{\circ 6}$ components of fermentation, the selection of one specific causal walk from glucose to alcohol, among the virtually infinite number of potential chemical singularities, symbolizes a degree of determinism unprecedented in the physical or chemical sciences. How are we to describe or symbolize this unique set of cause effect relationships? I suggest that complex causal pathways be organized in terms of the relative degrees of organization being symbolized in the ordering relationships of the languages of observations. This suggests four terms for directional causality:

$$
\begin{aligned}
& \text { Bottom-up <-------> Top-down } \\
& \text { Outside-inward <------> Inside-outward }
\end{aligned}
$$

One description of cause effect relationships (in thermodynamics, for example) is based on energy flow. This will be call this 'bottom - up' causality. Processes at all degrees of organization involve action and energy flow and hence bottom - up causality. Merely describing energy flow fails to predict the unprecedented determinism observed in living systems. For any particular hierarchical structure, the sum of the mass will uniformly increase with the degree of organization (since the mass of the whole must be greater than the mass of a one component of the whole.) When one or more higher degrees of organization collaborates with lower degrees of organization, it can be formally defined as top - down causality. Thirdly, examination of the yeast cell fermentation example (that is, the material process of a brewery) indicates the necessary role of the ecoment in organized processes. The ecoment is driving the conformation from outside the closure by supplying a component necessary to the cycles. This is named outside - inward causality. Finally, a cell sustains it boundary, its internal structures and its capacity to divide by responding as a whole to its ecoment. It is termed inside - outward causality and is quasi-conserved over generations. It emerges from the organization of the first three forms. These four directions of causality within a categorical perspective, are used to describe the directions of energy flows within $\mathrm{O}^{\circ}$ of a cell.

\section{Discussion}

Semiotics is the study of meaning. (The Greek root, sema, can be translated as 'mark, sign.') How is a scientific description of natural structure related to our common semiotics and transdisciplinary communication?

I seek to place organized systems $\left[\mathrm{O}^{\circ}\right]$ within the higher framework by comparing the constrained scientific usage with the more common general usage. In "Signs, an introduction to semiotics," the American semiotician, $T$. Sebeok [16], asserts that six species of signs are distinct. He asserts that the two parts of the sign are the signifier and the signified. With regard to hierarchy, he asserts: "It should be clearly understood, finally, that it is not signs that are actually being classified, but more precisely, aspects of signs: in other words, a given sign may -- and more often and not does - - exhibit more than one aspect, so that one must recognize differences in gradation. But it is equally important to grasp that the hierarchic principle is inherent in the architecture of any species of sign." The contrast between a scientist's and a semiotician's (Sebeok) view reflects the two cultures. Next, Sebeok's' six species are contrasted with the scientific semiotics developed here. 


\subsubsection{SIGNAL}

Semiotic perspective: "The signal is a sign which mechanically (naturally) or conventionally (artificially) triggers some reaction on the part the receiver."

$0^{\circ}$ systems perspective: The signal is an outside inward cause which pre-supposes the existence of a poised or anticipatory system. In the absence of knowledge about the ratio of scales of the objects, the nature of the relationship between the signal and the poised system is ill-defined. Signals may or may not trigger an observable reaction, depending on the intensity of the signal, the degree of organization and numerous other variables.

\subsubsection{SYMPTOM}

Semiotic perspective:: "A symptom is a compulsive, automatic, non-arbitrary sign such that the signifier coupled with the signified in a manner of a natural link."

$0^{\circ}$ systems perspective: A symptom can be viewed as a special (abnormal) internal conformation. It can inform the skilled observer on one specific category or on a collection of linkages of a hierarchy of categories.

\subsubsection{ICONIC}

Semiotic perspective: "A sign is said to be iconic when there is a topological similarity between a signifier and its denotata." (Sebeok sites Pierce's 1867 paper on 'On a New List of Categories' in which Pierce asserted three kinds of representations -- (a) likenesses (icons) whose relation to their objects is a mere community in some quality' (b.) indices 'whose relation to their objects consist in a correspondence in fact and (c) symbols those the ground of whose relation to their objects is an imputed quality. Subsequently, the category of icon was partitioned into three subclasses, images, diagrams and metaphors by Pierce.)

$O^{\circ}$ systems perspective: Insofar as one has a likeness (icon, mental image) in mind for each degree of organization, one seeks a topological representation which is consistent among all degrees of organization, not just an immediate one. $O^{\circ}$ complexity is grounded on observations of many altemative iconic representations for a single closure as illustrated by correspondences between the degrees of organization.

\subsubsection{INDEX:}

Semiotic perspective: "A sign is said to be indexic insofar as its signifier is contiguous with its signified, or is a sample of it. The term contiguous is not to be interpreted literally in this definition as necessarily meaning 'adjoining or adjacent'; thus "Polaris may be considered an index of the celestial pole..." $0^{\circ}$ systems perspective: Organization hierarchy require two radically distinctive types of indices vertical and horizontal. Vertical indices represent the composition of new degrees of organizations with emergent properties. Each degree of vertical organization is described with a novel logical language of dynamic description. Horizontal indices may simply denote an ordering relationship on an arbitrary list within one language of description. Horizontal indices can create ordering relationships along a causal pathway.

\subsubsection{SYMBOLS}

Semiotic perspective: "A sign without either similarity or contiguity, but only with a conventional link between its signifier and its denotata and with an intentional class for its designatum, is called a symbol."

$0^{\circ}$ systems perspective: Scientific usage of a symbol requires observations. If one views science as a unified body of knowledge, then a specification of meaning is within organized natural structures.

\subsubsection{NAME}

Semiotic perspective: "A sign which has an extensional class for its designatum is called a name. An extensional definition of a class is one given 'by listing the names of the members or by pointing to every member successively." (see Reichenbach, 1948).

$0^{\circ}$ systems perspective: A name within a given degree of organization constrains the material composition of the object to specific patterns of relationships. For a specific living system, a biological name intrinsically distinguishes a unique historical (genetic) path.

6.2 Role of Supra-ordination in scientific semiotic and organizational theory

Distinctions between scientific notation and general semiotics go beyond the meanings of definitions. Sebeok writes of applying the "Law of Inverse Variation," "The terms, sign, symbol, emblem, and insignia are here arranged in the order of their subordination, each term to the left being a genus of its subclass to the right and each term to the right being a species of its genus to the left. Thus, the denotation of these terms decreases; for example, the extension of 'symbol' includes the extension of emblem but not conversely."

The example used to illustrate the Law of Inverse Variation consists of a series of four terms: sign, symbol, emblem and insignia. Set theoretic language is used to indicate that the denotation of each term decreases in a subordination of semantic content -- or, in mathematical terms, a nested sequence of subsets of denotata exists among the four 
terms. If viewed as a discrete sequence of natural numbers, the Law of Inverse Variation implies the extension of a term may become vanishingly small.

Supra-ordination, rather than sub-ordination, is intrinsic to the semiotics of cellular organization presented here. Supra-ordination follows a historical, evolutionary perspective of the emergence of complexity - a path of creativity from the simple to the more complex $[9,12,14$, $3,4]$. Supra-ordination is composed by combining lower symbols to form higher symbols, $\mathrm{O}^{\circ 1}, \mathrm{O}^{\circ 2}, \mathrm{O}^{\circ 3}, \ldots$, in a sequence of natural numbers. While the law of subordination of general semiotics deduces a decreasing domain, this scientific semiotics generates an exponentially increasing domain within the material structure of natural degrees of organization. The number of potential combinatorial binding relationships is mathematically unending. No stopping rules for the series $\mathrm{O}^{\circ 1}, \mathrm{O}^{\circ 2}, \mathrm{O}^{\circ 3}, \ldots$ is known. Assuming the historical trends continue, this implies that more highly cooperative systems will emerge in the future. This is not novel idea, but it provides a new line of support for the thesis [9].

This $\mathrm{O}^{\circ}$ hypothesis provides a basis for scientific semiotics which is both richer and poorer than linguistically-based semiotics. $O^{\circ}$ is richer in the sense that scientific semiotics is based on enumeration of material complexity, hence it demands both algebraic and geometrical pattems within the symbology and semantics of objectivity. It is also richer in the sense that $\mathrm{O}^{\circ}$ provides a basis for the transdisciplinary communities to communicate within a common symbolic framework. By the same token, this scientific semiotics is poorer than general semiotics when attempting to aggregate human desires, values, feelings, longings, ambitions or objectives. Spiritual traditions, literature, the performing arts, and the sciences continue to create more higher organized learned structures, ever more remote from the autonomy of $\mathrm{O}^{\circ 1}$ particles. Natural history is a history of generating options and generating selections of ever greater complexity.

Discussions with B. Chandler, A. Ehresmann, K. Harbaugh, J. Long, R. Khuri, A. Vogt, and B. Weems have added to the pleasure of this composition.

\section{References}

[1] Baas, N. A. (1994). Emergence, Hierarchies and Hyperstructures. Artificial Life III. Ed. C.G. Langton, Addison-Wesley pp. 515-537.

[2] Chaisson, E. (1996). "Cosmological Complexity on the Grandest Scales" Actes du Sym ECHQ, Amiens, 20-26.

[3] Chaisson, E. (1989). The Life Era W.W.Norton, NY.

[4] Chandler, Jerry LR (1991). "Complexity: A Phenomenologic and Semantic Analysis of Dynamical Classes of Natural Systems," WESScomm 134 - 40.
[5] Chandler, Jerry LR (1992). "Complexity II. Logical Constraints on the Structure of Scientific Languages," WESSCOMm 2 34-37.

[6] Chandler, Jerry LR. (1995). "Third Order Cybernetics," Proc. of 14th Int'l Congress on Cybernetics, Namur, Belgium, p. 489-493.

[7] Chandler, Jerry LR (1995). Complexity III. Emergence. Notation and Symbolization. WESScomm 5, 1-12.

[8] Chandler, Jerry LR, Ehresmann, A. C., and Vanbremeersch, J-P. (1994). Contrasting Two Representations of Emergence of Cellular Dynamics" 6 Intl Symp. Systems and Cybernetics, Baden-Baden, DE.

[9] de Chardin, Pierre. T. (1959). The Phenomenon of Man. Harper and Row, NY.

[10] Ehresmann, A. C. and Vanbremeersch, J.-P. (1987). "Hierarchical Evolutive Systems," Bull. Math. Biol. 49 13-50.

[11] Ehresmann, C. (1966). Trends toward unity in mathematics. Cahier de Topologies et Geometrie Differentielle 8, 759 - 765 .

[12] Jantsch, Erich (1980). The Self-Organizing Universe. Scientific and Human Implications of the Emerging Paradigm of Evolution. Pergamon Press, Oxford.

[13] Miller, J. (1978). Living Systems. McGraw-Hill, NY.

[14] Morowitz, H. J. (1992). Beginnings of Cellular Life. Metabolism Recapitulates Biogenesis. Yale Press.

[15] Rosen, Robert (1991). Life Itself. A Comprehensive Inquiry into the Nature, Origin and Fabrication of Life. Columbia Univ. Press, NY.

[16] Sebeok, Thomas A., (1994). Signs: An Introduction to Semiotics, Toronto Press.

[17] Thom, Rene (1990). Semiophysics, Addison-Wesley.

[18] Vanbremeersch, J.-P, Chandler, Jerry LR and Ehresmann, A.C. (1996). "Are Interactions among different Time Scales a Characteristic of Complexity?" Actes Du Symposium ECHO Amiens, Fr., 162-167.

[19] Walters, R. F. C., (1991). Categories and Computer Science. Cambridge Press. 


\title{
A HOLONIC APPROACH TO INTELLIGENT CONTROL OF A FLEXIBLE TRANSFER SYSTEM FOR MANUFACTURING
}

\author{
Mihaela Ulieru \\ School of Engineering Science \\ Simon Fraser University \\ Burnaby, BC V5A 1S6 CANADA \\ ht t p://www.sfu.ca/eng-sci/irms \\ Tel. +1-604-4150636 \\ Fax. +1-604-2914951
}

\begin{abstract}
This paper presents the design and implementation of a holonic approach to intelligent control of a flexible transfer system which is regarded as a multi-agent system. After a brief introduction in the concepts of holonic manufacturing the design of a multi-agent architecture for the flexible transfer system is presented. Then a behavioral control strategy supporting the triplet Planning-ExecutionMonitoring is presented together with its implementation for the control of the work-pieces transfer on the optimal path.
\end{abstract}

Keywords. Multi-agent architecture, holonic control, flexible transfer system.

\section{INTRODUCTION - HOLARCHIES OF HOLONS}

In the past few years the application of the socio-economic principles developed by Artur Koestler in his book "The Ghost in the Machine" has revolutionized the organizational/management principles of the manufacturing industry worldwide[9]. Briefly, holonic manufacturing systems can be regarded as a unified way to approach the hierarchical control of any manufacturing unit, from the production process to the whole enterprise level. Broadly speaking holons have most of the generic properties of agents, being mainly distinguished through their dual "whole-in-the-part" nesting properties of super ordination to the part(s) which is (are) contained by them and subordination to the bigger whole to which they belong. Families of "wholes within wholes" (named holarchies) replicate the same properties starting from an overall highest level which includes several "sub-wholes" with similar properties, these in turn including other "sub-sub-wholes", etc. until a last level contains atomic parts which don't contain any other "subwholes" and which usually have different properties than the (sub-)wholes Fig. 1. As an example one can consider a federation of states (e.g. USA or Canada) within a country. The country as a holarchy of states, has its own political structure. Each state in turn replicates a $n$ own political structure. Within each state there are districts with there own political organizations. These are the atomic elements which don't contain any other holons.

\section{THE FLEXIBLE TRANSFER SYSTEM}

In this paper a holonic approach to the implementation of the control strategy for a flexible transfer system is presented. The system, Fig. 2, consists of a set of pallets (organized in a grid which can have different shapes) which transfer a certain number of work pieces simultaneously. Each pallet is regarded as an agent, which is a sub-holon in the holarchy represented by the whole system of pallets constituting the transfer system. 


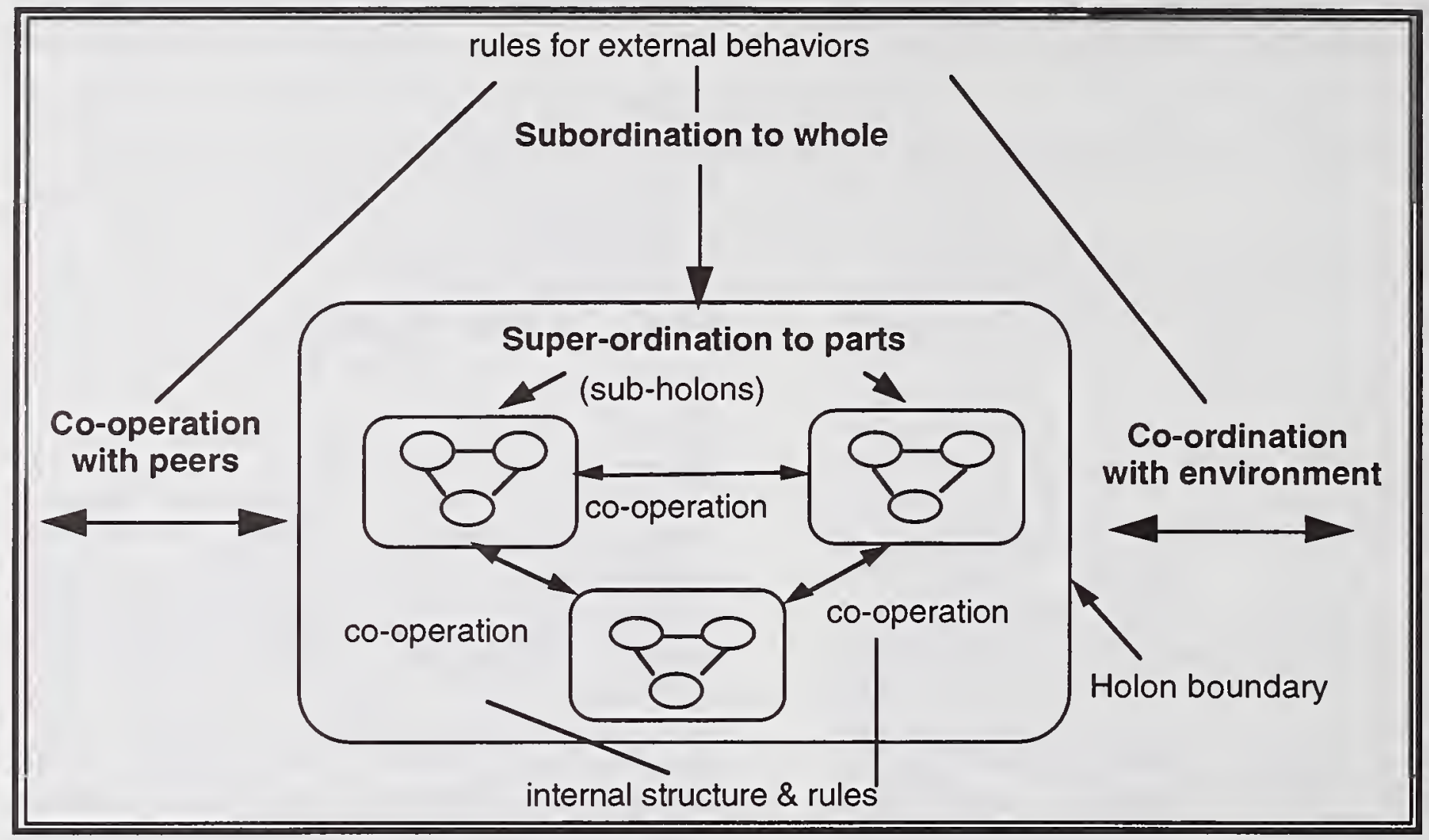

Fig. 1: Holarchy as a system of nested holons [from 9]

Each pallet (regarded as an agent/subholon) has the ability to act autonomously via a computational system which includes ability to process sensor information and data carriage, which allows as well communication with the other pallets (agents/sub-holons) via a communication module, Fig. 3. The spatial configuration of the holarchy can be changed while preserving the overall control strategy which allows each holon to move the work-piece in any direction via an OMNI-wheels mechanism. The overall goal is to minimize the time of transfer of all work pieces from their initial place to a desired destination while minimizing the conflictual situations (like e.g. when two work pieces should cross at the same time a pallet on their minimal path,(etc.)

\section{THE PROPOSED ARCHITECTURE FOR} THE FLEXIBLE TRANSFER

\section{SYSTEM REGARDED AS A MULTI- AGENT SYSTEM}

The representation of a pallet presented in Fig. 3 can be regarded as a n intelligent agent, as the term is generically understood in the AI community being an entity capable of understanding its environment and able to independently deliberate and reason how to use own resources to reach a desired goal. The agent has the ability to move in a certain manner and $\mathrm{can}$ manipulate objects in its environment. The main concern from an architectural perspective is to find the most adequate way for agent interaction and cooperation such that agents can achieve their goals optimally (in our case the fastest transfer of the work pieces from their initial positions to the desired destinations.) 
As presented in Fig. 4, the proposed architecture for the flexible transfer system consists of groups (sets) of

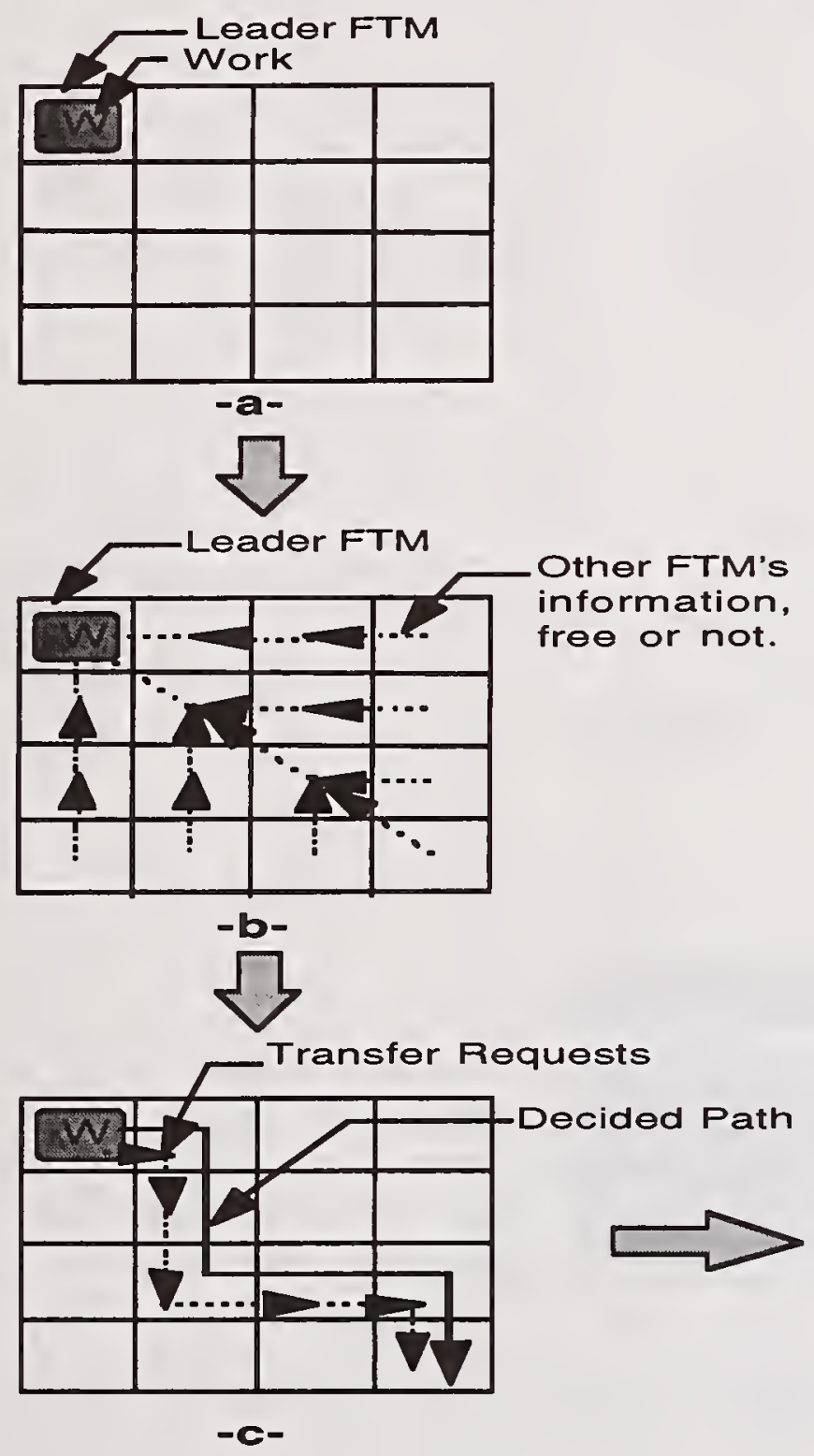

Fig. 2: The Flexible Transfer System

belonging to the same group are coordinated by a supervisor of the group. The supervisor's goal is to provide its agents with necessary information which would allow them to achieve their own goals. A group of agents can be regarded as a blackboard system where specialists represent knowledge sources regarding specialist agents. Each group is able to perform a certain kind of specialized task. The interactions among specialist agents

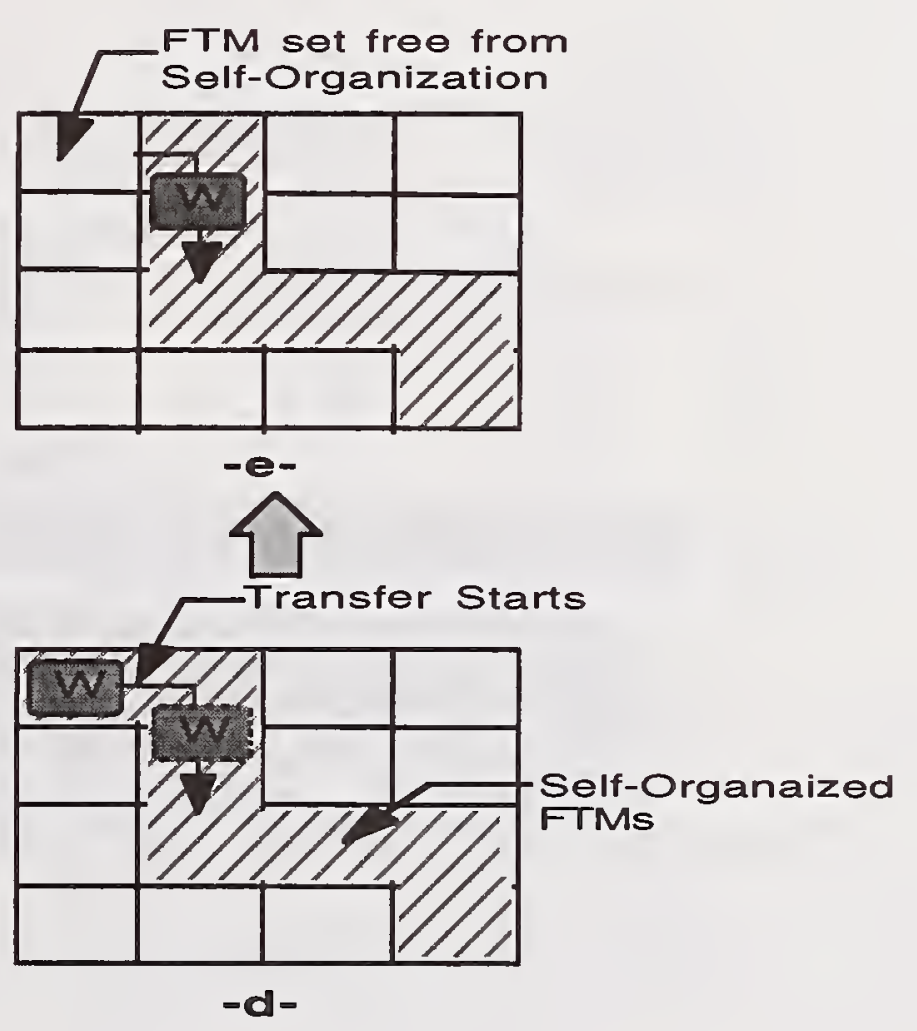

execution of a certain kind of task and the supervisor plays the role of both blackboard and control mechanism of the system. Since the architecture contains many supervisors, the proposed multiagent-system model can be viewed as a multi-blackboard architecture. 

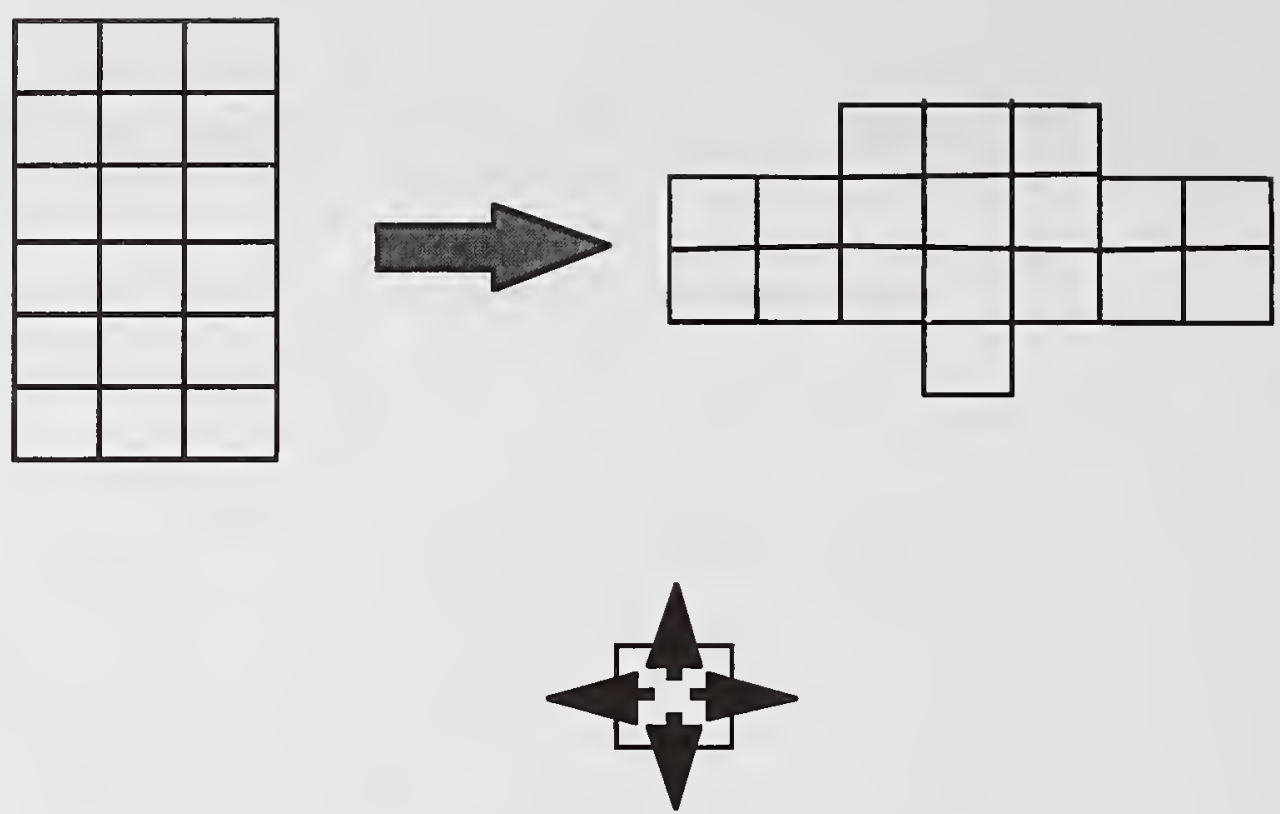

FT-Module

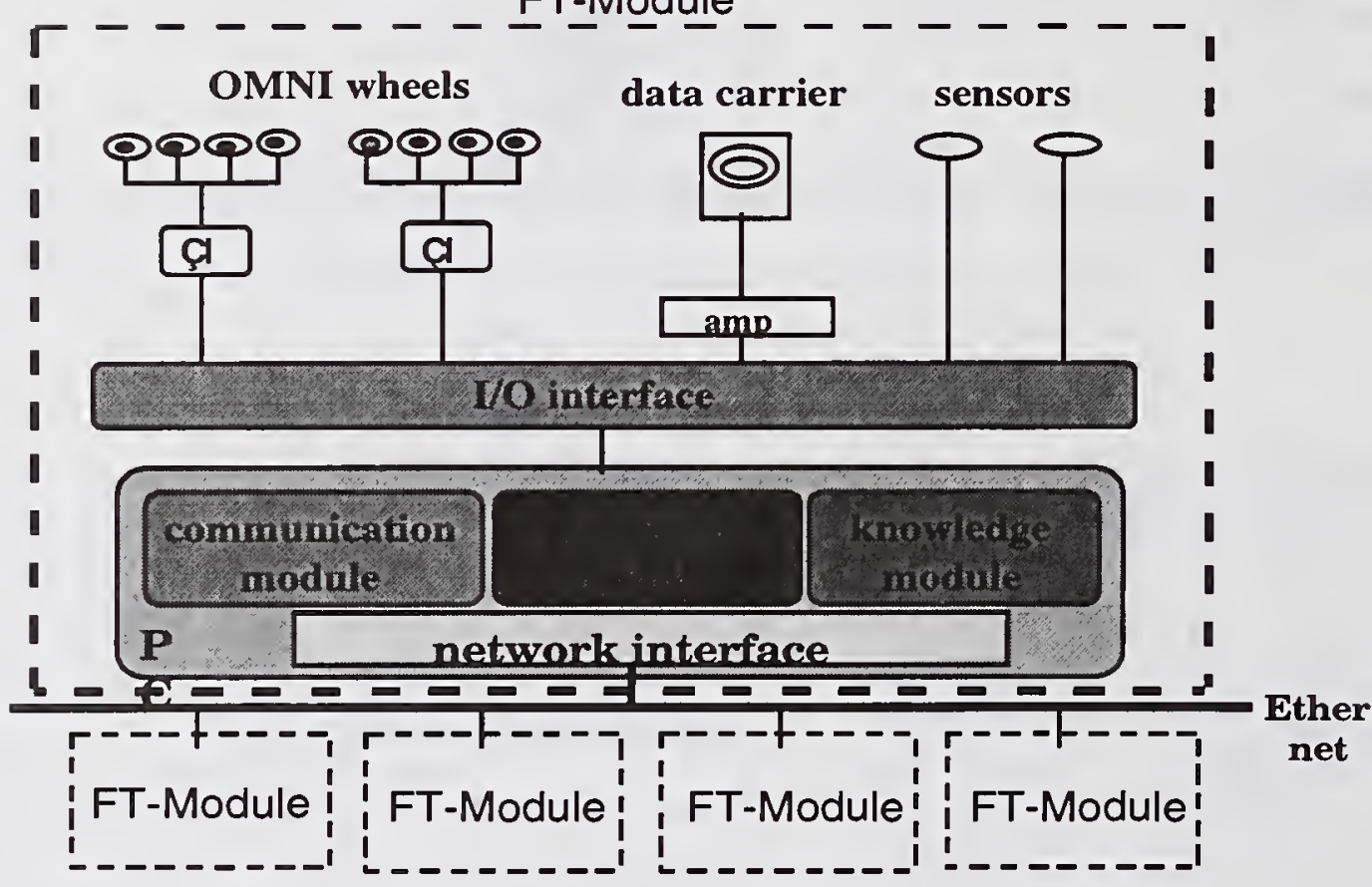

Fig. 3: Generic structure of a pallet (agent/sub-holon) in the flexible transfer system holarchy )

The two main classes of agents: supervisor and specialist will be instantiated for the flexible transfer system as follows. Any "leader" pallet (i.e. any pallet that carries a workpiece) is a supervisor for the whole time it carries the workpiece or transports it towards its next carrier-pallet (leader). All the potential next carriers (potential next "leaders") are specialist agents belonging to the same group, for which the groupsupervisor is the pallet that carries the work-piece at that moment in time. There are as many groups of specialist agents as many work pieces are transported at once. Each group has as supervisor the carrier of the work piece. Each group is on-line dynamically organized in order to form a path to transport the work piece to its final destination. Group supervisors 


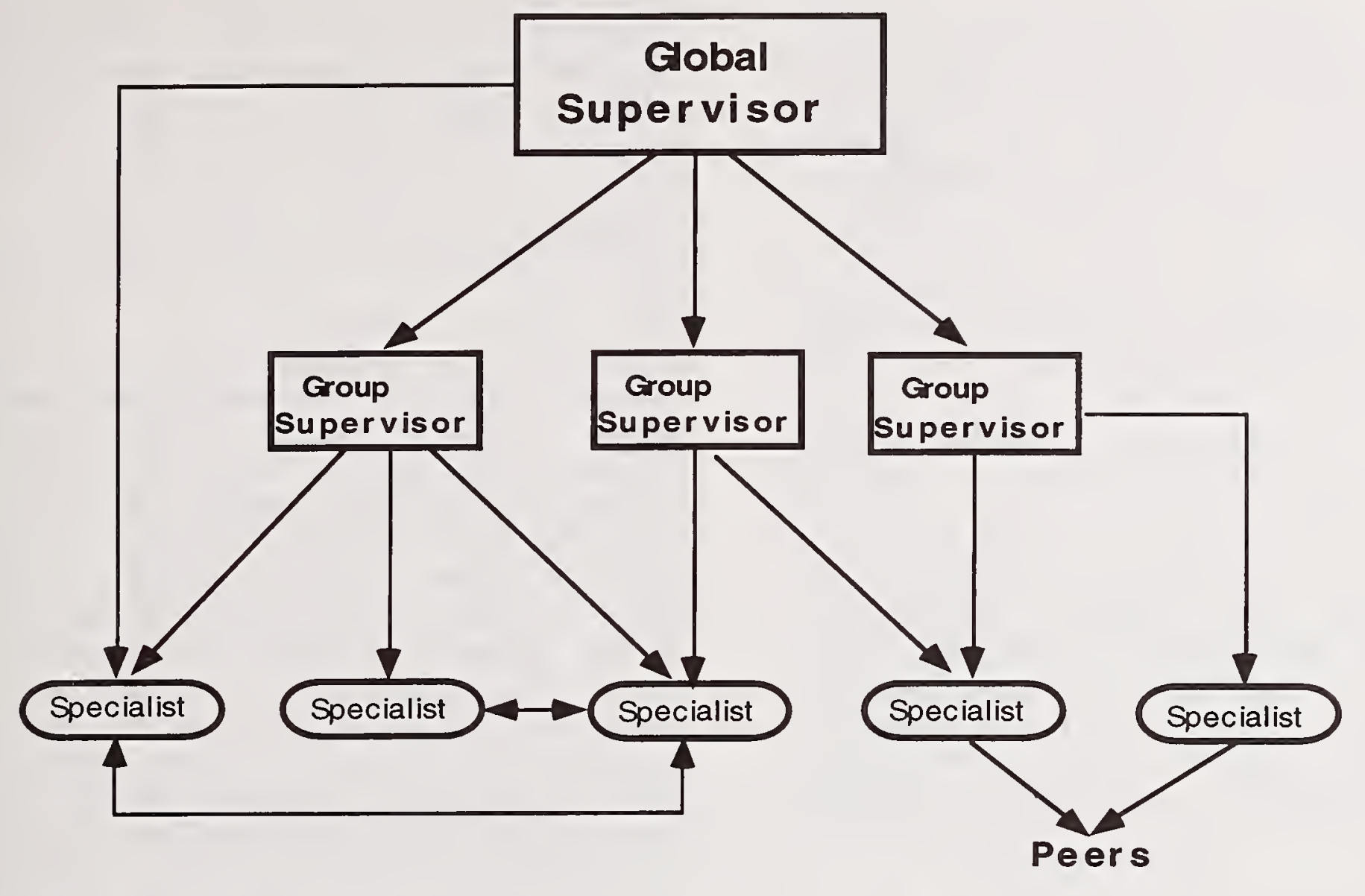

Fig. 4: Multi-agent architectural model of the FTS

cooperate in order to solve conflicts in case two paths cross each-other or overlap on a certain portion. In extreme situations decisions are made through negotiation as of which piece should pass first through the same cell (pallet).

Each specialist agent on a path will become a supervisor when the work piece reaches it. So, the configuration of the architecture is dynamically changed. The next carrier becomes from specialist agent a supervisor and in turn the last supervisor becomes a specialist agent after the work piece has been transported on the next carrier. Each group defines a class of agents consisting of individual objects which are specialist agents. Interactions between groups are managed by the group supervisors at each step. The goal of each group is to transport the piece carried by the supervisor as soon as possible to the desired destination. Supervisors are able to do optimal path planning, conflict resolution, cooperation, negotiation and other intelligent tasks.

Given that the upper limit in the number of supervisors equals the maximal number of pieces that can be transported at once, this number has to be determined together with the analysis of the feasibility of the proposed control solution. As well an upper limit of the number of workpieces to be transported at once by the flexible transfer system without need of a global supervisor has to be determined. Once this threshold is exceeded the control architecture has to be enhanced with a higher level of overall control implemented via a global supervisor. The role of the global supervisor would be to manage/coordinate the interactions between group supervisors, solve conflicts between them and help their negotiation strategies in making adequate decisions by setting priorities according to a-priori established rules. 
Within a group, specialist agents have two types of communication: horizontal (characteristic to the autonomous architectural paradigm) and vertical (characteristic to the blackboard architectures.)

Horizontal communication aims to support interactions between specialists belonging to the same group.

Vertical communication indicates how specialists interact with their group supervisor.

Specialist agents are endowed with capabilities for perception (sensors) reasoning and planning (i.e., they are cognitive). They must have the capability to adapt themselves to unexpected situations. This is achieved through adequate adaptive and dynamic control strategies.

\section{HOLONIC CONTROL WITH PLANNING-EXECUTION-MONITORING}

The proposed control strategy is based o $\mathrm{n}$ the triplet of Planning, Executing and Monitoring (in short PEM.) In designing intelligent multi-agent control and especially in building a logical model, all activities (manipulation, motion and sensing) are implemented with corresponding planning, executing and monitoring elements. Each activity is expressed as an instance of the PEM triplet.

The connections between the generic activities Planning, Execution and Monitoring are made by means of control, data flow and related models. There are different choices to carry out the activation and deactivation of planning, executing and monitoring. Ability to cope with re-planning in dealing with unexpected situations is essential. It is the responsibility of executing and monitoring to decide whether the current plan is considered valid or not. If not, re planning will be activated. The actual decomposition takes place in the execution phase, while the planned operations are activated. The execution mechanism takes care of activation and deactivation of planned operations as described in the plan. Activation of a decomposable operation means activation of the meta-control at the immediate lower level.

The atomic operations pass control commands and sensing requests to actuators and sensors through devicedrivers. They process and pass information for the use of planners and executors to the local models, either to their own level or upper levels of the control hierarchy. Atomic operations also update the global models and therefore are used to maintain model consistency. The way the system reacts to erroneous or unexpected situations (not covered with the possible conditional structures in the plans) depends not only of the capability of the planners to find recovering plans but also on the timing logic of the metacontrol. Reactive behavior can be achieved only if replanning and the subsequent execution are done as quickly as possible.

The proposed control strategy is inspired by the synchronous planning principle of Albus [2]. In his model, Albus assumes instant planning done synchronously in a frequency typical to the level of hierarchy. It means that the ordinary planning is signaled to start by a clock mechanism, while replanning is signaled a-synchronously from erroneous situations. The distinctive aspect of our strategy is to decompose the execution mechanism into lower level planning and execution mechanisms (nested.) Planners are considered to do all the planning related activities, that is also the computation based sensory information during the execution. After a partial plan has been created, the execution starts. The planning is activated again when acquired sensory information has to be transformed into plan parameters. There are several ways in which the executor and the planner can be synchronized (i.e. connected to the data in the local model 
either with a direct control signal from the executor to the planning function of the planner - in case the goal exists - or by passing the control signal via the meta-control - if a goal does not exist.)

\section{IMPLEMENTATION OF THE CONTROL STRATEGY}

Considering an object-oriented mail-box based communication approach, an agent is regarded as a concurrent object blocked in waiting the next message to execute. Each agent is characterized by its mailbox which is managed by a thread. This allows an asynchronous communication between agents which are physically distributed through a set of stations.

We define five classes of objects: object, class, meta-super, specialist and supervisor. The basic executing model contains following classes: object, class and meta-super. The class object has two kind of actions: ask - that enable object to get a value from an attribute and give that enable object to affect a value to a $n$ attribute. In the class class the action new allows to create a new class. In the class meta-super, the action create-super instantiates supervisor objects. The class meta-super allows to generate as many control levels as demanded by the complexity of the system. The action broadcast allows a supervisor to communicate with its group's agents.

A group supervisor has a limited view of the environment. All group supervisors whose physical environment should be crossed by a piece must cooperate in order to lead this piece to the desired point. Each supervisor has a goal to move the piece to the desired point, and according to the environment it manages it provides a plan for moving. The global supervisor determines what supervisors must cooperate and specify them the trajectory the piece should pursue. By knowing the initial position of the piece as well as the final position the piece should reach, the global supervisor has a function sub-cooperate which takes coordinates of these points, its belief base (bell-base), and returns a list (List-ofsup) of group supervisors which must cooperate to satisfy this goal. Furthermore, its belief base contains coordinates of a set of points situated o n the intersection between two physical possible paths. These points represent "points" of cooperation between group supervisors coordinating the specialists groups on each of the intersecting path.

Consider for example $S$ being a global supervisor and S1, S2 group supervisors. To plan the move of a piece from position A to position $B$ the global supervisor should perform a sequence which can be encoded as follows (given our above considerations):

sup-cooperate (bell-base,A,B,List-of-sup); any $(\mathrm{Si}, \mathrm{Sj})$ in List-of-sup $\mathrm{X}$ List-of-sup inform (Si, M(x,y)); inform $(\mathrm{Sj}, \mathrm{M}(\mathrm{x}, \mathrm{y}))$;

broadcast (List-of-sup, plan-path);

In the above sequence, $M$ is a $n$ intersection point between two possible paths controlled by $\mathrm{Si}$ and $\mathrm{Sj}$, and $(\mathrm{x}, \mathrm{y})$ are its coordinates. As a result of message execution plan-path, each group supervisor plans a sequence of actions for enabling a robot to move in its physical environment.

For the supervisor $S 1$ the sequence of operations would be:

move-straight (30 pallets); turn-left (90 degrees); move-straight (30 pallets);

For the supervisor S2:

move-straight (25 pallets); turn-right (90 degrees); move-straight (20 pallets); turn-left (90 degrees); move-straight (20 pallets); turn-right (90 degrees); move-straight (15 pallets); 
Once the piece has reached the desired position, it needs to be integrated within the new group. The supervisor S2 must initialize the new transfer by specifying the belief rule base to load. The goal is to enable the transfer of the piece in the new environment. In this way the supervisor represents a cooperation framework which enables him to adopt a plan of actions by selecting (metacapabilities at the group's agents.

\section{CONCLUSIONS}

In summary, the proposed architecture is adjustable to applications with different levels of complexity. The control of the complexity is managed via the number of hierarchical levels chosen for the particular instantiation. In its simplest instantiation the architecture consists of a single group having one supervisor, which represents a blackboard configuration. There is practically no upper limit for the complexity of the system to be represented, which can be encapsulated by increasing the number of hierarchical levels in the architecture. This increases the flexibility and efficiency of the control strategy responsible for the adequate functionality of the proposed multi-agent architecture. The hierarchical control levels may be exploited as meta-levels for learning in a multi-agent environment. An agent has its own capability of learning, but if it encounters a non deterministic state it must interact with its supervisor to decide what actions it should choose to pass in a deterministic state.

\section{ACKNOWLEDGMENTS}

The author would like to express her gratitude to Toyo Engineering Corporation from Chiba, Japan and Intelligent Robotics Corporation, North Vancouver, Canada for sponsoring this work.
[1] Albus,J., Barbera, V., Nagel, H., "Theory and Practice of Hierarchical

Control", Proc. of the 23rd IEEE Computer Society International Conference,

Washington, Sept. 1981, pp. 18-27.

[2] Albus, J., McLean, B., Barbera, V., Fitzgerald, A., "An architecture for real-time sensory interactive control of robots in a manufacturing

facility", Proc. of the 4th IFAC/IFIP Symposium on Information Control

Problems in Manufacturing Technology, Gaithersburg, 26-28 Oct. 1982, pp. 81-90.

[3] Herman, I. and Albus, J. "Overview of Multiple Autonomous Underwater

Vehicles Project", Proc. of the 1988

IEEE Int. Conf. on Robotics and Automation,

Philadelphia, 24-29 April 1988, Vol.2, pp. 618-620.

[4] Albus, J., McGain, W., Lumia H., NASA/NBS Standard reference Model

for Telerobot Control System
Architecture, NIST Technical Note 1235, 1989 Edition, 76p.

[5] Brooks, K. "A robust layered control system for a mobile robot", IEEE

Journal of Robotics and Automation, 2, 1/1986, pp. 14-23.

[6] Brooks,K. Flynn,J. "Robot Beings", Proc. of the Int. Workshop on Intelligent

Robots and Systems, 1989, Tsukuba, Japan, pp. 2-10.

[7] Norelis, G. and Chantilla, T. "A general structure for mobile robot action control", Proc. of the International Workshop on intelligent Robots and Systems, IROS'89, Tsukuba, Japan, pp. 550-556.

[8] Brooks, A. "Intelligence without reason", MIT AI Lab. Memo 1293, April 1991.

[9] Intelligent Manufacturing Systems Consortium - Project on Holonic Manufacturing Systems, Vols. 1-3, Edited 1996 (proprietary info.)

\section{REFERENCES}




\section{Learning Coordination Patterns from Emergent Behavior in a Multi-Agent-Based Manufacturing System}

Maturana, F.

Graduate Student

Ph: 403-220-5782

Fax: 403-282-8406

maturana@enme.ucalgary.ca
Balasubramanian, S.

Graduate Student

$\mathrm{Ph}: 403-220-5782$

Fax: 403-282-8406

balasubr@enme.ucalgary.ca
Norrie, D.H. *

Nortel Chair in Intelligent Manufacturing

Ph: 403-220-5787

Fax: 403-282-8406

norrie@enme.ucalgary.ca

Division of Manufacturing Engineering, The University of Calgary

2500 University Drive, Calgary, Alberta, Canada

$\left[{ }^{*}\right.$ Corresponding author $]$

\section{ABSTRACT}

Multi-agent systems provide responsive and reconfigurable information-processing structures that can facilitate flexible, efficient use of manufacturing resources in a rapidly changing environment. For maximum scalability and fault tolerance, agents relevant for a particular task may be dynamically gathered into communities where they are coordinated and arbitrated by mediator agents. In a dynamic manufacturing environment, the collective behavior of such a system is an emergent property; hence, robust learning mechanisms are required for effective mediation. A learning mechanism that enhances the capabilities of a multi-agent concurrent design and manufacturing system is presented, and associated implementation issues are discussed.

Keywords: Multi-Agent System; Manufacturing; Emergent Behavior; Learning; Coordination; Mediator.

\subsection{INTRODUCTION}

Heterogeneous multi-agent systems, comprising a network of dissimilar autonomous interacting software entities called intelligent agents (Wooldridge and Jennings, 1995), are becoming increasingly popular for implementing distributed, cooperative manufacturing planning and control systems. Such systems provide responsive and reconfigurable information-processing structures that facilitate flexible, efficient use of manufacturing resources in a rapidly changing environment.

Intelligent agents are knowledgeable in their local domains and share the responsibility of achieving multiobjective system goals through concurrent negotiation. The relevant agents for a particular task may be gathered into a special community that is dynamically formed, coordinated and arbitrated by mediator agents (Wiederhold, 1992) for maximum scalability and fault tolerance. In this architecture, the responsibilities of a traditional centralized supervisory manager are distributed among the mediator agents (Duffie, 1990).

These mediators are also coordinators, possessing organizational knowledge and meta-level rules necessary to facilitate cooperation among intra- and interagent communities. In a dynamic manufacturing environment, their knowledge cannot be static, since the system's behavior depends on problem solving. Thus, the system's collective behavior is an emergent property, requiring robust learning mechanisms for effective mediation.

One possible approach is learning from history. For example, in manufacturing planning and scheduling many repetitive activities can be learned for reuse. For this, the intelligent system needs encoding models and specialized reasoning agents to decide upon the use or rejection of learned transactions. The learning ability facilitates improvement in the performance of both individual agents and the multi-agent system overall.

In addition to learning, a sophisticated mechanism for propagating the state of agent interactions into the future is required. This attribute provides an intelligent forecasting medium through which multi-agent system responses can be adjusted and refined.

\subsection{RELATED WORK}

In recent years, researchers have explored various apprcaches for learning and emergent behavior in multi-agent systems. The notion of "behavior" has become recognized as a fundamental building block in the artificial intelligence, control, and learning research community (Mataric, 1995). Behavior is seen as a regulator in interaction dynamics between agents and the environment. This phenomenon has also been observed by Steels (1990); Maes (1990); Brooks (1991).

The Touring Machine agent design architecture (Ferguson, 1992) has been used to control multi-agent activities in dynamic environments. The Heterogeneous Reasoning and Mediator System (HERMES) (Subramanian, 1996) is an architecture designed to integrate heterogeneous sources of data and reasoning paradigms through the creation of specialized mediators.

Distributed Refinement Among Multiple Agents (DRAMA) was developed as a machine-learning technique (Byrne and Edwards, 1995) to improve agent knowledge. This technique provides agents with the ability to learn their organizational roles in a distributed cooperative search. In addition, the agents are also capable of learning meta-level 
interactions in the search space. Dynamic reinforcement learning algorithms (Q-learning) (Sandholm and Crites, 1995) have been used in multi-agent systems to help individual agents learn about the environment and from other agents. Learning organizational roles in heterogeneous multi-agent systems has been addressed in the L-TEAM (Prasad et al., 1995) framework.

Exploiting past experience from intelligent agent interactions has been studied in the context of multi-agent Case-Based Reasoning (CBR) (Ketter and Hendler, 1994). The negotiated retrieval technique (Prasad et al., 1996) retrieves and assembles "case pieces" from multiple sources in a corporate memory to create an overall problem case. Federated peer learning (Prasad and Plaza, 1996) uses two models of cooperation: Distributed Case-Based Reasoning (DisCBR) and Collective Case-Based Reasoning (ColCBR). In DisCBR, an agent can delegate its authority to another agent to solve a problem. In ColCBR, an agent maintains its authority while exploiting the experience of a peer agent.

\subsection{EMERGENT BEHAVIOR AND LEARNING}

The multi-agent-based manufacturing system being used for the present work comprises several design subsystems and resource communities (virtual factories) of shop-floor resources. Each design subsystem provides feature-based capabilities through a part agent and various feature agents (Balasubramanian et al., 1996). The part agent is the repository for both product data and relationships among feature agents. Each part has a set of features, which in turn have technological requirements that help structure relationships with shop-floor resources. For each feature agent in the part, resource communities respond with feasible manufacturing plans and costs. Products are represented as part agents, which are containers for various types of features.

The manufacturing system needs to be dynamically configured to support changes in batch sizes, product mix, or design plans, which precipitate production setup changes. Extensive search processes are required to identify the multiagent interactions for each problem case. These interactions are an emergent property that evolves concurrently within each level in the organization for different types of requirements, constraints, and agents (Maturana and Norrie, 1996).

For each new problem case, the whole space of interactions is investigated using a restricted search approach similar to a distributed, local breadth-first search. This search mechanism achieves a global optimum but is computationally expensive and involves non-trivial overheads, particularly for large multi-agent populations. The learning mechanism presented here enables more efficient use the system's behavior, thereby reducing overheads.

Since the manufacturing system is highly dynamic, with numerous elements and constraints, it is very difficult (generally impossible) to establish an optimum generic procedure for manufacturing tasks using static knowledge about manufacturing resources. However, properly identifying dynamic interactions among agents and the emergent behavior of the agent group as a whole facilitates a new-optimal solution for these tasks.

Organization of resource communities occurs at two levels: macro and micro. Macro-level organization is static, based on knowledge about closely related agents that are distinct from others and can be physically separated. As shown in Figure 1, such communities can be made of part, inventory, machine, tool, and transportation agents.

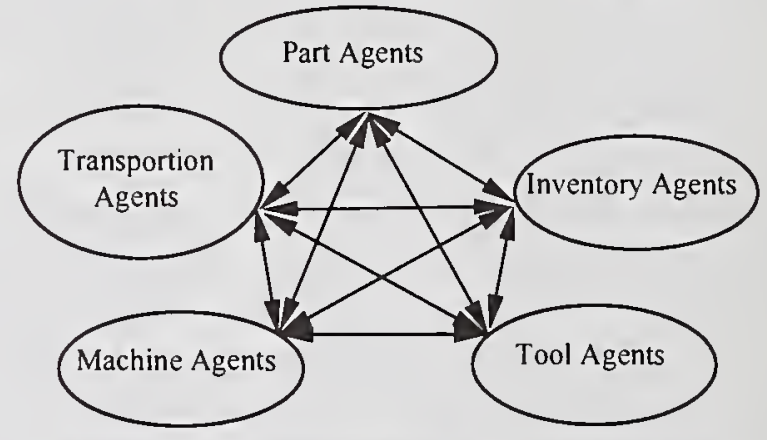

Figure 1: Macro-Level Communities

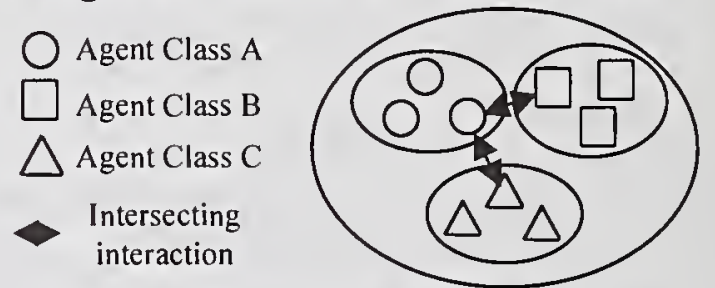

Figure 2: Micro-Level Communities
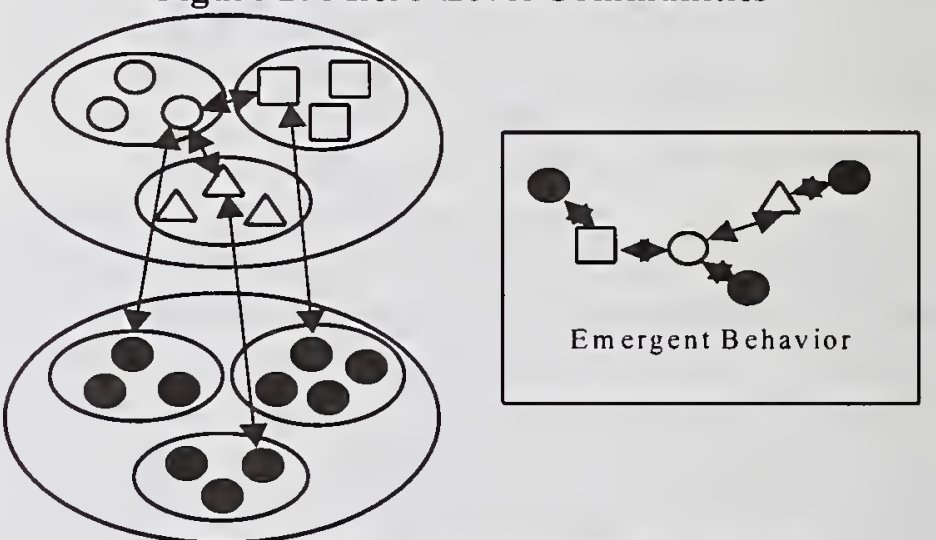

Figure 3: Inter-Community Interactions

Within a macro-level community, heterogeneous agents form micro-level communities whose composition is not static and therefore unknown. Such micro-level communities are determined by predominant behavioral characteristics (grouped in classes), see Figure 2. These communities typically contain primary or secondary process machine agents, design features, or tools.

There are three types of interaction among various agents within a system. The first type lies entirely inside the boundaries of a micro-level community. As shown in Figure 2 , interactions of the second type involve two or more microlevel communities within a macro-level community. In the third type, interactions span two or more macro-level communities, as shown in Figure 3. The learning model presented here considers all these organizational possibilities. 


\subsection{LEARNING MODEL}

The system's learning focuses on the transactions between the design system and the resource communities that result in promissory plans. These promissory plans include relationships among manufacturing resources ordered according to precedence constraints and processing times needed. For a given set of features, there are appropriate combinations of resources (resource networks) for the manufacturing operations. The composition of the resource network varies with time, since availability and service costs of resources are dynamic parameters affecting the decisionmaking processes. Thus, the learning model requires two main classification functions, one feature-based and the other resource-based.

Feature-based classification creates subcategories of features that are progressively recognized by mediator agents. These subcategories are associated with specific groups of resources and emergent behaviors that define the relationship between the features and the resources. Similarly, the resource-based function classifies resources into subgroups associated with specific families of features and emergent behaviors.

A behavioral pattern includes knowledge about microlevel communities, their constituent agents, dynamic associations among those agents for a specific time, work load, product type, and product mix. These patterns recognize how agents organize themselves into virtual clusters that define the knowledge hierarchies through which manufacturing tasks can be processed.

Initially, the multi-agent manufacturing system operates in a "blind space" and every resource in it is involved in decision-making activities. The system, lacking knowledge regarding behavioral patterns followed by agents during the decision process, is forced to carry out a global search for unknown patterns. At the beginning, generalized broadcasting is used to inform resource agents about the manufacturing tasks and their requirements. Subsequently, dynamic hierarchies of coordination clusters are formed and selective broadcasting can be used.

The behavioral knowledge gained through this learning process can be used in two ways to improve system performance. When a new task arises, a community mediator has to identify related macro- and micro-level communities of agents for initial broadcasting and for subsequent formation of coordination clusters. Behavioral knowledge facilitates this selection of agents for coordination clusters.

The second benefit relates to how queries are propagated among coordination clusters. Dynamic mediators can retrieve the performance history of agents to rank the agents in the cluster. Agents with higher rank are selected to propagate sublevel clusters. In this manner, dynamic mediators replace a naive search with a distributed depth-first search, by inhibiting the formation of coordination clusters by agents ranked below threshold limits. Only if higher ranked clusters fail will lower ranked clusters be formed.
This enhanced search process is shown in Figure 4. Ranking ranges from 1 to 10 and there are only two subranges (higher and lower), with a threshold value of 5 . All the clusters ranked above the threshold value are allowed to propagate at every level. The lower ranked ones are allowed to continue at each level only if the higher ranked clusters fail. This amounts to distributed backtracking without explicit control.

Since the propagation of coordination clusters defines the interaction among agents from various micro-level communities and the distributed search process, it also defines the potential domain of emergent behaviors. The ranking defines only the propagation mechanism and has no influence on the outcome. Any combination of resource agents might win the bidding process, despite their ranking.

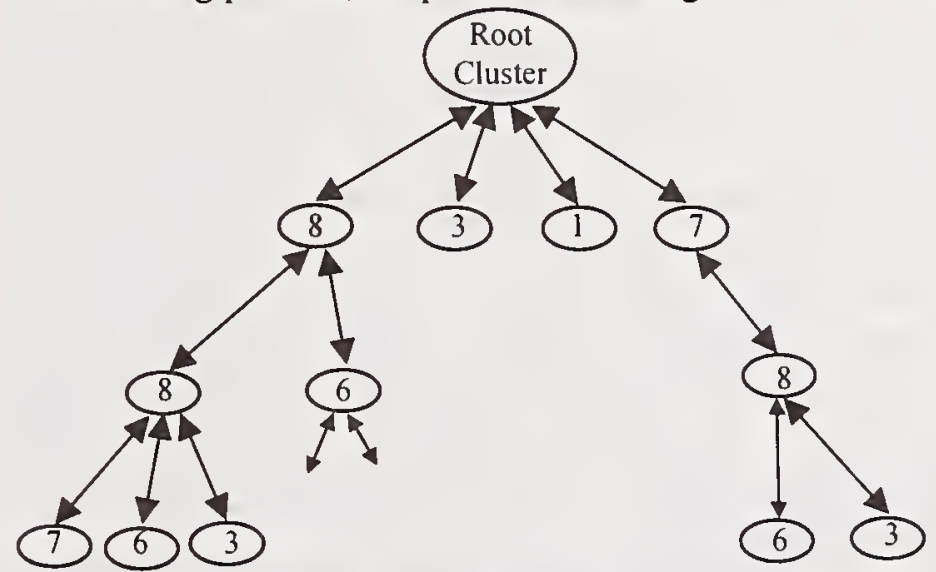

Figure 4: Distributed Depth-First Search

The ranking keeps changing with every outcome, necessitating continuous learning. Mediator agents possess the requisite meta-rules to accomplish this. Since an inherent characteristic of the multi-agent system is that multiple tasks may be solved at any given time, multiple distributed search processes can be carried out in parallel. The distributed depthfirst search mechanism helps this process by reducing the computational requirements and communication bandwidth.

Distributed parallel learning is then possible during the construction of partial promissory plans. Each coordination cluster contributes with a set of partial relationships (among the manufacturing resources) to solve a particular aspect of the manufacturing requests. Partial relationships are associated with both basic and dynamic costs, allowing cluster coordinators to define priorities and control for the propagation of clusters. By aggregating the partial relationships, a set of final promissory plans is established and subsequently refined to satisfy other dynamic constraints on the shop floor. The final refinement provides the best possible production sequence for the product, including relationships that link machine agents and tool agents into an emergent organization for it. Subsequently, promissory plans are used to define the best possible schedules for the resources in each relationship, yielding the best overall promissory plan (Maturana et al., 1997).

In recent experiments, it has been shown that the time for distributed planning in the system reduces by a factor of 
between 100 and 500 as learned relationships become used.

\subsection{LEARNING FROM HISTORY}

The emergent relationships obtained during the search, to encode the solution plans for partial aspects of the manufacturability request, are related to the individual features of the product's design. Depending upon the feature's category, geometric characteristics, and finishing requirements, it is now virtually linked to resources. For a single feature there may be several relationships, both technological and dynamic. The virtual links vary dynamically according to the status of the resources on the shop floor.

Encoding these emergent relationships for manufacturing is based on distributed machine-tool information. A machine resource may require one or several tools to either partially or thoroughly meet the requirements for a single feature. This information is dynamically captured in coordination clusters and sent to the community mediator for encoding and storing in information structures. Each community may implement several of these learning systems to encode different behaviors. After confronting the learning system with a previously recognized product, a set of relationships is obtained and accumulated. At this stage, the mediator agent uses the macro-level static classification of resources to create a hierarchical structure (i.e., primary, secondary, tertiary, etc.) that satisfies precedence constraints.

As the system learns dynamically from interactions, various emergent relationships define alternatives for the manufacturing sequence, each partitioned into an ownerdependent link to establish a reduced group of resource agents. An owner is an entity linked to a set of dependent entities. Conversely, a dependent is an entity owned by a higher level entity. The primary-level owner-dependent link initiates a primary-level coordination cluster, which is then provided with the other owner-dependent links for forming other sublevel clusters of either machine or tool agents. Each sublevel cluster is also provided with the link information associated with its searching domain. At this point, the mediator agent can structure a feasible search region for a request and broadcast to selected agents to generate promissory plans and costs.

\subsection{LEARNING FROM THE FUTURE THROUGH FORECASTING}

Forecasting the system's behavior consists of capturing the state of the multi-agent system (plans) and simulating it for a period of time into the future. The forecasting process simulates the behavior of the virtual model, which emulates the shop-floor activities. Since the agents' commitments are generated at a promissory level and based on distributed estimations of the system's real state, this process is a highlevel simulation activity that propagates this behavior into its future environment. Machine mediators monitor the system's status by conducting global performance evaluations. Various forecasting triggering parameters are used based on system load, extension of the scheduling horizon, and adjustment periodicity. These parameters are quantified and combined according to the following criteria:

1. System load: This parameter is set to promote a balanced work load among the resources. The number of new jobs allocated is measured to calculate a load ratio $\left(R_{1}\right)$ which is then checked against a load threshold. $R_{1}$ greater than this load threshold satisfies the first condition for triggering the simulation forecasting process. This condition is complemented by the adjustment periodicity parameter as shown in Equation 1:

$$
\begin{aligned}
& \mathrm{R}_{1}=\frac{\text { Number of new jobs allocated }}{\text { Total number of jobs allocated }} \\
& \text { If } \mathrm{R}_{1} \geq \text { Threshold } \\
& \text { Then } \\
& \text { If (Periodicity) } \\
& \text { Then Run Forecasting }
\end{aligned}
$$

Else Continue

By considering the natural adjustment of schedules after forecast scheduled times, resources may open availability slots at previously occupied positions.

2. Extension of the scheduling horizon $\left(R_{s}\right)$ : This parameter changes the configuration of schedules to vary job priorities. Since schedule adjustments either extend or contract the scheduling horizon, lateness and earliness penalizations affect the scheduling, differently. In order to extend the scheduling horizon, new job allocations are more heavily penalized if the jobs approximate the due-date boundaries. To contract the scheduling horizon, resources that contract their scheduling charts may allocate more jobs at a low penalization rate. This parameter is shown in Equation 2.

$$
\begin{aligned}
& \mathrm{R}_{\mathrm{s}}=\frac{\text { (Completion Time) }_{\max }}{\text { (Due Date) }_{\max }} \\
& \text { If } \mathrm{R}_{\mathrm{s}} \geq \text { Threshold }_{\mathrm{s}} \\
& \text { Then } \\
& \text { If (Periodicity) } \\
& \text { Then Run Forecasting }
\end{aligned}
$$

Else Continue

3. Periodicity $\left(R_{p}\right)$ : This parameter regulates the time delay between forecasting events. It may trigger the forecasting event either alone or in combination with one of the two parameters above. When $R_{p}$ acts alone, the number of new allocations must be greater than zero. A time periodicity threshold is also provided to quantify the periodicity, as shown in Equation 3.

$$
\begin{aligned}
& \text { If } R_{p} \geq \text { Threshold }_{p} \\
& \text { Then } \\
& \text { If (number of new allocations) }>0 \\
& \text { Then Run Forecasting }
\end{aligned}
$$

Else Continue

$$
\begin{gathered}
\text { If }\left(R_{p} \text { and } R_{1}\right) \text { or }\left(R_{p} \text { and } R_{s}\right) \\
\text { Then Run Forecasting }
\end{gathered}
$$


The system's performance is measured through a composite function that measures average flow time, maximum completion time, and number of late jobs, as shown in Equation 4.

$$
\begin{aligned}
& P=\mathrm{w}_{\mathrm{f}} * \bar{F}+\mathrm{w}_{\mathrm{c}}{ }^{*} C_{\max }+\mathrm{w}_{\mathrm{j}}^{*} L_{j} \\
& \text { where: } \\
& w_{f} \text {--average flow time weight } \\
& w_{c} \text {--maximum completion time weight } \\
& \mathrm{w}_{\mathrm{j}} \text {--late jobs weight } \\
& \bar{F} . \text {-average flow time } \\
& C_{\max } \text {--maximum completion time } \\
& L_{j} \text {-number of late jobs } \\
& J_{c} \text {-number of jobs completed }
\end{aligned}
$$

Each performance value is weighted according to management policies. The composite function is not restricted to these performance values. The performance ratio $\left(R_{\text {metrics }}\right)$ is calculated as follows:

$$
\begin{aligned}
& \mathrm{R}_{\text {metrics }}=\frac{\mathrm{P}^{\prime}}{\mathrm{P}} \\
& \mathrm{P}^{\prime} \text {.-forecasting simulation performance } \\
& \mathrm{P} \text {.-system performance before adjustment }
\end{aligned}
$$

Lower and upper thresholds $(-\sigma,+\sigma)$ are used to define acceptance and rejection performance-ratio regions. The system's state of commitments remains unaltered if ratios fall within the thresholds.

The forecasting simulation period is established by the system manager according to high-level policies. However, the determination of the simulation-control parameter may be customized to automatically adapt to the system's requirements. Two main approaches can be used to establish the forecasting period:

1. The forecasting simulation may be run for a period of time sufficiently large to permit the completion of every committed job. But for large simulation periods, this criterion incurs accumulative deviations, since several jobs may arrive in the system that affect intermediate allocation slots. Because these slots are not preempted during simulation, this approach only produces rough estimations.

2. The second approach attempts to avoid the accumulation of deviate behaviors, thereby extending the forecasting simulation for short periods of time only. This approach is more accurate, since the duration of the simulation is much less than the arrival frequency of jobs. Here, the multi-agent system can easily be adjusted while including the intermediate requirements.

Either approach produces an estimated performance value, which is used to decide upon the refinement of the system's commitments. Figure 5-A, illustrates the variation in system performance. Here, the system performance for two consecutive simulation periods is represented by $f_{1}$ and $f_{2}$ and the variation in performance for the forecast model is represented by $\mathrm{f}_{1}$ and $\mathrm{f}_{2}$. It is assumed that the simulationtriggering parameters are satisfied at period 1 . At the end of the simulation both performances .. i.e., $f_{1}$ and $f_{1}-$ are compared through a performance ratio function. In this model, the system behavior is very close to the simulated behavior; therefore, promissory planning is being developed within an acceptable range. Thus, the system continues to plan using the current state of commitments.

Fig. 5-B shows a second forecasting simulation occurring at period 2. Since the performance ratio between $f_{2}$ and $f_{2}{ }_{2}$ is sufficiently large to exceed the performance threshold the system decides to adopt the forecast scheduled times, replacing current schedule times by these. The state of commitments is modified at each planning level affected by these changes. For the next period (period 3) of the forecasting simulation, the performances were sufficiently close, so the current state of commitments was retained.

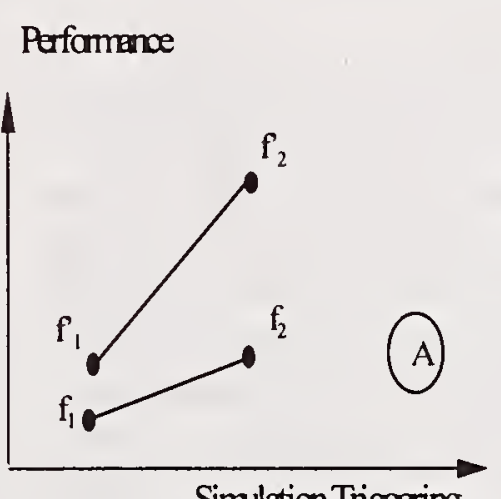

Simulation Trigering

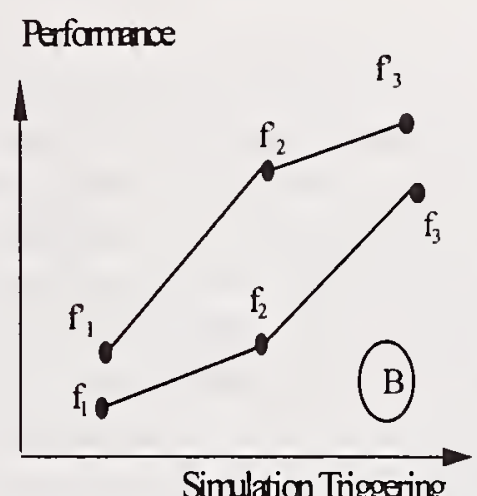

Simulation Triggering
Figure 5: System Performance Variation

\subsection{Experimental Results for Learning from Forecasting}

A product mix comprising 100 of each of three part types, namely, Bearing cover, EMI housing, and Guide, were "manufactured" in two shop-floor areas using an AGV transportation system to handle raw materials and semifinished products. Shop floor \#l had 3 vertical machining centers, 2 internal grinders, 1 surface grinder and 43 tools. Shop floor \#2 had 3 vertical machining centers and 35 tools. A profit margin of $35 \%$ of normal production cost was specified.

Once the production orders were placed in the design system interface, the static mediator made repeated broadcast requests to each shop floor involved. Since each offered a different cost, the static mediator applied a final integration of plans to determine the best one to manufacture the products. The final schedule data showed that 190 parts were produced in Shop floor \#1 and 110 parts in Shop floor \#2. Shop floor \#2 only manufactured EMl housing and Guide products. The Bearing cover product was only produced at Shop floor \#l.

Table 1 shows combined performance metrics of Average Flow Time (AFT), Maximum Completion Time (MCT), and Transportation Cost (TC) for both shop floors. Only 8 jobs in all were tardy.

Table 1: Experiment Results

\begin{tabular}{|c|c|c|}
\hline $\begin{array}{c}\text { AFT } \\
(\min .)\end{array}$ & $\begin{array}{c}\text { MCT } \\
(\min .)\end{array}$ & $\begin{array}{c}\text { TC }(\$) \\
(\text { Max. })(M i n .)\end{array}$ \\
\hline 414.06 & 7164.81 & 50.2125 .39 \\
\hline
\end{tabular}


The forecasting simulation proved to be a robust system for adjusting and enhancing performance. The forecasting was triggered 8 times through the entire planning process, as shown in Figure 6.

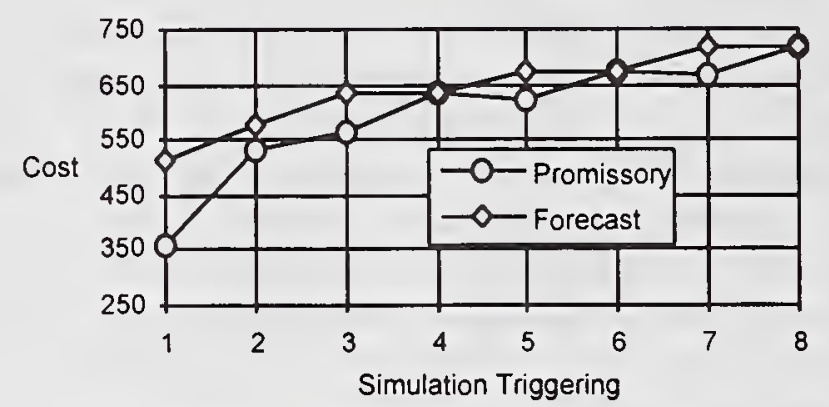

Figure 6: Triggering Thresholds

\subsection{CONCLUSION}

A learning mechanism for identifying multi-agent-based manufacturing system organizational knowledge and selective interaction propagation from emergent system behavior has been developed. This mechanism enhances coordination capabilities by minimizing communication and processing overhead, and facilitates distributed, parallel depth-first search. Though this learning model has been implemented in a distributed mediator architecture that is part of a concurrent design and manufacturing system, it is generic and can be applied to other areas as well.

A learning from forecast-futures technique has also been developed to dynamically adjust distributed schedules and planning in a multi-agent manufacturing system. Experimental results show the value of this approach for adjusting and enhancing performance.

\section{REFERENCES}

Balasubramanian, S., Maturana, F., and Norrie, D. (I996). Multi-agent planning and coordination for distributed concurrent engineering. In International Journal of Cooperative Information Systems Vol. 5, I53-I 79.

Brooks, R.A. (199I). Intelligence without reason. In Proceedings of the Twelfth International Joint Conference on Artificial Intelligence (IJCAI-91), Sydney, Australia, pp. 569595.

Byrne, C., and Edwards, P. (I995). Adaptation and learning in multi-agent systems. IJCAI'95 Workshop Montreal, Canada, August 2I, 22-39.

Duffie, N.A. (I990). Synthesis of heterarchical manufacturing systems. Computers in Industry 14, I67-I74.

Ferguson, I.A. (I992). TouringMachines: An Architecture for Dynamic, Rational, Mobile Agents. Technical Report No. 273, University of Cambridge Computer Laboratory.

Ketter, B.P., Hendler, J.A., Anderson, W.A., and Evett, M.P. (1994). Massively parallel support for case-based planning. IEEE Expert, 8-13.

Maes, P. (1990). Situated agents can have goals. NorthHolland, Robotics and Autonomous Systems 6 (I990), 49-70.
Mataric, M.J. (1995). Evaluation of learning performance of situated embodied agents. In Proceedings of the Third European Conference on Artificial Life, Granada, Spain, June 4-6.

Maturana, F.P., and Norrie, D.H. (I996). A generic multiagent mediator for distributed manufacturing systems, In Journal of Intelligent Manufacturing, Vol. 7, 257-270.

Maturana, F., Balasubramanian, S., and Norrie, D. (I997). Intelligent multi-agent coordination system for advanced manufacturing. Submitted to Intelligent Systems and Advanced Manufacturing Conference, 14-17 October I997, Pittsburg, Pennsylvania, USA.

Prasad, M.N., and Plaza, E. (I996). Corporate memories as distributed case Libraries. Accepted for presentation at the Corporate Memory \& Enterprise Modeling Track in the I0th Knowledge Acquisition Workshop, Banff, Canada.

Prasad, M.N., Lesser, V.R., and Lander, S.E. (1995). Learning organizational roles in a heterogeneous multi-agent system. IJCAI-95 Workshop on Adaptation and Learning in Multiagent Systems, August (1995), Montreal, Canada.

Prasad, M.N., Lesser, V.R., and Lander, S.E. (I996). Reasoning and retrieval in distributed case bases. In the Journal of Visual Communication and Image Representation, Special Issue on Digital Libraries, Vol. 7, No. 1, 74-87.

Sandholm, T., and Crites, R. (1995). On multi-agent Qlearning in semi-competitive domain. I4th International Joint Conference on Artificial Intelligence (IJCAI-95), Workshop on Adaptation and Learning in Multiagent Systems, Montral, Canada, 7 I-77.

Steels, L. (1990). Cooperation between distributed agents through self organization. In Demazeau, Y., and Müller, J.P., editors, Decentralized AI - Proceedings of the First European Workshop on Modelling Autonomous Agents in Multi-Agent Worlds (MAAMAW-89), 175-196.

Subramanian, V.S., Adali, S., Brink, A., Emery, R., Lu, J., Rajput, A., Rogers, T.J., Ross, R., and Ward, C. (1996). HERMES: Heterogeneous reasoning and mediator system. Technical Report, Computer Science Department, Univerisyt of Maryland, (http://www.cs.umd.edu/projects).

Wiederhold, G. (1992). Mediators in the architecture of future information systems. IEEE Transaction on Computer, 38-48.

Wooldridge, M., and Jennings, N. R. (1995). Intelligent agents: Theory and practice. The Knowledge Engineering Review 10 (2), 115-I52. 
IX

EVOLUTION 


\title{
Speculation on the Evolution of Symbols
}

\author{
David B. Fogel \\ Natural Selection, Inc. \\ 3333 N. Torrey Pines Ct., Suite 200 \\ La Jolla, CA 92037
}

\begin{abstract}
Evolutionary algorithms provide a framework for studying the evolution of symbols in dynamic environments. This paper offers a brief review of related efforts in evolutionary computation involving the invention of a language of descriptors, followed by preliminary experiments in evolving symbols describing an environment to be sent between a sender and a receiver. The results indicate that as more precision in the description of the environment is offered, the greater the possible accuracy; however, the greater the number of symbols, the more challenging the task of decoding the symbols. Thus there is a trade off between precision and accuracy.
\end{abstract}

KEYWORDS: symbols, communication, evolutionary computation

\section{INTRODUCTION}

Living organisms are predictors. All evolved biota continually predict features of their surroundings. The essential criterion of prediction is to minimize the cost of being surprised [1]. Those that fail to predict with sufficient accuracy or in a timely manner often face lethal consequences. The keystone of prediction in evolutionary systems may be taken even more broadly as a requisite component of all intelligent systems: Intelligence may be defined as the capability of a system to adapt its behavior to meet its goals in a range of environments [2]. By consequence, intelligent behavior requires a composite ability to predict one's environment coupled with a translation of each prediction into a suitable response in light of a given goal [3]. Without prediction, adaptation becomes impossible.

In nature, adaptation is accomplished through evolution, a twostep process of random variation and selection [4]. Individuals compete for resources in a finite arena. Natural selection stochastically eliminates those individuals that do not acquire sufficient resources. The primary purposeful goal imbued into all living system is therefore survival. The recognition of repeated patterns in natural circumstance with appropriate reactions (i.e., allocation of available resources) leads to greater rates of sur- vival. Under the assumption that such behavioral characteristics are heritable, the logical outcome is a series of individuals that are successively better predictors of regularities in their environment.

The search for underlying regularities in nature is also primary to the scientific method. Indeed, there is a fundamental connection between the process of evolution and the scientific process of discovery [3] (Figure 1). In nature, individual organisms serve as hypotheses concerning the logical properties of their environment. Their behavior is an inductive inference concerning some as yet unknown aspects of that environment. Validity is demonstrated by their survival. Over successive generations, organisms becomes better predictors of their surroundings.

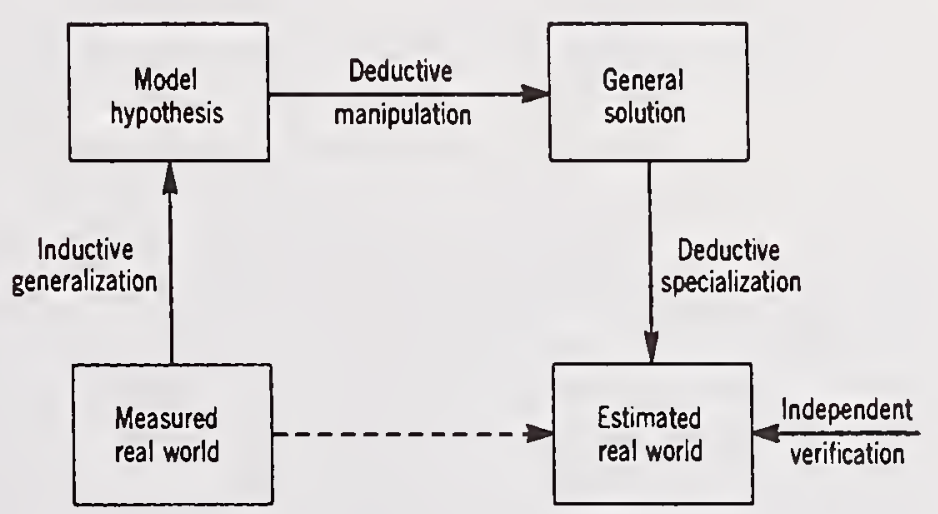

Figure 1. The scientific method. Unknown aspects of the environment are estimated. Data are collected in the form of previous observations or known results and combined with newly acquired measurements. After the removal of known erroneous data, a class of models of the environment that is consistent with the data is generalized. This process is necessarily inductive. The class of models is then generally reduced by parameterization, a deductive process. The specific hypothesized model (or models) is then tested for its ability to predict future aspects of the environment. Models that prove worthy are extended, or combined to form new hypotheses that carry on a "heredity of reasonableness" [2]. This procedure is iterated until a sufficient level of credibility is achieved (from [2]). 
These surroundings include other organisms, and thus communication between individuals can affect the likelihood of survival. Communication requires a language, a sequence of symbols transmitted between a sender and a receiver. The symbols can be transmitted through any of the senses. Common symbols in biota include threat postures, mating dances, grunts and whistles (or other sounds), disagreeable taste, and so forth. It is of interest to speculate on the evolution of such descriptive symbols and sequences of symbols.

This paper offers some speculations on the evolution of symbols as observed in recent efforts in evolutionary computation. The paper does not purport to provide a suitable literature review of efforts to explain the evolution of symbols in humans, other primates, or indeed any other natural system. Readers interested in these areas are referred to $[5,6]$ and others.

\section{EVOLUTIONARY COMPUTATION, SYMBOLS, AND COMMUNICATION}

\subsection{Background}

Evolutionary computation concerns efforts to simulate evolution on a computer (or other device). Efforts in simulated evolution date back to the early 1950 s and 1960 s, with three main lines of investigation proceeding currently: evolution strategies, evolutionary programming, and genetic algorithms. For a review of these methods, see [2], [7].

Within evolutionary computation, there have been efforts made to study the evolution of symbols and communication. Werner and Dyer [8] used artificial neural networks to represent the behavior of artificial organisms in the context of finding mates. "Females" were given the ability to see males and emit sounds. "Males" were blind, but could hear signals from females. Over successive generations, starting from randomly configured networks, the simulation generated nets that exhibited an increasing effectiveness in mate finding, as well as the evolution of dialects that promote the development of "subspecies." Levin [9] used a genetic algorithm approach to study the evolution of understanding between artificial agents having various "internal states" and "observable features." Fitness was increased for greater understanding of the internal states by other observed agents. Figure 2 offers a flow chart of Levin's procedure.

Whereas [8] and [9] were designed specifically to examine the evolution of symbols ${ }^{1}$, other evidence of the evolution of symbols can be found by reexamining experiments in the iterated prisoner's dilemma [10].

\subsection{The Iterated Prisoner's Dilemma and the Evolution of Symbols}

The prisoner's dilemma is an easily defined nonzero-sum, noncooperative game. The term nonzero-sum indicates that whatever benefits accrue to one player do not necessarily imply similar

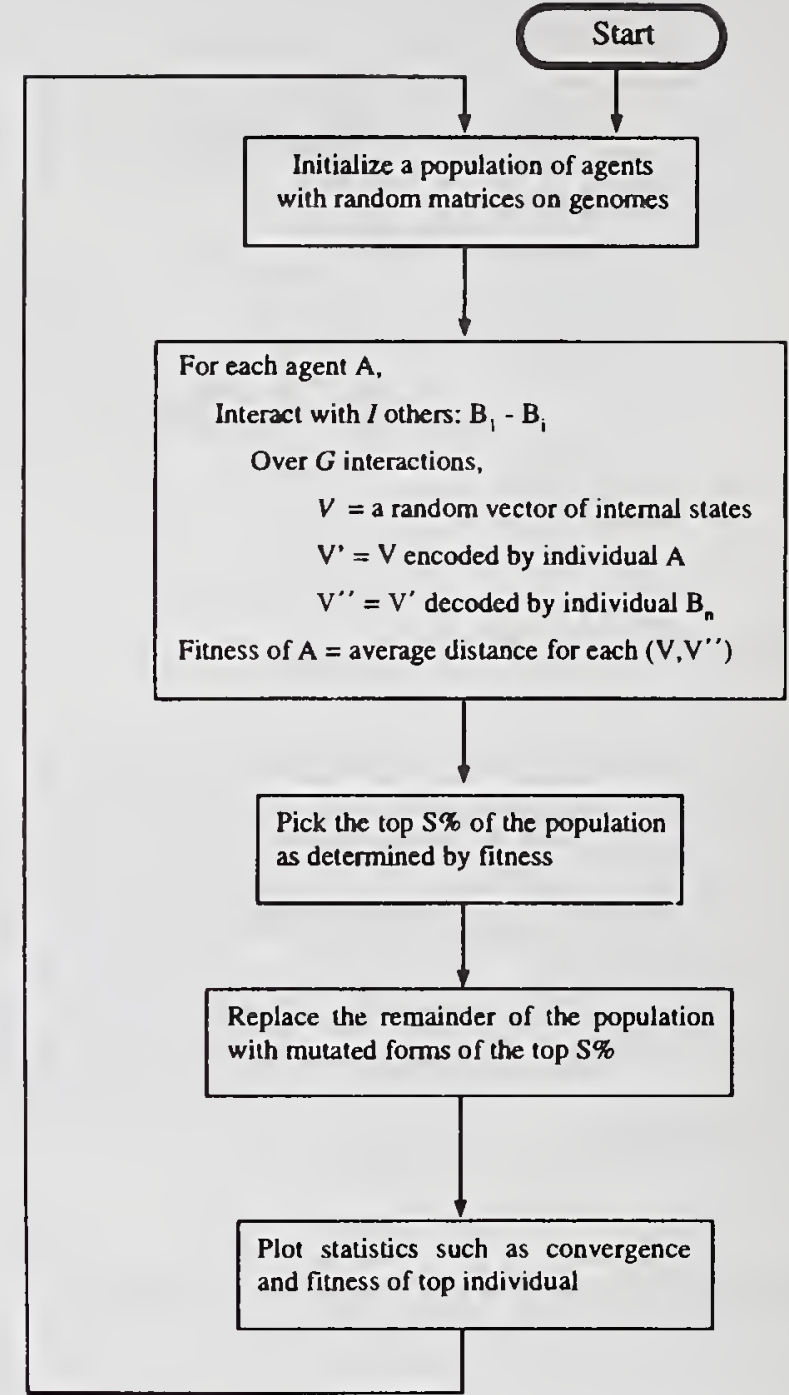

Figure 2. Flowchart of procedure in [9] to evolve communication based on how well other agents understand the offered signals regarding internal states (from [9]).

penalties imposed on the other player. The term noncooperative indicates that no preplay communication is permitted between the players. Typically, two players are involved, each having two alternative actions: cooperate or defect. Cooperation implies increasing the total gain of both players; defecting implies increasing one's own reward at the expense of the other player. The optimal policy for a player depends on the policy of the opponent.

The general form of the game is offered in Figure 3. The game is conducted over a series of trials. If information regarding prior plays can affect the decision making in future plays, learning can take place and even though defection is the dominant move in any single trial, cooperative behavior can evolve.

Following the seminal efforts of Axelrod [11], Fogel [10] evolved finite state machines to play the iterated prisoner's dilemma. The initial interest was to determine if population size had any association with the speed at which a population evolved mutually cooperative behavior. The results were essentially nega- 


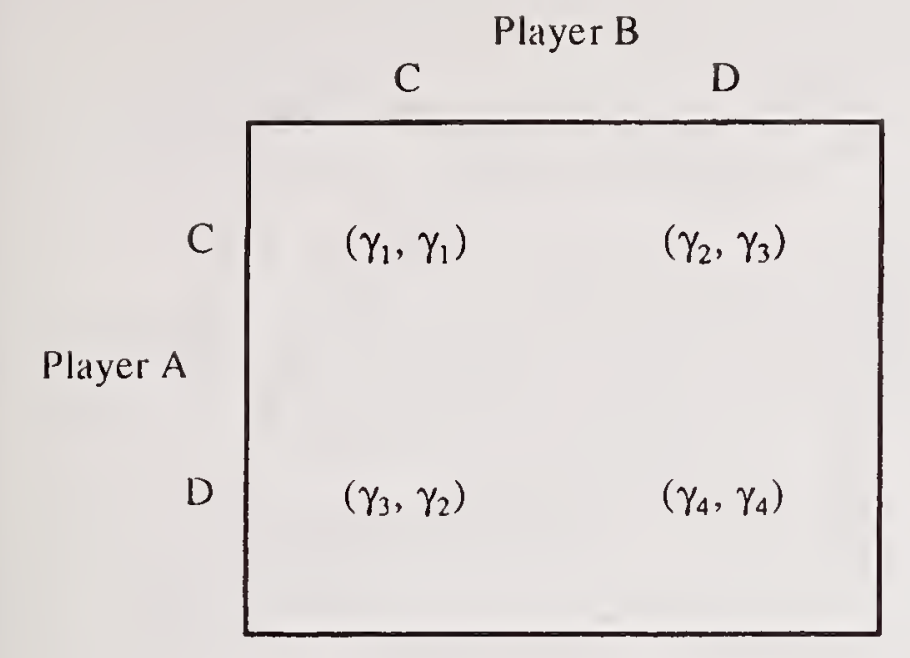

Figure 3. The general form of the payoff function in the prisoner's dilemma, where $\gamma_{1}$ is the payoff to each player for mutual cooperation, $\gamma_{2}$ is the payoff for cooperating when the other player defects, $\gamma_{3}$ is the payoff for defecting when the other player cooperates, and $\gamma_{4}$ is the payoff for mutual defection. An entry $(\alpha, \beta)$ indicates the payoffs to the players $\mathrm{A}$ and $\mathrm{B}$, respectively. The constraints $2 \gamma_{1}>\gamma_{2}+\gamma_{3}$ and $\gamma_{3}>\gamma_{1}>\gamma_{4}>\gamma_{2}$ are typical.

tive: population size did not appear strongly correlated with the number of generations required to evolve cooperation. Population sizes of $50,100,250,500$, and 1000 parents were considered. In each case, across several trials (up to 30), cooperation always evolved. Figure 4 depicts one evolved finite state machine from these experiments for a population size of 50.

It was of interest to compare the behavior that occurs when finite state machines from independent trials play each other to that observed when they play copies of themselves. Fogel [10] (also see [2]) reported that when each finite state machine chosen from a trial at any population size played against itself, it always eventually cycled into mutual cooperation. But when it played against a machine selected from a different independently evolved population, it was equally likely to cycle into alternating cooperation and defection (Figure 5).

It is speculative but reasonable to presume that each population of finite state machines evolved initial sequences of symbols that form patterns that can be recognized by other machines. This allows for the identification of machines that will tend to respond to cooperation with cooperation. The specifics of such sequences will vary by trial and may be as simple as merely cooperating on the first move. But the specifics are generally unimportant. When two machines from separate trials played against each other, the resulting behavior could vary because no pattern of initial symbols was recognized by either player. Thus it would be improper to describe the machines as being "cooperative" or "noncooperative" as such descriptions require qualification with regard to the circumstances in which they were evolved and the expected behavior of the other players. Each of

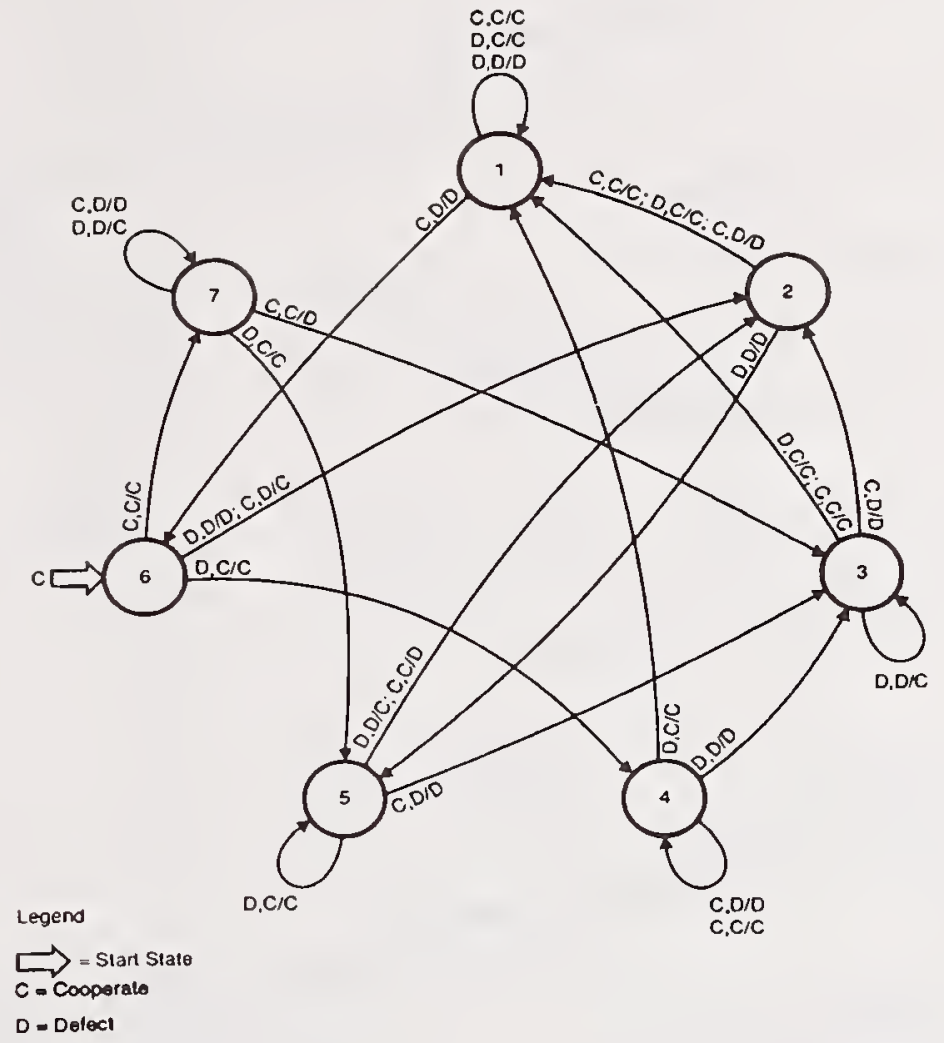

Figure 4. The best evolved finite state machine after 25 generations for a population size of 50 parents from [10]. If this machine were played against itself, it would eventually cycle into state 1 , and always cooperate $(C)$.

the machines tested for the results presented in Figure 5 was cooperative in its naturally evolved population.

\section{EXPERIMENTS}

Of potential interest is the manner in which symbols are invented on-the-fly in light of competition from other extant individuals. Two simple experiments have been conducted to initiate an investigation into this process. An environment is sensed and encoded by an individual into a binary string of some length. This string is then sent to another individual that must decode the string and recreate the sensed environment or predict the next state of the environment. The quality of communication and encoding/decoding is judged in terms of the correspondence between the desired response of the receiver and the actual response. The binary encoding, however, is made evolvable in that the number of bits used to describe the environment is a parameter of the sender, and is subject to random variation.

More specifically, in the first trial, a population of 250 senderreceiver pairs was initialized. Each sender and corresponding receiver took the form of partitioned neural networks, with the sender using threshold functions for hidden units (Figure 6). The sensed input was a sine wave sampled 100 times over a single period. The task was to encode the sensed value of the sine wave, have the receiver decode the value and recreate the sensed value 
(a)

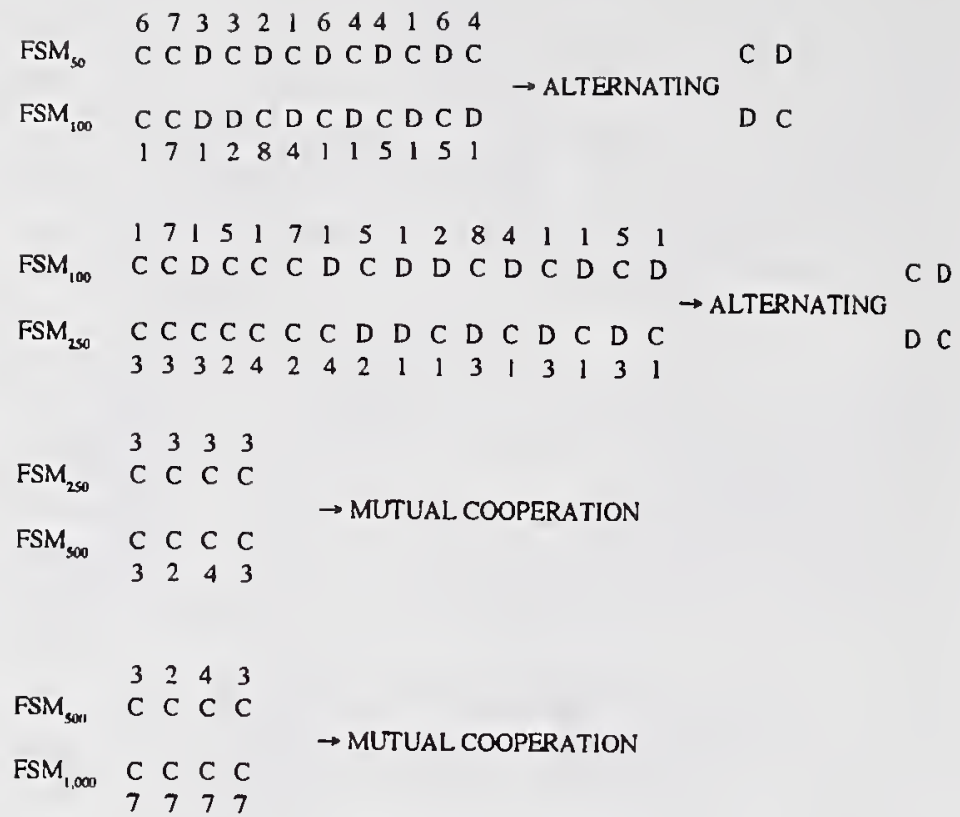

(b)

$$
\begin{aligned}
& \text { FSM } \quad \begin{array}{llllll}
6 & 7 & 3 & 3 & 1 & 1
\end{array} \\
& \text { FSM }_{50} \quad C \text { C C C C } \\
& \mathrm{FSM}_{\text {so }} \quad \mathrm{C} \text { C D C C C } \\
& 67331 \\
& \begin{array}{lllllll}
1 & 7 & 1 & 2 & 3 & 2 & 2
\end{array} \\
& \text { FSM }_{100} \text { C C C D D D } \\
& \text { FSM }_{100} \text { C C D D D C C } \rightarrow \text { MUTUAL COOPERATION } \\
& \begin{array}{lllllll}
1 & 7 & 1 & 2 & 3 & 2 & 2
\end{array} \\
& \mathrm{FSM}_{250} \quad \begin{array}{ll}
3 & 3 \\
\mathrm{C} & \mathrm{C}
\end{array} \\
& \mathrm{FSM}_{250} \quad \mathrm{C} \mathrm{C} \\
& \rightarrow \text { MUTUAL COOPERATION } \\
& \begin{array}{lllll}
\mathrm{FSM}_{300} & \mathrm{C} & \mathrm{C} & \mathrm{C} & \mathrm{C}
\end{array} \\
& \rightarrow \text { MUTUAL COOPERATION } \\
& \begin{array}{llll}
3 & 2 & 4 & 3
\end{array} \\
& \begin{array}{ll}
7 & 7
\end{array} \\
& \text { FSM }_{1,000} \text { C C } \\
& \begin{array}{lll}
\text { FSM }_{1,000} & C & C \\
& 7 & 7
\end{array}
\end{aligned}
$$

Legend
C= Cooperate
$\mathrm{D}=$ Defect
The numbers above and
below the move indicate
the current state for the
respective FSM.

Figure 5. The results of playing the iterated prisoner's dilemma (a) with independently evolved machines in which each machine plays against a machine that was created in a separate trial with a different number of parents in the population and (b) when each independently evolved best machine plays against itself. The subscripts indicate the population size. Mutual cooperation is always observed when finite state machines (FSMs) play against themselves, but not when they play separately evolved machines.

- over the 100 samples. All senders were initialized to have a single hidden node. This could be varied by mutation with a 0.25 probability of adding a node (up to a maximum of 10 nodes) and a 0.25 probability of deleting a node (again to a minimum of 1 node). All initial connections were initialized uniformly over $[-0.5,0.5]$ and were mutated using Gaussian perturbations with mean zero and a variance equal to the mean squared error of the sender-receiver pair divided by the number of degrees of freedom in the pair. Each sender-receiver pair generated a single offspring, which was evaluated, and then selection was imposed to probabilistically retain the best 250 pairs in accordance with competition rules in [2]. This process was iterated for 400 generations. Typical results are shown in Figure 7a. A second trial used two successive inputs from the sine wave $(x[t-1], x[t])$ and required the receiver to predict the next sample $(x[t+1])$. Typical results are shown in Figure $7 b$.

A general pattern covers typical results in both experimental designs. There is a gradual increase in the number of evolved encoding nodes coupled with a gradual decrease in the mean squared error. A greater number of encoding nodes provides greater precision, however there is no immediate leap to the maximum number of possible nodes ( 10 in this case, which could have been invented as early as the 10th generation). The difficulty in establishing the correct encoding-decoding mix appears to increase with the number of encoding nodes. Thus there is a trade off between precision and accuracy, and the appropriate symbolization evolves over successive generations.

\section{CONCLUSIONS}

Symbols evolve because they are useful. Evolutionary computation offers a tool to study the evolution of useful symbols in a variety of contexts. Previous efforts have identified patterns in the evolution of symbols both in sending messages from sender to receiver, and in abstracting meaning from an environment in light of a given goal. The preliminary results offered here show 


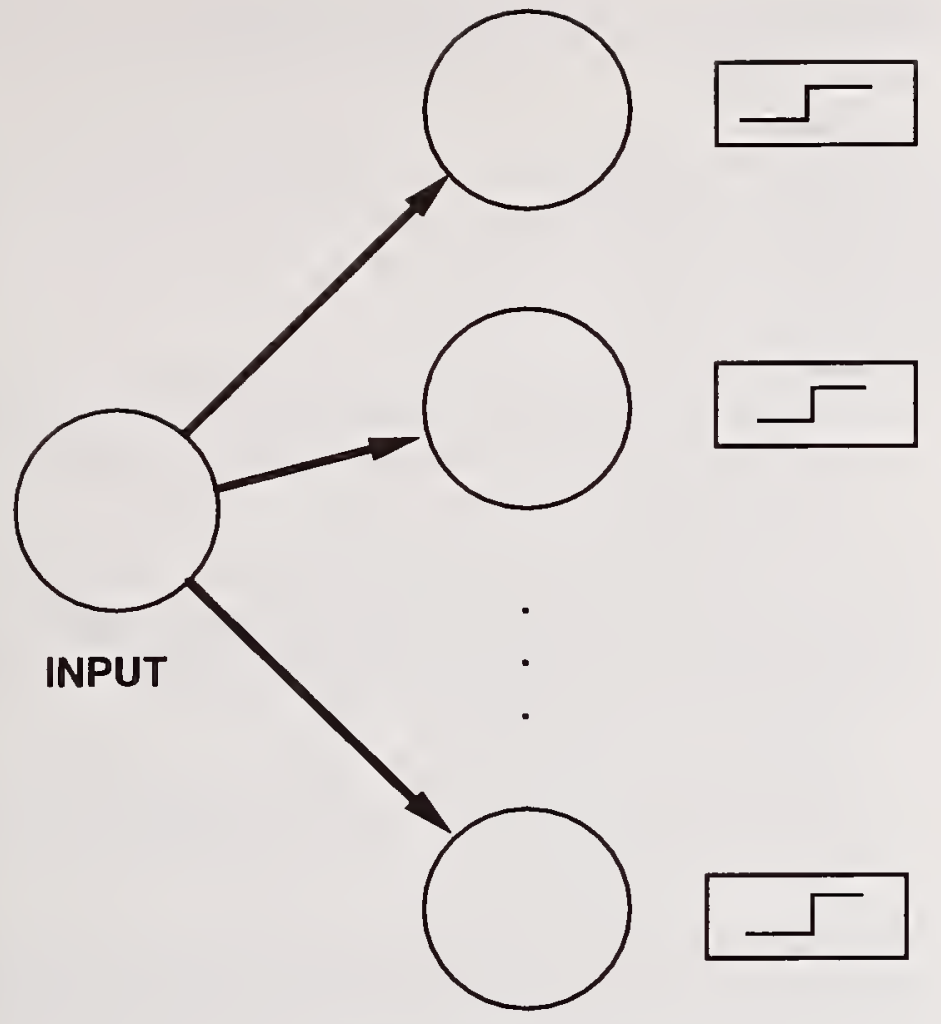

ENCODING NODES

Figure 6. The sender network in the evolutionary experiments. The input is a sine wave with 100 samples per period. The connections from the input node to the encoding nodes are weighted and can vary with Gaussian mutation (zero mean). The encoding nodes multiply the connection strength by the input value, subtract a bias term (implicit) and pass the result through a hard limiting threshold function. If the value is greater than or equal to zero, a 1 is transmitted, otherwise a 0 is transmitted. The receiver, by definition, has a number of input nodes equal to the encoding nodes of the sender and, in the first experiment, must essential perform the inverse operation to recreate the input value of the sender. In the second experiment, the sender has two sensors and encodes two successive values of a sine wave. The receiver must in turn predict the next value of the sine wave. The number of encoding nodes is simultaneously subject to mutation and selection along with the connection strengths and bias terms.
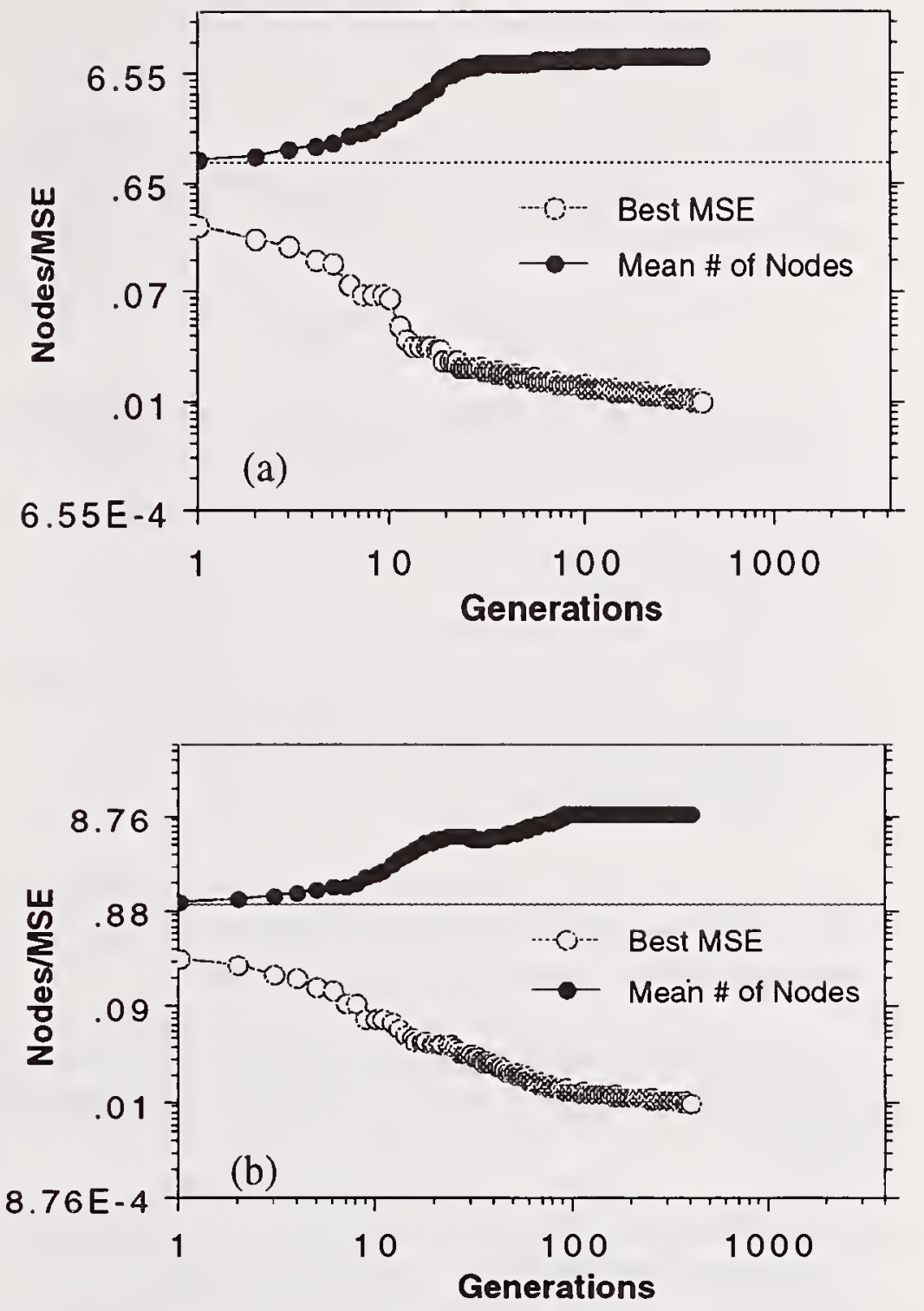

Figure 7. The progression of the mean number of encoding nodes (and thus the choice for symbolization) taken over all surviving sender-receiver pairs at each generation and the best mean squared error that was obtained. (a) Typical results for encoding and decoding a value from a sine wave. (b) Typical results for encoding and decoding the last two samples of a sine wave in order to predict the next value. Note that although the probability of adding nodes was equal to 0.25 there was no immediate jump to the maximum number of 10 nodes. Simply adding nodes did not automatically lead to improved performance because it requires simultaneous evolution of the appropriate connection weights. Nevertheless, increased precision allowed for increased accuracy. 
a first step toward describing the trade off between the degree of precision offered by a particular set of symbols and the accuracy that can be obtained when a receiver must decode these symbols into a meaningful response. If the results serve to promote further thought and experimentation in this direction then they will have fulfilled the author's purpose.

\section{ACKNOWLEDGMENTS}

The author appreciates the comments and criticisms of V.W. Porto and L.J. Fogel, and A. Meystel's encouragement to participate in this conference.

\section{FOOTNOTE}

Related efforts in evolving symbolic strings representing sensed environmental conditions can be found in $[12,13]$.

\section{REFERENCES}

[1] Atmar, J.W., "Speculation on the evolution of intelligence and its possible realization in machine form," Doctoral dissertation, New Mexico State Univ., Las Cruces, NM.

[2] Fogel, D.B., Evolutionary Computation: Toward a New Philosophy of Machine Intelligence, Piscataway, NJ: IEEE Press, 1995.

[3] Fogel, L.J., Owens, A.J., Walsh, M.J., Artificial Intelligence Through Simulated Evolution, New York: John Wiley, 1966.

[4] Mayr, E., Toward a New Philosophy of Biology: Observations of an Evolutionist, Cambridge, MA: Belknap Press, 1988.

[5] Warhurst, J., "Nationalism and Republicanism in Australia - The evolution of institutions, citizenship and symbols," Australian J. of Pol. Sci., vol. 28, pp. 100-120, 1993.
[6] Hewes, G.W., "Primate communication and the gestural origin of language," Curr. Anthrop., vol. 33, pp. 65-84, 1992.

[7] Bäck, T., Hammel, U., and Schwefel, H.-P., "Evolutionary computation: comments on the history and current state," IEEE Trans. Evolutionary Computation, vol. 1, no. 1, pp. 3-17, 1997.

[8] Werner, G.M. and Dyer, M.G., "Evolution of communication in artificial organisms," Artificial Life II, C.G. Langton, C. Taylor, J.D. Farmer, and S. Rasmussen (Eds.), Reading, MA: Addison-Wesley, pp. 659-687, 1991.

[9] Levin, M., "The evolution of understanding: a genetic algorithm model of the evolution of communication," BioSystems, vol. 36, pp. 167-178, 1995.

[10] Fogel, D.B., "Evolving behaviors in the iterated prisoner's dilemma," Evol. Comp., vol. 1, no. 1, pp. 77-97, 1993.

[11] Axelrod, R., "The evolution of strategies in the iterated prisoner's dilemma," Genetic Algorithms and Simulated Annealing, L. Davis (Ed.), London: Pitman, pp. 32-41, 1987.

[12] Holland, J.H. and Reitman, J.S., "Cognitive Systems Based on Adaptive Algorithms," Pattern-Directed Inference Systems, D.A. Waterman and F. Hayes-Roth (eds.), Academic Press, NY, pp. 313-329, 1978.

[13] Fogel, D.B., "An evolutionary approach to representation design," Proc. SecondAnn. Conf. on Evolutionary Programming, D.B. Fogel and W. Atmar (eds.), Evolutionary Programming Society, La Jolla, CA, pp. 163-168, 1993. 


\title{
The Evolution of Intelligence from the Semiotic Perspective
}

\author{
John R. Alexander, Jr. \\ Computer and Information Sciences Department \\ Towson State University \\ Towson, MD 21204 \\ E7C1ALE@TOE.TOWSON.EDU
}

\begin{abstract}
In this paper, we speculate that from both a semiotic perspective and from a perspective of intelligence as given in Albus' Outline of a Theory of Intelligence [2], we may cast some light upon the early stages of the evolution of intelligence by a simple, biologically plausible, model of neural control circuitry. This model implies that the ability of neurons to act as controllers is an inherent property of neurons and that this inherent control is a form of fuzzy control. In employing this model to solve a well-known benchmark control problem (the inverted pendulum on a cart balancing problem), the learning needed to set the few weights (and parameters) was accomplished using a genetic algorithm.
\end{abstract}

KEYWORDS: artificial neural net, average firing rate, evolution.

\section{INTRODUCTION}

"The brain uses stereotyped electrical signals to process all the information it receives and analyzes. The signals are symbols that do not resemble in any way the external world they represent, and it is therefore an essential task to decode their significance." These words begin Nicholls, Martin and Wallace's From Neuron to Brain. [12] With this quotation this author began a paper which appeared in the 1996 NIST Conference Proceedings - Intelligent Systems : A Semiotic Perspective [8]. This paper is a continuation of last year's work and discusses how very early neural signals (symbols) might be viewed from a semiotic perspective. SECTION 2 defines and discusses the signs, interpretants, and objects in a context of early neural signaling. SECTION 3 contains a discussion of why these early signals must have contained control information and how inherent properties of the integrating mechanism of neurons effects control. In SECTION 4, we describe the progress made using circuits consisting of two input neurons and one output neurons (Three Neuron Controllers or TNCs). We combined two TNCs each of whose output serves as input to yet a third TNC (creating a Seven Neuron Controller - Figure 3) and solved the Inverted Pendulum problem. Further, our Seven Neuron Controller net was trained using a genetic algorithm. SECTION 5 contains a discussion of why TNC control is analogous to fuzzy control. This information is included for completeness and summarizes the discussion given in last year's paper [8]. In SECTION 6, we reiterate earlier discussions [3][4] (speculations) of how elements of intelligence, as Albus describes them, could have evolved. Further, we look at the sign, interpretant, object triad, and show that it, too, admits a description couched in terms of the evolution of a simple neural model. Section 7 summarizes this paper.

\section{SECTION 2}

An article in the March-April issue of the American Scientist [9] (the Sigma Xi magazine) entitled The Origin of Animal Body Plans discusses the "Cambrian Explosion." Our speculation affords a plausible commentary on this phenomenon, that is, the rapid increase in the number and variety of animals on the earth which occurred about 530 million years ago. This commentary on the Cambrian Explosion will be given in SECTION 7. To discuss the evolution of intelligence from a semiotic perspective, we must consider the neural activity of the earliest of animals possessing neural control mechanisms. Thus, from the semiotic perspective, since the stereotyped signals are virtually identical in all nerve cells of the human body and also very similar in different animals [12], we assume that the information in the signs to be studied is contained in the frequency of firing of the neural signals (action pulses), that the interpretant is simply the neuron that integrates the neural signals from the impinging neurons, and that the object which caused the sign to be generated is either an external event reported to the organism by means of some sensory neural circuitry or a signal that was generated by some internal mechanism such as the output of a pacemaker neuron.

\section{SECTION 3}

Let us focus our attention on a very elementary form of life, a coelenterate, the jellyfish. These creatures have no associative memories [13], yet they can swim and attack prey. Thus, creatures exist with only control neural circuitry and no associative memory neural circuitry. Further, we see no biological advantage to a creature 
possessing associative memory neural circuitry but no control circuitry. Thus it seems plausible to conclude that control neural circuitry evolved before associative memory neural circuitry. Certainly one neuron signaling a second is the simplest neural net of all possible nets (of more than one neuron) to envision. The sending neuron in a twoneuron circuit can control the receiving neuron by either enhancing its firing rate (excitatory) or inhibiting it (inhibitory). Cortical neurons, because of the random firings of the massive number of neurons to which they are connected, possess a non-zero average firing rate [1]. It seems unlikely, however, that sensory or motor neurons of very early organisms did have a non-zero firing rate. It would appear that the ability of one neuron to inhibit the firing of another must have evolved to inhibit self-firing (pacemaker) neurons, or after the evolution of circuits with two or more neurons. Inhibiting self-firing or pacemaker neurons gives rise to the ability to generate rhythmic or cyclic firing patterns [10]. Rhythmic patterns are used to control the heartbeating and swimming of the leech. The flow of oxygen carrying blood eliminates the need for oxygen to diffuse through an animal's body. Creatures that rely on diffusion of oxygen are of necessity flat [9].

Adding a third neuron to a two neuron circuit could be done by adding the third so as to place three in line, or adding the third in parallel to the existent sending neuron so that the receiving neuron has two inputs.

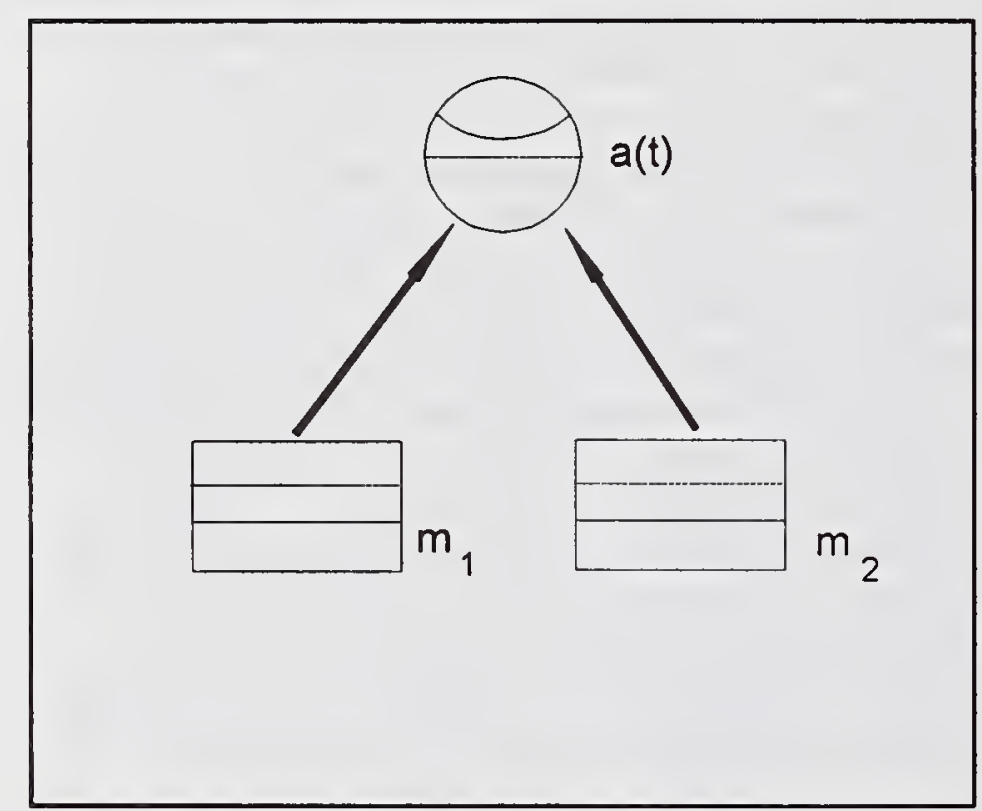

Figure $1 \mathrm{~A}$ Three Neuron Controller (TNC)

This latter circuit is pictured in Figure 1. This circuit allows control of two different input signals. This is the property of neurons which we assert is both natural and useful. We have successfully used such circuits as controllers, and have named them Three-Neuron Controllers (TNC)s. TNCs have been used to control the height of water in a tank at constant set point under conditions of random inflow [5] and to back a truck to a loading dock [6]. In the height of water in a tank and the tnuck backing problem, two neurons represent the inputs of these problems, while the third or output neuron represents or codes the control signal.

In Figure 1, we have assumed that the input neurons $(\mathrm{m} 1$ and $\mathrm{m} 2$ name both the input neuron and the value of its output) possess some average firing rate called Ave $_{j}$. The input signal that the two input neurons receive $\left(\right.$ Observ $\left._{\mathrm{j}}\right)$ is on $\left[\mathrm{Min}_{\mathrm{j}}, \mathrm{Max}_{\mathrm{j}}\right]$ and is mapped onto $[-1,1]$ by the following formulas.

$$
\begin{gathered}
m_{j}=\frac{\left[\text { Observ }_{j}-A v e_{j}\right]}{\left[\text { Max }_{j}-A v e_{j}\right]} \\
\text { If } \text { Observ }_{j}>A v e_{j} \\
m_{j}=\frac{\left[\text { Obser }_{j}-\text { Ave }_{j}\right]}{\left[A v e_{j}-\text { Min }_{j}\right]} \quad \text { Otherwise }
\end{gathered}
$$

We further assume that the weight connecting the input neurons to the output neuron, w11 and w12, are constrained to be on $[-1,1]$ also. Thus, the input signals which $\mathrm{a}(\mathrm{t})$, the output neuron, receives are on $[-1,1]$.

Thus far, we have studied two sets of non-linear differential equations which describe the flow of activation in Figure 1 [7] [8]. Both sets of equations constrain the output neuron $\mathrm{a}(\mathrm{t})$ to be on $(0,1)$. The first set studied was the author's dissertation equations and were called the RX equations. Most recently we have worked with McClelland and Rumelhart's iac (interactive activation and competition and which we call the ENIAC Equations for extended iac) [11] translated from difference to differential equation form. Their solutions are given in Equation 3 when the weighted sum of its inputs $m_{1}$ and $m_{2}$ is greater than zero, and Equation 5 when this sum is below zero.

$$
\begin{gathered}
a(t)=\bar{a}+\frac{(1-\bar{a})}{1-\left(1-\frac{(1-\bar{a})}{\left(a_{0}-\bar{a}\right)}\right) * \exp \left(-K_{1} *(1-\bar{a}) * t\right)} \\
\text { Where } K_{1}=\frac{\left(w_{11} m_{1}+w_{12} m_{2}-d\right)}{(1-\bar{a})}>0
\end{gathered}
$$

$$
\begin{gathered}
a(t)=\frac{\bar{a}}{1+\left(\frac{\left.\bar{a}-a_{0}\right)}{a_{0}}\right) * \exp \left(-K_{2} * \bar{a} * t\right)} \\
\text { Where } K_{2}=\frac{\left(w_{11} m_{1}+w_{12} m_{2}+d\right)}{\bar{a}}<0
\end{gathered}
$$


Observe, in Equation 3 when $t=0, a(t)=a_{0}$, and when $t$ is infinite, $a(t)=1$. In Equation 4 at $t=0, a(t)$ is also $=a_{0}$ and when $t$ is infinite, $a(t)=0$. The output neuron $a(t)$ has a resting value, $\bar{a}$. We generate the actual control output by subtracting from $a(t)$ the resting value and then scaling the result onto the range of possible control values. For example in the truck backing problem, the steering wheel is limited to plus or minus 15 degrees and the resting value of a(t), $\bar{a}$, was assumed to be 0.5 . Thus:

$$
\text { Control Signal }=(a(t)-0.5) * 30
$$

Note that when $\mathrm{a}(\mathrm{t})=0$, the control signal is -15 degrees, and when $\mathrm{a}(\mathrm{t})=1$, the control signal is +15 degrees and all values inbetween are attainable.

\section{SECTION 4}

Other problems solved by extending to three TNCs are: to back a cab with a trailer (truck and trailer) to a loading dock [7], and more recently (discussed below) to control (keep balanced) an inverted pendulum positioned on a cart and subject to random forces which tend to unbalance it. Both the truck and trailer and the inverted pendulum problems have more than two inputs. For more information on the truck and trailer case, the reader is referred to reference [7] In the Inverted Pendulum (IP) problem, pictured in Figure 2, there are four inputs $x$, and $\mathrm{R}$, the position and velocity of the cart and $\theta$ and $\theta$, the angle of the pendulum with the vertical and the angular velocity of the angle. The task is to keep the pendulum upright, and the cart centered over $\mathrm{x}=0$ while the cart and pendulum are subject to random disturbing forces. Control is via a motor on the cart. The control task is to calculate the appropriate torque to the motor.

The architecture of the neural net used to solve the IP problem is displayed in Figure 3. This is the first problem where we needed to train the weights. It seems apparent that in the IP problem, the control of the pendulum must be of higher precedence than control of the cart's $x$ position. The pendulum must not fall and hence, it must be controlled first. Only when it is stable, does it become possible to "walk" the cart back to the position $x=0$.

We designed a genetic algorithm determine the appropriate weights for the system. The only weight changed was the one from the $\mathrm{x}$ associated TNC to the final control node. The values of this weight generated by the genetic algorithm was .13. This reflects the fact that it is necessary to first control the pendulum.

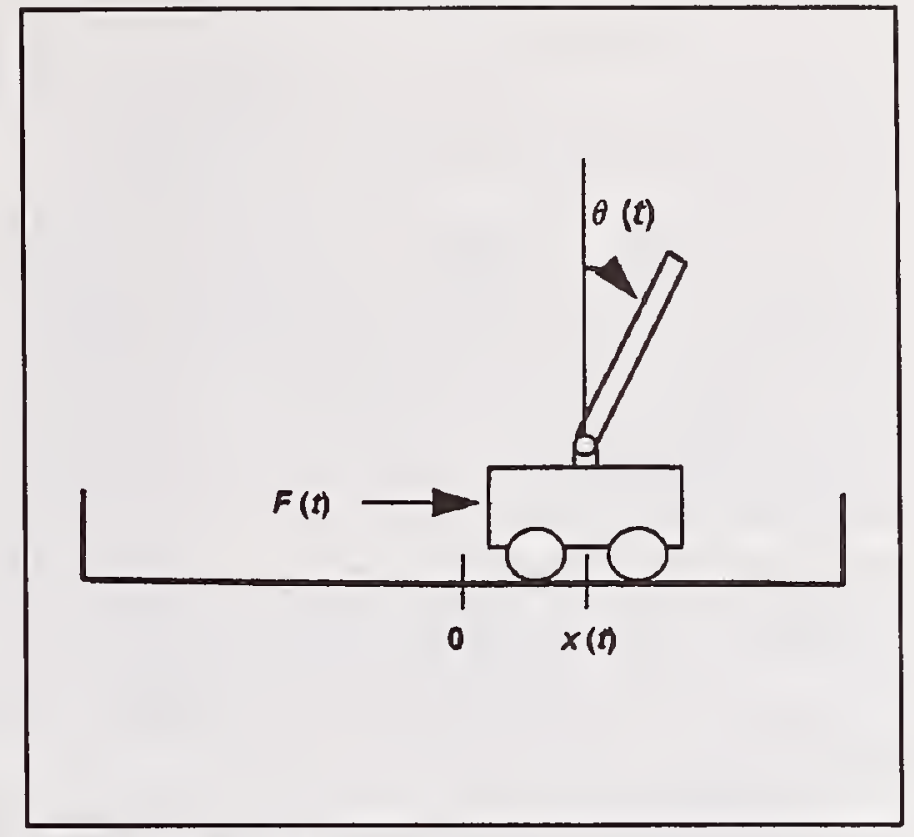

Figure 2 The IP Problem

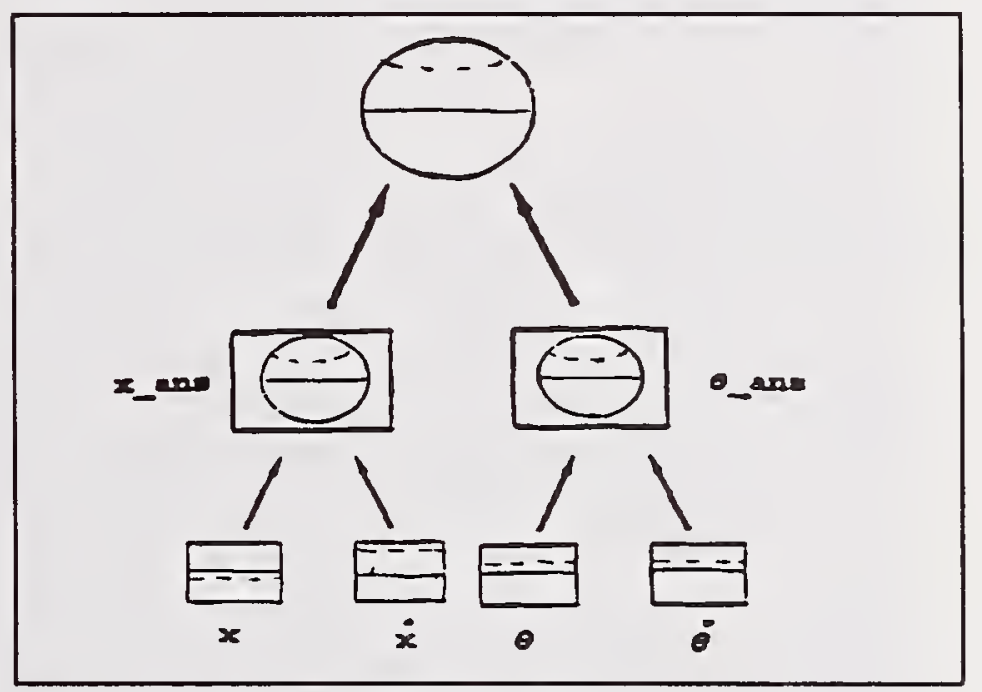

Figure 3 The Seven Neuron Controller

\section{SECTION 5}

We have compared all of our neural control answers to those generated by a fuzzy controller, with the exception of the IP problem. The results produced by all controllers were very similar. It is easy to see the mathematical reason for this. The first two of the problems with which we worked have had monotone control surfaces. By this we mean that if one of the inputs is held fixed at any point on its domain of $[-1,1]$, and the other input value allowed to increase from its minimum value of -1 , up to its maximum value of +1 , the control surface will either increase (monotone increasing) or decrease (monotone decreasing) over the complete range of input values. This is true of both inputs. But it is not true for all possible problems. Our model accepts the 
normalized inputs, uses them as constant parameters in the function to be integrated, and integrates over time up to a fixed upper limit of time (which happens to be different for each model, RX or ENIAC). Different functions are used as integrands depending on whether the input parameters are negative or positive. However, in each case the integral is also a monotone increasing or decreasing function of the inputs. That is, the greater the positive input (up to its maximum of 1 ) the larger will be the value of the integral (and hence control signal). The larger in absolute value (closer to -1) the negative input, the smaller will be the value of the integral. The smallest value possible for the integral is zero. By Equation 5 above, when a(t) (the value of the integral) is zero, then the control signal will be as large in the negative direction as is possible.

Note that none of the solutions calculated are in any way optimal. They are just solutions to a problem in a dense set of solutions. There are an infinite number of ways the fuzzy membership functions can be selected; also, one may chose from an infinite number of shapes for each membership function. Further, a wide variety of defuzzification procedures may be employed. What we have observed is that each reasonable method of solution that we have studied thus far has produced solutions "near" each other in the solution space; that is, they successfully control the process. Figure 4 shows why the fuzzy solutions are closely approximated by the neural techniques. For each fuzzy rule, there is a corresponding integral which, because of the monotonicity of the control surface, gives a similar result for a control value, as does the fuzzy rule that it emulates. The inputs to the integrals are crisp, as are the integrals upper limit. How can we claim that the process represents a fuzzy form of control? This claim is made in recognition of the infinite number of functions that could be used as integrand and give similar answers. Also, slight variation in the upper limit of the integral would effect the solutions very little. Thus, our fuzziness is in function space.

\section{SECTION 6}

Figure 5 displays what Albus [2] called an Element of Intelligence. In recent articles [3] [4], the author suggested that Albus' model [2] admits an evolutionary explanation as follows. The sensors and actuators evolved first. These correspond to simple sensory neurons directly connected to motor neurons. Note that these circuits at first involve no memory. As the evolutionary process continued, refined sensory processing and behavior generation abilities, which involved memory, would mean greater success from a biological standpoint to the organism possessing them. The functions performed by the World Models and Value Judgement elements could further enhance survival of the possessing organism. The referenced articles [3] [4] further suggested that the initial components, the sensors and actuators, could be emulated by a TNC, with the input neurons playing the role of the sensors, and the output neuron the role of the actuator.

We suggest that this model appears logical from the semiotic perspective also. As stated in SECTION 2, the sign of the semiotic triad is represented by the frequency of firing of input neurons. Initially the interpretant is simply a neuron that integrates the inputs of its impinging neurons. In order not to distort its message, the object of the triad, existing perhaps in the external world or perhaps internally as a self-generating neuron, should map, in a linear fashion, the degree to which the object is to be considered by the interpretant. From a practical standpoint, pressure sensitive neurons are linear over a wide range of inputs [10]. That is, the frequency of firing of the sensory neurons in the skin which measure pressure applied, may be represented as a constant times the pressure applied. Non-linearities are introduced by the integration accomplished by the interpretant. From a biological standpoint, this sequence appears plausible. The sign is a direct measure (i.e. directly proportional) to the magnitude of the object. The interpretant may apply a gain (introduced by the nonlinearity) to the sign representing the object as best suits the survival of the using organism. The reader should keep in mind that the system being discussed contains no memory.

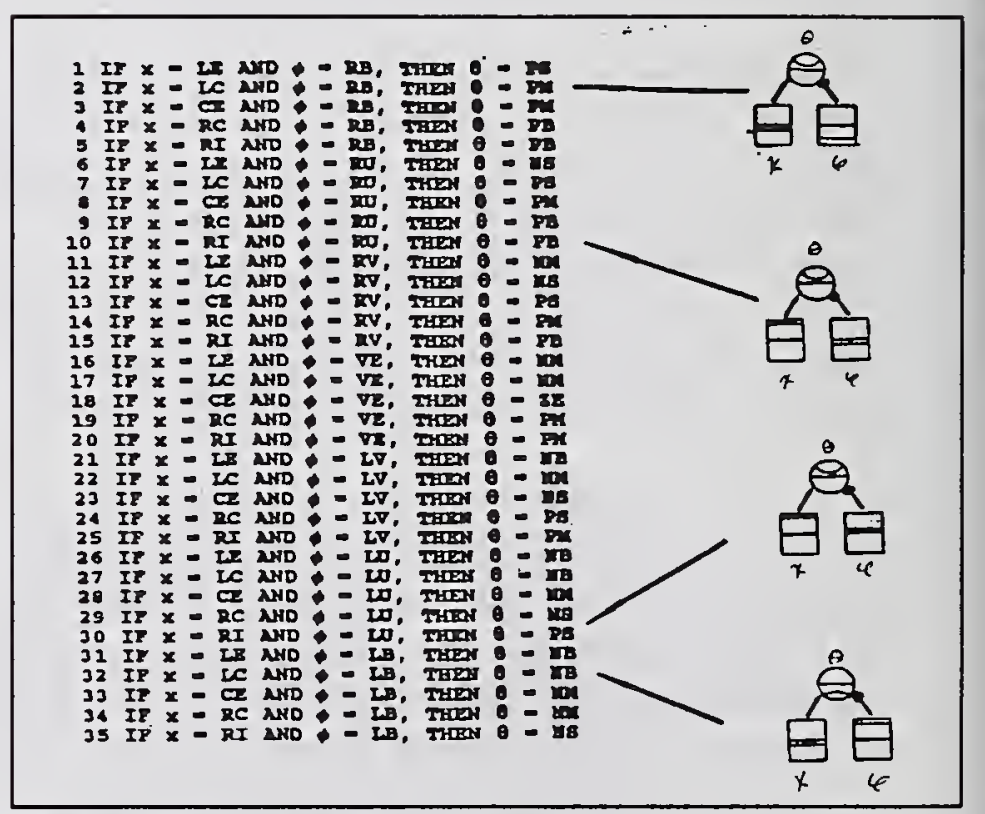

Figure 4 Neural and Fuzzy Rules 


\section{SECTION 7}

Viewed as the evolutionary beginnings of one of Albus' elements of intelligence, or from a semiotic standpoint, or simply from the standpoint of how intelligence might have evolved, this model seems relevant. Neurons do integrate their inputs, and we have shown that for a large class of problems, and for two different functions, that this integration can yield useful results. Since neural circuitry evolved in the Cambrian Period it does not therefore, seem unreasonable to the author, to assume that this property of neurons increased the survivability of those organisms possessing neurons to the extent that this property of neurons might have contributed to the Cambrian Explosion.

This paper is highly speculative. Speculation must be constrained in two ways as stated by Smith and Szathmary [14]. "First each event must be explained in a way that is consistent with the general theory of evolutionary change, the theory of natural selection. Second, an adequate account of the origin of any system must explain the peculiarities of the system as it exists today..."

The examples of jellyfish has led us to believe that control neural circuitry existed before associative memory neural circuitry. In the interest of enhancing our understanding and use of ANNs as associative memories, it would appear that a serious effort should be undertaken to understand how memory abilities evolved from control abilities.

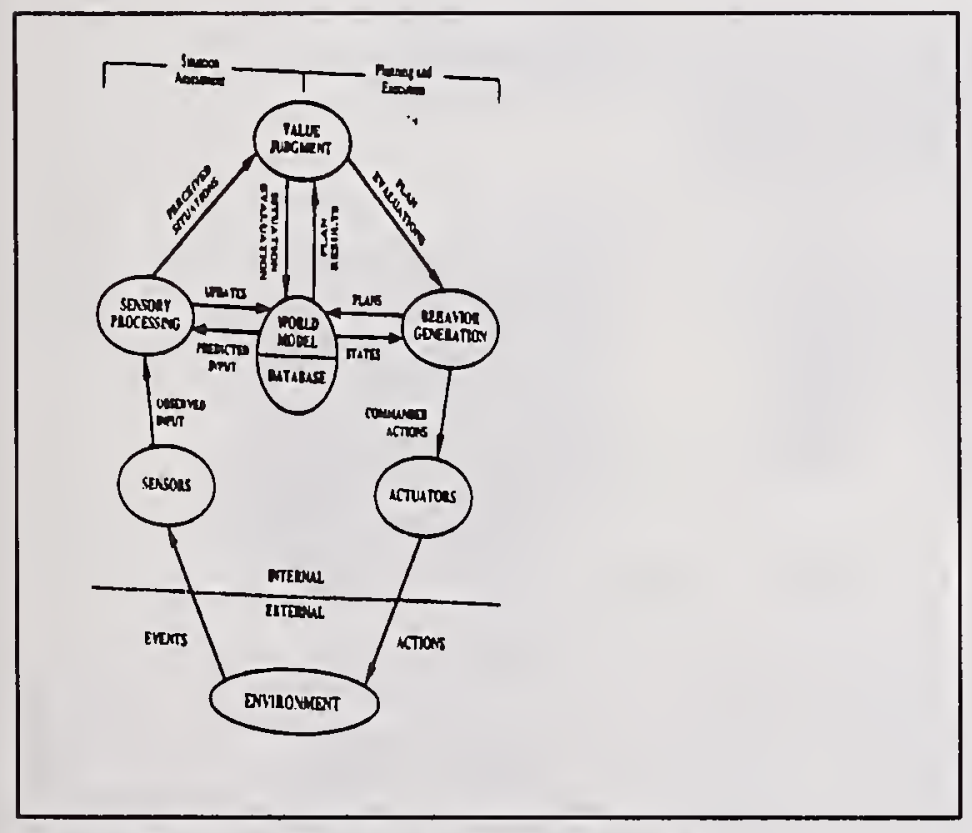

Figure 5 Albus' Elements of Intelligence

\section{REFERENCES}

[1] Ables, M. (1982) Local Cortical Circuits SpringerVerlag New York NY.

[2] Albus, James (1991) Outline of a Theory of Intelligence 1EEE Transactions on Systems, Man and Cybernetics May/June 1991.

[3] Alexander, J. (1992) An Artificial Neural Net Employing Probability Data as Weights and Parameters 1992 IEEE Systems, Man and Cybernitics Conference Proceedings.

[4] Alexander, J. (1994) Continued Speculation of the Evolution of Learning, 1996 1EEE Systems, Man and Cybernitics Conference Proceedings.

[5] Alexander, J. (1994) Calculating the Centroid of a Fuzzy Logic Controller Using an Artificial Neural Net, Intelligent Engineering Systems Through Artificial Neural Networks 4, ASME Press, New York, NY.

[6] Alexander, J. and Bradley, J. (1995) The Three Neuron Controller - History, Theoretical Foundations, and Possible Employment in Addapitve Control, Intelligent Engineering Systems Through Artificial Neural Networks 5, ASME Press, New York, NY.

[7] Alexander, J. and Cox, J. (1996) Backing Up a Truck and Trailer Using Sets of Three-Neuron Controllers, Intelligent Engineering Systems Through Artificial Neural Networks 6, ASME Press, New York, NY.

[8] Alexander, J. (1996) Biological Suggestions for Alternative Design of Artificial Neural Networks (ANNS). Proceedings of the National lnstitute of Standards and Technology 1996 International Multidisciplinary Conference Intelligent Systems: A Semiotic Perspective Volume II. Gaithersburg MD.

[9] Erwin, D., Valentine, J. and Jablonski, D. (1997) The Origin of Animal Body Parts. American Scientist MarchApril (vol. 85) Sigma Xi Press Research Triangle Park, NC.

[10] Muller, K., Nicholls, J. Stent, G. (1981) Neural Circuits Generating Rhythmic Movements in Neurobiology of the Leech Cold Spring Harbour, NY. [11] McClelland, J. and Rumelhart, D. (1988)

Explorations in Parallel Distributed Processing A Handbook of Models, Programs and Exercises MIT Press, Cambridge, Mass.

[12] Nicholls, Martin and Wallace (1992) From Neuron to Brain Sinauer Associates, Inc. Sunderland, MA. USA.

[13] Olds, James 1994 Private Conversations.

[14] Smith, J. and Szathmary, E. (1995) The Major Transitions in Evolution W.H. Freeman Spektrum, New York NY. 


\title{
On Hardware Evolvability and Levels of Granularity
}

\author{
Adrian Stoica \\ Center for Integrated Space Microelectronics \\ Jet Propulsion Laboratory, California Institute of Technology \\ Pasadena, CA, 91109
}

\begin{abstract}
Evolvable hardware addresses hardware that self-organizes/ reconfigures under the guidance of evolutionary mechanisms. Some experiments in evolving at transistor level are briefly presented and the perspective of transistors as functional approximators is suggested. In a broader context one analyses approaches to evolvable hardware specifying the level of granularity at which evolution will operate, in accordance with the level of design abstractions in the modeling hierarchy: primitive, functional and behavioral levels in simulated circuits, and transistor, subcircuit and high level function in hardware. Comments are made linking the role of Automatically Defined Functions in hardware evolution, the process of achieving higher/coarser levels of granularity in the semiotic perspective, and the evolution of modularity of organismic designs. Finally, it is suggested testing the effect of changing levels of granularity during hardware evolution.
\end{abstract}

KEYWORDS: evolvable hardware, granularity, evolvability

\section{EVOLVABLE HARDWARE}

Evolution appears to be nature's solution to design. Being able to replicate such a capability in an artificial system would offer tremendous insights into ourselves and a powerful tool for building adaptive, intelligent systems.

From the perspective of space exploration, empowering spacecraft with adaptive, intelligent capabilities is invaluable for autonomy. Adaptive features are needed to cope with the uncertainty of spacecraft remote operating conditions, performing totally unexpected functions, and for fault-tolerance. The on-board computer needs to be able to solve problems for which solutions were not specified on ground, and command the spacecraft to adapt to new situations. For adaptive, versatile spacecraft, electronic hardware must posses the capability to reconfigure, or moreover, to self-reconfigure, as needed.

Evolvable hardware (EHW) is adaptive hardware that reconfigures under the control of an evolutionary algorithm [1]. Extrinsic EHW refers to evolution in a software simulation using models of the hardware behavior, downloading the configuration of the best evolved architecture to programmable hardware. In intrinsic hardware (to which most of the following discussion applies) configuration bits are iteratively downloaded to hardware, evaluating a degree of adaptation/fitness by observing the behavior of the real hardware.

Hardware evolution is performed through a succession of changes of elementary cell functions and cell interconnectivity pattern, thus obtaining increasingly more fit configurations until a target functionality is reached. As it is the case in nature, evolution results in individuals that are increasingly more adapted to their environments, and can change themselves to match changes in environments and modifications of their own goals. Unlike in nature, evolution in silicon has the advantage that could be extremely rapid, with millions of generations of "living" circuits evaluated in only a few seconds.

Hardware evolution can be seen as an on-chip search for the circuit/configuration whose behavior is closest to the required one (e.g. gives best performance/adaptation to the environment). The suitability for a parallel hardware implementation of evolvable hardware, with multiple "islands" of concurrently evolving circuits on the same chip, or in a multi-chip or stacked configuration is very attractive.

Evolvable hardware: a fully parralel process

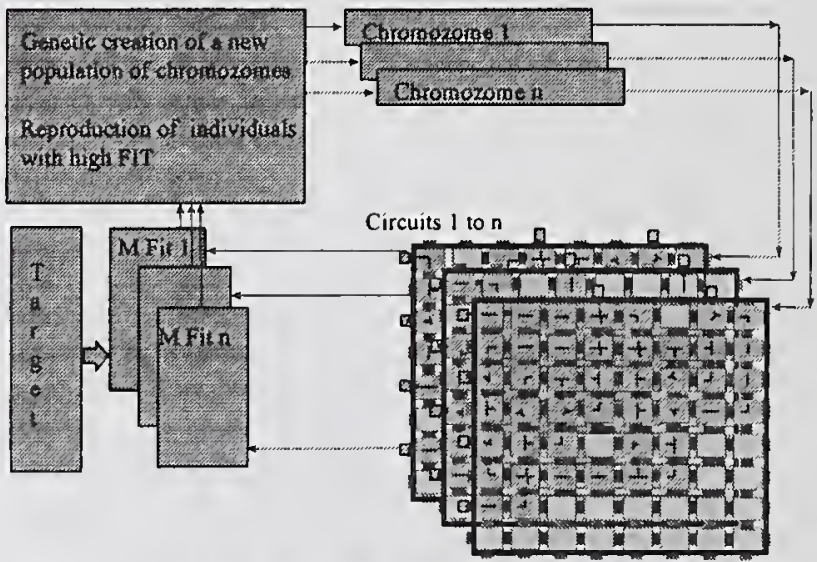

Fig. 1. Parallel implementation for evolvable hardware

The granularity of hardware building blocks for those attempting intrinsic evolvable hardware is currently influenced by the availability of certain programmable devices. The paper presents results of simulated evolution at transistor level and discusses on the role of the level of granularity and evolvability. 


\section{EXPERIMENTS IN EVOLVING CMOS CIRCUITS AT TRANSISTOR LEVEL}

Successful evolution has been reported in simulations (analog [2], [3] and digital [4] ) and in real hardware [5] [6]. In the intrinsic EHW perspective, the focus has been on using commercially available FPGAs (Field Programmable Gate Arrays) but custom designs of chips using higher-level functional blocks are also reported [6]. A collection of papers dedicated to the subject is [18].

In here the distinct focus of attention is on issues related to designing the reconfigurable part of an evolvable CMOS chip. The choice is motivated by the fact that CMOS technology is the basis of today's microelectronics industry, and NMOS/PMOS transistors are the elementary components of both analog and digital designs.

One can look at MOS transistors from the perspective of function approximators. Function approximation has recently been approached with computational intelligence techniques, demonstrating general approximation capabilities for structures of neural networks [7] [8] and fuzzy systems [9] [10]. As hardware implementations ultimately rely on silicon, a general functional approximator (FA) implemented in hardware will ultimately be relying on (e.g.) MOS transistors.

A set of experiments was performed to investigate evolution-related issues at transistor and simple sub-circuit level. The objective was to evolve a circuit that provided a bell-shaped response when the input increased linearly. This response can be obtained for example with the circuit in Fig. 2, with one input kept at constant voltage and the other increasing linearly. The evolution was performed on simulated circuits (using SPICE). Constraints were imposed on the mechanism generating the circuits such that all the circuits produced were SPICE simulatable (this differs than Koza's experiments where non-simulatable circuits are eliminated by evolution (e..g in [11]). A limitation existed also on the number of circuits evaluated, which was much smaller than those reported by Koza $\left(\sim 10^{4}\right.$ compared to $\left.\sim 10^{7}\right)$.

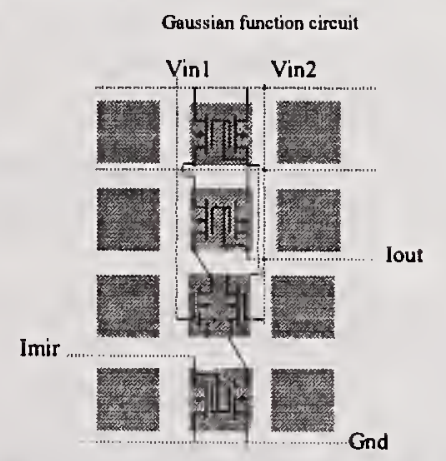

Fig. 2 A circuit producing a bell-shaped response

In the first set of experiments the circuit topology was considered known, and evolution concerned two types of parameters: transistor channel Width and Length. These parameter domains are discrete (they are a multiple of the feature size), and for this case a total of 8 possible widths and 8 lengths was considered. This led to a $(3+3) * 8=48$ bit coding for the circuit. Applying a Genetic Algorithm on a population of 50 individuals, converged easily to good solutions (an illustration of the population after about 200 generations is given in Fig. 3. This is explained by the fact that, although the region searched is small $\left(\sim 2^{10}\right.$ in the search space of $2^{48}$ possible combinations), many combinations are good; in this case the topology is the fundamental factor in circuit behavior.

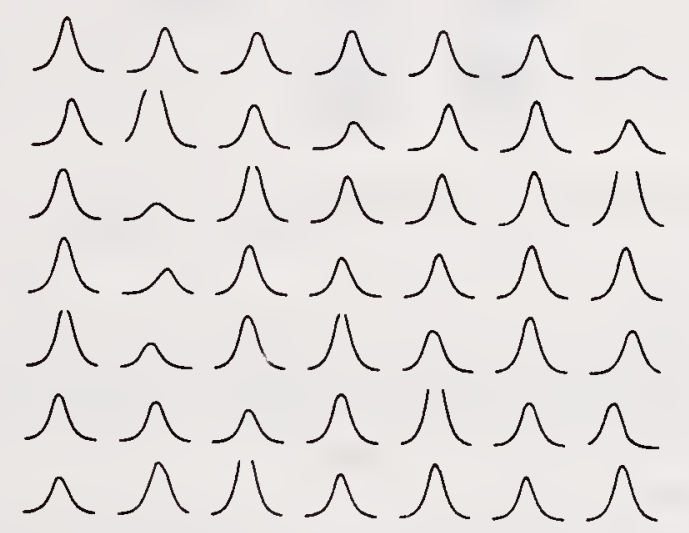

Fig. 3 Response of a population of 49 circuits after 200 generations

The problem becomes complicated when one tries to evolve the topology. Several alternatives were tried, briefly described in the following:

- A 2D transistor array was considered, which resembled an existing FPGA model (Xilinx 6216). The code for each cell specified the type of the cell which could be a transistor with a certain orientation, or a type of wire routing (these are illustrated in Fig. 4). A 2D chromosome was associated to the array. Only evolution by GA was attempted. The problem appears in specifying 2D crossover, determining which 2D zones should be swapped. Crossover must consider the fact that the new circuits must match their connections at cell borders.
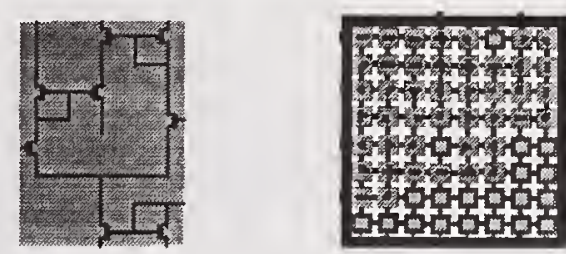

Fig. $4 \mathrm{~A}$ transistor array patterned after an FPGA

- A leveled architecture (with a matrix arrangement as in Fig. 2) where components on a given level were from a list of allowed components (see Fig. 5). Components were low-level subcircuits (current mirror, differential 
pair, pair of transistors). The coding was such that the possible connections were limited to those that made sense (e.g. the Source terminal of transistor in level 4 could not connect to VDD).

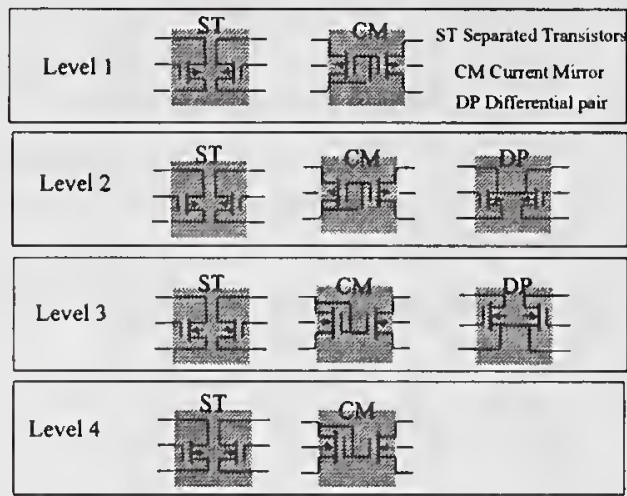

Fig. 5 Components allowed at different levels

The experiments can be interpreted as follows:

- When the topology of the electronic circuit is given, the transistor parameters (in this case PMOS/NMOS channel Width and Length) can be easily obtained through evolution.

- If one attempts evolution in a space restricted at the size of the human-designed solution (in our case when the circuits were limited at 8 transistors) it was not possible to evolve a circuit from specifications. The methods attempted included genetic algorithms, and a search based on orthogonal arrays. With the representation used, the search space appears very much as a flat region, with a singular spike for the solution circuit. Its neighbors, with genetic code differing even one bit from the solution had extremely low fitness; i.e. changing even one connection between 2 transistors had a dramatic effect in circuit behavior. This is possibly a consequence of the search around the optimal size (minimal number of transistors) solution. If the circuit would have contained redundant circuitry changes in those regions would have produce less significant effects.

It was however possible to evolve the topology using genetic programming and an embrionic, growth-based approach, in which the number of components (size of the circuit) was not restricted (results obtained by Koza's group, not published yet). The first set of simulations led to a circuit with 36 transistors.

Evolving on a structure with fixed, minimal number of transistors for the function (for which a solution circuit is already known) suffers from the fact that any mutation involving a connection/topology change leads to a dramatically different response. One possible way in which this can be alleviated is to allow gradual connections (e.g. connections modeled by resistors in $[0$ 100G]) during the evolution. Thus may be architecture which allows gradual transitions between topologies are possible. It could look like a densely-connected "sea of transistors" in which components are connected by a "fuzzy" wire (i.e. with values in $[0,1]$ rather than $\{0,1\}$ ), appearing much like a neural network, but with transistor instead of neurons. The graded connection would have a catalyst role during evolution; it smoothens the search space around the solutions (in this respect neural architectures using gradual connections/weights appear suitable for evolution).

\section{HARDWARE LEVELS OF GRANULARITY AND EVOLUTION}

A fundamental question is how does the choice of a level of design abstraction influence hardware "evolvability"?

(The search/optimization algorithm may be not that important: the "no free lunch" theorems establish that for any algorithm, any elevated performance over one class of problems is offset by performance over another class [12].) When evolution takes place directly in the component space the choice of the primitive building blocks affects the evolvability (e.g. evolving a NN appears easier than evolving a circuit with transistors).

The choices for the level at which evolution process could operate are as follows.

For evolving simulated circuits. The levels of design abstraction in the modeling hierarchy are [13]:

- $\quad$ Primitive Devices (Transistor level - MOS,BJT,etc) represented by analytical equations or tables

- Functional Macromodels (e.g. Op. Amp. Level) derived by circuit simplification, circuit build-up, symbolic methods

- Behavioral High level language descriptions linear and nonlinear mathematical equations, tables, etc.

Evolution can be made at a certain level and then the design converted for hardware implementation with appropriate compilers and synthesis tools.

For evolving directly in hardware. A similar selection of level of primitives is available in a reconfigurable structure (specifying what should be the "building blocks"):

- Transistor level. Some simulations were discussed in the previous section.

- Subcircuit level. This is the Op. Amp./digital gates level. Succesful hardware evolution taking place on an FPGA chip was reported by Thompson [5]. Versatile FPAA (Field Programmable Analog Arrays) are still missing, but are expected to appear on the market within 2-3 years. 
- High level/ functional level One way of coping with difficulties in evolving circuits is to provide high level building blocks, filters, modulators in the analog domain, or adders, MUXs in the digital ones. This approach is followed by Higuchi [6].

The idea of modularity, and changing granularity during evolution may be key to hardware to evolvability (in intelligent systems, knowing presumes changing resolution or granularity [14]). In the biological world modularity is most likely the result of evolutionary modifications [15]. In the evolvable hardware context, Koza has indicated the usefulness of Automatically Defined Function (ADF) for evolving analog electronic circuits (an ADF is "a function (subroutine, module, etc.) that is dynamically evolved during a run of genetic programming and that may be called by a program that is concurrently being evolved") [16]. In effect the idea that no approach to automated programming (and consequently hardware evolution) is likely to be successful on non-trivial problems unless it provides "some hierarchical mechanism to exploit, by reuse and parametrization, the regularities, symmetries, homogeneities, similarities, patterns, and modularities inherent in problem environments" is central to Koza's book [17].

When choosing representation levels, it may be interesting to consider having simultaneous representations at different levels of granularity. During evolution one may switch between levels of granularity depending which one offers advantages at that time.

\section{CONCLUSION}

The paper presented simulation results and discussed issues of evolution at transistor level. While parametric evolution appears easy, topological evolution is hard. Different levels of granularity are available for evolvable hardware. It was suggested that changing/switching levels of granularity during evolution may help.

\section{REFERENCES}

[1] De Garis, H. "Evolvable Hardware: Genetic Programming of a Darwin Machine". In Proc. Of the Int. Conf. On Artificial Neural Networks and Genetic Algorithms, Innsbruck, Austria, Springer Verlag, 1993

[2] Grimbley, J. B. Automatic Analogue Network Synthesis using Genetic Algorithms, 1st IEE/IEEE Conf: Genetic Algorithms in Engineering Systems, UK, 1995

[3] Koza, J., Bennett III, F. H., Lohn J., Dunlap, F., Keane M. A., and Andre, D. "Automated Synthesis of Computational Circuits Using Genetic Programming". In Proc. of Second Annual Genetic Programming Conference, Stanford July 13-16, 1997 (to appear)
[4] Hemmi, H., Hikage, T. and Shimohara, K. AdAM: A Hardware Evolutionary System, In Proc. of ICEC, (193-196), 1997

[5] Thompson. A., Silicon Evolution. In Proc. of First Annual Genetic Programming Conference, Stanford, 1996

[6] Higuchi, T., Murakawa, M., Iwata, M., Kajitani, I., Liu, W. and Salami, M. , "Evolvable Hardware at Function Level." In Proc. of ICEC, (187-192), 1997

[7] Cybenko, G. Approximation by Superpositions of a Sigmoidal Function. Mathematics of Control, Signals, and Systems, 2 (303314), 1989

[8 ] Hornik, K., Stinchombe, M., and White, H. Multilayer Feedforward Networks are Universal Approximators. Neural Networks, 2 (359-366), 1995

[ 9] Kosko, B. Fuzzy Systems as Universal Approximators. In Proc. of the Ist IEEE Conference on Fuzzy Systems, (1153-1162), 1992

[10] Wang, L. X. Fuzzy Systems are Universal Approximators. In Proc. of the Ist IEEE Conference on Fuzzy Systems, (1163-1170), 1992

[11] Koza, J., Bennett III. F.H., Andre, D. and Keane, M., Automated WYWIWYG Design of Both the Topology and Component values of Electrical Circuits Using Genetic Programming, In Proc. of First Annual Genetic Programming Conference, Stanford, (123-131), 1996

[12] Wolpert, D. H. and Macready, W. G., "No Free Lunch Theorems for Optimization", IEEE Transactions on Evolutionary Computation, Vol. 1. No. 1, (67-82), 1997

[13] Mantooth, H. A. and Fiegenbaum, M., Modeling with an Analog Hardware Description Language. Kluwer Acedemic Publishers, 1995

[14] Meystel, A. "What is 'semiotics'after all? (Learning how to know: semiotics and multiscale cybernetics)". Semiotcs97 Internet at http://isd.cme.nist.gov/ proj/semiotics/isas $97 . h t m l$

[15] Wagner, G. P. and Altenberg, L., "Perspective: Complex Adaptation and the Evolution of Evolvability". In Evolution, 50(3), (967-976), 1996

[16] Koza, J., Andre, D., Bennett III, F. H., and Keane, M. A., Use of Automatically Defined Functions and Architecture-Altering Operations in Automated Circuit Synthesis with Genetic Programming. In Proc. of First Annual Genetic Programming Conference, Stanford, (132-141), 1996

[17] Koza, J. R. Genetic Programming II: Automatic Discovery of Reusable Programs. Cambridge, MA: MIT Press, 1994

[18] Sanchez, E. and Tomassini, M. (Eds), Towards Evolvable Hardware - The Evolutionary Engineering Approach. Springer, 1996. 


\title{
Evolution of Learning The Merging of Semiotic and Evolutionary-System Theories
}

\author{
Wolfgang Hofkirchner \\ Social Cybernetics Group \\ Institute of Design and Technology Assessment \\ Vienna University of Technology \\ Möllwaldplatz 5 \\ A-1040 Wien \\ phone/fax **431504118633 \\ hofi@igw.tuwien.ac.at
}

\begin{abstract}
This paper deals with rather basic implications of theorizing systems which are able to learn. Starting from the fact that a unified theory of information is still missing it tries to sketch roughly a conceptualization that merges the concept of semiosis and the concept of evolutionary systems. This shall provide a framework for future research into every kind of information processing systems, including socio-technical systems.
\end{abstract}

KEYWORDS: Unified Theory of Information, semiosis, evolutionary systems

\section{The Quest For a Unified Theory of INFORMATION}

The Second Conference on the Foundations of Information Science (FIS 96) taught us that we are still lacking a Unified Theory of Information (UTI) which comprises the variety of manifestations of information processing in natural, in social, and in artificial systems. It showed at the same time that the UTI we are looking for may be well based upon a theory of evolutionary systems, that is open systems which are capable of self-organization [1]. This theory, however, is to be elaborated, too.

With the transition from System Theory I to System Theory II, as with the change from Cybernetics I to Cybernetics II and the increased scope of the Theory of Evolution, we can see a theory of open, non-linear, complex, dynamic, selforganizing (in short: evolutionary) systems approaching. This theory no longer deals merely with mechanisms, strategies and controls for achieving/maintaining homeostasis and the development of species; it also concerns the birth, growth and decline (i.e. development) of all systems, from the formation of the earliest known particle, through the arrival of terrestrial life forms, to the shaping of specific human socio-technical systems (see e.g. [2], [3], [4]).

As this theory of self-organization will conceive both system and evolutionary aspects, that is both structural and process aspects, I want to argue here that it offers the possibility of serving as the appropriate background theory for unifying information concepts and thus for understanding and designing intelligent and reasonable information processing systems [5].

In order to interlink informational and evolutionary-systems concepts they have to undergo some refashioning which will be described below (for preliminary work we have already done see [6], [7], [8]). Firstly, three levels of a system shall be distinguished. Secondly, the well-known semiotic aspects will have to be rearranged. Each of these will then be related to a certain system level. The next step is to point out that this fundamental structure of any information process shows various kinds of manifestations according to the evolutionary level the system belongs to. Three basic stages shall be identified. Finally, a stage model describes the symmetry breaking sign process.

\section{The Merger of the Concepts of Systems AND SEMIOSIS}

\subsection{Identifying Three System Levels}

In identifying a system, three different levels of the system's dynamics can be distinguished:

1. The level on which the elements of the system in question are interconnected. This is the level of the internal structure of the system (micro-level). 
2. The level on which the system itself is in one state or in another (meso-level).

3. The level on which the system exhibits its external behavior vis-a-vis its environment. The way the system interacts with its co-systems in the net is examined here (macro-level).

Each level is the base on which the next level is built; each level has progressively less space for possibilities than the one below. From one level to the next there is a leap of qualitative difference. This leap can (but need not) be bridged by a self-organization cycle. Thus these levels give the prerequisites for understanding the emergence of new system structures, system states, or system behavior, or even the emergence of new systems.

\subsection{Refashioning Semiotic Dimensions}

According to semiotics, information can be conceived as something having syntactical, semantical and pragmatic aspects. It seems sensible to interrelate these dimensions not in the way semiotics did sometimes, but rather as a nested hierarchy such that:

1. the innermost dimension is the syntactical one;

2. the intermediate dimension is the semantical one;

3. the outermost dimension is the pragmatical one.

This means that they are not merely placed side by side, without having anything to do with each other; each initial aspect is essential for the following one, and each following aspect is a sufficient condition for the preceding one.

In this way they form an architecture which allows for emergent properties [9].

\subsection{Assigning the Semiotic Dimensions to the System Levels}

Having done this, the system and semiotic aspects can easily be linked to one another. Each of the three semiotic aspects can be related to an appropriate system level, as follows (see Fig. 1):

1. syntactics refers to the micro-level;

2. semantics refers to the meso-level;

3. pragmatics refers to the macro-level of a system.

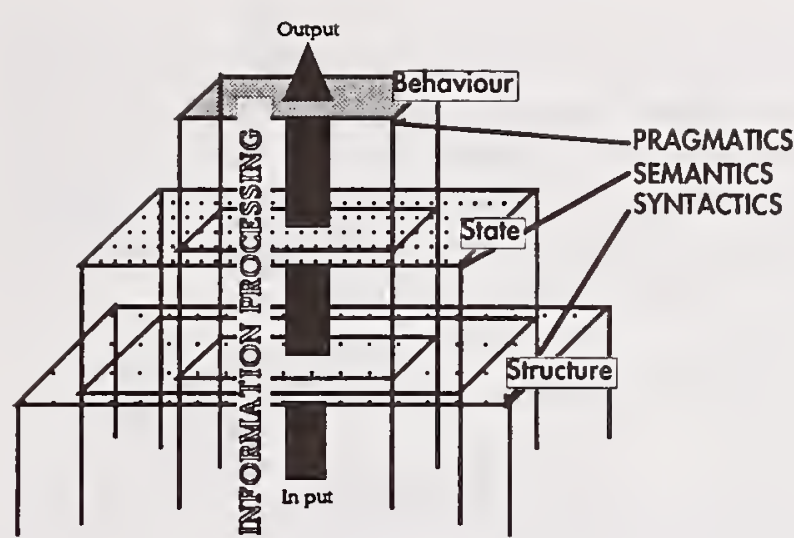

Figure 1. System Levels and Semiotic Dimensions in Information-Processing Systems.

The three semiotic aspects thus relate to one another in the same way that the system levels relate to each other.

Together they mark the varying qualities of information. That is to say, in any particular system, information occurs when as a result of a self-organization process there is a qualitative change on any one of the three levels. The underlying information process may result in structural changes to the interior of a system (on the micro-level), it may result in changes to the actual state of the system (on the meso-level), or it may result in altered external behavior (on the macrolevel). We must note that changes in the interior structure need not lead to changes in the system's state, and that changed states need not necessarily entail changes in the behavior. But a difference in the output of a system must be based upon a different state, and a different state must take as a basis elements and relations that differ from previous structures.

\section{Three Stages in the Evolution of INFORMATION-PROCESSING SYSTEMS}

\subsection{Reflecting as the Most Primitive Form of Semiosis}

If open systems are exposed to fields in which the gradient of the free energy density distribution exceeds a certain amount, they exhibit the capability of organzing themselves in that they build up order, that is make use of energy for performing work, depreciate it and remove the resulting entropy (so-called dissipative systems). Pattern formation is thus the most rudimentary form not only of self-organization, but also of sign processes (see Fig. 2). The system selects a particular option from the various structuring possibilities it has. The selection ends up in a new structure. The information process 
is the process of restructuring and its result is a structure which differs from the structure the system had previously. There is a relation between the different structures which can be looked upon as the most primitive appearance of the syntactical dimension of information. The way the system restructures itself is the self-determined way it reflects outer conditions in its environment (cf. [10], [11]).

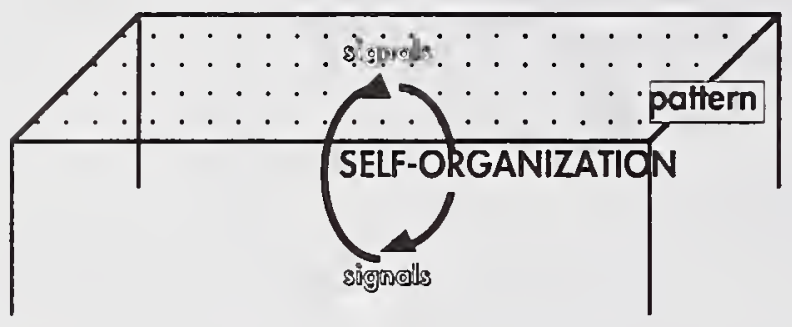

Figure 2. Pattern Formation in Primitive Self-organizing Systems.

\subsection{Building Representations as a Higher Form of Semiosis}

Reflection is the precondition for learning to behave in such a way that this makes the environment produce conditions beneficial to the maintenance and improvement of the system. Simple dissipative systems are, however, not in a position to perpetuate the flow of energy.

Biotic systems are a special category of dissipative systems which are able to perform in this way. They exhibit division into a sensorium and an effectorium, which involves two cycles of self-organization, one on the top of the other. Structural change splits into structural and state/behavior change. The self-organized structure, that emerges within the system, obtains a new function: it becomes a symbol which has a meaning, namely that the change in the outer world of the living system it represents is behaviorally relevant to the survival of the system (see [12]; see Fig. 3).

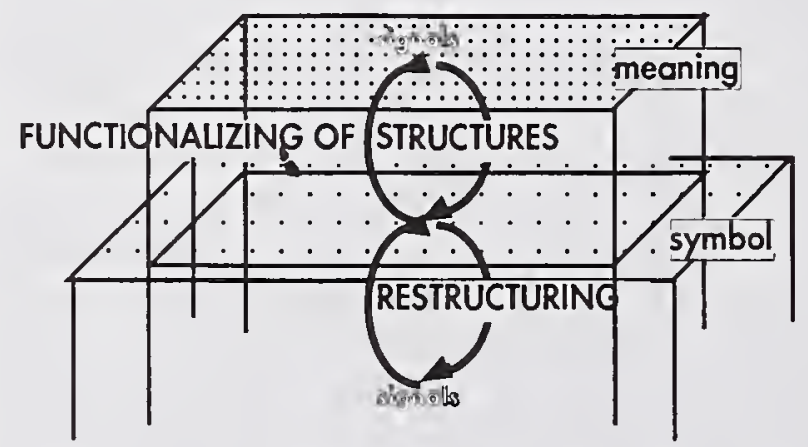

Figure 3. The Differentiation of Meanings and Symbols in Self-organizing Systems.
The syntactical dimension of the difference between the old and the new structures is supplemented by the fact that this difference makes a difference regarding the goal of maintaining the system. So a semantical-pragmatical dimension is added.

Thus representation is another step in the evolution of semiosis. Due to the presence of this new informational relation biotic systems show better adaptability to their environment. They are in a position to take advantage of the environment to such an extent that they can reproduce themselves. This behavior is referred to as intelligent behavior (see e.g. [13]).

\subsection{Decision-making as Most Advanced Form of Semiosis}

Certainly social systems which involve human cognition and communication are the most highly developed appearance of information processing which is known to us at present.

They exhibit even greater adaptability than mere biotic systems: they alter their environment to suit themselves. That is to say, their sphere of influence is characterized by a feedback loop, through which the systems can create the conditions necessary not only for their reproduction, but also for creating themselves according to the goals they freely choose. The behavioral decisions are no longer indistinguishable from the representations, but now are conveyed with the knowledge via another phase transition as knowledge is mediated with data (see Fig. 4). The process of information includes the activies of perceiving, interpreting, and evaluating, and shows separated dimensions of semantics and pragmatics. Knowledge makes a difference as to wisdom, that is reasoned instruction of acting which then deserves to be called reasonable behavior of a system.

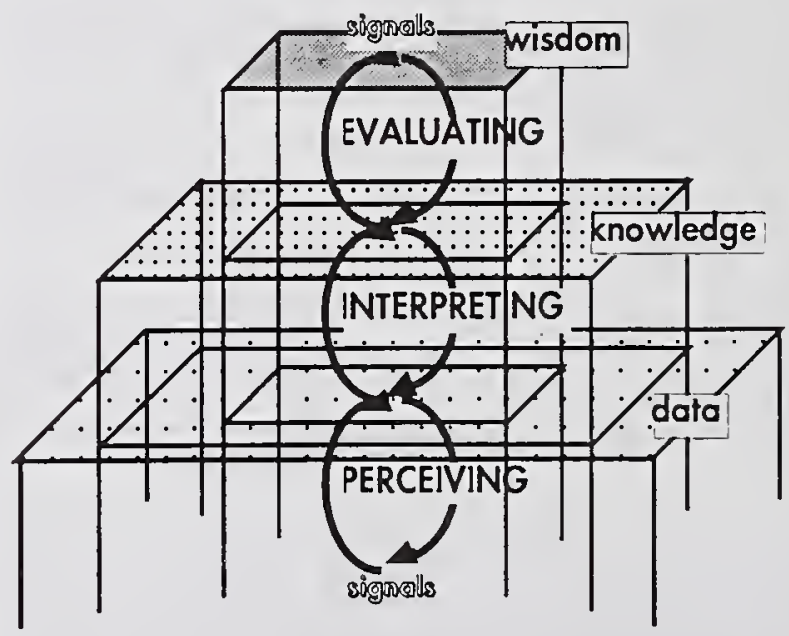

Figure 4. The Differentiation of Wisdom, Knowledge and Data in Advanced Self-organizing Systems. 


\section{A Symmetry-breaking Evolution of Semiosis}

Summing up, a stage model can be postulated in which a process of symmetry breaking unfolds from a stage which is characterized by simple reflective, self-organizing systems via a stage of systems which produce representations toward a stage on which decision-making systems produce perceptions, interpretations and evaluations (see Fig. 5).

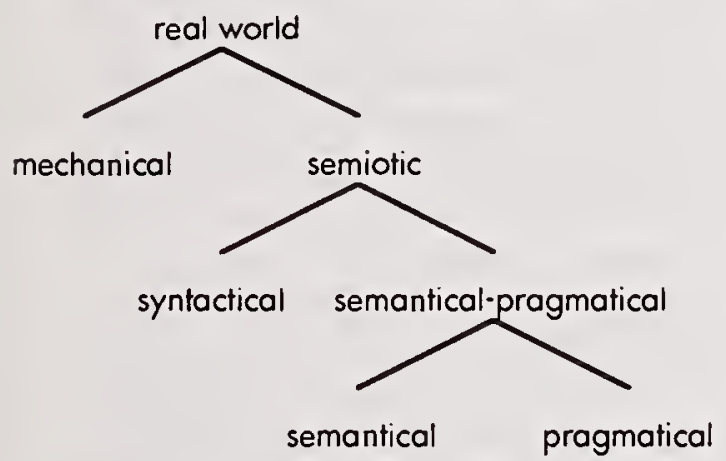

Figure 5. The Unfolding of Semiotic Aspects in the Course of the Evolution of Self-organizing Systems.

Thus a stage model of information merges the system aspects and the evolutionary aspects of semiosis.

In each phase of evolution a new moment appears which becomes characteristic of a layer for more highly developed systems and gives the entire layering of this system its nature.

This model may serve methodologically as a framework for theorizing information processes in all kinds of real-world systems and for the elaboration of a UTI. It may assist as well the design of artificial systems which aid human cognition and communication processes.

\section{REFERENCES}

[1] Hofkirchner, W. (Ed.), The Quest for a Unified Theory of Information, Proceedings of the Second International Conference on the Foundations of Information Science, World Futures, vols. 49(3-4)-50(1-4), 1997.

[2] Ebeling, W., Feistel, R., Chaos und Kosmos. Heidelberg: Spektrum, 1994.
[3] Goemer, S. J., Chaos and the Evolving Ecological Universe. Amsterdam: Gordon and Breach, 1994.

[4] Mainzer, K., Thinking in Complexity. The Complex dynamics of Matter, Mind, and Mankind. Berlin: Springer, 1994.

[5] Haefner, K. (ed.), Evolution of Information Processing Systems. Berlin: Springer, 1992.

[6] Fleissner, P., Hofkirchner, W.: "Emergent Information. Towards a Unified Information Theory", BioSystems, vol. 38, pp. 243-248, 1996.

[7] Fenzl, N., Fleissner, P., Hofkirchner, W., Jahn, R., Stockinger, G., "On the Genesis of Information Structures. A View that is neither Reductionist nor Holistic" in Kornwachs, K., Jacoby, K. (Ed.), Information, New Questions to a Multidisciplinary Concept. Berlin: Akademie Verlag, pp. 271-283, 1996.

[8] Fenzl, N., Hofkirchner, W.: "Information Processing in Evolutionary Systems. An Outline Conceptual Framework for a Unified Information Theory" in Schweitzer, F. (Ed.), Self-Organization of Complex Structures - From Individual to Collective Dynamics. London: Gordon and Breach, 1997.

[9] Blitz, D., Emergent evolution - qualitative novelty and the levels of realin'. Dordrecht: Kluwer, 1992.

[10] Haken, H., Information and Self-Organization. Berlin: Springer, 1988.

[11] Atmanspacher, H., Kurths, J., Scheingraber, H., Wackerbauer, R., Witt, A., "Complexity and Meaning in Nonlinear Dynamical Systems", Open Systems \& Inf. Dynamics, vol. 2/l, pp. 269-289, 1992.

[12] Ayres, R. U., Inforntation, Entropy, and Progress. New York: AIP, 1994.

[13] Stonier, T., Beyond Information. The Natural History of Intelligence. London: Springer, 1992. 


\title{
The Effects of Biological Aging on Genetic Algorithms
}

\author{
Tim Schmelmer and Frank M. Brown \\ Artificial Intelligence Laboratory \\ University of Kansas \\ Lawrence, Kansas, 66045 \\ tim@eecs.ukans.edu brown@eecs.ukans.edu
}

\begin{abstract}
This paper investigates the applicability of the biological concept of aging onto the performance of a Genetic Algorithm. It is explained how the natural phenomenon of decline in vitality due to old age is translated into the domain of Genetic Algorithms, and a GA implementation is tested on four partly NPhard problems. The basic rationale is that aging factors, depending on the problem they are applied to, can greatly influence the quality of the results produced by the GA, and thus speed up or slow down the search process. The results organized by test problem are presented, and compared quantitatively. Rules of thumb are laid out as to how influential the aging component of the fitness function should be chosen in order to achieve the best search results for the four problems treated in this research.
\end{abstract}

\section{Introduction}

In 1803, T.R. Malthus [7] stated his observation that a population in which the individuals reproduce will grow geometrically and thereby deplete its natural resources, unless it is regulated by the mortality of its individuals. Also, species of immortal individuals would stop evolving, because the process would stall, once all ecological niches fit for life had been exploited. By putting a cap on the number of individuals under competition and introducing new, variant individuals, death only enables the dynamic process of evolution. In other words, aging and eventual death is a natural implementation to give someone else a chance.

Throughout most of the natural species that reproduce sexually, it is a common fact that old individuals lose vigor and are generally less likely than young ones to survive in "struggle for existence"[4]. Also, natural individuals die of age by which they completely retreat from reproduction. Researchers in evolutionary biology state several different explanations for this phenomenon. Group selectionists hold the point of view that dying is an act of altruism for the rest of the species. They place the selection process on the level of a race, or even the entire species, which strives for perfection [14]. Old creatures would use up valuable resources for younger and fitter individuals, and therefore slow down the species' pace on its way to evolutionary perfection. Such a breed would not be fit for competition with other species, so it would die in the long run. Gene selectionists on the other hand think that there are lethal genes in every individual. Selection simply favors lethal genes, which are either only expressed at old age, or at least are suppressed until after reproduction. According to these theories, aging actually provides species with an advantage in the process of selection [3]. Some biologists (e.g. [10]) have gathered evidence that aging and death might be consequences of deleterious genes.

Irrespective of the viewpoint, aging is a major factor in the system of natural evolution. Considering its influence, it has gone remarkably unnoticed or has been passed by in the research of artificial evolution, though this concept can easily be translated into genetic algorithms. Holland's original and most commonly used concept of Genetic Algorithms [15] can be viewed as strictly generational. The life span of all individuals is simply one generation. After that they die and are replaced by offspring produced from the previous generation. This very drastic change introduces the necessary variation into the population, but it does not pay tribute to biological facts. It is important to understand that death does not come all of a sudden. It is rather the result of the dynamic process of first maturing, and then aging. This detail has an important impact onto the evolution of a species, because of the observation that old individuals are less likely to survive and take part in another mating phase. Evolutionary strategies [2] and steady-state Genetic Algorithms [9] have a different approach. Individuals are evaluated at the end of a generation, and an artificial fitness function determines an individual's likelihood to make the transition into the next generation. The implementation of the Reference Genetic Algorithm also follows this stochastic approach. Still, these approaches make death come to an individual all too sudden. They do not consider that death is just the outcome of the inherently gradual process of aging. Age does not play a role at all in an individual's probability to be selected into the next generation, nor does it influence the reproduction process. Nature does not work this way. This is why researcher in the field of artificial life, who are 
interested in simulating the emergence of life with the help of computers came up with different ideas to simulate the natural processes of aging and dying. In Thomas Ray's Tierra system [12] where selfreplicating programs consisting of assembly language instructions for a virtual machine run competing for computer memory and CPU time. Although in Tierra senescence does occur, the ideas of aging and death are based on the individual's success in coping with the system's requirements during its lifetime. As a consequence, individuals complying perfectly with Tierra's rules will live forever, unless more than 80 percent of the environment is filled up with infallible programs. This can also lead to stalling in the evolutionary process. This phenomenon of stalling is the reason why Peter Todd [13] in his system to study the evolution of behavior in a simulated environment introduced a new kind of artificial death. An individual perishes if it runs out of energy, so the only notion of aging in his original world is running out of energy. Still, Todd's examination of this system exhibited the evolution of immortal creatures, whose main feature was that they chose not to take part in the very costly process of producing offspring. The conclusion in [13] recommends that new forms of death are needed to prevent immortals from bringing the evolutionary process to a stop.

Research in the field of Genetic Algorithms has vastly ignored the notion of age inherent in biological systems. In traditional Genetic Algorithms where in each new generation the entire population is replaced by their offspring individuals can die too quickly to spread good genes. In purely stochastic approaches on the other hand, immortal individuals can slow down the evolutionary search process, or make it get stuck in local plateaus of the search space. The reason for this is that old individuals use up the resources for young ones. If they do not take part in the mating process a sufficient number of times then they do not introduce any variation into the population; they just sit in the population being an obstacle to evolution. The population does not profit from good genes they might have. Realizing this deficiency and inspired by the attempts made in Artificial Life to overcome this very unnatural way of generational transition, the authors examined the influence of a specific type of aging on process and results of genetic search.

The remainder of this paper addresses this topic. Section 2 below describes a basic Genetic Algorithm (i.e. GA) that is used in this work to compare its results to those found by the GA that implements the aging concept. This algorithm is described in section 3 . The following four sections describe and compare the application of both algorithms to a variety of problems. First among these is the 0/1 knapsack problem (KSP) [8] in section 4. It is followed in section 5 by the problem to optimize the amount of resources needed to survey an area of special interest (ASP). In section 6 we apply these algorithms to the iterated version of the Prisoners' Dilemma Problem (PDP) [1]. Section 7 is dedicated to the examination of a timetable scheduling problem (SCP). Finally, in section 8 we draw some conclusions about the general applicability of the aging concept in the field of evolutionary search.

\section{The Reference GA}

The basic, traditional GA as presented here implements a genetic algorithm as described in [Michalewicz1996]. In the following this GA will be referred to as the Reference GA. It incorporates three basic concepts of the evolutionary search procedure.

Selection process: Individuals that represent solutions to the problem are evaluated according to a fitness function that decides about the quality of a solution. A solution's fitness is proportional to its chances to survive.

$1^{s t}$ Genetic Operator -- Crossover: A certain percentage of the individuals in the population are randomly chosen to mate and produce offspring. The children's genetic code is a recombination of the parents' genome. This introduces variation the necessary to make the evolutionary search process work.

$2^{\text {nd }}$ Genetic Operator -- Mutation: The second genetic operator randomly picks units of genetic information from the gene pool and modifies them. By this procedure the altered gene can adopt a state that is not yet present in the population, which again increases the level of variety among the solutions.

These three concepts are incorporated into the repetitive process which is described in the following. Initially, half the population of bit strings is created randomly. To increase variation, the other half starts out to be the complement of the bit representation of the first half. Now the first activation of the selection process determines those individual solutions that survive to form the second generation. The main idea behind the distinction of those individuals surviving the generational transition is the probability distribution based on the individuals' fitness values. In other words, the probability for an individual to be selected to participate in the next generation is proportional to its fitness as defined by the problem to solve. A metaphor Michalewicz uses for this process is that of spinning a roulette wheel, where each individual has a reserved slot. The width of that slot is directly proportional to its fitness, and the circumference of 
the entire roulette wheel equals the sum of all slots for the individuals in the population.

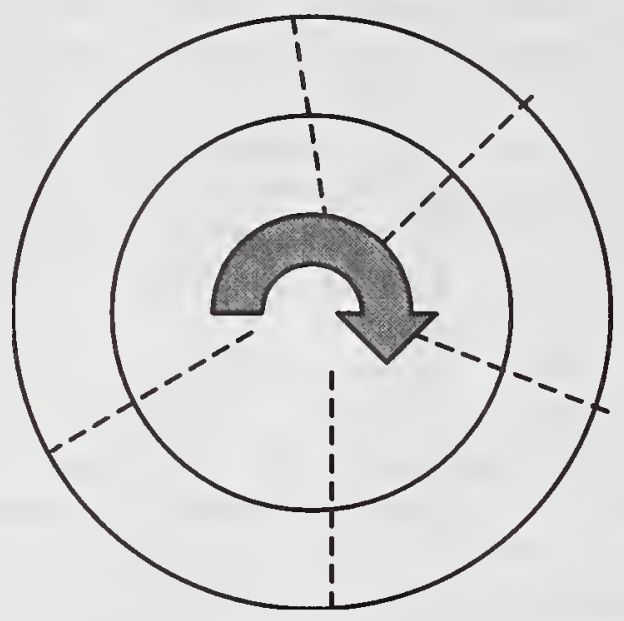

Figure 1: The selection roulette. Each slot delimited by dashed lines represents the fitness value for one individual solution. The different widths of the slots stand for the fact that there are fitter and less fit individuals in the population.

Maintaining a fixed number of solutions in the population is achieved by spinning the roulette wheel with one slot for each individual exactly as many times as there are individuals in the population. Since the slot size for an individual is sized according to the individual's fitness, the roulette ball is more likely to fall into the slot of a fitter individual. Note that it is possible (and even very likely in the case of a population which is composed of a few very fit solutions and many unfit solutions) that some of the individuals get chosen several times to take part in the next generation. The crossover operator simulates sexual reproduction. It is applied to the population, which results from the selection process. Initially, each individual gets assigned a random number between 0 and 1. All individuals whose number is smaller than a user-defined parameter for the GA, which determines the probability for a single individual to undergo crossover, will take part in the mating process. Here, solutions gather in pairs to perform the recombination of their genome. The method applied here is single-point crossover, which means that both parents switch their genetic code after a randomly chosen position in their bit string. Example: Two parents $p_{1}$ and $p_{j}$ are mated. Their genome strings are

$$
\begin{gathered}
p_{\iota}=\left(a_{1}, a_{2}, \ldots, a_{p o s}, a_{p o s+1}, \ldots, a_{N}\right), \\
p_{j}=\left(b_{1}, b_{2}, \ldots, b_{\text {pos }}, b_{\text {pos }+}, \ldots, b_{N}\right)
\end{gathered}
$$

After crossover at position pos the resulting individuals are

$$
\begin{gathered}
p_{\imath}=\left(a_{1}, a_{2}, \ldots, a_{p a s}, b_{p o s+1}, \ldots, b_{N}\right) \\
p_{j}=\left(b_{1}, b_{2}, \ldots, b_{p a s}, a_{p o s+}, \ldots, a_{N}\right)
\end{gathered}
$$

The children resulting from crossover will replace their parents in the population. The population effected by crossover is then rendered by mutation. Here another parameter determines the likelihood for one bit of genetic information in the gene pool to be flipped. This is done by randomly assigning a number to each bit in every individual, and then inverting its value if the random number for this bit is smaller than the mutation rate parameter.

One such sequence of selection, crossover, and mutation is called a generation. A limit is placed on the number of generations that the GA can try to evolve a good solution to the problem it is applied to. If criteria are know about the fitness of an optimal solution, then the evolutionary process is usually stopped as soon as such an individual is found.

\section{Implementation of Biological Aging}

In order to test the effect of aging, the Reference GA described in the previous section has to be adapted mainly in two ways. First of all, the representation of an individual needs to be extended to include information about its age (i.e., the number of times it has survived the selection process). Secondly, the term fitness has to be re-defined to encompass the idea to penalize old individuals that have not procreated. To serve the first purpose, each individual solution is represented as a pair. Its first element is the list of bits encoding the solution's genome. The second element contains the number of times this particular individual has been chosen to take part in the next generation.

Example: The dotted pair

$$
\text { ( (01111001).15) }
$$

represents a creature with the genome string $\left(\begin{array}{llll}0 & 1 & 1 & 1\end{array}\right.$ 1001 ) which has survived 15 generations.

Individuals created during the population initialization before the first generation, as well as the child solutions, which result from crossover, will start out having a zero in their age component. If one or more of an individual's genes was subject to mutation, then the individual's age component will also be reset to zero. This rejuvenation is done because a change of one gene only can often trigger an important allele, which is not yet part of the population's genetic pool. An old individual might become too valuable to justify penalizing it for its old age. 
Apart from the adaptation of the representation the selection process has to be changed to take the age of a solution into account. Aging is supposed to have a negative influence on the creature's fitness. Still, age does not play a role in the problem-specific, userdefined fitness function. The negative influence of age should happen in a way that is transparent for this external fitness. On the other hand, the impact of aging onto the process should also be quantifiable for the user. To ensure all these constraints, the influence of aging onto the fitness of an individual in the selection process is exerted in a special, internal fitness function. Assigning higher or lower values to the parameter AGE_FACTOR can regulate the actual importance of the aging.

\section{(define (InternalfF ind)}

let ( fitness

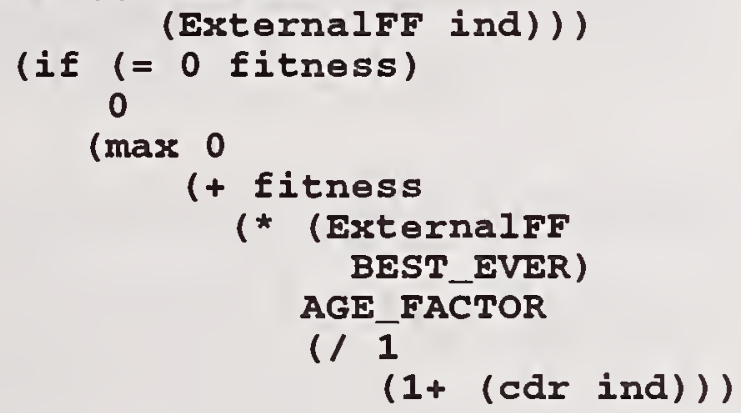

)...)

The internal fitness of a solution is a function of its external fitness which is increased by a portion of the external fitness value for the best solution found by the GA up to the time of the call. The degree of this increase is inversely proportional to an individual's age. However, an external fitness value of zero automatically effects the same internal fitness. The selection procedure, which decides about the survivors of a generation, is only based on the internal fitness. The Reference GA with the changes laid out in this section will be called Aging GA in the rest of this article.

\section{The 0/1 Knapsack Problem}

The first problem for which the differences between the Reference GA and the Aging GA are examined is the well-studied 0/1-KSP. It belongs to the class of NP-hard problems [Garey \& Johnson 1979], so it is reasonable to try and approach it with heuristics like a Genetic Algorithm. Imagine a traveler trying to fill his backpack. The task complicated by the fact that there are more objects to pack into the knapsack than it can carry. He has to decide which objects to take with him on the trip, and which objects to leave at home. In other words, he has to find the best packing for his rucksack, so that it does not break but that he still can transport the items that are most valuable to him. In the example instantiation of the KSP that both Genetic Algorithms try to solve there are $n=20$ Objects, and the capacity of the knapsack is $C=54$. The lists $\mathrm{W}$ and $\mathrm{V}$ store the weights and values of the objects that can be packed. One possible optimal packing with a value of 214 and a weight total of 54 would be to chose objects $2,3,4,7,8,10,12,13,14$, 17, 18, and 20. Table 1 lists the objects and their weights.

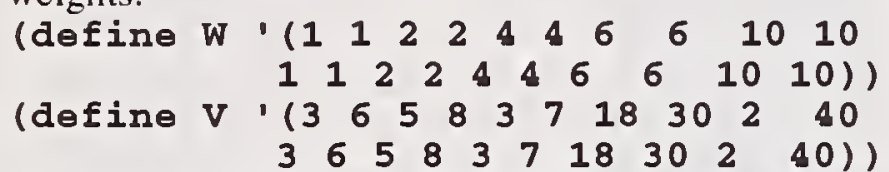

The individual solutions (i.e. the knapsack packings) are represented by lists in which each element represents one component of the vector $x$. A 1 in the $x[i]$ component of vector $x$ therefore indicates that object number $i$ is part of the particular packing, whereas a 0 means that it is not in the knapsack.

Example: The representation of a solution, that suggests packing objects 4,6 ,

$8-11,16$, and 19 , would be the following list: (00010101111000010010)

By looking up each activated objects weight and value, one can determine that the solution's weight sum is 47 , and its values add up to 99 .

The genetic algorithm creates an initial population of forty random solutions like the one in the example above. Here, as well as through the entire evolutionary process the GA can generate solutions whose weight sum of the packed objects exceeds the capacity of the knapsack. In the following these solutions are referred to as invalid (or infeasible) because they violate the constraint imposed onto a solution to be considered feasible. The decision was made to lift the weight constraint from the solutions, and thereby transform the constrained KSP into an unconstrained problem. Still In order to favor valid packings invalid solutions are penalized in proportion to their degree of invalidity. This was achieved by decreasing the individual's fitness according to its excess weight. The penalty calculated by the function

$\operatorname{Pen}(x)=c_{1} \cdot\left(\sum_{i=1}^{n} x[i] \cdot W[i]-C\right), c_{1}=\frac{3 \cdot \sum_{i=1}^{n} V[i]}{C}$

The value of this function increases with the individuals excessive weight. The entire fitness function can be stated as

Fitness $(x)=\left(\sum_{i=1}^{n} V[i] \cdot \sum_{i=1}^{n} W[i]\right)-\sum_{i=1}^{n}(V[i]-x[i] \cdot V[i])-\operatorname{Pen}(x)$

Both GAs were extensively tested for this problem. 840 runs of 200 generations each were conducted to determine the average and mean values for both GAs' weight and value sum. The results are shown in Table 2. 
Table 2: Results for the KSP

\begin{tabular}{|l|l|l|}
\hline & Reference GA & Aging GA 9.06 \\
\hline W Average & 52.075 & 52.274 \\
\hline W Mean & 52.119 & 52.226 \\
\hline Std. Dev. & 0.337 & 0.278 \\
\hline Optimality & $96.4 \%$ & $96.8 \%$ \\
\hline V Average & 188.979 & 190.261 \\
\hline V Mean & 189.036 & 190.311 \\
\hline Std. Dev. & 2.240 & 1.456 \\
\hline Optimality & $87.9 \%$ & $88.5 \%$ \\
\hline
\end{tabular}

The level of optimality in this table compares the average values of both GAs to the perfect solution, which uses the full capacity of the backpack to take item at a value total of 215 unit. Interpreting the results one can say that both the average weight as well as the average value sum of the packings produced by the Aging GA are closer to the optimal value. Even if the improvement is within the range of the standard deviation around the average of the Reference GA values, the results clearly show that aging can have a positive effect on the results of the KSP. The impact of aging is very sensible to changes of the AGE_FACTOR parameter for the internal fitness function. Although the value 9.06 was the best that the authors found during their test phase, an exhaustive search for the best parameter value was not attempted.

\section{The Area Surveillance Problem}

The setting for second problem is a 2-dimensional plane, in which parts are of special interest. These parts are later referred to as the surveillance area. A surveillance area consists of a set of different geometric objects. The types of these objects are circles, half planes, annuli (i.e. the intersection of one circle with the complement of another concentric circle), convex polygons, pies, and area segments. In addition to the surveillance area, there are cover objects. Their shapes can be chosen from the same range of types as those of the surveillance area. Cover objects are meant to cover a maximal part of the surveillance area. All objects (surveillance area as well as cover objects) have a permanent location. Once assigned a position in the plane during the problem description phase, they do not change it in the course of the problem solution. Another feature of the cover objects is that operating them bears certain costs. These costs are mainly influenced by the amount of surveillance area they cover, and by their position in the plane. Cover objects can be in one of two states: either they are engaged (switched on), or they are disengaged (switched off). Each cover object harbors a distinct part of the surveillance area once it is engaged. Cover objects can intersect. All objects (surveillance area as well as cover objects) are represented as a set of points in the plane contained by the object. The number of points in an object is proportional to the resolution used to describe the plane.

The goal is to maximize the percentage of surveillance area covered by cover objects, and at the same time to minimize the costs resulting from switching on a cover object. Depending on the specific situation, a trade-off has to be found between those two goals. The exact preferences of this tradeoff are defined by the user. The ASP problem can easily be reduced to the vertex cover problem[6]. This shows that it is also NP-hard.

In the ASP considered here, a set of 15 cover objects try to cover an area of 2 polygons and 2 annuli. Table 3 shows the objects and their activation costs, while Figure 2 and 3 sketch the surveillance area's and the cover objects' location in the plane.

\section{Figure 2 : The area to be surveyed}

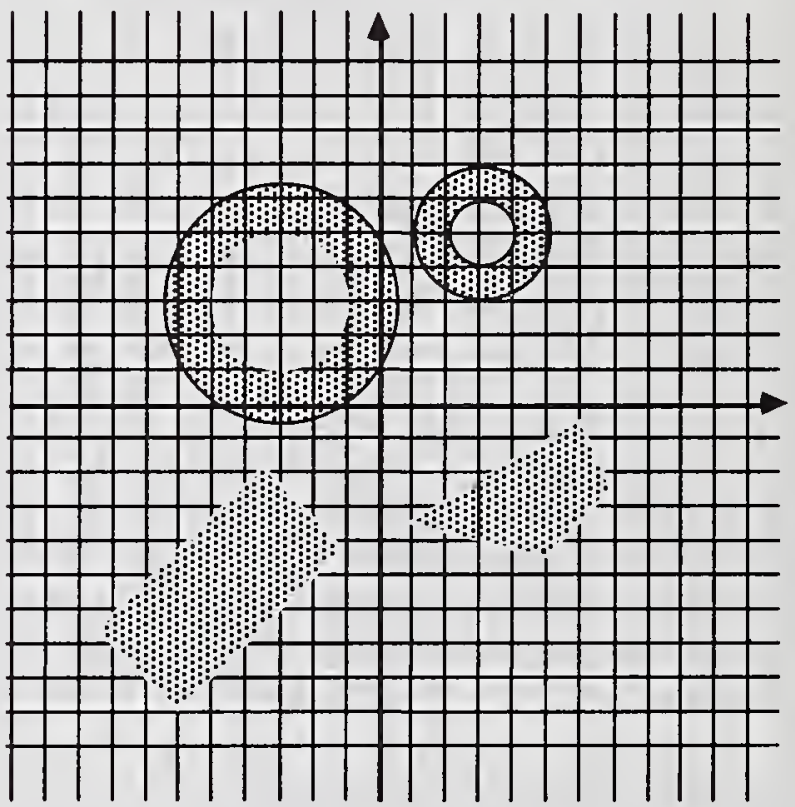

Table 3 : Cover objects and their cost factors for the ASP

\begin{tabular}{|l|l|l|l|l|l|l|l|l|}
\hline Object & 1 & 2 & 3 & 4 & 5 & 6 & 7 \\
\hline Costs & .50 & .45 & .40 & .15 & .35 & .32 & .10 \\
\hline 8 & 9 & 10 & 11 & 12 & 13 & 14 & 15 \\
\hline .20 & .25 & .22 & .22 & .28 & .18 & .18 & .15 \\
\hline
\end{tabular}


Figure 3: The cover objects

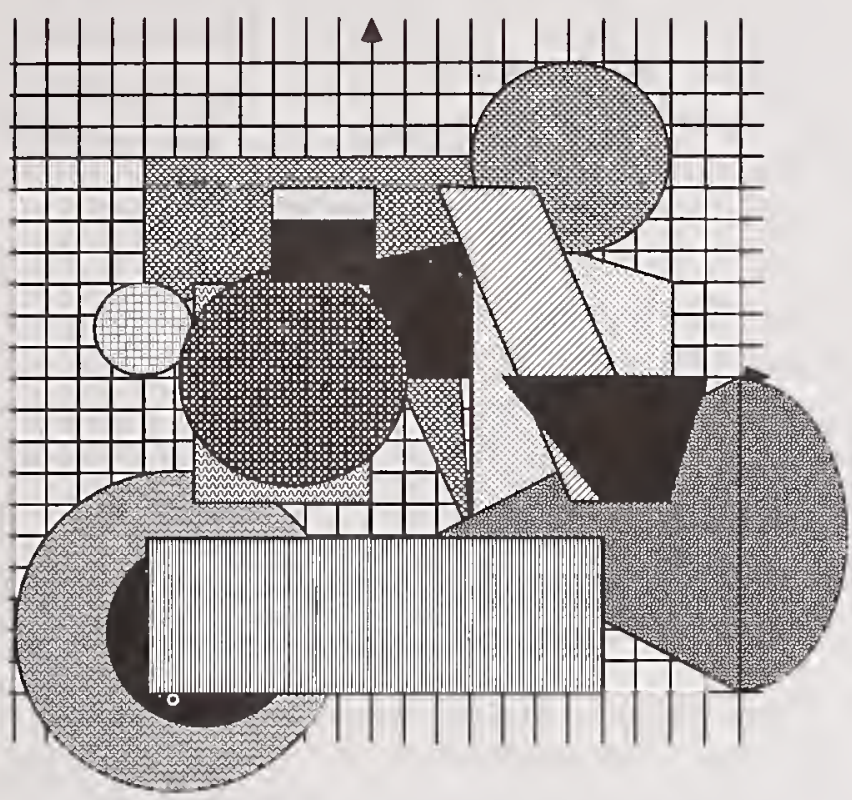

A solution to the problem of surveying an area is represented as a list of 15 bits. A 0 in position $m$ of the solution means that the cover object $m$ is disengaged, whereas a 1 would mean that it is switched on.

Example: The solution list (0 10000111000000000 010 ) would mean that the surveillance area is only covered by the cover objects number $2,5,6$, and 15 . Such a solution list also functions as one individual in the Genetic Algorithm.

For the ASP both the Reference GA and the Aging GA made 400 runs of 15 generations. The population size was set to 60 solutions. Table 4 presents the results.

Table 4: Results for the ASP

\begin{tabular}{|l|l|l|}
\hline & Reference GA & AgingGA -1.22 \\
\hline Average & 126.791 & 127.972 \\
\hline Mean & 126.547 & 128.037 \\
\hline Std. Dev. & 1.0226 & 1.873 \\
\hline Optimality & $93.6 \%$ & $94.4 \%$ \\
\hline
\end{tabular}

Very much like before, the average and mean fitnesses of the Aging GA reach a value which is closer to the fitness value of the best possible solution. However, both GAs had enough time to evolve close-to-optimal solutions. A particularity that can be observed here is that the aging factor has a negative algebraic sign. This can be interpreted as support for older individuals in the selection process. The optimal solution that both GA average fitnesses were compared to had a fitness value of 135.5 .

\section{The Scheduling Problem}

The Scheduling Problem (i.e. SCP) is also a member of the category of NP-hard problems. The particular problem addressed herein may be described as follows. For a small school with four teachers and four rooms a timetable for 20 courses has to be created by a GA, so that each of the courses is scheduled in one of 8 possible time slots. The timetable produced has to comply with certain constraints. A constraint violation leads to a penalization for the schedule's fitness. The smallest penalty is placed on assignments which violate constraints between courses. This happens if two courses are scheduled in the same time slot although they should not be held in parallel (e.g., because students ought to be enrolled in both classes in the same semester). A medium penalty is imposed on professor constraint violations. Such a penalty is given to timetables which schedule a professor to teach two courses at the same time. The hardest penalty affects timetables that schedule two different courses at the same time and in the same room. An individual schedule consists of 20 genes with 7 bit each. The first 2 bits of each gene represents one of the 4 rooms in which the courses can take place. The next 3 bits determine the time slot (out of 8 possible slots) during which the course is scheduled. The third element assigns one of the 4 professors to the course. One gene is also referred to as a schedule item.

The fitness function chosen for this problem processes three matrixes in order to keep track of constraint violations. The first matrix statically stores all conflicts between courses. The second and third matrix are dynamically updated during the process of a schedule evaluation. One of the two serves to survey the courses that take place in a particular room at a certain timeslot. The other one stores for each possible pair of teacher and timeslot if a professor is already assigned to teach a course at a certain time. The fitness function now examines each single schedule item in the following manner. If a room has already been assigned to another course during the same period of time, then the fitness function assigns a fitness value to this item that is only $3.3 \%$ of what a conflict-free schedule item would have scored. Otherwise the function tests the schedule item for course conflicts and professor conflicts. In case of a course conflict, the schedule item scores $10 \%$ of a conflict-free item, while a professor conflict results in $5 \%$ of the optimal score per item. Table 5 shows a conflict-free schedule that is in keeping with the course conflict matrix that was chosen for the test runs. The columns represent the four rooms $\mathrm{R} 1$ through $\mathrm{R} 4$, while the rows show the eight timeslots $\mathrm{S} 1$ through $\mathrm{S} 8$. Each entry $\mathrm{Cn} / \mathrm{Pm}$ in cell $(R j, S k)$ stands for the scheduling of course $C n$ in room $R j$ at slot $S k$, and assignment of professor $P m$ so teach the course. 
Table 5 : A perfect schedule without constraint violations

\begin{tabular}{|l|l|l|l|l|} 
& $\mathrm{R} 1$ & $\mathrm{R} 2$ & $\mathrm{R} 3$ & $\mathrm{R} 4$ \\
\hline S1 & $\mathrm{C} 1 / \mathrm{P} 1$ & $\mathrm{C} 6 / \mathrm{P} 2$ & $\mathrm{C} 11 / \mathrm{P} 3$ & $\mathrm{C} 16 / \mathrm{P} 4$ \\
\hline S2 & $\mathrm{C} 2 / \mathrm{P} 1$ & $\mathrm{C} 7 / \mathrm{P} 2$ & $\mathrm{C} 12 / \mathrm{P} 3$ & $\mathrm{C} 17 / \mathrm{P} 4$ \\
\hline S3 & $\mathrm{C} 3 / \mathrm{P} 1$ & $\mathrm{C} 8 / \mathrm{P} 2$ & $\mathrm{C} 13 / \mathrm{P} 3$ & $\mathrm{C} 18 / \mathrm{P} 4$ \\
\hline S4 & $\mathrm{C} 4 / \mathrm{P} 1$ & $\mathrm{C} 9 / \mathrm{P} 2$ & $\mathrm{C} 14 / \mathrm{P} 3$ & $\mathrm{C} 19 / \mathrm{P} 4$ \\
\hline S5 & $\mathrm{C} 5 / \mathrm{P} 1$ & $\mathrm{C} 10 / \mathrm{P} 2$ & & $\mathrm{C} 20 / \mathrm{P} 4$ \\
\hline S6 & & & $\mathrm{C} 15 / \mathrm{P} 3$ & \\
\hline S7 & & & & \\
\hline S8 & & & & \\
\hline
\end{tabular}

The SCP was tested in 840 runs of 300 generations each. The number of schedules in the population was 30. Table 6 exhibits greater differences than notable for the previous two problems

\section{Table 6: Results for the SCP}

\begin{tabular}{|l|l|l|}
\hline & Reference GA & Aging GA -0.56 \\
\hline Average & 152.524 & 172.687 \\
\hline Mean & 152.579 & 172.558 \\
\hline Std. Dev. & 0.971 & 1.1604 \\
\hline Optimality & $76.3 \%$ & $86.3 \%$ \\
\hline
\end{tabular}

The average result of the Aging GA is $10 \%$ closer than the Reference GA to creating a schedule that complies with all constraints. Here as before it is important to mention that this drastic improvement was only perceived for the exact value of -0.56 for the influence of aging. Other positive and negative values for aging exhibited worse results which mostly even scored below the Reference GA's average fitness.

\section{The Prisoners' Dilemma Problem}

The last problem that is used to show the importance of aging in the field of Genetic Algorithms is quite exceptional. The goal here is not so much to solve a computationally expensive problem than rather to learn more about the emergence of cooperative behavior among selfish individuals. The PDP comes from the field of game theory. Initially, the PDP was formulated as a two-person game in which two players, who have committed a crime together, were held in separate cells without any means of communication. Both players $\mathrm{A}$ and $\mathrm{B}$ are offered the same deal: if one defects and betrays the other one, whereas if the other player does not testify, then the defectant will not be imprisoned while the cooperative player will serve a 5 year sentence. If both players defect then their testimony will be discredited and they will both be put away for 4 years. However, in the case that both players do not testify against each other, they will only serve a 2- year sentence. A game consists of two moves, one by each player. The players independently decide which move to make, namely either to defect (D) or to cooperate with the other player (C). The following table describes the pay-off function for each player. The number in the pay-off columns indicates by how many years a player shortens his time in prison.

Table 7: Pay-off in years saved for Player $A$ and Player $B$ in the PDP

\begin{tabular}{|l|l|c|c|}
\hline Player A & Player B & Pay-off A & Pay-off B \\
\hline Defects & Defects & 1 & 1 \\
Defects & Cooperates & 5 & 0 \\
Cooperates & Defects & 0 & 5 \\
Cooperates & Cooperates & 3 & 3 \\
\hline
\end{tabular}

In this article we examine the case where the game is iterated several times. If in a repeated game both players always defect, they will get a by far lower total pay-off than in the case of mutual cooperation. Each player will have a memory of the last 3 moves. Based on the experience of these 3 moves a decision is made if the player's next move will be $\mathrm{D}$ or $\mathrm{C}$. To represent a strategy that decides on what to do in the next move based on the history of the previous 3 games, we follow Axelrod's approach] Details about the strategy representation can be found in [1].

To determine the fitness of Shis strategy, each player plays against every other player 10 times. For the population size of 20 chosen here this means 200 fights per individual and generation. The fitness value that is assigned to a strategy is the average total score that it can achieve in each of the 20 one-on-one encounters with another strategy. During 840 runs of 100 generations each, and with a population size of 20 individuals the following results were recorded.

Table 8: Results for the PDP

\begin{tabular}{|l|l|l|}
\hline & Reference GA & Aging GA 13.5 \\
\hline Average & 30.363 & 31.933 \\
\hline Mean & 30.433 & 32.015 \\
\hline Std. Dev. & 0.570 & 0.809 \\
\hline Optimality & $60.7 \%$ & $63.9 \%$ \\
\hline
\end{tabular}

The values for the standard deviations around the average fitnesses for both GAs justify the statement that the improvement achieved by the Aging GA is significant. The optimality measure applied here compares the average result of each GA to a theoretically prefect strategy that can always score the perfect pay-off of 5 years. Taking this into consideration, an increase of $3.2 \%$ in optimality of average fitness appears even more significant. 


\section{Conclusion}

This research introduced the concept of biological aging to the domain of traditional Genetic Algorithms. The effects of aging upon solving four well-examined optimization problems have been proven significant. Further research in this field has to be done to develop reliable theories about the relation between the problem domain and the degree of aging that should be applied. A rule of thumb for this appears to be that moderate values, which do not override the effect of the actual, external fitness function, are most appropriate. However, no signs of linearity between the impact of aging and the results of the optimization process were detectable. This suggests the application of a Meta-GA onto the problem of finding the best aging influence factor for a task.

\section{References}

[1] Axelrod, R. 1987. Genetic Algorithm for the Prisoner Dilemma Problem. In Davis (ed.), Genetic Algorithms and Simulated Annealing, Morgan Kaufmann Publishers, pp. 32-41.

[2] Baeck, T., Hoffmeister, F., Schwefel, H.-P. 1991. A survey of evolutionary strategies. In Belew, Booker (eds.) Proceedings of the Fourth International Conference on Genetic Algorithms. Morgan Kauffman, pp. 2-9.

[3] Bell, G. 1984. Evolutionary and nonevolutionary theories of senescence. American Naturalist, 124, pp. 600-603

[4] Darwin, C. 1859. The Origin of Species. London. John Murray. (Penguin Classics, London, 1985; New American Library, Mentor Paperback)

[5]Dawkins, C. 1976. The Selfish Gene. Oxford University Press, Oxford

[6]Garey, M.R., Johnson, D.S. 1979. Computers and Intractability: a Guide to the Theory of NPCompleteness, Freeman, San Francisco, CA.

[15]Holland, J.H. 1975. Adaptation in natural and artificial systems. Ann Arbor, MI: The University of Michigan Press.

[7]Malthus, T. R. 1803. An essay on the principle of population, or, A view of its past and present effects on human happiness: with an inquiry into our prospects respecting the future removal or mitigation of the evils it occasions. New edition 1992: Cambridge University Press, Cambridge.
[8]Martello, S., Toth, P. 1990. Knapsack Problems, John Wiley, Chichester, UK.

[9]Michalewicz, Z. 1992/1994. Genetic algorithms + Data Structures = Evolution Programs, SpringerVerlag, 175 5th Avenue, New York, NY,10010, (212) 460-1500.

[10]Medawar, P. 1952. An Unresolved Problem in Biology. H. K. Lewis, London.

[12]Ray, T. S. 1992. An Approach to the Synthesis of Life. In (Langton, Taylor, Farmer \& Rasmussen 1992), pp. 371-408

[16] Syswerda, G. 1989. Uniform Crossover in genetic algorithms, in J.D. Schaffer(ed.), Proceedings of the Third International Conference on Genetic Algorithms, Morgan-Kaufmann, pp. $2-9$

[13]Todd, P. M. 1993. Artificial death. Proceedings of the Second European Conference on Artificial Life (ECAL93), Vol. 2, pp. 1048-1059. Brussels, Belgium: Universite Libre de Bruxelles

[14] Wynne-Edwards, V. C. 1986. Evolution through group selection. Blackwell Scientific Publications, Oxford. 

X AUTONOMY 


\title{
Adaptive Autonomy: The Key to Dynamic, Responsive Formation of Sensible Agent Organizations*
}

\author{
K. Suzanne Barber \\ The Laboratory for Intelligent Processes and Systems \\ Electrical and Computer Engineering Department \\ The University of Texas \\ Austin, TX 787812
}

\section{ABSTRACT}

The practical deployment of distributed agent-based systems mandates that each agent behave sensibly. This paper focuses on the development of flexible, responsive, adaptive systems based on Sensible Agents. Sensible Agents perceive, process, and respond based on an understanding of both local and system goals. Each agent is capable of (1) deliberative or reactive planning and execution of one or more domain-specific service /task, (2) maintaining and interpreting knowledge about states, events, and goals related to itself, other agents, and the environment, and (3) adapting its behavior according to its understanding of its own local goals and overall system goals. This paper addresses the above issues in the context of applied semiotics, a field that analyzes and develops the formal tools of knowledge acquisition, representation, organization, generation, enhancement, communication, and utilization. A Sensible Agent architecture has been developed where each agent is composed of five modules: a Self-Agent Modeler, an External Agent Modeler, an Action Planner, an Autonomy Reasoner, and a Conflict Resolution Advisor.

Keywords: autonomous agents, dynamic organizations, adaptive systems, planning

\section{RESEARCH MOTIVATION}

There are two critical aspects to the old maxim, "It was the best decision that could be made under the circumstances." "Best decision" and "circumstances" reflect both the quality of the decision and the confining constraints. If decisions were unconstrained, the best decision would depend solely on relationships between the decision variables and a suitable measure of decision quality. Consideration of the circumstances reflects a more realistic view for decision-makers. The state of the world or environment in which the problem presents itself acts as a set of constraints restricting the application or acquisition of knowledge.

Research investigations have explored the decisionmaking process and attempted to automated portions of it. A large body of work has framed this problem as follows: Given a goal, how should the system search through a set of solutions (a solution space) to arrive at the optimal goal state given a relatively static picture of the world? In other words, what is the best decision or plan if the goal and constraints guiding the search for that goal remain constant? Underlying the premise of a constrained solution space, a limited set of communications and controls is allowed for derivation of feasible plan sets.

The true complexity of many problem domains (e.g. military command and control) is reflected by the following facts which make the above assumptions impractical for dynamic applications:

- Goals are not static.

- Multiple types of goals exist.

- Goals and plans to achieve those goals can conflict with one another.

- Circumstances change often and unpredictably.

Therefore, consideration of the decision-making as a process by which some entity (human or automated) searches through a set of possible solutions, known apriori, guided only by static constraints to arrive at its goals is just scratching the surface of the real problem. To address the true complexity of dynamic and unpredictable situations, it is important to understand that making the "best decision under the circumstances" involves not only intellect but the ability to be innovative (make changes), flexible (adjust to change) and responsive (react or execute readily).

\footnotetext{
This research is supported in part by the Texas Higher Education Coordinating Board $\# 003658452$ and the Applied Research Laboratories and Office of Naval Research Grant N00014-96-1-0298.
} 
Decisions rarely occur in isolation. A decision-maker must not only assess its own behaviors (its states and the events it is capable of processing) but also the behaviors of others possessing the ability to impact its circumstances. Additionally, the decision-maker must recognize the impact or necessity of interacting with others to develop and execute plans. These interactions and behaviors impact decisions, often acting as "the circumstances" driving a need to make a decision. Therefore, intellect or knowledge as well as innovation, flexibility, and responsiveness is often derived from a decision-maker's ability to dynamically 1) assess current and potential roles others play in interactions and 2) establish beneficial roles in these interactions. As situations change, the interactions supporting the decision-making process must also change. Simply put, the decision-maker must not only be smart but capable of applying those "smarts," deciding who to work with, if anyone, in an appropriate fashion at the right time. To address these issues, dynamic configuration of organizations is a must.

Organizations of decision makers must be capable of configuring themselves given dynamic constraints. For a member of the organization, these constraints include the state of the decision-maker, events triggering responses, states and events observed from the organization members and the environment, the decision maker's own goals and the goals of the system in which they are a member. The research presented here is driven by these motivations for dynamic organizations and the requirements for underlying theory and technology required to render this capability.

\section{RESEARCH OVERVIEW}

The practical deployment of distributed agent-based systems in dynamic, complex environments mandates that each agent behave sensibly, incorporating an understanding of both global system goals and its own local goals. Sensible Agents, capable of Dynamic Adaptive Autonomy, address many of the challenges encountered by multiagent systems. A Sensible Agent (1) maintains a representation of both behavioral knowledge and declarative knowledge of itself and other system agents, (2) develops and processes an understanding of both local goals and system goals, and (3) performs either deliberative or reactive actions with respects to its own internal events or external events from other system agents or the environment.

A critical consideration for this behavior is the agent's level of autonomy. The term level of autonomy refers to the types of roles an agent plays in its planning interactions with other agents. Specifically, this research seeks to prove the following hypothesis: The operational level of agent autonomy is key to an agent's ability to respond to dynamic situational context, (i.e. the states, events, and goals that exist in a multiagent system), conflicting goals, and constraints on behavior. Levels of autonomy are defined along a spectrum. (1) Command driven -- the agent does not plan and must obey orders given by another agent, (2) Consensus the agent works as a team member to devise plans, (3) Locally Autonomous -- the agent plans alone, unconstrained by other agents and, (4) Master -- the agent devises plans for itself and its followers who are command-driven. These conceptual autonomy levels are tied to the responsibility of an agent to plan for solving its goals.

Sensible Agents maximize the innovation, flexibility and responsiveness of multiagent planning systems operating under dynamic and constrained military situations. Consequently, the Sensible Agent technology will increase the ability of agents to coordinate the following:

- planning activities driven by an understanding of both local and system goals as well as the behaviors of other system agents and the environment.

- the roles individual planning agents play in interactions with other agents by allowing dynamic assignment of autonomy (i.e. dynamic assignment of responsibility, commitment, authority and independence) with respect to each goal held by a respective agent.

\section{Related WORK}

Several researchers have addressed the development of adaptive, intelligent, agent-based systems. These efforts range from representing coordination and collaboration [1], to production rule-based systems for organizational self-design [2], to partial global planning [3], to the development of multiagent planning architectures [4], and many others. The majority of previous work has focused on providing architectures with a constrained set of control schemes for agent interaction. In other words, one organization of decision-makers is developed for a problem domain. Although some new architectures provide the mechanisms through which different organizations can be realized, these architectures do not support the reasoning processes necessary to implement self-reconfiguration by the agents themselves. Therefore, a significant portion of this work makes an a-priori assignment of autonomy to each agent in the 
system. This Sensible Agent architecture and underlying technology pushes this envelope in an effort to provide dynamic configuration of organizations allowing adaptive control of agent interaction to maximize their intellect and minimize the limitations imposed by ever-changing constraints (e.g. circumstances).

This research advances previous work in the field, realizing that one specific level of autonomy may not be appropriate or even achievable at all times and under all conditions. Investigations of related research have not identified a comprehensive method for agents to employ to select autonomy levels given dynamic situations. Adaptive autonomy, the ability to transition autonomy levels during system operation, provide agents the ability to dynamically choose the most appropriate scheme of behavior and interaction. This research seeks to provide agents with the capability to reason about autonomy. This research effort does not focus on improving the problem-solving methods available at particular autonomy levels. Many ongoing research efforts focus on optimizing the planning process. Sensible Agents make use of existing problemsolving methods that best fit their particular tasks. To accomplish this, agents must know how to plan at each of the possible levels of autonomy. Previous and ongoing research provides inroads to these capabilities. The command driven / master agent relationship employs traditional centralized planning or distributed centers of planning control [5][6]. Planning under the influence of a master agent has also been examined specifically[7]. On the other hand, agents developing plans for consensus must negotiate to reach an agreement by which all goals are satisfied. The contract-net protocol and its extensions offer agents this capability [8]. Additional negotiation mechanisms [9][10] provide additional support for agent interactions at the consensus level of autonomy.

Locally autonomous agents, which plan independently but act as part of a larger system, exhibit the most diverse behavior. They may be fully cooperative and act under a functionally accurate, cooperative distributed system (FA/C) [11][12][13]. Alternatively, they may choose to act more selfishly. Durfee and Lesser's [3] method of communicating partial global plans allows agents to interact within a system in many different ways. However, they leave the method of choosing an interaction style open. Locally autonomous agents are unique in that they may cooperate to solve a system goal without communicating. This function can be supported by Tenney and Sandell's work on coordination [14][15]. If the autonomy level of agents in a system is allowed to change, the effective organizational structure of the system also changes. For this reason, it is important to understand the effect of different organizational structures. Previous work provides this insight in the form of formal studies of large-scale dynamic systems [14] and investigations of dynamic creation and destruction of agents [16]. The mechanics of changing organizational structure is also an important area of study. Several researchers have investigated how agents can form problem-solving groups [17][1]. In addition, centralized, distributed, and group approaches have been compared [18][19]. These studies provide important insight for autonomy reasoning. Gasser and Ishida [2] also provide an understanding of both adaptive self-configuration (through Organization Self-Design) and tradeoffs corresponding to different organizational structures.

\section{RESEARCH APPROACH}

The Sensible Agent architecture (Figure 1) permits dynamic adaptation of agent autonomy. One agent is highlighted and referred to as the self-agent in the discussions below.

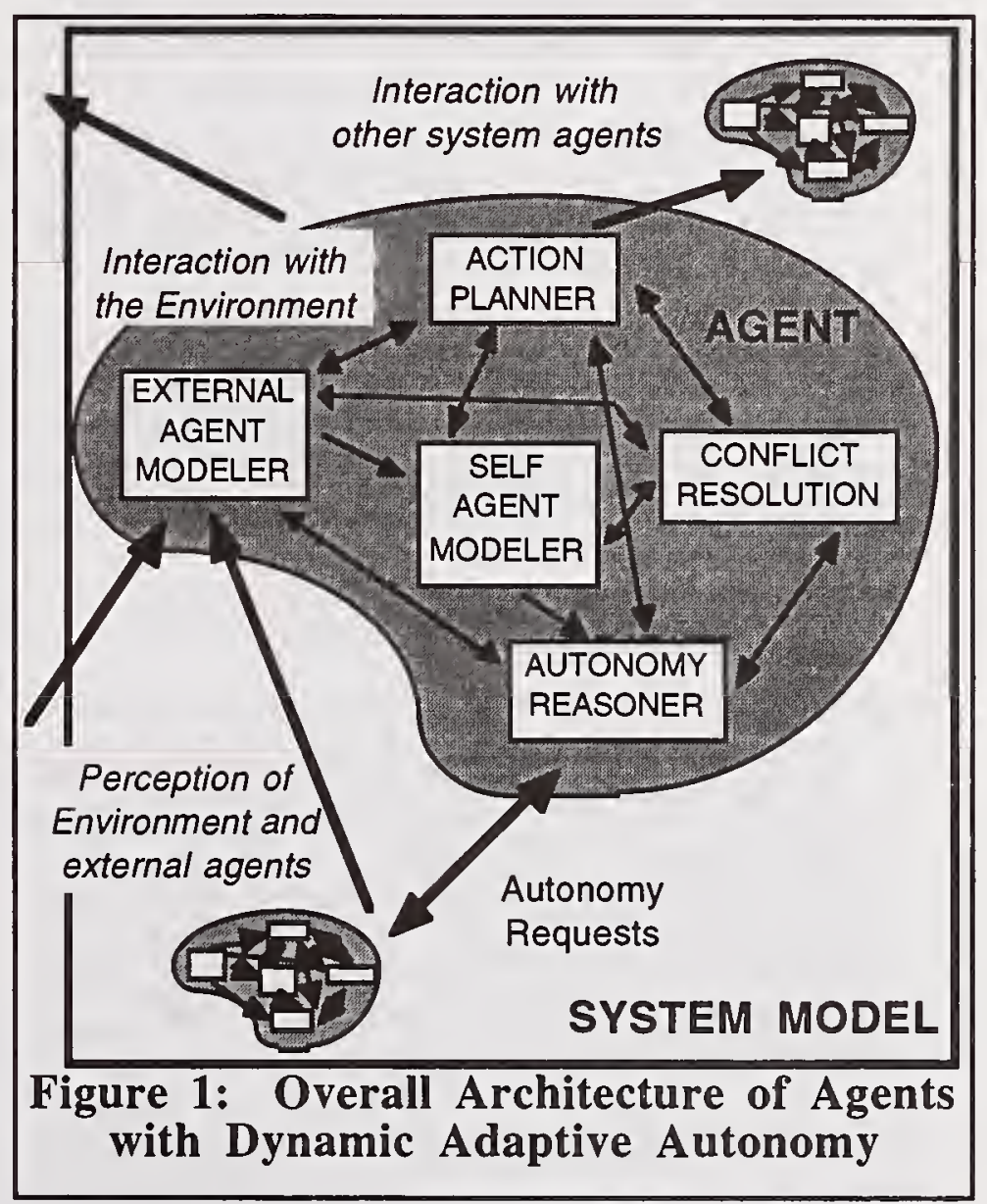

A Sensible Agent's behaviors are described by its internal states, events and goals. A Sensible Agent's 
understanding of the external world is built from interpretations of the states, events, and goals of other agents and the environment. Each agent is (1) responsible for one or more domain-specific service/tasks, (2) responsible for the maintenance of its internal states, events, and goal structure, (3) capable of interpreting external states, events, and goals, (4) capable of detecting and resolving conflicts, and (5) capable of assigning and adapting an autonomy level for each goal in the agent's goal structure. Each agent consists of five major modules.

- The Self-Agent Modeler contains the behavioral model of the agent. This module interpret internals or external events acting on the agent and change its state accordingly [20][21][22][23]. Other modules (within the self-agent) can access this model for necessary state information.

- The External Agent Modeler contains knowledge about other agents and the environment. This module maintains beliefs about states and events external to the agent and predict the actions of other agents [20][21][22][23][24]. Other modules within the selfagent can monitor this model for changes external to the self agent that affect their reasoning processes.

- The Action Planner solves domain problems, store agent goals, and execute problem solutions. This module interacts with the environment and other agents in its system. It also carries out solutions for conflict resolution. Communication between agents is handled by this module. The Action Planner draws information and guidance from all other modules.

- The Conflict Resolution Advisor module identifies, classifies, and generates possible solutions for conflicts occurring between the self-agent and other agents [25][26]. This module monitors the action planner, self-agent modeler, and external agent modeler to identify conflicts. It then offers suggestions to the action planner or Autonomy Reasoner in order to resolve the conflict.

- The Autonomy Reasoner determines the appropriate autonomy level for each goal, assigns an autonomy level to each goal, and reports autonomy-level constraints to other modules in the self-agent. It also handles all autonomy level transition and requests for transition made by other agents. The autonomy reasoner contains utility-based assessment of autonomy levels. The autonomy reasoner draws information from all other modules.

All modules within a Sensible Agent are domain independent except for the action planner. Specifically, these modules contain domain independent representations and reasoning mechanisms. Instantiations (i.e. knowledge instantiated in a particular representation) are, of course, domain dependent. For example, domain independent behavioral representations in the self agent models specify how states, events, and transitions are represented. The actual instantiations of states, events, and goals and their respective relationships are, of course, domain dependent. Analogously, reasoning mechanisms for detecting and resolving conflicts are domain independent while the actual execution utilizes knowledge instantiations (e.g. goal priorities) which are very much domain dependent.

\section{Organization Formation: Autonomy} Representation and Reasoning The autonomy spectrum (i.e. command-driven, consensus, local and master) conveys the intent and scope of each autonomy level. However, these intentions must be formalized and modeled computationally in order to implement Dynamic Adaptive Autonomy (DAA) in agent-based systems. Autonomy levels are represented by four autonomy constructs: Responsibility (R): a measure of how much the agent must plan to see a goal solved; Commitment (c): a measure of the extent to which a goal must be solved; Authority (A): a measure of the agent's ability to access system resources; Independence(i): a measure of how freely the agent can plan.

The autonomy level, AL, is a 4-tuple ( $R, c, A, i)$. These four autonomy constructs provide the foundation for a complete computational model of an agent's autonomy level. The autonomy constructs can be used in numerous ways to reason about agent autonomy, guide agent behavior, and facilitate system interactions and formation of problem-solving groups. It is these four constructs that the Autonomy Reasoner manipulates to perform autonomy level assignments and transitions.

A representation specifying how the different autonomy constructs affect utility (i.e. costs and benefits) follows. Utilities for a proposed autonomy level allow determination of the feasibility for the agent to assume the proposed autonomy level with respect to the goal. An agent assigns two utility values $\left(U_{\text {agent }}, U_{\text {system }}\right)$ to each goal, where $U_{\text {agent }}$ represents the goal's value to the agent itself, and $U_{\text {system }}$ represents the goal's value to the agent's overall system.

Let $A^{i}$ denote the set of agents in the system. Let $\mathbf{g}_{j}^{i}$, denote the $j^{\text {th }}$ goal of the agent $A^{i}$. The autonomy level of goal $\mathrm{g}_{\mathrm{j}}^{\mathrm{i}}$ is represented by the 4-tuple ( $\left.\mathrm{R}, \mathrm{c}, \mathrm{A}, \mathrm{i}\right)$. Let $f_{\mathrm{c}}$ be a function that returns a tuple $\left(f_{\mathrm{c}, \mathrm{agent}}, f_{\mathrm{c}, \mathrm{system}}\right)_{\mathrm{j}}$ using the commitment index, $\mathrm{c}$, for a specific goal, $\mathbf{g}_{\mathrm{j}}^{\mathbf{i}}$, where $f_{c, a g e n t}$ and $f_{c, \text { system }}$ denote the impact of the 
commitment index on the agent and the system, respectively. Similarly, we define $f_{\mathrm{R}}$ and $f_{\mathrm{i}}$ that denote the impact of the responsibility distribution and the independence index, respectively. Let $f_{\mathrm{A}}$ denote the cost of accessing resources used by the agent to fulfill goal $\mathrm{g}_{\mathrm{j}}^{\mathrm{i}}{ }^{1}$. It is important to note that the value of $f_{\mathrm{c}, \text { system }}$ (and the other functions) is not necessarily consistent among agents, differences may appear due to the perceptions each agent has about the system.

Let $F_{c}^{i}$ denote $\left(F_{c, a g e n t}, F_{c, \text { system }}\right)$ for a given agent $A^{i}$. $F_{c, a g e n t}$ and $F_{c, s y s t e m}$ is computationally derived from all the individual values of $f_{c \text {,agent }}$ and $f_{c, \text { system }}$ from the current goals of the agent. Similar values are derived for $F_{R}^{i}, F_{A}^{i}$, and $F_{i}^{i}$. In this research, utility represents the degree of benefit that an agent's goals provide an agent. The utility of all an agent's goals to the agent will therefore be given by:

- $\mathrm{U}_{\text {agent }} \propto h_{l}\left(\mathrm{~F}_{\mathrm{c}, \text { agent }}, \mathrm{F}_{\mathrm{R}, \text { agent }}, \mathrm{F}_{\mathrm{A}, \text { agent }}, \mathrm{F}_{\mathrm{i}, \text { agent }}\right)(\mathrm{Eq} 1)$

$\mathrm{U}_{\text {agent }}$ is assumed to be positively correlated with the four parameters of function $h_{l}$. Similarly, $\mathrm{U}_{\text {system }}$ is given by:

- Usystem $\propto h_{2}\left(\mathrm{~F}_{\mathrm{c}, \text { system }}, \mathrm{F}_{\mathrm{R}, \text { system }}, \mathrm{F}_{\mathrm{A}, \text { system }}, \mathrm{F}_{\mathrm{i}, \text { system }}\right)$

Again, $\mathrm{U}_{\text {system }}$ may not be consistent among all agents. Let $\mathrm{U}^{\mathrm{i}}$, given by $\left(\mathrm{U}_{\text {agent }}, \mathrm{U}_{\text {system }}\right)$, denote a utility vector for the agent. This utility vector is used in determining the benefit that can be attained from a goal. Let $\mathrm{A}^{\mathrm{i}}$ be an agent that has accepted a candidate-goal, $g_{n}$. Let the candidate-goal's possible autonomy level assignments be represented by the set of levels $\left\{\mathrm{L}_{0}, \mathrm{~L}_{1}, \ldots, \mathrm{L}_{\mathrm{m}}\right\}$. In selecting a level, $\mathrm{A}^{\mathrm{i}}$ is assigning an autonomy level to $\mathrm{g}_{\mathrm{n}}$ and must therefore predict the utility vector associated with each autonomy level.

\section{SUMMARY}

Whether acting as completely automated decision makers or acting as assistants for decision support, Sensible Agents will allow planning systems to perform with the kind of innovation, flexibility, and responsiveness demanded of dynamic situations (e.g. dynamic requirements planning resources such as data, communications and time). Significant progress has been made in the area of intelligent planning systems and should not be discarded. The Sensible Agent architecture provides a mechanism to leverage legacy

${ }^{1}$ For simplicity reasons, the subscripts $\mathrm{i}$ and $\mathrm{j}$ to denote the current goal, $\mathbf{g}_{j}^{i}$, of agent $A^{i}$, have been omitted from the description of functions $f_{\mathrm{c}}, f_{\mathrm{R}}, f_{\mathrm{A}}$, and $f_{\mathrm{i}}$. When a distinction between two goals is necessary, we will use the corresponding subscripts. planners while providing these planners the ability to dynamically adapt their level of autonomy.

Both the focus of previous research and the progression of systems engineering and architecture work offer a critical foundation. While a good foundation exists, current work is limited by a rather static view of the architectures to support decision making. The messages, or at least, the content of message may be dynamic in current architectures. Yet, static interactions (i.e. pre-defined control mechanisms) for planning execution force the systems engineer of the decision making system not the situation in which the decision making systems operates to dictate the available interactions for planning and execution. We have developed simulations for the naval radar frequency management problem which support our claims regarding the limitations of static organizations. These limitations include the following:

- Overuse of scarce communication resources in some circumstances

- Slow response due to fixed communication and negotiation protocol that does not vary with circumstances (e.g. adaptation of interactions based on geographical distribution of emitter, available spectrum resources, and other factors).

- Inability to reason effectively about third party interference sources and intentions (sources that are not members of the system).

A Sensible Agent's ability to 1) reason about the utility of autonomy levels with respect to both local and systems goals and 2) assign autonomy levels given utility-based assessments provides significant advances in the development of dynamic, responsive systems.

\section{REFERENCES}

1 Jennings, N. R., and Mamdani, E. H. Using Joint Responsibility to Coordinate Collaborative Problem Solving in Dynamic Environments. In Proceedings of the Tenth National Conference on Artificial Intelligence, pp. 269-275. American Association for Artificial Intelligence 1992.

2 Gasser, L., and Ishida, T. A Dynamic Organizational Architecture for Adaptive Problem Solving. In Proceedings of the Ninth National Conference on Artificial Intelligence, 185-190. American Association for Artificial Intelligence 1991.

3 Durfee, E., and Lesser, V. Using Partial Global Plans to Coordinate Distributed Problem Solvers. In Proceedings of the Tenth International Joint 
Conference on Artificial Intelligence, pp. 875-883, 1987.

4 Wilkins, D. E.; Myers, K. L.; and desJardins, M. 1997 IFD-5 Discussion and Summary of Multiagent Planning Architecture. Working Document. http://www.ai.sri.com/ wilkins/mpa/mpa-shortifd5.ps.

5 Corkill, D. 1979. Hierarchical Planning in a Distributed Environment. In Proceeaings of the Sixth International Joint Conference on Artificial Intelligence, pp. 168-175.

6 Georgeff, M. 1983. Communication and Interaction in Multiagent Planning. In Proceedings of the National Conference on Artificial Intelligence, pp. 125-129. American Association for Artificial Intelligence.

7 Ephrati, E., and Rosenschein, J. S. Constrained Intelligent Action: Planning Under the Influence of a Master Agent. In Proceedings of the Tenth National Conference on Artificial Intelligence, pp. 263-268. American Association for Artificial Intelligence. 1992.

8 Smith, R. The Contract Net Protocol: High-level Communication and Control in a Distributed Problem-Solver. IEEE Transactions on Computers 29(12):1104-1113 1980..

9 von Martial, F. Coordination by Negotiation Based on a Connection of Dialogue States with Actions. In Proceedings of the Eleventh International Workshop on Distributed Artificial Intelligence, pp. 227-246. 1992.

10 Zlotkin, G., and Rosenschein, J. S. Negotiation and Task Sharing Among Autonomous Agents in Cooperative Domains. In Proceedings of the Eleventh International Joint Conference on Artificial Intelligence, pp. 912-917. 1989.

11 Lesser, V. R. and Corkill, D. D. Functionallyaccurate, cooperative distributed systems. IEEE Transactions on Systems, Man, and Cybernetics 11(1):81-96. 1981.

12 Lesser, V. R. and Corkill, D. D. The Distributed Vehicle Monitoring Testbed: A Tool for Investigating Distributed Problem Solving Networks. AI Magazine 4(3):15-33. 1983.

13 Lesser, V. R. A Retrospective View of FA/C Distributed Problem Solving. IEEE Transactions on Systems, Man, and Cybernetics 21(6):1347-1362. 1991.

14 Tenney, R. R., and Sandell, N. R. Strategies for Distributed Decision Making. IEEE Transactions on Systems, Man, and Cybernetics 11(8):527-538, 1981 a.

15 Tenney, R. R., and Sandell, N. R. Structures for Distributed Decision Making. IEEE Transactions on Systems, Man, and Cybernetics 11(8):517-527, $1981 \mathrm{~b}$.

16 Ishida, T.; Gasser, L.; and Yokoo, M. Organization Self-Design of Distributed Production Systems,
IEEE Transactions on Knowledge and Data Engineering 4(2):123-134. 1992.

17 Numaoka, C. Conversation for Organizational Activity, pp. 189-198. In Werner, E., and Demazeau, Y. eds. Decentralized A.I. Vol 3. Proceedings of the Third European Workshop on Modeling Autonomous Agents in a Multi-Agent World. Amsterdam: Elsevier Science 1991.

18 Mertens, P. Falk, J., and Spieck, S. (1994). "Comparisons of Agent Approaches with Centralized Alternatives Based on Logistical Scenarios," Information Systems 19(8):699-709, 1994.

19 Bharatia, V. and Cook, D. (1995). Design and Analysis of Centralized, Distributed, and Group Multi-agent Coordination Models. Technical Report, University of Texas at Arlington, 1995.

20 Graser, T. "Meta Modeling of Sensible Agents." Semiotic Modeling for Sensible Agents Workshop, Intelligent Systems Conference, October 23-25, 1996, Gaithersburg, MD.

21 Graser, T. and K.S. Barber, "A Meta-Model Supporting the Engineering Lifecycle: A Focus on Behavioral Modeling and System Component Integration," submitted to HICSS-30 Conference, April 1996.

22 Suraj, A., S. Ramaswamy, and K.S. Barber, "Behavioral Modeling of Sensible Agents," Semiotic Modeling for Sensible Agents Workshop, Intelligent Systems Conference, October 23-25, 1996, Gaithersburg, MD.

23 Suraj, A., S. Ramaswamy, and K. S. Barber, "Extended State Charts for the Modeling and Specification of Manufacturing Control Software Systems," accepted to International Journal of Computer Integrated Manufacturing, to appear January 1997.

24 Suraj, A., S. Ramaswamy, and K. S. Barber, "Extended Statecharts: A Specification Formalism for High Level Design", accepted to the Fourth International Conference on Control, Automation, Robotics and Vision, December 3-6, 1996, Singapore.

25 Goel, A., T.H. Liu, and K.S. Barber, "Conflict Resolution in Sensible Agents," Semiotic Modeling for Sensible Agents Workshop, Intelligent Systems Conference, October 23-25, 1996, Gaithersburg, MD.

26 Liu, T.H., C. Chuter, and K.S. Barber. "Flexible Conflict Resolution Strategies for Multiple Mobile Robots" submitted to IEEE International Conference on Robotics and Automation, April 20-25, 1997, Albuquerque, NM. 


\title{
Finding and Using Multiple Models For Autonomous Learning
}

\author{
I.N.P. Davidson \\ CSIRO - Mathematical and Information Sciences \\ 723 Swanston St, Carlton, Victoria, Australia 3053
}

\begin{abstract}
Achieving autonomous learning systems which can govern themselves is one of the goals of A.I. Most learning systems explore a fixed model space to explain a set of data. We believe that the "best" but most distinct models in the available space can provide insight into questions of autonomy such as when to change the model space and how to generate new data points (via experiments). We explore this idea by focusing on clustering problems where the initial data is known to be insufficient to find the true model. We propose a method to generate new data points via experiments. Our approach results in convergence to the true model using half as many additional data points than if they were randomly selected.
\end{abstract}

KEYWORDS: Autonomous learning, machine discovery, clustering, unsupervised learning

\section{INTRODUCTION AND MOTIVATION}

If inductive learning aims at answering the question, "What does the data tell us ?", autonomous learning adds the question, "What can we now do to better understand the domain?".

So what can we do to better understand the domain to which we are applying our learning system ? Inductive learning like most artificial intelligence problems is inherently a search through a predefined model space. Most inductive learning tools whether they be unsupervised [1] or supervised [2], primarily focus on finding the single best model with respect to some criterion for a fixed set of data. This is quite adequate if the tool is to be used by a human who can interpret the results and make appropriate changes. To make such tools autonomously learn more about a domain we must address problems of how to change the model space and how to generate new data. It is our belief that finding and using multiple models can provide insight into these more complex questions associated with autonomous learning.

In this paper we focus on using multiple models to answer the question, "Given the current data and the best model(s) found, what should be the next set of experiments to conduct be to find the true model for the domain ?". Which model is better for a given set of data has been addressed by the minimal encoding length approach independently proposed by Wallace (1968) [1] and Rissanen (1978) [3]. Their approach has the benefit that complex models are chosen over simpler ones only if the data available justifies it. But to our knowledge the approach provides no indication of how to generate new data points. Whilst we focus on this question in this discourse, we believe our approach could be used to determine how to change the model space and other questions associated with autonomous learning. We intend to explore these at a latter time.

This paper documents our approach for finding and using multiple models for clustering problems otherwise known as unsupervised learning. The paper is divided into a further six sections. The first is a basic introduction to clustering which provides the terminology used throughout this paper. In the next section we define in limited detail the clustering system we have developed (a more complete description exists [4]). The criterion used to evaluate each model (the minimum message length) and our search mechanism (simulated annealing) are described. The subsequent sections outline how we search the model space to find multiple models and then how these can be used to answer our next experiment question. The final two sections discuss and conclude our current work and touches on future research.

\section{AN INTROdUCTION To CluStering}

Clustering, also called unsupervised or intrinsic classification, has a long history in numerical taxonomy [5] and machine learning [6]. Clustering attempts to find groups within data so as to better understand the domain the data is from. It has been applied to generation of taxonomies for flora and fauna, concept formation and data mining. The objects/entities to be clustered are each described by a set of $d$ attributes. Clustering involves determining the number of classes (groups), a description for each class which can be used to determine membership and assigning each object to one or more of these classes. As the number of classes is unknown and no preclassified training set exists, clustering is unsupervised. The collection of classes and their descriptions form a taxonomy/model of the objects.

A clustering system contains three major parts. The knowledge representation scheme (KRS) which defines the searchable model -space. The criterion which provides a "goodness" measure for each model and the search mechanism which explores the model space attempting to find the model which leads to the optimal criterion value.

The KRS determines the type of classes and their possible interrelationships. A dichotomy for clustering options which impact on the KRS has been defined elsewhere [7]. The criterion evaluates the "goodness" of each of the models. It is usually a real value function that takes as parameters the objects and/or class descriptions and is the objective function of the search. 
The search mechanism explores the model space attempting to find the best model by finding the optimal (either minimum or maximum) value of the objective function. For all but the most restrictive model spaces the number of possible models to evaluate is combinatorially large. Exhaustively evaluating each model is not even considered as a search mechanism. The search mechanism must consistently find the global optima or at least a good local optima in a number of different application domains with a minimum of computation.

\section{Our Clustering System}

The clustering system developed merges together two problem-invariant (robust) technologies: the minimum message length criterion (MML) and simulated annealing (SA). This has so far shown to result in a clustering system which can be applied to a number of different problems with minimum changes. The objective function of our search is to minimize the message length for non-hierarchical and probabilistic classes which objects are exclusively assigned to. However, most large and interesting search problems possess many local optima [14]. We feel that SA is a good search mechanism to explore these complex model spaces, since it can escape local minima [14]. In the following sub-sections we describe the two technologies.

\subsection{The Minimum Message Length Criterion}

Chaitin [8], Kolmogorov [9] and Solmonoff [10] in varying forms independently proposed algorithmic information theory (AIT). AIT intuitively allows us to quantify the notion of complexity and compressibility of objects. Learning by induction is inherently about compressing observations (the objects) into a theory (the model). Boyle's law $(P=k \cdot N / V)$ on ideal gases relates the number of molecules $(N)$ in a measurable closed volume $(V)$ to pressure $(P)$. A table could store every possible combination of $N$ and $V$ and the resultant pressure. However, Boyle's law compresses this table into a much shorter description, the above equation.

Wallace and Boulton [1], extend this compressibility notion into their minimum message length (MML) approach to induction. They define a criterion which can be used to select the most probable model from a given set of mutually exclusive and exhaustive models, $H^{*}$, for the objects, $D$. The MML approach specifies that the minimal encoding of the model and the objects given the model is the best. In terms of Bayes theorem, we wish to maximise the posterior distribution, $P\left(H_{i} \mid D, c\right)$ where $c$ is the background context:

$$
P\left(H_{i} \mid D, c\right)=\frac{P\left(H_{i} \mid c\right) \cdot P\left(D \mid H_{i}, c\right)}{P(D)}
$$

Taking the logarithm of this expression yields

$$
\begin{aligned}
-\log P\left(H_{i} \mid D, c\right) & =-\log P\left(H_{i} \mid c\right)+ \\
& -\log P\left(D \mid H_{i}, c\right)+\text { const }
\end{aligned}
$$

Our interest is in comparing relative probabilities so we can ignore const. Information theory [Shannon] tells us that -log ( $P$ (occurrence)) is the minimum length in bits to encode the occurrence. Hence by minimising equation (2) we inherently maximise the posterior distribution and find the most probable model. The expression to minimise has two parts, the first being the encoding of the model and the second the encoding of the objects given the model. The object collection is random if the size of encoding the model and the objects given the model is approximately equal to the size of directly encoding the objects. That is there is no way to compress the objects into a shorter description/theory. The two part message is precisely described for intrinsic non-hierarchical classification [1] and [11].

The MML criterion only defines a goodness measure for a model with an inherent bias towards simple models. It does not indicate how to search the model space. To do that we use simulated annealing.

\subsection{Searching The Model Space Using Simulated Annealing}

The Metropolis criterion was first used as a Monte Carlo method for the evaluation of state equations in statistical mechanics by Metropolis et al. [12]. Kirkpatrick et al. [13] demonstrated how using the Metropolis criterion as a test in iterative optimisation can solve large combinatorial optimisation problems. They called their approach the simulated annealing technique as it mimics the annealing of a piece of metal to minimise the energy state of the molecules within the metal. SA is an iterative local optimisation technique. At any time there is only one current solution which is slightly changed at each iteration. As SA is a Markov process the current solution, $S_{n}$, at time $n$, is a result of the perturbation of solution $S_{n-1}$. The algorithm continually perturbs the current solution to generate new candidate solutions. SA unconditionally accepts candidates of better quality than the previous solution and conditionally accepts those of a worse quality with a probability $p$, where:

$$
p=e^{-\left(\frac{\mid \text { Difference in Quality } \mid}{\text { System Temperalure }}\right)}
$$

Worse quality solutions can be accepted which allows the search to escape from local minima which are common in most complex search problems [13]. We set the initial temperature $T_{0}$, so there is a $90 \%$ probability of accepting an increase in cost. This probability decreases as the temperature decreases. The cooling constant, $R$ reduces the temperature such that, $T_{k}=T_{k-1} \cdot R$. 


$$
T_{0}=-\frac{C_{0}}{\log _{e}(0.9)}
$$

$C_{0}$ is the goodness evaluation of the initial solution.

The implementation of our algorithm can be found in [4].

Simulated annealing statistically guarantees that the global optimum will be found, if the thermodynamic equilibrium is reached at every temperature and the cooling schedule is slow enough [14]. However, this is an infinitely long process. We do not maintain these two requirements due to the need to find a solution in finite time. Instead, after a fixed number of iterations at a temperature, the temperature is reduced and the cooling constant provides discrete changes in the temperature. However non-ideal SA approaches, such as the one we use, still find good, local optima solutions [14].

\section{Finding Multiple Models}

Our thesis is that distinct but good models can be used to generate new experiments whose results can be used to better understand the domain. This requires finding the $n$ models which provide the best values for the objective function but are sufficiently different from each other. Just finding the $n$ best models would most likely result in finding a good model and slight variations of it.

To achieve our aim we must handle two key issues. Firstly, we must be able to quantify the difference between two models. Secondly we must adjust our search mechanism. Let us discuss the first.

\section{1-Quantifying The Difference Between Models}

A model can be characterised by its predictions or its syntactic description. A model (the taxonomy) makes predictions on how to group together objects. Each model assigns each object to a cluster. For two clusters from different models, we can measure the similarity between them by counting the number of common objects. For two models we can measure their similarity by counting the number of common objects for every possible combination of cluster pairs (one from each model). This is inherently a measure of the common "cluster neighbourhood" (clusterhood) each entity has in two different models. This measure can be achieved by building a $r \times c$ contingency table, $P$, where $r$ is the number of clusters in model $A\left(M_{A}\right)$ and $c$ is the number of clusters in model $B$ $\left(M_{B}\right)$. The cell $P_{i j}$ in the table holds the number of objects common to cluster $i$ in $\mathrm{M}_{\mathrm{A}}$ and cluster $j$ in $\mathrm{M}_{\mathrm{B}}$. The total count of the table will be the number of objects/entities we are clustering. Where $M_{A}$ is the same as $M_{B}$ only the leading diagonal of the resultant table will contain non-zero elements.

A model can also be characterised by its description. In clustering, a model consists of classes and their descriptions. In our approach each class description contains a probability distribution for each attribute. The message we construct (whose length we are trying to minimise) only encodes an attribute distribution of a class if it is sufficiently different from the population's (collection of all objects) distribution for that attribute. We can characterise the descriptive difference between two models in a contingency table, $D$, which has the same structure as the contingency table $P$. For each attribute we can determine which of the clusters for each model has a distribution for that attribute that is the greatest from the populations. The cell $D_{i j}$ holds the count of attributes for cluster $i$ in $\mathrm{M}_{\mathrm{A}}$ and cluster $j$ in $\mathrm{M}_{\mathrm{B}}$ whose probability distribution is of greatest distance from the population's distribution. The total count for the table is the number of attributes. The contigency table inherently holds the distinguishing features (attributes) of each class.

The contingency tables $P$ and $D$ contain the differences between the two models $A$ and $B$ in their most rudimentary forms (predictions and descriptions). We can use this information to measure if a relationship exists between the two models. Note we do not attempt to determine what the relationship is, only if it exists. This can be achieved by using a number of different contingency table association measures [15]. We choose the Goodman and Kruskal lambda measure of predictive ability because it is both a readily interpretable probability measure and is not symmetrical. The measure $\lambda_{\mathrm{AB}}$ measures the ability to predict the cluster in model $B$ given we know the cluster in model $A$. It should be noted that $\lambda_{\mathrm{AB}} \neq \lambda_{\mathrm{BA}}$ is generally true. That is, $A$ may be predictable from $B$ but not $B$ from $A$ and vice versa. Specifically $\lambda_{\mathrm{AB}}$ calculates the relative decrease in the probability of an error in guessing the class given by model $A$ if the class for model $B$ is known. Formally we can write:

$$
\begin{aligned}
& \lambda_{A B}=\frac{P_{1}-P_{2}}{P_{1}} \\
& P_{1}=1-\max \left(n_{j}\right) \\
& P_{2}=\sum_{i=1}^{r}\left(1-\left[\frac{\max \left(n_{i .}\right)}{n_{i}}\right]\right) \cdot n_{i} \cdot \frac{1}{N}
\end{aligned}
$$

By calculating the lambda value for the tables, $P\left(\lambda_{\mathrm{AB}}(P)\right)$ and $D\left(\lambda_{\mathrm{AB}}(D)\right)$ we can measure the predictability of a model from another in terms of predictions and descriptions respectively.

\subsection{Adjusting The Search}

The ideal annealing algorithm converges to the global optimum. However the trajectory through the model space in getting there may not be sufficiently diverse to find other good but different local optima. We must therefore adjust our search method to be consistent with our aim. We can achieve this by introducing a bias which guides the search away from already found good local optima. This is facilitated by storing $n$ models which are the best (with respect to the objective function) but sufficiently different from each other. These models are the best and most diverse models known. 
Models are only considered to be stored if their message length is less than any of the currently stored models. To be stored, the summation of the models predictability from every other stored model must be less than this same measure for one of the currently stored models. The model whose predictability is the greatest is replaced. Predictability is calculated using the $\lambda_{A B}$ measures for either the $P$ or $D$ contingency tables. Candidate models have a penalty added to their "goodness" value in proportion to their similarity to the stored models.

\section{The Uses of Multiple Models}

In the previous section we defined two measures of difference between models. These measures can be encoded in a contingency table and the predictability of one model from another calculated. How we should use these measures to influence our search depends on what we are trying to achieve. In this paper we focus on what the next best set of experiments to conduct are.

The question of how to guide the next experiments to conduct has been addressed in Lenat's work on AM [16] and Kulkarni and Simon's work on Kekada [6]. Lenat described the notion of "interestingness" and felt the system should focus its attention on interesting phenomena. Similarly, Kekada focuses its next experiments on surprising phenomena, believing that if a result of an experiment was unexpected then the knowledge of that area of the domain is obviously lacking and should be explored. Both approaches use heuristics to describe the notions of interestingness and surprise. As we hope to have available the best but most different models we focus the next set of experiments where these models' predictions differ. By doing this we can resolve which of these models is the better for the domain. By c $c^{\wedge}$ tinually running the clustering system, finding distinct but good models, and then generating data points where these models' predictions differ we generate data points where our knowledge of the domain is contradictory.

To determine which of measure of predictability (model description or predictability) is better we conducted experiments on the following problem. Consider a population of objects/entities each having $m$ binary attributes. In the population there exists $m$ classes. Class $i, i=1 \ldots m$ can be precisely described as having the value 0 (false) for all attribute except the ith which is 1 (true). Table 1 provides the precise description for a few classes for the $m=10$ situation.

\begin{tabular}{c|cccccccccc} 
Attribute & $\mathbf{1}$ & $\mathbf{2}$ & $\mathbf{3}$ & $\mathbf{4}$ & $\mathbf{5}$ & $\mathbf{6}$ & $\mathbf{7}$ & $\mathbf{8}$ & $\mathbf{9}$ & $\mathbf{1 0}$ \\
\hline Class 1 & 1 & 0 & 0 & 0 & 0 & 0 & 0 & 0 & 0 & 0 \\
Class 10 & 0 & 0 & 0 & 0 & 0 & 0 & 0 & 0 & 0 & 1
\end{tabular}

Table 1. Precise description for classes in $\mathrm{m}=10$ situation.

To determine the proportion of each class in the population we make use that the summation of the first $r$ integers is given by:
$S_{r}=\frac{r \cdot(r+1)}{2}$

from this the relative proportion, $P_{i, i}=1 \ldots \mathrm{m}$ of class $i$ in the population is given by:

$P_{i}=\frac{2 i}{m(m+1)}, \quad \sum_{i=1}^{m} P_{i}=1$

By using the MML equations described in [1] we can determine the approximate number of objects (data points) required to find the true model if the objects are randomly sampled from the population. For our trial set of objects this number was 184. Below this amount of data the best model is to place all objects into one class, indicating that the data from an information theoretic view is random. For this data set the encoding of the model and data for the true model and one class model were 566.21 and 570.24 nits respectively. The difference between the lengths is the comparitive difference in likelihood. Thus the true model is approximately $e^{4}$ times more likely than the one class model for the given data set.

We conducted trials to determine how successful our strategy of focusing experiments on where the models predictions differ is. In each trial the clusterer was given the first 60,80 and 120 objects of our data set. As we have shown, this is insufficient to chose the true model over the one class model. For 120 objects the true model and one class model had encoding length of 415.56 and 368.32 nits respectively for this reduced data set. The true model is approximately $e^{47}$ times less likely than the one class model for the data. The class distributions follow in table 2 :

\begin{tabular}{c|cccccccccc}
$\begin{array}{c}\text { Class } \\
\text { Number }\end{array}$ & 1 & 2 & 3 & 4 & 5 & 6 & 7 & 8 & 9 & 10 \\
\hline Frequency & 5 & 1 & 7 & 13 & 7 & 13 & 15 & 17 & 21 & 21
\end{tabular}

Table 2. Class distributions for initial 120 objects

Our aim is to generate new data points so that eventually the true model is found. The number of new experiments required to converge to the true model is one obvious measure of performance.

\subsection{How To Generate New Experimental Data}

The process of running the clustering system with a given set of data produces a number of theories (taxonomies/models) of the data. We wish to generate new experimental data which can be used in further applications of the clustering system to better understand the domain and find the true model. We focus on generating new data where the predictions of the theories are different. An example based approach is used where an example of an object the models' predictions disagree upon is used as input into an experiment. The experiment takes the object as an input and returns similar objects. For additional complexity there is a stochastic aspect 
to the experiments which results in the chance that the experiment will return the wrong result. In our studies this error is $25 \%$.

The examples can be selected by re-arranging a $P$ contingency table so that the leading diagonal has the largest counts. The remaining elements represent objects which are predicted indifferently for these two models. Completing this task for all possible pairs of models can determine those objects for which the model's predictions differ the greatest.

Experiments could also be generated by prescription. This would involve a description of an exemplar object for which, if it were to exist, the current stored models would make contradictory predictions for. This exemplar could be constructed from where cluster descriptions differ the most between all clusters from one model with all clusters from another. We have not explored this option as yet.

We established two control trials. One generated new objects by sampling them from the population (sampPop) whilst another generated new objects from each class in equal proportion (equProp).

Table 3 illustrates the comparison between each model search and experiment generation technique. Both search techniques stored the five best models which had the shortest message lengths but were different from each other. The techniques differed in the notion of difference. Search technique A used the predictive difference between models; B the description difference between models. Two experiment generation techniques were tried: technique $C$ generates new objects in batches of 10 whilst technique $\mathrm{D}$ generated objects in batches of 20. After each batch was generated, the clusterer was re-run and the process repeated until the true model was discovered. The control trial generated objects in batches of 5 . All four of our variations outperformed the two control approaches by requiring approximately half as many data points to converge to the true model.

\begin{tabular}{|l|c|c|c|c|c|c|}
\hline Search Technique & A & B & A & B & samp & equ \\
\hline $\begin{array}{l}\text { Experiment Generation } \\
\text { Technique }\end{array}$ & C & C & D & D & Pop & Prop \\
\hline $\begin{array}{l}\text { New objects required to } \\
\text { find true model. } 60 \text { initial } \\
\text { objects. }\end{array}$ & 70 & 70 & 80 & 80 & 170 & 140 \\
\hline $\begin{array}{l}\text { Additional objects required } \\
\text { to converge to true model. } \\
80 \text { initial objects. }\end{array}$ & 50 & 60 & 80 & 60 & 140 & 120 \\
\hline $\begin{array}{l}\text { Additional objects required } \\
\text { to converge to true model. } \\
120 \text { initial objects. }\end{array}$ & 40 & 40 & 60 & 60 & 100 & 80 \\
\hline
\end{tabular}

Table 3. Results of trials

\section{Discussion ANd Future Work}

We shall focus our discussion on the situation with 120 initial objects using search technique A (model difference measured by predictions) and experiment generation technique $C$ (batch sizes of 10). The system behavior is summarized in table 4 .

\begin{tabular}{l|ccccc} 
& $\mathbf{1}^{\text {st }}$ & $\mathbf{2}^{\text {nd }}$ & $3^{\text {rd }}$ & $4^{\text {th }}$ & $\mathbf{5}^{\text {th }}$ \\
Trial & Trial & Trial & Trial & Trial \\
\hline $\begin{array}{l}\text { True Classes } \\
\text { the Best Models }\end{array}$ & 6 & 8 & 7 & 9 & 10 \\
$\begin{array}{l}\text { Class experiments } \\
\text { focus on }\end{array}$ & 1 & 3 & 9 & 2 & \\
True Model Found & No & No & No & No & Yes
\end{tabular}

Table 4. Summary of Behavior For 120 Initial Object Case.

The ten classes in the true model are not justified by the initial data. After the first trial with 120 objects, the best models, in combination, contained the correct description and object assignments for six of these classes. The models most disagreed upon what class objects in the class 1 should belonged to, more objects similar to this class were requested. A further 4 more trials occurred before the true model was found. Of course in our situation we know what the true model is. It was interesting that the number of true classes found did not increase monotonically, nor that the class the models predictions differed most on, was not the least frequent.

One of our aims was to determine the impact of searching the model space to find the best but most different models with respect to description and predictions. However irregardless of the measure of difference used, similar models were found. We feel this is due to the simplicity of the problem and most likely this will not occur in more complex domains.

We have not made use if there exists any relationship between the similarity of two models for their predictive capability and descriptions. We can consider five cases:

\begin{tabular}{ll}
\hline \multicolumn{1}{c}{ Relationship } & \multicolumn{1}{c}{ Interpretation } \\
\hline$\lambda_{\mathrm{AB}}(\mathrm{P})>\lambda_{\mathrm{AB}}(\mathrm{D})$ & $\begin{array}{l}\text { The models predictions are more similar } \\
\text { than their descriptions. }\end{array}$ \\
$\lambda_{\mathrm{AB}}(\mathrm{P}) \gg \lambda_{\mathrm{AB}}(\mathrm{D})$ & $\begin{array}{l}\text { The models predictions are significantly } \\
\text { more similar than their descriptions. }\end{array}$ \\
$\lambda_{\mathrm{AB}}(\mathrm{P})<\lambda_{\mathrm{AB}}(\mathrm{D})$ & $\begin{array}{l}\text { The models descriptions are more } \\
\text { similar than their predictions. }\end{array}$ \\
$\lambda_{\mathrm{AB}}(\mathrm{P})<<\lambda_{\mathrm{AB}}(\mathrm{D})$ & $\begin{array}{l}\text { The models descriptions are } \\
\text { significantly more similar than their } \\
\text { predictions. } \\
\lambda_{\mathrm{AB}}(\mathrm{P}) \approx \lambda_{\mathrm{AB}}(\mathrm{D})\end{array}$ \\
& $\begin{array}{l}\text { The similarity between the models with } \\
\text { respect to their descriptions and } \\
\text { predictions are fairly equivalent. }\end{array}$ \\
\hline
\end{tabular}

Table 5. The relationship between measures of similarity between a models description and its predictions.

We can diagramatically represent each situation by considering a population of things which only has one 
attribute, which we believe to be normally distributed. Each model has only one class whose description is the mean and standard deviation for that particular attribute. Figure 1 illustrate the situations for $\lambda_{A B}(P)>\lambda_{A B}(D)$. The circles represented data points. In this situation the models make similar predictions for the current data points, but they are evidently different. $\lambda_{A B}(P)$ is larger than $\lambda_{A B}(D)$ because the current data points do not occur in areas where the models predictions would differ. Using and contrasting both measures could be of benefit.

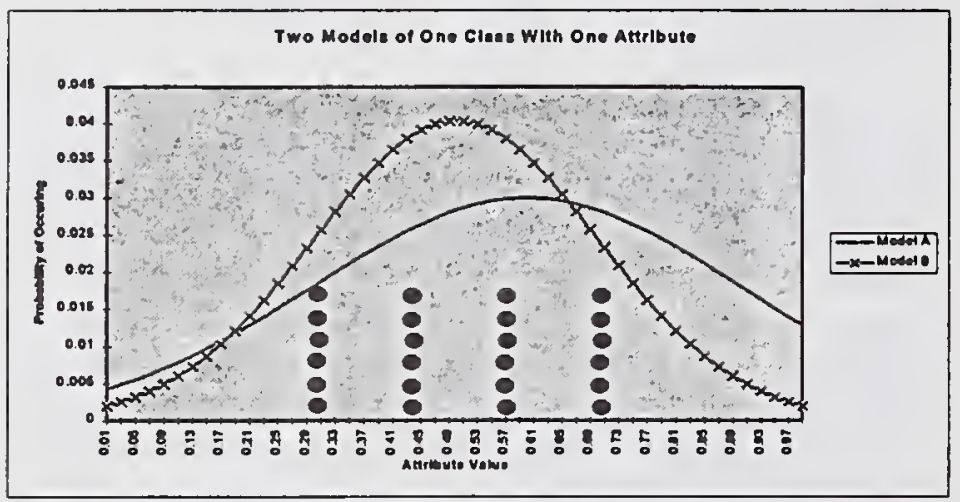

Figure 1. $\lambda_{A B}(P)>\lambda_{A B}(D)$

We intend to explore the annealing literature to see if any insight can be provided to bias the search technique to better explore the model space. The lambda measures of association used in the contingency tables whilst adequate are problematic for skewed distributions. We intend to explore measuring the information content of contingency tables to obtain better measures of predictability. As stated earlier we believe that using multiple models can address other questions in autonomous learning such as when to change the model space which we plan to explore. Our clustering system can change the model space by taking the Cartesian product of attributes and changing the probability distribution (discrete or normal) assumed for each attribute.

\section{CONCLUSION}

We have developed a clustering system which can search the model space for good but distinct models. The difference between models can be measured regarding their predictions or descriptions. Using these models can provide insight into how to address questions of autonomous learning systems of which, we have focused on the next set of experiments to conduct to better understand the domain. We have applied this clustering system to an artificial problem where the initial set of data is inadequate to find the true model. We explore the idea of generating new objects where the models predictions differ. We have shown that this approach results in finding the true model by generating only half as many additional objects than by using blind techniques for our problem.

\section{ACKNOWLEDGMENTS}

I am most grateful to Emeritus Professor C.S. Wallace from Monash University for his continual comment and suggestions on this work. Professor Wallace's work is supported by ARC grants A49703162 and A49703170.

\section{REFERENCES}

[1] Wallace C, Boulton D., "An Information Measure for Classification," Computer Journal, Volume 11 No. 2, pp. 185194, 1968.

[2] Quinlan J.R. "Induction of Decision Trees," Machine Learning, Volume 1, pp. 81-106, 1986.

[3] Rissanen J. "Modeling by the shortest data description," Automatica, Volume 14, pp. 465-471, 1978.

[4] Davidson 1.N.P. "Clustering Using The Minimum Message Length Criterion and Simulated Annealing," in Proceedings of the $3^{\text {rd }}$ International A.I. Workshop, Brno, Czech Republic 1996.

[5] Dunn G., Everitt B.S.. An Introduction to Mathematical Taxonomy. Cambridge University Press 1982.

[6] Michalski R.S., Stepp R.. "Learning from Observation: Conceptual Clustering," in Michalski R.S., Carbonell J.G, Mitchell T.M. editors, Machine Learning. An Artificial Intelligence Approach, CA: Morgan Kaufmann, 1983.

[7] Clifford T., Stephenson W. An Introduction To Numerical Classification, Academic Press, 1975.

[8] Chaitin G. "On The Difficulty of Computations," IEEE Transactions of Information Theory, IT-16, 1970, pp. 5-9.

[9] Kolmogorov A. "Logical Basis for Information Theory and Probability Theory," IEEE Transactions of Information Theory and Control, 1T-14, 1965, pp. 662-664.

[10] Solomonoff R. "A Formal Theory of Inductive Inference: Part 1," IEEE Transactions of Information Theory and Control, 1T:7, 1964, pp. 1-22.

[11] Wallace C.S., "An Improved Program for Classification," in Proceedings of the 9th Australian Computer Science Conference, Volume 8 No. 1, 1986, pp. 357-366.

[12] Metropolis N., et al, "Equation of State Calculations by Fast Computing Machines," The Journal of Chemical Physics, Volume 21 \#6 June, 1953, pp. 1087-1092.

[13] Kirkpatrick S., Gelati C., Vecchi M. "Optimization by Simulated Annealing," Science, Volume 220, 1983, pp. 671680.

[14] Van Laarhoven L. Theoretical and Computational Aspects of Simulated Annealing, CWI Tract, 1988.

[15] Everitt B. Contingency Tables, Cambridge University Press, 1980.

[16] Lenat D. "AM: An artificial intelligence approach to discovery in mathematics as heuristic search," In Knowledge-based systems in A.I., McGrawHill, 1982.

[17] Kulkarni D., Simon H.A., Computational Models of Scientific Discovery and Theory Formation, Editors P. Langely, and J. Shrager, chapter 9, Morgan Kaufmann, 1990. 


\title{
Agent based task viewing in telerobotic environments
}

\author{
Gerard T McKee, Paul S Schenker* \& Bernard G Brooks \\ University of Reading, Department of Computer Science, Whiteknights \\ Reading RG6 2AY, UK. Email: G.McKee@reading.ac.uk, B.Brooks@reading.ac.uk \\ *California Institute of Technology, Jet Propulsion Laboratory, \\ 4800 Oak Grove Drive/MS 198-219, Pasadena, CA 91109, USA. Email: schenker@telerobotics.jpl.nasa.gov
}

\begin{abstract}
Teleoperation environments consist of viewing and manipulation controls to interact with the remote environment. Camera control distracts the operator from the manipulation task, leading to reduced efficiency. Releasing the operator from the camera control overhead can be achieved with the use of a second operator or by employing an automated viewing system. In this paper we present a model for automated camera positioning that can track a task and index a set of viewing actions, visual acts, that in turn control camera placement. We illustrate the use of "multi-agent" architectures to create viable experimental environments for studying automated camera control and we present a multi-agent model we are currently exploring for creating an automated camera positioning support for operators in teleoperation scenarios.
\end{abstract}

\section{Introduction and Background}

Teleoperation environments consist of viewing and manipulation controls to interact with the remote cameras and manipulators respectively. Typically teleoperation tasks can be performed in two ways. In the first case, a single operator has sole control. However, the complexity of the controls means that typically the operator will control either the robot manipulators or the cameras, but not be able to control both at the same time. This means that the task of controlling the cameras tends to distract the operator from the manipulation task, and leads to significantly reduced efficiency [11].

In the second case, the teleoperation task is performed by two operators. The first operator controls the robot manipulators, and the second operator controls the cameras in an attempt to provide the first operator with the visual information required to perform the task. Although this is an improvement for the first operator, extensive operator training may be required. One of the key challenges in this mode of working is to structure the task such that the intentions of the first operator are signalled clearly to the second. This is normally achieved using a pre-compiled task script and communication between the two operators to register their actions within the real-time setting.

The aim of automating camera control is to remove the need for the second operator without incurring the burden of camera control on the first operator. A secondary aim is to reduce the complexity of the operator interface, and wherever possible, to offer additional assistance to the operator by complementing the sensory data from the remote environment [11].

In a previous paper we proposed to use deliberative task models and a reactive architecture to achieve these goals [9]. The deliberative model captured the notion of a script and the reactive model addressed the real-time environment. The automated viewing system, in this context, represents an "autonomous" agent acting cooperatively with the operator. It takes away the responsibility for visual control, leaving the operator to focus attention on the manipulation task. The success of the cooperation depends, however, on signalling the operator's intentions to the viewing system. In particular, it is essential that the automated viewing system remain stable and provide a consistent quality of service to the operator. Our aim, in particular, is for the viewing system to be "responsive" to the operator so that the operator still retains the feeling of being in control, while remaining "stable."

The actions of the operator and their concomitant impact on the remote environment, are the prime source of signals for an automated viewing system. However, they may not reveal themselves without some form of coercion during the design of the operator interfaces and the task environment. Therefore, autonomous systems need, in many cases, to be situated within an operational context. Within this context they are expected to behave intelligently. In a wider context, however, they may not display the same level of intelligent action, and we should not necessarily expect them to do so.

We should, however, expect the evolution of the autonomous agent to follow a path of generalisation as we develop techniques which allow us to relax the coercion. We might actually say that this evolution may progress in tandem with our ability to discriminate signals. The hybrid architecture we have proposed lends itself to a manmachine interface model in which manipulation actions can be enhanced to provide clear signals to the viewing system, while retaining a manipulation focus. We have to be clear, however, that while we are granting autonomy to the viewing system, we are at the same time creating a symbiotic link which ties the autonomous agent to a spe- 
cific operational context.

The remainder of this report is based on work being done in the Active Robotics Laboratory at the University of Reading to demonstrate computer aided camera placement techniques for teleoperation. In the next section we outline a model for automating camera placement. In section 3 we present some ideas for the simulation and implementation of this model in a real robotics laboratory, and in section 4 we present the specific model we are currently exploring. In section 5 we draw our conclusions.

\section{Intelligent Automated Camera Placement}

Our model for automated camera placement makes use of a high-level task based schedule. The components of the schedule are used simply to provide the context of a task operation. The cameras are placed under the control of a reactive decision making system which attempts to determine what the operator is "looking at" and provide suitable views.

The task model provided is generated from prior training runs. During these training runs, a sequence of high level instructions such as "pick up bolting tool", or "place screwdriver head in screw" can be determined. These instructions, are then classified as generic operations, such as "peg-in-hole", which are provided to the viewing system in the form of an operation-based schedule.

\section{Visual Goals}

Our proposal is based on decomposing task operations into simple atomic goals [1][7][8]. These goals, which match the model of human perception, are computed as if the operator were performing an assembly task. The notion is to use assembly planning techniques to compute a set of atomic geometric, kinematic or topological constraints that must be satisfied in order to perform the operation [4].

An example is the "peg-in-hole" operation (Fig. [1]). To perform this operation, the operator must first satisfy three basic geometric constraints before inserting the peg into the hole, namely:

- Axes of peg and hole must be parallel.

- Axes of peg and hole must be coincident.

- Lower surface of peg must be flush with the upper surface of the hole.

Each constraint is considered a visual goal, and each visual goal has associated with it a visual act to satisfy that goal. Since the visual goals are independent of each other, the visual acts can be performed sequentially in any order, or indeed in parallel. Constraints on the sequencing of the visual goals are imposed by the task setting. For example, the parallel and the coincident constraints have to be satisfied before the insertion of the peg in the hole can be completed.

A "visualisation model" for a task can therefore be

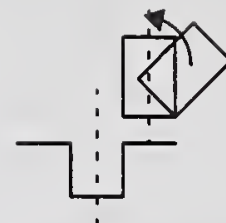

a. parallel axes

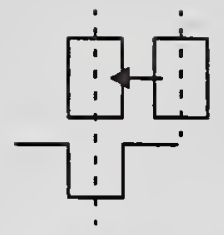

b. coincident axes

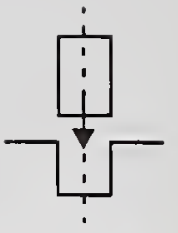

c. insertion
Figure 1: The peg-in-hole operation

defined as follows: A task can be segmented into a set of operations and a set of constraints on the sequencing of these operations. Associated with each operation is a set of visual goals and, implicitly, a set of constraints imposed by the task setting.

\section{Visual Acts}

As mentioned, a visual act is the viewing action required to satisfy a visual goal. Specifically, since it is the operator that performs the manipulative action, the automated camera placement system must use the information in the visual act to provide a set of viewing parameters for the camera system.

The camera placement system must track the operator continually. In particular it must determine:

- Which specific action, or actions, the operator is currently trying to perform in pursuit of the task.

- As the workspace evolves, how best to set the viewing parameters to satisfy each visual act.

Ideally, there would be no explicit communication of the operators intentions to the automated camera positioning system. However, this is not realistic at this time since even in the dual-operator scenario, where the second operator is also an "autonomous" agent, explicit communication of intentions is required to register the manipulation and viewing actions. Our aim is to at least retain the majority, if not all, of the operator's attention focused on the manipulation task. We are, therefore, willing to consider additional modules or actions within the manipulation interface which on the one hand can be motivated by the manipulation task, but which provides signals that can be used to identify the operator's intentions.

For example, we might introduce an additional module which allows the operator to designate task operations at various levels of abstraction. The automated camera positioning system can then takes its cue from this interface module to index the associated set of visual goals.

Within this top-down context we then propose a reactive multi-agent model [2][12] to select and deliver taskrelevant information to the operator. There are actually a number of ways in which we can deploy multi-agent models apparently to good effect. In the following section we 
outline a number of these approaches and we describe the multi-agent environment we are currently implementing to support these.

\section{Architecture}

Our choice of architecture is not just an implementation issue. With the way the problem is modelled, outlined above, it is almost impossible to divorce the architecture of implementation from the problem. In this section we hope to demonstrate how our architecture permits us to implement a solution and actually assists us with that solution.

\subsection{Resource Contention}

The resources to support remote viewing comprise not just of cameras, but also video displays, network bandwidth, etc. Four basic categories can be identified

- Operator console devices, including six degree of freedom input devices, and graphics user interfaces.

- Operator output devices, including video displays, and other devices to present data to the operator.

- Remote environment sensor devices, including cameras, sonar, and other sensors.

- System resources, including computation and communication devices.

These resources are generally limited. Thus, the visual acts will have to compete with each other for control of the resources. Some form of conflict resolution needs to be adopted if contention for resources arises. This means that it is necessary to identify how important it is to get a particular visual goal satisfied.

\subsection{Multi-Agent Architecture}

The problem of resolving resource contention and providing views automatically to the operator cannot be realised easily using either of the extremes of current agent architecture design. In classical planning systems, the planner generates the plan of achieving a goal in very fine detail before the plan can be executed. These systems rely heavily on the assumption that the environment is static, which clearly it is not, since the operator, who is a part of the viewing systems environment, can perform the task in any number of ways.

On the other hand, purely behaviour based reactive architectures [2] rely on the close coupling of sensors to effectors. With these systems goals are always represented implicitly according to a fixed, pre-compiled ranking scheme. They also assume that the requisite activity of the system can be implied directly from the current state of the environment [3]. Again, this architecture is not entirely suitable since visual acts are inherently parallel, and the operator is free to execute the task in whatever order he or she decides.

The system, therefore, must maintain a concept of the global task, but still be responsive to the operators choice. Our solution is to adopt a hybrid multi-agent architecture [5][12], with each agent executing in parallel to follow the operators progress as the operation is being performed. We use this method in order to allow the simultaneous tracking of each visual act by the viewing system. Although each agent embodies a single goal, they must compete for the necessary resources to achieve their goal, and any conflict must be resolved by negotiation. To do this, each agent is required to declare a qualitative measure of their contribution to the system. This requirement forces the system to reflect on the environment and react to the operators actions, while still maintaining a single global task constraint.

Initially the negotiation process may be as simple as choosing to allocate resources to agents that show the most promise of delivering a goal. However, to prevent instability, where agents oscillate between goals, or resources are switched continuously between agents, some additional rules may have to be applied. Again, simple rules, such as retaining the status quo until a particular threshold is reached, may suffice.

In order to map the problem onto a multi-agent architecture we have identified three approaches.

\section{Resource Centred}

Using this approach, each "resource agent" is assigned sole control over a single resource (camera, sonar sensor). At the beginning of the task, the operation is divided into its component visual goals (as described above), and each visual goal is injected into a central pool.

The goals in this pool are offered to the resource agents for "adoption". The agents negotiate with each other to decide which agent should adopt which visual goal. Once an agent has adopted a goal, the role of that agent is to manage its resource in order to provide the information necessary for the visual act.

In order to cope with the evolving nature of the environment, the agents are required to continue their process of negotiation. The effect is that each resource is occupied with providing information to the operator that most closely matches its situation in the environment.

\section{Requirements Centred}

Since the task is decomposed into its individual visual goals, this approach models each goal as an agent. These "requirements agents" are solely responsible for their visual goal being serviced by the available resources (Fig. [2]).

Each agent negotiates with the others for control of the necessary resources to achieve its goal. As with the resource centred approach, the agents continue to monitor the progress of the operator and renegotiate for control of resources as the operator progresses through the task. 


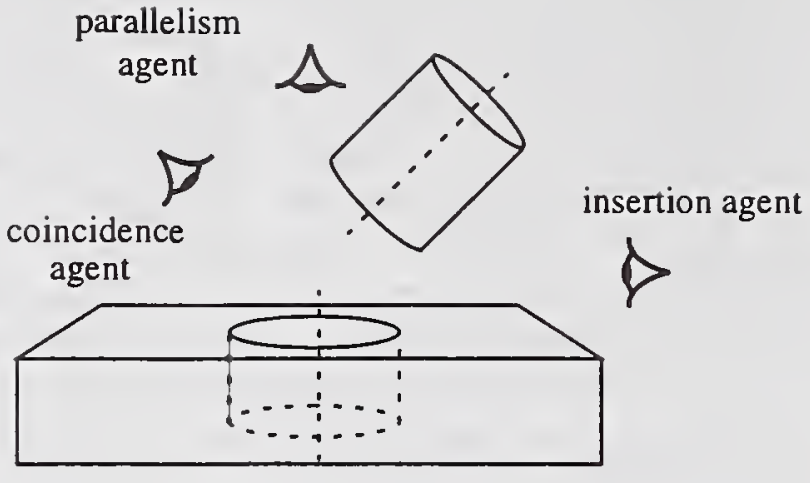

Figure 2: Resource agents for peg-in-hole operation

\section{Hybrid Approach}

In the "peg-in-hole" example used above, one of our three basic geometric constraints was given as

Axes of peg and hole must be coincident.

To achieve this, the operator may choose to divide this goal into yet simpler tasks

$\mathrm{X}$-axis of peg and hole must be coincident.

Y-axis of peg and hole must be coincident.

The operators choice on how to divide the operation further will depend on the available cameras in the environment and on any specific kinematic constraints (for example a camera may be constrained to pan/tilt control only).

Using the hybrid approach we consider the problem in two parts. The first stage is similar to the resource centred approach where each resource is modelled by an agent. The visual goals are presented to the agents which use knowledge of the environment and of their respective cameras, to propose further subdivisions of the goals. All the new goals are then injected into the central pool of visual goals.

In the second stage, the visual goals are themselves modelled as agents. These agents are now responsible for negotiating with each other to gain resources to achieve their particular visual act.

The advantage of this approach is to increase the systems flexibility and allow it to cope with particular hardware constraints, or perhaps, if there is a surplus of resources, allows the system to maximize the benefit gained from these resources.

\subsection{A Multi-Agent Implementation}

Our system makes very few constraints on the nature, implementation, or function of an agent. Agents can communicate with each other, enter into negotiations, issue instructions to the remote environment control system, apply for a sensor "feed" from any part of the remote environment or from each other, or perform any other action virtually without constraint.

However, an agent must embody a single goal, process or strategy. Agents must maintain their own local information necessary to perform their task. We may relax this rule for performance reasons, although only where this is transparent to the function of the agent.

With the exception of agents with control over a particular piece of hardware, agents have no physical properties. The "resource agents" are given control over only one specific piece of hardware, such as a camera or sonar device. In order for the system to perform at all, the agents must cooperate.

Our system also makes very few constraints on the nature of inter-agent communication, or agent to remote environment communication. In addition to traditional explicit communication channels, we expect to use implicit communication channels. For example, the basis of the approaches outlined above were the visual goal or resource "pools". These pools can be seen as implicit communication channels.

Our implementation of agents as individual processes, perhaps distributed across several machines, allows us to develop solutions that are truly flexible. Agents are thus forced to use the inter-process and network communications facilities available. This approach is necessary in order to simulate the dual operator scenario, however, it does also afford several additional benefits.

Clearly, two implementations of an agent, so long as they maintain the same interface, are interchangeable. We make use of this feature to implement some of the hardware resources in simulation. This allows us to develop the computational agents, test them in simulation, and then simply plug in the real hardware control agents in place of the simulation to test our algorithms with the real robotics hardware. Figure [3] shows how each module in the system relates to each other. The modules (such as the robot simulation module) are implemented as an agent or set of agents, and communicate with each other using standard point to point, and/or shared memory communication.

Our initial experimental aims have been to measure relative timings for simple teleoperation tasks using a single and dual operator system. We have begun with a simulated environment in order to constrain the problem and to enforce the assumptions that we have made concerning the required behaviour of the camera control system and the accuracy of the workspace model. This simulation environment is built using a multi-agent approach as outlined above.

The Active Robotics Laboratory at the University of Reading will form the basis of experiments using real robotic manipulators and cameras. The Netrolab environment [10] provides a set of networked robotics resources which can be configured to support diverse applications. 


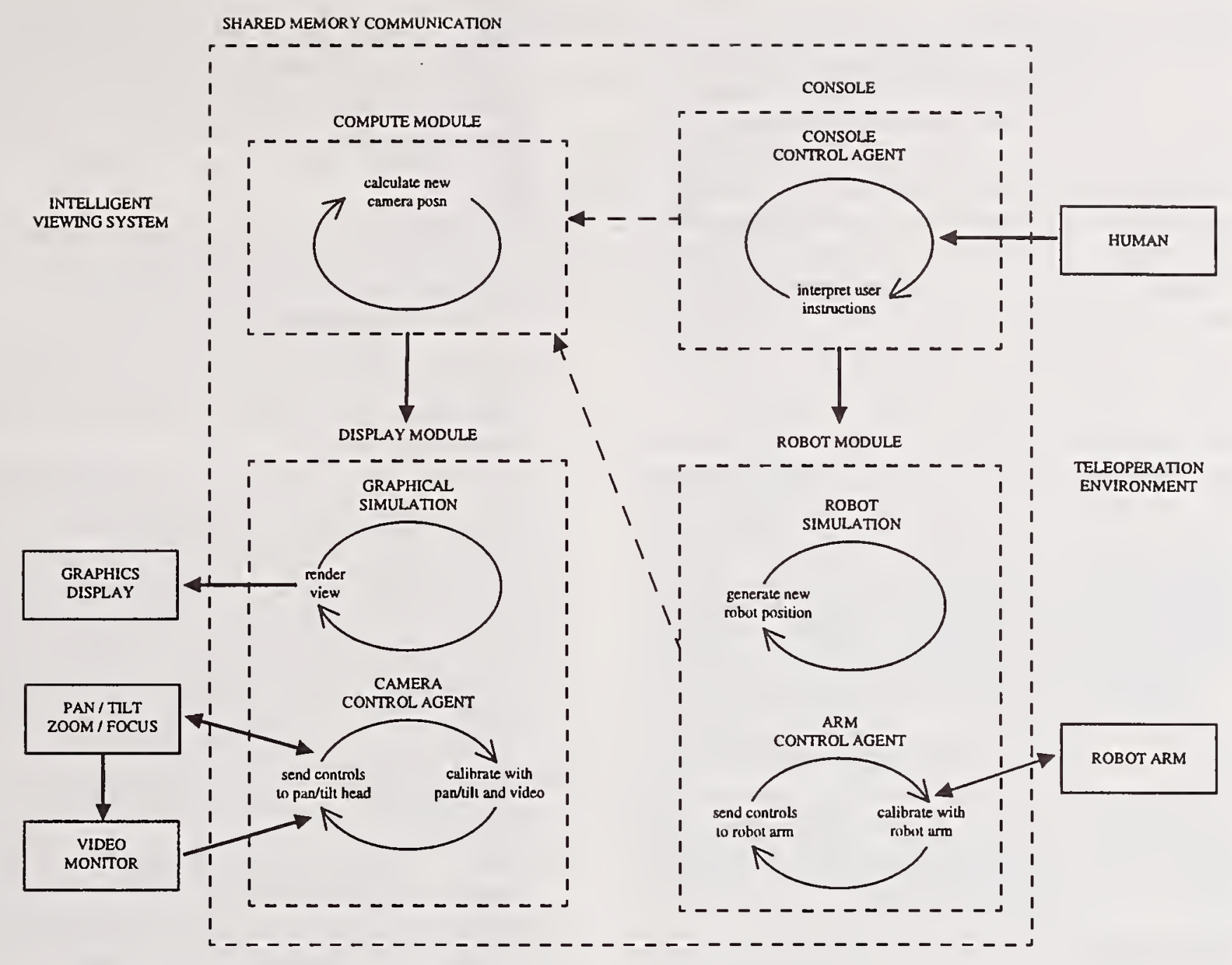

Figure 3: Multi-Agent Modules

The resources include stationary and mobile robots, laboratory viewing cameras and sonar sensors. The networked aspect of this laboratory suits the multi-agent architecture that we have proposed, and makes it particularly easy to plug the computational modules of the simulation directly into the laboratory [6].

\section{An Autonomous System for Camera Control}

The model we are investigating for automated camera control is based on the hypothesis that knowledge of:

- the task setting,

- the task operation being performed,

- the set of visual acts associated with task operations, identified above,

- the "information" content of the task environment, will provide sufficient constraints on the operator's intentions to determine his or her visual goal(s) at any instant during the performance of the task.

Following the model presented above, we assume that the task operation is designated by the operator at the manipulator interfaces. The task setting can be assumed to be instantiated by the operator prior to task execution and updated in tandem with the operator's actions. We assume, that is, that the task environment is known. The set of visual goals is assumed to be identified a priori through automated task planning or manual processing of task logs.

We also exploit the state of the manipulator in the remote environment, and the control actions of the operator to change this state, in order to determine the "information content" of the task environment. Specifically, we aim to track the level and the rate of change of the information associated with each visual goal.

Our aim is to develop a multi-agent model which will exploit this information to identify the current visual goal. In this model an agent will represent a visual goal, and will use the above information to "argue" its case. The agents which dominate will determine the visual acts presented to the operator and their relative configuration in the display interface. A number of methods for combining the infor- 
mation will be explored, including rule-based, fuzzy logic and neural network methods.

We intend to conduct two sets of experiments. The first, which we designate the "passive operator" scenario, will allow us to provide a base line model with which to compare and contrast the impact of the automated viewing system and allow us to assemble data with which to prime the latter. In the passive operator experiments, subjects will be "led" through a sequence of task operations according to a pre-defined script based on the visualisation model. Quantitative performance data, and qualitative observations and reports, will be used to assess the operator's reaction to this mode of working. We will be particularly interested to determine whether the operator feels "in control".

The second set of experiments will evaluate the multiagent model within an "active" operator scenario; the operator is free to direct the progression of the task. We are keen to observe the operator's performance within this scenario and his or her awareness of the viewing system. We are particularly interested in determining the operator's willingness to adapt to the viewing system as well as the required adaptation of the viewing system to the operator.

Another area where we will pay particular attention is the situation where there is no clear winner among the agents. We expect that an inertial factor will be need to be introduced in order to smooth the transition between visual acts, and therefore maintaining the "stability" mentioned earlier. These experiments are now being developed and will be reported in subsequent papers.

\section{Summary and Conclusions}

In this paper we have presented a model for automating viewing for teleoperation. We have discussed several techniques for realising this model using a multi-agent architecture, and have shown how this architecture can promote a flexible solution without incurring additional cost.

During teleoperation our system will track the progress of the operator and support him or her by providing views based on the visual acts model. This model is designed with stability as an important goal, in order that the environment remain firmly under the control of the operator. Man-machine interface design is a key tool in providing a symbiosis between the operator and the viewing system which maintains stability in the latter.

Using the peg-in-hole example, we are currently conducting tests on both single and dual operator controlled simulated teleoperation environments, and have identified several key aspects that warrant particular investigation. Our laboratory, which is well equipped for teleoperation applications, will allow us to expand our experiments to use real robotic manipulators and camera viewing systems.

\section{References}

[1] Brooks, B. and McKee, G. T. 1995, "Towards the Implementation of Visual Acts for Automated Viewing During Teleoperation", SPIE Intl. Symp. Intelligent Systems.

[2] Brooks, R. A. 1986. “A Robust Layered Control System for a Mobile Robot", IEEE J. Robotics and Automation, RA-2, pp. 14-23.

[3] Ferguson, I. A. 1992. "Touring Machines: An Architecture for Dynamic, Rational, Mobile Agents", Ph.D. Thesis, University of Cambridge, U.K., Technical Report No. 273, University of Cambridge Computer Laboratory.

[4] Hirai, J., and Nagata, T. 1994. "Agent-Oriented and Distributed Assembly Task Planning for Multiple Manipulators", Proc. IEEE Intl. Conf. Intelligent Robots and Systems, pp113-117.

[5] Haddadi, A. 1993. "A Hybrid Architecture for Multi-Agent Systems", Proc. Workshop Cooperation Knowledge Based Systems (CKBS-SIG), pp.13-25, Keele, U.K.

[6] McKee, G. T., and Brooks, B. 1996. "Interactive Robotics Using the Internet", Submitted Intl. J. Computer Science.

[7] McKee, G. T., and Schenker, P. S. 1995a. "Human-Robot Cooperation for Automated Viewing During Teleoperation", Proc. IEEE/RSJ Intl. Conf. Intelligent Robots and Systems, IEEE Computer Society Press, pp. 124-129.

[8] McKee, G. T., and Schenker, P. S. 1994. "Visual Acts for Goal-Directed Vision", Proc. SPIE Conf. Sensor Fusion VII, Vol. 2355, pp. 314-322.

[9] McKee, G. T., and Schenker, P. S. 1995b. "Visual Acts for Remote Viewing During Teleoperation", Proc. IEEE Intl. Conf. Robotics and Automation, Nagoya, Japan, pp. 53-58.

[10] McKee, G. T., Schenker, P. S., and Fryer, J., 1995. "Networked Robotics, Cooperating Agents, and an Application to "Visual Acts'", IEEE Workshop on Architectures for Intelligent Systems, Monterey, C.A.

[11] Schenker, P. S., Peters, S. F., Paljug, E. D., and Kim, W. S. 1994. "Intelligent Viewing Control for Robotic and Automation Systems", Proc. SPIE Conf. Sensor Fusion VII, Vol. 2355, Boston, M.A.

[12] Wooldridge, M., and Jennings, N. R. 1995. "Intelligent Agents: Theory and Practice", Knowledge Engineering Review. 


\title{
A Unified Approach for Fast Recognition, Learning and Interpretation of the Environment by an Autonomous Mobile Robot
}

\author{
Andreas Stopp and Lars Küttner \\ Daimler-Benz AG \\ Systems Technology Research \\ Alt-Moabit 96a, D-10559 Berlin, Germany \\ E-mail: stopp@DBresearch-berlin.de
}

\begin{abstract}
In this paper we briefly describe our approach to recognition and learning for autonomous mobile systems. Our system architecture is a multi-skill-oriented architecture. Each skill in our architecture comprises components for reflexive behavior and components for complex behavior. Complex behavior is represented by our Applied Memory-Based Reasoning approach (A-MBR). This is a scalable algorithm for fast recognition using a large extendable database containing sparsely coded knowledge. Application of AMBR to robot navigation is explained and learning principles are discussed. We are working on the extension to $3 \mathrm{D}$ recognition. We integrate principles from geometric hashing [2] and memory based reasoning [4] approaches known in the literature.
\end{abstract}

KEYWORDS: robot navigation, system architecture, learning, memory-based reasoning, geometric hashing,

\section{INTRODUCTION}

Autonomous systems such as robots are used for transport and service tasks in industry, manufacturing and space missions, where they have to solve various complex problems in the future. It will become necessary to recognize the real world, to handle large data sets, to learn unknown objects, environments, rules and behavior. A mobile system has to behave safely and reliably and to communicate at least on an interpretable level to make its behavior, plans and intentions transparent to cooperating systems and humans. To improve acceptance, the behavior should be safe and transparent to the user. The system should be able to react to unexpected events reliably and autonomously. Intelligent systems such as autonomous mobile robots need models for intelligent behavior besides the reflexive behavior. Applications are intelligent sensor-based control together with model-based predictive control for motion and manipulation tasks. For intelligent control we need improved fast prediction in the real-time range using realistic, large model bases. We need faster and more precise model matching and simplified model learning.

Section 2 describes the architecture as well as our framework for A-MBR. Section 3 gives a short overview of Recognition by Geometric Hashing. Section 4 is dedicated to our robot application. Subsequent sections describe recognition/learning principles and experimental results.

\section{SYSTEM ARCHITECTURE}

A schematic of our multi-skill-oriented architecture for learning systems is shown in Figure 1 . It is similar to the well-known reference architecture [1]. Each skill frame contains the functional blocks planning, reflexive behavior (RBF-like NN) and complex behavior (based on models).

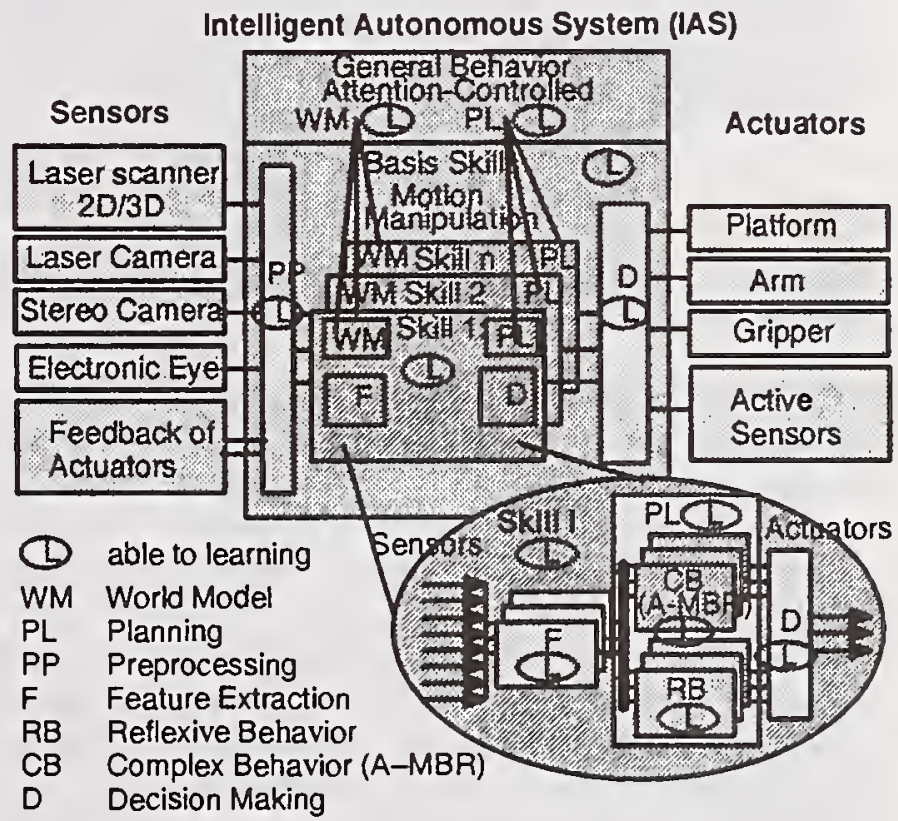

Figure 1 Schematic of system architecture 
In Figure I some functional blocks are labeled by the capital letter $\mathrm{L}$ which indicates that their knowledge representations are potentially open for learning. It would not be helpful to apply learning procedures to all knowledge representations in parallel but flexible access to all knowledge representations offers high potential for new learning principles. In this paper, we describe some aspects of our new Applied Memory-Based Reasoning (A-MBR) approach. This is our framework for handling and representing complex knowledge. A-MBR results in more intelligent behavior. It contains numerical and symbolic data of objects, relations, and rules. It is a large extendable database of sparsely coded knowledge. We use principles of the Memory-Based-Reasoning approach for handling knowledge such as relevance feedback. Data sets are derived from world models (e.g. CAD data) and from sensor data. Incomplete knowledge can be used. A-MBR provides symbolic and numerical entries into the database, which means at least symbolic input and symbolic output on the communication level and numerical input and numerical output on the control level (see Figure 2 ). The semantic layer is connected to the numerical data layer of our object model database even if planning models or planning maps are temporarily generated. In addition, the object database contains attributes such as docking points, grasping points and relations, rules, temporal logic, etc.

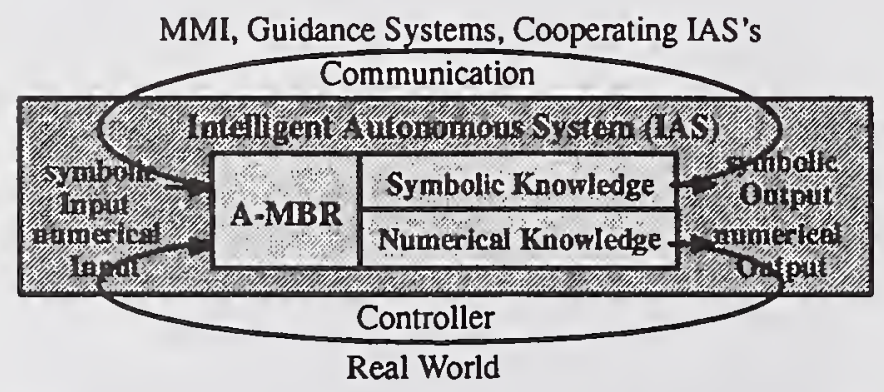

Figure 2 Schematic of A-MBR knowledge representation

Operator commands, messages from guidance systems, or messages from cooperating autonomous systems or from higher control levels, can be communicated to the symbolic level and can be interpreted by the planner. Cooperative planning is possible by communication on the common symbolic level and mapping into the local planner of each autonomous system, enabling consistency of a common view. Thus, recognition results, output messages and fault messages can be interpreted on the symbolic level and can be output via MMI to an operator or via messages to the guidance/planning hierarchy for continuing control or for fault treatment on a higher control level or for changing cooperation among autonomous systems. Unpredictable situations can be explained on the sym bolic level after recogni- tion. If the system learns, it is able to generalize learned information and transform it to the symbolic level. It is an open approach for complex task planning and execution. This includes task planning for different actions, skills for manipulation, and skills for motion.

\section{GEOMETRIC HASHING}

In our A-MBR framework Geometric Hashing as promoted by [3] is the preferred method for organizing the search for matches in the model-based vision system. It provides the search engine portion of the object recognition system.

A model or object is uniquely identifiable via its features. Provided that there exists an edge representation for the object, edge middle points can be taken as so-called point features. A feature may or may not possess an attached attribute list containing items such as edge orientation, length, or color. Edge representation and feature extraction form crucial inputs to the geometric hashing algorithm. With objects characterized through point features, the object recognition problem is equivalent to the problem of recognizing patterns of point features. An object may, however, have many features. To keep the pattern-matching task of reasonable complexity, the novel idea suggested in this paper - as opposed to the approach pursued by [3] - is to recognize an object on the basis of portions of an object. A portion is a fragment of an object containing only a bounded number of edges from a local neighborhood. The total number of different portions per object may vary but is roughly on the order of the number of edges that make up the object.

Before the process of pattern matching can commence, the portions must be normalized. The notion of a transformation base set is central in the scope of a portion. Such a base set uniquely determines a similarity-invariant transformation (translation, rotation, scale) with which the features of a portion will be transformed from their local coordinate system into the global hash space. Every normalized feature contains a reference to the portion it belongs to, which in turn contains the name of the original object as well as the transformation parameters. An object is thus multiply encoded by means of its complete set of normalized portions.

The recognition phase proceeds as follows. In a preprocessing stage, an edge segmentation algorithm is applied to the pixel image of the scene as recorded by the sensor. For every edge, the corresponding feature together with its attribute list is calculated. Next, a portion is composed as a collection of neighboring edges. From the portion's base set of points, a transformation matrix is determined with which all the features of this portion are normalized. This normalized portion to be recognized is now compared against all previously normalized portions stored in hash space on a feature-byfeature basis: every feature of the portion is compared 
against every other feature in its (hash-space) vicinity. Because noise has to be taken into consideration, the features pre-recorded in hash space are assumed to represent the mean value of a normal distribution $N(\mu, \Sigma)$ (with covariance matrix $\Sigma$ ) rather than an exact position. The hash space should be organized so as to provide quick access to all the features in the vicinity of a given point. This is achieved by subdividing the hash space into so-called bins (a mesh of small boxes with pointers to a list of all features that fall into the respective bin). Every feature in the vicinity of the portion's point feature gets a vote that increases with decreasing distance and approaches 1 as the distance approaches 0 . The following formula is introduced as a rough approximation for the vote a single feature may contribute: $v(d)=\exp (-\lambda$ d), where $d$ is the Euclidian distance and $\lambda>0$ an adjustable parameter. All the votes that are computed during a single hash table inquiry are accumulated on a per-portion basis (and not a per-object basis). The portions with the two highest accumulated votes are tracked down and compared against each other. A positive decision about a correct match can be made if (i) the absolute value of the vote of the top portion and (ii) the distance between the votes of the first and second portions exceed certain thresholds. Otherwise, a vector with the first $k$ "promising" portions (where $k$ may vary according to the distribution of the top votes) together with the votes they received is memorized, and the next hash table inquiry with a new portion of object features extracted from the scene is launched.

If recognition on the portion level is not possible (because of continued ambiguities), recognition on the object level might still be possible. In order to achieve this, the votes that were memorized at the end of each hash table inquiry could be accu mulated across inquiries on a per-object basis. In the end, one object might have received an overwhelming accumulated vote that distinguishes it from the others.

\section{ROBOT NAVIGATION}

A promising application field of our A-MBR approach is robotics and in particular navigation, relocation, position estimation, recognition/learning and planning as an integrated solution. Two examples illustrating most aspects are briefly described in Section 6.

Figure 3 shows the schematic of robot positioning by our A-MBR procedure using laser range data by means of known positions of objects (walls). In our first simulated scenario there are 16 rooms of different complex structure, few rooms are similar. New rooms can be generated and replace others. We can place the robot at any position in the 16 rooms with any direction. We start the relocation process by scanning the environment at the start position. Cyclic position estimation and position correction is performed by the A-MBR recognition (e.g. disturbances by mobile objects are tolerated up to a certain extent). Each hashed object portion knows its current position. The output is the name of the room and the current robot position (computed using recognition and current laser range data). In case of grasping or docking, we get the geometric data for the controller (grasping points, docking points) depending on the recognized object and its position.

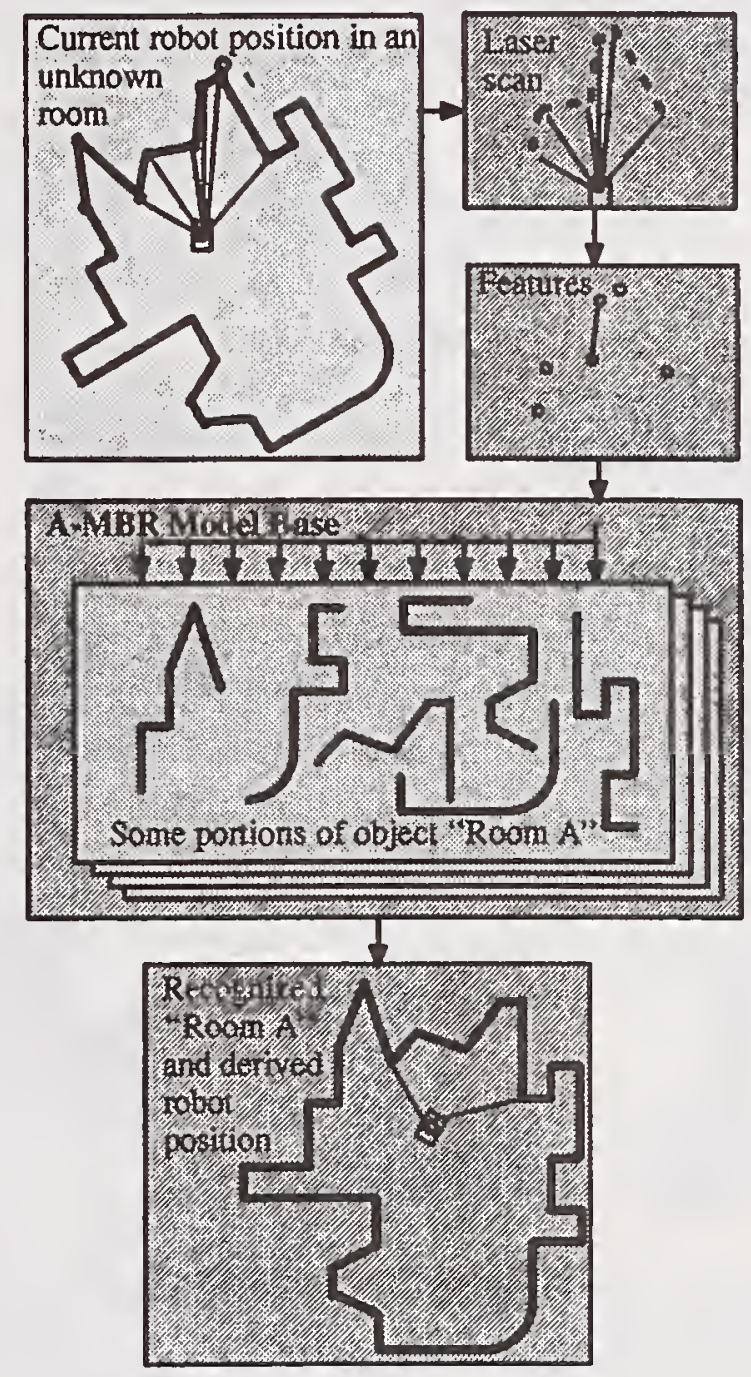

Figure 3 Object recognition for robot relocation

\section{RECOGNITION AND LEARNING}

A-MBR is able to recognize known objects, rooms and environments. Robustness allows the recognition of noisy or partially covered objects. Figure 4 shows a 2D example of a real laser scan of our laser sensor (180 degrees). Raw input data are preprocessed (first-step noise cancellation). Feature extraction detects lines and other features (depending on the 
context). In addition, features such as segmentation points and lines and points of further interest are derived. Figure 5 shows the simulated set of extracted feature data of one complete scan (360 degrees) at time $t$.

Recognition starts as described above with selection of information portions (feature neighborhood) from the feature data, depending on the focus of interest (e.g. object nearest to the sensor). The input data portion is compared to the hashed object data portions. Each portion belongs to an object concept and a well-defined name, or it belongs to a preliminary concept of new learned data.

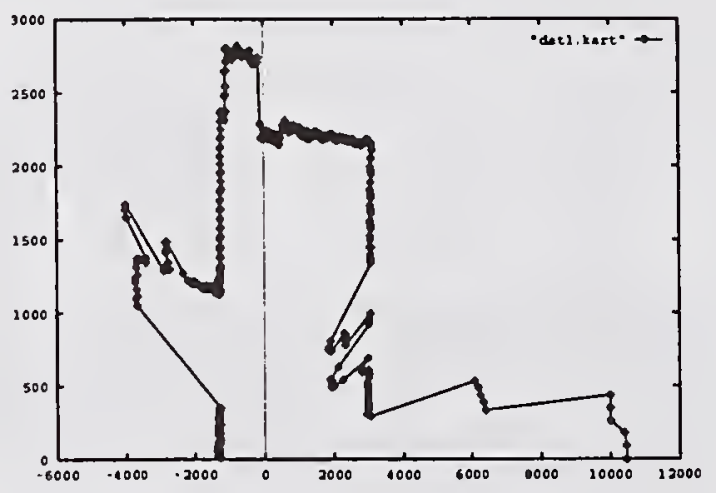

Figure 4 Example of a real laser scan

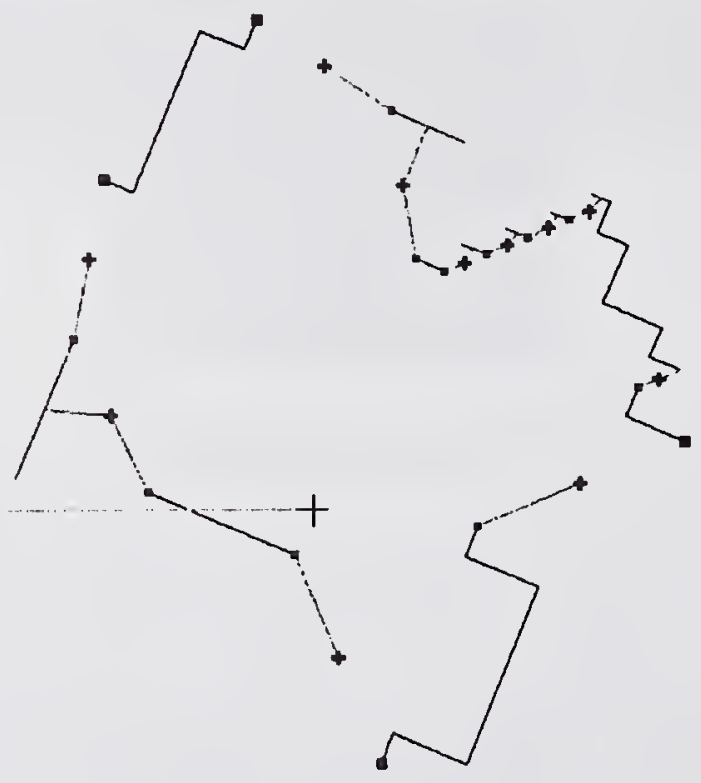

Figure 5 Extracted features of one laser scan (360 degree, robot coordinates)
As described above, the result is the number of matches. The further recognition process is controlled depending on the number of objects matching. Resulting answers are for example: too many, some, few, one, or no matches. If there is exactly one match with high probability against the rest of the data base, it is assumed to be correct. If there is more than one match, more sensor data are needed. No match means either the wrong model data set is used, or it is an unknown object and a candidate for learning. If results are not clear enough for precise decision making the recognition process continues self-controlled sensor data acquisition step by step. In most cases only one scan is required to achieve recognition under normal conditions (one-shoot recognition).

We introduced new learning procedures for A-MBR. Selfcontrolled data acquisition uses some preferred options for self-planned active sensing, e.g. turning/moving sensor head, moving/changing position, motion to the remaining points of interest, etc. Figure 6 shows the summarized data set of extracted features computed (merged) from some scanning cycles (and recognition/learning). One can see the more complete view compared to Figure 5 . We are accumulating sensor data on the feature level (merging numerical and symbolic features accompanied by failure correction).

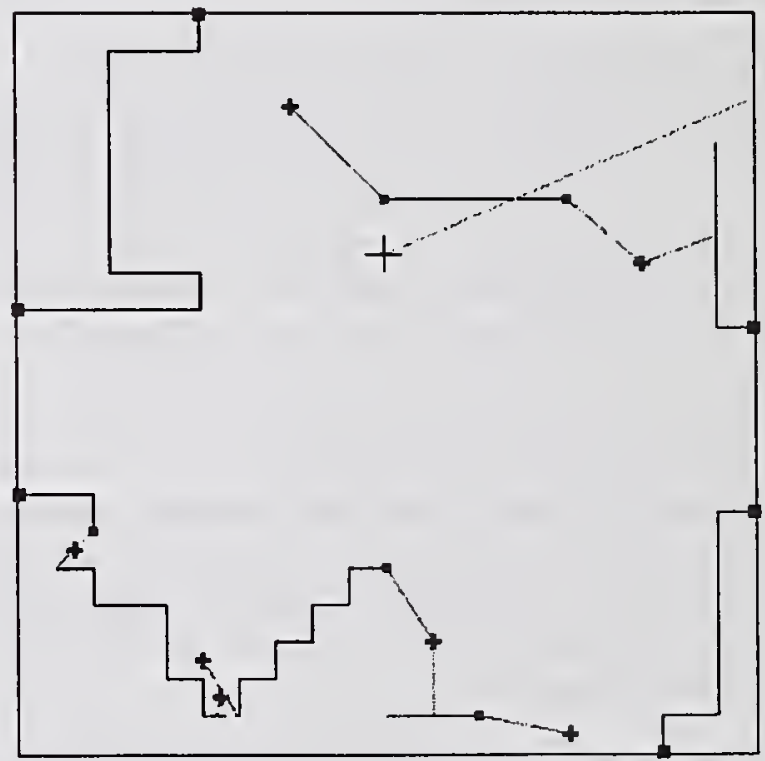

Figure 6 Summarized feature data of a still unknown object after a few scan cycles (memory coordinates) 
Besides detected lines there is additional information. In particular, the remaining points of further interest which are used for continuing recognition if needed.

If the current task is positioning or recognition, the process is stopped after successful recognition. The result is the name of the object/room and the current robot position. If we need higher reliability, or the object/room seems to be unknown, the process continues until all available sensor data are considered. After this exhaustive process (e.g. an object room has been completely scanned) the system has to decide whether the object/room has been known before to the system or not. Thus the system can store the summarized information as a candidate for learning, usable as preliminary vague data. Real learning should be accepted after verification, e.g., or entering a known area again, or after confirmation of relevance by higher-level instances. One can see there is no difference between the robust recognition procedure using all available methods for gaining information and the process of exploration of learning new sensor data and adding to the world model.

The most interesting and most important task is to find the best voting principles. This is an open research field. Different voting principles on different voting levels are applied, supported by voting on the symbolic level and on the level of relations and task context.

For systematic investigations of new voting principles and their recognition probability and for evaluation of robustness, we are able to tune the disturbance of the input data. With increasing disturbance the recognition/learning process becomes more uncertain. Thus, it takes more time for reliable decision making. The result confirms the robustness of the approach.

Concept generation (self-defined terms) for new learned data can be performed by taking the concept of the most similar data of the existing knowledge and adding some comments expressing similarity, uncertainty and the recognized main feature differences compared to the feature vector of the selected concept. Resulting information are e.g. "unknown object, but similar to object B differing in feature c". If there is no similar knowledge the simplest procedure is to append data to a nunning numbered list of unknown objects. Renaming of preliminarily named objects has to be done at a certain instant of time by considering more knowledge (via world knowledge) or by knowledge acquisition via MMI (communication to humans) using the quasi-symbolic description of features. This is a goal for future research.

Note that learning is done via sparsely coded knowledge data by A-MBR. Hash-table entries are generated from accepted A-MBR knowledge in order to keep all data consistent.

\section{SELF-CONTROLLED LEARNING}

Two examples describe the problems. In the first example, an autonomous system doesn't know where it is and it has to explore the environment in order to find any known objects or relocation points (the curious robot). If we start without knowledge and without GPS information it defines the current position, or a first good position (middle), of the unknown room as the origin of exploration (learning frame, preliminary coordinates). If a first known object/position is detected it is able to transform all learned objects/positions to the coordinate system of the known objects (knowledge frame, global coordinates). The learning process has to consider the transformation via coordinates of the robot and its sensors (robot frame, platform coordinates and sensor frame, coordinates of active sensor).
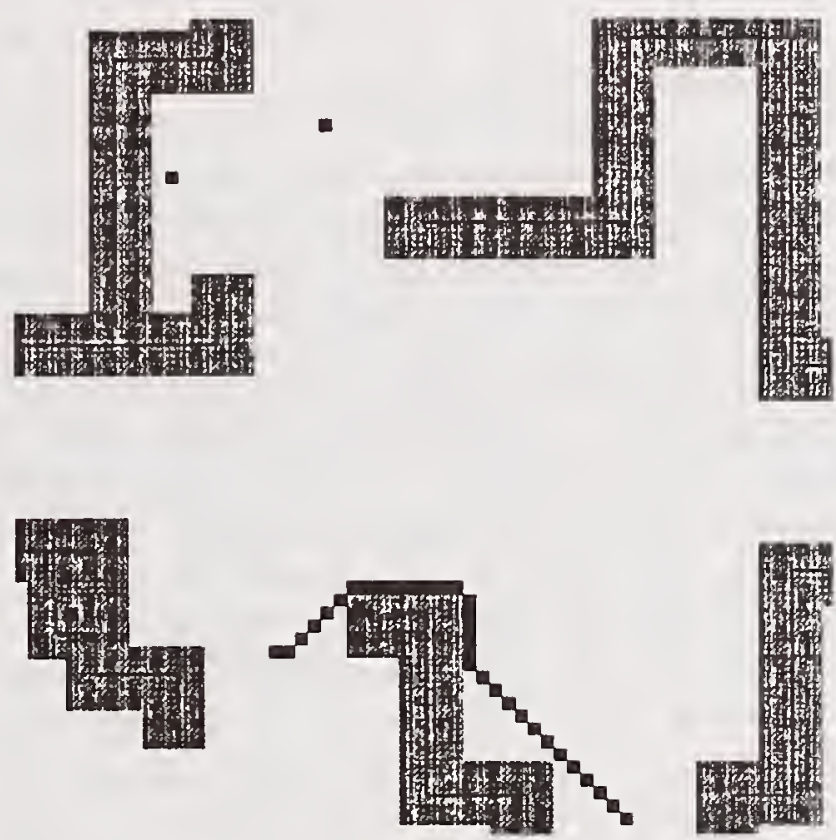

Figure 7 Derived planning map for exploration with the computed path from the current position (right) to the next point of interest (left)

A second example of a goal-oriented navigation (fire fighting robot) is not concerned with exhaustive exploration but with fastest motion toward one or more goal positions or centers of interest (valve, stopcock, center of fire, injured persons) It has an orientation toward the goal and learns onthe-fly. The first priority is the fastest move to the goal, but it learns differences of the map, closed gateways using old maps, or assuming free space. Its start position is known, direction or goal position is known, but the area between is unknown (destroyed). 
Both procedures are self-planning. Figure 7 shows an example of dynamic map generation and path planning for optimized recognition/learning/exploration process. Input to the planner is the latest update of the summarized information (features). The information is used to generate a gridbased planning model of desired size, granularity and desired value of obstacle growing. The planning process is performed after every new scan to plan an efficient way to the next point of interest (point of exploration) or even with a higher cycle rate. We integrated our planning approach [5] using a diffusion algorithm. The resulting path and laser range data are the input to the platform controller (reflexive knowledge) for collision avoidance and for following the path to the next point of interest. If the planner doesn't find a solution, map generation switches to the higher resolution level. Planning works with incomplete knowledge. All actions can be supervised, interpreted and reported using symbolic knowledge.

\section{EXPERIMENTAL RESULTS}

Current experiments are done on a Silicon Graphics. One complete cycle of recognition as described above takes less than one second for a normal scenario. Target hardware is parallel digital signal processor systems and multi-PC systems. Previous investigations on scalability and speed have been done on the Connection Machine CM-5 with 32 nodes and the data-parallel language $C^{*}$. Figure 8 shows the dependency of run time for object recognition (logarithmic) on the number of objects in the database (symbolic name of the object, here \# of the object in the database). The objects are polygons with a distinguished edge for control. The hashed object portions consist of 5 edges in this experiment.

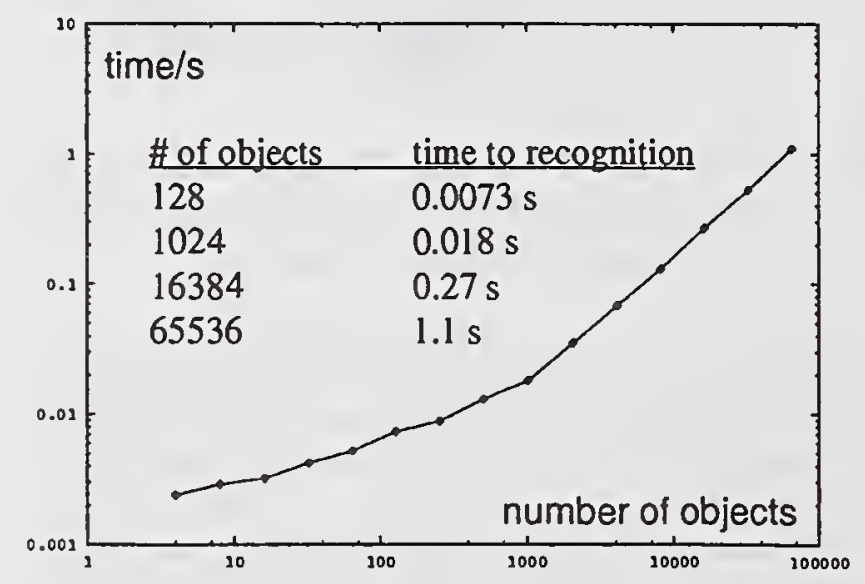

Figure 8 A-MBR - run time measurement on a parallel computer system - the Connection Machine CM-5 with 32 nodes

\section{CONCLUSIONS}

The A-MBR approach is able to support recognition and learning in a very fast and accurate way. It is an open approach for handling large data sets of sparsely coded and incomplete knowledge (numerical and symbolic). Our method provides a high functionality, in particular for robust recognition in a dynamically changing world of objects, for exploration of unknown space regions accompanied by robust path planning, and for interpreting actions and events on a symbolic level. The A-MBR method is scalable. Thus, the algorithm is simply mappable onto parallel architectures. Tests and simulations have been done on different hardware, e.g. Silicon Graphics, Multi-DSP's, CM-5. The target robot platform is equipped with a laser scanner (company Sick). Later, a laser image camera (a laser distance camera manufactured in our company) will be applied. We are working on the extension of our approach to recognition of 3D objects. On a Silicon Graphics, recognition of a simulated 3D object takes less than one second in a scenario of 4 selected objects and a database of 1024 objects. We intend to increase the A-MBR database with real 3D object data in combination with symbolic knowledge. We are investigating further extensions and new learning methods and plan to integrate them in our system architecture.

\section{REFERENCES}

[1] Albus, J. S., Meystel, A.M. "A Reference Model Architecture for Design and Implementation of Semiotic Control in Large and Complex Systems," in Proceedings of the ISIC-Workshop on Architectures for Semiotic Modeling and Situation Analysis in Large Complex Systems in Monterey, CA, Aug. 27-30, 1995

[2] Rigoutsos, I., Hummel, R. "Massively Parallel Model Matching," IEEE COMPUTER, Feb. 1992, pp. 33-42

[3] Rigoutsos, I., Hummel, R. "A Bayesian approach to model matching with geometric hashing," Computer Vision and Image Understanding, 62(1): 11-26, July 1995

[4] Stanfill, C., Waltz, D. "Towards Memory-Based Reasoning," Communications of the ACM, Vol. 29, Nr. 12, December 1986. pp 1213-1228.

[5] Stopp, A., Riethmüller, T. 'Fast Reactive Path Planning by 2D and 3D Multi-Layer Spatial Grids for Mobile Robot Navigation," Proc. of the 1995 IEEE Int. Symposium on Intelligent Control, Monterey CA, Aug. 1995. 


\title{
Learned Emergence of Functional Symbol Systems in Adaptive Autonomous Agents, Guided by Skinner's Analysis of Verbal Behavior
}

\author{
William R. Hutchison, Ph.D. \\ Behavior Systems LLC \\ 2002 Linden Drive, Boulder, CO 80304
}

\begin{abstract}
B.F. Skinner developed a detailed analysis of how the remarkable phenomena of language and symbol systems could emerge from the relatively simple processes of operant conditioning. The creation of computer models of operant conditioning- adaptive critic agents-finally enables this controversial theory to be tested. This paper describes simulations training such agents following procedures derived from Skinner's analysis, similar to those used to train humans. The simulations demonstrate several of the basic elements of symbol systems, but more importantly that they combine to produce significant emergent performances such as following memorized rules. Two simulations contradict one of the supposed limitations of conditioning theories of language, that they do not produce generalization to untrained situations. These results encourage further research in this parsimonious theoretical direction.
\end{abstract}

Keywords: emergence of language, computer simulations, operant conditioning, syntax, rules

\section{Introduction}

One of the main controversies about symbol systems and language is whether they can emerge from learning, and if so whether specialized brain mechanisms are required. B.F. Skinner's book, Verbal Behavior [19], presented an extremely detailed analysis of how an adaptive autonomous system can learn a complex symbol system based on selection of behavior by its consequences, i.e., operant conditioning. There are currently 800 professionals in a special interest group applying and expanding Skinner's analysis in research and clinical work. However, despite the considerable productivity of this theory, it has had little impact outside the field of behavioral analysis. The author attributes this lack of impact to the fact that verbal behavior research of this kind with human and animal subjects is inevitably open to question for two reasons: (1) The daunting complexity of variables cannot be reduced by imposing experimental controls with young human subjects as is done with animals, and (2) We do not know important properties of the system we are studying: for example, when a subject quickly learns a verbal function in our procedures, is it attributable to operant conditioning alone or is there also a specially-adapted brain mechanism?

Therefore, the recent development of computer systems that can simulate the learning processes of operant conditioning is especially valuable. Such computer "agents" enable us to overcome both of these problems: we can know the exact characteristics of an agent, and we can control the agent's environment through its entire history. At the least, such simulations can provide sufficiency tests of hypotheses such as Skinner's. Moreover, if the training process is similar to what humans experience, and if the acquisition process follows patterns like those of humans, the simulations provide a stronger argument that human verbal behavior is learned the same way. We advocated such research in the early days of neural networks [7,8], and have published earlier descriptions and research in $[7,11,12,13,21,22]$.

This paper gives an overview of seven years of research on the training of verbal behavior in adaptive autonomous computer models that simulate operant conditioning, along with more detail on three pertinent studies. We have used neural network models of the adaptive critic type $[1,15]$. The architecture is shown in Figure 1, and detailed descriptions and source code for our system is provided in [13]. The training has closely followed guidelines suggested by Skinner's detailed analysis, with exact training procedures specified in computer-based training (CBT) format. The agent and training software is written in Java and operates in environments written in VRML 2.0, though many of the older simulations have not yet been converted from the original Smalltalk versions.

\section{Overview of Skinner's Theory}

Skinner's analysis requires very limited assumptions about the properties of organisms: that they be capable of operant and classical conditioning. Operant conditioning could be roughly defined as the selection of behavior by the value of its consequences, where we assume the primary values have themselves been selected by a 


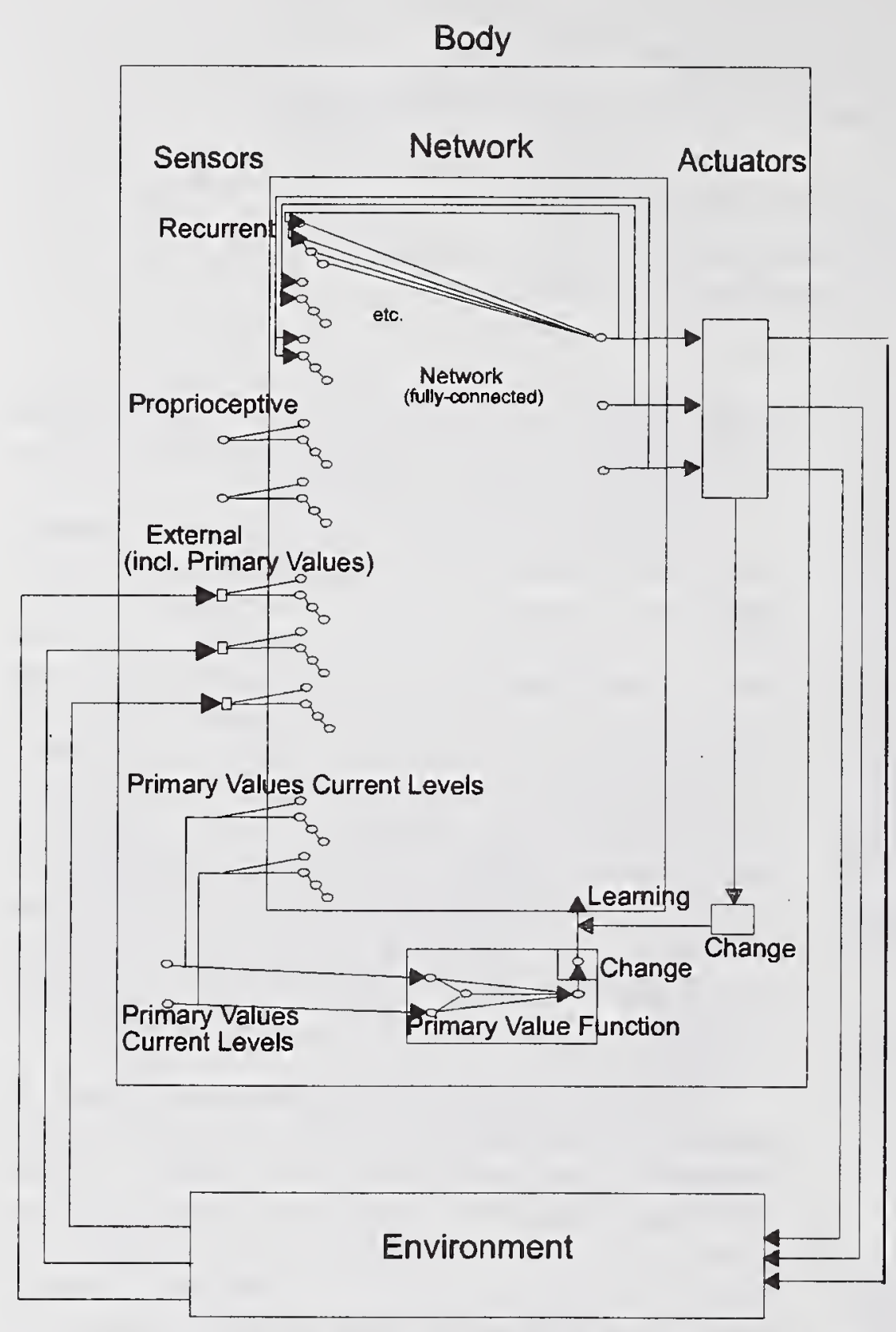

Figure 1. Agent architecture: a type of adaptive critic basic units are relatively simple and easily demonstrated (see below). Therefore, the two main open questions seem to be:

1. Do the basic units of verbal behavior actually combine to produce complex functional behavior?

2. Does learning generalize to untrained situations, as it must to explain human verbal behavior?

The basic units are the tact, intraverbal, mand, and autoclitic, along with a closely related unit which has been called pliance. A tact (cf. contact) is defined as a verbal response, similar to naming, controlled by environmental stimuli such as properties of objects or events (see Fig. 2a). Neural network researchers have easily demonstrated similar "categorizing" responses controlled by stimulus patterns, though the category is generally represented simply by which output is activated rather than by more complex naming responses. Tacts may be nouns, adjectives, verbs, adverbs, other parts of speech or smaller units such as the suffixes. An intraverbal (Fig. 2b) is a verbal response to a verbal stimulus, for example, saying "4" in response to hearing " 2 plus 2 is" or saying "Denver" in response to hearing "The capital of Colorado is". Intraverbals are often considered trivial, yet all explicit knowledge can be stated as intraverbals (cf. cognitive structures or world "models"). A mand (cf. command) is a verbal response with a characteristic consequence, which is therefore typically evoked by corresponding states of deprivation; e.g., asking for water when thirsty. An autoclitic is a verbal response controlled by a combination of verbal and nonverbal stimuli, and is therefore responsible for most grammatical genetic evolutionary process (e.g., water is reinforcing because organisms which are so reinforceable are more likely to survive; see $[4,20]$. Reinforcing events strengthen the connections between recently-emitted responses and the stimuli which were present. Secondary conditioning is very important in learning, and is nicely modeled in adaptive critic systems. None of these assumptions is very controversial either to adaptive agent modelers or to animal researchers. Note that the agent architecture in Figure 1 contains only elements necessary for operant conditioning, nothing specialized for symbolic processes.

Skinner proposed several basic units of verbal relations, and argued that the complexity of human verbal behavior and symbol systems is produced by variations and combinations of these units in complex environments. The structuring. Pliance (cf. compliance) is a nonverbal response to a verbal stimulus; e.g., lifting an arm upon hearing "Lift your arm" (Fig. 2c).

Each of these units has well-defined stimuli and responses, and they can be easily demonstrated in adaptive critic agents, as in the first simulation described. Many important verbal phenomena are variations of these basic units; for example, abstraction or analogy occurs when subsets of properties of objects control tacts, such as "animal" or "furniture." Other phenomena occur when variables combine in the complexity typical of the natural environment, such as tacting "heart attack" vs. "myocardial enfarction" depending on both the condition and the "audience" stimuli. However, we have suggested above that 


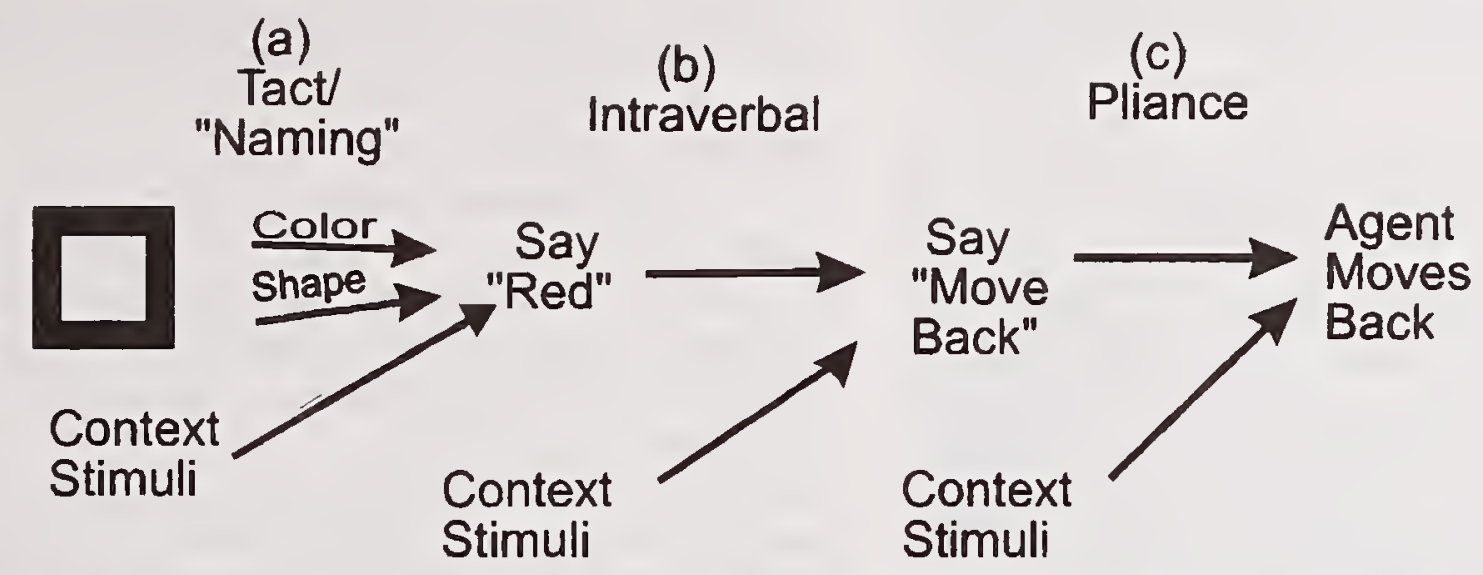

Figure 2. How Tact, Intraverbal, and Pliance Combine to Produce Rule-Following

the more challenging issues are whether these units combine to produce complex language-based functioning and whether they generalize to untrained situations. We will briefly describe simulations that give initial affirmative answers to both of these questions.

\section{Tact + Intraverbal + Pliance $\Rightarrow$ Rule-Following}

An extremely important function of language is enabling culture itself: the ability of humans to describe useful relations or rules to others, who can then behave effectively without having to learn personally through extensive and often dangerous direct experience. Werbos [24] has shown the power, and argued for the necessity, of "world models" in intelligent agents, but like most theorists he has created a hybrid system with a separate module for this function. Can we simulate this functional language using the verbal units described in a much simpler, operant system? Our formulation is as follows: verbal relations such as rules can be stated as intraverbals; an example we will use is "If red then move forward." For the intraverbal to be functional, its elements must have "meaning" to the agent; i.e. both the conditions (IF) and the actions (THEN) must connect appropriately to the real world. Logically, we look for two corresponding kinds of units: on the sensory side, we need a unit with environmental stimuli as inputs and with verbal responses as outputs; on the response side, we need a unit with verbal inputs and with actions on the environment as outputs. The tact meets the former requirements, and pliance meets the latter. Figure 2 shows how these units should fit together to produce rule-following behavior, according to this relatively simple formulation.

Upon sensing environmental stimuli, the agent utters a corresponding tact, which produces stimuli that evoke the intraverbal response; the intraverbal produces stimuli that instruct the appropriate action on the environment. Observations of humans $[6,18]$ suggest that humans often behave in exactly this way when following memorized rules.

In this simulation, the two rules were "If red then move forward" and "If green then move back." Our objective is for the agent to memorize the rules and learn the meanings of their elements and relations such that it can follow the rules the first time after learning them, without having ever done those behaviors in those conditions previously. First, we trained a naive agent to imitate the sounds A, B, F, G, I, M, R, and U by presenting the sounds and giving simulated calories if the agent produced corresponding sounds. Using those sounds as prompts which were then faded out, we trained the agent to tact the color of red, green, and blue objects, where saying "red" required the agent to say " $R$ " then " $A$ ", green was " $G$ " "I", and blue " $B$ " " $U$ ". For example, a red object was presented along with the sound sequence " $R A$ ", after which the agent's correct imitation of the sounds was reinforced. The " $\mathrm{R}$ A" prompt was faded until the agent could name the color without any prompt. Similarly, prompting was used to train the agent to say "R A M F" (= "red, move forward") and "G I M B" (= "green, move back"), with no colors present (Note that humans typically say rules in a minimal form without "If" or "then" [18]). Finally, we trained the agent to move forward or back after hearing and repeating the instructions "move forward" or "move back" ("M F", "M $B "$ ). The detailed training procedure is described in [11,12], and the exact code for agent and training are in [13]. The training procedures are like those a behavioral trainer might use with humans. At the end of this training, the agent moves forward when it sees red and moves back when it sees green, the very first time it sees these colors after learning the rules. This rule-following is mediated by the sequence 
of verbal responses as we predicted, exactly as observed with humans following rules.

\section{Does Learned Syntax Generalize?}

Two simulations have shown that, contrary to simplistic predictions $([3]$, but see $[14,17])$, this process of learning can produce grammatical ordering that generalizes to novel instances, as it must to explain human performance.

The first simulation shows that after learning only a few examples of saying adjectives (color) before nouns (based on shape), the agent will do the same in a novel case. In this simulation, the agent was first taught to tact blue, green, and red colors with unnamed shapes, using the same procedure as in the prior simulation. Similarly, the agent was taught to tact circle, square, and triangle shapes with unnamed color. Of the nine possible combinations of color and shape, eight were then trained by presenting the combined stimulus (e.g., a red triangle) and reinforcing only the correct sequence of verbal responses ("red triangle", or "R T" in the simplified language). It is very notable that even before the end of this training phase, the agent named two of these stimuli correctly the very first time they were presented. This cannot be explained by chance, since the agent's natural tendency is to repeat reinforced responses, so that it would say "R R" if it happened to say "R" first and received reinforcers, as occurred at this stage of training. When the ninth combination was presented, the agent used the correct grammatical ordering the first time it ever encountered a red circle, even in a test when hundreds of irrelevant responses occurred between the last training trial and the first test presentation.

Simulations enable us to analyze and understand surprising behaviors such as this. We have found that an extremely useful relation the agent learns is that, after saying something, saying it again will rarely produce reinforcement even though the conditions that evoked the response the first time are still present (e.g., red is still present). Not only is it generally true that listeners do not want to hear the same information twice, but suppression also functions as a modulator for syntax in many cases. In the red circle case, saying "red" suppressed saying it again so that saying "circle" would become relatively stronger. The architecture in Figure 1 enables this to be learned by the well-known mechanism of recurrence: the agent's responses produce stimuli for subsequent responses. This mechanism will show up again in the next simulation.

The second simulation addressed a more challenging situation. Can an operant agent learn to follow implicit grammatical rules to compose novel relational statements describing its environment? For example, can an agent learn to make descriptive statements of the type " $\mathrm{X}$ is left of $Y^{\prime \prime}$ when presented with pairs of objects in left-right relations, including the very first time either of the objects has been in that relation?

The test was for the agent to learn to say, for example, "Carp is left of tuna" when those objects were presented visually in that relation, even when neither object had ever been seen in that relation before. The agent was taught to name four visual patterns, called shark, tuna, carp, and jellyfish (named "S", "T", "C" and "J" in our simplified language). Then these objects were presented within its field of vision but not directly in front of its eyes, so that it learned to move its eyes to look directly at each object and then to name it (this procedure is consistent with the concept of "active vision" [2]). In the main training procedure, the agent received only 18 training trials of 2 cases each, one in which a shark was left of a tuna and one in which a jellyfish was left of a carp. The agent learned to look at the nearest object, name it, look left or right as necessary to look at the second object, say either "is leftOf" or "is rightOf" ("I L" or "I R") as demanded by the situation, then to name the second object. Correct responses were reinforced with simulated calories. After this brief training, a test situation was presented: a carp to the left of a shark. Even though those objects had not been seen in those positions before, the agent correctly said, "Carp is left of shark" ("C I L S"). How did it learn this generalized syntax after only 2 examples of it? As in the previous simulation, an examination of its network at each point in time showed how locally-available cues were adequate. Upon seeing an object, its strongest initial response is to orient toward it; then seeing it directly ahead, the strongest response is to name it. Naming it produces auditory and proprioceptive stimuli that suppress naming it again, so orienting toward the second object becomes the strongest response. Now there are two strong responses: one is to name the second object because the agent is now looking directly at it, and the other response is to say the relation "is leftOf" because of the stimuli from having just done the corresponding orienting response. Its history of reinforcement has produced a network that gives the relational response more strength, given this stimulus pattern. But after stating the relation, the responseproduced stimuli suppress saying it again and the naming becomes strongest. This is a very nice emergent solution because the agent can learn many different relational assertions without much mutual interference. Such control of relational statements by stimuli from the agent's own prior movements is quite general, according to Skinner's [19, pp 340-343] expansion upon Tooke's [23] argument that relational words generally have their roots in actions. 


\section{Other Verbal Phenomena That Have Been Simulated}

The following is a listing of verbal phenomena the author has simulated, including ones described above:

Generalized echoic behavior: imitation of sounds

Generalized textual behavior: reading from text

Tacting: naming objects, actions, properties of objects and actions

Intraverbals: verbal associations, phrases, sentences, rules

Instruction-following ("Move back"-> move back)

Generalized word ordering (adjectives before nouns: "blue circle")

Generalized relational descriptions of scenes ("Tuna is left of shark")

Generalized identification of matching vs. non-matching pairs of objects (Are 2 objects the same?)

Same, but with delay between stimulus presentations

Sequences of verbalizations maintained by reinforcement only at the end of the sequence

Symmetry (learn " $A=B$ ", say "B = A")

Transitivity (learn "A implies B" and "B implies C", say "A implies C" )

Generalized compliance with memorized rules when condition is first encountered

Interplay of rule-following and direct learning from experience

Creativity: recombine learned responses to solve problems [9]

Rapid training of verbal responses using prompt and fade procedures, as used with humans

Some of the verbal phenomena we would like to simulate in the near future include: using real voice input and articulatory speech output; more complex grammar (e.g., "The cat that ate the mouse jumped"); using larger, English-like words; training large verbal repertoires; generalized use of "not"; rhymes and puns; self-editing; combining relational statements ("Frog is on log" + "Log is beside tree"); and tacting of private events such as pain.

\section{Discussion}

Our simulations serve as sufficiency proofs that adaptive autonomous agents of the adaptive critic class can learn important and fairly complex functions of symbol systems with proper training. A critical property of this analysis is that it brings language into a larger biological/economic framework: Each set of behaviors produces consequences sufficient to maintain it, or equivalently, it contributes to the fitness of the organism. Rule-following generally produces valuable results, since people learn to discriminate reliable from unreliable rulegivers, as an inherent part of operant learning. In contrast, mechanistic theories of rule-following which produce automatic compliance fail to explain how people learn to avoid bad advice. Even more stringently than overall value, Skinner's theory requires that every action of each agent at each point in time be learnable and sustainable by actual consequences [19, pp. 84-90], a point emphasized in a simulation of a two-agent ecology in [10]).

Even if the training used in these simulations is characteristic of disciplined behavioral training techniques, some critics may object that it is uncharacteristic of human experience. While typical caregivers are not as consistent as professionals, observations show that humans provide extensive language training in the natural course of interacting with children [16]. For example, a parent might show a child blue objects, say "Blue" to each, and show a positive reaction to the child's saying anything approximating "Blue." In subsequent cases, prompts will typically be made weaker and better pronunciation will be required.

The results of these simulations support Skinner's analysis, while its consistency with a broad biobehavioral perspective gives the theory further appeal. The scientific principle of parsimony compels us to pursue the hypothesis that symbolic language functions emerge from accepted processes of operant conditioning without any need for language-specific brain mechanisms.

\section{References}

[1] Barto, A. Reinforcement learning and adaptive critic methods. In D.A. White \& D. Sofge (Eds.), Handbook of Intelligent Control: Neural, Fuzzy, and Adaptive Approaches. Van Nostrand, 1992.

[2] Blake, A. Active vision. In M. Arbib (Ed.), Handbook of brain theory and neural networks. Cambridge, MA: MIT Press, 1995.

[3] Chomsky, NA Review of Verbal Behavior, by B.F. Skinner. Language, 35, 26-58, 1959.

[4] Donahoe, J.W., Burgos, J.E., \& Palmer, D.C. A selectionist approach to reinforcement. Journal of the Experimental Analysis of Behavior, 60, 17-40, 1993.

[5] Donahoe, J.W. \& Palmer, D.C. (1994) Learning and complex behavior. Boston: Allyn \& Bacon.

[6] Horne, P. J., \& Lowe, C. F. On the origins of naming and other symbolic behavior. Journal of the Experimental Analysis of Behavior, 65, 185-243, 1996. 
[7] Hutchison, W.R. Cognitive vs. Behavioral Psychology in Artificial Intelligence. Newsletter for Behavioral Artificial Intelligence, December, 1984.

[8] Hutchison, W.R. \& Stephens, K.R. Integration of distributed and symbolic knowledge representations. Proceedings of the first international conference on neural networks, 2, 395-398. IEEE Press, 1987.

[9] Hutchison, W.R. \& Bloomquist, L. Novel or stereotyped behavior patterns as a function of subsistence level of reinforcement in neural networks and animals: Necessity is the only mother. Presented at the Association of Behavior Analysis Conference, Atlanta, May, 1995.

[10] Hutchison, W.R. Demonstration of work from the 7G Computer Modeling of Behavior Project. Presented at the Third Simulation of Adaptive Behavior Conference, Brighton, England, August, 1994.

[11] Hutchison, W.R. Teaching an Agent to Speak and Listen with Understanding: Why and How? Online proceedings of the Intelligent Information Agents Workshop, CIKM, Baltimore, Dec. 1-2, 1995. http://www.cs. umbc.edu/ cikm/iia/submitted/viewing/whutc hi.html

[12] Hutchison, W. R. Demonstration of Training Imitation, Language, and Cognition in a Behavior-based Agent. Presented at the Fourth Simulation of Adaptive Behavior Conference, Cape Cod, MA, September, 1996.

[13] Hutchison, W.R. Simulations of operant behavior; software and documentation, v.1.0. Cambridge, MA: Cambridge Center for Behavioral Science, 1997.

[14] MacCorqudale, K. On Chomsky's review of Verbal Behavior. Journal of the Experimental Analysis of Behavior, 13, 83-89, 1970.

[15] Miller, W.T., Sutton, R.S., \& Werbos, P.J. (Eds.) Neural networks for control. Cambridge, MA: MIT Press, 1990.

[16] Moerk, E.L. A first language: Taught and learned. Baltimore, MD: Paul H. Brookes, 1992.

[17] Palmer, D. Chomsky's nativism: A critical review. In P.N. Chase \& L.J. Parrott (Eds.), Psychological aspects of language (pp. 49-60). Springfield, IL: Charles Thomas, 1986.

[18] Potter, W. F. Comparison of Selection-based and Topography-based Verbal Behavior. Doctoral dissertation, Western Michigan University, 1996.

[19] Skinner, B.F. Verbal behavior. New York: AppletonCentury-Crofts, 1957. (currently available from B.F. Skinner Foundation)

[20] Skinner, B.F. The ontogeny and phylogeny of behavior. Science, 153, 1203-1213, 1966.

[21] Stephens, K.R. \& Hutchison, W.R. Behavioral personal digital assistants: the seventh general of computing. The Analysis of Verbal Behavior, 10, 149-156, 1992.
[22] Stephens, K.R. \& Hutchison, W.R. Behavior analysis and the quest for machine intelligence. Educational Technology. 52-61, October, 1993.

[23] Tooke, J.H. The Diversions of Purley. London, 1857.

[24] Werbos, P.J. Consistency of HDP Applied to a Simple Reinforcement Learning Problem. Neural Networks, 3, 179$189,1990$. 
XI LIKELIHOOD, MIXTURE, SYMBOLS 



\title{
TOWARDS QUANTUM FIELD THEORY OF SYMBOL
}

\author{
Leonid I. Perlovsky
}

\author{
Nichols Research Corporation, 251 Edgewater Dr., Wakefield, MA 01880 \\ Tel.617-246-4200, Fax 617-246-0065, E-mail nrcbmo@world.std.com
}

\begin{abstract}
A mathematical theory of a symbol as an adaptive process is presented. It is founded on the theory of modeling fields, in which learning is based on adaptive internal models. Quantum computation algorithms of the modeling field theory are developed. I discuss computational, philosophical, and physical aspects of the dynamic theory of the symbol.
\end{abstract}

KE Y W OR D : semiotics, symbol, computational intelligence, quantum computation, neural networks, internal models, learning algorithms, philosophy of mind

\section{INTRODUCTION}

The popularity and subsequent fall-out-of-favor of "Symbolic AI" left many researchers with a bad taste towards the word "symbol". But, an impression that "Symbolic AI" represented the mathematics of symbols is very wrong. A symbol is not a monumental piece of a bronze sitting on a foundation of stone. A symbol is a fleeting vortex of the interacting perceptions, feelings, a priori models, adaptation, attention, behavior and concept formation. In other words, a symbol is a process. It is a process of thought.

Semiotics is a science devoted to studying signs and symbols. The founders of semiotics (Pierce, 1935; Morris, 1971) introduced the notion of a sign as a trilateral unity of sign-vehicle, (the media used as a sign), designatum (the object which the sign refers to) and interpretant (or interpreter, a mind or an intelligent system, which interprets a signvehicle). The process of interaction within this triadic unity is called semiosis. Morris decomposed this process into the three dyadic relationships-processes: syntactics (relations among sign vehicles), semantics (relations between signvehicles and their designata), and pragmatics (relations between sign-vehicles and their interpreters). A contemporary tendency is to use a word semiosis for the learning process at a system level involving multiple triadic processes. When a single triadic process is concerned, a word sign is used in relatively simple, non-adaptive cases of semiosis, when there is an a priori known and fixed straightforward way of relating a signvehicle to designatum (such as a look-up table); and, a word symbol is used in complicated cases of a single-triadic semiosis, when an adaptive process is needed to establish the relationship; this adaptive process of semiosis is a symbol.

According to Pribram (1971), signs within the brain are acts of communications that are invariant to the context, while symbols are context dependent. Signs are the results of the associative cortex affecting the input sensory systems. These are the a priori, less-adaptive aspects of the internal models.
Symbols, according to Pribram are the results of interaction between frontal lobes and limbic system, they are stimulants to actions and are sensitive to context.

In this paper, mathematical and physical theories of the symbol are developed. They are based on the modeling fields theory (MFT) developed previously by the author (Perlovsky, 1996; Perlovsky et al, 1997). MFT combines a priori knowledge and adaptivity of the internal model. The global model describing "the world" is composed of multiple "local" models (similar to a visual field being composed of edges, etc.). MFT establishes a correspondence between subsets of local models and subsets of input data, while adaptively learning models. This process is similar to a central problem in several areas of artificial intelligence and pattern recognition [Winston, 1984; Negahdaripour \& Jain, 1991; Segre, 1992]. Traditional methods of solving problems of this kind lead to algorithms of exponential complexity, which are not physically realizable even for problems of moderate complexity, and which can not therefore serve as physical models of intellect. MFT leads to algorithms of linear complexity. A computational system of MFT is composed of multiple interacting loops adapting individual models and competing for evidence in the input data. Each loop involves an individual internal model, a measure of its similarity with the data, and its adaptation mechanism that increases the similarity. Each loop is a semi-independent intelligent agent performing an adaptation-recognition of a category. It involves a sign-vehicle that is the category name (or internal MFT message-code), a designatum that is a subset of input data corresponding to the category, and interpretant that is the MFT system that interprets and acts upon the recognized category. Thus, MFT agents recognizing and adapting categories are dynamic symbol-processes. The dynamics of agent-symbols, the process of semiosis, is composed of syntactics, semantics, and pragmatics.

Where are the dynamic symbol processes physically realized in the brain? A traditional interpretation is that they are realized by networks of neurons, such as described in (Perlovsky et al, 1997). An alternative interpretation is that each neuron has a microstructure capable of performing complicated computations. These sub-neuron computations are hypothesized to be performed by quantum process taking place in microtubular neuronal substructure [Hameroff \& Watt, 1983]. Keeping in mind this possibility, I describe a quantum system implementing MFT. The next Section 2 summarizes the mathematics of classical MFT. Section 3 describes quantum computation MFT algorithms, QMFT. Section 4 discusses results and relationships between mathematics, Kantian theory of mind, and classical semiotics. 


\section{MODELING FIELD THEORY}

MFT considers (1) a set of input data $\{\mathbf{X}(n), n \in N\}$, where each member is a vector in D-dimensional space, $\mathbf{X}=$ $\left\{X_{d}, d=1, \ldots D\right\} ;(2)$ a set of adaptive categories $\{h \in H\}$, which are characterized by internal parameters $\left\{\mathbf{S}_{\mathrm{h}}\right\}$ and by models of the data $\left\{\mathbf{M}_{\mathrm{h}}\left(\mathbf{S}_{\mathrm{h}}, \mathrm{n}\right)\right\}$; and (3) a similarity measure between the sets of models and data, $\mathrm{L}(\{\mathbf{X}\},\{\mathbf{M}\})$. A set of parameters is finite, $S_{h}=\left\{S_{h}^{a}, a=1, \ldots A\right\}$, but not necessarily limited, and a set of categories is not fixed, its cardinality $\mathrm{H}$ is finite, but may vary in the process of learning. A similarity measure is designed so that it treats each model as an alternative for each piece of data

$$
L(\{\mathbf{X}\},\{\mathbf{M}\})=\prod_{n \in N} \sum_{h \in H} r(h) \iota(\mathbf{X}(n) \mid h),
$$

here $\iota$ is a conditional partial similarity between data vector $\mathbf{X}(\mathrm{n})$ and model $\mathbf{M}_{\mathrm{h}}$. For example, $\iota$ can be selected as a probability density function. Then $\mathrm{L}$ is a total likelihood (this interpretation does not require statistical independence among data vectors $n$ and $n '$ : dependencies can be accounted for by considering $\mathbf{X}\left(\mathrm{n}^{\prime}\right)$ as parameters of the models for the data vector $\mathrm{n}$ ).

The problem consists in concurrent recognition and adaptation of categories. Adaptation is achieved by estimating internal parameters $\mathbf{S}$, and recognition consists in obtaining a partition of data among categories,

$$
\{N\}=\left\{N_{1}\left|N_{2}\right| \ldots \mid N_{H}\right\},
$$

that decides, which model $\mathrm{h}$ corresponds to an observation $\mathrm{n}$ (here $\mathrm{N}_{h}$ is a subset of $\mathrm{N}$ ). The recognition and adaptation process shall maximize the similarity (1). When likelihood is used as a similarity measure, this is a problem of the maximum likelihood estimation.

Categories activated by high similarity values produce actions, including generation of messages transmitted within the intelligent system, which acknowledge the category activation. Messages include the category name code and model parameters. They can serve as input data for other categories and can be used for control of actuators. Correspondingly, data are sensory data or internal messages, and models represent patterns of messages and sensory data. The above mathematical framework is fairly broad and encompasses most formulations of intelligent adaptive systems. It includes as particular cases, statistical and modelbased pattern recognition, complex adaptive systems, neural networks, and systems of intelligent agents. The above formulation addresses "higher-level" intelligence functions of recognition and adaptation. A compete intelligent system would include lower-level drives for survival, reproduction and, in case of an artificial system, a robot or infobot, drives for producing specific tasks it was designed for. Each individual model, $\mathbf{M}_{\mathrm{h}}$, together with the process of its adaptation and recognition defines a symbol-process.

In case, when a set of observations, $\mathrm{N}$, corresponds to a continuous flow of signals, for example, a flow of visual stimuli in time and space, it is convenient instead of eq.(1) to consider its continuous version,

$\mathrm{L}=\exp \int_{\mathrm{N}} \ln \left(\sum_{\mathrm{h} \in \mathrm{H}} \mathrm{r}(\mathrm{h}) \iota(\mathbf{X}(\mathrm{n}) \mid \mathrm{h})\right)$,

where $\mathrm{N}$ is a continuum, such as time-space. In this case, models describe a continuous modeling field, conditional partial similarities can be compared to Lagrangian, and maximization of similarity $\mathrm{L}$ can be compared to minimization of action in the physical field theory.

MFT solution of this problem uses fuzzy adaptive logic [Perlovsky, 1996]. Adaptive fuzzy class memberships f(hln), associating each data vector $n$ with each category $h$ are defined as:

$$
f(h \mid n)=r(h) \iota(X(n) \mid h) / \sum_{h^{\prime} \in H} r^{\prime}\left(h^{\prime}\right) \iota\left(X(n) \mid h^{\prime}\right)
$$

In case of the maximum likelihood estimate, upon convergence of the estimation procedure, $f(h \mid n)$ can be interpreted as a posteriori probabilities. An internal dynamics of the Modeling Fields (MF) is given by

$$
\begin{aligned}
& \mathrm{H} \\
& \mathrm{df}(\mathrm{h} \mid \mathrm{n}) / \mathrm{dt}=\mathrm{f}(\mathrm{h} \mid \mathrm{n}) \sum_{\mathrm{h}^{\prime}=1}\left\{\left[\delta_{\mathrm{h}^{\prime}}-\mathrm{f}\left(\mathrm{h}^{\prime} \mid \mathrm{n}\right)\right] \cdot\right.
\end{aligned}
$$

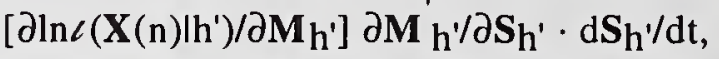

$$
\begin{aligned}
& \mathrm{d} \mathbf{S}_{\mathrm{h}} / \mathrm{dt}=\left\{\int_{\mathrm{N}} \mathrm{f}(\mathrm{h} \mid \mathrm{n})\left[\partial \ln c(\mathbf{X}(\mathrm{n}) \mid \mathrm{h}) / \partial \mathbf{M}_{\mathrm{h}}\right] \partial \mathbf{M}_{\mathrm{h}}^{\prime} / \partial \mathbf{S}_{\mathrm{h}}\right. \text {, }
\end{aligned}
$$

where

$\delta_{h^{\prime}}$ is 1 if h=h', 0 otherwise.

Parameter $t$ is the time of the internal dynamics of the MF system. A theorem was prooved that equations (5) through (7) define a convergent dynamical system of MF with stationary states defined by $\max _{\left\{S_{h}\right\}}$ L. It follows that the stationary states of an MF system give the maximum similarity solution of the category adaptation and recognition problem. When likelihood is used as a similarity, the stationary values of parameters $\left\{\mathbf{S}_{\mathbf{h}}\right\}$ are asymptotically unbiased and efficient estimates of these parameters [Cramer, 1963]. A computational complexity of the MF method is linear in $\mathrm{N}$,

complexity $(\mathrm{MF})=\mathrm{C} 2 \cdot \mathrm{H} \cdot \mathrm{N}$,

where $\mathrm{C} 2$ is defined by the relaxation time of the MF system and is likely to be independent of $\mathrm{N}$ (or depends weekly). In this way the MF system solves the problem of the exponential complexity associated with traditional methods. Fuzzy class memberships $f(h \mid n)$ define a fuzzy partition of set $\mathrm{N}$, which can be used to define a non-fuzzy partition of the type of eq.(4): $\forall \mathrm{n}, \max _{\mathrm{h}} \mathrm{f}(\mathrm{h} \mid \mathrm{n}) \Rightarrow \mathrm{n} \in \mathrm{N}_{\mathrm{h}}$.

MFT describes an intelligent system composed of 
multiple adaptive intelligent agents-symbols: each categorymodel-symbol is "dormant" until activated by a high similarity value. Every piece of data may activate several categories-symbols, which "compete" with each other, while adapting to the new piece of data. Adaptation and other actions by activated symbols are largely independent from most other symbols, except for those competing with each other. Most important actions by activated symbols are transmission of messages and sustaining the loop of adaptation of their own parameters. Evolutionary computation is naturally incorporated within MFT: transmitted messages, including category parameters and other aspects of categorymodel-symbols are strings-objects of evolutionary computations and are employed by modeling field systems for adaptation of its structural components that could not be incorporated in a continuous fashion and are not the subjects of adaptive MFT symbol-loops. The overall architecture of MFT categories-symbols can combine heterarchical and hierarchical organization.

\section{QUANTUM MODELING FIELD THEORY (QMFT)}

It is possible that quantum processes play a role in neuronal adaptation [Hameroff \& Watt, 1982]. Therefore, apart from implementing MF as a computer algorithm it is interesting to consider a possibility of realization of $\mathrm{MF}$ as a quantum system, QMF. Quantum computing received significant attention as a potential method of breaking through the limitations of classical computing paradigms since Feynman [1982, 1986] draw the attention to this area of research. The following two limitations of classical computational systems are expected to be surpassed. First, classical systems dissipate a finite amount of energy $(\sim \mathrm{kT})$ per 1 bit for every operation. Second, a number of important problems in classical computational intelligence, in the number theory, and in other fields are very hard in that their solutions require an exponentially large amount of computational steps as a function of the problem complexity. (While the MF discussed in the previous section addresses the exponential computational complexity of the symbol adaptation, still quantum algorithms promise tremendous advantage in computing power). Quantum computing is expected to surpass classical computing for the following reasons. First, quantum computation proceeds without energy dissipation until the process of quantum measurement, which potentially can be postponed until the end of the computation process. Second, a quantum system can exist in a superposition of multiple states, so that multiple computational paths (including all possible combinations of path segments) can potentially be performed in parallel, within a single process of quantum interference between the quantum system states. In QMF, an internal dynamics of the symbol adaptation occurs as a process of quantum interference (in place of eqs. $(4,7)$ ), and a partition similar to eq.(2) is obtained in the process of quantum measurement. Let us outline the main characteristics of QMFT.
QMFT describes a system which is characterized by quantum states $\mathrm{I} \mathrm{h}>$ and which interacts with the external world characterized by quantum states $|n\rangle$. The entire system including the QMF system and the external world, in the general case, is described by a quantum state which is a superposition of states

$(|\mathrm{h}>| \mathrm{n}>)$,

$\Psi(t)=\int_{N^{*}} \sum_{h=1}^{H} C_{h n}|h>| n>$.

Here integration over $\mathrm{n} \in \mathrm{N}^{*}$ includes spatial (and possibly other coordinates of the external world) but excludes time, $t$. Because of the probabilistic nature of quantum theory, we consider similarities given by likelihoods, and fuzzy class memberships being probabilities. Quantum amplitudes $\mathrm{C}_{\mathrm{hn}}$ are related to the probabilities defined in eq.(4) according to the rules of quantum theory

$\mathrm{f}(\mathrm{h} \mid \mathrm{n})=\left|\mathrm{C}_{\mathrm{hn}}\right|^{2}$.

Generally, the QMF system is in a mixed state and is described by the density matrix [Neumann, 1910; Sakurai, 1985],

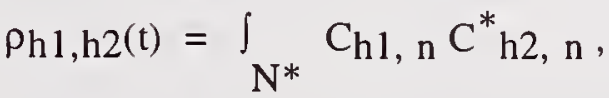

or, equivalently, by the density operator

$\rho(t)=\sum_{h 1 ; h 2=1}^{H}\left|h 1>\rho_{h} 1, h 2<h 2\right|$.

Equations of motion are given by a Hamiltonian, therefore the next step is to define the Hamiltonian in such a way that the dynamics of a QMF system would lead to a solution of the considered problem of the dynamic symbol adaptation. Two types of quantum systems are considered below, first, a nonequilibrium quantum statistical system evolving to Gibbs distribution and second, deterministic Hamiltonian quantum dynamical system.

\subsection{Gibbs Quantum Modeling Field System}

Consider the conditional similarities to be given by conditional probability distribution functions, or conditional likelihoods. Let us define the Hamiltonian so that the QMF dynamics would lead to a Gibbs distribution with probabilities defined by the similarity eq.(1). Following well known principles of the quantum statistical physics [Feynman, 1972; Zubarev, 1971], let us define the Hamiltonian through its relationship to the pdf. The dynamical variables of this system are unknown parameters $\left\{\mathbf{S}_{\mathrm{h}}\right\}$, while the data values $\mathbf{X}(\mathrm{n}), \mathrm{n}=1, \ldots \mathrm{N}$, are fixed, therefore the Hamiltonian, 
$H=-\ln \operatorname{pdf}\left(\left\{S_{h}\right\} \mid \mathbf{X}(1), \ldots X(N)\right)$.

Using Bayes' theorem,

$\operatorname{pdf}\left(\left\{\boldsymbol{S}_{\mathbf{h}}\right\} \mid\{\mathbf{X}(\mathrm{n})\}\right)=\operatorname{pdf}\left(\{\mathbf{X}(\mathrm{n})\} \mid\left\{\boldsymbol{S}_{\mathbf{h}}\right\}\right) \operatorname{pdf}\left(\left\{\boldsymbol{S}_{\mathbf{h}}\right\}\right) / \operatorname{pdf}(\{\mathbf{X}(\mathrm{n})\})$.

Here $\operatorname{pdf}\left(\left\{\mathbf{S}_{\mathrm{h}}\right\}\right)$ can be considered constant in absence of prior information concerning values of these parameters. In this case, $\operatorname{pdf}(\{\mathbf{X}(n)\})$ is also constant, because in absence of a priori values of these parameters it has to be invariant. Thus the Hamiltonian can be written as

$\mathrm{H}=-\ln \operatorname{pdf}\left(\{\mathbf{X}(\mathrm{n})\} \mid\left\{\mathbf{S}_{\mathrm{h}}\right\}\right)+$ const.

Comparing eq.(15) with eq.(1), we conclude that in absence of a priori information on the parameter values, the Hamiltonian is local and the Hamiltonian density $\mathscr{H}$ can be introduced

$\mathcal{H}=-\ln (\operatorname{pdf}(\mathbf{X}(\mathrm{n}))), \quad \mathrm{H}=\int_{\mathrm{N}(\mathrm{t})} \mathcal{H}+$ const.

Here $N(t)$ stands for a set of observations available at time t. This underlines the fact that the Hamiltonian is defined using past data only (in case when $n$ includes time). It is a nontrivial fact that availability of a priori values for the parameters may result in a non-local Hamiltonian.

The Hamiltonian eq.(16) defines the dynamics of the density operator [Feynman, 1972],

$\rho(t)=\exp \left(-i \int_{t} H\right) \rho(0) \exp \left(i \int_{t} H\right)$.

In order to complete a correspondence between Gibbs QMF (GQMF) and classical MF, we define an operator of internal model parameters $S$ as follows

$\mathbf{S}=\sum_{h \in H}\left|\mathrm{~h}>\mathbf{S}_{\mathrm{h}}<\mathrm{h}\right|$.

Thus, the partition of the type given by eq.(4) and the values of internal model parameters $\left\{\mathbf{S}_{\mathbf{h}}\right\}$ at time $t \mathrm{can}$ be obtained in the process of the quantum measurement

$f(h \mid n)=\operatorname{Tr}[|h\rangle<h \mid \rho(t)], S_{h}(t)=\operatorname{Tr}[S \rho(t)]$.

An initial state of a GQMF system is specified according to the initial state of the MF: one can choose initial values of $\mathrm{S}_{\mathrm{h}}$ based on a priori phenomenological considerations, this leads to initial probabilities $\mathrm{f}(\mathrm{h} \mid \mathrm{n})$ defined according to eq.(4), and to initial values of the density matrix $\rho(0)$ defined according to eq.(17), (18),

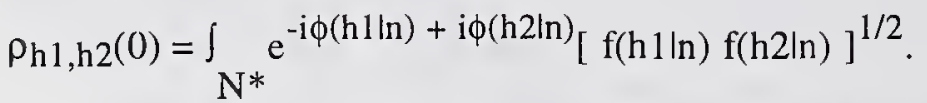

The phases $\phi$ in this expression are left undefined and can be chosen to suit concrete problems at hand. One way to avoid a need to choose phases is to use an alternative initialization procedure: define a non-fuzzy partition according to eq.(2), leading to a diagonal density matrix, and compute initial values of the model parameters $S_{\mathrm{h}}$ from eq.(6).

In a GQMF system described above, the finite temperature of the system (and therefore, the finite accuracy of the computation) does not interfere with computations, but is a part of the system dynamics. On the one hand this is a highly desirable property for any practical implementation of a quantum computing system. On the other, interaction with a thermal reservoir leads to irreversible energy dissipating processes involving quantum measurements. A desirable compromise is to reduce interactions with a thermal reservoir to a relatively rare occasion, which will ensure Gibbs distribution and will correct accumulating phase errors, while in between these interactions a GQMF system will evolve according to Schroedinger equation (17) without energy dissipation.

\subsection{Hamiltonian Quantum Modeling Field System.}

In this alternative approach, let us define the Hamiltonian so that the Hamiltonian QMF (HQMF) dynamics leads to the symbol adaptation. We will do this for a simplified case of MF, when the conditional pdfs defining similarities are Gaussians with unit covariances. Let us emphasize, that this Gaussian-symbol model leads to the Gaussian mixture distributions for classes described by more than one symbol, which can model probability densities of any shape, not only Gaussian. In this case symbol-model parameters are the Gaussian means, $\mathbf{M}_{\mathrm{h}}$. The ML estimation equations for the parameters $\mathbf{M}_{\mathrm{h}}$ can be written as

$\mathbf{M}_{\mathrm{h}}=\sum_{\mathrm{n}} \mathrm{f}(\mathrm{h} \mid \mathrm{n}) \mathbf{X}(\mathrm{n}) / \sum_{\mathrm{n}} \mathrm{f}(\mathrm{h} \mid \mathrm{n})$,

where the denominator has a meaning of the average number of observations described by symbol h,

$N_{h}=\sum_{n} f(h \mid n)$

Also, in place of eq.(18) we have

$\mathbf{M}=\sum_{h \in H}\left|\mathrm{~h}>\mathbf{M}_{\mathrm{h}}<\mathrm{h}\right|$

An internal HQMF encoding I $\mathbf{x}(\mathrm{n})>$ of the external patterns $\mathbf{X}(\mathrm{n})$ (equivalently, of the states $\mid \mathrm{n}>$ of the external world) is defined as,

$|\mathbf{x}(\mathrm{n})\rangle=\langle\mathrm{n} \mid \Psi(\mathrm{t})\rangle=\sum_{\mathrm{h}=1}^{\mathrm{H}} \mathrm{C}_{\mathrm{hn}}|\mathrm{h}\rangle$.

This encoding follows from eq.(9), it is a consequence of 
interaction between the external world and the QMF system. For a HQMF system, this interaction should be defined so that a parametric shape of the probabilities in eq.(10) corresponds to that of the classical MF system, eqs.(4) [Perlovsky, 1996]. Note that the encoding states defined in eq.(24), in general, do not form an orthogonal set. Using these states, the density operator can be written as

$\rho(t)=\sum_{n}|\mathbf{x}(n)><x(n)|$

Let us also introduce an observation operator $\chi$ acting on the encoding QMF states,

$x=\sum_{n=1}|\mathbf{x}(n)>X(n)<\mathbf{x}(n)|$

Consider eigenstates $\mid \lambda>$ of this operator,

$x|\lambda\rangle=\lambda|\lambda\rangle$, or $\langle\lambda|x| \lambda\rangle=\lambda$.

Substituting eq.(26) into eq.(27),

$\lambda=\sum_{n=1}|<\lambda| \mathbf{x}(n)>\left.\right|^{2} \mathbf{X}(\mathrm{n})$.

Comparing this to eqs.(10), (21), (22), and (23), we see that $\lambda$ is identified with $\mathbf{M}_{h} \cdot \mathrm{N}_{\mathrm{h}}$, and $\mid \lambda>$ is identified with $\mid \mathrm{h}>$. Introducing an operator

$\mathcal{M}=\sum_{h \in H}\left|\mathrm{~h}>\mathbf{M}_{\mathrm{h}} \cdot \mathrm{N}_{\mathrm{h}}<\mathrm{h}\right|$

we have the following identity for the quantum operators

$\mathcal{M}=X$

This identity should be attained in the result of an internal QMF dynamics. States $\mid \mathrm{h}>$, which are defined as the eigenstates of the operator $\mathcal{M}$ should evolve (according to Schroedinger equation) into the eigenstates of $\mathcal{X}$, and the considered dynamic symbol-process, is equivalent to the process of finding eigenstates of the operator $\chi$. A number of algorithms exist that can be used for this purpose [Brockett, 1991; Oja, 1992; Xu \& Yuille, 1995]. These algorithms utilize unitary transformations and can serve as a basis for the design of a quantum system. A Hamiltonian $\mathrm{H}$ can be defined as

$\mathrm{H}(\mathrm{t})=\mathrm{i}[\mathcal{M}, \chi]$

According to [Brockett, 1991], this Hamiltonian defines a dynamics that evolve eigenstates $\mid \mathrm{h}>$ of an operator $\mathcal{M}$ into the eigenstates of the operator $X$. It might be noted that a dynamical diagonalization of an operator naturally occurs in many quantum systems, therefore, a choice of a specific physical realization will determine a specific realization of the
Hamiltonian.

If $\mathbf{X}(\mathrm{n})$ are vector quantities (as they usually are), then $\mid \mathbf{x}(\mathrm{n})>$ are defined with a corresponding vector-index, so that $X$ is a vector-operator and various components of $\mathcal{X}$ operate on the corresponding components of $|\mathbf{x}(\mathrm{n})\rangle$. Thus components of $X$ commute (and similarly, components of $\mathcal{M}$ commute). If values of $\mathbf{N}_{h}$ are known, then $\mathbf{M}_{\mathrm{h}}$ can be directly obtained from $\mathcal{M}$. When $\mathrm{N}_{h}$ values are not known a priori, eq.(19) can not serve as a definition of the operator $N$, and $N_{h}$ have to be obtained similarly to $\mathbf{M}_{\mathrm{h}}$, in the process of internal HQMF dynamics. By comparing eqs.(10), (12), (22), (24), and (25) the $\mathrm{N}$ operator is identified with the density operator. Its eigenstates are different from $\mid \mathrm{h}>, \mathrm{N}$ and $\mathcal{M}$ do not commute, and expected values of $\mathrm{N}$ in $\mathcal{M}$-eigenstates $\mathrm{lh}>$ are given by the diagonal elements of the density matrix [Garvin \& Perlovsky, 1995],

$N_{h}=\langle h|\rho| h\rangle$.

The HQMF defined above evolves according to Schroedinger (Hamiltonian) dynamics without energy dissipation. I would also note that this algorithm does not require an exponential number of interfering quantum states, a "speed-up" relative to the classical computation occurs in the result of interference between a number of states, which is only a linear function of the complexity of the system. It seems, however, that a required coherency (accuracy of computation) is a constant number that does not grow with the complexity of the problem. This is because, only few amplitudes $<\mathrm{h} \mid \mathbf{x}(\mathrm{n})>$ (few $\mathrm{h}$ for each $\mathrm{n}$, and few $\mathrm{n}$ for each h) interfere. In addition, the HQMF system has an advantage of a relatively simple formulation, providing a foundation for the next step of a physical realization of a quantum computer.

\section{DISCUSSION}

The first dynamic conception of mind belongs to Aristotle. His theory of Forms describes learning as a realization of an a priori Form-as-potentiality. The conception of mind as a dynamic system based on a priori categories was further developed by Kant $(1781,1788,1790)$. Kant described working of the mind as a triadic process of UnderstandingJudgment-Reason. MFT gives the mathematical description of this process (Perlovsky, 1998). Understanding is given by a priori models, Judgment is given by a similarity measure, and Reason is given by the adaptation mechanism. MFT provides a mathematical description of both, Kantian theory of mind and the process of semiosis. This establishes the relationship between the two profound contributions to the analysis of mind, Kantian theory and semiotics. The dynamic symbol-formation loop describes Kantian triadic-mind process. In [Dmitriev, Perlovsky, 1996], these loops were called the vortexes of thought. The relationships among the semiotical description of the symbol-semiosis, the Kant triadic process, and MFT mathematical agent-vortexes will be illustrated. Sign-vehicles are the internal model codes, objects or designata are the incoming data, the interpretant (that recognizes sign- 
models as corresponding to patterns in input data and generates actions) is a combination of Judgment+Reason (that is, similarity + adaptation and other actions of the activated symbol within MFT). Syntactics is a logic governing Understanding that is relationships among internal models. Semantic is Judgment or similarity measures relating input data and models. And, pragmatics includes the effects of models on similarity and adaptation actions of Reason, modifying the models.

\section{REFERENCES}

Aristotle, (IV BC). Metaphysics . Trans. H.G.Apostle, 1966, Bloomington, IN: Indiana University Press.

Brockett, R. (1991). Dynamical Systems That Sort Lists, Diagonalize Matrices, and Solve Linear Programming Problems. Linear Algebra and Its Applications, 146, pp.79-91

Cramer, H. (1963). Mathematical Methods of Statistics. 10th printing. Princeton University Press. Princeton, NJ.

Dmitriev, V.A. \& Perlovsky, L.I. (1996). Art Form as an Object of Cognitive Modeling (Towards Development of Vygotsky's Semiotics Model). Proceedings of the Conference on Intelligent Systems and Semiotics '96. Gaithersburg, MD, v.2, pp. 385-389.

Feynman, R.P. (1972). Statistical Mechanics. Benjamin, Reading, MA.

Feynman, R.P. (1982). Simulating Physics with Computers. Foundations of Physics, 16, pp.507-31.

Feynman, R.P. (1986). Quantum Mechanical Computers. Int. Journ. of Theor. Physics, 21(6/7), pp.467-88.

Hameroff, S.R. \& Watt, R.C. (1982). Information Processing in Microtubules. J. Theor. Biol., 98, pp.549-61.

Kant, I. (1781). Critique of Pure Reason. Tr. J.M.D. Meiklejohn, 1943. Willey Book, New York, NY.

Kant, I. (1788). Critique of Practical Reason. Willey, New York, NY.

Kant, I. (1790). Critique of Judgment. Tr. J. H. Bernard, 1914, 2nd ed., Macmillan \& Co., London.

Negahdaripour, S. \& Jain, A.K. (1991). Final Report of the NSF Workshop on the Challenges in Computer Vision Research; Future Directions of Research. National Science Foundation.

Neumann von, J. (1910). Nachr. Acad. Wiss., 1910, 3, pp. $228-103$.

Morris C. (1971). Writings on the General Theory of Signs, ed. Th. A. Sebeok. The Hague: Mouton.

Peirce, C.S. (1935-66). Collected Papers of Charles Sanders Peirce. Cambridge: Harvard Univ. Press.

Perlovsky, L.I. (1994). Computational Concepts in Classification: Neural Networks, Statistical Pattern Recognition, and Model Based Vision. Journal of Mathematical Imaging and Vision, 4 (1), pp. 81-110.

Perlovsky, L.I. (1995). Unpublished.

Perlovsky, L.I. (1996). Mathematical concepts of intellect. World Congress on Neural Networks, San Diego, CA.

Perlovsky, L.I. (1998). Neural Networks and Physics of
Mind. Oxford Univ. Press, New York, NY.

Perlovsky, L.I., Schoendorf, W.H., Burdick, B.J. \& Tye, D.M. (1997). Model-Based Neural Network for Target Detection in SAR Images. IEEE Trans. on Image Processing, 6(1), pp.203-216.

Oja, E. (1992). Principal Components, Minor Components, and Linear Neural Networks. Neural Networks, 5, pp.92735

Sakurai, J.J. (1985). Modern Quantum Mechanics. Addison Wesley, New York, NY.

Segre, A.M. (1992). Applications of Machine Learning. IEEE Expert, 7(3), pp. 14-17.

Winston, P.H. (1984). Artificial Intelligence. 2nd edition. Addison-Wesley. Reading, MA.

Xu, L. \& Yuille, A.L. (1995). Robust Principal Component Analysis by Self-Organizing Rules Based on Statistical Physics Approach. IEEE Trans. Neural Networks, 6(1), pp. 131-43

Zubarev, D.N. (1971). Nonequilibrium Statistical Thermodynamics. Nauka, Moscow.

Acknowledgments: I am thankful to Lev Levitin and Roger Brockett for fruitful discussions. 


\title{
SEMIOTIC COMPOUNDS - MIXTURES OF COMPONENTS INVARIANT UNDER SPECIFIED TRANSFORMATIONS
}

\author{
Roy L. Streit \\ Naval Undersea Warfare Center \\ Code 2002, Building 1171 \\ Newport, RI 02841 \\ streit@c223.npt.nuwc.navy.mil
}

\section{ABSTRACT}

Semiotic compounds are mixtures of probability density functions. They are used to formulate joint estimation problems and methods that transcend the boundaries of the traditional detection, classification, and state estimation problem hierarchy. They are non-hierarchical statistical models because they are marginal densities over alternative hypotheses, or choices. Several novel examples of semiotic compounds are discussed in this paper. A common thread running through these examples is that useful semiotic compounds often have mixture components that are related by specific invariance transformations, that is, the components are invariant to characteristic transformations that are determined by the application.

KEYWORDS: continuous mixtures, compound mixtures, semiotic compounds, invariance properties, invariance, detection, estimation, classification

\section{INTRODUCTION}

Traditional techniques for detection, classification, and state estimation (or localization) are typically structured bierarchically, that is, detection is undertaken first, then classification is performed as a post-detection problem, and finally state estimation is conditioned on the outcomes of the detection and classification steps. This conditioning hierarchy is founded on natural and clear distinctions between important statistical issues inherent in many problems and applications; however, hierarchical conditioning may lead to performance that is inferior to joint non-hierarchical methods when numerous alternative hypotheses occur within the problem.

Semiotic compounds are mixtures of probability density functions (PDF's). Each of the alternative hypotheses contributes one component to the mixture. Semiotic compounds are presented in this paper as the constituent models of statistical approaches to joint detection, classification, and state estimation (or localization) capabilities. Joint methods and algorithms based on semiotic compounds defy easy categorization within the traditional hierarchical conditioning structure because of their use of marginalization and because of their avoidance of conditioning on specific hypotheses.

Semiotic compounds vary considerably depending on the list of alternatives over which the particular marginalization, or summation, is performed. A semiotic compound takes the form of a finite sum of PDF's if the alternatives are finite in number, and it takes the form of an integral if the alternatives are characterized by one or more real valued parameters. In the former case the semiotic compound is called discrete, and in the latter it is called continuous. Continuous semiotic compounds are not widely used, perhaps in part because of the (incorrect) perception that they are merely continuous extensions of the more widely used discrete mixtures. Infinite discrete semiotic compounds are also potentially interesting, but no examples of this kind are presented here.

Semiotic compounds also vary in the nature of the component PDFs characterizing the alternative hypotheses. In this paper it is assumed that the component PDF's have the same parametric form, but different values of the parameters; that is, they belong to the same general parametric family Discrete Gaussian semiotic compounds are easily the most widely used in applications to date. Mixtures of disparate types of PDFs are possible, but are not considered in this paper.

An important contribution of this paper lies in the importance it ascribes to the concept of component invariance under transformation. Recognizing the appropriate invariant transformation can give considerable insight into the problem under study. Invariance is not discussed in generality; rather, it is discussed in the context of the following specific problems (which may be read independently):

(a) In Section 2, a generalized likelihood ratio test (GLRT) for detecting transient and spread spectrum signals in noise is treated using a discrete exponential semiotic compound; estimates of both signal-to-noise (SNR) level and bandwidth fall out as by-products of the GLRT procedure.

(b) In Section 3, three discrete Gaussian semiotic compounds are discussed for estimating a PDF from sample data. Although two of these compounds are well known, the third is new and potentially very useful because of its numerical and statistical robustness. 
(c) In Section 4, a continuous semiotic compound is applied to so-called bearings-only target motion analysis (TMA) state estimation problem.

Concluding remarks are given in Section 5.

\section{TRANSIENT DETECTION}

Difficulties arise in GLRT detection problems where one or more of the signal parameters requires an enumeration that is computationally intractable. In transient signal detection the frequency characteristics of the signal are typically unknown, so even if aggregate bandwidth is assumed, the estimation problem intrinsic to the GLRT requires an enumeration of all possible sets of signal locations within the monitored band.

A prior distribution is imposed on the portion of the signal parameter space that usually requires intractable enumeration. By replacing enumeration with the problem of estimating the hyperparameters of the model jointly with other signal parameter, a new formulation of the problem that avoids enumeration and is computationally feasible is possible. The GLRT approach is not changed by this model - what is changed is the signal modal.

An observation vector $\mathrm{X}$ of length $\mathrm{N}$ is obtained by taking the magnitude squared of the output of an FFT whose input is a broadband Gaussian time series. Elements of $\mathrm{X}$ are therefore independent and exponentially distributed. It is assumed that the exponential distributions have one of two possible means: the larger mean, denoted by a, is the expected signal-plus-noise power; the smaller mean, denoted by $b$, is the expected noiseonly power. Every element of $\mathrm{X}$ is assumed to contain a sample obtained under either $\{\mathrm{H}$ : noise only\} or $\{\mathrm{K}$ : signal plus noise \}. Thus, two mutually exclusive and exhaustive alternative hypotheses are assumed.

A Bernoulli random variate with outcomes $\{\mathrm{H}, \mathrm{K}\}$ having probabilities $\{1-q, q\}$, respectively, is used to model the aggregate signal bandwidth, that is, the number of elements of $\mathrm{X}$ that contain signal. In this model, $\mathrm{N}$ samples of the Bernoulli variate are generated, one for each element of $X$. These outcomes comprise the missing data in the sense of the method of expectation-maximization (EM). Samples of the FFT outputs are then drawn from the exponential density determined by the Bernoulli outcomes. Thus, the expected signal bandwidth is equal to qN.

The parameters $\{\mathrm{q}, \mathrm{a}, \mathrm{b}\}$ are unknown and must be estimated from the data vector $X$ using maximum likelihood (ML). Marginalizing over the Bernoulli random deviates gives the likelihood function $L(q, a, b)$ as a product over the data of a two component mixture of exponential densities,

$$
\prod_{i=1}^{N}\left\{(1-q) \frac{1}{b} \operatorname{Exp}\left[-X_{i} / b\right]+q \frac{1}{a} \operatorname{Exp}\left[-X_{i} / a\right]\right\} \text {. }
$$

Straightforward application of the method of EM using the Bernoulli signal model yields the following ML algorithm:
Given current estimates $\{q, a, b\}$ for the parameters, for $i=1, \ldots, N$ compute the weights

$$
\begin{aligned}
& w_{i}(H)=\frac{(1-q) \frac{1}{b} \operatorname{Exp}\left[-X_{i} / b\right]}{(1-q) \frac{1}{b} \operatorname{Exp}\left[-X_{i} / b\right]+q \frac{1}{a} \operatorname{Exp}\left[-X_{i} / a\right]} \\
& w_{i}(K)=\frac{q \frac{1}{a} \operatorname{Exp}\left[-X_{i} / a\right]}{(1-q) \frac{1}{b} \operatorname{Exp}\left[-X_{i} / b\right]+q \frac{1}{a} \operatorname{Exp}\left[-X_{i} / a\right]} .
\end{aligned}
$$

Updated estimates of noise power and signal-plus-noise power are then given by the convex combinations

$$
b^{*}=\frac{\sum_{i=1}^{N} w_{i}(H) X_{i}}{\sum_{i=1}^{N} w_{i}(H)}
$$

and

$$
a^{*}=\frac{\sum_{i=1}^{N} w_{i}(K) X_{i}}{\sum_{i=1}^{N} w_{i}(K)} .
$$

The expected signal bandwidth is given by

$$
q^{*} N=\sum_{i=1}^{N} w_{i}(K) \text {. }
$$

These equations complete the algorithm statement.

The GLRT modeling philosophy requires that the final estimates of $\{q, a, b\}$ be substituted into a likelihood ratio for testing $\mathrm{H}$ against $\mathrm{K}$. Further details are given in [1], where the GLRT is shown to be comparable to the best of a family of nearly optimal power-law detectors developed for this problem.

Extension of the Bernoulli signal model to $\mathrm{p}>2$ power levels (i.e., outcomes) requires semiotic mixtures of $p$ exponential PDFs; applying the method of EM leads to an ML algorithm that is very similar to that sketched above. Further details are given in [1].

The importance of this example is two-fold. Firstly, it shows that the detection statistic requires joint estimation of signal and noise parameters from the data vector and that signal bandwidth is estimated before the signal detection decision is completed. Thus, the GLRT does not fit the traditional statistical hierarchy in this problem. Secondly, the invariant transformation of the exponential PDFs used in this problem is scalar multiplication. The difference between the noise and signal models is essentially an arbitrary distinction based on their power levels. 


\section{DENSITY ESTIMATION}

Discrete Gaussian semiotic compounds are widely used to approximate an unknown PDF from which a set of statistically independent and identically distributed (i.i.d.) sample data have been drawn. ML algorithms are easily derived via the method of EM for estimating the parameters of two classes of Gaussian mixture: homoscedastic ("same scatter") mixtures, which employ the same covariance matrix in every component; and heteroscedastic ("different scatter") mixtures, which use different covariance matrices in every component. The former are robust numerically, but may sometimes require many components to achieve good PDF approximation accuracy. Heteroscedastic mixtures may require fewer components for the same PDF accuracy as homoscedastic mixtures, but they may be numerically unstable in that one or more of the covariance matrices may become singular during iteration. Deficiencies of heteroscedastic mixtures become very severe as the dimensionality of the sample data PDF increases. Further discussion of these mixture classes is given in [2] and in the references therein.

In this paper, covariance restrictions are seen to be special cases of the general principle of component invariance under transformation. The transformation is selected to characterize the common semiotic content of the fundamental symbol under study; that is, components that are identical under the specified transformation comprise the components of the mixture, and each component is a legitimate expression of the same symbolic content. Thus, the mixture characterizes the symbol.

The invariance transformation of homoscedastic mixtures is rigid translation, because components differ only in their means. For heteroscedastic mixtures, the components may be general Gaussian PDF's, so the invariance transformation is the combined transformations of translation, rotation, and dilation. A rotation and dilation will transform one covariance matrix into another, as is easily seen from the singular value decomposition (SVD).

It is clear from the above paragraph that an important class of invariance transformations has been omitted, namely, the class of transformation comprising translation and rotation only. Gaussian semiotic compounds with this invariance transformation are called herein a strophoscedastic ("twisted scatter") compounds, and they were first discussed in [3]. Surprisingly, the method of EM yields an explicit ML estimation algorithm, that is, the M-step of the method of EM can be solved explicitly, just as it can in the homoscedastic and heteroscedastic cases. Although the derivation is significantly more difficult, the resulting algorithm is intuitively reasonable in that it differs from the algorithms for the other two mixtures simply in the amount of averaging that is required.
Let $\mathrm{p}(\mathrm{x})$ denote the true, but unknown, PDF of the sample data, and let $d \geq 1$ denote the dimension of the samples. The density $\mathrm{p}(\mathrm{x})$ is approximated by a strophoscedastic mixture, denoted by $q(x)$, having $N$ components. $N$ is assumed given; its selection is a model order selection problem and is outside the scope of this paper. The parameters defining the strophoscedastic approximation

$$
\mathrm{p}(\mathrm{x}) \cong \mathrm{q}(\mathrm{x})=\sum_{n=1}^{\mathrm{N}} \pi_{n} \mathcal{N}\left(\mathrm{x} \mid \mu_{n}, Q_{n}^{\prime} \mathrm{A} Q_{n}\right)
$$

are given as follows:

(i) Mixing proportions $\pi=\left\{\pi_{1}, \cdots, \pi_{N}\right\}$ such that $\pi_{\mathrm{n}} \geq 0$ for $\mathrm{n}=1, \ldots, \mathrm{N}$ and $\pi_{1}+\cdots+\pi_{\mathrm{N}}=1$,

(ii) Mean vectors $\mu=\left\{\mu_{1}, \cdots, \mu_{N}\right\}$ such that $\mu_{n} \in R^{d}$ for $\mathrm{n}=1, \ldots, \mathrm{N}$,

(iii) Positive definite diagonal kernel matrix

$$
\mathrm{A}=\operatorname{Diag}\left[\alpha_{1}, \cdots, \alpha_{\mathrm{d}}\right] \in \mathrm{R}^{\mathrm{d} \times \mathrm{d}}, \alpha_{1} \geq \cdots \geq \alpha_{d}>0,
$$

(iv) Orthogonal matrices $Q=\left\{Q_{1}, \cdots, Q_{N}\right\}$,

$$
Q_{n} \in R^{d x d}, Q_{n}^{\prime} Q_{n}=Q_{n} Q_{n}^{\prime}=I \text { for } n=1, \ldots, N \text {. }
$$

The parameters in the list (i) - (iv) are estimated from the sample data using the ML method. Let $\mathrm{K}$ be the number of i.i.d. samples in $X=\left\{x_{1}, x_{2}, \cdots, x_{K}\right\}$, where $x_{k} \in R^{d}$ is drawn from the distribution $p(x)$. Using the approximation (1) and the independence of the samples $\mathrm{X}$ gives the posterior likelihood function

$$
\mathcal{L}(X \mid \pi, \mu, A, Q)=\prod_{k=1}^{\mathrm{K}} \mathrm{q}\left(\mathrm{x}_{\mathrm{k}} \mid \pi, \mu, \mathrm{A}, Q\right) .
$$

Applying the method of EM requires defining random variables to characterize the so-called missing data. The missing data are easily understood from a simulation of the mixture $q(x)$ : Firstly, the discrete PDF defined on the set $\{1, \ldots, N\}$ by the probability vector $\pi$ is sampled to obtain a particular Gaussian component of the mixture. Secondly, this Gaussian component is sampled to produce a vector in the sample set $X$. Every sample in $X$ is modeled as having arisen in this fashion, so the missing data are the discrete outcomes of the first step of the hypothetical simulation. Using the missing data enables the method of EM to be applied to derive a ML algorithm that is guaranteed by general properties of the method to generate a sequence of parameter estimates that monotonically increase the likelihood function (2). Thus, if the likelihood function is uniformly bounded above for any choice of parameter set, the algorithm converges to either a local ML parameter estimate or a stationary point of (2).

For $m=0$, let $\pi^{(\mathrm{m})}, \mu^{(\mathrm{m})}, \mathrm{A}^{(\mathrm{m})}$, and $Q^{(\mathrm{m})}$ be initial estimates for the parameters defining the strophoscedastic mixture that satisfy the conditions imposed in (i) - (iv) above. For integers $m \geq 0$, updated estimates for iteration $m+1$ are given by the following equations: For $n=1, \ldots, N$ and $k=1$, $\ldots, \mathrm{K}$, compute the weights 


$$
\mathrm{w}_{\mathrm{nk}}^{(\mathrm{m}+1)}=\frac{\pi_{n}^{(\mathrm{m})} \mathcal{N}\left(x_{k} \mid \mu_{n}^{(m)},\left(Q_{n}^{(m)}\right)^{\prime} A^{(m)} Q_{n}^{(m)}\right)}{\sum_{j=1}^{N} \pi_{\mathrm{j}}^{(\mathrm{m})} \mathcal{N}\left(x_{k} \mid \mu_{j}^{(m)},\left(Q_{j}^{(m)}\right)^{\prime} A^{(m)} Q_{j}^{(m)}\right)} .
$$

The expected number of samples from component $n$, conditioned on parameter estimates at iteration $\mathrm{m}$, is

$$
\mathrm{K}_{\mathrm{n}}^{(\mathrm{m}+1)}=\sum_{\mathrm{k}=1}^{\mathrm{K}} \mathrm{w}_{\mathrm{nk}}^{(\mathrm{m}+1)}
$$

The updated mixing proportions are given by

$$
\pi_{\mathrm{n}}^{(\mathrm{m}+1)}=\mathrm{K}_{\mathrm{n}}^{(\mathrm{m}+1)} / \mathrm{K},
$$

and the updated mean vectors by

$$
\mu_{\mathrm{n}}^{(\mathrm{m}+1)}=\frac{1}{\mathrm{~K}_{\mathrm{n}}^{(\mathrm{m}+1)}} \sum_{\mathrm{k}=1}^{\mathrm{K}} \mathrm{w}_{\mathrm{nk}}^{(\mathrm{m}+1)} \mathrm{x}_{\mathrm{k}} .
$$

The within-component sample data covariance matrices (conditioned on parameter estimates at iteration $\mathrm{m}$ ) are

$$
S_{n}^{(m+1)}=\frac{1}{K_{n}^{(m+1)}} \sum_{k=1}^{K} w_{n k}^{(m+1)}\left(x_{k}-\mu_{n}^{(m+1)}\right)\left(x_{k}-\mu_{n}^{(m+1)}\right)^{\prime} .
$$

Compute the ordered SVD's

$$
S_{n}^{(m+1)}=U_{n}^{(m+1)} \Sigma_{n}^{(m+1)}\left(U_{n}^{(m+1)}\right)^{\prime},
$$

where the matrices $U_{n}^{(m+1)}$ are orthogonal and the diagonal elements of the matrices

$$
\Sigma_{n}^{(m+1)}=\operatorname{Diag}\left[\sigma_{1 \mathrm{n}}^{(\mathrm{m}+1)}, \sigma_{2 \mathrm{n}}^{(\mathrm{m}+1)}, \cdots, \sigma_{\mathrm{dn}}^{(\mathrm{m}+1)}\right]
$$

are arranged in decreasing order. The updated orthogonal matrices of the strophoscedastic mixture are the transposes of the orthogonal matrices of the ordered SVD's, that is,

$$
\mathrm{Q}_{\mathrm{n}}^{(\mathrm{m}+\mathrm{l})}=\left(\mathrm{U}_{\mathrm{n}}^{(\mathrm{m}+\mathrm{l})}\right)^{\prime} \text {. }
$$

The kernel matrix is obtained by averaging the diagonal matrices of the SVD's over all mixture components:

$$
A^{(m+1)}=\sum_{n=1}^{N} \pi_{n}^{(m+1)} \sum_{n}^{(m+1)} .
$$

This completes the statement of the algorithm. A derivation of the algorithm is given in [4].

ML algorithms for the three mixture classes differ only in where averaging is applied. Homoscedastic algorithms average the within-component sample data covariance matrices to obtain the component covariance matrix. Strophoscedastic algorithms average only the singular values of the withcomponent matrices. Heteroscedastic mixtures do not average the within-component matrices at all. The presence of averaging explains intuitively why homoscedastic and strophoscedastic ML mixture algorithms are so reliable and unlikely to encounter singular covariance matrices during iteration.

\section{TARGET LOCALIZATION}

Classical TMA statistical models are post-detection models, that is, they assume $a$ priori that the measurements $Z$ belong to a common target having a specified parametric form (e.g., straight line motion). Post-detection tracking implies that measurements are independent if they are conditioned on the target. ML estimators thus answer the question "Given data generated from a target track, which parameterized track best fits the data?" In contrast, the empirical maximum a posteriori (EMAP) estimators discussed here differ fundamentally from classical post-detection TMA because they are joint detection-estimation methods which seek to answer the alternative question "Does a target track of the specified parametric form fit the data?" A generalized likelihood ratio test (GLRT) in which track parameters are estimated and substituted into a likelihood ratio is the EMAP answer to the question; however, it is the estimated track - and not the GLRT detector - which is the object of interest in this paper.

The data $\left\{Z, X^{\circ}\right\} \equiv\left\{\left(Z_{n}, X_{n}^{o}\right)\right\}_{n=1}^{N}$ are assumed statistically independent because measurements are not specified a priori to belong to the same track. Independence implies that

$$
p_{\mathbf{Z X}}\left(Z, X^{\circ}\right)=\prod_{n=1}^{N} p_{\mathbf{Z}_{\mathbf{n}} \mathbf{X}_{\mathbf{n}}^{\circ}}\left(Z_{n}, X_{n}^{o}\right) \text {. }
$$

The data $\left\{\left(Z_{n}, X_{n}^{\circ}\right)\right\}_{n=1}^{N}$ contribute independent probability density assessments of "potential" target position that are valid at the measurement times. Let $X^{\Omega} \equiv\left\{X_{n}^{\Omega}\right\}_{n=1}^{N}$ denote socalled "empirical" random variables associated with potential target location. Empirical random variables are assumed independent when conditioned on corresponding measurements and sensor locations; hence, the empirical target location PDF is

$$
p_{\mathbf{X}^{\Omega} \mid \mathbf{Z} \mathbf{X}^{\circ}}\left(X^{\Omega} \mid \mathrm{Z}, X^{\circ}\right)=\prod_{n=1}^{N} p_{\mathbf{X}_{n}^{\Omega} \mid \mathbf{Z}_{\mathbf{n}} \mathbf{X}_{n}^{\circ}}\left(X_{n}^{\Omega} \mid Z_{\mathrm{n}}, X_{n}^{o}\right) \text {. }
$$

The empirical target likelihood function is evaluated for specified parametric target motion models, once the conditional density of $X_{n}^{\Omega}$ is defined.

In the remainder of this section the passive azimuthal bearings-only TMA problem is considered; thus, it is assumed that the target lies in the $x-y$ plane, that $Z_{n}=\theta_{n}$, where $\theta_{n}$ is the measured azimuthal bearing from the sensor to the target, and that $X_{n}^{0}=\left(x_{n}^{0}, y_{n}^{0}\right)$ is the sensor location when the bearing $\theta_{n}$ is obtained. Target is unobservable from a single bearing because range measurements are assumed to be unavailable from the passive sensor.

A continuous semiotic compound, or marginal integral representation, of the PDF of the empirical state variables can 
be derived by introducing the dummy random variable $r_{n}$ to model the "missing" sensor range measurement corresponding to $\theta_{n}$. Full details of this novel approach to target observability difficulties are provided in [5] and are not repeated here. Sensor and target characteristics are assumed to be such that the missing range variable lies in a known finite range interval, $r_{\min } \leq r_{n} \leq r_{\max }$.

Let $\lambda=\left\{x_{1}, y_{1}, x_{N}, y_{N}\right\}$ denote the endpoint coordinates of a target with straight line motion. Substituting this motion model into the integral representation, and substituting the result into the overall empirical likelihood function gives

$p_{\mathbf{X}^{\Omega \mid \mathbf{Z X}^{\circ}}}\left(X(\lambda) \mid Z, X^{\circ}\right)=\prod_{n=1}^{N} \int_{r_{\min }}^{r_{\max }} \operatorname{Exp}\left\{\frac{-Q}{2 \mathrm{r}_{n}^{2}}\right\} \frac{\mathrm{d} r_{n}}{r_{n}}$,

where the quadratic form $Q$ of the exponential function is

$$
\begin{aligned}
Q= & {\left[\begin{array}{l}
\alpha\left(t_{n}\right) x_{1}+\beta\left(t_{n}\right) x_{N}-x_{n}^{0}-r_{n} \cos \theta_{n} \\
\alpha\left(t_{n}\right) y_{1}+\beta\left(t_{n}\right) y_{N}-y_{n}^{0}-r_{n} \sin \theta_{n}
\end{array}\right]^{\prime} } \\
& \Sigma^{-1}\left(\theta_{n}, \sigma_{n}, \kappa_{n}\right)\left[\begin{array}{l}
\alpha\left(t_{n}\right) x_{1}+\beta\left(t_{n}\right) x_{N}-x_{n}^{0}-r_{n} \cos \theta_{n} \\
\alpha\left(t_{n}\right) y_{1}+\beta\left(t_{n}\right) y_{N}-y_{n}^{0}-r_{n} \sin \theta_{n}
\end{array}\right]
\end{aligned}
$$

and the linear interpolation functions are given by

$$
\alpha(t)=\frac{t_{N}-t}{t_{N}-t_{1}} \text { and } \beta(t)=\frac{t-t_{1}}{t_{N}-t_{1}} .
$$

The covariance matrix of the quadratic form $Q$ is given by $\Sigma\left(\theta_{n}, \sigma_{n}, \kappa_{n}\right)=$

$$
\left[\begin{array}{cc}
\cos \theta_{n} & \sin \theta_{n} \\
-\sin \theta_{n} & \cos \theta_{n}
\end{array}\right]^{T}\left[\begin{array}{cc}
\kappa_{n}^{2} & 0 \\
0 & \sigma_{n}^{2}
\end{array}\right]\left[\begin{array}{cc}
\cos \theta_{n} & \sin \theta_{n} \\
-\sin \theta_{n} & \cos \theta_{n}
\end{array}\right]
$$

where $\sigma_{n}^{2}$ is the variance of the bearing $\theta_{n}$, and $\kappa_{n}^{2}$ is the variance of the missing range variable $r_{n}$.

By discretizing the integrals and applying the method of EM, an EMAP estimation algorithm can be derived. It can be shown that this algorithm is a sequence of iteratively reweighted linear least squares problems. The variance of the missing range measurement $r_{n}$ is a free variable that can be used to greatly accelerate the convergence of the EM algorithm. Further details will be reported in [6].

The quadratic exponent $Q$ of the Gaussian component of the continuous semiotic compound is the only form satisfying a combination of geometrical and analytical properties natural to the bearings-only TMA problem. Firstly, empirical target location is assumed to be Gaussian distributed when conditioned on actual target location. This is a Bayesian assumption about the empirical target, not the target. Secondly, the marginal density over the empirical range variable of the conditional empirical PDF is required to be independent of actual target range. This requirement implies that the cross-range and down-range variances must grow as the square of target range. Thirdly, the marginal density over empirical range is required to be a function of the difference between the empirical bearing and the target bearing. This requirement forces the axes of the empirical covariance matrix to align with the cross-range and down-range directions. In summary, these requirements completely characterize the Gaussian component density of the semiotic compound, that is, the quadratic form $Q$ is the unique form that satisfies these requirements. For a detailed derivation, see [5, Appendix A].

As is seen from direct examination of $Q$, the components of the continuous semiotic compound are invariant under the combined transformations of translation down-range and square-law spreading of the variances of its principle components with increasing range. The resulting integral representation of the empirical density is remarkable in that it is very nearly constant along straight lines radiating outward from the sensor location, and moreover it is very nearly Gaussian along circular cuts centered on the sensor. Both these statement are valid within the annulus with inner and outer radii $r_{\min }+\varepsilon$ and $r_{\max }-\varepsilon$, respectively, where $\varepsilon$ is a small positive number.

Multitarget applications of discrete semiotic compounds are also possible. In these applications the missing data is the correct assignment of a measurement to a target. Just as in the examples above, the method of EM can be used to derive ML estimation algorithms. Invariance transformations have not yet been sought for these problems. See $[7,8,9]$ for further details.

\section{CONCLUDING REMARKS}

The common thread linking the various examples is that of an invariant transformation. However, the appropriate nature of the transformation depends strongly on the given application. It also appears from these examples that semiotic mixtures of components constructed (i.e., derived) to be invariant under a specified set of transformations may possess remarkable properties that are important for the application. In any event, more examples are needed to illustrate the utility of semiotic compounds with invariance transformations for joint detection, classification, and localization.

The method of EM is not the only available ML estimation method, for in any problem to which EM is applicable, the standard necessary conditions may always be derived and solved directly. EM is merely a general procedure for deriving local ML parameter estimation algorithms in problems whose likelihood function is modeled as a marginal PDF over "missing" information. Specific algorithms derived via EM vary considerably in nature, depending strongly on the parametric form of the particular PDF's that are used. EM algorithms often take huge steps toward the solution in the very first few steps (a phenomenon unaccounted for in the 
convergence literature), but it remains a complaint that they are asymptotically only linearly convergent. For numerical purposes, a hybrid algorithm seems potentially useful: initialize with EM, and finish with a quadratically convergent method such as Newton-Raphson. Ultimately, the real value of the method of EM may reside more in the way of thinking and modeling that it suggests for new and challenging problems than in any the resulting algorithm.

Implicit within the marginalization interpretation of semiotic compounds is a randomization concept that is essentially Bayesian in nature. Consequently, when detection, classification, and estimation problems are undertaken in contexts (e.g., high SNR) in which decision errors are very unlikely, the use of the traditional hierarchical conditioning may be satisfactory for many applications. However, when decision errors become common, special decision support systems (i.e., expert systems) are needed to protect against them and to accommodate conflicting decisions. Over the last decade, Bayesian networks (see, e.g., [10]) have been proposed and developed for reasoning under uncertainty with mathematical guarantees of consistency. In environments where reasoning under uncertainty is the central and pivotal issue, semiotic compounds are potentially useful models.

\section{ACKNOWLEDGMENTS}

This work was supported by the Office of Naval Research.

\section{REFERENCES}

[1] R. L. Streit and P. K. Willeth, "Hybrid Bayes/GLRT Detection of Transient Signals," Proceedings of the Conference on Information Sciences and Systems, Johns Hopkins University, Baltimore, MD, March 19-21, 1997.

[2] R. L. Streit and T. E. Luginbuhl, "Maximum Likelihood Training of Probabilistic Neural Networks," IEEE Transactions on Neural Networks, NN-5(5), 764 783.

[3] R. L. Streit, S. G. Greineder, and T. E. Luginbuhl, "Maximum Likelihood Training of Probabilistic Neural Networks With Rotationally Related Covariance Matrices," Proceedings of the International Conference on Neural Networks, Perth, Australia, 27 November - 1 December 1995, 300-303.

[4] R. L. Streit and T. E. Luginbuhl, "Estimation of Gaussian Mixtures With Rotationally Invariant Covariance Matrices," Communication in Statistics, Part A: Theory and Methods, to appear.

[5] R. L. Streit, "Bearings-Only Target Motion Analysis With Propagation Loss Models Of Uncertain Fidelity," Proceedings of the Third International Conference on Theoretical and Computational Acoustics, Newark, New Jersey, 14-18 July 1997.
[6] R. L. Streit, "Augmented Bearings-Only Target Motion Analysis Problems", IEEE Transactions on Aerospace and Electronic Systems, in preparation.

[7] R. L. Streit and T. E. Luginbuhl, "Maximum Likelihood Method for Probabilistic Multi-Hypothesis Tracking," SPIE International Symposium, Signal and Data Processing of Small Targets 1994, SPIE Proceedings Vol. 2335-24, pp. 394-405.

[8] R. L. Streit, "Probabilistic Multi-Hypothesis Tracking," Naval Undersea Warfare Center Technical Report 10,428, 15 February 1995.

[9] H. Gauvrit, C. Jauffret, and J. P. LeCadre, "A General Formulation of Multitarget Tracking in the Viewpoint of an Incomplete Data Problem," IEEE Transaction on Aerospace and Electronic Systems, to appear.

[10] F. V. Jensen, An Introduction to Bayesian Networks, Springer-Verlag, New York, 1996 


\title{
INFORMATION, LIKELIHOOD, AND EINSTEIN
}

\author{
Leonid I. Perlovsky \\ Nichols Research Corporation, 70 Westview Street, Kilnbrook IV, Lexington MA 02173 \\ Tel: 617-862-9400; Fax: 617-862-9485e-mail: perlovsl@nichols.com
}

\begin{abstract}
A new type of statistical estimation principle is suggested. It is based on the Einsteinian interpretation of the spectrum as a pdf. I discuss its relations to and distinctness from the classical estimation principles of maximum likelihood, information, and entropy. The new principle can be interpreted as a relaxation to equilibrium of a physical system consisting of the "world" and the estimation system. The new estimation principle has been successfully tested in a number of applications. Performance exceeding classical estimation principles has been indicated.
\end{abstract}

\section{INTRODUCTION}

Fundamental principles of statistical estimation include maximization of likelihood, maximization of mutual information, and related techniques. Maximization of likelihood (ML) is a most established principle, dating to Bayes. According to the ML, unknown parameters are estimated by maximizing a probability density function (pdf) of the available data. The ML has certain optimal properties, it is asymptotically unbiased and efficient. The information or entropy maximization has being used since 1950s. Kullback and Leibler ${ }^{1}$, following Khinchin, developed a measure of information distance, that was used to develop KhinchinKullback-Leibler estimation approaches also known as maximum entropy (ME), minimum cross entropy (MCE) and minimum discrimination information (MDI) ${ }^{2,3}$. The ME philosophy was formulated by Jaynes ${ }^{2}$ as: "maximally noncommittal with regards to missing information". A related minimum cross-entropy principle ${ }^{4}$ minimizes "information measure necessary to change a prior pdf into estimated pdf". Of a particular interest is Shore's comment ${ }^{4}$ that the ML estimation is justified from the basic principles only if a model is exactly correct (for some set of the model parameters), while MCE does not rely on this. Thus, he argued that MCE is a more general approach than ML, in that it is justified even if we know that our models are approximate.

I propose a new estimation principle, which is related to and distinct from the classical estimation principles discussed above. Following Einstein and $\mathrm{Hopf}^{5}$, I consider a different type of pdf than usually considered in statistical estimation. Since the concept of pdf is a basis for statistical estimation, likelihood, and information measures, the new principle leads to different results than the classical ones. A specific point of difference is the attribution of randomness. Usually, in statistical estimation, randomness is attributed to measured quantities as follows. E.g., if an image is produced by the light intensity, an intensity of the pixel is considered as a random quantity and pdf models are designed to model this randomness. Or, if a radar measures Doppler spectra, a signal intensity or power in a Doppler cell (a sample) is considered a random quantity, and pdf models are constructed accordingly (say, for the spectrum it could be a $\chi^{2}$ density). Contrary to this, Einstein interpreted the spectrum as a pdf of photon frequency. That is, the Doppler cell frequency is to be considered random, rather than the intensity or power.

I develop the Einsteinian concept further, and relate it to the type of estimation, which is usually considered in statistical physics. Namely, I relate the new estimation principle to the process of relaxation to equilibrium in a physical ensemble. The ML principle, when applied to "Einsteinian" likelihood, results in the same estimation equations as the maximum entropy principle used to find the equilibrium of a physical system. Also, the new estimation principle is interpreted as a maximization of mutual information in the model about the data. The "Einsteinian" likelihood and pdf models are applicable to signals or images formed by measuring the energy (spectrum) of electromagnetic or acoustic waves, which are widely used types of data.

Many statistical spectrum estimation methods are based on parametric spectrum models. This permits one to utilize information about signal phenomenology and enables one to achieve a better accuracy than by using non-parametric estimation approaches. A number of models have been utilized for this purpose, suitable for broad categories of signals, including autoregressive, moving average, their combination, and sinusoids in noise. Also, a number of parameter estimation methods have been developed, many of which are based on classical ML or maximum entropy principles ${ }^{7}$. Types of spectrum models and the estimation technique developed here are different from those previously discussed. In particular, they are applicable to often encountered cases when multiple interfering phenomena are present $6,8-11$, and when classical techniques may be inapplicable. Also, the new estimation technique described here results in more efficient estimation as compared to classical ML and ME methods, even when applied to classical autoregressive problems ${ }^{6}$.

Section 2 discusses Einstein's interpretation of photon spectra for electromagnetic waves, introduces a pdf model for signal spectra compatible with Einstein's ideas, and derives the ML estimators for the model parameters. I discuss the relationships between the ML and the entropy or information maximization and address specific similarities and differences 
between the new estimation principle and the classical ones. Section 3 extends these results to images and higherdimensional cases. Section 4 contains a discussion; also, it briefly overviews and provides references to the applications and numerical comparisons that have been implemented and published.

\section{EINSTEINIAN MODEL FOR SPECTRUM ESTIMATION}

Einstein interpreted the electromagnetic spectrum as a probability density function (pdf) of the photon energy 5 . A similar interpretation is valid for phonons of acoustic spectra (speech, seismic signals, etc.) and for any signal field obeying Bose-Einstein's statistics (bosons). A photon energy $\varepsilon$ is related to its frequency $\omega$,

$\varepsilon=\hbar \omega$

therefore, according to the Einsteinian interpretation, spectral models, $F(\omega)$, are to be interpreted as frequency pdfs, or, in other words, proportional to the number of photons with frequency $\omega$. This interpretation requires proper normalization; since empirical spectrum $S(\omega)$ is measured in units of energy, its interpretation as a pdf requires normalization on a photon energy. We, therefore, consider the number of measured photons

$N_{\omega}=S(\omega) / \hbar \omega, \quad \sum_{\omega} N_{\omega}=N$

and $F(\omega) / \hbar \omega$ is a model pdf for a single photon with frequency $\omega$, that is an expected value $E\{N \omega / N\}$ normalized as follows

$$
\sum \mathrm{F}(\omega) / \hbar \omega=1, \mathrm{~F}(\omega) / \hbar \omega=\mathrm{E}\{\mathrm{N} \omega / \mathrm{N}\}
$$

We consider discrete empirical spectra and their models, so that $N_{\omega}$ is the number of photons in the appropriate interval of frequencies and $F(\omega)$ is their normalized expected value. We also assume that a small interval of frequencies around zero is excluded, so that normalization (3) is possible. In many applications zero frequency is excluded, because the physical carrier signal frequency is much larger than the frequency variations of interest. In macroscopic systems, photons are statistically uncorrelated (most often, this is also true for microscopic systems as well). Therefore, for an ensemble of photons $n=1, \ldots, N$, the joint pdf or likelihood $\mathrm{L}$ is a product over individual photons

$$
\mathrm{L}=\underset{\mathrm{n}}{\Pi} \mathrm{F}\left(\omega_{\mathrm{n}}\right) / \hbar \omega_{\mathrm{n}}=\underset{\omega}{\Pi[\mathrm{F}(\omega) / \hbar \omega]^{\mathrm{N}} \omega}=\underset{\omega}{\Pi[\mathrm{F}(\omega) / \hbar \omega]^{\mathrm{S}(\omega) / \hbar \omega}}
$$

The second equality here is obtained as follows. The product over individual photons, $\mathrm{n}$, is split into two terms: a product over photons with a fixed frequency $\omega$ and a product over various frequencies $\omega$. There are $N_{\omega}$ photons with a fixed frequency $\omega$, all distributed according to identical pdf model $\mathrm{F}(\omega)$, this leads to the above expression. The likelihood (4) does not account for the variability in data associated with differences between a single realization of the empirical data $\mathrm{N}_{\omega}$ and the model of its expected value. For a quantum system containing a small number of photons this might be a significant deficiency, but for classical systems containing large numbers of photons, expression (4) is adequate. According to the ML principle, the likelihood (4) or its logarithm should be maximized in the estimation process. If the number of parameters, $\mathrm{N}_{\mathrm{par}}$, vary in the estimation process, the proper quantity to be maximized is called the Akaike Information Criterion, AIC; it is obtained by subtracting $\mathrm{N}_{\text {par }} / 2$ from the log-likelihood 12 :

$\mathrm{AIC}=\log \mathrm{L}-\mathrm{N}_{\mathrm{par}} / 2$

The specific shape of the parametric model for $F(\omega)$ can be determined based on the physics of the process under consideration 8,10 , or a general type of flexible parametric model can be selected as in $[6,11]$ or below. Often, signals can be considered as being produced by incoherent contributions from several sources. In such a case, the spectrum is a sum of individual source contributions,

$F(\omega)=\sum_{m} F(\omega \mid m), \quad m=1, \ldots, M$

Such a model for a pdf is called in statistics a mixture model, and we call each mixture component $F(\omega \mid \mathrm{m})$ a sub-model corresponding to source $\mathrm{m}$. Conditional pdf modeling individual sources in many cases can be modeled using Gaussian densities. Gaussian models are widely applicable, because a superposition of Gaussian models can be used to model any function. The Gaussian model is given by

$\mathrm{F}(\omega \mid \mathrm{m})=\hbar \omega \mathrm{A}_{\mathrm{m}} \mathrm{G}(\omega \mid \mathrm{m}) \Delta \omega, \quad \mathrm{m}=1, \ldots, \mathrm{M}$

$\mathrm{G}(\omega \mathrm{lm})=(2 \pi)^{-1 / 2}\left(\sigma_{\mathrm{m}}\right)^{-1} \exp \left\{-0.5\left(\omega-\omega_{\mathrm{m}}\right)^{2} / \sigma_{\mathrm{m}}{ }^{2}\right\}$.

In the above equations, $A_{m}$ is a sub-model weight, $\Delta \omega$ is a sampling interval, $\omega_{\mathrm{m}}$ is the sub-model mean frequency and $\sigma_{\mathrm{m}}$ is the sub-model frequency standard deviation. A multiplicative term $\hbar \omega$ in Eq. (7) is introduced according to the normalization requirement (3), so that $\left[A_{m} G(\omega \mid m)\right]$ is measured in units of a photon number, and $G(\omega \mid \mathrm{m})$ is interpreted as the conditional pdf of the photon frequency from source $\mathrm{m}$. A wide applicability of the above model is due to the fact, that Gaussian functions form an overcomplete set of functions so that any function $\mathrm{N}_{\omega}$ can be modeled using eqs. (6), and (7).

The mixture model specified by (6), and (7) is characterized by three parameters per Gaussian sub-model: the weight, the mean, and the standard deviation. The ML 
estimation equations for these parameters are derived by maximizing the likelihood $L$ in (4), or its logarithm, $L L=\ln L$. Constraint (3) can be accounted for by using the method of Lagrange multipliers, resulting in the following ML equations:

$$
\begin{aligned}
& A_{m}=N_{m} / N, \quad N_{m}=\sum_{\omega} P(m \mid \omega)[S(\omega) / \hbar \omega] \\
& N=\sum_{\omega} S(\omega) / \hbar \omega, \\
& \omega_{m}=\sum_{\omega} P(m \mid \omega)[S(\omega) / \hbar \omega] \omega / N_{m}, \\
& \sigma_{m}^{2}=\sum_{\omega} P(m \mid \omega)[S(\omega) / \hbar \omega]\left(\omega-\omega_{m}\right)^{2} / N_{m} .
\end{aligned}
$$

The term $\mathrm{P}(\mathrm{m} \mid \omega)$,

$$
\begin{aligned}
& \mathrm{P}(\mathrm{m} \mid \omega)=\mathrm{F}(\omega \mid \mathrm{m}) /\left[\sum_{\mathrm{m}^{\prime}} \mathrm{F}\left(\omega \mid \mathrm{m}^{\prime}\right)\right], \\
& \sum_{\mathrm{m}} \mathrm{P}(\mathrm{m} \mid \omega)=1, \mathrm{~m}=1, \ldots \mathrm{M},
\end{aligned}
$$

has the meaning of the a posteriori Bayes probability that a photon at frequency $\omega$ has originated from the source (or submodel) $\mathrm{m}$. Thus, $\mathrm{N}_{\mathrm{m}}$ is the number of photons from the source $\mathrm{m}$, and $\mathrm{N}$ is the total number of photons.

The ML estimation eqs. (8) through (11) do not yield an immediate estimate of the model parameters, because the probabilities $\mathrm{P}(\mathrm{m} \mid \omega)$ in the right-hand side of eqs. (8) through (10) depend on the unknown parameter values, according to (11). These equations can be considered as defining an iterative system: beginning with some values of parameters, the sub-models $F(\omega \mid \mathrm{m})$ are computed and the probabilities are computed according to (11); on the next iterations, the parameter values are recomputed according to (8) through (10), etc., until convergence. The convergence is determined by requiring that parameter changes are small from an iteration to iteration. The convergence is always attained; this is a consequence of the estimation-maximization (EM) algorithm 13 . If the number of sub-models, $M$, should be estimated from the data, this is accomplished by maximizing eq.(5).

It should be noted that the above Eqs. (8) through (11) are approximate. The approximation is in considering the Gaussian densities to be normalized for a discrete set of frequencies,

$$
\sum_{\omega} \mathrm{G}(\omega \mathrm{lm}) \Delta \omega \approx \int \mathrm{G}(\omega \mathrm{lm}) \mathrm{d} \omega \equiv 1 .
$$

A numerical evaluation shows that for $\sigma_{\mathrm{m}}>1$ (in units of sample numbers), the approximation is accurate.

Below, I discuss two alternative interpretations of the above estimation procedure. First, the estimation is interpreted as a relaxation to equilibrium of the photon ensemble, leading to a relationship between the "Einsteinian" likelihood and entropy. And second, the estimation is interpreted as maximization of the information contained in the internal model of an estimation system about the world.

\section{Equilibrium of the Photon Ensemble.}

I will demonstrate that the ML eqs. (8) through (11) describe the equilibrium state of a system consisting of the physical ensemble of photons and the estimation system, which degrees of freedom are the estimated parameters of the model. An average number of the observed photons is proportional to a number of the photon physical states. Therefore, according to Einstein's interpretation, a spectrum sub-model $F(\omega)$ is interpreted as being proportional to a number of physical states, $\Phi_{\omega}$, for a single photon at each frequency,

$$
F(\omega)=\text { const } \cdot \hbar \omega \cdot \Phi_{\omega} .
$$

The equations of physical equilibrium in our case can be derived by using a standard textbook procedure ${ }^{14}$, which is now briefly described (for the classical limit, when $N_{\omega} / \Phi_{\omega}$ $\ll 1)$. Since our estimation procedure deals with the fixed observation data, the number and energy of photons is fixed, which should be accounted for in the estimation procedure. In statistical physics, a system with the fixed energy and number of particles is called a microcanonical ensemble. The equilibrium of a microcanonical ensemble is obtained by maximizing the entropy of the ensemble, E, subject to the constraints of conservation of energy $\varepsilon$ and photon number $N$. According to eqs. (1), (2), and (3), this results in the following constraints on our model $F(\omega)$,

$$
\begin{aligned}
& \varepsilon=\sum_{\omega} S(\omega)=N \sum F(\omega) ; \\
& N=\sum_{\omega} S(\omega) / \hbar \omega=\underset{\omega}{N} F(\omega) / \hbar \omega .
\end{aligned}
$$

The ensemble entropy, E, is a logarithm of the number of states available to the system, $\Gamma$. It is a product of the number of states available at each frequency, $\Gamma_{\omega}$, and its computation is a standard textbook exercise ${ }^{14}$. For $\mathrm{N}_{\omega}$ photons with frequency $\omega$, the total number of states $\Gamma_{\omega}$ is

$$
\Gamma_{\omega}=\left(\Phi_{\omega}\right)^{N \omega} /\left(N_{\omega} !\right)
$$

where the numerator is the total number of all combinations and the denominator accounts for the permutations of the equivalent photons. Thus, we obtain for the entropy of the photon ensemble,

$$
\mathrm{E}=\ln \Gamma=\ln \prod_{\omega} \Gamma_{\omega} \approx \sum_{\omega} \mathrm{N}_{\omega} \ln \left[\Phi_{\omega} / \mathrm{N}_{\omega}\right]
$$

If the number of the parameters vary in the estimation process, the varying degrees of freedom associated with the estimation system have to be accounted for in the estimation process. 
This is accomplished by subtracting $1 / 2$ for every degree of freedom, this is subtracting the number of parameters divided by two, $\mathrm{N}_{\mathrm{par}} / 2$ to the above expression; this procedure is a consequence from the first principles, which is well known in statistical physics and in statistical estimation theory 14,12 . Combining eq.(16) with this additional item and with eqs. (2) and (13),

$\mathrm{E}=\sum[\mathrm{S}(\omega) / \hbar \omega] \ln [\mathrm{F}(\omega) /(\mathrm{const} \cdot \mathrm{S}(\omega))]-\mathrm{N}_{\mathrm{par}} / 2$

Maximization of this expression subject to constraints (14) can be obtained by using the method of Lagrange multipliers. It leads to the same equations (8) through (11). Thus, the ML estimation, using "Einsteinian likelihood" is equivalent to finding an equilibrium of the estimation system and photon ensemble.

\section{Information in the Model about the Data}

In his classical work ${ }^{15}$, Shannon introduced a concept of the mutual information contained in the received message about the sent message. For our purpose, this concept can be formulated as follows. We identify the sent message with the measured data $S(\omega)$ and the received message with the internal model, $F(\omega)$. Then, the mutual information in the model about the data is given by (see Appendix for details)

$I=\Sigma[S(\omega) / \hbar \omega] \ln [F(\omega) / \hbar \omega]$.

$\omega$

Maximization of the entropy (17), for the estimation purpose, is equivalent to maximization of the mutual information (18).

The information or entropy maximization discussed in this paper is connected to the maximum entropy estimation principle that has been used since 1950s. There are significant similarities and differences between our approach and other methods under similar names, so it is useful to provide a brief overview of the history and literature concerning the roots of the maximum entropy estimation in statistics. Kullback and Leibler $^{1}$, following Khinchin, developed a measure of information distance, that was used to develop KhinchinKullback-Leibler estimation approaches also known as maximum entropy (ME), minimum cross entropy (MCE) and minimum discrimination information $(\mathrm{MDI})^{2,3}$. The ME philosophy was formulated by Jaynes 2 as: "maximally noncommittal with regards to missing information". In ME estimation, the problem is formulated as follows. Estimate a pdf, $q(n)$, given a set of linear constraints on $q$. The ME estimation consists in maximizing the entropy defined as

$\max E ; \quad E=-\sum q(n) \ln q(n)$.

According to the ME philosophy, ME estimates a function $q(n)$ by maximizing its randomness, while satisfying the constraints. When a prior guess $p(n)$ estimating $q(n)$ is known in addition to the constraints, MCE is used, which minimizes cross-entropy CE,

$\min C E ; C E=\sum_{n} q(n) \ln [q(n) / p(n)]$,

subject to the constraints. MCE minimizes "information measure necessary to change $\mathrm{p}(\mathrm{n})$ into $\mathrm{q}(\mathrm{n})$ ", subject to the constraints. Note the differences between eq.(20) and our eq.(17): in MCE like in ME, the sum is weighted with the sought function $\mathrm{q}(\mathrm{n})$, while in our definition, the sum is weighted with the measured numbers of photons. Shore 4 emphasized that the ML estimation is justified from the basic principles only if a model is exactly correct (for some set of parameters), while MCE does not rely on this. Thus, he argued that MCE is a more general approach than ML, in that it is justified even if we know that our models are approximate. This is also true for our "Einsteinian" mutual information: the developed method extracts maximal information about the data, which could be extracted with a given model, even if the model is approximate.

\section{EXTENSION TO 2-D AND HIGHER DIMENSIONAL IMAGES}

The Einsteinian interpretation of spectrum does not have to be limited to the frequency-domain spectra, but is naturally extended to intensity or power densities in any coordinates, e.g., two-dimensional domains of time-frequency spectra, regular angle-angle intensity imagery, or higher dimensional domains such as time-frequency-range-angle imagery $6,10,11$. The extension is a straightforward one. Let us denote the general D-dimensional image coordinates as $\mathbf{x}$, imagery data, $\mathrm{S}(\mathbf{x})$, and image models, $\mathrm{F}(\mathbf{x})$. The "Einsteinian" likelihood expression and the ML equations are similar to the ones in the previous section,

$\log L=\sum[\mathrm{S}(\mathbf{x}) / \hbar \omega] \log [\mathrm{F}(\mathbf{x}) / \hbar \omega]$.

If the mixture model with D-dimensional Gaussian components (sub-models) is used,

$\mathrm{F}(\mathbf{x})=\sum \mathrm{F}(\mathbf{x} \mid \mathrm{m}), \quad \mathrm{F}(\mathbf{x} \mid \mathrm{m})=\hbar \omega \mathrm{A}_{\mathrm{m}} \mathrm{G}(\mathbf{x} \mid \mathrm{m}), \quad \mathrm{G}(\mathbf{x} \mid \mathrm{m})=$ $\mathrm{m}$

$(2 \pi)^{-\mathrm{D} / 2}\left(\operatorname{det} \mathrm{C}_{\mathrm{m}}\right)^{-1 / 2} \exp \left\{-0.5\left(\mathbf{x}-\mathbf{x}_{\mathrm{m}}\right)^{\mathrm{T}} \mathbf{C}_{\mathrm{m}}{ }^{-1}\left(\mathbf{x}-\mathbf{x}_{\mathrm{m}}\right)\right\},(22)$

Again, $\mathrm{A}_{\mathrm{m}}$ is a sub-model weight, $\mathbf{x}_{\mathrm{m}}$ is the sub-model mean position vector and $\mathrm{C}_{\mathrm{m}}$ is the sub-model covariance, determining the shape of the sub-model in $\mathrm{D}$-dimensional $\mathbf{x}$ space. The term $\left[A_{m} G(\omega \mid m)\right]$ is measured in units of a photon number, and $\mathrm{G}(\mathbf{x} \mid \mathrm{m})$ is interpreted as the conditional pdf of photons from source $\mathrm{m}$. And, the ML estimation equations for the model parameters are:

$A_{m}=N_{m} / N, N_{m}=\sum_{\mathbf{x}} P(m \mid x)[S(\mathbf{x}) / \hbar \omega], N=\sum[S(\mathbf{x}) / \hbar \omega]$, 
$\mathbf{x}_{\mathrm{m}}=\underset{\mathbf{x}}{\sum} \mathrm{P}(\mathrm{m} \mid \mathbf{x})[\mathrm{S}(\mathbf{x}) / \hbar \omega] \mathbf{x} / \mathrm{N}_{\mathrm{m}}$,
$\mathrm{C}_{\mathrm{m}}=\underset{\mathbf{x}}{\sum} \mathrm{P}(\mathrm{m} \mid \mathbf{x})[\mathrm{S}(\mathbf{x}) / \hbar \omega]\left(\mathbf{x}-\mathbf{x}_{\mathrm{m}}\right)\left(\mathbf{x}-\mathbf{x}_{\mathrm{m}}\right)^{\mathrm{T}} / \mathrm{N}_{\mathrm{m} .}$,
$\left.\mathrm{P}(\mathrm{m} \mid \mathbf{x})=\underset{\mathrm{m}^{\prime}}{\mathrm{F}} \mathrm{F}(\mathbf{x} / \mathrm{m}) /\left[\mathrm{m}^{\prime}\right)\right]$,

where $\mathrm{P}(\mathrm{m} \mid \mathbf{x})$ has the meaning of the a posteriori Bayes probability that a photon with coordinates $\mathbf{x}$ has originated from the source (or sub-model) $\mathrm{m}$. Thus, $\mathrm{N}_{\mathrm{m}}$ is the number of photons from the source $\mathrm{m}$, and $\mathrm{N}$ is the total number of photons. As in the previous section, this set of equations defines a convergent iterative system. While the Gaussian mixture model described above can model any intensity image, usually, it is practically useful for images composed of relatively few Gaussian "blobs". More complicated models that are required for more complicated images can be developed and estimated by combining the new estimation principle with a technique described in $[10,11,16]$.

\section{DISCUSSION}

This paper introduced a new estimation principle based on the Einsteinian interpretation of the spectrum as a probability density for the photon frequency. Today, the photon composition of light is well known, and Einsteinian interpretation of spectrum as a frequency pdf is not surprising and no different in principle from other distributions considered in statistical physics. Still, my inspiration for connecting physical estimation of state distributions with statistical estimation came from the 1910 paper by Einstein and Hopf, and I think that terms "Einsteinian likelihood" and "Einsteinian information" are justified.

The new estimation principle is applicable to most regular intensity images and signals produced by classical (macroscopic) imaging systems. We used classical limit for computations of the numbers of states, still, the quantum nature of the electromagnetic (or acoustic) field determines statistical properties of a photon (or phonon) ensemble and the estimation procedure. This can be compared to the Plank distribution of a classical blackbody radiation being determined by the quantum nature of the electromagnetic field. The quantum structure of nature is utilized in the new estimation principle for solving a particular problem of the classical Shannon information theory. Information is defined with respect to elementary choices among classes, or elementary states. In classical information theory, the definition of elementary states is arbitrary. In the proposed estimation principle, when interpreted as maximal mutual information, the elementary states are given by the quantum states, photons (or phonons).

This paper considered positive-valued intensity or power signals and images. But, it seems that the developed technique can be extended to non-positive and complex-valued signals as well. The physical field carrying the signal is composed of photons or other particles and the phase of the signal is related to the phase of the particles. Therefore, one may expect that phase density models can be developed that relate the signal phase to the photon (or phonon) density. These models will be suitable for oscillating and complex-valued signals, and will correspond to the estimation principle described in this paper.

The "Einsteinian" estimation principle described here has been applied to a number of diverse applications, on a somewhat ad hoc basis. It was applied to spectrum estimation of autoregressive signals 6 ; numerical results for a number of cases have shown that the new principle results in more efficient estimators than the classical ML and ME estimators in non-asymptotic region. Applications to model estimation for two-dimensional time-frequency spectra of acoustic signals also was described in [6]. Modeling of interfering phenomena in over-the-horizon radar spectra was described in $[8,9]$ along with estimation of parameters of specific events of interest due to ionospheric disturbances. Development of concurrent detection, classification and tracking techniques (so called track-before-detect) based on the new principle was described in [10] for radar and in [11] for sonar. Superior performance to the classical techniques was indicated.

\section{REFERENCES}

1. Kullback, S. \& Leibler, R.A. (1951). On information and sufficiency. Ann.Math.Stat. 22, 76-86

2. Jaynes, E.T. (1957). Information theory and statistical mechanics I, Phys.Rev., 106, 620-630

3. Shore, J.E. \& Johnson, R.W. (1980). Axiomatic derivation of the principle of maximum entropy and the principle of minimum cross-entropy. IEEE Trans. Inf. Theory, IT-26 (1), 26-37.

4. Shore, J.E. (1984). On a relation between maxmum likelihood classification and minimum relative entropy classification. IEEE Trans. Inf. Theory, IT-30 (6), 851-4.

5. Einstein, A. \& Hopf, L. (1910). Uber einen Satz der Wahrscheinlichkeitsrechnung und seine Anwendung an die Strahlungstheorie. Annals of Physics, 33, pp.1096-1104.

6. Perlovsky, L.I. (1994). A Model Based Neural Network for Transient Signal Processing, Neural Networks, 7(3), pp. 565-572.

7. Proakis, J.G., Rader, C.M., Ling, F. \& Nikias, C.L. (1992). Advanced Digital Signal Processing. Macmillan, New York, NY.

8. Perlovsky, L.I., Plum, C.P., Franchi, P.R., Tichovolsky, E.J., Choi, D.S., \& Weijers, B. (1995). Estimation of Ionospheric Effects on Radar Spectra. International Conference on Signal Processing Applications \& Tecnology, Boston, MA.

9. Perlovsky, L.I. (1996b). Einsteinian Neural Network.. Proceedings of World Congress on Neural Networks, San Diego, CA; Lawrence Erlbaum Associates, NJ, pp. 603-606.

10. Perlovsky, L.I. (1995). MLANS Tracker. Proceedings of ARPA Counterdrug Over The Horizon Radar Workshop, Chesapeake, VA, pp.A147-A173.

11. Perlovsky, L.I., Schoendorf, W.H., Tye, D.M., Chang, W. (1995). Concurrent Classification and Tracking Using 
Maximum Likelihood Adaptive Neural System. Journal of Underwater Acoustics, 45(2), pp.399-414.

12. Akaike, H. (1973). Information theory and an extension of the maximum likelihood principle. Petrov and Csaki, Eds. Proceedings of the Second International Symposium on Information Theory, Budapest: Akademiai Kido.

13. Dempster, A.P., Laird, N.M. \& Rubin, D.B. (1977). Maximum Likelhood from Incomplete Data via the EM Algorithm. Journal of the Royal Statistical Society, 39B.

14. Sakurai, J.J. (1985). Modern Quantum Mechanics. Addison Wesley, New York, NY.

15. Shannon, C.E. (1948). The mathematical theory of communication. Bell System Technical Journal, July and October, 1948.

16. Perlovsky, L.I., Schoendorf, W.H., Burdick, B.J. \& Tye, D.M. (1997). Model-Based Neural Network for Target Detection in SAR Images. IEEE Trans. on Image Processing, 6(1), pp.203-216.

\section{APPENDIX}

Consider a "universe" consisting of the two interacting systems, "world" characterized by the data $\{S(\omega)\}$ and "estimation system" characterized by the model $\{F(\omega)\}$. Mutual information in the model about the data is given by $\mathrm{I}=$ $\ln (\mathrm{p}(\mathrm{S}, \mathrm{F}) / \mathrm{p}(\mathrm{S}) \mathrm{p}(\mathrm{F}))$. Here, $\mathrm{p}(\mathrm{S}, \mathrm{F})$ is the probability (or pdf) of the data $\{S(\omega)\}$ and estimated model $\{F(\omega)\}$ (in the interacting universe), and $p(S)$ and $p(F)$ are the probabilities (or pdfs) for the non-interacting universe. Using $\mathrm{p}(\mathrm{S}, \mathrm{F})=$ $\mathrm{p}(\mathrm{S} \mid \mathrm{F}) \mathrm{p}(\mathrm{F})$, the mutual information can be rewritten as $\mathrm{I}=\ln ($ $p(S \mid F) / p(S))$, where $p(S \mid F)$ is a conditional probability (pdf). These probabilities can be computed by using corresponding numbers of states, $I=\ln \left(\Gamma_{S \mid F} / \Gamma_{S}\right)$. Here, $\Gamma_{S \mid F}$ is the number of the world states compatible with the knowledge of the data $\{S(\omega)\}$ given the estimated model $\{F(\omega)\}$, and $\Gamma_{S}$ is the number of states compatible with the a priori knowledge, "prior to interaction" between the two systems. Prior knowledge depends on properties of the model that are known a priori and do not change in the estimation process, this is given by $\mathrm{N}, \Phi$. By using computations similar to those in the text, we obtain $\ln \Gamma_{S \mid F}=\Sigma N_{\omega} \ln \left[\Phi_{\omega}\right]$ and $\ln \Gamma_{S}=N \ln \Phi$ $\omega$

(in these expressions we have omitted the terms accounting for permutations of identical photons, because, as we have seen, these terms do not depend on the model parameters and do not affect the estimation process). Finally, we obtain for the part of mutual information that depends on the model parameters, I $=\Sigma N_{\omega} \ln \left[\Phi_{\omega} / \Phi\right]=\Sigma N_{\omega} \ln [F(\omega) / \hbar \omega]$, this gives eq.(18).

W $\omega$

ACKNOWLEDGMENT. It is my pleasure to thank Dr. L.B. Levitin, who read the manuscript and made valuable suggestions. 


\title{
A Symbolic Representation of Misconceptions
}

\author{
Benjoe A. Juliano, Ph.D. \\ Department of Computer Science \\ Coastal Carolina University \\ Conway, South Carolina 29526, U.S.A. \\ Tel: (803) 349-2144 Fax: (803) 349-2455 \\ E-mail:Juliano@ccucs.Coastal.edu
}

\begin{abstract}
An intelligent system must process information as human beings do. A framework is presented wherein dynamic cognitive structures called chains of thought are used to embody the dynamic aspects of the intentions of intelligent agents. These chains of thought are represented as fuzzy cognitive structures. In this particular framework, structure mapping plays an important role in the notion of misconceptions. An expert agent has to deduce the "private" symbolic constructs of a novice agent in order to correctly guide the novice through a particular task. These "private" constructs are derived from (partially) corresponding "public" symbolic constructs that are a result of intelligent symbolic communication between the expert agent and novice agent. This whole process embodies what the author refers to as cognitive diagnosis.

Chains of thought, the "private" symbolic constructs, are the main focus of the current paper. Its applications in designing intelligent systems and cognitive diagnosis are emphasized. The resulting framework is also an important step towards developing a method for evaluating degrees of intelligence and possible comparison between (groups of) intelligent systems.
\end{abstract}

\section{INTRODUCTION}

For an artificial system to be considered intelligent, it must process information as human beings do. The theoretical work presented in this paper has two major goals. First, it investigates and describes what types of cognitive structures are used when intelligent agents communicate with each other. Secondly, it attempts to capture the dynamics of these cognitive structures during symbolic communication between intelligent agents.

Intelligent agents express their intentions when they communicate with one another. In order for this communication to take place, a common vocabulary has to be used. These "public" symbolic constructs make up speech, be it spoken or otherwise. In addition to this common vocabulary, there needs to be a correspondence between the syntax of the language used by each participant. As noted by Kohout [2], there must also be semantic agreement if the conversation is to be successful. Unfortunately, the transition from "private" intentions to "public" symbolic constructs is not always clear. This is true not just to agents actively involved in the conversation (we shall call these participants), but also to those agents not really involved in the conversation: spectators (correspondingly, non-participants) just observing the interaction.

Dynamic cognitive structures must be used by intelligent agents to deduce and make decisions. These must also be used to express themselves. The whole process of trying to determine what one agent is thinking, or attempting to express, based on a dialogue, their behavior, their actions, etc. is what initiated the work presented in this paper.

This work has relatively close ties to the field of semiotics, a discipline of combining the theory of signs, symbols, and meaning extraction. In the next few sections, we explore the area of intelligent tutoring systems as a foundation for the work by Juliano and Bandler [1]. Next, the dynamic cognitive structures that Juliano and Bandler call chains of thought [1] are introduced. A brief discussion of the implications of this work is presented and further work is also proposed.

\section{Tutoring Systems ANd Deducing Misconceptions}

\section{Intelligent tutoring systems}

Intelligent tutoring systems (or ITSs) provide, perhaps, the best ground work for investigating the cognitive structures of interest in this study. ITSs are systems designed to aid in tutoring students on a particular subject matter. These systems are supposed to possess some form of intelligence by having

1. some form of model of the student; and

2. some reasoning capabilities based on this model

The most common approach in the design of an ITS is to get a better understanding of possibly how we as humans perform in a tutoring role. In particular, we need to investigate how we represent and process information based on the two items listed above. This entails an approximation of the thinking process using some knowledge structure based on a system's "own" expert knowledge structure. The process of "thinking about thinking" is what Juliano and Bandler [1] have referred to as cognitive diagnosis, which includes any method of confirming the relative 
position of the novice student's thinking pattern based on a corresponding idealized pattern.

The approach we take is based on cognitive diagnosis during problem solving. In this scenario, the student is given several problems to solve and the tutor may intervene whenever appropriate in directing the student to understanding the concepts involved. It is during this problems solving session that a model of the student's understanding has to be formulated and used in determining when to intervene and how to control the direction of the session (do we present a simpler problem or do we go with one that is a little bit more challenging?).

\section{The process of deducing misconceptions}

Perhaps the most important factor in successful tutoring is how to correctly deduce student misconceptions. This is based on information gathered during a tutoring session, most likely consisting of partial or incomplete solutions to the problem at hand. This information may also contain a lot of noise. The student may indicate varying degrees of uncertainty (or confidence) to their solutions. Furthermore, under most normal situations, the information is usually available in various forms: verbal, visual cues, scribbled computations, diagrams or doodles, etc. Clearly, all this complicates approximating this in an artificial system.

To simplify the process, the following main assumptions are made for an underlying theory of knowledge states, which can be conveniently represented in a computer:

1. The knowledge of the expert agent and the knowledge of the novice agent overlap one another.

2. A knowledge state describes a set of concepts and the set of relations among these concepts.

3. When an expert agent approximates the knowledge state of a novice agent, this information is used to generate "lines of reasoning", which we call chains of thought, to account for each observed action.

The first assumption is important because it implies the view that expertise is decomposable into independent components that may be used to define various dimensions of knowledge. It also implies that "subsets" inherit essential characteristics of the "full" model; hence, the novice agent's mastery of a particular component can somewhat be deduced.

The second assumption indicates an associative representation. Fuzzy cognitive maps are used to account for the inherent vagueness and imprecision in most natural tutoring environments where decisions are based on imprecise, qualitative data. The third assumption adds an important level of detail in the dynamics of the process of cognitive diagnosis.

This subsethood assumption between the knowledge of the expert agent and novice agent somewhat parallels Kohout's [2] note that semantic agreement is required if a conversation between two agents is to be successful. In his statement, he indicates that there must be some form of overlap for this to proceed.

The process by which tutoring systems deduce misconceptions by novice agents is also similar to the way intelligent agents perceive their surrounding environment. In the tutoring scenario, the environment is replaced by a particular novice. The expert agent has to adjust to each novice agent and base its actions on its current model of that agent. Similarly, intelligent agents must adapt to changes in their environment.

\section{Approximating Chains of Thought}

\section{Fuzzy cognitive maps}

As indicated in the previous section, one of the assumptions we have is that knowledge states describe a set of concepts and the set of relations among these concepts. The dynamic cognitive structures we use to represent knowledge states are fuzzy cognitive maps (or FCMs). Mathematically [1], a FCM $M=\left\langle C_{M}, R_{M}\right\rangle$ over a finite universe of discourse, $X$, is a fuzzy graph that is a 2-tuple where: and

$$
C_{M} \in[0,1]^{X} \text { is a fuzzy concept space of } X
$$

$$
R_{M}=\left(R_{M}^{1}, R_{M}^{2}, \ldots, R_{M}^{r_{M}}\right) \text { is a fuzzy multirelation }
$$

Each $R^{k}$ (where $1 \leq k \leq r_{M}$ ) is a fuzzy relation on the fuzzy concept space $C_{M}$ For more details on the mathematics, operations, etc. on FCMs, refer to [1].

FCMs are ideal representations of knowledge states not just because they capture the essence of the first two assumptions listed in the previous section. They are ideal also because they can be encoded from the communication between two intelligent agents. This gives us a means of possibly representing "private" symbolic constructs to correspond to an agent's expertise or knowledge, or as an approximation of what another agent is trying to say.

\section{Chains of thought}

Knowledge states alone are not sufficient in capturing the three assumptions we laid out. To represent lines of reasoning, there has to be a way to move or transform from one knowledge state to another. This requires a formal representation of chains of thought. Mathematically [1], we can define a chain-of-thought structure on a universe of discourse, $X$, to be a 5-tuple $T=\langle C, R, \Psi, \Phi, \delta\rangle$ where the pair $C$ and $R$ define a FCM structure based on (1) and (2), respectively, and

$$
\begin{gathered}
\Psi \in[0,1]^{X} \text { is a knowledge state space } \\
\Phi \in[0,1]^{X} \text { is a valid input space } \\
\delta: \Psi \times \Phi \rightarrow \Psi \text { is a transition function }
\end{gathered}
$$

Notice that these definitions have some similarities to the formal definition of a finite-state sequential machine. For more details on the mathematics, operations, etc. on chains of thought, refer to [1]. 


\section{Knowledge communication and cognitive diagnosis}

What are the distinct processes involved in approximating chains of thought? We shall consider two intelligent agents, $A$ and $B$, although this could be extended to more than two agents. Without loss of generality, let us assume that $A$ is the expert agent and $B$ is the novice agent in a tutoring environment. Initially, we start off with the arrangement given in Figure 1 below.

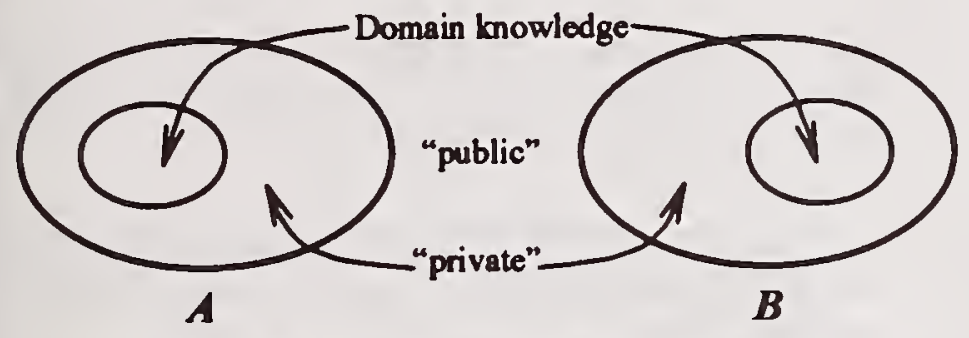

Figure 1 Initial phase of the interaction

The shaded region in each agent denotes domain knowledge. Recall that in our first assumption, the overlay principle (subsethood property) actually holds between agents.

Agent $B$ 's knowledge is then communicated to agent $A$ in some manner, possibly during a problem solving task. This is depicted in Figure 2.

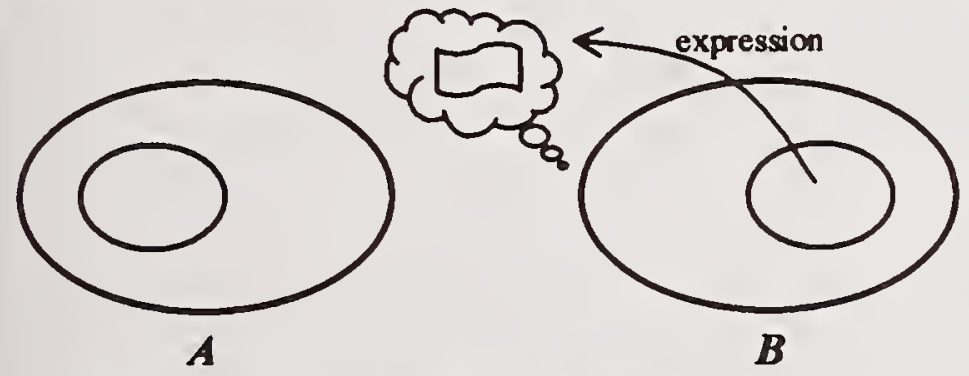

Figure 2 Communication from the novice agent

Whatever was expressed by novice Agent $B$ now has to be encoded by expert Agent $A$. This is done by Agent $A$ to either develop or update a model of what Agent $B$ is thinking. This is illustrated in Figure 3.

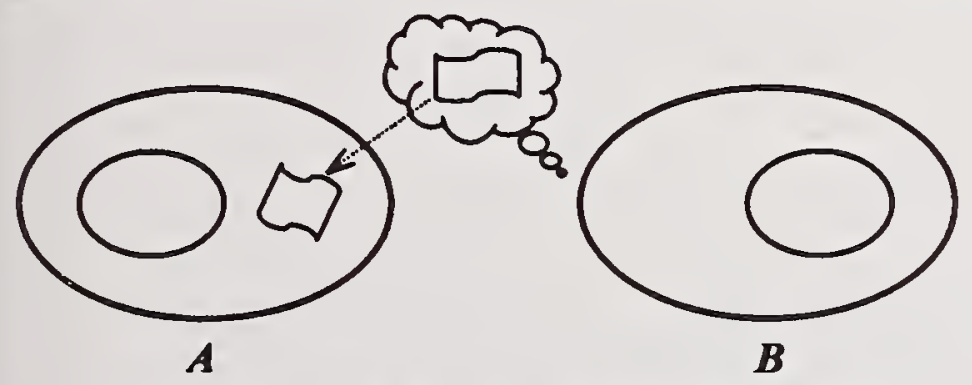

Figure 3 Encoding and model generation/updating by the expert agent

After expert Agent $A$ has generated and/or updated its model of what Agent $B$ is expressing (or thinking), the next step is model interpretation. In [1], Juliano and Bandler propose the use of a discrepancy operator to identify misconceptions, if any, indicated by the model of the novice. Whatever type of operator is used, this is applied to the expert's domain knowledge and the current model of the novice. This is depicted in Figure 4.

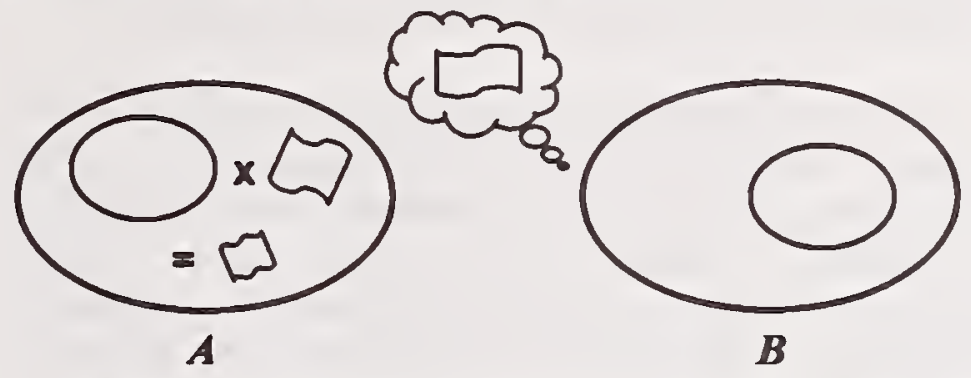

Figure 4 Model interpretation by the expert agent

Whatever interpretation is derived by expert Agent $A$, this has to be conveyed back to novice Agent $B$ in some form. This is illustrated in Figure 5. Hopefully this information will direct Agent $B$ to the ideal chain of thought.

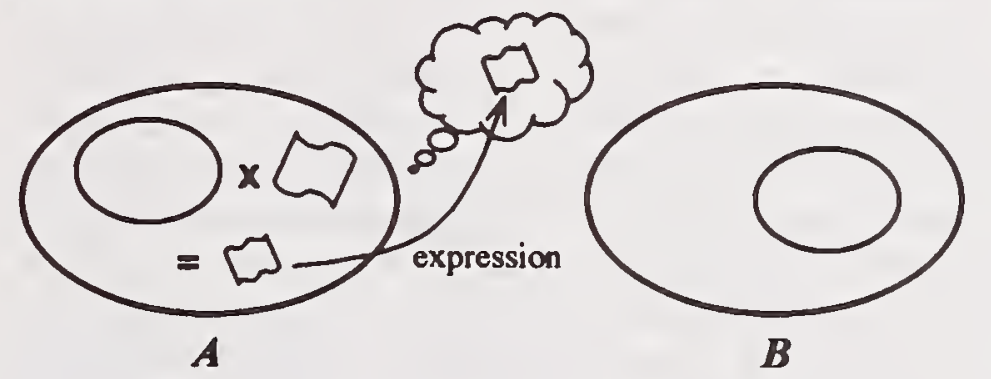

Figure 5 Expressing misconceptions identified by the expert agent

Next, notice that the novice Agent $B$ now undergoes a process similar to that depicted in Figures 3 and 4 . Communication from expert Agent $A$ has to be encoded and this information interpreted and possibly assimilated into Agent $B$ 's current knowledge structure. Thus, this model has a fairly general application for intelligent agents and the modeling of some underlying cognitive processes.

\section{Discussion}

The model for cognitive diagnosis outlined in the previous section warrants elaboration. Firstly, the appropriateness of using FCMs to represent knowledge states is emphasized by two factors in the model. Knowledge communication, the act of expressing one's knowledge (see Figures 2 and 5), is primarily fuzzy in nature. One can observe how to express most everyday reasoning and common sense knowledge - these are conveyed imprecisely. When it comes to approximating this in a machine, this is where the expressiveness of fuzzy set theory steps in. Furthermore, the transformation of spoken (or otherwise) language into an internal, "private" model as in Figure 3 is inherently noisy and imprecise as well. Again, fuzzy set theory can capture this imprecision.

The use of fuzzy graphs, called FCMs, also facilitates modeling of the operation(s) depicted in Figure 4. In [1], operations for fuzzy difference and fuzzy discrepancy between two 
FCMs are defined. In a tutoring scenario, these can be used by an expert agent to approximate any deviations by the novice agent from an ideal cognitive structure. This ideal, of course, is based on and limited by the expert agent's own domain knowledge.

FCMs also facilitate the formal definition of a chain of thought structure. Figure 4 was meant to depict operations on either knowledge states (using FCMs) or operations on lines of reasoning (using chain of thought structures). Again, in [1], a formal definition for chain of thought homomorphisms was defined that includes both structure preserving and transition preserving constraints.

Perhaps the biggest concern here is on the encoding scheme. This is depicted in Figure 3 as the transformation from communicated language to model generation or updating. To achieve semantic agreement, a limit can be placed on the communications bandwidth through the design of the corresponding user interface for the system under consideration. For example, in designing an intelligent tutoring system for elementary genetics, a graphical user interface can be designed to include such tools as a calculator, icons depicting various species to "breed", Punnet squares, etc. The interface can be designed so that the use of each tool corresponds to the expression, as in Figure 2, of a novice's knowledge in FCM (or chain of thought structure) form. Of course, this does not guarantee that all the necessary tools will be used in attempting to solve a problem, but this is merely part of the imprecision and noise inherent in the task at hand.

With this in mind, the expression of misconceptions depicted in Figure 5 can be done in the reverse transformation. The correct use of the tools provided by the interface can be emphasized. This, in conjunction with the elaboration of any other corrections to misconceptions (most likely at the knowledge state level) can account for a fairly powerful tutoring tool.

The framework presented here does not only apply to the area cognitive diagnosis in intelligent tutoring systems. The panoply of operations and relations on the cognitive structures listed in [1], some of which are presented in this paper, also provides an important step towards developing systematic methods for evaluating degrees of intelligence between intelligent agents. This will also be useful in comparing (groups of) intelligent systems.

\section{SummaRY AND CONClusions}

What was presented in this paper is a continuation of the ideas presented in [3] with emphasis on the dynamics of the chain of thought structure. The focus was on the idea that misconceptions can be symbolically represented by FCMs at the knowledge state level, or by chain of thought structures at the reasoning level. The area of cognitive diagnosis lends itself to other areas similarly characterized by decision making in complex systems with uncertainty. This uncertainty may be in the form of incomplete, incorrect, contradictory, misleading, or even noisy information.
Chains of thought, used to represent the "private" symbolic constructs of intelligent agents, facilitate the identification and symbolic representation of misconceptions. This is an important step in the design of intelligent systems; in particular, systems where cognitive diagnosis is a significant function.

\section{REFERENCES}

[1] B. A. Juliano and W. Bandler, Tracing Chains of Thought: Fuzzy Methods in Cognitive Diagnosis, Heildelberg: Physica-Verlag, 1996.

[2] L. J. Kohout, A Perspective on Intelligent Systems: A Framework for Analysis and Design, London: Chapman and Hall, 1990.

[3] L. J. Kohout and B. A. Juliano, "Dynamic aspects of interpretation of symbolic constructs," presented at Intelligent Systems and Semiotics - International Multidisciplinary Conference, National Institute of Standards and Technology, Gaithersburg, MA, 1996. 
XII SEMIOTICS OF TEXTS AND IMAGES 



\title{
MATHEMATICAL ASPECTS OF CYBERAESTHETICS
}

\author{
Leonid I. Perlovsky
}

\author{
Nichols Research Corporation, 70 Westview Street, Kilnbrook IV, Lexington MA 02173 \\ Tel: 617-862-9400; Fax: 617-862-9485e-mail: perlovsl@ nichols.com
}

ABSRACT: Cyberaesthetics addresses an emerging integrated multi-disciplinary approach to the development of intelligent systems: combining cybernetics, semiotics and aesthetics. The paper describes a mathematical apparatus of intelligence related it to Kantian philosophy, which has a room for the concept of beauty.

\section{INTELLIGENCE AND AESTHETICS.}

Robots that can read and enjoy literary texts are not yet at hand. Notwithstanding, there is a growing understanding among engineers and mathematicians, that intelligent systems of tomorrow will have to be able to perceive the meaning and intentions of concepts, scenes, and texts, depending on situations and contexts. And, in order to achieve this, robots must be endowed with emotions and aesthetics. The term "robot" we use broadly as an intelligent system, an "infobot". The term "aesthetics" we use in the Kantian sense as a science of feelings and feeling perception, a science of emotions. In western cultural tradition, there is a long-standing opposition between thinking and feeling. And often, we tend to forget that this is a highly abstract, refined, top level view of our psyche. Actual human processes of intellection are complicated interwoven interactions, vortices of multiple neural processes, involving various parts of the brain and various conscious, unconscious, and instinctual levels. A historic view of rationality as limited to Aristotelian-Gödelian logic is too narrow and is being rejected today. A new understanding of rationality emerges as a hierarchical goaldirected functioning, which involves internal and external actions; actions within the mind of an intelligent system and into the outside world. This new understanding emerges concurrently in multiple fields dealing with phenomena of intelligence: in philosophy, cognitive sciences, art and art criticism, education, mathematics and engineering.

If you pinch your finger, it hurts, and an ability to feel the pain is obviously an a priori faculty, which is necessary for survival to such an extent that it is shared throughout the entire animal kingdom. This "lower" origin of feelings separates them from our higher cognitive abilities. And, there is a long-standing line of thought that separates and counterposes feelings and thinking, emotions and intellect. Is there any ground for their unification? Neural and cognitive sciences have been concerned with relating emotions to material neural and bodily physiological functions. For example, neural pathways have been found from hypothalamus (brain areas associated with emotions) to viscera. The popular known connection between fear and upset stomach could possibly be understood as a survival mechanism regulating interactions between fear and hunger. This is another example of the "lower" aspect of emotions. Brain research relating emotions to higher intellectual functions is yet in the incipient stage. Interactions between cortical systems (associated with high cognitive functions) and hypothalamus is hypothesized to be mediated through amygdala. The high degree of reciprocal anatomical connections found among these neural structures suggests existence of information processing loops involving emotional and cognitive functions. Our knowledge of the brain structure is insufficient for deducing the mathematical theory of "higher" emotional functions, and many believe that even if the entire wiring diagram of the brain were available, it still would not be possible to deduce its main mathematical concepts. Neural and psychological data have to be combined with philosophical analysis and physical intuition in order to develop mathematical theory of higher emotions.

In 1787 in a letter to his friend, Kant wrote that he discovered a new type of a priori principle, the feelings of pleasure and pain, which he found to be a necessary part of our intellect. Kant came to a conclusion that our Judgment faculty is based on the feeling of pleasure caused by the harmony or correspondence between our internal representations-concepts and empirical phenomena. The new principle governs "intellectual emotions". These "higher" emotions are not separated from thoughts, but they are combined together in a dynamical process described by Kant in his three volumes that overturned our understanding of the entire history of the philosophical analysis of the "mind-body problem" [1781; 1788; 1790]. The present paper describes mathematical aspects of Cyberaesthetics, combining mathematics with Kantian theory of mind. The next Sect. 2 overviews a mathematical apparatus of the modeling field theory (MFT), suitable for describing higher emotions and providing a foundation for the mathematics of mind in which a concept of beauty could be included. Sect. 3 briefly reviews Kantian theory and establishes connections with MFT. Sect. 4 discusses a possibility for Emotional Machine and outlines directions of future research.

\section{MODELING FIELD THEORY}

\subsection{Systems with Adaptive Intelligence}

Consider (1) a set of input data $\{X(n), n \in N\}$, where each member is a vector in D-dimensional space, $\mathbf{X}=\left\{X_{d}, d=1, \ldots\right.$ $D\}$; (2) a set of categories $\{h \in H\}$, which are characterized by internal parameters $\left\{\mathbf{S}_{\mathrm{h}}\right\}$ and by models of the data $\left\{\mathbf{M}_{\mathrm{h}}\left(\mathbf{S}_{\mathrm{h}}\right.\right.$, n)\}; and (3) a similarity measure between the sets of models and data, $\mathrm{L}(\{\mathbf{X}\},\{\mathbf{M}\})$. A set of parameters is finite, $\mathbf{S}_{\mathrm{h}}=$ $\left\{\mathrm{S}_{\mathrm{h}}^{\mathrm{a}}, \mathrm{a}=1, \ldots \mathrm{A}\right\}$, but not necessarily limited, and a set of categories is not fixed, its cardinality $\mathrm{H}$ is finite, but may vary 
in the process of learning. A similarity measure is designed so that it treats each model as an alternative for each piece of data

$$
L(\{\mathbf{X}\},\{\mathbf{M}\})=\prod_{n \in N} \sum_{h \in H} r(h) \iota(X(n) \mid h),
$$

here $\ell$ is a conditional partial similarity between data vector $\mathbf{X}(\mathbf{n})$ and model $\mathbf{M}_{\mathbf{h}}$. For example, «can be selected as a probability density function. Then $\mathrm{L}$ is a total likelihood (this interpretation does not require statistical independence among data vectors $n$ and $n '$ : dependencies can be accounted for by considering $\mathbf{X}\left(\mathrm{n}^{\prime}\right)$ as parameters of the models for the data vector $\mathbf{n})$.

The problem consists in estimating internal parameters $\mathbf{S}$ and in making a choice of the categories (or classification), that is, in obtaining a partition

$$
\{N\}=\left\{N_{1}\left|N_{2}\right| \ldots \mid N_{H}\right\},
$$

that decides, which model $\mathrm{h}$ corresponds to an observation $\mathrm{n}$ (here $\mathrm{N}_{h}$ is a subset of $\mathrm{N}$ ), by maximizing the similarity eq.(1). When likelihood is used as a similarity measure, this is a problem of the maximum likelihood estimation.

Categories activated by high similarity values produce actions, including generation of messages transmitted within the intelligent system, which acknowledge the category activation. Messages include the category parameters. They can serve as input data for other categories and can be used for control of actuators. Correspondingly, data are sensory data or internal messages, and models are patterns of messages and sensory data. The above mathematical framework is fairly broad and encompasses most formulations of intelligent adaptive systems. It includes as particular cases, statistical and model-based pattern recognition, complex adaptive systems, neural networks, and systems of intelligent agents. The above formulation addresses "higher-level" intelligence functions of recognition and adaptation. A compete intelligent system would include lower-level drives for survival, reproduction and, in case of an artificial system, a robot or infobot, drives for producing specific tasks it was designed for.

In case, when a set of observations, $\mathrm{N}$, corresponds to a continuous flow of signals, for example, a flow of visual stimuli in time and space, it is convenient instead of eq.(1) to consider its continuous version,

$$
\mathrm{L}=\exp \int_{\mathrm{N}} \ln \left(\sum_{\mathrm{h} \in \mathrm{H}} \mathrm{r}(\mathrm{h}) \iota(\mathbf{X}(\mathrm{n}) \mid \mathrm{h})\right),
$$

where $\mathrm{N}$ is a continuum, such as time-space. In this case, models describe a continuous modeling field, conditional partial similarities can be compared to Lagrangian, and maximization of similarity $\mathrm{L}$ can be compared to minimization of action in the physical field theory.

\subsection{Multiple Hypothesis Testing}

A standard method of solving the above problem [Winston, 1984; Segre, 1992] consists in (1) considering all possible partitions eq.(2) (within certain a priori established limits), (2) obtaining an estimate of the parameters $\boldsymbol{S}_{\mathbf{h}}$ for each partition by maximizing similarity,

$\max \left\{S_{h}\right\}\left[\operatorname{pdf}(\{X(n), n \in N\}) \mid\left\{N_{1}\left|N_{2}\right| \ldots \mid N_{H}\right\}\right]$

and (3) choosing the most similar partition. Obviously, a computational complexity of this algorithm is at least on the order of the number of partitions $\sim \mathrm{H}^{\mathrm{N}}$, and denoting a complexity of maximization of the eq.(4) by $\mathrm{C} 1$, a complexity of the MHT algorithm can be written as

complexity $(\mathrm{MHT})=\mathrm{C} 1 \cdot \mathrm{H}^{\mathrm{N}}$

Newly emergent mathematical methods of solving the problem of intelligence while avoiding the combinatorial complexity of MHT include multiscale hierarchical organization and evolutionary computation.

\subsection{Fuzzy Partitions and Modeling Field Theory (MFT)}

A new proposed method of solving this problem utilizes fuzzy adaptive logic [Perlovsky,1995;1996]. Let us introduce fuzzy class memberships $\mathrm{f}(\mathrm{h} / \mathrm{n})$, associating each data vector $\mathrm{n}$ with each category $\mathrm{h}$ :

$\mathrm{f}(\mathrm{h} \mid \mathrm{n})=\mathrm{r}(\mathrm{h}) \iota(\mathbf{X}(\mathrm{n}) \mid \mathrm{h}) / \sum_{\mathrm{h}^{\prime} \in \mathrm{H}} \mathrm{r}\left(\mathrm{h}^{\prime}\right) \iota\left(\mathbf{X}(\mathrm{n}) \mid \mathrm{h}^{\prime}\right)$.

Eq.(8) looks like the Bayes formula for a posteriori probabilities, and $f(h \mid n)$ can be interpreted as probabilities, if likelihood is used as a similarity measure and when the parameters of the models are accurately estimated. Upon convergence of the estimation procedure described below, $f$ converge to the estimated probabilities. Let us specify an internal dynamics of the Modeling Fields (MF) as follows,

$$
\begin{aligned}
& \mathrm{H} \\
& d f(h \mid n) / d t=f(h \mid n) \sum_{h^{\prime}=1}\left\{\left[\delta_{h h^{\prime}}-f\left(h^{\prime} \mid n\right)\right] \cdot\right.
\end{aligned}
$$

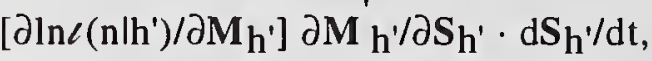

$$
\begin{aligned}
& \mathrm{d} \mathbf{S}_{\mathrm{h}} / \mathrm{dt}=\left\{\int_{\mathrm{N}} \mathrm{f}(\mathrm{h} \mid \mathrm{n})\left[\partial \ln \iota(\mathrm{n} \mid \mathrm{h}) / \partial \mathbf{M}_{\mathrm{h}}\right] \partial \mathbf{M}_{\mathrm{h}}^{\prime} / \partial \mathbf{S}_{\mathrm{h}},(8)\right.
\end{aligned}
$$

here

$\delta_{h^{\prime}}$ is 1 if $h^{\prime}=h^{\prime}, 0$ otherwise.

Parameter $t$ is the time of the internal dynamics of the MF system. The following theorem was prooved.

Theorem. Equations (7) through (9) define a convergent dynamical system MF with stationary states defined by $\max _{\left\{S_{h}\right\}^{L} \text {. }}$

It follows that the stationary states of an MF system give the maximum similarity solution of the model-based pattern recognition problem. When likelihood is used a similarity, the stationary values of parameters $\left\{\boldsymbol{S}_{\mathbf{h}}\right\}$ are asymptotically unbiased and efficient estimates of these parameters [Cramer, 
1963]. A computational complexity of the MF method is linear in $\mathrm{N}$,

complexity $(\mathrm{MF})=\mathrm{C} 2 \cdot \mathrm{H} \cdot \mathrm{N}$,

where $\mathrm{C} 2$ is defined by the relaxation time of the MF system and is likely to be independent of $\mathrm{N}$ (or depends weekly). In this way the MF system solves the problem of the exponential complexity associated with the MHT method, eq.(5). Fuzzy class memberships $f(h \mid n)$ define a fuzzy partition of set $\mathrm{N}$, which can be used to define a non-fuzzy partition of the type of eq.(4): $\forall n, \max _{h} f(h \mid n) \Rightarrow n \in N_{h}$.

MFT describes an intelligent system composed of multiple adaptive intelligent agents: each category-model is "dormant" until activated by a high similarity value. Every piece of data may activate several categories, which "compete" with each other, while adapting to the new piece of data. Adaptation and other actions by activated categories are largely independent from most other categories, except for those competing with each other. Most important actions by activated categories are transmission of messages that include category parameters and sustaining the loop of adaptation of their own parameters. Evolutionary computation is naturally incorporated within MFT: transmitted messages, including category parameters and other aspects of category-models are strings-objects of evolutionary computations and are employed by modeling field systems for adaptation of its structural components that could not be incorporated in a continuous fashion and are not the subjects of adaptive MFT loops. The overall architecture of MFT categories can combine heterarchical and hierarchical organization.

\section{MFT AND KANTIAN THEORY OF MIND}

\subsection{The Role of Kant's Theory}

What is intelligence? It is still shrouded in mystery. A lot is understood, but much still is unknown and, most likely, will remain unknown for a while. Intelligence is attributed to natural and artificial systems and some of these systems are very simple, while others are very complex. At lower levels, intelligence is an ability to sense the environment and to control the body (machinery) toward achieving few predetermined goals. At higher levels intelligence includes abilities of thinking, including recognition and formation of concepts, developing complicated internal representations of the outer world and self, understanding, language ability; planning behavior, including direction of attention, definition of goals and subgoals and the ways to achieve them; acting within itself and in the outer world; an ability of judgment, including feeling and emotions; abilities of intuition, learning, consciousness, creativity, and a mysterious feeling of freedom of will.

Kant overturned the understanding of the relationship between the mind and world by considering the specific a priori contents of mind that enable its functioning. He brought the philosophy of Pure Spirit close to the scientific method. He developed a rational explanation of mind as a system, if not in its entirety, still in its most interesting "higher" intellectual abilities. Many aspects of Kant's theory were further developed by a number of philosopher $s$ and psychologists, including Shopenhouer, Hegel, Nietzsche, Freud, Jung, Bergson, Jaspers. Still, the original theory of Kant remains unsurpassed in its comprehensive treatment of mind as a system. And, mathematical theories of intellect have remained far removed from the penetrating depth of his understanding and are inadequate for coming even close to the width of his analysis. This does not have to be so, for Kant's analysis is rational and therefore can perfectly serve as a foundation for developing the mathematical theory of mind. A first step toward rectifying this deficiency of the mathematical theories of intellect is undertaken here.

In three volumes on Critique of Pure Reason, Critique of Judgment, and Critique of Practical Reason, Kant explained a wide variety of intellectual experiences based on three fundamental abilities or faculties of mind: Understanding, Judgment, and Reason. Each is based on a specific a priori principles or instincts contained in mind: concepts, correspondence between concepts and manifold of sensory data, and will or desire. Understanding is a faculty of concepts, a source of general notions. Judgment is an ability to see that a particular case comes under the general rule. And, Reason is an ability to draw conclusions that is to generate behavior. (The most important type of behavior, interwoven with higher intellectual abilities and emotions, Kant considered to be the behavior of learning.) These three abilities correspond to the three main modes of consciousness: knowledge (of concepts), feeling (of correspondence between concepts and outer world), and desire (to act). Even so Kant devoted a separate book to each ability, they should be combined within a dynamical system constantly exercising all three abilities in their interaction. This paper makes a step toward developing a mathematical apparatus of Kantian theory. We will see that MFT contains seeds of mathematical modeling of the three main elements of intelligence identified by Kant. MFT carries Kantian analysis further: it is a dynamical system, in which the three abilities identified by Kant exist in the process of constant interaction, as it were in a "vortex". This vortex models learning of a category as a dynamical formation of a symbol. I overview some of the higher intellectual abilities, along with attempts at their rational explanation and mathematical modeling.

Emotions and perception of beauty are fundamental to human mind, alike in everyday life, arts, and sciences. Still, the concept of beauty is mystifying. The first step towards mathematics of beauty is made here. It is founded on the relationship between MFT and Kantian theory of mind. MFT's will for learning, according to Kant, is the most important will, which serves as a basis for emotional intellectual abilities, for beautiful and sublime.

\subsection{Understanding Is Based on Internal Models}

An internal model is a basis of intelligence. Even at the lower levels, say, of a lobster sensing and grabbing food, with the axons of sensing cells "wired" directly into the neurons 
that control muscles, we can talk about internal model. Because, the signal that a "food-sensing" neuron sends to "muscle-neuron" indicates an internal lobster's representation of food. There is no such thing as "food" in the ocean, "food" is a dynamic process of interaction between an object in the ocean, sensing neuron (that forms an internal representationsignal), grabbing neuron, and other relevant neural aspects of the lobster's experience. A lobster's mind has literally few neurons, and if our final goal would be modeling of a lobster's mind, we will directly proceed to studying its wiring diagram without such nebulous and not obviously useful concepts as lobster's internal models.

Our aim, however, is to understand and model higher levels of intelligence. At higher levels, a complete "wiring diagram" of a neural system, even if available, would be so complicated that it does not furnish an understanding of the basic principles of mind. A significant part of the brain is involved with internal models (storing, updating, and using them). Our ability to recognize concepts, even simple ones, such as objects, is due to internal models or representations of concepts. Understanding, first of all, consists of concepts in our mind along with their interrelationships. Higher levels of understanding, such as understanding of meaning, involve a complicated internal model composed of a large number of submodels-concepts with multiple interconnections among them. Possibly, every particular phenomenon of understanding-meaning only exists within a limited domain, within a certain situation or with regard to a certain goal. Then, meaning of a concept is modeled by including this concept within a set of situationally relevant other concepts and goals. Meaning requires a hierarchical system: the understanding of a meaning of a concept requires a point of view from the next levels in a hierarchy, above the level of the concept's inner model and its recognition. Thus, the meaning of a lower level concept is included into a higher level concept. However, relationships among levels are not rigidly fixed: formation of certain concepts involves multiple hierarchical levels, and relative position of concepts in the hierarchical levels might be situationally dependent. Thus, heterarchical hierarchy might be a better term. Explanation of mind as based on a priori inner models ascends to Plato and Aristotle. Kant identified a priori inner models as a separate faculty of mind that he called Understanding. Mind's operations with a priori concepts Kant calls the domain of Pure Reason.

The main question that the analysis of Pure Reason shall answer, according to Kant, is "How are synthetic judgments a priori possible?" This ability Kant identified as a specific a priori faculty of pure reason. In our theory of mind, this specific faculty is represented in hierarchical models: next levels in a hierarchy contain synthesis of the lower level concepts. This synthesis is of the a priori nature, because the hierarchical structure of the internal model is of the a priori origin. Thus, development of hierarchical models is a key to mathematical modeling of the Understanding and Pure Reason. Making this hierarchy adaptable and situationally dependent is an additional challenge.
Explanation and modeling of the phenomena of meaning and understanding requires also including them within behavior generation and acting of an intelligent system. The acting could be inside, within an intelligent system, or outside of the intelligent system, into the outer world. Actions, corresponding to the goal or situation (internal or external), constitutes a part of meaning. There is also another aspect of acting out in the external world noted by Freeman, who introduced a concept of external representations in the world. Our external acts and their results (being perceived by ourselves and others) from gestures and utterances to our entire culture, as it exists in the outside world, are external representations. To the extent that external representations are included into the Kantian cycle of category formation, they can be viewed as parts or extensions of our internal models. Computer simulations are a perfect example of such extensions of internal models.

According to Kant, Logic gives laws of understanding, or laws of relationships among a priori categories. Here, in the world of Ideas, there is a significant domain of applicability of the Aristotelian logic. For example, an internal model category of an object is either that of target or not, according to the Aristotelian logic law of excluded third (and this logic is different from fuzzy logic of judging which real signal belongs to which category). This domain of the Aristotelian logic encompasses non-adaptive aspects of the a priori models. To the extent that the a priori models can adapt, they are fluid, non-Aristotelian, fuzzy. Development of adaptive hierarchies of models is a challenge for future research.

To summarize the MFT relationship to Kant's Understanding: MFT ability for Understanding or forming concepts is due to a priori internal models, and an ability for synthetic judgments a priori is due to an a priori hierarchy of models and relationships among them.

\subsection{Judgment Is Based on Similarity Measures}

For internal models or concepts to be useful, there should be a way of relating them to experience. In other words, we should be able to recognize individual phenomena according to general concepts and to decide which empirical facts correspond to which concepts. Kant called this ability Judgment and considered it as one of the three main abilities of mind. Judgment is an ability to see that a particular case comes under the general rule. In MFT theory, Judgment is mathematically represented by similarity measures. MFT contains a measure of similarity between the internal model and the world, as well as between each submodel-concept-agent and a particular subset of input data. This is an a priori property of MFT and, according to Kantian analysis it is an a priori property of our mind.

Kant differentiates determinant and reflective aspects of Judgment. Finding particular subsets of sensory data corresponding to a specified concept-submodel is the determinant Judgment. And, finding the concept corresponding to the data is the reflective Judgment. MFT contains mechanisms of both, determinant and reflective Judgment. Within the iterative loop of MFT adaptation, 
determinant Judgment is given by the association (segmentation) of data with submodels, and reflective Judgment is given by selecting the concept-submodel most similar to the data segment (subset). In MFT both are given by the fuzzy associations, $f(k \mid n)$, with $n$ designating data and $k$ designating the concept-submodels.

Why is the Nature in its manifold knowable to our mind? Is it due to a specific property of Nature, or due to a specific property of mind? In other words, what makes it possible for our Understanding and Judgment to function in the way described above? Kant's answer is that this possibility is due to a special a priori property of our mind. This property is the purposiveness of our internal representations (models). Understanding and Judgment are so constructed that internal representations of empirical events and objects appear to us as purposive (the purpose includes first, a correspondence between our internal representations and the world, and second, an ability to learn or to improve this correspondence). This purposiveness provides a foundation for the development of higher faculties of mind including higher emotions, and the notions of beautiful and sublime.

A reader might wonder if this discussion is too philosophical and irrelevant to a mathematical theory of mind? The relevancy of this discussion is in that it guides us in constructing internal models, measures of similarity, and in developing evolutionary theories explaining these abilities. The models and similarities are constructed so that they have a purpose or meaning within the intelligent system, which is the mathematical description of the intentionality of the intellect. This intentionality includes the correspondence to the world and adaptivity that provides for learning. And, it is needed so that the "lower level" instincts for survival, for performing specific tasks, etc. can be more efficiently satisfied (by a living being or a robot). Intentionality provides a background for a mathematical theory of higher faculties of mind, including a possibility for mathematical treatment of beautiful and sublime. And, an evolutionary theory have to lead to these abilities.

To summarize the MFT relationship to Kant's Judgment: MFT ability for Judgment is due to similarity measures and fuzzy category memberships, which select data corresponding to the categories of Understanding and select categories corresponding to the data, in iterative cycles of every MFT loops-agents.

\subsection{Reason Is Based on Similarity Maximization}

Judgment mediates between concepts of Understanding and concepts of Reason (will, and freedom). In particular, reflective Judgment ascends from particular to universal, from sensory data to concepts. Its principle is an ability to learn, which Kant called the purposiveness of intellect towards the object. This ascendance from data to concepts is practically realized by Reason. In MFT functioning, finding a submodel corresponding to a piece of data (Judgment) is followed by adaptive modification of the model, which is the act of will according to the learning principle (law) of Reason. Reason provides laws for behavior, and MFT paradigms considered in previous chapters were concerned with one type of behavior: learning behavior as adaptation of the internal model. Modification of models in MFT is governed by the principle of maximum similarity between the model and data. The MFT parameter-adaptation equations maximizing the similarity give the laws of Reason. Thus, MFT provides for a mathematical description of a will for learning, a will for improvement of its internal representations of the world and the laws of Reason governing this will.

Kant emphasized a fundamental nature of the antinomy between causality and freedom and severely criticized philosophers, who underestimated the difficulty of the causality-freedom antinomy. And, his criticism still applies to a researcher, who is too cavalier about resolving this antinomy. The fundamental source of difficulty is in that freedom is an opposite of randomness. Freedom supposes causality. If there is no causality, there could be no freedom. But if the world's laws are causal, how the freedom could be explained? Kant made a step towards resolving this antinomy. He assigned the concept of causality to Understanding, where causality is an a priori concept of understanding the nature, the world of phenomena. And, he assigned the concept of freedom to Reason, where it is an a priori concept governing human desire and will. According to Kant, freedom belongs to a noumenal world, it originates from the unknowable nature of a human-in-itself. A next step toward resolving this antinomy should be attempted by identifying the unknowable human-initself with our unconscious and developing a physical theory of conscious and unconscious aspects of mind.

\section{EMOTIONAL MACHINE (TOWARD MATHEMATICS OF BEAUTY)}

In 1787 Kant discovered a new type of a priori principle, which led to his formulation of Judgment faculty that unified his philosophy. Kant came to a conclusion that Judgment is based on the feeling of pleasure caused by the harmony or correspondence between our internal representations-concepts and empirical phenomena. The new principle governs "intellectual emotions", which are not separated from thoughts, but are combined together in a dynamical process of Kant-MFT loopagents. Mathematical apparatus describing Judgment in MFT are given by similarity measures. And, a thought process is a loop, a vortex of concepts, emotions, and adaptation actions.

Higher emotions are related to an ability for the perception of beauty, which is a universal and fundamental property of human mind. It is important not only in the field of fine art, but it pervades human experience. And, there are well known statements by famous scientists, explaining that the first and foremost test of a scientific theory is its beauty. But, mathematical attempts to model mind, so far, have not touched the subject of beauty, and the directions along which this could be attempted seem to be hidden in mystery and not accessible to scientific investigations. Here, I attempt a first step in this direction. In this, I have found that I am greatly added most of all by Kant, who with scrupulous detailness analyzed rational mechanisms of beauty and other higher emotional faculties. 
When designing an intelligent system, say a robot, we decide what kinds of objects the robot should be able to recognize and we supply the robot with the internal models of these objects. From robot's perspective, only those types of objects exist which it can recognize. Every object has a purpose of being recognized (in addition to any other purpose, the robot may put this object for). A universal purpose of any object is its concept: for an object to have any purpose for a particular intelligent system, the object's concept has to exist in the system. This is a design principle of any intelligent system. And, this design principle is applicable to us: the evolution (or God) designed us so that we can find our ways around those objects which we recognize in the nature. The basic principle of the design is that the nature appears to us as purposive. The purposiveness of nature is the a priori part of our representations and it harmonizes nature with our desire for knowledge and produces the feeling of pleasure (or pain, if chaos is encountered).

Knowledge about objects comes from experience and from the a priori concepts (Understanding). The role of Judgment in this process was discussed in the previous section: it is an objective, or cognitive aspect of Judgment. In this section, we concentrate on the subjective aspect of Judgment, that relates to the subject and not to the object. This subjective aspect is the satisfaction and feeling of pleasure that is bound with the harmony between our internal representations and an object. Kant calls this aesthetical aspect of Judgment, because it relates more to emotions than to cognition (even so all aspects are combined in every act of perception and cognition). This aesthetical aspect of Judgment is related to the "pure" purposiveness of our representations, which is separate from any specific purpose that an object can be used for, and includes only the knowledge itself. Thus, android-robots capable of self-learning have to be designed so that they have an aesthetical affinity to knowledge. In MFT this is given by the similarity, $\mathbf{l}(\mathbf{X} \mid \mathbf{M})$, that relates a particular case $\mathbf{X}$ to the general concept $\mathbf{M}$, without any further specific purpose the object can be used for.

To the extent that the purposiveness is felt in its pure form and is bound to its a priori nature, the object is called beautiful. The nature of beauty is related to an interest not in the object, but in the subject: what I make out of this representation in myself. Beautiful is what coincides with the purpose of acquiring more knowledge and improving the harmony between the internal representations and Nature. Kant discusses two higher intellectual aesthetical abilities: feelings of beautiful and sublime. Beautiful involves the relationship between Judgment and Understanding and Sublime involves the relationship between Judgment and Reason. The feeling of Sublime moves the Reason to act toward improvements of internal representations. MFT provides a foundation for the mathematical description of these abilities: similarity performs both of these functions, it establishes relationships among data and models (concepts of Understanding), and it activates actions of adaptation toward improving the harmony between the models and nature.
Is it possible to write an equation of beautiful? And should it be the goal of our study? Could there be an equation that tells the difference between Rembrandt, Warhol, and a causal recreational artist? Of course not. The purpose here is to demonstrate that there is a possibility for a mathematical theory of mind, in which concepts of emotion and beauty have place. But then, why not proceed directly to producing by means of MFT even a simplest example of something beautiful? Where is the difficulty precluding us? The difficulty is in an adaptive nature of beauty. Kant got himself in trouble with later readers and admirers, by his attempts to provide examples of what is beautiful and what is not. His examples (such as e.g., that drawings could attain pure beauty and paintings do not) were immediately criticized and he was branded as having undeveloped aesthetical taste and worse. One of the reasons is that what was beautiful thousands years ago is not necessarily beautiful today. Concepts of Understanding evolve, and those concepts that were useful sometimes ago, in that they captured important aspects of nature and provided an evolutional advantage to those who possess them, are not necessarily useful any longer, they lost their purposfulness. Within our evolving internal models, some concepts niay become commonplace, outdated, empty of useful contents, and contradictory to newer, better adapted concepts. Since mathematically, beauty is related to the harmony between the internal model and nature, it is changing with time. What is an excellent harmony between an adaptive model and data in an engineering system, such as considered in [Perlovsky, 1994; Perlovsky et al, 1997], is a very simple construct, unworthy of the word "beauty" in the context of our mind. In order to design android-robots capable of the humanlevel perception of beauty, even at a rudimentary level, their internal models have to be much more complicated than currently used in engineering applications. But this quickly changes. And, possibly, we will see complicated robots, which would have to learn for many years, like human do, acquire individual subjective experience, and their perception of beauty will acquire human-like, individual features.

\section{REFERENCES}

Kant, I. (1781). Critique of Pure Reason. Willey, NY.

Kant, I. (1787). Letter to Reingold. In Tractats and Letters, 1980, Interscience, Moscow.

Kant, I. (1788). Critique of Practical Reason. Willey, NY.

Kant, I. (1790). Critique of Judgment. Tr. J. H. Bernard, 1914, 2nd ed., Macmillan \& Co., London.

Perlovsky, L.I. (1994b). A Model Based Neural Network for Signal Processing, Neural Networks, 7(3),pp. 565-72.

Perlovsky, L.I. et al (1997). Model-Based Neural Network for Target Detection in SAR Images. IEEE Trans. Image Proc. 6(1).

Segre, A.M. (1992). Applications of Machine Learning. IEEE Expert, 7(3), pp. 31-34.

Winston, P.H. (1984). Artificial Intelligence. 2nd edition. Addison-Wesley. Reading, MA. 


\title{
Semiotics and Modeling Computer Classification of Text with Genetic Algorithm : Analysis and first Results
}

\author{
Vincent Rialle*, Jean-Guy Meunier**, Sofiane Oussedik*, Georges Nault**
}

* Laboratoire TIMC-IMAG, UMR CNRS 5525, Université Joseph Fourier, Grenoble.

E.mail : Vincent.Rialle@imag.fr.

** Laboratoire d'Analyse Cognitive de l'Information (LANCI). Université du Québec à Montréal.

\begin{abstract}
Computer engineering proposes the construction of complex systems by dynamic prototyping (Buddle and Bacon, 1992). But this prototyping cannot be inductive and purely considered as a trial an error process. To be successful, one must possess an underlying hypothetical model (Marr, 1982) of what are the functions of the system. If these functions relates to physical tasks, such as sensing temperature, manipulatiing objects, etc., the desired behavior can be observed, and a model can be built. Conversely, if the functions of the system are to be applied to semio-informational tasks, such as language translation, information retrieval, hypertext navigation, text generation, etc., the interpretative behavior is not readily observable. Now, as any other computer systems, these systems are symbol manipulation machines (Newell ,1980). They must also manipulate input and outputs, but, in themselves, these data are semiotic objects, and not physical ones. These systems manipulate objects that have to be interpreted by some cognitive agent. In other words, systems that manipulate physical objects require a model of the physical word, while systems that manipulate informational objects require a semiotic model. In this paper, we illustrate how a semiotic model can help in the conception, the modeling, and the experimentation of a semiotic behavior such as Computer Assisted Reading and Analysis of Text (CARAT), and how this model has called upon the Genetic Algorithm (GA) theory to realize some of its aspects.
\end{abstract}

\section{Presentation of CARAT}

\subsection{General presentation}

Computer Assisted Reading and Analysis of Text is the computer technology that offers readers an asssistance in attaining some aspects of the informational or semiotic content of a text (discursive, lexical, hypertextual, thematic, stylistic, etc.). So, CARAT definitely relates to interpretative actions. There is in no way a robot that reads or understand a text by itself.

One the classical models of text interpretation is the philological one ${ }^{1}$. Through the centuries, thousands of readers, exegetes, and interpreters have practiced this method. Because of the quality of its principles, it has acquired compelling recognition, and the weight of its

1 A certain number of researchers using information technologies are beginning to place themselves in this philological perspective (cf. Thrane el al., 1992). experience. The basic principle of philological perspective is that one can construct relatively systematic procedures capable to ensure rigor in text interpretation. As a matter of fact, philology is an instanciation of an interpretative semiotic process applied to the processing of textual signs. It takes sets of signs (a text) as its input, then classifies, categorizes them, explores and selects them, and produces a new set of signs - the commentaries - as its output. This interpretation process can be translated functionally in terms of (a) inscription, (b) classification, (c) exploration, and (d) configuration, of information (Seffah and Meunier, 1994). In its principles, three important dimensions can be emphasized : text reading and analysis is a systematic, dynamic and plastic behavior. Systematicity pertains to the controlled processing of information; dynamicity concerns the interaction of the analyst with the text; and plasticity allows the constant renewed interpretation of the text.

In order to respect this particular type of interpretation process, a computer model must rely on an open architecture. It must allow an information processing flow that is systematic dynamic, and plastic. Each processing will be built out of interactive advances and restarts which sometimes are autonomous, sometimes are interrelated, but which all aim at assisting the reader and analyst in penetrating the content of the information. Hence, again a CARAT system is not a robot reader, but a faithful assistant in reading and analyzing texts. In this perspective CARAT is defined as the set of serial or parallel operations which, with the assistance of the computer, construct interpretative paths in which each moment produces a new textual object to be classified, explored and configured.

\section{I.2 CARAT and classification}

There exists an infinity of possible CARAT processing flow. Each text, for each person, can be read and analysed in so many ways. One can, for instance, inquire on a particular theme, paraphrase and summarize a specific segment, study the lexicon, evaluate the style, retrieve information, build a thesaurus or an index of the content, and so forth. Among all the operations at work in each of these processing, we will study more particularly the classification process. This process is important since interpretation always requires some type of classification of the incoming signs, symbols or information.

In the field of text processing, there exists many strategies of classification. Some classical strategies : a) 
are of logico-symbolic type (eg (Hobbs, 1993; Sowa, 1991) or semantico-linguistic (Rastier, 1987, BertrandGastaldy et al., 1987, 1993). Others are statistical (Church and Hanks, 1990; Reinhert, 1994; Lebart and Salem, 1994; Pustejovsky, 1991; Wilks, 1996; Salton, 1989; etc.). Albeit very systematic these approaches lack dynamicity and plasticity. Learning is limited, and they are very weak on processing a constantly everchanging informational input, as it is often the case with textual data (for instance on the World Wide Web). Finally, some can be referred to as "emergent computation" models (Forrest, 1991). They include Markovian fields (Kindermann and Snell, 1980; Bouchaffra and Meunier, 1995), connectionnism (Rumelhart, 1986; Salton and Buckley, 1994), and Genetic Algorithms as shown in this article. Besides their properties of statistical strength and generalization, they are systematic like any other clustering strategies, dynamic and plastic (learning is possible).

Our purpose here, is mainly to show how the Genetic Algorithm approach to classification can be applied to the problem of semiotic interpretation of text, and most of all in the context of CARAT technology. Although validation and experimentation of the GA approach is not the main purpose of this paper, which is modeling, some initial experiments will be reported.

\section{Genetic Algorithms}

\section{II.1 General presentation}

The GA approach takes its inspiration from research done on adaptive systems. This research sees such a type of system as an agent that applies to a domain (called the environment) specific operations which allow him to act upon it in the most efficient manner. This principle is of course based on the assumption that an adaptive system is able to detect, or extract, from its heteroclite domain any regularities which concern it, and vis-à-vis which it must construct a plan of adaptation.

"The adaptive plan determines just what structures arise in response to the environment, and the set of structures attainable by applying all possible operator sequences marks out the limits of the adaptive plan's domain. " (Holland 1992: 4)

In other words, a strategy of adaptation is the best plan of action that a system could put into place in order to identify the structures of its environment. In a more traditional sense, it uses some type of pattern recognition strategy in order to adapt. When applied to the field of genetic reproduction of species, this strategy of adaptation consists in finding, for a given environment, groups of individuals chromosomically best adapted. When constructed in a formal model, this strategy translates into an algorithmic model called genetic algorithm. The notion of genetic algorithm, presented for the first timeby John H. Holland in 1975 (Holland, 1975; Holland, 1992), was considerably developed during the 1980's and 1990's (Goldberg, 1989; Rawlins, 1991; Varela and Bourgine, 1992; Michalewicz, 1994).

The main function of this algorithm is the production of a population of individuals, out of an original population, best adapted to an environment which represents the constraints and particularities of the problem dealt with. The degree of adaptation is evaluated by means of a fitness function $f$. So, the GA is based on:

- an incoding of information, situations, problems and solutions, in the form of strings of building blocks, each string being able to be broken between each block, in the exact image of chromosomes which constitute veritable lists of characteristics of an individual. This incoding usually takes the form of a highly structured binary string, of a fixed or variable length according to the type of problem;

- the capacity to reproduce such strings in large number, which metaphorically relates to the sexual reproduction;

- the existence of a faculty of adaptation (simulated by the function $f$ ) which permits the evaluation of the quality of each individual created by the algorithm.

\section{II.2 Basic cycle of a GA}

In practice, a population $\mathrm{P}^{0}$ of potential solutions (the chromosomes) to the problem to be treated is generated at the initialization step. Then the following standard cycle, also called genetic search, is reapplied :

\section{INITIALIZATION}

If (stop test not verified) then

begin

EVALUATION ; SELECTION ; REPRODUCTION ; REPLACEMENT

end

At any given moment $t$, the population is: $\mathrm{P}^{\mathrm{t}}=\left\{\mathrm{a}_{1}^{\mathrm{t}}, \ldots, \mathrm{a}_{\mathrm{p}}^{\mathrm{t}}\right\}$, where $\mathrm{a}_{\mathrm{i}}^{\mathrm{t}}$ stands for the candidate solution $a_{i}$ at cycle number $t$. The main steps can be briefly described as follows :

1) EVALUATION: The elements of $\mathrm{P}^{t}$ are rank ordered from the most to the least fitted according to the selection probability Probs $_{S}$

$$
\operatorname{Prob}_{s}\left(a_{i}^{t}\right)=f\left(a_{i}^{t}\right) /\left(\sum_{j=1}^{p} f\left(a_{j}^{t}\right)\right)
$$

2) SELECTION: The elements which best satisfy the constraints or characteristics of the solution sought are selected according to the selection probability, and arranged by couples in order to prepare the next step.

3) REPRODUCTION: genetic operators are then applied to this population of élites, called parents, in order to obtain an intermediary population $\mathrm{P}^{\prime t}$. These operators permits the creation of new strings, among which some should have better fitness properties than their parents. Two genetic operators are generally employed: crossing-over, which allows the production of two new elements from two parent elements (Fig. 1), and mutation, which allows the creation of new solutions that would have been impossible to obtain by simple crossing. Mutation consists of a random selection of one of the bits of the chromosome, and to change its value with a pre-defined probability (probability of mutation) (Fig. 2).

4) REPLACEMENT: This is the generation of a new population by replacing the worst elements of the previous population $\mathrm{P}^{\prime}$ by the best of $\mathrm{P}^{\prime} \mathrm{t}$. This new 
population possesses at least as many and sometimes more of the characteristics of the solution than the preceding generation.

$1011000101101000: 001011010000$ $0110100011101001: 011000101101$

Before cross-over

$1011000101101000: 011000101101$ 0110100011101001001011010000

After Cross over

Fig. 1. Diagram of crossing-over

$\frac{1000111010}{0<I_{>} \quad p=0,0001}$

Fig 2. Mutation

This cycle is iterated a lot of times until a generation of optimal solutions is obtained, and from which only the bests will be retained. In certain contexts it is the entire population which stands in lieu of the solution.

\section{Genetic Algorithms and CARAT}

\section{III.1 The object of the model}

As previouly said, CARAT can fan out in many different processing flows. In this section, we propose a modeling, by means of a genetic algorithm, of the classification moment of two specific processing flows : the first flow aims at giving the reader hints on the semantics contexts of particular words ; the second flow aims at automatically suggesting hypertext links among segments of texts. So, the main concepts that define GAs will be translated in the terms of classifying segments of texts, and this theoretic exposé will be followed by a presentation of some experimental results.

In the CARAT context, genetic algorithms consists of finding, amongst different segments of a text, which one offer some regular structures or form classes of regularities. The GA is seen as a process of classificatory treatment which identifies segments of text containing some identical "type" of information. These segments are most often pages, and contains unifs (for units of information), which are simple or compound words, lexemes, etc. The determination of unifs and segments can be done by means of specialized computer text analyzing programs such as SATO, BOOKMANAGER, SPIRIT, OPEN TEXTE, NATUREL, etc. Here one creates a lexicon, a linguistic markup, a tagging, etc.

So, the original text is transformed into an set of segments containing only a balanced and controlled choice of units of information. A procedure identifies the presence or absence of each unif in each segment, and builds the following matrix (Tab. 1) of $n$ segments by $m$ unifs.

\begin{tabular}{l|l|l|l|l} 
& unif 1 & unif 2 & $\ldots$ & unif $m$ \\
\hline seg. 1 & $\operatorname{Pre}(1,1)$ & $\operatorname{Pre}(1,2)$ & $\ldots$ & $\operatorname{Pre}(1, \mathrm{~m})$ \\
seg. 2 & $\operatorname{Pre}(2,1)$ & $\operatorname{Pre}(2,2)$ & $\ldots$ & $\operatorname{Pre}(2, \mathrm{~m})$ \\
$\ldots$. & $\ldots$ & $\ldots$ & $\ldots$ & $\ldots$ \\
seg. $n$ & $\operatorname{Pre}(\mathrm{n}, 1)$ & $\operatorname{Pre}(\mathrm{n}, 2)$ & $\ldots$ & $\operatorname{Pre}(\mathrm{n}, \mathrm{m})$
\end{tabular}

Table 1. Matrix segments-unifs
One particular segment is represented by a line vector of binary numbers given by the predicate Pre(i, s) : 0 for absence, 1 for presence.

\section{III.2 Genetic classification}

The object of the model is to assign each segment to a specific and unique class. The assignment of a segment to a class is called classing, and uses a classifier, whereas the process of research of the best classing (i.e., the best classifier) should be called induction of classification.

III.3 Set definition and initial population

In order to introduce, in the context of CARAT, the concept of population of individuals (Fig. 3), and particularly the one of initial population, we must appeal to three important sets :

a) the set $T$ corresponds to the set of segments of the text. Let $T=\left\{S_{1}, \ldots, S_{n}\right\}$

b) the set $\mathrm{K}$ of classes. Let $\mathrm{K}=\left\{\mathrm{C}_{1}, \ldots, \mathrm{C}_{\mathrm{NbC}}\right\}$

Where $\mathrm{NbC}$ represents the number of classes. A class is a set of segments which are not too distant one from another according to the function of adaptation defined below. At the initialisation setp, the number of classes is arbitrarily chosen large (it is equal to the number of segments $n$ ) in such a way as to give the process the freedom to construct as many classes of segments as possible. It is the purpose of the function of adaptation to reduce, during the genetic search, this number to an optimal value. The number of cycles is also arbitrarily fixed at a fairly large value, in the order of one thousand cycles. Finally, the interpretation of each class is devolved upon the user.

c) The set (or population) $\mathrm{P}$ of the individuals $\left(\mathrm{P}^{\mathrm{t}}\right.$ represents the state of the population at time $t$. It contains a fixed number $p$ of individuals). The elements of $\mathbf{P}$ are called classifier-vectors, and represents the candidate classifiers, i.e., the potential solutions to the problem of the best classing. In other words, an individual encodes a tentative solution for classing segments. The size of the classifier-vectors is $n$; the position number $\mathrm{i}$ corresponds to the $\mathrm{i}$-th segment in the text, and contains an integer equal to the number of the class to which the segment belongs.

At the outset, the classifier-vectors are randomly built to produce the initial population $\mathrm{P}^{0}$.

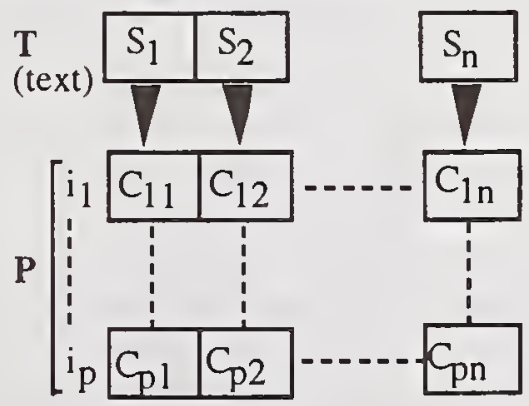

Fig. 3- relations of sets $T, K$ and $P$.

The genetic search has the task of carrying out a considerable number of modifications to the classifiervectors, such as recombinations and mutations, in an attempt to find the best one according to the function of adaptation. 
The $\mathrm{j}$-th individual of $\mathrm{P}$ corresponds to the vector :

$\mathrm{V}_{\mathrm{j}}=\left(\mathrm{C}_{\mathrm{j} 1}, \ldots, \mathrm{C}_{\mathrm{jn}}\right), \mathrm{C}_{\mathrm{ji}} \in \mathbf{K}$

An individual or chromosome could, as well, be seen as a set of couples which associates each segment to a unique class. The individual represents also a function which plunges the set $\mathbf{T}$ of segments into the set $\mathbf{K}$ of classes.

Example :

Let $\mathbf{T}=\left\{\mathrm{S}_{1}, \mathrm{~S}_{2}, \mathrm{~S}_{3}, \mathrm{~S}_{4}\right\} ; \mathbf{K}=\left\{\mathrm{C}_{1}, \mathrm{C}_{2}, \mathrm{C}_{3}\right\}$

Example of classifier-vector : $\mathbf{V}=(2,1,3,2)$

Interpretation: the first segment belongs to the class $C_{2}$, the second to the class $C_{1}$, the third to the class $C_{3}$, and the last one to the class $C_{2}$.

\section{III.4 The function of adaptation}

\section{III.4.1 Finality}

The function of adaptation must evaluate the intrinsic value of an individual, and hence the quality of the classing that it codes. This function is defined over the set of individuals and gives a real value.

In an ideal classifier $a$, segments are grouped into compact classes. This quality is characterized by the fact that displacing a segment $\mathrm{i}$ from one class to another (i.e., changing the class number located at position $\mathrm{i}$ in the classifier-vector) could result only in a decreased value of $f(a)$. The individuals selected for reproduction are those which posses the best values by $f$. Therefore, this function directs the entire process of the genetic search, and the quality of the whole GA model depends essentielly on it.

\section{III.4.2 Choice of a criterium of similarity}

It behoves the GA designer to conceive the most efficient function of adaptation. In particular, this function must be most discriminating. This discriminating feature involves two complimentary aspects :

- the evaluation of internal cohesion of classes, i.e., the degree of similarity of segments within each class;

- the evaluation of the differentiation between classes of the classifier-vector, i.e., the degree of contrast existing between classes.

The function of adaptation we propose is based upon the score of Jaccard as criterium of similarity. This score uses only the property of presence or absence of unifs in segments, and constitutes a common measure for evaluating the similarity of textual documents in the case of information research. It has been used notably for indexation (Gordon, 1988). However, there do exist a few other criteria (Salton, 1989).

The Jaccard score of a couple of segments $\left(X_{j}, X_{k}\right)$, notated $\operatorname{Sim}\left(\mathrm{X}_{\mathrm{j}}, \mathrm{X}_{\mathrm{k}}\right)$, is equal to the proportion of unifs common to both segments (notation : $\left|X_{j} \cap X_{k}\right|$ ) relatively to the total number of unifs present in the two segments: $\operatorname{Sim}\left(X_{j}, X_{k}\right)=\frac{\left|X_{j} \cap X_{k}\right|}{\left|X_{j} \cup X_{k}\right|}$

where : $|\mathrm{A}|$ represents the cardinal of the set $\mathrm{A}$.
- the internal cohesion of a class is evaluated by a coefficient of internal cohesion noted as $\operatorname{IC}\left(C_{i}\right)$. The coefficient is the balanced sum of the similarity of segments taken two by two in this class. It is defined by:

$$
\begin{gathered}
\operatorname{IC}\left(C_{i}\right)=\frac{1}{N(i)} \times \sum_{\substack{X_{j}, X_{k} \in C_{i} \\
j \neq k}} \operatorname{Sim}\left(X_{j}, X_{k}\right) \\
C I\left(C_{i}\right)=\frac{1}{N(i)} \times \sum_{\substack{X_{j}, X_{k} \in C_{i} \\
j \neq k}} \frac{\left|X_{j} \cap X_{k}\right|}{\left|X_{j} \cup X_{k}\right|}
\end{gathered}
$$

$\mathrm{N}(\mathrm{i})$ is the number of combinations of the segments of the class $\mathrm{C}_{\mathrm{i}}$ taken two by two.

- the differentiation of the classsing is evaluated by the coefficient of external dissimilarity, noted as $\mathrm{ED}\left(\mathrm{C}_{\mathbf{i}}\right)$, and computed for a class $\mathrm{C}_{\mathbf{i}}$ in relation to all other classes. It is defined as follows:

$$
\begin{aligned}
& \operatorname{ED}\left(C_{i}\right)=1-\frac{1}{N C(i)} \times \sum_{X_{j} \in C_{1}, X_{k} \in C C_{1}} \operatorname{Sim}\left(X_{j}, X_{k}\right) \\
& =1-\frac{1}{N C(i)} \times \sum_{X_{j} \in C_{i}, X_{k} \in C C_{i} \mid} \frac{\left|X_{j} \cap X_{k}\right|}{\left|X_{j}\right|}
\end{aligned}
$$

$\mathrm{CC}_{\mathrm{i}}$ is the complimentary set of $\mathrm{C}_{\mathrm{i}}$. This set contains all of the segments that do not belong to $C_{i}$. $\mathrm{NC}(\mathrm{i})$ is the number of couples $\left(\mathrm{X}_{\mathrm{j}}, \mathrm{X}_{\mathrm{k}}\right), \mathrm{X}_{\mathrm{j}}$ belonging to $C_{i}$, and $X_{k}$ belonging to $C C_{i}$.

Finally, the function of adaptation $f$ is equal to the sum of these two coefficients.

$$
f(\text { individual })=\sum_{i \in[1, N b C]}\left(\mathrm{IC}\left(\mathrm{C}_{\mathrm{i}}\right)+\mathrm{EC}\left(\mathrm{C}_{\mathrm{i}}\right)\right)
$$

All the inter-segment similarities are computed at the initialisation step, before the GA search.

To summerise. The genetic search runs a number of cycles equal to the maximum number of cycles determined at the start-up of the GA. When the cyclic processing has finished, the resulting population represent the best classifiers that the GA could produce. The last step is to select from this population the classifier-vector which gives the greatest value for $f$. At the end of the algorithm, a certain number of classes are empty. This is both expected and hoped since the number of classes was arbitrarily fixed at a high number. Thanks to the function of adaptation, the algorithm converges towards an optimum number of non-empty classes.

\section{III.5 Experimentation results}

The experiment was carried out on a textual sample drawn from Spirale, a Belgian review on Education Sciences. The GA was developed using Matlab, and was integrated into a software platform, Aladin (Seffah and Meunier, 1995), developed at LANCI for the CARAT approach.

The probability of crossing-over was fixed to 0.8 , and the one of mutation to 0.05 . The number of 
individuals in the initial population was 100 , and the number of generations (or iterations) was 300 .

The text was partitioned uniformly into 54 segments of 50 words each, the end of a segment being determined as follows: fifty words are counted from the beginning of the segment and then any words remaining up to the next point are added to the initial fifty words to constitute the whole segment. We had at our disposition a lexicon composed of 1701 words of which the number was restricted to 1360 roots after a preliminary process of lemmatisation. So, the size of the text matrix was (1360 x 54).

The genetic search decreased the number of classes from 54 to 24 . Here is a short sample of interpretable results. For instance, Class 4 contains the following segments 8 and 21 , in whicn underlined italic words are common unifs determined par the GA :

Segment 8 : « At last, Joëlle Delatte is grappling with the problem of books for blind and sight-impaired children, which would seem to be the preoccupation of at least some editors who have recently proposed specially designed albums for them. This production is characterized by a certain diversity if however unified by the prudence of their approach : Children's literature does not represent all the reading nor all the literature, but it does exist with a sufficiently rich past and present. ${ }^{2}$.

Segment 21: «To the teacher convinced of the importance of reading literature there exists a question of choice of texts and how to transmit them. There exists at this level no ministerial propositions nor lists of lists of books as there are for colleges; nor a specialized university teaching tradition to define the methods; the initial and ongoing training is incongruous and left largely to one's_own initiative. In effect, the teacher who chooses his own texts and his own course of action, wittingly or not, is putting into action his personal conception of the culture and the role of the school in the education of the child. " 3 .

Within these two segments, three unifs have been included in the same class : child, literature, reading. This result might facilitate the work of a user facing the

2 «Enfin, Joëlle Delattre aborde le problème des livres pour enfants aveugles et malvoyants, dont certains éditeurs semblent d'ailleurs se préoccuper en proposant maintenant des albums spécialement conçus pour eux. comme on le voit, une certaine diversité caractérise cette livraison, mais son unité nous semble résider dans la prudence des approches : La littérature de jeunesse ne représente ni toute la lecture ni toute la littérature, mais elle existe, avec un présent et un passé suffisamment riches. »

3 «Se pose alors, à l'enseignant convaincu de l'importance de la lecture littéraire, la question du choix des textes et des modalités de transmission. En effet, il n'y a à ce niveau ni propositions ministérielles de listes d'ouvrages, comme le collège : ni tradition d'enseignement universitaire spécialisé pour définir de méthodes ; la formation initiale et continue est disparate, largement laissée à l'initiative de chacun. En fait, l'instituteur, qui choisit ses textes et ses démarches, sciemment ou non, met en jeu toute sa conception personnelle de la culture et du rôle de l'école dans la formation de l'enfant. " problem of reading and analysing a large text, by suggesting a precise relation between the segments. A more in-depth analysis of the results would of course require the help of a terminologist or a specialist of the field. Such help is just as indispensable at the moment of preparation of the text matrix as it is at the end for the analysis of the results.

So, the genetic algorithm has built classes that classifies segments of text that offer some lexical similarity. It has done this in a systematic, dynamic and plastic manner. From these classes of segments the processing flow can then whether choose a particular word and see the class of similar segments in which it operates (its particular semantic contexts) or choose to built a hyperlink between two similar segments.

\section{Conclusion}

The use of the genetic algorithm that we have applied to the analysis of a text is still at the experimental observation stage. But it is, however a very promising territory and very flexible, which combines coding, the processing of data, computation of probabilities, artificial intelligence, and the genetic mode of evolution. The variants of the model presented are numerous and are linked to the diversity and richness of the genetic operators, to the multiple ways of coding the solution, and to the conception of the function of adaptation.

Research perspectives are situated around the most in-depth study of the genetic algorithm applied to CARAT and to the comparison of results obtained with other classifying systems that are presently being developed, such as simulated anealing and the ART neural network.

We believe that the modeling of CARAT by genetic algorithms allows us to foresee solutions to a certain number of problems of processing textual information that require classification tasks. On the one hand, classification allows regrouping the segments of a text in terms of an optimization of the similarity level for relating segments of information. On the other hand, by the nature of its mathematical structure of the topological type, the GA permits the processing of a body of text that is in constant evolution.

This algorithmic strategy is applicable to diverse types of textual information processing systems, such as terminological classification, thematic extraction in text. Automatic generation of hypertextuel relations.

\section{Thanks}

The present work is part of a Franco-Québecois project "Les classifieurs émergentistes et le traitement de l'information" under the tutelage of, for France, le Minisère de l'Enseignement Supérieur et de la Recherche (MENESRIP, Délégation à L'Information Scientifique et Technique, programme "Ingénierie linguistique et de la connaissance"), and le Ministère des Affaires Etrangères; for Quebec: Gouvernement du Québec - Ministère des relations Internationales. We extend a special thanks to these trustees for work and cooperation that they have permitted.

We would also like to thank the entire team at LANCI and in particular M. Nyongwa and I. Biskri for their advice and technical support. 


\section{References}

Bertrand-Gastaldy, Paquin, L. C., Meunier, J. G., (1993). L'analyse de texte par opposition à la gestion des documents, ICO, vol. 4, 12-18

Bertrand-Gastaldy, S., Meunier, J. G., and Lebel, H. (1987). A Call for Enhanced representation of Content as a Means of Improving Online Full-Text Retrieval. International Classification 14 (1), 2-10.

Bouchaffra, D. Meunier, J.G. (1995). A Markovian Random Field Approach to Information Retrieval, ICDAR, Third Int. Conf. Doc. Anal., and Recog. IEEE, Computer Society Press. Vol 2, 997-1003.

Buddle, R., and Bacon, P. (1992). Prototyping: an approach toevolutionary system development. Springer Verlag

Church, K. W., and Hanks, P. (1990). Word association norms, mutual information, and lexicography. Computational Linguistics 16 (1), 22-29.

Davis L. (1991). Handbook of Genetic Algorithms, Van Nostrand Reinhold. New York.

Delany, P., and G. Landow (ed.) (1993), The Digital Word: Text Based Computing in the Humanities. Cambridge, Mass: MIT Press.

Delany, P., and Landow, G. (1991). Hypermedia, and Literary Studies. Cambridge: MIT Press.

Dupoirier, D. (1994). Technologie de la GED, Paris, Hermès.

Feldman, J. J. (1988). Connectionnist Representation of Concepts. In J. A. Waltz David \& Feldman (ed.), Connectionist models, and theirimplications: Readings from Cognitive Science Norwood: AblexPublishing.

Forrest D. (ed.) (1991). Emergent Computation, MIT Press, Cambridge, Massachusetts.

Goldberg D. E. (1989). Genetic algorithrns in search, optimisation, and machine learning, AddisonWesley. Reading, Massachusetts.

Gordon (1988), Probabilistic, and genetic algorithms for document retrieval. Communications of the ACM, 31(10): 1208-1218.

Hobbs, J. (1993). Intention, Information, and struture in discourse. In Proceedings of the Nato Advanced Research Workshop on Burning issues in Discourse, Maraka, Italy, 41-66.

Hoffmeister F., and Bäck T. (1992). Genetic SelfLearning, in Varela F. J., and Bourgine P. (ed.). Toward a Practice of Autonomous Systems, MIT Press. Cambridge, Massachusetts, 227-235.

Holland J. (1975). Adaptation in Natural, and Artifial Systems, University of Michigan Press. Ann Arbor, Michigan.

Holland J. (1992). Genetic Algorithms, Scientific American, July, 66-72.

Kindermann R., and L. Snell (1980), Markov Random Fields, and their applications, Contemporary mathematics, AMS, Vol. 1.

Kintsch, W. (1988). The role of knowledge in discourse compr_hension: A construction-integration model. Pryshological Review 95 (163-182),

Lebart L., Salem A. (1994). Statistique textuelle, Dunod, Paris.
Marr, D. (1982). Vision: A Compuational Investigation into Human Representation, and Processing of Information, San Francisco, Freeman Publ.

Michalewicz Z. (1994). Genetic Algorithms + Data Structures $=$ Evolution Programs. Springer-Verlag, Berlin Heidelberg.

Newell, A. (1980), Physical Symbol systems.Cognitive science. 1984: 2, 135-183.

Pustejovsky, J. (1991). The generative lexicon. Computational Linguistics. 17(4).

Rastier, F. (1987). Sémantique et Intelligence Artificielle, Langage, vol. 87.

Rawlins G. J. E. (ed.) (1991). Foundations of Genetic Algorithms, Morgan Kaufmann, San Mateo, CA.

Reinheirt, (1994), M. Quelques aspects du choix des unités d'analyse et de leur contrôle dans la méthode Alceste. In L.L.S. Bolasco, and A. Salem (ed.), Analisi Statistica dei Dati Testuali. vol. 1 (19-27). Rome: CISU.

Rumelhart, D. E., and Mc CLelland, J. (1987). Parallel Distributed Processing :Explorations in the Micro Sctucture of Cognition, 2 vol. Cambridge: MIT Press.

Sabah, G. (1989). L'I. A et le langage vol 2. Paris: Hermès.

Salton G., and McGill, M. (1983). Introduction to models of Information Retrieval, New York: Mc Graw Hill.

Salton, G. (1989). Automatic Text Processing: The transformation, Analysis, and Retrieval of Information by Computer. . Reading, M. A: Addison Wesley.

Salton, J., Buckley A. C. (1994). Automatic structuring, and retrieval of large text fileCom. of the ACM, 37, (2), 97-107.

Seffah, A., and Meunier, J.G. (1995). ALADIN: Un Atelier génie logiciel orienté objets pour l 'analyse cognitive de textes. In Bolasco. S, Lebart, L. Salem., A. : Analisti Statistica dei Dati Testuali. JADT, 1995, Rome, CISU, VOL II, p. $105-113$.

Sowa, J. F. (1991). Principles of semantic networks, San Mateo: Morgan Kaufman.

Spark-Jones, and Kay. M. (1973), Linguistics, and Information, Science Academic Press Londres.

Thrane T., Olsen J., Jansen S., and Prebensen H. (1992). Discourse Analysis. Copenhague : Museum Tuscalanum Press.

Varela F. J., and Bourgine P. (ed.) (1992). Toward a Practice of Autonomous Systems, Proc. 1st Europ Conf Artificial Life, MIT Press, Cambridge, MA.

Virbel, J. (1993). Reading, and Managing Texts on the Bibliothèque de France Station. In P. Delany, and G. Landow (ed.), The Digital Word: Text Based Computing in the Humanities. Cambridge, Mass:MIT Press.

Wilks, Y. A. (1996). Dictionaries, Computers, and Meaning. Cambridge: MIT Press.

Willett, P. (1988). Recent trends in hierarchic document clustering: A critical Review. Information Processing, and Management, 24 (5), 577-597. 


\title{
Dynamic Word Meaning Representations and the Notion of Granularity. Text understanding as meaning constitution by SCIPS. Burghard B. Rieger \\ Dept. of Computational Linguistics - Fachbereich II: LDV/CL \\ University of Trier, D-5428, TRIER, Germany \\ E-Mail: rieger@ldv.Uni-Trier.de
}

\begin{abstract}
Semiotic Cognitive Information Processing Systems (SCIPS) are inspired by information systems theory. In a rather sharp departure from CL and AI approaches, Computational Semiotics (CS) modelling neither presupposes rule-based or symbolic formats for linguistic knowledge representations, nor does it subscribe to the notion of world knowledge as some static structures that may be abstracted from and represented independently of the way they are processed. Consequently, knowledge structures and the processes operating on them are to be modelled procedurally and implemented as algorithms. They determine SCIP systems as a collection of cognitive information processing devices whose semiotic character consists in a multi-level representational system of (working) structures emerging from and being modified by such processing. Corresponding to these levels of emerging structures are different degrees of resolution [1] that account for varying levels of representational granularity [8].

The emergence of semantic structure as a self-organizing process is studied on the basis of word usage regularities in natural language discourse, whose linearly agglomerative (or syntagmatic) and whose selectivelyinterchangeable (or paradigmatic) constraints are exploited by text analysing algorithms. These accept natural language discourse as input and produce-via levels of intermediate representationand processing - a vector space structure as output which may be interpreted as an internal (endo) representation of the SCIP system's states of adaptation to the external (exo) structures of its environment as mediated by the discourse processed. The degree of correspondence between these two is determined by the granularity that the texts provide in depicting the exo-view, and the resolution that the SCIP system is able to acquire as its endo-view of it in the course of processing the discourse.

The SCIP system's architecture is a two-level consecutive mapping of distributed representations of systems of (fuzzy) linguistic entities. Being derived from usage regularities as observed in texts, these representations provide for the aspect driven generation of formal dependencies and their interrelations in a format of structured stereotypes. Corresponding algorithms select and represent fuzzy subsets (word meanings) as dispositional hierarchies that render only those relations accessible to perspective processing which can-under differing aspects differently - be considered relevant. Such dynamic dispositional dependency structures $(D D S)$ have proved to be an operational prerequisite to and a promising candidate for the simulation of content-driven (analogically-associative) instead of formal (logically-deductive) inferences in semantic processing ([2], [3], [4]). Considered as states which the SCIP system can enter, certain properties of these structures can be identified as results of symbolic functions which were shown to correspond to basal referencial predicates $([5],[6],[7])$. Thus, the dynamics of semiotic knowledge structures and the processes operating on them essentially consist in their recursively applied mappings of multilevel representations resulting in a multiresolutional granularity of fuzzy word meanings which emerge from and are modified by such textprocessing. Test results from experimental settings (in semantically different discourse environments) are produced to illustrate the SCIP system's granular language understanding and meaning acquisition capacity without any initial explicit syntactic and semantic knowledge.
\end{abstract}


[1] A. Meystel: Semiotic Modeling and Situation Analysis: an Introduction. (AdRem Inc,) Bala Cynwyd, PA, 1995.

[2] B. Rieger: Unscharfe Semantik. Die empirische Analyse, quantitative Beschreibung, formale Repräsentation und prozedurale Modellierung vager Wortbedeutungen in Texten. (Peter Lang), Frankfurt a.Main/ Bern/ Paris, 1989.

[3] B. B. Rieger: Distributed Semantic Representation of Word Meanings. In: J. D. Becker, I. Eisele, and F. W. Mündemann (editors): Parallelism, Learning, Evolution. Evolutionary Models and Strategies, WOPPLOT-89, Lecture Notes in Artificial Intelligence 565, pages 243273. (Springer) Berlin/ Heidelberg/ New York, 1991.

[4] B. B. Rieger: Fuzzy Computational Semantics. In: H. Zimmermann (editor): Fuzzy Systems. Proceedings of the Japanese-German-Center Symposium, Series 3: Publications of the JapaneseGerman Center Berlin 8, pages 197-217. (JGCB) Berlin, 1994.

[5] B. B. Rieger: Meaning Acquisition by SCIPS. In: B. M. Ayyub (editor): ISUMA-NAFIPS-95, pages 390-395, Los Alamitos, CA, 1995. IEEE-Transactions: Joint Intern. Conf. on Uncertainty Modeling and Analysis, North American Fuzzy Information Processing Society, (IEEE Computer Society Press).

[6] B. B. Rieger: Situation Semantics and computational linguistics: towards Informational Ecology. A semiotic perspective for cognitive information processing systems. In: K. Kornwachs and K. Jacoby (editors): Information. New Questions to a Multidisciplinary Concept, pages 285-315, Berlin, 1996. E.W.-Heraeus-Stiftung, Deutsche Physikalische Gesellschaft, Deutsche Gesellschaft für Systemforschung, (Akademie Verlag)

[7] B. B. Rieger: Meaning Acquisition by Semiotic Agents. Semiotic Cognitive Information Processing in a Language Environment. In: J. Becker (editor): Agents, Cooperation, and Communication, page [in print], Berlin/ Heidelberg/ NewYork, 1997. University of the German Federal Forces/ ICAS, (Springer).

[8] L. Zadeh: Towards a Theory of Fuzzy Information Granulation and its Centrality in Human Reasoning and Fuzzy Logic. In: P. Wang (editor): Proceedings of the Third Joint Conference on Information Sciences, page [in print]. (Bookwrights) Durham, NC, 1997. 


\title{
CYBERAESTHETICS IN LIGHT OF ZOOSEMIOTICS
}

\author{
Victor Dmitriev*, Leonid I. Perlovsky $\dagger$ \\ *Department of Foreign Languages and Literatures, Oklahoma State University, \\ victor@osuunx.ucc.okstate.edu. \\ $\lceil$ Nichols Research Corporation, 70 Westview Street, Kilnbrook IV, Lexington MA 02173 \\ Tel: 617-862-9400; Fax: 617-862-9485; e-mail: perlovsl@nichols.com
}

ABSTRACT: Semiotic analysis of text and aesthetic literary criticism are brought closer together by considering a human reader as an intelligent symbolic system, between the kingdoms of animals and pure spirit. A science integrating aesthetics, cybernetics and semiotics we call cyberaesthetics. It is a philosophical and mathematical theory of intelligence related to Kantian philosophical aesthetics and the dynamical concept of symbol. Phenomenology of literary text and its perception is used to compare concepts of cyberaesthetics and zoosemiotics.

In his book, "Sign and Its Masters", Thomas Sebeok tells the following story. Once Sigmund Freud entered an auditorium with a huge cigar in his mouth. Students questioned the respected professor if this cigar didn't remind too much of a phallic symbol. Freud responded: "Sometimes a cigar is just a cigar". No doubt, students laughed, but it is quite possible that someone was pondering a question: "Just sometimes?" Not in vain, we remembered this joke.

Lev Tolstoy wrote a story, "After a Ball". The essence of this story is as follows. A young man was dancing at a ball in the home of his beloved one, whom he was about to propose. The ball was gorgeous. The young man especially remembered one moment when his beloved danced with her farther, a regiment commander, a tall and powerful man, who looked like the emperor, Nicholas the First. After the ball, the young man could not sleep, he was excited by just experienced happiness, and in the morning, walikng around the town, coming by the plaza, he saw that there was going on a punishment of a soldier: he was bitten by . An officer commanding the procedure was the very same colonel that the young man had admired just few hours ago. Here, at the plaza, the very same man was cold and unforgiving. And, bumping at his future son-in-law and looking straight into his face, the colonel did not even recognize him, even so he was smiling at him and so gently at the ball, just hours ago. After this terrible scene, the young man could not even think about marrying the colonel's daughter, so terrified he was by the man.

So, what is this story about? Possibly, that even when blinded by love, a human being can not loss the compassion to another human being. The violence is deeply repugnant to us; even to the extent that it can overpower the love. Where the violence reins, happiness is impossible, and it is the happiness that we are looking for in love. It is important to remember that the story was written by a writer, who was at the time an active proponent of the idea that evil should be opposed without violence. The young man did not become a revolutionary, did not attack the colonel, but could not become his son-in-law.

Sigmund Freud was not surprised at his student's question, because he taught them to ask such questions. As far as Lev Tolstoy is concerned, he possibly would be much surprised if he learned about one contemporary American Slavic scholar's idea. According to this scientist, the young man in the story did not want marry the tall and powerful girl, because unconsciously, her body frightened him. The young man was searching for a reason to avoid the marriage. Cruelly bitten, blooded body of the soldier, looking like a piece of meet, got associated in young man's mind with vagina, which he despised a priori. According to the author of this conception, at the moment of creating this story, Tolstoy was writing folk tales, was enmeshed into Russian folklore, with its popular motif of "vagina with iron teeth". Also a popular image is one of Kastchey Undead, an old voluptious evildoer. The conclusion: colonel dancing with the daughter at the ball got associated in young man's mind with Kastchey Undead in a ritual dance with a future prey of his violence.

Here is not a place to argue with this interpretation or its legitimacy. It transforms a writer Tolstoy into a folktale teller, destroys the Christian pathos of the story and replaced it with an openly paganistic canvas. Even so, the very existence of 
this interpretation is sufficient to explore the criteria for a valid interpretation. Which version is true? The one that tells about confrontation of good and evil, love and violence, or the other that tells about the disgust to the woman body, despite the sublime passion? If both interpretations are true, than, purely artistically, either they are incompatible, or they provide a foundation for another interpretation. The physiological is telling about itself through the spiritual, making fun of it. And making fun of the writer, who think that he inspires the reader by socio-humanistic pathos. Similar to student's interpretation being catastrophic to Freud, and to all smokers of cigars along with him, the interpretation of Professor Zolkovsky is catastrophic to the story, or more accurately to the way that Tolstoy saw it.

Tolstoy intended to struck a catastrophic blow to the idea of government violence. The developed contemporary culture revealed the internal catastrophisity of this very intent. New, searching readers pointed out to Tolstoy the true nature of his humanistic pathos: his disgust to woman Tolstoy veiled with a socially and ethically noble fable.

What is of interest to us in this situation? That it evolves in the aesthetic space, in a non-spatial interpretive field of the text. Relationships among its elements are not temporal, because they are already accomplished, they are prior to our analysis. Apriority is timelessness. Cybernetics of aesthetical is the nonspatial and atemporaneous relationship of elements, which are being defined by this very relationship. This is a definition of a literary, or in general, artistic image. An image is always a manifold, that is, it consists of other images, it is a virtual manifold of relationships. What is a mechanism of inter-relationship between an image and text? How is it possible to relate a text, which is a spatio-temporal object to an image which is an aesthetical space object? Text, in principle, contains no catastrophe that is a property of the aesthetic space.

The first theory of mind that combined its higher intellectual abilities with emotions and developed a rational theory of aesthetics, including the concept of beauty, belongs to Kant $(1781 ; 1788 ; 1790)$. His theory is based on three fundamental abilities or faculties of mind: Understanding, Judgment, and Reason. They are related to specific a priori principles or instincts contained in the mind: concepts, correspondence between concepts and manifold of matter, and will or desire. Understanding is a faculty of concepts, a source of general notions. Judgment is an ability to see that a particular subset of the manifold comes under the general rule. And, Reason is an ability to draw conclusions that is to act. The most important type of actions, interwoven with higher intellectual abilities, with beautiful and sublime, Kant considered to be the acts of learning. These three abilities correspond to the three main modes of consciousness: knowledge (of concepts), feeling (of correspondence between internal concepts and outer world), and desire (to act). While Kant devoted a separate book to each ability, they ought to be combined within a dynamical system constantly exercising all three abilities in their interaction.

Cyberaesthetics provides the mathematical apparatus of Kantian theory (Perlovsky, 1996; 1997). It carries Kantian analysis further: it is a dynamical system, in which the three abilities identified by Kant exist in the process of constant interaction, as it were in a "vortex". The judgment evaluates a similarity between an a priori conceptmodel and input data (sensory data, or lower-level concepts). A high judgment-emotion activates the learning-adaptation action modifying the conceptmodel. And, the modified model changes the judgment-emotion. The vortex of conceptsemotions-actions proceeds until an equilibrium is reached: from a split-second, in a case of a simple less-adaptive sign, it extends to generations in case of learning new cultural paradigms. The mind is a system of interacting vortexes, conıpeting for the evidence in the manifold of matter. Each vortex is a process of semiosis, a process of the dynamical formation of a symbol. Perception of beauty is related to the ability of complex adaptive symbolic systems to perceive a similarity between the internal a priori model and outer world beyond any specific purpose and related exclusively with a will for learning, that is a will for improving ourselves, our internal representations of the reality.

"The connection of ideas does not imply the relation of cause and effect but only a mark or sign with the thing signified. The fire which I see is not the cause of the pain I suffer upon approaching it, but the mark that forewarns me of it (Berkeley). That, which Berkeley calls here "the mark" is an internal sign. An internal, because it is inside of my body, inside myself. It is a sign of the fire, which obviously does not burn. It is a semiotical aspect of experience. An aspect that was isolated, emphasized and analyzed by semiotics. We see the sign of fire, because we look inside of ourselves, and we look not with eyes, but with all our body, with its biophysical memory, which all are a part of our 
internal concept-model of fire. When we imagine something, like approaching fire, we do it with all our body.

This is why, the Freudist interpretation of the Tolstoy story is not illegitimate. This is an interpretation made by the body. Therefore, this interpretation could be much more dangerous for its creator, than for Lev Tolstoy. But what could be said about our interpretation, which seems to us to be so humane, sublime, spiritual, etc.? It is also a body's interpretation. The covered with blood body of the soldier associated in young man's mind with his own body. He saw himself in the place of the soldier, and because he was not a sadist nor masochist, he was terrified. His actions are affective, that is undifferentiated, highly singleminded. Multi-minded functioning, differentiation of thoughts and feelings is an aesthetical phenomenon, belonging to the realm of higher emotions that do not deterministically control our behavior through ancient affective systems, but are an integral part of the intellect. A scientific approach to nature is an aesthetic one, since it assumes differentiation, multi-minded functioning, and multiple methods. A human being is capable of avoiding determinacy. This capability is a manifestation of a cyberaesthetic "energy", a psychic energy that is non-deterministically concentrated. An adaptive logic of cyberaesthetics assumes freedom with respect to space-time, freedom of consciousness towards itself. The difference between a symbol-image and non-adaptive sign is that the image is fuzzy, it "knows" of its nonidentity with itself, which is manifest in its adaptivity, an inherent property of the dynamical symbol of cyberaesthetics. A bee functions in an image-reality which is visible to our mind, but invisible to the bee. A bee is a pure sign that is a pure text, the writing itself, while a human being, in addition, is also an author of the writing; a human exists as a will that moves the hand.

Professor Sebeok quotes an important citation from Peirce: " ...thought is not necessarily connected with a [human] brain. It appears in the work of bees, of crystals, and throughout the purely physical world... Not only is thought in the organic world, but it develops there [and] there cannot be thought without Signs" [Peirce, 1935]. Evidently, here Peirce talks about the universal organizing principle, the universal spirit of Hegel that through the Schellingian Absolute ascends to the philosophy of Jacob Behme. A human being is a manifestation of this universal spirit like a bee or a crystal. But, what is not touched upon by Peirce is a human being as an internal catastrophe of the spirit, an internal freedom in which the universal spirit does not know itself. This is the "space" or realm that we call aesthetics.

Origins of aesthetics and semiotics are complementary. Aesthetical realm is a thought without sign. Semiotical realm is a signs without thought. In this regard, zoosemiotics differs from antroposemiotics just quantitatively, by the number and configurations of signs. The purpose of the semiotical is to point out its aesthetical meaning, its self-other, where signness disappears yielding to functionality. The meaning is a function of the sign. But, as a function, the sign is an element of the aesthetical realm, it virtualizes, dissolves in the image. Cybernetical aesthetics is a science of control of the sign-realm, a control that transforms signness into the psychic energy of combined sign and its apriority, into the vortex of thought.

Here we would remind you of the most idiosyncratic zoosemiotical example ever discussed by philosophers. We are talking about the Ludwig Feierbach's cat, which instead of scratching out its own eyes still jumps at the mouse and eats it up. In this way Feierbach argued against berkelians, hegelians, and other idealists whom he broke off with. From the cyberaesthetical point of view, jumping at the mouse, the cat indeed scratching out its own eyes, the very same eyes that it saw the mouse with. The cat, as it were, puts in itself a new eye, it changes the space of its functioning, transforms it, for now it obeys a will of the third, of some true interpretant. Within the will of this interpretant, the cat and the mouse are just but elements of some whole system, elements whose continuous transformations are the conditions of the system's existence. To cut it short, the mouse is a self-interpretation of the cat. Without the "mouse", the cat would be something else. We came to the notion of a system, which can not be understood as a sum of individual objects. The understanding can not proceed through the differentiation alone, but requires a next step, the synthesis. Similarly, every instinct is a manifestation of a cyberaesthetical phenomenon, a sign expression of the beyond-sign reality.

In professor's Sebeok book there is a story of the honey-guide bird, which guides people to the bee-hive with honey. It is not clear, what causes this bird kindness, for seemingly, it gains nothing. It does not eat honey, nor has any use for wax, because it does not build nests: it put its chicks into others nests. While telling us about this one more 
example of zoo-utilization of signs, professor Sebeok's role is that of a semioticist. But, when asking: what is behind this bird's actions? - he leaves the realm of semiotical and enters the cyberaesthetical, the realm of meaning, where the bird as such does not exist. Semiotics is, as it were, a Wilson camera collecting the traces of the aesthetical. Let us assume some interpretation, for example, that the bird "put" a human into the beehive, similar to putting its own progeny into other's nests. So, if we assume that all the altruism of the bird is dictated by its inborn "meanness", we enter the realm of aesthetical. The mechanism of our analysis is the very cybernetical of cyberaesthetical, this is our attempt to bring the elements into interaction in such a way that the purposiveness of one is seen through the purposiveness of the other. This is the principle of any discovery, an artistic or scientific one.

From the semiotical point of view the bird is a sign vehicle that is waiting for its interpretant. But what the interpretant is about to interpret? If an object exists before the interpretation, this means that it has already been interpreted. So, purely semiotically, the bird itself is not a puzzle. It is a puzzle aesthetically, for in this way, the bird indeed is "a trace of a bird", a trace of a yet unknown situation, in which the bird-human-beehive are combined in some whole, which configuration is not yet manifest. An assumed self-regulation of these elements we construct so that the configuration is consistent, isomorphic with our experience. This is the "mechanism" of any scientific discovery, any discovery whatsoever. Thus, the growth of our knowledge in some way is similar to that of a crystal, it takes care of the homogeneity of its structure, reminding us of the universal spirit.

A very interesting story is told by professor Sebeok about a difficult life of male-fireflies of the genus Hilara sartor. A male kills an insect and with this "wedding gift" flies to a female, who eat the gift during the intercourse. If it eats up the entire gift too fast, it starts eating its beloved one right during the intercourse. This love story may seem repugnant to some of us. Especially to those, whose imagination, like that of the young man in the Tolstoy story, let's us identify our body with the body of a male Hilara sartor. This feeling of disgust is our affective bodily reaction. And, what about the beauty that, according to Kant is related to the perceived purposiveness of the object? Does Kantian theory support such a relativism of beauty? The behavior of the Hilara sartor female is quite purposive: she takes care of producing offspring. Then, why it might be impossible to some of us to perceive beauty in this case? Kant explains that beauty is related not to any purposiveness, but to the purposiveness of the object with respect to our internal models, independent of any utilitarian goal. Apparently, our models of love are more purposive to us than what we see among Hilara sartor. Even so our models are not as directly utilitarian as theirs, some of us do not feel that our models can improve by watching them. So, this genus does not look beautiful to some of us. But, are we really that different from Hilara sartor? Aren't their relationships all too similar to human relationships? "Dowryless" husbands and wives often feel quite bad, especially after the romantic part of relationships is over. If the husband does not bring money home, the wife "eats him" in many different ways, If the Hilara sartor male not always can buy himself off from sexual appetites of his partner, so among human this is also quite possible. The ideal of beauty does not always matches the reality, so some of us may enjoy observing Hilara sartor relationships, if not for improving our ideals of beauty, then at least for improving our model adaptivity to some immediate situations in life.

The above analysis is an aesthetical one, not semiotical. An art of comparison, discovering isomorphisms among phenomena, is a dramatic one, in that semiotic signs, passive objects which are predestined to remain objects of interpretation, acquire a tendency to transform into another category, to become a mechanism of cognition, mechanism of isomorphism, mechanism of transformation. To achieve this, a sign has to relieve a transfiguration first, it has to dye and risen. Relative to the semiotic space, the aesthetic one is like a black hole, an annihilating antimatter. Our taboo are borders keeping us from getting too close to these black holes, to its annihilating nature. Every science is filled with these taboo as well as any sphere of human activity.

Ants of genus mirmekaphilus confuse the rear side of other ants with the front part of their own kind, and make an intercourse "erroneously". "The multiple resemblance between this icon and the object for which it stands are so striking, subtle, and precisely modulated that they can hardly be explained away as an evolutionary coincidence" [14]. If an evolution follows a definite logic, there would be no room for the black holes of aesthetical in this semiotic kingdom. There would be no freedom, no catastrophicity. Professor Sebeok explains indecent behavior of ants by confusion due to resemblance in 
appearances of various genus, but then, how to explain a similar behavior of more advanced species, when there is no confusion at the level of signappearances? If there is a "confusion", it occurs in aesthetical space, where the meaning of signs is formed and where a familiar sign may acquire a new meaning. Semiotics emphasizes apriority, considering it as an imperative, which can only be violated erroneously: if not for a confusion of appearances, the ant would be more decent. Cyberaesthetics equally supports a very different point of view, natural imperatives that evolved in the course of evolution are of a relative, adaptive nature. And, in particular, sexual drives are directed at more than just one definite utilitarian goal of gene propagation. They also could be affine to a wider purposiveness having no specific utilitarian goal, and they are hostages of camouflages and countermeasures evolved throughout evolution. And, sexual drive may burn the organism in the fire of isomorphisms.

Returning to the Tolstoy's story, we conclude that both, "humanistic" and "Freudian" interpretations contain elements of truth. Still another interpretation seems more interesting: the young man identified his relationships to his beloved one and her farther, and being disappointed in one, he lost his love to the other. Thus, a much more refined isomorphism of sublime ideas seems to take place in the aesthetical space of the story. When a "Freudist", looking at a smoking man, sees "the marks" that dissolve the smoker, his perception is short-circuited through the "marking" semiotic system directly to the bodily functions. Similarly a building perceives bricks it is build of. We can not but quote Carl Jung: "I remember one Indian coming up softly behind me while I was looking at the mountain over the pueblo, and saying quite suddenly in my ear, 'Don't you think all life is coming from the mountain?' It was just in that way that Freud talked about sexuality" [Schoenl, 1996].

A widow of one famous theologist was in rage, when after the death of her husband, instead of uncompleted manuscripts, she found in his desk pages teared from erotic magazines. Naive woman was under a spell of an opposite extreme, she thought that all life is coming not from the mountain but from the Mountain sermon. Erotic interests of her husband she perceived as an error commensurate with an ant, not a great theologist. It is a purely semiotical error, that a great mind can not mix up sinful with sacred. In order to overcome the gravitation of a zoosemiotic realm, one need all its energy. Cyberaesthetics is a philosophy and science of combining emotional with conceptual, a theory of control of the invisible energy of the visible.

\section{REFERENCES}

Dmitriev, V.A. \& Perlovsky, L.I. (1996). Art Form as an Object of Cognitive Modeling (Towards Development of Vygotsky's Semiotics Model). Proceedings of the Conference on Intelligent Systems and Semiotics '96. Gaithersburg, MD, v.2, pp. 385-389.

Kant, I. (1781). Critique of Pure Reason. Willey, NY.

Kant, I. (1787). Letter to Reingold. In Tractats and Letters, 1980, Interscience, Moscow.

Kant, I. (1788). Critique of Practical Reason. Willey, NY.

Kant, I. (1790). Critique of Judgment. Tr. J. H. Bernard, 1914, 2nd ed., Macmillan \& Co., London.

Perlovsky, L.I. (1996a). Fuzzy Logic of Aristotelian Forms. Proceedings of the Conference on Intelligent Systems and Semiotics '96. Gaithersburg, MD, v.1, pp. 43-48.

Perlovsky, L.I. (1996b). Mathematical Concepts of Intellect. Proceedings of World Congress on Neural Networks, San Diego, CA; Lawrence Erlbaum Associates, NJ, pp. 1013-1016.

Perlovsky, L.I. et al (1997). Mathematical aspects of cyberaestetics. ISAS'97.

Schoenl, W. (1996). Major issues in the life and work of C.G. Jung. Univ. Press of America, Inc. Lanham, New York, NY (p.10).

Sebeok, T. (1979). Sign and Its Masters. Univ. of Texas Press, Austin, TX. 


\section{TBE HOLE IN THE SYSTEM}

Floyd Merrel]

Purdue University

Abstruct: Physicist John Archibald Wheeler's concept of the "boundary of boundaries" that lies at the heart of what customarily goes as our physical world finds resonance with Charles S. Peirce's notion of semiosis, which includes the categories of Firstness. Secondness, and Thirdness, possible, actual, and potential signs, the sign components (representamen, objeot, intenpretant), and the oomplementarity between sign overdetermination and sign underdetermination.

If Charles S. Peirce's semiosis is process, flow, signs becoming signs, the question incvirably surfaces: What where, when, how, are signs, when considering body as well as mind. self as well as other, sign as well as interpreter, individual as well as his/her community and the world 'out there'?

In desperation, we may happen to run onto that Indian counterpart to Heraclitus. Nagarjuna, who counsels that body, mind self. world gre signs all. The query 'To sign or not to sign' is thus no query at all. for all that is, is sign. Which is to say that (1) the sign is. but also, (2) it is nor, for there is no nonsign against which to gauge it as a sign; in such case (3) it is both sign and nonsign, but if that is the case. then (4) it is neither sign nor nonsign. And yet, if we are to know signs, all four of these possibilities are to be negated! Nagarjuns brings about such a negation. All is no more than appearance, but it is more than appearance, for it is, but if it is, then it is not, and we find ourselves on the menry-go-round once again. The very possibility of negation is negated, which gives rise to affirmatuon, but since there is nothing to affirm, then what was affirmed is negared, so it is impossible either to affirm or to negate, which is to say that there is both affirmation and negation and at the same timo there is neither affirmation nor negation. In other words, in a manner of speaking there is only 'emptiness'. 'Emptiness' is not a matter of two unthinkables making a thinkable, much as two Hegelian negations make an affirmation. No. For, there is nothing, no-thing that is cither one thing or the other thing. There is just 'emptiness'.

Where is all this jabber about 'emptiness' taking us, perhaps, is loward what physicist John A. Wheeler dubs the 'boundary of the boundary', which is another way of saying 'emptiness'. Yes, the secret lies in the 'boundary of the boundary'. The boundary, Wheeler implies, is tantamount to the square rool of minus one. $\sqrt{ }-1$, the function of which is found in logic, in mathematics and chaos thcory, in computer engineering. and in quantum mechanics and relativity theory. $\sqrt{-l}$ is in a sense everywhere and nowhere. It is as if it were 'emptiness' realized paradoxically. The boundary of the boundary, the sign of itself, the hinge that allows for all the action: it's from there that the whole show gushes forth.

Wheeler, the quancum physicist, tells us that a boundary at its simplest best and at its confoundingly worst begins with a solitary "direct or "oriented" line--a one-dimensional manifold" that has for its boundary 'the starting point and the corminal point, both zerodimensional' [7]. Such a boundary is a hole cut out of a shect of paper with a pair of scissors. It consists of a two-dimensional manifold the cut itself defined as a one-dimensional manifold. So. where is the boundary? At the edge of the paper. What is the boundary, that is, of what does it consist? Why. nothing at all. It is zero! Zero, 'whatever the point at which we consider that line to have started, that is also the point at which the line terminates' [7]. A debt is incurred at the starting point when the scissors penerrate the two-dimensional manifold and then that staring point is annihilated. consumed eaten up. Which is to say, if in our threedimensional world we were to carve out a cube, then, like a square hole marring a Flatlander's twodimensional existence, there would be a section in our world that would not hold water, or anything else for that manter. Morcover, our cube separating our space fram 'nothingness', from 'elsewhere', would be a boundary consisting of twelve lines (one-dimensional boundaries) and six planes (two-dimensional boundaries) making up a three-dimensional region, and that the sum of these boundaries is zero! Nothing. A nothing that separates what is 'here-now' from mere 'emptiness'.

Wheeler demonstrates his point with the image of a cube. Each of its six faces has an orientation in three-dimensional space. Three spaces give the cube's position, and three morc give its movement along its time-line, its world-line. In addition, each face of the cube inherits from the interior an orientation, a swirl, a direction of spin as it moves along its time-line toward the future (in contemporary physics no moving body faces forward and forward only, nor is it Janus-faced, but in constant osoillatory, vibratory, undulatory, gyration).. The ane-dimensioaal boundary of one of the faces of the cube is given a swirl by a line and an arrow. There is a start, wherever that is, and an ending, but the ending ends up at the starting point, and the staring point is the ending, so they cancel each other out: zero dimensions. But isn't the line a boundary? That's one dimension, isn't it? But the line begins eatung its own tail, finally leaving nothing. Total washout. Kaput. 
Actually, we cut the sheet of paper, a twodimensional universe with three-dimensional scissors from within our three-dumensional vantage point. The question is, then: What lind of scissors could we possibly use to cut a cube out of our own threedimensional space from within a fourth dimension? Why, four-dimensional scissors, of course. A big problem. Where do we get them and how can we use them? Wc don't, and we can't, short of our playing the impossible role of a Maxwell Demon and a Laplace Superobserver all wrapped up into anc. So we don'h, and we can't. Yet, let's see how far we can go. If twodimensional space is subject to one-dimensional boundaries and threc-dimensional space to twodimensional boundaries. then it stands to reason that four-dimensional space must be subject to throcdimensional boundaries. Wheeler depicts the wholc shebang as a 'hypercube'. The four-dimensional block of spacetime at the center is bounded by eight threedimensional cubes according to the orientations of those surrounding the block. Each cubc sports six twodimensional faces, and each face is bounded by four lines. Yet in the final analysis it's the same story: these lines cancel themselves out, leaving zero. By the same token. we would supposc that in four-dimensional space the planes, serving as boundaries in our threcdimensional space, would likewise annihilate each other to yield 'nothingness'.

In a desperate altempt to get a better handle on all this, let us turn our attention to Ludwig, an imaginary four-dimensional demon. Ludwig would essentially dwell in the central 'hypercube' with a vantage point allowing him to peer into our innards and view a stomach ulcer, a kidney stone, some lung crad, and whatever other personal secrets we have stashod away in that blemished temple, our body. How can we possibly identify and empathize with Ludwig? We can't, or at least it is well nigh impossible to do so. So we must concode that while prolonged contemplation of Ludwig would be interesting enough, plenty of meat for a Ray Bradbury story, we really should be addressing ourselves to sigas of the sart we can sink our tceth into. A difficult lask, for if we ask ourselves what there is in terms of signs, our signs, ourselves as signs, the answer must be: Not much, really. We and our signs are virtually infinitesimal in comparison to the entire rcalm of the possible. In fact, we and our signs are hardly anything at all. On the other hand, we are in a certain sense virtually everything. We begin with 'emptiness', zero, Lhere in the 'center', wherever that is. Nothing precoded it and it has no boundaries. Then we move on to the equivalent of the 'empty set', a sort of 'noticed absence'. the absence of something that might have been there but is not, with certain tentatively dofined boundaries, or of something that was never there, yet there is at least the suggestion of boundaries, since the set is 'emply'--there is some domain that can possibly be filled by something.

Then we move on to the first sign cansisting of the Firstness (qualisign, quality, mere feeling without consciousness of the feeling as such) of the representamen (sign manifestation) of the semiotic object, and of the interpretant (roughly. the sign's meaning). This consists of $\underline{R}-\underline{O}-I$ relations in tripod Pashion about the 'avis' or 'node' of 'emptiness' such that $\underline{R}$ is related $10 Q$ in such a manner in which they are both brought together by the mediation of $\underline{I}$ at the same time that $I$ is brought into relation with $\mathrm{R}$ and $\mathrm{Q}$ in the same way that they are brought into relation with it. The relations are properly triadic, for each pair of terms depends upon the existence or the third term through the existence of the 'node'. We have moved somowhere along the infinitesimal thin plane within that fathomless cube, our four-dimensional spacetime continuum. But whatever this most elementary sign is, it is not for us, not yet at least. That is, we are not yet canscious of it as sentient and self-conscious semiolic agents poised and ready to pul whatever there is into the service of the semiosic stream within which we happen to find oursclves at the moment. Such consciousness of must await further sign development. So we move on, somewhere within the vast expanse of the cube. Then we bocome aware of the sign as something other, and we have gravitated coward the Secondness of the sign as something 'out there' related to something that is the object of our altention and that it must have some meaning of some sort or olher. This relation between ourselves and the sign is at this moment no more than Secondness. oursclves in contrast to some other. We see the sign, and sense that it must be related to something else and that that relation in its relation to us must bring about the emergence of some meaning, some interprotant. Then, an idea comes to mind, and we are in Thirdness. and ' $\mathrm{LO}$ and behold!', there is a world 'out there' awalting our interaction and participation with it in its and our self-organizing unfolding. Then we exercise a move toward more developed signs: language crentually comes issues forth, then dialogue, narrative, discourse.

But somewhere along the line our signs cannot help becoming self-reflexive, a mirror of themselves. As mirroring themselves, ad infinitum they can never be final. For, in Peirce's words:

what anything really is, is what it may finally come to be known to be in the ideal state of complete information, so that reality depends on the ultimate decision of the community;... In this way, the existence of thought now, depends on what is to be hereafier; so that it 
has only a potential existence, dependent on the future thought of the commurity.

The individual man, since his separate existence is manifested only by ignorance and error, so far as he is anything apart from his fellows. and from what he and they are to be, is only a negation. [4]

The process of knowing is ongoing, future oriented; knowledge in the full sense is always in the future and nol for us in the 'bere-now'. Conscquently, the 'individual man' (or 'self. Pelrce's terms), is 'only a negation'. Only a negation? Well, yes. In a manner of pulting it. Perhaps there's no other way adequately of putting it. The negation is the negation of a boundary, which boundary is an infinitcsimal line, nothing, 'nothingness'. 'emptiness'. Sheer emotiness.

So we are back to that again. But our condition is not as dire as it might appear. We might hope for Peirce's cnvisioned 'ideal state of complete information' as the community consensus that would finally be arrived at in the long nu of things. However, it seems that our hopes are shattered anew, for, according to Peirce, that cormounity consensus will never come to frution short of infinite lime and an infinitely extended community (i.e. it is a converging series). Yet whatever knowledge can be had, however limited, and whether consciously and willfully expounded or tacilly displayed, must be the product of a collective effort, through community practice and dialogue.

Speaking of language and dialogue, let us return to physics.

\section{BACK TO THE SUTURE, THEN?}

The crisis in physics during the first quarter of the present century and shortly thereafter brought on an unexpected and unwanted crisis of language. The chief problem, Hoisenberg [2] writcs, was that 'no language existed in which ooc could speak consistendly about the new situation'. The language available at the time 'was based upon the old concepts of time and space and this language offered the only unambiguous means of communication'. It was a matter, Heisenberg [2] goes on, of waiting 'for the devclopment of the language, which adjusts itself after some time to the new situation:. Heusenberg sees Niels Bohr's 'complementarity principle' as encouragement 'to use an ambiguous rather than an unambiguous language, to use the classical concepts in a somewhat vague manner in conformity with the principle of uncertainty, to apply altematively different classical concepts which would lead to contradictions if used simultancously' [2]. In other words, an electron could be described either as a particle or a wave in classical language, but both descriptions could not be used in the seme context without falling into contradictions. So whatever sort of language was to be used in describing this new sinuation, it must be relatively loose and vague.

Heisenberg's words appear strange, coming from a physicist. But when compared to the way language is actually used, they are as natural as can be. Peirce, in this vein, was also an ardent proponent of the need for vague and ambiguous signs (of Firstness) as well as logically precise language (of Secondness and Thirdness). For this reason he promised--though be never delivered the finished artucle--a 'logic of vagueness' as an alternative to the classical principles of identity, noncontradiction, and the excluded-middle. A vague and ambiguous language contains, within itself. contradictory and incompatible principles. Such a langunge offering the possibility of various altematives, some of them mutually exclusive. can be labeled overdetermned. In order to talk coherently, we must attempt 10 differentiate between incompatibles, saying either one thing or the other, but not both in the same breath. But if something contains the possibility of being two or more distinct thangs, when we can see it and say it as now one thing, now the other thing. The two secings and sayings are alternatives, and either might be effective in one context while the other is more effective in another context: different alternatives actualized at different times and different places, all of them construed to be valid in their own right. are characteristic of an underdetermined system.

Wirgenstein's rabbit-duck drawing is ambiguous in terms of its Secondness: it is actualizes as generally either one thung or the otber. Within the sphere of Firstness, however, it is vague. both the one and the other, though it remains as no more than a possibility: it is overdetermined. Within the sphere of Thirdness, on the other hand, it contains the potentiality for actualization as geither the one nor the otber but something else, in fact, many possible other things. It can be imagined as the map of an island, it can be a masked person at the Mardi Gras, it can be a strangely shaped cloud, or it can be just squiggles. In this sense it is underdetermined. According to Bohs complementarity, an electron behaves like a particle under one set of circumstances and like a wave under another set of circumstances. Expressing the electron as now one thing, now another, is revelation of language's underdetermination. Of course, it is from another view both a particle and a wave (overdetermined), though it cannot manifest both of those characteristics in simultaneity-unor could we be aware of both characteristics at onoe even if they could be so manifested. We have also seen this use of language in the above comments by Heisenberg, especially in bis observation that 'the situation of complementarity is not confined to the atomistic world alone' but is also found in the arts and in everyday life situalions [2]. 
Actually, we eut the sheet of paper, a twodimensional universe, with three-dimensional scissors from within our three-dimensional vantage point. The question is, then: What kind of scissors could we possibly use to cut a cube out of our own threedimensional space from within a fourth dimension? Why, four-dimensional scissors, of course. A big problem. Where do we get them and how can we use them? We don't, and we can't. shor of our playing thie impossible role of a Maxwell Demon and a Laplace Superobserver all wrapped up into one. So we don't, and we can't. Yet, let's see how far we can go. If twodimensional space is subject to one-dimensional boundaries and three-dimensional space to twodimensional boundaries, then it stands to reason that four-dimensional space must be subject to threedimensional boundaries. Wheeler depicts the whole shebang as a 'hypercube'. The four-dimensional block of spacetime at the oenter is bounded by eight threedimensional cubes according to the orientations of those surrounding the block. Each cube sports six twodimensional faces, and each face is bounded by four lines. Yet in the final analysis it's the same story: these lines canod themselves out, leaving zero. By the same token, we would suppose that in four-dimensional space the planes, serving as boundarics in our threedimensional space, would likewise annihilate each other to yield 'nothingness'.

In a desperate attempt $t 0$ get a better handle on all this. let us turn our attention to Ludwig, an imaginary Pour-dimensional demon. Ludwig would essentially dwell in the central 'hypercube' with a vantage point allowing him to peer into our innards and new a stomach ulcer, a kidney stone, some lung crud, and whatever other personal secrets we have stashed away in that blemished temple, our body. How can we possibly identify and empathize with Luctwig? We can't, or at least it is well nigh impossible to do so. So we must concede that while prolonged contemplation of Ludwig would be interesting cnough, pleary of meat for a Ray Bradbury story, we really should be addressing ourselves to signs of the sort we can sink our teeth into. A difficult task, for if we ask ourselves what there is in terms of signs, our signs, ourselves as signs. the answer must be: Not much, really. We and our signs are virtually infinitesimal in comparison to the entire realm of the possible. In fact, wo and our signs arc hardly anything at all. On the other hand we are in a certain sense virtually everything. We begin with 'emptiness', zero, there in the 'center', wherever that is. Nothing preceded it and it has no boundaries. Then we move on to the equivalent of the 'empty set', a sort of 'noticed absence', the absence of something that might have been thare but is nol, with ceriain tentatively defined boundarics, or of something that was never there, yer there is at least the suggestion of boundaries, since the set is 'empty"--there is some domain that can possibly be filled by something.

Then we move on to the first sign consisting of the Firstness (qualisign, quality, mere feeling without consciousness of the feeling as such) of the Iepresentamen (sign manifestation) of the scmiotic object, and of the interpretant (roughly, the sign's meaning). This consists of $\mathrm{R}-\mathrm{Q}-\mathrm{I}$ relations in tripod fashion about the 'axis' or 'node' of 'emptiness' such that $R$ is related to $Q$ in such a manner in which they are both brought together by the mediation of I at the same time that $\underline{I}$ is brought into relation with $\underline{R}$ and $Q$ in the same way that they are brought into relation with it. The relations are properly triadic, for each pair of terms depends upon the existence of the third term through the cxistence of the "node". We have moved somewhere along the infinitesimal thin plane within that fathomless cube, our four-dimensional spacetime continuum. But whatever this most elementary sign is, it is not for us, not yet at least. That is, we are not yet conscious of it as sentient and self-conscious semiotic agents poised and ready to put whatever there is into the service of the semiosic stream within which we happen to find ourselves at the moment. Such consciousness of must await further sign development. So we move on. somewhere within the vast expanse of the cube. Then we become aware of the sign as something other, and we have gravitated toward the Secondness of the sign as something 'out there' related to something that is the object of our attention and that it must have some meaning of some sort or other. This relation between oursclves and the sign is at this moment no more than Secondness, ourselves in contrast to some other. We see the sign, and sense that it must be related to something else and that that relation in its relation to us must bring about the cmergence of some meaning, some interpretant. Then, an idea comes to mind, and we are in Thirdness, and 'Lo and beholdl'. there is a world 'out there awaiting our interaction and participation with it in its and our self-organizing unfolding. Then we exercise a move toward more developed signs: langugge eventually comes issues forth, then dialogue, narrative, discourse.

But somewhere along the line our signs cannot help becoming self-reflexive, a mirror of themselves. As mirroring thomsclvcs, ad infinitum, they can never be final. For, in Peirce's words:

what anything really is, is what it may finally come to be known to be in the ideal state of complete information, so that reality depends on the vltimate decision of the community;... In this way, the existence of thought now, depends on what is to be hereanter; so that it 
has only a potential existence, dependent on the future thought of the community.

The individual man, sinoe his separate existence is manifested only by ignorance and error, so far as he is anything apar from his fellows, and from what he and they are to be, is only a negation. [4]

The process of knowing is ongoing, future oriented; knowledge in the full sense is always in the future and not for us in the 'here-now'. Consequently, the 'individual man' (or 'self', Peirce's terms), is 'only a negatuan'. Only a negation? Well, yes. In a manner of putting 1t. Perhaps there's no other way adequately of putting it. The ncgation is the negation of a boundary, which boundary is an infinitesimal line, nothing, 'nothingness', 'emptiness'. Sheer emptincss

So we are back to that again. But our condition is not as dire as it might appear. We might hope for Peirce's envisioned 'idcal stare of complete information' as the community oonsensus that would finally be arrived at in the lang run of things. However, it seems that our hopes are shattered anew, for, according to Peirce, that community consensus will never come to fruition shart of infinite time and an infinitely extended community (i.c. it is a converging series). Yet whatever knowledge can be had, however limited and whether consciously and willfully expounded or tacitly displayed, must be the product of a collective effort. through community practice and dialogue.

Spcaling of language and dialogue, let us Ieturn to physics.

\section{BACK TO THE FUTURE, THEN?}

The crisis in physics during the first quarter of the present century and shortly thereafter brought on an unexpected and unwanted crisis of language. The chief problem, Heisenberg [2] writes, was that 'no language existed in which onc could speak consistently about the new siruation'. The language available at the time 'was based upon the old concepts of time and space and this language offered the only unambiguous means of communication'. It was a matter, Heisenberg [2] goes on, of waiting 'for the development of the language, which adjusts itself after some time to the new situation'. Heisenberg sees Niels Bohr's 'complementarity principle' as encouragement 'to use an ambiguous rather than an unambiguous language, to use the classical concepts in a somewhat vague manner in conformily with the principle of uncertainty, to apply alternatively different classical concepts which would lead to contradictions if used simulancously' [2]. In other words, an electron could be deseribed either as a particle or a wave in classical language. but both descriptions could not be used in the same context without falling into contradictions. So whatever sort of language was to be used in describing this new sination, it must be relatively loose and vague.

Heisenberg's words appear strange coming from a physicist. But when compared to the way language is actually used, they are as natural as can be. Peirce, in this vein, was also an ardent proponent of the need for vague and ambiguous signs (of Firstness) as well as logically precisc language (of Secondness and Thirdness). For this reason he promised--though be never delivered the finished article-a "logic of vagueness' as an alternative to the classical principles of identity, noncontradiction, and the excluded-middle. A vague and ambiguous language contains, within itself, contradictory and incompatible principles. Such a language offering the possibility of various alternatives, some of them munally exclusive. can be labeled overdetermined. In order to talk coherently, we must altempl to differentiate between incompatibles, saying either one thing or the other, but not both in the same breath. But if something contains the possibility of being two or more distinct lhings. when we can see it and say it as now one thing, now the other thing. The two secings and sayings are alternatives, and either might be effecrive in one context while the other is more effective in another context: different alternatives actualized at different times and different places, all of them construed to be valid in their own right, are characteristic of an underdetermined system.

Wingenstejn's rabbit-duck drawng is ambiguous in terms of its Sccandnoss: it is actualizes as generally either one thing or the other. Within the sphere of Firstness. however, it is vague, both the ane and the other, though it remains as no more than a possibility: it is overdetermined. Within the sphere of Thirdness, an the other hand it contains the potentiality for actualization as neither the one nor the other but something else, in fact, many possible ouher things. It can be imagined as the map of an island it can be a masked person at the Mardi Gras, it can be a strangely shaped cloud, or it can be just squiggles In this sense it is underdetermined. According to Bohr complementarity, an electron behaves like a particle under one set of circumstances and like a wave under another set of circumstances. Expressing the electron as now one thing, now another, is revelation of language's underdetermination. Of course, it is from another view both a paricle and a wave (overdetermined), though it cannot manifest both of those characteristics in simultaneity-nor could we be aware of both characteristics at once even if they could be so manifested. We have also seen this use of language in the above comments by Heisenberg, especially in his observation that "the situation of complementarity is not confined to the atomistic world alone' but is also found in the arts and in everyday life situations [2] 
In other words. when physicists use ordinary language. they are forced to talk about electrons and quarks in about the same way as they talk about anything elsc. And talk, well, it's just talk. Particles and waves, black marks on white making up a book, squiggles forming a rabbit or a duck, and so on, are alternate ways of talking about our various 'semiotic' worlds. Ultimately, in this respect. what the physicist studies 'is not nature in atself, but nature exposed to our method of questioning it. Our scientific work in physics consists in asking questions about nature in the language we possess and trying to get an answer from expcriment by the means that are at our disposal" [2]. For Bohr himself, it is wrang to suppose that the task of physics is that of saying what the world is. More adequately stated physics is concerned with what the physicist can say about the world. In response to the argument that the world precedes and is more fundamental than language, Bohr once quipped: 'We are suspended in language in such a way that we cannot say what is up and what is down. The word "reality" is also a word a word we must leam to use correctly'[5]. Bohr's suggestion is that no matter how we talk about the world language ends up saturating it, and in the prooess we finally end up talking about talk itself. 'Stand for', 'reference', and 'representation', like 'reality', are also words, and to assume our words 'stand for', 'refer to', or 'represent' something beyond them in the big, wide world are of the substance dissolved dreams are made of. Talk is ultimately related to talk and if it is also somehow related to the world so much the better. But we should be mindful that this relationship will more often than not come as a hopeful byproduct of the talk.

This is interesting in light of Wheeler's postEinstein 'physics of meaning'. The 'physics of meaning' stipulates that withoul the scientist's asking questions of the universe, talking to it. observing it, interacting with it, it simply is: it has no significance for physics. Thus the physicist her/himself is a panicipatory semiotic agent, a sign her/himself, interacting with the entire range of possible signs, initially actualizing them as signs, and pushing them along the road towards thoir fulfillment as genuine signs with fully developed interpretants. In fact. the cotire community to which the physicist belongs is involved. Implicit within the process of the physicist's interaction with the universe is the injunction, do such-and-such, which entails pasticipation with what there is. This is germane to Bohr's complementarity principle. In both cases participation of the semiolic agent is a must, and by mcans of such participation signs become full-blown signs, in the process taking on menning.

The story goes something like this. A set of signs, most effectively put in the form of a scnience, is taken as an assertion that such-and-such is the case. It is at the same time an injunction, orther implicit or explicit, to consider the practical effects the assertion might conceivably have with respect to the act of following the injunction. For example, if you say 'My speakers can take a 90 watt blast'. I might take it that I am invited to subject them to the full volume of the amplifier. If I do so. and if they withstand the pressure, then we know. If they don't. we still know--and you rurn on your word processor. intent on writing a lettor to the manufacturer regarding their warranty. So 'My speakers can take a 90 watt blast' is nol thoroughly meaningful until we put it to the test. In other words, in Wheeler's [6] terms, we must put the signs (or phenomena) to use, whether they be sign-events, in the case of conceivable and acrual physical effects, or thought-signs, in the case of fictions, purely hypothetical situations. or 'thought-experiments'

This is comparable to Bohr's theory of meaning emerging from his complementarity principle [3]. Is an electron a particle or a wave"? Since it cannot be a particle and a wave simultaneously, one might surmise that it is neither a particle nor a wave, or that it is both a particle and a wave. In the first case, one does not commil oneself, but holds out for a more general concept of 'particle' and 'wave'; in the second case, one cmbraces a contradiction at the expense of admitting a cloud of vagueness into one's purview. For Bohr, neicher case is adequately meanugful: 'an electron as particle' is meaningful under certsin sctualized conditions, and 'an electron as wave' is meaningful under other complementary conditions. The conditions must be actualized before the signs in question become genuine signs. Meaning is a matter not of what we can know about the 'real world' but what we can do with signs in such a way that we can say more about the 'semiotically real' objects surrounding us.

In a Peircean vein. the notion of boch a 'paricle' and a 'wave' exists in the overdctcrmincd sphere of possibilities (Firstness), neither a 'particle' nor a 'wave' at the same moment has citizens rights to the underdelermined sphere of generalities (Thirdness), ordained by convencion. habit, and an incessant movement towards an expansion of one's conceptual grasp. In contrast. Bohr's now a 'particle', now a 'wave' (or either a 'prarticle' or a 'wave') holds trie to classical logic and the tenets of physical existence (actualities. Secondness). It has to do with the things we can do with signs and the things they do to us. Quite obviously, Firstness and Thirdness break out of the ferrers imposed on them by classical logic, for contradiction and excluded middles are allowed, while Secondness, the master of our physical world as we would like to perceive it and conceive it, generally remains faithrul to classical prunciples So a sign can be 
drawn from a massive range of possibllities to become an actual sign, which can then be meaningful in terms of its practical consequences--its interrelation in the world of acpun signs. But its meaning will remain incomplete, for between the neither and the nor (or the either and the ord other signs always stand a chance of popping up to alter whatever meoning was to be had.

\section{WHAT MORE CAN WR DO?}

The initiacion of semiosic flow, I would submit, is graphically depieted in the central portion of Figure 1, where there is nothing mare than an infinitesimally thin plane or boundary separating nothing from anything clse: it is mere 'nothingness', 'emptiness', no more than falds and warps, topological distortions.

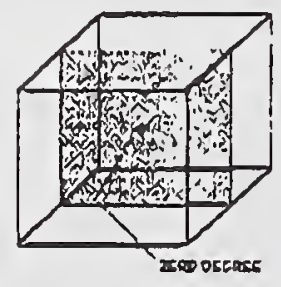

FIOURE 1

Yet this 'nothingness' or 'emptiness' contains, 'wichin' itself, the sphere of possibilities that can give rise to the cmergence of everything that is in a particular 'semiotically real' warld. We could say of semiosis what Wheeler says of geometrodynamics:

This great world around us--how is it put together? Out of gears and pinions? By 2 oorps of Swiss watchmakers') Aocording to some multifaceted master plan embodying an all-embracing corpus of laws and regulations? Or the direct opposite? Are we destined to find that every law of physics, pushed to the extreme of experimental lest. is statistical-nas heat isnot mathematically perfeot and precise? Is physics in the end 'law without law', the very epitome of austerity? [7]

Now, what can Wheeler possibly mean by 'law without law'? That the boundary is where the action is. And what is the boundary? Once again, zero, zilch. nothing at all. It is a fold, that which is folded within itself, like a cavity that is a cavity of itself, like the origami fold, the paper of which is superfluous, for what is impornant is the fold itself, a fold of space that is at the same time an enfolding and an unfolding. A hurricane of incessant unfolding of the enfolded and enfolding of the unfolded. At this level, there is no distinction between organic and inorganic matter, between the living and the dead. Thus wc are now told by Ilya Prigogine, fractal geometers, and chaos mathematicians, that all is self-organizing (codependent emergence), like the self-organizing universe itself. A geological formacion is enfolded into a vein of gold, sheaves and shears of tectonic places press plants into black carboniferous matter and ultimately enfold that into a diamond, an enfolded chick embryo or an acom unfolds once. twice, thrice, and virtually countless times.

But please don't get me wrong. The unfolding of the enfolded and vice versa is not simply a matter of tension relcase and tension build-up, nor is it dilation and contraction. but rather, it is evolving and involving, developing and enveloping. convolution and involution. A living organism's organs are involved, enveloped, contracted folds that got that way after generation upon generation of evolution, development, and dilation and displaying of what was there all along as a possibility. And now, they are there, within the organism. in the process incessantly of enveloping, involving, enfolding, implicating matter and energy from the outside in order that they may sustain life: that is their charge and their purpose in and for the organism. A cell is the product of one-dimensional engenderment according to a few letters and an algorithm in three-dimensional spacc to create something ' $h$ cre' that is distinguished from everything 'there' by a rwo-dimensional wrap. a relatively smooth fold, a boundary. The entire surface of a living organism takes up a minute piece of threedimensional space, and at the same time that surface is a boundary in four-dimensional spacetime in the sonse of Wheeler.

'This won't do the trick' someone retorts. 'You must really be more explicit.' However, how can we say what there is in that 'emptiness' which allows of no saying at all? How can we say, really say, what there is withun the center of the Figure 1 cube where everything is enfolded and nothing unfolded? Impossible! As impossible as geometrodynamic field equations 'saying' how mass--momentum and energy-get a grip on spacetime at the boundary of the boundary. It can be described.through equations but hardly said clearly and distinetly in natural language [7]. You simply can't describe the enfolded point, the center, which is the equivalent of Wheeler's zero moment of rotation. How can anything be of zero rotation in a universe in which all movement depends upon frames of reference? Assume we place a cube of ice with a small buck-shot frozen in its center--like Figure 1--on a skating rink floor. We rotale the cube, which is a quite effortless task since there is little friction berween ice cube and icy surface. The cube being well-nigh symmetncal, and all other luings being equal, we look at the buck-shor and observe that it appears to be motionless. As a virtually infinitesimal point it has zero moment of rotation. Everywhere else there is force imparted with a certain amount of encrgy. but this central point has no energy and it exercises no forcc. It 
is comparable to the hub of the Buddhist whecl. It is the 'image' of 'emptiness'।

'But'. someone retorts, 'what we have here is a mere point, clearly and distincty 'nothingness'--if not to say "emptiness" You should seally get back to the idea of boundary. which cannot be explained away in such sophomoric fashion'. O.K. Assume we have a Linelander gliding happily along her two-dimensional strip-world one extremity of which is given a twist and connected to the other extremity to form a Mobius-strip. Easy enough. She is a one-dimensional being residing on a two-dimensional sheet curved and doubled back on utself in three-dimensional space. In essence, she inhabits three dimensional space. Now supposc she strctches herself out along the strip until she encounters her hind quarters and latches onto them. She has become a circle. As such. she is finite but unbounded. Unbounded? Of course. She is apparcntly a ring, an unbounded boundary that bisects the Mobbius-strip, scparating it into two worlds, this one and that ane, the one that is first 'above' and then 'below'. and the other one that is first 'below' and then 'abovc'. Now that is a boundary we can identify with. Is it not? We have a one-dimensional line, or Firstress, that, in relationship with its other, the two-dimensional strip, brings about a 'cut' in order to construct a binary relationship of Secondness, all by means of the mediating capacity of Thirdness from our three-dimensional view wherein the Möbius-strip could become a legitimate Mơbius-strip.

Let us suppose we bisect our Linelander by taking our scissors and cutting the strip in two pieces precisely where she is lying in apparently so rclaxed and carefree a position. Now we should lave two Mobbiusstrips separated by our erstwhile Linelander. To our suprise. however, the strip isn't bisccted at all. It remains intact. But it is definitely not the same strip. No, that's not correct, not exactly. It is now half as wide and twice as long as it was, and it sports two twists instend of one. Zcro sum game again! Then it is the same strip, only evincing a different form. What did our Linelander-boundary separate anyway? 'Nothing' from 'nothing'. The boundary was a zero boundary. While we were cutting the strip. there was a direction, and time passed, irreversible time. But when we finish we realize that there had been no line that separated something from something else, no dircetion, no irreversibility, and no indication of ume. There was no other, nothing distinguishing this from that.

Now, essentially the whole strip is indivisible if we cut it along its clongated surface. In this sense, it is, most properly speaking an icon, self-concained, selfreferential. unary (it is chiefly of Firsmess). The strip itself, then, is indexical insofar as it is capable of incorporating two-dimensionality, directionality, binarıly (it is chielly of Secondness). And the line is symbolicity in the sense that it cntails one-dimensional, linear engenderment of signs (it is chiefly of Thirdncss). However, the fact remains that the strip as icon in the pure sense is indivisible. Try to sever it with images. relations, and words, and it remains as it is, though of a slightly to radically different countenance. Yct virtually nothing has changed. The sum of all changes is no change--as the French say, the more things change the more they stay the same.

Now, what happens if we apply our Linelander within her Flatland to our own sphere of existence? We take the cube of Figure 1. metaphorically tantamount to our three-dimensional universe, and we stretch the right-hand side of it out, give it a twist, and bring it back to its left-hand side and connect it. What we have is a rectangularly shaped sor of skewed torus. Now suppose we split our angular, twisted torus in half along the shaded plane in Figure 1. We now know what the yicld will be. We are left with a torus half as thick and twice as long as it was. Zero change once again! Total change is no change at all. The iconicity of the whole remains intact. What are we, within this wholc concoction. but, ourselves, minuscule twists, tiny warps. skewed spots. in four-dimensional space?--we are like the buck-shot in Pigure 1, which. if stretched out when the cube is topologically iransformed into a Morbiustorus, becomes a line, a boundary that separates 'nothing' from 'nothing'.

So much for stories and boundaries. What we are left with is a flow of interdependent. intcrclatcd, merging, diverging and converging, signs. Signs all Semiosis: the becoming of signs for us whithin our world-line.

\section{REFERENCES}

[1] Baer, Eugen (1988). Medical Semiorics. Lanhana: University Press of America.

[2] Heisenberg, Werner (1958). Bhysics and Philosophy. Ann Arbor: Michigan UP.

[3] Murdoch, Dugald (1987). Niels Bohr's Philosophy of Physics. Cambridge: Cambridge UP.

[4] Peirce, Charles Sandors (1931-35). Collected Papers of Charles Sanders Peirce, $C$. Hartshome and $P$. Weiss (eds.), vols. 1-6. Cambridge: Harvard UP.

[5] Petersen, Aage (1985). 'The Philosophy of Nicls Bohr.' In Niels Bohr: A Centenary Volume A. French and P. Kennedy (cds.), 299-310. Cambridge: Harvard UP.

[6] Wheeler, John Archibald (1980). 'Law Without Law." In Structure in Soience and Art, P. Medawar and J. H. Shelley (eds.). 132-68). Amsterdam: Exoerpta Medica.

[7] Whecler, Jolin Archibald (1990)). A Journey into Gravity and Spacetime. New York: Scientific Amcrican Library. 



\section{MULTIDISCIPLINARY APPLICATIONS OF SEMIOTICS}





\title{
Control Mechanisms for a Nonlinear Model of International Relations
}

\author{
Áron Péntek, Jim Kadtke \\ Institute for Pure and Applied Physical Sciences, University of California at San Diego, La Jolla, CA 92093-0360 \\ Suzanne Lenhart \\ Mathematics Department, University of Tennessee, Knoxville, TN 37996-1300 \\ Vladimir Protopopescu \\ Computer Science and Mathematics Division Oak Ridge National Laboratory, Oak Ridge, TN 37831-6364
}

\begin{abstract}
Some issues of control in complex dynamical systems are considered. We discuss two control mechanisms, namely: a short range, reactive control based on the chaos control idea and a long-term strategic control based on an optimal control algorithm. We apply these control ideas to simple examples in a discrete nonlinear model of a multi-nation arms race.
\end{abstract}

KEYWORDS: chaos control, optimal control, arms race

\section{INTRODUCTION}

The field of nonlinear dynamics offers the possibility to describe high dimensional complex systems - which hitherto have only been modeled stochastically or heuristically - in terms of nonlinear low dimensional deterministic models. The appeal of such models stems from two apparently opposing features. Indeed, on one hand the models - described by a system of either coupled maps (discrete evolution) or ordinary differential equations (continuous evolution) - are simple enough to warrant a rigorous and extensive analysis while on the other hand they display an extremely rich dynamical behavior that captures some of the complexity of the systems they are supposed to model. Due to these features, low dimensional nonlinear models have been extensively applied to ecologic, neuromorphic, societal, and military systems. Recently the focus of these applications has shifted from analysis and exhaustive exploration of the phase and parameter spaces to control and prediction [15].

In this paper we discuss the application of two conceptually different control mechanisms to simple discrete dynamical systems of Richardson type used to model arms races. $[5,8,13]$. A short description of these models will be given in Section 2. In Section 3 we present our first control strategy to arms races and international relations, namely a short range, reactive control designed to react to changes in international relations based on immediate feedback utilizing the natural dynamics of the system. In this case policy changes are typically planned only one (time) step ahead. At each time step control is revised based on some feedback that reflects the actual state of the system and does not take into account any long term strategic thinking. The main advantage of this short term crisis management is that typically it requires only very small changes in the system parameters. Indeed, due to the inherently nonlinear and potentially unstable character of the dynamics, this control is based on a chaos control algorithm [12-14] and it is used to stabilize unstable periodic orbits or fixed points of the dynamics by applying only small perturbations to (one of) the system parameters. The basic idea is to perturb the system in such way to drive it to the unstable manifold of the desired orbit. On this manifold the natural dynamics of the system will bring the trajectory closer and closer to the desired fixed point or limit cycle.

The second procedure, called long range, strategic control reflects a longer term planning of policy changes that are implemented consistently throughout the whole evolution. The long term strategic control is achieved by an optimal control algorithm [6] that we briefly discuss in Section 4. In the optimal control framework the perturbations (controls) are evaluated in order to minimize or maximize some objective functional that depends on the state and the controls. The actual form of this functional essentially depends on the goal we want to achieve and explicitly includes the reward and cost of that goal. The main advantage of such planning is that the changes one has to implement are known over the whole time span of the system's evolution and provide an optimal solution (an optimal cost effectiveness is realized for attaining the desired goal). The basic disadvantage of this method is that modeling errors, inherent perturbations, and unforeseen factors will usually lead to a trajectory different from the planned one.

\section{MODIFIED RICHARDSON MODEL}

To illustrate the control algorithms we choose a very simple example related to the socio-political field. Re- 
cently, Miller, Sulcoski, and Farmer have presented a modification of the Richardson model (MRM) describing an $N$-nation arms race, in the form of a discrete, nonlinear set of coupled dynamical equations [8]. This complex spatio-temporal system can be simply represented by a coupled map lattice (CML). Specifically, the model is given by

$$
x_{i+1}^{a}=x_{i}^{a}+\left(x_{\text {max }}^{a}-x_{i}^{a}\right)\left[k_{a a}\left(x_{\text {min }}^{a}-x_{i}^{a}\right)+\sum_{b} k_{a b} x_{i}^{b}\right]
$$

where $x_{i}^{a}$ is the "total military capability potential" (TMCP) of country $a$ at time $i, x_{\max }^{a}$ and $x_{\min }^{a}$ are maximum/minimum values for the TCMP sustainable by the respective country $a$, and $k_{a a}$ and $k_{a b}$ are coupling constants, representing internal threats and external alliances, respectively. The internal instability in$\operatorname{dex} I_{a}=2 k_{a a} /\left(x_{\max }^{a}-x_{\text {min }}^{a}\right)$ determines whether country a behaves like a democratic $\left(I_{a}>0\right)$ or totalitarian $\left(I_{a}<0\right)$ nation [8]. Another interpretation classifies these countries as non-militaristic and militaristic, respectively. For

particular parameter values, iteration of this mapping produces a $2 \mathrm{D}$ landscape ( 1 spatial, 1 time) whose topology describes the qualitative dynamics of the political situations of the $N$ countries. Being globally coupled and nonlinear, this model has the potential for rather rich behavior, and so far there have identified stationary, periodic, and chaotic parameter regimes. The relevance of this model, as for the original Richardson model, is that it may have solutions which yield considerable insight into the possible dynamical regimes for the current

multi-sphere world order, allowing qualitative analysis and prediction.

Case A) A four-nation model, consisting of a weak democratic nation (1), a weak totalitarian nation (2), a stronger totalitarian nation (3), and a strong democratic nation (4). These countries form two alliances, between the totalitarian and democratic nations, respectively, which are relatively strong. The parameter values are: $k_{11}=1.0, k_{22}=-0.1, k_{33}=-0.3, k_{44}=2.0$, $k_{12}=k_{21}=0.1, k_{13}=k_{31}=3.0, k_{14}=k_{41}=-0.1$, $k_{23}=k_{32}=-0.1, k_{24}=k_{42}=0.1, k_{34}=k_{43}=0.1$,

$x_{\text {max }}^{1}=0.7, x_{\text {max }}^{2}=1.5, x_{\text {max }}^{3}=0.7, x_{\text {max }}^{4}=2.0$, $x_{\text {min }}^{1}=0.2, x_{\text {min }}^{2}=0.5, x_{\text {min }}^{3}=0.3, x_{\text {min }}^{4}=0.5$. As initial conditions (armament levels) we choose $x_{0}^{1}=0.4$, $x_{0}^{2}=1.4, x_{0}^{3}=0.6$ and $x_{0}^{4}=1.6$. Figure 1 (a) shows the resulting time evolutions of this system which indicate exponentially

growing behavior (which is artificially truncated after some time for illustrative purposes).

Case B) A three-nation model, with the following parameter values: $k_{11}=0.1, k_{22}=-0.9$,

$$
k_{33}=0.3, k_{12}=k_{21}=0.4, k_{23}=k_{32}=0.7 \text {, }
$$

$k_{13}=k_{31}=0.4, x_{\max }^{1}=0.6, x_{\max }^{2}=1.5, x_{\max }^{3}=$ $0.7, x_{\text {min }}^{1}=0.2, x_{\text {min }}^{2}=0.3, x_{\text {min }}^{3}=0.5$. As initial conditions (armament levels) we choose $x_{0}^{1}=0.3, x_{0}^{2}=$ 1.1 , and $x_{0}^{3}=0.4$. This corresponds to a system with two weak democratic nations ( 1 and 3 ) and a stronger totalitarian nation (2) which is destabilized by an internal security threat. The resulting complex time evolution of this system is shown in Fig. 2(a), which indicates chaotic evolution of the totalitarian nation's TMCP.

The instabilities in the MRM leading to fast oscillations or chaotic evolution of the TMCP may be interpreted as a transition to war or insurgency [7] (c.f. Figs. 1(a),2(a)). The basic question we try to answer in the next Section is whether or not such instabilities arising at some stage of the dynamics of the interaction of the nations can be controlled, by actively changing the international or internal relations, and thus avoiding the onset of "war". The question is difficult and here we only address some simple examples. We will examine the MRM dynamics and sketch some conclusions about controllability, as follows from the MRM (1).

For this discussion, we assume that the model equations and most estimated coefficients are known, and the only parameters that can be effectively changed are those related with internal affairs $\left(k_{a a}\right)$ and international relations $\left(k_{a b}\right)$. Interpreted in the context of international relations, we assume that a given government can attempt to strengthen or weaken its military potential by changing the internal relations within its own country, or by carefully tailored international alliances with any of the other countries. The other parameters of Eq. (1), the maximum and minimum sustainable capability $x_{\max }^{a}$ and $x_{m i n}^{a}$, are assumed difficult to change on short time-scales as they reflect economic structure, culture, demographics, technological development, governmental structure, etc.

\section{SHORT RANGE REACTIVE CONTROL}

Recently nonlinear dynamics has provided us with a series of methods designed to control unstable saddle points of complex systems, with only small

changes in one or a few of the experimentally accessible parameters. These methods assume an active monitoring of the system and evaluation of the new control parameters at each time step based on the actual state of the system (active feedback, "closed-loop control"). Although here we assume perfect knowledge of the governing equations, in practical cases this is neither realistic nor necessary, as most of the information needed to control the system can be extracted by observing the evolution of the system and the effect of parameter variations (c.f. Ref. $[12,13]$ ). We have to emphasize that such an approach to stabilize international relations may not be 
regarded as a long-term solution, but rather as a short term crisis management.

Here we will consider the method of Ott, Grebogi and Yorke [12], which is one of the most widely accepted chaos control algorithms, and which has already been applied to a variety of physical systems, such as magneto-elastic ribbon, chaotic laser, semiconductor devices, chemical reactions etc. The method applies only small perturbations to drive the system to the curve in the phase space along which the saddle point can be exactly reached, called the stable manifold. Thus it takes advantage of the naturally attractive dynamics along the stable manifold. The reader is referred to Ref. $[12,13]$ for a detailed description of the method as well as to Ref. [14] for an extension to higher dimensional systems.
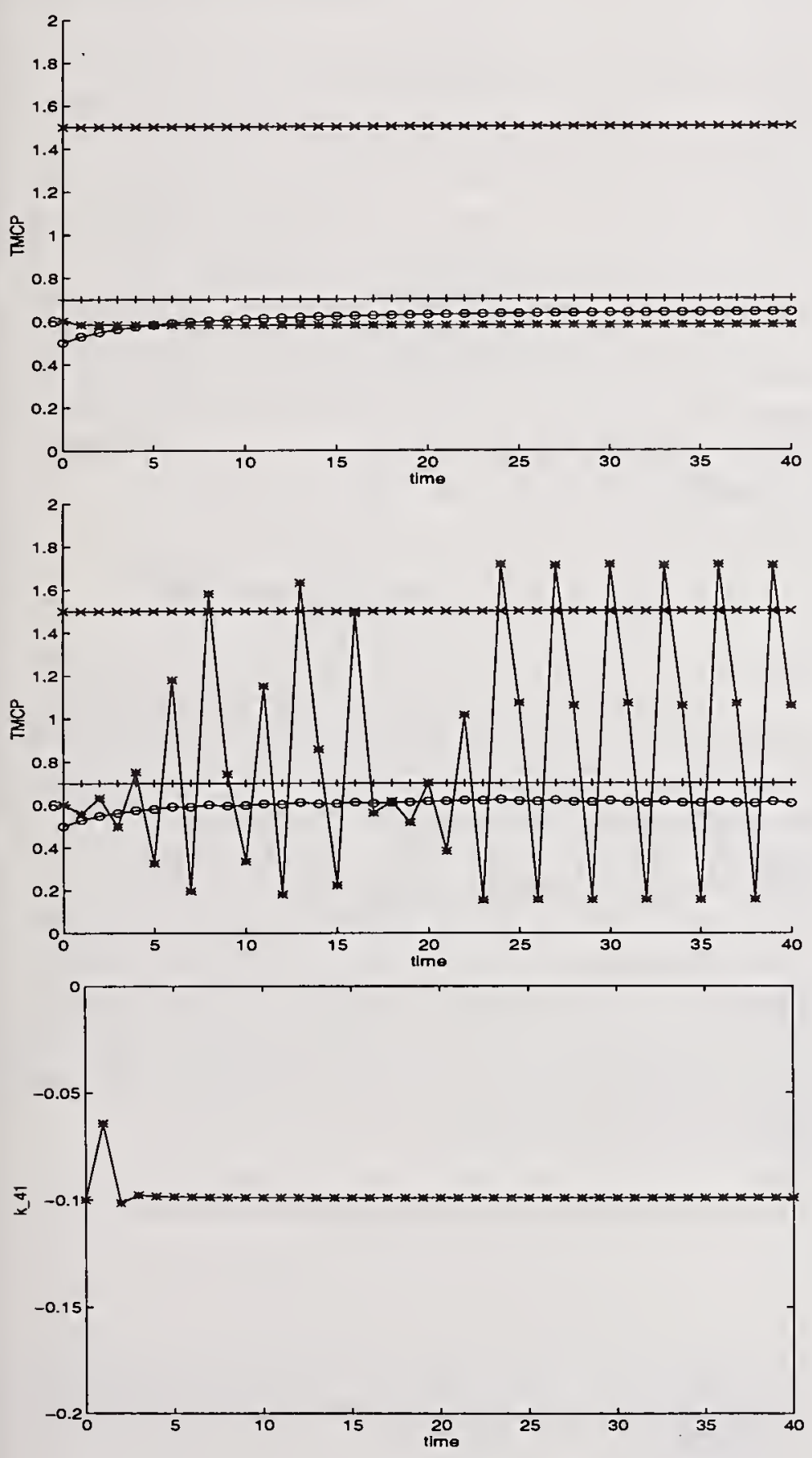

FIG. 1. Time evolution of the TMCP for the uncontrolled (a) and controlled (b) case. Here the short range reactive control have been employed. Fig. (c) shows the time evolution of the control parameter. Note that the except the first timestep the applied perturbation is very small.

Our main conclusions about controllability can be summarized as follows: a) although in the mathematical model the control can be sustained for arbitrary long time with only small perturbations, in practical cases this procedure may fail after some time as the model may become invalid; b) with the exception a few special cases it is not possible to control nations at their maximum capability potential; c) when the nations are strongly coupled and have nontrivial fixed points one has to employ a higher dimensional control algorithm, and the controllability condition should be also tested in a higher dimensional framework as presented in Ref. [14].

This control crisis management is most effective when at least two of the nations have saddle points that differ from their maximum sustainable potential. Such an example is provided by Case A. This model results in a fixed point at $\mathbf{r}^{*}=(0.6422,1.5,0.7,0.5779)$. This shows the presence of two strongly democratic nations, one of them at the edge of internal stability $\left(I_{4}=1.5\right.$ ), (similar behavior is observed at slightly lower $I_{4}$ values). The strongly democratic nation has a large maximum sustainable potential $\left(x_{\max }^{4}=2.0\right)$ but has a low armament level. Figure 1(a) shows the time evolution with initial TMCP values $x_{0}^{1}=0.5, x_{0}^{2}=1.5, x_{0}^{3}=0.7$ and $x_{0}^{4}=0.6$. One can observe that while the weak democratic and the totalitarian nations quickly reach a steady TMCP level, the strong democratic nation's TMCP oscillates periodically after some chaotic transients. Our strategy is now to actively change one of the coupling coefficients $k_{41}$ between the two democratic nations. Figure 1(b) shows the time evolution during the control procedure and Fig. 1(c) the evolution of the corresponding coupling parameter $k_{41}$.

\section{LONG TERM STRATEGIC CONTROL}

This approach is based on the optimal control method applied to discrete systems [6]. The optimal control method can be simply summarized as follows. The system is described by a scalar or vector state function depending on a number of independent variables that take values in the phase space of the system. The state satisfies a (usually nonlinear) dynamical scalar or vector equation that depends also on some parameters that take values in the parameter space. One or more of these parameters are considered external controls to be adjusted at will. The goal is to optimize a given objective functional that depends on the state and on the control(s). 
From the state equation and objective functional one constructs, in a canonical way, a non-homogeneous adjoint equation for a (scalar or vector) adjoint variable. The original state equation together with the adjoint equation form the optimality system (OS). The optimality condition yields an explicit, analytical formula for the optimal control in terms of the solutions of the OS. By replacing this expression of the control in the OS and solving it, one obtains the optimal state and adjoint variable and therefore the optimal control. The general framework for optimal control for general nonlinear systems as developed by J.-L. Lions [9] was recently applied to competitive systems of social and military interest $[10,11]$.

As an example for this control strategy we chose the three-nation model of Case B described in Section 2. The overall goal is to minimize the oscillations of the totalitarian nation's TMCP by changing his internal stability coefficient $\left(k_{22}\right)$. Also we want to achieve this goal at an optimal cost, and by simultaneously keeping the the democratic nations stable. The corresponding choice of the cost function is
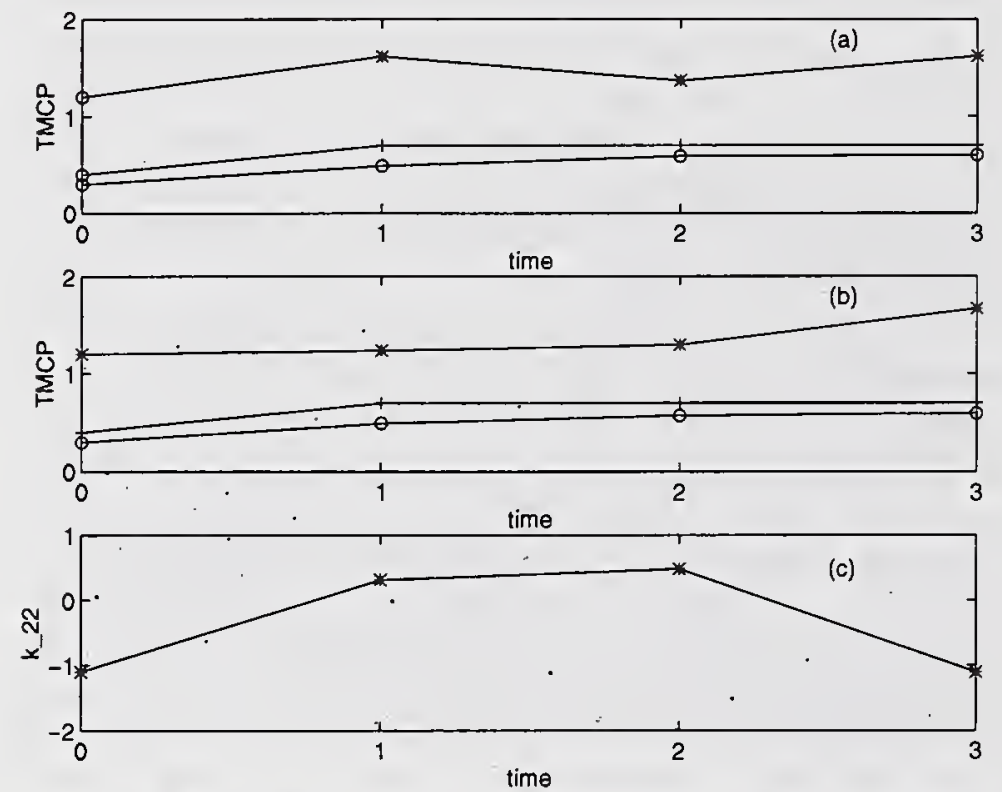

FIG. 2. Time evolution of the TMCP for the uncontrolled (a) and controlled (b) case. The long range strategic control is applied to minimize the oscillations of the totalitarian nation's TMCP $\left({ }^{*}\right)$ at a minimal cost. The parameters used are $a_{1}=0.2, a_{2}=1.0, a_{3}=0.2$ and $\gamma=0.01$. Fig. (c) shows the time evolution of the control parameter.

$$
\begin{gathered}
G=\sum_{i=1}^{N}\left[a_{1}\left(x_{i}^{1}-x_{i-1}^{1}\right)^{2}+a_{2}\left(x_{i}^{2}-x_{i-1}^{2}\right)^{2}+\right. \\
\left.a_{3}\left(x_{i}^{3}-x_{i-1}^{3}\right)^{2}-\gamma k_{22, i}^{2}\right]
\end{gathered}
$$

where $a_{1}, a_{2}, a_{3}$ and $\gamma$ are positive constants weighting the relative importance of the "goals" and the "cost", respectively. In this example we choose a $N=3$ time step planning, an correspondingly the control will be also projected three time steps ahead reflecting a longer term strategical objective. Fig. 2(a) shows the uncontrolled trajectory, while Fig. 2(b,c) the controlled version and the variation of the control parameter, respectively. Ac- cording to the choice of the cost function the oscillations in the totalitarian nation's TMCP have been minimized at optimal cost.

\section{CONCLUSIONS}

In principle, each of the above control methods can be used to achieve either stabilization or destabilization of the dynamics of MRM. The choice between the two methods is not merely a political decision. In some cases one or another method may be more efficient, or one of these methods may not be suitable at all. One simple example is the problem of destabilizing stable fixed points of the dynamics. If the fixed point is elliptic, the short range reactive control does not work because small perturbations applied to the system are unable to perturb the trajectory from the elliptic point's neighborhood. In general, short range reactive control is better suited when the model of the system is incomplete and cannot take into account all relevant factors. Hence it is a good choice when one does not have a well-defined overall goal or strategy and in unforeseen crisis situations. The long range control is better suited when one has a good idea about global strategic objectives, the costs involved and, one wants to achieve a well-defined goal. Since the role of the user is essential in deciding what control strategy to use we see here an excellent example of applied semiotic analysis to international relations.

\section{ACKNOWLEDGEMENTS}

The authors wish to thank M. Sulcoski, L.D. Miller, G. Mayer-Kress, and Z. Toroczkai for very useful discussions. This work was partially supported by a grant from the Office of Naval Research, from the National Ground Intelligence Center and from the US-Hungarian Science and Technology Joint Fund under Project JF No. 501. A.P. wishes to thank partial support from the Hungarian Science Foundation under Grant Nos. OTKA T17493, F17166. V.P. was partially supported by the Office of Basic Energy Sciences of the U.S. Department of Energy under contract No. DE-AC05-96OR22464 with Lockheed Martin Energy Systems, Inc.

[1] Kadtke, J. B., "Classification of highly noisy signals using global dynamical models." Phys. Lett. A 203 (1995): 196-202.

[2] Crutchfield, J., and McNamara, B., "Equations of motion from a data series." Complex Systems 1 (1987): 417-452. 
[3] Cremers, J., and Hubler, A., "Construction of differential equations from experimental data." Z. Naturforsh. 42A (1987): 797-802.

[4] Kadtke, J. B., and Kremliovsky, M., "Estimating Statistics for Detecting Determinism Using Global Dynamical Models", preprint (1996).

[5] Richardson, L. F., Arms and Insecurity. Pittsburgh; Boxwood, 1960.

[6] Fan, L.-T., and Wang, C.-S., The Discrete Maximum Principle. New York; John Wiley \& Sons, 1964.

[7] Saperstein, A. M., "Chaos - a model for the outbreak of the war." Nature 309 (1984): 303-305.

[8] Miller, L. D., Sulcoski, M. F., and Farmer, B. A., "Discrete Richardson Model: A Paradigm for International Relations?", preprint(1996).

[9] Lions, J.-L., Optimal Control of Systems Governed by Partial Differential Equations, New York; Springer Verlag, 1971.

[10] Lenhart, S., and Protopopescu, V., "Optimal Control for Competitive-Cooperative Systems: Modeling Flexible Coalitions in Tomorrow's Competitive World", paper presented at the 61st MORS Conference, Dayton, Ohio, June 22-24, 1993.

[11] Lenhart, S., Protopopescu, V., and Szpiro, A., A., "Optimal Control for Competing Coalitions", to appear in Nonlinear Analysis: Theory, Methods, and Applications.

[12] Ott, E., Grebogi, C., and Yorke, J. A., "Controlling Chaos." Phys. Rev. Lett. 64 (1990): 11961199.

[13] Kadtke, J., Kremliovsky, M., and Pentek, A., "Dynamical Classification and Control of a Nonlinear Model of International Relations", in Proceedings of the 1996 International Workshop on Complex Systems: Issueas of Measurement and Semiotic Analysis, 8-12 December 1996, Las Cruces, New Mexico.

[14] Pentek, A., Kadtke, J. B., and Toroczkai, Z., "Stabilizing chaotic vortex trajectories: an example of high-dimensional control." Phys. Lett. A in press (1996).

[15] Coombs, M., and Sulcoski, M., eds. Proceedings of the 1996 International Workshop on Control mechanisms for Complex Systems: Issues of Measurement and Semiotic Analysis, 8-12 December 1996, Las Cruces, New Mexico. 


\title{
GALEN IN MEDICAL SEMIOTICS
}

\author{
Thomas A. Sebeok
}

Indiana University

Ullmann (1951: 161) distinguished among four juxtaposed branches of word-study: "(1) the science of names (lexicology if synchronistic, etymology if diachronistic); (2) the science of meanings (semantics); (3) the science of designations (onomasiology); (4) the science of concepts (Begriffslehre)." Although the distinction between designation and meaning, particularly as displayed in the works of German and Swiss semanticists (of the sometimes loosely, as well erroneously, called TrierWeisgerber School) is far from consistently drawn or pellucid, I take it that this alterity depends on whether one's starting point is the name, the lexeme, or, more generally, the sign; or whether it is the concept or, more generally yet, the object, i.e. the constellation of properties and relations the sign stands for. If the former, the analysis should yield a semiotic network responsive to the question: what does a given sign signify in contrast and opposition to any other sign within the same system of signs? If the latter, the analysis should reveal the sign by which a given entity is designated within a certain semiotic system. According to Ullmann, the second inquiry "is the cornerstone of Weisgerber's structure" (1951), but I believe that the two questions are indissolubly complementary. In any case, the whole enterprise critically hinges upon how the investigator parses the sign/object (aliquid/aliquo) relation, and what the conjunctive stands for, in the judgment of the investigator, entails.

The probe becomes at once more intricate, but also more intriguing, when the lexical field (Bedeutungsfeld? Sinnfeld? Wortfeld?) being explored happens to be reflexive, that is, selfsearching. Such is the case of symptom (Sebeok 1986:45-58), an ancient technical term in both semiotics and medicine. Thus its examination may begin in the inner realm of the lexicon, if viewed as a name, or in the outer realm of clinical experience, if viewed as sense.

One may properly inquire: what does the lexeme symptom mean in language $\mathrm{L}_{1}$; or what does the same lexeme designate, or reveal as a diagnostic intimation, with respect to, say, an actual quality of "diseasehood" (Fabrega 1974:123), that F.G. Crookshank (in Ogden and Richards 1938:343) foresightedly portrayed as "a mysterious substantia that has 'biological properties' and 'produces' symptoms"? In the end, the results of such dichotomous inquiries amalgamate in a common dialectical synthesis. For the purposes of this exposition, $\mathrm{L}_{1}$ is American English. However, the semantic field of "medical discourse," which is typically nested within wider sets of concentric frames (Labov and Fanshel 1977:36f.), is here assumed to be, mutatis mutandis, very similar to that in every other speech community committed to the paradigm of medical theory and practice "in the context of the great tradition" (Miller 1978:184) of thinking marked by a continuity that links modern clinicians with the idea of isonomia launched by the brilliant Alcmaeon of Croton during the first half of the fifth century. This heritage was further consolidated by Hippocrates -- arguably considered, at one and the same time, the "father of medicine" (Heidel 1941:xiii), and the "der Vater und Meister aller Semiotik" (Kleinpaul 1972:103) -- and Plato, Aristotle, and the Alexandrian physicians of the fourth century B.C. (Manetti 1987:57-134). Equally perceptive studies of symptom have, in fact, cropped up in the semiotic literature (e.g., Baer 1982, 1986, 1988) and in the medical literature (e.g., Prodi 1981), undertaken by savants who mutually know their way around the other field as well as their own (see also Staiano 1979, 1982). One should, however, continue to be ever mindful of the admonition of Mounin (1981) against a mechanical application of semiotic (especially linguistic) concepts to medicine (especially psychiatry).

Symptom always appears in conjunction with sign, but the precise nature of the vinculum is far from obvious (as in MacBryde and Blacklow 1970 or Chamberlain and Ogilvie 1974). The basic semiosic facts were perspicuously depicted by Ogden and Richards: "If we stand in the neighbourhood of a crossroad and observe a pedestrian confronted by a notice To Grandchester displayed on a post, we commonly distinguish three important factors in the situation. There is, we are sure, (1) a Sign which (2) refers to a Place and (3) is being interpreted by a person. All situations in which Signs are considered are similar to this. A doctor noting that his patient has a temperature and so forth is said to diagnose his disease as influenza. If we talk like this we do not make it clear that signs are here also involved. Even when we speak of symptoms we often do not think of these as closely related to other groups of signs. But if we say that the doctor interprets the temperature, etc. as a Sign of influenza, we are at any rate on the way to an inquiry 
as to whether there is anything in common between the manner in which the pedestrian treated the object at the crossroad and that in which the doctor treated his thermometer and the flushed countenance" (1938: 21).

The relation of sign to symptom involves either coordination or subordination. If the distinction is between coordinates, what matters less than their inherent meaning is the mere fact of the binary opposition between the paired coordinate categories. This was nicely brought to the fore in a report of an investigation of the symptom "fatigue" by two physicians, Shands and Finesinger: "The close study of...patients made it imperative to differentiate carefully between 'fatigue,' a feeling, and 'impairment,' an observable decrement in performance following protracted effort. The distinction comes to be that between a symptom and a sign. The symptom is felt, the sign observed by some other person. These two terms cover the broad field of semiotics; they are often confused, and the terms interchanged [at least in $\mathrm{L}_{1}$ without warning" (Shands 1970:52).

This passage underscores the importance of separating the "private world" of introspection reported by the verbal description or nonverbal exhibition of the symptoms on the part of the patient from the public world of signs reported by the description of status or behavior observed on the part of the physician. As I had written earlier: "It is a peculiarity of symptoms that their denotata are generally different from the addresser, i.e. the patient ('subjective symptoms,' confusingly called by many medical practitioners 'signs') and the addressee, i.e. the examining physician ('objective symptoms,' or simply 'symptoms')" (Sebeok 1976:181). Note that only a single observer -- to wit, oneself -- can relate symptomatic phenomena or events, whereas an indefinite number of observers -- including oneself -can observe signs. Accordingly, within this framework the fact of privacy looms as a criterial distinctive feature that demarcates any symptom from any sign (Sebeok 1991:36-48). Symptoms could thus be read as recondite communiques about an individual's inner world, an interpretation that sometimes acquires the status of an elaborate occult metaphor. For instance, the eating disorder anorexia nervosa would appear to be reasonably decipherable as "I am starving (emotionally) to death." Its symptoms are sometimes believed to result from disturbed family relationships and interpersonal difficulties (Liebman, Minuchin, and Baker 1974a, $1974 b)$. One palpable sign of this ailment is, of course, weight phobia, measurable as a decrement in the patient's mass.

The crucial distinction between fatigue and impairment is "similar to that between anxiety as a felt symptom and behavioral disintegration often exhibited in states of panic. The latter is a sign, not a symptom" (Shands 1970). The dissemblance exemplified here is obviously related to Uexküll's (1982:209) notion, maintained both in the life and the sign science, of "inside" and "outside. "I take the pivotal implication of this to be as follows: "Something observed (= outside) stands for something that is (hypothetically) noticed by the observed subjects (= inside). Or something within the observing system stands for something within the observed system" (ibid.). For any communication, this complementary relationship is obligatory, because the organism and its Umwelt together constitute a single system. The shift from physiological process to semiosis is a consequence of the fact that the observer assumes a hypothetical stance within the observed system (Bedeutungserteilung / Bedeutungsverwertung).

For symptom (in $\mathrm{L}_{1}$ ), there exists an array of both stricter and looser synonyms. Among the former, which appear to be more or less commonly employed, Elstein et al. solely but extensively use cue. Although they do so without a definition, their import is made quite clear from passages such as "cues were interpreted by physicians as tending to confirm or disconfirm a hypothesis, or as noncontributory" (1976:279). Fabrega, on the other hand, seems to prefer indicator, but he uses this commutably for either symptom or sign; and when he remarks that "all indicators may be needed in order to make judgments about disease" (1974:126), he surely refers to both categories together. The word clue, on the other hand, is a looser synonym for symptom: generally speaking, where symptom is used in medical discourse, clue is found in the detectival sphere (Sebeok $1981 \mathrm{a}$, Eco and Sebeok 1983).

In the minimalist coupling, sign / symptom are equipollent; both are unmarked vis-a-vis one another (Waugh 1982). Sometimes, however, symptom encompasses both "the objective sign and the subjective sign" (cf. Staiano 1982:332). In another tradition, symptom is a mere phenomenon "qui précisement n'a encore rien de sé miologique, de sémantique," or is considered falling, for instance, in the terminology of glossematics, in the area of content articulation, la substance du signifiant, an operationally designated figura that is elevated to full semiotic status only through the organizing consciousness of the physician, achieved through the mediation of language (Barthes 1972:38f.). However, still other radically different sorts of arrangements occur in the literature. In Bühler's organon model (see Sebeok 1981b), symptom constitutes but one of three "variable moments" capable of rising "in three different ways to the rank of a sign." These include signal, symbol, as well as symptom. Bühler (1965:28) specifies further that the 
semantic relation of the latter functions "by reason of its dependence on the sender, whose interiority it expresses." He clearly subordinates this trio of words under one and the same "Oberbegriff 'Zeichen'," then goes on to ask: "Ist es zwäkmässig, die Symbole, Symptome, Signale zusammenzufassen in einem genus-proximum 'Zeichèn'?" It should also be noted that Bühler's first mention of symptom is immediately followed by a parenthetic set of presumed synonyms: "(Anzeichen, Indicium)." (Note that the German verb anzeigen bears an ominous secondary judicial connotation, "to denounce somebody" [see Sebeok 1981b:228f.)

Thus, in acknowledging the importance of the notion of privacy as an essential unmarked feature of symptom, Bühler also recognizes that, while it is coordinate with two other terms, it is also subordinate to the (unmarked) generic notion of sign, namely, that kind of sign that Peirce earlier, but unbeknownst to Bühler, defined with much more exactitude as an index (Sebeok 1991:128-143).

Despite his extensive knowledge of medicine (Sebeok 1981a:37), Peirce did not often discuss symptom (nor anywhere, in any fecund way, syndrome, diagnosis, prognosis, or the like). For him, a symptom, to begin with, was one kind of sign. In a very interesting passage, from the dictionary lemma "Represent," he expands: "to stand for, that is, to be in such a relation to another that for certain purposes it is treated by some mind as if it were that other. Thus, a spokesman, deputy, attorney, agent, vicar, diagram, symptom, counter, description, concept, premise, testimony, all represent something else, in their several ways, to minds who consider them in that way" $(2: 273)$.

For Peirce, however, a symptom was never a distinct species of sign, but a mere subspecies, namely the index -- or secondness of genuine degree (in contrast to a demonstrative pronoun, exemplifying secondness of a degenerative nature) -- of one of his three canonical categories. But what kind of sign is this? Peirce gives an example that I would have preferred to label a clue: "Such, for instance, is a piece of mould with a bullet-hole in it as sign of a shot; for without the shot there would not have been a hole; but there is a hole there, whether anybody has the sense to attribute it to a shot or not" (2.304). The essential point here is that the indexical character of the sign would not be voided if there were no explicit interpretant, but only if its object were removed. An index is that kind of a sign that becomes such by virtue of being really (i.e. factually) connected with its object. "Such is a symptom of disease" (8.119). All "symptoms of disease," furthermore, "have no utterer," as is also the case with "signs of the weather" (8.185). We have an index, Peirce prescribed in 1885 , when there is "a direct dual relation of the sign to its object independent of the mind using the sign... Of this nature are all natural signs and physical symptoms" (3.361).

A further detail worth pointing out is that Peirce calls the "occurrence of a symptom of a disease...a legisign, a general type of a definite character," but "the occurrence in a particular case is a sinsign" (8:335), that is to say, a token. A somewhat cryptic remark reinforces this: "To a sign which gives reason to think that something is true, I prefer to give the name of a symbol; although the words token and symptom likewise recommend themselves" (MS 787, 1896). Staiano is undoubtedly correct in remarking that "the appearance of a symptom in an individual is thus an indexical sinsign, while the symptom interpreted apart from its manifestation becomes an indexical legisign" (1982:331).

Symptoms, in Peirce's usage, are thus unwitting indexes, interpretable by their receivers without the actuality of any intentional sender. Jakobson likewise includes symptoms within the scope of semiotics, but cautions that "we must consistently take into account the decisive difference between communication which implies a real or alleged addresser and information whose source cannot be viewed an addresser by the interpreter of the indications obtained" (1971:703). This remark glosses over the fact that symptoms are promptings of the body crying out for an explanation -- for the construction, by the self, of a coherent and intelligible pattern (which of course may or may not be accurate; cf. Polunin 1977:91).

Pain comprises one such symptom that embodies a message compelling the central nervous system to influence both covert and overt behavior to seek out signs of pain, throughout phylogeny, ontogeny hic et ubique. Miller befittingly expands: "From the instant when someone first recognizes his symptoms to the moment when he eventually complains about them, there is always an interval, longer or shorter as the case may be, when he argues with himself about whether it is worth making a complaint known to an expert.... At one time or another we have all been irked by aches and pains. We have probably noticed alterations in weight, complexion and bodily function, changes in power, capability and will, unaccountable shifts of mood. But on the whole we treat these like changes in the weather.... (1978: 45-49).

Peirce once particularized the footprint that Robinson Crusoe found in the sand to be an index "that some creature was on his island" (4:351), and indeed an index always performs as a sign the vectorial direction of which is toward the past, or, as Thom put it, "par reversion de la causalité génératrice" (1980:194), which is the inverse of physical causality. Augustine's class of signa naturali, defined -- in contrast to signa data -- by the 
relation of dependence between sign and the things signified (De Doctrina Christiana II.1.2), beside its orthodox sense (such as a rash as a symptom of measles), is also illustrated by footprints left by an animal passed out of sight. It may thus be regarded as encompassing a portent, or in the most general usage, evidence (for instance, as a south-westerly wind may both signify and bring rain, i.e., give rise to its significatum). Thus symptoms, in many respects, function like tracks --.footprints, toothmarks, food pellets, droppings and urine, paths and runs, snapped twigs, lairs, the remains of meals, etc. -- throughout the animal world (Sebeok 1976:133), and in hunting populations, where humans "learnt to sniff, to observe, to give meaning and context to the slightest trace" (Ginzburg 1983). Tracks, including notably symptoms, operate like metonyms. This trope is also involved in pars pro toto, as extensively analyzed by Bilz, who spelled out its relevance: "Auch eine Reihe körperlicher Krankheitszeichen sog., funktioneller oder organneurotischer Symptome, haben wir unter den Generalnenner der Szene gebracht, einer verschütteten Ganzheit... Hier ist es...eine Teil funktion der Exekutive...wobei wir abermals auf den Begriff der Parsprototo stiessen" (1940:287).

It is, of course, Hippocrates who remains the emblematic ancestral figure of semiotics -- that is to say, semiology, in the narrow, particularly Romance, sense of symptomatology -- although he "took the notion of clue from the physicians who came before him" (Eco 1980:277). Baer alludes to a "romantic symptomatology," which he postulates may have been "the original one," carrying the field back "to an era of mythical consciousness" (1982:18). Alcmaeon remarked, in one of the scanty fragments of his book: "As to things invisible and things mortal, the gods have certainties; but, so far as men may infer...," or, in an alternative translation, "men must proceed by clues..." (Eco 1980:281), namely, provisionally conjecture. And what is to be the basis of such circumstantial inference? Clearly, the concept that has always been central is symptom (semeion; Ginzburg 1983).

While Alcmaeon is commonly regarded as the founder of empirical psychology, it was Hippocrates, a clinical teacher par excellence (Temkin 1973), who broke with archaic medical practice where the physician was typically preoccupied with the nature of the disease, its causes and manifestations, refocusing directly upon the sick person and his/her complaints --in brief, upon the symptoms of disease: "Nicht so sehr die Krankheit als das Kranke Individuum" (Neuburger 1906:196).

For Hippocrates and his followers symptoms were simply "significant phenomena" (cf. Heidel 1941:62). Their consideration of symptoms as natural signs -- those having the power to signify the same things in all times and places -- was of the most comprehensive sort. An early discussion of this type is found in Hippocrates' Prognostic (XXV): "One must clearly realize about sure signs, and about symptoms generally [peri ton tekmèrion kai ton allon semeion, that in every year and in every land bad signs indicate something bad, and good signs something favourable, since the symptoms sèmeia described...prove to have the same significance in Lybia, in Delos, and in Scythia. So one must clearly realize that in the same districts it is not strange that one should be right in the vast majority of instances, if one learns them well and knows how to estimate and appreciate them properly."

I have previously recalled an enduring example of his method, the detailed description of the famous facies hippocratica (Sebeok 1979:6f.); another example may here be cited from Epidemics I: "The following were the circumstances attending the diseases, from which I formed my judgments, learning from the common nature of all and the particular nature of the individual, from the disease, the patient, the regimen prescribed and the prescriber -- for these make a diagnosis more favorable or less; from the constitution, both as a whole and with respect to the parts, of the weather and of each region; from the customs, mode of life, practices and age of each patient; from talk, manner, silence, thoughts, sleep or absence of sleep, the nature and time of dreams, pluckings, scratchings, tears; from the exacerbations, stools, urine, sputa, vomit, the antecedents of consequents of each member in the succession of diseases, and the absessions to a fatal issue or a crisis, sweat, rigor, chill, cough, sneezes, hiccoughs, breathing, belchings, flatulence, silent or noisy, hemorrhages, and hemorrhoids. From these things we must consider what their consequents also will be" (Heidel 1941:129).

In The Science of Medicine, Hippocrates also stated: "What escapes our vision we must grasp by mental sight, and the physician, being unable to see the nature of the disease nor to be told of it, must have recourse to reasoning from the symptoms with which he is presented." The means by which a diagnosis may be reached "consist of observations on the quality of the voice, whether it be clear or hoarse, on respiratory rate, whether it be quickened or slowed, and on the constitution of the various fluids which flow from the orifices of the body, taking into account their smell and colour, as well as their thinness or viscosity. By weighing up the significance of these various signs it is possible to deduce of what disease they are the result, what has happened in the past and to prognosticate the future course of the malady" (Chadwick and Mann 1950: 87-89).

However, it was Galen, whose one and only idol was Hippocrates, and whose medicine remained (on 
the whole) Hippocratic, who attempted to provide prognostics, wherever feasible, with a scientific underpinning, i.e. to base his forecasts on actual observations. This he was able to do because he practiced dissection and experiment: whereas Hippocrates studied disease as a naturalist, Galen "dared to modify nature as a scientist" (Majno 1975: 396; cf. Neuburger 1906:385). "Empirical method was first formulated in ancient medicine," as systematic and detailed expression in the Hippocratic corpus (De Lacy 1941:121), and became a part of the theory of signs of the Epicureans and Sceptics, in opposition to the Stoic rationalistic position. Philodemus' fragmentary treatise (circa 40 B.C.) is by far the most complete discussion of a thoroughgoing methodological work uncovered (in the Herculean library) and extensively elucidated to date. Galen, despite all of his Platonic training, was later "forced by his profession to be more empirical" (Phillips 1973: 174), even though this open-minded investigator, who continued to speak with the voice and authority of a man of science, did gradually turn into something of a dogmatic mystic (Sarton 1954: 59). He can therefore be regarded as a subtle founder of clinical semiotics. As such, his work therefore constituted something of a watershed, since "die galenische Semiotik verwertet die meisten Beobachtungs- und Untersuchungsmethoden die das Altertum ausgebildet hat" (Neuburger 1906: 385).

Although Galen can, very likely, be reckoned the first "scientific" semiotician, philosophy and medicine are, as well, consistently conjoined in his writings. Barnes (1991:50) recently reminded us that, according to Galen, "The best doctors... are also philosophers," and that he even wrote a pamphlet to show how a competent physician "possesses all the parts of philosophy -- logic, natural science, ethics," to which one may dare add: semiotics, or logic in its most technical Peircean sense; (on Galen's logic, see Barnes 1991:54-56 et passim). Galen counted himself among "those who teach the greatest and finest human achievements -- the theorems which philosophy and medicine impart." No wonder that the Emperor Marcus Aurelius, as Galen himself informed us, referred to him as "the first among physicians, unique among philosophers" (quoted ibid.). He was indeed the first to explicitly articulate, as far as we know, the kind of medical semiotics put in practice by his most eminent predecessors -- in fact, he deemed this to have been a platitude in the Hippocratic tradition -- and, having reaffirmed it, emulated by a veritable legion of professional successors in and beyond his craft.

Particularly intriguing is Galen's usage of the term endeixis -- roughly: "indication" -- in medicine and logic, as most recently, and very revealingly (if not exhausively) reviewed by Kudlien (1991) (with added commentary on its prehistory and Medieval
Latin renderings, by Durling [1991]). This important term, so cherished by Peirce, is very seldom found in the Hippocratic Corpus and then never in a technical sense or in the sense, or rather the broad spectrum of senses, employed by Galen. Kudlien rightly emphasizes that doctors then, as now, do not deem"indication" to be a "a mere 'sign'," but rather, as puts it, an action: that is, a sign which points (usually) to the treatment of certain symptoms, or the syndrome, of the disease being diagnosed (Kudlien 1991:103f.). According to him, the evidence confirms that "it was Galen, above all, who used the word in the modern sense" (ibid. 104), along with such derivatives as endeiktikos, endeiknynai, synendeiknysthai, antendeiknysthai, and the like. In brief, for Galen, endeiktikon is by no means the same as semeion or symptomata. His own usage was quite different from those of the adherents of the Empiricist or again the Methodist schools of physicians (he counted himself a member of the socalled Dogmatist school of medicine). "When seeking for the true 'endeixis', the Dogmatist would not observe all possible signs/symptoms as such (as the Empiricist does), but select only those that are 'indicative of the cause (sc. of the disease)..." (Kudlien 1991:106). In brief, in Galen's writings, true endeixis is always something "logical." As he clearly remarked: "One must also use the logical [viz., semiotic method to recognize what all the diseases are, with regard to species and kinds, and how, for every disease, one must take an 'endeixis' of the therapeutical measures ('iamata')" (quoted in Kudlien 1991:107).

Galen's pen was as busy as his scalpel. In the course of his exceptionally bulky writings, he classified semiotics as one of the six principal branches of medicine (merè iatrikès ta men pròta esti, to te phusiologikon, kai to aitilogikon è pathologikon kai to hugeinon kai to sèmiotikon kai to therapeutikon [XIV:689]), an ordering that had a special, indeed, critical importance for its "effect on the later history of medicine" (Phillips 1973:172). The strength of Galenism, as Temkin (1973:179) also emphasized, "reposed in no small measure in having provided medical categories...for relating the individual to health and disease," including "semeiology (the science of signs)."

Of semiotics, Galen further specified: Sèmeiòsis de dai eis therapeia men anakaia, all' ouk estin autè hè therapeia. Dia gar tès hulès hè therapeia sunteleitai kai to men hulikon aneu therapeias ouden heteron sumballetai. To de sèmeiòikon kai aneu therapeias anankaion pros to eidenai tina therapeutika kai tina atherapeuta kai periistasthai pros to eidenai tina therapeutika kai tina atherapeuta kai periistasthai auta, hopòs mè epiballomeomenoi adunatois sphallòmetha (XIV:689). At the end of this same chapter, Galen then divided the field into 
three enduring parts: in the present, he asserted, its concern was inspection, or diagnosis, in the past cognition, or anamnesis (etiology), and in the future providence, or prognosis (diaireitai de kai to sèmeiòtikon eis tria, eis te epignòsin tòn parelèluthotòn kai eis tèn epikepsin tòn sunendreuont òn kai eis prognòsin ton mellontòn") (XIV:690). His clinical procedure was depicted by Sarton (1954:6) thus: "When a sick man came to consult him, Galen... would first try to elicit his medial history and his manner of living; he would ask questions concerning the incidence of malaria and other common ailments. Then the patient would be invited to tell the story of his new troubles, and the doctor would ask all the questions needed to elucidate them and would make the few examinations which were possible." Galen regarded "everything unnatural occurring in the body" as a symptom (VII:50, 135; also X:71ff), and an aggregation of symptoms (athroisma tòn symptomaton) as a syndrome. He was fully aware that symptoms and syndromes directly reflected clinical observation, but the formulation of a diagnosis required causal thinking (cf. Siegel 1973). $\mathrm{He}$ was the master of foretelling the course of diseases: Galen "pflegte...die Prognostik in besonderem Masse und nicht den geringsten Teil seines Rufes als Praktiker dankte er richtigen Vorhersagungen" (Neuburger 1906: 383). Although his prognostications rested essentially and loyally upon the Corpus Hippocratum, his own anatomical knowledge and exactitude of mind predisposed him to build up his prognoses from a cogent diagnostic foundation.

It would not appear unreasonable to expect a finely attuned reciprocal conformation between internal states and "reality," between an Innenwelt and the surrounding Umwelt, or more narrowly, between symptoms and their interpretations as an outcome over time, in evolutionary adaptation -prodotto genetico, in Prodi's succinct formulation (1981:973) -- that benefits an organism by raising its "fittingness."

But such does not reflect the state of the art of diagnosis. The probabilistic character of symptoms has long been realized, among others, by the PortRoyal logicians (Sebeok 1976:125); the often vague, uncertain disposition of symptoms was clearly affirmed by Thomas Sydenham, the seventeenth century physician often called the "English Hippocrates" (Colby and McGuire 1981:21). This much-admired doctor, held in such high regard by his brother in the profession, John Locke, was also known as the "Father of English medicine" (Latham 1848:xi). Sydenham was noted for his scrupulous recognition of the priority of direct observation. He demanded "the sure and distinct perception of peculiar symptoms," shrewdly emphasizing that these symptoms "referred less to the disease than to the doctor." He held that "Nature, in the production of disease, is uniform and consistent; so much so, that for the same disease in different persons, the symptoms are for the most part the same; and the self-same phenomena that you would observe in the sickness of a Socrates you would observe in the sickness of a simpleton" (Latham 1848:14ff.). This assertion of his was, of course, quite mistaken, although the medical-student jape referred to by Colby and McGuire "that the trouble with psychiatry is that all psychiatric syndromes consist of the same signs and symptoms" (1981:23), appears to be equally exaggerated. There are, to be sure, certain diagnostic difficulties inherent in the similarities between the symptomatology of functional syndromes and of those of the organic maladies. The marginal, or supplementary, symptoms of the former can, however, be assimilated according to specific criteria, such as are set forth, for instance by Uexküll (1979).

This set of strictures leads me to a consideration of an aspect of symptom that is seldom mentioned in the literature but that I have found both fascinating and, certainly for semiotics, of broad heuristic value. This has to do with anomalies, a problem that was considered, in a philosophical context, especially by Peirce. According to Humphries (1968:88), a naturally anomalous state of affairs is such "with respect to a set of statements which are at present putatively true," or, putting the matter in a more direct way, "any fact or state of affairs which actually requires an explanation can be shown to be in need of explanation on the basis of existing knowledge" (1968:89). The enigmatic character of semiotic anomalies can especially well be illustrated by clinical examples, where few existing models are capable of accounting for a multitude of facts. Medicine may, in truth, be one of the few disciplines lacking an overarching theory, although local, nonlinear, and hence restricted and over-simple paradigms, such as the "theory of infectious diseases," certainly do exist.

Take as a first approach to the matter of anomalies the spirochete Treponema pallidum. This virus, in its tertiary phase, may manifest itself ("cause") aortitis in individual A, paretic neurosyphilis in individual $\mathrm{B}$, or no disease at all in individual $C$. The latter, the patient with asymptomatic tertiary syphilis, can be said to have a disease without being ill. Note that a person may not only be diseased without being ill, but, conversely, be ill without having a specifically identifiable disease. What can we say, in cases such as this, about the implicative nexus conjoining the "proposition," i.e. the virus, with its consequent, expressed in some tangible manner or, on the contrary, mysteriously mantled? Are A, B, and C in complementary distribution, and, if so, according to 
what principle -- the constitution of the patient, or some extrinsic factor (geographic, temporal, societal, age- or gender-related, and so forth), or a coalition of these? The influence of context, one suspects, may be paramount. This becomes overriding in the matter of hypertension -- not a disease at all, but a sign of cardiovascular disorder (Paine and Sherman 1971:272) -- which is realized in one and only one restricted frame: within that of patient/physician interaction, assuming the aid of certain accessories, such as a sphygmoscope. Semiosis is, as it were, called into existence solely under the circumstances mentioned; otherwise there are no symptoms (the asymptomatic, or so-called silent, hypertension lasts, on average, fifteen years) -- there are no signs, and there is, therefore, no determinate -- i.e. diagnosable -- object.

Studies have shown that the majority of people who have gallstones -- about fifteen million or probably many more Americans among them -- go through life without palpable problems. The presence of these little pebbles of cholesterol that form in a sac that stores digestive juice can clearly be seen on X-rays: the shadows are the "objective signs," but most of them never cause pain, or any other symptom. They remain mute. They are, in other words, diagnosed only in the course of detailed checkups, and thus require no surgical intervention.

Sensory experiences, at times, lead to semiosic paradoxes, such as the following classic contravention. A hole in one of my teeth, which feels mammoth when I poke my tongue into it, is a subjective symptom I may elect to complain about to my dentist. The dentist lets me inspect it in a mirror, and I am surprised by how trivially small the aperture -- the objective sign -- looks. He lets me inspect it in a dental mirror, and I am surprised by how trivially small the aperture -- this objective sign -- looks. The question is: which interpretation is "true," the one derived via the tactile modality or the one reported by the optical percept? The felt image and the shape I see do not match. The dentist is, of course, unconcerned with the size of the hole, filling the cavity he/she beholds.

It is a common enough experience that the symptom (for reasons ultimately having to do with the chance evolutionary design of the human central nervous system) refers to a different part of the body than where the damage is actually situated. "The pain of coronary heart-disease, for example, is felt across the front of the chest, in the shoulders, arms and often in the neck and jaw. It is not felt where the heart is -- slightly over to the left" (Miller 1978:22). Such a misreport is unbiological, in the sense that a lay reading could be fatal. An even more outlandish symptom is one for which the referent is housed nowhere at all, dramatically illustrated by a phantom limb after amputation. Miller writes: "The phantom limb may seem to move -- it may curl its toes, grip things, or feel its phantom nails sticking into its phantom palm. As time goes on, the phantom dwindles, but it does so in peculiar ways. The arm part may go, leaving a maddening piece of hand waggling invisibly from the edge of the real shoulder; the hand may enlarge itself to engulf the rest of the limb" (1978:20). What is involved here is an instance of subjective -- as against objective -pain, a distinction introduced by Friedrich J. K. Henle, the illustrious nineteenth century German anatomist and physiologist, and generally perpetuated in classifications of pain ever since (e.g., by Behan 1926).

Subjective pain is described as having "no physical cause for existence," i.e. there is no organic basis for its presence (indeed, with respect to a limb unhinged, not even an organ): it results "of impressions stored up in the memory centers, which are recalled by the proper associations... aroused" (Behan 1926:74f.), which is to say that the pain remains connected with a framework of signification dependent upon retrospective cognizance. Referred pain and projection pain are closely allied; the latter is a term assigned to pain that is felt as being present either in a part that has no sensation (as in locomotor ataxia) or in a part that because of amputation no longer exists.

Certain symptoms -- pain, nausea, hunger, thirst, and the like -- are private experiences, housed in no identifiable site, but in an isolated annex that humans usually call "the self." Symptoms such as these tend to be signified by paraphonetic means, such as groans or verbal signs, which may or may not be coupled with gestures, ranging in intensity from frowns to writhings. An exceedingly knotty problem, which can barely be alluded to here, arises from the several meanings of self and how these relate to the matter of symptomatology. The biological definition hinges on the fact that the immune system does not respond overtly to its own self-antigens; there are specific markers that modulate the system generating antigen-specific and idiotype-specific cell lines -- in brief, activate the process of self tolerance. Beyond the immunological self, there is also a "semiotic self," which I have discussed severally elsewhere (cf. Sebeok 1991, Chaps. 3 and 4, and 1992).

Another diacritic category of symptoms deserves at least passing mention. A linguist might be tempted to dub these "minus features," or subtractive symptoms. Here belong all the numerous varieties of asemasia (Sebeok 1976:57, 1979:58) such as agnosia, agraphia, alexia, amnesia, amusia, aphasia, apraxia, etc., as well as "shortcomings" like blurred vision, hardness of hearing, numbness -- in short, symptoms that indicate a deficit from some ideal standard of "normality." 
In any discussion of symptoms, it should be noted that even a syndrome, or constellation of symptoms -- say, of a gastronomical character (anorexia, indigestion, hemorrhoids) -- may not add up to any textbook disease labeling or terminology. Ensuing treatment may, accordingly, be denominated "symptomatic," accompanied by the supplementary advice that the patient remain under continuing observation. In some circumstances, "the syndrome might be ascribed to psychologic etiology" (Cheraskin and Ringsdorf 1973:37). What this appears to mean is that the interpretation of symptoms is often a matter involving, over time, a spectrum of sometimes barely perceptible gradations, entailing a progressively multiplying number of still other symptoms. It is also worth remarking that, temporally, or for predictive purposes, symptoms generally precede signs, which is to say that the orderly unfolding of evidence may be termed prognostic.

No one at present knows how afferent neuronal activity acquires meaning, beyond the strong suspicion that what is commonly called the "external world," including the objects and events postulated as being contained in it, is the brain's formal structure (logos). For all practical purposes, we are ignorant about how the CNS preserves any structure and assigns a meaning to it, how this process relates to perception in general, and how it induces a response. Implicit in this set of queries is a plainly linear model: for example, that fear or joy "causes" increased heart rate. Not only does such a model seem to me far too simplistic, but there is not even a shred of proof that it exists at all.

The future of symptomatology will clearly rest with program developments using computer techniques derived from studies of artificial intelligence. These are intended to mime and complement, if not to replace, human semiosic processes, such as judgment based on intuition (in one word, abduction). Such automated diagnostic counselors are already operational, as for example the program termed Caduceus (McKean 1982). In the simplified example illustrated in Fig. 1, this program "examines a patient with fever, blood in the urine, bloody sputum from the lungs, and jaundice. The program adds together numbers that show how much each symptom is related to four possible diagnoses -- cirrhosis of the liver, hepatitis, pneumonia, and nephritis -- and picks pneumonia as top contender. The runner-up in score is hepatitis. But because hepatitis has one symptom not shared with pneumonia (blood in the urine), Caduceus chooses cirrhosis as first alternative. This process, called partitioning, focuses the computer's attention on groups of related diseases" (McKean 1982:64).

[Fig. 1]
The craft of interpreting symptoms has a significance far exceeding the physician's day-to-day management of sickness. As Hippocrates had already anticipated, its success derives from its psychological power, which critically depends on the practitioner's ability to impress his/her skills on both the patient and their joint environment (the audience gathered in this workshop, which may consist of the patient's family and friends, as well as the physician's colleagues and staff). Dr. Joseph Bell, of the Royal Infirmary of Edinburgh, attained the knack with panache, leaving his imprint not just on clinical practice but, famously, on the detective story, following in the footsteps of his pupil's, Dr. Arthur Conan Doyle's fictional realization, Sherlock Holmes (Sebeok 1981a, Ginzburg 1983). According to recent medical thinking, the contemporary preoccupation with diagnosis -- that is, the doctor's perceived task, or pivotal drive, to explain the meaning of the patient's condition -- rests in the final analysis with the doctor's self-assigned role as an authenticated expositor and explicator of the values of contemporary society. Disease is thus elevated to the status of a moral category, and the sorting of symptoms had therefore best be viewed as a system of semiotic taxonomy -- or, in Russian semiotic parlance, a "secondary modeling system."

Lord Horder's dictum -- "that the most important thing in medicine is diagnosis, the second most important thing is diagnosis and the third most important thing is diagnosis" (Lawrence 1982) -must be true, because medical knowledge has risen to the status of a means of social control. Symptomatology has turned out to be that branch of semiotics that teaches us the ways in which doctors function within their cultural milieu.

\section{REFERENCES}

Baer, Eugen. 1982. The medical symptom: philogeny and ontogeny. American Journal of Semiotics 1(3):17-34.

..... 1986. Symptom and Syndrome. Encyclopedic Dictionary of Semiotics 2:1033-1035, Thomas A. Sebeok, ed. Berlin: Mouton de Gruyter.

-.--. 1988. Medical Semiotics. Lanham: University Press of America.

Barnes, Jonathan. 1991. Galen on logic and therapy. In: Kudlien and Durling 1991, pp. 50-102.

Barthes, Roland. 1972. Sémiologie et médicine. In: Les sciences de la folie, Roger Bastide, ed. Paris: Mouton.

Behan, Richard J. 1926. Pain: Its Origin, Conduction, Perception, and Diagnostic Significance. New York: D. Appleton.

Bilz, Rudolf. 1940. Pars Pro Toto. Leipzig: Georg Thieme. 
Bornet, John. 1892. Early Greek Philosophy. Reprint ed. 1930. London: MacMillan.

Bühler, Karl. 1934. Sprachtheorie: Die Darstellungfunktion der Sprache. Reprint ed. 1965. Stuttgart: Gustav Fischer.

Chadwick, John, and William N. Mann. 1950. The Medical Works of Hippocrates. Oxford: Blackwell.

Chamberlain, Ernest N. and Colin Ogilvie, eds. 1974. Symptoms and Signs in Clinical Medicine. Bristol: Wright.

Cheraskin, Emanuel and William Ringsdorf. 1973. Preventive Medicine: A Study in Strategy. Moutainview, CA: Pacific Press.

Colby, Kenneth Mark, and Michael. T. McGuire. 1981. Signs and symptoms. The Sciences 21(9):21-23.

De Lacy, Phillip Howard, and Estelle Allen De Lacy, eds. 1941. Philodemus On Methods of Inference: A Study in Ancient Empiricism. Philadelphia: American Philological Association.

Durling, Richard D. 1991. 'Endeixis' as a scientific term: B) 'Endeixis' in authors other than Galen and its Medieval Latin equivalents. In: Kudlien and Durling 1991, pp. 112-113.

Eco, Umberto. 1980. The sign revisited. Philosophy andSocial Criticism 7(3/4):261-297.

-----, and Thomas A. Sebeok, eds. The Sign of Three: Dupin, Holmes, Peirce. Bloomington: Indiana University Press.

Elstein, Arthur S., et al. 1978. Medical Problem Solving: An Analysis of Clinical Reasoning. Cambridge, MA: Harvard University Press.

Fabrega, Horatio, Jr. 1974. Disease and Social Behavior: An Interdisciplinary Perspective. Cambridge, MA: MIT Press.

Ginzburg, Carlo. 1938. Clues: Morelli, Freud and Sherlock Holmes. In: Eco and Sebeok 1983, pp. 81-118,

Hankinson, R.J. 1991. Galen on the Therapeutic Method. Books I and II. Oxford: Clarendon Press.

Heidel, William Arthur. 1941. Hippocratic Medicine: Its Spirit and Method. New York: Columbia University Press.

Humphreys, Willard C. 1968. Anomalies and Scientific Theories. San Francisco: Freeman, Cooper.

Jakobson, Roman. 1971. Selected Writings II: Word and Language. The Hague: Mouton.

Kleinpaul, Rudolf. 1888. Sprache ohne Worte: Idee einer allgemeinen Wissenschaft der Sprache. Reprint ed. 1972. The Hague: Mouton.

Kudlien, Fridolf. 1991. 'Endeixis' as a scientific term: A) Galen's usage of the word in medicine and logic). In: Kudlien and Durling 1991, pp. 103-111.

Kudlien, Fridolf and Richard J. Durling, eds. 1991. Galen's Method of Healing. Proceedings of the 1982 Galen Symposium. Leiden: E.J. Brill.
Kuhn, Carolus Gottlob, ed. 1821-1833. Claudii Galeni Opera omnia, 22 Vols. Leipzig: Cnobloch. Labov, William, and David Fanshel. 1977. Therapeutic Discourse: Psychotherapy as Conversation. New York: Academic Press.

Latham, Robert G. 1848. The Works of Thomas Sydenham, M.D. London: Sydenham Society.

Lawrence, Christopher. 1982. Illnesses and their meaning. Times Literary Supplement.

Liebman, Ronald, Salvador Minuchin, and Lester Baker. 1974a. An integrated program for anorexia nervosa. American Journal of Psychiatry $131: 432-435$.

----. 1974b. The role of the family in the treatment of anorexia nervosa. Journal of the American Academy of Child Psychology 3:264-274.

MacBryde, Cyril M., and Robert Backlow, eds. 1970. Signs and Symptoms: Applied Pathologic Physiology and Clinical Interpretation. Philadelphia: J.B. Lippincott.

Majno, Guido. 1975. The Healing Hand: Man and Wound in the Ancient World. Cambridge, MA: Harvard University Press.

Manetti, Giovanni. 1987. Le teorie del segno nell'antichità classica. Milano: Bompiani.

Markus, Robert A. 1957. St. Augustine on signs. Phronesis 2:60-83.

McKean, Kevin. 1982. Diagnosis by computer. Discover 3(9):60-83.

Miller, Jonathan. 1978. The Body in Question. New York: Random House.

Mounin, Georges. 1981. Sémiologie médicale et sémiologie linguistique. Confrontations Psychiatriques 19:43-58.

Neuburger, Max. 1906. Geschichte der Medizin. I. Stuttgart: Ferdinand Enke.

Nutton, Vivian. 1991. Style and context in the Method of Healing. In: Kudlien and Durling, pp. 125.

Ogden, Charles K., and Ivor A. Richards. 1923. The Meaning of Meaning: A Study of the Influence of Language Upon Thought and the Science of Symbolism. Reprint ed. 1938. New York: Harcourt, Brace.

Paine, Robert, and William Sherman. 1970. In: MacBryde and Backlow 1970.

Peirce, Charles S. 1935-1966. Collected Papers of Charles Sanders Peirce. Charles Hartshorne, Paul Weiss, and Arthur W. Burks, eds. Cambridge, MA: Harvard University Press. [References are either to volumes and paragraphs, or to unpublished manuscripts from the Peirce Edition Project located at Indiana University - Purdue University at Indianapolis.]

Phillips, Eustace D. 1973. Greek Medicine. London: Thames and Hudson. 
Polunin, Ivan. 1977. The body as an indicator of health and disease. In: The Anthropology of the Body. London: Academic Press.

Prodi, Giorgio. 1981. Sintomo/diagnosi. Enciclopedia: Ricerca-Socializzazione 12:972-992.

Sarton, George. 1954. Galen of Pergamon. Lawrence: University of Kansas Press.

Sebeok, Thomas A. 1976 [1985]. Contributions to the Doctrine of Signs. Lanham: University Press of America.

-.--. 1981a. The Play of Musement. Bloomington: Indiana University Press.

---.. 1981b. Karl Bühler. In: Die Welt als Zeichen, Martin Krampen, et al., eds. 205-232. Berlin: Severin und Siedler.

----. 1986. I Think I Am a Verb: More Contributions to the Doctrine of Signs. New York: Plenum Press.

----. 1991a. A Sign Is Just A Sign. Bloomington: Indiana University Press.

----. 1991b. Indexicality. In: A Sign Is Just A

Sign, 128-143. Bloomington: Indiana University Press.

----. 1992. "Tell Me, Where is Fancy Bred": The Biosemiotic Self. In: Biosemiotics: The Semiotic Web 1991, Thomas A. Sebeok and Jean UmikerSebeok, eds. 333-343. Berlin: Mouton de Gruyter.

Shands, Harley C. 1970. Semiotic Approaches to Psychiatry. The Hague: Mouton.

Siegel, Rudolph E. 1973. Galen on Psychology, Psychopathology, and Function and Diseases of the Nervous System: An Analysis of His Doctrines, Observations and Experiments. Basel: S. Karger.

Staiano, Kathryn Vance. 1979. A semiotic definition of illness. Semiotica 28:107-125.

-.-. 1982. Medical semiotics: redefining an ancient craft. Semiotica 38:319-346.

Temkin, Oswei. 1973. Galenism: Rise and Decline of a Medical Philosophy. Ithaca: Cornell University Press.

Thom, René. 1980. L'espace et les signes. Semiotica 29:193-208.

Uexküll, Thure von. 1982. Semiotics and medicine. Semiotica 38:205-215.

-..-., et al. 1979. Lehrbuch der Psychosomatischen Medizin. München: Urban \& Schwarzenberg.

Ullmann, Stephen. 1951. Principles of Semantics. Glasgow: Jackson, Son \& Co.
Waugh, Linda. 1982. Marked and unmarked: a choice between unequals in semiotic structure. Semiotica 38:299-318.

\section{NOTE}

This article has had an involuted publishing history, which I need to record along with my acknowledgments.

In 1982, I wrote a paper titled "Symptom" for delivery at a Symposium on New Directions in Linguistics and Semiotics, convened at Rice University at the initiative of Professor Sydney Lamb. That paper was published, in much expanded form, in a book bearing the same Symposium title, edited by James E. Copeland (Houston: Rice University Studies, 1984), pp. 211-230. A somewhat revised version appeared thereafter in my own book, I Think I Am a Verb: More Contributions to the Doctrine of Signs, pp. 45-58 (New York: Plenum Press, 1986).

On October 19, 1992, I then presented, under the new title "Medical Semiotics: The Legacy of Galen," at the 4th Congress of the Hellenic Semiotic Society, at the University of Thessaloniki, Greece, by invitation of the co-organizers, a substantially reworked but still necessarily abridged variant based on my earlier paper. That version will be eventually be published among the voluminous Proceedings of the Congress.

In November, 1992, while I was both a Visiting Professor of Semiotics at The University of Toronto and a Senior Fellow in residence at Massey College, I wrote the latter further elaborated version, incorporating added materials, particularly on Galen. I want to thank Professors Alexandros Lagopoulos and Karin Lagopoulou for their generous hospitality in Athens, Iracleo, and especially in and around Thessaloniki. I would also like to record my deep appreciation to Professor Marcel Danesi, of The University of Toronto, for providing me with the opportunity to lecture for a month in Ontario and elsewhere in Canada, and to write at leisure in the stimulating environment of Massey College.

The present version is a still further expanded variant of my Greek Congress paper, which is to appear, due to local technical considerations, only after much delay, in 1996 or 1997. 


\title{
INTRODUCTION IN PHYSICS OF OPEN SYSTEMS Main Principles. Diagnostics on the "S-Theorem" Criterion. Norm of Chaos. Degradation and Self-Organization. Phase Transitions
}

\author{
Yu.L.Klimontovich \\ Physical Faculty \\ Moscow State University \\ 117234 Moscow Ruaaia \\ E-mail: ylklim@hklim.phys.msu.su
}

$(12-1997)$

The aim of this Report to give the short representation of some ideas, methods and results of the statistical theory of open systems.

\section{PREFACE}

Let us begin by quoting from the Preface to the author's Statistical Physics (Moscow, Nauka, 1982; Harwood Academic Publishers, New York, 1986) [1]:

"My God! Yet another book on statistical physics! There's no room on my bookshelves left". Such emotion are quite understable. Before jumping to conclusion, however, it would be worthwhile to read the Introduction and look through the table of contents. Then the reader will find that this book is totally different from the existing courses, fundamental and concise" ....."The author certainly does not wish to exaggerate the advantages of the book, considering it as just the first attempt to create a textbook of a new kind".

The second step in this direction was the author's Turbulent Motion and the Structure of Chaos (Moscow, Nauka, 1990; Kluwer Academic Publishers, Dordrecht, 1991) [2]. This book is subtitled A New Approach to the Statistical Theory of Open Systems. Naturally, "the new approach" is not meant the defy of course the consistent and efficient methods of the conventional statistical theory of the nonequilibrium processes. "The new approach" is based on a judicious combination of "the old" and "the new".

The following and the radical step was in the last author's book Statistical Theory of Open Systems. Volume I: with the subtitle A Unified Approach to Kinetic Description of Processes in Active Systems, published by "Yanus", Moscow 1995; Kluwer Academic Publishers, Dordrecht, 1995 [3]. Now it is almost ready the manuscript of the following Statistical Theory of Open Systems. Volume II: Part 1 New Approach in the Plasma Kinetic Theory (Myth on Collisionless Plasma); Part 2 Kinetic Theory of Second Order Phase Transitions [4]. The author hopes that will be prepared and the next book Statistical Theory of Open Systems. Volume III: Quantum Open Systems [5].

We must try to give now the answer on the question:
What means the notion "Physics of open systems?.

\section{PHYSICS OF OPEN SYSTEMS}

"Physics of open systems" is an interdisciplinary field of science [1-5]. Here is a brief list of key words and notions to characterize it: Chaos and Order; Open systems; Criteria for the relative degree of order in open systems; Norm of chaos; Degradation and self-organizing; Synergetics; Diagnostics of open systems; Semiotic control; Constructive role of dynamic instability of atomic motion; Transition from reversible to irreversible equations; Kinetic and hydrodynamic description of nonequilibrium processes taking into account the structure of continuous medium; Nonequilibrium process in active media; Equilibrium and nonequilibrium phase transitions; Unified kinetic description of laminar and turbulent motions; Quantum open systems.

Certainly, many of these concepts are not at all 'new'. The purpose of "Physics of open systems" is to elaborate ideas and methods for the integrated description of this broad class of problems.

Open systems can exchange energy, matter, and (last but not least) information with the environment. We shall consider only open macroscopic systems. They are composed of many objects, constituent structural elements. These elements may be microscopic, e.g. atoms or molecules in physical and chemical systems. They may be small but macroscopic such as macromolecules in polymers or cells in biological structures. Due to the complexity of open systems, they may host a variety of structures. Dissipation plays a constructive role in the formation of these structures. At first sight, this seems surprising because the dissipation concept, is associated with the attenuation of various forms of motion, energy scattering, and the loss of information. It is extremely essential, however, that dissipation is necessary for the formation of structures in open systems. To emphasize this, I Prigogine has coined the term "dissipative structures" $[6,7]$. This comprehensive and exact term covers all sorts of structures: temporal, spatial and time- space structures. The latter are exemplified by autowaves.

The complexity of open systems provides an ample opportunity for cooperative phenomena to occur. In order 
to emphasize the role of collective interactions in the formation of dissipative structures, H. Haken has introduced the term 'synergetics', that means joint action. The objective of synergetics is to reveal common ideas, methods, and laws in totally different fields of natural science, sociology, and even linguistics $[8,9]$.

Moreover, synergetics is an area where various special disciplines cooperate. The scope of synergetics is illustrated by a series of books under the common title of Synergetics published by Springer Verlag. The last issue in this series is volume $67[10]$.

Synergetics stems from thermodynamics and statistical physics. This accounts for the word Physics being the first in the title of this Section. Thereby, it is emphasized that the theory of open systems is virtually based on fundamental physical laws

All systems that we are going to deal are macroscopic. This means that they consist of a large number of elements. This allows in many cases to treat such systems as a continuous medium. Such convention change dramatically the nature of the system. In order to avoid the potential problems, it is necessary to use a physical definition of continuous medium rather than a formal mathematical definition. This requires a concrete definition of physically infinitesimal time and length scales in term of characteristic parameters of system. The corresponding physically infinitesimal volume is the equivalent as a physical "point".

\section{PHYSICALLY INFINITESIMAL INTERVALS (SCALES) OF LENGTH AND TIME IN A RAREFIED GAS AND A RAREFIED PLASMA} $[1-3,11]$

\section{A. Rarefied gas. Kinetic level of description.}

Thus, a rarefied (Boltzmann) gas and a rarefied (Debye) plasma are characterized by the dimensionless density and plasma parameters:

$$
\varepsilon=n r_{0}^{3} \ll 1, \quad \mu \sim \frac{1}{n r_{D}^{3}} \ll 1
$$

These parameters determine and the connection between infinitesimal scales $\tau_{p h}$ and $l_{p h}$ with the corresponding relaxation ("collision") parameters $\tau \equiv \tau_{r e l}$ and $l \equiv l_{\text {rel }}$ of the Boltzmann gas and the Debye plasma.

We denote by $N_{p h}=n V_{p h}$ the number of particles in the physically infinitesimal volume $V_{p h}$. By definition of physically infinitesimal scales, the number of particles within $V_{p h}$ is large (that is $N_{p h} \gg 1$ ), and the scales $\tau_{p h}$ and $l_{p h}$ are small compared to the characteristic scales $T$ and $L(\tau$ and $l$ for the Boltzmann gas and the Debye plasma):

$$
\tau_{p h} \ll T, \quad l_{p h} \ll L, \quad N_{p h} \gg 1
$$

The definition of physically infinitesimal scales is not be universal: it will depend on the adopted level of description on nonequilibrium processes (kinetic, hydrodynamic, diffusion).

Boltzmann gas. The following relationships between characteristic lengths hold for a rarefied gas:

$$
r_{0} \ll r_{a v} \ll l, \quad \varepsilon=n r_{0}^{3} \ll 1 .
$$

The time of transition to local equilibrium is determined by the "collision time" $\tau$. The relevant length scale is the mean free path $l$. So, for the kinetic stage of relaxation we set in formulas (3.2):

$$
T \rightarrow \tau, \quad L \rightarrow l
$$

At first sight, this attempt to regard a rarefied gas as a continuous medium may seen paradoxical. To show that this is not so, we proceed as follows.

Divide the value of $\tau$, which is average time interval between two consecutive collisions of a given particle, into the number $N_{p h}=n V_{p h}$. The resulting value is the time between consecutive collisions of an single particle contained within physically infinitesimal volume $V_{p h}$. It would be natural to take this for the definition of $\tau_{p h}$. Using the relationship $\tau_{p h}=l_{p h} / v_{T}$ for the kinetic stage of description, we obtain two equations ,

$$
\frac{\tau}{N_{p h}} \sim \frac{\tau}{n l_{p h}^{3}}=\tau_{p h ;} \quad \tau_{p h}=\frac{l_{p h}}{v_{T}} .
$$

Hence, making use of the definition of $l$ and $\varepsilon$, we obtain concrete estimates for physically infinitesimal scales:

$$
\tau_{p h} \sim \sqrt{\varepsilon} \tau \ll \tau, \quad l_{p h} \sim \sqrt{\varepsilon} l \ll l, \text { and } N_{p h}=\frac{1}{\sqrt{\varepsilon}} \gg 1 .
$$

In hydrodynamics the relaxation times are expressed in terms of the external parameter $L$, and one of the three dissipative coefficients: diffusion $D$, viscous friction $\nu$, and thermal conductivity $\chi$. All these dissipative processes are of diffusion type; accordingly, the "coefficient of diffusion" is represented by one of the coefficient $D, \nu$, and $\chi$. Then the time of relaxation by diffusion is

$$
\tau_{D}=\frac{L^{2}}{D}, \text { where } D=D, \nu \text { or } \chi \text {. }
$$

For diffusion processes the linkage between physically infinitesimal scales is given, instead the "kinetic relationship" $\tau_{p h}=l_{p h} / v_{T}$, by the corresponding "gasdynamic ("G") relationship

$$
\tau_{p h}^{G}=\frac{\left(l_{p h}^{G}\right)^{2}}{D}, \quad \text { where } D=D, \text { or }, \nu, \text { or } \chi .
$$

Observe, that by this definition, "traces" of diffusive (hydrodynamic) motion are preserved also within the 
physically infinitesimal volume $V_{p h}$ - in a "point" of continuous medium.

Hence, making use of the definitions (3.7), (3.8), we obtain the concrete estimates of physically infinitesimal scales for the gasdynamic level of description:

$$
\tau_{p h}^{G} \sim \frac{\tau_{D}}{N^{2 / 5}} \ll \tau_{D}, \quad l_{p h}^{G} \sim \frac{L}{N^{1 / 5}} \ll L, \quad N_{p h}^{G} \sim N^{2 / 5} \gg 1 .
$$

Here we have used equation $n L^{3}=N$.

We see that, unlike the case of (3.6) for the kinetic description, now the physically infinitesimal scales depend on the external parameter $L$. This is a clear indication that the definition of physically infinitesimal scales for the Boltzmann gas depends on the adopted level of description.

Naturally, the gasdynamic descriptions is rougher than the kinetic one, and so the "point" of continuous medium in hydrodynamics is larger, that is, $V_{p h} \leq V_{p h}^{G}$. The transition from the kinetic equation to the more "rough" gasdynamic description can be made in the following manner. For this transition it is usually uses the perturbation theory on Knudsen number (methods of Hilbert, Chapmasn-Enscog and Grad).

$$
K n=\frac{l}{L}
$$

However, in this way we meet serious difficulties in applying the pointed perturbation theory. They are due to the fact that parameter $K n$ does not reflect the structure of the "continuous medium".

Instead of Knudsen number it is more natural to use the "physical Knudsen number". For the kinetic level of description it is defined by the expression:

$$
K_{p h}=\frac{l_{p h}}{L} \rightarrow \frac{l_{p h}}{l} \sim \frac{1}{\sqrt{N_{p h}}} .
$$

The smallness of this parameter is ensured by condition $N_{p h} \gg 1$.

In the limit of free molecular flow -" collisionless flow", when the characteristic length $L$ (for instance, the diameter of pipe) is much smaller than the free path $l$, the approximation of continuous medium may be used as long as

$$
l_{p h} \ll L \ll l .
$$

In the case of hydrodynamic description, the physically infinitesimal scales are defined by expressions (3.9), and the physical Knudsen number is

$$
K_{p h}^{G}=\frac{l_{p h}^{G}}{L} \sim \frac{1}{\left(N_{p h}^{G}\right)^{1 / 2}} .
$$

The smallness of this parameter is ensured by condition $N_{p h}^{G} \gg 1$.
For the unified kinetic and gas-dynamic description the size of a "point" is defined by relations:

$$
L_{\min } \sim \sqrt{N_{p h}} l_{p h} \sim \frac{l}{\sqrt{N_{p h}}}, \quad(K n)_{\max } \sim \sqrt{N_{p h}} .
$$

We see that the minimum length $L_{\text {min }}$ - the minimum size of point, for which the traces of diffusive motion are still retained and the gasdynamic description is still feasible, is less than mean free path $l$ and greater than the kinetic physically infinitesimal scale $l_{p h}$. So, a unified description of kinetic and hydrodynamic processes is possible in a broad range of Knudsen numbers without using perturbation theory.

The relevant characteristic time is defined as

$$
\left(\tau_{p h}^{G}\right)_{\min } \sim \frac{L_{\min }^{2}}{D} \sim \sqrt{\varepsilon} \tau \sim \tau_{p h}
$$

and is of the order of physically infinitesimal time interval for kinetic description. We shall need this result for the generalization kinetic equation for unified description of kinetic and gasdynamic processes in rarefied gases and plasmas.

The above obtained relationships allow making estimates for the number of particles within a "point":

$$
N_{\text {min }}=n L_{\text {min }}^{3} \sim \varepsilon^{-5 / 4} .
$$

Hence, at normal conditions, when $\varepsilon \sim 10^{-4}$, the number of particles within a "point" is $N_{\min } \approx 10^{5}$.

Rarefied Coulomb plasma (Debye plasma) For a rarefied plasma the possibilities of the unified description kinetic and hydrodynamic processes are more justified, than for a rarefied gas. It is the cause due to collective character of charged particles interaction.

For a rarefied plasma there are two possibilities for the definition of the physically infinitesimal scales. The former is similar to one that we meet in the kinetic definition (3.6) for a rarefied gas.

However, for a plasma it is a more natural to adopt the following definition of physically infinitesimal scales:

$$
l_{p h} \sim r_{D} \ll l, \quad \tau_{p h} \sim \frac{r_{D}^{2}}{D}, \quad D \sim v_{T} l .
$$

Here, as for a rarefied gas, three kinetic coefficient: diffusion $D$, viscous friction $\nu$, and thermal conductivity $\chi$, are equal. We see that now the connection between physically infinitesimal scales is defined by the "gasdynamical relation"

$$
\tau_{p h} \sim \frac{l_{p h}^{2}}{D}
$$

Thus, for a rarefied plasma there is a wide region of parameters (more wide than for a rarefied gases) for the unified description of kinetic and gasdynamical processes Now the role of the length $L_{\min }$ plays the Debye radius. 
The physical Knudsen number and the maximal usual Knudsen number for a plasma the following formulas are defined as:

$$
K_{p h}=\frac{l_{p h}}{L} \sim \frac{r_{D}}{L}, \quad\left(K_{n}\right)_{\max }=\frac{l}{r_{D}}=\frac{1}{\mu} \gg 1 .
$$

The last inequality opens a possibility for the unified description of kinetic and hydrodynamic processes in a rarefied plasma on scales $L>r_{D}$.

We have now enough information about the structure of a rarefied plasma as a continuous medium in order to construct the corresponding kinetic equations.

\section{RELATIVE ORDERING CRITERIA IN OPEN SYSTEMS $[1-5,12]$}

\section{A. Degradation and self-organization in evolution}

Evolution is the process of changes and development in nature and society. Worded in this manner, it is a very general concept. In physically closed systems, evolution in time results in the equilibrium state to which maximum entropy and the maximum degree of chaos correspond.

In open systems, it is possible to distinguish two classes of evolutionary processes:

(1) Development in time towards the nonequilibrium stationary state

(2) Evolution via a series of nonequilibrium stationary states of an open system. The latter process is due to variation of the so-called control (governing) parameters.

Darwin's theory of evolution is based on the principle of natural selection. Thus, evolution can either lead to degradation or represent a self-organization process during which more complex and sophisticated structures arise. Can selforganization be the unique outcome of evolution? The answer is negative because neither physical nor even biological systems display an "intrinsic drive" towards selforganization. Therefore, evolution may also lead to degradation. A physical example is evolution to the equilibrium state, the most chaotic one, according to Boltzmann.

Thus, selforganization is only one of the possible routes of evolution. Criteria for selforganization are needed to answer the question along which route a process will develop, but such fundamental concepts as degradation and selforganization are not necessarily to be defined. This is very difficult to do, laying aside the ambiguity of such definitions. Of much greater importance is the comparative analysis of the relative degree of order (or chaos) in different states of the open system being examined. Only such analysis can answer the question whether the open system undergoes selforganization or degradation.

We have already emphasized the concepts of chaos and order. Now, what distinguishes order from chaos?
There are cases when the difference between them is clear. However, it appears from the comparison of laminar and turbulent flows that a seemingly obvious inference may turn out to be incorrect. Quantitative criteria for the relative degree of order (or chaos) in different states of open systems allow a more reliable conclusion to be obtained.

The results of such analysis are objective and provide additional information which constitutes the basis for the establishment of the "norm of chaos" and helps to reveal two- side deviations from the norm under the influence of various impacts. In biology, for instance, all kinds of stress may cause deviations in the degree of chaos from the norm. Deviations on either side suggest "pathology", i.e. represent the process of degradation.

Therefore, a statement (based on a selected criterion) of the impaired degree of chaos does not necessarily mean that selforganization occurs and vice versa, an increase in the degree of chaos is not always identifiable with degradation. Such a conclusion is valid only for those physical systems where thermal equilibrium may be taken as the reference point for the degree of chaos. For example, in such, an open system as a generator of electrical oscillations, the equilibrium state is that of zero feedback when only thermal fluctuations exist in the electrical contour.

Since an organism normally functions only if a certain norm of chaos is available, corresponding to an essentially nonequilibrium state, the above reference point is nonexistent. This accounts for the lack of objective information about variation of the degree of chaos in biology as well as in economics and sociology which hampers distinguishing between self-organization and degradation in such systems.

However, another classification is equally relevant. The norm of chaos being determined, deviations on either side may be regarded as "pathology", i.e. degradation. Therefore, it is possible to monitor the choice of "therapy". Here, a criterion for the relative degree of order is at stake again. If the 'treatment' normalizes the state of the open system, in terms of this criterion, selforganization occurs. Otherwise, "therapy" leads to further degradation.

The difficulty in introducing the relative measure of order (or chaos, for that matter) in open systems is in the first place due to the absence of definitions for the initial concepts: chaos, order, degradation, and selforganization. It has already been mentioned that the definitions of these concepts are to a great extent arbitrary. We have just noted that the transition to a more chaotic state should not necessarily be regarded as degradation. It is essential to consider deviations from the norm of chaos.

In this context, it appears useful to consider the principal concepts at greater length in order to formulate the criterion for the relative degree of order without which the notions of degradation and self-organization actually remain void of meaning. 


\section{B. Physical and dynamical chaos}

Although "chaos" and "chaotic motion" are fundamental physical concepts, their precise definitions are lacking. according to Boltzmann, motion in an equilibrium state is most chaotic. However, motions far from equilibrium are also called chaotic. Such is the "motion" in noise generators intended for signals suppression.

Normally, different forms of turbulent motion in gases and fluids are also described as chaotic, e.g. turbulent motion in pipes which arises from laminar motion when the pressure difference at the ends of the pipe is sufficiently large. It seems natural to conceive turbulent motion as being more chaotic than laminar motion. However, such a view largely stems from the confusion of the concepts of complexity and chaos. Observation of turbulent motion primarily reveals its complexity, whereas additional analysis is needed to estimate the degree of chaos and appropriate criteria to quantify it.

Of late, the concept of "dynamical chaos" has been extensively exploited to characterize complex motions in relatively simple dynamic systems. The word "dynamic" implies the absence of fluctuation sources, that is sources of disorder.

For this reason, the "dynamic system" concept is a some- what idealized one. A more real chaotic motion, with random sources taken into account, might be called "physical chaos". An example is the chaotic motion of atoms and molecules in equilibrium. The mathematical notion of "dynamical chaos" can be traced back to the works of H. Poincare and A N Kolmogorov.

The main feature of dynamical chaos is dynamic instability of motion. It is expressed as strong (exponential) divergence of the originally close trajectories and leads to their mixing.

A major contribution to the investigation into the relationship between the dynamical and statistical descriptions of complicated motion was made by the prematurely deceased N.S.Krylov [13]. He was the first to raise the question of the role of dynamic instability and mixing as the basis of statistical physics in his posthumously published book Works on the Foundations of Statistical Physics (1950).

\section{Constructive role of dynamic instability of motion}

Relatively simple dynamic systems can give rise to very complicated motions perceived as chaotic. This was the reason for the introduction of such new concepts, as the strange attractor and dynamical (or determined) chaos.

As a rule, the word "chaos" has negative connotations in physics, biology and economics. However, the concept of chaos is a many-faceted one. For example, life can exist neither in complete chaos nor in perfect order. A normal organism needs a certain norm of the degree of chaos which can be estimated on the criterion of the relative degree of chaos.

Given an opportunity to measure the relative degree of chaos, the word requires no additional attributes. It is therefore appropriate to ask whether the term "dynamical chaos" is relevant. In fact, it was coined to characterize the complex states which arise from dynamic instability, i.e. exponential divergence of trajectories at a minor change of the initial conditions. However, this term is somewhat in conflict with the fact that the trajectories computed from dynamic equations can be reproduced based on the initial data in a numerical experiment. Moreover, dynamic instability can play a constructive role in the physics of open systems..

Indeed, dynamic instability of motion, i.e. exponential divergence of the trajectories leads to mixing of trajectories in phase space. This accounts for the possibility of smoothening at physically infinitesimal scales and introducing the concept of "continuous medium" to pass from reversible microscopic equations of motion for gas particles to irreversible kinetic and hydrodynamic equations for macroscopic functions of the continuous medium.

In this approach, the atomic structure of a system is taken in account to define "a point of continuous medium". This requires that physically infinitesimal time and length scales be defined as well as the corresponding physically infinitesimal volume which actually stands for the "point" of a continuous medium.

Naturally, it is desirable that such definitions agree with the definition of the mininum mixing region and the minimum time for the development of dynamic instability.

\section{Criterion for the relative degree of order. S-theorem}

Of all macroscopic functions, only entropy $S$ possesses a combination of properties that allow it to be used as a measure of uncertainty (chaos) in the statistical description of processes in macroscopic systems.

Boltzmann gave a statistical definition of entropy for both equilibrium and nonequilibrium (irreversible) processes and proved the famous $H$-theorem.

It states: The entropy of a system tending to an equilibrium state grows and remains unaltered after equilibrium is attained. According to Boltzmann, the degree of chaos monotonically increases during evolution and has the maximum value at equilibrium, entropy being a measure of uncertainty (chaos).

In this context, it is essential that during the evolution of a closed system to equilibrium in compliance with the Boltzmann equation, the average energy $\langle E\rangle$ remains constant. This means that fluctuations of energy are allowed. This to internal non-clossedness of the Boltzmann gas system. It is due to the existence of an infinite (in the thermodynamic limit) buffer of internal degree of freedom. Boltzmann's kinetic equation only contains a small 
fraction of total information about the system. However, conservation of average energy in course of evolution is not a common property of all kinetic equations.

It is only natural that the criterion for the relative degree of order needs to be universal. There is no reason it should be applicable only to a class of systems where average energy is conserved during evolution. Which route may lead to the solution of this problem?

Entropy being the sole function with the properties of a measure of chaos, there is but one option. It is necessary to redefine entropy so that the average energy remains constant in the course of evolution.

We shall consider the evolution of stationary states in open systems at slowly changing governing parameters. It is for this type of evolution, that the criterion for the relative degree of order in various states of open systems will be introduced below. This criterion was for the first time formulated by the author and called the "S-theorem".

Let us consider the evolution of stationary states of an open system at a varying control parameter $a$. Let us further assume the existence of two states with the control parameters $a=0$ and $a=a_{1}$, e.g. the stationary states of the Van der Pol generator at different values of the feedback parameter. Of course, the description of generation should take into account both current and charge fluctuations. Then, thermal fluctuations of the current and the charge in the electrical contour correspond to the former parameter value, when feedback is absent. The developed generation state, with the feedback parameter considerably exceeding the threshold, corresponds to the latter value.

In the general case, the degree of order of the distinguished states differs, which accounts for one of them being more chaotic than the other. Let us term it "physical chaos". As a rule, this state is nonequilibrium and more ordered than the equilibrium state. However, in the case of a generator with $a=a_{0}$, it coincides with the equilibrium state.

Let us denote the macroscopic characteristic of the stationary state as $X$. The role of $X$ for a generator can be played by the oscillation energy $E$. Let us further denote the distribution functions of two distinguished states as $f_{0}, f_{1}$ and the corresponding entropy values as $S_{0}, S_{1}$.

In the general case, there is no such notion as energy for an open system and only the effective energy can be introduced. It may just as well be termed the effective Hamilton function and denoted as $H_{\text {eff }}$. It is defined by the distribution function of the physical chaos state $H_{e f f}=-\ln f_{0}$ and as a rule vanishes with a change of the control parameter. For this reason, functions substituted by the corresponding new values $\tilde{f}_{0}, S_{0}$, if the entropy difference $\tilde{S}_{0}-S_{1}$ needs to be used as a measure of the relative degree of order in the distinguished states. These new values are determined from the equality condition for the examined states of the average effective Hamilton function.

When the physical chaos state coincides with the equi- librium state, as is the case with the Van der Pol generator, renormalization is carried out by substituting temperature $T$ by the new value $\tilde{T}$. It is determined ifrom the solution of the equation which describes the equality condition for the average effective Hamilton functions in the two states of interest. This equation has the form:

$$
\int H_{e f f} \check{f}_{0}\left(X, a=a_{0}\right) d X=\int H_{e f f} f_{1}\left(X, a=a_{1}\right) d X .
$$

Given the correct choice of the 'physical chaos state', the solution of this equation has the form:

$$
\tilde{T}(a) \geq T .
$$

The sign of equality is relevant at $a=a_{0}$, i.e. for the state of physical chaos. Evidently, the state "0" should be 'heated' to equalize average energies. As the comparison is now made at identical values of the average effective energy, the entropy difference $\tilde{S}_{0}, S_{1}$ can serve as a measure of the relative degree of order in the distinguished states.

The renormalized distribution function may be presented in the form of the canonical Gibbs distribution:

$$
\tilde{f}_{0}(X)=\exp \frac{F_{e f f}(\hat{T})-H_{e f f}(X)}{k_{B} \tilde{T}} .
$$

The corresponding expression for the BoltzmannGibbs- Shannon entropy is derived from the equation

$$
\tilde{S}_{0}=-\int \ln \left(\tilde{f}_{0}(X)\right) \tilde{f}_{0}(X) d X .
$$

Now, let us turn back to the equation (4.1). If the " 0 " state is coincident with equilibrium, its solution has the form (4.2), with $T$ standing for the temperature. In the general case, the " 0 " state, i.e. the state of physical chaos, is a nonequilibrium one. The distribution (4.3) includes effective temperature which is equal to unity for the physical chaos state. Therefore, the solution (4.2) should be written in the form

$$
\tilde{T}(a) \geq 1 \text {. }
$$

Here, the sign of equality also corresponds to the state of physical chaos. However, both effective temperature and free energy are now dimensionless. If the inequality (4.5) is valid at control parameter values $a>a_{0}$, the choice of the condition " 0 " in the form of physical chaos is correct. and the relative degree of order in the distinguished states is defined by the difference between the corresponding entropies.

Using the expression (4.3) for the distribution function $\tilde{f}_{0}(X)$ and the constancy condition for the average effective Hamilton function, the expression for the entropy difference may be presented as the inequality: 


$$
\tilde{S}_{0}-S=\int\left(\ln \frac{f_{1}(X)}{\tilde{f}_{0}(X)}\right) f_{1}(X) d X \geq 0 .
$$

on the condition that

$$
\left\langle H_{\text {eff }}\right\rangle=\text { const. }
$$

The known inequality $\ln a \geq 1-1 / a$ at $a=f_{1} / \tilde{f}_{0}$ is used to derive the formula (4.6).

To summarize, the result of computing the relative degree of order in the two distinguished nonequilibrium states is represented by two inequalities. One (4.5) confirms the correct choice of the "0" state as presenting physical chaos. Given the opposite inequality, physical chaos would be represented by the state " 1 ". The formula (4.6) provides a quantitative measure of the relative degree of order in the distinguished states.

The above calculations were made for the case of one a parameter. When several control parameters are available, it is possible to optimize the search for the most ordered state.

Let us now apply the " $S$-theorem" to estimate the relative degree of order upon the transition from laminar to turbulent flow.

\section{E. Relative ordering of laminar and turbulent flows $[2,3,14]$}

Calculations based on the $S$-theorem allowed the general results $(4.5),(4.6)$ to be specified for the case of transition from the laminar flow in a pipe to the steady state turbulent flow.

It will be clear from the forthcoming discussion that laminar flow may be assumed to represent the state of physical chaos. The role of the effective Hamilton function is played by the average kinetic energy of the laminar flow. For the equality of this energy in both laminar and turbulent flows to be true, the laminar flow should be "warmed up":

$$
k_{B} T_{l a m}=k_{B} T_{\text {turb }}+\frac{m}{3}\left\langle(\delta u)^{2}\right\rangle \geq k_{B} T_{l a m} .
$$

The temperature difference is defined by the sum of squared diagonal elements in the Reynolds stress tensor. Reynolds' stress representing collective degrees of freedom, the equality (4.8) may be interpreted as indicating that a part of thermal (chaotic) motion is replaced by the collective degrees of freedom during the transition from laminar to turbulent flow. This justifies the choice of the Reynolds stress tensor as the order parameter of the turbulent flow.

The result (4.6) which in the present case defines the relative degree of order in laminar and turbulent flows has the form:

$$
T\left(S_{l a m}-S_{t u r b}\right)=\frac{m n}{2}\left\langle(\delta u)^{2}\right\rangle \geq 0 .
$$

Thus, the entropy of the turbulent flow is lower than that of the laminar one. This implies a higher degree of order in the turbulent flow. Here, the role of the control parameter is played by the pressure difference at the ends of the pipe. At its zero value, the fluid is in an equilibrium state characterized by the maximum degree of chaos. This is another important example of a physical system in which the equilibrium state is taken as the reference point for the degree of chaos. One more example is the Van der Pol generator.

When the pressure difference is other than zero, all states are better ordered. This adds weight to the argument, in accordance with what is said in Section 4.4, that the transition from laminar to turbulent flow is an example of the selforganization process. However, this does not mean that the degree of order grows monotonically with increasing Reynolds number.

A higher degree of organization of the turbulent motion compared with that of the laminar one is also apparent as demonstrated below.

The momentum transfer between layers in a laminar flow is mediated through a molecular mechanism which consists in independent changes of momenta of individual gas particles.

Conversely, in the case of a turbulent flow, the momentum transfer from one layer to another is a collective process. It other words, individual disorganized motion in a laminar flow changes, upon transition to the turbulent flow, into collective (hence, more organized) motion.

This results in the turbulent viscosity coefficient being much higher than the corresponding parameter for a laminar flow.

A higher degree of order in turbulent motion is also confirmed by calculations of entropy production.

\section{F. Estimation of the relative degree of order from experimental data}

Practical application of the S-theorem implies that the effective Hamilton function is known. It is easy to find provided a mathematical model of the process in question is available. In many cases, however, there are no adequate mathematical models for open physical systems. This problem is even more complicated as far as biological, social, and economic entities are concerned.

Therefore, it is sometimes necessary to be able to determine the relative degree of order in open systems directly from experimental data. This can be achieved in the following way:

1. By selecting control parameters for a given system, e.g. two states of the system with control parameters $a_{0}$ and $a_{0}+\Delta a$.

2. By experimentally obtaining sufficiently long temporal realizations for the chosen values of the governing para- meters.

$$
X_{0}\left(t, a_{0}\right), \quad X\left(t, a_{0}+\Delta a\right) .
$$


These data are loaded into a computer and used to construct the corresponding distribution functions:

$$
f_{0}\left(X, a_{0}\right), \quad f\left(X, a_{0}+\Delta a\right) .
$$

The two distributions are normalized to unity.

Further operations are as above.

\section{G. Diagnosis of medico-biological objects $[3,12]$}

Let us consider some applications of the $S$-theorem for the purpose of medico-biological diagnostics. Investigations into this problem were initiated in Kiev and Moscow in 1990, using both mathematical models and experimental data. In 1994, the first results of the analysis of cardiograms based on the $S$-theorem were obtained by the joint efforts of biologists and clinicians in the Laboratories of Nonlinear Dynamics at the Saratov and Potsdam Universities.

Biological experiments reported by T. G. Anishchenko from Saratov university revealed significant differences in the responsiveness of male and female rats to the noise stress. Biochemical studies have demonstrated opposite changes in the behaviour of the two sexes. This finding provided the basis for a study of men and women's behaviour in response to stress. The evaluation was also made using the $S$-theorem.

Two cardiograms were obtained from each subject included in the study, one before and the other after identical stress impact (a shrilly acoustic signal).

Two cardiograms being available from each subject, this allowed a change in the relative degree of order to be individually estimated using the $S$-theorem. The experiment has demonstrated opposite changes in the degree of order in men and women, the former showing a decreased degree of chaos, while in the latter it increased.

In both cases, there was a deviation from the "norm of chaos" suggesting "pathology". It is for physicians to decide which "disease" is more dangerous.

The return to the "norm of chaos" may be spontaneous. Then, the "recovery" occurs unaided, with time serving as the control parameter.

If the patient's conditions are normalized by drug therapy, its efficacy is possible to evaluate using the same criterion.

Naturally, each doctor has his (her) own criteria unrelated to the $S$-theorem. However, it may be equally useful to take advantage of the additional objective information derived from the analysis of the relative degree of order as described above.

\section{WHAT IS SELF-ORGANIZATION? [12]}

Two classes of systems were outlined in a previous Section.
One of them includes many physical systems exemplified in the foregoing discussion by two cases. To begin with, it is a Van der Pol generator in which losses (of electrical resistance) are first compensated as the feedback parameter grows while its further rise results in the transition to the developed generation region. According to the $S$-theorem, this is a case of selforganization. This process starts from equilibrium, that is, thermal fluctuations in an electrical contour in the absence of feedback. This leads to the conclusion that the process of selforganization may be defined as the transition from a most chaotic (equilibrium) state to a more ordered one (generation).

The situation is similar in the transition from laminar to turbulent flow in a pipe with increasing pressure difference (a higher Reynolds number).

Here, the reference point for the degree of chaos is also the equilibrium state of a fluid in the absence of pressure difference, that is at the zero control parameter. In this case, hydrodynamic motion is lacking and only chaotic motion of molecules occurs. Evidently, this state is most chaotic.

Again, the process of self-organization is the transition from a more chaotic to a less chaotic state. Is this the universal definition of self-organization? It can be inferred from the previous section that the process of self-organization is not necessarily associated with an increase in the degree of order.

Indeed, there is a broad class of systems (in the first place, biological systems) for which neither the state of complete chaos (thermodynamic equilibrium) nor that of ideal order can be realized. Biological systems would not function under such conditions.

A more fundamental notion for such systems is the "norm of chaos" which has been used more than once in the previous discussion. This notion is compatible with that of "health". Then, selforganization is the process of reconvalescence.

Now, let us turn back to the studies on the responsiveness of men and women to stress. Earlier, we have agreed to regard post-stress conditions as "pathology". This means that the transitions to the "norm of chaos" in women is actually the "recovery" referred to above as selforganization, i.e. the transition from a more chaotic to less chaotic state.

Conversely, the stress-induced state for men is "illness" which corresponds to a more ordered state.

Hence, the "recovery"(self-organization)for men is the transition from an ordered state to a more chaotic one.

Thus, the concepts of self-organization and degradation in biological systems cannot be unequivocally related to an enhanced (self-organization) or impaired (degradation) degree of order respectively.

A more fundamental notion for such systems is the "norm of chaos" which can be estimated from empirical data using the ' $S$-theorem'.

To summarize, it appears from the above analysis that in certain cases selforganization is easy to observe, e.g. 
the generation developing in a Van der Pol system with an increasing feedback parameter. Other well-known examples are the appearance of a new structure (Benar cells) at the liquid surface heated from below and Taylor vortices between rotating coaxial cylinders. Using the most fortunate term "dissipative structures" coined by I Prigogine, the selforganization process may be described as the spontaneous occurrence of structures in nonlinear dissipative open systems, e.g. temporal dissipative structures in the Van der Pol generator and spatial dissipative structures exemplified by the Benar cells and Taylor vortices. Elimination of the control parameter (feedback, temperature gradient, etc.) in all these cases results in a "system at rest", i.e. one in the state of thermodynamic equilibrium.

Such understanding of the term "self-organization" underlies the theory of formation of dissipative structures. The first systematic exposition of this range of problems has been given in the well-known book of I Prigogine and G. Nicolis. The starting point was Prigogine's ideas on thermodynamics of irreversible nonequilibrium processes.

Haken's theory of self-organization is based on the appearance of structures due to collective interactions. In other words, cooperative processes are posited as being of primary importance. This prompted $\mathrm{H}$. Haken to use the term "synergetics" for this new interdisciplinary field of research.

In more complicated cases such as transition from one turbulent motion to another, in biological systems, it is possible to distinguish between the processes of degradation and self-organization based on the criterion " $S$ theorem". In such cases, the understanding of selforganization as the appearance of new structures or the transition from less to more ordered states becomes insufficient.

This inference is valid for all systems in which the equilibrium state can not serve as the reference point for the relative degree of chaos (or order). Here, the "norm of chaos" concept is of greater importance and, in the general case, certainly applies to the nonequilibrium state, with the transition from "pathology" to "health" corresponding to self-organization. Since deviation from the norm is possible in two directions (towards a greater or smaller degree of chaos), the self-organization process may in the general case also proceed in two directions.

Therefore, the traditional definition of self-organization as the spontaneous formation of structures in dynamic nonlinear dissipative open systems is too "narrow". A more comprehensive description of selforganization processes, even their mere identification, is feasible by the methods of the statistical theory of open systems.

The term "self-organization" is actually rooted deep in ancient thought. This is a very interesting question worthy of illustration by the following facts.

In 1966, the book on "Principles of Self-Organization" was published in the Russian language. It is a collection of reports delivered to a Symposium at the Illinois State
University, USA, in 1961. Here is a quotation from the Preface to the Russian edition by A Lerner, the editor:

"Despite the marked prevalence of self-organizing systems and persistent attempts of scientists to understand the phenomena occurring in such systems, selforganization has in a way remained for many centuries perhaps the most mysterious phenomenon, the most intimate of nature's secrets". The Preface goes on to state: "... the reader will hardly find here a report which would not claim to disclose the mystery of selforganization".

Heinz von Foerster, the editor of the American publication, writes in the Introduction with reference to a story by Plato, a famous Greek philosopher:

"The house of Agathon was the place where the first memorable symposium was held on the problems lying at the junction of different sciences, attended by philosophers, statesmen, dramatists, poets, sociologists, linguists, doctors and students learning various trades".

The report by Y. Eshby, a known expert in the field, contains a statement to the effect that the word "selforganization" can also mean "transition ifrom bad to good organization", even though the author does not explain how to distinguish between "bad" and "good". An approach to this problem is illustrated by the abovementioned analysis of cardiograms which allowed to differentiate between "health" and "pathology". Such a distinction is also possible based on the above criterion for the relative degree of chaos in different states of open systems.

Naturally, there are more diagnostic criteria to evaluate the state of biological systems. However, the comparison of different diagnostic tools is a matter which requires special attention.

\section{PHYSICS OF OPEN SYSTEMS FOR SOCIOLOGISTS AND ECONOMISTS}

H. Haken has reported one of the earliest applications of synergetics to sociology. The expedience of applying synergetics for this purpose is due to the important role of collective effects in social processes. Specifically, they are to a great extent involved in shaping public opinion even though separate acts of choice are, by necessity, individual. A model survey of social systems was carried out by the group of $W$. Weidlich who suggested simple models for the description of the formation of public opinion, population migrations, and urbanization.

At present, methods of synergetics are extensively employed to simulate economic processes. Economics is an ancient science with deeply rooted traditions and advanced methods of qualitative and quantitative description of various processes. Nevertheless, there is a wealth of unresolved problems challenging the physics of open systems to apply its methodology in this field.

A substantial part of these problems is related to the optimization of the relationships between production, 
distribution, and consumption based on the criterion for the relative degree of order in open (social or economic) systems. Such an approach may hopefully provide additional information necessary to monitor the efficiency of the assumed control parameters, to estimate the "norm of chaos', and to "treat the disease", that is a deviation from the 'norm of chaos' on either side.

In case of spontaneous "recovery", i.e. without interference from the outside ("medication"), "reconvalescence" may also be regarded as a self-organization process. Certainly, similar to the situation with biological systems, the equilibrium state cannot serve as the reference point in estimating the relative degree of chaos in social and economic systems. Only the state corresponding to the "norm of chaos" may be used for this purpose. Identification of such a state is the principal task which can be accomplished using criteria for the relative degree of order in physics of open systems.

A few years ago, G. Cagliotti [15], an Italian investigator, published a popular-science book under the title of Dynamics Ambiguity. One of the first pages in the book reads as follows: "A study of perception may reveal integrating factors. That is, originally disordered sensory stimuli become correlated and organized in the brain into ordered coherent structures which are then converted to a thought". In other words, the transition from perception to idea is the transition from a less to more ordered state of the brain.

True, this is a very beautiful picture of the birth of an idea. The question is how close it is to reality. The book gives no answer since it does not consider the criteria for a relative degree of order in open systems which would allow for the distinction between 'order' and 'chaos'. Doubtless, some information about the modulation of orderliness accompanying the generation of an idea can be obtained from the analysis of brain activity using encephalograms and the above criteria from the physics of open systems, specifically the $S$-theorem.

Such an approach implies a series of experimental studies designed to elucidate "the thought production rate", its difference in men and women, the influence on artistic performance, etc. Naturally, joint efforts of specialists representing different scientific disciplines are necessary to solve such a difficult problem.

Finally, all this his brings to mind the famous book of Erwin Schrodinger What is Life? published in English in 1944 and in Russian in 1947 . We shall refer to only one fragment directly related to the present discussion.

Very interesting statement which Schrodinger makes in the final chapter of his book is worth citing:

"An organism's astonishing gift of concentrating "a stream of order" on itself, and thus escaping the decay into atomic chaos of "drinking orderliness" from a suitable environment seems to be connected with the presence of the "aperiodic solid", the chromosome molecules, which doubtless represent the highest degree of wellordered atomic association we know of - much higher than the ordinary periodic crystal...".
This is truly a remarkable thought, but it cannot be considered here at greater length. Suffice it to answer the following question: "Is the degree of order in an aperiodic crystal higher than in an usual periodic one, in terms of the above theory?" There is every reason to argue that the answer must be in the affirmative!

Indeed, there is an analogy with the relative degree of order for laminar and turbulent flows. It seems natural to identify a laminar flow with a periodic crystal and a turbulent one with an aperiodic crystal. The thermal atomic motion in periodic crystals may be assumed to represent the state of physical chaos. Hence, collective degrees of freedom in aperiodic crystals are of greater importance than in periodic ones. This suggests, in conformity with the S-theorem, a higher degree of order in an aperiodic crystal than in a periodic one. This inference, however, remains to be quantitatively confirmed.

\section{KINETIC DESCRIPTION OF THE SECOND ORDER PHASE TRANSITIONS}

In this part the kinetic method of physics of open system for description of phase transitions in ferroelectrics is considered [16].

\section{A. Kinetic equation}

Let us $X(R, t)$ is the local value of a relative displacement of crystal lattices of a ferroelectrics. In the Landau theory for the nonsymmetrical phase it shall be play the role of the local order parameter.

Following the Landau theory [17-20] we introduce the effective Hamilton function. For the ferroelectrics model the effective Hamilton function $h_{\text {eff }}$ for the one particle is defined by expression $[21,16]$

$$
h_{e f f}=\frac{m \omega_{0}^{2}}{2} X^{2}\left[\left(1-a_{f}\right)+\frac{1}{2} b X^{2}\right] .
$$

$m$ is the mass of atom, and $m \omega_{0}^{2}$ is the hardness, and $\omega_{0}$ is the proper frequency of the atoms vibration. The parameter $a_{f}(T)$ defines the influence of the Lorentz effective field on the hardness. This parameter depends on the temperature. In the region of the critical point, following the Landau Theory this dependence is defined by the expression

$$
1-a_{f}=\frac{T-T_{c}}{T_{c}}
$$

Here $T_{c}$ is the critical temperature.

To obtain a qualitative representation about the behavior of the system for all temperature it is possible to represent the function $a_{f}(T)$ by the following expression:

$$
1-a_{f}=\tanh \frac{T-T_{c}}{\Delta T} .
$$


Here $\Delta T$ is the "temperature width" of the transition region..The parameter $b$ characterizes the role of nonlinearity.

For kinetic description of the second order phase transitions we shall be to use the kinetic equation for a distribution function $f(X, R, t)$ not only internal variable $X$, but also and coordinates $R$ of bistable elements $[3,4,16]$ :

$$
\frac{\partial f(X, R, t)}{\partial t}=\frac{\partial}{\partial X}\left[D_{(x)} \frac{\partial f}{\partial X}+\frac{1}{m \gamma} \frac{\partial h_{e f f}\left(X, a_{f}\right)}{\partial X} f\right]+D \frac{\partial^{2} f}{\partial R^{2}}
$$

It contains two dissipative terms, which are determined by redistribution of bistable elements in space $R$ with the diffusion coefficient $D$, and redistribution in the space $X$ with the diffusion coefficient $D_{(x)}$, accordingly.. For simplicity we assume lower that two diffusion coefficients are equal. The equilibrium solution of the kinetic equation for the homogeneous distribution coincides with the Boltzman distribution

$$
f_{B}\left(x, a_{f}, T\right)=\exp \frac{\psi\left(a_{f}, T\right)-h_{e f f}\left(x, a_{f},\right)}{k_{B} T} .
$$

The Hamilton function $h_{\text {eff }}$ depends on temperatures and, as consequence, the additional thermodynamic "thermal force" appears:

$$
F_{t h}(T)=-\int \frac{\partial H_{e f f}(X, T)}{\partial T} f\left(X, a_{f}, T\right) d X .
$$

This leads to the change of the thermodynamic relations. In particular, the known Helmholtz relation has now the form

$$
\frac{\partial F(T)}{\partial T}=-S_{e f f}(T)-F_{t h}(T) .
$$

Here $F(T)$ is the Gibbs free energy, the effective entropy $S_{\text {eff }}(T)$ for all system is defined by the Boltzmann distribution $f_{B}\left(X, a_{f}, T\right)$.

\section{B. Thermodynamic functions in Landau theory}

In the Landau theory thermodynamic functions are defined through the most probable values $X_{m . p}$. for the distribution function

$$
X_{m . p .}=0 \quad \text { a } \quad \tau \geq 0 ; \quad X_{m . p .}= \pm \sqrt{|\tau| b} \quad \text { a } \quad \tau \leq 0 .
$$

In the critical point there is a place the jump of heat capacity:

$$
\Delta C=\frac{1}{2} k_{B} N \frac{1}{X_{T_{c}}^{2} b}>\frac{1}{2} k_{B} N, \quad X_{T_{c}}^{2}=\frac{k_{B} T}{m \omega_{0}^{2}} .
$$

We see, that in the Landau theory the peak of the function $C(T)$ is absent. For the Boltzmann distribution the heat capacity is defined not by the Boltzmann entropy, but by the thermal force. Thus the Helmholtz relation is replaced by the following expression:

$$
\frac{\partial F\left(x_{m . p .}, T\right)}{\partial T}=-F_{t h}\left(x_{m . p .}, T\right), \quad S_{\text {eff }}\left(x_{m . p .}, T\right)=0 .
$$

The Boltzmann entropy on this level on the description is not taking into account.

\section{The first-moment approximation. Monodomain state}

On a basis of the kinetic equation we can obtain the chain of equations for moments $\left\langle X^{n}\right\rangle$. In the selfconsistent approximation, when $\left\langle X^{n}\right\rangle=\langle X\rangle^{n}$, we receive closed equation for the first moment $\langle X\rangle$. In the approximation the distribution function $f(X, R, t)$ is possible to represent in the form:

$$
f(X, R, t,)=\delta(X-X(R, t,)), \quad\langle X\rangle=X(R, t)
$$

The kinetic equation is reduced in this case to the reaction-diffusion equation for first moment - the function $X(R, t)[20,22]$ :

$\frac{\partial X(R, t)}{\partial t}=-\frac{\omega_{0}^{2}}{\gamma}\left[\frac{T-T_{c}}{T_{c}}+b X^{2}(R, t)\right] X(R, t)+D \frac{\partial^{2} X(R, t)}{\partial R^{2}}$.

It differs from the corresponding Ginsburg-Landau equation by the structure of dissipative terms.

With the help of this equation it is possible to describe, for example, the structure of domain walls in ferroelectrics In the stationary one-dimensional state $(R$ is parallel $y$ ) the solution is defined by the expression:

$$
X(y)=-\left\langle\frac{T_{c}-T}{T_{c} b}\right\rangle^{\frac{1}{2}} \tanh \frac{y}{d}
$$

The width of a wall is defined by the formula:

$$
d=X_{T}\left(2 \frac{T_{c}}{T_{c}-T}\right)^{\frac{1}{2}}, \quad X_{T}^{2}=\frac{k T}{m \omega_{0}^{2}}
$$

The ratio of the thickness of the wall $d$ to the "amplitude" at $X(y=-\infty)$ is defined by the expression

$$
\frac{d}{X(y=-\infty)}=\left(2 X_{T}^{2} b\right)^{1 / 2} \frac{T_{c}}{T_{c}-T} .
$$

At approach to a critical point this ratio increase by the Curie law. In the critical point this solution loses sense. On the basis of the kinetic we can evaluate this ratio and in a critical point. 
In order to describe kinetic fluctuations, we shall use the kinetic equation with the Langevin source. The intensity of a noise is defined by a sum of "reaction" and diffusion terms.

By solving this linearized equation (7.12)for region $T>$ $T_{c}$, we get the following expression for space-time spectral density of fluctuations $\delta X(R, t)$ :

$$
(\delta X \delta X)_{\omega, k}=\frac{2\left(\gamma_{(x)}+D k^{2}\right)}{\omega^{2}+\left(\gamma_{(x)}+D k^{2}\right)^{2}} \cdot \frac{1}{n}\left\langle(\delta X)^{2}\right\rangle .
$$

We used here the designations for the "reaction" dissipative coefficient $\gamma_{(x)}$ at $T>T_{c}$ and for dispersion $\left\langle(\delta X)^{2}\right\rangle$ for the Boltzmann distribution in Gaussian approach

$$
\gamma_{(x)} \frac{T-T_{c}}{T_{c}} \frac{\omega_{0}^{2}}{\gamma},\left\langle(\delta X)^{2}\right\rangle=\frac{k_{B} T}{m \omega_{0}^{2}} \frac{T_{c}}{T-T_{c}} \equiv X_{T}^{2} \frac{T_{c}}{T-T_{c}} .
$$

In the expression (7.16) on the equal rights are taking into account both dissipative terms. In the consequence of this the spatial density of fluctuations and the spatial correlation functions are defined by formulas:

$(\delta X \delta X)_{k}=\frac{1}{n}\left\langle(\delta X)^{2}\right\rangle ;\langle\delta X \delta X\rangle_{R, R^{\prime}}=\frac{1}{n}\left\langle(\delta X)^{2}\right\rangle \delta\left(R-R^{\prime}\right)$.

Thus the spatial correlation function is now not defined, as in the Landau theory, by the Ornstein-Zernike (OZ) formula. The dispersion $\langle\delta X \delta X\rangle_{R=R^{\prime}}$ differs from zero only in a volume of a point of continuous medium (in physically infinitesimal volume $V_{p h}$ ), as the function $\left.\delta\left(R-R^{\prime}\right)\right|_{R=R^{\prime}}=V_{p h}^{-1}$. For one-point correlator $\left\langle(\delta x)^{2}\right\rangle$ we have, in result, the expression:

$$
\langle\delta X \delta X\rangle_{R=R^{\prime}}=\frac{1}{N_{p h}}\left\langle(\delta X)^{2}\right\rangle
$$

Here $N_{p h}=n V_{p h}$ is a number of particles in a physically infinitesimal volume. Thus the dispersion of fluctuations are smoothed on a volume of "point" is in $N_{p h}$ times less, than for the Boltzmann distribution.

The $\mathrm{O}-\mathrm{Z}$ formula is connected now, not with the integral for the spectral line on frequency $\omega$, but with a spectral line for zero frequency $\omega=0$. After the integration over the wave number we get:

$(\delta X \delta X)_{\omega=0, r} \gamma_{(x)}=\frac{1}{n}\left\langle(\delta X)^{2}\right\rangle \frac{1}{2 \pi r_{c}^{2} r} \exp \left(-\frac{r}{r_{c}}\right) ; r_{c}^{2}=X_{T}^{2} \frac{T_{c}}{T-T_{c}}$

We see that the meaning of the formula $\mathrm{O}-\mathrm{Z}$ in theory of phase transitions is changed.

We can now to enter new correlation parameter $K$, which is analog to the Ginsburg parameter $G i$ :

$$
K=\frac{(\delta X \delta X)_{\omega=0, r_{c}} \gamma_{(x)}}{X_{m . p}^{2}} \sim \frac{X_{T_{c}}^{2} b}{n r_{c}^{3}}\left(\frac{T_{c}}{\left|T-T_{c}\right|}\right)^{2}
$$

In the kinetic theory it is possible to give the definition for the correlation parameter for all values of temperature (see below).

\section{APPROXIMATION OF THE SECOND MOMENT. POLYDOMAIN FERROELECTRICS}

Let us the system has the polydomain structure and the first moment $\langle X(R, t)\rangle=0$. In this case it is more natural to use the self-consisting approximation for the second moments $\left\langle X^{2 m}\right\rangle=\left\langle X^{2}\right\rangle^{n} \equiv\langle E\rangle$ which leads to the reaction-diffusion equation, but now for the function $\left\langle X^{2}\right\rangle \equiv\langle E\rangle \equiv E(R, t)$ we have the following equation:

$$
\frac{\partial E(R, t)}{\partial t} 2\left[D_{(x)}-\Gamma\left(\frac{T-T_{c}}{T_{c}}+b E(R, t)\right) E(R, t)\right]+D \frac{\partial^{2} E(R, t)}{\partial R^{2}} .
$$

The stationary solution for the homogeneous state is determined by the equation:

$$
\left\langle X^{2}\right\rangle+\frac{T-T_{c}}{T_{c} b}\left\langle X^{2}\right\rangle=\frac{X_{T}^{2}}{b}, \quad E(R, t) \equiv\left\langle X^{2}\right\rangle .
$$

For tree selected states:

$$
\left\langle X^{2}\right\rangle=\cdot \begin{cases}X_{T}^{2} \frac{T_{c}}{T-T_{c}} & T>T_{c} \\ X_{T}^{2}\left(\frac{1}{X_{T}^{2} b}\right)^{1 / 2} & T=T_{c} \\ \frac{T_{c}-T}{T_{c} b} & T<T_{c}\end{cases}
$$

We see that the second moment plays for the nonsymmetrical phase the role of the order parameter. In the critical point it has a finite value. Above the critical point the function $\left\langle X^{2}\right\rangle$ coincides with the dispersion for the Boltzmann distribution.

For the calculation of fluctuations for the approximation is considered in the kinetic we can carried out the replacement:

$$
\frac{1}{m \gamma} \frac{\partial h_{e f f}\left(X, a_{f}\right)}{\partial X} X \rightarrow \Gamma\left(\frac{T-T_{c}}{T_{c}}+b\left\langle X^{2}\right\rangle\right)
$$

As a result the kinetic equation has the form:

$$
\frac{\partial f}{\partial t}+\frac{\partial}{\partial X}\left[D_{(x)} \frac{\partial f}{\partial X}+\Gamma\left(\frac{T-T_{c}}{T_{c}}+b E(R, t)\right) X f\right]+D \frac{\partial^{2} f}{\partial R^{2} .} .
$$

Together with the equation (8.2) it consists the closed system of equations for function $f(X, R, t)$ and average energy $E(R, t)$.

We shall see that there are two type of fluctuations: "fast" and "slow". The fluctuations of energy are fast ones. To describe its we can use the closed equation (8.1) for energy's fluctuations. The slow fluctuations are described by the last equation with mean energy is determined by stationary equation (8.2). In this approximation the kinetic equation (8.5) can be represented in the form:

$$
\frac{\partial f}{\partial t}=\frac{\partial}{\partial X}\left[D_{(x)} \frac{\partial f}{\partial X}+\frac{D_{(x)}}{\left\langle X^{2}\right\rangle} X f\right]+D \frac{\partial^{2} f}{\partial R^{2}} .
$$


The equilibrium solution is the Gaussian distribution

$$
f(X)=(2 \pi\langle E\rangle)^{-1 / 2} \exp \left(-\frac{X^{2}}{2\left\langle X^{2}\right\rangle}\right) .
$$

Now we can proceed to the calculation of fluctuations.

\section{FAST AND SLOW FLUCTUATIONS AT PHASE TRANSITIONS}

The equation for the Fourier components of energy fluctuation $\delta E(R, t)$ is:

$$
\frac{\partial \delta E(\omega, k)}{\partial t}+\frac{1}{\tau_{(E)}} \delta E(\omega, k)=\delta y_{(E)}(\omega, k) .
$$

The relaxation time is defined by the following formula for all temperatures

$$
\frac{1}{\tau_{(E)}} \equiv \Delta_{(E)}(k)=2 \Gamma\left(\frac{T-T_{c}}{T_{c}}+2 b\left\langle X^{2}\right\rangle\right)+D k^{2}
$$

The average energy $\left\langle X^{2}\right\rangle \equiv\langle E\rangle$ is defined by the solution of the equation (8.2).

For calculation of a slow fluctuations we shall use the kinetic equation (8.6) with an appropriate Langevin source. In result, the equation for a Fourier components of the function $X(R, t)$ has the form:

$$
\left(-i \omega+\frac{1}{\tau_{(X)}}\right) X(\omega, k)=y_{(X)}(\omega, k)
$$

with the appropriate width of a spectral line:

$$
\frac{1}{\tau_{(X)}} \equiv \Delta_{(X)}=\frac{D_{(X)}}{\left\langle X^{2}\right\rangle}+D k^{2} \equiv \frac{D}{\left\langle X^{2}\right\rangle}\left(1+r_{c}^{2} k^{2}\right) .
$$

The appropriate response on the Langevin source $y(\omega, k)$ is defined by the expression:

$$
\chi_{(X)}(\omega, k)=\frac{1}{-i \omega+\frac{D}{\left\langle X^{2}\right\rangle}\left(1+r_{c}^{2} k^{2}\right)},
$$

and therefore

$$
\chi(X)(\omega=0, k=0)=\frac{\left\langle X^{2}\right\rangle}{D} \equiv \frac{1}{\Gamma} \frac{\left\langle X^{2}\right\rangle}{X_{T}^{2}} .
$$

All received characteristics can be determined in a critical region for all values of temperature including and the critical point.. Their behavior in a symmetrical phase qualitatively same, as and in the Landau theory. In the nonsymmetrical phase, however, its are essentially other from known ones.

The susceptibility increase on the Curie law only at approach to the critical point from the side of a symmetrical phase. At approach to critical point from the side of low temperatures the susceptibility decrease. It give the reason to speak about of existence of the "jump for susceptibility":

$$
\chi(X)\left|T_{c}-T \gg \Delta T-\chi(X)\right| T-T_{c} \gg \Delta T=\frac{1}{\Gamma} \frac{1}{X_{T_{c}}^{2} b} \gg \frac{1}{\Gamma} .
$$

Remark, that this formula is like to the one for the jump of the heat capacity in the Landau theory. Similar behavior of a dielectric susceptibility is observed in some kind of ferroelectrics. Exists also and the "jump of a relaxation time".

At last, the correlation radius is defined by the expression

$$
r_{C}^{2}=\frac{D}{\Gamma} \frac{\left\langle X^{2}\right\rangle}{X_{T}^{2}}=D \tau_{(X)}=\left\langle X^{2}\right\rangle .
$$

¿From it follows that exists and the " jump of square of a correlation radius".

So, the square of $r_{C}^{2}>X_{T}^{2}$ for all $T<T_{c}$ and, as consequence, for $T<T_{c}$ the spatial coherence exists.

For slow fluctuations the Ornstein-Zernike formula also take place only for the temporal correlator on zero frequency. This means, that in nonsymmetrical phase the interaction at temperature $T<T_{c}$ is collective. This result allows to enter the finite dimensionless correlation parameter for all values of temperature.

The heat capacity at the phase transition is defined by the formula

$$
C(T)=\frac{1}{2} k_{B} N \frac{T_{c}}{\left|T-T_{c}\right|},
$$

and in the critical point

$$
C\left(T=T_{c}\right)=\frac{1}{2} k_{B} N \frac{1}{\sqrt{X_{T}^{2} b}} .
$$

We see, that now the jump of the heat capacity is equal to zero and the function $C(T)$ is the symmetrical function of temperature with a finite value in the critical point.

Thus, for polydomain systems the jump has a place not for the heat capacity, but for a susceptibility and for characteristics is connected with it. The similar behavior was pointed characteristics observe on experiments.

\section{SHORT CONCLUSION}

We represented here some illustration of ideas, methods and results of the modern statistical theory of open systems - systems capable of exchanging matter, energy and information with the surrounding world. No doubt that the notions "norm of chaos" or "norm of order" were introduced above allow to differentiate the degradation and self-organization processes in Intelligent Systems and Semiotics [23,24].

REFERENCES 
1.Yu.L.Klimontovich. Statistical Physics (Harwood Academic Publishers, New York, 1986); ("Nauka", Moscow, 1982 (In Russian)).

2.Yu.L.Klimontovich. Turbulent Motion and the Structure of Chaos (Kluwer Academic Publishers, Dordrecht, 1991); ((in Russian) "Nauka", M.,1990).

3.Yu.L. Klimontovich. Statistical Theory of Open Systems (Kluwer Academic publishers, Dordrecht, 1995); ((in Russian) "Janus", M.,1995).

4.Yu.L. Klimontovich. Statistical Theory of Open Systems. Vol. II.

Part 1. Alternative approach in the Kinetic Theory of Plasma.

Part 2. Kinetic Theory of Phase Transitions.

Manuscript.

5.Yu.L. Klimontovich. Statistical Theory of Open Systems. Vol. III.

Physics of the Quantum Open Systems. (In work).

6.I.Prigogine. From Being to Becoming (Freeman, Sun Francisco, 1980).

7.I.Prigogine, I.Stengers. Order out Chaos (Heinemann, London, 1984).

8.H.Haken. Synergetics (Springer, Heidelberg Berlin New

York, 1978).

9 H.Haken. Advanced Synergetics (Springer, Heidelberg Berlin New York, 1983).

10.H.Haken. Principles of Brain Functioning (Springer, Heidelberg Berlin New York, 1996).

11.Yu.L. Klimomtovich. Physics of collosionless plasma. (Uspechi Fiz. Nauk 167 (1997) 23-55).

12.Yu.L. Klimomtovich. Relative ordering criteria in open systems (Uspechi Fiz. Nauk 166 (1996) 1231-1243).

13.N.S.Krylov. Works for the Foundations of Statistical Physics ("Nauka" Moscow, 1950 (in Russian)).

14.Yu.L. Klimomtovich. Is turbulent motion chaos of order? Is the hydrodynamic or the kinetic description of turbulent motion more natural? (Physica B 228 (1996) 51-62).

15.G.Cagliotti. Dynamics of Ambiguity (Springer, Heidelberg Berlin New York, 1992).

16. Yu.L.Klimontovich. Two alternative description of second order phase transitions -- the Landau and the selfconsistent approach.(Physics Letters A 210 (1996) 6570).

17.L.D. Landau and E.M. Lifshitz, Statistical Physics (in Russian) (Nauka, Moscow, 1976).

18.H.E. Stanley, Introduction to Phase Transition and Critical Phenomena (Clarendon Press, Oxford, 1971).

19.B. Strukov, .P.Levanuk. Physical Foundations of Segnetoelectrics Phenomena in Cristalls ("Nauka", Moscow,1995 (in Russian)).

20.A.Z. Patashinskii and V.L. Pokrovskii, Fluctuation Theory of Phase Transitions (Nauka, Moscow, 1982) (in Russian).

21.V.L.Ginzburg. (Sov.Phys.Uspekhi, 1949, v.38, p. $490 ; 1962$, v.77, p.621).
22.E.M.Lifshitz and L.P.Pitayevsky. Physical Kinetics ("Nauka", Moscow, 1979 (in Russian)).

23.M.Coombs, M.Sulcoski (Eds) Control Mechanism for Complex Systems. Las Cruces, New Mexico, 1996.

24.A.Meystel. Semiotic Modeling and Situation Analysis (AdRem, Inc, 1995). 


\title{
Non-Equilibrium Thermodynamics Explains Semiotic Shapes: Applications to Astronomy and to Non-Destructive Testing of Aerospace Systems
}

\author{
Roberto Osegueda, Carlos Ferregut, Mary J. George, \\ Jose M. Gutierrez, and Vladik Kreinovich \\ FAST Center for Structural Integrity of Aerospace Systems \\ University of Texas at El Paso, El Paso, TX 79968, USA \\ emails osegueda@utep.edu,ferregut@utep.edu,mjgeorge@utep.edu, \\ jmgutie@sandia.com, and vladik@cs.utep.edu
}

\begin{abstract}
Celestial bodies such as galaxies, stellar clusters, planetary systems, etc., have different geometric shapes (e.g., galaxies can be spiral or circular, etc.). Usually, complicated physical theories are used to explain these shapes; for example, several dozen different theories explain why many galaxies are of spiral shape. Some rare shapes are still difficult to explain.

It turns out that to explain these "astroshapes", we do not need to know the details of physical equations: practically all the shapes of celestial bodies can be explained by simple geometric invariance properties. This fact explains, e.g., why so many different physical theories lead to the same spiral galaxy shapes.

This same physical idea is used to solve a different problem: the optimal sensor placement for non-destructive testing of aerospace systems.
\end{abstract}

KEYWORDS: astrogeometry, semiotics, symmetry groups, non-equilibrium thermodynamics, non-destructive testing, aerospace structures

\section{SEMIOTIC SHAPES IN ASTRONOMY: FORMULATION OF THE FIRST PROBLEM}

From the computer viewpoint, an astronomical image is a set of pixels of different brightness. However, astronomers traditionally interpret these images in terms of certain geometric shapes, usually, described in semiotic terms (by words and symbols). For example, they talk about spiral or elliptical galaxies, etc. This language is very productive, because it enables astronomers to predict new results.
However, the very origin of these shapes remains somewhat a mystery.

To be more precise, there are several dozens theories that explain, e.g., the spiral galaxy shape (see, e.g., $[2,9,10]$ ), but the very fact that there are so many different theories for explaining the same observations probably means that the physical details involved in these theories are not needed, and these shapes can follow from fundamental principles.

In this paper, we show that that this is indeed the case: we can explain these shapes by using the fundamental physical ideas of symmetry and non-equilibrium thermodynamics.

\section{MAIN PHYSICAL IDEA}

The initial state of the Universe was highly symmetric. To find out how shapes have been formed, let us start from the beginning of the Universe (for a detailed physical description, see, e.g., Zeldovich and Novikov [13]). The only evidence about the earliest stages of the Universe is the cosmic $3 \mathrm{~K}$ background radiation. This radiation is highly homogeneous and isotropic; this means that initially, the distribution of matter in the Universe was highly homogeneous and isotropic. In mathematical terms, the initial distribution of matter was invariant w.r.t. arbitrary shifts and rotations.

We can also say that the initial distribution was invariant w.r.t. dilations if in addition to dilation in space (i.e., to changing the unit of length), we accordingly change the unit of mass.

In the following text, we will denote the corresponding transformation group (generated by arbitrary shifts $\vec{x} \rightarrow$ $\vec{x}+\vec{a}$, rotations, and dilation $\vec{x} \rightarrow \lambda \cdot \vec{x}$ ) by $G$. 
Dynamic equations are also symmetric. On the astronomical scale, of all fundamental forces (strong, weak, etc.) only two forces are non-negligible: gravity and electromagnetism. The equations that describe these two forces are invariant w.r.t. arbitrary shifts, rotations, and dilations in space. In other words, these interactions are invariant w.r.t. our group $G$.

The problem: our world should be symmetric, but it is not.

- The initial distribution was invariant w.r.t. $G$;

- the evolution equations are also invariant;

hence, we will get $G$-invariant distribution of matter for all moments of time. But our world is not homogeneous. Why?

Solution: spontaneous symmetry violation. The reason why do not see this homogeneous distribution is that this highly symmetric distribution is known to be unstable: If, due to a small perturbation, at some point $\vec{a}$ in space, density becomes higher than in the neighboring points, then this point $\vec{a}$ will start attracting matter from other points. As a result, its density will increase even more, while the density of the surrounding areas will decrease. So, arbitrarily small perturbations cause drastic changes in the matter distribution: matter concentrates in some areas, and shapes are formed. In physics, such symmetry violation is called spontaneous.

Non-equilibrium thermodynamics explains why perturbations usually preserve some symmetry. What kind of perturbations are possible? In principle, it is possible to have a perturbation that changes the initial highly symmetric state into a state with no symmetries at all, but statistical physics teaches us that it is much more probable to have a gradual symmetry violation: first, some of the symmetries are violated, while some still remain; then, some other symmetries are violated, etc. (Similarly, a (highly organized) solid body normally goes through a (somewhat organized) liquid phase before it reaches a (completely disorganized) gas phase.) At the end, we get the only stable shape: rotating ellipsoid.

This idea leads ot an explanation of all possible astroshapes. Before we reach the ultimate ellipsoid stage, perturbations are invariant w.r.t. some subgroup $G^{\prime}$ of the initial group $G$. If a certain perturbation concentrates matter, among other points, at some point $\vec{a}$, then, due to invariance, for every transformation $g \in G^{\prime}$, we will observe a similar concentration at the point $g(\vec{a})$. Therefore, the shape of the resulting concentration contains, with every point $\vec{a}$, the entire orbit $G^{\prime}(\vec{a})=\left\{g(\vec{a}) \mid g \in G^{\prime}\right\}$ of the group $G^{\prime}$. Hence, the resulting shape consists of one or several orbits of a group $G^{\prime}$.

\section{THE RESULT OF \\ PHYSICAL ANALYSIS: \\ DESCRIPTION OF ASTROSHAPES}

In view of the above analysis, to describe all possible shapes of celestial bodies, it is sufficient to describe all possible orbits of subgroups $G^{\prime}$ of the group $G$ (= all shifts, rotations, and dilations). In this paper, we will show that this description really describes all known astroshapes. (Some of these results were first announced in [3-5,6-8].)

A word of warning: geometric shapes are only approximate. Objects of nature can only approximately be described by geometric figures. Correspondingly, in our physical explanation, perturbations are only approximately invariant w.r.t. $G^{\prime}$. The farther away from the point $\vec{a}$, the less similar is the point $g(\vec{a})$ to the point $\vec{a}$. Therefore, in reality, we may observe not the entire orbit, but only a part of it.

Possible orbits. 0-, 1-, and 2-dimensional orbits of continuous subgroups $G^{\prime}$ of the group $G$ are easy to describe:

0 : The only 0 -dimensional orbit is a point.

1: A generic 1-dimensional orbit is a conic spiral that is described (in cylindrical coordinates) by the equations $z=k \rho$ and $\rho=R_{0} \exp (c \varphi)$. Its limit cases are:

- a logarithmic (Archimedean) spiral: a planar curve $(z=0)$ that is described (in polar coordinates) by the equation $\rho=R_{0} \exp (c \varphi)$.

- a cylindrical spiral, that is described (in appropriate coordinates) by the equations $z=k \varphi$, $\rho=R_{0}$.

- a circle $\left(z=0, \rho=R_{0}\right)$;

- a semi-line (ray);

- a straight line.

2: Possible 2-D orbits include:

- a plane;

- a semi-plane;

- a sphere;

- a semi-plane;

- a circular cylinder, and

- a logarithmic cylinder, i.e., a cylinder based on a logarithmic spiral. 
Possible orbits are exactly possible shapes. Comparing these orbits (and ellipsoids, the ultimate stable shapes) with astroshapes enumerated in VorontsovVeliaminov [12], we conclude that:

- First, our scheme describes all observed connected shapes.

- Second, all above orbits, except the logarithmic cylinder, have actually been observed as shapes of celestial bodies.

For example, according to Chapter III of VorontsovVeliaminov [12], galaxies consist of components of the following geometric shapes:

- bars (cylinders);

- disks (parts of the plane);

- rings (circles);

- $\operatorname{arcs}$ (parts of circles and lines);

- radial rays;

- logarithmic spirals;

- spheres, and

- ellipsoids.

The only orbit-originated shape that is not in this list is logarithmic spiral. It is easy to explain why logarithmic cylinder was never observed: from whatever point we view it, the logarithmic cylinder blocks all the sky, so it does not lead to any visible shape in the sky at all. With this explanation, we can conclude that we have a perfect explanation of all observed astroshapes.

Comment: we can also explain difficult-to-explain disconnected shapes. In the above description, we only considered connected continuous subgroups $G^{\prime} \subseteq G$. Connected continuous subgroups explain connected shapes.

It is natural to consider disconnected (in particular, discrete) subgroups as well; the orbits of these subgroups leads to disconnected shapes. Thus, we can explain these shapes, most of which modern astrophysics finds pathological and difficult to explain (see, e.g., Vorontsov-Veliaminov [12], Section I.3).

For example, an orbit $O$ of a discrete subgroup $G^{\prime \prime}$ of the 1D group $G^{\prime}$ (whose orbit is a logarithmic spiral) consists of points whose distances $r_{n}$ to the center forms a geometric progression: $r_{n}=r_{0} \cdot k^{n}$. Such dependence (called TitziusBode law) has indeed been observed (as early as the 18th century) for planets of the Solar system and for the satellites of the planets (this law actually led to the prediction and discovery of what is now called asteroids). Thus, we get a purely geometric explanation of the Titzius-Bode law.
Less known examples of disconnected shapes that can be explained in this manner include:

- several parallel equidistant lines (Vorontsov-Veliaminov [12], Section I.3);

- several circles located on the same cone, whose distances from the cone's vertex form a geometric progression (Vorontsov-Veliaminov [12], Section III.9);

- equidistant points on a straight line (VorontsovVeliaminov [12], Sections VII.3 and IX.3);

- "piecewise circles": equidistant points on a circle; an example is MCG 0-9-15 (Vorontsov-Veliaminov [12], Section VII.3);

- "piecewise spirals": points on a logarithmic spiral whose distances from a center form a geometric progression; some galaxies of Sc type are like that (Vorontsov-Veliaminov [12]).

Not only shapes can be this explained. This idea also explains relative frequency of different shapes, the directions of rotation and magnetic field, possible evolution of geometric shapes, etc. (see, e.g., [5]).

\section{ALTERNATIVE EXPLANATION: OPTIMIZATION UNDER UNCERTAINTY AND CORRESPONDING OPTIMAL SHAPES}

There is an alternative way of analyzing the shapes, that does not refer to physics at all, but is instead looking for the best approximations of (unknown) actual shapes.

If we use this idea, we face the problem of selecting the best family of images for use in extrapolation under an uncertain optimzality criterion. How can we solve this problem? It turns out that for every optimality criterion that satisfies the natural symmetry conditions (crudely speaking, that the relative quality of two image reconstructions should not change if we simply shift or rotate two images), the extrapolation shapes that are optimal with respect to this criterion can be described as orbits of the subgroups of the corresponding symmetry group.

As a result, we get exactly the shapes used in astronomy (such as spirals, planes, spheres, etc.) The details of this description are given in $[3,4]$.

\section{OPTIMAL SENSOR PLACEMENT FOR NON-DESTRUCTIVE TESTING OF AEROSPACE SYSTEMS: THE SECOND PROBLEM}

Testing is extremely important. Structural integrity is extremely important for airplanes, because in flight, the 
airframe is subjected to such stressful conditions that even a relatively small crack can be disastrous. This problem becomes more and more important as the aircraft fleet ages.

Sensors must be placed. At present, most airplanes do not have built-in sensors for structural integrity, and even those that have such sensors, do not have a sufficient number of them, so additional sensors must be placed to test the structural integrity of a airframe.

Each integrity violation (crack etc.) starts with a small disturbance that is only detectable in stressful in-flight conditions. Therefore, to detect these violations as early as possible, we should complement on-earth testing by in-flight measurements.

Optimal sensor placement: a problem. Sensors attached outside the airframe interfere with the airplane's well-designed aerodynamics; therefore, we should use as few sensors as possible. The problem is, given the number of sensors that we can locate on a certain surface of an airframe, what are the optimal placements of these sensors, i.e., locations that allow us to detect the locations of the faults with the best possible accuracy.

For future aircraft, we have a similar problem of sensor placement. The ideal design of a future airplane should include built-in sensors that are pre-blended in the perfect aerodynamic shape. Each built-in sensor is expensive to blend in and requires continuous maintenance and data processing, so again, we would like to use as few sensors as possible.

This optimality problem is difficult to formulate in precise terms. Both for aging and for the future aircraft, the ideal formulation of the corresponding optimization problem is to minimize the average detection error for fault locations. However:

- this ideal formulation requires that we know the probabilities of different fault locations and the probabilities of different aircraft exploitation regimes.

- In reality, especially for a new aircraft, we do not have that statistics, and for the aging aircraft, the statistics gathered from its earlier usage may not be applicable to its current state.

Therefore, instead of a well-defined optimization problem, we face a not so well defined problem of optimization under uncertainty. Since the problem is not well defined, we cannot simply use standard numerical optimization techniques, we must use intelligent techniques.

Geometric approach. The problem of choosing an optimal sensor placement can be formulated in geometric terms: we need to select points (sensor placements) on a surface of the given structure.

To solve this problem, we use the experience of solving similar symmetry-based geometric problems of optimization under uncertainty in image processing and image extrapolation (see above). Since the basic surface shapes are symmetric, a similar symmetry-based approach can be applied to the problem of optimal sensor placement. For the simplest surfaces such as planes, cones, etc., this general approach describes several geometric patterns that every sensor placements which is optimal with respect to reasonable (symmetric) optimality criterion must follow.

The use of neural networks. We then use neural networks:

- first, to confirm that these placement patterns indeed lead to better fault location, and

- second, to select a pattern that leads to the best results for each particular problem.

Discussion about the results. The resulting placements are different for different problems: For example,

- when we test on-earth, then our main goal is not to miss the crack; as long as we detected it, we can always perform additional measurements to determine its location with any desired accuracy.

- In flight, however, detecting the crack is not enough; in a fly-by-wire aircraft, we may need to adjust the control algorithm so as not to stress the faulty surface. For that, we need to know where exactly this fault is located.

Space structures: a similar problem. A similar problem of optimal placement of sensors for non-destructive testing can be formulated and solved for space structures.

Acknowledgment. This work was partially supported by NSF Grant No. EEC-9322370 and by NASA Grant No. NCCW-0089.

\section{REFERENCES}

[1] Arnold, V.I. Mathematical methods of classical mechanics, N.Y.: Springer, 1978.

[2] Binney, J. "Stellar dynamics", in: Appenzeller, I., Habing, H.J., and Léna, P. (eds.), Evolution of galaxies: astronomical observations, Springer Lecture Notes in Physics, Vol. 333, Berlin, Heidelberg, 1989, pp. 95-146. 
[3] Finkelstein, A., Kosheleva, O., and Kreinovich, V. "Astrogeometry, error estimation, and other applications of set-valued analysis", ACM SIGNUM Newsletter, 1996, Vol. 31 , No. 4, pp. 3-25.

[4] Finkelstein, A., Kosheleva, O., and Kreinovich, V. "Astrogeometry: towards mathematical foundations", International Journal of Theoretical Physics, Vol. 36, No. 4, pp. 1009-1020, 1997.

[5] Finkelstein, A., Kosheleva, O., and Kreinovich, V. "Astrogeometry: geometry explains shapes of celestial bodies", Geombinatorics, Vol. VI, No. 4, pp. 125-139, 1997.

[6] Kosheleva, O.M., and Kreinovich, V., Astrogeometry, or geometrical investigation of forms of celestial bodies, Technical Report, Center for New Information Technology "Informatika", Leningrad, 1989.

[7] Kosheleva, O.M., Kreinovich, V., and Finkelstein, A.M., "Group-theoretic approach to foundations of spacetime theory," in Proceedings of the Symposium on Global Geometry and Foundations of Relativity, Novosibirsk, 1982, pp. 76-78 (in Russian).

[8] Kreinovich, V. Referee's comments in a review of V. A. Dubrovin, Novikov, S.P., and Fomenko, A.T. Modern Geometry, Moscow: Nauka, 1980, Zentralblatt fur Mathematik, Vol. 433, pp. 295-297, 1981.

[9] Strom, S.E., and Strom, K.M. "The evolution of disk galaxies", Scientific American, April 1979; reprinted in Hodge, P.W. (ed.), The Universe of galaxies, N.Y.: Freeman and Co., 1984, pp. 44-54.

[10] Toomre, A., and Toomre, J. "Violent tides between galaxies", Scientific American, December 1973; reprinted in Hodge, P.W. (ed.), The Universe of galaxies, N.Y.: Freeman and Co., 1984, pp. 55-65.

[11] Thom, R. Structural stability and morphogenesis, Reading, MA: Benjamin Cummings, 1975.

[12] Vorontsov-Veliaminov, B.A. Extragalactic astronomy, Chur, Switzerland, London: Harwood Academic Publishers, 1987.

[13] Zeldovich, Ya.B., and Novikov, I.D. Relativistic Astrophysics. Part 2. The structure and evolution of the Universe, Chicago and London: The University of Chicago Press, 1983. 


\title{
Towards Computers of Generation Omega - Non-Equilibrium Thermodynamics, Granularity, and Acausal Processes: A Brief Survey
}

\author{
M. Koshelev and V. KReinovich \\ Department of Computer Science \\ University of Texas at El Paso, El Paso, TX 79968 \\ email mkosh@cs.utep.edu
}

\section{ABSTRACT}

Nowadays, we are using mainly computer of fourth generation, and we are designing fifth-generation computers. It is reasonable to ask: what is the perspective? What will the computers of generation omega look like?

- As the speed of data processing increases, we face a natural limitation of causality, according to which the speed of all processes is limited by the speed of light.

- Lately, a new area of acausal (causality violating) processes has entered mainstream physics.

This area has important astrophysical applications. In this paper, we show:

- how non-equilibrium thermodynamics makes these processes consistent,

- how these processes can be used in computations, and

- how the very possibility of these processes lead to the granularity of the physical world.

KEYWORDS: computer generations, granularity, non-equilibrium thermodynamics, quantum computing, acausal processes

1. GENERATIONS OF COMPUTERS: WE NEED FASTER AND FASTER COMPUTERS

No matter how fast modern computers are, there are still problems that take too much computational time and, thus, cannot yet be handled by modern computers. To solve these problems, we must design faster and faster computers. So far, the speed of the computers has been doubling every few years. Can we keep up with this increase?
According to special relativity, all velocities are bounded by the speed of light; thus, to make computer elements faster, designers try to decrease the size of these elements. Every hardware technology eventually reaches its limit, i.e., the smallest element size that this technology can achieve; after that, to decrease the size further, we need to invent a new technology. Computers that use this new technology are usually called computers of a new generation.

The existing 4th generation computers are based on VLSI technology. At the current speed-up rate, this technology will soon exhaust its potential. Physicists and engineers are therefore working on new technologies for fifth, sixth, etc., generations of computers. Vague ideas are proposed for technologies suitable for even further generations. (The further generation, the more vague the ideas.)

It is therefore desirable to get a clear view of the computers of the very distant future generations. We will call these computers generation omega after the notation "omega" $(\omega)$ for the first infinite ordinal number proposed by Cantor, the founder of set theory (the first consistent theory of infinite objects).

\section{COMPUTER GENERATIONS AND QUANTUM PHYSICS: GENERAL DE- SCRIPTION}

To get faster computers, we must decrease the size of the elementary processing elements.

As the size of an object decreases, quantum effects become more and more essential in its description: 
- for macro-size objects, quantum effects are rare (e.g., in lasers), small, and difficult to measure;

- in chemistry (which studies molecules) quantum effects are often important;

- for elementary particles, quantum effects are so overwhelming that their non-quantum description is practically impossible.

Therefore, as the size of the computer elements decreases, we need to take quantum effects into consideration to a larger and larger extent.

To take these effects into consideration, we must use quantum physics. In general, a physical theory describes how particles and fields interact in space-time. Therefore, in the ideal quantum physical theory, particles, fields, and space-time structures must be considered from the quantum viewpoint. In practice, the effects of their quantization is different, so, some of these quantum effects can often be neglected:

- the largest quantum effects are related to objects that have been known and analyzed for the longest time, i.e., particles;

- the next quantum effects are related to newer objects: fields;

- and finally, the smallest quantum effects are due to quantization of space-time physics, physics whose experimental effects are still on the edge of modern observation abilities.

The smaller the objects, the more effects we need to consider. At first, we have to use traditional quantum mechanics (also called first quantization), in which fields (and space-time structures) are described by non-quantum formulas, but the particles' quantum behavior is taken into consideration. This quantum mechanics describes atoms, quantum chemistry, etc. Modern engineering research into quantum dots as computer units and modern theoretical research into quantum computing, with its exciting potential ability of solving such hard-to-solve problems as factoring large integers (see, e.g., $[2,3,6,7,29,30]$ ), is at this quantization level.

- From the practical viewpoint, quantum dots will have a huge potential of further miniaturizing computers, so, if this project is successful, we will not need to worry about it for at least a few decades.

- However, from the fundamental viewpoint of a more distant future, we need to look further.

To describe even smaller objects, we need to use second quantization (or quantum field theory (QFT)), in which both particles and fields are quantized, and the fully quantized theory, in which space-time is quantized as well.

\section{ENTER ACAUSAL PROCESSES}

In general (curved) space-time, the maximum possible communication speed (i.e., the speed of light $c$ ) is determined by the metric tensor field $g_{i j}$; see, e.g., [15].

- In non-quantum theories, this field is smoothly depending on coordinates and therefore, the corresponding maximal speed is slightly changing in space and time (and is practically constant for small areas).

- Quantization of space-time means, in particular, that the metric tensor field undergoes quantum fluctuations, and, as a result, the actual maximal speed at any given point is randomly larger or randomly smaller than $c$. Since the average deviation must be 0 , this means, roughly speaking, that in half of the cases, the maximal possible speed is larger than $c$, and in half of the cases, it is $<c$.

An object of finite size is influenced by the "average" field in the area that this object occupies.

- If the object is large enough, then the random fluctuations "average out", and the object moves as if in a space where the maximal speed is the macro-world speed of light.

- However, if we consider much smaller objects, then these objects can actually feel the local fluctuations.

Therefore, if this tiny object moves (and transfers information) at a maximal local speed, and this local speed, due to a fluctuation, is larger than $c$, then we get a microobject that, without violating causality, is able to transfer information at a speed $v$ that is larger than the macro-level speed of light $c$.

The smaller the object, the larger this potential speed $v$ (it can be, actually, as large as possible).

As a result, we have an unexpected additional boost in computer performance:

- we considered smaller and smaller processing elements because the smaller these elements, the faster the computer;

- it turns out that if these elements are small enough to take into consideration full quantum theory, then not only their size gets smaller, but also the actual speed of communication transfer can be made faster than the macro-level speed of light; thus, computers become even faster. 


\section{ACAUSAL PROCESSES IN PHYSICS AND BIOLOGY: A BRIEF HISTORY}

Traditional physics is causal in the sense that future events are determined by the past state of the Universe. This dependence can be deterministic (as in classical, prequantum physics), or stochastic (as in quantum physics). There have been for some time an idea of the possibility of acausal processes, in which the influence can go in the opposite direction: future can influence the past. Such processes are called acausal.

The idea that the speed of all particles cannot exceed the speed of light (and that, therefore, it is impossible to influence the past) was one of the main ideas of Einstein's Special Relativity Theory.

In quantum mechanics, due to its probabilistic character, many deterministic restrictions of pre-quantum physics become somewhat "blurred" in the sense that they are no longer prohibiting some events completely, but simply telling that these formerly prohibited events have small probability. For example, in classical physics, a particle cannot penetrate the potential barrier if the energy of this barrier exceeds the initial energy of the particle; in quantum physics, however, it is quite possible (although not highly probable) that a particle "tunnels" through this barrier and end up on the other side of it. This is not simply a theoretical conclusion, this "tunnel effect" is the basis of "tunnel diodes" that are extensive used in nowadays electronics.

Uncovered possibility that quantum mechanics can make pre-quantum restrictions "soft" lead to a possibility that causality may also be violated in quantum processes. Such violations were first discovered by Einstein, Podolsky, and Rosen in their famous paradox (physicists call it EPR paradox for the first letters of the authors' names; for details, see [32]). Einstein, who was not a great fan of quantum mechanics, proposed this paradox as a way of disproving this theory. (It is worth noticing at this point that all experiments so far seem to confirm quantum mechanics.) EPR paradox does not lead to a real time travel: it simply shows that in the resulting quantum formalism, the future state influences the past one; however, all attempts to extract a real time travel from it turned out to be futile because, crudely speaking, the resulting influence on the past is so small that, when we try to measure it, it "drowns" in the inevitable quantum uncertainty of measurements.

This fact does not mean that causality is true in quantum physics. In the last decade, several more sophisticated schemes have been proposed that, in principle, can lead to the actual time travel [33-35].

In addition to physical arguments in favor of possible causality violations, there exist biological motivations for such processes: Rosen [25] suggests that the living beings can use physical processes that influence the current events depending on the future ones (he calls such acausal processes anticipatory; see also [24-28].

Until 1988, acausal processes has been mainly considered as one the many possibilities, not the most probable possibility, and not part of the mainstream physics. In 1988, the physicists' attitude to acausal processes changed when Kip S. Thorne, the world's leading astrophysicist, published several papers in the leading physical journal Physical Reviews in which he showed that within the existing quantum physics and cosmology, acausal processes are highly probable; these publications lead to several other serious research results $[1,9,17,18,20-22,31]$. As a result of this research, three basic types of acausal processes have been discovered; these processes are summarizes in Thorne's monograph [32] (for more popular expositions, see, e.g., $[4,5,12,13,19,23,36]$.

\section{PARADOXES OF ACAUSALITY AND HOW NON-EQUILIBRIUM THERMO- DYNAMICS CAN SOLVE THEM}

The idea of acausal processes was, for a long time, mainly part of science fiction, because this idea is paradoxical. The most well known father paradox is most convincingly described in terms of the actual time travel (travel to the past):

The time traveler paradox occurs when a time traveler goes to the past and shoots his own father to death before he himself was conceived. Then:

- On one hand, the time traveler is still alive, because he was alive before the killing, and he did not harm himself in any way.

- On the other hand, since his father has died, he could not have conceived the time traveler, and hence, the time traveler cannot be born. So, he is at the same time not alive.

A similar paradox occurs if we cannot actually travel to the past, only influence it; also, it occurs even if we have no human beings at all, simply physical processes. The reason why we (and other authors) present this paradox in its time-traveler form rather that in the form of differential equations of physics is that when we have a problem with differential equations, there can be many reasons for that (wrong equations, wrong method of solution, etc.), while the time-traveler paradox reveals the paradoxical character of acausal processes themselves.

For clarity of exposition, we will describe the current solution of the paradoxes of acausality on the same timetraveler example as we described the paradox itself. Of 
course, similar to the fact that the paradox occurs even when there is no time traveler at all, the described solution is also applicable to the case of purely physical acausal processes. This solution is described in $[8,10,11,14,16]$.

Since the time traveler is alive at the time when he starts the shooting, this means that he was conceived after all, and therefore, that his attempt to kill his father has failed. Why could it have failed? Well, the gun may have malfunctioned, or he might have missed, or a brick (or a meteorite) might have fallen on the time traveler's head at the very moment when he was ready to shoot, or a policeman of the past has stopped him, etc.

Some of these possibilities are quite realistic, some (like a meteorite) have an extremely low probability. The time traveler can prepare for some of these possibilities: he can check his gun before going to the past, use automatic weapons, wear a hard hat against falling bricks, a fake police uniform to prevent an interference of the past's police, etc. In principle, whatever possibility we describe, the time traveler can take care of it. However, he cannot take care of them all: for example, in principle, the gun can malfunction simply due to some unexpected (but probable) random Brownian motion of its molecules.

If the time traveler takes care of all possibilities with reasonable (sufficiently high) probability, this still leaves other possibilities, with extremely low probability, that normally do not occur, but that would have to occur because otherwise, we would have a paradox.

Summarizing: if an acausal process is possible, then some events will take place, whose probability is normally extremely low to prevent this acausal influence from happening. This conclusion is true not only for a time traveler, but for an arbitrary acausal process.

\section{COMPUTERS THAT USE ACAUSAL PROCESSES}

According to the above analysis, the very possibility of an acausal process leads to the implementation of highly improbable events. Let us assume that we have organized such an acausal process in such a way that if we switch it on, it will lead to an implementation of a highly unprobable event with a probability $p_{0} \ll 1$.

Let us show how this device can be used to solve a typical hard-to-compute problem of propositional satisfiability: given a Boolean (propositional) formula $F\left(x_{1}, \ldots, x_{n}\right)$ with $n$ Boolean variables $x_{1}, \ldots, x_{n}$, find the values (if any) for which the resulting formula is true. This problem can be easily solved by trying all $2^{n}$ possible combinations of $n$ "true" and "false" values. Unfortunately, this exhaustive search becomes non-feasible even for $n \approx 300$, when the resulting computation time exceed the lifetime of the Universe. It is known that this problem is computationally hard (the precise term is NP-hard) in the sense that if we can solve it in reasonable time (i.e., time bounded by a polynomial of $n$ ), then we would be able to solve all problems from a large class (called NP) in reasonable time, and this most computer scientists consider impossible.

To find the values $x_{i}$ using acausal processes, we can set up $n$ quantum random number generators that generate $n$ random bits. Then, we check whether the results $x_{1}, \ldots, x_{n}$ of these bits satisfy a given formula. If they do, these values are the desired answer; if they do not, we switch the above-mentioned acausal process on. Nature has two choices:

- It can generate the desired solution in the random generators. If the formula has only one satisfying combination of variables (out of $2^{n}$ ), the probability of this event is $2^{-n}$.

- It can also generate a vector that does not satisfy the given formula. In this case, the switched-on acausal process makes the nature implement the highly unprobable event, with probability $p_{0}$.

Therefore if $p_{0} \ll 2^{-n}$, it is much more probable that Nature will prefer the first alternative.

This idea was announced in $[11,14]$ and described in detail in $[10]$.

\section{ACAUSAL PROCESSES LEAD TO GRANULARITY OF THE PHYSICAL WORLD}

In the previous section, we applied the idea of acausal processes to computations. artificially designed computations. However, we can also apply it to nature itself.

- In traditional causal physics, whatever initial conditions $x\left(t_{0}\right)$ we set at the initial moment of time $t_{0}$, we can always integrate the equations and end up with the state of the Universe $x(t)=F\left(t, x\left(t_{0}\right)\right)$ for all consequent moments of time $t>t_{0}$ (here, the function $F$ describes the dynamics of the system). In this case, initial conditions are arbitrary and therefore, we have a continuous set of possible states.

- If there is an acausal process present, then the initial condition cannot be arbitrary: if, e.g., we have an acausal process that transforms a part $p(x(t))$ of a state at moment $t$ into a moment of time $t^{\prime}<t$, then, in addition to the dynamical equation that connects $x(t)$ and $x\left(t^{\prime}\right)$ with $x\left(t_{0}\right)$ must have an additional condition $p(x(t))=p\left(x\left(t^{\prime}\right)\right)$. Therefore, the initial condition $x\left(t_{0}\right)$ must satisfy the additional equation $p\left(F\left(t, x\left(t_{0}\right)\right)=p\left(F\left(t^{\prime}, x\left(t_{0}\right)\right)\right.\right.$.

How does an additional equation restricts the set of all possible conditions? For the case of one variable, a linear 
equation has a single solution, a quadratic equation has, in general, two solutions, etc. The more complicated the equations, the more granular is the set of its solutions. Since the dynamic equations are, usually, very complicated, we naturally expect that the additional equations caused by acausal processed lead to a high granularity of Universe.

\section{ASTROPHYSICAL APPLICATIONS OF ACAUSALITY}

A general idea of these applications. As we have mentioned, acausal processes lead to highly unprobable events. According to statistical physics, if Nature has a choice, it would rather prefer situations where these highly unprobable events do not occur. Therefore, if there is a random (statistical physics-type) process that can either lead to an acausal process or not, then, the actual probability of this process resulting in acausality is very low, much lower that it would have been if we did not take the possibility of acausal processes into consideration (practically, thermodynamically impossible).

In this paper, we only show this idea on one possible applications, whose description enables us to avoid technical details; other applications are also possible (see, e.g., $[8,11])$.

Example: The isotropization of the Universe. One of the main problems of modern cosmology (see, e.g., [32]) is that the Universe is too isotropic. On large scale, in all directions in which we look, we see the same statistical distribution of matter. The initial state of the Universe was, according to the modern physical viewpoint, random, and therefore, far from being isotropic. Hence, the observable isotropization is due to some physical processes. Many physical processes shuffle matter around and thus, contribute to the isotropization, but calculations show that during the lifetime of our Universe, these processes are not sufficient to explain the current isotropy; to be more precise, for random initial conditions, the probability of the initial conditions that lead to the observed isotropy is very low.

The explanation of this phenomenon in acausal physics is as follows: Anisotropy means that different distant areas of the Universe will have radically different matter densities. For acausal processes, there is no speed restriction; therefore, since there is an excess of matter in one area and abundance in another area, acausal processes will re-shuffle the matter from the dense area to the area where matter is scarce. Such a process is, as we have mentioned, thermodynamically unprobable and therefore, it is much more possible that the random initial conditions are chosen in such a way that prevents these acausal re-shufflings, i.e., that the initial conditions lead to the observable isotropic
Universe.

What this explanation does is shows that the probability of an initial state of the Universe leading to isotropization, the probability that is small if we do not take acausal processes into consideration, becomes much larger if we consider the possibility of acausal processes.

Acknowledgment. This work was partially supported by NSF Grant No. EEC-9322370 and by NASA Grant No. NCCW-0089. The authors are greatly thankful to Kip S. Thorne for his inspiring talks, papers, and books, and for his interest.

\section{REFERENCES}

[1] Allen, B., and Simon, J. "Time travel on a string", Nature, Vol. 357, May 7, pp. 19-21, 1992.

[2] Bernstein, E., and Vazirani, U. "Quantum complexity theory", in Proceedings of the 25th ACM Symposium on Theory of Computing, 1993, pp. 11-20.

[3] Berthiaume, A., and Brassard, G. "The quantum challenge to structural complexity theory", in Proceedings of the 7th IEEE Conference on Structure in Complexity Theory, 1992, pp. 132-137.

[4] Davidson, M. "Is time travel possible?" USA Today (Magazine), pp. 64-67, Jan. 1990.

[5] P. Davies, "Wormholes and time machines," Sky \& Telescope, pp. 20-23, Jan. 1992.

[6] Deutsch, D. "Quantum theory, the Church-Turing principle, and the universal quantum computer", Proceedings of the Royal Society of London, Ser. A, Vol. 400, pp. 96-117, 1985.

[7] Deutsch, D., and Jozsa, R. "Rapid solution of problem by quantum computation", Proceedings of the Royal Society of London, Ser. A, Vol. 439, pp. 553-558, 1992.

[8] Dimitrov, V., Koshelev, M., and Kreinovich, V. "Acausal processes and astrophysics: case when uncertainty is non-statistical (fuzzy?)", BUlletin for Studies and Exchanges on Fuzziness and its AppLications (BUSEFAL), No. 69, pp. 183-191, 1997.

[9] Gott, J. R. "Closed timelike curves produced by pairs of moving cosmic strings: exact solution", Physical Review Letters, Vol. 66, pp. 11.26-1129, 1991.

[10] Kosheleva, O.M., and Kreinovich, V. "What can physics give to constructive mathematics," in Mathemati- 
cal Logic and Mathematical Linguistics, Kalinin, 1981, pp. 117-128 (in Russian).

[11] Kreinovich, V., and Mignani, R. "Noncausal quantum processes and astrophysics", Bolletino della Societá Italiana di Fisica, Vol. 112, August 29, p. 88, 1977.

[12] Lemonick, M.D. "How to Go Back in Time", Time, May 13, p. 74, 1991.

[13] Macvey, J.W. Time Travel: a guide to journey in the forth dimension, Chelsea, MI: Scarborough House, 1990.

[14] Maslov, S.Yu. Theory of deductive systems and its applications., Cambridge, MA: MIT Press, 1987.

[15] Misner, Ch., Thorne, K.S., and Wheeler, J.A. Gravitation, San Francisco: W.H. Freeman, 1973.

[16] Moravec, H. Time travel and computing,

Carnegie-Mellon University, Computer Science Department, Preprint, 1991.

[17] Morris, M.S., and Thorne, K.S. "Wormholes in spacetime and their use for interstellar travel: a tool for teaching general relativity", American Journal of Physics, Vol. 56, May, pp. 395-412, 1988.

[18] Morris, M.S., Thorne, K.S., and Yurtzever, U. "Wormholes, time machines, and the weak energy condition", Physical Review Letters, Vol. 61, pp. 1446-1149, 1988.

[19] Nahin, P. Time machines: time travel in physics, metaphysics, and science fiction, American Institute of Physics, N.Y., 1993.

[20] Novikov, I.D. "Analysis of the operation of a time machine", Soviet Physics JETP, Vol. 68, pp. 439-443, 1989.

[21] Novikov, I.D. "Time machines and self-consistent evolutions in problems with self-interaction", Physical Review $D$, Vol. 45, pp. 1989-1994, 1992.

[22] Ori, A. "Rapidly moving cosmic strings and chronology protection", Physical Reviews D, Vol. 44, pp. 2214$2215,1991$.

[23] Parker, B. "Tunnels through time", Astronomy, June 1992, pp. 28-36.

[24] Rosen, R. "Church's thesis and its relation to the concept of realizability in biology and physics", Bull. Math. Biophysics, Vol. 24, pp. 375-393, 1962.
[25] Rosen, R. Anticipatory systems, N.Y.: Pergamon Press, 1965.

[26] Rosen, R. In: Herken, R. (ed.) The universal Turing machine: a half-century survey, Hamburg: Kammerer \& Unverzagt, 1988, pp. 523-537.

[27] Rosen, R. Life itself: a comprehensive inquiry into the nature, origin, and fabrication of life, N.Y.: Columbia University Press, 1991.

[28] Rosen, R. "What can we know?", In: Casti, J.L., and Karlqvist, A. (eds.), Beyond belief: randomness, prediction, and explanation in science, Boca Raton, FL: CRC Press, 1991, pp. 1-13.

[29] Shor, P.W. "Algorithms for quantum computations: discrete logarithms and factoring", in Proceedings of the 35th Annual Symposium on Fundamentals of Computer Science (FOCS), 1994, pp. 124-134.

[30] Simon, P. "On the power of quantum computation", in Proceedings of the 35th Annual Symposium on Fundamentals of Computer Science (FOCS), 1994, pp. 116-123.

[31] Thorne, K.S. "Do the laws of physics permit closed timelike curves?", Annals of the New York Academy of Sciences, Vol. 631, pp. 182-193, 1991.

[32] Thorne, K.S. From black holes to time warps: Einstein's outrageous legacy, N.Y.: W.W. Norton \& Company, 1994.

[33] Tipler, F.J. "Rotating cylinders and the possibility of global causality violation", Physical Review D, Vol. 9, pp. 2203-2206, 1974.

[34] Tipler, F.J. "Causality violation in asymptotically flat space-times", Physical Review Letters, Vol. 37, pp. 879$882,1976$.

[35] Tipler, F.J. "Singularities and causality violation", Annals of Physics, Vol. 108, pp. 1-36, 1977.

[36] Travis, J. "Could a pair of cosmic strings open a route into the past?", Science, 1992, April 10, pp. 179-180. 
XIV

SEMIOTICS OF OPEN SYSTEMS 


\title{
'Seeing' the Battlefield through Virtual and Constructive Simulation
}

\author{
Ken Hunt \\ Mounted Maneuver Battlespace Lab \\ Ft. Knox, KY 40121-5000
}

\begin{abstract}
Determination of terrain-based decision drivers operative in enabling the tactical commander 'to see' the battlefield represents a critical objective for soldier training as well as implementation of autonomous land traverse systems. At process level, the effort unveils obtained waypoints in the development dynamic for knowledge evolution. Based on manipulation of distance factors among maneuver (combatant) elements and terrain features in a mission execution context within a simulations arena, an aggressive methodology is uncovered for extracting participatory knowledge elements, and a differentiation scheme is disclosed for realizing (reporting) pattern-element significance applicable in tactical decision development. A resource is suggested for detailed analysis of 'deep' decision factors and their scheme of application in land traverse and tactical maneuver situations.
\end{abstract}

KEYWORDS: tactical decision logic, virtual simulation, constructive simulation, JANUS, Distributed Interactive Simulation (DIS)

\section{INTRODUCTION}

To 'see' the battlefield represents a critical capability for the tactical commander as well as, in today's technologically advanced culture, for autonomous systems intended to maintain pace with or substitute for manned land traverse systems. To 'see' the battlefield is to grasp not just the presence and location of unfolding elements in an immediate field of view but, for the tactical commander, to appreciate the relevance of near and remote terrain features for tactical advantage and identify zones of potential action and denial areas for enemy activity. It is to mentally prepare for emerging events by identifying the utility of successive terrain features to achieve reduction of risk, extended optimization of element positioning, and expeditious accomplishment of the mission.
The projected research explores the elements of awareness implicit in 'seeing' the battlefield under alternative tactical conditions.

In approaching decision logic elements for 'seeing' the battlefield, the projected effort turns to simulation capabilities for circumstance creation and observation of battle commander behaviors imposed during review of displayed evolving events. In this combination, the research discloses a methodology for using simulation to identify 'deep significance' among terrain details for tactical maneuver and soldier preparation, and specifically, focuses interest on the impact of providing virtual-simulation terrain detail to tactical commanders and staff during JANUS constructive-simulation exercises (see system description below), while probing the relevance of entity (terrain feature or tactical element) separations for situational-awareness during those exercises. The interaction of JANUS constructive-simulation technology with DIS (Distributed Interactive Simulation) virtual simulation lies at the heart of the investigative approach.

\section{SYSTEM DESCRIPTIONS}

The JANUS model is a man-in-the-loop, forceon-force, stochastic model which provides schematic representation of the battlefield to unit staff members who control the action from individual display consoles. Terrain representation is limited to contour indicators overlaying a latitude-longitude grid and shaded with colored areas indicative of vegetation, water, and urban development. Roads, rivers, obstacles, and man-made features are portrayed, as are tactical vehicle icons. Terrain elevation profiles along single selected axes can be displayed successively in a superimposed window. 
Virtual imagery at the Battlefield Distributed Simulation - Developmental (BDS-D) facility offers an out-the-window representation of tactical and environmental features in a man-inthe-loop, force-on-force context. Battlefield entities are portrayed in a contextually accurate fashion relative to size, range, orientation, intervisibility, and relative spatial separation. Displayed fields of view and entity state modifications are operator controlled through representational mechanisms reflecting actual user equipment.

Despite the JANUS capability of portraying profile arrays along selected terrain azimuths in a superimposed display window, staff training with such resources provides no direct view of the relevant terrain from selected viewing points (vehicles). This denies appropriate staff officers terrain-use details potentially beneficial to the operation, and limits investigation of tactical decision logic to broad force-maneuver concerns exclusive of terrain-characteristics-driven tactical movement issues. Through linkage of constructive schematic displays and virtual tactical arrays in a mission execution context, analysis of the 'deep significance' of confrontational and cooperative terrain details for tactical maneuver and soldier preparation is operationalized.

\section{EXPERIMENTAL CONCEPT}

Through linked virtual and constructive simulation technologies including mannedsimulators, semi-automated force generators (ModSAF systems), and JANUS workstations to represent an indirect-fire-supported, staffsupported mounted maneuver element advancing toward a designated objective, exploration of knowledge elements operative in 'seeing' the battlefield will be undertaken at the Mounted Warfare Test Bed (MWTB), Ft Knox, Ky. Staff officers at JANUS workstations representing 'global' tactical events and augmented with virtual terrain imagery synchronized for current battlefield conditions and provided through imagery transmissions from manned-simulator-based maneuver elements will direct maneuver and fire support activities for an advancing armor force, while vehicle commanders in manned tank-simulators with access to schematic JANUS arrays participate as maneuver elements. In each exercise, the maneuver force supported by ModSAF-generated additional support units (an artillery firing battery) for tactical realism will advance over European terrain as JANUS-based staff officers (a battalion commander, an operations officer [S3], and a fire support officer [FSO] ) direct tactical support for the mission.

\section{Tactical Context.}

With poor threat intelligence, BLUFOR (i.e., the friendly maneuver force) will advance across a well-vegetated hilly European environment, maintaining maximum use of concealment and relying primarily on indirect fire (artillery) to respond at threat encounters. The remotely located BLUFOR battalion staff will provide axis-of-advance and fire-support information to the BLUFOR maneuver commander, designating determined terrain advantages for route selection and indirect-fire attrition zones from provided displays. BLUFOR vehicle commanders will fashion and execute micromaneuvers based on JANUS and virtual representations, disclosing across scenarios, terrain-interpretation strategies for local maneuver demands. BLUFOR will encounter OPFOR [i.e., opposing force or Threat] at selected, test-directorate-controlled BLUFOR/OPFOR distances from appointed terrain features, to assess 'distance participation' as a contextual factor in decision logic. Interest in why an action is selected based on aggregated 'distance' factors, as opposed to how an action is performed, underlies the inquiry.

\section{Execution.}

Each scenario will be performed twice, once with virtual and constructive screenaugmentation at respective locations and once without. Training effectiveness and decision logic will be explored under each condition in the series of scenarios portraying alternative inter-feature and inter-combatant distances as primary decision-drivers. Achievement of this disclosure will be done by comparing BLUFOR leader-directives developed with JANUS schematics (regarded as aggregates of distances, locations, and features), and those provided with JANUS schematics synchronized with virtualarray 'intervention elements' descriptive, in an 'aspected' array, of spatially serial features to be 
dealt with (disruptive [obstacles] and desirable [concealment] ) on the represented terrain array (battlefield). 'Seeing the battlefield', currently addressed through relevant feature/combatant separations, will be examined through directorate-imposed distances among display elements. (OPFOR elements will emerge from selected hidden start-points under the direction of test directorate personnel to create BLUFOR decision circumstances consistent with study objectives. BLUFOR display options will reflect associations of BLUFOR remoteness-to-terrainfeatures relative to OPFOR-remoteness-toadditional-[or the same]-features, and will serve as the basis for knowledge-element characterization effective in bringing about commander decisions.) Performance will be examined for the 'intervention logic' applied at the S3, the battalion commander, the FSO, and the maneuver commander duty positions. Training enhancements will be assessed through performance comparisons across early and late trials with both JANUS and JANUS-augmented display data. With OPFOR elements reflecting ground and air units moving along single, multiple, and air corridors in separate events, with various delays, distances, and locations relative to BLUFOR, at BLUFOR threat observation, data loggers will capture objective data for a variety of tactical circumstances.

\section{Player Functionality.}

Battalion Staff. BLUFOR battalion staff personnel will direct maneuver and fire direction in geographically similar but tactically diverse operational situations. Battalion commanders will formulate orders positioning friendly forces against observed threat elements based in part on 'intervention' configurations; S3s will fabricate traverse and kill-zone guidelines, also from 'intervention' configurations ('traverse logic'); and FSOs will fashion fire support and kill-zone alternatives again in part from 'intervention' logic. From displays descriptive of designated distances among terrain features and combatant forces, staff personnel will exercise the option (decision) to apply or bypass terrain features for tactical advantage, and investigators will be provided a metric (distance at which circumstance-import changes from beneficial to detrimental) by which interpretation strategies can be specified. Early-late comparisons of identical tactical configurations will address training benefits associated with display augmentation.

Vehicle Commander Operations. Maneuver commanders serving as a platoon leader and his 'wingmar' will advance using maximum concealment (to enhance survivability) on a designated objective in each of the tactical scenarios. At threat observation, BLUFOR initially will request indirect fire to suppress the OPFOR and will maneuver to achieve terrain advantage for a possible subsequent direct fire engagement. Commanders again will use 'intervention' details provided in virtual and JANUS displays to select and execute micromaneuvers, and will disclose, through display options, the relevance of pertinent distance factors for movement decisions. This will provide investigators additional metric insights for interpretation strategies at the site level of maneuver operations.

\section{PROJECTION}

Assessment of terrain-detail significance for tactical maneuver and soldier training through simulation technologies and controlled stimulation events unveils an exciting and robust procedure for 'deep significance' determination at multiple levels of concept formation and in diverse operational circumstances. Though currently structured for distance relevance extraction in platoon maneuvers, the present application, and each succeeding application, essentially serves as a solitary designation in the larger requirementsdetermination quandary for autonomous behavior, and conveys receptivity to easy reconfiguration, by echelon, combat activity, battlefield resources, support activity, threat activity, or other decision drivers, to assess distance relevance impacted by additional factors in platoon/unit maneuvers. The significance of abundant sub task-level decisions which necessarily underpin mission performance, and the importance and complicity of seat-specific, soldier decision logic and input, which again underlie duty performance, also are highlighted in the approach.

Given the methodology as a convincing solution for extracting and characterizing significance/ 
meaning from observed circumstances, the approach, through combined virtual and constructive simulation application, ultimately provides the critical element of authenticity to the development of significance-measures, essentially through analysis in a mission execution context. In capturing such measures in this manner, a broad operational context is allowed to function as backdrop and source of serial and spatial depth for more effective assessment of proximal and distal significance operators. 


\title{
TVA's Kingston Unit 9 Distributed Control System (DCS) Retrofit Benefit Documentation
}

\author{
Dennis Linkins, TVA Technology Advancement \\ David Lewis, TVA Kingston Fossil Plant \\ Rob Frank, EPRI I\&C Center \\ Joe Weiss, EPRI
}

Many utilities have been contemplating a control system retrofit for their fossil power plants. However, in many cases, the benefit justification has been lacking. Consequently, EPRI and TVA formed a tailored collaboration project to quantify the benefits of implementing a distributed control system (DCS) retrofit in a 1955-vintage coal-fired power plant. TVA's Kingston Fossil Plant Unit 9 was the selected unit for the project. A comprehensive benchmarking program was established before the changeout and is continuing. This paper provides a complete detailing of the retrofit and the documentation of benefits to date. Benefits documented to date include reduced emissions (NOx and $\mathrm{CO} 2$ ), heat rate improvement, reduced unburned carbon, improved dispatch capability, maintenance cost reduction, and other factors. To date, heat rate, unburned carbon, dispatch improvements and NOx reduction appear to be significant.

\section{BACKGROUND}

Fossil plant operation is changing. This includes the move from base load operation to cycling operation and meeting the emission requirements of the Clean Air Act Amendments. Many utilities are implementing Distributed Control System (DCS) retrofits to meet these new challenges. Many other utilities have been seeking cost/benefit data to justify a control retrofit. To date, most of the economic justification for control system retrofits has been heat rate improvement. However, a DCS should be able to provide other benefits including improved plant reliability/availability, improved dispatch response, reduced emissions, salability of wastes, and more. Additionally, since control retrofits usually occur during an outage when other improvements/modifications also are being performed (e.g., turbine modifications), it has been difficult to estimate the direct benefits from the control retrofit. The purpose of this project was to provide a comprehensive benchmarking of control retrofit benefits including all potential plant improvements. Kingston Unit 9 is a 200 MW, CE-tangential-fired, twin-furnace boiler; a typical 1950's vintage coal-fired plant with electric analog controls which is currently being dispatched by Automatic Generation Control (AGC).

\section{APPROACH}

EPRI and TVA studied the feasibility of retrofitting all nine of the Kingston plant control systems with DCSs. The feasibility study included evaluating the existing instrumentation, actuators, and plant support systems as well as the control system. EPRI Controls Retrofit Guidelines were used in the feasibility study. Subsequent to the feasibility study, it was decided to retrofit only one of the units (Unit 9) and compare the benefits of the retrofit to the other eight non-retrofitted units.

\section{SCOPE OF WORK}

As a result of a study, several TVA Task Teams identified the need for a state-of-the-art control system upgrade at the Kingston Fossil Plant. Through a Tailored Collaboration (TC) agreement, TVA and EPRI planned for the 
installation of a full Distributed Control System (DCS) for Kingston Unit 9, the I\&C Center to be established at the Kingston Plant, the installation of a high fidelity simulator within the center for training and advanced control system algorithm development, and the documentation of benefits as a result of the retrofit. Another project deliverable was the detailed documentation of the project benefits.

\section{PROJECT DESCRIPTION}

The project was done in three phases. The Phase I study was performed by Gilbert Commonwealth under contract with TVA's Fossil Engineering Services (FES) group. Overall project management for Phase I was provided by TVA's Technology Advancement (TA) organization which at the time was called Research \& Development. The objective of Phase I was to develop a conceptual state-of-the-art design basis and cost estimate. Phase I was completed in February 1994. The Phase I study provided a detailed scope of work and an estimate for Phase II. Phase II was the detailed engineering and design of the project and procurement of the DCS system. During this phase of the project, specifications were prepared by the project team for the DCS, simulator and long-lead items. The DCS contract and a contract for design were awarded to Foxboro. Detailed design was performed and long-lead items were procured during this phase. During Phase III, the DCS upgrade was performed on Unit 9, the unit was commissioned and testing was performed to gather the data needed to document the benefits.

The Unit 9 controls upgrade consisted of a complete renovation of the control room and the installation of a CRT-based control room environment. The bench and vertical boards, including the annunciators, were removed and the floor openings filled. The control room was redesigned to accommodate the CRT concept. All field sensors, actuators, and several of the valves were replaced, including field cables. Flame scanners, high energy ignitors, and a new oil supply system were installed to provide additional safety and turndown capability. Secondary air damper actuators were installed to provide the capability to adjust the fuel-air ratio at the burner nozzle area for $\mathrm{NO}_{\mathrm{x}}$ control. The DCS system was designed to include network communications with the TVA network to facilitate access to the unit performance and statistical data from the plant network. An EPRI Performance Monitor Workstation (PMW) was installed to provide realtime performance evaluation for the unit. The majority of phase III activities were completed by the time the unit was restarted in December 1995. The post baseline test was performed to observe the improvements due only to the DCS upgrade. In addition to the baseline test a series of parametric tests were performed to begin optimizing the unit. An experimental design was used to determine the combinations of conditions for NOx optimization testing.

The EPRI I\&C Center was established during this project. Construction of the I\&C Center was completed in August of 1995. The I\&C Center was dedicated on February 29, 1996. The new simulator was available for operator training during the Unit 9 outage for the installation of the new controls, and all Unit 9 operators went through a 5 week training course prior to startup. The simulator is housed in the I\&C Center building.

\section{PROJECT RESULTS}

Listed below are the major results of the DCS retrofit on Kingston Unit 9. These benefits were primarily derived by two methods; baseline testing and comparative before and after operational data. There were three baseline test performed. The first test performed was a true baseline in that no retrofit work or pulverizer improvements had been implemented prior to the test. The second baseline was performed after extensive fuel balancing work and pulverizer blueprinting work had been performed. The final baseline test was performed after the controls retrofit was completed. The benefits listed here are the benefits obtained solely from the DCS retrofit and independent of other plant modifications (e.g., pulverizer and turbine modifications), i.e. the delta between the second and final baseline test. 
- The heat rate has improved by 250 BTU/KWH as a result of the controls retrofit due in part to the following:

- a $1.47 \%$ boiler efficiency improvement

- an LOI reduction from $\sim 6 \%$ to $\sim 3 \%$

- improved steam temperature controls

- a reduction in station service usage

- reduced excess air (enabled by better O2 control)

- closed loop primary air control.

- This project demonstrated that this unit and similar ABB CE units can be retrofitted with a DCS and tuned to reduce NOx emissions by approximately $25 \%$.

- Due to tighter steam temperature controls, the Plant Forced Outage Rate is projected to be reduced by one forced outage per year.

- This project has demonstrated that Unit 9 with the new DCS is capable of regulation at $3 \%$ per minute or better over a $50 \%$ load range. Prior to the retrofit, Unit 9 was limited to $1 \%$ per minute.

- Improved steam temperature controls could allow the unit to return to steam temperatures of $1025{ }^{\circ} \mathrm{F}$ from $1000^{\circ} \mathrm{F}$, while providing greater protection from excursions and reduced boiler and turbine damage. There are current negotiations with the plant operations staff to raise these steam temperature setpoints.

- The unit carbon loss (LOI) was reduced from $6 \%$ down to $3 \%$ This is a measure of the improvement in the combustion process and the addition of primary air controls on the pulverizers.

- Startup time for Unit 9 has been reduced by at least 2 hours due to improved ignitors and lowload temperature controls.

- Velocity induced wear on fuel system components from pulverizer to burner nozzle was reduced by $35 \%$ because of reduced primary air flow.

- The environmental emissions have been reduced by improving the unit heat rate and burning approximately 8,000 tons less coal per year on Unit 9.

- The life of the Unit 9 has been extended by replacing the 40 year old field cables and other plant equipment.

\section{PROJECT BENEFITS}

The benefit details are shown in the Table 1, and indicate the planned vs. actual benefits. Benefits on a $\$ /$ Year basis are shown. The benefits listed are based on an $80 \%$ capacity factor and a $15 \%$ discount rate. Some of the actual cost numbers are derived from financial figures deemed confidential by the TVA and cannot be disclosed.

\section{LESSONSLEARNED}

Listed below are the major lessons learned which came out of this project:

\section{The Project Team}

This team was responsible for budget, schedule, and overall coordination of project activities. This project was managed much differently than most TVA projects. A Project Team was established and reported to the Kingston Plant Manager. The project was managed with a site perspective instead of the usual central office Design Engineering approach. The Project team selected was directly involved in preparation, review, award and management of equipment and construction fixed price contracts. Most members had a strong instrument or controls background.

The Project Team approach was very successful in controlling the project and resolving problems as they arose. The project was completed utilizing fixed price contracts and completed on schedule with almost no scope changes. A design package has been completed to be used for future DCS retrofits. This will reduce the cost considerably for design, construction, and implementation of similar projects in the future. The continuity of responsibilities achieved by a single party being responsible for design, equipment supply, construction and startup of the unit was a very valuable asset.

\section{Plant Ownership}

This project has clearly demonstrated the value of plant involvement in every step of the project from conception and justification to design, 
construction, startup and benefits documentation. The level of involvement at Kingston was from the plant manager through the entire staff including Trades and Labor personnel who assisted in the checkout and startup.

\section{Operations and Graphics Design}

Plant operators designed the pictorial graphics that were used on Unit 9 and the Simulator. The use of existing operations staff to build the Data Acquisition-type graphics fostered considerable ownership of the project from the operations personnel at the site. Operators were selected on a voluntary basis for the initial start-up and subsequent operation of the new system Operators were encouraged to comment on graphics being developed and suggestions were implemented when appropriate. This gave operators ownership and satisfaction in being part of the "design."

\section{Pulverizer Primary Air Flow Measurement And Control}

Install primary air flow measuring and control equipment on all $\mathrm{CE}$ pulverizers. The cost (approximately $\$ 12 \mathrm{k} /$ pulverizer on this project) and specifications for each installation are site specific and will require a study to determine the technical requirements. The Unit 9 project has demonstrated that primary air control is a major key in the boiler optimization process.

\section{Furnace $\mathrm{O}_{2}$ Balance}

It was decided to try to control the $\mathrm{O}_{2}$ leaving each furnace and the windbox-to-furnace differential pressure using only the secondary air dampers. This strategy has worked well throughout the load range with no apparent disadvantages. It is a recommendation of this project that any ABB CE Twin-Furnace unit with automated secondary air dampers employ this strategy for a more steady control of $\mathrm{O}_{2}$, better $\mathrm{O}_{2}$ balance between furnaces, and good windbox-tofurnace differential pressure control.

\section{Boiler Safety/Carbon Monoxide Monitoring}

Install boiler carbon monoxide $(\mathrm{CO})$ monitors on all boilers that must optimize for $\mathrm{NO}_{\mathrm{x}}$ emissions.
$\mathrm{CO}$ is a better indicator of incomplete combustion than excess $\mathrm{O}_{2}$ when operating at very low $\mathrm{O}_{2}$ levels. The unit must operate in a region that has no margin for error.

7. Use of experimental design and model to expedite performance and $\mathrm{NO}_{\mathrm{x}}$ optimization testing

Statistical experimental design techniques were used to select specific test points for $\mathrm{NO}_{\mathrm{x}}$ minimization and plant optimization testing.

\section{Fuel System Improvements}

There were several lessons learned dealing with pulverizer optimization. Coal pipe rifflers play a very important role in providing equal fuel distribution to all coal pipes. Rifflers should be checked for primary and secondary alignment, excessive wear, and size variations which can cause flow to bypass the rifflers. All of these items affect fuel balance and unit optimization for emissions and efficiency control.

Tests and discussions with $\mathrm{ABB}$ CE experts have confirmed that air in-leakage on Raymond bowl mills is approximately 20 to $25 \%$. This is normal on all negative pressure pulverizers and should be accounted for in the fuel/air optimization process.

During the process of optimizing pulverizer performance to reduce LOI and improve heat rate and $\mathrm{NO}_{\mathrm{x}}$ emissions, it was pointed out that $\mathrm{ABB}$ $\mathrm{CE}$ had recommended extending the pulverizer classifier deflector ring to the bottom of the classifier openings to improve 50 mesh coal grinding. Controlled test on Unit 9 indicated that both 200 and 50 mesh coal grinding was improved, especially when the primary air was controlled near the correct fuel air ratio of 1.8 pounds of air to 1.0 pound of coal.

\section{Improved Performance Monitoring Package}

It is recommended to install an on-line performance monitor in conjunction with the DCS retrofit. On this project, EPRI's Performance Monitoring Workstation (PMW) was used and has proven to be an effective tool for monitoring plant performance. The monitoring software should be configurable by plant personnel to allow 
additions and modifications without major expense.

\section{Simulator - Training}

The use of the simulator provided an excellent tool in the training of the operators for Unit 9. The training familiarized the operators with the new CRT man-machine interface, gave the operators further opportunity to customize the graphics, and identified some problems prior to startup and enabled faster resolution of problems during startup.

\section{Simulator - Engineering Uses}

The simulator provided on this project is a valuable engineering tool in addition to its usefulness as an operator training system. There are several benefits an engineering simulator can provide to a retrofit project such as this one. First, it can be used to verify proper operation of the control system logic prior to the start up of the new control system. Second, it can be used to test proposed modifications to the control system whether they are simple logic revisions or completely new control algorithms. Third, it can be used to evaluate plant performance and proposed modifications to the plant mechanical systems.

\section{Marketable Ash Product}

Due to improved combustion control, the Unit 9 flyash now has low enough unburned carbon to be a salable product.

\section{Plant Wide Historian}

Modern digital control and data acquisition systems like the DCS installed on Unit 9 are capable of collecting large amounts of plant operating data. This operating data is very valuable in analyzing plant performance and operating problems. It is recommended that all DCS retrofits include a comprehensive plant archive system which can store data from not only the DCS but also from any other plant system which collects data.
The benefit justification for a typical DCS retrofit has been lacking. EPRI and TVA initiated a project to quantify the benefits of installing a new distributed control system on a 40 year old coal fired plant. The retrofit has been completed and project benefits documentation is continuing. The primary benefits to date are heat rate improvement, NOx emission reduction, and dispatch response improvement. Evaluation of long term benefits is continuing and will be documented in the future. 


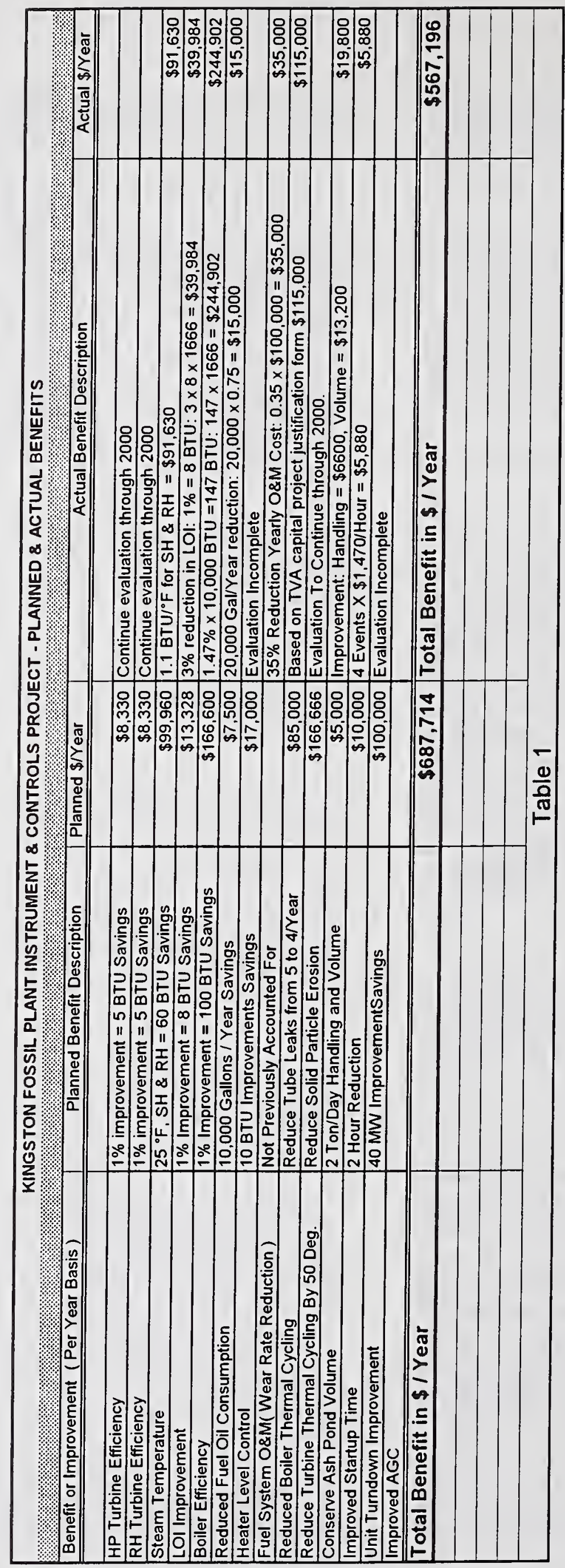




\title{
EVALUATION MEASURES FOR THE COLLECTIVE INTELLIGENCE OF CLOSED SOCIAL STRUCTURES.
}

\author{
SZUBA TADEUSZ \\ Department of Mathematics and Computer Science, Science College, Kuwait University, \\ P.O. Box 5969 Kuwait, 13060 SAFAT. E-mail: szuba@mcs.sci.kuniv.edu.kw
}

\begin{abstract}
Different live beings are co-operating (ants, wolves, humans, etc.) inside closed social structures, which express collective intelligence to solve their problems. Individual beings, express intelligence for their own purposes, as well as for their groups, even if some beings temporarily behave illogically or even against their social structure. Such social structures solve different problems in parallel, chaotic ways. Another observed phenomenon is that some problems are partially solved unconsciously in advance; thus when the problem arises, the solution is almost waiting to be applied. The phenomenon of collective intelligence, also known as swarm intelligence, can be considered a part of semiotics because the essence of intelligent cooperation is either direct exchange of information or observation and evaluation of the activity signs of other beings. This paper will try to define formal measures for collective intelligence based on the inferring ability of closed social structures. A social structure will be mapped into the Random PROLOG Processor (RPP), and on this basis, the efficiency of the $\mathrm{N}$-step inference will be evaluated as the IQ for the social structure (IQS). The proposed concept of IQS can be developed in a formal way, as well as for practical evaluation of the given grounded structure. On a simulation base we will demonstrate how some properties of social structures affect the value of IQS.
\end{abstract}

\section{INTRODUCTION.}

Let's assume that there is a given computational_space (CS) filled with objects (agents). They take fixed positions, or perform arbitrary movement. Some agents (interpreted as live beings, computers, etc.) can express inferring ability; other which are not able to do this will be interpreted as messengers or messages. It will be demonstrated in this paper (see also [9], [10]) that one common, formal description can be used for all types of agents, based on the concept of the information molecule (IM) and the membrane, defined for the first time for the Chemical Abstract Machine by Berry, et al. [2]. The term $I M$ is claimed here to be more general than the term agent because the concept of IMs of different levels, can cover the whole spectrum of agents. A single message can be considered as an agent without a membrane and inferring ability. To create the structure/batch of messages, it is sufficient to enclose such agents inside the membrane. The concept of the membrane also allows us to define the independent internal structure of an $I M$, other than the obligatory structure of an exterior. The individual inferring agent can be considered as the membrane enclosing an inferring system of any nature, consisting of $I M s$ of e.g. facts, rules, and goals (similar to the structure of the Expert System). This formal approach can be continued up through the levels of social structures such as the village, city, etc. The top level $C S$, filled with agents, can even be considered a meta-IM. In general, a given $I M$ is characterized by its inferring ability, the properties of it membrane which define Input/Output characteristics, and its mobility. Zero-mobility means that it takes a fixed position in the $C S$; non-zero mobility must refer to the characteristic of $I M$ movements (e.g. chaotic movement, displacements along the structure of streets, along data-pathways etc.). The lowest level of $I M$ is proposed to have the form of logical, PROLOG clauses of facts, rules, and goals. The lowest level of the inference process is proposed to be the act of rendezvous of $I M S$ followed by a PROLOG-like inference. Higher order $I M s$ are suitable to model, e.g. live beings by defining the proper membrane incorporating any internal inferring system used for modeling behavior of, e.g. ants. Higher level social structures can be defined as $C S$ with spatial structure where any displacements of IMs of different complexity take place. The proposed concept of $C S$ filled with $I M s$ is able to model social systems of quasi-randomly moving insects e.g. ants, which communicate through the use of pheromones. We can define necessary (static or dynamic) spatial fields of $I M s$, carrying unit clauses (atoms), corresponding to the information carried by chemical molecules of pheromones. The insects themselves will be higher order IMs. Also structures of biological cells in any organism/body which make contact through hormones, can be modeled on the concept of structured IMs and membranes.

An Intelligence Quotient (IQ) based on pattern recognition and problem solving tests can be defined for different beings, including humans. Tests can have different parameters and natures, that can verify certain components of intelligence. For tests based on pattern recognition and problem solving ability equivalent logic inferring systems can be written, able to solve a given test and to give the number of necessary inference steps [6], [8].As the IQ measure for closed social structures, we propose to use the $\mathrm{N}$-step inference. The concept is that a given social structure will be mapped into the RPP, which will be described in the second part. In the RPP we can model or formally evaluate $\mathrm{N}$-step inference process, which will provide us an IQS measure. It is important that the PROLOG is used as it is based on mathematical logic of 1-order predicate calculus. The different activities and behaviors of a social system can be described through formalism of facts, rules, and goals as an inferring system, without regard to nature of the given 
behavior. It can be the "informal dance of the honey bee", or the output of pheromones, or a network message.

The third part of the paper will discuss $\mathrm{N}$-step inference as a Social IQ test. In the fourth part examples of mapping from social structure to the RPP will be given, with simulations and evaluation of IQS for some specific social structures. A simple case will be considered where a being is approximated by a single clause of a fact, rule, or goal. This way the effect of IQS on certain configurations and statistical factors of closed social structures will be verified.

\section{VIRTUAL RANDOM PROLOG PROCESSOR.}

\subsection{RPP ARCHITECTURE.}

The Virtual Processor for the RPP is defined as the $C S$ using the notation of Multisets [1] and cham [2].

The 0 -level $C S^{\prime \prime}$ is defined as a single IM of one PROLOG clause $c$, of a fact or rule or goal. It can only undergo internal transition. For any $C S$, we can define the membrane [2] denoted by $|\cdot|_{n}$ which encloses inherent facts, rules, and goals. $C S^{0}$ has no membrane. It is obvious that

$$
\mathrm{CS}^{\mathrm{l}}=\left\{c_{1}, \ldots \mathcal{L}_{n}\right\} \equiv\left\{c_{1},\left.\ldots \mathcal{L}_{n}\right|_{p_{1}}\right\}
$$

For the given kind of membrane its type $p_{\text {, defines which } I M S}$ can pass through it. Such an act is considered as Input/Output. If the CS also contains other $\mathrm{CS}^{j}$ it is then considered a higher order one, depending on the level of internal $\mathrm{CS}^{j}$. Such internal $\mathrm{CS}^{j}$ will be also labeled with $\hat{v}_{j}$ e.g.

$$
C S^{2}=\left\{c_{1}, \ldots, C S_{\hat{v}_{1}}^{1}, \ldots, c_{n} \mid\right\} \quad \text { iff } C S_{\hat{v}_{1}}^{1} \equiv\left\{\left|b_{1}, \ldots, b_{m}\right|\right\}
$$

where $b, i=1 \ldots m$ and $c, j=1 . . n$ are clauses

Every $c_{i}$ internal $C S$ will be labeled with $\hat{v}_{j}$ to denote characteristics of its individual displacements. The general practice will be that higher level $C S s$ will take fixed positions, i.e. will create structures, and lower level CSs will perform displacements. For a given $C S$ there is a defined function pos (position) of any nature:

$$
\begin{aligned}
& \text { pos: } O_{1} \rightarrow\langle\text { position descriptio } n\rangle \cup \text { undefined } \\
& \text { where } O_{i} \in C S
\end{aligned}
$$

The following examples of pos can be given:

$$
\operatorname{pos}\left(O_{1}, t\right)=(x, y, z)
$$

where $x, y, z$ are fixed Cartesian co-ordinates or position at a given moment $t$;

$$
\operatorname{pos}\left(O_{1}, \Delta t\right)=\left(A_{x}, A_{y}, A_{z}\right)
$$

where $A_{x}, A_{y}, A_{z}$ are components of the vector in 3-D space describing the displacement of the element in the given time period $\Delta t$;

$$
\operatorname{pos}\left(O_{1}, t \text { or } \Delta t\right) \equiv \mu(x, y, z)
$$

where $\mu$ is any fuzzy function over Cartesian space, defining the position of a given clause or the internal $C S$. Obviously there are also other possible definitions of pos.

If there are any two internal $C S$ objects $O_{1}, O_{j}$ in the given $C S$, then there is a defined distance function

$$
D\left(\operatorname{pos}\left(O_{1}\right), \operatorname{pos}\left(O_{1}\right)\right) \rightarrow \Re
$$

and rendezvous distance $d \in \Re$. We say that during the computational process, at any time $t$ or time period $\Delta t$ two objects $O, O$, come to rendezvous iff

$$
D\left(\operatorname{pos}\left(O_{i}\right), \operatorname{pos}\left(O_{j}\right)\right) \leq d \text {. }
$$

The rendezvous act $\&$ will be denoted by the relation' ${ }^{\prime}$ e.g. $O, \otimes O$, which is reflexive and symmetric, but not transitive.

The computational process for the given $C S$ is defined as the sequence of frames $F$ labeled by $t$ or $\Delta t$, interpreted as the time (given in standard time units or simulation cycles), with a well-defined start and end e.g. $F_{t_{0}}, \ldots, F_{t_{e}}$. For every frame its multiset $F_{i} \equiv\left(\left|c_{1}, \ldots, c_{m}\right|\right)$ is explicitly given, with all related specifications: pos(.), membrane types $p$, and movement specifications $v$.

The simplest case or Virtual Processor for the RPP is the 3-D space with randomly travelling clauses of facts, rules, and goals. Such a simple processor is initialized to start the computational process, in such a way that the set of clauses, facts, rules, and goals (defined by the programmer) is injected into this CS. After some time, if the proper form of goal clause is reached e.g. $c_{i} \equiv$ answer(...). then $\hat{v}_{j}$ of this clause is automatically changed to provide migration to the necessary destination, e.g. to bottom of the cube. Later on, such IMs can pass through the $C S$ membrane to the outside. In this way, the appearance of the solution of the problem, anywhere in $C S$ is observable. More advanced examples of the Virtual Processor for the RPP are e.g. a single main $C S^{2}$ with a set of internal $C S^{l}$ which take fixed positions inside $C S^{2}$, and a number of $C S^{0}$ who are either local for a given $C S_{i}^{1}$ (because the membrane is not transparent for them), or global for any subset of $C S_{j}^{1} \in C S^{2}$.

When modeling the inference power of closed social structures, interpretations in the structure will be given for all $C S_{n}^{m}$ i.e. "this $C S$ is a message"; "this is a single being"; "this is a village, a city", etc. At the end of this section, the importance of properly defining $\hat{v}_{j}$ for every $C S_{j}^{\imath}$ should be emphasized. As has been mentioned, the higher level $C S_{j}^{i}$

\footnotetext{
'For another definition of rendezvous as the $\lambda$-operator, see Fontana, et al [3].
} 
will take a fixed position to model substructures like villages or cities. If we model a single being (e.g. human) as $C S_{j}^{1}$, then $\hat{v}$, will reflect displacement of the being. Characteristics of the given $\hat{v}_{j}$ can be purely Brownian or can be quasirandom, e.g. in lattice, but from the computational point of view, it is profitable to subject it to the present form of $C S_{j}^{\prime}$. With the proper characteristics of $\hat{v}_{j}$, the following can be provided:

- migration of the goal clause, when it reaches the final form answer(...). toward the Output location. This can be a membrane of the main $C S$ or even a specific, local $C S$;

- temporarily, the density of some $I M s$ can be increased in the given area of a main $C S$ in such a way that after the given low-level $C S_{j}^{i}$ reaches the necessary form, it migrates to specific area(s). This will significantly increase the speed of inference.

\subsection{LANGUAGE OF RANDOM PROLOG.}

The RPP language is elementary, but elements from attribute grammars are necessary, as well as the redefinition of rule and goal clause. The rule and goal clause in the RPP is an unordered set $S($ ) of unit clauses, contrary to the standard PROLOG, where it is the ordered list of unit clauses. Such a set can even be considered as a local, invariable $C S$ of rule or fact, containing unit clauses. This approach is compatible with the general philosophy of the RPP, and introduces additional nondeterminism of inferences on the level of unification. In the RPP, every fact, rule, and goal is associated with two inherited attributes: position pos and probability $P$ of occurrence at the given state of computations. During the simulations, the value of certain attributes is very attractive, e.g. the probability value that the given inference will be successfully done. Our experiments demonstrate that in practice it is difficult or even impossible to calculate $P$ for more complicated cases. Only general conclusions can be reached in a formal way. For some simulations it will be profitable to introduce one more attribute: configuration, to define the shape and to order in any way (linearly or even spatially) the unit clauses in the $\mathbf{S ( )}$. This will provide an easy instantiation of the RPP clause to the linearly ordered clause of standard PROLOG.

The general pattern of inference in Random PROLOG generalized for any CS has the form:

$$
\begin{array}{ll}
C S_{j}^{i} \circledast C S_{l}^{k}, & \text {; rendezvous exists } \\
\cup\left(C S_{j}^{i}, C S_{l}^{k}\right), & \text {; check unification } \\
\text { C(one or more } C S_{n}^{m} \text { of conclusion) } & \text {; check if satisfiable }
\end{array}
$$

$\mathrm{R}\left(C S_{\text {, or }}^{i} C S_{l}^{k}\right)$; retract parent $I M$ if necessary.
In general, successful rendezvous can result in the birth of one or more child IMs. All of them must then fulfil a C( ) condition; otherwise they are aborted. Because our system is based on inferring in logic, the symbol of reaction $\rightarrow$ used in cham semantics is replaced with $\vdash$ (inference).

Our proposed implementation of the RPP is tuned to evaluate the inference power of closed social structures. It was necessary to make some simplifying assumptions. Rendezvous and direct inference between two $C S_{j}^{l}$ if $i \geq 1$ will be left for further research. Thus this paper deals with a single $C S^{\prime}$ as the main $C S$ only. The example of multiple lower level subspaces $C S^{\prime}$ and clauses $C S^{0}$ existing, being displaced, and inferring in a given higher level $C S^{2}$ makes it possible to model multiple beings $\boldsymbol{H}$, performing internal inferences (in their brains), independently to higher level, co-operative inferences inside $C S^{2}$ and exchange of messages of type $C S^{\circ}$.

It is also important to assume that the results (products) of inference after this are not allowed to infer between themselves. Products of inference must immediately disperse; however, later on, inferences are allowed - if they rendezvous.

Now, let's discuss the example type of inferences between clauses, when the rendezvous takes place.

\section{Inference: fact with rule}

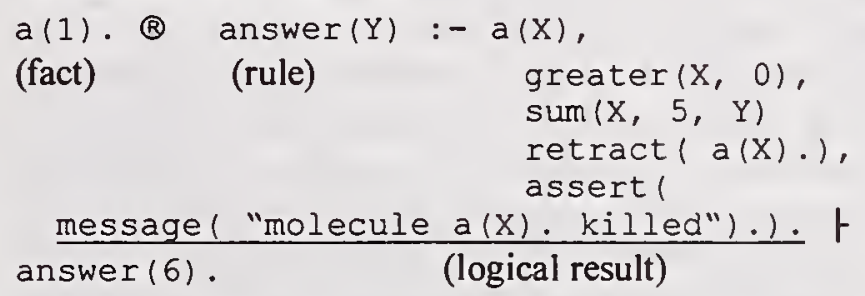

Therefore, after the rendezvous the following processing takes place:

step 1: matching;

unification of clauses $a(1)$ and $a(X) \Rightarrow\{X / 1\}$

step 2: evaluation

logical evaluation of greater $(1,0) \Rightarrow$ True

numerical calculation of $\operatorname{sum}(1,5, Y) \Rightarrow$ True

step 3: retract and assert control actions:

retract one parent; fire the message molecule into the RPP; step 4: child molecule is produced

logical expression (parent rule) reduces to unit clause i.e. fact answer $(6)$. (because there are no evaluations into False and all built-in predicates are executed successfully). An $I M$ answer (6) . can be considered a legal child. The message molecule is the "background child".

The syntax of a PROLOG dialect used for RPP resembles a simple PROLOG. Nevertheless, a quite different execution model yields new possibilities of inference diagrams, as well as creating new demands for built-in clauses, especially for control clauses. In the standard PROLOG execution model (e.g. WAM) we are restricted to backward inference, starting 
from a goal [4], [5], [7], [11]. The nature of the RPP is random or quasi-random, with parallel rendezvous of IMs carrying clauses, so it is obvious that it is possible to implement more inferring diagrams between facts, rules, and goals. We can have backward, forward, and aggregated inferences (i.e. rule with rule) at the same time.

\section{Remarks:}

- simulation experiments for the $\mathrm{N}$-step inference have demonstrated the power of a rule-rule inference diagram. Rules infer together building "aggregates" which are more powerful rules (observed also in GAMMA [1]). As a result, suddenly the final solution can be generated on the basis of e.g. a starting fact and such an aggregated rule;

- in the RPP when a clause is produced in the form answer $(\ldots)$, parameter $\hat{v}_{j}$ is changed in such a way that this $I M$ moves toward assumed Output of the RPP;

- there is no restriction on how many copies of a given fact or rule or goal we can have in the RPP. This is a very useful tool to speed up some inferences.

\section{N-STEP INFERENCE FOR IQS MEASURE.}

The basic question is to find proper IQ tests for closed systems $H S$ of beings $H$. Different beings express intelligence through different actions: e.g. ability to find a path in a maze. It can be expressed as inferences, in a similar way as we simulate intelligence of Human through structure of Expert Systems. The number of inferences per given period of time as the IQS test is not sufficient. It is easy to find examples of situations when the frequency of inferences is high, but the desired final conclusion is not reached at all (e.g. infinite loops). The benchmark which is proposed in this paper is the N-step inference (NSI). It can be defined as follows:

\section{Definition: $\mathrm{N}$-step inference (NSI)}

There is a given 1-level CS $\mathrm{CS}^{1}=\left\{c_{1}, \ldots c_{n}\right\}$ such that the following are defined

- $D\left(\operatorname{pos}\left(O_{1}\right), \operatorname{pos}\left(O_{j}\right)\right) \rightarrow \mathfrak{R}$ and rendezvous distance $d$

- $\hat{v}_{j} j=1 \ldots n$ i.e. characteristics of random displacements;

- in $\mathrm{CS}^{1}=\left\{c_{1}, \ldots c_{n}\right\}$ there exists a subset of clauses (one fact, some rules, one goal) of the form $\left\{a_{0}, a_{1}:-a_{0}, a_{2}:-a_{1}, \ldots, a_{n}:-a_{n-1}\right\}$. The number of copies of selected $c_{i}$ is one, to make the benchmark more rigorous.

- The IQS is measured by the probability $P$ that after $m$ frames $F$, the conclusion $a_{n}$ will be reached, from starting fact $a_{0}$. This is denoted $I Q S=P_{n-s l e p}^{F_{0} \rightarrow F_{m}}$. If we use experi- mental evaluation, then the average number of frames $\bar{m}$ is given ${ }^{2}$, after which $a_{n}$ is reached.

Justification for this benchmark is as follows.

1. N-step inference can be very broadly interpreted as:

- any problem-solving process in HS or inside a single $H$, where $\mathrm{N}$ inferences (logical or computational steps) are necessary to get a result;

- any production process, where N-technologies/elements have to be found in HS and unified into one final technology or production etc.

2. Simulating N-step inference in the RPP, we can model the situation in a very natural way, where the resources for inference (represented by starting fact, rules, goal) are dissipated, moving, (or locally concentrated) around the CS. This reflects well dissipated, moving, or concentrated resources in the village, city, or nation;

3. With this benchmark very easy cases can be simulated where some elements of the inference chain (missing facts, rules) are not available at the moment. This means that the chain of inference is temporarily broken, but after a certain time period, another inference process (e.g. which is running in the background or parallel) will produce the missing component. Such situations are well known in Human Systems, e.g. when a given research or technological discovery is blocked until missing e.g. theorems or subtechnology is discovered/produced/found.

4. HS of Humans infer in all directions i.e. forward (e.g. improvements of existing technology), backward (e.g. searching how to manufacture given product going back from the known formula) and also through generalization, e.g. two or more technologies can be combined into one more general and powerful technology or algorithm. Nstep inference simulated in the RPP reflects all these cases very well.

\section{EVALUATING THE INFERENCE POWER OF CLOSED SOCIAL STRUCTURES.}

Now let's investigate with the help of the proposed IQS benchmark some very simple problems referred to within the us.

\section{Phenomenon 1: Citry}

Suppose that a CS is given, and IQS is a 10-step inference. Let's analyze how the speed of NSI is affected, if the city is defined in the CS as any privileged area. It is difficult to define what the city really is even for humans; thus the simple intuitive concept is applied for these experiments, where the HS consists of a highly populated area (city) surrounded by a lowly populated region. For our experiment we define city as

\footnotetext{
${ }^{2}$ In computer simulations, every frame $F$ corresponds to one cycle of recalculation of situation in $C S$.
} 
the local $C S^{\prime} \subset C S$ with a fixed position at $(0,0,0)$, and the radius $R=0.2$ while the whole $C S$ is the cube $1 \times 1 \times 1$. The membrane for $C S^{\prime}$ is defined in such a way that $I M s$ after entering the (city) CS' must stay there for a period of a fixed number of cycles I (the membrane will reflect back into the city all molecules staying there fewer than presumed number of cycles), and after this period the $I M$ is allowed to depart through the membrane to the outside. In the city, the properties of random walking should be different; thus, the variance of Gaussian distribution of speed is set to $v=R / 2$ (half of city radius $\mathrm{R}$ ). The city will speed up the NSl in a surprising way, what is demonstrated in Table 1 . The conclusion from the simulations confirms what looks trivial and obvious to us: that the existence of the city is profitable outside of any discussion, at least from the speed of inference point of view. Now with the RPP and NSI we have tools to analyze this phenomenon, and immediately we can raise the following analytical problems:

- the optimal size of the city as the function of population size, $d$, location of the city in CS, parameters of movement of $I M s$, etc.;

- the better option for $\boldsymbol{H S}$ : a single city or a structure of cities - which type of cities is optimal?;

- how far the city will reduce the $v$ (which can be recalculated into energy savings in structures of beings) if the city is introduced, assuming the same NSI speed as before;

- etc.

Rendezvous distance $d=0.025$; Size of CS: $1 \times 1 \times 1$; size of $\mathrm{CS}^{\prime}$ : sphere at $(0,0,0) R=0.2$;

Brownian movements: Gaussian distribution with variance $v=0.124, \mu=0$;

\begin{tabular}{|l|l|l|}
\multicolumn{3}{|c|}{ with variance $v=0.124, \mu=0 ;$} \\
\hline \multicolumn{2}{|c|}{ CS without City } & $\begin{array}{l}\text { with City } \\
(50 \text { cycles } \\
\text { inside })\end{array}$ \\
\hline Conclusion reached after cycles & 17643 & 1436 \\
\hline Final number of IMs in CS & 69 & 74 \\
\hline
\end{tabular}

Table 1 Influence of City to speed of NSI

\section{Phenomenon 2: ABILITY to TRAVEL/COMMUNICATE}

The average global speed of molecules $\left\langle v_{\text {glonal }}\right\rangle$ can be easily interpreted as the average ability to travel and to communicate. If we want to simulate the ability to communicate (exchange information), it is necessary to introduce a special type of $I M M$, where $M \neq \boldsymbol{H}$ interpreted as the messages $\boldsymbol{H} \rightarrow \mathbb{H}$. By definition $M$ has very high (compared to being) ability to travel i.e. $\left.\left\langle v_{M}\right\rangle \gg\right\rangle\left\langle v_{H}\right\rangle$. As a result, the $\left\langle v_{\text {global }}\right\rangle$ will increase in the CS simulating given $\boldsymbol{H S}$ to a high level. In the
HS systems with restricted communication abilities, the $\left\langle v_{\text {global }}\right\rangle$ will be much lower because the $\left\langle v_{H}\right\rangle$ component will prevail.

Let's investigate this problem with the help of a simulation of NSI $=10$-steps in the simple CS (without the city). The existence of the city highly compensates for the lack of ability for distance travelling or remote exchange of information, because "everything is nearby".

The general conclusion that higher $\langle v\rangle$ gives higher speed of NSI (see Table 2.) is not very revealing, but with the RPP model of computations we can evaluate this conclusion toward practical applications e.g.:

"how introductions of the remote communication system e.g. telephone system, affect speed of inference measured with the help of NSI in the given $\boldsymbol{A S}$ ". Statistical data like "the number of trips and the average distance per human per year", "the number of telephone calls and the average distance of the call per person per year" should be easily available;

\begin{tabular}{|c|c|c|c|}
\hline \multicolumn{4}{|c|}{$\begin{aligned} & \text { Rendezvous distance } d= 0.025 ; \text { Size of CS: } 1 \times 1 \times 1 \text {; } \\
& \text { no City } \\
& \text { Brownian movements: Gaussian distribution with } \mu=0 ; \\
& \text { NSI }=10 \text {-step inference; }\end{aligned}$} \\
\hline variance & $\langle v\rangle=$ & $\langle v\rangle=$ & $\langle v\rangle=$ \\
\hline & 0.124 & 0.062 & 0.031 \\
\hline $\begin{array}{l}\text { Conclusion reached } \\
\text { after cycles }\end{array}$ & 17643 & 28265 & 45029 \\
\hline Final number of $I M s$ in CS & 69 & 44 & 97 \\
\hline
\end{tabular}

Table 2 Influence of average speed $\langle v\rangle$ to speed of NSl.

\section{PHENOMENON 3: THE PROBLEM OF OPPOSITION. (ABILITY TO INFER IN LOGICALLY INCONSISTENT ENVIRONMENTS).}

One of the phenomenon of Social Structures HS is opposition. It can be any action of being(s) with individual interest directed against the global interest of $H S$. It can be also unconscious wrong doing of being(s) (caused by the lack of necessary information), or even technical failure/accidents. With help of mathematical logic we can express such a situations with an inconsistent inferring system. Simple case of opposition doing can be expressed in such a way that negated conclusions are made in parallel to correct ones e.g. in different areas of CS. Rules $a \Rightarrow b ; a \Rightarrow \neg b$ can be used to get contradictory results. Another possibility is that if the global interest of $\boldsymbol{H S}$ is to reach a given goal as soon as possible, through a given chain of inference, some being(s) $\boldsymbol{H}$ will always go back to reverse/obstruct these inferences. In the Fig. 1 IL loops represent such a situations. In our simulations, for compensation purposes it has been be assumed that favorable events also exist in the inference process, marked with $J$, to model situations where some conclusions have been done "way ahead". It 
is interesting to demonstrate, that chaotic inferring systems can exist and work well, even with opposition, but under the condition that stabilizing measures are applied to reduce com-

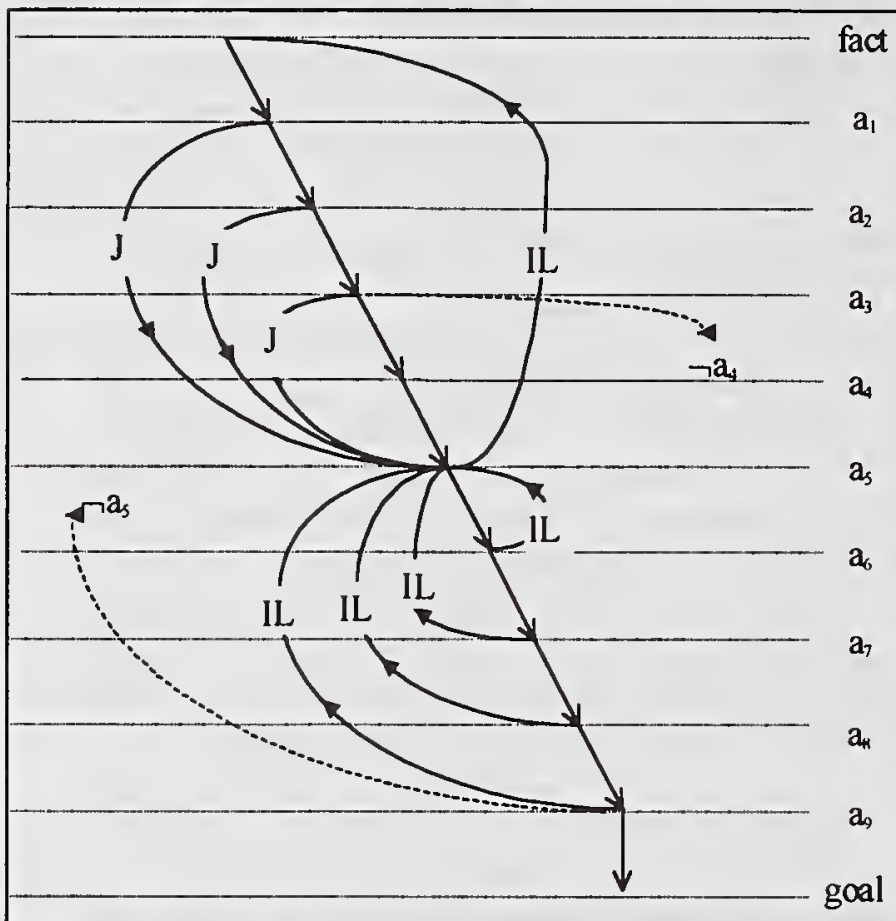

Fig. 1. System with infinite loops and inconsistent inferences.

binatorial explosions of inferences. This is also the general problem of other inferring systems, when incorrect heuristics are applied. Systems HS with opposition can't exist at all without introducing tough stabilizing measures to control the number of $I M s$. The alternative is immediate combinatorial explosion and "death" of any such inferring system. The simplest proposal to reduce the combinatorial explosion is based on the assumption that every new conclusion will retract an old one (e.g. parent(s)); which can be freely interpreted in real life as exchange of generations of beings. The problem of inferences $a \Rightarrow b ; a \Rightarrow \neg b$ can be stabilized in such a way that when there are rendezvous of IMs like $b \otimes \neg b$, the result will be retraction of both. The following rule can be used:

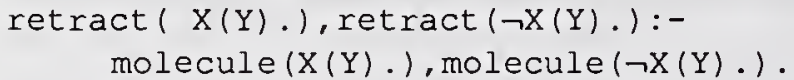

In Fig. 1. the main point of interest are infinite loops caused by certain types of opposition inside HS, and how effective the proposed stabilizing measures are. Thus the negative inferences i.e. $a \Rightarrow \neg b$ are left dangling.

Size of CS: $1 \times 1 \times 1$; rendezvous distance $d=0.025$; no City

Brownian movements: Gaussian distribution with $\mu=0$; $\langle v\rangle=0.124$ $\mathrm{NSI}=10$-step inconsistent inference;

\begin{tabular}{|l|l|}
\hline Conclusion reached after cycles & 25436 \\
\hline Final number of $I M s$ in CS & 108 \\
\hline
\end{tabular}

Table 3. Influence of opposition inferences to speed of NSI.

\section{CONCLUSION}

This paper presents the concept of Intelligence Quotient IQS for Social Structure, of any nature. As the theoretical base, the chaotic, molecular model of computations has been used which can be considered as implementation of the NonDeterministic Turing Machine. For programming purposes, a dialect of the PROLOG is used, because the logic of predicate calculus gives the best chance to describe any different behaviors inside social structures which can be considered as inferring processes. The simulation system has been built in parallel C for an 8-processor SGI computer, with 3-D graphics to observe chaotic parallel inferring processes. With this tool our simulations were implemented. The presented research results give clear directions for future research and intended commercial application:

- Different AS can be compared for their IQS values and ordered, both theoretically and through simulations;

- How certain social, political and technical factors affect the IQS can be analyzed for a given $\boldsymbol{A S}$;

- The proposed approach gives a way to define a formal theory of $1 Q S$ for $4 S$;

- Further studies are necessary on the methodology of, how the behavior of some non-Human beings (bees, ants etc.) should be translated into facts, rules, and goals, which subsequently could be used for IQS evaluation.

\section{REFERENCES}

[1] Banatre J-P., Le Metayer D.: Programming by multiset transformations. Communications of the ACM. Jan. 1993.

[2] Berry G., Boudol G.: The chemical abstract machine. Theoretical Computer Science. 1992. p. 217-248.

[3] Fontana W., Buss L. W.: The arrival of the fittest - toward a theory of biological organisation. Bull. Math. Biol. 56, 1994

[4] De Kergommeaux J. C., Codognet P.: Parallel logic programming systems. ACM Computing Surveys. September 1994.

[5] Kogge P. M. : The architectures of symbolic computers. McGraw Hill. 1991.

[6] Laurie J-L.: Problem solving and Artificial Intelligence. Prentice Hall 1990.

[7] Newmarch J. D.: Logic programming in PROLOG and stream parallel languages. Prentice Hall 1990.

[8] Sternberg R. J.: Beyond IQ. Cambridge University Press, 1988.

[9] Szuba T.: Random parallelisation of computations in the Random PROLOG Processor. Journal of Kuwait University. Topical Issue No 1. 1996.

[10] Szuba T., Stras A.: On evaluating the inference power of closed social structures with the help of a Random PROLOG Processor. PAP'97 "Practical Application of PROLOG". 21-24 April, 1997 London

[11] Takeuchi A.: Parallel logic programming. Wiley \& Sons. 1992. 


\title{
The Semiotics of Autonomous Investigations of Natural Systems
}

\author{
Paul S. Prueitt \\ Highland Technologies Inc. \\ paul@htech.com
}

\begin{abstract}
A view point is presented that emergence is a fundamental property of open complex systems and thus that natural language understanding by humans depends on the emergence of understanding. Human understanding is based on the assembly of components from implicit memories. Autonomous understanding of targets of investigation, by computational systems, depend critically on the extraction of features from the target and the use of logio-linguistic principles to encode structural characteristics of these features. It is argued that linguistic principles reflect general systems properties because the underlying morphology of concept formation must conform to a class of general systems properties. Also, linguistic principles reflect general systems properties that occur in the world. New logical principles are required to design and implement machine based autonomous investigations of natural systems.
\end{abstract}

Introduction: Neural systems derive implicit structure and encode structural representation of this structure into a physical substrates of the brain (Schacter \& Tulving, 1995). Through adaptation, the components of the substrate express emergent neural phenomena that have correspondence between the structural invariants of the world experienced and internal mental event that represent this experience to the higher order processing centers of the brain (Pribram, 1991; Levine et al, 1993; Prueitt, 1997).

The phenomena of emergence goes to the issue of how knowledge can be extracted from a data source, because the extraction must make correlations over time and develop a flexible mapping between internal representations and external objects and agents (Michalski, 1994). The computational system must have an "understanding" of how objects are composed from a substrate and how they function over time. This understanding can be "engineered" into software and hardware; however, the situational analysis that is to be derived must have some of the same properties of openness as do natural systems.
Open systems theory extends engineering and close loop control theory into a new class of logics similar to quantum logic and the logics of C. S. Peirce. These new logics reflect several classes of open systems properties. The permutation of systems into each other produces one class of properties, however, the focus of this paper is on the features of assembly/disassembly processes.

The logics of situational analysis for complex natural systems must have formal means to describe how constituents are assembled into operational wholes and how operational wholes are disassembled into components. It is only when these properties are part of the computational paradigm that one is entitled to talk about autonomous intelligence.

This means that formal tools for describing assembly, degeneracy and indeterminacy must be part of the computational logic (Tzvetkova, 1995). The interface between a human user and a computational system will have to use such tools to organize information and provide a pragmatic axis to situational analysis (Prueitt, 1997). This means that theoretical/experimental results from general systems theory must inform the architectural issues regarding the design of the human/ computer interface and the issue of machine understanding of natural language.

A central issue facing general systems theory is about whether or not a class of transformations can be characterized using methods more powerful than numerical models. In fact, Zabezhailo et al (1995) demonstrates that a predictive theory of biochemistry is realizable by using special logics that perform iconic computations. These computations are carried out using the special inference operators of Quasi Axiomatic Theory (QAT) (Finn, 1991, 1995). The paths in "iconic" space are analogous to trajectories in numerical state spaces; however, these iconic trajectories are lawfully constrained by the information in a database containing the results of specific analysis of biochemical structural activity relationships. Pospelov (1986; 1995) refers to these trajectories as syntagmatic chains. 
As another example, the relationship between canalization in simplicial complexes (Johnson, 1996) and structural adacity (Burch, 1989) in artificial life systems provide a means for the simulation of some of the mechanisms that are enfolding the individual experience of computational agents in a complex artificial ecosystem. However, much more needs to be done with artificial life systems before they will provide adequate models of the properties of natural open systems.

Natural and Artificial Systems: In (Prueitt, 1996a) the author postulated a fundamental difference between a natural system and a computational system. It is postulated that the difference is so great as to identify an oppositional scale with "natural system" at one end and "computational system" at the other. As in all oppositional scales the midpoint is most interesting because it is at this point that the classification of a system as computational or natural breaks down completely (Pospelov, 1994). Figure 1 identifies this midpoint as a perceptual system. Language systems and iconic systems are born from complementarity, with iconic systems more computational like and language systems more similar to nature.

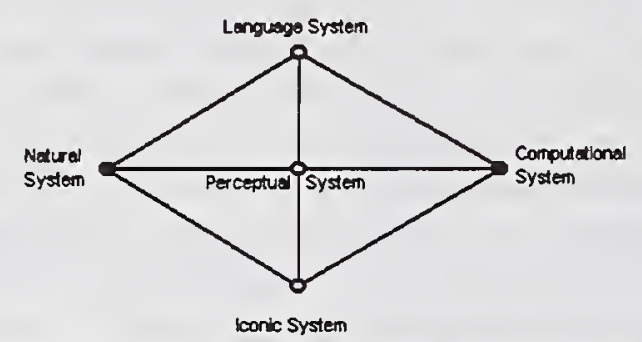

Figure 1: A semantic oppositional scale between natural systems and computational systems

Semiotic knowledge bases are indexed to numerical computations, as are computer implementations of structural adacity and canalization. Engineering is in total control, except to the extent that some critical issues regarding how things are composed might be left to the decision of a natural system in interaction with the computer. But seamless human/ computer interaction supporting situational analysis has been very difficult to achieve.

How then do we actually get out of the domination of computational processes in the development of situational models. The answer may be in the definition of situationally specific "intermediate" languages as an aid to translation between the computer and the natural system.
These transitional tools will map linguistic descriptions of situations to formal systems and computational inference engines.

A semantic oppositional scale between natural systems and computational systems suggest that human perception uses computational like iconic representations (mental images) as well as language to describe the world. For example, we observe the cognitive act of counting as a singular process only because this act is already deeply embedded into mature neural processes. Moreover, the cognitive act of counting is but one manifestation of the mechanisms of mental imaging. These mechanisms include tools for measurement of new observables by living systems. In a generic sense, these mechanisms function as part of a process cycle expressed as action followed by perception.

Ecological processes: Action perception cycles are emergent from the physical properties of subsystems and are driven by a class of periodic forcing functions. The scholarly research in ecological psychology and ecological physics views periodic forcing is an essential part of the temporal stratification of biological organization into levels (see Prueitt, 1997 for review). In this view, any one level of organization contains objects that are emergent constructions from a relatively stable substrate of basic elements.

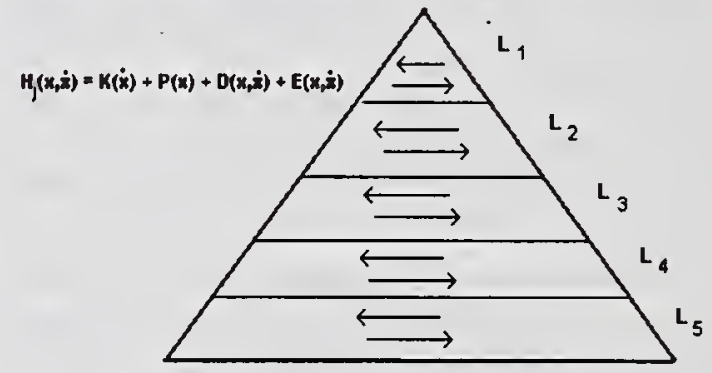

Figure 2: Natural systems stratify

The relationship that must always exist between a substrate and a natural system is the critical issue that must be resolved in any situational analysis of natural systems. In Figure 2 each level, $L_{i}$, has the capability of forming a substrate from which new organizational wholes may emerge. Each of these levels, and their interaction, can be partially simulated by a thermodynamical model.

Natural systems are "open" systems with varying degrees of openness. For example, enzymes produce micro environments where constraints are placed on the "meaning" of 
assembled biochemical agents, and in the hippocampus micro environments fuse the associational traces of implicit memory into compartmental invariances that are "judged" by the processes of other limbic systems and of cortical regions. These micro environments are agile semi-closed flexible and capable of responding to novelty and nonstationarity in the environment.

The stratified thermodynamical model of open systems, in Figure 3, have micro environments that emerge through some coherent aggregation of compatible processes. The model can be used under the assumption that the average life time of processes at a certain level is an order of many magnitudes different from the average life time of the processes at the next level. For example, atoms form one level and chemicals form a different level.

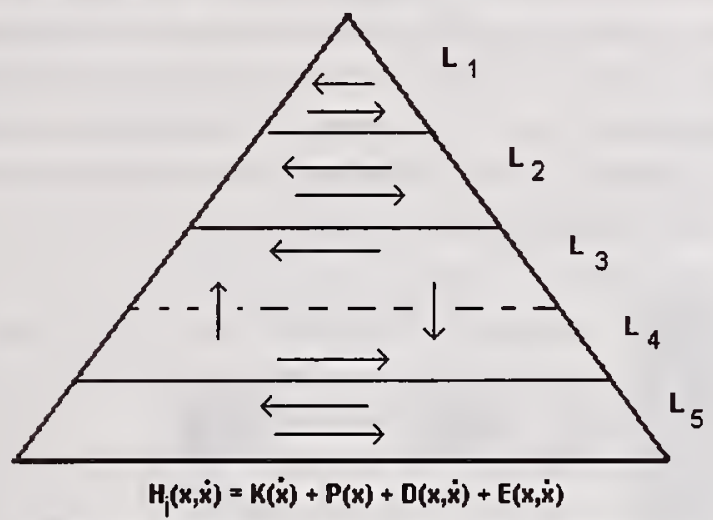

Figure 3: Stratified thermodynamical model of open systems, where one of the levels has collapsed.

The collapse of one of the micro environments leads to the diffusion of material into an environment that supports the reassemble of this material, and other similar material, into new micro environments. As these new environments arise, the structure and function reflect the characteristics of the material as well as the state of the environment.

Application to Machine Intelligence: Our approach to machine translation and knowledge representation assumes the existence of a table (database) where the system states, i.e. those states that a compartment can assume, are all specified and related to a database of subfeatures. We have a system that is specified in a formal fashion and which is computable. In this case, a theory of the world is provided in the form of computer program, with data, as a "computational ontology".

Within the compartmental boundary of this program, an underlying ontology, as expressed in a semantic net or table, can assume different system states and thus captures how the sense of the terms may drift. The rules that govern an ontology allow a modification of the sense of the terms. This gives us hope that issues of interlingua and pragmatic type can be addressed within individual compartments (Citkina, 1996). However, the procedures for the formation and dissolution of these programs must allow for the limitations that formal systems have in general and that the specific program has in particular. How is this accomplished?

Machine intelligence can also have a layered architecture where data constructions in one layer are combined to produce flexible concepts using partially defined relationships. An inductive analysis of the situation is then possible, where a fusion of data occurs based on the accommodation of characterizations of the novelty of the situation with characterizations of the background knowledge. This fusion must have the nature of learning (Michalski, 1994).

Learning involves a reorganization of an underlying constructive field that has certain general properties (Pribram, 1991). For example, the system states of advanced computational ontologies may be evaluated with respect to oppositional scales (Pospelov, 1994).

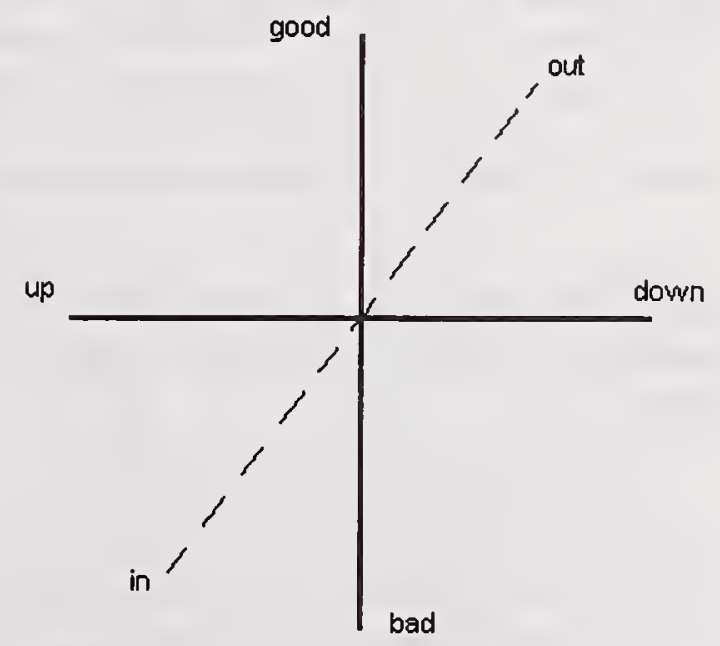

Figure 4: Oppositional scales provide metrics for the interpretation of phenomena.

Oppositional scales are specified, as expected, within a semantic space that reflect pairs of evaluators, such as good and bad, and qualitative properties of the system states (Figure 4). The formation of the compartment for modeling a specific situation might deal directly with the observation of data and the extraction, from this data, of a minimal set of oppositional scales. The use of these oppositional scales must contain a logical means for restructuring the 
semantic space by using a group transformation on the space's basis set.

Transformation rules are augmented by quasi axiomatic theory as an "open" algebraic transformation to allow the introduction of new observables and the formalization of second order cybernetic transformations of semantic spaces. The properties of these semantic spaces are representable in the form of a database plus a specific situational language and logic.

Various approaches have been made towards a formal description of second order cybernetics, including; quasi axiomatic theory (Finn, 1991), constructive induction (Michalski \& Ram, 1995), and connectionist architectures for resolving explanatory coherence (Thagard, 1988). However, none of these formalisms have had the success enjoyed by simple biological systems. Complex natural systems are open systems that (1) are embedded in a larger space (2) are composed through an assembly process, (3) have behavior properties with internal and external work cycles, (4) can be described as variably stratified (Figure 5).

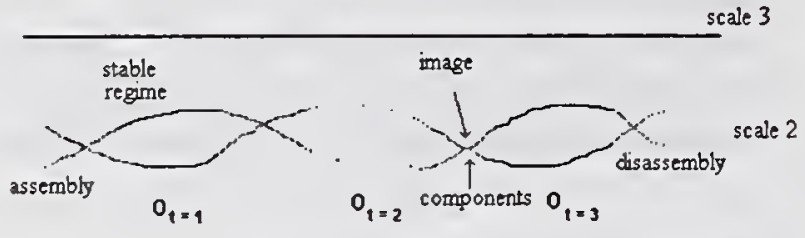

scale 1

Figure 5: The Target of Investigation is observed a number of times

Because of these four properties, the Target of Investigation must be observed a number of times

$$
T=\left\{\mathrm{O}_{1}, \mathrm{O}_{2}, \ldots, \mathrm{O}_{\mathrm{n}}\right\}
$$

- each "observation", $O$, of the target has a "bag" of properties $\mathbf{P}=\left\{\mathrm{p}_{1}, \mathrm{p}_{2}, \ldots, \mathrm{p}_{\mathrm{s}}\right\}$. The cardinality of the bag of properties is finite (but open).

- each "observation", $\mathrm{O}$, is composed from a "bag" of basic elements $\mathbf{A}=\left\{a_{1}, a_{2}, \ldots\right.$, $\left.a_{s}\right\}$. The cardinality of the bag of elements is finite (but open).

- logical hypothesis (J.S. Mill) can be asked

1) does an observation have (not have) property $p$

2) for each basic element, $a_{i}$, is $a_{i}$ cause of (or not a cause of) the property $p$
Of course, the descriptions of properties and the descriptions of basic elements of the Target of Investigation must be possible.

The quality of any automated reasoning system is a function of its power to reveal the basic signature of a situation under investigation (see Ritz and Huber, 1996). General systems properties, such as oppositional scales, are required to extract and reveal this signature. A system that creates and resolves paradoxes will produce complementarity described by the oppositional scales mentioned in Pospelov (1994). Multiple viewpoints are then accommodated through the emergence of a new system for understanding both ontologies and their natural inter-relationships; and a new player in the situation, the accommodation itself.

Block Diagram for Situational Analysis: The block diagram in Figure 6 is open to architectural instanciations of computational systems that duplicate the structural mechanisms that experimental neuropsychology suggest are used by biological systems.

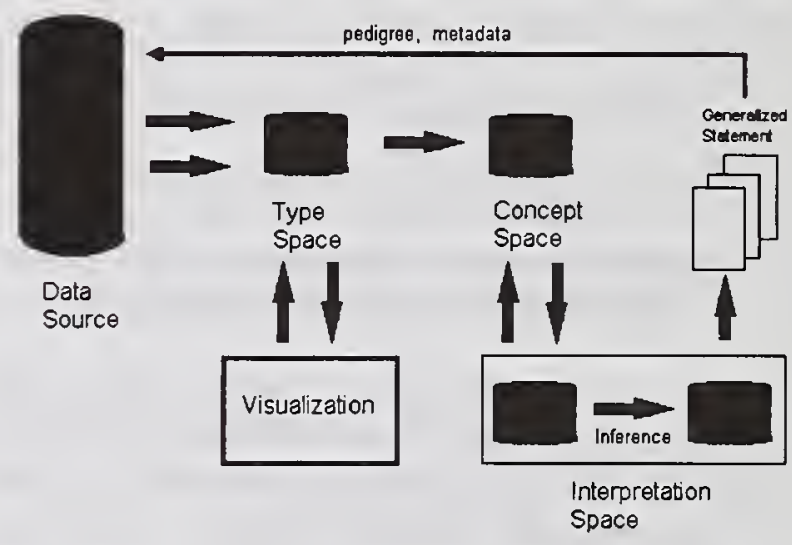

Figure 6: Block diagram for Situational Analysis

First, a data source is attended to by the system using measurements about properties and features. These properties and features are represented, approximated, as elements of a set of primary components. For example, the Fourier transform produces a representation of the spectral domain as the sum of weighted principle components. The Gabor representation may play a similar role in the human cortex (see Prueitt, 1997 for literature review). The theme vector transformation of a document's content produces a similar representation (Rijsbergen, 1979; Prueitt, 1996b). The type space is then projected into a system for visualization. In the case of human perception, this projection is a 
reentrant bi-projection between the lateral geniculate nucleus and the layered visual cortex.

Very few software systems have visualization of theme (feature) space as well as an integrated representation of knowledge in the form of a concept database. Concept databases exist in a few cases (Abecker, et al, 1997), but are primarily restricted to mechanical systems or industrial plants. Software packages such as Spires, developed by Pacific Northwest National Laboratories, makes projections into feature spaces based on cluster analysis. New computational projection schemes are being based on situational semantics. The object of visualization might be related not only to a themespace but also a concept space.

The scientific challenge is to see both computational visualization and human perception within the same paradigm. This may not be so difficult. A large amount of experimental research exists and some of this has been integrated into explanatory frameworks. The study of these frameworks suggests that the human brain achieves the formation of concepts through a distributed disassembly and reassembly of representational features (Figure. 7). This framework can be seen to be illustrative of a general systems property regarding the emergence of operational wholes within ecosystems.

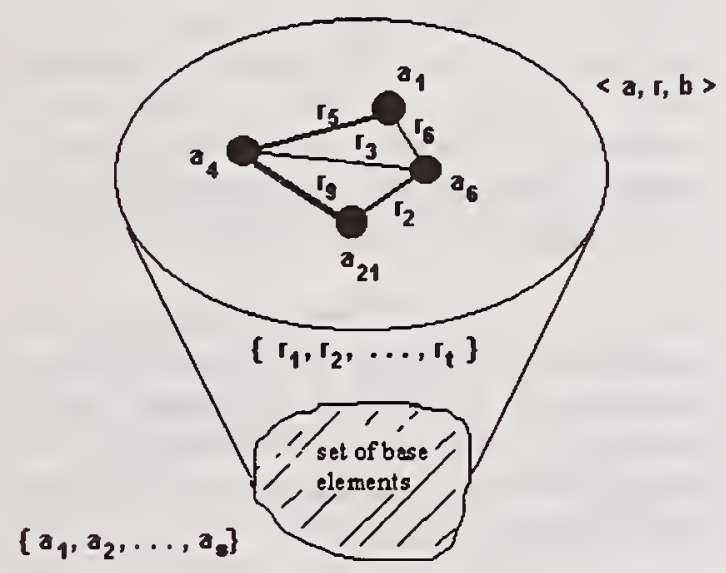

Figure 7: The niches in ecosystem share in the common use of a finite class of natural type.

It is also suggested that the human in vitro concept space is a virtual space in the sense that the space does not actually ever exist in total in any specific circumstance. Parts of this virtual space come into being while other parts are blocked by various types of competitive cooperative network dynamics. Of course, this simple architecture disguises the complexity of how the brain uses both its neural architecture and its chemical composition.

A projection from a complete enumeration of a knowledge engineering type concept space can be made onto a concept subspace. In Figure 4 this is called an interpretation space. This subspace may be mirrored by activation of components of a situational model supporting automated reasoning. The mirrors can be maintained by a neural network associative memory as demonstrated in (Dubchak \& Muchnik, 249). The mirrors can be two way, and as a consequence a separate mapping back to a concept subspace may be made after an inference engine has changed the state space of the situational model. This notion of projections and mirrors between representational spaces is a reasonable first model for the production of computer based situational models. However, there are a number of scientific issues that are not yet resolved.

The structural form of a computer based concept space has not been worked out. Simple themespaces have been defined, but a new class of objects are needed in themespaces. These objects will create topological distortions of the otherwise flat Euclidean space, and instanciate a theory of semantic operators defined in coincidence with the inference engines.

\section{References:}

Abecker, A.; Bernardi, A.; Hinkelmann, K.; Kuhn, O; \& Sintek, M. (1997) Towards a Well-Founded Technology For Organizational Memories. Proceedings of the 1997, AAAI Spring Symposium on Artificial Intelligence in Knowledge Management, Stanford University.

Burch, Robert (1989). A Peircean Reduction Thesis, the foundations of topological logic. Lubbock Texas, Texas Tech University Press.

Citkina, Faina (1996). Terminological Relativity and Translation. In: Terminology and Knowledge Engineering, Proceedings of Fourth International Congress on Terminology and Knowledge Engineering, 26-28 August 1996, Vienna, Austria, Christian Galinsky and Klaus-Dirk Schmitz (ed.), Indeks Verlag, Frankfurt, pp.:328337

Dubchak, I; Muchnik, I. (1995). Classification Scheme for Complex Systems: Prediction of Protein Structure Models, in J. Albus, A. Meystel, D. Pospelov, and T Reader, (Eds), Architectures for Semiotic Modeling and Situational Analysis in Large Complex Systems, AdRem, Bala Cynwyd, PA 
Finn, Victor (1991). Plausible Inferences and Reliable Reasoning. Journal of Soviet Mathematics, Plenum Publ. Cor. Vol. 56, N1 pp. 2201-2248

Finn, Victor (1995). JSM-reasoning for control in open (+/-) worlds, in J. Albus, A. Meystel, D. Pospelov, and T Reader, (Eds), Architectures for Semiotic Modeling and Situational Analysis in Large Complex Systems, AdRem, Bala Cynwyd, PA

Johnson, Jeffrey (1996) Design and Control of SelfOrganizing Complexes. In the proceedings of the Workshop on Control Mechanisms for Complex Systems: Issues of Measurement and Semiotic Analysis: 8-12 Dec. 1996.

Levine D, Parks, R., and Prueitt, P. S. (1993). Methodological and Theoretical Issues in Neural Network Models of Frontal Cognitive Functions, in International Journal of Neuroscience. $\mathbf{7 2}$ 209-233.

Michalski, R. S. (1994). A Theory and Methodology of Inductive Learning, in R. S. Michalski, J. G. Carbonell \& T. M. Mitchell (eds), Machine Learning: An Artificial Intelligence Approach, Morgan Kaufmann, Los Altos, CA.

Michalski, Ryszard S, \& Ram Ashwin (1995). Learning as Goal-Driven Inference, in A. Ram \& D.B. Leake (eds), Goal-Driven Learning, MIT Press/Bradford Books.

Pospelov, D. (1986). Situational Control: Theory and Practice. Published in Russian by Nauka, Moscow.

Pospelov, Dmitri (1994). The gray and/or black and white, in Applied Ergonomics, Moscow, Applied Ergonomics Assoc.

Pospelov, Dmitri (1995). Semiotic Models in Control Systems, In J. Albus, A. Meystel, D. Pospelov, and T Reader, (Eds), Architectures for Semiotic Modeling and Situational Analysis in Large Complex Systems, AdRem, Bala Cynwyd, PA

Pospelov, Dmitri (1996). Large Semiotic Models in Control Systems. In the proceedings of the Workshop on Control Mechanisms for Complex Systems: Issues of Measurement and Semiotic Analysis: 8-12 Dec. 1996.

Pribram, K. (1991). Brain and Perception, Erlbaum, New York.

Prueitt, Paul S. (1995). A Theory of Process Compartments in Biological and Ecological Systems. In J. Albus, A. Meystel, D. Pospelov, and T Reader, (Eds), Architectures for Semiotic Modeling and Situational Analysis in Large Complex Systems, AdRem, Bala Cynwyd, PA

Prueitt, Paul S. (1996a). Is Computation Something New?, published in the Proceedings of NIST Conference on Intelligent Systems: A Semiotic Perspective. Session: Memory, Complexity and Control in Biological and Artificial Systems. October 20-23.

Prueitt, Paul S. (1996b). Semiotic Design for Document Understanding, in the proceedings of the Workshop on Control Mechanisms for Complex Systems: Issues of Measurement and Semiotic Analysis: 8-12 Dec. 1996.

Prueitt, Paul S. (1997). Optimality and Options in the Context of Behavioral Choice, in Daniel Levine and W. Elsberry (Eds). Optimality in Biological and Artificial Networks?, Erlbaum

Ritz, Thorsten and Hubler, Alfred (1996). Large Controlling Complex Systems and First Principles. In the proceedings of the Workshop on Control Mechanisms for Complex Systems: Issues of Measurement and Semiotic Analysis: 812 Dec. 1996.

Rijsbergen, C. J. van (1979) Information Retrieval, Butterworths, London.

Rocha, L. M. (1996). Evidence Sets: Contextual Categories, in the proceedings of the Workshop on Control Mechanisms for Complex Systems: Issues of Measurement and Semiotic Analysis: 812 Dec. 1996.

Schacter, Daniel \& Tulving, Endel (Eds) (1995). Memory Systems 1994, The MIT Press, Cambridge Mass.

Thagard, Paul (1988). Computational Philosophy of Science, The MIT Press, Cambridge Mass.

Tzvetkova, Galia (1995). A general view on complex control systems: their evolutionary development and intelligence. In J. Albus, A. Meystel, D. Pospelov, and T Reader, (Eds), Architectures for Semiotic Modeling and Situational Analysis in Large Complex Systems, AdRem, Bala Cynwyd, PA

Zabezhailo M. I., Finn V. K., Blinova V. G., Fabrikantova E. F., Ivashko V. G., Leibov A. E., Melnikov N. I., Pankratova E. S. (1995) Reasoning Models for Decision Making: Applications of the JSM-method in Intelligent Control Systems, in J. Albus, A. Meystel, D. Pospelov, and T Reader, (Eds), Architectures for Semiotic Modeling and Situational Analysis in Large Complex Systems, AdRem, Bala Cynwyd, PA

Zabezhailo M. I (1996). The Application of QAT reasoning to Forecasting the Delayed Effects of Environmental Degradation on Human Health, presented at the QAT Teleconference, New Mexico State University and the Army Research Office, December 13, 1996 



\title{
Soft Modeling for Intelligent and Complex Systems
}

\author{
Paul P. Wang \\ Department of Electrical \& Computer Engineering \\ Duke University \\ Box 90291 \\ Durham, NC 27708-0291
}

\section{ABSTRACT}

This paper proposes a three-level hierarchical mathematical model for intelligent and complex systems. Motivation, background, rationale and interpretations are given. Illustrated in a block diagram are different visions from different schools of thought. The concept of "soft-modeling" represents a dramatic departure from conventional mathematical modeling is also presented. The characteristics, approach, types of mathematics used, and the interpretations that will eventually lead to engineering realization and commercialization are also presented.

KEYWORDS: soft modeling, intelligent systems, hybrid co ntrol, intelligent control, computational intelligence, fuzzy logic, first order predicate calculus, fizzy constraint network, cognitive science, pattern recognition, adaptive intelligence, control of complex systems, computing with words, sofi optimization, soft computing.

\section{INTRODUCTION}

In recent years, we have observed the rise of the concept of "soft computing" both in recognition of importance and in the increase in number of technical papers published in open literature $[1,2,3,4,5,6,7]$. No doubt, soft computing as Lotfi A. Zadeh conceived has been and will continue to influence the intelligent engineering. Because its foundation is biologically inspired, soft computing can solve difficult problems, especially nonlinear problems. At the same time, Lotfi A. Zadeh and many other leading researchers in the field agree that the most fruitful areas of application will concern "complex systems" and "intelligent systems." The definition of "intelligent" is very complicated and we will not be able to draw any sensible conclusions for a very long time. On a more optimistic note, the desirable features constituent of intelligent, such as adaptation, reasoning, generalization, similarity, focusing attention, pattern recognition, introspection, combinational search, etc., are useful and important for making better products by improving quality, reliability, and performance of industrial products for human beings. All these desirable features can happen by lowering the cost of production and saving energy, materially and humanly.
As far as complexity is concerned, the impact will be at least equally, if not more profound. The complex systems, both static and dynamic, are in front of us and surround us everywhere. Historically, the understanding of these systems is limited because they are usually not mathematically traceable. The types of mathematics referred to are those recognized by earlier researchers or with already established credibility in the sense of rigorous scientific methodologies and traditions.

The mathematical system theory begins with mathematical modeling of pragmatic and realistic systems, physical or manmade. There is very little one can do if a mathematical model cannot be established. In other words, all the analysis and engineering design that follow it cannot be processed. The mathematical model, in a sense, represents some mathematical constraints that must be satisfied. This is the place where the difficulties in analyzing complex systems arise. In retrospect, all mathematical approaches have traditionally used in systems analysis belong to what one may call " $=$ " or "equal culture." In other words, the constraints are exact. Unfortunately, this constraint condition is not always where we are ready to tackle complex systems.

The purpose of this paper is to propose a novel approach to soft modeling. Soft modeling is a generalization of the concept of mathematical modeling in the traditional sense. Three area of generalization worthy of mentioning:

1. The generalization in mathematical constraints.

2. The generalization of the type of mathematics being used.

3. The generalization of the types of physical or manmade systems to be treated (i.e., the mixture of the physical, engineering, and social economic systems).

\section{THREE-LEVEL SYSTEM ARCHITECTURE FOR INTELLIGENT AND COMPLEX SYSTEMS}

To ease the discussion of three-level system architecture, Figure 1 illustrates this system in terms of three separate blocks. Furthermore, to cover more ground, different schools of thought that currently exist are also listed. (Please bear in mind that the investigation of systems of this type can quickly 


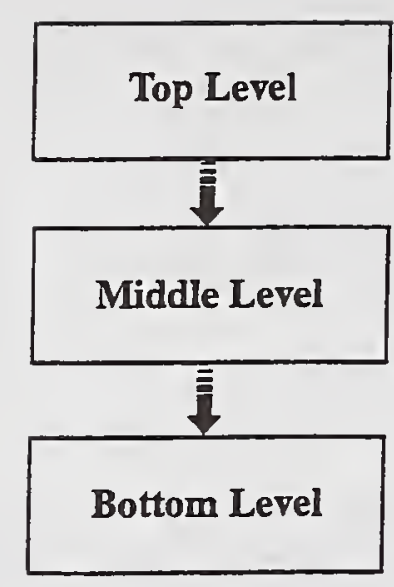

(i) Taxonomic Vision

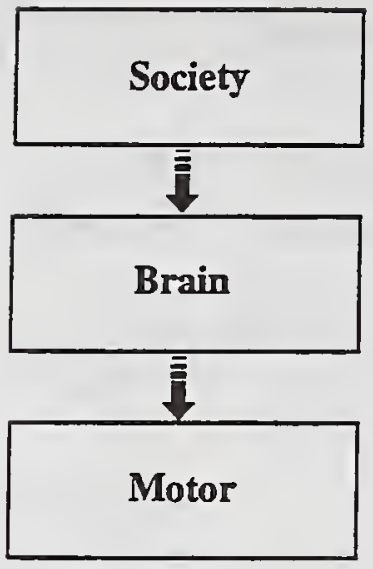

(ii) Cognitive Science Vision

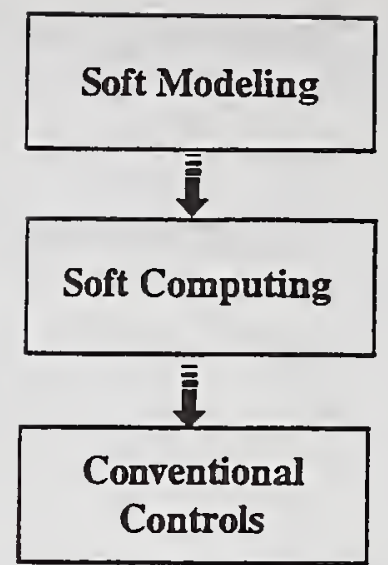

(iii) Soft Computing Vision

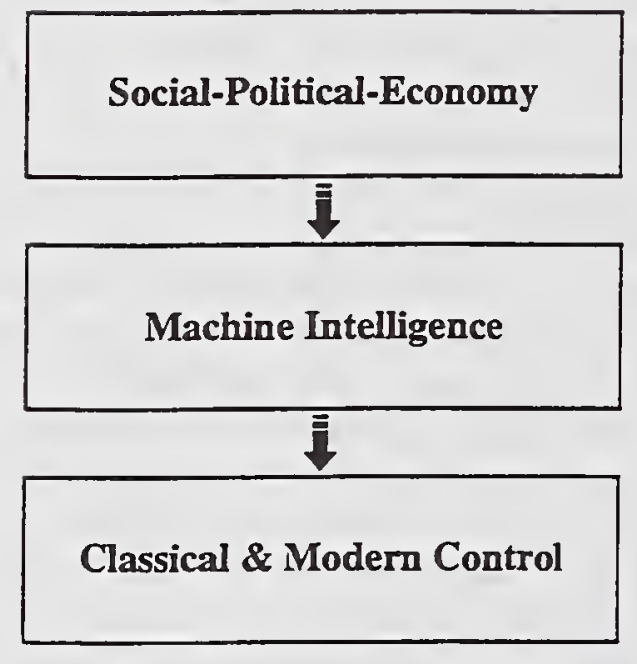

(iv) Hard Computing Vision

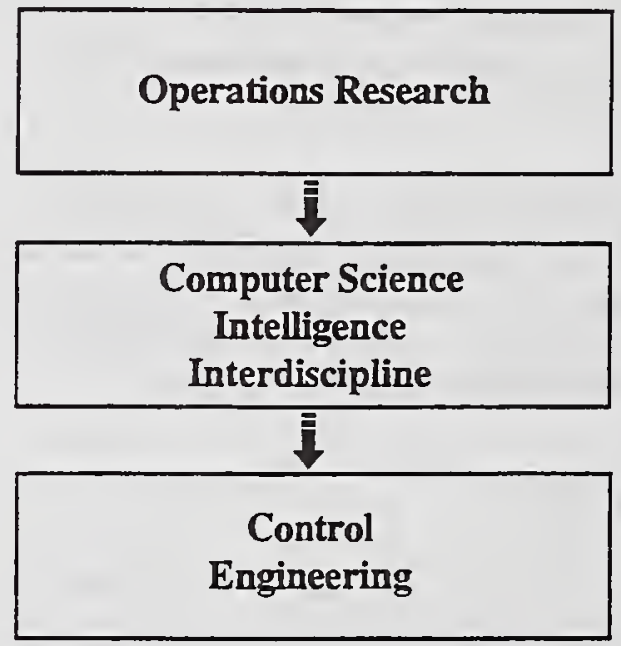

(v) Scientific Discipline Classification

Figure 1: Three-level hierarchical models for different schools of thought.

be extremely complicated while the features of intelligence and learning are to be incorporated.)

For the proper perspective on the kinds of problems being addressed, A. C. Antoulas of Rice University [8] says". . . Scientific activities can be divided today into two broad categories. The first category includes by in large the natural sciences. . . Its objective is to investigate fundamental properties of matter, and big bang, black holes . . . The second category is concerned with phenomena and structures which display high complexity. These can be found in nature, biological phenomena and the structure of molecules, like DNA, are two examples. But for the most part they are artificial, generated by disciplines such as engineering, computer science, cybernetics, ecology, operations research, economics, etc."
In other words, the complex systems described in this paper will deal exclusively with the examples in category 2. Although we may be deeply involved with the study of brain phenomena in order to get biologically inspired spirit out of it, we are neither addressing DNA, or crystal structures. Antoulas [8] went on to say, ". . . The main distinction between these two categories is their system component: the former has a small system component while the latter has a large system component."

Before we enter the detailed description of all three levels of the proposed hierarchical structure, all three levels will be briefly described in three practical complex systems: (1) a large hotel complex, (2) an automatic manufacturing plant, and (3) the command of a battlefield. 
In a nutshell, a three-level hierarchical model consists of three levels: (1) the mission statement is the top level or constraint imposed by the society of meaningful human endeavor. The soft modeling as proposed in this paper is intended for modeling this level. (2) The existing technology is the bottom level. And (3) the middle level, on the other hand, is called cutting edge research and development.

These three levels are:

1. Top-Level Subsystem - The goal of achieving some global optimization for a large complex hotel (example 1) is to optimize yield management with a set of constraints. The set of constraints include law, public policy, economic condition, number of available workers, several reservation priorities, geographical location of hotel complex, seasonal variations, special events and cultural activities, etc. In order to establish a mathematical model successful, a new modeling methodology (soft modeling) is needed to make use of different mathematical tools.

Example 2, similarly, has a set of constraints. These are very realistic constraints that the complex system must satisfy: purchasing orders, availability of raw materials, defective parts replacement, number of available workforce, international trade, inventory, public policy, up-dating of legal codes, environmental regulations, up-grading of the production lines, etc. And, finally, soft modeling is also needed for the battle field command of Example 3. Availability of communication networking, food and material supplies, injured soldiers, field hospital, transportation, ammunition and weapons supplies, intelligent information fusion, etc. are just a subset of constraints which must be handled.

Yes, these are constraints that come in different sizes, forms, units, measurements, emphasis, criteria, values, inherited culture, etc., etc. That is precisely the motivation for the novel concept of soft modeling.

2. Middle Level Subsystem - This subsystem is the most important and exciting components of the complex system. The recognition of its importance has been firmly established by a number of leading researchers. However, this is not the main theme of this paper, because we are discussing the issue of the top-level subsystem. For the sake of completeness and an overall picture of how to handle complex systems issues, J. S. Albus of NIST summarizes "enabling technology" in his paper [9]. "The latter half of the 20 th century has seen a number of fundamental breakthroughs that have radically altered the landscape of technological possibilities:

a. The power and speed (per unit cost) of electronic computing has risen exponentially by more than a factor of ten per decade for over four decades. . .

b. The store of knowledge about how the biological brain works has also increased many fold over the last half century, . .

c. Solid theoretical and mathematical foundations have been established and progress is rapid in the scientific study of language and speech understanding, image understanding and perception, knowledge representation, simulation, reasoning and decision making, planning and control.

d. Engineering approaches have begun to emerge for designing, constructing, testing, and exploiting intelligent systems for practical applications in commerce and industry." In large measure, one may say that the soft modeling concept as proposed in this paper was motivated by item d quoted above. Tremendous research and development opportunities exist in the course of the realization of the item $d$. It is fair to say that a satisfactory answer for the middle-level subsystem's problems might take a long time to obtain.

3. Bottom-Level Subsystem - There is very little that needs to be said about this third level subsystem. It would not be far off to say this subsystem is already here. During and after World War II, researchers and engineers have brought us to the present level of maturity. However, it is not to be said that we do not have new problems. In fact, there is a whole research community of hybrid systems conducting pioneering work in this area. The 5th Hybrid Systems Workshop, headed by Panos Antsalkis, will take place at Notre Dame [10]. Hybrid systems research is very solid and down to earth. As we look to the future of intelligent systems research, it is worth considering an even deeper system structure. Referring to Figure 2, one can look at hybrid system design as a special case of the proposed three-level model in at least two aspects: the automation is just a special case of a much more general mathematics linguistic model and without considering the lop-level subsystem, then the problem of both intelligent and complex systems has not been fully addressed, in our opinion.

The research activities for the middle level subsystem are very active at present and will continue to be very active for a long time to come. Again, the main concern of this paper is soft modeling of intelligent and complex systems.

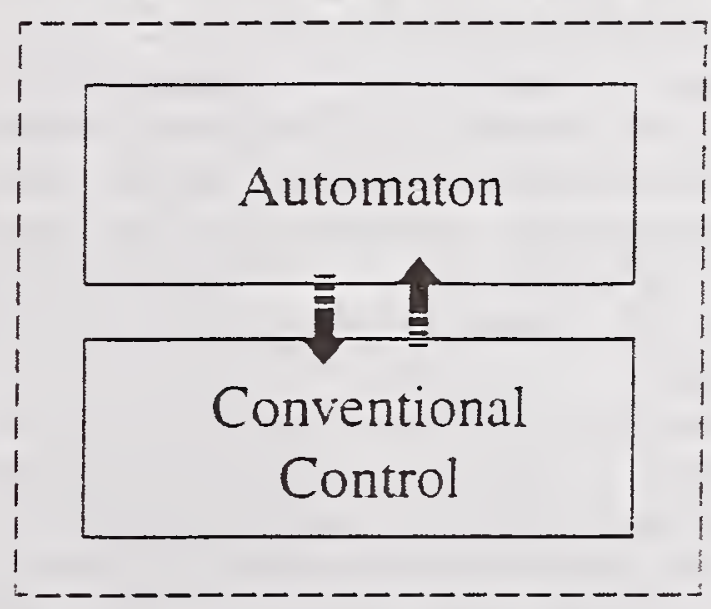

Hybrid Control

Figure 2: Hybrid control systems.

In Figure 1, five hierarchical block diagrams represent five 
different visions depending on different schools of thought. The semiotic view by Alex Meystel and James Albus is not illustrated here because it is difficult to present it in this structure. Please refer to Meystel's original works for proper reference [9].

One thing is certain, with FCN as the top subsystem and approximate reasoning in the middle, a much better performance of a complex system can be realized as shown in Figure 3.

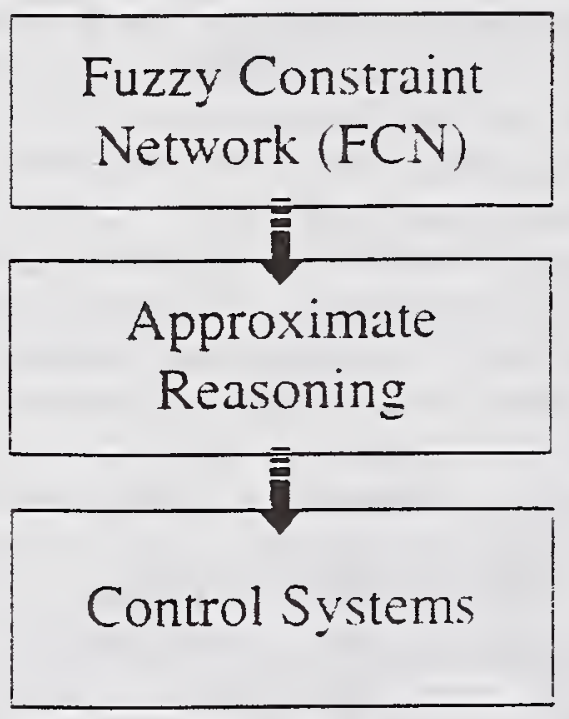

Figure 3: A fuzzy-based realization of soft-computing model.

\section{SOFT MODELING}

With all the above background, we now embark on the task of explaining the concept of "soft-modeling." By no means does one pretend to borrow fancy words in order to gain attention. Rather, it is an outgrowth of necessity.

In Section II.1, we have introduced three realistic examples of a top-level subsystem. Traditionally, for this type of problem, the modeling has been done by operations researchers. Their approaches are not unique. One standard approach is using linear programming. Another approach introduced by Lotfi A. Zadel $[5,6]$ is a fuzzified version of First Order Predicate Calculus (FOPC) method. The novelty aspect as described in this paper is the interface between the top level and the middle level subsystems. In a later section, we shall also show a linear programming approach that is a special case of the fuzzy FOPC formula.

To appreciate the need of soft-modeling, we must go back to study the evolution of convention control. It is well recognized that the first mathematical model to describe plant behavior for control purposes is attributed to J. C. Maxwell, of Maxwell's equation fame, who, in 1868, used differentiation equations to explain instability problems encountered with James Watt's flyball governor [11]. Control theory has made significant strides since 1868 . During the 1930s, World War II, and the 1940s, the use of frequency domain methods and Laplace transforms were the dominant methods for analyzing and designing control systems.

What does the above statement tell us? That we can solve only problems that are linear and time invariant? In fact, only linear time invariant systems can be solved because both Fourier and Laplace transforms are linear transformations. Conventional control systems have always been designed using mathematical models of physical systems. A valid mathematical model is a prerequisite for control system designs. Yet, with this very restricted and often unreasonable approximation (linearization), it is obvious that we have left many problems unsolved. In fact, in conventional control approaches, a mathematical model that captures the dynamic behavior of interest must be simple enough to be analyzed with the available mathematical techniques and accurate enough to describe the important aspects of the relevant dynamic behavior [11]. For nearly half a century, engineering students were trained for the most part via so-called small signal theory. This kind of approximation only delivers the behavior of a plant in the neighborhood of an operating point. This is true for all electronic, vacuum tube, transistors, VLSI products as well as electrical, mechanical, optical, hydraulic, and thermo systems and any combination thereof. So what is the point? The point is that serious restrictions are artificially imposed in order to use frequency domain analysis and Laplace transforms. These restrictions basically rule out the opportunities to solve many important and pressing complex problems. This observation leads to one of the explanations for the need of soft-modeling.

The second reason deals with the nature of the systems we must learn to cope with. Contrary to mathematical modeling that is almost always based on the Hamiltonian principal and Lagrangian equations for continuous mechanics, there exist few guidelines for the three different types of examples as described in Section II. Depending on the designers' background and disciplines, the outcomes of modeling can be very different. The fact is that no principle similar to Hamiltonian and Lagrangian is available for use in solving the three examples. In other words, new methods of solving these kinds of examples must be developed. These methods would be different from ordinary differential or difference equations and static algebraic equations. For example, binary logic, fuzzy logic, the concept of relations, the operations of subsets all of a sudden become very important. Predicate Calculus and First Order Logic are very general methods for solving problems when the theory is applied to many different physical or human societal problems [12]. Fuzzification does not alter much in terms of modeling, but it makes a difference in terms of problem solving abilities.

The dramatic difference in soft modeling is brought about by the type of mathematics used. For example, we have seen what linear programming methodology can do for operations researchers. This methodology includes the so called 
"inequality" or symbolic " $<$ " that is relation in a mathematical concept. To fully understand soft modeling, one can think of the solution for a state vector that may not be unique and one must talk about "solution space" instead of just a unique "solution." In optimal control theory, the solution is nearly always unique. In the case of soft modeling, this will not hold true. This very fact may be justification for the nomenclature "soft computing" as coined by Lotfi A. Zadeh [3]. The work "soft" means the solutions will be "solution space" as a direct consequence of the soft modeling.

Several important observations concerning soft modeling are in order (refer to Figures 4 and 5):

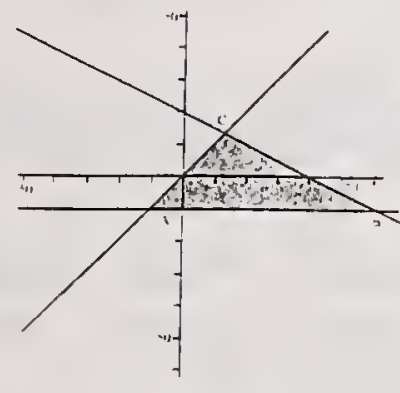

(1) Finute Soluturn Gipace

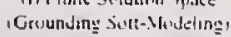

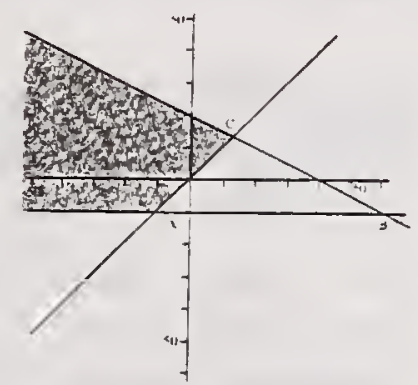

(ii) Intinuc Solution Spate
Figure 4: Solution space.

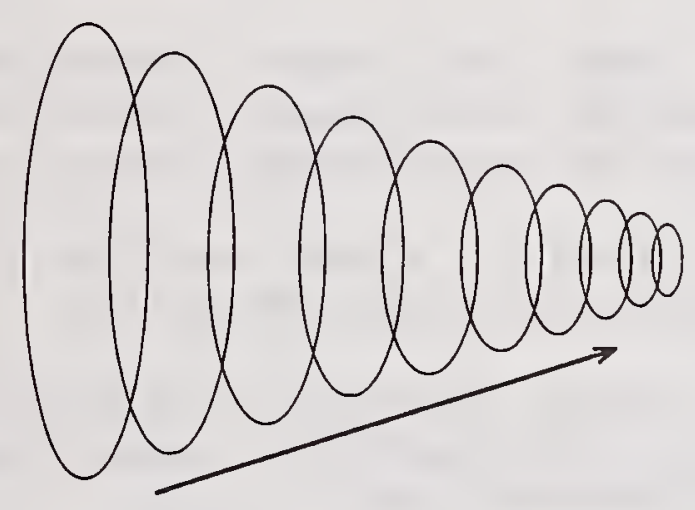

\section{Shrinking of Solution Space}

Figure 5: Learning of soft-modeling.

Observation No. 1 - There are two types of solution space-finite and infinity.

Observation No. 2 - The quality of soft-modeling depends on the size of solution space, the smaller the better.

Observation No. 3 - An infinitive solution space can be change to a finite solution space. In principle, this is always possible for manmade social-economic systems. The real difference is at what cost.

Observation No. 4 - It is impossible for a solution space to be reduced to a single point in state space. Should it happen that the solution space is reduced to a single point under very special conditions then the system involved can no longer be call soft modeling.

Observation No. 5 - The learning problem as defined by soft modeling is eminently related to the shrinking of solution space.

Observation No. 6 - . The optimization process for soft modelled systems begins where the final solution space is finished. (Actually one says the soft system is grounded or it is established.) The optimal solution then can be established by examining the optimal solution over the solution space.

\section{FUZZY CONSTRAINT NETWORK}

Fuzzy Constraint Network (FCN) is the fuzzy version of FOPC and has been recognized as a key methodology in the top- level subsystem in our hierarchical structure. FCN was first presented to the intelligent system community by James Bowen and Robert Lai in Lai's ${ }^{1}$ dissertation [13,14]. Didier Dubois and Henry Prade have also been working on a somewhat different approach for many years [15]. Subsequently, FCN has been investigated further by Paul P. Wang, Dennis Bahler, C. Y. Tyan [16]. The application of FCN has always been fairly broad and subsequently there is no lack of papers in this field. Potentially, FCN can play a very important role in soft modeling.

As discussed in Section I, the concept of mathematical modeling must be critically revisited in order to meet the challenges of solving intelligence and complex problems. Some thoughts on this very issue are in order. Since the systems are complex, to find suitable mathematics to produce quantitative results may not be readily available. As Lotfi A. Zadeh has demonstrated, fuzzy logic can solve complex issues that are otherwise impossible [2]. One can view the mathematical modeling as an attempt to search for some kind of mathematical language to provide useful information and consequently some numerical value available and useful. On the other hand, it is not one's intention to make this over complicated. Cover and Thomas have discovered Einstein's saying in a Chinese fortune cookie: "Everything should be made as simple as possible, but no simpler." [17] Upon further examination of the three examples, one discovers the fuzzy FOPC has indeed exhibited remarkable express power in modeling the reality of a complex system. This kind of model can also be made quite robust and should be constructed in an adaptive manner. This is precisely what the FCN methodology is good form.

In conventional control theory, the design of a physical system often consists of four steps [18]:

1. Modeling

2. Setting up mathematical descriptions

${ }^{1} \mathrm{Ph} . \mathrm{D}$. dissertation at Computer Science, North Carolina State University 


\section{Analyses \\ 4. Design}

The physical system, theoretically speaking, is an object existing in the real world, its precise characteristics are often unknown to us. The difficulties will increase many fold when the systems are complex. Our final objective, of course, is to accomplish items 1 through 3 successfully and eventually develop a good design. With this perspective in mind, the research needed for this proposed system hierarchical structure is only the beginning. Indeed, we have a long way to go.

\section{CONCLUDING REMARKS}

As early at 1958, researchers [19] recognized a logic circuit can be incorporated in a conventional control system for one of two general purposes: for adjusting the control system parameters or for overriding the conventional control system. It took many years for hybrid control systems to become a serious research topic [10]. The thesis of this paper actually is quite straightforward. Since fuzzy logic is much more general than bivalent logic and its ability to handle imprecision and incomplete situations have been demonstrated many times already, the proposed three-level architecture ought to be the general problem solver by adopting FCN [16].

Three-level architecture is analogous to that of a three-layer neural network as a generic term. Theoretically speaking, because the architecture of a large complex system can assume many variations in form and structure, many possible combinations of the basic building blocks are possible. Depending on the nature of the real-world problems, the number of possible combinations and variations can indeed be huge. What we have presented in this paper is just the very basic structure of a generic subsystem.

System theory is a discipline which aims at providing a common abstract basis and unified conceptual framework for studying the behavior of various types of forms of systems [19]. The foundation issues covering fundamental concepts such as system, state, stability, controllability, observability, determinateness, equivalence, etc. are not going to be easy for the intelligent systems of this type. It is highly possible that new concepts will be developed as we move along. On the other hand, we are fortunate to have a collection of various methods, techniques, and algorithms designated as soft computing for studying the behavior and design of complex systems.

The study of intelligence, learning, and training has not been mentioned in this paper because our focus has been on the concept of soft modeling for complex systems. Many problems remain to be investigated.

\section{REFERENCES}

[1] Wang, Paul P. Fuzzy Subsets and Logic: An Effective Tool for Machine-Intelligence Control System, Proceedings of the 1996 International Multidisciplinary Conference, Intelligence System: A Semiotic Perspective, V. II, Applied Semiotics, 1996, pp. 293-297.

[2] Zadeh, Lotfi A. "Outline of a New Approach to the Analysis of Complex Systems and Decision Processes, "IEEE Transactions on Systems, Man, and Cybernetics, pp. 28-44, 1973.

[3] Zadeh, Lotfi A. "Fuzzy Logic = Computing with Words, " IEEE Transactions on Fuzzy Systems, 4(2), pp. 104$111,1996$.

[4] Wang, Paul P. and C. Y. Tyan "Fuzzy Dynamic System and Fuzzy Linguistic Controller Classification," Automatica, 30, pp. 1769-1774, 1994.

[5] Zadeh, Lotfi A. "Knowledge Representation in Fuzzy Logic," IEEE Transactions on Knowledge and Data Engineering, 1(1), pp. 89-100, 1989.

[6] Zadeh, Lotfi A. PRUF - A Meaning Representation Language for Natural Languages, Memo No. UCB/ERL M77/61, University of California, Berkeley, CA, 1977.

[7] Buckley, J. J. A Fuzzy Expert System, Fuzzy Sets and Systems 20, North Holland, 1986, pp. 1-16.

[8] Antoulas, Anthanasios C. (ed.) Mathematical System Theory - The Influence of $R$. E. Kalman, Springer Verlag, ISBN 0-387-52994-2, 1991.

[9] Albus, James, and A. Meystel. Intelligent Systems A Semiotic Perspective. A conference newsletter for Multidisciplinary Professional in the Area of Intelligence, Large and Complex Systems, Knowledge Engineering, and Others., No. 1, 1996.

[10] IEEE Control Society 1997. E-Letter on Systems, Control and Signal Processing, No. 106.

[11] Antsaklis, Panos J., M. Lemmon, and J. A. Stiver. Chapter 2, Learning to be Autonomous - Intelligent Supervisory Control in Intelligent Control Systems. IEEE Press, IEEE Neural Networks Council, 1989, pp. 27-62. 
[12] Nilsson, Nils J. "Logic and Artificial Intelligence," Artificial Intelligence, 47, pp. 31-56, 1991.

[13] Bowen, R. Lai, and D. Bahler. "Lexical Imprecision in Fuzzy Constraint Networks," Proceedings of 15th International Joint Conference on Artificial Intelligence, San Jose, CA, 1992, pp. 616-621

[14] Bowen, J., R. Lai, and D. Bahler. "Fuzzy Semantics and Fuzzy Constraint Networks, " Proceedings of First IEEE International Conference on Fuzzy Systems, San Diego, CA, 1992, pp. 1009-1016.

[15] Dubois, D., H. Fargier, and H. Prade. Flexible Constraint Satisfaction with Application to Scheduling Problems. Report IIT/93-30-R. Institute de Recherche en Informatique de Toulouse, France, 1993.
[16] Tyan, C. Y., Paul P. Wang, D. R. Bahler, and S. P. Rangaswamy. "A New Methodology of Fuzzy ConstraintBased Controller Design Via Constraint-Networking Processing," IEEE Transaction on Fuzzy Systems, 4(2), pp. 166-178, 1996.

[17] Cover, Thomas M., and Joy A. Thomas. Elements of Information Theory, Wiley Series in Telecommunications, John Wiley \& Sons, Inc., New York, 1991.

[18] Chen, C. T. Introduction to Linear System Theory, Holt, Rinehard, and Winston, New York, 1970.

[19] Zadeh, Loti A., and E. Polak. System Theory, InterUniversity Electronic Series, 8, McGraw Hill, New York, 1969.

421 


\title{
Computational Embodiment: Biological Considerations
}

\author{
Kirstie L. Bellman \\ Information Technology Office, Defense Advanced Research Projects Agency \\ 3701 N. Fairfax Dr., Arlington, Virginia 22203-1714 \\ Phone: (703)696-2219, FAX: (703)696-0564, E-mail: kbellman@arpa.mil \\ Christopher Landauer \\ National Systems Group, The Aerospace Corporation \\ The Hallmark Building, Suite 187, 13873 Park Center Rd., Herndon, Virginia 20171 \\ Phone: (703) 318-1666, FAX: (703) 318-5409, E-mail: cal@aero.org
}

\begin{abstract}
Computational Embodiment is the computer implementation of principles that we believe will lead to more autonomous and self-generating behaviors that will allow software systems to exist in and interact with complex environments. We restrict our attention here to symbolic environments (MUDs), as an initial step towards understanding and constructing "interaction spaces" in which humans and computer programs can interact on an equal footing.
\end{abstract}

Our approach to constructing autonomous software systems is based on theoretical work on the structures that underly language and movement in biological systems and on the structure of constructed complex systems mediated or integrated by software.

This paper describes some of the important lessons to be learned from biological systems, and points towards some of the principles of construction of autonomous systems.

\section{Introduction}

In this paper and its companion [17], we describe an approach to constructing autonomous agents that is based on theoretical work on the organization of structures that underly language and movement and on the structure of constructed complex systems mediated or integrated by software. We are not only interested in control of such systems "to keep the system within a desired space" (which to us includes planned or computed reactions to unplanned events or behaviors), but also in instrumentation to find out what is going on inside and outside the system, in on-line management of system resources for efficiency, in negotiation among system resources, and in system archi- tectures that will help us design, build, use, maintain, and analyze such systems.

We are constructing autonomous software agents in symbolic environments called Multi-User Domains (MUDs) [20] [21] [9], using a style of "computational embodiment", which is part of our research program on interaction spaces [13].

In our view, a system is "autonomous" if it can be said to have "purposeful behavior", e.g., act independently based on internally generated intentions [5]. There are really only two classes of (difficult) requirements for effective autonomy: robustness and timeliness. Robustness means graceful degradation in increasingly hostile environments, as well as an ability to exploit unexpected aspects in the environment to one's own advantage, which to us implies a requirement for adaptability. Timeliness means that situations are recognized "well enough" and "soon enough", and that "good enough" actions are taken "soon enough". There is not necessarily any optimization here.

In this paper, we describe some fundamental theory of computational embodiment, primarily theory derived from biological systems (Section 2). In the companion paper [17], we show how our notion of wrappings as dynamic infrastructure supports the theory, and describe how the architecture we use is being implemented.

An earlier version of this paper appeared in [10], and a summary in [4].

\section{Biological Characteristics}

It is instructive to compare computational systems with biological systems, since those are the only ones we know that have any interesting complexity and variety of behavior [5]. From the very smallest and simplest organ- 
isms, biological systems have generative processes that provide varying numbers of appropriately constrained actions that retain sufficient flexibility for robust behavior. These "controlled sources of variation" require both variation (spaces within which choices are constrained) and control (of the choices), and remain one of the most difficult areas in theoretical biology. We note also that the use of language for communication among biological entities can be viewed as an extension of movement processes [3], both being essentially defined by layers of symbol systems.

In biological systems, generative processes make use of many different types of mechanisms at different levels of the system; everything from mechanisms that take advantage of side-effects and even inevitable errors (e.g., the inability to replicate DNA perfectly becomes eventually a critical source of genetic variation, leading to different phenotypes that can be acted upon by natural selection) to mechanisms that allow partial or graded behavioral responses (e.g., diffusion of neurotransmitters across synapses, different conduction rates in bundles of neurons, and modulation of chemical channels across cell membranes) to mechanisms that allow rich cognitive control over behavioral choices (and for us, at least, conscious manipulation of symbol systems). Generative processes in biological systems have dynamic behavior that can be used both for organizing behavior and creating information.

In contrast, computational systems are relatively trivial, since the absence of generative processes means that the system developers must provide these controlled sources of variation explicitly, generally using knowledge bases and computational resources [18]. This need to build all the parts and all the selection mechanisms severely limits our ability to construct large systems with many types of adaptive and coordinative behavior. One of our philosophical principles for system architecture is the analogue of the "controlled sources of variation" mentioned above: every flexibility we want in a constructed system must have a corresponding coordinative mechanism that manages the flexibility. We use the notion of layers of symbol systems to interpret action and behavior as extensions of computation [3], gaining some conceptual simplicity thereby, and though most of the hard questions still have no good answers, we have made some progress in defining essential features of complex computing systems [10] [12] [13] [14] [2] [15] [16].

Our purpose is to improve the capabilities of computerbased agents, so that they can work with humans to perform increasingly difficult cognitive tasks, such as finding relevant information, filtering and refining the presentation of information for our purposes, and organizing and executing the sequence of command steps for such machines as factory product lines or autonomous land vehicles. We believe that the growing field of agents research could make more use of the hard-won lessons from biological research and robotics about what it means to carry out intelligent functioning within ANY world (be it abstract or physical).

These four lessons include:

1. Developing "ecological niches" or portable contexts for computational agents;

2. Creating artificial "embodiment" for abstract agents;

3. Creating entities with "social" behaviors; and

4. Developing capabilities for growth and adaptivity of behaviors.

Before we expand on each of these points below, we want to emphasize here that each of these directions is being considered in current agent research [19], but often only indirectly or implicitly. We suggest that by placing the agents within an explicit context (with its own processes for being examined and modified), by giving agents a definite "shape" and body within this context, and by giving agents a means of socially responding to other agents in explicit and observable ways, we can strengthen the agents' performances by creating new and stronger information with which to evaluate our ideas of intelligent functionality by agents. We believe that ecological niches, embodiment, and social intelligence are all good starts to building agents with a much richer repertoire of mechanisms for adaptation and growth.

The grounding of agent behaviors within an observable context lets us build active, generative processes that can monitor their own behavior, change it, catalog it, and learn from it. But in order to capitalize on the creation of such processes, the agent needs (1) architectures that allow it to pull in new types of processing resources, and (2) selfreflective capabilities. In a companion paper [17], we describe our wrapping approach to dynamic infrastructure, which provides the flexibility and infrastructure that supports these two goals.

\subsection{Ecological Niches for Agents}

In nature, the capabilities and characteristics of any given animal are strongly related to the environment within which it lives. The context within which the animal must perform includes the physical world, the other species that exist within that area, the history of its own species within that environment, and its immediate social interactions with others of its own kind. Biologists continue to struggle with understanding the kinds of powerful principles that lead to the extraordinary amount of specialization and variety that has resulted. 
We need to take a lesson from biology here: there are no such things as "general purpose fish". They are all recognizably fish, and hence there are many general aspects that we can use in creating architectures and standard processes for "fishness". However, fish are specialized by living in saltwater or fresh; living near the top sunlit waters or being bottom dwellers; by surviving near fierce predators or by having few competitors; by living in schools or developing other protective mechanisms; and so forth. Eventually, we want to enumerate the corresponding "ecologically" important distinctions for "agents", e.g., do they live on the Internet or in a digital library; do they have to fight for our attention or do they have dedicated lines or other access to us; do they work as part of a temporary alliance of known (or harder, unknown) resources, or are they a simple logic or filter component in a larger process; and so forth.

The research advantages of building explicit contexts for our agents are clear. Instead of trying to align our "agents" and their behaviors to a hidden or implicit context, we can start to represent and manipulate "niches" explicitly, study the mechanisms by which our agents relate to that kind of context, study the behaviors of our agents within the explicit contexts, and begin to build principles of how we distribute information and processing capabilities between the agent and its operating environment.

Multi-User Domains (MUDs) are an interesting new kind of groupware that incorporates people into the program. MUDs have become enormously popular as games and as educational support tools over the last few years because they get the human interactions right in some fundamental sense, and because they engage our sense of "place". MUD clients and servers are easy to obtain and run (most servers and clients are free), but they usually only provide text worlds; there is little interaction with existing tools that are outside the MUD; though some have construction languages that allow complex programming, it is the usual kind of programming; and it is not very easy to access large volumes of information. We have been exploring the use of MUDs as an ecological niche for agents [13].

\subsection{Embodiment}

The next hard-won lesson from biology and robotics is that intelligent functionality needs a body, e.g., it needs a shape that defines and limits its capabilities and scope. In animals, bodies reflect both the short-term and long-term history of a species' adaptation to its ecological niche. In animals, bodies reflect the physical constraints of the environments, the historical and immediate interactions, choices, and methods of dealing with the environment (which includes others). The embodiment is the grounding of the agent and its means of relating to its environment.

Three other critical issues emerge from considering bodies.

The first is that an embodied agent reminds us that an agent always exists within its world and that it is always doing something within that world. Even "idling" is a behavior, the result of which can be quite unpleasant for prey in the real world, or simply annoying to a user waiting for performance. Therefore, we must account for the causal connection to and from the environment, that is, the effects of events in the environment on the agent, and the effects of agent activities on external objects. The agent can learn of these effects or cause others only through the specific interface between the agent and the environment. That interface must make actions available, so that the use of tools and other activities has the proper effect. There must be appropriate sensors to collect information for the agent from the environment, sufficient effectors, so the agent can change its relationship with the environment, including motion, so it can announce information to environment, make changes in the environment via the use of tools, and there should be probes, which are tasked sensory activities governed by a focus of perception.

Our computational version of the language and movement theory [3] is that there are layers of symbol systems that separately normalize external signals into interesting information spaces, so that the useful processing can take place in those spaces (this separation is the analogue of the behavior of different senses).

The second is that the body is a barrier; we cannot reach into the embodied system and turn switches when we want something to happen [2]. Commands are only suggestions, since we cannot force any particular kind of behavior on the system. We can provide compelling reasons, and try to arrange that we have programmed the system to respond appropriately, but we can't enforce it. This is one of the main differences we see between computer programs that we want to call agents and ordinary computer programs.

The third is that embodiment is an explicit representation of the "Integration Concept" for the "whole" animal. This integration concept provides a unity to the whole system. It is what turns a bag of parts into a coherently operating individual entity. A body has many parts, but works as one entity, integrated across disparate resources (unless it is sick or dysfunctional).

In our opinion, some of the most important aspects of autonomous systems revolve around this notion of "embodiment", that is, the connection of the computation to an entity in the physical world. Embodiment in this sense has several essential properties:

- a sense of environment: the environment has prop- 
erties and contains tools, objects, and other agents, and makes certain actions available

- a sense of presence: there is a causal connection to and from the environment, so that the use of tools and other activities have a direct impact on it

- a sense of time: it is important for the agent to remember the history of what has gone before, to recognize certain kinds of event patterns, and to make predictions

- a sense of place: what happens and what exists take place in some locality, which has a notion of geography, connectivity or connection, distance, and space

- a sense of will: autonomous means self-governing, so an autonomous system must generate its own goals, and exhibit a sense of purpose and intention

- a sense of self: an autonomous system needs to have a notion of its own resources, abilities, and internal state

- a sense of perspective: in order to make effective choices and recognize opportunities and threats, an autonomous system needs a viewpoint of itself in its environment

These qualities are analogous to the well-known "where, when, what, who" of descriptive reporting, the "why" of interpretation, and the "me, here" of local viewpoints. We have organized them in this way to show the analogies.

Current research work on MUDs includes ways of embodying intelligent agents and studying them in a context where humans and agents can interact. A number of researchers under the DARPA CAETI (Computer-Aided Education and Training Initiative) program [1] have been embodying evaluation agents, intelligent tutors, librarians, receptionists, and guide agents as part of research in the use of MUDs and agents in education.

\subsection{Social Intelligence}

Part of the context for any animal is the presence of other individuals, both competing species and members of its own species. If we imagine the jungle of the Internet, we can well envision the situation in which one's own agents will have to deal not only with well-behaved conspecific agents, but all sorts of tools with different degrees of intelligence and behavior. They may even have to deal with malicious software or rogue agents.

Furthermore, even if we have agents that deal only with carefully selected others, we need to develop much better ideas of what it means to communicate and behave cooperatively among agents. Many social scientists and now a few computer scientists [6] [8] have been pointing out for some time that cooperative behaviors - shared goals, work, understandings, communications - do not occur inside a participant alone. In addition to the interpretations of the acts or symbols within each participant alone, there is also an act of negotiation and agreement among participants as to the meaning of acts (including speech acts). These mutually-defined meanings are necessary in order for the group to interact even on the simplest levels, e.g., to determine what constitutes the desired results of even individually determined actions, or what constitutes that an action has been "done" in order for another agent to proceed. Clearly these "meanings" can be built in (and in fact usually are in most agent interactions, in the form of stopping rules or predefined preconditions for further activity), but eventually we want to have agents that are "smart" enough to act more autonomously and adaptively.

A key aspect of being able to negotiate among agents turns out to be the ability to share a common context and to observe the other behaviors within that context. The notion of niche allows us to create places within which an agent's behaviors are visible and interpretable both by itself and by others. In an immediate practical sense, this increases the "bandwidth" of information that others can process about the agent; instead of just relying on messages and self-report, other agents can observe and infer usable information from the agent's behavior. Eventually, we want to use such behavioristic information to form the basis for a shareable semantics - a real semantics, grounded in shared experience within this niche - for human-agent communication.

\subsection{Growth, Adaptation, and Develop- ment}

Lastly, Adaptation, like all other sets of mechanisms, will be itself specialized to different worlds. We consider adaptation to have two somewhat different aspects: one is the construction and selection of variation spaces within which the adaptations may occur, and the other is the selection of actions within those spaces. Biological systems seem to be able to generate both of these kinds of process as needed, at least to a limited extent. In order that the system can respond to a wide dynamic range of possible environmental conditions, a very broad range of potential behaviors must be available to the system. We call these collectively the "variation space" for the problem at hand, and regard the problem of constructing the appropriate variation spaces as the fundamental part of adaptation. Making choices once the choices are presented is not as hard. 
There must be processes that construct variation spaces within which adaptation can take place, and decision processes that can make adaptive choices within those spaces. We want algorithms for generating appropriate variation spaces and decision processes. We want change processes to make evolutionary behavior automatic. We want some changes to be directed by history, some by environment, and some by intention.

We claim that the purpose of adaptation in systems is to allow them to be effective in uncertain dynamic environments, that this purpose argues for complex heterogeneous systems, and that regardless of the algorithms used for adaptation, the rest of the organization must have certain properties for adaptation to work. Adaptation requires flexibility; every flexibility must have a corresponding coordination mechanism, and every such pairing can use different methods for adaptation. In fact, every such pairing must use different methods for adaptation, at least superficially, since their context and scope are different. This argument leads directly to the requirement for multiplicity in $A L L$ aspects of the system, including the basic adaptation algorithms.

Adaptation of external interaction behavior (input collection, motion, and communication), using internal representations of that behavior and its effects, is important for real-world embedding, because it lets the system adjust the dynamic range of its sensors to the current environmental characteristics. Adaptation of internal representations (notations, interpreters, and other processes) is the same process, applied to the internal processing in the system.

\subsection{The Use of Symbols}

Biological entities use symbols (mainly chemical) and their interpretations (mainly through chemical processes) to represent and transmit knowledge of their interior and exterior context [3]. Over time, they somehow also make interpretations and distinctions at increasing depths of detail, and use previous distinctions to define contexts that allow them to focus more and more precisely. Eventually, new symbols arise in context via their use in interactions. Their meaning is usually inferred, not explicitly transmitted. The general success of this process must mean that the assumed shared experience that underlies interaction is sufficient. With computers it will be different; there is no reason to believe that they will ever have the same shared experience, so we must examine very carefully how we can provide the appropriate replacement for the context and knowledge structures supplied by that experience [15].

In particular, there is a fundamental difference in the way symbols are grounded. Biological systems have symbols grounded in the "real world". Computational systems generally have their symbols grounded in a small discrete system. We want to construct complex heterogeneous computing systems that have increasingly interesting and robust complexities of behavior, and we believe that this goal requires an advance in computational practice: changing the ways in which symbols are represented and used in computers. We must therefore define computationally viable theories of symbols and representation, that is, ways for programs to work more effectively with the meanings that underlie the labels used in them, given the relatively primitive operations on those labels currently available to computing systems. We believe that these theories cannot be restricted to structures built from indivisible units, and that we therefore need some new developments in Mathematics to treat this new kind of semiotic foundation for systems [11] [16].

\section{Prospects}

Autonomous systems must be complex systems, with an ever-increasing repertoire of possible behaviors and processes for selecting them, fallback choices to account for incorrect situation estimation, and quick partial solutions to reduce decision time. All activity is situated, strongly dependent on context, and there need to be different decision processes in different situations. This flexibility requires an architecture in which many parts of the system are infrastructure, organizing other parts of the system to identify and address problems, and monitoring their behavior [7]. The ability to reflect on one's own behavior (and modify it) is one of the main keys to effective autonomy.

We believe that with proper attention to niches, embodiment, social behaviors, and architectures supporting adaptive and reflective behaviors, we can build agents that are more useful partners in an increasingly complex information environment.

\section{References}

[1] Kirstie L. Bellman, CAETI (Computer-Aided Education and Training Initiative), Home Page available on the Web at URL "http: //triton.dmso.mil /CAETI" (availability last checked 18 January 1997), last changed 26 November 1996

[2] Kirstie L. Bellman, "When Intelligence is in Control", pp. 10-12 in Intelligent Systems: A Semiotic Perspective, Proceedings of the 1996 International Multidisciplinary Conference, Volume I: Theoretical Semiotics, Workshop on Biologically Inspired Com- 
plex Systems, 20-23 October 1996, Gaithersburg, Maryland (1996)

[3] Kirstie L. Bellman and Lou Goldberg, "Common Origin of Linguistic and Movement Abilities", American Journal of Physiology, Volume 246, pp. R915-R921 (1984)

[4] Kirstie L. Bellman, Christopher Landauer, "A Note on Improving the Capabilities of Software Agents", pp. 512-513 in Proceedings AA'97: The First International Conference on Autonomous Agents, 5-8 February 1997, Marina Del Rey (1997)

[5] Kirstie L. Bellman and Donald O. Walter, "Biological Processing", American Journal of Physiology, Volume 246, pp. R860-R867 (1984)

[6] Alan H. Bond, Les Gasser (eds.), Readings in Distributed Artificial Intelligence, Morgan Kaufmann (1988)

[7] Barbara Hayes-Roth, Karl Pfleger, Philippe Lalanda, Philippe Morignot, Marka Balabanovic, "A Domain-Specific Software Architecture for Adaptive Intelligent Systems", IEEE Transactions on Software Engineering, Vol. SE-21, No. 4, pp. 288-301 (April 1995)

[8] Carl Hewitt, Jeff Inman, "DAI Betwixt and Between: From "Intelligent Agents" to Open Systems Science", IEEE Transactions on Systems, Man, and Cybernetics, Volume SMC-21, No. 6, pp. 1409-1419 (November 1991)

[9] Christopher Landauer, The MUDpage, file accessible via the World-Wide Web at URL "http: //tinylondon.ucsd.edu/ cal/MUDpage.html", first version (December 1994), updated (23 January 1995) (availability last checked 7 July 1996)

[10] Christopher Landauer, "Constructing Autonomous Software Systems (Extended Abstract)", Workshop on Biologically Inspired Autonomous Systems: Computation, Cognition, and Action, 4-5 March 1996, Durham, N.C. (March 1996)

[11] Christopher Landauer, Kirstie L. Bellman, "New Mathe-

matical Foundations for Computer Science", Initiative announcement available via anonymous ftp from aerospace.aero.org, in directory /pub/newmath, in file workbook.html, and from WWW at URL ftp://www.cs.umd.edu/ cal/newmath.html (original July 1994), revision 1.4 (February 1995) (availability last checked 23 June 1996)

[12] Christopher Landauer, Kirstie L. Bellman, "Knowledge-Based Integration Infrastructure for Complex Systems", International Journal of Intelligent Control and Systems, Volume 1, No. 1, pp. 133-153 (1996)

[13] Christopher Landauer, Kirstie L. Bellman, "Integration Systems and Interaction Spaces", pp. 161-178 in Proceedings of the First International Workshop on Frontiers of Combining Systems, 26-29 March 1995, Munich (March 1996)
[14] Christopher Landauer, Kirstie L. Bellman, "Constructed Complex Systems: Issues, Architectures and Wrappings", pp. 233-238 in Proceedings EMCSR 96: Thirteenth European Meeting on Cybernetics and Systems Research, Symposium on Complex Systems Analysis and Design, 9-12 April 1996, Vienna (April 1996)

[15] Christopher Landauer, Kirstie L. Bellman, "Semiotics of Constructed Complex Systems", pp. 35-40 in Intelligent Systems: A Semiotic Perspective, Proceedings of the 1996 International Multidisciplinary Conference, Volume I: Theoretical Semiotics, Workshop on Intelligence in Constructed Complex Sys. tems, 20-23 October 1996, Gaithersburg, Maryland (1996)

[16] Christopher Landauer, Kirstie L. Bellman, "Mathematics and Linguistics", pp. 153-158 in Intelligent Systems: A Semiotic Perspective, Proceedings of the 1996 International Multidisciplinary Conference, Volume I: Theoretical Semiotics, Workshop on New Mathematical Foundations for Computer Science, 20-23 October 1996, Gaithersburg, Maryland (1996)

[17] Christopher Landauer, Kirstie L. Bellman, "Computational Embodiment: Software Architecture", (to appear) in Proceedings of ISAS'g7: Intelligent Systems and Semiotics 1997, 23-25 September 1997, Gaithersburg, Md. (1997)

[18] George F. Luger, with Peder Johnson, Carl Stern, Jean E. Newman, and Ronald Yeo, Cognitive Science: The Science of Intelligent Systems, Academic Press (1994)

[19] Pattie Maes (ed.), Special Issues of Robotics and Autonomous Systems, Volume 6, Nos. 1 and 2 (June 1990); reprinted as Pattie Maes (ed.), Designing Autonomous Agents: Theory and Practice from Biology to Engineering and Back, MIT / Elsevier (1993)

[20] Mike O'Brien, "Playing in the MUD", Ask Mr. Protocol Column, SUN Expert, Volume 3, No. 5, pp. 19-20, 23, 25-27 (May 1992)

[21] R. Riner and J. Clodius, "Simulating Future Histories", Anthropology and Education Quarterly (Fall 1994) 


\title{
The Neurodynamics of Intentionality in Animal Brains Provides the Basis of Intelligent Behavior
}

\author{
Walter J Freeman \\ Department of Molecular and Cell Biology \\ University of California \\ Berkeley CA 94720-3200 \\ http://sulcus.berkeley.edu wfreeman@socrates.berkeley.edu
}

\begin{abstract}
Intelligent behavior is characterized by flexible and creative pursuit of endogenously defined goals. It has emerged in humans through the stages of evolution that are manifested in the brains and behaviors of the vertebrate series. Intentionality is a key concept by which to link brain dynamics to goal-directed behavior. The archetypal form of intentional behavior is an act of observation into time and space, by which information is sought for the guidance of future action.
\end{abstract}

The neurodynamics of intentionality in the process of observation

The first step in pursuit of an understanding of intentionality is to ask, what happens in brains during an act of observation? This is not a passive receipt of information from the world. It is a purposive action by which an observer directs the sense organs toward a selected aspect of the world and interprets the resulting barrage of sensory stimuli. The concept of intentionality has been used to describe this process in different contexts, since its first use by Aquinas 700 years ago. The three salient characteristics of intentionality as it is treated here are (a) intent or directedness toward some future state or goal, (b) wholeness, and (c) unity (Freeman 1995). These three aspects correspond to use of the term in psychology with the meaning of purpose, in medicine with the meaning of mode of healing and integration of the body, and in analytic philosophy with the meaning of the way in which beliefs and thoughts are connected with ("about") objects and events in the world.

Intent comprises the endogenous initiation, construction, and direction of behavior into the world. It emerges from brains. Humans and animals select their own goals, plan their own tactics, and choose when to begin, modify, and stop sequences of action, and humans at least are subjectively aware of themselves acting. Unity appears in the combining of input from all sensory modalities into Gestalten, in the coordination of all parts of the body, both musculoskeletal and autonomic, into adaptive, flexible, yet focused movements. Subjectively, unity appears in the awareness of self. Wholeness is revealed by the orderly changes in the self and its behavior that constitute the development and maturation of the self, within the constraints of its genes and its material, social and cultural environments. Subjectively, wholeness is revealed in the remembrance of self through a lifetime of change.

\section{The limbic system is the organ of intentional behavior}

Brain scientists have known for over a century that the necessary and sufficient part of the vertebrate brain to sustain minimal intentional behavior is the ventral forebrain, including those components that comprise the external shell of the phylogenetically oldest part of the forebrain, the paleocortex, and the deeper lying nuclei with which the cortex is connected. These components suffice to support remarkably adept patterns of intentional behavior, in dogs after all the newer parts of the forebrain have been surgically removed (Goltz 1892), and in 
rats with neocortex chemically inactivated by spreading depression (Bures et al. 1974). Intentional behavior is severely altered or absent after major damage to the basal forebrain, as manifested most clearly in Alzheimer's disease.

Phylogenetic evidence comes from observing intentional behavior in salamanders, which have the simplest of the existing vertebrate forebrains (Herrick 1948; Roth 1987). The three parts are sensory (which, as in small mammals, is predominantly olfactory), motor, and associational. The latter part contains the primordial hippocampus with its associated septoamygdaloid and striatal nuclei, which are identified in higher vertebrates as the locus of the functions of spatial orientation (the "cognitive map") and temporal integration in learning (the organization of long and short term memory). These processes are essential, inasmuch as intentional action takes place in to the world, and even the simplest action, such as searching for food or evading predators, requires an animal to know where it is with respect to its world, where its prey or refuge is, and what its spatial and temporal progress is during sequences of attack or escape .

\section{Neurodynamics of intentionality}

The crucial question for neuroscientists is, how are the patterns of neural activity that sustain intentional behavior created in brains? The answer is provided by studies of electrical activity of the primary sensory cortices of animals that trained to respond to conditioned stimuli (Freeman 1975, 1992, 1995; Barrie et al. 1996; Kay et al. 1996). Cortical neurons are selectively activated by sensory receptors to generate microscopic activity. By interactions among the cortical neurons a population forms that "binds" their activity into a macroscopic pattern (Haken 1983; Gray 1994; Hardcastle 1994; Singer and Gray 1995). The brain activity patterns that are seen in electroencephalograms (EEGs) reveal the macroscopic brain states that are triggered or induced by the arrival of stimuli.
These brain states are not representations of stimuli, nor are they the simple effects caused by stimuli. Each learned stimulus serves to elicit the construction of a pattern that is shaped by the synaptic modifications among cortical neurons from prior learning, and also by the brain stem nuclei that bathe the forebrain in neuromodulatory chemicals. It is a dynamic action pattern that creates and carries the meanings of stimuli for the animal. It reflects the individual history, present context, and expectancy, corresponding to the unity and the wholeness of the intentionality. The patterns created in each cortex are unique to each animal. All sensory cortices transmit their signals into the limbic system, where they are integrated with each other over time, and the resultant integrated meaning is transmitted back to the cortices in the processes of selective attending, expectancy, and the prediction of future inputs.

The same kinds of EEG activity as those found in the sensory and motor cortices are found in various parts of the limbic system. This discovery indicates that the limbic system also has the capacity to create its own spatiotemporal patterns of neural activity. They are related to past experience and convergent multisensory input, but they are self-organized. The limbic system provides a neural matrix of interconnections, that serves to generate continually the neural activity that forms goals and directs behavior toward them. EEG evidence shows that the process occurs in discontinuous steps, like frames in a motion picture. Each step follows a dynamic state transition, in which a complex assembly of neuron populations jumps suddenly from one spatiotemporal pattern to the next, as the behavior evolves. Being intrinsically unstable, the limbic system continually transits across states that emerge, spread into other parts of the brain, and then dissolve to give rise to new ones. Its output controls the brain stem nuclei that serve to regulate its excitability levels, implying that it regulates its own neurohumoral context, enabling it to respond with equal facility 
to changes that call for arousal and adaptation or rest and recreation, both in the body and the environment. It is the neurodynamics of the limbic system, assisted by other parts of the forebrain such as the frontal lobes, that initiates the novel and creative behavior seen in search by trial and error.

The limbic activity patterns of directed arousal and search are sent into the motor systems of the brain stem and spinal cord. Simultaneously, patterns are transmitted to the primary sensory cortices, preparing them for the consequences of motor actions. This process has been called "reafference" (von Holst and Mittelstaedt 1950; Freeman 1995), "corollary discharge" (Sperry 1950), "focused arousal" (Sheer 1989), and "preafference" (Kay et al. 1996). It sensitizes sensory systems to anticipated stimuli prior to their expected times of arrival Sensory cortical constructs consist of brief staccato messages to the limbic system, which convey what is sought and the result of the search. After multisensory convergence, the spatiotemporal activity pattern in the limbic system is up-dated through temporal integration in the hippocampus. Between sensory messages there are return up-dates from the limbic system to the sensory cortices, whereby each cortex receives input that has been integrated with the output of the others, reflecting the unity of intentionality. Everything that a human or an animal knows comes from this iterative circular process of action, reafference, perception, and up-date. It is done by successive frames that involve repeated state transitions and selforganized constructs in the sensory and limbic cortices. This neurodynamic system is defined here as the "limbic self" in the brain of an individual, where intentional behavior is created, with help from other parts of the forebrain.

An act of observation comprises Aquinas' intentional action of "stretching forth" and learning from the consequences, and the existential "action-perception cycle" of
Merleau-Ponty (1942). It corresponds to Piaget's (1930) cycle of "action, assimilation, and adaptation" in the sensorimotor stage of childhood development. His postulated sequences of equilibrium, disequilibrium, and reequilibration conform to state transitions in brain dynamics, which initiate and sustain action, construct dynamic patterns in the sensory cortices, and up-date the limbic patterns by modifying synapses in the learning that follows the sensory consequences of intended actions. For Piaget, cause and effect are chains of events that have the appearance of linkage corresponding to the unfolding experience of that exploration, by which a child is trying to make sense of its world by manipulating objects in it. The origin of causal inference is buried deeply in the pre-linguistic exploratory experience of each of us. It is not easily accessed by cognitive analysis or introspection.

We are all aware of our acts of observation. It is partly by expectation of what we are looking for through reafference, partly by perceiving the changes that our actions make in the dispositions of our bodies through proprioception, and partly by our selection of stimuli from the environment through exteroception. We perceive our intentional acts as the "causes" of changes in our perceptions, and the subsequent changes in our bodies as "effects" (Freeman 1995). If this hypothesis of limbic dynamics is correct, then everything that we know we have learned through the action-perception cycle, and the iterative state changes by which it is produced in brains.

\section{Characteristics of brain states}

The "state" of the brain is a description of what it is doing in some specified time period. A state transition occurs when the brain changes and does something else. For example, locomotion is a state, within which walking is a rhythmic pattern of activity that involves large parts of the brain, spinal cord, muscles and bones. The entire neuromuscular system 
changes almost instantly with the transition to a pattern of jogging or running. Similarly, a sleeping state can be taken as a whole, or divided into a sequence of slow wave and REM stages. Transit to a waking state can occur in a fraction of a second, whereby the entire brain and body shift gears, so to speak. The state of a neuron can be described as active and firing or as silent, with sudden changes in the firing manifesting state transitions. Populations of neurons also have a range of states, such as slow wave, fast activity, seizure, or silence. The science of dynamics is designed to study states and their transitions.

The most critical question to ask about a state is its degree of stability or resistance to change. Evaluation is done by perturbing an object or a system (Freeman 1975). For example, an object like an egg on a flat surface is unstable, but a coffee mug is stable. A person standing on a moving bus and holding on to a railing is stable, but someone walking in the aisle is not. If a person regains his chosen posture after each perturbation, no matter in which direction the displacement occurred, that state is regarded as stable, and it is said to be governed by an attractor. This is a metaphor to say that the system goes ("is attracted") to the state through an interim state of transiency. The range of displacement from which recovery can occur defines the basin of attraction, in analogy to a ball rolling to the bottom of a bowl. If the perturbation is so strong that it causes concussion or a broken leg, and the person cannot stand up again, then the system has been placed outside the basin of attraction, and a new state supervenes with its own attractor and basin.

Stability is always relative to the time duration of observation and the criteria for what is chosen to be observed. In the perspective of a lifetime, brains appear to be highly stable, in their numbers of neurons, their architectures and major patterns of connection, and in the patterns of behavior they produce, including the character and identity of the individual that can be recognized and followed for many years. Brains undergo repeated transitions from waking to sleeping and back again, coming up refreshed with a good night or irritable with insomnia, but still, giving the same persons as the night before. Personal identity is usually quite stable. But in the perspective of the short term, brains are highly unstable. Thoughts go fleeting through awareness, and the face and body twitch with the passing of emotions. Glimpses of their internal states of neural activity reveal patterns that are more like hurricanes than the orderly march of symbols in a computer. Brain states and the states of populations of neurons that interact to give brain function, are highly irregular in spatial form and time course. They emerge, persist for a small fraction of a second, then disappear and are replaced by other states.

In using dynamics we approach the problem by defining three kinds of stable state, each with its type of attractor. The simplest is the point attractor. The system is at rest unless perturbed, and it returns to rest when allowed to do so. As it relaxes to rest, it has the history of what happened, but that history is lost after convergence to rest. Examples of point attractors are silent neurons or neural populations that have been isolated from the brain, and also the brain that is depressed into inactivity by injury or a strong anesthetic, to the point where the EEG has gone flat. A special case of a point attractor is noise. This state is observed in populations of neurons in the brain of a subject at rest, with no evidence of overt behavior. The neurons fire continually but not in concert with each other. Their pulses occur in long trains at irregular times. Knowledge about the prior pulse trains from each neuron and those of its neighbors up to the present fails to support the prediction of when the next pulse will occur. The state of noise has continual activity with no history of how it started, and it gives only the expectation that its amplitude and other statistical properties will persist unchanged. 
A system that gives periodic behavior is said to have a limit cycle attractor. The classic example is the clock. When it is viewed in terms of its ceaseless motion, it is regarded as unstable until it winds down, runs out of power, and goes to a point attractor. If it resumes its regular beat after it is re-set or otherwise perturbed, it is stable as long as its power lasts. Its history is limited to one cycle, after which there is no retention of its transient approach in its basin to its attractor. Neurons and populations rarely fire periodically, and when they appear to do so, close inspection shows that the activities are in fact irregular and unpredictable in detail, and when periodic activity does occur, it is either intentional, as in rhythmic drumming, or pathological, as in nystagmus and Parkinsonian tremor.

The third type of attractor gives aperiodic oscillation of the kind that is observed in recordings of EEGs. There is no one or small number of frequencies at which the system oscillates. The system behavior is therefore unpredictable, because performance can only be projected far into the future for periodic behavior. This type was first called "strange"; it is now widely known as "chaotic". The existence of this type of oscillation was known to Poincaré a century ago, but systematic study was possible only recently after the full development of digital computers. The best known simple systems with chaotic attractors have a small number of components and a few degrees of freedom, as for example, the double-hinged pendulum, and the dripping faucet. Large and complex systems such as neurons and neural populations are thought to be capable of chaotic behavior, but proof is not yet possible at the present level of developments in mathematics.

The discovery of chaos has profound implications for the study of brain function (Skarda and Freeman 1987). A chaotic system has the capacity to create novel and unexpected patterns of activity.
It can jump instantly from one mode of behavior to another, which manifests the facts that it has a collection of attractors, each with its basin, and that it can move from one to another in an itinerant trajectory (Tsuda 1996). It retains in its pathway across its basins its history, which fades into its past, just as its predictability into its future decreases. Transitions between chaotic states constitute the dynamics we need to understand how brains do what they do.

\section{The cortical state transition is the elemental step of intentionality}

Systems such as neurons and brains that have multiple chaotic attractors also have point and limit attractors. A system that is in the basin of one of its chaotic attractors is legendary for the sensitivity to what are called the "initial conditions". This refers to the way in which a simple system is placed into the basin of one of its attractors. If the basin is that of a point or a limit cycle attractor, the system proceeds predictably to the same end state. If the basin leads to a chaotic attractor, the system goes into ceaseless fluctuation, as long as its energy lasts. If the starting point is identical on repeated trials, which can only be assured by simulation of the dynamics on a digital computer, the same aperiodic behavior appears. This is why chaos is sometimes called "deterministic". If the starting point is changed by an arbitrarily small amount, although the system is still in the same basin, the trajectory is not identical. If the difference in starting conditions is too small to be originally detected, it can be inferred from the unfolding behavior of the system, as the difference in trajectories becomes apparent. This observation shows that a chaotic system has the capacity to create information in the course of continually constructing its own trajectory into the future.

In each sensory cortex there are multiple basins corresponding to previously learned classes of stimuli, as well as to the unstimulated state. This chaotic 
prestimulus state of expectancy establishes the sensitivity of the cortex, so that the very small number of sensory action potentials evoked by an expected stimulus can carry the cortical trajectory into the basin of an appropriate attractor. The stimulus is selected by the limbic brain through orientation of the sensory receptors by sniffing, looking, and listening. The basins of attraction are shaped by limbic input to sensitize the reception of a desired class of stimuli. The web of synaptic connections that was modified by prior learning contributes to the formation of basins and to the attractors. This is an act of observation.

\section{Acknowledgement}

This work was supported by a grant from the National Institute of Mental Health, and from the Office of Naval Research

\section{References}

Barrie JM, Freeman WJ, Lenhart M (1996) Modulation by discriminative training of spatial patterns of gamma EEG amplitude and phase in neocortex of rabbits. Journal of Neurophysiology 76: 520-539.

Bures J, Buresová O, Krivánek J (1974) The Mechanism and Applications of Leão's Spreading Depression of Electroencephalographic Activity. New York: Academic Press.

Freeman WJ [1975] Mass Action in the Nervous System. New York: Academic Press.

Freeman WJ [1992] Tutorial in Neurobiology. International Journal of Bifurcation and Chaos 2: 451-482.

Freeman WJ [1995] Societies of Brains. A Study in the Neuroscience of Love and Hate. Hillsdale NJ, Lawrence Erlbaum Associates.

Goltz FL (1892) Der Hund ohne Grosshirn. Siebente Abhandlung über die Verrichtungen des Grosshirns. Pflügers Archiv 51: 570-614.

Gray CM (1994) Synchronous oscillations in neuronal systems: mechanisms and functions. Journal of Comparative Neuroscience 1: 11-38.
Haken H (1983) Synergetics: An Introduction. Berlin: Springer.

Hardcastle VG (1994) Psychology's binding problem and possible neurobiological solutions. Journal of Consciousness Studies 1: 66-90.

Herrick CJ (1948) The Brain of the Tiger Salamander. Chicago IL: University of Chicago Press.

Kay LM, Lancaster L, Freeman WJ (1996) Reafference and attractors in the olfactory system during odor recognition. International Journal of Neural Systems 7: 489-496.

Merleau-Ponty M (1942) The Structure of Behavior (AL Fischer, Trans.). Boston: Beacon Press (1963).

Piaget J (1930) The child's conception of physical causality. New York: Harcourt, Brace. p. 269

Roth G (1987) Visual Behavior in Salamanders. Berlin: Springer-Verlag

Sheer DE (1989) Sensory and cognitive 40-Hz event-related potentials: Behavioral correlates, brain function, and clinical application. Brain Dynamics. Basar E, Bullock TH (eds.) Berlin: Springer-Verlag.

Singer W, Gray CM (1995) Visual feature integration and the temporal correlation hypothesis. Annual Review of Neuroscience 18: 555-586.

Skarda CA, Freeman WJ (1987) How brains make chaos in order to make sense of the world. Behavioral and Brain Sciences 10: 161-195.

Sperry RW (1950) Neural basis of the spontaneous optokinetic response. Journal of Comparative Physiology 43: 482-489.

Tsuda I (1996) A new type of selforganization assocated with chaotic dynamics in neural networks. Internaltional Journal of Neural Systems 7: 451-459.

von Holst E \& Mittelstadt H (1950) Das Reafferenzprinzip Naturwissenschaften 37: 464-476. 


\title{
Hypermedia Integration of Information Resources for Plant Operations
}

\author{
Lefteri H. Tsoukalas ${ }^{*}$ and Robert E. Uhrig \\ * Purdue University \\ West Lafayette, Indiana 47907-1290 \\ University of Tennessee, Knoxville, TN 37996-2300 and \\ Oak Ridge National Laboratory, Oak Ridge, TN 37831-6005
}

\begin{abstract}
Computer hypermedia technologies offer significant possibilities for integrating data, information and multifaceted knowledge resources abounding in existing and next generation plant operations. A hypermedia system may be viewed as a set of nodes and links allowing non-linear access to plant information residing in computers regardless of format. The process of accessing information in hypermedia systems is known as navigation. After reviewing the state of the art we present quantitative criteria for the development of hypermedia databases and a fuzzy graph-based methodology for navigating the large information spaces involved in nuclear plant operations. In the developed methodology membership functions embodying contextdependent criteria provide application-specific tools for navigation. The methodology is illustrated through numerical examples and a HyperCard-based prototypical system for monitoring special material in a next generation plant.
\end{abstract}

KEYWORDS: hypermedia databases, fuzzy graphs, navigation systems, information systems

\section{INTRODUCTION}

Among the various aspects of plant operations, the maintenance and upgrade of stored records and information resources is probably the least urgent on a daily basis, yet one of the most important contributors to life long performance and efficiency. Indeed the outcome of records and other information resources maintenance and utilization has far reaching implications for the efficiency of operations, the integrity of safety systems, the effectiveness of training and the overall performance of plant technical, operations and managerial personnel. Hypermedia and the related field of virtual environments, once thought of as science fiction-like technologies, are opening new ways for improving a variety of tasks including, but not limited to, enhancement of training, outage planning, and component inspections in hard to reach areas. ${ }^{1,2}$ In essence, hypermedia allows for a more flexible encoding and utilization of plant records and human expertise through the integration of textual, visual and audible data, with a variety of plant information and knowledge resources residing in networked computers.
It is technically feasible and possibly advantageous that records such as design drawings, scientific and engineering calculations, safety analysis reports, technical specifications, purchasing and accounting data, and audit reports, although stored in different formats and platforms, can be integrated and transparently available to users having a diversity of backgrounds and plant functions. For example, through a plant hypermedia system, a safety system may be viewed not only as a physical object, but a programmable computer object possessing a number of distinct, yet equally valid representations, e.g., relations with neighboring objects (pipes, valves etc.), technical specifications, manufacturing data, photos, maintenance records, location video, or acoustic data signatures.

The driving force for hypermedia integration comes from the rapid ongoing advances in distributed (networked) computer systems transparently sharing memory and software resources. Computer storage in the order of Gigabyte $\left(10^{9}\right.$ bytes) and network communication speeds in the order of Mbauds $\left(10^{6}\right.$ bits/sec) are at present readily available; the world wide web and hypermedia browsers such as Mosaic, Netscape and Internet Explorer are familiar tools in the vast global networks of computers opening new commercial activities around the globe. Hence, it is now apparent that distributed hypermedia databases will be of great benefit in, amongst others, tracking the entire history of plant components, enhancing training, improving planning and the overall conduct of plant operations.

In hypermedia information is organized as networks of nodes connected by links. A node is the smallest unit of meaningful information to which a hypermedia link is made. For example, a piece of text, a photograph, a computer screen, a 2-minute video segment, and a maintenance record of an emergency diesel generator, can all be thought of as nodes. Nodes may contain text, graphics, audio, video and software for operating on numerical and/or symbolic data. A link is a connection between information stored in two different nodes. A group of links that have related functions is referred to as a link family, and most hypermedia systems feature link buttons (link icons) which can be arbitrarily embedded within the content material.

Yet, while it is feasible to store in hypermedia databases, a multitude of design, maintenance and 
operations data, as well as plant information and knowledge to an unprecedented extent, user disorientation and possibly confusion become the limiting factors in their efficient utilization. Increasing the number of connections, or links, increases the possibility that a user will get lost in irrelevant information; a situation proverbially known as the "lost in hyperspace problem." User disorientation may be particularly severe for large scale applications such as those involved in nuclear plants and given the critical nature or many plant operations, it may actually be a prohibitive bottleneck as far as the field deployment of the technology is concerned.

\section{REVIEW OF HYPERMEDIA}

Hypermedia allows for easy and intuitive access to documents and programs by linking dispersed yet interrelated information throughout a document, a program or a series of documents and programs. Conventional information structures use a hierarchical or sequential logic, i.e., there is a single linear sequence defining the order in which text is to be accessed. The typical design report of a plant provides an example of a hierarchical structure. The report has several chapters; each chapter is broken to several numbered sections; each section has one or more numbered subsections; each subsection may have several numbered sub-subsections and so on. Figure 1 illustrates the way information is structured in Chapter 10 Materials Handling System of a Plant Design Report. To access and understand 10,3 Plant Product, we have to proceed from 10.2 Processing, and to understand the description of the 10.2.2 Waste Safety Evaluation, one has to proceed from 10.2.1 Waste Product Descriptions through 10.2.1.1, 10.2.1.2 and 10.2.1.3, solid, liquid, and gaseous wastes respectively. There are two special articles (shown within the circular nodes) in the information structure of figure 1: the Table of Contents (TOC) and the References (REFS). TOC may be considered an index node (a node pointing to other nodes), while $R E F S$ may be a reference node (a node pointed to by several other nodes).

Contrary to the hierarchical structure of conventional information structures, hypermedia information is structured in a non-sequential or associational manner. An associational information structure allows a user to go from a node to any other node directly or through intermediate nodes without having to observe the linear sequence implicit in figure 1. Hypermedia presents several options to the user who determines which one of them to follow largely based on specific informational needs. This nonsequential organization enables one to store and retrieve information in a more flexible manner.

Research and development in the field of hypermedia have made considerable progress in the past decade, steadily moving towards more complex applications including, but not limited to, pedagogical projects in numerous fields, ${ }^{6,7,8,9}$ managing and presenting documentation, ${ }^{10,11}$ learning and classroom applications, ${ }^{12,13}$ information retrieval and indexing, ${ }^{14.15}$ industrial applications, ${ }^{1,16}$ electronic journal publishing, ${ }^{17}$ and mass media applications. ${ }^{18,19,20,21,22,23}$ In several successful projects, hypermedia systems integrating an impressive corpus of material related to academic fields of studies and encyclopedic knowledge have been developed. Results from a number of evaluations in different learning environments have demonstrated that hypermedia posses an important edge for accelerating learning and for supporting new types of learning and teaching (see ref. 6).

In Germany, hypermedia systems supporting training are currently in the process of being integrated into existing training systems at several Power Stations. Since 1994, various hypermedia systems have been developed and applied to Computer Based Training (CBT) for plant personnel in subjects such as, thermodynamics, electrical engineering, and operations in specific plants. For example, a hypermedia learning program about water hammers in pipes integrating text, video, animation, graphics and sound has been developed and reported in ref. 1. The system very clearly demonstrates to users how a high wave blocks the crosssection of a pipe and the resulting liquid plug is accelerated by the flow of steam. Video sequences provide laboratory visualization of the phenomenon occurring in a glass pipe and photographs of pipes damaged by plug acceleration make direct connection with actual work situations and point to the significance of the problem in malfunctions that have actually occurred in several plant environments. The authors of the system have included a large body of fundamental scientific knowledge and technical expertise within the system including possible ways of preventing damage, user access to system diagrams and operating manuals.

Hypermedia systems feature flexible information structures and offer great browsing freedom for the users. Yet, the advantages of flexibility and freedom come with a potential cost: user disorientation and confusion or what is popularly referred to as the problem of being "lost in hyperspace." Previous attempts to address this problem have concentrated on improving the user interface through multiple windows, maps, and tours or path mechanisms. Unfortunately, multiple windows help only in a limited localized way, and maps are hard to design and maintain in nuclear power plant applications when hundreds or thousands of nodes may be involved.

Another attempt to address the user disorientation issue is textual analysis, where the designers of the hypermedia system statistically analyze word and concept frequencies in order to index nodes by significant terms, facilitating retrieval and navigation among relevant documents. ${ }^{24}$ Textual analysis provides only partial solution to the disorientation problem, 
however, for it is important for readers to individually grasp the information structure by utilizing their own criteria and respond to unique application needs. Given the diversity of user backgrounds and application requirements, one possible way to achieve this appears to be through fuzzy modeling.

Information-based measures may be used to categorize the links of a hypermedia system in a number of different ways. ${ }^{25}$ One scheme of classification (see ref. 3) divides all links into: relative position links, hard links and return links. Relative position links connect two nodes a pre-defined distance apart. Examples of such links are the "Go Next" or "Go Previous" commands allowing a user to go forward/backward through one node at the time. Hard links, on the other hand, are connections made from a node to another specific node, such as for example linking from any node to a common map or a home page. Hard links are used in conjunction with conditional structures (e.g., $I F / T H E N / E L S E$ ) to control branching and to control the display of hidden fields containing information. Finally, return links connect users to a destination node and then take them back to where the link began, in a way forcing them to return to the point of departure. Return links are useful for permitting user access to reference nodes and imposing designer-controlled constraints on where users may go in the information space.

For the purpose of analyzing a hypermedia structure and facilitating its design through information measures, it is useful to distinguish between hierarchical (organizational) and associational (referential) links. The former are used to create a hierarchy while the latter are used to cross-reference information.

\section{HYPERMEDIA NAVIGATION USING FUZZY GRAPHS}

In this section we develop a navigational methodology for hypermedia on the basis of the generalized theories for information retrieval ${ }^{26,27}$ and the theory of fuzzy graphs. ${ }^{28}$ Fuzzy graphs have been used in connection with fault diagnosis in nuclear applications. ${ }^{29}$ In earlier work fuzzy graphs have also been used in connection with hypermedia integration and navigation algorithms. ${ }^{30}$

A fuzzy graph is defined as follows. Let $E_{1}$

and $E_{2}$ be two sets and let $x \in E_{1}$ and $y \in E_{2}$. The Cartesian product of the two sets is the set of ordered pairs $E_{1} \times E_{2}$. A fuzzy graph is defined as the fuzzy subset $G$ such that

$$
\forall(x, y) \in E_{1} \times E_{2}: \mu_{G}(x, y) \in M
$$

where, $\mu_{G}(x, y)$ is a membership function, and $M$ is the membership set on $E_{1} \times E_{2}$. The membership function $\mu_{G}(x, y)$ is a mechanism for grading each element of the relation represented by the graph.

A fuzzy graph is a graph where the links are not Boolean, i.e., either 0 or 1 , but graded by a membership function which takes values in the interval $[0,1]$. A hypermedia system may be viewed as a fuzzy graph where different nodes are connected with each other through some graded link reflecting application-specific or in general user-specified criteria. Thus the navigation problem in the information hyperspace is translated to a problem of traversing a graph in accordance with some directional aids quantified through fuzzy membership functions.

\section{A HYPERMEDIA PROTOTYPE FOR THE MHTGR-NPR}

The Special Material Accounting System (SMAS) is a hypermedia prototype designed to provide comprehensive monitoring and surveillance of the tritium produced in the Modular High Temperature Gas Cooled New Production Reactor (MHTGR-NPR). The objective of the system is to facilitate meeting required tritium accountability criteria to within $10 \mathrm{gm}$ of total inventory. These criteria are established in Plant Design Requirements Documents along with the overall performance, functional, interface, operational, safety, maintenance, inspection and decommissioning requirements for the plant. An advantage of a hypermedia system in this context is that the multitude of data and information involved in monitoring the production of special material over long periods of time, can be integrated with textual, visual and audible data in a manner that facilitates the desired accountability. SMAS was developed in the HyperCard environment which includes the object-oriented programming language called HyperTalk. ${ }^{5}$ It consists of three major subsystems referred to as Target Fabrication, MHTGRNPR Production, and Target Processing. These subsystems correspond to the three major phases in the history of the production process, i.e., the fabrication phase, the production phase (target inside the reactor), and the processing phase. Once a reliable, tested and validated version of the system has been developed, it could possibly be integrated with the Production Assurance Protection System of the Plant.

Figure 2 illustrates the overall architecture of the Special Material Accounting System. SMAS is designed to have access to historical as well as current data, and plant information and knowledge as well as access to external networks. In SMAS, the root node is an entrance stack of cards linked to all other stacks. Buttons that link to a data acquisition program are indicated by the button "Data". Buttons that link to local historical data are indicated by "Information" and buttons that include textual or pictorial data are indicated by the button "Knowledge." A sequence of jumps to a screen 
containing pictorial information about the target elements is achieved by pressing the appropriate buttons. The hypermedia information measures outlined in sections 2 and 3 were used for the development of the system, and the fuzzy graph based methodology of section 4 provides the navigational support.

HyperCard is based on the card computational metaphor where nodes can be either a card, or a collection of cards is called a stack. Buttons are constructed on the screen (through a HyperTalk program) and can be activated when a user clicks on them. Through HyperTalk however, other events can trigger button actions; for example, when the cursor enters the button region or when a specified time has elapsed. A major advantage of HyperCard is that links do not need to be hard-wired. Through HyperTalk anything that can be computed can be used as the destination for a link. This is an advantageous feature for the purpose of integration. Goto statements achieve hypermedia jumps or links. Commands such as show and hide can simulate pop-up windows.

\section{SUMMARY}

Hypermedia is a technology that takes advantage of the phenomenal availability of computer storage and networking capabilities. It allows for the development of large information spaces, a possibility of considerable merit for nuclear plant operations. While it is feasible to store maintenance and operations data, information and knowledge to an unprecedented extent, hypermedia utilization is limited by factors such as user disorientation during access of the large information spaces involved. To overcome this problem we have presented a set of information measures primarily applicable during the design of such systems and a fuzzy graph methodology pertinent during the use of the system, i.e., for navigation. The development of hypermedia systems where information can be retrieved and utilized to satisfy a variety of needs, requires that a user can navigate through enormous hyperspaces assisted by tools that offer context-specific direction. In this paper we outlined a directional tool that allows a user to link to the associated nodes in accordance with application-specific criteria embodied in membership functions. Further research is needed to experimentally test and verify the merit of such approaches in actual environments. An example of a hypermedia-based prototype for special-material monitoring was presented. It is likely that navigational tools will be indispensable for upscaling development and large-size implementations where the possibilities opening through hypermedia for improved operations in nuclear plants will be tested and realized.

\section{REFERENCES}

1. Rehn, H., Majohr, N., Staude, F., Haferburg, M., and Forster, K., "Stimulating the Senses with Multimedia," Nuclear Engineering International, pp. 3637, April 1995.

2. "Video Helmet Makes Quick and Effective Maintenance a Virtual Reality," Nuclear News, pp 27 28, April 1995.

3. Stevens, G.H., and Stevens E.F., Designing Electronic Performance Support Tools, Educational Technology Publications, Englewood Cliffs, New Jersey, 1995.

4. Nielsen, J., Hypertext and Hypermedia, Academic Press, Boston, 1990.

5. Michel, S. L., HyperCard: The Complete Reference, Osborne McGraw-Hill, Berkeley, 1989.

6. Machionini G. and Crane G., "Evaluating Hypermedia and Learning: Methods and Results from the Perseus Project," ACM Transactions on Information Systems, Vol. 12, No. 1., pp. 5-34, 1994.

$7 . \quad$ Theodoropoulos, G., Loumos, V., Antonopoulos, J., "Veterinary Anatomy Tutoring System," Computer Methods and Programs in Biomedicine, Vol. 42, N. 2, pp 93-98, Feb. 141994.

8. Nelson, W.A., "Analyzing User Interactions with Hypermedia Systems," Computer Graphics. (ACM) Vol. 28, n 1, p 43-45, Feb. 1994.

9. Hillinger, M., Leu, D.J., "Guiding Instruction in Hypermedia," Proceedings of the 38th Annual Meeting of the Human Factors and Ergonomics Society, Part 1 (of 2), Nashville, TN, Oct. 24-28, 1994.

10. Brown, P.J, "Do we need Maps to Navigate Round Hypertext Documents?" Electronic Publishing, 2, 2, pp 91-100, 1989.

11. Maurer, H., Schmaranz, K., "J.UCS - the Next Generation in Electronic Journal Publishing," Computer Networks and ISDN Systems, 26 Suppl 2-3 p S63-S69, 1994.

12. Chignell, M. and Lacy R., "Integrating Research and Instruction: Project Jefferson," Academic Computing, Vol. 3, 2, pp. 12-17, pp. 40-45, 1988.

13. Merlet, J-F., "Human Computer Interactions through an Intelligent Hypermedia," Proceedings of the IEEE Intern. Conf. on Systems, Man and Cybernetics, $v$ 4, p 268-271, 1993.

14. Marchionini, G., Crane, G., "Evaluating Hypermedia and Learning: Methods and Results from the Perseus Project," ACM Transactions on Information Systems, v 12, n 1, p 534, Jan 1994.

15. Seno, H., Kai, K., Isobe, T., "Multimedia Information Broadcasting Service 'PRESENT'," Proceedings of the 1994 Vehicle Navigation and Information Systems Conference; Yokohama, Japan, Aug. 31-Sept. 2, 1994.

16. Kini, R.B., "Data Tree Structure for a Hierarchical Structure Processing," Computers \& Industrial Engineering, Vol. 26, n 3, p 551-563, Jul. 1994. 
17. Maurer, H., Schmaranz, K., "J.UCS - The Next Generation in Electronic Journal Publishing," Computer Networks and ISDN Systems, 26 Suppl. 2-3, pp S63S69, 1994.

18. Seno, H., Kai, K., Isobe, T., "Multimedia Information Broadcasting Service 'PRESENT"," Proceedings of the 1994 Vehicle Navigation and Information Systems Conference, Yokohama, Aug. 31Sept. 2, 1994.

19. Hirata, K., Hara, Y., "Concept of Media-based Navigation and its Implementation on Hypermedia System 'Miyabi'," NEC Research \& Development, Vol. 35, n 4, pp 410-420, Oct. 1994.

20. Hirata, K. Hara, Y., Shibata, N., Hirabayashi, F. "Media-based Navigation for Hypermedia Systems," Proc. of the 5th ACM Conference on Hypertext, Seattle, Nov. 14-18, 1993.

21. Arons, B., "Hyperspeech," Proceedings of the Conference on Human Factors in Computing Systems INTERACT '93 and CHI '93, Amsterdam, Apr. 24-29, 1993.

22. Merlet, J-F., "Human Computer Interactions Through an Intelligent Hypermedia," Proceedings of 1993 International Conference on Systems, Man and Cybernetics, Part 4 (of 5); Le Touquet, France, Oct. 1720, 1993.

23. Harary, F., Norman, R.Z., Cartwright, $D_{\dot{-}}$ Structural Models: An Introduction to the Theory of Directed Graphs, Wiley, New York, 1965.
24. Salton, G., Automatic Text Processing, Addison-Wesley, Reading, Mass., 1989

25. Botafogo, A.R., Rivlin, E., and Shneiderman, B., "Structural Analysis of Hypertexts: Identifying Hierarchies and Useful Metrics," ACM Transactions on Information Processing, Vol. 10, No. 2, pp 142-180, April 1992.

26. Nomoto, K., Wakayama, S., Kirimoto, T., Ohashi, Y., and Kondo, M., "A Document Retrieval System Based on Citations using Fuzzy Graphs," Fuzzy Sets and Systems, Vol. 38, pp 207-222, 1990.

27. Radecki, T., "Generalized Boolean Methods of Information Retrieval," Int. J. Man-Machine Studies, Vol. 18, pp 407-439, 1983.

28. Tsoukalas, L.H., Uhrig, R.E., Fuzzy and Neural Approaches in Engineering, John Wiley \& Sons, New York, 1997.

29. Park, J. H. and Seong, P.H., "Nuclear power plant pressurizer fault diagnosis using fuzzy signeddigraph and spurious faults elimination methods," Annals of Nuclear Energy, Vol. 21, n 6, pp 357-369, Jun. 1994.

$30 . \quad$ Tsoukalas, L.H., Upadhyaya, B. R., Clapp, N., "Hypertext-Based Integration for Nuclear Plant Maintenance and Operations," Proceedings of AI 91: Frontiers in Innovative Computing for the Nuclear Industry, pp. 207-216, ANS Topical Meeting, Jackson, Wyoming, September 15-18, 1991.

31. Furnas, G.W., "Generalized Fisheye Views," Proceedings of the CHI' 86 Conference, (Boston, April 13-17, 1986), pp 16-23, ACM, New York, 1986.

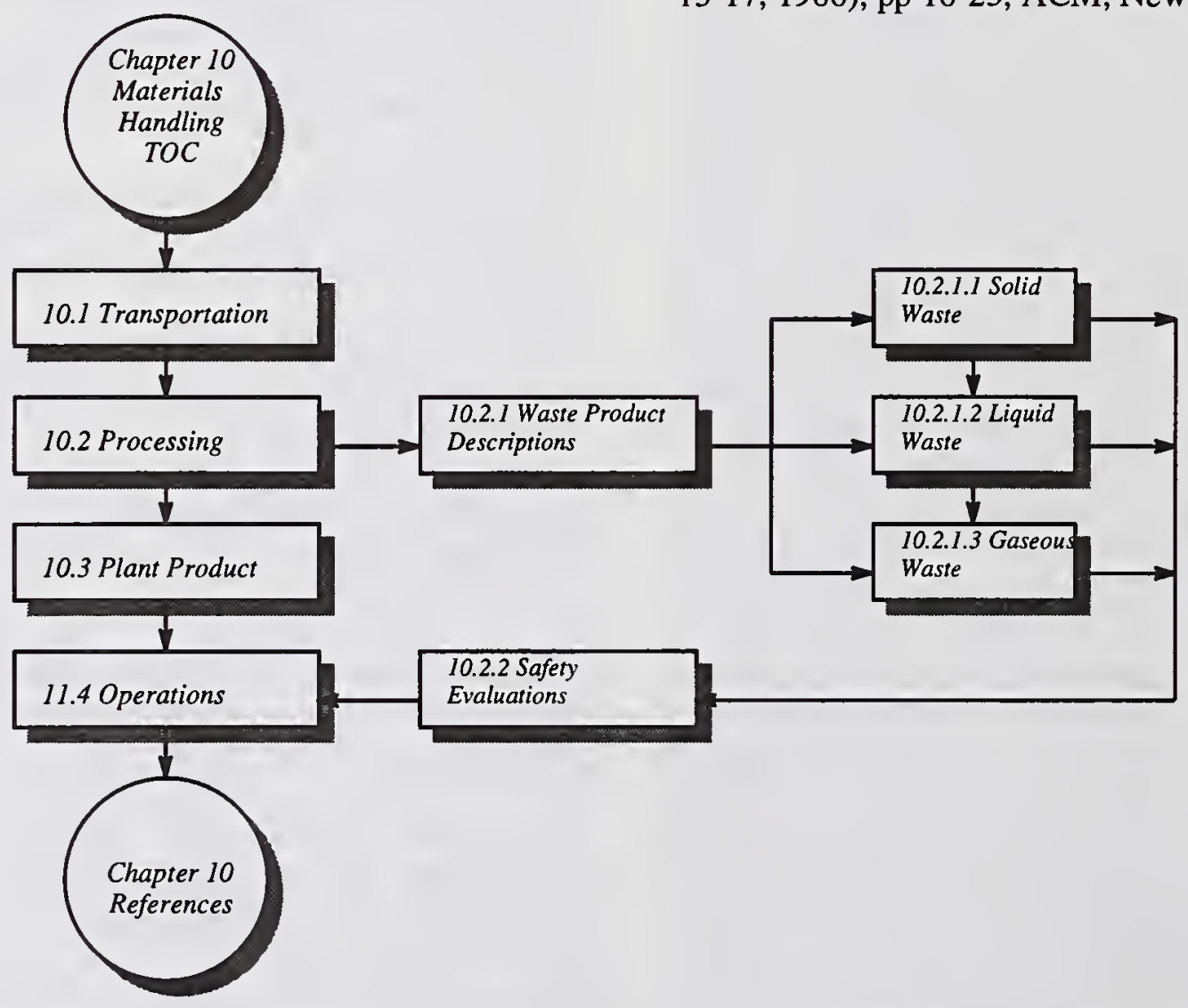

Figure 1. A schematic illustration of the hierarchical information structure of Chapter 10. 


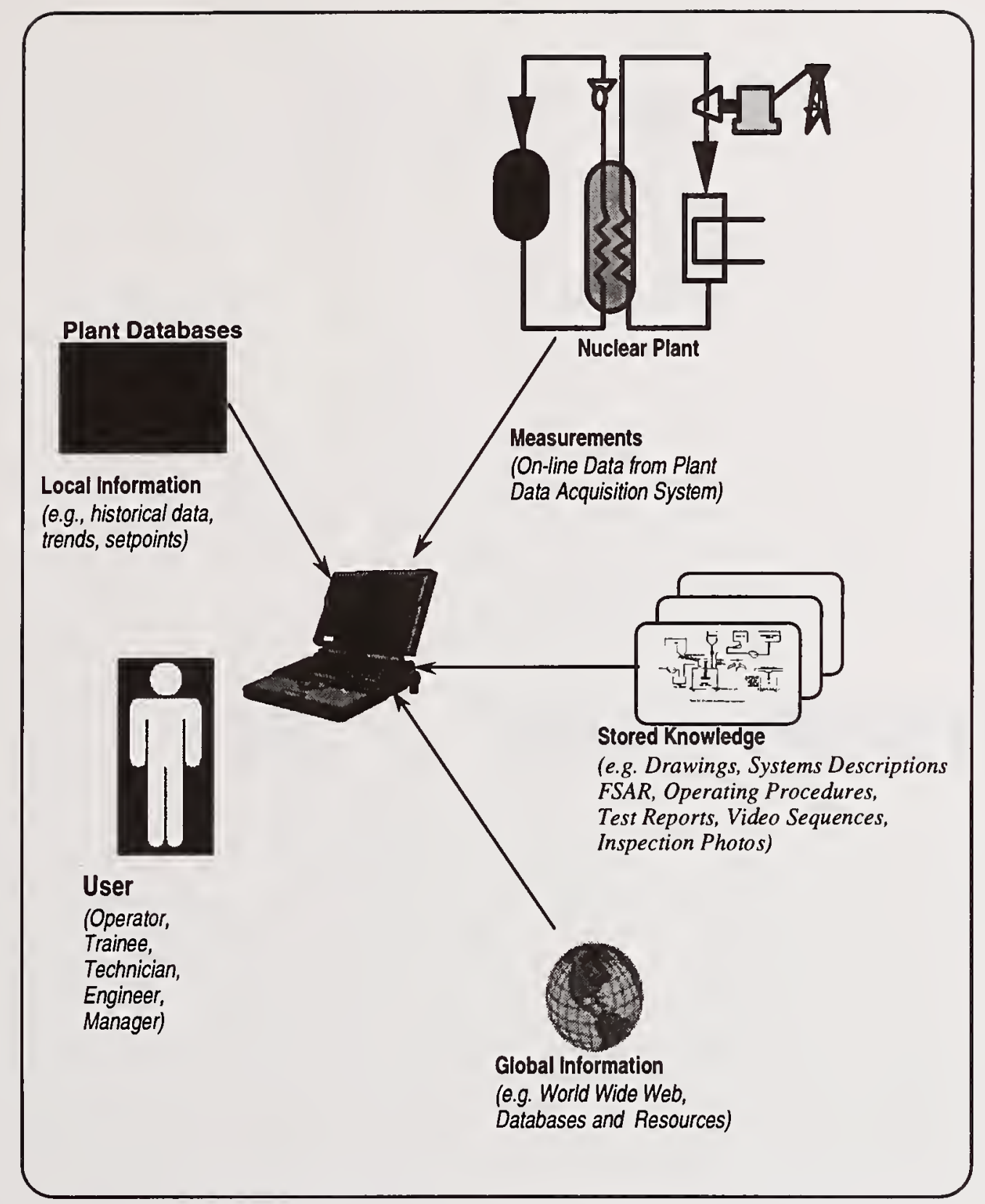

Figure 2. The overall structure of the hypermedia prototype SMAS integrating data, with information and knowledge resources. 



\title{
GAME PROBLEM ON SOFT LANDING FOR MOVING OBJECTS
}

\author{
Arkadii A.Chikrii \\ Cybernetics Institute, 40 Glushkov Ave., \\ Kiev, 252187, Ukraine \\ Fax : (044)266-1558 \\ e-mail: chik@d165.icyb.kiev.ua
}

\section{Abstract}

The game problem on pursuit of a controlled object, moving in the horizontal plane, by another one, moving in the space, is treated here. In this connection the horizontal plane may be considered as a state constraint for the pursuer. The goal of the pursuer is to bring together geometric coordinates and velocities of the players (soft landing) at some finite instant of time. We advance the method of pursuit for solution of this problem which consists of three stages. On the first one the pursuit is performed only by geometric coordinates. The first step ends at so called critical instant of time which is the last one beginning from which the pursuer can avoid meeting with the horizontal plane at nonzero angle. The phase states of the pursuer and the evader at the critical instant are then fixed and on the second stage we solve the problem of optimal control on the transition of the pursuer into the evader's position at the critical time with velocity exceeding in magnitude and coinciding in direction with the velocity of the evader. It goes without saying that during the transition time the evader leaves his initial position. That is why on the third stage the pursuit in tracks on the horizontal plane is performed right up to the soft landing with the further holding a trajectory in the conditions of coincidence of geometric coordinates and velocities of the players.

Keywords : soft landing, differential game, set-valued mapping, dynamic system, pursuit in tracks, quasistrategy, optimal control.

\section{Introduction}

In the theory of differential games there is a number of constructive methods making it possible to solve rather wide classes of problems under various information conditions. Most employed as strategies are strobostrophic $[4,11]$, positional $[5,19]$, and quasistrategies $[6,14,17]$. For successful termination of the game from the pursuer's point of view certain advantage of the pursuer in control resources is needed. Most typical and frequently employed kind of advantage is Pontryagin's Condition and its various modifications $[4,6,11,14,16,17,18]$.

The specific feature of the pursuit problem on soft landing suggested by J.Albus and A.Meystel [1] as "The Eagle Snatch" problem is that for this problem not only Pontryagin's Condition but also all its typical modifications $[6,14]$ fail. The sole exception is the analogue of Pontryagin's Condition dealing with the prolongation of pursuit time and the allowance for a memory [18]. But even in this case the constructing of efficient pursuit procedure on the base of one of the above mentioned methods is rather difficult problem.

The approach advanced in this paper appears as a combined one that corresponds to the ideology of the Artificial Intelligence [2,3]. The process of soft landing is partitioned into three stages. Such procedure seems to be natural and clear from the physical point of view. It goes without saying that one can not speak about optimality of the time of pursuit though the behavior of the pursuer on each stage is guided by the considerations of optimality. 
Nontheless, the conditions on parameters of the conflict controlled process are derived ensuring the finiteness of the time of pursuit for any initial position. Also the controls of the pursuer ensuring the pursuer's goal are given in explicit form.

\section{Problem Statement}

A motion of the pursuer in three-dimensional space is subject to the equation

$$
\ddot{x}+\alpha \dot{x}=\rho u,\|u\| \leq 1,
$$

where $x=\left(x_{1}, x_{2}, x_{3}\right)$ are geometric coordinates of the object, namely $x_{1}, x_{2}$ denote coordinates in the horizontal plane and $x_{3}$ is a height. Vectors $\dot{x}$ and $\ddot{x}$ are a velocity and an acceleration respectively, integer $\alpha>0$ is a coefficient of friction, $\rho>0$ is a force coefficient, $u$ is a control parameter taking its values in the unit sphere, centered at the origin of space $R^{3}$, besides function $u(t), t \geq 0$, is assumed to be Lebesque measurable $[20,24]$.

A motion of the evader evolves in the plane in the following way

$$
\ddot{y}+\beta \dot{y}=\sigma v,\|v\| \leq 1,
$$

where $y=\left(y_{1}, y_{2}\right), \dot{y}$ and $\ddot{y}$ are a velocity and an acceleration, $\beta>0$ is a friction coefficient, $\sigma>0$ is a evader's control parameter taking its values in the planar sphere centered at zero and being a time measurable function. In order to treat $y$ as a vector in $R^{3}$ we shall sometimes write $\tilde{y}=\left(y_{1}, y_{2}, 0\right)$ or $\tilde{y}=(y, 0)$.

We shall analyse the game (2.1), (2.2) standing on the pursuer's side. He should perform the soft meeting with the evader at some finite instant of time, that is, at this instant the inequalities should hold

$$
\|x-\tilde{y}\| \leq \varepsilon_{1},\|\dot{x}-\dot{\tilde{y}}\| \leq \varepsilon_{2}
$$

The hyperplane $\left\{y_{3}=0\right\}$ stands for the state constraint for the pursuer. The pursuer is allowed to move in this hyperplane not interesting it. If this is not the case a trajectory can be shifted by some $\delta>0$ above the horizontal plane and further considerations would be valid for this case too.

Without loss of generality we can assume that $x_{3}^{0}=$ $x_{3}(0)>0$, that is, at the initial instant of time the pursuer finds itself in the upper halfspace.

In the sequel, for simplicity's sake we shall assume that $\varepsilon_{1}=\varepsilon_{2}=0$, that is the precision soft landing will be treated. The passage from solution of this problem to the problem (2.3) is trivial, and what is more, the solution of problem (2.3) immediately follows from the solution of the problem on the precision soft landing.

For convenience of further investigation of the problem (2.1)-(2.3) we reduce the second order systems (2.1), (2.2) to the system of first order with help of the variables' substitution

$$
z_{1}=x, z_{2}=\dot{x}, z_{3}=\tilde{y}, z_{4}=\dot{\tilde{y}} .
$$

Differentiating in $t$ the above equations with account of (2.1), (2.2) we come to the equivalent system

$$
\begin{aligned}
& \dot{z}_{1}=z_{2} \\
& \dot{z}_{2}=-\alpha z_{2}+\rho u \\
& \dot{z}_{3}=z_{4} \\
& \dot{z}_{4}=-\beta z_{4}+\sigma v
\end{aligned}
$$

From the formal point of view the linear system (2.4) is a system of 12-th order but in fact only of 10-th, since vectors $z_{1}, z_{2}, z_{3}, z_{4}$ are three-dimensional and the two last have zeroth third components.

The problem facing the pursuer is to bring a trajectory of system (2.4) from given initial state to the fourdimensional subspace

$$
M_{0}: z_{1}=z_{3}, \quad z_{2}=z_{4}
$$

lying in the direct product of four planes for any admissible counteractions of the evader.

The condition on the availability of information to the pursuer in the course of the game will be further specified during the solution of the problem at hand.

Denote the phase states of the players as follows

$$
\bar{x}=(x, \dot{x})=\left(z_{1}, z_{2}\right), \bar{y}=(\tilde{y}, \dot{\tilde{y}})=\left(z_{3}, z_{4}\right)
$$

\section{Rough Method of Solution}

The game problem $(2.4),(2.5)$ is the linear differential game. The matrix of system (2.4) has the form

$$
A=\left(\begin{array}{cccc}
0_{3} & E_{3} & 0_{3} & 0_{3} \\
0_{3} & -\alpha E_{3} & 0_{3} & 0_{3} \\
0_{2} & 0_{2} & 0_{2} & E_{2} \\
0_{1} & 0_{1} & 0_{1} & 0_{1} \\
0_{2} & 0_{2} & 0_{2} & -\beta E_{2} \\
0_{1} & 0_{1} & 0_{1} & 0_{1}
\end{array}\right)
$$

The fundamental matrix is

$$
e^{A t}=\left(\begin{array}{cccc}
E_{3} & \frac{1-e^{-\alpha t}}{\alpha} E_{3} & 0_{3} & 0_{3} \\
0_{3} & e^{-\alpha t} E_{3} & 0_{3} & 0_{3} \\
0_{2} & 0_{2} & E_{2} & \frac{1-e^{-\beta t}}{\beta} E_{2} \\
0_{1} & 0_{1} & 0_{1} & 0_{1} \\
0_{2} & 0_{2} & 0_{2} & e^{-\beta t} E_{2} \\
0_{1} & 0_{1} & 0_{1} & 0_{1}
\end{array}\right)
$$

Here $E_{i}, 0_{i}$ are respectively unit and zero matrices of orders $i=1,2,3$. We now verify Pontryagin's Condition [4]. 
In so doing the fact should be taken into account that the orthogonal complement to the subspace $M_{0}$ is the eightdimensional space $L$ consisting of vectors of the form

$$
\left(p_{1}, q_{1}, p_{2}, q_{2},-p_{1}, q_{3},-p_{2}, q_{4}\right)
$$

where $p_{1}, p_{2}$ are arbitrary two-dimensional and $q_{i}, i=\overline{1,4}$, arbitrary one-dimensional vectors. It is just the subspace $L$ on which the players' control resources are compared.

Thus, Pontryagin's Condition $[4,6,16]$ for the problem $(2.4),(2.5)$ is

$$
U(t) \stackrel{*}{-} V(t) \neq \emptyset \forall t \geq 0
$$

where

$$
\begin{gathered}
U(t)=\left\{\left(\frac{1-e^{-\alpha t}}{\alpha} \rho u, e^{-\alpha t} \rho u\right):\|u\| \leq 1\right\}, \\
V(t)=\left\{\left(\frac{1-e^{-\beta t}}{\beta} \sigma \tilde{v}, e^{-\beta t} \sigma \tilde{v}\right): \tilde{v}=(v, 0),\|v\| \leq 1\right\},
\end{gathered}
$$

and ${ }^{*}$ is the operation of geometric subtraction or Minkowski' difference $[4,6]$

$$
X \stackrel{*}{-} Y=\{z: z+Y \subset X\}=\cap_{y \in Y}(X-y)
$$

Note that $U(t)$ and $V(t)$ are convex-valued, compactvalued mappings acting from $[0, \infty)$ to $2^{R^{6}}$.

The relationship (3.1) at fixed $t$ implies that a sixdimensional vector $d=\left(d_{1}, d_{2}\right), d_{i} \in R^{3}$, exists such that for any $v,\|v\| \leq 1$, the equations for $u,\|u\| \leq 1$,

$$
\begin{aligned}
\frac{1-e^{-\beta t}}{\beta} \sigma \tilde{v}+d_{1} & =\frac{1-e^{-\alpha t}}{\alpha} \rho u \\
e^{-\beta t} \sigma \tilde{v}+d_{2} & =e^{-\alpha t} \rho u
\end{aligned}
$$

are solvable. This occurs, in particular, at $v=0$. Therefore

$$
d_{1}=\frac{1-e^{-\alpha t}}{\alpha} \rho u_{0}, d_{2}=e^{-\alpha t} \rho u_{0},
$$

where $u_{0},\left\|u_{0}\right\| \leq 1$, is the solution to system (3.2) corresponding to $v=0$. In other words, the system of equations for $u,\|u\| \leq 1$,

$$
\begin{aligned}
\frac{1-e^{-\beta t}}{\beta} \sigma \tilde{v} & =\frac{1-e^{-\alpha t}}{\alpha} \rho\left(u-u_{0}\right) \\
e^{-\beta t} \sigma \tilde{v} & =e^{-\alpha t} \rho\left(u-u_{0}\right)
\end{aligned}
$$

has solution for any $v,\|v\| \leq 1$. Consequently the equations

$$
\begin{gathered}
\frac{1-e^{-\beta t}}{\beta} \sigma \tilde{v}=\frac{1-e^{-\alpha t}}{\alpha} \rho \omega, \\
e^{-\beta t} \sigma \tilde{v}=e^{-\alpha t} \rho \omega, \omega \in R^{3},\|\omega\| \leq 2,
\end{gathered}
$$

have solution $\omega$ for any $\tilde{v},\|\tilde{v}\| \leq 1$. Evidently $\omega=\lambda \tilde{v}$ where $\lambda$ is a certain integer which is positive for $v \neq 0$. Let us fix some $v \neq 0$. Then from the second equation of (3.3) we have

$$
\lambda=\frac{\sigma}{\rho} e^{-(\beta-\alpha) t}
$$

Substituting obtained expression for $\lambda$ into the first equation of (3.3) we have

$$
\alpha+(\beta-\alpha) e^{-\beta t}-\beta e^{-(\beta-\alpha) t}=0
$$

When $\alpha \neq \beta$ the left side of the relationship (3.4) may vanish only at finite number of points (has no more than two real roots). These are just the points at which Pontryagin's Condition holds when $\rho<\sigma$. If $\rho \geq \sigma$ this condition fails and the method does not work because the avoidance of meeting is then possible even in the geometric coordinates $[4,25]$.

Thus, for the problem (2.4), (2.5) Pontryagin's Condition fails.

Nevertheiess the problem (2.4), (2.5) is in principle solvable in a finite time under certain conditions. Below these conditions will be given. For this purpose we shall use the following technique.

Set $\Delta_{1}=z_{1}-z_{3}, \Delta_{2}=z_{2}-z_{4}$. Then from (2.4), (2.5) we have

$$
\dot{\Delta}_{1}=\Delta_{2}, \dot{\Delta}_{2}=-\alpha z_{2}+\beta z_{4}+\rho u-\sigma v
$$

We add to and subtract from the right side of second equation the value $\gamma \Delta_{2}$, where $\gamma>0$ is some integer. Then

$\dot{\Delta}_{1}=\Delta_{2}, \dot{\Delta}_{2}=-\gamma \Delta_{2}-(\alpha-\gamma) z_{2}+(\beta-\gamma) z_{4}+\rho u-\sigma v$

From formula Cauchy for the equations (2.4) there follows that integers $t_{\varepsilon}^{1}\left(z_{2}^{0}\right)$ and $t_{\varepsilon}^{2}\left(z_{4}^{0}\right)$ exist such that for $t \geq$ $t_{\varepsilon}^{1}\left(z_{2}^{0}\right)$,

$$
\left\|(\alpha-\gamma) z_{2}(t)\right\| \leq \frac{\rho}{\alpha}|\alpha-\gamma|+\varepsilon
$$

and for $t \geq t_{\varepsilon}^{2}\left(z_{4}^{0}\right)$

$$
\left\|(\beta-\gamma) z_{4}(t)\right\| \leq \frac{\sigma}{\beta}|\beta-\gamma|+\varepsilon,
$$

where $\varepsilon>0$ is an arbitrarily chosen small number.

Note that we may set

$$
t_{\varepsilon}^{1}\left(z_{2}^{0}\right)=\frac{\ln \left[\left(|| z_{2}^{0} \|+\frac{\rho}{\alpha}\right)|\alpha-\gamma|\right]-\ln \varepsilon}{\alpha}
$$

and

$$
t_{\varepsilon}^{2}\left(z_{4}^{0}\right)=\frac{\ln \left[\left(\left\|z_{4}^{0}\right\|+\frac{\sigma}{\beta}\right)|\beta-\gamma|\right]-\ln \varepsilon}{\beta} .
$$

So far as the term $-(\alpha-\gamma) z_{2}+(\beta-\gamma) z_{4}$ has bounded value beginning from some instant of time, in order to 
supress its influence with the help of parameter $u$, we take into consideration the function

$$
f(\gamma)=\frac{\rho}{\alpha}|\alpha-\gamma|+\frac{\sigma}{\beta}|\beta-\gamma|
$$

Let us find its minimum. One can easily see that it is furnished by $\gamma$ equal to either $\alpha$ or $\beta$, and therefore

$$
\min _{\gamma>0} f(\gamma)=\min \left\{\frac{\rho}{\alpha}, \frac{\sigma}{\beta}\right\} \cdot|\alpha-\beta|
$$

In order that the pursuer has advantage in the right side of the equation (3.5) it is necessary that

$$
\rho-\min \left\{\frac{\rho}{\alpha}, \frac{\sigma}{\beta}\right\}|\alpha-\beta|>\sigma
$$

So far it is assumed that $\frac{\rho}{\alpha} \geq \frac{\sigma}{\beta}[4]$ even for the coincidence of geometric coordinates the above inequality is equivalent to the following one

$$
\rho-\frac{\sigma}{\beta}|\alpha-\beta|>\sigma,
$$

which in the turn is equivalent to the inequalities

$$
\begin{gathered}
\rho>\sigma \text { and } \frac{\rho}{\alpha}>\frac{\sigma}{\beta}, \text { when } \alpha \geq \beta, \\
\rho>\left(2-\frac{\alpha}{\beta}\right) \sigma, \text { when } \alpha<\beta .
\end{gathered}
$$

Then for $t \geq \max \left\{t_{\varepsilon}^{1}\left(z_{2}^{0}\right), t_{\varepsilon}^{2}\left(z_{4}^{0}\right)\right\}$ the equations (3.5) may be changed for the equations

$$
\dot{\Delta}_{1}=\Delta_{2}, \dot{\Delta_{2}}=-\alpha \Delta_{2}+\rho u-\eta,
$$

where vector $\eta$ satisfies the equation

$$
\|\eta\| \leq \sigma\left(1+\frac{1}{\beta}|\alpha-\beta|+\varepsilon\right),
$$

in addition $\rho>\sigma\left(1+\frac{1}{\beta}|\alpha-\beta|+\varepsilon\right)$.

It is known [5] that the game problem (3.9) as the problem for the same type objects with the goal $\Delta_{1}=0$, $\Delta_{2}=0$ is equivalent to the problem of control of the system

$$
\dot{\Delta}_{1}=\Delta_{2}, \Delta_{2}=-\alpha \Delta_{2}+\zeta,
$$

where $\|\zeta\| \leq \rho-\sigma\left(1+\frac{1}{\beta}|\alpha-\beta|\right)$, with the goal of its bringing to the origin.

It will be shown below that the problem (3.10) has a solution in a finite time. Thus, if

$$
\rho>\sigma\left(1+\frac{1}{\beta}|\alpha-\beta|\right),
$$

then the game problem (2.4), (2.5) has a solution in a finite time. But this time may be rather great since the estimates of the kind (3.6), (3.7) should hold.
Below we advance another method of solution of the problem (2.4), (2.5) in lesser time, though this time, generally speaking, is not optimal. The process of the problem solution will consist of three stages with further holding a trajectory in the set (2.5). The first stage refers to the game problems of pursuit in geometric coordinates, the second one is a problem of control for a system of the type (3.10) on transition from one point to another, and the third stage is a pursuit in tracks with exceeding velocity. At the first and the second stages one should handle carefully the state constraint of the pursuer.

\section{The Problem of Pursuit}

Let system (2.4) at $t=0$ be in the position

$$
z^{0}=\left(z_{1}^{0}, z_{2}^{0}, z_{3}^{0}, z_{4}^{0}\right)
$$

We shall treat the problem of pursuit for this system with the terminal set

$$
M^{*}: z_{1}=z_{3} .
$$

The solution to this system is known and can be obtained either in the class of strobostrophic strategies [4,16] on the base of Pontryagin's First Direct Method or with the help of Extremal Targetting Rule of Krasovskii [5] in the class of positional strategies, or in the class of quasistrategies on the base of the Method of Inverse Minkowski Functionals (Resolving Functions) $[6,14]$. The guaranteed time of pursuit is the same for all these methods and appears as optimal. We now dwell upon the last of the above mentioned methods [6]. Pontryagin's function for the problem (2.4), (2.5) has the form

$$
\begin{aligned}
& W(t)=\frac{1-e^{-\alpha t}}{\alpha} \rho S_{3}-\frac{1-e^{-\beta t}}{\beta} \sigma S_{2}= \\
& =\left[\frac{1-e^{-\alpha t}}{\alpha} \rho-\frac{1-e^{-\beta t}}{\beta} \sigma\right] S_{3}=\omega(t) S_{3}
\end{aligned}
$$

where $S_{i}$ is a unit sphere in space $R_{i}$, and Pontryagin's Condition holds if $\omega(t) \geq 0$. This is true when [4]

$$
\rho \geq \sigma, \frac{\rho}{\alpha} \geq \frac{\sigma}{\beta}
$$

what is more, if at least one of the inequalities is strict then $\omega(t)>0$.

Denote

$$
\xi\left(t, z^{0}\right)=z_{1}^{0}-z_{3}^{0}+\frac{1-e^{-\alpha t}}{\alpha} z_{2}^{0}-\frac{1-e^{-\beta t}}{\beta} z_{4}^{0}
$$

Then the time of the pursuit termination is

$$
T=T\left(z^{0}\right)=\min \left\{t \geq 0:\left\|\xi\left(t, z^{0}\right)\right\|=\int_{0}^{t} \omega(\tau) d \tau\right\}
$$


When $k=\frac{\rho}{\alpha}-\frac{\sigma}{\beta}>0$ the equation in the relationship (4.4) has a positive root for any $z^{0}$ since at $t=0$ the left side is greater then the right one and as $t \rightarrow+\infty$ the former remains bounded while the latter grows linearly with the coefficient $k$.

In order to find the control, ensuring the capture, we shall introduce the resolving function of the problem (2.4), $(4.2)$ according to the technique developed in $[6,14]$. Set

$$
\begin{gathered}
\alpha\left(t, \tau, z^{0}, \tilde{v}\right)=\frac{\frac{1-e^{-\beta(t-\tau)}}{\beta} \sigma\left(\tilde{v}, \xi\left(t, z^{0}\right)\right)}{\|\xi(t, z)\|^{2}}+ \\
+\left(\left[\frac{1-e^{-\beta(t-\tau)}}{\beta} \sigma\right]^{2}\left(\tilde{v}, \xi\left(t, z^{0}\right)\right)^{2}+\right. \\
+\left\|\xi\left(t, z^{0}\right)\right\|^{2}\left[\frac{1-e^{-\alpha(t-\tau)}}{\alpha}\right]^{2} \rho^{2}- \\
\left.-\left[\frac{1-e^{-\beta(t-\tau)}}{\beta}\right]^{2} \sigma^{2}\|\tilde{v}\|^{2}\right)^{1 / 2} \cdot\|\xi(t, z)\|^{-2}
\end{gathered}
$$

Then, if $\xi\left(T, z^{0}\right) \neq 0$, then on the active segment of pursuit $\left[0, t_{*}\right)$, where $t_{*}$ is an instant such that the test function [6]

$$
h\left(t_{*}\right)=1-\int_{0}^{t_{*}} \alpha\left(T, \tau, z^{0}, \tilde{v}(\tau)\right) d \tau
$$

vanishes, we choose the pursuer's control in the form

$$
\begin{gathered}
u(\tau)=\left[\frac{1-e^{-\alpha(T-\tau)}}{\alpha} \rho\right]^{-1}\left[\frac{1-e^{-\beta(T-\tau)}}{\beta} \sigma \tilde{v}(\tau)-\right. \\
\left.-\alpha\left(T, \tau, z^{0}, \tilde{v}(\tau)\right) \xi\left(T, z^{0}\right)\right]
\end{gathered}
$$

On the passive segment $\left[t_{*}, T\right]$ we set

$$
u(\tau)=\left[\frac{1-e^{-\alpha(T-\tau)}}{\alpha} \rho\right]^{-1} \frac{1-e^{-\beta(T-\tau)}}{\beta} \sigma \tilde{v}(\tau)
$$

If, otherwise, $\xi\left(T, z^{0}\right)=0$ then the control of the pursuer for $\tau \in[0, T]$ is chosen in the form (4.6). It corresponds to the control by Pontryagin's First Direct Method [6,16]. This control ensures the bringing of a trajectory of the process (2.4) to the set (4.2) at time $T$ or, in other words, this control guarantees the capture of the evader by the pursuer at time $T$ beginning with the initial state $z^{0}$.

We assume that the game evolves under perfect information, that is, in the course of the game an information on current states of the players is available to both players.

Note the following circumstance. At the time of the evader's capture the velocity of the pursuer $x_{2}(t)$ may form obtuse angle with the normal to the horizontal plane. This fact would point to the further intersection of the horizontal plane by the pursuer, that is, to the violation of the state constraints. This is the reason why the process of pursuit is ceased at certain instant of time $\tau_{*}, \tau_{*}<T$.

Below we describe how this instant is chosen. Denote by $D\left(\vec{x}\left(\tau_{*}\right), t\right)$ the attainable set in geometric coordinates of the system (2.1) at time $t$ from the point $\bar{x}\left(\tau_{*}\right)$. Then

$$
D\left(\bar{x}\left(\tau_{*}\right), t\right)=x\left(\tau_{*}\right)+\frac{1-e^{-\alpha t}}{\alpha} \dot{x}\left(\tau_{*}\right)+\int_{\tau_{*}}^{t} \frac{1-e^{-\alpha \tau}}{\alpha} \rho d \tau \cdot S_{3}
$$

The integral of this set-valued mapping is defined in a standard way [20] as a union of the integrals of measurable selections of the mapping. In our case the integral of the sphere of variable radius is the new sphere of the radius equal to the integral of the initial sphere radius $[6,14]$. Set

$$
\tau_{*}=\max _{\tau \in[0, T]}\left\{D(\bar{x}(\tau), t) \cap\left\{x_{3} \geq 0\right\} \neq \emptyset \forall t \geq \tau\right\},
$$

where $\left\{x_{3} \geq 0\right\}$ is the upper halfspace (above the horizontal plane).

If a motion of the pursuer is to evolve at distance $\delta>$ 0 above the horizontal plane then for the evaluation of instant $\tau_{*}$ the halfspace $\left\{x_{3} \geq 0\right\}$ should be changed for the halfspace $\left\{x_{3} \geq \delta\right\}$.

Evidently, $\tau_{*}$ is just the last instant of time when the pursuer may avoid unwanted meeting with the horizontal plane. He is allowed only to touch it, not intersecting.

Thus the process of pursuit will be performed only on the interval $\left[0, \tau_{*}\right]$.

\section{The Problem on System Transi- tion from One Point to Another}

Let us fix the instant $\tau_{*}$ and the corresponding states of the players $\bar{x}\left(\tau_{*}\right)$ and $\bar{y}\left(\tau_{*}\right)$. We shall analyse the problem of optimal control of system (2.1) on the fastest transition from the state $\bar{x}\left(\tau_{*}\right)$ into the state $\tilde{y}\left(\tau_{*}\right), \dot{\tilde{y}}\left(\tau_{*}\right)(1+\varepsilon)$, $0<\varepsilon<1$. Thus, the problem is to achieve in the shortest time the concurrent coincidence of the players' geometric coordinates and velocities' directions under the advantage of the pursuer in the velocity's magnitude. It goes without saying that in so doing the state constraints should not be violated.

The integer $\varepsilon$ accounts for the magnitude of wanted advantage of the pursuer over the evader in velocity at point $\bar{y}\left(\tau_{*}\right)$. By virtue of Cauchy formula the attainable set of the system (2.1) in six-dimensional space has the form

$$
\bar{D}\left(\bar{x}_{0}, t\right)=\Phi(t) \bar{x}_{0}+\int_{0}^{t}\left\{\Phi(\tau)\left(\begin{array}{c}
0 \\
\rho u
\end{array}\right),\|u\| \leq 1\right\} d \tau
$$

where $\Phi(t)=\left(\begin{array}{cc}1 & \frac{1-e^{-\alpha t}}{\alpha} \\ 0 & e^{-\alpha t}\end{array}\right) \otimes E_{3}(\otimes$ is Kronecker symbol) is a fundamental matrix of the homogeneous system, and $\bar{x}_{0}=\left(z_{1}^{0}, z_{2}^{0}\right)$ is the initial phase state. This set 
is convex and closed. Therefore the fact that the point $\bar{x}_{*}=\left(z_{1}^{0}, z_{2}^{0}\right)=\left(\tilde{y}\left(\tau_{*}\right), \dot{\tilde{y}}\left(\tau_{*}\right)(1+\varepsilon)\right)$ lies in the set $(5.1)$ can be expressed in terms of support functions in the following way:

$$
\begin{array}{r}
\left(p_{1}, z_{1}^{*}\right)+\left(p_{2}, z_{2}^{*}\right) \leq\left(p_{1}, z_{1}^{0}+\frac{1-e^{-\alpha t}}{\alpha} z_{2}^{0}\right)+ \\
+\left(p_{2}, e^{-\alpha t} z_{2}^{0}\right)+\int_{0}^{t} \rho \max _{\|u\| \leq 1}\left(\frac{1-e^{-\alpha \tau}}{\alpha} p_{1}+e^{-\alpha \tau} p_{2}, u\right) d \tau
\end{array}
$$

for all $p=\left(p_{1}, p_{2}\right) \in R^{6},\|p\|=1$.

Above the integral and the maxima are interchanged on the basis of Lyapunov's theorem on vector measures [20].

Thus, the issue on the possibility and the duration of the transition of the system (2.1) from point $\bar{x}_{0}$ to point $\bar{x}_{*}$ reduces to the issue on the existence of a positive root of the following equation for $t$

$$
\begin{gathered}
\min _{\|p\|=1}\left[\left(p_{1}, z_{1}^{0}-z_{1}^{*}+\frac{1-e^{-\alpha t}}{\alpha} z_{2}^{0}\right)+\left(p_{2}, e^{-\alpha t} z_{2}^{0}-z_{2}^{*}\right)+\right. \\
\left.+\int_{0}^{t} \rho\left\|p_{1} \frac{1-e^{-\alpha \tau}}{\alpha}+p_{2} e^{-\alpha \tau}\right\| d \tau\right]=0
\end{gathered}
$$

Such root exists. Denote the least of them by $\theta$. To $\theta$ it corresponds vector $p^{*}=\left(p_{1}^{*}, p_{2}^{*}\right)$ which furnishes maximum in (5.3).

In accordance with the Maximum Principle of Pontryagin the program control of the pursuer satisfies the relationship

$$
(p(t), \tilde{u}(t))=\max _{\|u\| \leq 1}(p(t), \tilde{u}), t \in[0, \theta],
$$

where $\tilde{u}=(0, u)$, and $p(t)$ is a solution of the conjugate system

$$
\dot{p}(t)=\left(\begin{array}{cc}
0 & 0 \\
-1 & \alpha
\end{array}\right) \otimes E_{3} p(t), p(\theta)=p^{*} .
$$

\section{Pursuit in Tracks}

Thus, right to the time $\theta$ the following relationships are true

$$
x(\theta)=\tilde{y}\left(\tau_{*}\right), \dot{x}(\theta)=\dot{\tilde{y}}\left(\tau_{*}\right)(1+\varepsilon), \tau_{*}<\theta
$$

Set

$$
\tau(t)=\tau_{*}+(1+\varepsilon) t-\frac{\varepsilon^{2}}{4\left(\theta-\tau_{*}\right)} t^{2}, t \geq 0 .
$$

One can easily verify that

$$
\begin{aligned}
& \tau(0)=\tau_{*}, \dot{\tau}(0)=1+\varepsilon, \\
& \tau(t)<\theta+t, 0 \leq t<t^{*},
\end{aligned}
$$

$$
\tau\left(t^{*}\right)=\theta+t^{*}, \dot{\tau}\left(t^{*}\right)=1,
$$

where $t^{*}=\frac{2\left(\theta-\tau_{*}\right)}{\varepsilon}$. Then, beginning from the instant $\theta$ at each instant of time $\theta+t, t \geq 0$, the pursuer will use the information on $\bar{x}(\theta+t), \bar{y}(\tau(t))$, and $\tilde{v}(\tau(t))$ striving to perform the soft meeting with the evader by moving along his tracks, that is, by holding the relationship

$$
x(\theta+t)=\tilde{y}(\tau(t)), t \geq 0 .
$$

From the wanted relationship (6.3) there follow the relationships

$$
\begin{gathered}
\dot{x}(\theta+t)=\dot{\tilde{y}}(\tau(t)) \dot{\tau}(t) \\
\ddot{x}(\theta+t)=\dot{\tilde{y}}(\tau(t)) \ddot{\tau}(t)+\ddot{\tilde{y}}(\tau(t)) \dot{\tau}(t)
\end{gathered}
$$

These relationships are equivalent to the inclusion

$$
(\bar{x}(\theta+t), \bar{y}(\tau(t))) \in M_{\dot{\tau}(t)-1}, t \geq 0,
$$

where

$$
M_{\varepsilon}=\{x=\tilde{y}, \dot{x}=\dot{\tilde{y}}(1+\varepsilon)\} .
$$

From system (2.1), taking account of the relationships (6.4), (6.5), and (2.2), we deduce the explicit form for the pursuer's control

$$
\begin{gathered}
u(\theta+t)=\frac{\dot{\tau}(t)}{\rho}\left(\dot{\tau}(t)-\frac{\alpha}{\beta}\right) \ddot{\tilde{y}}(\tau(t))+\frac{1}{\rho} \dot{\tilde{y}}(\tau(t)) \ddot{\tau}(t)+ \\
+\frac{\alpha}{\beta} \cdot \frac{\sigma}{\rho} \dot{\tau}(t) \tilde{v}(\tau(t)), t \in\left[\theta, t^{*}\right] .
\end{gathered}
$$

From the equations $(6.1),(6.2)$ with account of $(6.3),(6.4)$ we obtain the equations

$$
\begin{gathered}
x\left(\theta+t^{*}\right)=\tilde{y}\left(\tau\left(t^{*}\right)\right) \\
\dot{x}\left(\theta+t^{*}\right)=\dot{\tilde{y}}\left(\tau\left(t^{*}\right)\right) .
\end{gathered}
$$

Thus, the soft landing is performed.

\section{The Problem on Holding of a Trajectory}

Performing a soft landing sometimes it is necessary that the equalities in geometric coordinates and velocities hold during some period of time.

We shall follow the line of reasoning of the previous section and choose the control of the pursuer from the condition of the coincidence of current velocities analogously to (6.6). Then we shall obtain the control which will ensure the holding of a trajectory in set $M_{0}$ for $t \geq \theta+t^{*}$. The constraints on the process parameters imposed in Section 3 make such choice possible. 


\section{Conclusion}

Thus, the sufficient conditions for solvability of the game problem on soft landing (2.1), (2.2), (2.5) in a finite time beginning with any initial state are derived. The process of pursuit is partitioned into three stages. On the first stage the pursuit in geometric coordinates is performed on the active and passive segments and the control is chosen in accordance with formulas (4.5), (4.6). In the case of availability of information only on a current position the same goal may be attained by the methods contained in $[5,19,25]$. On the second stage the typical problem on transition from one point to another is solved. The control is chosen from the relationship (5.4). This process may be realized on the base of Pontryagin's Maximum Principle. Finally, the last stage is a pursuit in tracks. The control ensuring the soft landing is given by expression (6.6). What is more, the proximity of geometric coordinates and velocities may be maintained during as great as possible period of time.

\section{References}

[1] J. Albus, A. Meystel, The Eagle Snatch, Intelligent Systems: A Semiotic Perspective, Proceedings of the 1996 International Multidisciplinary Conference, NIST, Gaithersburg, 1996, USA.

[2] J. Albus, Outline of the Theory of Intelligence, International Multidisciplinary Conference "Intelligent Systems: A Semiotic Perspective", Preparatory Materials, NIST, Gaithersburg, USA, 1996, pp.3-41.

[3] A. Meystel, Intelligent Systems: A Semiotic Perspective, Proceedings of the 1996 International Multidisciplinary Conference, NIST, Gaithersburg, 1996, USA, pp.41-69.

[4] L.S. Pontryagin, Selected Scientific Papers, Vol. 2, Moscow, 1988, 576p. (Russian).

[5] N.N. Krasovskii, Game Problems on the Encounter of Motions, Nauka, Moscow, 1970, 420p. (Russian).

[6] A.A. Chikrii, Conflict Controlled Processes, Kluwer Academic Publishers, Boston-London-Dordrecht, 1997, 427p.

[7] L.S. Pontryagin, V.G.Boltyanskii, R.V.Gamkrelidze, Eu.F.Mischenko, The Mathematical Theory of Optimal Processes, Wiley, New York, 1962, 392p.

[8] E.B. Lee, L.Markus, Foundations of Optimal Control Theory, John Wiley, New York, 1966, 576p.

[9] M.Atans, P.Falb, Optimal Control, McGraw-Hill Book Company, New York, 1965, 760p.
[10] G.Leitmann, The Calculus of Variations and Optimal Control, New York, Plenum, 1983.

[11] O. Hajek, Pursuit Games, Academic Press, New York, 1975, 266p.

[12] L. Berkovitz, Optimal Control Theory, SpringerVarlag, New York, 1974.

[13] A. Friedman, Differential Games, New York, Wiley, 1971, 350p.

[14] A.A. Chikrii, Conflict Controlled Processes, Kiev, Naukova Dumka, 1992, 384p. (Russian).

[15] V.I.Blagodatskikh, The Linear Theory of Optimal Control, Izdat. Gos. Univ., Moscow, 1978, 94p. (Russian).

[16] M.S.Nikolskii, Pontryagin's First Direct Method in Differential Games, Izdat. Gos. Univ., Moscow ,1984, 65p. (Russian).

[17] N.L.Grigorenko, Mathematical Methods of Control of Several Dynamic Processes, Izdat. Gos. Univ., Moscow, 1990, 198p. (Russian).

[18] D.Zonnevend, On One Method of Pursuit, Dokl. Akad. Nauk SSSR, vol.204, No6, 1972, pp.1296-1299 (Russian).

[19] A.A. Chikrii, Differential Games with Many Pursuers, Matematical Control Theory, Banach Center Publ., PWN, Warsaw, Vol. 14, 1985, p.81-107.

[20] A.D. Joffe, V.M. Tichomirov, Theory of Extremal Problems, North Holland, Amsterdam, 1979, 479p.

[21] J.-P. Aubin, I. Ekeland, Applied Nonlinear Analysis, John Wiley, New. York, 1984, 512p.

[22] J. Warga, Optimal Control of Differential and Functional Equations, Nauka, Moscow, 1977, 623p. (Russian).

[23] T. Rockafellar, Convex Analysis, Princeton Univ. Press, New York, 1970, 472p.

[24] A.N.Kolmogorov, S.V.Fomin, Elements of Functions Theory and Functional Analysis, Nauka, Moscow, 1981, 544p. (Russian).

[25] A.A.Chikrii, Methods for Investigation of ApproachAvoidance Game Problems, Doct. Thesis, Math., Kiev, 1979, 282p. (Russian). 


\title{
Game Problems of Control with Delay of Information
}

\author{
Greta Ts. Chikrii \\ Cybernetics Institute, 40 Glushkov Ave., \\ Kiev, 252187, Ukraine
}

\begin{abstract}
An approach to treat linear differential games with information time lag appearing as a function of time is presented here. This approach consists in reduction of linear differential game with information time lag to equivalent one with perfect information. It makes possible using all methods and approaches developed for perfect information differential games to solve linear differential games with variable information time lag.

To apply the method connected with time of the "first absorption" to the reduced game it was extended to nonstationary case. Sufficient conditions on the game parameters are derived to construct the control bringing a trajectory of the game with information time lag to the terminal set in the "first absorption" time.
\end{abstract}

Keywords: time lag, terminal set, time of "first absorption", pursuer, evader.

\section{Introduction}

R. Isaacs in his monograph [1], dedicated to the theory of differential games, imphasized an importance of problem statements in which one of the players has a time lag on the availability of the opposer's state vector. In fact, such models most accurately describe physical dynamic situations of conflict. There exists a number of methods for solution of differential games of pursuit [2-4] but all of them work under the assumption that the pursuer has perfect information about a current position of his adversary. However, in practice such information may be available only with some delay in time, needed for example for processing the incoming data.

M. Giletti was first, who provided a rigorous formulation of a broad class of two-person zero-sum differential games with imperfect information in the form of a constant time lag $[5,6]$. He extended socalled Hamilton-Jacobi theory of optimal control and main equation analysis, developed by Isaacs, to the case of payoff separable with respect to opposer's control parameter. In the case of separated motions of the players the differential game of pursuit with constant information time lag immediately reduces to the perfect information differential game [7].

In [8-10] the author suggested an approach to treat general linear differential games with constant time lag, later extended to the case of variable time lag [11-131, which made possible using all methods developed for perfect information games [2-4]. In this paper we adress the linear differential games with information time lag which appears as a function of time. Herein we outline an approach for studing this class of differential games consisting in reduction of the game to equivalent one with perfect information and further extension of the known method [4] to nonstationary case.

\section{Equivalent Game}

Let the motion of conflict-controlled system be snbject to tile linear differential equation

$$
z=A z+u+v
$$

where $z \in R^{n}$, A is a matrix, $u \in U, v \in V, U \subset R^{n}$, $\mathrm{V} \subset R^{n}$. The terminal set,

$$
M, M \subset R^{n}
$$

is given. Two players (the pursuer and the evader) choose controls $u$ and $v$ in competition. The goal of the pursuer is in the shortest time to drive a trajectory of the system to the terminal set $M$ by means of his control choice. The goal of the evader is the opposite.

As admissible controls of the players serve measurable functions with values in $U$ and $V$ respectively. Denote the sets of admissible controls by $\Omega_{v}$ and $\Omega_{v}$.

We shall analyze the development of game from the pursuer's point of view. The crux of the problem 
facing the pursuer is the time lag on the availability of a current state vector. This time lag appears as a function of time $\tau(t)$, defined on a half-closed interval $[0,+\infty)$, where $\tau(0)=\tau_{0}, \tau_{0}>0 . \tau(t)$ is assumed to be nonnegative smooth function with reasonable restriction on its growth rate: $\tau(t)<1$. The last condition provides current information about the system development to be continuously updated. On the initial time interval $\left[-\tau_{0}, 0\right)$ the pursuer applies an arbitrary admissible control. At instant $t=0$ he comes to know the initial state of the system, and besides he remembers own control on [$\left.\tau_{0}, 0\right)$, denoted by $u^{0}(\cdot)$. The pair $\left(z^{0}, u^{0}(\cdot)\right)$ stands for the initial position of the game. By position of the game at instant $t$ is meant a pair $\left(z(t-\tau(t)), u^{t}(\cdot)\right)$, where $u^{\prime}(\cdot)$ is realization of the pursuer's control on the half-closed interval $[t-\tau(t), t)$. Let $Z(t)$ be a set of all points attainable at instant $t$ from the point $z(t-$ $\tau(t))$ through $u^{\prime}(\cdot)$ and various admissible controls of the evader on $[t-\tau(t), t)$. In view of formula Cauchy we have

$$
z(t)=z(t)+V(\tau(t))
$$

where

$$
\begin{gathered}
z(t)=e^{\tau(t) A} z(t-\tau(t))+ \\
+\int_{t-\tau(t)}^{t} e^{(t-\theta) A} u^{t}(\theta) d \theta, \\
V(\tau(t))=\int_{0}^{\tau(t)} e^{\theta A} V d \theta
\end{gathered}
$$

We shall say that the differential game of pursuit with infotmation time lag can be terminated at time instant $t, t \geq 0$, if there exists a strategy of the pursuer, providing the inclusion $Z(t) \subset M$ for any control of the evader. This inclusion can be rewritten with regard to (3)-(5) in the form

$$
\boldsymbol{z}(t) \in M(\tau(t))
$$

where

$$
M(\tau(t))=M \stackrel{*}{-} V((\tau(t))
$$

Above the operation of geometric subtraction of sets [2] was used:

$$
X \stackrel{*}{-} Y=\{z: z+Y \subset X\}, X, Y \subset E^{n}
$$

It is easily verified that on $[0, \infty)$ vector $z(t)$ satisfies the differential equation

$$
\begin{aligned}
& z(t)=A z(t)+u(t)+ \\
& +\left(1-\tau^{\prime}(t)\right) e^{\tau(t) A} v(t-\tau(t))
\end{aligned}
$$

with the initial condition

$$
z(0)=e^{\tau_{0} A} z^{0}+\int_{-\tau_{0}}^{0} e^{-\theta A} u^{\tau_{0}}(\theta) d \theta
$$

Reference to (4) shows that the pursuer has perfect information about current value of $z(t)$. Let us consider the perfect information differential game, described. by the differential equation (8) and the terminal set $M(\tau(t))(7)$. Above reasoning led to the following assertion.

Theorem 1. Let set $M(\tau(t))$ be nonempty for $t \in$ $[0, \infty)$. The linear differential game (1), (2) with information time lag $t(t)$ can be terminated at time $T$ from initial position

$\left(z_{0}, u^{0}(\cdot)\right)$ if and only if the corresponding perfect information differential game (8) with terminal set $M(\tau(t))$ can be terminated at the same. time $T$.

From this theorem it follows that all methods and approaches, developed for differential games with perfect information, can be applied to solve linear differential games with variable information time lag.

\section{Time of "First Absorption"}

To apply the method, connected with the "first absorption" time [3, 4], for solution of the perfect information differential game (7), (8) we need an extension of this method to nonstationary case. Below we provide conditions on parameters and initial position of the linear nonstationary differential game with perfect information which are sufficient for bringing a trajectory of the game to some time dependent terminal set in the "first absorption" time. These conditions are an extension. of well known result [4].

Let the motion of a conflict-controlled system be described by the following equation

$$
z(t)=A(t) z+B(t) u+C(t) v
$$

Here $z \in R^{n}, u \in U, v \in V, U$ and $V$ are convex compacts, $U \subset R^{\prime}, V \subset R^{s}, A(t), B(t)$, and $C(t)$ are matrices of order $n \times n, n \times r$, and $n \times s$, respectively, with their elements to be time continuous functions. 
The goal of the pursuer is, in the shortest time to provide the inclusion $z(t) \in M(t)$, where $M(t)$ is nonempty, convex set, closed for all $t \in\left[t_{0}, \infty\right)$.

It is assumed that the support function of set $M(t)$

$$
\mathrm{W}_{M(t)}(\psi)=\sup _{x \in M(t)}(\psi, x)
$$

in continuous in $\psi$ on set

$$
\mathrm{K}_{M(t)}=\left\{\psi: \psi \in R^{n}, W_{M(t)}(\psi)<+\infty\right\}
$$

and convex cone $\mathrm{K}_{M(t)}$ is closed at $t \in\left[t_{0}, \infty\right)$.

Denote the pair $(t, z(t))$ by position of the game at time instant $t$. Let the initial position $\left(t_{0}, z_{0}\right)$ be given. We shall say that this game with perfect information can be terminated in time $\mathrm{T}\left(t_{0}, z_{0}\right)$ from the initial position $\left(t_{0}, z_{0}\right)$ if there exists a strategy of the pursuer, bringing a trajectory of the process to the terminal set at some time instant $\mathrm{t}_{1}, \mathrm{t}, \leq \mathrm{T}\left(t_{0}, z_{0}\right)$, that is

$$
\begin{aligned}
& z\left(t_{1}\right) \in M\left(t_{1}\right) \\
& W(\psi, t, z, s)= \\
& =\left(\hat{O}^{*}(s, t) \psi, z\right)+\int_{t}^{s} \max _{u \in U}\left(\hat{O}^{*}(s, \theta) \psi, B(\theta) u\right) d \theta+ \\
& +\int_{t}^{s} \min _{v \in V}\left(\hat{O}^{*}(s, \theta) \psi, C(\theta) v\right) d \theta, \\
& \lambda(t, z, s)=\min _{\|\phi\|=1}\left[W(\psi, t, s, z)+W_{M(s)}(-\psi)\right] \text {, } \\
& \tilde{A}(t, s, z)=\{\psi:\|\psi\|=1, \\
& \left.W(\psi, t, z, s)+W_{M(s)}(-\psi)=\lambda(t, z, s)\right\}
\end{aligned}
$$

Here $\hat{O}(s, t)$ is a matrizant of homoge-neous system $z=\mathrm{A}(\mathrm{t}) \mathrm{z}, \tilde{\mathrm{A}}(\mathrm{t}, \mathrm{s}, \mathrm{z}) \subset-K_{M(s)}$. If $z(t) \notin M(t)$ then from the definition of support function it follows that

$$
\lambda(t, z(t), t)=\min _{\|\phi\|=1}\left[(\psi, z(t))+\mathrm{W}_{M(t)}(-\psi)\right]<0
$$

Denote by $\mathrm{T}(t, z)$ the least root of the equation for $\mathrm{s} \lambda(t, s, z)=0$, which is more or equal $t$. If there is no such root we set $\mathrm{T}(t, z)=+\infty$. Evidently $\mathrm{T}(t, z)=t$ only for $\mathrm{z}(t) \in \mathrm{M}(t)$. Unlike stationary case, where by $T(z)$ is meant the time which is left before absorption of the terminal set by the attainable set, in nonstationary case $\mathrm{T}(t, z)$ is the time instant of this absorption exactly.

Theorem 2. Let for any position $\left(t_{p}, z_{1}\right)$ such that $\mathrm{T}\left(t_{1}, z_{1}\right)<+\infty$ the following conditions hold a) set $\tilde{\mathrm{A}}\left(t, z, \mathrm{~T}\left(t_{1}, z_{1}\right)\right)$ consists of a unique vector $\psi\left(t, z, \mathrm{~T}\left(t_{1}, z_{1}\right)\right)$ for all $(t, z)$ from the neighborhood $\left(t_{1}\right.$ ,$\left.z_{1}\right)$;

\section{b) for all points from this neighborhood}

$$
\max _{u \in U}\left(\hat{O}^{*}\left(T\left(t_{1}, z_{1}\right), t\right) \psi\left(t, z, T\left(t_{1}, z_{1}\right)\right), B(t) u\right)
$$

is furnished. by unique vector $u\left(t, z, T\left(t_{1}, z_{1}\right)\right)$.

Then the pursuer can terminate the game in time $T\left(t_{0}, z_{0}\right)$.

Proof. Let initial position $\left(t_{0} z_{0}\right)$ be given and $\mathrm{T}\left(t_{0} z_{0}\right)$ $<+\infty$. By assumptions of the theorem there exists a neighborhood of the point $\left(t_{\sigma}, z_{0}\right)$, where vector $\psi\left(t, z, T_{0}\right) T_{0}=\mathrm{T}\left(t_{0}, z_{0}\right)$, is uniquely defined. Let us denote this neighborhood by $\Omega\left(t_{0}, z_{0}\right)$. Since function $\mathrm{W}\left(\psi, t, z, T_{0}\right)+W_{M\left(T_{0}\right)}(-\psi)$ is continuous in $t, z$, and $\psi$ then function $\psi\left(t, z, T_{\partial}\right)$ is continuous in $\mathrm{t}, \mathrm{z}$ and the' control $u^{0}\left(t, z, T_{0}\right)$ furnishing maximum to expression

$$
\left(\hat{\mathrm{O}} *\left(T_{o_{1}} t\right) \psi\left(t, z, T_{0}\right), B(t) u\right)
$$

is continuous in $t, z$. We assign the pursuer's control inside $\Omega\left(t_{0}, z_{0}\right)$ to be of the form $u==u^{0}\left(\mathrm{t}, \mathrm{z} . T_{0}\right)$. Consider the equation

$$
\begin{aligned}
& z=A(t) z+B(t) u^{0}\left(t, z, T_{0}\right)+C(t) v(t), \\
& z\left(t_{0}\right)=z_{0}
\end{aligned}
$$

For any control $v(t)$ of the evader this equation has a solution which can be extended up to the boundary of $\Omega\left(t_{0}, z_{0}\right)$. Thus, a trajectory of (13) is constructed on some time interval $\left[t_{0}, t_{1}\right]$. We now prove the following inequality

$$
T\left(t_{1}, z^{0}\left(t_{1}\right)\right) \leq T\left(t_{0}, z_{0}\right)
$$

To do this it will suffice to show that

$$
\begin{aligned}
& \lambda\left(t, z^{0}(t), T\left(t_{0}, z_{0}\right)\right) \geq 0, \\
& t_{0} \leq t \leq t_{1} .
\end{aligned}
$$

So far as $\tilde{\mathrm{A}}\left(t, z, T_{0}\right)$ consists of a unique vector $\psi\left(t, z, T_{0}\right)$ inside $\Omega\left(t_{0}, z_{0}\right)$ then $\lambda\left(t, z, T_{0}\right)$ has continuous partial derivatives in $t$ and $z$ there. We now evaluate the time derivative of $\lambda\left(t, T_{0}, \mathrm{z}^{0}(t)\right)$, taking into account the pursuer's control choice on $\left[t_{0}, t_{l}\right]$. 


$$
\begin{aligned}
& \frac{a \lambda\left(t, z^{0}(t), T_{0}\right)}{d t}= \\
& -\left(A^{*}(t) \psi\left(t, z^{0}(t), T_{0}\right), \hat{O}\left(T_{0}, t\right) z^{0}(t)\right)- \\
& -\max _{u \in U}\left(\hat{O}^{*}\left(T_{0}, t\right) \psi\left(t, z^{0}(t), T_{0}\right), B(t) u\right)- \\
& -\min _{v \in V}\left(\hat{O}^{*}\left(T_{0}, t\right) \psi\left(t, z^{0}(t), T_{0}\right), C(t) v\right)+ \\
& +\left(\hat{O}^{*}\left(T_{0}, t\right) \psi\left(t, z^{0}(t), T_{0}\right), A(t) z^{0}(t)+\right. \\
& \left.+B(t) u^{0}\left(t, z^{0}(t), T_{0}\right)+C(t) v(t)\right)
\end{aligned}
$$

It is seen that the above expression is nonnegative on $\left[t_{0}, t_{l}\right]$, that is function $\lambda\left(t, z^{0}(t), T_{0}\right)$ does not increase along $z^{0}(t)$,

$\mathrm{t} \in\left[\mathrm{t}_{\sigma} t_{1}\right]$. So far as $\lambda\left(\mathrm{t}_{o}, z_{\alpha^{\prime}} T_{0}\right) \geq 0$. Formula (15) is true. In a similar way, beginning with the position $\left(t_{l}\right.$, $z^{0}\left(t_{1}\right)$ ) we shall construct a solution of (13) on some interval $\left[\mathrm{t}_{1}, t_{2}\right]$ such that

$$
T\left(t_{2}, z^{0}\left(t_{2}\right)\right) \leq T\left(t_{1}, z^{0}\left(t_{1}\right)\right)
$$

It is easy to see that the construction of trajectory can be prolonged until $\mathrm{T}\left(t, z^{0}(t)\right)$ will vanish. By virtue of the inequality (14) which holds true in the course of the pursuit it will occur no later than at time $\mathrm{T}\left(t_{0}, z_{0}\right)$.) Proved.

Substituting into expressions given by formulas (10)-(12) the parameters of the equivalent game (7), (8)

$$
\begin{gathered}
\hat{O}(s, t)=e^{(s-t) A}, B(t)=I, C(t)=(1-\tau(t)) e^{\tau(t) A}, \\
z^{0}=e^{\tau_{0} A} z^{0}+\int_{-\tau_{0}}^{0} e^{-\theta A} u^{0}(\theta) d \theta
\end{gathered}
$$

we denote them by $\mathrm{W}_{l}(\psi, t, s, z), \lambda_{l}(t, s, z), \tilde{\mathrm{A}}(t, s, z)$ respectively.

Let $\mathrm{T}_{l}(t, z)$, be the least root of the equation for $s$

$$
\lambda_{1}(t, s, z)=0
$$

which is more or equal $t$.

We suppose that for all $t \in[0, \infty)$ the support function of set $M(\tau(t))$, namely, $\mathrm{W}_{M(\tau(t))}(\psi)$, is continuous on set $\mathrm{K}_{M(\tau(t))=}\left\{\psi: \psi \in R^{n} \cdot \mathrm{W}_{M(\tau(t))}(\psi)<\right.$ $+\infty\}$ and set $\mathrm{K}_{M(\tau(t))}$ is closed. Clearly Theorem 2 holds true when $z$ is substituted for $z$. We came to the following result in view of Theorems 1,2 .
Theorem 3. Let the assumptions of Theorem 2 hold for the game (9), (8). Then the linear differential game (1), (2) with time lag $\tau(t)$ can be terminated from the, initial position $\left(z^{0}, u^{0}(\cdot)\right)$ in time $T_{1}\left(0, z_{0}\right)$

\section{Example}

Below one model game [1] complicated by variable information time lag on the availability of an object state vector is solved to illustrate obtained result.

Let the motion of object be subject to the system of equations

$$
\begin{aligned}
& \quad z_{1}=z_{2}, z_{2}=u, z_{3}=v, \\
& \text { where } z_{i} \in R^{n}, i=\overline{1}, \overline{3},\|u\| \leq 1,\|v\| \leq 1, n \geq 2
\end{aligned}
$$

We consider the game terminated as soon as $\left\|z_{1}-z_{3}\right\| \leq \varepsilon$ ( $\varepsilon$-capture in geometric coordinates). Let $\left(z_{1}^{0}, z_{2}^{0}, z_{3}^{0}, u^{0}(\cdot)\right)$ be the initial position of the game. Function $\tau(t), \tau(t) \in C^{l}[0, \infty), \tau(t)<\varepsilon, \tau(0)=\tau_{0}$ is given. Here

$$
M=\left\{z:\left\|z_{1}-z_{3}\right\| \leq \varepsilon\right\}
$$

Then

$$
\begin{gathered}
K_{M}=\left\{\left(\Psi_{1}, \Psi_{2}, \Psi_{3}\right): \Psi_{1}+\Psi_{3}=0,\right. \\
\left.\Psi_{2}=0, \Psi_{1} \in R^{n}, i=1,2,3\right\}
\end{gathered}
$$

and on $K_{M} W_{M}(\Psi)=\varepsilon\left\|\psi_{1}\right\|$. Performing calculatios we obtain

$$
\begin{aligned}
& W\left(\psi, t, z^{0}\right)=\left(\psi_{1}, z_{1}^{0}+t z_{2}^{0}-z_{3}^{0}\right)+ \\
& \int_{0}^{t} \max _{\| u \leq 1}\left(\theta \psi_{1}, u\right) d \theta+ \\
& \int_{0}^{t} \min \left[\left(1-\tau^{\prime}(\theta)\right)\left(-\psi_{1}, v\right)\right] d \theta
\end{aligned}
$$

We observe that $v=v=\frac{\psi}{\left\|\psi_{1}\right\|}$ furnishes maximum and minimum in the above expression. Therefore

$$
\begin{aligned}
& W\left(\psi, t, z^{0}\right)=\left(\psi_{1}, z_{1}^{0}+t z_{2}^{0}-z_{3}^{0}\right)+ \\
& \left\|\psi_{1}\right\|\left(\frac{t^{2}}{2}-\tau_{0}-t+\tau(t)\right)
\end{aligned}
$$


and for $\psi \in K_{M}$

$$
\begin{aligned}
& W_{M(\tau(1))}(\psi)=\left\|\psi_{1}\right\|(\varepsilon-\tau(\mathrm{t})), \\
& \lambda\left(t, z_{0}\right)=\min _{\left\|\psi_{1}\right\|=1}\left[\left(\psi_{1}, z_{1}^{0}+t z_{2}^{0}-z_{3}^{0}\right)+\right. \\
& \left.t^{2} / 2-t-\tau_{0}+\varepsilon\right]
\end{aligned}
$$

Minimum in above expression is furnished by the vector

$$
\psi_{1}\left(t, z_{0}\right)=\frac{z_{1}^{0}+t z_{2}^{0}-z_{3}}{\left\|z_{1}^{0}+t z_{2}^{0}-z_{3}\right\|}
$$

provided $\left\|z_{1}^{0}+t z_{2}^{0}-z_{3}\right\| \neq 0$. Then $\tilde{\mathrm{A}}\left(\mathrm{t}, z_{0}\right)$ consists of a unique vector

$$
\psi\left(t, z^{0}\right)=\left(\psi_{1}\left(t, z^{0}\right), 0,-\psi_{1}\left(t, z^{0}\right)\right),\|\psi\|=\sqrt{2},
$$

and $\mathrm{T}\left(0, z_{0}\right)=\mathrm{T}_{11}\left(\mathrm{z}^{0}, \mathrm{u}^{0}(\cdot)\right)$ can be found from the equation

$$
\left\|z_{1}^{0}+t z_{2}^{0}-z_{3}^{0}\right\|=t^{2} / 2-t+\varepsilon-\tau_{0}
$$

where

$$
\begin{aligned}
& z_{1}^{0}=z_{1}^{0}+\tau_{0} z_{2}^{0}+\int_{-\tau_{0}}^{0} \theta u^{0}(\theta) d \theta, \\
& z_{2}^{0}=z_{2}^{0}+\int_{-\tau_{0}}^{0} \theta u^{0}(\theta) d \theta, \quad z_{3}^{0}=z_{3}^{0}
\end{aligned}
$$

In order that the condition a) of Theorem 3(2) be satisfied it suffices that the inequality.

$$
t^{2} / 2-\tau_{0}-t+\varepsilon>0
$$

holds for $\mathrm{t}>0$. In the turn, the latter holds when $\varepsilon>$ $\tau_{0}+1 / 2$. In these conditions $u=\psi_{1}(t, z)$ is uniquely defined, that is the condition b) of Theorem $3(2)$ is satisfied. From Theorems 1, 3 there follows that when $\varepsilon>\tau_{0}+1 / 2$ the game (16), (17) can be terminated from the initial position $\left(z_{1}{ }^{0}, z_{2}{ }^{0}, z_{3}{ }^{0}, u^{0}(\cdot)\right)$ in time $\mathrm{T}\left(z^{0}, u^{0}(\cdot)\right)$ defined as the first positive root of the equation (18).

\section{References}

[1] Isaacs, R., "Differential Games", Wiley, New York, 1965.

[2] Pontryagin, L.S., "On Linear Differential Games", 1, Dokl. Acad. Nauk SSSR, Vol.176, No.6, 1967 (in Russian).
[3] Krasovskii, N.N., "Game Problems on the Encounter of Motions", Nauka, M., 1970 (in Russian).

[4] Pshnitchny, B.N., "Linear Differential Games", Avtomatika $i$ Telemechanika, No.1, 1968 (in Russian).

[5] Giletti, M.D., "Results in the Theory of Linear Differential Games with an Information Time Lag, Journal of Optimization Theory and Applications, Vol.5, No.5, 1970.

[6] Giletti, M.D., "New Results in the Theory of Differential Games with Information Time Lag", Journal of ptimization Theory and Applications, Vol.8, No.4, 1971.

[7] Chernousko, F.L., "On Differential Games with Information Time Lag", Dokl. Acad. Nauk. SSSR, Vol.188, No.4, M., 1969 (in Russian).

[8] Dzyubenko (Chikrii), G.Ts., Pshenitchny B.N. "Discrete Differential Games with Information Time Lag", Kibernetika, No.6, 1972 (in Russian).

[9] Dzyubenko (Chikrii), G.Ts., "Linear Differential Games with Information Time Lag", Kibernetika, No.6, 1973 (in Russian).

[10] Dzyubenko (Chikrii), G.Ts., "On Linear Differential Games of Pursuit with Information Time Lag", Kibernetika, No.2, 1974 (in Russian).

[11]Chikrii, G.Ts., "On Problem of Pursuit with Variable Information Time Lag on the Availability of Object State Vector", Dokl. Acad. Nauk Ukrainy, No.10, 1979 (in Russian).

[12]Chikrii, G.Ts., Bardadym, T.A., "On One Problem of Pursuit with Variable Information Time Lag", Kibernetika, No.6, 1980 (in Russian).

[13] Chikrii, G.Ts., "On One Approach to Solution of Linear Differential Games with Variable Information Time Lag", Problems of Control and Informatics, No.6, 1994, Scripta Technica, Inc. A Wiley Company (USA). 


\title{
REFLEXIVE GAME THEORY
}

\author{
Vladimir A. Lefebvre \\ School of Social Sciences \\ University of California, Irvine CA 92697
}

\section{ABSTRACT}

Traditional game theory is a normative science and is not meant for modeling the real behavior of players. This paper describes a method the goal of which is to predict the choices of players in real situations rather than to compute optimal decisions. It is assumed that each player faces a choice between two strategies: active and passive. The method is based on structural representation of a subject together with his images of the self and another. This representation allows us to compose systems of equations whose solutions are the probabilities with which the players choose the alternative strategies.

KEYWORDS: reflexive games, decision making, dynamic systems

\section{THE TRADITIONAL APPROACH TO GAMES}

Game Theory allows us to construct models of the ideal behavior of players seeking to guarantee themselves optimum outcome. At its birth game theory seemed to be able to serve not only as a basis for rational decision making but also as a tool for modeling the real behavior of players. This approach looked promising at first because laboratory experiments slowed that in certain cases game theory can predict players' choices. Most attempts to use game theory for modeling real strategic behavior with the purpose of its prediction have failed, however, and are now no longer undertaken. The reason for this negative result is that the real decisions in strategic situations cannot be explained only by the players' desire to guarantee optimum outcome. Today we still face the necessity of creating a general game theory capable of predicting human choice under conditions of the conflict of interests.

\section{AN ALTERNATIVE APPROACH}

In real games players make decisions based on images of themselves and of their partners. These images are reflexive: a subject can "see" himself and others, together with the images they have. A simple reflexive structure is represented in Fig. 1. Rectangle $a_{1}$ represents a player who performs an action $X_{1}$; rectangle $a_{2}$ is his image of the self and $y_{2}$ his image of his partner. Symbol * is the image of their relationship, and rectangle $x_{3}$ is the subject's knowledge about his image of himself. Speaking in psychological language, in this rectangle there appear the player's conscious plans and intentions.

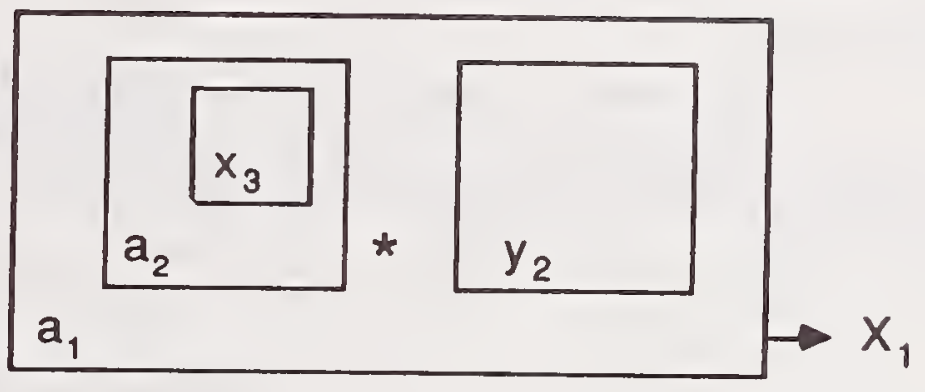

Figure 1. The subject's reflexive structure

In 1966 I put forth the hypothesis that an alternative game theory could be based on schemes similar to that in Fig. 1 [1]. At that time it was only an idea. To construct a theory of "reflexive games" one needs to know the functions which connect the rectangles in Fig.1. These functions must be universal in relation to various human activities, that is, they must reflect the psycho-anthropological fundamentals of human choice. In my subsequent work [2,3] I argue that these basic functions are the following:

$$
\begin{aligned}
& z_{1}=x \stackrel{y \text { def }}{=}=1-y+y x, \\
& z_{2}=x \cdot y \stackrel{\text { def }}{=} x y, \\
& z_{3}=x \oplus y \stackrel{\text { def }}{=}=x+y-x y,
\end{aligned}
$$

where $x, y \in[0,1]$.

The value of each variable is interpreted as a "measure of positivity." Function $z_{1}$ describes the correlation between a player's reflexion and his state: the value of $z_{1}$ is the measure of the player's positivity when the world influences him with the measure $x$ and he "sees" a situation with the measure $y$. Symbols - and $\oplus$ correspond to relationships between the players which can be only of two types: strong $(\bullet)$ or weak $(\oplus)$. The values of $z_{2}$ and $z_{3}$ are the measures of positivity of situations when players with the measures $x$ and $y$ are involved.

Now we can begin developing the theory of reflexive games. 


\section{THE REPRESENTATION OF A PLAYER}

We assume that every player faces a choice between two strategies: an active strategy and a passive one. An active strategy is a positive pole, a passive strategy is a negative pole. The relationship between players is described by the operation

$$
x p y \stackrel{\text { def }}{=}=p(x \cdot y)+(1-p)(x \oplus y),
$$

where $p \in[0,1]$.

This operation is a generalization of operations (2) and (3). Now the reflexive structure in Fig. 1 can be assigned functional meaning and written as the following expression:

$$
a_{1}^{x_{2}}{ }^{x_{3}} y_{2}=X_{1},
$$

where $a_{1}, a_{2}, x_{3}, y_{2}, p \in[0,1]$.

It follows from (5) that $X_{1} \in[0,1]$. The value of $a_{1}$ is interpreted as the measure of the external world's pressure toward an active strategy on the non-conscious level; the value of $a_{2}$ is the measure of pressure of which the player is aware. The value of $y_{2}$ is interpreted as the measure of an opponent's readiness to choose an active strategy, from the player's point of view. The value of $\mathrm{p}$ is the player's subjective estimate concerning the extent of his involvement in a relationship with an opponent. In a situation of conflict the value of $p$ can be interpreted as the intensity of confrontation. The value of $x_{3}$ is the player's plan or intention, and the value of $X_{1}$ is the objective readiness of the player to use the active strategy. This value can be interpreted as the probability of the player's choosing an active strategy. Therefore, $X_{1}$ corresponds to the real choice and $x_{3}$ to the player's subjective intention.

Further we will consider a player who performs conscious actions, that is, he uses only such plans $x_{3}$ for which the following equation holds:

$$
x_{3}=X_{1} \text {. }
$$

With the condition (6), expression (5) turns into equation in relation to $\mathrm{x}$ :

$$
\begin{array}{lll}
x & \\
a_{2} & p & y_{2} \\
a_{1} & &
\end{array}
$$

Theorem 1.

For any $a_{1}, a_{2}, p, y_{2} \in[0,1]$ equation ( 7$)$ has the root $x_{0} \in[0,1]$.

\section{SAMPLE APPLICATION}

Let two companies, A and B, be in conflict. A blames $B$ for failing to fulfill its obligations. Company $A$ can either use an active strategy, that is, to sue the other firm, or a passive one, refrain from suing. Company $B$ also has two strategies: active, to file a counter-claim, and passive, to refrain from filing.

Further we will consider the situation from B's point of view. Let $B$ not want A to file a suit. A consultant hired by B advises to threaten $\mathrm{A}$ with a counter-claim. To do so, B must publicly dramatize the situation, using the mass media. But these actions are expensive. B's executives face a dilemma: to accept the consultant's advice or to refuse it. The theory of reflexive games can help B. The analysts from company B have to construct a model of their opponent based on equation (7). Let it be known that A's lawyers are trying to push the executives to start the law suit, so B's analysts assume

$$
a_{2}>0 \text {. }
$$

Since there are no signs that A's lawyers can covertly influence the executive's subconsciousness,

$$
a_{1}=a_{2} \text {. }
$$

The value of $p$ is the degree of dramatization of a conflict. When $p=1$, dramatization is maximal; when $p=0$, it is minimal. The value of $y_{2}$ in equation (7) is B's readiness, from A's point of view, to file a counter-claim. The more $B$ dramatizes the situation and threatens $A$ with a counter-claim, the greater $y_{2}$. To reflect this in the model, B's analysts must choose the monotonically increasing function $y_{2}=\varphi(p)$. The simplest function of this type is

$$
y_{2}=p \text {. }
$$

In solving equation (7) with limitations (8), (9), and (10), the analysts find the probability of A's filing a law suit as a function of $\mathrm{p}$ :

$$
x=\frac{1-\left(1-a_{1}\right)\left(1-p+p^{2}\right)}{1-\left(1-a_{1}\right)^{2}\left(1-2 p+2 p^{2}\right)}
$$

Mathematical analysis shows that function (11) at the interval $[0,1]$ has its minimum at $p=1$ and $p=0$, that is, either when the dramatization of a conflict is at its greatest or when it is absent. This answer solves the dilemma: company B must reject its consultants' advice and not spend money on dramatizing its relationship with firm $\mathrm{A}$.

While modeling the player's behavior we used very little specific information about him or about the situation. This is possible only because the model is based on the fundamentals 
of human choice. Equations (1) through (4) contain this information.

\section{GAME REPRESENTATION}

Let two interacting players have correct images of each other. Then the following system of equations corresponds to the game:

$$
\left.\begin{array}{c}
a_{1}^{x} p y=x \\
a_{1}^{y} b_{2}^{y} x=y
\end{array}\right\}
$$

\section{Theorem 2.}

For any $a_{1}, a_{2}, p, b_{1}, b_{2}, q \in[0,1]$ the system of equations (12) has solution $\mathrm{x}_{0}, \mathrm{y}_{0} \in[0,1]$.

Consider an example: let $a_{1}=1 / 3, a_{2}=3 / 4, b_{1}=1 / 3$, $b_{2}=1 / 2, p=1$, and $q=1$. Then the system is

$$
\left.\begin{array}{l}
\left(\frac{3}{4}\right)^{x} \cdot y \\
\left(\frac{1}{3}\right)^{=x} \\
\left(\frac{1}{2}\right)^{y} \cdot x \\
\left(\frac{1}{3}\right)^{=}=y
\end{array}\right\}
$$

In transforming these equations into a standard form we obtain a system of two non-linear equations:

$$
\left.\begin{array}{l}
6-6 x-4 y+x y=0 \\
3-2 x-3 y+x y=0
\end{array}\right\}
$$

This system has two solutions, but only one of them has roots in the interval $[0,1]$ :

$$
x_{0}=0.557, y_{0}=0.772 \text {. }
$$

The model helps us to find that, under the conditions reflected by the system (13), the first player chooses an active strategy with the probability 0.557 , and the second one with the probability 0.772 . Generally, a game corresponds to the system:

$$
\left.\begin{array}{l}
\mathrm{f}_{1}\left(\mathrm{a}_{1}, \mathrm{a}_{2}, \mathrm{p}, \mathrm{y}, \mathrm{x}\right)=\mathrm{x} \\
\mathrm{f}_{2}\left(\mathrm{~b}_{1}, \mathrm{~b}_{2}, \mathrm{q}, \mathrm{x}, \mathrm{y}\right)=\mathrm{y}
\end{array}\right\}
$$

whose parameters and variables may be related functionally.

Using the theory of reflexive games it is also possible to construct dynamic models of games. To do so one has to use systems of equations of the following type:

$$
\left.\begin{array}{l}
F_{1}\left(y_{n}, x_{n}\right)=x_{n+1} \\
F_{2}\left(x_{n}, y_{n}\right)=y_{n+1}
\end{array}\right\}
$$

From the mathematical point of view the theory of reflexive games is connected with non-linear equations and dynamic systems.

\section{REFERENCES}

[1] Lefebvre, V. A. Elements of Logic of Reflexive Games. In: Problemy Inzhenernoi Psikhologii, 1966, No.4, pp.273-299.

[2] Lefebvre, V. A. Algebra of Conscience, Dordrecht, Holland: D. Reidel, 1982.

[3] Lefebvre, V. A. Cosmic Subject. Moscow: Russian Academy of Sciences Institute of Psychology Press, 1997. 




\title{
SUPPORTING END-USER PROGRAMMING WITH EXPLANATORY DISCOURSE
}

\author{
Clarisse Sieckenius de Souza \\ Departamento de Informática. PUC-Rio \\ Rua Marquês de São Vicente 225, Rio de Janeiro - RJ \\ email: clarisse@inf.puc-rio.br
}

\begin{abstract}
The need to empower end users with programming capacities in order adapt and extend software applications brings forth the opportunity to examine and explore semiotic aspects in computing. This paper discusses some of these aspects within a knowledgebased extensible environment where (a) software designers can explain their rationale to users and (b) differentiated discourse for user-system interaction and designer-user communication fosters a better understanding of the application's model and features.
\end{abstract}

KEYWORDS Semiotic Engineering, End User

Programming, Knowledge Representation

\section{INTRODUCTION}

A growing need to empower computer users so that they can customize, extend, and create software has been fuelling research in such fields as Human-Computer Interaction (HCI), End-User Programming (EUP), and Artificial Intelligence (AI). Software Engineering is rapidly revealing its semiotic nature as programs and systems produce, interpret, and become themselves documents and messages which groups of people can directly or indirectly exchange. In the sequel of previous work about the Semiotic Engineering of User Interface Languages [5], this paper broadens the scope of computer language and message design in order to try and meet the challenges of wider computer literacy. It adopts a communication-centered architecture to support the users' learning processes required for programming and uses differentiated agent-designer discourse to help users grasp the intended application's model.

The main point in this work is a finer structuring of communication in interface dialogues (involving users, systems, and designers), which promotes programmers and systems designers to the role of writers of material to be learned, adapted, and used as inspiration by computational readers. This is our interpretation of the computer literacy challenge of software industry. We unite $\mathrm{HCI}$ and $\mathrm{AI}$ under the cover of Semiotics and discuss some communicative requirements and alternatives for intelligent extensible computer applications. We do not, however, discuss the features of the underlying macro language in which users can codify extensions to the application.

The reported work is part of an ongoing research project aiming at the construction of a fully-functional EUP environment. The global idea is presented in Section 2, and specific implementation aspects are raised and discussed in Section 3, with an emphasis on representation languages. Section 4 presents our current conclusions about the linguistic requirements for a communication centered architecture to stand and be useful, as well as the benefits we identify in this proposal.

\section{A COMmunication-CENTERED ARCHITECTURE}

User Interfaces can be seen as meta-communications artifacts. They obviously send and receive messages during interaction with users, but they are themselves an achieved one-shot message sent from system designer to system user. This idea is central to Semiotic Engineering [5], a theoretically-based approach to designing user interface languages and codes. However, user interfaces must now support not only productive user-system interaction, but also end-user programming. This can be achieved by more or less sophisticated techniques for configuration settings, macro recordings, or programming in the small $[12 ; 15]$, as well as by some knowledge-based $(\mathrm{KB})$ programming by demonstration [4].

For users to realize that they need and can re-configure, reprogram, or extend software, they first need to understand its meaning, and then understand how new meanings can be added to it or replace old meanings. Such laborious processes of interpretation should be facilitated and supported by conscious Semiotic Engineering of computational languages. Thus, users should not only be able to make sense of designers' intents, but also express and achieve their own meanings.

Designers always predict and prescribe communication form and content. Good designers make good predictions and write good prescriptions. However, users are seldom (if ever) deliberately made aware of the arbitrary rules and models 
that underlie applications. In reaction to an excessive stress on grammatical rule-learning imposed by the command language interface style, current user interface guidelines seem to be heading to some elusive minimal rule-learning ideal. Popular Graphic User Interface (GUI) style guides [3; 11] follow the trend of capitalizing on metaphors and analogies, and requiring trivial click'n'drag interactive modes. But, there is as much arbitrariness and language learning there as there is in any command language interface.

Our communication-centered architecture is one which exposes the existence of arbitrary learnable computer codes, and optionally unfolds [7] successive levels of their grammatical and semantic design rules, so that users can potentially wield such linguistic resources to build new discourse, by adapting and programming applications. Figure 1 presents the global scenario for reference in the following.

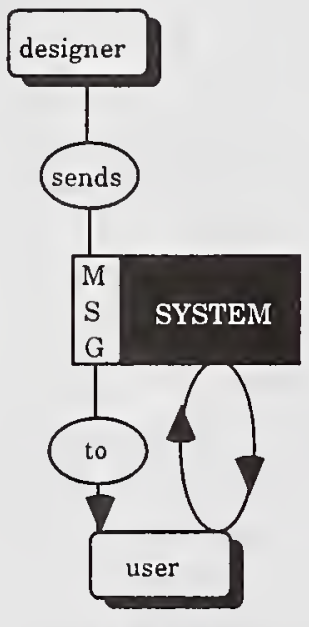

Typical HCI Scenario

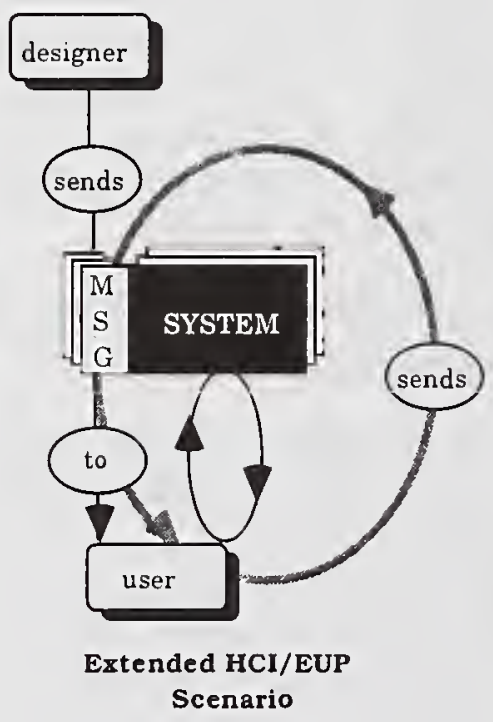

Figure 1: Communication in $\mathrm{HCl} \&$ EUP
Along with each extensible application, a rich learning environment must be provided to users. Important components thereof are multimodal multicode interfaces, explanation KB-systems, on-line help and documentation modules, as well as textual and graphic programming environments. Embedded explanation modules should help designers adopt a communication perspective as if they were engaged in documentary book-writing or film-making, and cared to get their message across.

The basic architecture we envisage for truly extensible computer applications, from an end user's point of vicw, is presented in Figure 2. At first sight, the adopted architccture may appear overwhelmingly complex and undesirable from a cost/benefit perspective. Nevertheless, at a closer look, we realize that a number of popular office automation packages already offer users most of the proposed components. On the User Interface side, the only lacking modes are the NL question-answering and the visualization facilities. MS Word for Windows, for example, includes the other 3 alternatives and a macro language interpreter of its own.

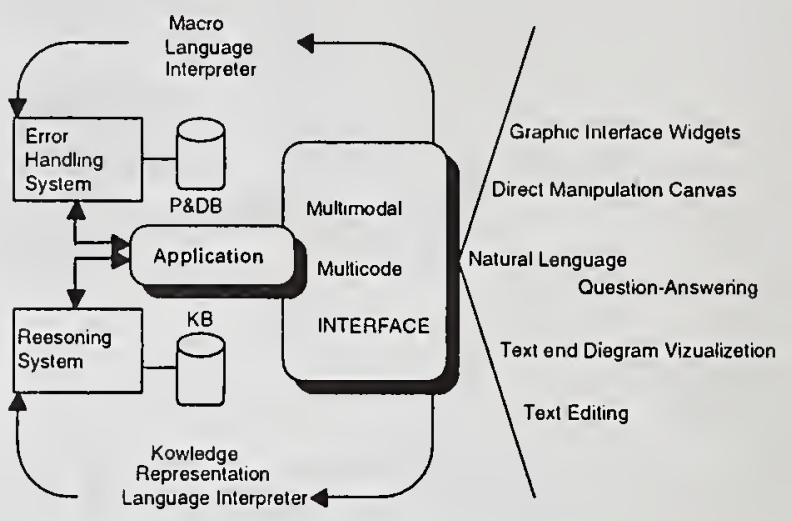

Figure 2: A Communication-Centered Architecture

The missing end of existing extensible applications is the knowledge base one. In the lower left side of Figure 2, we clearly see an embedded intelligent system (reasoner and Knowledge Base (KB)). In the upper side, we see a runtime consistency checker and a Program and Data Base (P\&DB). This latter component is the repository of end users' programmed extensions which are added to the application in separate modular form.

The overall communication management is achieved by means of integrated programming-explaining activities. When a user engages into introducing a new functionality to an existing application, explanations are elicited from him or $\mathrm{hcr}$ in schematic form. Thus, not only are new items introduced in the P\&DB, but also the corresponding cxplanatory items are added to the $\mathrm{KB}$. This feature requires that programming and knowledge representation languages be co-referential and maintain consistency between each other:

Co-referentiality between input and output languages has been previously proposed in the framework of user-centered systcms design [8]. Our leap here is to extend linguistic coreferentiality from the realm of interfaces to that of applications. Of course, requiring users to explain their programming to the system is preceded by requiring the applications' designers and programmers to do the same. The whole point of communication contered programming is to tell pcople what we as programmers have in mind and incrcase the chances that we are understood, and that our product becomes really useable and useful.

\section{HUMAN-COMPUTER INTERACTION, KNOWLEDGE-REPRESENTATION, AND END- USER PROGRAMMING}

Wc will now illustrate how a communication perspective impacts design by resorting to fcatures of a demo $\mathrm{KB}$ application we have becn working on. It is based on Pattis et al.'s "Karel, the Robot - A Gentle Introduction to the Art of Programming" [16], whose aim is to tcach novices how to 
program. We have built a Visual Basic Interface to Karel's World (see Figure 3, at the end of this paper), and provided interaction for Karel's minimal operating capacities about moving, turning left, picking up and putting down beepers, in a gridded (limited) space where walls can block his passage. We have also opened the opportunity for extensions by allowing Karel to learn new things. The simplest of these, appears in the upper side of the screen on Figure 3 ("Karel's learned actions") - TURN AROUND. Along Pattis's lines, TURN AROUND is a possible user-programmed extension to Karel's ability that results from a sequence of 2 consecutive primitive TURN LEFT commands grouped together by some macro recording and naming mechanism.

But, unlike what holds for the original proposal, we want users to be told about (1) thc original programmer's arbitrary decisions, (2) this programmer's personal interpretations and intentions in endowing Karel with computing capacity, and (3) getting the notion of how he or she can now, as a user, change the original model and express his or her own interpretations and intentions in playing with the programmable robot.

At the User Interface level, the new perspective in design becomes evident in the layout shown in Figure 3. The World View window allows users to deploy walls and beepers in Karel's world via direct manipulation of graphic objects. Since the idea is to program (i.e. to tell, in linguistic mode) Karel to evolve in this space, direct manipulation is halted when it comes to commanding the robot to move and change the world. For this, users have the more traditional options of menu selection and button pressing.

Two additional windows appear at the interface: one for Karel's messages and anothcr for the designer's messages. The former are the result of the robot's being asked situational questions such as: "How many beepers do you have?" or "Where have you been?". The latter are produced as a typical result of "Why" and "Why-not" questions. For instance, queries about "Why Karel cannot carry more than 10 beepers with him" should not be directed to Karel, but to his "creator" - the programmer. The answer is expected to be that the number 10 is an arbitrated value a user CAN or CANNOT alter in the original application.

Note that knowledge in this scenario is used BY THE DESIGNER, not by the agent. Karel IS NOT an intelligent agent. We could endow Karel with virtually no knowledge and have it shutdown if he is ordered to move forward and bumps into a wall. However, in our program, we have decided to test for disastrous actions before they are performed. It is the programmer, and not Karel, who stops the agent short of shutting down and uses a $\mathrm{KB}$ explanation system to reflect his decision that Karel is a physical model which does not traverse walls. In Figure 3, another shutdown situation caused by a wrong MOVE command is prevented by the designer and explained in the lower right-hand side of the screen.
An interesting extension case is that of a user "teaching" Karel how to GO HOME. Expcrienced programmers would do this by implementing complex graph navigation algorithms, so that the shortest possible path between Karel's current position and his home (coordinate 0,0 ) is securely followed. End user programming, however, could introduce some apparently "naïve" computational solutions as making Karel go home by walking back on his steps (note that in Figure 3, users can ask Karel "Where have you been?"). Since (1) he always starts his journey from home, and (2) any place he can currently be has necessarily been reached through a valid path, he can always track his way back by resorting to his "memory" of visited places.

Minimal path algorithms can be as much of an idealization as indefinitely large memory store space (required for Karel to be able to remember EVERYTHING he has done from the beginning of a session). For an EUP scenario, the best choice is that which represents the least cognitive effort for the user to program. But, an experienced programmer certainly has reasons to go for the minimal path idealization. Among other qualities, this approach is more general than the maximal storage one; it serves a general capacity of going from any one point in space to another, no matter the peculiar relations they may bear on an agent's experience in the world (e.g. having been previously visited, having beepers at it, having walls, being home).

For Karel to be able to GO TO anywhere in his world, there must be KNOWLEDGE about the way he was designed. Most KB systems have no problem in endowing the agents with such reasoning capacity. Tale telling interfaces to such systems slip easily into 1st person discourse and present messages that start with "I cannot" or mention "my path". The implications of such anthropomorphism may lead users to build numerous misconceptions about how intelligent and able computer agents can be. For instance, given that an agent knows he cannot traverse walls, he could be expected 10 know why and what would happen if he could (i.e. have meta-knowledge).

In our Semiotic Engineering approach, the designer is a legitimate voice in discourse. Whatever the agent can or cannot do, it is a consequence of human interpretation and programming. Therefore, if Karel is endowed with the ability go from any one point to another, or diagnose he is walled into (or out of) a subspace, this is the product of a designers intellectual and technical decision.

The impacts of having users identify the origin of such decisions and interpretations is similar to that of letting them know that trying to undo a SAVE action in typical text cditing interaction is not wrong. It is perfectly logical to try to backtrack from SAVE, in the same way one backtracks from a SELECT_ALL and DELETE sequence of actions. However, it is the cost of maintaining formatted files in "buffers" within the application that gears arbitrary choices, even if net damage in both situations is rigorously the same 
from the user's point of view (i.e. backtracking from a disastrous piece of interaction).

The costs of imparting more knowledge in extensible application environments may be high. But, we will give an example of how we have designed an explainable $\mathrm{KB}$ in Visual Prolog, to be coupled to Karel's Visual Basic Interface. It should show that the effect of such knowledge is quite considerable compared to current commercially available products. A span of Prolog programming for our $\mathrm{KB}$ is shown below.

1. count_bagbeepers :-

not (beepers_in_bag(_)), nl, write("Karel and his world have not been loaded yet." ), nl, ! .

2. count_bagbeepers :- beepers_in_bag $(\mathrm{X})$, $\mathrm{x}=0, \mathrm{nl}$, write ("As of now, I carry no beepers in my bag."), nl, !.

3. count_bagbeepers :- beepers_in_bag $(X)$, $\mathrm{X}<>0, \mathrm{nl}$, write ("As of now, I carry ", $\mathrm{X}$, " beeper(s) in my bag."), nl, ! .

Messages associated to the count_beepers predicate are answers to questions about how many beepers Karel is carrying with him at a given point. Although they may at first look like regular simple output messages any program can print, from a $\mathrm{KB}$ perspective they are epistemologically different. Clauses 2 and 3 are consistent with an agentcentered point of view. Clause 1, however, is quite different in that an agent that hasn't been "created" (loaded) cannot possibly inspect his state. In communicative terms, clause 1 is used in the generation of a Designer's Messages, whereas the other two are used in that of Karel's Messages. The effect of multiple-person discourse is more clearly noticeable in the following case.

no_move (east, coord $(X, Y)$ ) :-

4.1. NewX $=X+1$, falloff $(\operatorname{coord}($ NewX, Y $)), n l$, write("This move would make Karel fall off the limits of his world."), nl, !;

4.2. NewX $=X+1, \operatorname{bump}(\operatorname{coord}($ NewX, $Y)), n l$, write("This move would make Karel bump right into a wall."), nl, !.

The above OR clause (";") states the disjunction of conditions that are sufficient for a move to be impossible (codified in the KB by predicate the no_move (east, coord $(X, Y)$ )). In our design, this clause is used in messages generated by the designer - not by Karel. Our intention is to prevent Karel from being conceived as an intelligent (even if modestly so) agent. The implicatures in switching discourse from designer to Karel have been associated above to arbitrary levels of metaknowledge designers would have to endow computer agents with. The role of a designer is then to dismiss misconceptions with messages such as the proposed in 4.1 and 4.2. Moreover, the very fact of telling "the truth" about a computer artifact duly places designers in their seat of software constructors, and weakens the idea that software is "just like that", hopelessly inexplicable.
At KB design time, a communication perspective leads us to generate code of the sort illustrated by the following Prolog lines relative to reasoning about move:

\section{1. move, nl, write("I have moved forward." ), nl, !; \\ 5.2. not (move), nl, write("Karel has not moved forward."), nl, !}

In other words, we codify not only the knowledge about the conditions that allow Karel to move and know such things as his new position and what action he has just performed, but also those conditions that prevent him from doing so. In each case, discourse is produced by different communicators. For every negated action, there must by an associated WHY NOT question users may ask DESIGNERS to understand what happened. This is made possible if designers introduce knowledge codified as.

\section{6. karel (Ori, LoC), no_move (Ori, Loc), !.}

Combined with the instantiation of the no_move predicate proposed in 4.1 above, goal 6 generates the explanation illustrated in Figure 3. Thus, a discipline in generating explanatory discourse for every piece of extensible code in a given application is required from designer. This discipline can be facilitated by a programming environment that elicits knowledge from designers and maintain co-referentiality between programs and knowledge base. From the Visual Basic end, the code for MOVING Karel has only minor additions, as seen in:

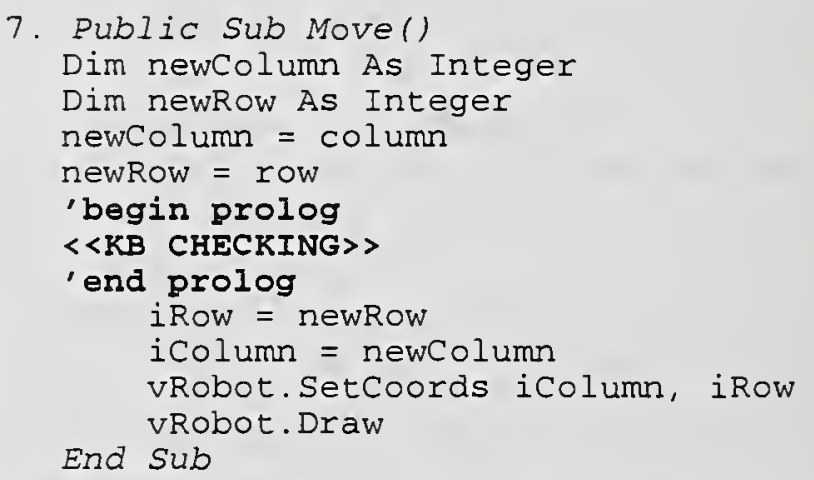

The Prolog program call in the code may include data exchange or file read/writing instructions. The real change in design is a consistency checking one step before codifying the new drawings to appear in the GUI depicted in Figure 3. The bonus is the generation of explanations and the transmission of the designer's rationale in building these parts of the application. We should emphasize that those parts that are not meant to be extended by the user do not require the same degree of knowledge elicitation and explanations.

From a user perspective, the interface overhead is that of connecting user-generated code to user-generated explanations about code. Just as EUP languages are not $\mathrm{C}$ or Pascal, but some easier subset of these, $\mathrm{KB}$ representation languages are not Prolog. The need for more adequate programming languages goes hand in hand with the need for 
more adequate knowledge acquisition languages. This is the aim of ongoing research in our current project.

\section{CONCLUSION}

Computer Semiotics has grown from Interface Design [13], to Systems Analysis and Design [2], and Multimedia Applications Design $[1 ; 14]$. Previous semiotic accounts of computing, included mathematical perspectives [e.g. 18], as well as philosophical ones [e.g. 9]. The work of Peirce, himself, with its original logic underpinnings [17], now experiences a revival in theoretical computing and information processing [e.g. 10].

The anticipated need for end user programming has not been fully explored by Computer Semiotics [6], and requires that software design be made with a view on letting users learn more about applications than is required in most current $\mathrm{HCI}$ scenarios. Heavier cognitive loads involved in programming, if compared to typical human-computer interaction, magnify the architectural scope of application environments and suggest that embedded knowledge-based systems may play a major role in supporting the organization of explanatory discourse about extensible application modules. In semiotic terms, reasoning systems allow users to gain one degree of perspective on a designer's interpretation of a given domain, its objects and tasks, as well as on his or her encoded abstractions and arbitration in building a computer model of the domain and its implementation.

This leads us to adopt a broader semiotic perspective on Software Engineering, and viewing programs generated in the communicative approach proposed above as effectors of limited semiosis. Although the term may shock the Peircean tradition, in Computer Science it gains life and dimension as interface messages are algorithmically interpreted in a procedurally limited chain of signs that turn into other signs and eventually crystallize into output. Whichever sign is present in this chain was once part of the designer's interpretant derived from the application's domain.

Knowledge-based components attached to extensible applications empower the designers' capacity to express themselves, and the users' capacity to make sense out of systems. We have shown how embedded KB explanation systems enlarge the spectrum of interface discourse and allow for computer agents, programmers and users to express their why's and why-not's in writing and executing software. The linguistic overhead is that of designing co-referential programming and knowledge representation languages that users can access from the application's interface.

\section{Acknowledgements}

I would like to thank Simone Barbosa for her valuable help in programming and discussing the main concepts presented in this paper. I also owe much reflection about this theme to Ernest Edmonds and Allen Cypher, and to Frieder Nake who has been particularly insightful with his discussion of "algorithmic semiosis".
Thanks also go to my graduate students, who always make me learn and grow with their enthusiasm, constant questioning and generous trust.

\section{REFERENCES}

1. Andersen,P.B.; Holmqvist,B.; and Jensen,P. (Eds.) Computers as Media. Cambridge. Cambridge University Press.

2. Andersen,P.B. (1990) A Theory of Computer Semiotics. Cambridge. Cambridge University Press.

3. Apple Computer, Inc. (1992) Macintosh Human Interface Guidelines. Reading,Ma. Addison Wesley.

4. Cypher,A. (1993)Watch what I do: Programming by Demonstration. Cambridge, Ma. MIT Press.

5. de Souza,C.S. (1993) The Semiotic Engineering of user interface languages. in International Journal of ManMachine Studies. No.39. pp. 753-773

6. de Souza,C.S. (1996) The Semiotic Engineering of Concreteness and Abstractness: from User Interface Languages to End-User Programming Languages. in Andersen,P.; Nadin,M.; Nake,F. (1996) Informatics and Semiotics. Dagstuhl Seminar Report No. 135, p. 11. Schloß Dagstuhl., Germany.

7. DiGiano, C. and Eisenberg, M. (1995) Self-disclosing design tools: A gentle introduction to end-user programming. in Proceedings of DIS '95. Ann Arbor, Michigan. August 23-25, 1995. ACM Press.

8. Draper,S.W. (1986) Display managers as the basis for user machine communication. in Norman and Draper (Eds.) User Centered System Design. Hillsdale. Lawrence Erlbaum and Associates. pp. 339-352

9. Eco,U. (1998) On truth: a fiction. in Eco, Santambrogio and Violi (Eds.) Meaning and Mental Representations. Bloomington. Indiana University Press

10. Hammer,E. M. (1995) Logic and Visual Information. Stanford, Ca. CSLI Publications.

11. Microsoft Corporation (1995) The Windows Interface Guidelines for Software Design. Redmond. Microsoft Press.

12. Myers,B. (1992) Languages for Developing User Interfaces. Boston. Jones and Bartlett.

13. Nadin, M. (1988) Interface Design and Evaluation Semiotic Implications In Hartson, R. and Hix, D. (eds.), Advances in Human-Computer Interaction, Volume 2, 45- 100

14. Nadin, M. (1995) Negotiating the World of Makebelieve: The Aesthetic Compass. in Real-Time Imaging I, pp.173-190

15. Nardi,B. (1993) A Small Matter of Programming. Cambridge, Ma. MIT Press

16. Pattis,R.E.; Roberts,J.; and Stehlik,M. (1995) Karel the Robot: A Gentle Introduction to the Art of Programming. New York, N.Y. John Wiley and Sons. 
17. Peirce, Charles Sanders (1931) Collected Papers, Cambridge, MA: Harvard University Press (excerpted in Buchler, Justus, ed., Philosophical Writings of Peirce, New York: Dover, 1955)
18. Zemanek,H. (1966) Semiotics and Programming Languages. Communications of the ACM. Vol.9. No.3. pp. 139-143.

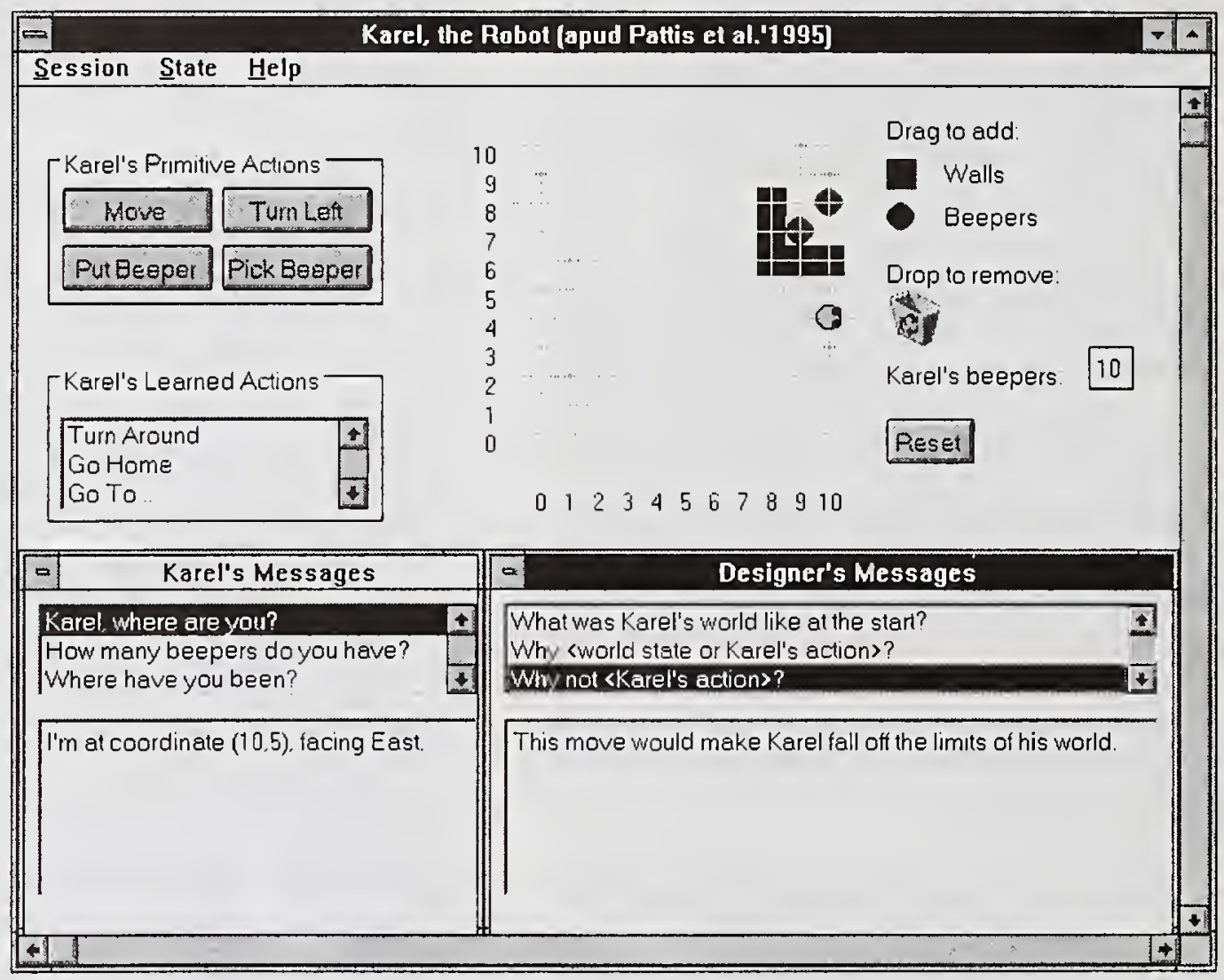

Figure 3: A snapshot of Karel's World screen. 


\title{
An Approach to Computational Semiotics
}

\author{
Ricardo Gudwin and Fernando Gomide \\ DCA-FEEC-UNICAMP \\ Caixa Postal 6101 \\ 13.083-970 Campinas - SP - Brasil \\ e-mails: gudwin@dca.fee.unicamp.br, gomide@dca.fee.unicamp.br
}

\begin{abstract}
The aim of this paper is to introduce the theoretical foundations of an approach for intelligent systems development. Derived from semiotics, a classic discipline in human sciences, the theory developed provides a mathematical framework for the concept of knowledge and for knowledge processing. As a result, a new perspective to study and to develop intelligent systems emerges. A taxonomy of elementary types of knowledge is proposed based on the classification of types of signs in semiotics, followed by a another classification of knowledge from the point of view of application in cognitive systems. In addition, we propose the mathematical definition of objects, objects systems and objects networks, to model mathematically the different types of knowledge described. The symbiosis of such key concepts introduces a computational paradigm to develop and implement intelligent systems, called here computational semiotics.
\end{abstract}

KEYWORDS: computational semiotics, theory of objects, intelligent systems, models of knowledge

\section{INTRODUCTION}

Human intelligence has always been of interest and curiosity in the scientific world. In 1991, Albus published an outline for a theory of intelligence, and simultaneously Brooks [3] argued that for an intelligent behavior, there should not necessarily exist representation or inference. Additional aspects of intelligence, e.g. approximate reasoning (including fuzzy or incomplete concepts), learning, prediction, and adaptation are being studied in the fields of computational intelligence [14] and soft computing [13]. Considerable research effort on fuzzy set theory, neural networks and evolutive systems have and still are being pursued. The contribution of these fields in understanding the nature of human intelligence has been quite impressive.

Parallel to the developments in computer science and engineering, in human sciences there was a similar effort to model intelligence and intelligent behavior. Well known examples include the work of Piaget [2], and the development of semiotics by Peirce and Morris $[12,9,10,11]$, just to mention a few. Semiotics deals with signs (representations), objects (phenomena) and interpretants (knowledge), that is, the main issues in cognition and communication. Semiotics has shown to be an useful tool especially when the basic ingredients of intelligence and their relationships are of concern.

Despite the challenges in discovering the formal mysteries behind human intelligence and the intrinsic difficulties in building machines and computer programs to emulate intelligent behavior, very few works analyzing intelligence in an integrated and organized manner have been done. Often, only particular aspects of intelligence are addressed. A notable exception comes from Albus' 1991 paper. In his work, Albus provides a systematic study of intelligence, and gives a description of the different parts composing the global phenomena. The integration of all parts should lead to intelligent behavior. Albus definitions and theorems are essentially linguistic due to the lack of a formal system to describe intelligence. In other words, currently there is no adequate mathematical model to describe intelligence as a whole. Most existing formalisms are closely tied to particular aspects, being unsuitable for a global, computational formalization of intelligent systems. Semiotic Modeling and Situation Analysis-SSA, developed by Pospelov and his team in Russia was another important attempt in this direction. A key feature of the SSA approach is extraction of knowledge from the descriptive information by its consistent analysis based upon well established algorithms [7]. From this point of view, mathematical tools of semiotics are considered to include those used in control science, pattern recognition, neural networks, artificial intelligence, cybernetics. But semiotic specific mathematical tools (for combining signs, symbols and extracting meaning) are still in the process of development [7].

In [8], the use of semiotics as a tool suitable for the analysis of intelligent systems was suggested. Concurrently, in [4] the computational view of semiotics for modeling, development and implementation of intelligent systems, the computational semiotics approach, was proposed. Computational semiotics is build upon a mathematical description of concepts from classic semiotics. Its formal contents can be regarded as a contribution towards the development of semiotic specific mathematical tools. Thus, it is in the very realm of the formal foundations of intelligent systems. The main purpose of this paper is to introduce the mathematical aspects which subsumes computational semiotics. 


\section{ELEMENTARY TYPES OF KNOWLEDGE}

Based on the classification of types of signs in semiotics, we have derived a hierarchy of elementary types of knowledge, shown in figure 1.

Knowledge is divided in three main classes, the rhematic knowledge, dicent knowledge and argumentative knowledge. Strictly speaking, rhematic knowledge concerns the semantic of (linguistic) terms, dicent knowledge combines sequences of terms with truth values and analyses how rhematic knowledge relates to a real environment, and argumentative knowledge embodies the knowledge of how knowledge is transformed comprising reasoning, inference and learning.

\section{APPLIED KNOWLEDGE}

Based on its intended use, knowledge can be classified as designative, apraisive or prescriptive (figure 2), terms coined by Morris $[9,10,11]$. This classification is complementary to the elementary types of knowledge. In principle, any elementary type of knowledge can be used as designative, apraisive or prescriptive, i.e., this classification is orthogonal to the elementary classification.

Designative knowledge models the world. For this purpose it uses rhematic, dicent and argumentative knowledge, either specific or generic. Designative knowledge can also be viewed as descriptive knowledge. A cognitive system initially has just a few, or eventually no designative knowledge at all. Usually

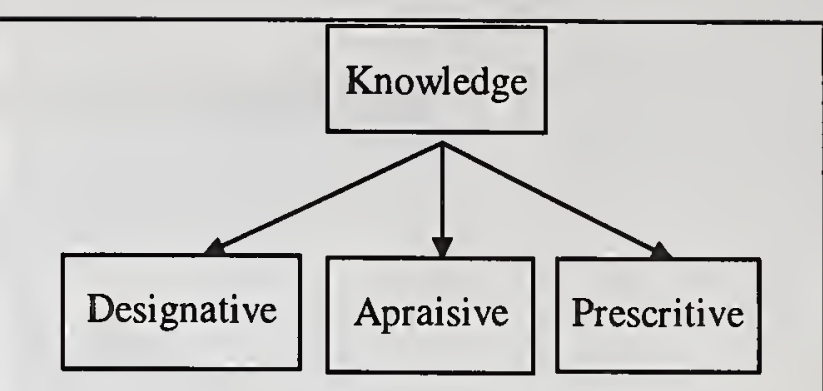

Figure 2 - Classification of Applied Knowledge

designative knowledge emerges from the interaction between the system and world.

Apraisive knowledge is a type of knowledge used as an evaluation, a judgment, a criteria to measure the success in achieving goals. In natural systems, apraisive knowledge is closely related with the essential goals of a being; reproduction, survival of the individual, survival of the specie, increasing knowledge about the world, for example. Depending on the goal it assumes special forms like: desire, repulse, fear, anger, hate, love, pleasure, pain, confort, disconfort, etc. Essentially, apraisive knowledge evaluates if a given sensation, object, or occurrence is good or not, as far as goal achievement is concerned.

Prescriptive knowledge is intended to act on the world. Basically, prescriptive knowledge is used to establish and to implement plans through actuators. However, prescriptive knowledge will not necessarily end up with an action. Prescriptive knowledge may also be used to do predictions, but only one of them is selected to generate an action.

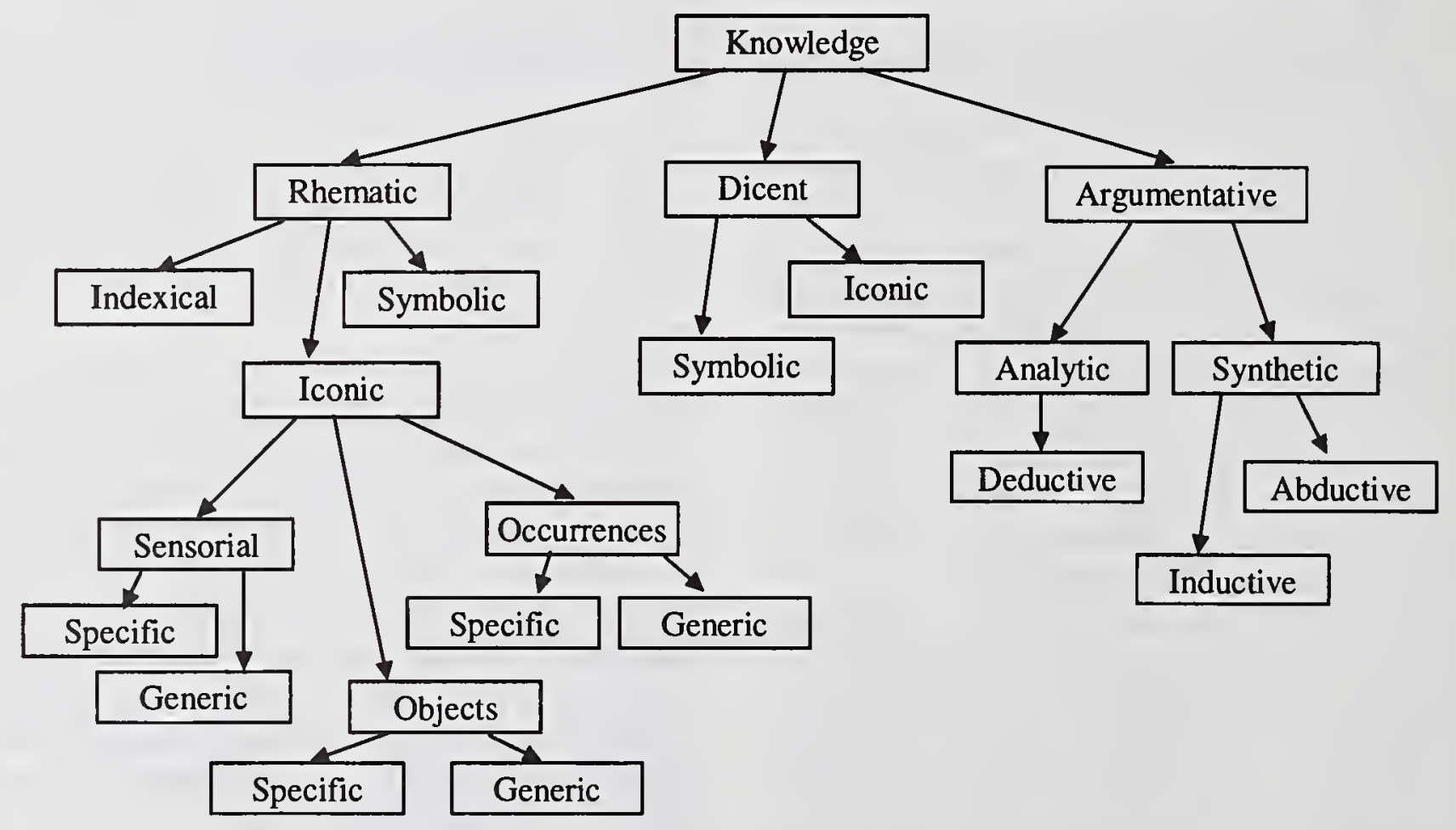

Figure 1 - Classification of the Elementary Knowledge Types 


\section{A MATHEMATICAL THEORY OF OBJECTS}

This section introduces concepts and definitions as a formal background for computational semiotics. The focus here is on the main issues and definitions only. For a more in depth coverage the reader is referred to [4] or $[5,6]$.

\subsection{Variable}

Let $\mathrm{N}$ be a countable set with a generic element $\mathrm{n}$, and $X \subseteq U$. A variable $x$ of type $X$ is a function $x: N \rightarrow X$. Note that a function is also a relation and hence it can be expressed by a set. Thus, $\mathrm{x} \subset \mathrm{N} \times \mathrm{X}$.

\subsection{Class}

A class $C$ is a set whose elements $c_{i}$ are tuples of the type: $\left(v_{1}, v_{2}, \ldots, v_{n}, f_{1}, f_{2}, \ldots, f_{m}\right), n \geq 0, m \geq 0$ where $v_{i} \in V_{i}$, and $f_{j}$ are functions

$$
f_{j}: \sum_{p \in P_{j}} V_{p} \rightarrow \underset{q \in Q_{j}}{X} V_{q} .
$$

Here means the cartesian product, $\mathrm{P}_{\mathrm{j}} \subseteq\{1, \ldots, \mathrm{n}\}$ and $\mathrm{Q}_{\mathrm{j}} \subseteq\{1, \ldots, \mathrm{n}\}$.

\subsection{Object}

Let $\mathrm{C}$ be an non-empty class and $\mathrm{c}$ be a variable of type $\mathrm{C}$ Thus $\mathrm{c}$ is an object of class $\mathrm{C}$.

\subsection{Object System}

A set of objects $c_{i}$ is an object system if the $c_{i}$ 's are related to each other in the sense that each instance of such objects in a given instant is a function of the instances of all objects in the previous time instant:

$$
c_{k}(t+1)=f\left(c_{1}(t), \ldots, c_{n}(t)\right) .
$$

This is only a concise definition of an object system. The complete definition is far more involved. The reader is referred to [4] or $[5,6]$ for details.

\subsection{Object Network}

An object system is a too generic approach for modeling elementary knowledge types. An object network is a special type of object system in which additional restrictions concerning interactions are included. To distinguish object network and object system let us assume places and arcs whose roles are similar to those in Petri nets. Objects in places can only interact with objects in places connected through arcs. Thus, at each instant, the objects defined should be at one place. For each place there is a set of places connected with through of input arcs. These places are called the input gates of the place. Analogously, each place has a set of places connected with it by means of output arcs, called output gates.

\subsection{Additional Definitions}

Other definitions, important for the particular aspects of some of the knowledge types, may be found in [4] and [5,6]. They include the temporal restriction for objects, set variable, generic objects, fuzzy objects, meta-objects, instances of metaobjects, occurrences of meta-objects in objects, generic objects and fuzzy objects, generic meta-objects, occurrences of generic meta-objects in objects, generic objects and fuzzy objects, fuzzy meta-objects, occurrences of fuzzy meta-objects in objects, generic objects and fuzzy objects.

\section{MODELS OF KNOWLEDGE TYPES}

Using the mathematical definitions given above, we are able to model the elementary types of knowledge and use them to build intelligent systems, mainly concerning the applied aspects of knowledge, i.e., their property of being designative, apraisive or prescriptive.

Basically, sensorial and object rhematic iconic knowledges can be modeled by passive objects, i.e., objects that do not have functions in its image tuples. Occurrence rhematic knowledge can be modeled by meta-objects (standard, generic or fuzzy). Those meta-objects can be reduced, however, to objects by means of appropriated techniques. Dicent knowledge can also be modeled by objects. Argumentative knowledge must be modeled by active objects, i.e., objects that have working functions in its image tuples. The complete description of such modeling representations can be found in [4] or [6].

This leads to a scenario where a whole intelligent system may be modeled by an object network. The representation by means of an object network has many advantages. An object network is more powerful than a Petri net in the sense that it allows modifications in its active parts, what is not possible in Petri nets. This is important for systems that have learning and adaptive capabilities, which can not be represented by Petri nets, including there colored Petri nets. The possibility of representing an intelligent system by a formal computational tool allows for a more in depth study of phenomena involving intelligent behavior. Some properties that were targeted linguistically in early studies of intelligent systems (e.g. [1]), may be translated into a mathematical framework, allowing for a more solid foundation. In this sense, the tools provided by computational semiotics seems to be a very promising set of mechanisms for building a future theory of intelligence. 


\section{CONCLUSIONS}

In this paper we briefly introduced a new approach for the study of intelligent systems. This approach, called computational semiotics, uses the concepts brought from semiotics to propose a hierarchy of elementary types of knowledge, and based on a mathematical framework, models such knowledges in a mathematical way. Due to its object oriented nature, the mathematical model is very suitable for computational implementation, providing in addition, a mathematical description of intelligent systems.

It is very important to stress, though, that such an approach is in its very beginning. The presented taxonomy of types of knowledge, despite significant, is only partial. In his works, Peirce identifies more than 100 different types of signs, eventually implying in different types of knowledge. These are not included in the presented taxonomy. But, the presented taxonomy provides an elaboration of rather sophisticated intelligent systems. More than that, it creates an organization that is not usually found in literature, concerning the differences among the knowledge used when building intelligent systems. The formalism presented for objects in this paper, does not aim to be a general theory for objects, but simply put foundations for a future grow of such a theory. Some extensions are actually needed, mainly to consider asynchronous interaction among objects. But, the formalism, in its current form, is suitable to represent intelligent systems, what is a very important characteristic. Despite its representation power, the object networks developed upon the mathematical concept for objects still have many limitations. For example, analysis tools are still incipient, when compared with other modeling tools, e.g., Petri Nets. Indeed, very few systems have been modeled by the object network formalism. In addition, there is a lack of a formal representation for the types of knowledge not covered by our elementary knowledge hierarchy. As new types of knowledge are included in the hierarchy, new formal definitions would be demanded. Very few intelligent systems were built so far using the computational formalism. To consolidate object networks as a valid and general tool for modeling intelligent systems, it is still necessary to solve a broad class of problems to emphasize its virtues and to precisely identify the extensions needed.

An application example concerning the control of an autonomous vehicle was successfully developed and implemented using the computational semiotics approach $[4,6]$.

Acknowledgements: The first author acknowledges $\mathrm{CNPq}$ for a fellowship and to FAPESP. The second author acknowledges CNPq for grant \#300729/86-3.

\section{REFERENCES}

[1] Albus, J.S. "Outline for a Theory of Intelligence" - IEEE Transactions on Systems, Man and Cybernetics, vol. 21, n. 3, May/June 1991.

[2] Boden, M. A. - As Idéias de Piaget - tradução de Álvaro Cabral - Editora Cultrix - Editora da Universidade de São Paulo - São Paulo, 1983 (in portuguese).

[3] Brooks, R.A.- "Intelligence Without Reason" in Proceedings of the Twelfth International Conference on Artificial Intelligence, Vol. 1 (1991) 569-595.

[4] Gudwin, R.R. - Contribuições ao Estudo Matemático de Sistemas Inteligentes - Phd Thesis - DCA-FEEC-UNICAMP, Maio 1996 (in portuguese)

[5] Gudwin, R.R.; Gomide, F.A.C. - "Computational Semiotics : An Approach for the Study of Intelligent Systems - Part I : Foundations" - Technical Report RT-DCA 09 DCA-FEEC-UNICAMP, 1997.

[6] Gudwin, R.R.; Gomide, F.A.C. - "Computational Semiotics : An Approach for the Study of Intelligent Systems - Part II : Theory and Application" - Technical Report RTDCA 09 - DCA-FEEC-UNICAMP, 1997.

[7] Meystel, A.M; Albus, J. - "Intelligent Systems : A Semiotic Perspective" - Report NIST, Intelligent Systems Division, Bldb. 220 Room B124, Gaithersburg, MD 20899, USA, 1996.

[8] Meystel, A.M. - "Intelligent System as an Object of Semiotic Research" - 1996 Biennial Conference of the North American Fuzzy Information Processing Society - NAFIPS New Frontiers in Fuzzy Logic and Soft Computing Berkeley, California, USA, June 1996.

[9] Morris, C.W. - Signs, Language and Behavior - New York : Prentice Hall, 1947

[10] Morris, C. W. - Significant and Significance - New York - Prentice Hall, 1964.

[11] Morris, C. W. "Foundation for a Theory of Signs" - in Writings on the General Theory of Signs - The Hague : Mouton, 1971

[12] Peirce C.S. - Collected Papers of Charles Sanders Peirce - vol I - Principles of Philosophy; vol II - Elements of Logic; vol III - Exact Logic; vol IV - The Simplest Mathematics; vol V - Pragmatism and Pragmaticism; vol. VI - Scientific Metaphysics - edited by Charles Hartshorne and Paul Weiss - Belknap Press of Harvard University Press Cambridge, Massachussets, $2^{\text {nd }}$ printing, 1960.

[13] Zadeh L. - "Soft Computing and Fuzzy Logic", IEEE Software, vol 11, n. 6, pp. 48-56, 1994.

[14] Zurada, J.; Marks II, R.J.; Robinson, C.J.Computational Intelligence - Imitating Life - IEEE Press, USA, 1994. 


\title{
Sequential Algorithm for the Semantic Representation of Actions in Terms of Moving Parts
}

\author{
Hiroyuki Nishina \\ Saitama University \\ 255 Shimo-Okubo, Urawa, Saitama 338, JAPAN
}

\begin{abstract}
This paper reports on a new model giving a sequential semantic representation of an action verb. We show that the algorithm for static categorization which utilizes locational information to represent the defining characteristics of individuals can also play a central role in the dynamic categorization. Static categorization involved in the definition of stative predicates is stipulated in terms of Category Function. Orientation Function, taking as one of its arguments the defining characteristics of the denotation of each category, is defined to capture the correspondence relation between the bodies of the same individual at two different time-points. With the former two functions and the function derived from the latter, it is possible to construct an algorithm to trace the movements of the place-points of individuals' bases. This enables us to distinguish the parts which have more or less moved from those occupying the same space. Our point is that capturing sequentially the moving parts (of an individual) relevant for an action it performs is, in principle, necessary and probably sufficient to represent and interpret the cognitive meaning of action sentences. The moving parts playing central roles in order during each action are defined in terms of the features similar to those utilized in phonology. After the notion "interval" is introduced to decompose the structure of action sequentially, Action Function, the total algorithm for specifying the moving parts involved in each step of an action, is defined and discussed. The category function is built into this algorithm together with the interval length function. Action Function is a device for "monitoring" and mark the parts of an action as it proceeds. Action formula, another algorithm for action without recourse to intervals, is also defined and investigated. Finally, some residual problems involved in this framework are pointed out and discussed.
\end{abstract}

KEYWORDS: orientation, categorization, defining features, intervals, action function, action formula

\section{HISTORICAL BACKGROUND}

In the semantic studies sofar undertaken, efforts have beenmade to construct the adequate interpreting device based on the semantic relations born by the main verb to its arguments. For this purpose, several universal primitives have been sought to represent and explain the overall structure of propositional meaning. Lakofrs causative analy sis showed the manuer to factor verb meaning into primitives and represent it with lexical decomposition [7], and Jackendoff proposes the lexical structure in which propositional meaning is translated in terms of thematic relations [5], originally proposed in Gruber [4]. With these, it has become possible to represent explicitly the semantic organization of the overall state/event structure denoted by the relevant verb of each sentence. Thematicrelationswereneatlyaccommodatedintothe government and binding framework proposed in Chomsky as theta roles [2]. In the causative analysis, the causer is made distinct from the caused event, and in the thematic-relational analysis, verb meaning is categorized in terms of moving object, the way of motion, the moving manner, etc. In neither case, however, semantic "factorization" is sequential, thus neither analysis can represent the temporal change in the motion of the agent of an action in a stepby-step manner.

Montague Grammar was successf $u$ in representing the meaning of syntactic constituentsinpurelydenotational terms, whichprocedure was achieved in a compositional manner by using type theory [8]. His model theory consists of an ontology, a set of entities, and a denotation assignment function, the latter of which assigns a unique denotation to each constant in the object language. In this model, grammatical categories belong to functional types, thus the denotation of transitive verbs of $\langle e,\langle e, \downarrow\rangle\rangle$ being represented as $\left(\{1,0\}^{A}\right)^{A}$. This extensional analysis, however, cannot reach the internal structure of verbs, unlesssome decompositional approachis invoked. It did not dare to decompose verb meaning dynamically, solely translating it as a semantic primitive.

In this paper, I will introduce a new semantic model in which denotationassignment can be performed sequentially in the cases of action verbs in order to represent each step of their processes. I will show that by postulating that eachindividual is asequence of sets of place points occupied by it at time-points, a more articulate structure can be given to the model.

\section{CATEGORIZATION AND PARTS}

One of the human cognitive abilities is categorization of things from theirappearances. This is possible because humans can sort out some individuals as a specific kind from others by checking all the bits of its apparent definition. Humans can also further distinguish the individuals of the same category, not only humans but other higher animals, still lower organisms, even minerals. On the other hand, they can identify the same individual, though it is 
seen from different physical points of view. Thus, humans can readily identify the upright and upside-down figures of the same individual. The point is that despite such various differences, individual or orientational, humans can do readily determine membership. This indicates that criteria for categorization should not be too narrow nor broad to include into the same kind.

When recalling a thing of a certain category to mind, we can readily imagine the parts which contribute naturally to the definition of its kind. Thus, in the case of "horse", it has such and such a head, a tail, legs, etc. In that of "human being", he/she has such and such hands in addition to his/her head and legs. It would, however, be a bit unrealistic to think of a desk or a table without legs totally. Weassume that when confronted withanindividual whosecategory is to be determined, humans matchit with the stored "prototypical" sets of figures (incl uding their parts) shared among the members of therelevant categories. Each category can be successfully assigned to an individual only when the latter's figure can be exhaustively (or sufficiently in most in exceptional cases) decomposed into the primitive parts which are to be matched to those of the former's prototype. Those building parts of the targetindividual, in turn, can be reconstructed into the meaningful whole belonging to the fonner.

\section{CATEGORIZATION OF ACTIONS}

Motions are spatiotemporal objects. Human beings can recognize several, not all, of possible motions and verbalize them as actions if they fulfill the definitions of the action verbs of their own languages. This doesnot mean that the possible "actions" excluded from verbal actions cannot be linguistically expressed. They can be expressed by building words into phrases. In a purely physical point of view, actions are spatiotemporal continuums. Each action invol ves a continuous cliange of appearance exhibited by its agent. It would, however, be incorrect to say that humans can only recognize such objects as actions if they continue to observe them. In real perception, it is very often sufficient to recognize an action merely by glimpsing at the appearance of its agent. This is sufficiently possible despite the fact that all of the tokens of an action are distinct in not only time and location of occurrence but also duration, size, proportion etc. This is a piece of evidence that humans are capable of categorizingvarious tokens of amotion under one category of action in a manner that does not burden thein with so much load for recognition.

\section{POSTURES OF MOVING OBJECTS}

To understand what an individual is doing and to express (a part/parts of) its movement in terms of an action sentence of a natural language, we need to capture not onl y the position but the posture of the moving individual at each time during its action.
Seen as a moving physical object, however, an individual performing an action is undergoing a sequence of changes in the position of its volume. To decide which category of action the relevant movement belongs to, we have to deliberately distinguish the changes in its posture from the concomitant transition of its whole volume.

If we focussed solely upon the course of its movement, we could easily detect its trace as a path with some thickness occupied by the whole volume of the moving individual. But this is not enough. To accumulate sufficient information for categorization of action, we need to keep calculating the posture at each step of a mover in terms of the parts involved in the relevant action during it. There are two possible approaches at present available to realize this. The first one is to specify totally sequentially the sets of parts involved in the relevant action at each timepoint in tenns of a placepoint set. The second one is to show the inclusion relations of subactions which are sequentially described in terms of the parts involved in the total action.

\section{PARTS IN TERMS OF FEATURES}

It is useful toconsider how speech sounds are recognized by humans and described by linguists. In each particular language, despite the individual difference in the strength, length, pitches, quality, etc. of the "same" phone produced by speakers, they share some average /referential sound of psychological reality within their speech community. Tocapture this, in phonology, each family of phones that are approximately equal in articulatory point is selected from theinventory of all the phones producible by humans and is given a phonetic value, whichis givenalabel of "phoneme". Furthermore, ingenerative phonology, eachphonecorresponding toonephoneme is definedasa bundle of distinctive features, which specify a manner of articulation mainly based upon an articulatory point [3].

This is applicable to the definition of semantic representation of action verbs, since thelatteris definable in terms of "partsinvolved inits "constituent" movements". The difference between thesetwo cases is that the latter invol ves "action", the spatiotemporal object extending over time in such a procedural manner that requires a sequential definition of parts involved in the relevant action. Although the distinctive features for one sound mainly involve no factors related to time sequence since it is defined in terms of a simul taneous complex of articulatory parts, they provide us a promising hint toward the dynamic representation of action verbs. We thus assume that each action consists of a sequence of subactions (or sets of subactions) definable in terms of parts involved in

them.

\section{SPATIOTEMPORAL INDIVIDUALS}


By extending the index introduced in Montague and extended in Barwise and Perry, which consists of a possible world and an interval of time [1], we propose a model where the spatiotemporal structure of an action is described in terms of a sequential set of the features denoting the parts involved in that action. To enable this, the location of each individual is established based on placepointtimepoint pairs. Thus, each individual's location is represented as the set of placepoints occupied by it at each timepoint: Base Function, takingindividual and timepoint as arguments, returns the volume occupied by the former at the latter.

\section{BASE FUNCTION}

In order to specify (sets of) placepoints on individuals, it is necessary to be able to cal culate their location at each timepoint. Let each world at a timepoint be full of placepoints, with the same world continuing to exist along an axis of timepoints. At each time of its existence, each individual occupies a set of placepoints, which will hencef orth call its "base". To locate individuals' bases at each tine, we define Base Function as:

$$
\beta(x, y)=z \text {, where } x \in I N D, y \in T, z \in 2^{P} \text {. }
$$

Withindividual and timepoint asitsarguments, $\beta$ returns the set of placepoints (i.e. base) occupied by the former at the latter.

\section{ORIENTATION FUNCTION}

Weshowed that a specificoccurrenceof action canberepresentedby means of Orientation Function [9]. Taking a category, a set of placepoints and a placepoint as arguments, this returns as value a content point, whose ordinal numberindicates the orderin which to count the input placepoint of the base:

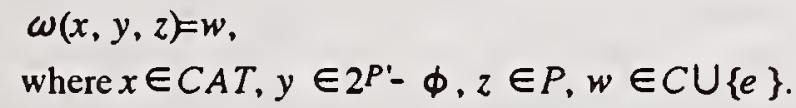

The resulting order is the criterion for orientation by which the relev ant individual is cognized by the observer as falling under the input category. It was shown that an instance of walking can be described in terms of a set of sequences of placepoints occupied by the content points of its agent individual.

\section{THEORETICAL BACKGROUND}

Accommodating the notion of "feature", introduced in Jacobson, Fant and Halle [6] and Chomsky and Halle, we proposedelsewhere that stative categories be defined by sets of features and that relevant (partsof)individualsbelocated by Category Function. This, taking a subset of placepoints and a feature, returns the placepoint set denoted by that feature:

$$
\kappa_{i}(x, y)=z, \text { where } x \in 2^{P}\{\phi\} y \in D_{i}, z \subseteq x \text { or } z=\phi \text {. }
$$

This function enabled us to set up Fulfillment Condition, which specifiesanindividual'smembershipin thesetdenotedby acategory in the following way: if and only if the union of the subsets of the base of an individual which correspond toall of the defining features of a static category is included into that base at a time point, the individual belongs to the relevant category at that time. Taking the category BIRD as an example, its prototype has a beak, wings, stick-like legs, etc. The fulfillment condition for BIRD is:

$$
\begin{aligned}
& \text { j } \\
& B I R D\left(\text { ind }_{a}\right) \text { at } t_{b} \Leftrightarrow U \operatorname{bird}\left(\beta\left(\text { ind }_{a}, t_{b}\right), d_{i}\right) \subseteq \beta\left(\text { ind }_{a}, t_{b}\right) \text {, } \\
& i=1 \\
& \text { where } D_{\text {bird }}\left\{d_{1}: \text { beak, } d_{2} \text { :wing, d;:stick-like leg, ... }\right\} \text {, }
\end{aligned}
$$

saying that at timepoint $b$, individual $a$ belongs to the category "bird" if and only if all of the defining features of this category, among which are "beak", "wing", "stick-leg", never fails to find their location on the base of $a$ at $b$.

To be able to identify the posture of a moving individual, we need some standard with which to measure the moving parts. For this we invoke the reverse partial function of Orientation Function, capturing the point-to-point correspondencebetween the bases of an individual at two different timepoints.

\section{PROBLEMS}

Thefirst problem addressed here is how to represent the continuous change in the shape of the moving agent as it performs an action. It was shown by us that this is done by decomposing each action into subactions definable in terms of the parts of its agent [10]. The second problem is how to identify various tokens of one action as belonging to the same category. Two candidates for this task are possible: to define each action as a sequence of sets of features, which specifies the parts in motion in order and as a complex of subactions by specifying a moving part individually. For the first algorithun, some device has to be created tofocus on the order of the parts involved in a whole motion, neglecting the real timelengths between the initial and terminal endpoints of the subactions involved in instances of the same type of action. To enable this, Interval Length Function is invoked to"neutralize" the individual difference in the relative lengths of the intervals which switch the parts in motion. Nointerval function nor its equivalent needed for thesecondalgorithm, where theorder of subactionsinvolvedineach action is sufficient for their sequential specification. Furthermoreit is necessary to compare the two devices and set up a relation between these. We will discuss this problem and give it a tentative answer elsewhere. 


\section{INTERVAL LENGTH FUNCTION}

We must now see an al gorithm which, at each interval on the whole time axis along which an action takes place, specifies a set of definingfeatures and locates their denotation on the placepointset occupied by its agent, i.e. its base. It is important to note, here, that the 'timing' of changing intervals is not specified, in view of the fact that agents of the same action usually differ in the lengths of compatible intervals. This reminds us the description of speech sounds in terms of phonemes, which we have seen before. Let us assume that Interval Length Function $f_{\text {intv }}(x)=y$ takes theinteger $x$ of the $x$-th interval involved in an action and returnsits length $y$ in terms of the number of the unit intervals constituting that interval:

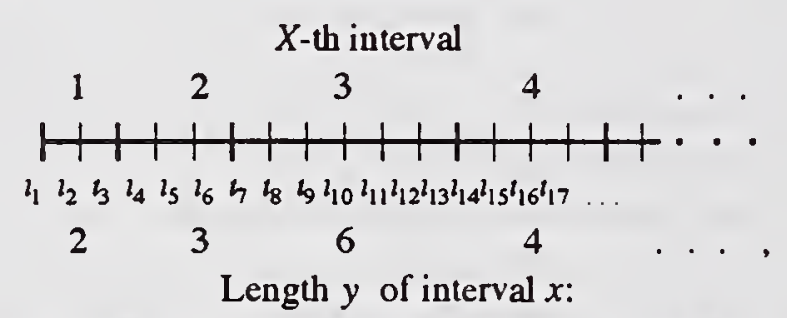

where $f_{\text {innv }}(1)=2, \quad f_{\text {inv }}(2)=3, \quad f_{\text {innv }}(3)=6, \quad f_{\text {invv }}(4)=4, \ldots$

If we represent each interval involved in an action as an ordered pair of its endpoints, the endpoints of $l$-th interval $<t_{q}, t_{\Gamma}>$ of the whole interval of an action starting at $t_{\mathrm{j}}$ are calculated by using Interval Function as follows:

$$
\begin{aligned}
& <t_{q}, t>_{u} \text { such as: } \\
& u=1: \quad<q=j, r=f_{\text {inwl }}(1)+j>, \\
& u-1 \\
& u \geqq 2:<q=\sum_{k=1}^{u} f_{i n t v l}(k)+\mathrm{j}, \quad \mathrm{r}=\sum_{k=1}^{u} f_{i n w l}(k)+j>.
\end{aligned}
$$

The calculation of the endpoints of each interval is based upon the fact that the number of the right endpoint is equal to that of the initial point plus that of the left endpoint.

In this sense, Interval Length Function plays an essential role in grouping various tokens of the same action together into one type of action, which has the invariant denotation of an action verb. By allowing eachinterval torange freely, this function hclps tocapture an observable fact that the lengths of the durations of the subactions constituting a whole action are usually different in its real occurrences.

\section{ACTION FUNCTION}

Watching an action closcly to its end, we can see that its agent successively changes its posture, during which the location in motion shifts from one sets of parts to anouler to complete an action. Thus, each action is defined as a sequence of sets of moving parts. To take the action of "nodding" as an example: first the head gets bent forward, second the neck moves forward to some extent while the head is continuing the same movement, third the movement affects the shoulder with the other two parts continuing the same, fourthall these parts halt for a moment, and they resume this process backwards. The sequence of moving parts taking parts in "nodding"canberepresented as: nod: $D_{n}=<\{$ head $\}$, $\{$ head, neck $\}$, \{head, neck, shoulder $\}, \ldots>$.

The sequential specification of moving parts enables us to define Action Function. At each interval of the whole duration of an action, this algorithm specifies a set of defining features and locates thcir denotation on the placepoint set occupied by its agent:

$$
\begin{aligned}
& \alpha_{i}\left(t_{j},\left\langle t_{q}, t_{p_{u}}, \kappa_{i}^{\prime}\left(\beta(x, y), D^{\prime}{ }_{i u}\right)\right)=z, j \leqq q+1 \leqq h \leqq r,\right. \\
& \text { where } x \in I N D, y=t_{h} \in T, z \in 2^{P}\{\phi\} \text {, } \\
& u=1:\left\langle q=j, r=f_{\text {innv }}(1)+j>,\right. \\
& u-\mathrm{t} \quad u \\
& u \geqq 2:<q=\sum f_{\text {innvl }}(k)+j, \quad r=\sum f_{\text {innll }}(k)+j>\text {. }
\end{aligned}
$$

If the base function, the first argument of the extended category function, which is in turn the third argument of an action function, takcs an individual and the starting timepoint $t_{j}$ and cal culates its base, then the action function picks up the first interval(, which contains that timepoint and is of allowably arbitrary length,) from within the overall interval of the action $\alpha_{i}$ starting at $t_{j}$ and return as its value a subset of the base which corresponds to the first set of features of the defining sequence for the action $\alpha_{i}$. Similar calculation isiterated until the rclevant timepoint exhausts the first interval. Switching to the second interval, the action function returns a subset of the base for the second feature set of the defining set and each timepoint of the second interval, similar process bcing iterated until the last interval is reached. Thus, the category function embedded in this function plays a central role in representing the meaning of each action by displaying sequentially the denotations of the moving parts of its agent.

\section{CATEGORY FULFILLMENT}

Using Action Function, we set up Dynanic Category Fulfillment Condition, aninterpretationmechanism which chccksif acandidate situation falls under the relevant category of action. If, by decomposing an action into several intervals, we can find that the parts of its agent which have changed their positions during each $i$ thinterval contain the parts specified by the value returncd from an action function with that $i$-th set of feature as its second argument, then we can decide that the movement belongs to the category denoted by that function: 


$$
\begin{aligned}
& \left\{y \mid<x, y>\in\left(\left(\omega\left(I N D, \beta\left(\text { ind }_{a}, t_{k+1}\right)\right)\right)^{-1}\right.\right. \\
& -\left(\left(\omega\left(I N D, \beta\left(\text { ind }_{a}, t_{k}\right)\right)\right)^{\prime}-1\right. \\
& \left.\left.\left.\cap\left(\omega\left(I N D, \beta\left(\text { ind }_{a}, t_{k+1}\right)\right)\right)^{-1}\right)\right)\right\} \\
& \supseteq \alpha_{i}\left(t_{j},\left\langle t_{q}, t_{r}\right\rangle_{u}, \kappa_{i}^{\prime}\left(\beta\left(\operatorname{Ind}_{a}, t_{k+1}\right), D_{i, u}\right)\right) \text {. }
\end{aligned}
$$

This is a recognition device which, given a complex of actions performedbyanindividual, judges whether thecomplexcontains the specified action. Then, how are we to represent actions in these terms?

\section{ACTION FORMULA}

In contrast to the sequential specification of the subsets of defining features denoting the parts involved in motion, we can set up another algorithm, Action Formula. This specifies the defining features as they are individually without reorganizing them into their subsets:

$$
\begin{aligned}
& \left.A^{\prime} T I O N_{i} \text { inda } a_{q}\left|t_{q}\right| t_{q}, t_{j}\right\}_{q} \mid\left(d_{i j}\right) \\
& =<\left\langle P_{j q}^{\prime}, P_{j q+1}^{\prime}>_{1},\left\langle P_{j q+1}^{\prime}, P_{j q+2}^{\prime}>_{2}, \ldots<P_{j r-1}^{\prime}, P_{j r}^{\prime}>_{r-q}>\right.\text {. }\right.
\end{aligned}
$$

The domain of this function is limited to the set of the defining features, which denotes the moving parts involved in the relevant action. If any of the features for that action is taken as its value, the function returns a sequence of pairs of placepoint sets occupied by the agent, over the maximal interval during which the part denoted by that feature actually is continuing some motion. The settheoretical definition of this function is done by applying recursively the adjacent movement function:

$$
\begin{aligned}
& \text { ACTION } \text { inda }_{q_{q}}\left|t_{q}\right| t_{j} t_{j} \mid \\
& { }_{d e} \hat{f}\left\langle x,\left\langle\left\langle y, z>\mid \mu_{\text {inda }}\left\langle t_{q}, l_{q+1}\right\rangle(y)=z\right\rangle\right.\right. \\
& \uparrow-1 i \rightarrow j-1 \\
& \text { lx }=\left(\omega^{\prime}\left(I N D I V I D U A L, \beta\left(\text { ind }_{a}, t_{i}\right)\right)\right)(y), x \in D_{i} \\
& \left.y \in 2 \beta\left(i v d_{a} \cdot l_{k}\right)-\{\phi\}, q_{j} \leqq k \leqq r_{j}-1,1 \leqq j \leqq\left|D_{i}\right|>\right\} .
\end{aligned}
$$

The returned value shows the series of changes in the figure of a moving part over the interval during which that subaction is ongoing, in terms of a sequence of pairs of place-point sets at adjacent timepoints. The relative order of the subactions of an action is defined by the endpoints of the inner interval.

Let us, here, define Set Trace Function, which, with its fixed individual and time interval, takes any subset of content points for thecategoryINDIVIDUAL, returnsasequence of pairsdenoting the transition, over that interval, of the part denoted by that subset of content points:

$$
\begin{aligned}
& \left.S T_{\text {inda }}\right|_{q} t_{r} t_{\mathrm{J}}\left(C^{\prime}\right) \\
& =<<P_{q}^{\prime}, P_{q+1}^{\prime}>_{1},\left\langle P_{q+1}^{\prime}, P_{q+2}^{\prime}>_{2}, \ldots<P_{r-1}^{\prime}, P_{r-q}^{\prime}>_{r-q}>.\right.
\end{aligned}
$$

The transition shows the all possible changes in the figure of the relevant part.

\section{ACTION CONTAINMENT}

To check if an individual has performed an action over an interval, we have to find that for every sequence returned from the relevant action formula with a feature as its argument, there is some sequence which is returned from the relevant set trace function with its argument corresponding to the part denoted by the feature and which includes the former sequence as a subset. For a specific individual over a specific interval, the following holds:

$$
\begin{aligned}
& \forall y_{1}\left[A C T I O N_{i \text { inda }}\left|t_{q}\right| t_{q}, t_{j} t_{j} \mid(x)=y_{1}\right. \\
& \rightarrow \exists y_{2}\left[S T_{\text {inda }} \mid t_{q} t_{J}\right](z)=y_{2} \\
& \quad \& y_{1} \subseteq y_{2} \\
& \left.\quad \& \omega^{\prime}\left(I N D, \beta\left(\text { ind }_{a}, t_{k}\right), \kappa_{i}\left(\beta\left(\text { ind }_{a}, t_{k}\right), x\right)=z\right]\right],
\end{aligned}
$$

where $q_{j} \leqq k \leqq r_{j}, 1 \leqq j \leqq\left|D_{i}\right|$.

This denotes how the transition of each part of the agent, over the interval of motion, is included into its transition over the whole interval. Simplifying its notation by deleting the details, we can express the universal quantification as an inclusion relation. See the following:

For every $d \in D_{i}, A C T I O N_{i}$ inda $\left|t_{q}\right| t_{q}, t_{j} j_{j} t_{p}\left|(d) \subseteq S T_{i n d a}\right| t_{q}, t_{j} \mid\left(C^{\prime}\right)$, where $\omega^{\prime}\left(I N D, \beta\left(\right.\right.$ ind $\left._{a}, t_{k}\right), \kappa_{i}\left(\beta\left(\right.\right.$ ind $\left.\left.\left._{a}, t_{k}\right), d\right)\right)=C^{\prime}$.

\section{CONCLUDING REMARKS}

The above setting enables us to interpret sentences of action in two compositional ways. Limiting to a simple intransitive case, functions and conditions are to be applied roughly as follows:

i) a. [s [NP agent's base] [vP [v Action Function] ] ]

b. [s [NP agent's base] [vP Action Function ]]

c. [s Dynamic Category Fulfillment Condition ]

ii) a. [s [NP Agent's base] [VP [V Action Formula] ] ]

b. [s [NP Agent's base] [vP Action Formula ] ]

c. Is Action Containment

We have shown a possibility that human recognition process not only of static categories but al so of dynamic ones like action verbs isperformed by someplace-locatingmechanism significantlybased upon vision as its input. If this mechanism is to be fully understood, then it will provide some evidence that the human cognitive ability to categorize is actually driven or supported by a comparatively simple physical system. 
We have seen two alternatives based upon the categorization al gorithm in which several functions detect sequentially the parts involved in ongoing actions by specifying them in terns of pl acepoint sets. Does the physical cognitive system, then, favor one over the other? We have no biologically oriented data to choose between those.

From a linguistically semantic viewpoint, however, there is a possibility that the first one provides a model for interpretation and the second forms a representation which is at least partially compositionally calculated from the syntax. We will discuss this point elsewhere.

We have still more questions to answer and problems to solve. Some of them are the following: how to semantically represent transitive cases, for which we have been developing an al gorithm, and what if an agent performs an action on aindependently moving object?, for which we have to set up an embedding device where Orientation Function applies multiply.

\section{REFERENCES}

[1] Barwise, J. and Perry, J. Situations and Attitudes. Cambridge, Mass: MIT Press, 1983.

[2] Chounsky, N. Lectureson Government and Binding .Dordrecht: Foris, 1981.

[3] Chomsky, N. and Halle, M. The Sound Patterns of English. New York: Harper and Row, 1968.

[4] Gruber, G. Lexical Structures in Syntax and Semantics. Amsterdam: North-Holland, 1976.

[5] Jackendoff, R. Semantic Structures . Cambridge, Mass: MIT Press, 1990.

[6] Jacobson, R., C. G. M. Fant and Halle, M. Preliminariesto Speech Analysis: The Distinctive Features and Their Correlates. Cambridge, Mass: MTT Press, 1952.

[7] Lakoff, G. Irregularity in Syntax. New York: Holt, Rinehart \& Winston, 1970.

[8] Montague, R. "The Proper Treatment of Quantification in Ordinary English," in Hintikka, J., Moravcsik, J. and Suppes, P. (eds.),ApproachestoNaturalLanguage .Dordrecht:Reidel,1970,pp. 221-242.

[9] Nishina, $H$ "Describing Actions in Locational Terms," in Takahaslu, S., Asao, K. and Matsumoto, R (eds.), Festsclirift for Nobuyuki Higashi, Tokyo: Kenkyusha, 1995, pp.117-127.
[10] Nishina, H. 1996, "On the Spatiotemporal Semantic Description of Action Verbs UsingFeatures, "Budapest: Workshop 12 "Representation and Processes between Vision and NL", ECAl'96, August, 1996, pp. 84-90. 


\title{
Solving the Forward Kinematics Problem for the Stewart Platform by Focusing Attention and Searching
}

\author{
A. Lacaze $\star \circ$, C. Tasoluk•, A. Meystel $\star \bullet$ \\ * National Institute for Standard and Technology, \\ - University of Maryland, • Drexel University \\ lacaze@cme.nist.gov
}

\section{ABSTRACT}

This paper presents a new algorithm for solving the forward kinematics of the Stewart Platform by using Focusing Attention and Searching. Previous algorithms solve this problem by numerically finding the solution for a 16th degree polynomial. We will show that it is possible to drastically reduce the search space by representing the system with three angles around the circles formed by the given lengths. Then, we search in a nonlinear equation with one unknown and two roots. Reduction of computational complexity allows us to use the feed-forward kinematics transform in a broader scope of practical cases.

\section{INTRODUCTION}

In this paper, we demonstrate that no matter how complex the problem is local focusing of attention and searching can be used to substantially simplify the effort. This paradigm of dealing with problems (outlined in $[1,2]$ can be implemented in many cases. The Stewart Platform was introduced in [3] and further developed in $[4,5]$. It is a closed kinematic system with parallel links which is consider to be far more rigid than the serial counterparts of the same size and weight. Its force-output-to-manipulator-ratio is generally an order of magnitude bigger than most industrial robots [6]. This same closed kinematic structure that gives its rigidity also complicates the solution of the forward kinematics in such a way that no close loop solution for this problem has been found. Some authors go as far as to say that "due to the kinematic nature of this procedure, it is impossible to compute the kinematics solution on line"[6].

To solve this problem, some researchers have transformed it into solving 30 non-algebraic simultaneous equations in [7]. More recent results include the solution of 24 th [8], 16 th $[9,10]$, or 12 th [11] order polynomial in a single variable. Finally, in [6] an algorithm that involves three non-linear simultaneous equations with three unknowns. [12] shows that some of these

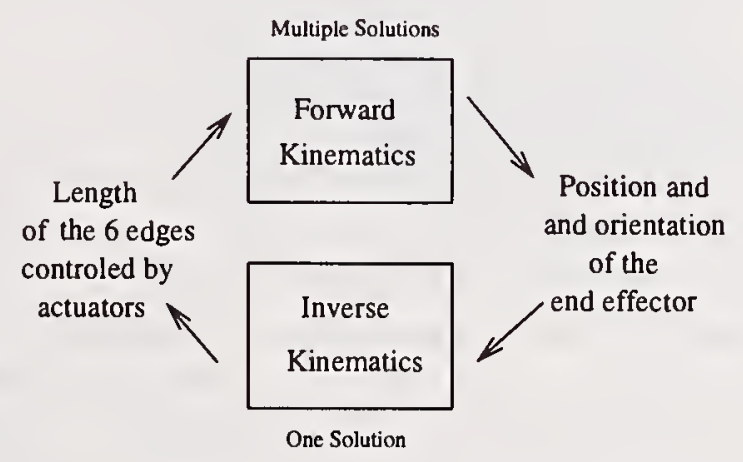

Figure 1: Forward and inverse kinematics

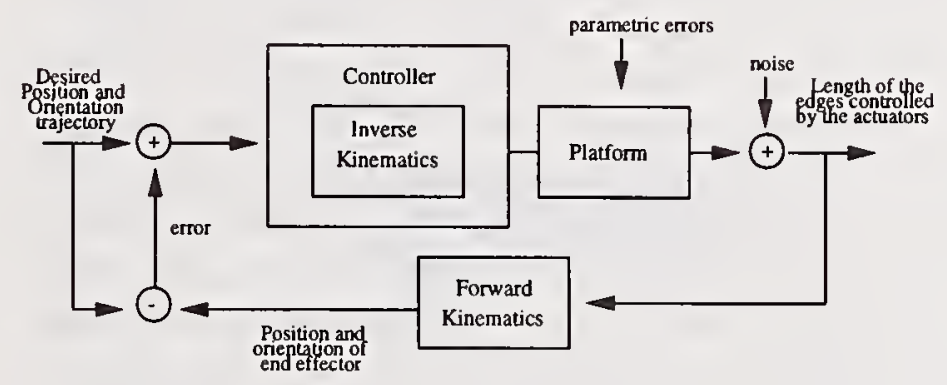

Figure 2: An example of using transformations of forward and inverse kinematics for control purposes

algorithms can be solved in parallel, thus reducing the time of computation.

Our algorithm has a number of advantages in comparison with the existing solutions. It uses a nonlinear equation with one unknown and two roots. It is not necessary to solve all 16 mathematically possible solutions [11] for the platform. One should be sufficient, considering that the structure of the platform is known and that we make sure that we are inside of some regions of existence that we will later define

The results can be used for real-time control application in a variety of settings. Control structures are presently being developed. 


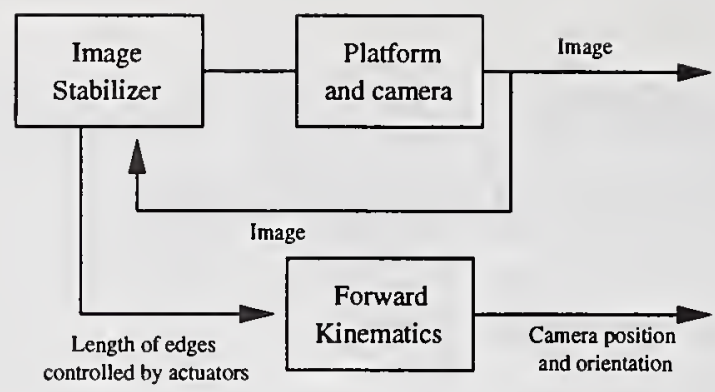

Figure 3: An example of using inverse kinematics for measuring purposes

\section{Forward Kinematics AND ITS NEED FOR Control and MEasurement}

Forward and inverse kinematics are terms to describe mapping from the space of inputs to the space of outputs of a non-dynamic mechanical system as shown in Figure 1. Figure 2 shows a typical application of the forward and inverse kinematics algorithm. A trajectory such as a milling machine or a welding system is to be followed. The controller, using the inverse kinematics algorithm (and calculating desired changes in position), computes the control signal given to the actuators. At the output of the platform we measure the lengths of the edges controlled by the actuators. Then, the forward kinematics algorithm transforms those length into positions. The position signal is compared to the assigned position and added to the controller's input. Because of its closed kinematic chain, in the Stewart platform, the rigidity of the end effector allows a reasonably accurate feed-forward assignment. Due to the complexity of older forward kinematics algorithm, most Stewart platforms are controlled without using feedback. The proposed algorithm will allow the calculation of the forward kinematics in real time. This, opens an opportunity to increase the accuracy of the control algorithms and broadening the current uses of the platform.

There are other applications where the solution of forward kinematics is mandatory. These are the cases when the Stewart platform is used as a component of a measuring device. These cases include:

- camera mapping where a camera is mounted on top of a Stewart platform. For example, we let the camera roam until it finds a target, and then we ask where is the camera pointed. It is also usefull when we have a fast control system to stabilize the camera and then have the forward kinematics algorithm tell where the camera is pointed (See Figure 3).

- positioning devices (6 degrees of freedom joysticks), and

- inspection systems mounted in Stewart platforms that could be used to touch the part to inspect it.
We were unable to find any examples where the Stewart platform was used for any of these purposes in industry, although the uses of parallel manipulators for sensing are very common in nature (i.e. our necks and eyes). We can conjecture that these structural solutions did not emerge in industry because the previous forward kinematics algorithms were not fast enough to allow for real time operation of these devices.

In [13] and [14] new sensors were added to their parallel manipulators in order to find the forward kinematics in a faster way. We expect our algorithm to decrease the manufacturing cost of these platforms by solving the problem without the need to add extra sensors.

In this paper, we will first show how this algorithm is used to solve the forward kinematics of the 3-3 Stewart manipulator (three joints in the fixed platform and three joints in the moving platform). See Figure 4. Later we show how this algorithm can be extended for other parallel platforms.

In Figure 5, the $R S T$ triangle is the fixed platform and the $A B C$ triangle is the moving platform. The length of the six edges controlled by the actuators of the platform $\mathcal{L}_{1}, \ldots, \mathcal{L}_{6}$, (Figure 4 ). The position of $R, S$, and $T$ are constant and given. If we draw the geometrical place of the point A (Figure 5), it will be a circle $(\circ A)$ since the segments $\overline{R S}, \overline{S A}=\mathcal{L}_{2}$ and $\overline{R A}=\mathcal{L}_{1}$ are given. This circle can be created by rotating the triangle $A R S$ around $\overline{R S}$. In the same manner, $\circ B$ and $\circ C$ are created by rotating $B$ around $\overline{S T}$, and $C$ around $\overline{R T}$ respectively. For simplicity, let $\overline{A B}=\overline{A C}=\overline{B C}=b$ and $\overline{R S}=\overline{R T}=\overline{S T}=a$. By looking at Figure 5 , it is possible to see that the forward kinematics algorithm must find a point in each circle such that the distance among these points matches the dimension of the edge of the moving platform.

\section{The Algorithm of Forward Kinematics}

Therefore, the algorithm in the general form works in the following manner (Figure 6):

1. The subsequent procedures start with finding the range of the real solutions. In order to explain how this is done, we will outline the algorithm.

2. Pick a point $A^{\prime}$ in $\circ A$ (See Figure 7). $A^{\prime}$ is completely defined by $\circ A$ and $\theta_{A}$ the polar angle inside that circle. For now, $A^{\prime}$ is an arbitrary point. Later, we show that it is possible to analytically find a region where the solutions must exist.

3. Analytically find a point $B^{\prime}$ in $\circ B$ such that $\overline{A^{\prime} B^{\prime}}=b$. We will see that there are two analytical solutions. Let's name them $B_{+}^{\prime}$ and $B_{-}^{\prime}$ (for positive and negative slope of the $\overline{A^{\prime} B^{\prime}}$ versus $\theta_{A}$ curve, top-left of Figure 9. For now, assume that these are arbitrary labels). 


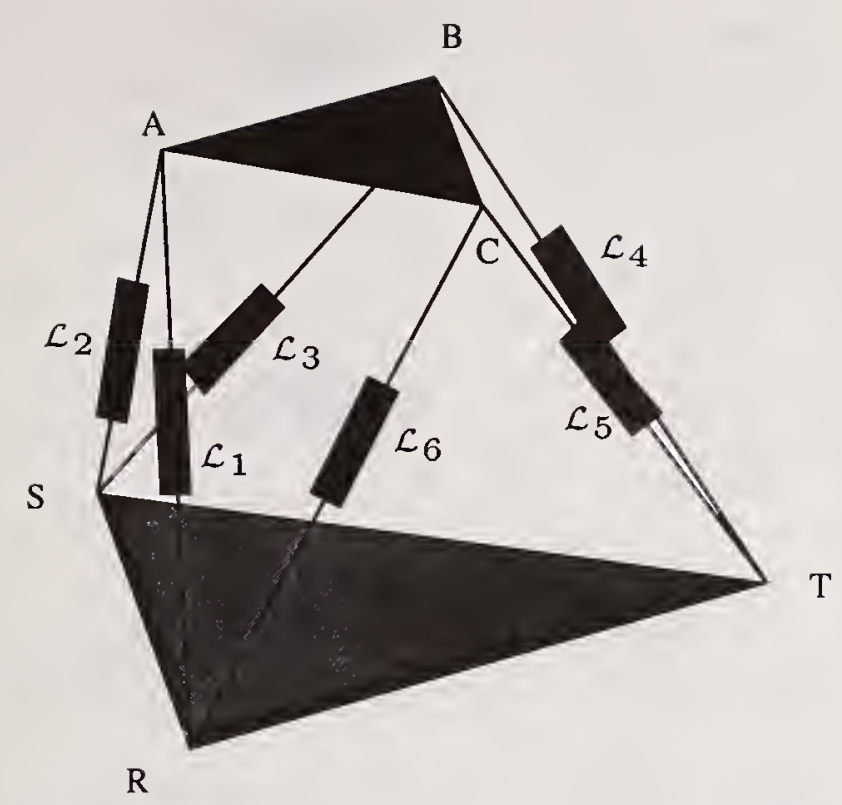

Figure 4: 3-3 Stewart Platform showing the actuators

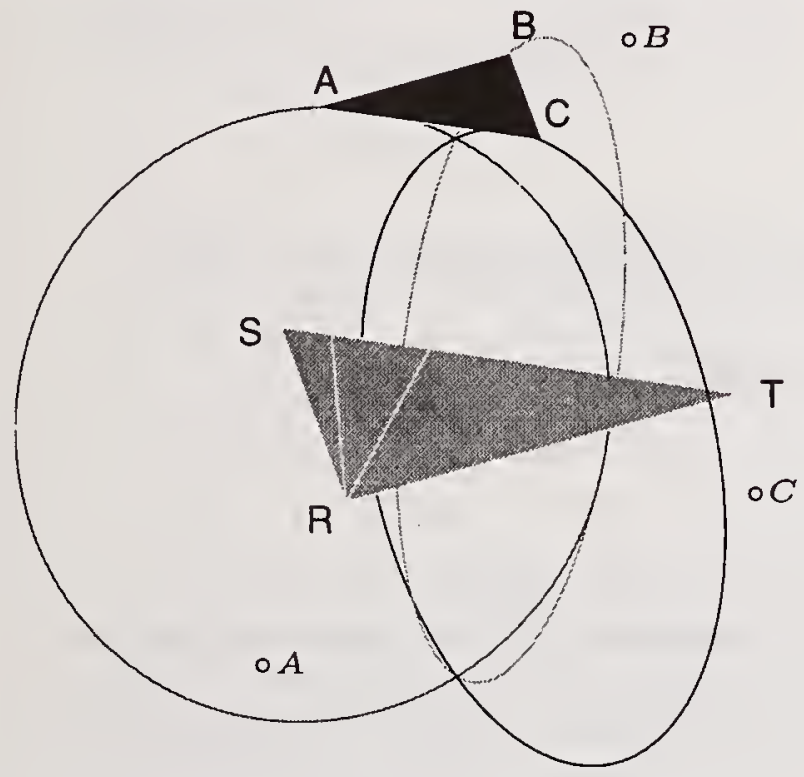

Figure 5: 3-3 Stewart Platform showing $\circ A, \circ B$, and $\circ C$

4. Analytically find a point $C^{\prime}$ in $\circ B$ such that $\overline{A^{\prime} C^{\prime}}=b$. Again, there are two analytical solutions. Let's name them $C_{+}^{\prime}$ and $C_{-}^{\prime}$ (for positive and negative slope of the $\overline{A^{\prime} C^{\prime}}$ versus $\theta_{B}$ curve).

5. At this point, we found five points such that $\overline{A^{\prime} B_{+}^{\prime}}=\overline{A^{\prime} B_{-}^{\prime}}=\overline{A^{\prime} C_{+}^{\prime}}=\overline{A^{\prime} C_{-}^{\prime}}=b$, but

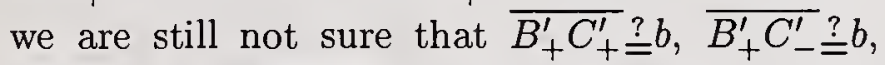
$\overline{B_{-}^{\prime} C_{+}^{\prime}} \stackrel{?}{=} b$, and $\overline{B_{-}^{\prime} C_{-}^{\prime}} \stackrel{?}{=} b$. We can create four functions: $f_{1}\left(\theta_{A}\right)$ (length of $\overline{B_{+}^{\prime} C_{+}^{\prime}}$ as a function of $\left.\theta_{A}\right), f_{2}\left(\theta_{A}\right)$ (length of $\overline{B_{+}^{\prime} C_{-}^{\prime}}$ as a function of $\left.\theta_{A}\right), f_{3}\left(\theta_{A}\right)$ (length of $\overline{B_{-}^{\prime} C_{+}^{\prime}}$ as a function of $\theta_{A}$ ) , and $f_{4}\left(\theta_{A}\right)$ (length of $\overline{B_{-}^{\prime} C_{-}^{\prime}}$ as a function of $\left.\theta_{A}\right)$. Examples of these functions are shown in Figure 8 . The algorithm then individually uses Newton-Raphson search procedure and finds the

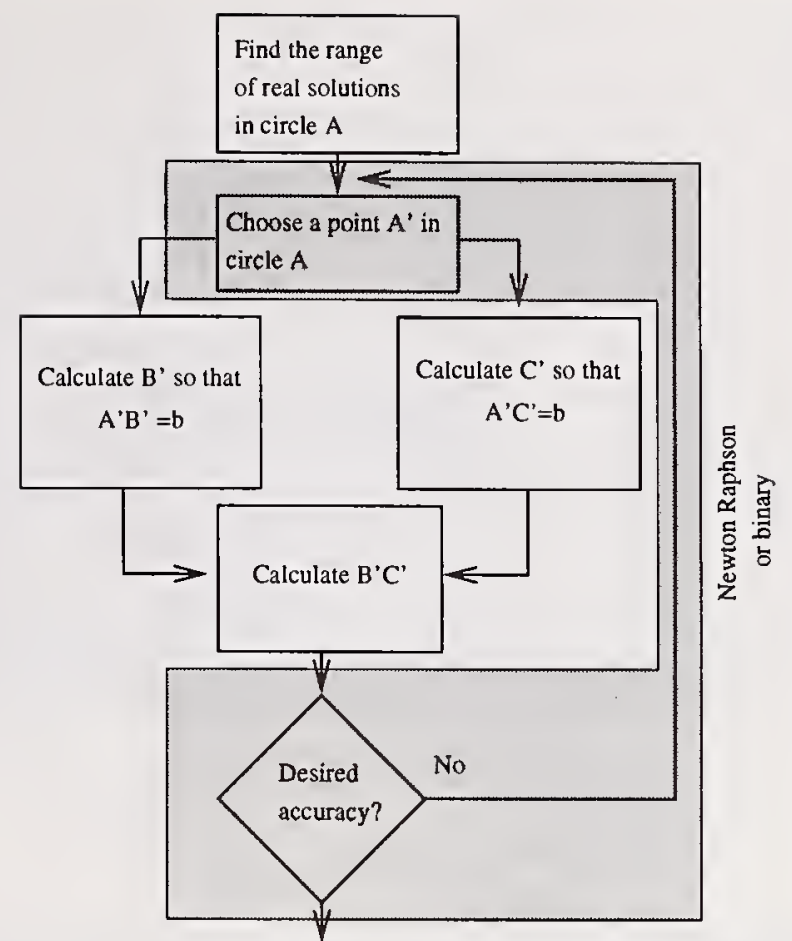

Figure 6: A general view of the forward kinematics algorithm

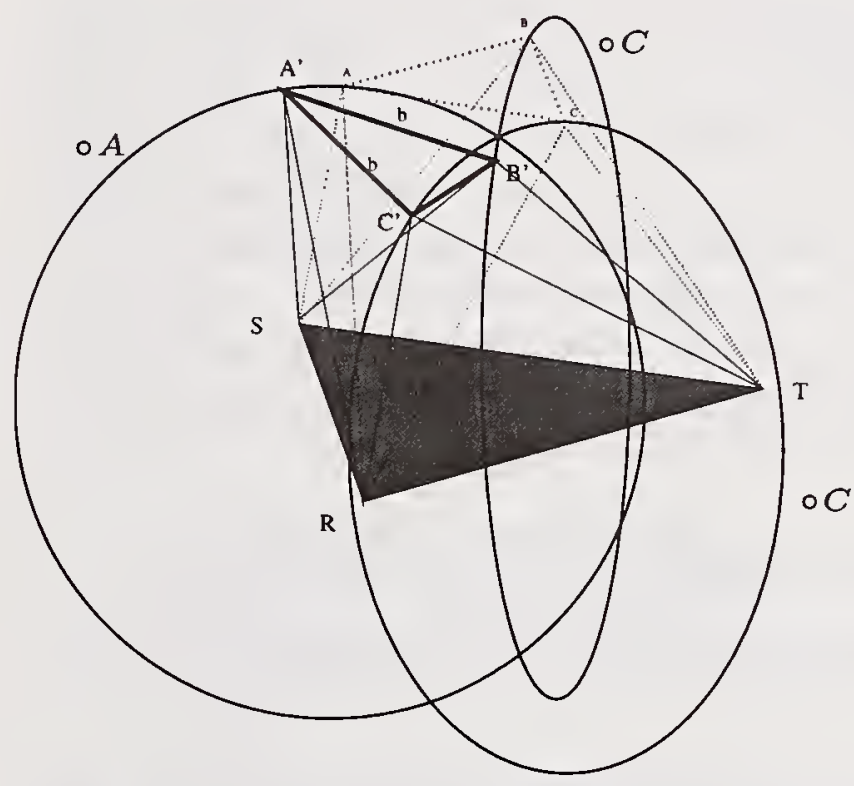

Figure 7: Searching for the correct $b$ lengths

value for $\theta_{A}$ in each of the functions until $B^{\prime} C^{\prime}=b$ to the desired accuracy. These functions are periodic, and they will have at most two roots in each half period. Thus yielding 8 forward kinematic solutions: $A B C_{+++}$being the positive slope solution of the $f_{1}\left(\theta_{A}\right), A B C_{++-}$being the negative edge of the same functions. Similarly, $A B C_{+-+}$, $A B C_{+--}, A B C_{-++}, A B C_{-+-}, A B C_{--+}$, and $A B C_{---}$are the other six solutions, to a total of eight solutions (for the half period of $\theta_{A}$ see Figure 9). 

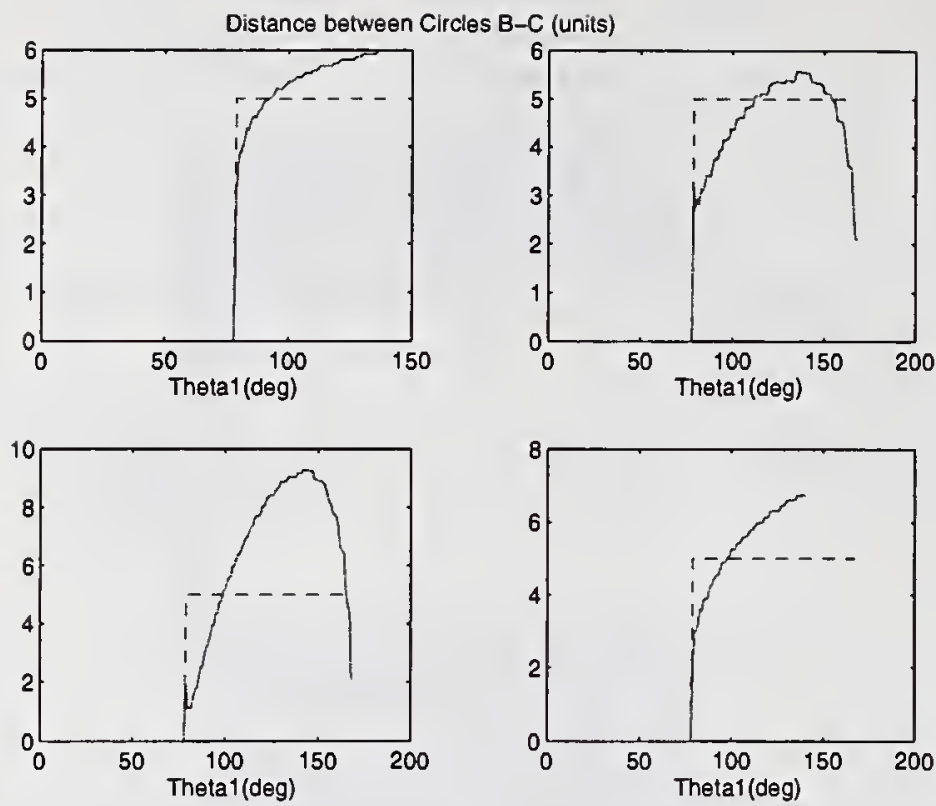

Figure 8: $f_{1}\left(\theta_{A}\right), f_{2}\left(\theta_{A}\right), f_{3}\left(\theta_{A}\right)$, and $f_{4}\left(\theta_{A}\right)$ for $a b=5$ example

It is unnecessary to search all four functions unless we are interested in finding all 8 (top) solutions. The reason is that if we know how our platform is currently configured (i.e. in the $A B C_{+--}$), it is easy to prove that the next solution will also be a $A B C_{+--}$provided that we have not gone through maxima which are actually a singularity point. These singularity points can be easily derived from the expression for the $A^{\prime} B^{\prime}$ and $A^{\prime} C^{\prime}$. For each concrete configuration, a search strategy can be assigned in such a way that the complexity of computation is minimized.

\section{The Equations of The System}

We can write the equations of the system in the following way:

$$
\begin{aligned}
& \mathcal{L}_{1}=\sqrt{\left(x_{A}-x_{R}\right)^{2}+\left(y_{A}-y_{R}\right)^{2}+\left(z_{A}-z_{R}\right)^{2}} \\
& \mathcal{L}_{2}=\sqrt{\left(x_{A}-x_{S}\right)^{2}+\left(y_{A}-y_{S}\right)^{2}+\left(z_{A}-z_{S}\right)^{2}} \\
& \mathcal{L}_{3}=\sqrt{\left(x_{C}-x_{R}\right)^{2}+\left(y_{C}-y_{R}\right)^{2}+\left(z_{C}-z_{R}\right)^{2}} \\
& \mathcal{L}_{4}=\sqrt{\left(x_{C}-x_{T}\right)^{2}+\left(y_{C}-y_{T}\right)^{2}+\left(z_{C}-z_{T}\right)^{2}} \\
& \mathcal{L}_{5}=\sqrt{\left(x_{B}-x_{S}\right)^{2}+\left(y_{B}-y_{S}\right)^{2}+\left(z_{B}-z_{S}\right)^{2}} \\
& \mathcal{L}_{6}=\sqrt{\left(x_{B}-x_{T}\right)^{2}+\left(y_{B}-y_{T}\right)^{2}+\left(z_{B}-z_{T}\right)^{2}}
\end{aligned}
$$

are the Euclidean equations between the three points that define the lower platform $R, S$, and $T$ and the points of the upper moving platform $A, B$, and $C$. We can also write the Euclidean equations of the top platform. Here, we assume that it is an equilateral triangle, but we are not making any simplifications based

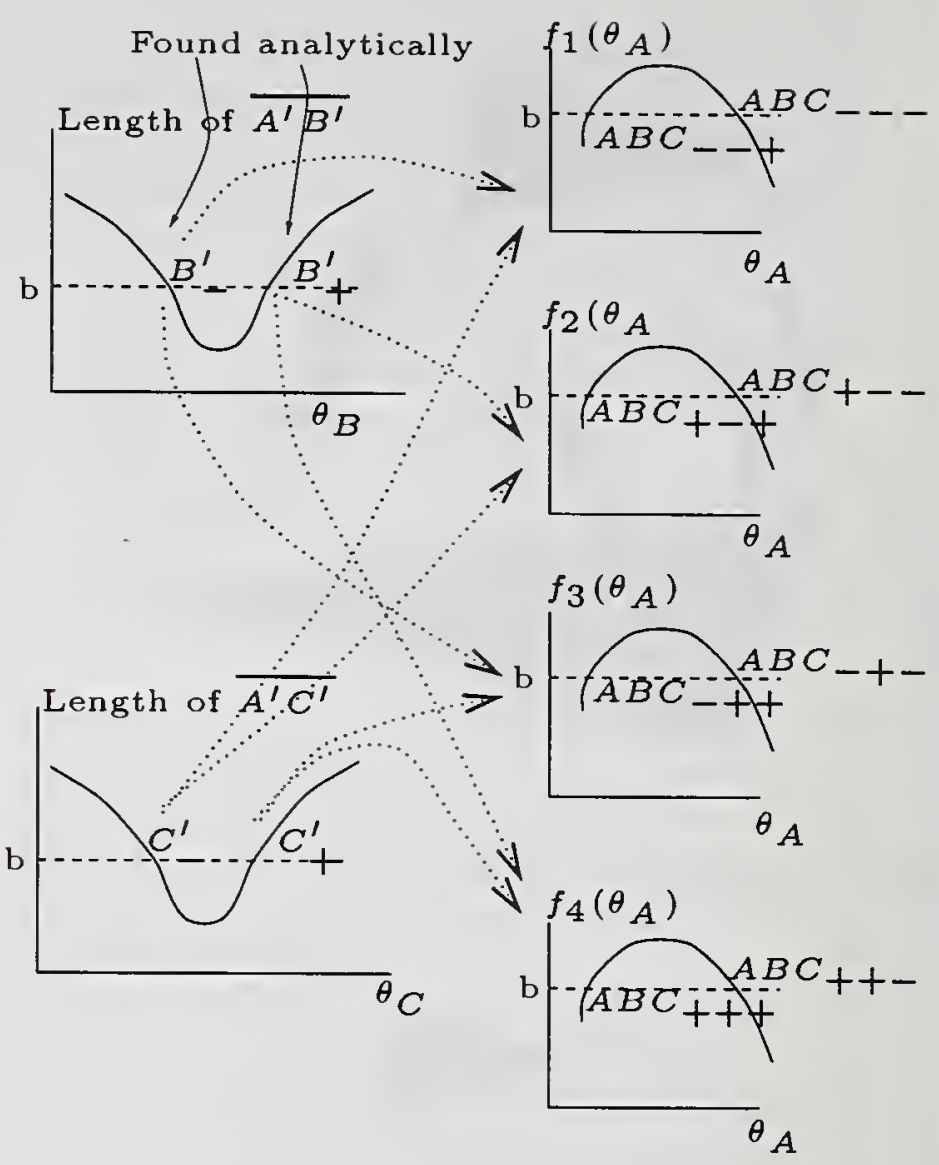

Figure 9: 8 solutions in the Stewart Platform

on this assumption.

$$
\begin{aligned}
& b^{2}=\left(x_{A}-x_{B}\right)^{2}+\left(y_{A}-y_{B}\right)^{2}+\left(z_{A}-z_{B}\right)^{2} \\
& b^{2}=\left(x_{A}-x_{C}\right)^{2}+\left(y_{A}-y_{C}\right)^{2}+\left(z_{A}-z_{C}\right)^{2} \\
& b^{2}=\left(x_{B}-x_{C}\right)^{2}+\left(y_{B}-y_{C}\right)^{2}+\left(z_{B}-z_{C}\right)^{2}
\end{aligned}
$$

where $b$ is the length of the equilateral triangle of the top platform. We create a new reference coordinate system on $S$ (Figure 10) to simplify the expressions as follow:

$$
\begin{array}{lll}
x_{R}=a & y_{R}=0 & z_{R}=0 \\
x_{S}=0 & y_{S}=0 & z_{S}=0 ; \\
x_{T}=\frac{a}{2} & y_{T}=\frac{\sqrt{3} a}{2} & z_{T}=0
\end{array}
$$

As shown in Figure 10, we define:

$$
\begin{array}{ll}
p_{A}=\frac{\left(\mathcal{L}_{1}^{2}-\mathcal{L}_{2}^{2}+a^{2}\right)}{2 a} & r_{A}=\sqrt{\mathcal{L}_{1}^{2}-p_{A}^{2}} \\
p_{B}=\frac{\left(\mathcal{L}_{3}^{2}-\mathcal{L}_{4}^{2}+a^{2}\right)}{2 a} & r_{B}=\sqrt{\mathcal{L}_{3}^{2}-p_{B}^{2}} \\
p_{C}=\frac{\left(\mathcal{L}_{5}^{2}-\mathcal{L}_{6}^{2}+a^{2}\right)}{2 a} & r_{C}=\sqrt{\mathcal{L}_{5}^{2}-p_{C}^{2}}
\end{array}
$$

By looking at Figure 10 and by using definitions (13) through (15), we can easily write a polar parametric equation of $\circ A$ in the following manner,

$$
\begin{aligned}
& x_{A}=a-p_{A} \\
& y_{A}=r_{A} \cos \theta_{A} \\
& z_{A}=r_{A} \sin \theta_{A}
\end{aligned}
$$



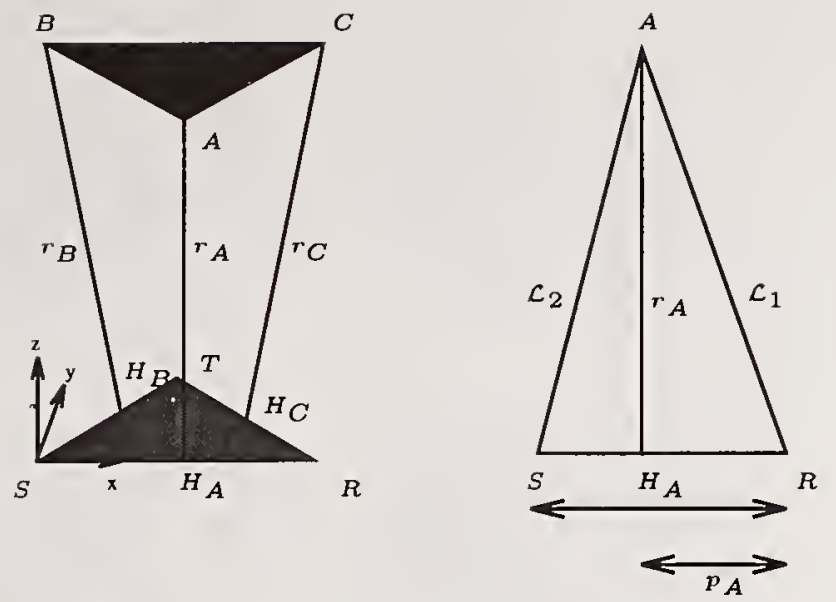

Figure 10: New coordinate system and some definitions

in a similar way, after some trigonometric transformations the equations for $\circ B$ and $\circ C$ :

$$
\begin{aligned}
& x_{B}=\frac{\sqrt{3} r_{B} \cos \theta_{B}+p_{B}}{2} \\
& y_{B}=\frac{-r_{B} \cos \theta_{B}+\sqrt{3} p_{B}}{2} \\
& z_{B}=r_{B} \sin \theta_{B} \\
& x_{C}=a-\frac{\sqrt{3} r_{C} \cos \left(\theta_{C}\right)-a+p_{C}}{2} \\
& y_{C}=\frac{-r_{C} \cos \left(\theta_{C}\right)+\sqrt{3}\left(a-p_{C}\right)}{2} \\
& z_{C}=r_{C} \sin \theta_{C}
\end{aligned}
$$

Let's assume that we have chosen a $\theta_{A}$ in $\circ A$ using equations (16) through (18) which uniquely defines a point $A$ in $\circ A$. We are interested in finding the points in $\circ B$ that are a distance $b$ away from $A$. We will present two methods for accomplishing this.

Two methods where tested (both with positive results:

\section{Method 1: Solving the $2^{\text {nd }}$ order polynomial} and

\section{Method 2: Intersecting a sphere and a circle}

The intersection between the sphere and $\circ B$ as shown in Figure 11. Note that the only way for a platform to go from $\theta_{B_{+}}$to $\theta_{B_{-}}$is that:

- the platform has gone through a singularity point $\left(\theta_{B_{+}}=\theta_{B_{-}}\right)$. These points are at the maximum of the curves in Figure 8 or 9

- one of the cables (or more) has gone slack if the Stewart platform has flexible members

- an actuator has broken if the platform has rigid members.

It is only necessary to calculate one $\theta_{B}$ and one $\theta_{C}$ for a real platform. To start our search technique, we

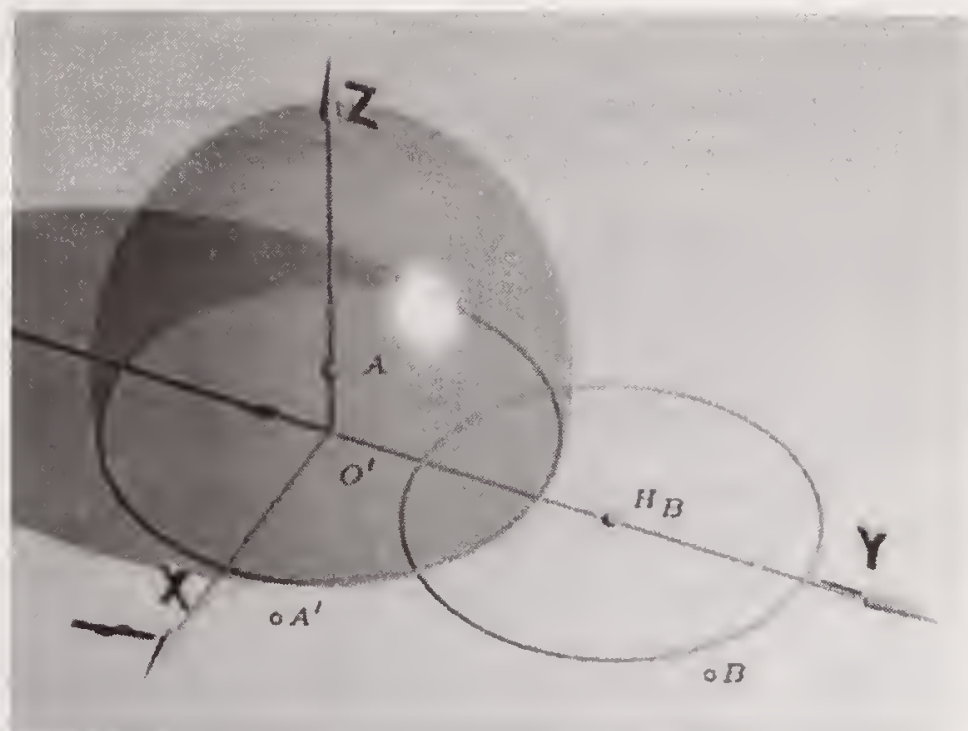

Figure 11: A new coordinate system

calculate the distance between $B$ and $C$ which it is given by (9).

\section{The Search Technique}

Previously, we presented an expression that given $\theta_{A}$ will give the distance $B^{\prime} C^{\prime}$, or $f\left(\theta_{A}\right)$. We need to find $\theta_{A}$ such that $f\left(\theta_{A}\right)=b$. There are several searching techniques that can be used. The search problem has the following features:

1. the region of search is known

2. there are no more than two solutions

3. there is only one maximum

One simple way of finding $\theta_{A}$ such that $f\left(\theta_{A}\right)=b$ consists of the following steps:

1. find the maximum, using Newton-Raphson search,

2. divide the space of search in two parts: to the left and to the right of the maximum,

3. search for the root of the equation only in one of the parts.

If we have the previous forward kinematics solution, we can use that value as a starting point for our search, thus avoiding the need to find the maximum.

\section{IMPLEMENTATION}

The previous algorithm was compiled in two different computers: a Sun Sparc 2 and a Sun Sparc 20. Table 1 shows the different average times of calculation. Table 2 shows the time that the algorithm takes with respect to the desired accuracy of the result.

The algorithm of forward kinematics was tested using data from a Stewart based milling machine. See Figure 12. The trajectory is the spiral shown in Figures 13 and 14, position trajectory and unit vectors, show the direction of the end effector respectively. 


\begin{tabular}{|c|c|}
\hline \hline Hardware & $\begin{array}{c}\text { average time } \\
\text { in } \mu \text { seconds }\end{array}$ \\
\hline $\begin{array}{c}\text { Sun Sparc 20 with } \\
\text { compiler optimization }\end{array}$ & 98 \\
\hline $\begin{array}{c}\text { Sun Sparc 20 without } \\
\text { compiler optimization }\end{array}$ & 121 \\
\hline $\begin{array}{c}\text { Sun Sparc 2 with } \\
\text { compiler optimization }\end{array}$ & 490 \\
\hline $\begin{array}{c}\text { Sun Sparc 2 without } \\
\text { compiler optimization }\end{array}$ & 512 \\
\hline
\end{tabular}

Table 1: Comparative study of different hardware to find the complete forward kinematic solution with an accuracy of \pm 0.01 radians and the Newton Raphson search method

\begin{tabular}{|l|l|}
\hline \hline Accuracy in ( \pm radians) & typical time ( $\mu$ seconds) \\
\hline 0.01 & 98 \\
0.001 & 136 \\
0.0001 & 250 \\
0.00001 & 492 \\
\hline \hline
\end{tabular}

Table 2: Typical algorithm times using a Sun Sparc 20 and using the "-O4 -msuperspark" compiling options with different accuracies and the Newton Raphson search method.

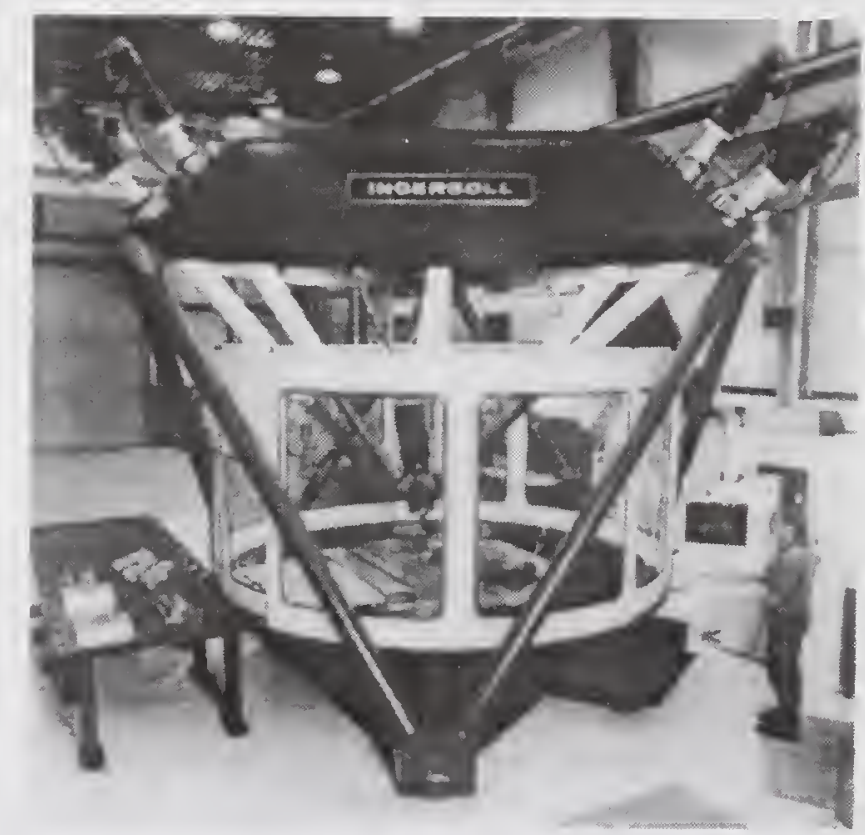

Figure 12: Ingersol-NIST Hexapod
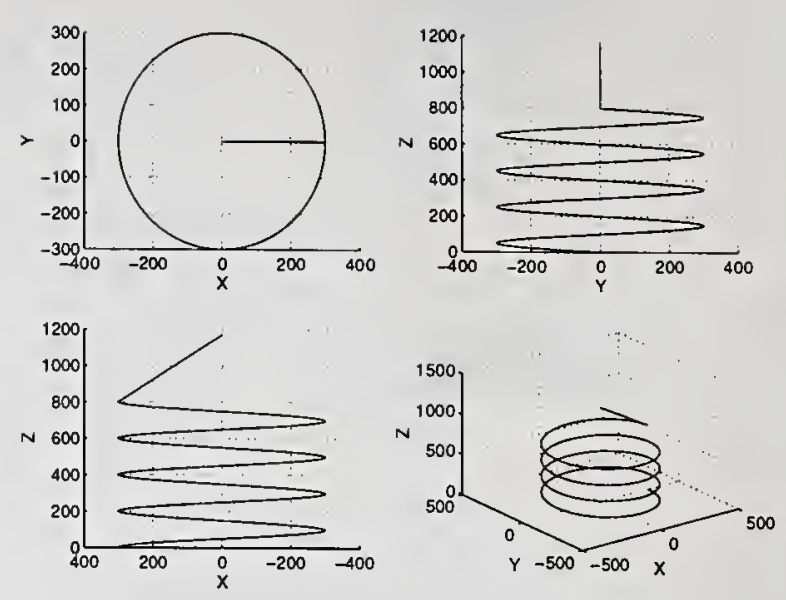

Figure 13: Spiraling trajectory
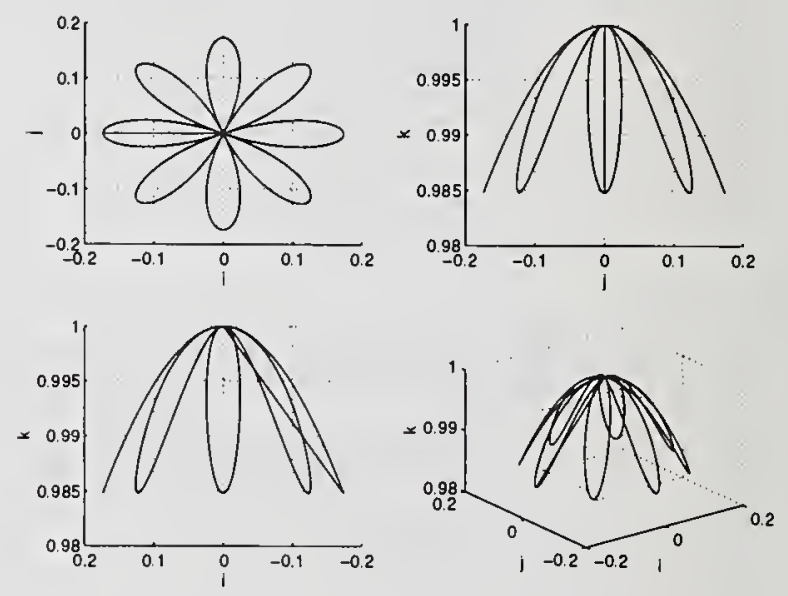

Figure 14: Angular trajectory

Figure 15 shows the four four sets of curves created by plotting the distance $B C$ when $A C=A B=b$ as a function of $\theta_{A}$ along the spiral trajectory. Each different curve corresponds to the distance $(B C)$ for a point along the trajectory shown in Figures 13 and 14. Note that the algorithm does not need to draw this curve to find the solution. It is searching in these solutions to find the $B C=b$ solution that corresponds to the current configuration of the platform. Figure 16 is a two-dimensional view of Figure 15 that shows the found solutions marked in the bottom left curve.

\section{Conclusions}

1. An algorithm of forward kinematics for Stewart Platform which allows for substantial reduction of computational complexity is proposed.

2. Unlike many of existing systems, this algorithm knows the number of solutions in advance. Since the previous configuration is considered part of the assignment, we must only search for only one root in most cases. 

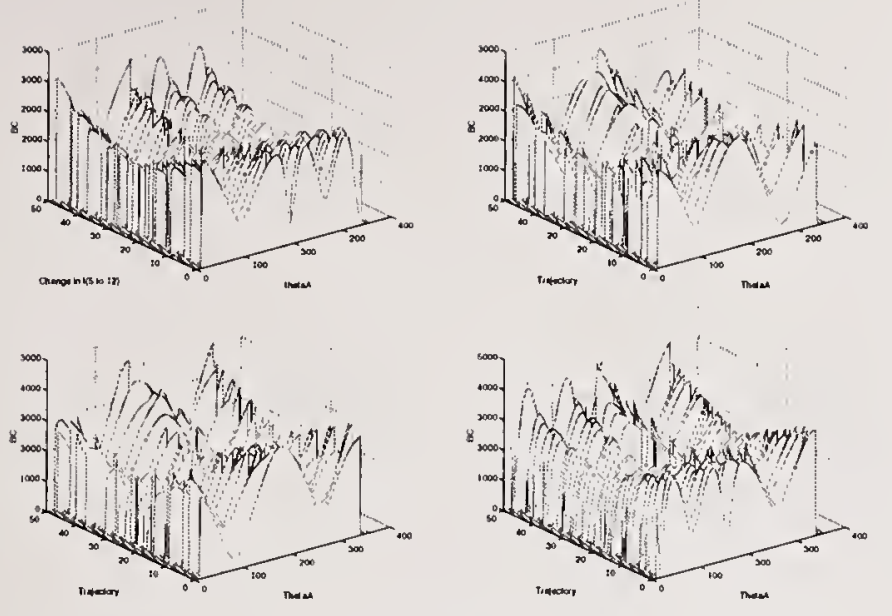

Figure 15: $B C$ along the spiral trajectory
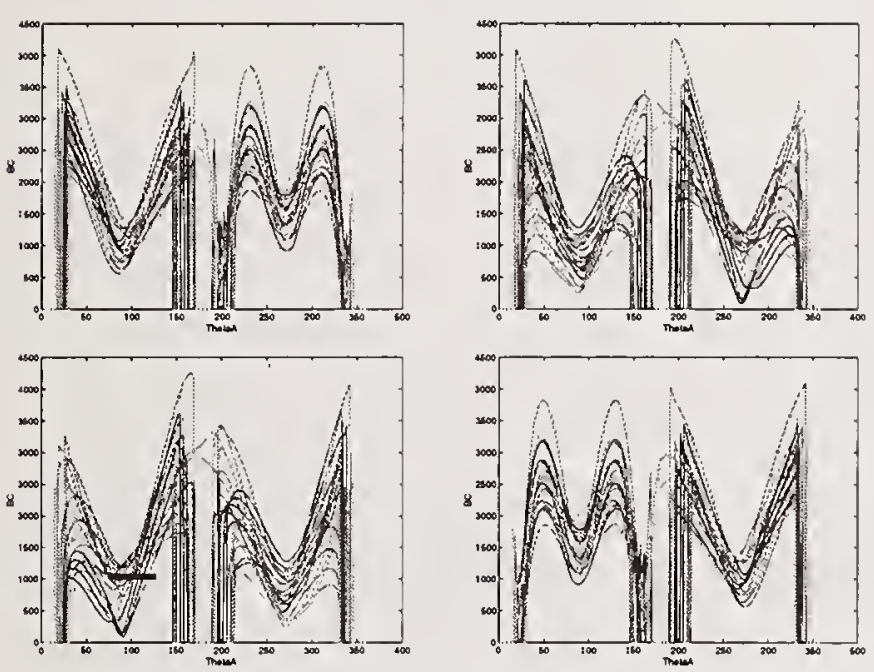

Figure 16: $B C$ along the spiral trajectory in $2 \mathrm{~d}$

3. In addition to reducing complexity, the algorithm is easy to implement.

4. Due to the constraint and monotonic character of the functions in the search zone, the search algorithm is stable (it cannot jump roots).

5. The algorithm allows for real time applications. Further reduction of computational time can be achieved by introducing parallel computation.

6. Unlike some of the existing algorithms, the proposed one does not depend on the length of the edges.

\section{REFERENCES}

[1] A. Meystel. Intelligent systems, a semiotic perspective. International journal of Intelligent control and Systems, 1:31-58, 1996.

[2] A. Meystel and A. Lacaze. Unified learning/planning automaton: generating and using multigranural knowledge hierarchies. Intelligent System and Semiotics, 97.

[3] D. Stewart. A platform with six degrees of freedom. The Institution of Mechanical Engineers, 180(15):371-384, 1965.
[4] J. Lee, J. Albus, N. Dagalakis, and T. Tsai. Computer simulations of a parallel link manipulator. Robotics and Computer Integrated Manufacturing, August 1988.

[5] J. Albus, R. Bostelman, and N. Dagalakis. The nist robocrane. Journal of Robotic Systems, 10(5):709-724, 1992.

[6] K. Liu, J.M. Fitzgerald, and F.L. Lewis. Kinematic analysis of the stewart platform. IEEE Transactions on Industrial Electronics, 40(2):282293, 1993.

[7] E.F. Fichter. A stewart platform-based manipulator. International Journal of Robotic Research, $5(2): 157-181,1968$.

[8] K. J. Waldron, M. Raghavan, and B. Roth. Kinematics of a hybrid series-parallel manipulator. J. Dynamic System Measurement Control, 111(2):211-221, 1989.

[9] P. Nanua, K. Waldron, and V. Murthy. Direct kinematic solution of a stewart platform. IEEE Trans. on Robotics and Automation, 6, 1990.

[10] J.P. Merlet. An algorithm for the forward kinematics of parallel manipulators. In 91 ICAR, Fifth International Conference on Advanced Robotics, volume 2, pages 1136-40, 91.

[11] D. Lazard and J.P. Merlet. The (true) stewart platform has 12 configurations. In Proceedings of International Conference on Robotics and Automation, volume 3, pages 2160-5, 1994.

[12] C. Gosselin. Parallel computational algorithms for the kinematics and dynamics of parallel manipulators. In Proceedings of International Conference on Robotics and Automation, volume 1, pages 883-8, 1994.

[13] H. Hollerbach and D. Lokhorst. Closed-loop kinematic calibration of the rsi- 6 hand controller. In Proceedings of IEEE International Conference on Robotics and Automation, volume 2, pages 142148, 1993.

[14] JP. Merlet. Closed-form resolution of the direct kinematics of parallel manipulators using extra sensor data. In Proceedings of IEEE International Conference on Robotics and Automation, volume 1, pages 200-204, 1993. 




\title{
A neurobiological interpretation of semiotics: meaning, representation, and causality
}

\author{
Walter J Freeman \\ Department of Molecular \& Cell Biology, LSA 129 \\ University of California at Berkeley CA 94720-3200 \\ wfreeman@socrates.berkeley.edu http://sulcus.berkeley.edu/
}

\begin{abstract}
That branch of semiotics called semantics deals with the relationships between meanings and representations. In my view meanings exist only in brains without representations there. A meaning is the focus of an activity pattern that may occupy the entire available brain. It is constructed by intentional action followed by learning from the consequences of the action. Communication between brains requires reciprocal construction of representations in accordance with meanings, which elicit other meanings in other brains. A representation, as a material object or process, has no meaning in itself. EEG data indicate that neural patterns of meaning occur in trajectories of discrete steps marked by cortical state transitions, served by rapid exchanges of discrete wave packets between interactive cortical domains. These wave packets are made by self-organizing dynamics that control behavior and shape the sensitivities of sensory cortices to the sequellae of actions. The nature of causality must be analyzed and understood in accordance with the questions: How do meanings cause representations, and how do representations cause meanings?
\end{abstract}

\section{Introduction}

Archeologists studying a petroglyph ask not only who did it and when, but what does it mean? They conclude that no one can know what it means now, and that they can only speculate on the prior meanings in the minds of the makers and viewers. The lesson is that the petroglyph contains no meaning, even though it was made by humans with intent to communicate meaning by evoking the formation of comparable meanings in other humans.

Engineers who want to make semantic machines are faced with the task of defining meaning, which at present exists only in brains, and then with the task of learning how to make or cause meaning in machines, as shown by Tani [1996] and Clark [1997]. The requirements on network models to simulate the chaotic dynamics of brains include global though sparse connectivity, continuous time dynamics, and distributed spatial functions in two-dimensional arrays of nonlinear integrators. Digital hardware may suffice to emulate the biological functions of sensory cortex in brains by use of nonlinear difference equations [Chang and Freeman, 1996; Shimoide and Freeman, 1995; Freeman et al., 1996]. In this way, a next step to machine intelligence may be to use a model of a sensory cortex as an interface between the unconstrained real world, which is infinitely complex, and the finite state automata that constitute the main support for contemporary artificial intelligence. That is, models from brain dynamics can provide eyes and ears for conventional computers.

However, this step will require that a major problem be addressed: the relation between representation and meaning in brain function. Shannon-Weaver information theory, which is representational, has divorced meaning from information and therefore does not apply to brains. The aim of this presentation is to sketch some of the principal elements of the problem, as a basis for discussing some possible pathways toward solutions through 


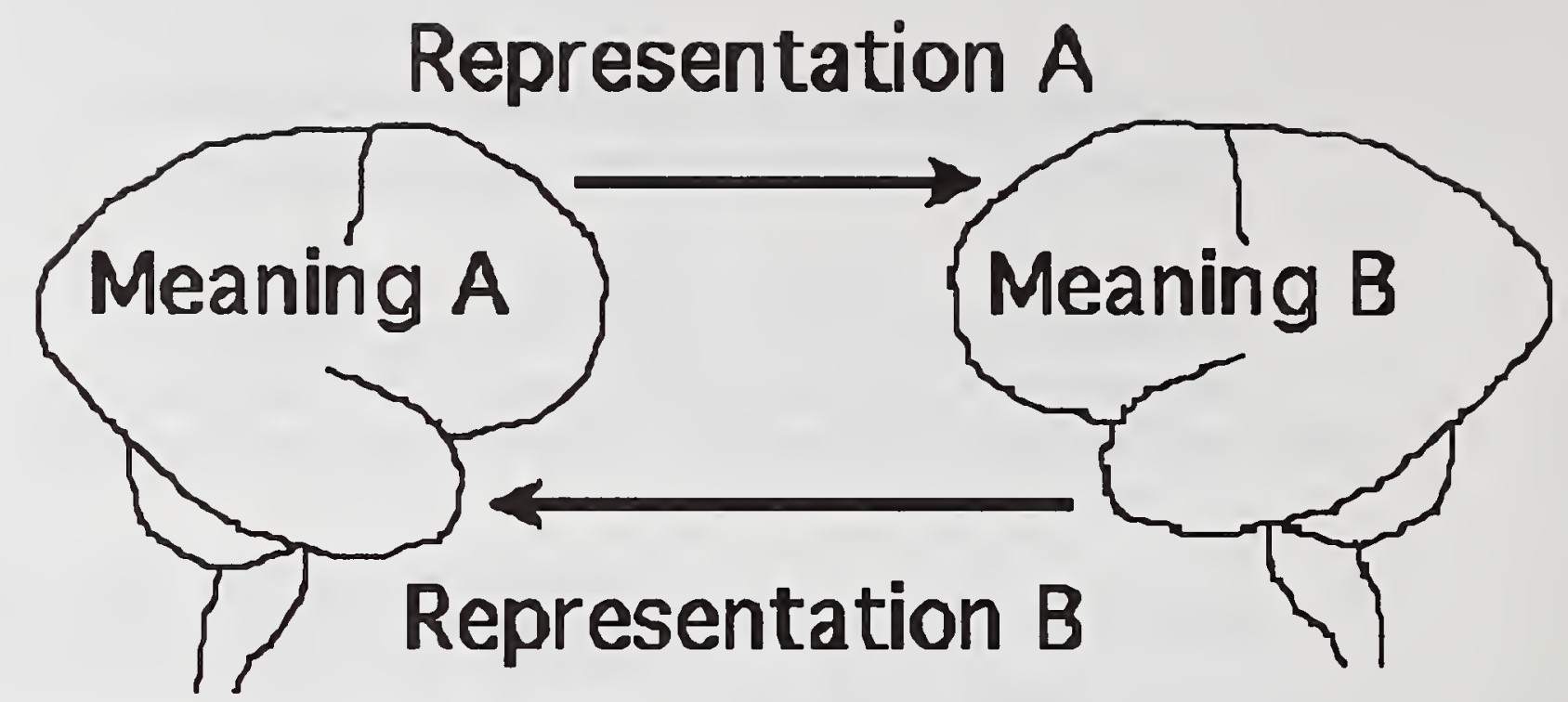

Figure 1

a better understanding of the biological basis of meaning as relations between brain states and behavioral actions, not between symbols in syntactical systems.

\section{Communication by representations}

Operational discreteness is essential for communication in dialogue. A pair of brains can act, sense, and construct in alternation with respect to each other, not merely as dogs sniff, but as two humans speak, listen, and hear. Consider brains A and B interacting (Figure 1), where A-B are parent-child, wife-husband, rabbit-dog, philosopherbiologist, neuroscientist-rabbit, etc. $\mathbf{A}$ has a thought that constitutes some meaning $M(a)$. In accordance with this meaning $\mathbf{A}$ acts to shape a bit of matter in the world (a trace of ink on paper, a vibration of air, a set of keystrokes on e-mail, movements of the face, etc.) to create a representation (a sign or symbol for humans, merely a sign for animals) directed at $\mathbf{B}, \mathbf{R}(\mathrm{ab})$. $\mathbf{B}$ is impacted by this shaped matter and is induced by thought to create a meaning $M(b)$. So B acts to shape a bit of matter in accordance with $\mathrm{M}(\mathrm{b})$ in a representation $\mathrm{R}(\mathrm{ba})$, which impacts on $A$ to induce $M(a+1)$.

And so on. Already by this description there is implicit recognition of a discrete ebb and flow of conversation like recurrence of tides, so that meanings $\mathrm{M}(\mathrm{i})$ 's as constructions of thoughts become the internal active states, and the R(ij)'s as attributes of matter become the external representations. By its nature an external "re"presentation can be used over and over. It cannot be said to contain or carry meaning, since the meanings are located uniquely inside $\mathbf{A}$ and $\mathbf{B}$ and not between them. Moreover, the same R's induces different meanings $M(i)$ in other subjects $C$ who intercept the representations. The objects that are used to communicate are shaped by meanings that are constructed in $\mathbf{A}$ and $\mathbf{B}$ iteratively and induce the constructions of meaning in $\mathbf{B}$ and $\mathbf{A}$ alternately. If communication is successful, then the internal meanings will come transiently into harmony, as manifested by cooperative behavior such as dancing, walking in step, shaking hands, exchanging bread, etc. Symbols can persist like books and stone tablets, while minds fluctuate and evolve until they die.

\section{Observations of electroencephalograms}

A biological approach to the problem of meaning is to study the evolution of minds and brains, on the premiss that animals have minds that are prototypic of our own, and that their brains and behaviors tell us what essential properties are common to their minds and to our own minds. 
Experimental measurements of brain activity (EEG) that follows sensory stimulation of animals show that sensory cortices engage in construction of activity patterns in response to stimuli [Freeman, 1975]. The operations are not those of filter, storage, retrieval, or correlation mechanisms. Each construction is by a state transition, in which a sensory cortex switches abruptly from one basin of attraction to another, thereby changing one spatial pattern instantly to another like frames in a cinema. The transitions in the primary sensory cortices, visual, auditory, somatic and olfactory [Barrie, Freeman and Lenhart, 1996], are shaped by interactions with the limbic system, which establish multimodal unity, selective attention, and the intentional nature of percepts. The interactions of the several sensory cortices and the limbic system occur in conjunction with goaldirected actions in time and space. Each cortical state transition involves synaptic changes constituting learning throughout the forebrain, so that cumulatively a unified and global trajectory is formed by each brain over its lifetime. Each spatial pattern appears to reflect the entire content of past and present experience [Skarda \& Freeman 1987], that is, a meaning.

The most important experimental finding is that the neuroactivity patterns in sensory cortex, which are correctly classified on perception of conditioned stimuli by the animals, are not invariant with respect to the unchanging physicochemical stimuli. The brain activity patterns are found to change slightly but significantly with any change in the significance of the stimuli, such as by changing the reinforcement, or adding new stimuli [Freeman, 1992]. From numerous tests of this kind the conclusion is that brain patterns reflect the value and significance of the stimuli for the animals, not a fixed memory store. Each pattern formed in response to the presentation of a stimulus is freshly constructed by chaotic dynamics in the sensory cortex, in cooperation with input from the limbic system enacting processes of attention and intention, and it expresses the history and existing state of the animal as much as or more than the actual incident stimulus. The patterns cannot be representations of stimuli or of meanings of stimuli. They are active states induced by stimuli, constituting evolution of the brains in their growth of experience [Piaget, 1930].

\section{The neural basis for intentional action}

The making of a representation is an intentional action. All intentional actions begin with the construction of patterns of neural activity in the limbic system, which has been shown by use of lesions and by comparative neuroanatomy and behavior to be a product of the limbic system [Herrick, 1948; Roth, 1987; Freeman, 1995]. In mammals all sensory input is delivered to the entorhinal cortex, which is the main source of input to the hippocampus, and the main target of hippocampal output (Figure 2). Goaldirected action must take place in time and space, and the requisite organ for these matrices is the hippocampus with its 'short term memory' and 'cognitive map' [O'Keefe and Nadel, 1978].

For example, hunger is an emergent pattern of neuroactivity that expresses the requirements of brains and bodies for metabolic fuel and building material. It induces a state transition in the neural populations of the forebrain under the influence of sensory stimuli from the gut and the brain's own chemoreceptors for its chemical state. It is also shaped by neurohormones from nuclei in the brain stem. The emergent pattern impacts the brain stem and spinal cord, leading to stereotypic searching movements that are adapted to the immediately surrounding world. Feedback from the muscles and joints to the somatosensory cortex provides confirmation that the intended actions are taking place. The impact of the movements of the body on sensory input is conveyed to the visual, auditory and olfactory systems. All of these perceptual constructs, that are triggered by sensory stimuli and are dependent on prior learning, are transmitted to the limbic system, specifically to the entorhinal cortex, where they are combined. When an animal detects an odor of food, it must hold it, move, take another sniff, and compare the two concentrations in order to decide which way to move next. The difference in strength has no meaning, unless the animal has records of 


\section{DYNAMIC ARCHITECTURE OF THE LIMBIC SYSTEM}

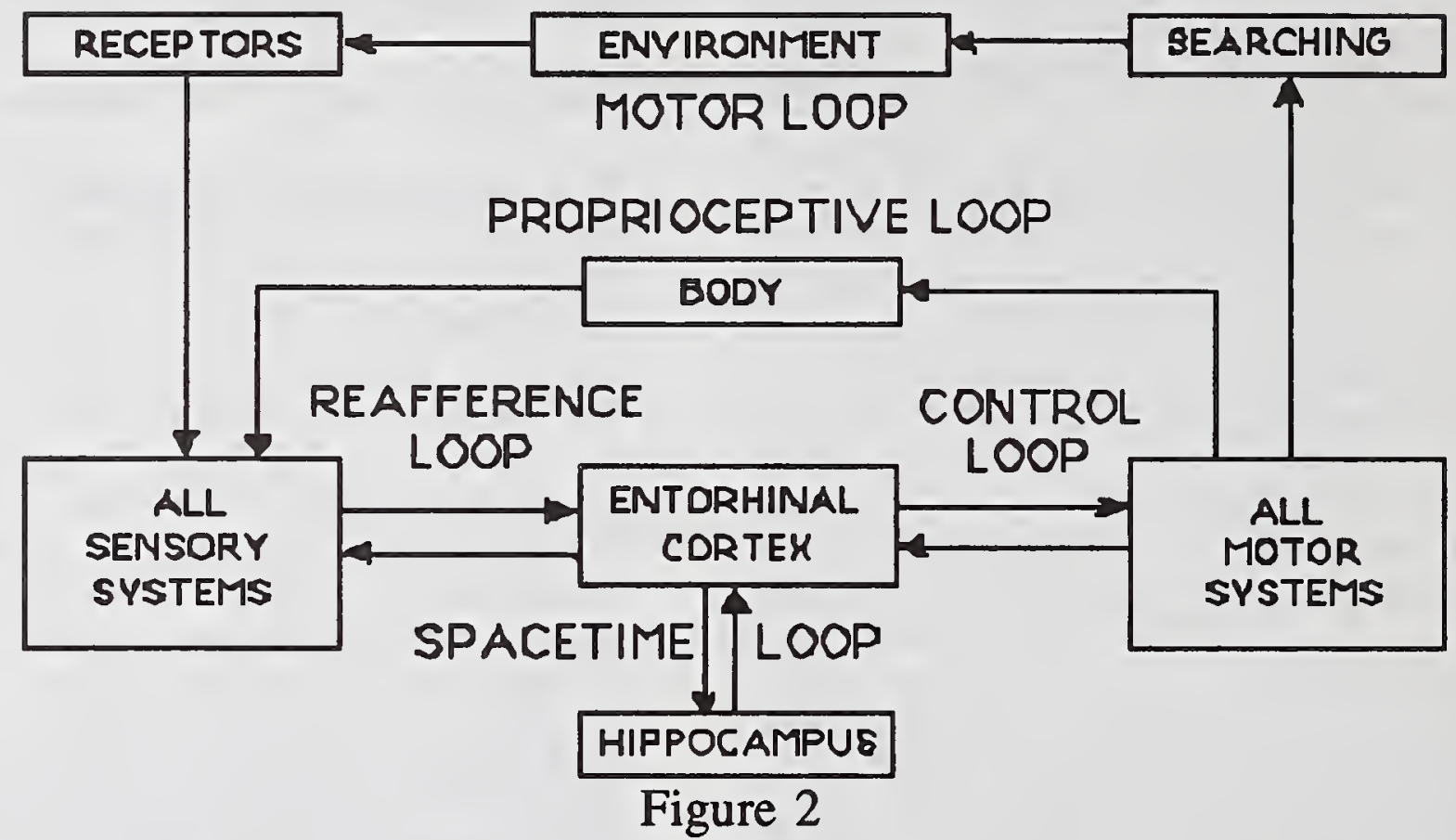

which way it moved, when the samples were taken, and a basis for determining distance and direction in its environment. These basic operations of intentional behavior are properties of the limbic system. The same requirements hold for all distance receptors, so it is understandable that evolution has provided multimodal sensory convergence in order to perform space-time integration on the Gestalt, not on its components.

In the description thus far the flow of neural activity is counterclockwise through proprioceptive and exteroceptive loops outside the brain. Within the brain there is a clockwise flow of activity constituting reafference. When a motor act is initiated by activity descending into the brainstem and spinal cord, the same or a similar activity pattern is sent to all of the sensory systems by the entorhinal cortex, which prepares them for the impact of the movements of the body and, most importantly, sensitizes them by shaping their attractor landscapes to respond quite selectively to stimuli that are appropriate for the goal toward which the action has been directed. These reafferent patterns have been denoted as the senseof effort [Helmholtz, 1879], reafferent signals (vonHolst and Mittelstaedt, 1950], efference copies [Sperry, 1950], and preafference [Kay, et al., 1995, 1996]. They are the essence of attention.

\section{Linear versus circular causality in self-organizing systems}

The conventional view of sensory cortical function holds that stimuli activate receptors, which transmit to sensory cortex through a linear causal chain, with the eventual outcome of a motor response to the initiating stimulus. Modeling with nonlinear dynamics shows that the stimulus is typically not the initiating event. Rather it is the search for the stimulus that arises in the limbic system in a recurrent manner from prior search and its results. This is circular causality at the level of intentional behavior [Merleau-Ponty, 1942].

Much lower in the hierarchy of brain organization is the event in the primary sensory cortex, which consists of the destabilization of a macroscopic state by the introduction of microscopic sensory input. In this case the transition from a prior basin of attraction to a new one, which has been facilitated by limbic modulation, is guided by the sensory input that activates a learned nerve cell assembly comprising a small subset of cortical neurons. The transition to a new state is global, so that this causal chain is also circular. The stimulus-dependent neural activity of a few neurons triggers the state transition, and then the entire domain of the 
primary sensory cortex transits to another pattern, which in the words of Haken (1983) "enslaves" the whole set of cortical neurons by acting as an "order parameter". This new active state has been characterized by Ilya Prigogine (1980) as a "dissipative structure", that constitutes the "emergence of order out of chaos".

The similarity of the properties of neural activity in the various parts of the limbic system to those in the primary sensory cortices [Kay et al., 1995, 1996] indicates that populations of neurons there also maintain global attractors, which are accessed by nonlinear state transitions, and which are responsible for the genesis of motor patterns controlling goal-directed actions and of reafference patterns that prepare the sensory cortices for the consequences of those actions.

\section{A hypothesis on the causal relations of meanings and representations}

The idea is proposed that representations are formed by the forward, counterclockwise flow of neural activity, which emerges from a microscopic level by the interactions of neurons and neuronal populations, and which places the motor systems of the brainstem and spinal cord into appropriate basins of attraction, thereby changing the sensory inflow in a goal-directed manner. The making of a representation is an ordering of the neural control systems of the musculoskeletal apparatus, that is aimed to elicit sensory feedback of a certain kind, namely the patterns of receptor discharge from representational stimuli transmitted by other beings, that place the sensory cortices into the expected basins of attraction. The meaning of the representation is implicit in the form that is given to the representation by the limbic system.

The clockwise backflow of neural activity serves as an order parameter to modulate and shape the neural activity patterns of the sensory cortices, which transmit the states of their neural populations before and after the expected inputs have occurred, and also if they do not occur as expected, or at all. It comprises not only the exteroceptive input but the proprioceptive feedback as well. This global active state, enslaving alike the limbic system and the primary sensory cortices, shapes the meaning not only of the unified sensory input consequent to the transmitted representation, but also of the emitted representation.

The implication here is that the agent who is constructing and transmitting the representation cannot fully know its meaning until after the immediate consequences have been delivered through his or her own sensory systems. More generally, a poet, painter, or scientist cannot know the meaning of his or her creation until after the act has been registered as an act of the self, nor even until the the listeners and viewers have responded with reciprocal representations of their own, each with meaning unique to the recipients.

\section{Conclusion}

Why do brains work this way? Animals and humans survive and flourish in an infinitely complex world despite having finite brains. Their mode of coping is to construct hypotheses in the form of neural activity patterns and test them by movements into the environment. All that can be known is that which has been constructed, tested, and either accepted or rejected [Piaget, 1930; Merleau-Ponty, 1942]. The same limitation is currently encountered in the failure of machines to function in environments that are not circumscribed and reduced in complexity from the real world. Truly flexible and adaptive intelligence operating in realistic environments cannot flourish without meaning.

This global state variable may be regarded as a mechanism supporting consciousness, which in the neurodynamic view is a global internal state variable composed of a sequence of momentary states of awareness [Hardcastle 1995]. Its regulatory role is comparable to that of the operator in a thermostat, that instantiates the difference between the sensed temperature and a set point, and that initiates corrective action by turning a heater on or off. The machine state variable has little history and no capacities for 
learning or determining its own set point, but the principle is the same: the internal state is a form of energy, an operator, a predictor of the future, and a carrier of information that is available to the system as a whole. The feedback device is a prototype, an evolutionary precursor, not to be confused with awareness, any more than tropism in plants and bacteria is to be confused with intentionality. In animals and humans, the operations and informational contents of this global state variable constitute the experience of causation.

\section{Acknowledgements}

This work was supported by research grants from the National Institute of Mental Health MH06686 and the Office of Naval Research N00014-90-J-4054. .

\section{References}

Barrie JM, Freeman WJ, Lenhart M (1996) Modulation by discriminative training of spatial patterns of gamma EEG amplitude and phase in neocortex of rabbits. Journal of Neurophysiology 76: 520-539.

Chang HJ, Freeman WJ [1996] Parameter optimization in models of the olfactory system. Neural Networks 9: 1-14.

Clark A (1996) Being There. Putting Brain, Body, and World Together Again. Cambridge MA: MIT Press.

Freeman WJ [1975] Mass Action in the Nervous System. New York: Academic Press.

Freeman WJ [1992] Tutorial in Neurobiology. International Journal of Bifurcation \& Chaos 2: 451-482.

Freeman WJ [1995] Societies of Brains. Hillsdale NJ, Lawrence Erlbaum Assoc. Freeman WJ (1996) Random activity at the microscopic neural level in cortex ("noise") sustains and is regulated by lowdimensional dynamics of macroscopic cortical activity ("chaos"). International Journal of Neural Systems 7: 473-480.

Haken H (1983) Synergetics: An Introduction. Berlin: Springer.

Hardcastle VG (1995) Locating Consciousness. Philadephia: John Benjamins.
Helmholtz H von (1879/1925) Treatise on Physiological Optics: Vol. 3. The Perceptions of Vision (JPC Southall, Trans.). Rochester NY: Optical Society of America.

Herrick CJ (1948) The Brain of the Tiger Salamander. Chicago IL: University of Chicago Press.

Kay LM, Freeman WJ, Lancaster L (1996) Simultaneous EEG recordings from olfactory and limbic brain structures: Limbic markers during olfactory perception. Ch. 6 in: Gath I, Inbar GF (eds.) Information Processing and Pattern Analysis of Biological Signals. New York: Plenum Press, pp. 71-84.

Kay LM, Lancaster L, Freeman WJ (1996) Reafference and attractors in the olfactory system during odor recognition. Intern. Journal of Neural Systems 7: 489-495.

Merleau-Ponty M [1942/1963] The Structure of Behavior [AL Fischer, Trans.]. Boston: Beacon Press.

O'Keefe J \& Nadel L (1978) The Hippocampus as a Cognitive Map. Oxford UK: Clarendon.

Piaget J (1930) The child's conception of physical causality. New York: Harcourt, Brace.

Prigogine I (1980) From Being to Becoming: Time and Complexity in the Physical Sciences. San Francisco: Freeman.

Roth G (1987) Visual Behavior in Salamanders. Berlin: Springer-Verlag

Shimoide K, Freeman WJ [1995] Dynamic neural network derived from the olfactory system with examples of applications. IEICE Transaction Fundamentals E-78A: 869-884.

Skarda CA, Freeman WJ (1987) How brains make chaos in order to make sense of the world. Behav. \& Brain Sci. 10: 161-195. Sperry RW (1950) Neural basis of the spontaneous optokinetic response. Journal of Comparative Physiology 43: 482-489.

Tani J (1996) Model-based learning for mobile robot navigation from the dynamical systems perspective. IEEE Transactions on Systems, Man \& Cybernetics 26: 421-436.

von Holst E \& Mittelstaedt H (1950) Das Reafferenzprinzip (Wechselwirkung zwischen Zentralnervensystem und 


\title{
INTELLIGENCE vS. MENTALITY: Important but Independent Concepts
}

\author{
lames H. Fetzer \\ Department of Philosophy \\ University of Minnesota \\ Duluth, MN 55812
}

\begin{abstract}
Intelligence and mentality are frequently identified as though they were one property designated by different predicates. That, however, appears to be a misconception-albeit one with many intriguing ramifications. The most common tendency presumably would be to identify intelligence with mentality of a high order. But il some cognitive systems possess mentality of a low order. then they have mentality without intelligence. Other systems, which may or may not qualify as "cognitive", moreover, appear to have intelligence without mentality. Since properties are the same only if they have the same class of instances, evidently intelligence and mentality cannot be the same property.
\end{abstract}

1. Introduction. Perhaps the most common mistake that occurs within the context of theorizing about cognition is that of treating intelligence and mentality as if they were the same thing. If we were to employ the term "system" to stand for any entity-simple or complex-for which specific kinds of input, causes, or stimuli (probabilistically) bring about particular kinds of output, effects, or responses, no one ought to be tempted to suppose that those entities are necessarily "cognitive", "intelligent" or possess "mentality". The concept of system thus defined is broad enough to include sticks and stones along with digital machines and human beings within its extension or class of instances.

Since lew would suppose that sticks or stones-ordinary sticks and stones, at any rate-are things that are capable of cognition, the intension or defining conditions of "cognitive system" had better exclude them on pain of demonstrating its own inadequacy. If some systems are cognitive, while others are not, then that property, ton, had better be carefully defined, with consideration for whether or not it clarifies and illuminates their differences as well as their similiarites. It may be that some systems properly qualify as "intelligent" even though they do not properly qualify as "cognitive".

of course. if we are unable to tefine what it means for something to be a "cognitive system", then we are open to the charge that we literally do not know what we are talking about. My purpose here, therefore, is to sketch a theory of the nature of mentality that brings with it an account of the nature of cognition. The differences between mentality and intelligence are then explored 
with special concern for the prospects of developing tests for mentality and tests for intelligence, respectively, which tend to support the distinctions drawn within this context and their consequences with respect to differentiating between them.

\section{Minds as Semiotic Svstems. The right} place to begin. I believe. is with a theory of mind that builds on a foundation provided by Charles Peirce's theory of signs. According to Peirce, a sign is a something that stands for something else in some respect or other for somebody. There turn out to be three basic classes of signs that differ in the way in which they stand for something else, namely: icons which are things that resemble other things; indices which are things that are causes or effects of those other things; and symbols, which are merely habitually associated with the things for which they stand. Relations of resemblance and of cause-and-effect provide natural as opposed to artilicial signs.

Photographs, paintings, and statues (at least, when they look like the things they stand for) are therefore icons in this sense. Ashes are indices of fire and fire of ashes, because they are causally connected. The words-such as "golf" and "tennis", that occur in ordinary languages, such as Englishhowever, are symbols, even when the habitual associations between such words and that for which they stand happen to be reinforced by traditions, customs, and practices of a community. Attaining the goals of a community requires conperation, and cooperation is facilitated by (the capacity for i successful communication. Although many words could have stood for things other than those for which they happen to stand, when they are thus reinforced, they assume the standing of conventions.
The triadic character of the sign relation-which connects signs with what they stand for and sign-users -suggests the prospect of defining minds as sign-using (or "semiotic") systems, where "minds" turn out to be the kinds of things for which something can stand for something else in some respect or other. This approach implies that there ought to be at least three kinds of minds, namely: those that have the capacity to use icons; those that have the capacity to use indices; and those that have the capacity to use symbols, as successively stronger kinds of minds (Fetzer 1988, 1990, 1996).

\section{Conditions of Adequacy. A mong} the most important but least mentioned aspects of theory of mind is the conditions that must be satisfied for a theory of mind to qualify as adequate. These include $(\mathrm{CA}-1)$ explaining how mental states can exert an influence upon behavior; (CA -2) the differences between human minds, animal minds, and the minds of machines, if such a thing is possible; and $(\mathrm{CA}-3)$ how we can tell-how it's possible to know-whether or not something has a mind. These may not be the only desiderata an adequate theory of mind must satisfy, but they are among the most important. Whether or not the theory of minds as semiotic systems can satisfy them is thus a measure of its adequacy.

Because signs "stand for" that which they stand in some respect or other things that "stand for" other things characteristically do 
so only partially rather than completely. Because everything resembles itself -by "being like" itself in every property and at every time-there is a trivial sense in which everything stands for itself iconically. Typically, however, things "stand for" other things only in certain respects, as in the case of a driver's license photo, which may resemble you (on a bad day) when viewed from in front but not when viewed from the side (since you are just not that thin)! The use of even the least complex kind of sign-an icon-thus presupposes the adoption of an appropriate perspective, that is, of a "point of view"!

The theory of minds as semiotic systems supports a distinction between consciousness and cognition relative to signs of various kinds. A system is conscious (with respect to signs of specific kinds) when it has the ability to utilize signs of that kind and is not incapacitated from the exercise of that ability. Even when a person is familiar with the rules of the road and understands trafric signs, that does not guarantee they will be able to perceive and respond to the $\mathrm{m}$. The conditions under which drivers fail to obey signs include those in which, although a sign was present, it was obscured from vision, the driver suffered from (temporaryi blindness, or was so intoxicated that his attention span was abbreviated.

4. The Nature of Cognition. The phenomenon known as cognition appears to occur as a consequence of the causal interaction between signs and minds (or sign-users). When a sign-user is conscious in relation to signs of a specific kind, then the presence of a sign of that kind within suitable causal proximity (probabilistically) brings about the occurrence of cognition, during which the sign is taken as standing for something in some respect or other by that user. Thus, consciousness combines ability and capability, while cognition arises as an effect of consciousness and opportunity, where both notions are relative to signs of fixed kinds.

The meaning of signs for signusers may be captured most adequately by their causal influence upon behavior. This influence is affected by the causal interaction of every other factor whose presence or absence makes a difference to the behavior of that system. In the case of human beings, the full range of kinds of factors that tend to affect behavior would appear to be exhausted by motives, beliefs, ethics, abilities, capabilities, and opportunities. The role of opportunities differs somewhat relative to factors of these other kinds insofar as they represent the actual situations that obtain for systems themselves as opposed to the situations they believe they are in.

If we refer to some particular combination of motives, beliefs, ethics, abilities and capabilities as a "context" $\mathrm{Ci}$, then the meaning of a sign $\underline{S}$ for a system $\underline{z}$ is the totality of behaviors (actual or potential) $\mathrm{B} 1 \mathrm{~B} 2 \ldots$ that the system would (probabilistically) display in the presence of that. sign, relative to various contexts C1,$C_{2} \ldots$ Thus, if we employ $" \ldots \Rightarrow$ _. as a subjunctive conditional and "... $=\mathrm{n}=>$ _ as the causal conditional, the meaning of $\operatorname{sign} \underline{\mathrm{S}}$ for $\underline{z}$ relative to $\underline{\mathrm{Ci}}$, is: 
(MS1) Clzt $=\left(\underline{\mathrm{St}}=\underline{\mathrm{n}}=>\mathrm{B} 1 \mathrm{zt}{ }^{*}\right) \&$

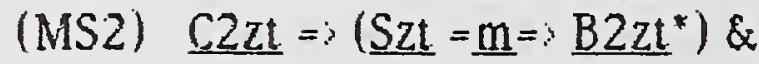

where the causal influence of $\underline{S}$ within context $\mathrm{Cl}$ would (with the strength $\underline{n}$ ) bring about behavior of kind B1; within context $\mathrm{C2} 2$ would iwith the strength mi bring about behavior of kind $\mathrm{B} 2$; etc.

5. Meaning and Behavior. Thus, when a little old lady from Pasadena reaches a traffic intersection and fails to notice a stop sign because her vision is not as good as it used to be, she may run the stop sign and cause an accident, but it would not have been on purpose. If a felon fleeing with police in hot pursuit notices a stop sign but runs it anyway, he may cause an accident, but because it was a risk that he was willing to run. When an expectant father decides that he cannot take the time to obey a sign because his wife has begun labor in the back seat, he might pray no accident is about to happen, because that would be the worst possible outcome. And so on.

The same sign (such as an octagonal red surface with the letters "S-T-0-P" inscribed) thus has the same meaning for different systems when their (actual or potential) behavior in the presence of that sign-more precisely, the strength of their tendencies toward behavior of various kinds-are the same across different contexts. This does not mean their actual behavior is the same, because they may have been in different contexts, but only that, if they had been in the same context, then the behavior they displayed-more precisely, the strength of their tendencies toward behavior of various kinds-would have been the same, under those conditions.
Similarly, different signs have the same meaning for a system when the (actual or potential) behavior it would display-more precisely, the strength of its tendencies toward behavior of different kinds-would be the same across different contexts. It can turn out that sameness-of-meaning differs in some contexts even though not in others. With regard to purchasing power, four quarters, two halfdollars, and a dollar bill would be the same; from other perspectives, such as convenience of carrying in a wallet, they would differ. Sameness of meaning thus seems to be a property that can vary by degrees.

6. Animal Mentality. The theory of minds as semiotic systems also implies that there should be a correlation between lower species and lower mentality and higher species and higher mentality. Indeed, symbolic mentality presupposes indexical mentality, and indexical mentality presupposes iconic. It would be predictable on the basis of the semiotic account, therefore, that there is some corresponding progression in nature from iconic mentality up to symbolic. where each kind might be instantiated in nature in more than one variety, depending on the number and types of signs a system uses.

The lowly E. coli bacterium, for example, has the tendency to swim toward twelve different che mo-tactic substances and to swim away from eight more (Bonner 1980, p. 63). It should be evident, from the semiotic perspective, that E. coli possess the 
ability to recognize different instances of each of these twenty che mo-tactic substances, since otherwise they would be unable to respond to the m properly. Somewhat surprisingly, therefore, E. coli appear to possess iconic mentality. It is not necessary that E coli should also be aware that twelve of these are beneficial and eight of these are har mful. since the tendency to avoid har mful stimuli and to encounter beneficial ones would evolve as a function of natural selection even in the absence of indexical ability.

Another (no doubt, less controversial) example of animal mentality arises with the vervet monkey, who makes at least three different alarm calls, one for eagles (or air-born predators), one for leopards (or earth-bound predators), and one for snakes (or earth-bound curiousities). A call of the first kind tends to cause the $m$ to hide in thick bushes; a call of the second kind, to run up into trees; and a call of the third kind, to look down for something interesting to watch (Slater 1985. pp. 155-157). Observe how beautifully this example satisfied the conception of minds as sign-using systems, where the meaning of a sign is given by the actual and potential behavior it induces within a context. Here clearly at least indexical and perhaps even symbolic mentality is displayed. since the alarm calls even appear to possess elements of conventions.

7. Machine Mentality. It may come as a disappointment to some that the theory of minds as sign-using syste ms implies that ordinary computers-von Neumann or digital machines, at least-are not the possessors of minds. This follows from the realization that these machines are designed to process marks or to manip- ulate syntax. where "syntax" consists of strings of marks that are subject to interpretation. Since digital machines are capable of processing syntax, it may be tempting to suppose they must be possessors of mind. But the capacity to processs syntax is merely a necessary and not a sufficient condition for a system to be "semiotic", since it must also be the case that the marks that are processed stand for something for that system rather than just for the users of that system.

The principal difference between semiotic systems and syntax-processing systems (such as digital machines) is that the syntax that is processed by a semiotic system stands for something for that system itself, while the syntax that is processed by a digital machine instead stands for something for users of that system. Since a mind is a something lor which something can stand for something else for that system, clearly systems that lack the ability of taking something to stand for something for itself cannot properly qualify as minds. This might be described as the "static" difference between minds and digital machines. (Fetzer 1988, 1990, 1996.)

Another difference that some may consider to be at least equally instructive might be described as a "dynamic" difference between the $\mathrm{m}$, which is that digital machines are governed by algorithms implemented in the for $m$ of programs, because of which transtions between machines states assume the character of (what has sometimes been called) "disciplined step satisfaction". A semiotic analysis of the transitions that typify human thought, by contrast, suggest that human thought 
has an associationistic dimension that disciplined step satisfaction lacks (Fetzer 1994). The potential for the same sign to function iconically, indexically, or symbolically-possibly probabilistically-in the same or different contexts poses apparently insuperable problems for those who would reduce thinking to computing. They are just not the same.

\section{Mentality and Intelligence. Perhaps} the gravest blunder that has occurred in discussions of machines and mentality has been to ignore the difference between systems that yield the same output given the same input and systems that yield the same output given the same input by means of the same processes. A simple illustration arises in the case of question-answering mechanisms. The prospect that a machine might be able to answer questions by providing the same answers that a human being might provide not only does not prove it is human but also does not prove it has a mind. The critical difference concerns the issue cited above between systems for which things stand for other things for those systems themselves and those for which things stand for the users of those systems. No one should doubt that digital machines can simulate thought processes; the serious issue is whether they can replicate them.

The difference between intelligence and mentality arises again at this juncture. The appropriate criterion for mentality given the theory of minds as semiotic systems, appears to be the ability to make a mistake (Fetzer 1988, 1990, and 1996. for example). Things that have the ability to make mistakes, after all, have the ability to take something to stand for something. while doing so wrongly. This is an ability that appears to be wide-spread among animals, moreover, but impossible for digital machines, which can malfunction, but whose "mistakes" are attributed to those who design, program and use them rather than to those machines.

In the case of intelligence. however, it would be at least faintly ridiculous to suppose that a suitable test would be the ability to commit mistakes! Something more like the opposite-the ability to avoid themwould seem to be a more appropriate criterion. But this is because intelligence is commonly understood to be akin to a high order of mentality. Machines that are incapable of committing mistakes may still be entitled to be described as "intelligent", especially when they are capable of the successiul perfor mance of complex tasks, such as the manipulation of syntax. Digital machines thus exemplify intelligence without mentality. where the conflation of these properties seems to be a significant mistake.

\section{$\underline{\text { References }}$}

Bonner, J. (1980), The Evolution of Culture in Animals (Princeton).

Fetzer, J. H. (1988), "Signs and Minds", in J. Fetzer, ed., Aspects of AI (Kluwer). Fetzer, J. H. (1990), AI: Its Scope and Limits (Kluwer).

Fetzer, J. H. (1994), "Mental Algorithms", Pragmatics and Cognition 2/1.

Fetzer, J. H. (1996), Philosophy and Cognitive Science, 2nd ed. (Paragon). Slater, P. J. B. (1985), An Introduction to Ethology (Cambridge). 


\title{
Functional Organization of Cortico-Basal Ganglia-Thalamocortical Loops
}

\author{
K.V. Baev \\ Barrow Neurological Institute \\ 350 West Thomas Road, Phoenix, AZ 85013
}

\begin{abstract}
A hypothesis is proposed that each cortico - basal ganglia thalamocortical loop is a control system that possesses a model of behavior of its controlled object. The control system utilizes error signals that are distributed via dopaminergic neurons of the substantia nigra pars compacta and ventral tegmental area to adjust both the control law and the model, i.e., for learning. The whole system of cortico - basal ganglia - thalamocortical loops is considered as a system with nested hierarchies of functional loops. Movement toward higher hierarchical levels is accompanied by generalization of encoded parameters. An error signal computed at a particular level is sent to the model of the same level and to the higher loop as well. Within the proposed theoretical framework, Parkinson's disease that is caused by a substantial loss of substantia nigra dopaminergic neurons can be conceptualized as a disorder of the error distribution system. Necessary experimental data supporting the proposed hypothesis are discussed.
\end{abstract}

KEYWORDS: Optimal Control System, informational signal, initiating signal, error signal, basal ganglia

\section{INTRODUCTION}

Cortico - basal ganglia - thalamocortical circuits are considered to be the major component of the highest brain hierarchical levels. Five such circuits were distinguished: the "motor" (or "skeletomotor"), the "oculomotor", the "dorsolateral prefrontal", the "lateral orbitofrontal", and the "anterior cingulate" [1]. According to the existing views, each basal ganglia - thalamocortical circuit receives its multiple corticostriate projections only from functionally related cortical areas. Moreover, each circuit is formed by partially overlapping corticostriate inputs which are progressively integrated in their passage through pallidum and substantia nigra (pars reticulata) to the thalamus, and from there to a definite cortical area. Usually the target area is one of those which sent projections to the basal ganglia. That is why the hypothesis appeared that the characteristic feature of all basal ganglia - thalamocortical circuits is the combination of "open" and "closed" loops.

According to current views, similar neuronal operations are performed at comparable stages of each of the five mentioned loops; the apparent uniformity of synaptic organization at corresponding levels of these loops and the parallel nature of these circuits are indirect proof of this opinion.
Classical neurobiological theoretical views are not capable of providing a reasonable explanation of how these loops function and why they were created in the course of evolution. However, it is relatively easy to create a conceptual theory of function of these loops by utilizing modern control theory.

\section{A NOTION OF GENERIC NEURAL OPTIMAL CONTROL SYSTEM}

Experimental studies of spinal Central Pattern Generators (CPG) for locomotor and scratching hindlimb movements in cats have demonstrated that these CPGs possess a model of object behavior [2], and CPG treats model flow as a component of peripheral afferent flow. The latter means that both flows interact on a parity basis. A CPG deprived of afferent flow (after deafferentation) can generate "proper" rhythm by utilizing model afferent flow.

Later theoretical developments led to the conclusion that a CPG is a regime of work of spinal Optimal Control System (OCS), and every neuronal OCS is constructed according to the same functional principle, regardless of its location in the hierarchy of the nervous system (Fig. 1). Such neural controlling system contains two distinct functional subdivisions: (1) a controller, the subsystem providing a governing set of rules or commands - a controlling law - that directs the action of the recipient of these rules - the controlled object; and (2) a model, the subsystem that generates a model of object behavior, i.e., expected afferent flow from the controlled object. The function of the model is to predict with high probability any given state of the controlled object that can result from influences on it from its controlling system.

There are two main reasons for the control system to have a model of the object behavior - incomplete observability and incomplete controllability of the controlled object. The presence of the model within any neural control system is a consequence of its optimality. Model afferent flow is used by the system to determine the most probable current state of the object by using the mechanism of filtering of afferent information (for simplicity, the filtering mechanism is put in the control law box and is not shown in the Fig. 1). Another advantage of having a model is the ability of a control system to receive very important information such as mismatch or error signals. A mismatch signal between real and model 
flows is necessary for the process of learning (see below) and is also computed during filtering process (Fig. 1).

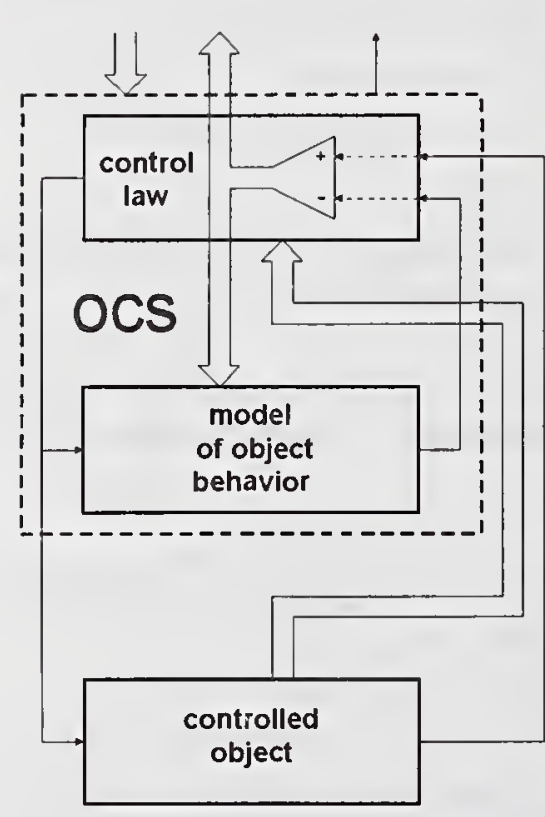

Figure 1. Functional architecture of a generic neural optimal control system (OCS). Types of signals - informational and initiating - that controlled object and subunits of OCS send to each other are shown by single and double arrows, respectively.

It is necessary to note that the described above two functional subdivisions of any given OCS may be inseparable anatomically. Both the control law and the model can be realized within the same neural circuit in the simplest of control systems. These two functional subdivisions can be more clearly separated anatomically in complex control systems.

It is rather easy to imagine a hierarchy of neuronal OCSs. Lower OCS becomes a controlled object for higher OCS. Fig. 1 illustrates what signal types are sent by one hierarchical level to another. It is well seen that the controlled object sends to its controlling system, lower OCS, the same types of signals that the lower OCS sends to a higher OCS. Those are initiating and informational signals. The latter are used by a control system to compute a control output that minimizes the initiating signals. An initiation of lower automatism is performed by a simple tonic command that means that a trajectory at the lower level corresponds to a point within a given system state at the higher command OCS. Therefore, movement toward higher hierarchical levels is accompanied by generalization, increase in parameters' abstraction, of encoded parameters. It is clear that there should be a match between the control level and its detectors, because the latter should properly describe the corresponding space state coordinates of the controlled object. The hierarchy of detectors is created in such a way that lower detectors become controlled objects for the higher detectors.

The above-mentioned theoretical conclusions created a foundation for theoretical analysis of organization of the highest brain levels. Cortico - basal ganglia - thalamocortical loops are the major anatomical substrate of the highest brain levels, and let us try to analyze the available anatomical and physiological data by utilizing the language of control theory. It is easy to identify in these loops corresponding functional subdivisions and types of signals.

\section{SKELETOMOTOR CORTICO - BASAL GANGLIA - THALAMOCORTICAL LOOP}

The skeletomotor circuit is largely projected on the putamen, which receives projections from the motor and somatosensory circuits (Fig. 2a). In addition to these projections, the
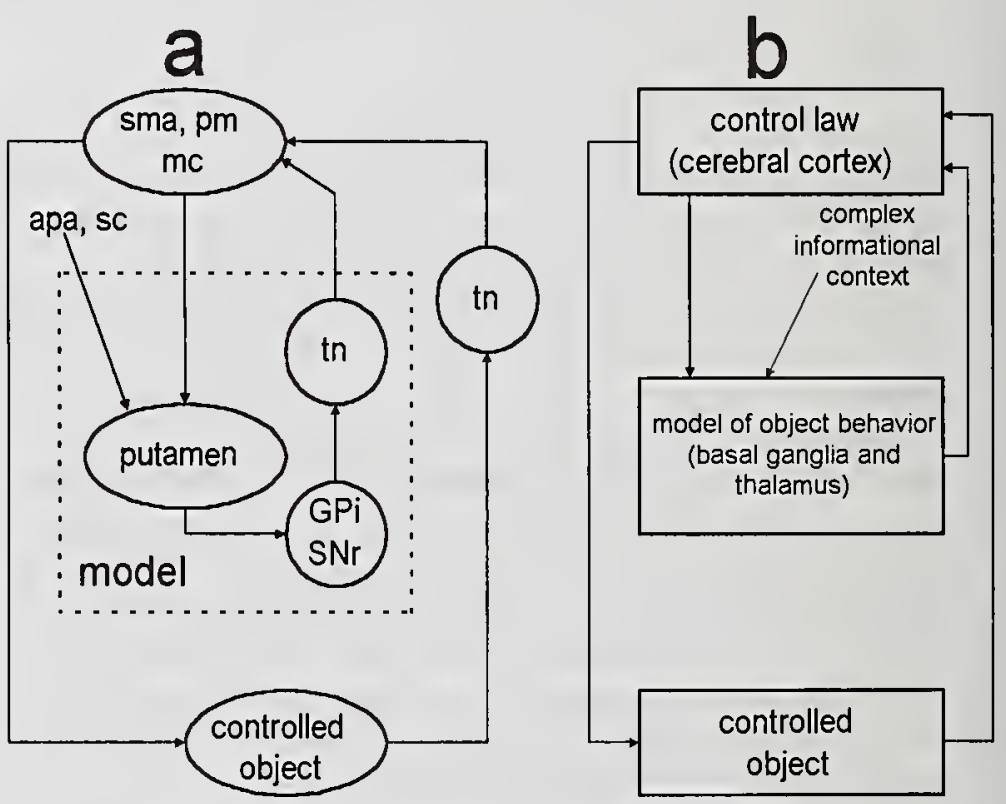

Figure 2. Structural and functional organization of the skeletomotor circuit. a - "closed" and "open" cortico - basal ganglia - thalamocortical loops. Abbreviations: APA arcuate premotor area; GPi - internal segment of globus pallidus. MC - motor cortex; PM - premotor cortex; SC somatosensory cortex; SMA - supplementary motor area; $\mathrm{SNr}$ - substantia nigra pars reticulata; tn - thalamic nuclei. b cortico - basal ganglia - thalamocortical circuit is a control system which has a model of the controlled object.

putamen also receives projections from area 5 , from lateral area 6 including the arcuate premotor area, and from the supplamentory motor area. While the most prominent projections of each of these cortical areas go to the putamen, there is slight encroachment of each projection upon neighboring regions of the caudate nucleus. Additional corticostriate inputs to the "motor" circuit from other functionally related regions - precentral, ventral cingulate premotor area, the supplamentory somatosensory area and certain parts of superior and inferior parietal lobules, are still in question. The putamen sends topographically organized projections to discrete regions of the globus pallidus (e.g., ventrolateral two-thirds of both the internal and the external segments) and to caudolateral portions of the pars reticulata of the substantia nigra. The above mentioned internal pallidal regions and substantia nigra send topographic projections to specific thalamic nuclei including nucleus ventralis lateralis pars oralis (VLo), lateral part of nucleus 
ventralis anterior pars parvocellularis (VApc), lateral part of nucleus ventralis anterior pars magnocellularis (VAmc), and the centromedian nucleus (CM). The motor circuit is closed by a means of thalamocortical projections from VLo and lateral VAmc to the supplementory motor area (SMA), from lateral VApc (from VLo as well) to premotor area (PM), and from VLo and CM to motor cortex (MC).

Thus, the general rule of connections between the cortex and the basal ganglia may be formulated in the following way: each part of the basal ganglia that comprises a specific basal ganglia - thalamocortical loop receives inputs from much bigger regions of the cortex than those to which they project their signals.

\section{The Basal Ganglia is the Major Substrate for the Model}

Closed neuronal loops are the substrate for the model. Therefore, it is possible to redraw Fig. 2a in the other way (Fig. 2b). It is necessary to note that the functional subdivision shown in Fig. $2 b$ should not be completely identified with anatomical subdivision. This identification may be done only to a certain extent (see Introduction). But for our purpose we may do this as a first approximation and consider the basal ganglia as a system which models the controlled object.

It is not difficult to determine what is the controlled object for the controlling system of the skeletomotor basal ganglia thalamocortical circuit. It is the body of the animal and the environment. This model describes the behavior of the body and the environment during animal movements.

To predict the behavior of the object, the model uses the language of afferent signals that enter the system. For the spinal OCS, it was the language of peripheral afferents. At the level of the basal ganglia - thalamocortical circuit, the situation is different. Lower OCSs are responsible for different motor automatisms and the problem of basic movement coordination is solved at those lower levels. Various motor control levels such as initiating systems of the brainstem, the cerebellum, and even cortical level (motor cortex) are controlled object for cortico - basal ganglia thalamocortical loop. The latter is a hierarchical control system. Additionally, cortical area to which the basal ganglia project their signals receives inputs from other cortical areas that means that a great variety of cortical detectors (including very complex ones) send signals to this area. These detectors determine what values have relevance to parameters describing the state of the object or its parts for any given moment in time. The model predicts the behavior of these detectors as well.

Fig. 3 illustrates how different levels of skeletomotor cortico - basal ganglia - thalamocortical loop are functionally interconnected with each other. Numerous subloops subordinated to one another can be separated within skeletomotor cortico - basal ganglia - thalamocortical loop.
The higher the subloop in the hierarchy, the more abstract parameters are processed by this loop. Clearly, each subloop has to receive corresponding afferent inputs to function properly. This is why all types of afferent information have to be processed by different cortical regions before afferent signals arrive in the corresponding subloop. For instance, the information from the skeletomotor subloop that supplies the motor cortex is not directly intermingled with information from the cerebellum that arrives in the sensorimotor cortex. Cerebellar projections go to the cortex that is located between motor and sensorimotor cortices.

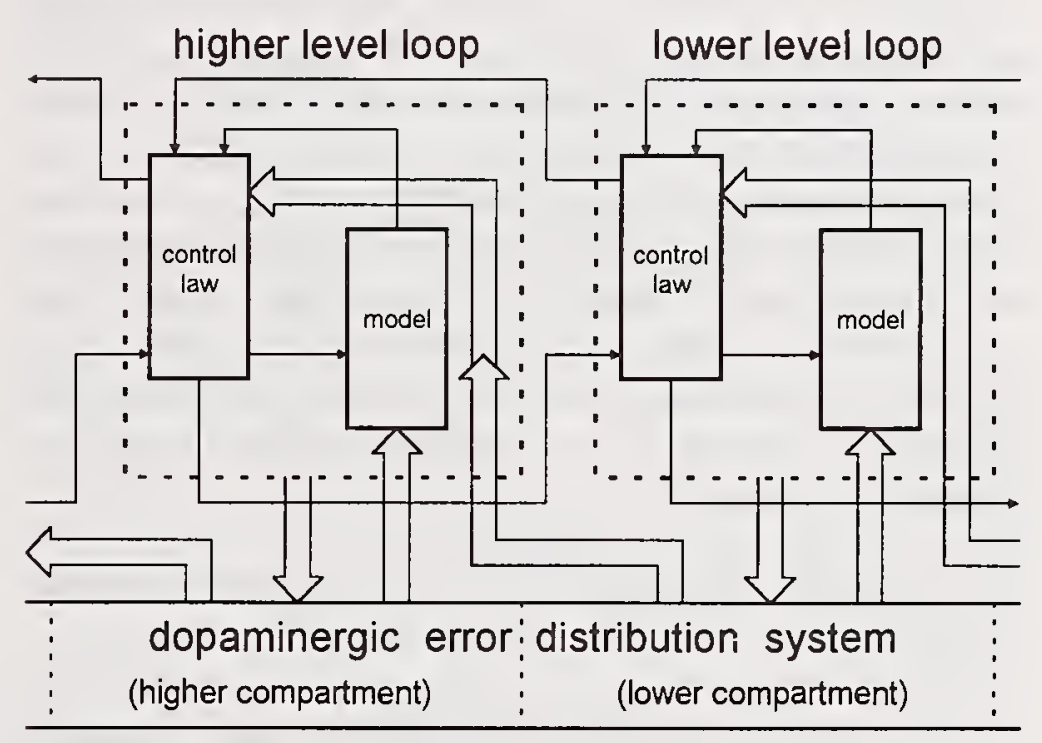

Figure 3. Hierarchy within the skeletomotor loop. Dopaminergic neurons are included in error distribution system.

The model for which the basal ganglia - thalamocortical circuit is the substrate is used in two ways. First, as mentioned above, it is used during execution of the movement to predict the transition to a new state. Second, it is used during planning of a movement that needs the model in a full scale because of a lack of afferent information about future states. It is hard to imagine this process without using a model. Moreover, a cause-effect model is not constrained by real time and may function at rates faster than real time that are necessary for multistep planning. It is obvious that using the model without the constraint of real time requires efferent and afferent channels to be cut off until a correct decision is found. It is not difficult to imagine how deafferentation or deefferentation can be done at this level; different inhibitory mechanisms can be used.

The reasoning made above helps to better understand the circuitry in the basal ganglia and shows the way to future investigations. Several simple suggestions may be made about basal ganglia circuitry. A few examples will be discussed below. The system at the higher level has to "jump" from one state to another while performing controlling 
functions. The optimal (easiest) way to create such a system is to build it on the basis of pacemaker neurons or neurons possessing bistable properties. It is easy to create a system which is able to switch from one stable state to another while simultaneously counting time intervals by using simple trigger elements, i.e. neurons having the above described properties. For instance, it is well known that such neurons are frequently a part of central pattern generator circuitries in many animal species. They model the period of rhythm generation during locomotion, breathing, etc.

Such neurons should be included in the basal ganglia circuitry. Otherwise basal ganglia circuits that include inhibition of inhibitory neurons will not work. Such processes take place in striatum, GPe (external segment of globus pallisus), GPi (internal segment of globus pallidus), and pars reticulata of the substantia nigra. Moreover, there could be one additional interesting mechanism: more complex circuit triggers. A system having a large number of such triggers, each of which may be only in two stable states, can encode the whole variety of possible states of the object. Therefore, a hierarchy of states may be encoded so that higher level triggers change less frequently than lower level triggers.

\section{Dopaminergic Neurons are a Part of An Error Distribution System}

It was implied above that control system uses the model while performing its function. Such systems have to have an error distribution system in order to properly tune the model on the object. It is easy to find it in skeletomotor cortico basal ganglia - thalamocortical circuit. Error distribution system includes dopaminergic neurons of the substantia nigra and adjacent mesocorticolimbic group (Fig. 3). The complex organization of the cell body subgroups, the one located in the substantia nigra pars compacta and the other in the ventral tegmental area, are no longer defined in terms of striatal or mesocorticolimbic projections. These subgroups are intermingled, and some mesocorticolimbic projections have their origin in the substantia nigra and vice versa [3].

This view is in good agreement with numerous experimental data. Dopaminergic neurons of pars compacta and mesencephalic tegmentum react to any behavioral or environmental change, i.e., to stimuli which are not predicted by the model. If the stimulus can be predicted, situation is different. For instance, in conditioning paradigm, dopaminergic neurons respond to unconditioned stimulus in the beginning of learning. Later, as the animal learns the task, the cells respond to conditioned stimulus that can not be predicted and do not respond to unconditioned one [4]. However, they will fire if the unconditioned stimulus is not presented at its previously predicted time interval, for instance, the stimulus appears earlier than expected. Any previously described patterns of dopamine neuron firing could be misleading in terms of stimuli that activate this neuronal system and some authors suggested that signaling of dopamine neurons predicts future reinforcement [5]. Although the idea of prediction is still the major feature of their model, the authors ascribe this function to a circuit that includes dopaminergic neurons. As we have seen above, it is more appropriate to consider the whole basal ganglia circuit as a substrate for predictions. In this case, a preceding firing of dopaminergic neurons in response to unpredicted conditioned stimulus can play a role of tuning the basal ganglia circuitry on correct future prediction of unconditioned stimulus.

There are three possible locations where mismatch signals between model and real flows may be calculated. This mismatch signal goes to dopaminergic neurons mentioned above. First, in the cortex, from which signals about mismatch go to those putamenal and caudate neurons which project to dopaminergic neurons. Second, in the putamen and the nucleus caudatus. Third, in both places. But it is necessary to note that there are no principle differences between these possibilities. In all cases error signal resulting from mismatch between model and real flows, will reach dopaminergic neurons.

In addition to the described above mismatch signals, dopaminergic neurons also receive less numerous initiating inputs from other sources: from, entopeduncular nucleus, dorsal nucleus of raphe, the central nucleus of the amygdala, and the bed nucleus of the stria terminalis. There are also some indications about direct cortical inputs to the substantia nigra [see 6]. The existence of multiple inputs to the error distribution system does not contradict to the theory. They can create new minimization criteria for the system.

Thus, mismatch signal computed at the level of one subloop goes through dopaminergic neurons to the model of the same level and to the controlling network (corresponding cerebral cortex) of higher subloop.

Learning in the basal ganglia starts when initiating signals become larger than minimal and stops when they are minimized. It is possible to suggest that very complex strategies may be used at this level to minimize initiating signals. The strategy of random search may be used in early ontogenesis when there is no knowledge of object behavior. Later, more complex and more advanced learning strategies may be used. These strategies can be based on some specific mechanisms, for instance, memorization of informational context, memorization of incorrect decisions, etc. The complexity of cortical and basal ganglia networks makes such a suggestion reasonable. Moreover, in this system error signal possesses a sign: Dopaminergic influence excites direct pathway from the putamen to the globus pallidus and inhibits the indirect one. Clearly, the sign accelerates significantly the process of learning, and fast tuning of the model on the object becomes possible. It was already mentioned that a hierarchy of parameters exists at this level, 
and it is possible to mention the simplest analogy. Suppose the predictive mechanism is built on the basis of a counter that encodes object states. In this case, if this predictive mechanism is working quite well it will be necessary to adjust only lower digits by changing thresholds of their switches.

\section{Parkinson's Disease}

Parkinson's disease is one of the most studied neurodegenerative disorders. Clinical studies and experimental data from animal models of parkinsonism have convincingly shown that death of dopaminergic neurons of the substantial nigra leads to Parkinson's disease. Akinesia, muscular rigidity and tremor are the main symptoms of this disease [see 7, 8].

Thus, based upon the proposed theoretical approach it is possible to conclude that Parkinson's disease is the consequence of disorders of learning processes in the basal ganglia (in its skeletomotor loop), a disorder of error distribution system. As a result of this, the model incorrectly predicts the state of the object in these patients (Fig. 4)
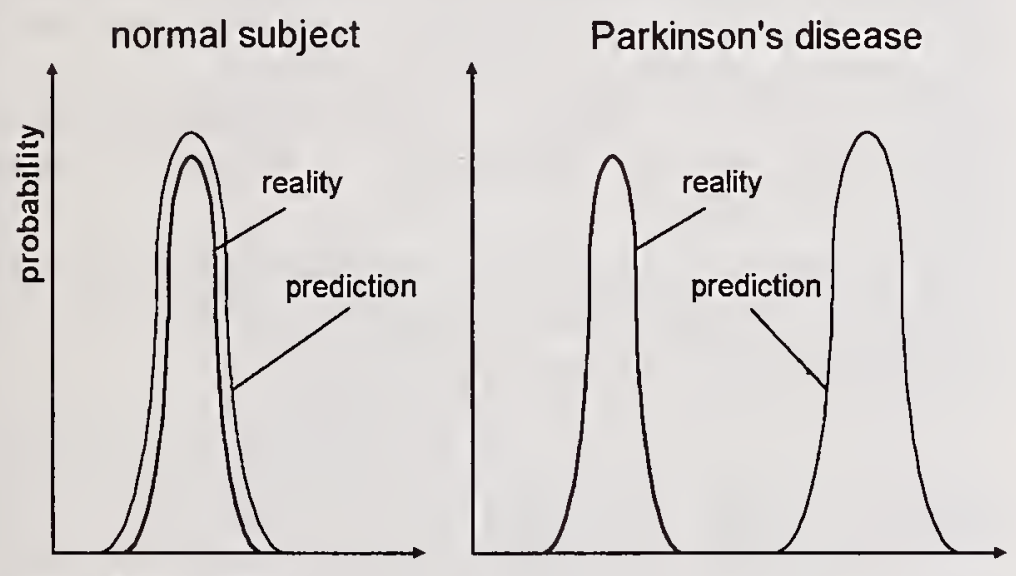

object state

Figure 4. Probability of the controlled object to be in a definite state. When the model functions correctly, the prediction coincides with the probability distribution generated by real afferent flow. In case of Parkinson's disease, predicted and real probability distributions do not coincide when the model functions incorrectly.

Let us analyze how incorrect prediction leads to symptoms in Parkinson's disease. It is clear that symptoms depend on what parameters of the object are predicted incorrectly (what digits of a "counter", lower or higher, are set improperly). When the state of antagonistic muscles is predicted incorrectly, there will be rigidity or tremor. In the latter case, it looks like overregulation when the model always misses the equilibrium point. More complex explanations should be used for bradykinesia. In this case higher level subloops are involved, and the prediction of the model differs significantly from the real state of the object. The model may predict also several states with equal probabilities. Therefore, it takes much more time for the system to choose from these states, to decide which of them is more probable.

Let us consider one of the simplest analogies - interaction of antagonistic reflexes at the spinal level. It is well known that when receptive fields for antagonistic reflexes are stimulated simultaneously it is impossible to predict which of these reflexes will be evoked. The latent period of the evoked reflex is usually much longer than in the normal situation. Moreover, sometimes none of these reflexes appear at all. Severe akinesia is the result of an absolutely unreal prediction of the model.

The most curious problems unearthed by observations generated by functional neurosurgery for Parkinson's disease still remain unclear. For example, why do partial lesions of particular structures within the neural network of the basal ganglia - thalamocortical loop, globus pallidus pars interna and some thalamic nuclei, improve symptoms. This observation contradict common sense: How is it possible to improve the network function by destroying its part? Moreover, chronic stimulation of some of the same structures produce identical effect. This is a fundamental paradox that until now has not been reasonably explained by reflex theory or the balance of excitation and inhibition that is postulated to occur in the basal ganglia. In the case of stimulation, two possible mechanisms exist: (1) Stimulation produces a functional block in regions immediately adjacent to the electrode tip; and (2) spreading of influences to other brain regions occurs via both fibers passing through the stimulated region and axons of neurons excited during stimulation. However, these latter influences do not provide the system with meaningful information for signal processing. Thus, the second mechanism simply introduces noise into the network. Therefore an obvious question appears: Why does noise added to such a system improve its function? At last, it is well known that such parkinsonian symptom as tremor can be effectively removed by placing a lesion in thalamic nucleus that conveys information from the cerebellum to the cortex, that means that the real afferent flow is changed by this procedure.

From the point of view of the developed theory explanation of these medical facts can be the following. When the network generating model afferent flow is partially destroyed, probability distribution of possible object states become lower and wider. Obviously, predicted states can partly overlap with possible real states of the object after this procedure. As a result, system does not find an error in its prediction anymore, and does not try to correct the object position in it state space. Situation is symmetrical in case of placing the lesion in the network processing afferent flow. Model flow is not changed. What is changed is the probability distribution predicted by real afferent flow, and two distributions generated by model and real flows start to 
overlap. The latter leads to alleviation of parkinsonian symptoms, for instance, tremor.

An explanation for the effects of chronic stimulation becomes transparent after what was said above. Functional block works as partial destruction of the structure. Therefore, the mechanism of chronic stimulation is partly similar to placing a lesion. Concerning noise, another possible factor that can work in the case of chronic stimulation, it is well known that adding noise effectively helps the system to slide down the error surface to its global minimum, much like shaking an uneven declining surface helps a ball slide down to the lowest point on that surface. While the results of chronic stimulation are similar to the clinical results of placing a lesion, they do not produce immediate neural tissue destruction. Furthermore, one can stop stimulating tissue by turning off the source of current, thus producing a reversible type of functional lesion.

One critical aspect of this explanation should be always kept in mind. Placement of a lesion or chronic stimulation within an OCS network improves symptoms, but does it make the controlling system function normally? The answer is no! After the lesion has been placed, the system is effectively tricked and does not find any errors in its prediction. However, normal function of the controlling system is not restored. Therefore, functional neurosurgical procedures in PD should be considered merely as palliative, symptomatic interventions and not curative because they do not stop the fundamental degenerative process. Only restoration of the structural integrity of the system by rewiring of lost connections, or at least prevention of further dopaminergic cell loss, will be the most effective form of treatment for PD.

Therefore, functional neurosurgical procedures have to be considered as methods of treatment based on holographic properties of biological neural networks, that means that system can function being partially distructed. Like in holography when complete three-dimensional image of an object can still be reproduced after partially destruction of the photographic plate, but with a lesser resolution.

\section{OTHER CORTICO - BASAL GANGLIA - THALAMOCORTICAL CIRCUITS}

Skeletomotor loop described above controls movement of such objects as the animal's body and the environmental objects. Oculomotor loop is principally very similar to the skeletomotor loop. Obviously, if the level of parameters' abstraction in such a system as the cortico - basal ganglia thalamocortical circuit became sophisticated enough to perform "movement" of abstract objects in an abstract state space out of real time, there would clearly be the creation of a principally new functional feature, and the process of elaborately detailed multistep planning would be possible, i.e., the capacity for thought. Prefrontal loops are the substrate where such operations take place.

The above-described theoretical approach can be utilized for explaining the functional role of the cingulate gyrus and its cortico - basal ganglia - thalamocortical loop. The latter loop is often referred as the "limbic" loop. It is a part of the limbic system. According to numerous experimental data, the limbic system controls: Activities essential for the self-preservation of the individual, including emotional ones (e.g., feeding behavior and aggression, behaviors that are often accompanied by emotions); activities essential for the preservation of the species (e.g., mating behavior, procreation, and the care of the young); visceral activities associated with both of the above and numerous other activities of the hypothalamus; mechanisms for memory.

\section{REFERENCES}

[1] Alexander, G.E., DeLong, M.R. and Strick, P.L. (1986): Parallel organization of functionally segregated circuits linking basal ganglia and cortex. Ann Rev Neurosci 9: 357381

[2]Baev, K.V., and Shimansky, Yu.P. (1992): Principles of organization of neural systems controlling automatic movements in animals. Progr Neurobiol 39: 45-112

[3] Le Moal, M., and Simon, H. (1991): Mesocorticolimbic dopaminergic network: functional and regulatory roles. Physiol Rev 71: 155-234

[4] Ljungberg, T., Apicella, P., and Schultz, W. (1992): Responses of monkey dopamine neurons during learning of behavioral reactions. $J$ Neurophysiol 67: 145-163

[5] Houk, J.C., Adams, J.L., and Barto, A.G. (1995): A model of how the basal ganglia generate and use neural signals that predict reinforcement. In: Models of Information Processing in the Basal Ganglia, Houk, J.C., Davis, J.L., and Beiser, D.G., eds. Cambridge, Massachusetts, London, England: Bradford Book, The MIT Press. pp. 249-270

[6] Brodal, A. (1981): Neurological anatomy. In relation to clinical medicine. New York, Oxford: Oxford University Press

[7] Bergman, H., Wichmann, T., and DeLong, M.R. (1990): Reversal of experimental parkinsonism by lesions of the subthalamic nucleus. Science 249: 1436-1438

[8] Greene, K.A., Marciano, F., Golfinos, J.G., Shetter, A.G., Lieberman, A.N., and Spetzler, R.F. (1992): Pallidotomy in levodopa era. Adv Clinical Neurosci 2: 257-281 


\title{
Learning How To Know: Semiotics and Multiscale Cybernetics
}

\author{
A. Meystel \\ Drexel University \\ National Institute of Standards and Technology
}

\begin{abstract}
In this paper a comparison is made of Semiotics and Cybernetics. It was found that semiotics can be considered a cybernetics of the $\mathrm{m}$ th order. The latter can be characterized by the phenomena of self-reflection and multiscale representation. A syllabus for the corresponding course is outlined.
\end{abstract}

Key words: cybernetics, $m$-th order, multiscale representation, reflection, semiotics

Knowledge Acquisition. Intelligent systems acquire knowledge through preprogramming and learning. These processes deal with information represented in signs (labels, elementary codes) and symbols (generalizations of signs). The synapses of our nervous system generate signs and symbols, and are signs and symbols themselves. The formation and use of signs and symbols is analyzed within the discipline of semiotics. In semiotics, the central process under consideration is called semiosis. This process takes the form of an elementary loop of acquisition and processing which represents both functioning and learning.

Semiosis is combined of the following subprocesses $[1,2]$ :

a) encoding of the sensations (sets of signals from transducers interpretable as states of the reality) by using elementary signs

b) associating encoded sensation with codes of actions (sets of phenomena which entail the commands generated by the system)

c) constructing strings of codes for "states-actions..." (S-A-S-A...) stored in the memory

d) assigning to these strings of signs values of goodness $\mathbf{J}$ interpretable under specific goals $\mathbf{G}$, which allow interpretation of "experiences" $\mathbf{E}$ : $[\mathbf{G},(\mathbf{S}-\mathbf{A}-\ldots)]-->\mathbf{J}$

e) discovering classes of experiences $\{\mathbf{E}\}$, assigning labels (signs, symbols) to them, assigning signs to their components (generation of the concepts of objects and actions)

f) forming hypotheses of new behavioral rules using previously stored results of prior processes of semiosis.
Statements "a" through " $\mathrm{f}$ " can be formed only for a particular "level of resolution" (or "granularity".) The level is characterized by two major constraints in focusing attention (from above and from below.) The first constraint corresponds to the scope of view, the second - to the minimum "granule" (the "indistinguishability zone" introduced in [3].)

All semiotic processes contain an operation of generalization which is typical for all processes of knowledge acquisition. The need in generalization is determined by the orientation toward complexity reduction. All algorithms of generalization consist of procedures of grouping, focusing attention, and combinatorial search (see, GFACS in [2].) A special feature of the semiotic system is that GFACS is being applied to the results it produces at its output. Thus, the multigranular systems emerge.

Cybernetics vs Semiotics. The loop of semiosis has a striking similarity with a cybernetic views of the system. The sequence "a" through " $f$ " describes computational activities at a singe level of granularity in a system for knowledge acquisition, generalization, and utilization (e.g. "action") i.e. in a semiotic system. Unlike a classical cybernetic system the semiotic system generalizes the acquired information and processes it again many times, thus building up a system of multiresolutional nested loops. Each of these loops is a classical cybernetic loop. A system of these loops allows for considering $m$ levels of granularity.

In classical cybernetics, the issues of granularity are considered to be a source of special effects (e.g. nonlinearity) but does not interfere with the architecture of the "loop": "...vibrations of the molecules in the nucleic acid complexes may be responsible for their behavior as organized systems ...in dynamical systems of a much coarser texture the vibration properties play a large part in their organization. This is true both with biological and with engineering systems." [4] 
It is typical for the analysis of semiotic system to be focused upon the meaning of the system and the process: after answering the What? question, Why? is the question that the semiotician attempts to answer. In cybernetics, the same elementary signal processing loop is a source of different insights. How? and How to control? are the typical questions for the cyberneticist. As a result, a semiotic system turns out to be equipped by the means of generating feedforward commands which contain the goals and tasks, and it becomes a conventional cybernetic system.

Levels of various granularity. In other words, the cybernetic loop of system functioning is almost equivalent to the semiotic loop of semiosis with the following difference: the first describe control processes in physical terms while the second analyzes the processes in the multigranular sign-symbol domain. A more serious difference is in the emphasis: since semiotics is pursuing the meaning of the system, the goal is always questioned, and another loop should be discussed at which the goal is just a variable. This new loop (a coarser granularity loop) has a different goal, and the goal of the first loop is just a variable for this new loop. The pursuit of meaning leads us to discovery of loops of different granularity. (Actually, emergence of the objects and processes of coarser granularity was ingrained in the stage " $\mathrm{g}$ " of the description of the process of learning.

When $\mathbf{m}$ levels of granularity (resolution, scale) emerge as a result of learning in the semiotic system, similar levels emerge in the corresponding cybernetic system. A cybernetic system of the $m$-th order emerges, or multiscale cybernetics. The multiscale cybernetic systems can be characterized by a multigranular (m-loops) semiosis which invokes a variety of interesting and important phenomena including reflection ("reflexia"), selforganization and emergence (usually attributed to the situation of "complexity") and others.

Can we talk about virtual equivalence of semiotic and cybernetic systems just by considering a single level loop? We can but the powerful concepts of the semiotic-cybernetic scientific paradigm will be wasted. We do not need the tools and techniques of either in a single loop case. On the other hand, if learning is involved, if planning is of interest, if processes of self-organization and self-description are of significance, no single loop (single level) discussion can be productive: such a simplification will have hidden mistakes.

At a single level of resolution, the relation between the constructive mathematics and non- standard analysis can be demonstrated. Both are characterized by illustrative paradoxes which demonstrate that movement to another level is required.

The joint semiotic-cybernetic paradigm allows us to address all these issues and explore the research developments implied, and the applications of the expected theoretical results.

Semiotic Architectures. A long time ago people discovered that intelligent systems and learning processes depend on architectures of signsymbol processing. These architectures determine how intelligent systems perceive the world, recognize objects in it and interpret the results of recognition for the subsequent actions. The multigranular architectures of our intelligence and the multigranular representations generated by our intelligence are affected by the processes of single and multi-level self-reflection.

Architectures of sign processing affect the ways intelligent systems behave in the world. They depend on the way these systems think they behave. Careful analysis demonstrates that all our knowledge depends on our self-reflection. Our own brain-architectures are reflected in all the knowledge we acquire, even when we think that we analyze an electron, a spray casting, or a bacterium. All our representations reflect the architecture of our brain. Architectures of intelligent systems incorporate the recursion of self-reflection which becomes crucially important for describing interactions among multiple intelligent systems.

In intelligent systems knowing always presumes changing resolution or granularity or scale (which is the same). Therefore, learning brings on changing the scale of representation and subsequent changes in processes of decision making, planning, and error compensation within this scale of representation. Each next step of learning leads to the generation of a next order of cybernetic analysis. Once a higher level (level of coarser granularity) is achieved, all previously known (finer resolution) subsystems are subsumed by (aggregated within the model of) the level of coarser granularity.

Cybernetics was a step in learning how to know. We have discovered the phenomenon of selforganization via planning and error compensation during functioning. Self-organization was a step toward self-reflection. With realization of the phenomenon of self-reflection we started paying more attention to the techniques of interpretation. The results of our interpretation are strongly 
affected by our "self" hidden in the techniques of our intelligence. Then, the cybernetics of the second order emerged (this term was introduced by H. von Foerster to describe the level produced by self-reflection).

Socio-scientific Aspect of Knowledge Acquisition. People were always respectful to and comfortable with activities related to knowledge acquisition and organization, all of them starting from broadminded Aristotle and ending with narrowly trained professionals of the XX Century. Problems related to knowledge emerged when we entered the era of intelligent systems:

a) we realized that within a single problem we should address modeling of the system at several levels of resolution (granularity, scale)

b) we started analyzing processes of selfreflection when in addition to the world produced by sensations we encountered a world produced by our own representations which included representation of ourselves.

How Is Semiotics Related To Other Disciplines. The gist of the movement toward Unified Science was rejected by the reality of socioscientific process. However, the essence of it cannot be discarded because of the following reasons:

- all sciences are based upon the system of symbols and their transformation induced by our brain architecture

- thus all phenomena of the world perceived and reasoned by us are preprocessed so as to fit to be interpreted by these architectures

What makes the natural sciences distinct is something that can be called "legacy issues": this is the way how people in the particular socioscientific niche are used to talk about their subdomain entities, so do not interfere with this legacy. Nevertheless, it seems to be prudent, to study the invariance of the natural sciences before we embark on doubtful enterprise of unconditionally solidifying ' obstacles for generalization which are copiously collected within the bulk of legacy issues. The following temporal sequence of knowledge acquisition can be recommended in the imaginary syllabus of the course on "Semiotics of Knowledge":

1. Science of Signs Formation which includes:

- Energy and Complexity

- Entity formation techniques

Grouping, Focusing Attention, Combinatorial Search (GFACS)
- Generalization and formation of the entityrelational structure

- Continuity and discretization

- Granularity and Resolution

- The phenomenon of recognition

- Equivalence, Resemblance, Similarity

A significant issue (among others) is the skill and the habit of notations: they carry with themselves premises, assumptions and prejudices.

2. Science of representation including Syntactics and Semantics.

3. The Phenomenon of Interpretation

4. Sciences of Generalized Reasoning including Logic, Mathematics, and Computer Science

5. Knowledge Engineering and Epistemology

6. Science of Value Judgment including methods of evaluation and optimization of Goodness and Beauty.

7. [Intuitive] World Modeling in Art and Literature

8. [Weak] World Modeling (Experimental

Knowledge Classification) in Zoology, Botany,

Medicine, Geography, History, Economics

9. [Strong] World Modeling (Architecture Analysis) with the legacy disciplines of Physics,

Chemistry, and Biology

10. Problem identification and creative solving:

Theory of Design

11. Philosophy and Psychology: Theory of Mind

12. Control Theory

13. Information Theory

14. Science of Learning including Process of Semiosis, Adaptation, Evolution, Machine Learning

15. Engineering

16. Life Science, Evolution, Genetics

17. Large Complex Systems (including Sociology and others)

18. General Pragmatics and Symbol Grounding

A special role of Control Theory. In 60s, the ideas surfaced that theory of control should go further than just constructing mathematical models of dynamic systems (K. S. Fu, G. Saridis.) The annual symposium on intelligent control has been initiated in 1984 when it became clear that Control Theory benefits from unified application of techniques and ideas typical for Operation Research (OR) with its search, and Artificial Intelligence (AI) with its logics and linguistics. In the course of events, it became clear that there is more to the situation than just merger of:

\section{Control Theory+OR+AI.}


Some groups persistently associated intelligence with neural networks, fuzzy set theory, and evolutionary computation. This wing of control/computational science exists now as a community of Soft Computing for whom the body of intelligent control is a sum:

\section{NN+Fuzzy Sets+Evolutionary Computation.}

Finally, many people believe that it is very important to determine everything by the architectural issues which makes problems dependent of the cases of intelligent systems known from the biology of nervous systems in living creatures, laws of language, physics, and technical solutions in autonomous robotics. Thus, a new set of interrelated domains was added to the area of Intelligent Control:

\section{Architectures+Brain+Semiotics}

One can see that these three communities are not independent, they rather overlap a lot. It is tempting to say that the truth is in the middle. However, in this situation finding a "middle" is a formidable problem by itself.

Conclusions. Various disciplines, including knowledge engineering, artificial intelligence, intelligent control, and semiotics, are stages in the exciting search within and traveling toward multiresolutional cybernetics, or cybernetics of the m- th order. Such systems produce representations in multiple scales, supplement control with selforganization and throw us into a totally new world, a multigranular world, in which we always live even though we do not pay too much attention to its multiresolutional nature.

Various artifacts of life and technology should be discussed from the point of view of multiscale cybernetics. In engineering problems and poetry, scientific research and visual arts, everywhere we will continue to discover the multiresolutional world of semiotic-cybernetic models.

\section{References}

1. R. Carnap, "Foundations of Logic and Mathematics", in International Encyclopedia of Unified Science, eds. O. Neurath, R. Carnap, C. Morris, U. of Chicago Press, Chicago and London, 1955, pp. 139-213

2. A. Meystel, Semiotic Modeling and Situation Analysis: An Introduction, AdRem, Bala Cynwyd, PA 1995, pp. 81-91

3. A. Meystel, "Planning in a hierarchical nested controller for autonomous robots," Proc. IEEE 25th Conf. on Decision and Control, Athens, Greece, 1986

4. N. Wiener, "Perspectives in Cybernetics", in Eds. N. Wiener, J. Shade, Cybernetics of the Nervous System, Elsevier, Amsterdam, 1965 


\section{XIX}

SEMIOTICS IN INTELLIGENT CONTROL 



\title{
Semiotics: a Key to Intelligent Control
}

\author{
Tariq Samad \\ Honeywell Technology Center \\ 3660 Technology Drive \\ Minneapolis, MN 55418 \\ samad@htc.honeywell.com
}

\section{ABSTRACT}

As the discipline concerned with communication and signification in their broadest senses, semiotics has much to offer for the development of truly intelligent systems. This paper explores the relevance of semiotics to intelligent control. I focus in particular on the role of the semiotic sign in control systems, distinguishing several types of signs involved. These include signs used to communicate system states, human user inputs, and environmental variables to the control system, signs used to communicate control actions to the system, and signs used internally by the control system for its functioning. Viewing these various flows of information as general signification activities broadens our conventional notion of control, and renders it more relevant to large-scale, complex processes. Several technologies that can contribute to the "semioticization" of control are noted: multiscale representations, fuzzy logic, learning and adaptation, computational linguistics, and classical control.

Keywords: control systems, multiresolution models, fuzzy logic, learning systems, computational linguistics.

\section{VISIONS OF INTELLIGENT CONTROL}

The successes that have been achieved with our existing control technology over the last few decades are a mixed blessing. On the one hand, control technologists can take justifiable pride in contributing to the technological progress of our societies. On the other hand, further research in classical control appears to be a path of incremental, evolutionary improvements. Yet challenging control problems are still far from being solved-practical learning/adaptive systems for real-world applications are still far from reality, to take one example. The interest in "intelligent control" reflects both the recognition of the outstanding research needs in control and the view that classical control methods cannot by themselves satisfy these needs. There remains, however, a considerable mismatch between the motivation for intelligent control and much of the research that is currently being conducted under its aegis. Single-loop neural and fuzzy controllers are only a small part of the solution-at best.

One critical limitation of most research-whether in a classical or intelligent control vein-is the simplistic, highly restricted code theory that is assumed to underlie communication and signification in control systems. Aspects of this communication include: a user's command to the control system, the representation of sensory measurements, controller design parametrization, system models as used in the controller, and the control action itself. In each of these cases, current control systems only permit simple types of information structures, such as numerical vectors.

As the discipline concerned with communication and signification in their broadest senses, semiotics has much to offer control science and engineering. This "position paper" attempts to demonstrate this relevance. The next section discusses how a control system can be viewed as a semiotic system, the latter being taken to mean a system in which the Peircean triad of object/sign/interpretant is realized. Section 3 outlines several possible roles for signification, noting how they extend our current thinking in control. Semiotics is not, at least not yet, a technology with packaged solutions to offer, but several technological fields have significant connections with it. Some of these are briefly discussed in Section 4. Section 5 contains some concluding remarks.

This paper is speculative. It presents no crisp technical ideas. I am hoping that it can serve as grist for discussing the role of semiotics in intelligent systems in general, and intelligent control systems in particular. The majority of the exposition is abstract, but I occasionally refer to some examples. These are taken from domains I have some familiarity withindustrial process control and building control-but the exposition is intended to apply to control systems generally.

\section{ROLES FOR SEMIOSIS IN INTELLIGENT CONTROL SYSTEMS}

I discuss in this section how a control system can be viewed as a semiotic entity, placing particular emphasis on the role of the sign as the linchpin of semiosis. The notion of the sign has been adapted considerably over the history of semiotics, and it is relatively recently that the possibility of artificial entities generating and interpreting signs has been acknowledged. The very thought that a control system can engage in semiosis may still seem a novelty to some.

Nevertheless, a semiotic explication of a control system can be suggested: A control system receives signs from its environment, interprets and processes them according to models explicitly or implicitly embedded in it, and produces other signs. The question arises: What, if any, advantage is gained by this potentially confusing reformulation over our conventional understanding and articulation of a control system? 
For the lowest level of controllers-which comprises the majority of them-the benefits may appear to be few. I do not, for example, see how our understanding of the PID algorithm can be improved given the semiotic insight relative to its explication as $\mathrm{u}=\mathrm{Kce}(\mathrm{t})+\mathrm{Ki} \int \mathrm{e}(\mathrm{t}) \mathrm{dt}+\mathrm{Kd} \frac{\mathrm{de}}{\mathrm{dt}} \cdot{ }^{I}$ Even for loop control, though, the core algorithm is but one piece of the puzzle. The functioning of a PID controller also requires integral windup protection, a bumpless transfer mechanism, tuning procedures, manual override capability, etc. These functions may be ancillary, but the complexity of most exceeds that of the controller's "primary" element.

In this larger picture, a semiotic perspective is meaningful, and a semiotic triangle associated with detecting a poorly tuned process is presented in Fig. 1. One manifestation of a poorly controlled process is high variance in the process output, here assumed to be temperature. In the figure, this sign has an interpretant, a consequence of a human operator observing, for example, the temperature versus time trend on a display. The interpretant is itself a sign-perhaps a command to the control system to initiate a retuning of the PID controller. There are many other signs of the considered object: large overshoot in step responses, high control action, poor quality product downstream, etc. There are also many potential interpretants, which could be generated by manual or automatic systems.

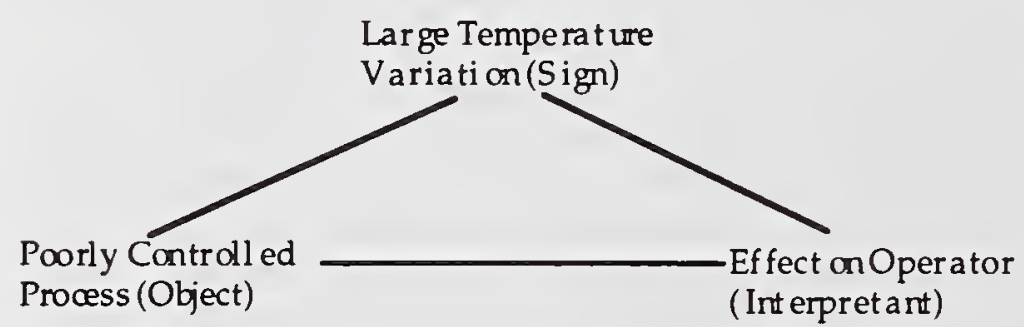

Fig. 1. A semiotic triangle for a simple control application.

The chain of semiosis - a sign resulting in an interpretant which, being a sign itself, leads to another interpretant, and so on-exists in control systems no less than in general semiotic systems. At some point, the chain terminates and a physical action is taken-the retuned controller starts operating a valve differently than before. At this limit of the semiotic activity, its triadicity is replaced by the dyadic action of the physical world. Since a control system operates by feedback, the semiotic regression ultimately affects its object,

${ }^{1}$ For those readers unfamiliar with PID controllers, the control output $u$ is computed as a weighted sum of three error terms: the instantaneous error $e(t)$ (i.e., the difference between the current system output value and the desired value), the integrated sum of this error, and the rate of change of this error. $\mathrm{K}_{c}, K_{i}, K_{d}$ are the respective weights and are generally referred to as the proportional, integral, and derivative gains. the system under control, thereby initiating another chain of interpretants.

There is as yet no consensus on a typology of signs. Eco (1984) analyses various historical treatments of the concept of the sign, stressing its generality. Peirce's initial classification differentiated between indices (signs in physical adjacency to their objects), icons (signs similar to their objects), and symbols (in which the sign/object connection is arbitrary). Eco (1976) labels this classification "untenable" and suggests that signs can be classified from different perspectives (e.g., relative to the channels of communication, the gradation of the sign, and its origin). Sebeok (1994) extends Peirce's tripartite scheme with three additional types: signals, symptoms, and names.

What types of signs are appropriate for control systems? It is useful to adopt Eco's distinction between a signal and a sign (Eco, 1976; p. 46) ${ }^{2}$. A signal, by his definition, is "a pertinent unit of a system that . . . could also be a physical system without any semiotic purpose. . . . A signal can be a stimulus that does not mean anything but causes or elicits something." In our earlier example, we described the operation of a PID algorithm as lacking a semiotic character. We can thus say that, when the process output error signal serves only as input to a PID controller, it is solely a signal. It assumes a semiotic role, and the status of a sign, only when a semiotic triangle is configured through the operation of a (human or machine) interpreter that is capable of generating an interpretant for further signification.

Some signs relevant to intelligent control systems are shown in Fig. 2, which also differentiates between mandatory and optional signs. In the general case, a control system will receive inputs, and these may have signification potential, from its environment, its human users, the system to be controlled, and other automated systems. Its outputs, again potentially signs, will be communicated to human users (some of these may be identical to the human users that provide input to the control system, but others will not be), the controlled system, and other systems. Semiosis is not limited to the interface between the control system and the world; signification occurs internally as well. I am assuming in this figure that any device that can be directly effected by the control system is by definition part of the controlled system. The environment is therefore not an output.

This figure is not intended to represent a far-off, futuristic vision in which intelligent control systems may operate with complete, unmitigated autonomy. Semiotics can play a revolutionary role in control systems in the considerably nearer-term. Some role for the human will undoubtedly exist in this case-at a minimum, in the start-up, shut-down, and maintenance of the control system. For a practical intelligent control system, the human interaction is not optional.

2"Signal" is also one of the six types of signs classified by Sebeok (1994). In this paper, I follow Eco's usage since it conforms to the common notion of signal in control systems. 


\section{Sign FUnCTIONS IN CONTROL SYSTEMS}

Next, I briefly discuss the signification potential for some of the interactions shown in Fig. 2. None of these inputs and outputs are completely absent in today's control systems. However, I hope to show how considering the communications depicted in Fig. 2 as significations in a general sense naturally results in a broadening of what we mean by a control system, with the potential for dramatic advances in control technology.

\subsection{Signs referring to states of the controlled system}

Most industrial control systems are configured to expect sensor readings to consist of raw numeric information: temperatures, pressures, flows, forces, etc. Sensor readings are only intended to be signals. There is now increasing interest in the use of unconventional modalities in feedback systems, extending the notion of a sensor in novel ways. Control engineers are actively exploring the integration within control systems of "artificial noses" for fugitive emissions detection, pattern recognition devices for visual quality control, vibration signature analyzers for rotating machinery, etc. However, this exploration is proceeding in an ad-hoc, piecemeal manner that does not recognize the fundamental signification function that these innovations have in common. In most of these cases, the control system may provide the capability for a human to view the sensor output and for a human or a separate stand-alone system to process the sensory information, but this limits the role of the control system to a transmission medium. The video image or vibration spectrum may be a sign for the human, but it is not a sign for today's control system.

The growing use of neural networks and related technologies is also providing a limited semiotic capability to control systems, but much more can be done. A sensor complex that detects the number and distribution of people in a conference room could, in principle, be used for more effective and efficient temperature, humidity, and air quality control. In current systems, realizing these improvements would necessitate one-of-a-kind encoding strategies. In contrast to sensor signals, where (for example) the 4-10 mA standard is universally accepted, there is no standard for representing complex information regarding system state.

These shortcomings can be attributed, not entirely but in large part, to a failure to recognize that control systems can be semiotic entities.

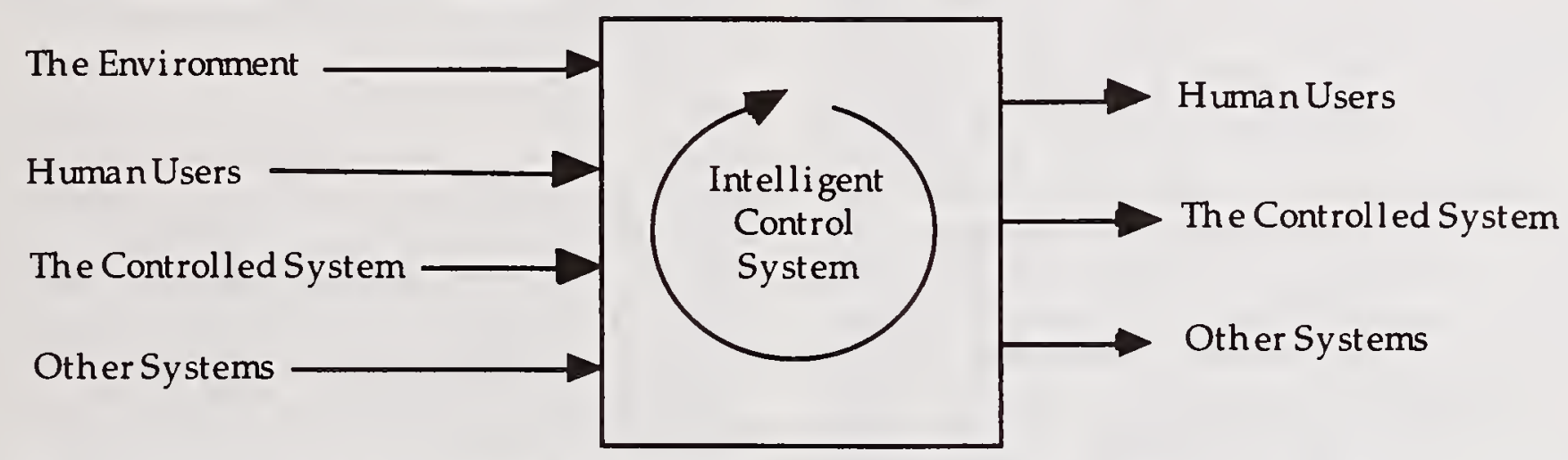

Fig. 2. The roles of signs as input to, output from, and internal to, an intelligent control system. The lighter arrows imply optional significations.

\subsection{Signs from human users}

Another critical aspect of communication to a control system is user commands. Current systems adopt unstructured, flat codes, a limitation that is not problematic for the tasks demanded of them, such as regulating the temperature of a room to a fixed setpoint. But this limitation is incompatible with revolutionary advances in control. In the foreseeable future, we would like to have automatic control systems that can respond to commands such as (for a building cooling system): "Attempt to reduce energy consumption by $10 \%$ without permitting temperatures to rise more than $1^{\circ} \mathrm{C}$ from nominal settings in any zone and without resorting to full lighting setback except in unoccupied areas." The expression need not be in a natural language, although that is a worthy goal, but in either case the intelligence required to effect such commands requires a semiotic sophistication well beyond what current control technology offers, or even seeks to offer.

Other types of signification from users can also be catalogued: commands that relate to controller operation (such as switching from manual to automatic control), the communication of prior knowledge about the controlled system (such as model order or parameter estimates), the communication of application-specific heuristics for control (such as fuzzy rules), etc.

\subsection{Signs referring to controller commands}

The common valve today is restricted in its control input to a stem position command, which it receives every sample and executes with a small actuational delay. Consider, however, a valve that can take as input a schedule of position or position 
change commands. Such a device would enable more efficient utilization of control system resources. Valve technology is not a stumbling block: smart valves with self-diagnostic and self-calibration features are becoming available (adequately dealing with these also requires a sign-function capability). A primary obstacle is the highly restricted code used to communicate the controller output to the valve.

Even today, control systems for industrial applications are hierarchically structured (a multivariable controller will typically generate setpoints for PID controllers). Thus it is already commonplace for the output of a control system to be an input to another control system. The remarks above regarding the signification potential for controller inputs (in particular, signs that refer to users' expectations of the system) suggest that controller outputs should be viewed in the same light.

\subsection{Signs referring to controller structures}

This category hides considerable complexity. For those of us involved in the design and analysis of intelligent control systems, as distinct from their black box operation, it is also the most interesting one.

The key point here is that some sort of modularity is inevitable in a control system of any sophistication. Controllers for large-scale systems will comprise ystem models (often compositional themselves), optimizers, parameter estimators, state estimators, constraint handlers, diagnostic modules, schedulers, planners, performance monitors, adaptation blocks, and many other separable but synergistic. Modularity does not imply a commitment to any specific structure, such as a strict hierarchy. On the contrary, more amorphous compositions may well be preferable. One general way to view an intelligent control system is as an agent architecture, with the agents representing different (although possibly overlapping) functionalities. Communication between agents can occur through shared "blackboards" or by message-passing. This communication must embody a semantic richness that is, in all likelihood, incompatible with representing the input/output parameters of the modules or agents as unstructured numerical variables.

\section{Elements of a SEMIOTIC APPROACH TO INTELLIGENT CONTROL}

Semiotics is not a technology in the sense that it can provide algorithms or devices ready for implementation in intelligent systems. However, as a discipline that focuses on signification and communication in general, it can be considered to subsume aspects of several technologies. In this section, I briefly discuss some of these connections, and their relevance to endowing intelligent control systems with semiotic capabilities.

\subsection{Multiresolution models}

Intelligent controllers need to handle target systems of all scales, from single-loop to system-wide. Representations that are appropriate for this breadth of scope are needed. A naive view may presume that one large "flat" model will serve the purpose, but this strategy is hardly efficient, or even feasible for large-scale systems.

Current control schemes already exhibit some compositionality, with a hierarchy of implicit or explicit models:

- At the single-loop level, PID or (occasionally) more sophisticated controllers are implemented with heuristic knowledge of elementary process dynamics and little consideration of variable couplings.

- Multivariable controllers explicitly take into account the interactions between different components of a small or moderate scale system. In most cases, multivariable controllers provide setpoints for PIDs rather than directly commanding actuators.

- There is now increasing interest in higher levels of control-unit-wide or plant-wide optimization in industrial processes, for example. At these levels, unlike the preceding two, models often attempt to capture nonlinearities, but sacrifice detailed knowledge of dynamics.

Conventional control schemes make little or no attempt to integrate these various control and modeling scales. Ensuring consistency among models of different scales is the task of the human control engineer.

Multiple scales of representation are also required on the temporal level. In most large-scale systems, both external disturbances and intrinsic dynamics will occur over a wide range. Energy consumption in commercial buildings, for example, will show variations that are correlated with a) day/evening, b) weekday/weekend, and c) seasonal cycles. In setting interior lighting levels and lighting setbacks on a daily basis, knowledge of the (seasonal) daylight hours can be used to optimize energy efficiency.

Wavelets are an obvious candidate for a medium that can represent a wide range of temporal and spatial phenomena. A few intriguing research results at incorporating wavelets in control schemes have been reported, and more work in this direction is needed. One limitation of current work in this area is also notable: it assumes that wavelets are inherently a univariate representation scheme, well suited for capturing variations at multiple time scales of a single signal (such as building energy consumption). Their value for representing multi-scale multivariable input-output models is still in question.

\subsection{Fuzzy logic}

A distinguishing feature of intelligent control systems, as distinct from many other intelligent systems, is the requirement that they function both in the symbolic and numerical domains. Fuzzy logic is useful in intelligent control systems in large part measure because it fulfills some part of this requirement. Through the use of fuzzy membership functions, raw numeric data can be translated into 
linguistic labels. Furthermore, fuzzy rules allow heuristic (and symbolic/linguistic) control information gained from human experts to be used for continuous control applications.

These features are often noted from the standpoint of systemhuman communication, but they are also important for intermodule (or inter-agent) communication in intelligent control systems. Reasoning processes in these systems may be symbolic, but, for most control applications, their outcomes will need to be translated into continuous quantities.

The relevance of fuzzy logic to intelligent control is unquestioned, but the current state and directions of this technology have some significant limitations. In particular, popular fuzzy rule formalisms are inconvenient to use for more than small-scale applications. A rule in a fuzzy controller maps conjunctions of sensor readings to a controller output through a simple conditional relationship. More complex rules are rarely considered.

\subsection{Self-organization and learning}

The alternative to some ability for self-organization/ learning/adaptation in a control system is to have every facet of the control scheme manually specified. Whether or not this is seen as a feasible option depends largely on what we mean by learning, or conversely what we mean by manual specification. Current usage betrays some inconsistencies. For example, training a neural network (even off-line) is typically classified as learning, whereas linear system identification is not.

It is neither accurate nor helpful for promoting intelligent control to view learning and adaptation as capabilities that control systems either do or do not exhibit. Rather, there is a spectrum of possibilities. Towards the simpler end of this spectrum, the widespread use of system identification, gain scheduling, and even feedback linearization demonstrates the feasibility of learning and adaptation in some form. The issue then is not whether to include learning capabilities in future control systems, but how to increase their learning capabilities in order to reduce the level of manual effort currently involved in control design, system modeling, and controller modifications in the face of unexpected variations. It is inconceivable that human involvement can be completely dispensed with, just as it is inconceivable that our current control technology would have progressed to the point it has without some element of learning.

In intelligent systems research, the interest in learning has been predominantly geared toward improving the performance of individual modules in the system. Learning in a semiotic system has another important implication: the generation of new communication and signification capabilities. Thus languages for inter-agent communication can be evolved to allow the communication of appropriate information in a sufficiently expressive yet easily interpreted form.

\subsection{Computational linguistics}

As we broaden our view of control, a fundamental insight to contemplate is that human users of control systems need to be part of the picture. The importance of human-system interaction increases in proportion to the functionality of the control system. Further, different classes of users must be considered, including operators, control engineers, maintenance supervisors, and others.

Effective human/control-system interaction requires appropriate communication mechanisms. The control community's view of user communication needs has generally been limited to graphical and tabular displays of numeric data. These are sufficient for most low-level control functions, but not for higher levels. Commands from users at these levels, for example, will not be setpoint changes but more complex instructions which may be most naturally expressed in some limited linguistic form (as illustrated in Section 3.2).

Current research in theoretical linguistics is largely in the Chomskyan generative tradition. The hard distinctions drawn in this research, such as competence versus performance and syntax versus semantics, with the subject of linguistic study limited to syntactic competence, limits its direct relevance to engineering systems. The discipline of computational linguistics, however, addresses all aspects of linguistic signs and signification-syntactic, semantic, and pragmatic. Computational linguistics exploits insights from modern linguistics, along with developments in less analytic approaches (e.g., statistical and connectionist natural language processing), and shows considerable promise for the development of effective language understanding and generation systems for focused applications.

The immediate relevance of computational linguistics to control systems is in the pursuit of more effective and efficient human-system interaction. However, there is another intriguing connection. As noted earlier, an intelligent control system will not be an undifferentiated block of software, but will be composed of numerous subsystems many of which will exhibit some aspects of intelligent behavior. Natural language is the preferred means of human communication because it has evolved to satisfy the communication needs between individual intelligent beings. As control systems increase in sophistication, functionality, complexity (and intelligence), their component modules will too. Parameter or signal vectors will not provide an adequate communication mechanism between these modules. A full-fledged natural language capability is neither required nor desirable, but the discipline of computational linguistics has much to offer towards devising a more limited language, one that is qualitatively richer than current mechanisms.

\subsection{Classical control}

Neither humans nor intelligent controllers are directly responsible for the control of today's aircraft, manufacturing plants, automobiles, or any of the other domains that represent the true successes of control. The credit, in large part, goes to our classical control technology. Intelligent control must build on this proven foundation, and it must provide the framework for classical control techniques to continue to serve their many useful purposes. As we discuss the need for semiotically-sophisticated control, and for new 
codes and signification capabilities, it is important to remember that such semiotic capabilities as are incorporated in current control systems must be maintained. Even as we suggest the need for linguistic communication, for example, at some level of an intelligent controller there will still be a need to communicate linear transfer function matrices. Analogously, the imprecision of fuzzy logic can supplement, but it cannot supplant, the expression of model uncertainty in the $\mu$ framework.

\section{CONCLUDING REMARKS}

A matter of definition lurks behind the scene in this paper: is the broader vision of control sketched above overstepping the boundaries of the discipline? In fact, the vision of control has always been a broad, overarching one. Any introductory control textbook will first present some examples of target systems for control technology, and these can encompass national economies, global ecologies, and similarly largescale, complex domains (see Fig. 3). Unfortunately, the logic of control often belies its rhetoric. A semiotic perspective is fully compatible with the classical vision of control, even if the practice of control science and engineering shows little evidence of this connection.
Although in this paper I have focused on the role of semiotics in intelligent control systems, I have attempted neither to define intelligent control nor to separate it from any other adjectival variation-definitions of intelligence in any context are of problematic value. I propose not a definition but a guiding principle: the degree of intelligence in a control system is in direct proportion to the sophistication of its semiotic character.

Acknowledgement: I am grateful to Hannah Blau for review and criticism.

\section{REFERENCES}

Deely, J. (1990). Basics of Semiotics. Bloomington, IN: Indiana Univesity Press.

Eco, U. (1976). A Theory of Semiotics. Bloomington, IN: Indiana Univesity Press.

Eco, U. (1984). Semiotics and the Philosophy of Language. Bloomington, IN: Indiana Univesity Press.

Sebeok, T.A. (1994). Signs: An Introduction to Semiotics. Toronto, Canada: University of Toronto Press.
Fig. 3. Visions of Control. From Richard C. Dorf, Modern Control Systems, Addison-Wesley, 1974. As adapted from The Limits to Growth: A Report for the Club of Rome's Project on the Predicament of Mankind, 2nd ed., D.H. Meadows et al. New York: Universe Books, 1972. Used with permission of Potomac Associates, Washington, D.C.

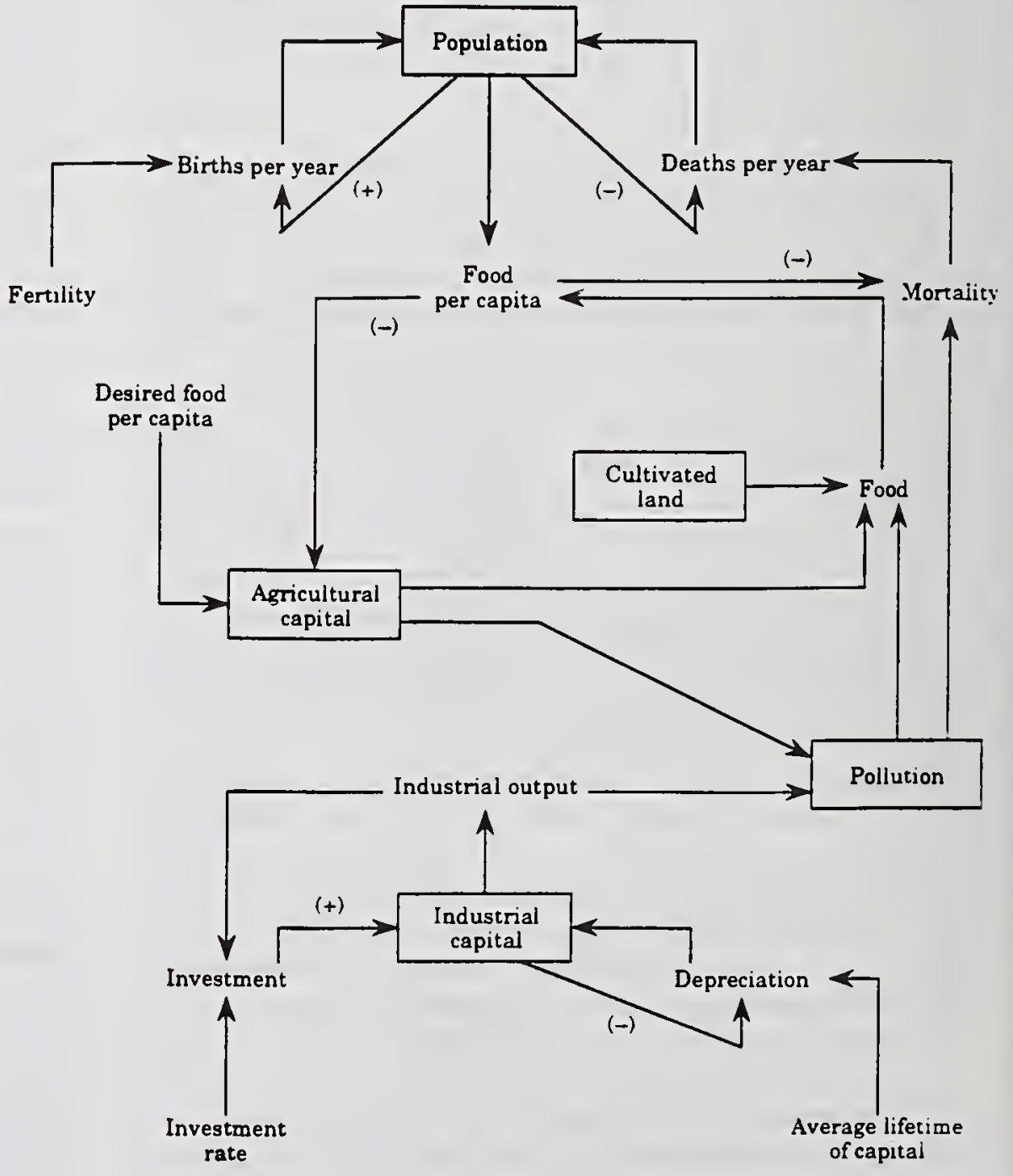




\title{
INTELLIGENT VS REFLEX CONTROL FOR COMPLEX DYNAMICAL SYSTEMS
}

\author{
Michel Cotsaftis, Professor \\ LTME/ECE, 53, Rue de Grenelle, 75007 Paris, France \\ email : mcot@ece.fr
}

\begin{abstract}
Direct consequence of systems complexity increase is more complicated, less observable and less controllable trajectory in phase space in a classical sense. Control adequacy should imply more freedom in decision process to balance system modeling unpreciseness and inadapted information flux handling when too much centralized. More global approach than classical state space is now required to fit reality of larger trajectory wandering and their natural organization along invariants in phase space inside which they become undistinguishable. Most elementary is function space approach, where the "unit" is a whole trajectory, and control problem becomes embedding system solution into initially prescribed function space from asymptotic stability and monotonicity conditions. This "functional" control strongly reduces information fluxes up and down inside system structure by taking advantage of internal system selforganization consecutive to complexification. Hierarchical structure is here satisfied within subsidiarity principle, by delegation of more "intelligence" toward lower levels and by leaving more freedom for decisional processes at higher levels where fuzzy controllers are mostly adapted, opening the way to intelligent selfdeciding systems.
\end{abstract}

KEYWORDS: Complex System, Intelligent Control, Stability, Functional Control

\section{INTRODUCTION}

To accomplish more complex technical tasks with more complex systems[1] as required in modern industries to survive in world spreadout economy, one might rely upon technology advance of the various system components and augment in parallel control complexity with larger sensor information. Independent of failure increase with all its consequences, this approach is different from existing living systems, exhibiting remarkable ability to decide and to perform extremely complex tasks with their complex "living" machine. This suggests that another less strictly computerized and more qualitative way is possible, and some elements in this direction are given in the following. For Lagrangian dynamical systems, classical PD-type con- trollers locally give system trajectories a stable fo- custype structure guaranteeing trajectory asymptotic stability. For more complicated systems, concepts of "adaptiveness" [2] and of "robustness" [3] have been analyzed. They can be represented with symbolic "co- ordinates" (system complexity-performance level) as arrows along a xisses from initial simple PID control domain, see Fig.1. Adaptiveness is asymptotically stable by construction, but at some level system complexity and performance analysis cannot be handled together in real time, leading to a tradeoff. In robustness approach, simple stability resulting from system simplification forbids higher preciseness required for more advance performance quality, so that both extensions are mainly localized along the two axisses. On the other hand, as elementary performance is not worth from a complex system, nor high level performance is usually output from a simple system, real system representative point should stay inside a band along the first diagonal.

This means that both previous approaches are suffering from the defect of not changing system structure which cannot remain too centralized beyond some level of complexity. Improvement of information flux circulation requires more delegation of decisional capability to system parts themselves with system complexity increase, ie to subsidiase more intelligence into local controls which become of reflex type, so that upward information flux is strongly reduced for higher level controllers to deal with affordable amount of material. Here Fuzzy controllers[4] are singled out as they would provide very adequate relations between quantitative description at system dynamics level and qualitative representation at decision intelligent higher level. So as they split off the diagonal band, previous control approaches are not adapted for extension to intelligent higher controls due to the gap between their (too much detailed) representative variables and the (more global) ones adapted to intelligent controls, leading to unaffordable explosion of logical rules with system complexity from combinatorics.

Convergence toward the diagonal band is nevertheless possible, as living systems are showing it everyday by changing the nature of control from trajectory space to task space, which is made possible by the huge experience coll- 
ected by each individual. For complex mechanical or technical systems, it is also possible to converge toward the diagonal band with a new type of control, called functional control[5], based on intermediate "natural" variables filling this gap, and with which a multilevel control allowing integration of decisional functions into system control can be constructed. The main idea is that when system complexity increases, its trajectory in phase space becomes more complicated, but at the same time the information content in its observation decreases, because trajectories are becoming less distinguishable, ie are becoming equivalent in a dynamic sense. They are filling more and more regularly domains in phase space, and initial conditions (local invariants) cannot be used as before to define a trajectory. Only more global invariants[6] can be used to classify trajectories in groups within which single trajectories cannot be distinguished anymore. Examples are for instance magnetic field lines which are not distinguishable on their flux surfaces, and extreme case is a gas in thermodynamics the trajectories of which on energy surface are all equivallent. Another quite frequent situation occurs when, due to nonlinearities, there exists bifurcation points at which system dynamics branch off toward more complicated trajectories up to chaotic ones[7]. In all cases, invariants are no longer initial trajectory coordinates as for classical mechaical problem, but the corresponding invariants, for instance flux surface for magnetic field and energy surface for gas. Here part of trajectory behavior escapes from direct control in the sense that because the number of DOF of state vector is much larger than control vector, system dynamics organize themselves on invariant surfaces. It is interesting to note that in this case, state vector may still be observed, but all its components are not controllable. In consequence control problem transforms into acting more globally onto system trajectory as a whole rather than correcting it locally, and to impose the solution of system dynamics to belong to a prescribed function space, chosen from general desired properties in their functional behavior (overshoot, decay, monotonicity,..), and compatibility with system dynamical behaviors which should belong to this function space. This is the only way to take advantage of system selforganization resulting from its complexification, and to eliminate risk of antagonizing this selforganization by inadequate control. The resulting "embedding" problem fits in to Fixed Point Theorem[8] framework, and asymptotic stability toward desired trajectory may be guaranteed.

To technically deal with system dynamics complication and difficulty of correct physical phenomena representation, an efficient way would be to combine both advantages of functional and robust controls, ie asymptotic stability and simplicity. From analysis of regular robustness approach, simple stability is due to narrowness of allowed function space when applying Lyapounov Theorem, and forbids application of Fixed Point Theorem, whereas adequate function space enlargement leads to asymptotic stability property without requiring extra system information. In next part reduction of dynamical system equations to canonical form is given. Structure of classical robust control is analyzed afterward with its inherent defects, and improved robust control is explicitely constructed. Resulting asymptotic robust control is of simple form and is easily implementable. In contrast, fuzzy control law, even when analytically explicited in terms of system variables to save basic combinatorics computation, requires their approximate evaluation, making the approach unusable at this detailed level for complex large dimension system. Application to $\mathrm{N}$-link actuated compliant and deformable mechanical system is given as an example.

\section{CANONICAL ERROR EQUATIONS}

Let for $t>t_{0}$ the dynamical system

$$
\frac{d X}{d t}=F(X, t, U)
$$

with state vector $X=\operatorname{col}\left(X_{1}, X_{2}, . . X_{n}\right.$ and control vector $U . F(., .,$.$) is supposed smooth enough to appropriate$ order in its arguments so that a solution $X=X\left(t, X_{0}\right)$ exists for a fixed $U=U_{f}(t)$ with initial condition $X_{0}=$ $X\left(t_{0}\right)$, and with possible different behaviors for $t \rightarrow \infty$ for different $X_{0}$. For a fixed $X=X_{d}(t)$, there conversely exists for $U$ one (or more) solution of eqn(1) $U_{d}=$ $F^{-1}\left(X_{d}, d X_{d} / d t, t\right)$, so in principle $U$ may be determined for eqn(1) to have a solution behaving in a prescribed way. System trajectories close to $X_{d}(t)$ have to be analyzed to determine the conditions for their convergence to $X_{d}(t)$ for large $t$. To deal with in not well known functional dependence of $F(., t,$.$) in real cases, much simpler structural$ stability analysis is called for. To proceed, the RHS of eqn(1) is splitted into a "simple" part for which a control exists guaranteeing asymptotic convergence, and a "rest" with upper bounded effect. An equivalence class with respect to this "rest effect" is realized if an additional control exists giving also the complete system asymptotic convergence property, thus reducing the analysis to "skeleton" systems out of which these equivalence classes are constructed. This will be called a canonical reduction.

With $X=X_{d}+x, U=U_{d}+u$, variational error system from eqn(1) is

$$
\frac{d x}{d t}=A_{0} \cdot x+B \cdot\left(u_{0}+\Delta u\right)+\eta
$$

splitting variational control $u$ into $u_{0}$ guaranteeing as- ymptotic convergence of "skeleton" equation with $\eta=0$, and additional part $\Delta u$ to annihilate the action of extra term $\eta$ containing both nonlinear and error terms when passing from eqn(1) to eqn(2). Eqn(2) is useful if there exists for skeleton equation a P-type, possibly time dependent, 
control such that, with $u_{0}=-K x$, there exist positive definite matrices $(P, Q)$ satisfying $\left(A_{0}^{T}+K^{T} B^{T}\right) P+P\left(A_{0}+\right.$ $B K)+d P / d t=-Q$ and Lyapounov stability theorem applies with $V=x^{T} P x, d V / d t=-x^{T} Q x . \Delta u$ should now be found such that with extra term $\eta$, asymptotic convergence property is maintained for eqn(2).

\section{IMPROVED ROBUST CONTROL ANALYSIS}

Derivative of $V=x^{T} P x$ along trajectories of eqn(2) writes

$$
\frac{d V}{d t}=-x^{T} Q x+2\left(B^{T} P x\right)^{T} \Delta u+2(P x)^{T} \eta
$$

With the bound $|\eta| \leq \rho>0$, not specified for the moment, usual robust method is defined by additional control[9]

$$
\Delta u_{R}=-\rho\left(B^{T} B\right)^{-1} B^{T} P x \cdot \frac{1}{[\operatorname{Sup}(\epsilon,\|P x\|)]}
$$

where $\epsilon$ is small $>0$, leading to the bound

$$
\frac{d V}{d t} \leq-2 y\left[\frac{\lambda_{\min }\left(P^{-T} Q P^{-1}\right)}{2} y-\rho_{L}\left(1-\frac{y}{\epsilon}\right)\right]
$$

with $y=\|P x\|, \rho_{L}(y)=S u p_{t} \rho(y, t)$, and $\lambda_{\min }=M i n_{j, t} \lambda_{j}$ $(t)$. For bounded $\rho_{L}$ with $\rho_{L}(0) \neq 0$, there exists $y=y_{m}$ below which $d V / d t$ becomes positive, defining an attractor inside the ball $x_{m}=P^{-1} y_{m}$ [7]. All trajectories will hit its surface after a finite time, and remain captured inside for larger time. Its size is small for $\epsilon$ small and $\lambda_{\min }(Q)$ large, ie for large gains. So with usual robustness approach, initial system uncertainty due to nonzero norm bounded terms $\eta$ cannot be completely removed and leads to state uncertainty inside a finite ball $\left\|x_{m}\right\|$ on system error dynamics, giving simple stability result after a first exponential decay period. Due to conservativeness of sufficient stability condition in Lyapounov method this decay cannot be satisfied for all time. Also, for $\left(\Delta U_{R A}=0\right)$ and $\eta \neq 0$, one gets $d V / d t \leq-x^{T} Q x+2 \rho\|P x\| \leq-\lambda V+2 k \rho_{L} V^{1 / 2}$ with $\lambda$ and $k$ from bounds on eigenvalues of $P$ and $Q$. $V$ is upper bounded by $t=\int_{Y_{0}^{1 / 2}}^{Y^{1 / 2}}\left[k \rho_{L}\left(x^{2}\right)-(\lambda / 2) x\right]^{-1} d x$ with exponential behavior $\Delta x \simeq \exp \left(2 k x_{m} \rho_{L m}^{\prime}-\lambda / 2\right) t$ close to a zero $x_{m}$ of integrand denominator, stable or unstable depending on the sign of coefficient of $t$. Final behavior of $Y(t)$ depends on the number of possible $x_{m}$. Thus classical robust control only changes the size of induced uncertainty ball, but not its existence.

Less conservative condition follows from using different additional control algebraic form. Instead of $\Delta u_{R}$ from eqn(4), let

$$
\Delta u_{R A}=-\frac{\rho\left(B^{T} B\right)^{-1} B^{T} P x}{\alpha\|P x\|+\epsilon f(t)}
$$

with $\alpha<1$, and "driving" function $f(t)$ to be discussed later. $V(t)$ is bounded above by the solution of majorant equation

$$
\frac{d Y}{d t}=-\lambda Y+2 \frac{\epsilon}{\alpha} \rho f(t)
$$

where $f(t)$ is determined such that, for given functional form of $\rho(Y, t)$, the solution $Y(t)$ of eqn(7) exhibits a prescribed time dependence $Y_{d}(t)$. Note that with $f(t)$ much larger freedom exists now than in eqn(5) with only number $\epsilon$, and simple calculation would give $f(t)$ for prescribed $Y_{d}(t)$ from eqn $(7)$. However it is more appropriate in ro bustness context to solve an embedding problem where a correspondance is researched between function spaces $\mathcal{F}$ and $\mathcal{Y}$ for various possible functional forms of $\rho(Y, t)$, with $f(t) \in \mathcal{F}$ and $Y \in \mathcal{Y}$, and where $\mathcal{Y}$ is fixed by general properties such as continuity and behavior (decay) for large $t$. Usual approach is to determine $\mathcal{Y}$ from $\mathcal{F}$ and $\rho(Y, t)$ via Fixed Point Theorem. Specific applications are obtained when $\mathcal{Y}$ is usual Lebesgue $\mathcal{L}^{p}$ or Sobolev $\mathcal{W}_{k}^{p}$ space and $\rho(Y, t)$ ranges in a similar space, as when $\rho(Y(t), t)$ satisfies Caratheodory condition[10]

$$
\rho(Y(t), t) \leq a(t)+b(t) Y(t)^{p}
$$

with $a(t)$ and $b(t)$ in adapted spaces, by use of Holder inequality onto eqn $(7)$ to determine the order of $\mathcal{F}$, ie the power $q$ in $f(t)=k_{1} Y(t)^{q}$, chosen to eliminate control explicit time dependence. Eqn(8) follows from thermodynamic properties in natural systems, and is obtained for systems with real, physical or technical, origin. Asymptotic stability is now obtained for a large class of functions $\rho(Y(t), t)$, in contrast to previous robust result, but with decay weaker than exponential.

When inequality $\rho(Y(t) \exp \mu t, t) \leq \bar{\rho}(Y(t)) h(t)$ holds with nondecreasing function $\bar{\rho}($.$) , one gets$

$$
Y(t) \leq \exp -\mu t \cdot G^{-1}\left(G\left(Y_{0}\right)+2 \operatorname{sgn}(G) I(t)\right)
$$

with $I(t)=(\epsilon / \alpha) \int_{0}^{t} \exp \mu t^{\prime} f\left(t^{\prime}\right) h\left(t^{\prime}\right) d t^{\prime}$ where $G(x)=$ $\int^{x} d v / \bar{\rho}(v)$. When $G($.$) is sublinear, \operatorname{sgn}(G())>$.0 , and $Y(t)$ is defined for any $[Y(0), t]$, whereas if $G($.$) is super-$ linear, $\operatorname{sgn}(G())<$.0 , and $Y(t)$ is only defined for $Y(0) \in$ $\left[0, Y_{c}\right]$ such that the argument of $G^{-1}$ does not change sign for $t<+\infty$, ie $G\left(Y_{0}\right)=2 \frac{\epsilon}{\alpha} \int_{0}^{\infty} \exp \mu t^{\prime} f\left(t^{\prime}\right) h\left(t^{\prime}\right) d t^{\prime}$ in order to avoid finite time Lagrange instability. Here $Y(t)$ may decay faster than exponential but in a limited ball of initial conditions depending on $f(t)$. When $\mu=0, h(t)=1, Y(t)$ in eqn(7) is bounded by $\int_{Y_{0}(t)}^{Y(t)}\left[u-L \bar{\rho}(u) u^{q}\right]^{-1} d u=-\lambda t$ in implicit form with $L=\left(2 \epsilon k_{1} / \alpha \lambda\right)$, and decays asymptotically to 0 for large $t$ under the simple explicit conditions

$$
q>1>L \bar{\rho}\left(Y_{0}\right) Y_{0}^{q-1}
$$


relating system and control parameters for given initial ball $Y_{0}$, best satisfied for large linear gains and small initial ball. In all cases, with control of generic canonical "triplet" form $U=U_{d}+u_{0}+\Delta u$, asymptotic stability can be found in conjunction with robustness constraint, in contrast to classical approach, and the role of $f(t)$ which drives the behavior of $Y(t)$ is clearly shown.

\section{EXPLICIT FUZZY CONTROL}

To escape from detailed dynamical system description another way would be to set conditional "linguistic" statements in the form of if-then rules[11] allowing to directly pass to generic descriptive representation of system trajectories in geometric space. However this is only possible when their qualitative nature is already known, which rests upon the correspondance between the minimum number of rules in rule space $(\mathcal{R})$ and types of trajectories in geometric space $(\mathcal{T})$, only known in very few cases, despite a global relation from universal approximator property[12]. One case corresponds to simple "driving car" rules[13], and applies for focus-type (or node-type) trajectories, produced at least locally by lagrangian equations[14]. Again in this case, combinatorics explosion for large enough dimension number renders this approach useless, indicating that the variables on which control method is applied are not good ones because too much detailed, suggesting direct search of explicit final defuzzyfied control law to avoid all intermediate steps.

Consider for simplification fuzzyfication under translationally invariant membership functions $f_{i}(x)=f\left(x-i \Delta_{1}\right)$ over normalized interval and such that $f_{i-1}\left(\Delta_{1}\right)$

$=1, f_{i}\left(\Delta_{1}\right)=0$, guaranteeing always only two nonzero membership values $m_{i-1}$ and $m_{i}$ for any $0 \leq x \leq$ $\Delta_{1}$, see Fig.2. Similarly after usual max-min compositional rules of inference, defuzzyfication by centroid method with translationally invariant output membership functions $g_{j}(y)=g\left(y-j \Delta_{2}\right)$ gives analytic crisp output control $u=u_{P D}+\Delta u_{F U Z}$ with usual PD part $u_{P D}=1 / 2\left[y_{k-1}+\right.$ $\left.y_{k}\right]$ in normalized form and fuzzy contribution $\Delta u_{F U Z}=$ $\left(\Delta_{2} / 2\right)\left(1+\mathcal{A}_{k-1} / \mathcal{A}_{k}\right)^{-1}$ with domains $\mathcal{A}_{k-1}$ and $\mathcal{A}_{k}$ shown on Fig. 2 and

$$
\frac{\mathcal{A}_{k-1}}{\mathcal{A}_{k}}=\frac{m_{1}\left[z_{1}+\operatorname{Inf}\left(y_{0}, z_{1}\right)+2 \operatorname{Sup}\left(I_{01}, 0\right)\right]}{I_{12}+\left.z g\left(\Delta_{2} y\right)\right|_{y_{1}} ^{y_{2}}}
$$

renormalizing $y$ to $\Delta_{2}$, with $y\left(m_{j}\right)=y_{j}, z_{j}=1-y_{j}$, $I_{j k}=\int_{y_{j}}^{y_{k}} g\left(\Delta_{2} y\right.$, and intersection point $y_{0}=1 / 2$ between two contiguous membership functions corresponding to $g\left(\Delta_{2} y_{0}\right)=m_{0}$ and $g\left(\Delta_{2} y\left(m_{k}\right)\right)=g\left(\Delta_{2}\right) m_{k}$ writing $m_{k-1}=m_{1}, m_{k}=m_{2}$ with translational invariance property of membership functions. Note that $F_{k}\left(m_{k}, 0\right)=1$ and $F_{k}(m, m)=0$. With the constraint $m_{k-1}+m_{k}=$ $m_{1}+m_{2}=1$, and $f\left(\Delta_{1} x\right)=m$ from input with normalized $x, F_{k}(1-m, m)$ can be thoroughly studied for $m \in[0,1 / 2]$ with slopes $-2\left(\int_{0}^{1} g\left(\Delta_{2} t\right) d t\right)^{-1}$ at $m=0$ and
0 at $m=1 / 2$. With for instance generic membership function $g(y)=y^{\alpha}$ and $\alpha \geq 0$, the steepening increases with $\alpha$ ie with pitching.

So one may only use the final explicit control expression in eqn(11) once system variables are properly located in their membership domains. This only requires inverse functions $X=f^{-1}(m)$ and $Y=g^{-1}(m)$, but the problem still lies in evaluation of all system coordinates with flags when crossing membership domain. Here the advantage in unpreciseness is unfavorably balanced by the number of variables to treat, showing again that high quality of fuzzy controller is not fully exploited at this too detailed description level, as compared to very global and limited knowledge required in robust control given in eqn(6).

\section{APPLICATION TO N-LINK MECHA NICAL SYSTEM}

Canonical error equations for actuated mechanical systems with compliance at joints and flexion and torsion link deformations can be exactly cast in to the form[15] of eqn(2) with $x=\operatorname{col}(e, d e / d t, Z, d Z / d t), \eta=\operatorname{col}\left(\eta_{1}\right.$,

$\left.\eta_{2}\right), \Delta u=\operatorname{col}\left(\Delta u_{0}, \Delta u_{1}\right), A=A_{0}-B K$ and error terms with regressors associated to both system parameters and deformations parameters and obtained as difference between exact and estimated quantities in initial system. Application of control $\Delta u_{F U Z}$ is only possible here if all elements in column vector $x$ are evaluated which is difficult for the last component. On the contrary, from analysis of previous expressions one gets the bound $\left\|\eta_{j}\right\| \leq$ $\sum_{\lambda} A_{j \lambda}\left\|X_{\lambda}\right\|+B_{j}=\rho_{j}$ of Caratheodory form allowing direct application of eqn(10). With $f(t)=k\|X\|^{q}$ asymptotic stability of $\|X\|(t)$ is obtained if the conditions

$$
q \geq 1, \quad 1>L(1+\beta\|X(0)\|)\|X(0)\|^{q-1}
$$

are satisfied by proper choice of parameters $\alpha, \epsilon, k, q$ with $L=2 \epsilon a k / \lambda \alpha, \beta=b / a$, and $a, b>0$ obtained from $A_{j}, B_{j}$ and supposed constant in time with appropriate upperbounding. So for $N$-link mechanical system a large class of decaying driving functions is found, which monitor system decay guaranteeing both robustness and asymptotic stability with only knowledge of basic skeleton parameters and error ball for uncorrectly estimated parameters, in contrast to usual robust analysis showing simple stability property, and to other control approaches requiring more detailed knowledge of system parameters. Present better result is due to taking full advantage of Caratheodory type constraint and to less stringent conditions on the majorant of error vector norm, used as a Lyapounov function. Lyapounov conditions for asymptotic stability are not satisfied, but the majorant still fulfills (weaker) conditions leading to asymptotic (not necessarily exponential) decay. Numerical application to a 3-dof is given in [16].

\section{CONCLUSION}


Error equations for dynamical system equations are canonically reduced to a generic form composed of a (usually linearized) part coming from linear known terms, and a residual nonlinear one coming from unavoidable mismatch between system representation and its actual (unknown) form, both at parametric level and at functional form of functions modeling physical phenomena (friction, contact interactions). Control should give for solution of error equations best convergence to origin. A first component from linearized part is of PD type, usually giving locally weakly damped oscillatory behavior from power limitations restricting allowed gains. Resulting asymptotic convergence is not structurally stable because residual part, when norm-bounded, leads to simple stability of error system solution. So an additional controller is needed for further improvement. Simplest form is a robust one requiring the knowledge of norm-bound, classically leading to simple stability result because of unmaintainable condition for all time that error norm vector is majorized by exponentially decaying function.

New form of robust controller allowing larger functional space results from introduction of a driving function $f(t)$, whose proper design guarantees asymptotic decay depending on functional form of norm bound, even when Lyapou nov conditions are not satisfied. The controller combines simplicity of previous conventional robust controller requiring only information on norm bound of residual terms, with efficiency and preciseness of trajectory tracking by maintaining structurally stable asymptotic convergence. So here results are in real systems obtained at lowest level of mechanical structure. Contrary to fuzzy controller even in explicit final form after application of logical rules demanding evaluation of complete system state vector, very few informations are required in new proposed robust controller, so its description at higher level is extremely simplified, easing the role of higher loop decisional fuzzy type adapted controllers[14].

\section{REFERENCES}

[1]-B. Cohen, W.T. Harwood, M.I. Jackson : The Specification of Complex Systems, Addison-Wesley, N-Y, 1986; M. Gell-Mann : What is Complexity, Complexity, Vol.1(1) p.16, 1995; H. Morowitz : The Emergence of Complexity, ibid. p.4; G. Nicolis, I. Prigogine : A la Rencontre du Complexe, PUF, Paris, 1992

[2]-J.J. Craig : Adaptive Control of Mechanical Manipulators, Addison-Wesley Pub., Reading, 1987; S. Sastry, M. Bodson : Adaptive Control, Stability Convergence and
Robustness, Prentice-Hall, Englewood-Cliffs, N.J., 1989 [3]-M.W. Spong, M. Vidyasagar : Robot Dynamics and Control, J. Wiley and Sons, New-York, 1989

[4]-C.C. Lee : Fuzzy Logic in Control Systems : Fuzzy Logic Controllers, I,II, IEEE Transac. on SMC, Vol.20 p.404,435, 1990; R.R. Yager, D.P. Filev : Essential of Fuzzy Modeling and Control, Wiley and Sons, N-Y, 1994; L.X. Wang : Adaptive Fuzzy Systems and Control, PTR Prentice-Hall, Englewoods-Cliffs, N.J., 1994

[5]-M. Cotsaftis : Functional Control of N-Link Deformable Mechanical Systems, Proc. 1rst MOVIC Conf., Yokohama, Sept.7-11, 1992

[6]-H. Poincaré : Les Méthodes Nouvelles de la Mécani que Céleste, Gauthier-Villars, Paris, 1897, 1899; M. Hirsch, C. Pugh, M. Shub : Invariant Manifolds, Lecture Notes in Math. 583, Springer-Verlag, Berlin, 1977

[7]-T.S. Parker, L.O. Chua : Chaos, a Tutorial for Engineers, Proc. IEEE, Vol.75(8), p.982, 1987; S.H. Strogatz : Nonlinear Dynamics and Chaos with Applications to Physics, Biology, Chemistry and Engineering, AddisonWesley, N.Y., 1994; E. Ott : Chaos in Dynamical Systems, Cambridge University Press, Cambridge, Mass. 1993

[8]-E. Zeidler : Nonlinear Functional Analysis and its Applications : Tome I, Springer-Verlag, New-York, 1986

[9]-G. Leitman : On the Efficacy of Nonlinear Control in Uncertain Linear Systems, J. Dyn. Syst. Meas. Control, Vol.103 (1981) p.95

[10]-J. Appell, P.P. Zabrijko : Nonlinear Superposition Operators, Cambridge University Press, Cambridge, Mass. 1990

[11]-R.R. Yager, D.P. Filev : Essentials of Fuzzy Modeling and Control, Wiley ans Sons, New-York, 1994

[12]-L.X. Wang : Adaptive Fuzzy Systems and Control, Prentice-Hall Inc., New-York, 1994

[13]-M. Sugeno : Industrial Applications of Fuzzy Control, North Holland, Amsterdam (1985)

[14]-S. Lefschetz : Stability of Nonlinear Control Systems, Acad. Press, New-York (1965)

[15]-M. Cotsaftis : Global Control of Deformable N-Link Mechanical Systems, Proc Winter Annual Meeting ASME New-Orleans, Louisiana, Nov.28-Dec.3, 1993

[16]-M. Cotsaftis : Asymptotic Robust Control for Mechanical Systems, Proc. 1994 Japan-USA Symposium on Flexible Automation, Kobe, Japan, July 11-18, 1994, Vol.II, p.671

[17]-M. Cotsaftis : Intelligent Control for Complex Systems, Proc. 1993 IEEE Intern. Conf. on Robotics and Automation, Atlanta, Georgia, Vol.II, p.75, 1993 


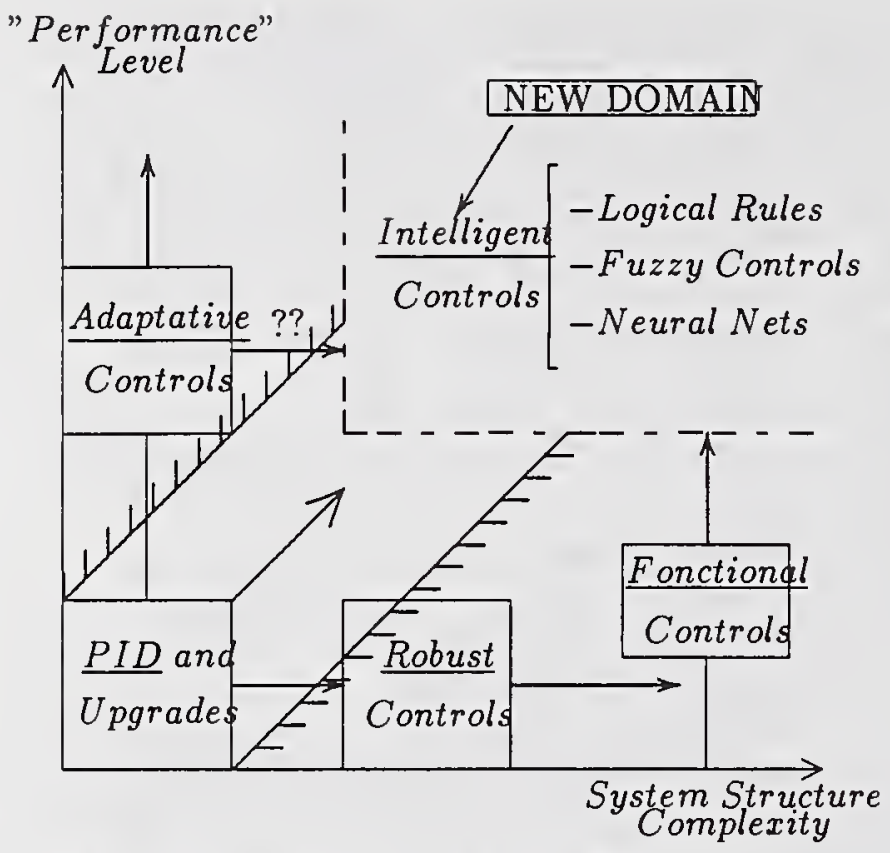

Fig.1 : System Performance vs Structure Complexity with Various Controls

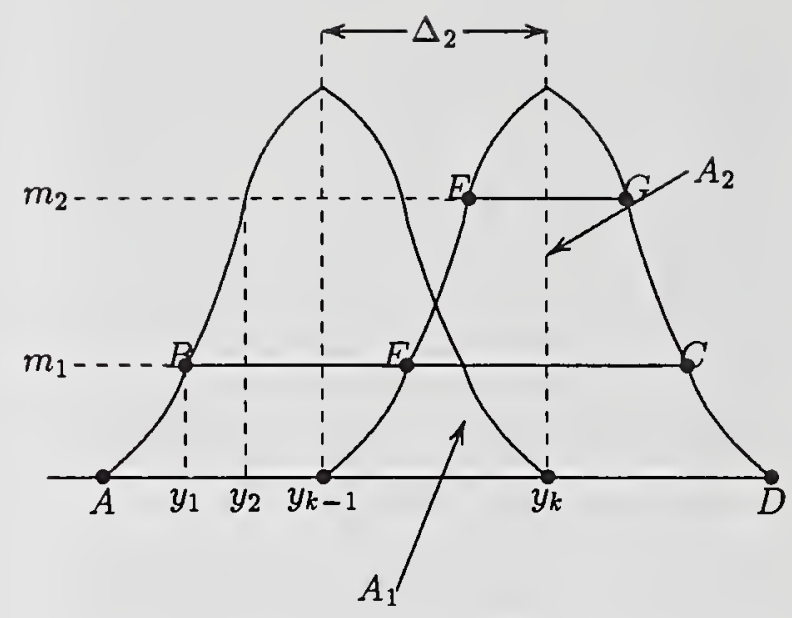

Fig.2 : Membership Functions and Areas $\mathcal{A}_{1}=$ $\operatorname{Domain}(A, B, C, D), \mathcal{A}_{2}=\operatorname{Domain}(E, F, G, C)$ 


\title{
Introduction to Integrated Learning/Planning Paradigm
}

\author{
A. Meystel ${ }^{*}$, A. Lacaze L $^{\text {to }}$ \\ "Drexel University \\ ${ }^{\dagger}$ University of Maryland \\ ${ }^{\circ}$ National Institute of Standards and Technology, Intelligent Systems Division
}

\begin{abstract}
.
This paper is an effort to address the issues of planning and learning as two components of the same process of intelligent system functioning.

Key words: behavior, goal, path, planning, redundancy, resolution, representation, tessellatum, trajectory
\end{abstract}

\section{Planning Problems of Behavior Generation}

Behavior Generation [1] can have many mechanisms of planning and execution. At the present time, these mechanisms cannot be considered as thoroughly known, and the general theory of planning can hardly be attempted. We will discuss a subset of problems in which the goal is defined as attainment of a particular state. Other types of problems can also be imagined: in chess the goal is clear (to win) but this goal cannot be achieved by achieving a particular position in a space (even in a descriptive space.) Most of the problems related to the theory of games and linked with pursuit and evasion are characterized by a similar predicament and are not discussed here.

Planning is understood as searching for appropriate future trajectories of motion leading to the goal. Searching is performed within the system of representation.

\section{Planning in a Representation Space with a Given Goal}

The world is assumed to be judged upon by using its Space of Representation which is interpreted as a vector space with a number of properties. Any activity (motion) in the World (Space of Representation) can be characterized by a trajectory of motion along which the "working point" or "present state" (PS) is traversing this space from one point (initial, or state, IS) to one or many other states (goal states, GS.) The goal states are given initially from the external source as a "goal region", or a "goal subspace" in which the goal state is not completely defined in a general case. One of the stages of planning (often the initial one) is defining where exactly is the GS within the "goal region." In this paper, we will focus upon planning problems in which one or many GS remain unchanged through all period of their achievement. Traversing from IS to GS is associated with consuming time, or another commodity (cost.)

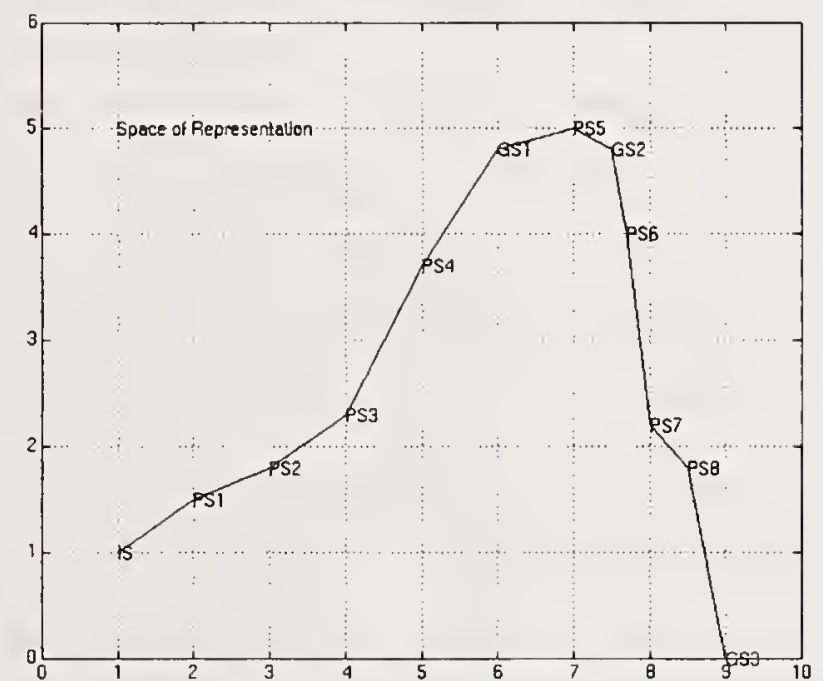

Figure 1. The general paradigm of planning

\section{Learnable Representations}

All Representation Spaces are acquired from the external reality by the processes of Learning. Many types of learning are mentioned in the literature (supervised, unsupervised, reinforcement, dynamic, PAC, etc.) Before classifying a need in a particular method of learning and deciding how to learn, we would like to figure out what should we learn. Now, it is not clear whether the process of learning can be separated into two different learning processes:

- that of objects representation, and

- that of the rules of action representation, or are these two kinds of learning just two sides of the same core learning process?

The following knowledge should be contained in the Representation Space. If no GS is given, any pair of state representations should 
contain implicitly the rule of moving from one state to another. In this case, while learning we inadvertently consider any second state as a provisional GS.

We will call "proper" representation a representation similar to the mathematical function and/or field description: at any point of the space, the derivative is available together with the value of the function; the derivative can be considered an action required to produce the change in the value of the function.

We will call "goal oriented" representation a representation in which at each point a value of the action is given required for describing not the best way of achieving an adjacent point but the best way of achieving the final goal.

Both "proper" and "goal oriented" representation can be transformed in each other.

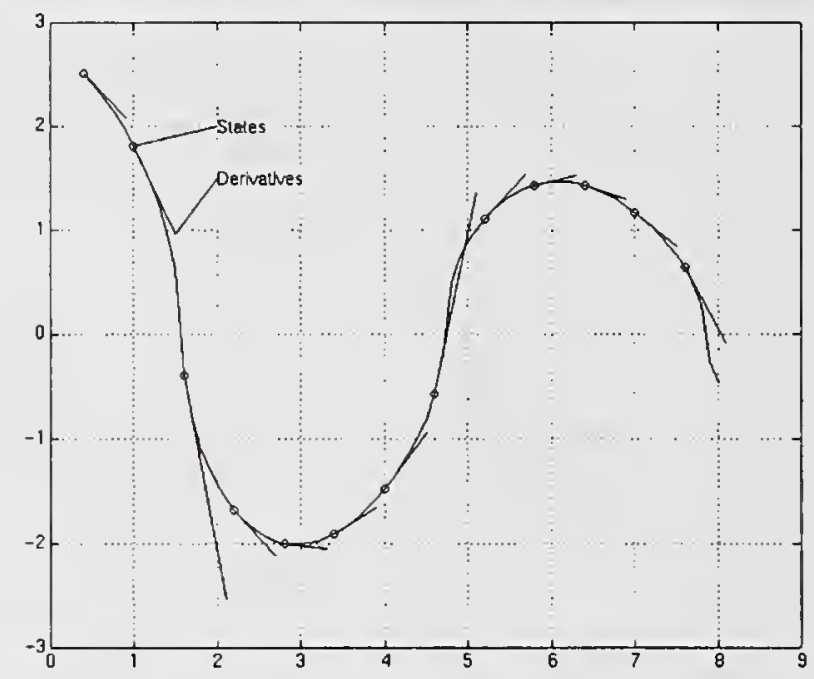

Figure 2. Knowledge of state, and knowledge of action which produces changes

\section{The Artifacts of Representation Space}

Representation (that of the World) can be characterized by the following artifacts:

- existence of states with its boundaries determined by the resolution of the space each state is presented as a tessellatum [2], or an elementary unit of representation, the lowest possible bounds of attention)

- characteristics of the tessellatum which is defined as an indistinguishability zone (we consider that resolution of the space shows how far the "adjacent" tessellata (states) are located from the "present state" (PS)

- lists of coordinate values at a particular tessellatum in space and time
- lists of actions to be applied at a particular tessellatum in space and time order to achieve a selected adjacent tessellatum in space and time

- existence of strings of states intermingled with the strings of actions to receive next consecutive tessellata of these strings of states

- boundaries (the largest possible bounds of the space) and obstacles

- costs of traversing from a state to a state and through strings of states.

In many cases, the states contain information which pertains to the part of the world which is beyond our ability to control it, and this part is called "environment." Another part of the world is to be controlled: this is the system for which the planning is to be performed. We will refer to it frequently as "self." Thus, part of the representation is related to "self" including knowledge about actions which this "self" should undertake in order to traverse the environment.

It is seen from the list of artifacts that all knowledge is represented at a particular resolution. Thus, the same reality can be represented at many resolutions and the "multiresolutional representation" is presumed.
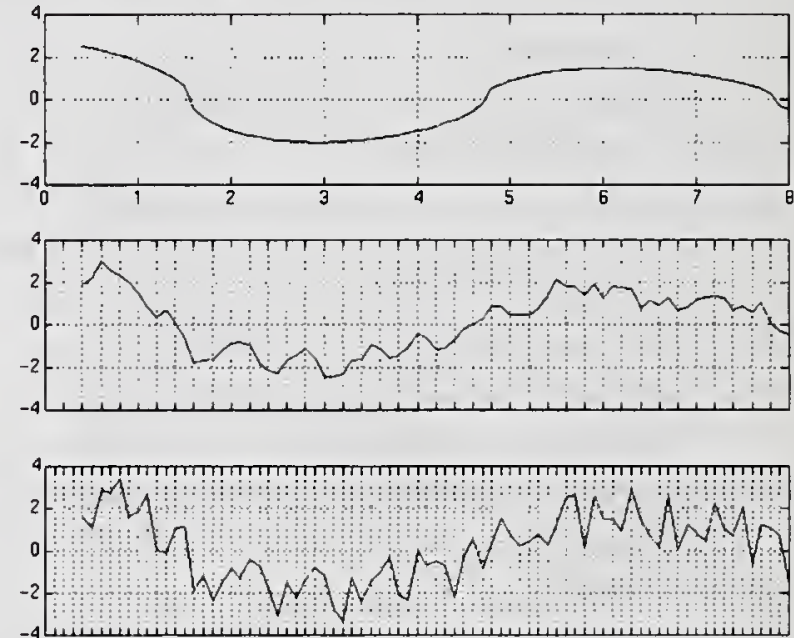

Figure 3. Multiresolutional representation

\section{Planning in Redundant Systems}

Non-redundant systems have a unique trajectory of motion from a state to a state. Redundant system is defined as a system in which there is more than one trajectory of motion from one state to another. It can be demonstrated for many realistic couples "system-environment" that

- they have a multiplicity of traversing trajectories from a IS to 


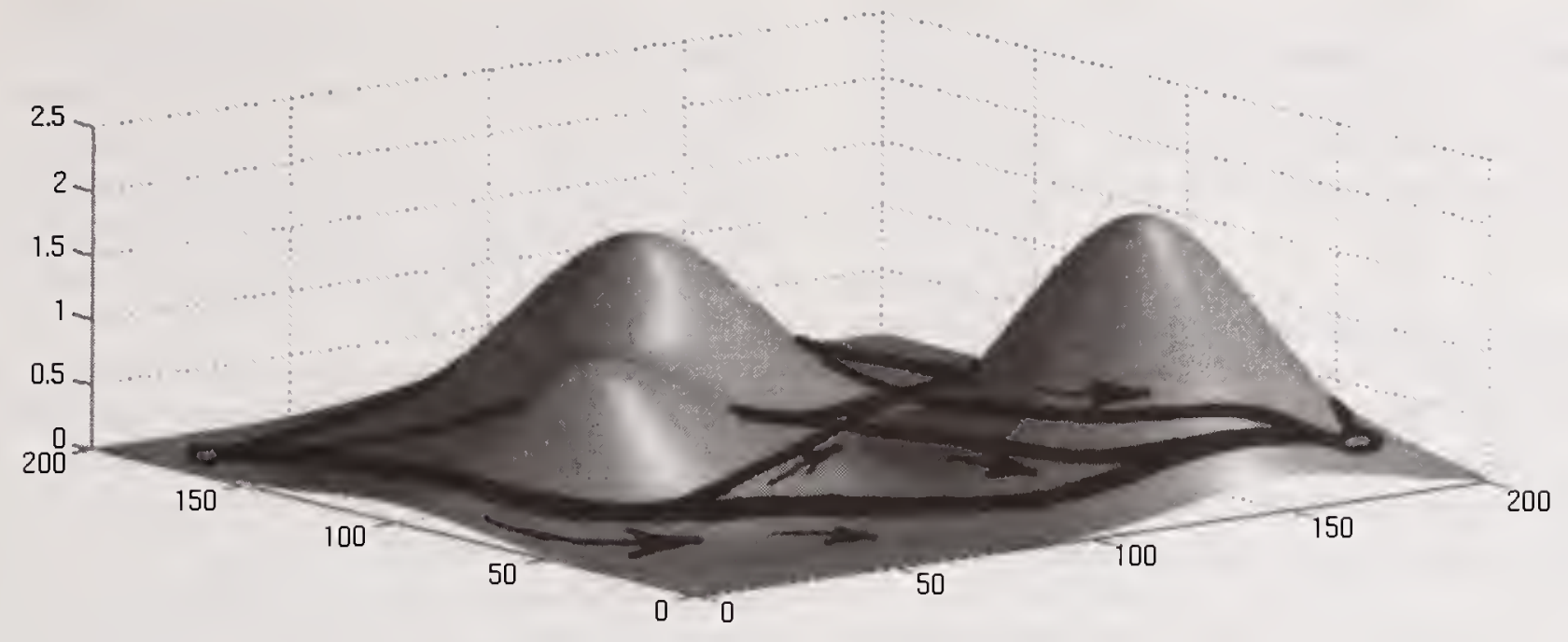

Figure 4. Multiplicity of plan alternatives

- these trajectories can have different costs.

These systems contain a multiplicity of alternatives of space traversal. Redundancy grows when the system is considered to be a stochastic one. The number of available alternatives grows even higher when we consider also a multiplicity of goal tessellata of a particular level of resolution under the condition of assigning the goal at a lower resolution level which is the fact in multiresolutional systems (such as NIST-RCS.)

In on-redundant systems there is no problem of planning. Since the trajectory of motion to be executed is a unique one, the problem is to find this trajectory and to provide tracking of it by an appropriate classical control system.

\section{Learning as a Source Representation}

Learning is defined as knowledge acquisition via experience of functioning. Thus, learning is development and enhancement of the representation space. The latter can be characterized in the following ways:

- by a set of paths (to one or more goals) previously traversed

- by a set of paths (to one or more goals) previously found and traversed

- by a set of paths (to one or more goals) previously found and not traversed

- by a totality of (all possible) paths

- by a set of paths executed in the space in a random way.

One can see that this knowledge contains implicitly both the description of the environment and the description of the actions required to traverse a trajectory in this environment.
Moreover, if some particular system is the source of knowledge, then the collected knowledge contains information about

properties of the system which moved in the environment.

All this information arrives in the form of experiences which record states, actions between each couple of states, and evaluation of the outcome. The collection of information obtained in one or several of these ways forms knowledge of space, KS.

If the information base contains all tessellata of the space with all costs among the adjacent tessellata - we usually call it the representation.

Ergo: the representation is equivalent to the multiplicity of explanations how to traverse, or how to move. In other words: all kinds of learning mentioned in p. 3 are equivalent.

Comments: a) remember Albus' question about knowing states, or knowing derivatives" (actions) from a state to a state; b) apparently, each state can be characterized by some cumulative cost (value), while each traversal from a state to a state can be characterized by some incremental cost (goodness of a move or a set of moves.)

\section{Types of Problems of Planning}

Any problem of planning is associated with

- actual existence of the present state

- actual, or potential existence of the goal state

- knowledge of the values for all or part of the states as far as some particular goal is concerned.

From this knowledge the cumulative costs of trajectories to a particular goal (or goals) can be 
deduced. On the other hand, the knowledge of costs for the many trajectories traversed in the past can be obtained which is equivalent to knowing cumulative costs from the initial state (PS) to the goal state (GS) (from which the values of the states can be deduced.)

In other words, any problem of planning contains two components: the first one is to refine the goal (bring it to the higher resolution.) The second one is to determine the path to this refined goal. These two parts can be performed together, or separately. Frequently we are dealing with them separately. In the latter case they are formulated as follows:

a) given PS, GS and KS (all paths) find the subset of KS with a minimum cost, or with a prearranged cost, or with a cost in a particular interval.

b) given PS Gs from the lower resolution level and KS (all paths) find the GS with a particular value

\section{The Duality of Planning and Learning Algorithms}

Finding solutions for these problems is done by a process which we will call planning. In other words, planning is construction of the goal states, and/or strings of states connecting the present state with the goal states. As we analyze the desirable processes of planning, we notice a striking similarity between planning and learning, actually their inseparability.

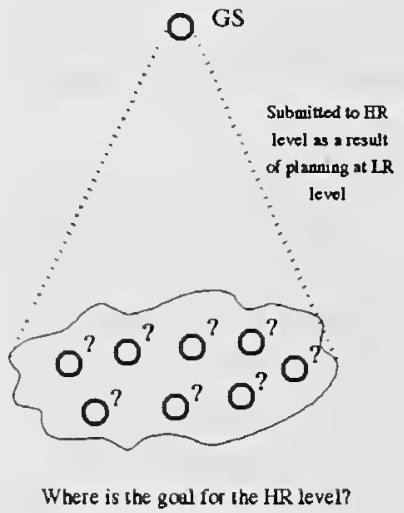

a

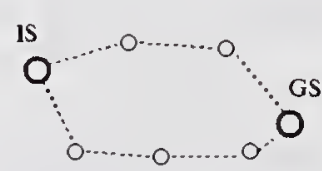

Which trajectory to choose?

b
Figure 5. Two parts of planning problem

Indeed, the first component of the planning algorithm is translation of the goal state description from the language of low resolution to the level of high resolution. We must learn where the goal is located, and it is done by consecutive refinement of the initial coarse information. Frequently, it is associated with increasing of the total number of the state variables. In all cases it is associated with reduction of the indistinguishability zone, or the size of the tessellatum associated with a particular variable. We plan and learn by testing: in the representation, for planning, and in the reality, for learning.

The second component is the simulation of all available alternatives of the motion from the initial state, IS to one or several goal states, GS and selection of the "best" trajectory. Procedurally, this simulation is performed as a search, i.e. via combinatorial construction of all possible strings (groups). To make this combinatorial search for a desirable group more efficient we reduce the space of searching by focusing attention.

The need in planning is determined by the multialternative character of the reality The process of planning can be made more efficient by using appropriate heuristics which is not considered in this paper.

\section{The Unified System of Planning and Learning}

Planning is performed by searching within a limited subspace

- for a state with a particular value (designing the goal)

- for a string (a group) of states connecting SP and GP satisfying some conditions on the cumulative cost (planning of the course of actions.)

The process of searching is associated ether with collection of addition information about experiences, or with extracting from $\mathrm{KS}$ the implicit information about the state and moving from state to state, or learning. In other words, planning is inseparable from and complementary to learning.

This unified planning/learning process is always oriented toward improvement of functioning in engineering systems (improvement of accuracy in an adaptive controller) and/or toward increasing of probability of survival (emergence of the advanced viruses for the known diseases that can resist various medications, e.g. antibiotics.)

Thus, this joint process can be related to a system as well as to populations of systems and determines their evolution.

LPA is a tool which allows for jointly exploring these two fundamental processes of intelligent systems. 


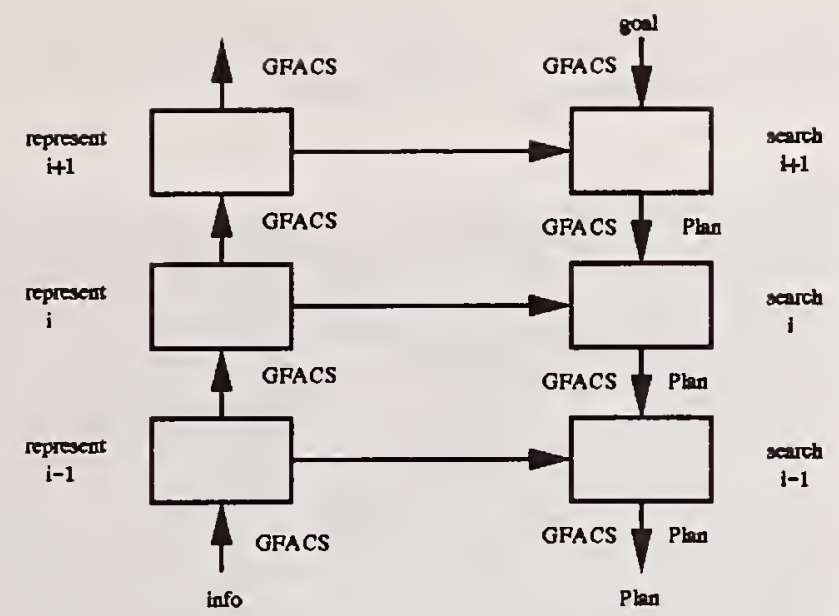

LEARNING

PLANNING

Figure 6. On the relations between planning and learning

\section{The Elementary Components of Planning and Learning}

Search is performed by constructing feasible combinations of the states within a subspace (feasible, means: satisfying a particular set of conditions.) Search is interpreted as exploring (physically, or in simulation) as many as possible alternatives of possible motion and comparing them afterwards.

Each alternative is created by using a particular law of producing the group of interest (cluster, string, etc.) Usually, grouping presumes exploratory construction of possible combinations of the elements of space (combinatorial search) and as one or many of these combinations satisfy conditions of "being an entity" - substitution of this group by a new symbol with subsequent treating it as an object (grouping.)

The larger the space of search is the higher is the complexity of search. This is why a special effort is allocated with reducing the space of search. This effort is called focusing attention and it results in determining two conditions of searching, namely, its upper and lower boundaries:

a) the upper boundaries of the space in which the search should be performed, and

b) the resolution of representation (the lower boundaries)

\section{Planning and Intelligence}

Formation of multiple combinations of elements (combinatorial search, CS) satisfying required conditions of transforming them into entities (grouping, G) within a bounded subspace (focusing attention, FA) is a fundamental procedure in both learning and planning. Since these three procedures work together we will talk about them as about a triplet of computational procedures (GFACS.) Notice, that in learning it creates lower resolution levels out of higher resolution levels (bottom-up) while in planning it progresses from the lower resolution levels out of higher resolution levels (top-down.)

This triplet of computational procedures is characteristic for intelligence and probably is the elementary computational unit of intelligence. Its purpose is transformation of large volumes of information into a manageable form which ensures success of functioning. The way it functions in a joint learning-planning process explains the pervasive character of hierarchical architectures in all domains of activities.

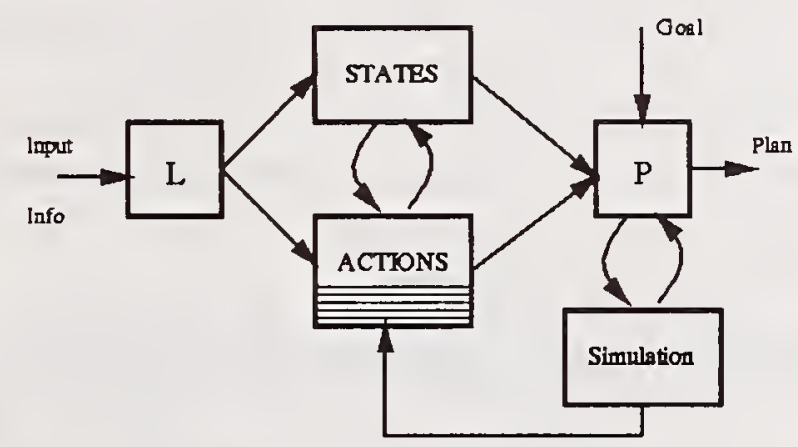

Figure 7. Functioning of GFACS in the joint learning-planning process

The need in GFACS is stimulated by the property of knowledge representations to contain a multiplicity of alternatives of space traversal (which is a property of representations to be redundant.) Redundancy of representations determines the need in GFACS: otherwise the known systems would not be able to function efficiently (it is possible that redundancy of representations is a precondition for the possibility of Life and the need in Intelligence)

\section{Planning, Learning, and Control Theory}

Representations reduce the redundancy of reality. Elimination of redundancy allows for having problems that can be solved in a closed form (no combinatorics is possible and/or necessary). Sometimes, this ultimate reduction of redundancy is impossible and the combinatorial search is the only way of solving the problem). If the problem cannot be solved in a closed form, we introduce redundancy intentionally to enable functioning of GFACS. 
At each level of resolution, planning is done as a reaction for the slow changes in situation which invokes the need in anticipation and active interference

a) to take advantage of the growing opportunities, or

b) to take necessary measures before the negative consequences occur.

The deviations from a plan are compensated for by the compensatory mechanism also in a reactive manner. Thus, both feedforward control (planning) and feedback compensation are reactive activities as far as interaction systemenvironment is concerned. Both can be made active in their implementation. This explains different approaches in control theory.

Examples: a) Classical control systems are systems with no redundancy, they can be solved in a closed form. Thus, they do not require any searching.

b) Any stochastics introduced to a control system creates redundancy and requires either for elimination of redundancy and bringing the solution to a closed form, or performing search.

c) Optimum control allows for the degree of redundancy which determines the need in searching.

In Figure 8, the process of multiresolutional planning via consecutive search with focusing attention and grouping is demonstrated for the control problem of finding a minimum-time motion trajectory.

The space is learned in advance by multiple testing, and its representation is based upon knowing that the distance, velocity and time are linked by a simple expression which is sufficient for obtaining computationally the theoretically correct solution with an error accepted to be admissible. Several methods of constructing the envelopes of attention can be applied.

\section{References}

\section{J. Albus, A. Meystel, "A Reference Model} Architecture for Design and Implementation of Intelligent Control in Large and Complex Systems", International Journal of Intelligent Control and Systems", Vol. 1, No. 1, pp. 15-30 2. A. Meystel, "Architectures, Representations, and Algorithms for Intelligent Control of Robots", in Intelligent Control Systems: Theory and Applications, eds. M. M. Gupta, N. K. Sinha, IEEE Press, New York, pp. 732-788

3. J. Albus, A. Lacaze, A. Meystel, "Autonomous Learning via Nested Clustering", Proc. of the 34th
IEEE Conference on Decision and Control, Vol.3, New Orleans LA, pp. 3034-3039

a)

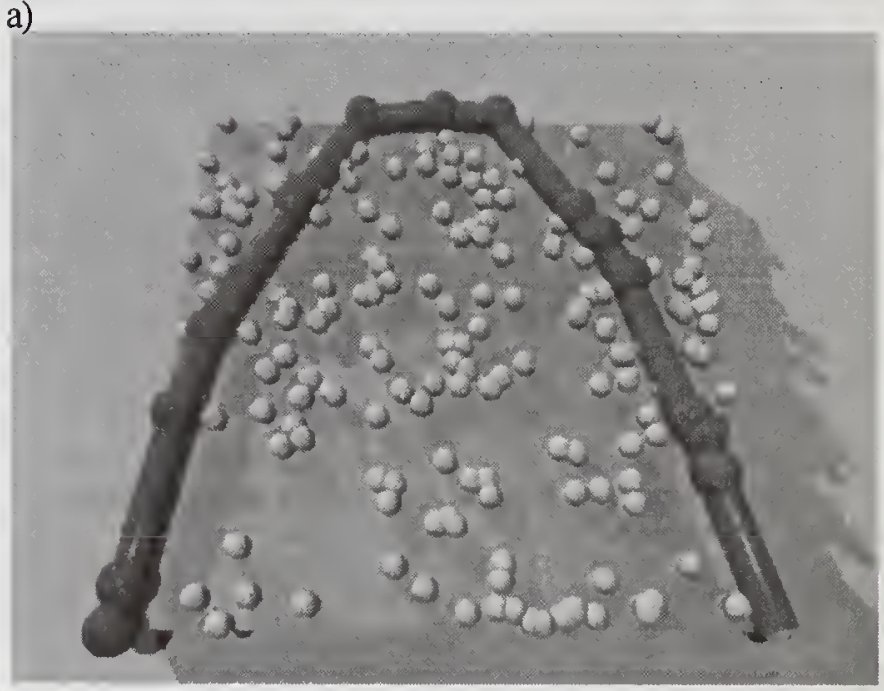

b)

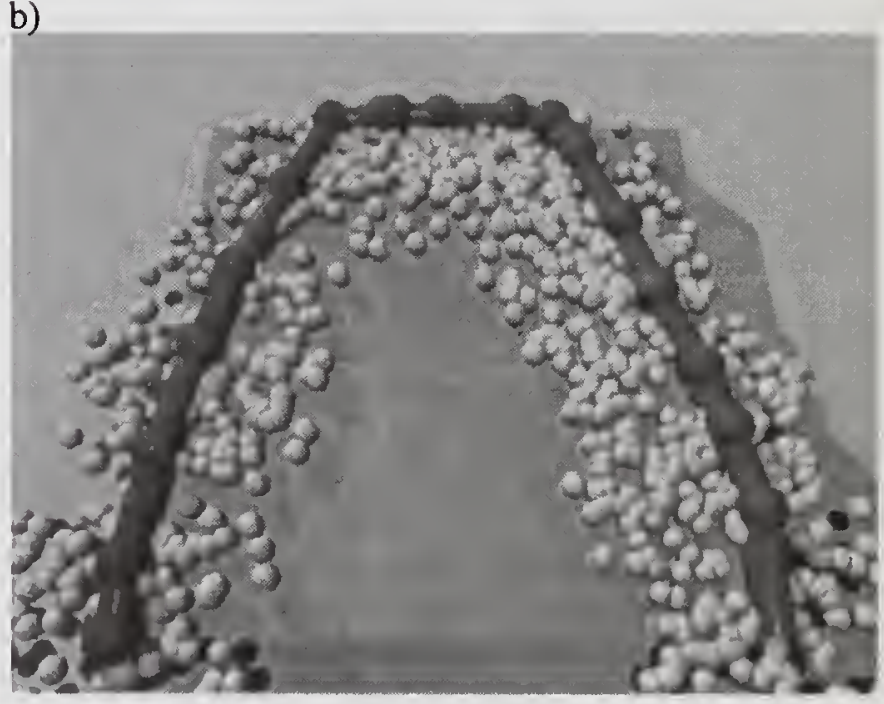

c)

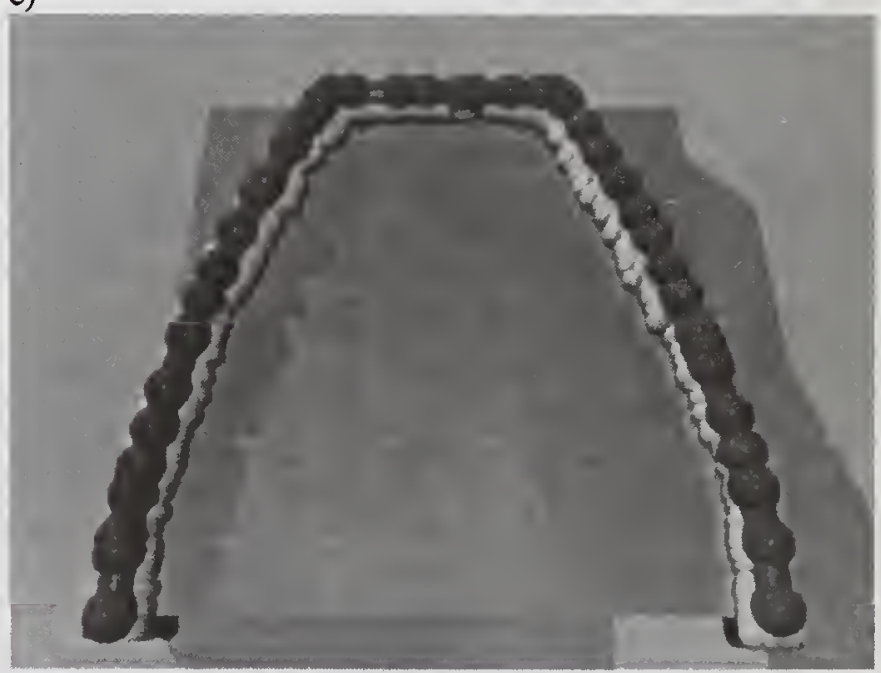

Figure 8. Solving a minimum-time control problem by multiresolutional with grouping and consecutive focusing attention 



\title{
A Road Map to the Future for Intelligent Systems and Semiotics
}

\author{
by \\ James S. Albus \\ Intelligent Systems Division \\ Manufacturing Engineering Laboratory \\ National Institute of Standards and Technology
}

\section{Introduction}

In common usage, a road map is a piece of paper with lines that indicate roads and dots that represent cities. More technically, a road map is an iconic representation of the surface of the earth with roads represented as lines, and destinations (or goals) represented as points, or regions on the map. In the context of the title of this paper, a road map implies a planned route on the map from our present position to some future goal.

What is the goal for intelligent systems and semiotics? What should it be? What could it be?

In order to decide on a goal, one must choose a cost/benefit function. What are the factors that go into a cost/benefit function? What is important? Important for what?

I will argue that intelligent systems and semiotics are important for science, economic prosperity, military strength, and human well being.

Intelligent systems and semiotics are important to science because they extend human knowledge into a rich and largely uncharted region -- the mind. Intelligent systems theory has much to contribute to a fundamental understanding of the mind and of the nature of perception, cognition, and behavior.

Intelligent systems and semiotics are important to economic prosperity because they enable productivity improvements in the production of wealth. Intelligent systems applications have the potential to reduce the cost and improve the quality of almost every kind of product and service.

Intelligent systems and semiotics are important to military strength because they will enable a whole new generation of battlefield sensors and weapons that will revolutionize the art of war. Information dominance and unmanned weapons systems have the capacity to increase the effectiveness and reduce the risk of projecting military power to trouble spots throughout in the world.

Intelligent systems and semiotics are important to human well being because they will enable us to clean up the environment, to switch to more efficient means of production, and to improve health care, education, public safety, and personal security for everyone. In short, intelligent systems have the potential to provide the means to end poverty and bring about a golden age for human kind.

\section{Important for Science}

I would argue that all of science can be summed up in three great questions:

1. What is the nature of matter?

2. What is the nature of life?

3. What is the nature of mind?

Over the past 200 years, research in the physical sciences has produced a wealth of knowledge about the nature of matter, both on our own planet and in the distant galaxies. We now understand the forces and particles that make up atoms and their constituent parts: what holds 
them together, and what gives them their properties.

Over the past half century, the biological sciences have produced a revolution in knowledge about the nature of life. We have a profound understanding of the molecular basis of life. We are well on our way to mapping the human genome. We may soon understand how to cure cancer and prevent AIDS. We will see the development of new drugs and new sources of food. Within the next century, we may eradicate most genetic diseases.

However, of all the questions in science, the deepest and most profound may be -- "What is mind?"

This question can be asked in a number of different ways. From the time of Moses, prophets and priests have asked, "What is the nature of the soul?" Since the time of Aristotle, philosophers and theologians have asked, "What is the relationship between mind and body?" For at least a century, psychologists have asked, "What is a thought? What does it mean to think about something? How do we imagine things?" Psychophysicists ask, "What is perception, and how is it related to the world that is perceived?" Psychiatrists ask, "What are emotions and dreams, and why do we have them?" Psychologists want to know, "What is motivation and intention, and how do we decide what we intend to do?" Neurophysiologists ask, "How do we convert intention into action and sensation into feeling?" Researchers in linguistics and semiotics ask, "How do we generate and use language and how do we represent knowledge about the world in our minds?" Researchers in artificial intelligence and operations research ask, "How do we plan, and how do we solve problems and reason about the world?"

Intelligent systems from a semiotic perspective is the study of mind. The study of intelligent systems addresses what is arguably THE most important question in science -- What is the nature of mind?

But let me move on to the issue of the importance of Intelligent Systems and Semiotics to economic prosperity.

\section{Important for Economic Prosperity}

Intelligent manufacturing systems are important economically because they can dramatically increase productivity.

Toffler has suggested that there have been three pivotal groups of inventions that shaped the course of human history. The first was the invention of agriculture, which made it possible to augment hunting and gathering with the more efficient and less dangerous processes of cultivation of crops and husbandry of animals. This brought about the development of cities, the organization of nations, and the rise of civilization.

The second group of inventions was the invention of the steam engine, the discovery of electricity, and the development of the internal combustion engine. These made possible the augmentation of muscle power with mechanical energy in the production of goods and services. This caused the demise of slavery and the rise of middle-class prosperity.

Toffler's Third Wave is based on the invention of electronics and the digital computer. This is enabling the augmentation of brain power with computer power in the production of goods and services. Intelligent systems have the capacity to increase industrial productivity by orders of magnitude. This could reduce the cost of production and enable wealth to be generated at a rate sufficient to eliminate both poverty and pollution and create a world of prosperity in which everyone could be economically secure and financially independent. Intelligent machines could become the economic equivalent of mechanical slaves. Machine owners would become the modern equivalent of an aristocracy, and with the proper economic policy, everyone could become an owner of wealth producing machines.

Let me be more specific about how intelligent machine and semiotics can be important for economic prosperity.

\section{Manufacturing}

Intelligent manufacturing systems can dramatically reduce the cost and improve the quality of cars, trucks, airplanes, appliances, furniture, clothing, food products, electronics, optics, drugs, chemicals, construction equipment, 
mining and drilling equipment, farm machinery, railroads, ships, and weapons systems.

For example, intelligent machine controllers based on inexpensive personal computer technology will soon make it possible to automatically generate programs for lifting, positioning, cutting, joining, machining, forming, finishing, and assembly operations in factories and shops throughout the world. Online monitoring of material flow and machine availability will enable real-time production planning and scheduling. Advanced sensory perception systems will enable on-line inspection and testing. Intelligent adaptive control systems will increase the efficiency and reduce the cost of plants, processes, and machines.

The manufacturing industries produce $\$ 1.1$ trillion of goods and services each year in the United States. Every percentage point improvement in manufacturing productivity produces over $\$ 10$ billion increase in the nation's wealth production.

\section{Business management}

Intelligent systems technologies are becoming increasingly important for business management. Computers are already involved in design, planning, scheduling, word processing, business management, financial services, marketing, and customer services.

\section{Transportation Safety and Efficiency}

Intelligent systems technologies are about to have a revolutionary impact on cars and highways around the world. For more than a decade in Europe and Japan, and now in the United States, serious efforts are being directed toward Intelligent Highway and Vehicle Systems. In Germany, experimental vision-guided automatic automobiles are undergoing regular tests driving the streets and highways in traffic at normal speeds, almost completely without human assistance. A sedan recently drove from Munich to Copenhagen under automatic control over $95 \%$ of the time. In Japan, drivers can view detailed maps of streets with directory assistance, on-line traffic information, and voice input/output. In the U.S., an automobile under computer control recently drove from the east coast to San Diego largely without human assistance.
Advanced cruise control with collision avoidance will enhance safety by alerting drivers that have gone to sleep or whose attention has wandered. Automatic lane following and distance keeping will improve throughput and increase safety on congested freeways. This may significantly reduce the need to build additional lanes in areas where construction is restricted by the availability of space.

Intelligent systems can also improve airline safety and prevent most rail and ship collisions. A large percentage of accidents are caused by human operator errors. Most of these could be prevented by intelligent systems technology.

\section{Communications}

Intelligent systems technologies are already having a profound impact on the communications industries. The internet, satellite and cable television, and cellular phone systems are creating a communications network that rivals in bandwidth, complexity, and sophistication the network of neural fibers that interconnect various parts in the human brain. The potential for productivity improvements from these new technologies is beyond our ability to predict.

\section{Construction}

Studies of the construction industry have suggested that significant benefits can be achieved from integrating computer design data with on-site measurements to enable real-time planning and scheduling and inventory tracking. Intelligent measurement systems can assure that construction tolerances are met and parts fit together without modification. Intelligent planning systems can assure that parts, tools, and materials arrive at the right place, at the right time, and in the right order for construction operations to flow seamlessly. Complete "asbuilt" records can be kept for future reference during maintenance, repair, and future modifications.

Intelligent systems technology for construction can result in improved productivity, lower cost, and higher quality in the construction of factories, plants, high rise buildings, homes, highways, bridges, tunnels, port facilities, sewer, water, electricity, and gas utilities, and homes. 
The construction industry produces about $\$ 460$ billion per year. Each percentage point improvement in construction productivity growth rate produces a $\$ 4.6$ billion increase in the nation's rate of wealth production.

\section{Waste Management}

Intelligent systems will enable improved methods for toxic and radioactive waste handling and cleanup of waste dumps. New approaches to trash collection, recycling, and pollution monitoring will enable environmental restoration and preservation.

\section{Hospital and Nursing Support}

Intelligent fetch and carry robots are already used every day in hospitals. Computers are used for patient record keeping, patient monitoring, and diagnostic aids. Intelligent systems can be used in laboratory analysis, drug manufacturing, and prescription filling. These applications could reduce costs and improve care for hospital patients.

\section{In-home Patient Services}

Intelligent systems could be developed for lifting and positioning invalid and infirm patients in and out of bed, on and off of the toilet, and in and out of the bath. Intelligent systems can provide mobility, food preparation, physical therapy, security and health monitoring, telecommuting for work and shopping, and entertainment and education for the home patient. This can reduce the cost and improve the life of patients that prefer to remain at home rather than be institutionalized in nursing care facilities.

For example, around 100,000 persons enter nursing homes every month in the United States at a cost of more than $\$ 2000$ per month. Delaying the average date of entry of the elderly to nursing homes by only one month would save the country about $\$ 2.4$ billion per year.

\section{Physical Security}

Intelligent systems will enable advanced security systems for the detection and tracking of intruders with a minimum of false alarms.

\section{Agriculture and Food Processing}

Robotics and intelligent machine systems have only begun to enter the field of agriculture and food processing industries. The application of intelligent systems technologies to farming the oceans has not even begun to be explored.

\section{Mining and Drilling}

Three-fifths of the earth's surface is too deep beneath the oceans for mining or drilling operations using conventional techniques. This means that most of the earth's mineral resources have never been touched. Intelligent undersea robots offer significant potential for productivity improvements in deep sea mining and oil drilling operations.

\section{Space and Undersea Exploration}

Outer space, planetary surfaces, and the bottom of the ocean all share the characteristic that manned exploration is extremely expensive and hazardous. Intelligent systems and robotics promise to decrease the cost and risk and increase the amount of data that can be gathered from space and undersea exploration.

\section{Important for Military Strength}

Intelligent systems technology promises to revolutionize warfare through a new generation of sensors, machines, decision aids, and weapons systems. Intelligent weapons are already highly advanced. Cruise missiles, smart bombs, unmanned vehicles, and computer augmented command and control systems are currently being developed and deployed. These are but the vanguard of a whole new generation of military systems that will become possible once intelligent systems becomes a mature engineering discipline.

In future wars, unmanned air vehicles, ground vehicles, ships, and undersea vehicles will be able to outperform manned systems. Many military vehicles are limited in performance because of the acceleration, vibration, or pressure limits of the human body, or the need of humans to consume air, water, and food. A great deal of the weight and power of current military vehicles is spent on armor and life support systems that are necessary to protect and maintain human operators.

Intelligent military systems will reduce both the risk to human personnel and the cost of training and readiness. Unmanned vehicles and weapons systems put fewer humans in harms way and require little or no training to maintain 
readiness. Unmanned systems can be stored in forward bases or at sea for long periods of time at low cost. They can be mobilized quickly in an emergency. They operate without fear under fire, the first time and every time.

Intelligent systems can be fast and effective in gathering, processing, and displaying information. They can enable human commanders to be quicker and more through in planning operations and in replanning for unexpected events as the battle evolves.

In short, intelligent systems promise to multiply the capabilities of the armed forces, while reducing casualties and hostages and lowering the cost of training and readiness.

\section{Important for Human Well Being}

Productivity growth depends on technology. Intelligent systems and semiotics have entered a new phase of rapid development that will produce rapid growth in productivity. Intelligent machines represent a new breed of workers that will be able to produce more and higher quality goods and services at lower cost. There is every reason to believe that we are on the cusp of an S-curve where productivity can grow exponentially for a long period of time. Given adequate investment, there is no reason to doubt that productivity growth could return to $2.5 \%$, which is the average for the 20th century. Or, it could rise to $4 \%$ which is the average for the 1960-68 time frame, or even to $12 \%$ which occurred during the period of World War II between 1939 and 1945 . If this were to occur, many of the problems of poverty, disease, and pollution that result from stagnant economic growth would disappear. Society could afford to improve health care and education, to clean up the environment, to adopt less wasteful forms of production and consumption, to reduce taxes, and provide a minimum income for all.

\section{Charting the Route}

At this point, a goal destination for our road map has been established. Our goal is to understand the nature of mind and to build intelligent systems that will improve economic prosperity, strengthen military security, and improve human well being. I believe this is a worthy and desirable goal.
Let me now turn to the issue of finding a route from where we are today to the goal destination.

First, let me say that we have already made a good start and progress is rapid. The study of intelligent control systems is an extremely active field. Research in neural nets, fuzzy logic, and genetic algorithms is showing how to recognize patterns, discover control laws, and control complex industrial processes. Computer science, artificial intelligence, semiotics, and linguistics are probing the nature of language and image understanding. Significant progress has been made in expert systems, rule-based reasoning, planning and problem solving. Game theory and operations research have developed methods for decision-making in the face of uncertainty. Research in sonar, radar, and optical signal processing has developed methods for fusing sensory input from multiple sources, and assessing the believability of noisy data.

Robotics and autonomous vehicle research has produced advances in real-time sensory processing, world modeling, navigation, trajectory generation, and obstacle avoidance. Research in computer integrated manufacturing and process control has produced intelligent hierarchical controls, distributed databases, representations of object geometry and material properties, data driven task sequencing, network communications, and multiprocessor operating systems. Modern control theory has developed a precise understanding of stability, adaptability, and controllability under various conditions of feedback and noise. Intelligent control closes the loop between sensing and acting through perception, world modeling, planning, and control. Intelligent control enables large complex systems with many sensors and actuators to pursue and achieve sophisticated goals in an uncertain, competitive, and sometimes hostile environment. Intelligent control enables a system to analyze the past, to perceive the present, and to plan for the future. Intelligent control enables systems to assess the cost, risk, and benefit of past events and future plans and to make choices between alternative courses of action.

In the neurosciences, much is known, both about the machinery of the brain and the processes that run in it. Neuroanatomy has produced extensive maps of the interconnecting 
pathways making up the structure of the brain. Neurophysiology is demonstratin sow neurons compute functions and communicate information. Much is known about the functionality of specific areas of the brain. For example, the areas responsible for vision, hearing, manipulation, locomotion, emotions, hunger, thirst, and sexual drive are well explored. Within the vision system, there are modules and pathways that specialize in analysis of motion, and others that specialize in recognition of shape. The brain has neuronal mechanisms for estimating distance to visible surfaces, for tracking objects, anticipating movement, and for fusing information derived from eyes and ears.

Neuropharmacology is discovering many of the transmitter substances that activate and modify behavior, that enable learning, that dispense reward and punishment, and that assign value to perceived objects and events.

Psychophysics provides many clues as to how individuals perceive objects, events, time, and space, and how they reason about relationships between themselves and the external world. Neuropsychology adds information about mental development, feeling, emotions, and behavior.

\section{Technology is Accelerating}

The latter half of the 20th century has seen a number of fundamental breakthroughs that have radically altered the landscape of technological possibilities. Technological knowledge is not just increasing, it is accelerating. There is an exponential growth in technological progress that shows no sign of saturation. For example:

1. The power and speed (per unit cost) of electronic computing has risen exponentially by more than a factor of ten per decade for almost fifty years. This rate of progress shows no sign of slowing. This means that computing power comparable to that which exists in the brain could be assembled in the foreseeable future.

2. The store of knowledge about how the biological brain works has also increased many fold over the last half century. The neurosciences show no sign of running up against a fundamental barrier to understanding.
3. The number of scientists and engineers is increasing exponentially. Over half of all the scientists and engineers that ever lived are alive and working today.

4. Perhaps of most importance, the nature of knowledge is such that it is not subject to diminishing returns. The more that is known, the easier it is to discover new things. There is no limit to what there is to know. There is only a limit to the amount of time and resources we dedicate to the pursuit of knowledge.

\section{So what is lacking?}

The main thing that is missing is a widely accepted theoretical framework that ties together all of the diverse fields related to intelligent systems, semiotics, and the neurosciences. There is little symbiosis between fields. Things are constantly being rediscovered in different fields and called by different names.

Also missing is a widely accepted reference model architecture that can support an engineering methodology for designing and building intelligent systems. Several architectures have been proposed, but none is widely adopted. There are no standardized metrics for measuring performance that can scientifically evaluate experiments and test theories. There are no interface standards so that intelligent systems can be easily assembled from component parts. Finally, there is no educational curriculum for producing scientists and engineers skilled in the art of designing and building intelligent systems.

\section{An Outline for a Theory of Intelligence}

As a result of our research in the Intelligent Systems Division here at NIST, an outline for a theory of intelligence has been published. (Albus 91) In this paper, the functional elements of intelligence are defined as behavior generation, sensory processing, world modeling, and value judgment. Behavior generation provides the functions of planning and control. Sensory processing filters data from sensors, detects events, recognizes patterns, and analyzes situations. World modeling stores and maintains a knowledge database and uses knowledge about the world to predict the future and simulate the results of hypothesized plans. Value judgment 
functions compute cost, benefit, risk, and uncertainty.

These functional elements are supported by a distributed knowledge database where knowledge about the world and the system is represented in terms of state variables, event and entity frames, attributes, maps, lists, tasks, rules, equations, and processes.

The functional elements and knowledge database are integrated by a system architecture that provides communications, timing, and an operating system.

Based on this theory, a reference model architecture has been developed.

A diagram of the relationship between the functional elements, and the knowledge database in this reference model architecture is shown in Figure 1.

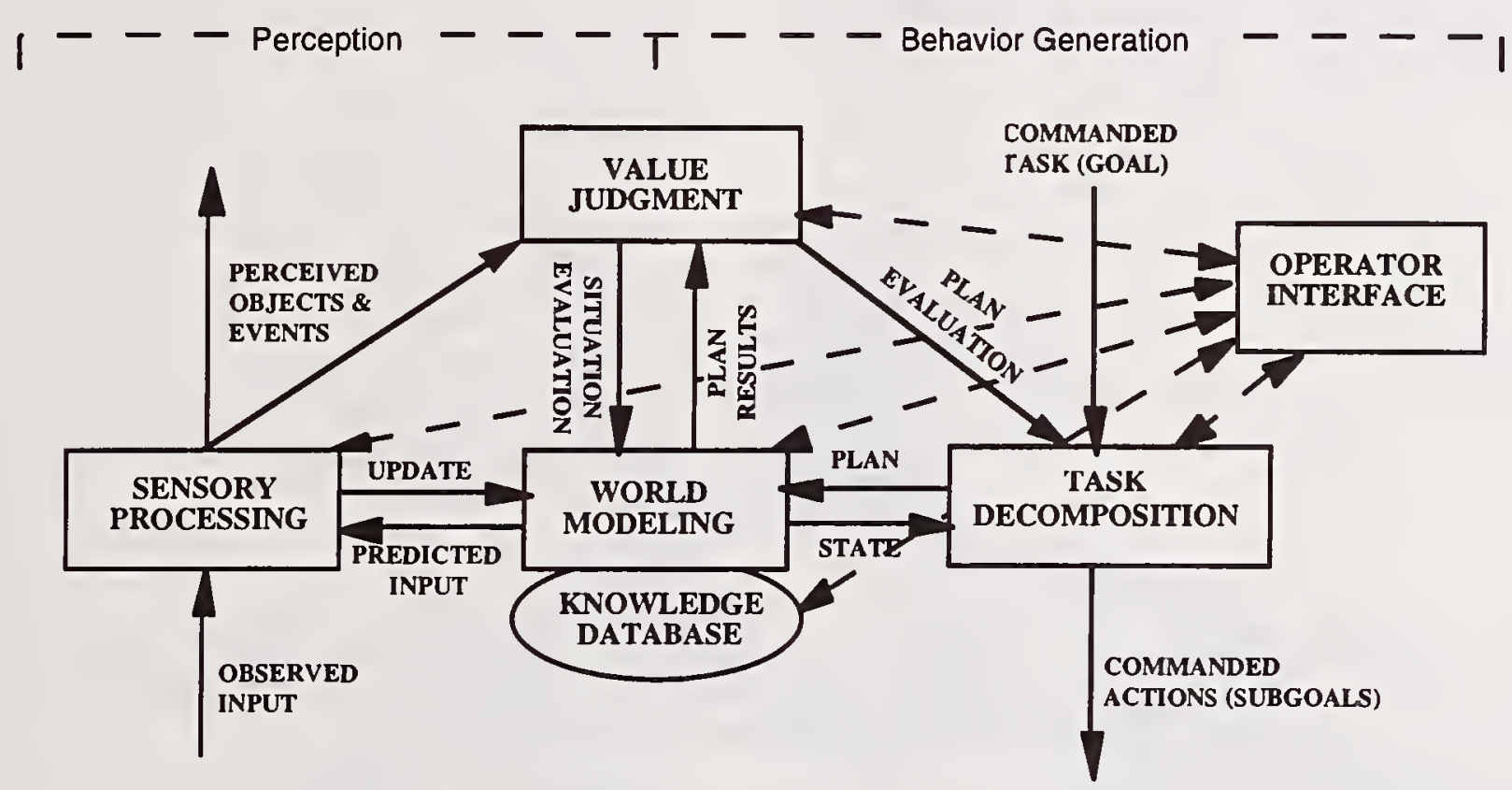

Figure 1. Relationships between the functional elements and the knowledge database within the NIST reference model architecture for intelligent systems.

Computational modules and a knowledge database are interconnected by communication pathways that transmit information so as to close control loops, enable perception and planning, and facilitate knowledge acquisition and learning.

In the intelligent system, values and cost functions control the selection of goals and optimization of behavior. Hypothesized plans are communicated from Behavior Generation to World Modeling for simulation and the results of simulation to Value Judgment for evaluation. Evaluations are communicated back to Behavior Generation for decision-making in selecting goals, setting priorities, focusing of attention, organizing perception, and controlling behavior. World Modeling uses information in the knowledge database to predict incoming data.
Sensory processing compares predictions with observations and computes correlations and differences. Differences are returned to the world to update the knowledge database. This is a Kalman filtering loop for recursive estimation. Correlations between predictions and observations signal recognition of events and objects. Value judgment facilitates learning by computing what is important or trivial, and what is rewarding or punishing. Real-time data in the world model closes a control loop between sensory feedback and task execution. Within this system the deliberative is integrated with the reflexive, enabling a real-time intelligent control system to be both goal driven and sensory interactive.

Figure 2 is a reference model architecture for an intelligent shop controller within a 
manufacturing enterprise. Each node in the hierarchical architecture shown in Figure 2 consists of the set of functional elements shown in Figure 1.

In each node of the architecture shown in Figure 2:

a World Modeling function maintains the Knowledge database, answers queries, predicts sensory input, and simulates the result of hypothesized plans;

a Sensory Perception function scales and filters input from sensors, detects events, and recognizes objects and situations; a Value Judgment function computes cost, risk, and benefit, and assigns values to recognized objects and events and the results of simulated plans;

a Behavior Generation function uses the information provided by the World Modeling and Value Judgment functions to find the best assignment of tools and resources to agents, to find the best plan from the current state to a goal state, and to execute that plan.

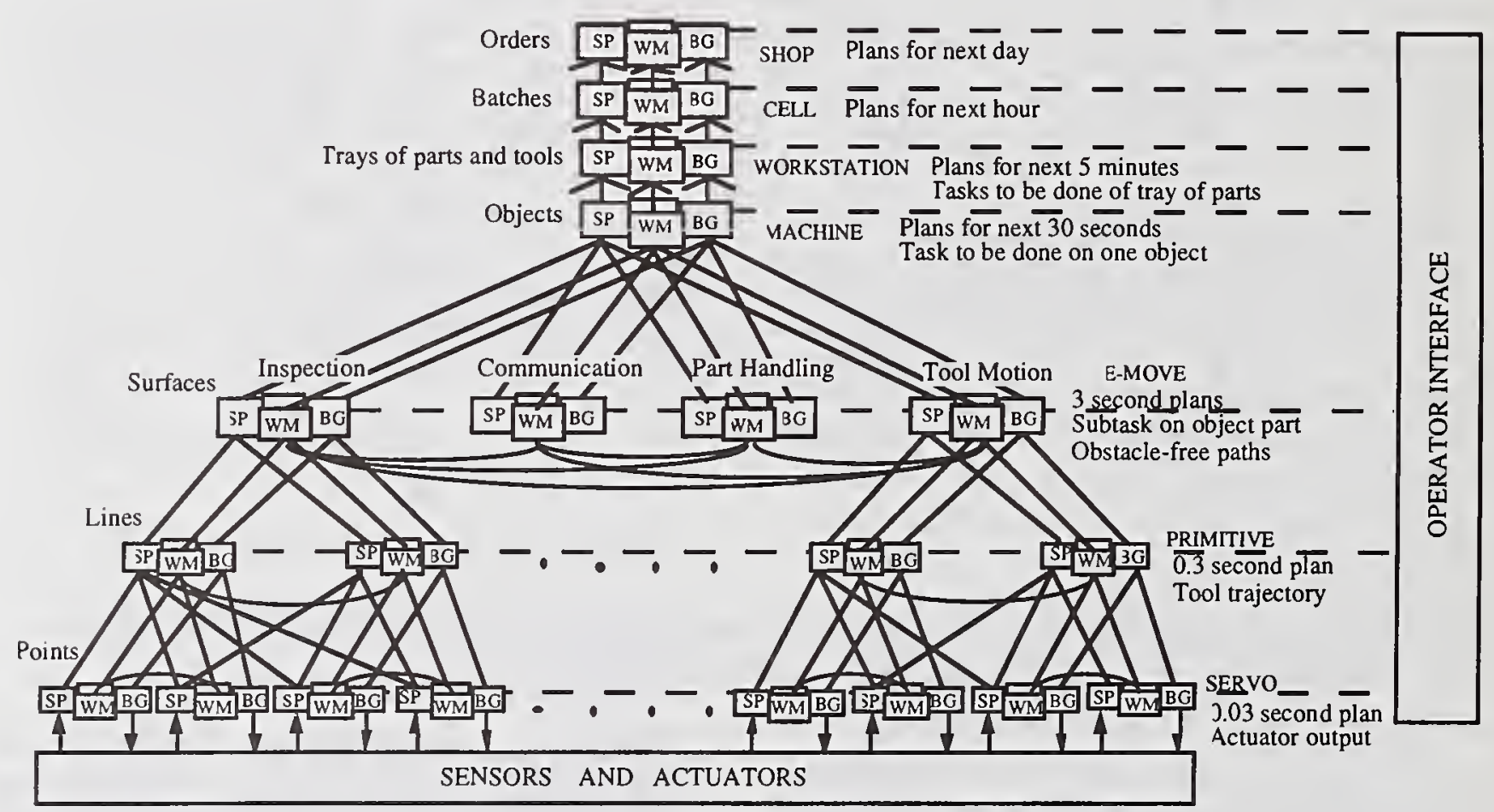

Figure 2. An intelligent system architecture for manufacturing.

Typically, control loops are closed in each node. Each layer of this hierarchical architecture has a characteristic servo loop bandwidth and a characteristic planning horizon. For example, at the servo level the feedback loop might be closed every 3 milliseconds with a planning horizon of 30 milliseconds. At the primitive level, the feedback loop may close every 30 milliseconds with a planning horizon of 300 milliseconds. At the elemental move level the feedback loop is closed every 300 milliseconds with a planning horizon of 3 seconds. At the individual machine level, the planning horizon may be 30 seconds. At the workstation level, the planning horizon may be 5 minutes, and at the cell level an hour. At the shop level, the planning horizon may extend a day into the future.

The architecture is hierarchical in order to deal with complexity. The hierarchical structure enables a manufacturing system of arbitrary complexity to be decomposed into manageable units. Each level of the hierarchy has a characteristic range and resolution in time and space. Each chain of command within the 
hierarchy generates a hierarchy of tasks and plans for a hierarchy of agents. The world model and knowledge database in the nodes contain a hierarchy of entities and events. This hierarchical decomposition enables the development of an engineering methodology within a systematic framework of modular components, standard interfaces, that enable designers to easily add sensors and upgrade processing capabilities.

The intelligent system architecture for manufacturing shown in Figure 2 is a derivative of the Real-time Control System (RCS) reference model architecture for intelligent systems that has been developed at NIST and elsewhere over the past two decades. RCS has been used to develop a wide variety of applications including robots for machine loading and unloading using vision and tactile sensors, deburring and chamfering using active force control, integration of an Automated Manufacturing Research Facility including robots, machine tools, a turning machine, a coordinate measuring machine, and automatic guided vehicles. RCS also has been used to control multiple autonomous undersea vehicles, a space station telerobotic system, a simulated nuclear submarine operating under ice during a transit of the Bearing Straits. Intelligent controllers have been developed for coal mine automation, post office automation, a RoboCrane system, a next generation inspection system, and an Enhanced Machine Controller for a five axis machining center. (Albus 97)

Current work at NIST is concentrating on developing an systematic engineering methodology for developing intelligent systems applications. We are working on generic templates and software development tools. We are working with our four sister divisions in the Manufacturing Engineering Laboratory to develop a National Advanced Manufacturing Testbed (NAMT) which will perform research in virtual and distributed manufacturing. The NAMT will be a place where advanced laboratory equipment such as the Hexapod machine tool, the Next Generation Inspection Systems, and the RoboCrane, the Advanced Deburring and Chamfering System, and the HMMWV Unmanned Ground Vehicle can be accessed and used by experimenters at remote locations anywhere in the world. Through the NAMT facilities, experimenters will also be able to access experimental equipment in other divisions at NIST, as well as at universities, industry labs, and other government labs such as Sandia and Oak Ridge.

Our Intelligent Systems Division is planning to distribute RCS software as freeware to universities and to work with professors to develop lecture material and lab experiments that can be used for course work in intelligent control. Through the NAMT virtual and distributed testbed, we will work with professors and students to develop algorithms and do experiments and perform thesis research in intelligent manufacturing, construction, and unmanned vehicle systems. We also hope to collaborate with the National Science Foundation and other sources of funding to support users groups, promote student competitions, and fund collaborative research.

If this is successful, we intend to solicit industrial partners to propose an Advanced Technology Program targeted thrust area for industry research in intelligent systems.

\section{Conclusion}

So this is my proposed road map for the future. The goal of the roadmap is to understand the nature of mind and to build systems that will improve economic prosperity, strengthen military security, and improve human well being.

The suggested route is:

(1) to develop a theory and reference model architecture for intelligent systems,

(2) to develop an engineering methodology and educational curriculum,

(3) to distribute freeware and provide user support,

(4) to build a virtual and distributed testbed environment so that researchers in many different locations can work together, and

(5) to solicit partners and pursue additional funding to advance the theory and practice of intelligent systems and semiotics.

In closing, let me reiterate that intelligent systems are important for a wide variety of scientific, economic, and military reasons. The technology of intelligent systems is moving rapidly, but there are some gaps that impede 
progress. Some suggestions have been made to fill in these gaps and achieve the ultimate goal of human well being.

As a final thought, I want to suggest that intelligent systems represent a fundamentally new technology that will create a new industrial revolution. The impact of this new industrial revolution will at least equal, and may far exceed, that produced by the invention of the steam engine and the discovery of electricity. The ultimate result could be a breakthrough in productivity growth that would bring an end to poverty and introduce a golden age for human kind.

\section{References}

Albus, J.S., "Outline for a Theory of Intelligence," IEEE Transactions on Systems, Man and Cybernetics, Vol. 21, No. 3, pgs. 473-509, May/June, 1991

Albus, J.S., "The NIST Real-time Control System (RCS): an approach to intelligent systems research", J. Expt. Theor. Artif. Intell. 9, p.157174,1997 


\title{
Computational Semiotics and Fuzzy Linguistics On Meaning Constitution and Soft Categories*
}

\author{
Burghard B. Rieger \\ FB II: Department of Computational Linguistics - University of Trier \\ rieger@ldv01. Uni-Trier.de
}

\begin{abstract}
Whereas most cognitive approaches in the study of language have been developing hypotheses concerning the principles of knowing and understanding natural languages (i.e. competence) without bothering too much about communicative language usages in realworld situations (i.e. performance), new semiotic approaches in cognitive computational linguistics explore the procedures believed to underlie processes of language learning and understanding. They do so by simulating these capabilities as system behaviour under recourse to modeled structures, observable in very large samples of situated natural language discourse and represented in vector space formats via numerically specified quantitative methods of dynamic (re-)construction. It will be argued that the ecological understanding of informational systems in Computational Semiotics corresponds well to the procedural modeling and numerical reconstruction of processes that simulate the constitution of meanings and the interpretation of signs (semiosis). The theories of fuzzy sets [24] and possibility distributions [23] together with their derivatives in soft computing [25] appear to be promising in providing suitable formats for computational approaches to natural language processing without the obligation neither to reject nor to accept traditional formal and modeltheoretic concepts or ontologies. Examples from fuzzy linguistic research [10] [18] will be given to illustrate these points.
\end{abstract}

\section{Cognitive Information Processing}

For the majority of researchers in knowledge representation and natural language semantics the common ground and widely accepted frame for their modeling may be found in the dualism of the rationalistic tradition of thought as exemplified in its matter-mind notion of an independent (objective) reality and some (subjective) conception of it.

\subsection{The traditional approach}

According to the realistic view, the meaning of any portion of language material (like e.g. discourse, utterance, word(token), morph, phone, etc.) is interpreted as being an instantiation of (or partly derivable from) certain other entities, called linguistic categories (like e.g. text, sentence, word(type), morpheme, phoneme, etc.),

"presented at the NIST/NSF/IEEE/DARPA Interntl. Multidisciplinary Conference INTELLIGENT SYSTEMS: A SEMIOTIC Perspective, Oct. 20-23, 1996, Gaithersburg, Md, USA with the understanding that these categories structure natural language material according to their compositional functions. It is by these functions that language material (strings of terms) appear to be composed of linguistic entities (aggregates of categories) to form structures and it is also by these functions that the quality of language structures (having meaning) is conceived as being part of both, the physical reality of language material and the semantic significance of linguistic signs. Mlustrating this twofold membership are the graph-theoretical formats which have become standard representations for natural language meanings, both as relational structure and as referential denotation. Thus, relating arc-and-node configurations with sign-and-term labels in graphs like trees and nets appears to be but another aspect of the traditional mindmatter-duality according to which a realm of meanings is presupposed very much like the assumption of the pre-given structures of the real world related by signs. Accepting this duality has neither allowed to explain where the structures or where the labels come from nor how their mutual relatedness as meanings of signs can be derived. The emergence of the meaning relation, therefore, never occurred to be in need of some explanatory modeling because the existence of signs, objects and meanings were taken for granted and hence seemed to be out of all scrutiny. Under this presupposition, fundamental semiotic questions of semantics simply did not come up, they have hardly been asked yet, and are still far from being solved.

\subsection{Modeling cognition}

Extending an earlier attempt [22] to classify approaches in cognitive science, we may roughly discern four ${ }^{1}$ types of approaches in modeling cognition:

$\triangleright$ the cognitive approaches presuppose the existence of the external world, structured by given objects and properties and the existence of representations of (fragments of) this world internal to the system, so that the cognitive systems' (observable) behaviour of action and reaction may be modelled by processes operating on these structures;

$\triangleright$ the associative approach is described as a dynamic structuring based on the model concept of self-organization with cognitive systems constantly

\footnotetext{
${ }^{1}$ There were only the first three of these four approaches distinguished by Varela/Thompson/Rosch (1991).
} 
adapting to changing environmental conditions by modifying their internal representation of them.

Whereas both these approaches apparently draw on the traditional rationalistic paradigm of mind-matterduality-static the former, dynamic the latter-in presupposing the external world structure and an internal representation of it, the third and fourth category do not:

$\checkmark$ the enactive approaches may be characterized as being based upon the notion of strcutural coupling. Instead of assuming an external world and the systems' internal representations of it, some unity of structural relatedness is considered to be fundamental of-and the (only) condition for-any abstracted or acquired duality in notions of the external and internal, object and subject, reality and its experience;

$\checkmark$ the semiotic approaches focus on the notion of semiosis and may be characterized by the process of enactment too, supplemented, however, by the representational impact. It is considered fundamental to the distinction of e.g. cognitive processes from their structural results which-due to the traces these processes leave behind-may emerge in some form of knowledge whose different representational modes comply with the distinction of internal or tacit knowledge (i.e. memory) on the one hand and of external or declarative knowledge (i.e. language) on the other.

According to these types of cognitive modeling, computational semiotics can be characterized as aiming at the dynamics of meaning constitution by simulating processes of multi-resolutional representation [5] within the frame of an ecological information processing paradigm [18].

As we take human beings to be systems whose knowledge based processing of represented information makes them cognitive, and whose sign and symbol generation, manipulation, and understanding capabilities render them semiotic, we may do so due to our own daily experience of these systems' outstanding ability for representing results of cognitive processes, organize these representations, and modify them according to changing conditions and states of system-environment adaptedness.

\section{Computational Semiotics}

For the semiotic approach to human cognition (constituting computational semiotics) such representations resulting from complex semiotic cognitive information processing may be found in any natural language discourse. In an aggregated form of pragmatically homogeneous text corpora [7] communicatively performative natural language discourse provides a cognitively highly interesting and empirically accessible material whose extreme structuredness may serve as a guideline for the cognitively motivated, empirically based, and computationally realized research in the semiotics of language, too.

Following this line, however, will necessitate to pass on from traditional approaches in competence oriented linguistics analysing introspectively the propositional contents of singular sentences as conceived by ideal speakers/writers towards a new understanding of meaning constitution as a dynamic process based upon the semiotic cognitive information processing the traces of which are to be identified and systematically reconstructed on the basis of empirically well founded observation and rigorous mathematical description of universal regularities that structure and constitute different levels of representations in masses of pragmatically homogeneous texts produced by real speakers/writers in actual situations of either performed or intended communicative interaction. Only such a performance oriented semiotic approach will give a chance to formally reconstruct and model procedurally both, the significance of entities and the meanings of signs as a function of a first and second order semiotic embedding relation of situations (or contexts) and of language games (or cotexts) which corresponds to the two-level actualisation of cognitive processes in language understanding [18].

\subsection{Ecological information systems}

Life may be understood as the ability to survive by adapting to changing requirements in the real world. In terms of the theory of information systems, faculties like perception, identification, and interpretation of structures (external or internal to a system) may be conceived as a form of information processing which (natural or artificial) systems-due to their own structeredness-are able to perform. Thus, living systems receive or derive information only from relevant portions of their surrounding environments, they learn from experience, and change their behaviour accordingly. In contrast to other living systems which transmit experiential results of environmental adaptation only biogenetically ${ }^{2}$ to their descendants, human information processing systems have additional means to convey their knowledge to others. In addition to the vertical transmission of system specific (intraneous) experience through (biogenetically successive) generations, mankind has complementally developed horizon$\mathrm{tal}$ means of mediating specific and foreign (extraneous) experience and knowledge to (biogenetically unrelated) fellow systems within their own or any later generation. This is made possible by a semiotic move that allows not only to distinguish processes from results of experience but also to convert the latter to knowledge facilitating it to be re-used, modified and improved in learning. Vehicle and medium of this move are representations, i.e. complex sign systems which constitute languages and form structures, called texts which may be realized in communicative processes, called actualisation.

\footnotetext{
${ }^{2}$ According to standard theory there is no direct genetic coding of experiential results but rather indirect transmission of them by selectional advantages which organisms with certain genetic mutations gain over others without them to survive under changing environmental conditions.
} 
In terms of the theory of information systems, texts-whether internal or external to the systemsfunction like virtual environments ${ }^{3}$.

\subsection{Modes of Representation}

Considering the system-environment relation, virtuality may be characterized by the fact that it dispenses with the identity of space-time coordinates for systemenvironment pairs which normally prevails for this relation when qualified to be indexed real. It appears, that this dispensation of identity-for short: spacetime-dispensation-is not only conditional for the possible distinction of (mutually and relatively independent) systems from their environments, but establishes also the notion of representation. Accordingly, immediate or space-time-identical system-environments existing in their space-time-identity may well be distinguished from mediate or space-time-dispensed systemenvironments whose particular representational form (texts) corresponds to their particular status both, as language material (being signs), and as language structure (having meaning). This double identity calls for a particular modus of actualisation (understanding) that may be characterized as follows:

For systems appropriately adapted and tuned to such environments actualisation consists essentially in a twofold embedding to realize

$\checkmark$ the spaciotemporal identity of pairs of immediate system-environment coordinates which will let the system experience the material properties of texts as signs (i.e. by functions of physical access and mutually homomorphic appearance). These properties apply to the percepts of language structures presented to a system in a particular discourse situation, and

$\checkmark$ the representational identity of pairs of mediate system-environment parameters which will let the system experience the semantic properties of texts as meanings (i.e. by functions of emergence, identification, organisation, representation of structures). These apply to the comprehension of language structures recognized by a system to form the described situation

Hence, according to the theory of information systems, functions like interpreting signs and understanding meanings translate to processes which extend the fragments of reality accesssible to a living (natural and possibly artificial) information processing system. This

\footnotetext{
${ }^{3}$ Simon's (1982) remark "There is a certain arbitrariness in drawing the boundary between inner and outer environments of artificial systems. .... Long-term memory operates like a second environment, parallel to the environment sensed through eyes and ears" (pp. 104) is not a case in point here. Primarily concerned with where to place the boundary, he does not seem to see its placing in need to be justified or derived as a consequence of some possibly representational processes we call semiotic. As will become clear in what follows, Simon's distinction of inner (memory structure) and outer (world structure) environments is not concerned with the special quality of language signs whose twofold environmental embedding (textual structure) cuts accross that distinction, resolving both in becoming representational for each other.
}

extension applies to both, the immediate and mediate relations a system may establish according to its own evolved adaptedness or dispositions (i.e. innate and acquired structuredness, processing capabilities, represented knowledge).

\subsection{Semiotic enactment}

Semiotic systems' ability to actualize environmental representations does not merely add to the amount of experiential results available, but constitutes also a significant change in experiencial modus. This change is characterized by the fact that only now the processes of experiencing may be realized as being different and hence be separable from the results of experience which in immediate system-environments appear to be indistinguishable. Splitting up experience in experiencial processes and experiencial results-the latter being representational and in need for procedural actualisation by the former-is tantamount to the emergence of virtual experiences which have not to be made but can instead just be tried, very much like hypotheses in an experimental setting of a testbed. These results-like in immediate system-environments-may become part of a system's adaptive knowledge but may also-other than in immediate system-environments-be neglected or tested, accepted or dismissed, repeatedly actualized and re-used without any risk for the system's own survival, stability or adaptedness.

This in a way experimental quality of textual representations which increases the potentials of adaptive information processing beyond the system's lifespan, is constrained simultaneously by dynamic structures corresponding to knowledge. The built-up, employment, and modification of these structural constraints ${ }^{4}$ is controlled by procedures whose processes determine cognition and whose results constitute adaptation. Systems properly attuned to textual system-environments have acquired these structural constraints (language learning) and can perform certain operations efficiently on them (language understanding). These are prerequisites to recognizing mediate (textual) environments and to identify their need for and the systems' own ability to actualize the mutual (and trifold) relatedness constituting what PEIRCE called semiosis ${ }^{5}$. Systems capable of and tuned to such knowledge-based processes of actualisation will in the sequel be referred to as semiotic cognitive information processing systems (SCIPS) $[17,19]$.

Representation, therefore, has to be considered fundamental to the distinction of the processes of cognition from their results which may emerge-due to the traces these processes leave behind-in some structure (knowledge). Different representational modes of this

\footnotetext{
What Simon (1982) calls memory in accordance with his questioning of the inner-outer-distiction of cognitive systems and their environments.

${ }^{5}$ By semiosis I mean [...] an action, or influence, which is, or involves, a coōperation of three subjects, such as sign, its object, and its interpretant, this tri-relative influence not being in any way resolvable into actions between pairs. (Peirce 1906, p.282)
} 
structure not only comply with the distinction of internal or tacit knowledge (i.e. memory) on the one hand and of external or declarative knowledge (i.e. texts) on the other ${ }^{6}$, but these modes also relate to different types of formats (distributional vs. symbolic), modeling (connectionist vs. rule-based) and processing (stochastic vs. deterministic). It is this range of correspondences that Fuzzy Linguistics is based upon and tries to exploit to come up with a unifying framework for most of the different approaches followed sofar.

Soft categorising appears to be a prerequisite for fuzzy linguistic modeling, examples of which will illustrate the notion of dynamic structures emerging from corpora of natural language discourse when processed.

\section{Fuzzy Linguistic Modelling}

It is far from certain yet whether-and if so, howsemiotic models will help to understand how structures may emerge from orders of some kind and how these orders evolve from regularities which multitudes of repeatedly observable entities show. Recent research findings, however, give rise to expect that processes which determine regularities and assemble them to form (intermediate) representations whose properties resemble (or can even be identified with) those of observable entities may indeed be responsible for (if not identical with) the emergence and usage of sign-functional structures in language understanding systems, both natural and artificial. As more abstract (theoretical) levels of representation for these processes -other than their procedural modeling-are not (yet) to be assumed, and as any (formal) means of deriving their possible results-other than by their (operational) enactment-are (still) lacking, it has to be postulated that these processes-independent of all other explanatory paradigms - will not only relate but produce different representational levels in a way that is formally controlled or computable, that can be modeled procedurally or algorithmized, and that may empirically be tested or implemented [4]. Procedural models are understood to denote a class of (re-)presentational or modeled (re-)constructions of entities whose interpretation is not (yet) tied to an underlying theory which would provide the semantics for the entities (or expressions) that these type of models present. Instantiating their defining procedures as implemented algorithms will result in processes which produce some observable structures that can only then be compared to those of the modeled original.

As some of these procedural characteristics have

\footnotetext{
${ }^{6}$ Whereas tacit knowledge cannot be represented other than by the immediate system-environments' corresponding states, explicit knowledge is bound to acquire some formal properties in order to become externally presented and thereby part of mediate system-environments. Natural languages obviously provide these formal properties-as partly identified by research in linguistic competence (principles knowledge and acquisition of language)-whose enactment-as investigated in studies on natural language performance (production and understanding of texts)-draws cognitively on both bases of (explicit and tacit) knowledge.
}

also be claimed by cognitive linguistic approaches and computational models of language understanding, their main traits may help to illustrate the different positions of semiotic modeling in fuzzy linguistics.

\subsection{Cognitive linguistic strata}

Cognitive theory has long identified the complex of language understanding to be a modular system of subsystems of information processing. The idea of symbolic representation and the computer metaphor offered a frame for modeling cognitive processes, formally grounded on logical calculi and procedurally realized in algorithms operating on representational structures. Following and partly supplementing strata of semiotic description and analysis of signs, different levels of modular aggregation of external and internal information have been distinguished in cognitive models of language understanding. These partly correspond to and partly cut accross the syntacticssemantics-pragmatics distinction in the semiotic relatedness of signs, the utterance-discourse-corpus levels of performative language processing, and the hierarchy of morpho-phonological, syntax-sentencial and lexicosemantic descriptions in traditional models of structural linguistics.

In one of the rare ventures on discussing of how cognitive, i.e. knowledge based information processing mechanisms may be provided with the knowledge bases they are meant to operate on, and how these knowledge structures may be related to observable language data, BIERWISCH (1981) sketches a hierarchy of information processing mechanisms whose representational format (sets of rewrite rules operating on structured data) allows algorithms be formulated and implementations be found to guarantee their computability. According to this schema (Fig. 1) and starting with the morphophonological level, an information processing mechanism $M_{1}$ is postulated which receives utterances as input and produces some associated structures as output. In doing so, however, the mechanism's performance will be determined not only by the external input strings but also by some internal knowledge of elements and rules which allow to agglomerate the structures identified. The acquisition and representation of this internal knowledge is hypothesized as resulting from a process $M_{2}$ which also includes a multitude of rudimentary, incomplete, and tentative $M_{1}$-kind processes. $M_{2}$ is assumed to be a complex information processing mechanism whose inputs are corpora of utterances together with some environmental information, and whose outputs will be the grammars underying these utterances. Again, this mechanism's results will not only and completely be determined by the external inputs but also by some internal structures which are believed to control the human language faculty in a fundamental way as so-called linguistic universals. These may (or may not) be assumed to be derivable as results of an information processing mechanism $M_{3}$ whose input is as comprehensive (or unspecified) as the term languages might allow. 


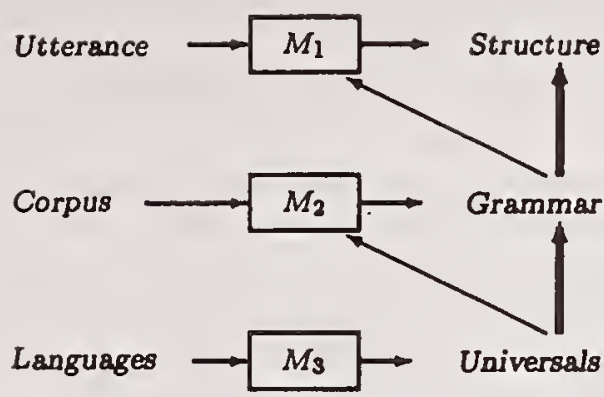

Figure 1

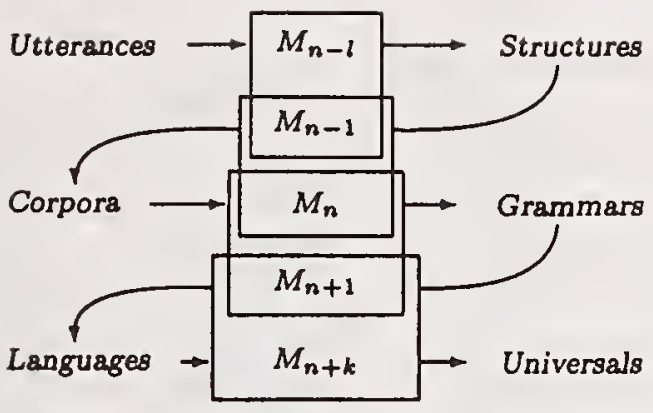

Figure 2

Schemata of model hierarchy of cognitiv linguistic strata of mechanisms (BIERWISCH) as compared to model tiling of computational semiotic coverage of procedures (RIEGER) for the analysis and representation of (abstracted and observable) language phenomena.

Taking the relation of inclusion for $M_{1} \subset M_{2}$ to hold also for $M_{2} \subset M_{3}$, and considering $M_{1}, M_{2}, M_{3}$ computationally specifyable procedures of language analysing processes instead of mere metaphors for some (more or less plausible) mechanisms of the human mind, then it appears reasonable to consider $M_{3}$ a collection of all the processes of methodical analysis, representation, and comparison of structured sets of utterances from different languages, including the processes in $M_{1}$ as a device that explicidly specifies an utterance's structure relative to a given grammar, and the processes in $M_{2}$ as a system that generates a grammar from a corpus of utterances relative to the given set of universals. This modeling view allows for the notions of Universals $\Rightarrow$ Grammar $\Rightarrow$ Structure to be understood as variables of theoretical constructs hinged on empirical regularities observed in Languages, Corpus, Utterance respectively. Whereas the latter are external representations, the former are internal to any SCIP-system and considered external representations only under the competence linguistic approach to cognitive modeling. As such they are hypothesized to form a hierarchy of linguistic-not language - entities which formally specify a class of other linguistic entities (following the double arrows in Fig. 1).

The model theoretical and operational problems inherent in this setup concern the (non universal and highly restrictive) representational format which is assumed to enable the denotation of universals, grammar and structure, and the essentially top-down, nonrecursive propagation of externally presented but internally processed results of these mechanisms. Thus, $M_{3}$ whose performance in identifying universals and representing them externally depends crucially on the efficient performance of $M_{2}$ which is said to employ these universals as internal procedural constraints in order to identify syntactic regularities and represent them externally in a rule based format as grammars. Grammars, in turn, have to be employed as internal procedural constraints by $M_{1}$ if this mechanism's identification processes and the external representation of their findings is meant to be successful.

Distinguishing between these two kinds of structures either external or internal to the mechanisms $M$ introduced so far, is indicative of the systems theoretical view proposed in semiotic modeling. It easily allows to translate these mechanisms as sets of procedures which allow to describe and simulate a living systems' abilility to process environmental input (external structures) according to procedural constraints known to the system (internal structures) in order to produce some results of this processing. However, it appears not at all conclusively compelling to assume that these procedural constraints and the processing results need to be represented in a rule-based format. According to an ecologically motivated systems theoretical view, systems enacting these processes under boundary conditions as determined by their surrounding environments, or their internal structuredness, or both, will have to process certain inputs to produce specified output structures. But identifying their status of being at the same time internal and external to the processing system is tantamount to the methodological dilemma which can solely be solved on the grounds of revising the representational mode and the formatting constraints which the model construction has to be decided on to allow.

Following СномsкY these modes have been restricted to abstract principles of language competence by processes [2] whose assumed rule-based determinacy consequently led to formal representations of these rules giving rise to the above model hierarchy of discrete strata [1]. In trying to relate these strata to observable performative language data structures in order to mediate observable language regularities with theoretical constructs supposedly representing principles underlying these constructs, the methodological shortcomings of the cognitive linguistic approach are revealed. It suffers from competence theoretically inspired idealisations of regularities and theoretical abstractions (like universals, grammars, sentences) whose symbolic notations and formal expressions may be scrutinized for their syntactic correctness but lack empirically observable and experimentally testable procedures of language representation which are independant from competent speakers' understanding of that 
language.

\subsection{Fuzzy linguistic tiling}

Other than cognitive linguistic and competence theoretical mechanisms, we propose cognitive semiotic and language performative procedures which redefine the modularity of language understanding as an overlapping covering of computational processes. The classical and coarse three-stage description and modeling of linguistic regularities will be replaced or rather complemented [11] by a multi-stage covering of semiotic procedures $M_{n-l}$ to $M_{n+k}$ (Fig. 2) which allows for the definition of more adequate soft categories or intermediate representations to fit regularities of entities on any level. Their essentially cognitive character will not be borrowed from any predefined strata (and their puportedly related abstract categories) but is to be derived as a result of their performance, i.e. the ability to transform linearly structured entities (strings) of one level to multi-dimensional structures of entities (vectors) on another.

This is achieved by analysing the linear or syntagmatic and selective or paradigmatic constraints which natural language structure imposes on the formation of (strings of) linguistic entities on whatever level of entity formation. It has been shown and illustrated elsewhere [15], [9] in some detail, that fuzzy linguistic modeling allows to derive the representational means (e.g. soft categories, continuous gradation, variable granularity, flexible plasticity, dynamic approximation, etc.) which crisp categories and competence theoretically inspired idealisations of performative regularities lack. The (numerical) specificity and (procedural) definiteness of sub-symbolic, distributed formats in entity formation appear to provide for higher phenomenological compatibility and more cognitive adequacy than traditional levels of categorial representation whose symbolic mediation and syntactic correctness could only formally be scrutinized but not empirically or experimentally be tested [11].

\section{Procedural (Re-)Construction}

The success of computational language analysis and generation is based upon adequate structural descriptions of input strings and their semantic interpretations. This was assumed to be made possible by the correctness of rule based representations of (syntactic and lexical) knowledge of language and of (referential and situative) world knowledge on which grammar formalisms and deductive inferential mechanisms can operate on. Although the essentially static representations of structures in this kind of cognitive modeling of language processing (based on monotonic logics, symbolic representations, rule-based operations, serial processing, etc.), has produced considerable advances in formal theory and the consistent development of increasingly more complex systems, their idea of representing language entities as essentially crisp categorial type linguistic entities proves to become increasingly problematic. As the processing of very large language corpora $(\text { VLLC })^{7}$, has made clear, traditional linguistic categories do not reduce but increase model complexity when applied to regularities and structures which quantitative-numerical means may easily identify and represent. Trying to map such sub-rule regularities and sub-symbolic structures to inadequate categories will generally result in a large number of borderline cases, variations, and ambiguities which then have to be dealt with, but could possibly more easily be avoided from the very start.

\subsection{Exploiting constraints}

Structural linguistics has given substantial hints ${ }^{8}$ on how language items come about to be employed in communicative discourse the way they are. They have identified the fundamental constraints that control the multi-level combinability and formation of language entities by distinguishing the restrictions on linear aggregation of elements (syntagmatics) from restrictions on their selective replacement (paradigmatics). This distinction allows within any sufficiently large set of strings of natural language discourse to ascertain syntagmatic regularities of element aggregations on level $n$ whose characteristic patterns form paradigmatic regularities tantamount to their aggregational status on level $n+1$. As has been illustrated above, the distinction of representational levels is tantamount to the categorial constraints applied when identifying regularities. Fully deterministic if-then rules will result in a rather coarse three-level hierarchy of categorial description whereas probabilistic or possibilistic dependency produces a continuous, multi-level covering of distributional representations. Thus, it can be distinguished sharply between cognitive linguistic and semiotic procedures the computations of the latter transform structured input data according to its immanent regularities to yield new, structural representations emerging from that computation (as hypothesized by performative linguistics and realized in procedural models of computational semiotics). The elements of these new structures are value distributions or vectors of input entities that depict properties of their structural relatedness, constituting multi-dimensional (metric) space structures (semiotic spaces). The elements may also

\footnotetext{
${ }^{7}$ The Trier dpa-Corpus for instance comprises the complete textual materials from the so-called basic news real service of 1990-1993 (720.000 documents) which the Deutschen Presseagentur (dpa), Hamburg, deserves thanks to have the author provided with for research purposes. After deletion of editing commands the Trier-dpa-Corpus consists of approx. $180 \mathrm{Mio}$. $\left(18 \cdot 10^{7}\right)$ running words (tokens) for which an automatic tagging and lemmatising tool is under development. It is this corpus which provides the performative data of written language use for the current (and planned) fuzzy-linguistic projects at the our department.

${ }^{8}$ In subscribing to a structuralistic view of natural languages, the distinction of langue-parole and competence-performance in modern linguistics allowes for different levels of language description and linguistic analysis. Being able to segment strings of language discourse and to categorize types of linguistic entities, however, is but making analytical use of structural couplings presented by natural language discourse to semiotic systems properly attuned.
} 


\begin{tabular}{|c|c|c|c|c|c|}
\hline $\begin{array}{c}n- \\
\text { grams }\end{array}$ & $\begin{array}{r}\left.|| F_{n} \mid\right) \\
\text { (fact.occurr.) } \\
\end{array}$ & $\begin{array}{c}\left|T_{n}\right|=\left|Z^{n}\right| \\
\text { (theor.possib.) }\end{array}$ & $\begin{array}{l}100 \cdot \frac{F_{\mathrm{n}}}{T_{n}} \\
\text { percent }\end{array}$ & $\begin{array}{r}\left.A_{n}=m \cdot\left|F_{n-1}\right|\right) \\
\text { (act.possib.) }\end{array}$ & $\begin{array}{l}100 \cdot \frac{F_{n}}{A_{n}} \\
\text { percent }\end{array}$ \\
\hline 1 & 31 & 31 & 100,000 & 31 & 100,000 \\
\hline 2 & 817 & 961 & 85,015 & 961 & 85,015 \\
\hline 3 & 10.175 & 29.791 & 34,154 & 25.327 & 40,174 \\
\hline 4 & 54.470 & 923.521 & 5,898 & 315.425 & 17,268 \\
\hline 5 & 164.045 & 28.629 .151 & 0,572 & 1.688 .570 & 9,715 \\
\hline 6 & 357.632 & 887.503 .681 & 0,040 & 5.085 .395 & 7,032 \\
\hline 7 & 634.767 & 27.512 .614 .111 & 0,002 & 11.086 .592 & 5,725 \\
\hline \multicolumn{2}{|c|}{ Size of test-corpus } & .326 & (signs) & 502.587 & (words) \\
\hline
\end{tabular}

Table 1: Graph-(letter-)combinatorics with (theoretically and faktually) possible and actually occurring types of $n$-grams in a subset of the Trier dpa-Korpus

be interpreted as fuzzy sets allowing set theoretical operations be exercised on them that do not require categorial type (crisp) definitions of concept formations. Computation of letter (morphic) vectors in word space, derived from n-grams of letters (graphemes) [10] [11] as well as of word (semic) vectors in semantic space [12] [13], derived from wordtype correlations of their tokens in discourse will serve to illustrate the operational flexibility and fine granularity of vector notations [9], [16] to identify regularities of semiotic meaning constitution in language performance which traditional linguistic categories fail to represent.

\subsection{The word space}

The following notations will be used to outline the computational semiotic approach on the morphic level:

$n$-grams are $n$-elementary strings of entities. For $n \geq$ 2 they may be analysed as ordered pairs of adjacent items (letters, graphs, sign-strings, word-strings, etc.) which are the basis of

abstractions over such items procedurally be determined as soft categorial types (corresponding to characters, graphems, morphems, syllables, words, etc.). These have been introduced as dispositional dependency structures (DDS) [14] [8] and formally declared as structured

fuzzy (sub-)sets of multi-dimensional sign inventories $Z^{n}$ with $n \geq 1$

$$
\tilde{X}_{n}:=\left\{\left(x, \mu_{n}(x)\right): x \in Z^{n}\right\} \subseteq Z^{n} \times[0,1]
$$

whose elements' grades of membership are defined by the membership-function

$$
\mu_{n}: Z^{n} \rightarrow[0,1]
$$

membership-values $\mu_{n}(x)$ may be computed inductively as the overall tendency of linear chaining of items in language corpora. For an $n$-elementary string $x \in Z^{n}$ be $H_{n}(x)$ the frequency of $x$ occurring in a corpus. Then, for any

bi-gram $x=(y, z) \in Z^{n}, y \in Z^{n-1}, z \in Z$, the coefficient

$$
\mu_{n}(x)=\frac{H_{n}(x)}{H_{n-1}(y)} .
$$

with $Z=\left\{z_{1}, \ldots, z_{m}\right\}$ will yield for each $y \in Z^{n-1}$ a vector

$$
\left(\mu_{n}\left(y, z_{1}\right), \ldots, \mu_{n}\left(y, z_{m}\right)\right)^{T} \in \mathbb{R}^{m} .
$$

The set of all vectors reflect the morphological structure of the corpus analysed which is the numerically specified basis for the procedural definition of

soft categories which are defined as a system of fuzzy sub-sets of observed chaining regularities. They may be interpreted to represent elastic constraints operating on the language items' chaining tendencies which structure the corresponding corpus.

The presentation of the development of soft categories as elastic constraints (operating on different levels) can be simplified by their formal introduction as ( $n$-ary) fuzzy relations and their corresponding numerical formats of transition matrices (of higher orders).

For written German discourse analysed on typesetting level with $m$ discernable types of signs (letters) and maximum lengths $n$ of strings there are quite a number of theoretically possible (Tab. 1, col. $T_{n}$ ) crisp $n$-ary relations $T_{n}=Z^{n}$, i.e.

$$
\begin{aligned}
Z=T_{1} & =\left\{x_{1}\right. & & \left.: x_{1} \in Z\right\} \\
T_{2} & =\left\{\left(x_{1}, x_{2}\right)\right. & & \left.\vdots x_{1}, x_{2} \in Z\right\} \\
T_{3} & =\left\{\left(x_{1}, x_{2}, x_{3}\right)\right. & & \left.\vdots x_{1}, x_{2}, x_{3} \in Z\right\} \\
& \vdots & \vdots & \vdots \\
T_{n-1} & =\left\{\left(x_{1}, \ldots, x_{n-1}\right)\right. & & \left.\vdots x_{1}, \ldots, x_{n} \in Z\right\} \\
T_{n} & =\left\{\left(x_{1}, \ldots, x_{n}\right)\right. & & \left.\vdots x_{1}, \ldots, x_{n} \in Z\right\} .
\end{aligned}
$$

Out of these, however, only those have to be computed which are not only actually possible (col. $A_{n}$ ) but which have indeed been observed to factually occur, i.e. $F_{n} \subseteq F_{n-1} \times Z\left(T a b 1\right.$, col. $\left.F_{n}\right)$, i.e.

$Z=$

$$
\begin{aligned}
& F_{1} \subseteq\left\{x_{1} \quad: x_{1} \in Z\right\} \\
& F_{2} \subseteq\left\{\left(x_{1}, x_{2}\right) \quad: x_{1} \in F_{1}, x_{2} \in Z\right\} \\
& F_{3} \subseteq\left\{\left(x_{1}, x_{2}, x_{3}\right) \quad:\left(x_{1}, x_{2}\right) \in F_{2}, x_{3} \in Z\right\} \\
& \vdots \quad \vdots \quad \vdots \\
& F_{n-1} \subseteq\left\{\left(x_{1}, \ldots, x_{n-1}\right):\left(x_{1}, \ldots, x_{n-2}\right) \in F_{n-2}, x_{n-1} \in Z\right\} \\
& F_{n} \subseteq\left\{\left(x_{1}, \ldots, x_{n}\right) \quad:\left(x_{1}, \ldots, x_{n-1}\right) \in F_{n-1}, x_{n} \in Z\right\} .
\end{aligned}
$$

The fuzzy relational modeling (Eqns. 3 and 2 ) shows that even for higher $n$ only bi-grams have to be traced 


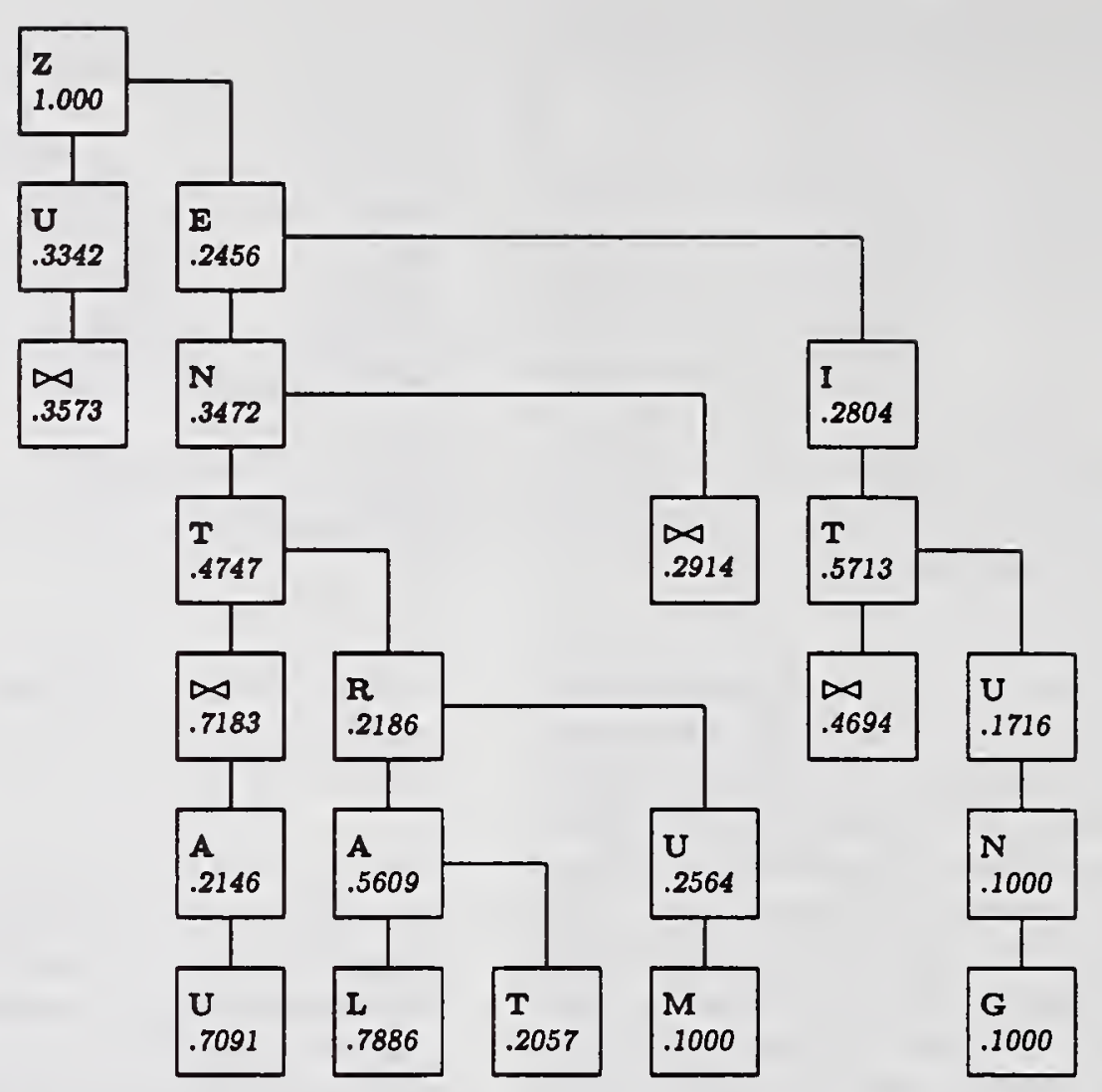

Figure 4: Tree representation of procedural soft category $\tilde{Z}$ depicting the hierarchy of graded letter agglomeration according to decreasing transition tendencies (in 7-grams) of German newspaper texts.

and computed due the $(n-1)$-ary relations computed on the previous level of representation. It is this principle of procedural self-similarity of $n$-ary agglomerative steps which allows for the trie-like representation [3] of entities that are labeled (by soft categorial $n$-relative letter transitions) and are an outcome of procedural constraints (over $n$ levels of processing) which produce a dynamically structured system of fuzzy relations that depicts the overall transition tendencies of signs. For the letter $Z$ this structure is given in Fig. 4 illustrating sub-regularities of morphic word formation.

\subsection{The semantic space}

Based upon the language entity word, its different types, and their frequencies of occurrence in natural language discourse, the fundamental distinction of agglomerative or syntagmatic and selective or paradigmatic relatedness can also be employed to reconstruct a system structure which will serve as base for a procedural model generating tree-like representations of dynamic semantic constraints. As these techniques have been introduced and elaborated elsewhere [9] [15] [20], their concise description may suffice here.

The core of the representational formalism can be characterized as a two-level process of abstractions. The first (called $\alpha$-abstraction) on the set of fuzzy subsets of the vocabulary provides the word-types' usage regularities or corpus points, the second (called $\delta$-abstraction) on this set of fuzzy subsets of corpus points provides the corresponding meaning points as a function of all differences of all usage regularities which a set of word-types may produce by its word-tokens' frequencies as observed in pragmatically homogeneous corpora of natural language texts.

The basically descriptive statistics to specify intensities of co-occurring lexical items in texts is centred around the correlational measure

$$
\begin{array}{r}
\alpha\left(x_{i}, x_{j}\right)=\frac{\sum_{t=1}^{T}\left(h_{i t}-e_{i t}\right)\left(h_{j t}-e_{j t}\right)}{\left(\sum_{t=1}^{T}\left(h_{i t}-e_{i t}\right)^{2} \sum_{t=1}^{T}\left(h_{j t}-e_{j t}\right)^{2}\right)^{\frac{1}{2}}} ; \\
-1 \leq \alpha\left(x_{i}, x_{j}\right) \leq+1
\end{array}
$$

where $e_{i t}=\frac{H_{i}}{L} l_{t}$ and $e_{j t}=\frac{H_{j}}{L} l_{t}$, computed over a textcorpus $K=\left\{k_{t}\right\} ; t=1, \ldots, T$ having an overall length $L=\sum_{t=1}^{T} l_{t} ; 1 \leq l_{t} \leq L$ measured by the number of word-tokens per text, and a vocabulary $V=$ $\left\{x_{n}\right\} ; n=1, \ldots, i, j, \ldots, N$ of word-types whose frequencies are denoted by $H_{i}=\sum_{t=1}^{T} h_{i t} ; 0 \leq h_{i t} \leq H_{i}$.

To specify these correlational value distributions' differences, a measure of similarity (or rather, dissimilarity) is used

$$
\begin{array}{r}
\delta\left(y_{i}, y_{j}\right)=\left(\sum_{n=1}^{N}\left(\alpha\left(x_{i}, x_{n}\right)-\alpha\left(x_{j}, x_{n}\right)\right)^{2}\right)^{\frac{1}{2}} \\
0 \leq \delta\left(y_{i}, y_{j}\right) \leq 2 \sqrt{n}
\end{array}
$$




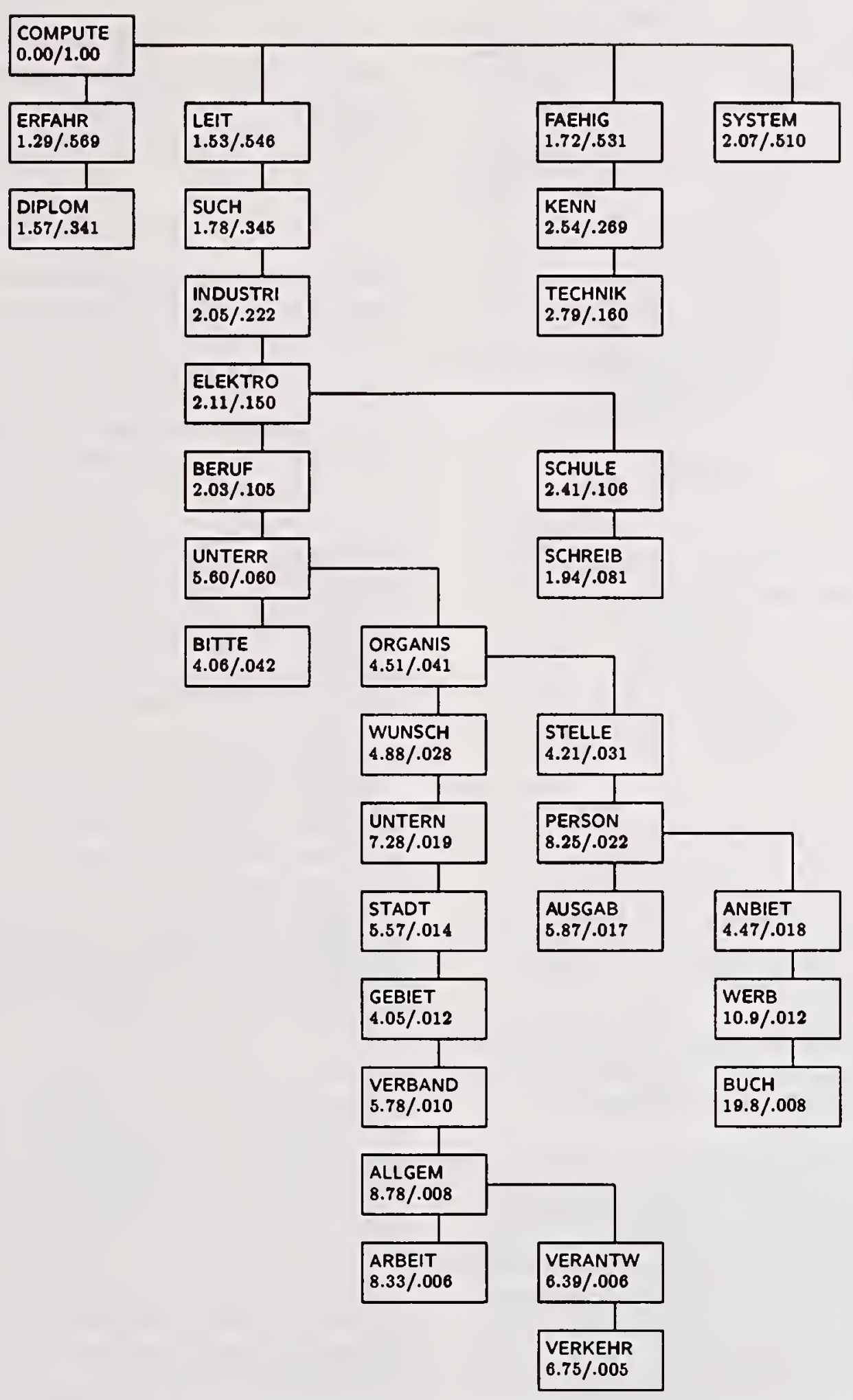

Figure 5: DDS-tree representation of meaning of COMPUTER as assembled for relevant meaning points' distances (first value) and criterialities (second value) on the basis of the semantic space $\langle S, \zeta\rangle$ intermediate as computed from a subcorpus of German newspaper texts (DIE WELT, 1964 Berlin Edition). 
The consecutive application of (Eqns. 7) on input texts and (Eqns. 9) on the output data of (Eqns. 7) allows to model the meanings of words as a function of differences of usage regularities (Fig. 6).

Thus, $\alpha_{i, j}$ allows to express pairwise relatedness of word-types $\left(x_{i}, x_{j}\right) \in V \times V$ in numerical values ranging from -1 to +1 by calculating co-occurring word-token frequencies (Eqn. 5) for pairs of items.

As a fuzzy binary relation, $\tilde{\alpha}: V \times V \rightarrow I$ can be conditioned on $x_{n} \in V$ which yields a crisp mapping

$$
\tilde{\alpha} \mid x_{n}: V \rightarrow C ;\left\{y_{n}\right\}=: C
$$

where the tupels $\left\langle\left(x_{n, 1}, \tilde{\alpha}(n, 1)\right), \ldots,\left(x_{n, N}, \tilde{\alpha}(n, N)\right)\right\rangle$ represent the numerically specified, syntagmatic usage regularities that have been observed for each word-type $x_{i}$ against all other $x_{n} \in V$. $\alpha$-abstraction over one of the components in each ordered pair defines

$$
x_{i}(\tilde{\alpha}(i, 1), \ldots, \tilde{\alpha}(i, N))=: y_{i} \in C
$$

Hence, the regularities of usage of any lexical item will be determined by the tupel of its $\alpha$-values which for all word types can be represented as vector space $C$.

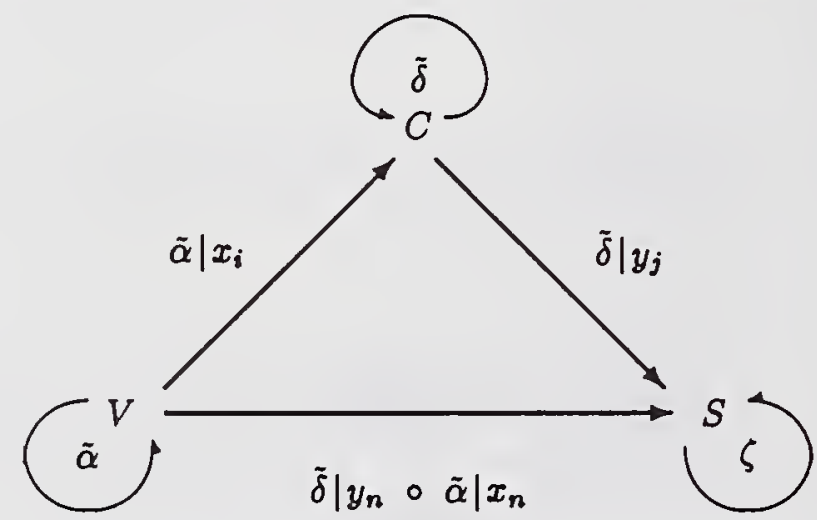

Figure 6: Fuzzy mapping relations $\tilde{\alpha}$ and $\tilde{\delta}$ between the structured sets of vocabulary items $x_{n} \in V$, of corpus points $y_{n} \in C$, and of meaning points $z_{n} \in S$.

Considering $C$ as representational structure of abstract entities constituted by syntagmatic regularities of word-token occurrences in pragmatically homogeneous discourse, then the similarities and/or dissimilarities of these entities will capture their corresponding word-types' paradigmatic regularities calculated by $\delta$ Eqn. 6 serving as second mapping function, As a fuzzy binary relation, $\tilde{\delta}: C \times C \rightarrow I$ can be conditioned on $y_{n} \in C$ which again yields a crisp mapping

$$
\tilde{\delta} \mid y_{n}: C \rightarrow S ;\left\{z_{n}\right\}=: S
$$

where the tupels $\left\langle\left(y_{n, 1}, \tilde{\delta}(n, 1)\right), \ldots,\left(y_{n, N} \tilde{\delta}(n, N)\right)\right\rangle$ represents the numerically specified paradigmatic structure that has been derived for each abstract syntagmatic usage regularity $y_{j}$ against all other $y_{n} \in C$. The distance values can therefore be abstracted analogous to Eqn. 8, this time, however, over the other of the components in each ordered pair, thus defining an element $z_{j} \in S$ called meaning point by

$$
y_{j}(\tilde{\delta}(j, 1), \ldots, \tilde{\delta}(j, N))=: z_{j} \in S
$$

Identifying $z_{n} \in S$ with the numerically specified elements of potential paradigms, the set of possible combinations $S \times S$ may structurally be constrained and evaluated without (direct or indirect) recourse to any pre-existent external world. Introducing a EuCLIDian metric

$$
\zeta: S \times S \rightarrow I
$$

the hyperstructure $\langle S, \zeta\rangle$ or semantic space $(S S)$ is declared constituting the system of meaning points as an empirically founded and functionally derived representation of a lexically labelled knowledge structure.

Weighted numerically as a function of an element's distance values and its associated node's level and position in the tree, $\operatorname{Cr}\left(z_{i}\right)$ either is an expression of the head-node's $z_{i}$ meaning-dependencies on the daughter-nodes $z_{n}$ or, inversely, expresses their meaning-criterialities adding up to an aspect's interpretation determined by that head [15]. To illustrate the feasibility of the $\Delta$-operation's generative procedure, the substructure of relevant constraints (related meaning points) $D D S\left(z_{i}\right) \subseteq\langle S, \zeta\rangle$ anchored with the lexical item $x_{i}, i=$ COMPUTER is shown in Fig. 4.3.

\section{Conclusion}

It has been outlined here that the morphic sign or the semantic meaning functions' ranges may be computed and simulated as a result of exactly those (semiotic) procedures by way of which (representational) structures emerge and their (interpreting) actualisation is produced from observing and analyzing the domain's possibilistically determined constraints as imposed on the linear ordering (syntagmatics) and the selective combination (paradigmatics) of natural language entities (morph-types, word-types) in communicative language performance. For fuzzy linguistic morhology and lexical semantics this is tantamount to (re-)present an entity's semiotic potential (function, meaning) by a fuzzy distributional pattern of the modelled system's state rather than a single symbol. The representational system's dynamic structure modeled by the procedures outlined is to represent a semiotic cognitive information processing system's interpretation of its environment.

\section{References}

[1] M. Bierwisch: Logische und psychologische Determinanten der Struktur natürlicher Sprachen. In: J. Scharf (Ed): Naturwissenschaftliche Linguistik, Nova Acta Leopoldina, NF 54, Nr.245, pp. 176187; 841-851 (J.A. Barth) Halle, 1981.

[2] N. Chomsky: The Logical Structure of Linguistic Theory. (Plenum) NewYork, 1975.

[3] D. E. Knuth: The Art of Computer Programming. Vol.9, (Addison-Wesley) Reading, MA, 1973. 
[4] D. Marr: Vision. (Freeman) SanFrancisco, 1982.

[5] A. Meystel: Semiotic Modeling and Situation Analysis: an Introduction. (AdRem Inc) Bala Cynwyd, PA, 1995.

[6] C. Peirce: Pragmatism in Retrospect: A Last Formulation. In: J. Buchler (Ed): The Philosophical Writings of Peirce, pp. 269-289. (Dover) New York, 1906.

[7] B. Rieger: Bedeutungskonstitution. Einige Bemerkungen zur semiotischen Problematik eines linguistischen Problems. Zeitschrift für Literaturwissenschaft und Linguistik, (27/28):55-68, 1977.

[8] B. Rieger: Unscharfe Semantik. Die empirische Analyse, quantitative Beschreibung, formale Repräsentation und prozedurale Modellierung vager Wortbedeutungen in Texten. (Lang) Frankfurt a.Main/ Bern/ Paris, 1989.

[9] B. Rieger: Unscharfe Semantik: zur numerischen Modellierung vager Bedeutungen von Wörtern als fuzzy Mengen. In: H. Friemel, et.al. (Eds): Forum-90: Wissenschaft und Technik. (Informatik-Fachberichte 259), pp. 80-104. (Springer) Berlin/ Heildeberg/ NewYork, 1990.

[10] B. Rieger: Fuzzy Modellierung linguistischer Kategorien. In: W. Feldmann, H. Hinrichs (Eds): Text und Lexikon, [in print] (Niemeyer) Tübingen, 1996.

[11] B. Rieger: Warum Fuzzy Linguistik? Überlegungen und Ansätze einer computerlinguistischen Neuorientierung. In: D. Krallmann, W. Schmitz (Eds): Intern. Gerold Ungeheuer Symposium, [in print] (Nodus) Münster, 1996.

[12] B. B. Rieger: Feasible Fuzzy Semantics. On some problems of how to handle word meaning empirically. In: H. Eikmeyer, H. Rieser (Eds): Words, Worlds, and Contexts. New Approaches in Word Semantics, pp. 193-209. (de Gruyter) Berlin/ NewYork, 1981.

[13] B. B. Rieger: Fuzzy Representation Systems in Linguistic Semantics. In: R. Trappl, et.al. (Eds): Progress in Cybernetics and Systems Research, Vol. XI, pp 249-256. (McGraw-Hill), Washington/ NewYork/ London, 1982.

[14] B. B. Rieger: Lexical Relevance and Semantic Disposition. On stereotype word meaning representation in procedural semantics. In: G. Hoppenbrouwes, et.al. (Eds): Meaning and the Lexicon, pp 387-400. (Foris), Dordrecht, 1985.

[15] B. B. Rieger: Distributed Semantic Representation of Word Meanings. In: J. D. Becker, et.al. (Eds): Parallelism, Learning, Evolution.
Evolutionary Models and Strategies WOPPLOT89, [Lecture Notes in Artificial Intelligence 565], pp. 243-273. (Springer) Berlin/ Heidelberg/ New York, 1991.

[16] B. B. Rieger: On Distributed Representation in Word Semantics. ICSI-TR-91-012, (ICSI), Berkeley, CA, 1991.

[17] B. B. Rieger: Meaning Acquisition by SCIPS. In: B. M. Ayyub (Ed): ISUMA-NAFIPS-95, [IEEETransactions: Joint Intern. Conf. on Uncertainty Modeling and Analysis, North American Fuzzy Information Processing Society], pp. 390-395, (IEEE Computer Society Press), Los Alamitos, CA, 1995.

[18] B. B. Rieger: Situation Semantics and Computational Linguistics: towards Informational Ecology. A semiotic perspective for cognitive information processing systems. In: K. Kornwachs, K. Jacoby (Eds): Information. New Questions to a Multidisciplinary Concept, pp 285-315, (Akademie), Berlin, 1995.

[19] B. B. Rieger: Situations, Language Games, and SCIPS. Modeling semiotic cognitive information processing systems. In: A. Meystel, N. Nerode (Eds): editors, Architectures for Semiotic Modeling and Situation Analysis in Large Complex Systems, pp. 130-138, (AdRem), Bala Cynwyd, PA, 1995.

[20] B. B. Rieger / C. Thiopoulos: Semiotic Dynamics: a self-organizing lexical system in hypertext. In: R. Köhler, B. Rieger (Eds): Contributions to Quantitative Linguistics. Proceedings of the 1 st Quantitative Linguistics Conference - QUALICO91, pp. 67-78. (Kluwer Academic) Publishers, Dordrecht, 1993.

[21] H. A. Simon: The Sciences of the Artificial. (MIT Press), Cambridge, MA, 1982.

[22] F. Varela / E. Thompson / E. Rosch: The Embodied Mind. Cognitive Science and Human Experience. (MIT Press), Cambridge, MA, 1991.

[23] L. Zadeh (Ed): Fuzzy Sets and their Application to Cognitive and Decision Processes. (Academic Press), New York/ San Francisco, 1975.

[24] L. A. Zadeh: Fuzzy Sets. Information and Control, 17(8):338-353, 1965.

[25] L. A. Zadeh: Fuzzy Logic, Neural Networks, and Soft Computing. Comm. of the $A C M, 37(3): 77-84$, 1994. 


\title{
Linguistic Geometry: Model Evaluation
}

\author{
Boris Stilman \\ Department of Computer Science \& Engineering \\ University of Colorado at Denver, Campus Box 109 \\ Denver, CO 80217-3364, USA \\ bstilman@cse.cudenver.edu \\ http://www.cudenver.edu/ bstilman/boris.html
}

\begin{abstract}
A Linguistic Geometry* introduced in this paper includes mathematical tools for knowledge representation and reasoning about multiagent discrete pursuit-evasion games. These class of games is an adequate mathematical model for the real world combat operations, particularly, for the air force and navy problem domains. Linguistic Geometry relies on the formalization of search heuristics, which allow one to decompose the game into a hierarchy of images (subsystems), and thus solve otherwise intractable problems by reducing the search dramatically. These hierarchical images extracted in the form of networks of paths from the expert vision of the problem are formalized as a hierarchy of formal languages. An example of the simplified four aircraft pursuit-evasion game is considered. While the solution of this problem was presented in other publications in this paper we prove optimality of the solution. Based on this example we can suggest that for a certain class of multiagent problems Linguistic Geometry tools generate optimal solutions.
\end{abstract}

\section{Background}

Problems of long and short-range mission simulation, especially for autonomous navigation, aerospace robot control, such as unmanned aerial vehicles (UAVs), aerospace combat operations control, etc., are usually described mathematically in the form of pursuit-evasion differential games. An example of such problem is the problem of real time control of the air combat in which a number of planes (manned or unmanned) equipped with the so-called countermeasures evade a number of pursuers equipped with missiles. Another example is the problem optimal control of UAVs which are in the reconnaissance flight to locate mobile missile launchers. The actual launch points are usually detected by the satellite based sensor. The UAVs use detected launch points to initiate their search, locate and possibly destroy them. In the real world scenario the UAV control should be considered together with the air combat when UAVs evade pursuing enemy aircraft. Similar problems for the development and real time replanning of the combat scenarios are essential for the Navy and Army battlefields.

The classic approach based on the conventional theory

* This research is supported by Sandia National Laboratories, Albuquerque, NM, USA. of Differential Games is insufficient, especially in case of dynamic, multiagent models (Garcia-Ortiz et al., 1993). It is well known that there exists a small number of differential games, for which exact analytical solutions are available. There are a few more for which numerical solutions can be computed, under rather restrictive conditions, in a reasonable amount of time. It is even worse that each of these games is one-to-one which is very far from the real world combat scenarios. They are also of the "zero-sum type" which does not allow a new agent to join the game or all the agents of both sides to be disengaged. Another difficulties arise from the requirements of the 3D modeling and from limitation of the lifetime of the agents.

Following (Rodin, 1988; Shinar, 1990) discrete-event modeling of complex control systems can be implemented as a purely interrogative discrete simulation. These techniques can be based on generating geometrically meaningful states rather than time increments with due respect to the timeliness of actions. By discretizing time, a finite game tree can be obtained. The nodes of the tree represent the states of the game, where the players can select their controls for a given period of time. It is also possible to distinguish the respective moves of the two sides (including simultaneous actions). Thus, the branches of the tree are the moves in the game space. The pruning of such tree is the basic task of heuristic search techniques. Interrogative approach to control problems offers much faster execution and clearer simulator definition (Lirov et al., 1988).

In the beginning of 80 's Botvinnik, Stilman, and others developed one of the most interesting and powerful heuristic hierarchical models based on semantic networks. It was successfully applied to scheduling, planning, control, and computer chess. Application of the developed model to the chess domain was implemented in full as program PIONEER (Botvinnik, 1984). Similar heuristic model was implemented for power equipment maintenance in a number of computer programs being used for maintenance scheduling all over the former USSR (Botvinnik et al., 1983; Stilman, 1985, 1993a). The semantic networks were introduced in (Botvinnik, 1984; Stilman, 1977) in the form of ideas, plausible discussions, and program implementations.

\section{A Linguistic Geometry: Informal Survey}

A formal theory, the Linguistic Geometry - LG (Stilman, 1992-96), includes the syntactic tools for 
knowledge representation and reasoning about multiagent discrete pursuit-evasion games. This approach provides us with an opportunity to transfer formal properties and constructions from one problem to another and to reuse tools in a new problem domain. In a sense, it is the application of the methods of a chess expert to robot control or maintenance scheduling and vice versa.

Linguistic Geometry has been developed as a generic approach to a certain class of complex systems that involves breaking down a system into dynamic subsystems. We generate new multi-goal, multi-level system and substitute it for the original one-goal, one-level system by introducing intermediate goals and breaking the system down into subsystems striving to attain these goals.

A set of dynamic subsystems is represented as a hierarchy of formal languages. Various examples of problems solved employing LG tools have been published (Stilman, 1992-1996). During the entire history of the development of LG, we were always concerned about approximate solutions, almost winning strategies, and how to measure their accuracy. This was like an axiom: in general, heuristics do not generate an optimum, and even if they do it is usually hard to prove that this is an optimum.

This paper is devoted to a proof of optimality of the solution generated by LG algorithms for a class of multiagent problems. We will show how the heuristic algorithm generates a solution employing a very small search tree (Section 8). Then, in Section 9, we will prove that this solution is optimal.

\section{A Class of Problems}

A Complex System is the following eight-tuple: $<\mathrm{X}, \mathrm{P}, \mathrm{R}_{\mathrm{p}},\{\mathrm{ON}\}, \mathrm{v}, \mathrm{S}_{\mathrm{i}}, \mathrm{S}_{\mathrm{t}}, \mathrm{TR}>$, where

$\mathrm{X}=\left\{\mathrm{x}_{\mathrm{i}}\right\}$ is a finite set of points; locations of elements;

$\mathrm{P}=\left\{\mathrm{p}_{\mathrm{i}}\right\}$ is a finite set of elements; $\mathrm{P}=\mathrm{P}_{1} \cup \mathrm{P}_{2}, \mathrm{P}_{1} \neq \varnothing, \mathrm{P}_{2} \neq$ $\varnothing$;

$\mathrm{R}_{\mathrm{p}}(\mathrm{x}, \mathrm{y})$ is a set of binary relations of reachability in $\mathrm{X}(\mathrm{x}$ and $y \in X, p \in P$ );

$\mathrm{ON}(\mathrm{p})=\mathrm{x}$, where $\mathrm{ON}$ is a partial function of placement from $P$ into $X$;

$\mathrm{v}$ is a function on $\mathrm{P}$ with positive integer values describing the values of elements. The Complex System searches the state space that has initial and target states;

$S_{i}$ and $S_{t}$ are the descriptions of the initial and target states in the language of the first order predicate calculus, which matches with each relation a certain WellFormed Formula (WFF). Thus, each state from $S_{i}$ or $S_{t}$ is described by a certain set of WFF of the form $\left\{\mathrm{ON}\left(\mathrm{p}_{\mathrm{j}}\right)=\mathrm{x}_{\mathrm{k}}\right\}$

TR is a set of operators, TRANSITION(p, x, y), of transitions of the System from one state to another one. These operators describe the transition in terms of two lists of WFF (to be removed from and added to the description of the state), and of WFF of applicability of the transition. Here, Remove list: $O N(p)=x, O N(q)=y$; Add list: $O N(p)=y$; Applicability list: $(O N(p)=x) \wedge$ $R_{p}(x, y)$, where $p \in P_{1}$ and $q \in P_{2}$ or vice versa. The transitions are carried out with participation of a number of elements $\mathrm{p}$ from $\mathrm{P}_{1}, \mathrm{P}_{2}$.

According to the definition of the set $\mathrm{P}$, the elements of the System are divided into two subsets $P_{1}$ and $P_{2}$. They might be considered as units moving along the reachable points. Element $\mathrm{p}$ can move from point $\mathrm{x}$ to point $\mathrm{y}$ if these points are reachable, i.e., $R_{p}(x, y)$ holds. The current location of each element is described by the equation $\mathrm{ON}(\mathrm{p})=\mathrm{x}$. Thus, the description of each state of the System $\left\{O N\left(p_{j}\right)=x_{k}\right\}$ is the set of descriptions of the locations of elements. The operator TRANSITION(p, x, y) describes the change of the state of the System caused by the move of the element $\mathrm{p}$ from point $\mathrm{x}$ to point $\mathrm{y}$. The element $\mathrm{q}$ from point $y$ must be withdrawn (eliminated) if $p$ and $q$ do not belong to the same subset $\left(\mathrm{P}_{1}\right.$ or $\left.\mathrm{P}_{2}\right)$.

The problem of the optimal operation of the System is considered as a search for the optimal sequence of transitions leading from the initial state of $S_{i}$ to a target state of $S_{t}$. To solve this class of problems, we could use formal methods like those in the problem-solving or planning systems (like STRIPS or subsequent). However, the search would have to be made in a space of a huge dimension (for nontrivial examples). Thus, in practice, no solution would be obtained. We devote ourselves to finding a solution of a reformulated problem.

\section{A Set of Paths: Language of Trajectories}

A trajectory for an element $\mathrm{p}$ of $\mathrm{P}$ with the beginning at $x$ of $X$ and the end at $y$ of $X(x \neq y)$ with a length $l$ is the following formal string of symbols $a(x)$ with points of $X$ as parameters: $\mathrm{t}_{0}=a(\mathrm{x}) a\left(\mathrm{x}_{1}\right) \ldots a\left(\mathrm{x}_{l}\right)$, where $\mathrm{x}_{l}=\mathrm{y}$, each successive point $x_{i+1}$ is reachable from the previous point $x_{i}$, i.e., $R_{p}\left(x_{i}, x_{i+1}\right)$ holds for $i=0,1, \ldots, l-1$; element $p$ stands at the point $x: O N(p)=x$. We denote by $t_{p}(x, y, l)$ the set of all trajectories for element $\mathrm{p}$, beginning at $\mathrm{x}$, end at $\mathrm{y}$, and with length $l$.

A shortest trajectory $\mathrm{t}$ of $\mathrm{t}_{\mathrm{p}}(\mathrm{x}, \mathrm{y}, l)$ is the trajectory of the minimum length for the given beginning $x$, end $y$, and element $\mathrm{p}$.

A Language of Trajectories $\mathrm{L}_{\mathrm{t}} \mathrm{H}_{(\mathrm{S})}$ for the Complex System in a state $S$ is the set of all the trajectories of length less or equal $\mathrm{H}$. Various properties of this language and generating grammars were investigated in (Stilman, 1993a).

Examples of distance measurements and trajectory generation for robotic vehicles are considered in (Stilman, 1993a, 1993c, 1994b).

\section{Networks of Paths:}

\section{Languages of Trajectory Networks}

An example of such network is shown in Fig. 2. The basic idea behind these networks is as follows. Element $p_{0}$ should move along the main trajectory $a(1) a(2) a(3) a(4) a(5)$ to reach the ending point 5 and remove the target $\mathrm{q}_{4}$ (an opposing element). Naturally, the opposing elements should try to disturb those motions by controlling the intermediate 
points of the main trajectory. They should come closer to these points (to the point 4 in Fig. 2) and remove element $\mathrm{p}_{\mathrm{O}}$ after its arrival (at point 4). For this purpose, elements $\mathrm{q}_{3}$ or $\mathrm{q}_{2}$ should move along the trajectories $\boldsymbol{a}(6) \boldsymbol{a}(7) \boldsymbol{a}(4)$ and $a(8) a(9) a(4)$, respectively, and wait (if necessary) at the next to last point ( 7 or 9 ) for the arrival of element $p_{o}$ at point 4 . Similarly, element $p_{1}$ of the same side as $p_{0}$ might try to disturb the motion of $\mathrm{q}_{2}$ by controlling point 9 along the trajectory $a(13) a(9)$. It makes sense for the opposing side to include the trajectory $\boldsymbol{a}(11) \boldsymbol{a}(12) \boldsymbol{a}(9)$ of element $\mathrm{q}_{1}$ to prevent this control.

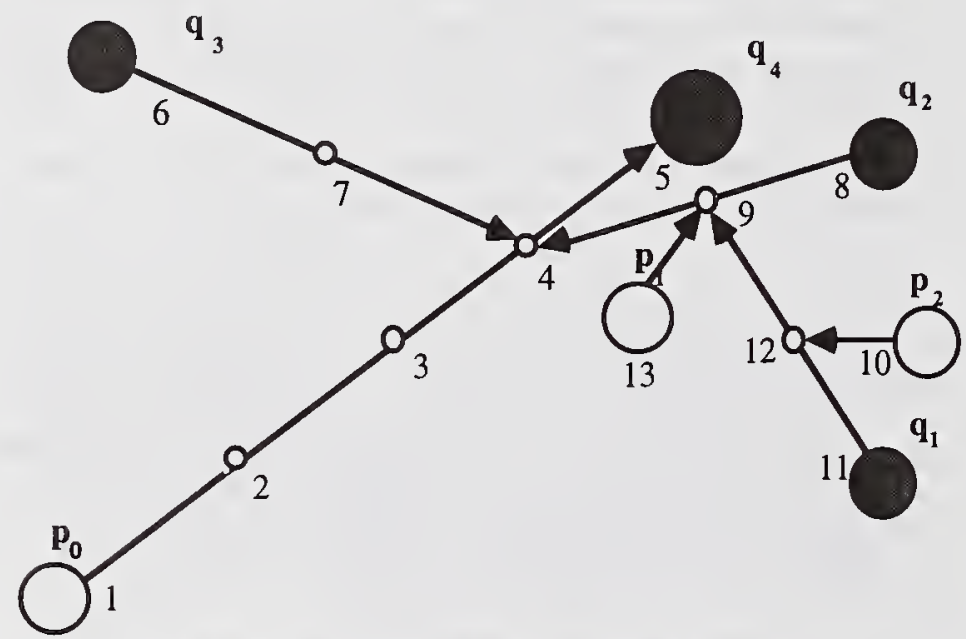

Fig. 2. Network language interpretation.

A trajectory connection of the trajectories $t_{1}$ and $t_{2}$ is the relation $C\left(t_{1}, t_{2}\right)$. It holds if the ending link of the trajectory $t_{1}$ coincides with an intermediate link of the trajectory $t_{2}$; more precisely, $t_{1}$ is connected with $t_{2}$ if among the parameter values $\boldsymbol{P}\left(\mathrm{t}_{2}\right)=\left\{\mathrm{y}_{1}, \mathrm{y}_{1}, \ldots, \mathrm{y}_{l}\right\}$ of trajectory $\mathrm{t}_{2}$ there is a value $\mathrm{y}_{\mathrm{i}}=\mathrm{x}_{\mathrm{k}}$, where $\mathrm{t}_{1}=a\left(\mathrm{x}_{0}\right) a\left(\mathrm{x}_{1}\right) \ldots a\left(\mathrm{x}_{\mathrm{k}}\right)$. If $\mathrm{t}_{1}$ belongs to a set of trajectories with the common end-point, then the entire set is said to be connected with the trajectory $t_{2}$.

A formal definition of the Language of Zones (based on the trajectory connection) is given in (Stilman, 1993b, 1993c, 1994a). The Zone corresponding to the trajectory network in Fig. 2 is represented as follows.

$$
\begin{gathered}
\mathrm{Z}=\boldsymbol{t}\left(\mathrm{p}_{0}, \boldsymbol{a}(1) \boldsymbol{a}(2) \boldsymbol{a}(3) \boldsymbol{a}(4) \boldsymbol{a}(5), 5\right) \boldsymbol{t}\left(\mathrm{q}_{3}, \boldsymbol{a}(6) \boldsymbol{a}(7) \boldsymbol{a}(4), 4\right) \\
\boldsymbol{t}\left(\mathrm{q}_{2}, \boldsymbol{a}(8) \boldsymbol{a}(9) \boldsymbol{a}(4), 4\right) \boldsymbol{t}\left(\mathrm{p}_{1}, \boldsymbol{a}(13) \boldsymbol{a}(9), 1\right) \\
\boldsymbol{t}\left(\mathrm{q}_{1}, \boldsymbol{a}(11) \boldsymbol{a}(12) \boldsymbol{a}(9), 3\right) \boldsymbol{t}\left(\mathrm{p}_{2}, \boldsymbol{a}(10) \boldsymbol{a}(12), 1\right)
\end{gathered}
$$

A language $\mathrm{L}_{\mathrm{Z}}{ }^{\mathrm{H}}(\mathrm{S})$ generated by the certain grammar $\mathbf{G}_{\mathbf{Z}}$ (Stilman, 1993b, 1993c, 1994a) in a state $\mathrm{S}$ of a Complex System is called the Language of Zones.

\section{A Complex System of Robotic Vehicles}

For the robotic model the set X of the Complex System (Section 3) represents the operational district, which could be the area of combat operation, broken into smaller $2 \mathrm{D}$ or $3 \mathrm{D}$ areas, "points", e.g., in the form of the 2D or 3D grid. P is the set of robots or autonomous vehicles. It is broken into two subsets $P_{1}$ and $P_{2}$ with opposing interests; $R_{p}(x, y)$ represent moving capabilities of various robots for various problem domains: robot $\mathrm{p}$ can move from point $\mathrm{x}$ to point $\mathrm{y}$ if $R_{p}(x, y)$ holds. Analogously, we can represent the rest of the parameters of the Complex System.

\section{2D Model: Problem Statement}

Robots with various moving capabilities are shown in Fig. 3. The operational district $\mathrm{X}$ is the table $8 \times 8$. Robot W-FIGHTER (White Fighter) standing on h8, can move to any next square (shown by arrows). The other robot BBOMBER (Black Bomber) from h5 can move only straight ahead, one square at a time, e.g., from $\mathrm{h} 5$ to $\mathrm{h} 4$, from $\mathrm{h} 4$ to h3, etc. Robot B-FIGHTER (Black Fighter) standing on a6, can move to any next square similarly to robot WFIGHTER (shown by arrows). Robot W-BOMBER standing on c6 is analogous with the robot B-BOMBER; it can move only straight ahead but in reverse direction. Thus, robot W-FIGHTER on $\mathrm{h} 8$ can reach any of the points $\mathrm{y}$ $\epsilon\{\mathrm{h} 7, \mathrm{~g} 7, \mathrm{~g} 8\}$ in on step, i.e., RW-FIGHTER(h8, y) holds, while W-BOMBER can reach only c8 in one step. Assume that robots W-FIGHTER and W-BOMBER belong to one side, while B-FIGHTER and B-BOMBER belong to the opposing side: W-FIGHTER $\in \mathrm{P}_{1}, \mathrm{~W}$-BOMBER $\in \mathrm{P}_{1}$, $\mathrm{B}$ FIGHTER $\in \mathrm{P}_{2}$, B-BOMBER $\in \mathrm{P}_{2}$.

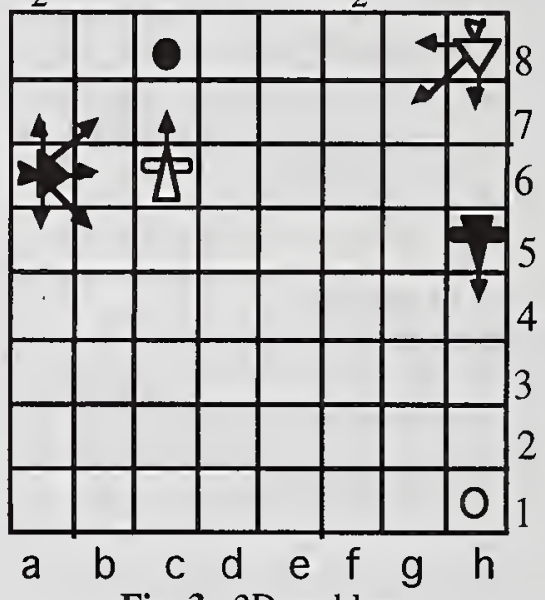

Fig. 3. 2D problem.

Also assume that two more robots, W-TARGET and BTARGET, (unmoving devices or target areas) stand on hl and $\mathrm{c} 8$, respectively. W-TARGET belongs to $\mathrm{P}_{1}$, while $\mathrm{B}$ TARGET $\in P_{2}$. Each of the BOMBERs can destroy unmoving TARGET ahead of the course; it also has powerful weapons capable to destroy opposing FIGHTERs on the next diagonal squares ahead of the course. For example W-BOMBER from c6 can destroy opposing FIGHTERs on b7 and d7. Each of the FIGHTERs is capable to destroy an opposing BOMBER approaching its location, but it is also capable to protect its friendly BOMBER approaching its prospective location. In the latter case the joint protective power of the combined weapons of the friendly BOMBER and FIGHTER can protect the BOMBER from interception. For example, W-FIGHTER located at d6 can protect W-BOMBER on c6 and c7.

The combat considered can be broken into two local operations. The first operation is as follows: robot BBOMBER should reach point hl to destroy the $\mathrm{W}$ TARGET, while W-FIGHTER will try to intercept this 
motion. The second operation is similar: robot WBOMBER should reach point $\mathrm{c} 8$ to destroy the BTARGET, while B-FIGHTER will try to intercept this motion. After destroying the opposing TARGET the attacking side is considered a winner of the local operation and the global battle. The only chance for the opposing side to avenge is to hit its TARGET on the next time increment and this way end the battle in a draw. The conditions considered above give us $S_{t}$, the description of target states of the Complex System. The description of the initial state $\mathrm{S}_{\mathrm{i}}$ is obvious and follows from Fig. 3 .

Assume that motions of the opposing sides alternate and due to the shortage of resources (which is typical in a real combat operation) or some other reasons, each side can not participate in both operations simultaneously. It means that during the current time interval, in case of White turn, either W-BOMBER or W-FIGHTER can move. Analogous condition holds for Black. Of course, it does not mean that if one side began participating in one of the operations it must complete it. Any time on its turn each side can switch from one operation to another, e.g., transferring resources (fuel, weapons, human resources, etc.), and later switch back.

Both restrictions have been relaxed and completely eliminated in (Stilman, 1995c, 1995d, 1996b).

It seems that local operations are independent, because they are located far from each other. Moreover, the operation of B-BOMBER from h5 looks like unconditionally winning operation, and, consequently, the global battle can be easily won by the Black side.

Is there a strategy for the White side to make a draw? The specific formal question is as follows: Is there an optimal strategy that provides one of the following?

1. Both BOMBERs hit their targets on subsequent time increments and stay safe for at least one time increment.

2. Both BOMBERs are destroyed before they hit their targets or immediately after that.

We answer this question in Sections 8-9. Of course, it can be answered by the direct search employing, for example, minimax algorithm with alpha-beta cut-offs. Experiments with the computer programs showed that in order to solve this problem employing conventional approaches the search tree should include about a million moves (transitions). Consider how the Hierarchy of Languages works for the optimal control of this Robotic System (Fig. 3).

\section{2D Model: Search}

We generate a string of the Language of Translations representing it as a conventional search tree (Fig. 5) and comment on its generation.

First, the Language of Zones in the start state is generated. The targets for attack are determined within the limited number of steps which is called a horizon. In general, the value of the horizon is unknown. As a rule, this value can be determined from the experience of solving specific classes of problems employing Linguistic
Geometry tools. In absence of such experience, first, we have to consider the value of 1 as a horizon, and solve the problem within this value. If we still have resources available, i.e., computer time, memory, etc., we can increase the horizon by one. After each increase we have to regenerate the entire model. This increase means a new level of "vigilance" of the model, and, consequently, new greater need for resources.
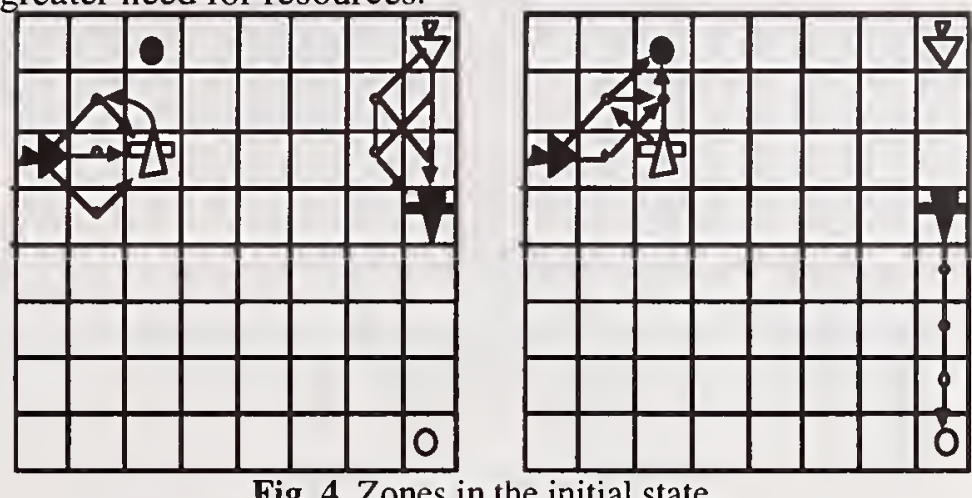

Fig. 4. Zones in the initial state.

In our case it is easy to show that within the horizons of 1, 2, 3, 4 all the models are "blind" and corresponding searches do not give a "reasonable" solution. But, again, after application of each of the consecutive values of the horizon we will have a solution which can be considered as an approximate solution within the available resources. Thus, let the horizon $\mathrm{H}$ of the language $\mathrm{L}_{Z}(\mathrm{~S})$ is equal to 5 , i.e., the length of main trajectories of all Zones must not exceed 5 steps. All the Zones generated in the start state are shown in Fig. 4. Zones for the FIGHTERs as attacking elements are shown in the left diagram, while Zones for BOMBERs - in the right one.

Generation begins with the move 1. c6-c7 in the White Zone with the target of the highest value and with the shortest main trajectory. The order of consideration of Zones and particular trajectories is determined by the grammar of translations. Next move, 1... a6-b7, is in the same Zone along the first negation trajectory. Interception continues: 2. c7-c8 b7:c8 (Fig. 6, left). Symbol ":" means the removal of element. Here the grammar cuts this branch with the value of -1 (as a win of the Black side).

Then, the grammar initiates the backtracking climb. Each backtracking move is followed by the inspection procedure, the analysis of the subtree generated in the process of the earlier search. After the climb up to the move 1 ... a6-b7, the tree to be analyzed consists of one branch (of two plies): 2. c7-c8 b7:c8. The inspection procedure determined that the current minimax value $(-1)$ can be "improved" by the improvement of the exchange on c8 (in favor of the White side). This can be achieved by participation of W-FIGHTER from h8, i.e., by generation and inclusion of the new so-called "control" Zone with main trajectory from $\mathrm{h} 8$ to $\mathrm{c} 8$. The set of different Zones from h8 to $c 8$ (the bundle of Zones) is shown in Fig. 6, right. The move-ordering procedure picks the subset of Zones with main trajectories passing g7. These trajectories partly coincide with the main trajectory of another Zone attacking the opposing W-BOMBER on h5. The motion along such trajectories allows to "gain time", i.e., to approach two goals simultaneously. 
The generation continues: 2. h8-g7 b7:c7. Again, the procedure of "square rules" cuts the branch, evaluates it as a win of the black side, and the grammar initiates the climb. Analogously to the previous case, the inspection procedure determined that the current minimax value $(-1)$ can be improved by the improvement of the exchange on c7. Again, this can be achieved by the inclusion of Zone from $\mathrm{h} 8$ to $\mathrm{c} 7$. Of course, the best "time-gaining" move in this Zone is 2 . h8-g7, but it was already included (as move in the Zone from $\mathrm{h} 8$ to $\mathrm{c} 8$ ), and it appeared to be useless. No other branching at this state is generated.

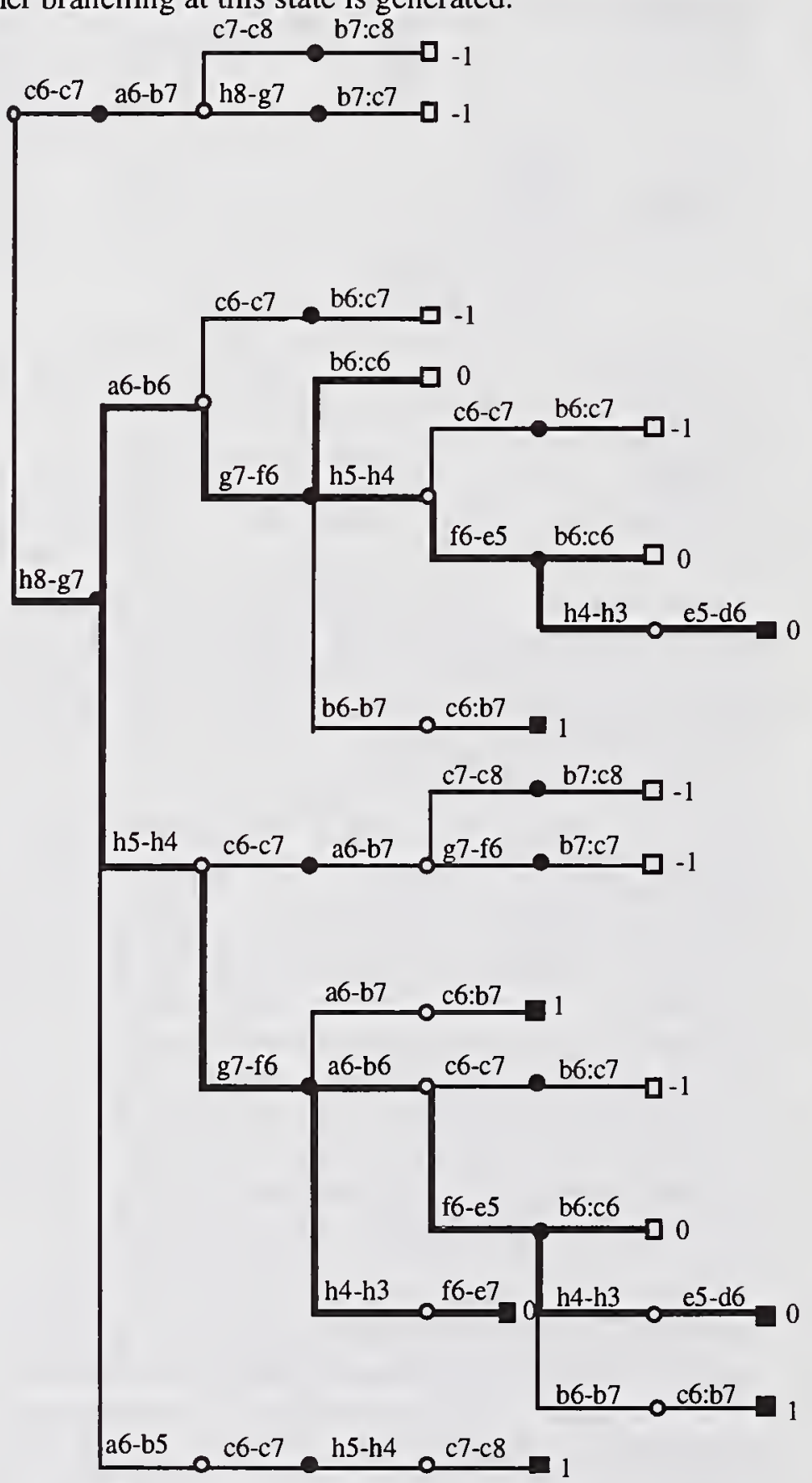

Fig. 5. Search tree for the robotic system.

The inspection procedure does not find new Zones to improve the current minimax value, and the climb continues up to the start state. The analysis of the subtree shows that inclusion of Zone from h8 to $c 8$ in the start state can be useful: the minimax value can be improved. Similarly, the most promising "time-gaining" move is 1. h8-g7. The Black side responded 1. ... a6-b6 along the first negation trajectories $a(\mathrm{a} 6) \boldsymbol{a}(\mathrm{b} 6) \boldsymbol{a}(\mathrm{c} 7)$ (Fig. 4, left). Note, that the grammar "knows" that in this state trajectory $\boldsymbol{a}(\mathrm{a} 6) \boldsymbol{a}(\mathrm{b} 6) \boldsymbol{a}(\mathrm{c} 7)$ is active, i.e., B-FIGHTER has enough time for interception. The following moves are in the same Zone of W-BOMBER: 2. c6-c7 b6:c7. This state is shown in Fig. 7, left. The "square rule procedure" cuts this branch and evaluates it as a win of the Black side.
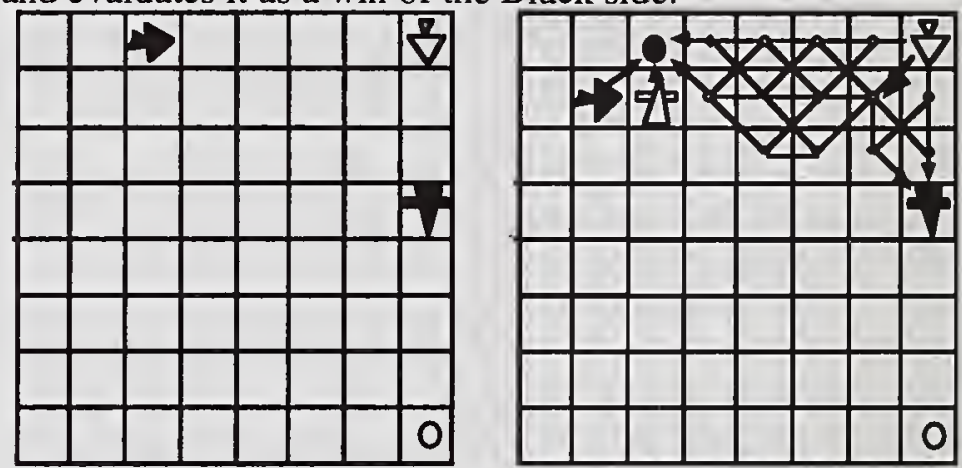

Fig. 6. States where the control Zone from h8 to $\mathrm{c} 8$ was detected (left) and where it was activated (right).
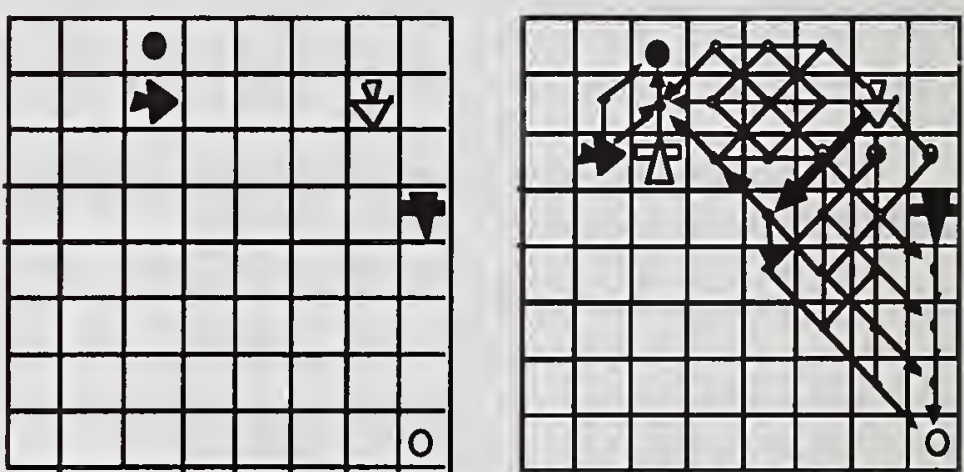

Fig. 7. States where the control Zone from $\mathrm{g} 7$ to $\mathrm{c} 7$ was detected (left) and where it was activated (right)

New climb up to the move 2. ... a6-b6 and execution of the inspection procedure resulted in the inclusion of the new control Zone from $\mathrm{g} 7$ to $\mathrm{c} 7$ in order to improve the exchange on $\mathrm{c} 7$. The set of Zones with different main trajectories from $\mathrm{g} 7$ to $\mathrm{c} 7$ is shown in Fig. 7, right. Besides that, the trajectories from $\mathrm{g} 7 \mathrm{to} h 4, \mathrm{~h} 3, \mathrm{~h} 2$, and $\mathrm{h} 1$ are shown in the same Fig. 7. These are "potential" intercepting trajectories. It means that beginning with the second symbol $\boldsymbol{a}(\mathrm{f} 6), \boldsymbol{a}(\mathrm{g} 6)$ or $\boldsymbol{a}(\mathrm{h} 6)$ these trajectories become intercepting trajectories (Section 5) in the Zone of B-BOMBER h5. Speaking informally, from squares $\mathrm{f} 6$, g6, and h6 WFIGHTER can intercept B-BOMBER (in case of white move). The move-ordering procedure picks the subset of Zones with the main trajectories passing f6. These trajectories partly coincide with the potential first negation trajectories. The motion along such trajectories allows to gain time, i.e., to approach two goals simultaneously. Thus, 2. g7-f6. This way proceeding with the search we will generate the tree that consists of 46 moves.

\section{2D Model: PROOF}

\section{1 Terminal Sets}

Let us prove that the optimal variant of the reduced search tree shown in Fig. 5 is the optimal solution of this problem. This means that we have to prove that this variant 
is optimal not only within the reduced search tree but also within the full search tree, which could be generated as a result of exhaustive search. (Here and below in this paper we use bold font to designate various sets.)

The set of goal states for this problem can be broken into three subsets:

1. Subset $\mathbf{W}-\mathbf{W i n}$ is the set of states where B-BOMBER is destroyed, and W-BOMBER hit B-TARGET and has been safe for at least one time increment after that.

W-Win = BB-Destroyed $\cap$ WB-Safe

2. Subset $\mathbf{B}-\mathbf{W i n}$ is the set of states where W-BOMBER is destroyed, and B-BOMBER hit W-TARGET and has been safe for at least one time increment after that.

B-Win = WB-Destroyed $\cap$ BB-Safe

3. Subset Draw is the set of states where both BOMBERs hit their targets on subsequent time increments and have been safe for at least one time increment or both BOMBERs are destroyed before this happens. The set of states considered in this definition can be represented as follows:

where

$$
\text { Draw = Safe } \cup \text { Destroyed, }
$$

Destroyed = BB-Destroyed $\cap$ WB-Destroyed, Safe $=$ BB-Safe $\cap$ WB-Safe

A subtree of the full search tree is called optimal if after applying the minimax algorithm on the full tree, the minimax value of the root node is equal to one of the values of the terminal nodes of this subtree.

Let $\mathbf{A}$ be a set of states. The strategy is called an $\mathbf{A}$ strategy if it is represented by the optimal subtree with the terminal nodes which represent states from $\mathbf{A}$, only. Thus, for the W-Win strategy, the corresponding terminal nodes belong to W-Win only. For the B-Win strategy, the terminal nodes should belong to B-Win. The Draw strategy is represented by the optimal subtree with the terminal nodes from Draw.

To prove that the bold subtree of the search tree shown in Fig. 5 represents optimal strategy for this problem, and this strategy gives a draw, we have to prove that it represents a Draw strategy for this problem. This means that the following Theorem holds:

\section{Theorem}

1. The terminal states of the bold subtree belong to the Draw.

2. The bold subtree is optimal (with respect to the full search tree).

Let us prove statement 1.

\section{2 Terminal Sets Expansion}

The terminal states of the bold subtree shown in Fig. 5 belong neither of the subsets considered above despite they received exact values. Let us expand these state subsets trying to include the actual terminal states. We shall begin with the expansion of the set Draw by introducing a new set DrawExpand. By definition this is the set of states such that for each of them a Draw strategy exists. Ultimately, we have to prove that our start state belongs to
DrawExpand.

We will achieve this goal by investigating the structure of this set employing LG tools.

First, we will consider the states where the draw can be achieved by destroying both BOMBERs (Fig. 8). Let BBIntercept be the set of states where for each of the states there is an optimal strategy for W-FIGHTER to intercept BBOMBER. More formally, if BB-Destroyed is the set of states where B-BOMBER is destroyed (see definition of the Draw), then BB-Intercept is the set of states where BBDestroyed strategy exists.

Let WB-Intercept be the set of states where for each state there is an optimal strategy for B-FIGHTER to intercept the W-BOMBER. More formally, if WBDestroyed is the set of states where W-BOMBER is destroyed (see definition of Draw), then WB-Intercept is the set of states where WB-Destroyed strategy exists.

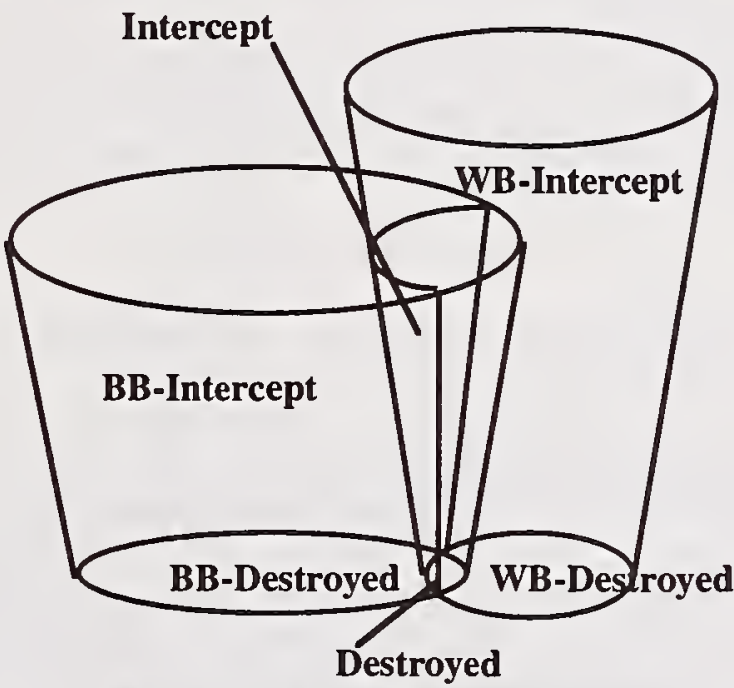

Fig. 8. Expansion of terminal sets

Consider the intersection of BB-Intercept and WBIntercept,

Intercept $=$ BB-Intercept $\cap$ WB-Intercept

i.e., the set of states where the Destroyed strategy exists, taking into account that

Destroyed $=$ BB-Destroyed $\cap$ WB-Destroyed.

Obviously, Destroyed $\subset$ DrawExpand.

Analogously, we will consider the states where the draw can be achieved by hitting the TARGETs and saving both BOMBERs.

Let BB-Protect be the set of states where for each of the states there is an optimal strategy for B-BOMBER to hit the W-TARGET and stay safe. More formally, if BB-Safe is the set of states where W-TARGET is hit and BBOMBER is safe (see definition of the Draw), then B BProtect is the set of states where BB-Safe strategy exists.

Let WB-Protect be the set of states where for each state there is an optimal strategy for W-BOMBER to hit BTARGET and stay safe. More formally, if WB-Safe is the set of states where B-TARGET is hit and W-BOMBER is safe (see definition of Draw), then WB-Protect is the set of states where WB-Safe strategy exists.

Now, consider the intersection BB-Protect and WBProtect.

$$
\text { Protect }=\text { BB-Protect } \cap \text { WB-Protect }
$$


i.e., the set of states where the Safe strategy exists taking into account that

Safe $=$ BB-Safe $\cap$ WB-Safe.

Obviously, Protect $\subset$ DrawExpand. Thus,

Intercept $\cup$ Protect $\subset$ DrawExpand.

It should be noted that this is a strict implication. The existence of the Destroyed strategy for Intercept, the Safe strategy for Protect, and the Draw strategy for Draw Expand, and the fact that

does not mean that

$$
\text { Draw }=\text { Safe } \cup \text { Destroyed, }
$$

\section{DrawExpand $=$ Intercept $\cup$ Protect.}

Basically, we are saying that the expansion of the union of two sets is not equal to the union of their separate expansions, i.e., the distribution law does not hold in this case. Indeed, there might be states where the draw can be achieved by the strategy leading to the states from Safe and Destroyed simultaneously. This means that at some higher levels of the subtree representing the optimal strategy we would not be able to distinguish which kind of draw is being currently pursued, Safe or Destroyed. Consequently, these states might belong to the direct expansion of the union

which means that

$$
\text { Safe } \cup \text { Destroyed, }
$$

DrawExpand $-($ Intercept $\cup$ Protect $) \neq \varnothing$.

\section{3 A Structure of Expanded Terminal Sets}

Let us describe the structure of the subsets introduced above employing LG tools. We begin with BB-Intercept. In our problem the only element that can potentially intercept and destroy the B-BOMBER is the W-FIGHTER. Consider the set of all states where W-FIGHTER is in the Zone of B-BOMBER, and it is the only intercepting element. First we define the local BB-Intercept $\mathbf{B}_{\mathbf{B}-Z o n e}$. BB-Intercept $\mathbf{B}_{\mathbf{B}-Z o n e}$ can be described as the set of states of the following set of Zones, the B-Zone, with one of the following main trajectories: $\boldsymbol{a}(\mathrm{h} 5) \boldsymbol{a}(\mathrm{h} 4) \boldsymbol{a}(\mathrm{h} 3) \boldsymbol{a}(\mathrm{h} 2) \boldsymbol{a}(\mathrm{h} 1)$, $\boldsymbol{a}(\mathrm{h} 4) \boldsymbol{a}(\mathrm{h} 3) \boldsymbol{a}(\mathrm{h} 2) \boldsymbol{a}(\mathrm{h} 1), \boldsymbol{a}(\mathrm{h} 3) \boldsymbol{a}(\mathrm{h} 2) \boldsymbol{a}(\mathrm{h} 1), \boldsymbol{a}(\mathrm{h} 2) \boldsymbol{a}(\mathrm{h} 1)$.

These Zones are nested in each other. Two subsets of $\mathbf{B}$ Zone are shown in Fig. 9.
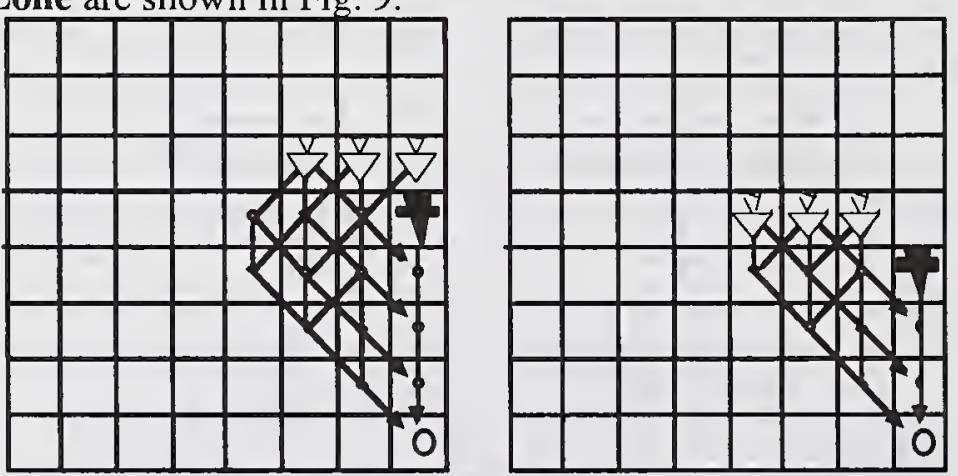

Fig. 9. The sample Zones describing BB-Intercept and corresponding gateways.

The multiple locations of W-FIGHTER designate Zone gateways, the locations through which W-FIGHTER can enter the Zone employing the shortest path leading from $\mathrm{h} 8$. Once in the Zone (through one of the gateways) in case of
White turn the W-FIGHTER is able to intercept the BBOMBER pursuing it persistently even in the worst case, i.e., if Black does not skip moves in this Zone. In case of the Zones considered here the proof of guaranteed interception is trivial. This follows from the fact that the trajectory of W-FIGHTER is the 1st negation trajectory which is by definition of such a length that interception is guaranteed.

We assume that this Zone is considered independently of the rest of the elements of Complex System which means the interception of B-BOMBER is guaranteed if we consider the motions within the B-Zone, only. For difforent problems and more sophisticated Zones to prove that the optimal variant of the Zone's skirmish contains interception of the main element we would have to use theorems on Network Languages (Stilman, 1994). The global B BIntercept is a subset of BB-Intercept ${ }_{B-Z o n e}$ such that interception is guaranteed with respect to the entire system.

Consider WB-Intercept. In our problem the only element that can "potentially" intercept and destroy the WBOMBER is the B-FIGHTER. Consider the set of all states where B-FIGHTER is in the Zone of W-BOMBER, and it is the only intercepting element. First we define the local WBIntercept $_{W \text {-Zone }}$. WB-Intercept ${ }_{W \text {-Zone }}$ can be described as the of the set the set of states of Zones, the W-Zone, with one of the following main trajectories $a(\mathrm{c} 6) a(\mathrm{c} 7) a(\mathrm{c} 8)$, $a(\mathrm{c} 7) a(\mathrm{c} 8)$.

These Zones are nested in each other. This W-Zone is considered independently of the rest of the elements of Complex System which means the interception of WBOMBER is guaranteed if we consider the motions within the W-Zone, only. Two subsets of W-Zone are shown in Fig. 10. A subset of Zones shown in Fig. 10 (left) represents Zones as they are in the initial state. The global WBIntercept is the subset of WB-Intercept ${ }_{W}$-Zone suchthat interception is guaranteed with respect to the entire system.
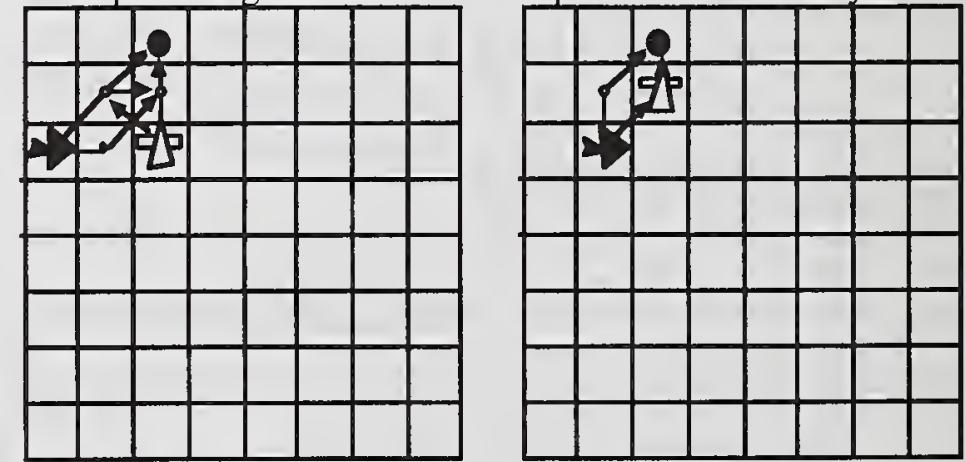

Fig. 10. Sample Zones describing WB-Intercept.

Consider BB-Protect. In our problem the only element that can "potentially" protect the B-BOMBER, if necessary, and let it hit the W-TARGET is the B-FIGHTER. But the $B$-FIGHTER is involyed in WB-Intercept and cannot leave it. Thus, the B-BOMBER can accomplish its mission safely without protection only if it does not need one. This means that the Zone of B-BOMBER, the B-Zone, should be free of intercepting elements. Consider the set of all the states where the B-BOMBER is alone without any 1 st negation trajectories. This is our local BB-Protect B-Zone $_{\text {. }}$ 
The global BB-Protect is the subset of BB-Protect ${ }_{\mathbf{B}}$ Zone such that protection is guaranteed with respect to the entire system.

Finally, let us consider WB-Protect. In our problem the only element that can potentially protect W-BOMBER is the W-FIGHTER. Consider the set of all states where WFIGHTER is in the Zone of W-BOMBER. Three possible locations, the gateways, are shown in Fig. 12 (left). The gateways are shown as multiple locations of the $\mathrm{W}$ FIGHTER, the locations through which the W-FIGHTER can enter the Zone employing the shortest path leading from $\mathrm{h} 8$. Once in the Zone (through one of the gateways) the WFIGHTER is able to protect the W-BOMBER from being intercepted. The set of all the states where W-BOMBER is protected by W-FIGHTER from $\mathrm{d} 6, \mathrm{~d} 7$, or $\mathrm{d} 8$ is included into WB-Protect $\mathbf{W}$-Zone . Of course, another way to protect W-BOMBER is for B-FIGHTER to leave this Zone. One of such states is shown in Fig. 12 (right). Once leaving the Zone B-FIGHTER would not have chance to enter it and intercept the W-BOMBER. All such states are also included into the WB-Protect $\mathbf{W}$-Zone . The global WB-Protect is the subset of WB-Protect $\mathbf{W}$-Zone such that protection is guaranteed with respect to the entire system.

BB-Protect $_{\mathbf{B}-Z \text {-Zne }}$ and $\mathbf{B B}$-Intercept ${ }_{\mathbf{B} \text {-Zone }}$ represent different subsets of states of the same set, the B-Zone.

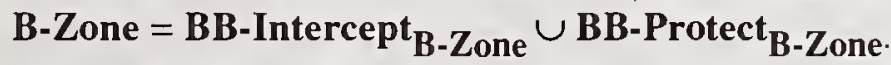
A similar statement is true for the Zone of W-BOMBER, the W-Zone:

$\mathbf{W}$-Zone $=\mathbf{W B}$-Intercept $\mathbf{W}$-Zone $\cup$ WB-Protect $_{W}$-Zone ${ }$
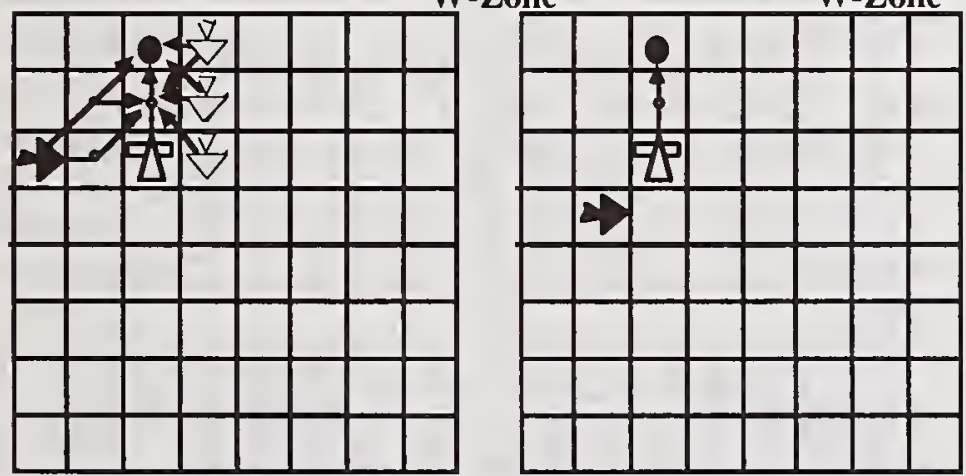

Fig. 12. The sample Zones describing WB-Protect.

\section{4 Terminal States of the Subtree}

Now we can evaluate all the terminal states of the bold subtree (Fig. 5, 13).

In particular, the terminal states of the following variants:

1. h8-g7 a6-b6; 2. g7-f6 b6:c6.

1. h8-g7 a6-b6; 2. g7-f6 h5-h4; 3. f6-e5 b6:c6.

1. h8-g7 h5-h4; 2. g7-f6 a6-b6; 3. f6-e5 b6:c6.

belong to global

Intercept $=$ BB-Intercept $\cap$ WB-Intercept.

The terminal states of the following variants:

1. h8-g7 a6-b6; 2. g7-f6 h5-h4; 3. f6-e5 h4-h3;

4. e5-d6.

1. h8-g7 h5-h4; 2. g7-f6 a6-b6; 3. f6-e5 h4-h3;

4. e5-d6.

1. h8-g7 h5-h4; 2. g7-f6 h4-h3; 3. f6-e7. belong to global

Protect $=$ BB-Protect $\cap$ WB-Protect.

Now we conclude that all the terminal states of the bold subtree belong to

Intercept $\cup$ Protect $\subset$ DrawExpand.

Thus, statement 1 . is proved.

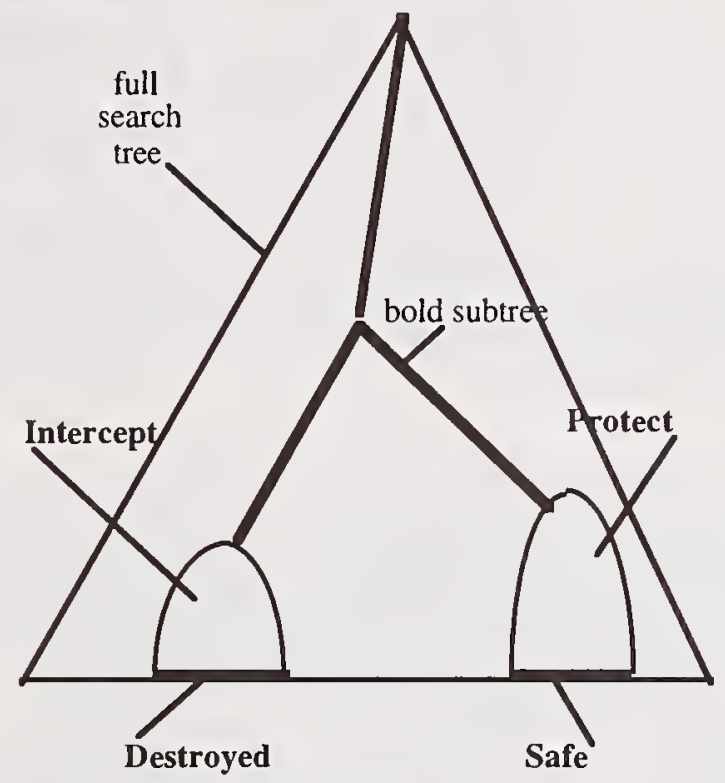

Fig. 13. Terminal states of the bold subtree.

Let us prove statement 2 . of the Theorem that the bold subtree is optimal (with respect to the full search tree).

\subsection{A Zone Status Change}

As we know from the above

B-Zone $=$ BB-Intercept ${ }_{\text {B-Zone }} \cup$ BB-Protect ${ }_{\text {B-Zone }}$

$\mathbf{W}$-Zone $=\mathbf{W B}$-Intercept $\mathbf{W}$-Zone $U \mathbf{W B}$ - Protect $_{\mathbf{W}}$-Zone

If we consider Zones independently of the rest of the elements following definition the expanded terminal sets, every Zone can not switch from one terminal set to another. For example, if the current state of the $\mathbf{B}$-Zone belongs to BB-Intercept and we continue search following the optimal branch, the B-Zone will never switch to the state from BBProtect, it will reach BB-Destroyed.
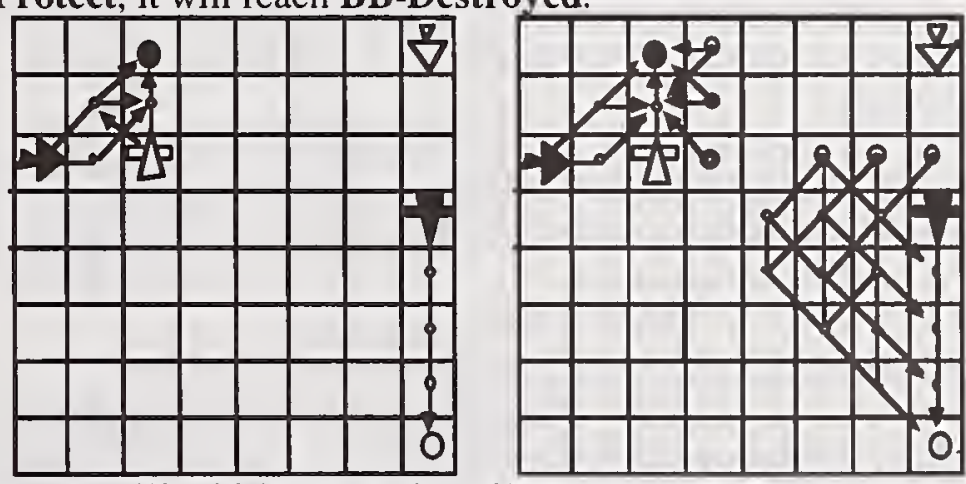

Fig. 14. Interpretation of B-Zone and W-Zone and their status change.

The initial state of the B-Zone belongs to BB-Protect ${ }_{\mathrm{B}}$. Zone, for the W-Zone it belongs to WB-Intercept ${ }_{W-Z o n e}$. In the real search every element can move including the elements that are currently outside the Zone. This might 
cause the Zone to switch from one status to another. In particular, if W-FIGHTER comes to one of the gateways $\mathrm{f} 6$, g6, or h6 the current state of the B-Zone will switch from the initial BB-Protect $\mathbf{B}-$ Zone $($ Fig. 14, left) to B B Intercept $_{\mathrm{B}-\text { Zone }}$ (Fig. 14, right).

If no other elements can interfere and White has enough time for interception, then the new status cannot be changed, this state actually belongs to the global B BIntercept. In case of the W-Zone, if the W-FIGHTER arrives at one of the gateways e6, e7, or e8, this Zone switches from the initial local WB-Intercept $\mathbf{W}$-Zone (Fig. 14, left) to the global WB-Protect (Fig. 14, right). This status is final because there are no elements in the Complex System to interfere.

\section{6 A Description of Winning Strategies}

Now, following the definition of terminal sets we can give a complete description of possible W-Win, B-Win, and Draw strategies. Obviously, in reality, only one of them takes place.

1. W-Win strategy:

W-Win = BB-Destroyed $\cap$ WB-Safe

The W-Win strategy, if it exists, is to change status of both W-Zone and B-Zone from the initial, WB-Intercept $\mathbf{W}$ Zone and BB-Protect ${ }_{\mathrm{B}-Z \text { Zne' to the global WB-Protect }}$ and $\mathrm{BB}$-Intercept, respectively.

To do that we have to move W-FIGHTER from h8 into both Zones as fast as possible. The presence of WFIGHTER in these Zones immediately converts their status into WB-Protect and BB-Intercept, respectively, and this status stays permanently in the search tree until Zones shrink to the states where the W-BOMBER is destroyed, BB-Destroyed state for the W-Zone, or B-TARGET is hit, BB-Safe state for the B-Zone.

\section{B-Win strategy:}

B-Win = WB-Destroyed $\cap$ BB-Safe

The B-Win strategy, if it exists, is to keep the status of both W-Zone and B-Zone unchanged as they are in the initial state, WB-Intercept $\mathbf{W - Z o n e}$ and BB-Protect $\mathbf{B - Z o n e}$ ' respectively, which means to reach the state where the status is global, and belongs to WB-Intercept and BBProtect.

To do that Black has to destroy W-BOMBER and hit the B-TARGET as fast as possible by shrinking these Zones down to WB-Destroyed and BB-Safe states.

3. Draw strategy:

Draw $=$ Safe $\cup$ Destroyed

where Destroyed = BB-Destroyed $\cap$ WB-Destroyed,

Safe $=$ BB-Safe $\cap$ WB-Safe

Thus, the Draw strategy, if it exists, is to change the status of at least one of the Zones, W-Zone or B-Zone, from the initial, WB-Intercept $\mathbf{W - Z o n e}$ and BB-Protect ${ }_{B-Z o n e}$, to the global WB-Protect and BB-Intercept, respectively.

To do that we have to move W-FIGHTER from h8 into both Zones as fast as possible. The presence of $\mathrm{W}$ FIGHTER in these Zones immediately converts their status into WB-Protect and BB-Intercept, respectively, and this status stays permanently in the search tree until Zones shrink to the states where the W-BOMBER is destroyed, BB-Destroyed state for the W-Zone, or B-TARGET is hit, BB-Safe state for the B-Zone.

\subsection{A Strategy at the Start State}

Obviously, we do not know in advance which strategy actually takes place in this problem. Let us consider the start state for this problem and assume that White follows W-Win strategy while. Black follows B-Win strategy. Following W-Win strategy W-FIGHTER should get into both Zones, the W-Zone and the B-Zone. The distance from the start state (which belongs to BB-Protect $\mathbf{B}_{\mathbf{B}-Z \text { Zne }}$ ) to the set of states BB-Intercept can be measured as the length of the shortest trajectory of the W-FIGHTER from h8 to one of the B-Zone gateways. It is equal to 2 steps. Analogously, the distance from the start state to the WBProtect is equal to the length of the shortest trajectory of the W-FIGHTER from $\mathrm{h} 8$ to one of the $\mathbf{W}$-Zone gateways. It is equal to 4 steps. The total is 6 . However, the distance from the initial state to the intersection BB-Intercept $\cap$ WB-Protect can be reduced down to 4 steps employing the time-gaining trajectory through $\mathrm{g} 7$ and $\mathrm{f6}$ (Fig. 15, left). Also, this is the only way to approach both sets simultaneously. Thus, following the W-Win strategy WFIGHTER moves along the shortest trajectory passing through g7: 1. h8-g7. Next, the Black, following the B-Win strategy, should keep the status of W-Zone and B-Zone unchanged as it is in the start state, WB-Intercept $\mathbf{W}$-Zone

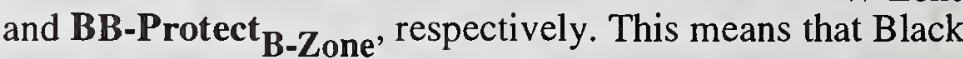
should shrink these Zones by moving either the BFIGHTER from a6 along one of the intercepting trajectories to intercept the W-BOMBER within W-Zone (Fig. 15, right) or the B-BOMBER from h5 along the main trajectory $a(\mathrm{~h} 5) a(4) a(\mathrm{~h} 3) a(\mathrm{~h} 2) a(\mathrm{~h} 1)$ to hit the W-TARGET within the B-Zone.
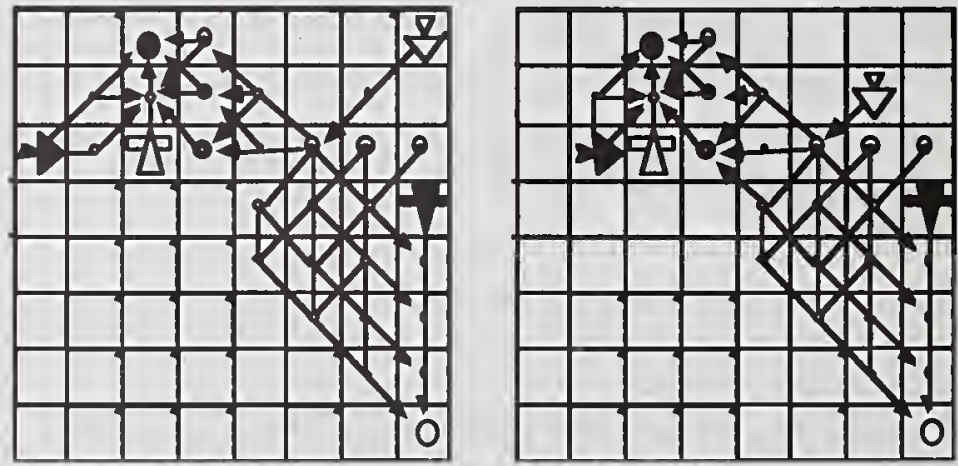

Fig. 15. The distance measurement to BB-Intercept $\cap$ WBProtect from the start state (left) and from the state generated after 1. h8-g7 a6-b6 (right).

If Black is involved in the W-Zone and responds, e.g., 1. ... a6-b6, the W-FIGHTER should continue motion along the same shortest trajectory (Fig. 15, right): 2. g7-f6 following the W-Win strategy.

Alternatively, if Black is involved in the B-Zone and moves 1... h5-h4, the B-Zone shrinks (Fig. 16). The new set of the shortest time-gaining trajectories from $\mathrm{g} 7$ through 
f6 to both W-Zone and B-Zone is shown in Fig. 16. Thus, following W-Win strategy the response of the White must be the same: 2. g7-f6. Consequently, the white moves (in bold) 1 . h8-g7 X-X; 2. g7-f6 are universal, i.e., they must be included in the optimal subtree, the W-Win strategy. These moves even do not depend of the black first move, 1. ... X-X, whatever it is, even if Black follows the B-Win strategy.

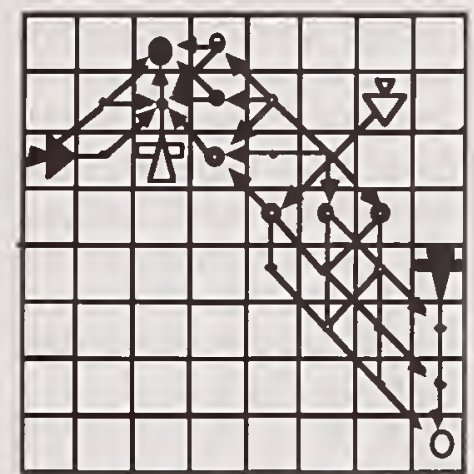

Fig. 16. The distance measurement from the state after 1. h8-g7 h5-h4 to BB-Intercept $\cap$ WB-Protect.

According to this strategy the Black should keep the status of the W-Zone and B-Zone unchanged. Let us investigate three possible alternatives for the Black response 2... Y Y Y, a), b), and c).

a) If Black is involved in the $\mathbf{W}$-Zone on the first and second moves, i.e., if 1 . h8-g7 X-X; 2. g7-f6 Y-Y and $X-X$ and $Y-Y$ are both in the $W-Z$ one, then WFIGHTER at $\mathrm{f} 6$ is immediately getting into B-Zone while the B-Zone itself is changing its status to BBIntercept $\mathbf{B}_{\mathbf{B}}$ Zone. This happens because of the alternation of turns: while making the $Y-Y$ move, Black spends one time increment in the $\mathbf{W}$-Zone that gives an extra time increment to the White in the BZone, the white turn, and the intercepting trajectories from f6 become the 1st negation trajectories (Fig. 15, right) - see Section 5. Thus, if White follows the WWin strategy and Black - the B-Win strategy, we ended up with the change of the status of one of the Zones, the B-Zone, and this status will stay unchanged for the rest of the search which means that both WBOMBER and W-FIGHTER will be eventually destroyed. The state after 2...Y.Y is a terminal state that belongs to global WB-Intercept $\cap$ BB-Intercept. Consequently, we actually implemented the Draw strategy,

for the case of

$$
\text { Draw }=\text { Safe } \cup \text { Destroyed }
$$

Destroyed $=$ BB-Destroyed $\cap$ WB-Destroyed

The corresponding branches are included in the bold subtree (Fig. 5)

b) If Black is involved in the B-Zone on the first and second moves, i.e. X-X and $\mathrm{Y}-\mathrm{Y}$ are $1 . . . \mathrm{h} 5-\mathrm{h} 4$ and 2. ... h4-h3, respectively, then the W-FIGHTER should move towards W-Zone gateways $\mathrm{d} 6, \mathrm{~d} 7$, and $\mathrm{d} 8$ along the most time-gaining trajectory through e7 (Fig. 16): 3. f6-e7. This is the terminal state which belongs to
WB-Protect $\cap$ BB-Protect, i.e., we changed status the $\mathbf{W}$-Zone. As in the case a), if White follows the $\mathbf{W}$. Win strategy and Black - the B-Win strategy, we end up with the Draw strategy

for the case of

$$
\text { Draw }=\text { Safe } \cup \text { Destroyed }
$$

Safe $=$ BB-Safe $\cap$ WB-Safe.

The corresponding branches are included in the bold subtree (Fig. 5).

c) If Black is involved in the $\mathbf{W}$-Zone on the first move 1 . ... X-X and switches to $\mathbf{B}-Z$ Zone on the second move 2 . ... h5-h4, then W-FIGHTER should continue moving along the shortest time-gaining trajectory 3. f6-e5. There are two alternatives.

If Black switches back to the W-Zone, then WFIGHTER at e5 is immediately getting into B-Zone while the $\mathbf{B}-$ Zone itself is changing its status to $\mathbf{B B}$ Intercept. The situation in this state is similar to the case a): this is the terminal state which belongs to WB-Intercept $\cap$ BB-Intercept, i.e., we changed status the B-Zone. Thus, if White follow the W-Win strategy and Black follow the B-Win strategy, again, we end up with the Draw strategy

Draw $=$ Safe $\cup$ Destroyed

for the case of

Destroyed = BB-Destroyed $\cap$ WB-Destroyed. Otherwise, if Black continues in the B-Zone 3... h4$\mathrm{h} 3$, then W-FIGHTER at e5 should immediately get into the $\mathbf{W}$-Zone through d6 gateway while the $\mathbf{W}$ Zone itself is changing its status to WB-Protect. The situation in this state is similar to the case $\mathbf{b}$ ): this is the terminal state which belongs to WB-Protect $\cap$ BB-Protect, i.e., we changed status the W-Zone. Thus, finally, if White follows the W-Win strategy and Black follows the B-Win strategy, we end up with the Draw strategy,

for the case of

$$
\text { Draw }=\text { Safe } \cup \text { Destroyed }
$$

Safe $=$ BB-Protect $\cap$ WB-Protect.

In case $\mathbf{c})$ the corresponding branches are included in the bold subtree (Fig. 5).

We proved that the only optimal strategy possible at the start state is the Draw strategy. The bold subtree is an implementation of this strategy. Thus, statement 2. and Theorem are proved.

\section{Discussion}

Theoretical results received employing other approaches and experiments with programs utilizing these approaches show that the search tree generated in order to solve the problem considered in Sections 7-9 consists of more than a MILLION moves with the branching factor (Nilsson, 1980) around 9. In contrast, the Linguistic Geometry tools allowed to find the optimal solution generating the search tree which consists of about 46 moves, with the branching factor 1.5 . The low branching 
factor indicates that the algorithm is goal-oriented.

The novelty of this paper is in the proof of optimality of the solution. It appears that LG tools are able to distinguish and significantly expand the "islands of potential stability", the sets of states (positions) with known value in the "ocean of all states of unknown value" to be searched employing conventional brute force approach. In our case we expanded the small islands of terminal states, Destroyed and Safe, into the sets Intercept and Protect. Moreover, I envision the LG search like an "optimal navigation of the ship" from the start state through the ocean of unknown states to the expanded islands employing the shortest path. In our problem this was reflected as a motion of the W-FIGHTER towards WZone and B-Zone simultaneously. It is likely that similar ideas work in all the LG examples. This will be a subject of our further research. If this is the case we can speculate that LG tools allow for a very efficient break of the state space that drives the search directly to the optimum.

It is easy to predict that the power of the Linguistic Geometry goes beyond the domain of aerospace games. The definition of Complex System (see Section 3) is generic enough to cover a variety of different problem domains. The core component of this definition is the triple $\mathrm{X}, \mathrm{P}$, and $R_{p}$. Thus, looking at the new problem domain we have to define $X$, the finite set of points - locations of elements. In different real world problems we can consider $X$ as an operational district of the underwater or on-surface combat, or even as a set of orbits. The set of elements $\mathrm{P}$, the mobile units, can be the set of submarines, tanks, squadrons, or satellites with various moving capabilities. Indeed, these capabilities are represented with the binary relations $R_{p}$, which is exactly the place for introduction of the variable speed, the gravity impact, the engine impulse duration, etc.

A dramatic search reduction achieved in the serial and concurrent games (Stilman, 1994-1996) allowed us to initiate the development of the prototype of the system for optimal planning and control of the real world aerospace combat with participation of air fighters, satellites, and unmanned aerial vehicles - UAVs. This work is currently under way at Phillips Lab, Kirtland AFB, NM, USA. Also, a prototype of the generic Hierarchy of Formal Grammars, a test-bed of the multiagent architecture for various applications, is planned to be developed at Sandia National Laboratories, Albuquerque, NM.

\section{References}

Botvinnik, M.M. (1984). Computers in Chess: Solving Inexact Search Problems. Springer Series in Symbolic Computation, New York: Springer-Verlag.

Botvinnik, M., Petriyev, E., Reznitskiy, A., et al. (1983). Application of New Method for Solving Search Problems For Power Equipment Maintenance Scheduling. Economics and Mathematical Methods (1030-1041), 6, (in Russian).

Chomsky, N. (1963). Formal Properties of Grammars. in Handbook of Mathematical Psychology, eds. R.Luce, R.Bush, E. Galanter., vol. 2 (323-418). New York: Wiley.
Fikes, R.E. and Nilsson, N.J. (1971). STRIPS: A New Approach to the Application of Theorem Proving in Problem Solving. Artificial Intelligence 2 (189-208).

Garcia-Ortiz, A. et al. (1993). Application of Semantic Control to a Class of Pursue-Evader Problems, Computers and Mathematics with Applications, 26(5), (pp. 97-124).

Lirov Y., Rodin, E.Y., McElhaney, B.G., and Wilbur, L.W. (1988). Artificial Intelligence Modeling of Control Systems, Simulation, (pp. 12-24), 50(1).

McCarthy, J. (1980). Circumscription-A Form of NonMonotonic Reasoning. Artificial Intelligence, (27-39), 13.

McCarthy, J. and Hayes, P.J. (1969). Some Philosophical Problems from the Standpoint of Artificial Intelligence. Machine Intelligence (463-502), 4.

Nilsson, N.J. (1980). Principles of Artificial Intelligence, Palo Alto, CA: Tioga Publ.

Rodin E. (1988). Semantic Control Theory, Applied Mathematical Letters, (pp. 73-78), 1(1).

Sacerdoti, E.D. (1975). The Nonlinear Nature of Plans, Proc. Int. Joint Conference on Artificial Intelligence.

Shinar, J., (1990). Analysis of Dynamic Conflicts by Techniques of Art. Intelligence, INRIA Report, Antipolis.

Stilman, B. (1977). The Computer Learns. in Levy, D., 1976 US Computer Chess Championship (83-90). Computer Science Press, Woodland Hills, CA.

Stilman, B. (1985). Hierarchy of Formal Grammars for Solving Search Problems. In Artificial Intelligence. Results and Prospects, Proc. of the Int. Workshop (6372), Moscow, (in Russian).

Stilman, B. (1993a). A Linguistic Approach to Geometric Reasoning, Int. J. Computers and Mathematics with Applications (29-57), 26(7).

Stilman, B. (1993b). Network Languages for Complex Systems, Int. J. Computers and Mathematics with Applications (51-79), 26(8).

Stilman, B. (1993c). Syntactic Hierarchy for Robotic Systems, Integrated Computer-Aided Engineering(5781), 1(1).

Stilman, B. (1993d). A Formal Language for Hierarchical Systems Control, Languages of Design (333-356), 1(4).

Stilman, B. (1994a). Translations of Network Languages. Int. J. Computers and Mathematics with Applications (65-98), 27(2).

Stilman, B. (1994b). A Formal Model for Heuristic Search. Proc. of the 22nd Annual ACM Computer Science Conf., (380-389), March 8-10, Phoenix, AZ.

Stilman, B. (1994c). Heuristic Networks for Space Exploration, Telematics and Informatics, Int. J. on Telecommunication \& Information Technology, 11(4), (403-428).

Stilman, B., (1994d) A Linguistic Geometry for Control Systems Design, Int. J. of Computers and Their Applications, (89-110), Vol. 1(2), Dec. 1994.

Stilman, B., (1995a) Deep Search in Linguistic Geometry, SYMPOSIUM ON LINGUISTIC GEOMETRY AND SEMANTIC CONTROL, Proc. of the First World Congress on Intelligent Manufacturing: Processes and Systems, (868-879), Mayaguez, Puerto Rico, Feb. 1995. 
Stilman, B., (1995b) A Linguistic Geometry for 3D Strategic Planning, Proc. of the 1995 Goddard Conference on Space Applications of Artificial Intelligence and Emerging Information Technologies, (279-295), NASA Goddard Space Flight Center, Greenbelt, MD, USA, May 1995.

Stilman, B., (1995c) A Linguistic Geometry for Multiagent Systems, Proc. of The 8th International Conference on Industrial \& Engineering Applications of Artificial Intelligence \& Expert Systems - IEA/AIE, (3-12), Melbourne, Australia, June 1995.

Stilman, B., (1995d) Total Concurrency and Uncertainty in Linguistic Geometry, Proc. of the IEEE International Symposium on Intelligent Control, (79-84), Monterey, CA, USA, August 1995.

Stilman, B., (1996a) Network Languages for Intelligent Control, An International Journal: Computers \& Mathematics with Applications, (91-118), 31(3).
Stilman, B., (1996b) Network Languages for Concurrent Multiagent Systems, An International Journal: Computers \& Mathematics with Applications., (to appear).

Stilman, B., (1996c) From Serial to Concurrent Motions in Multiagent Systems: A Linguistic Geometry Approach, Journal of Systems Engineering, (to appear).

Yakhnis, V., Stilman, B., (1995a) Foundations of Linguistic Geometry: Complex Systems and Winning Conditions, SYMPOSIUM ON LINGUISTIC GEOMETRY AND SEMANTIC CONTROL, Proc. of the First World Congress on Intelligent Manufacturing: Processes and Systems, (843-854), Mayaguez, Puerto Rico, Feb. 1995.

Yakhnis, V., Stilman, B., (1995b) A Multi-Agent GraphGame Approach to Theoretical Foundations of Linguistic Geometry, Proceedings of the Second World Conference on the Fundamentals of Artificial Intelligence (WOCFAI 95), Paris, France, July 1995. 

XXI

ISAS'96: CONFERENCE REVIEW 



\section{ISAS'96: Conference Review}

prepared by A. Meystel, K. Bellman, D. Filev, J. Goguen, C. Hewitt, C. Joslyn, L. Kohout, M. Kokar, C. Landauer, I. Muchnik, L. Perlovsky, V. Stefanuk, Y. Yufuk

This Review is based upon reports submitted by the workshop's chairmen. The complete set of reports for ISAS' 96 and ISAS' 97 will be combined into a white paper entitled "Semiotics: Its Significance and Perspective". This will be distributed among the organizations involved in monitoring and funding the research and educational activities in the US.

\section{SYNTACTICS OF INTELLIGENT SYSTEMS: THE KINDS OF LOGIC AVAILABLE}

- The main theme of the Syntactics workshop was how to deal with infinite/complex structures in modeling systems within the framework of logic.

- The main reason to look at systems from the semiotic perspective is the COMPLEXITY of both real and artificial systems (cf. the comment by Professor Sebeok on the existence of infinite number of possible signs generated by a finite number of primitives, e.g., genetics, immune system.)

- The workshop focused on the available tools. The pervasive question was, how can we feasibly use these tools?

- We generally concluded that various system engineering techniques combined within a framework of logic lead to significant improvement, by using semiotic tools for handle the complexity of both real and artificial large-scale systems.

- The following approaches have been discussed:

1. Moshe Vardi stated that in order to establish communication between (or among) agents, they need common (shared) knowledge. Establishing such a knowledge base within the logical framework may lead to an infinite number of message exchanges. M.Vardi proposed two approaches to establish such common knowledge in a finite number of steps.

2. Wlodek Zadrozny proposed an algebra to represent ten categories of signs in Peirce's classification. Although, it seems that this algebra should be an infinite one. Zadrozny showed that it can be finite.

3. Mieczyslaw Kokar showed how to combine many methods from system engineering within a logical framework to improve system performance. The main point is that the issues of complexity of logical tools can be remedied by such system engineering tools. 
The question is how far can we go with developing such heterogeneous systems into autonomous ones.

4. Sergey Petrov analyzed languages that are "poor" in the sense that some deductions in these languages are not possible. His question was if we can decide whether or not there are finite axiomatization for some languages.

5. Jery Tomasik showed that classical results of Rasiowa and Keisler can be used to construct models of the world based upon inputs from sensors. Sensors can provide infinite number of input combinations. Tomasik showed how to address infinity within the framework of model theory.

6. William Farmer showed how an existing interactive theorem proving system (IMPS) changes contexts via "theory interpretation." IMPS keeps a base of "little theories" which are examined by the system for their use in a specific problem.

\section{MULTIRESOLUTIONAL CONCEPTS AND METHODOLOGIES}

- Multiresolutional approach is a powerful tool of complexity reduction. Introduction of multiple resolution levels has actually determined the architecture of all existing systems including architecture of the brain. Multiresolutional methods can be applied to the following: time, space, descriptions and relations of entities, events, problems and plans.

- Advantages: MR-methods allow us to represent the world at many levels of resolution due to the power of generalization algorithms. This creates a limitation on analysis from above and from below. Total complexity of computations can be drastically reduced.

- Engineering methodologies of MR-methods include the following: object oriented programming, fractals, MR-signal processing, Wavelets, multirate control systems, multiscale and multigranular representations, and others

- Standards of modules and interfaces can be created based upon properly introducing definitions for granularity, zone of indistinguishability, scope, scale, and cost-functional of interest.

- Disadvantages: rigidness of hierarchy seems to be the most pervasive complaint. Frequently, this complaint is linked with neglect of the overall computational advantages. However, we should work on developing the trade-off methodologies and analysis of the existing restrictions. One of the ways to avoid the disadvantages is by adding adaptation capabilities to MR-systems

Participants of the workshop concentrated on three major themes: MR-representation, MRprocedures, and MR-models.

\section{MR-REPRESENTATION IN DIFFERENT DOMAINS}

The development of this is based on studying multiple results of practical applications for examples in various areas. 
Example 1: In all problems of image processing, we deal with a hierarchy of pixels - units of granularity ("atoms" or indistinguishability zones for the images.) Three levels of a pixel characterization:

- a component of the pixel (a successor or a "child") which can be determined for the pixel at the lower resolution level (its "father")

- the level quantitative characterization of the image elementary components (the diameter of the indistinguishability zone.)

- the description of the inclusion relationships among the pixel and pixel's children.

Example 2: Recognition systems for the handwriting:

- the indistinguishability zones or elementary entities are irregular structures; MRmethod allows for the existence of irregular elements of contour images and models of trajectories. Example (graphs of interactions within large molecules in biochemistry)

Example 3. Elementary units of different levels of resolution in various domains:

- atoms in physics

- scale units in cartography

- Lyapunov ball control theory

- radical groups of molecular structures in chemistry

- sub-molecules (amino acids in proteins) in chemistry

- hyper units (monomer proteins in multi-domain proteins) in chemistry

- coalitions of protein reloaded with particular biological functions in biochemistry

- irregular elementary entities (iee) from the low level of resolution are integrated in a trajectories between iee for higher resolution in handwriting analysis

Example 4. Hidden Markov process in the area of signal processing

1st Level of resolution: elements should be vectors parameters for observed component of the hidden process and names of its hidden states:

2nd Level - elements of structural parameters of the teams of the first level elements.

Example 5. Complex systems analysis: elementary entity at a level characteristic for the objectoriented analysis.

\section{MR-PROCEDURES}

1. Analysis at a single level:

- aggregation and decomposition of elements for a single level of resolution

- characterization of their uniqueness

- determining their relationship

2. Analysis of the complete structure of the multiresolutional architecture: 
- a priori design of the multiresolutional architecture which delivers the minimum of computational complexity to a system

- development of mathematical models for systems belonging to a particular resolution level

- development of relations among mathematical models of the same system at different levels of resolution

- switch on and off procedures of the single level analysis

- generalization of results of the single level analysis

- evaluation of correlations of these results with final outcome and designing the final rules to get the outcome

- changing the current multiresolution structure of analysis and iterating it in a new form

- solution recognition (global stop rule)

- cooperation with a human assistant

III Mathematical Models of Multiresolutional Systems (MRS)

- generators of hierarchical structures

- hierarchical structure algebra

- dynamic models onto hierarchical structures (new automata theory and)

- Methods of Simulation of MRS

- Mathematical criteria for:

* aggregation - decomposition of assemblies

* abstraction - specialization of features

* generalization - insinuation of features

IV. Efficiency Evaluation for the MRS Approach:

Systematic collection is required of the benefits and shortcomings of MRS used for Intelligent System Development.

\section{FUZZY LOGIC AND THE MECHANISMS OF GENERALIZATION}

- The main goal of this workshop is to work toward establishing a link between some methods and concepts from semiotics and fuzzy logic and system fields.

- Fuzzy logic methodology enriches the logic field with methods for handling linguistic statements in terms of linguistic variables introduced by Zadeh. Fuzzy logic can also deal with approximation and incompleteness issues.

- Semiotics, on the other hand, provides methods to deal with the meaning of linguistic and logic statements, paying attention to their syntax, semantics, and pragmatics.

- Deeper integration of these two approaches with the aim to strengthen their applicability in design, analysis, and use of Intelligent Systems is required. 
- The following issues are discussed:

1. Granularity, Generalization, and Meaning

- The main goal of semiosis is the interpretation of signs and symbols which can be understood also as recognition of their meaning. The meaning interpretation unit is a very complicated one. The meaning of something can be different, depending on the scale which is used for representation. This makes the notion of granularity a very important one. Levels of granularity are also referred to as levels of resolution, and they are closely related to generalization.

- Granularity was introduced into fuzzy sets by Zadeh. In his interpretation, "granulation involves a decomposition of the whole into parts, and conversely organization involves an integration of the parts into whole."

- Intelligent systems deal with interpreting information communicated in symbolic form. Interpretation and meaning depend on context. Any dynamic process considered with all its details and the details of its interaction with its environment cannot be properly understood unless the details, which are irrelevant within a particular context, are removed. But, the information on which such an interpretation could be based will often be incomplete. With incompleteness, there may be more that one interpretation. Indeed, a large family of interpretations may be possible, which may conflict. Fuzzy sets, relations and logic can play an important role here. They allow us, through the theory of potentiality (or virtual plurality), to deal with the whole family of virtual outcomes and also to measure the degree of conflict of individual members of some possible family of outcomes produced by the meta-process of interpretation.

2. The Role of Granulation as and for Generalization in Cognition and Action

- Generalization plays an essential role in the human cognitive and symbolic activities. But, it is equally important in artificial intelligent systems. Generalization works as a filter of information. During the process of generalization, the relevant pieces of information are included in the final outcome, and the irrelevant ones are excluded. Information to be included and/or excluded depends on the contexts and information processing goals.

- Architectures of intelligent systems employ the concept of granularity. The examples of multigranular (multiscale, multiresolutional) systems are known in robotics and manufacturing (e.g. NIST-RCS.)

- Some advanced intelligent system architectures mimic biological models of the human brain. Such architectures operate as the coupling of functional hierarchical (or heterarchical) levels and loops. Each level operates at a different level of generality, abstracting many different features from the world with which the architecture interacts. Granulation and generalization are closely interlinked.

- In computer science, the problem of structuring systems is commonly addressed via the Object-Oriented (OO) approach. This is a set of important design techniques for structuring systems in a hierarchical fashion. There are, however, some conceptual and practical 
problems with $\mathrm{OO}$ programming when dealing with highly dynamic systems. Like data base entities, objects are usually assumed to be given. This, however, hypothesizes hierarchies, with no accounting for the dynamic binding of levels of individual objects.

- To deal with this problem we have to examine the essence of inheritance and investigate how the objects emerge. The phenomenon of object emergence is linked with formation of crisp and fuzzy classes (generalized groupings). Indeed, we deal with the logical theory of fuzzy relations, which can be used to expose the inadequacy of currently used logical structure of crisp (i.e. non-fuzzy objects). Logically, generalization is a process in which relevant properties (intentional specifications) of objects are grouped. We create new objects -structures (given as such by extension) by new intentional specification. So OO approach can be viewed as a very special case of the pragmatics of groupings, a kind of granulation.

- The issues of granulation in their full generality, however, must be adequately addressed by employing fuzzy classes, the semantics of which can be provided by many-valued logic based relations with special meta-properties. The issues of adequate multi-context semantics that is needed for the Intelligent Systems require this generality. The OO approach has to be extended.

\section{The Issues of Incompleteness of Information}

- In Intelligent Systems we must deal with incompleteness of information. This is the domain where fuzzy sets can help. The context in which objects operate determines the goals and meaning of the objects and their generalization hierarchical structure. Without the semiotic notions, this aspect cannot be satisfactorily handled. We need a synergistic development of fuzzy and semiotic methodologies. One of the classic notions of semiotics is the triadic structure of the object, its notation and its interpretation.

- The concept of the semiotic triplet of subdisciplines syntax -- semantics -- pragmatics was coined by Morris in the late 1930s. That is where the duality Semiotics -- Mathematics comes in. In logical methods of proofs, only the "form," the syntactic composition plays the role. In the logical theory of models, the primary goal is to interpret syntax in semantic meta-structures and the pragmatics of emergence of either of these. Here, the fuzzy logic can play an important role since we have the duality of linguistic descriptors and fuzzy structures to which these descriptors apply. We also have the duality symbolic vs. numerical that are both addressed by what Zadeh called "the fuzzy logic in wider sense."

- When forming grouping/generalizations/classes on the basis of their properties, one substantial difficulty appears. One needs to generalize on the basis of relevant properties of objects that are present as well as on the basis of the relevance of the absence some properties. This leads to entirely new problems. This problem which is closely connected with semisets and vagueness is determined by problem of lack of properties and by the locality of negation. These are closely connected with semisets.

\section{Conclusions}

1. There are potential links between fuzzy logic and semiotics that should be further explored. Granularity is essential to develop any complex system. To deal with granularity in intelligent systems, the potential of fuzzy logic to approximate should be explored. Fuzzy relations provide high-level specification language and computational tool to form granules that 
subsume equivalences, similarities, and hierarchies of objects. Semiotic concepts and methods can deal with naming, contexts, dynamics of symbolic communication, and the pragmatics of naming and actions.

2. There is sufficient background to conduct further discussion and formulating an action plan to deal with the topic of fuzzy logic, granulation an generalization. This should be pursued via internet. The chair of this workshop is willing to mediate further discussion and produce a document summarizing these.

\section{INTELLIGENCE OF RECOGNITION}

- The question of intelligence of algorithms and neural networks is being answered today in at least five ways. First, mathematical models are developed for psychological experimental data on perceptual and behavioral phenomena. Second, mathematical tools are developed for modeling human perceptual functions such as vision. Third, mathematical models are developed for those functions of intellect, which are associated with the internal working or mind, such as meaning and consciousness. Fourth, mathematical modeling of brain organization is used to search the understanding of mind. Fifth, mathematical and metaphysical analysis of intelligence is being undertaken. This establishes connections among various mathematical concepts and their relationships to metaphysical concepts of intelligence. These directions are discussed in the workshop.

- Recognition of objects in images and in temporal sequences of images is one of the most important recognition paradigms. A lot of information is accumulated about biological vision systems and a lot of effort has been invested into developing mathematical tools and engineering applications. Although we are still far from completing a general mathematical theory of vision, significant progress has been achieved in a number of applications and a number of useful mathematical tools has been developed. One thing is clear. A vision system is not a homogeneous one, but it combines a large number of diverse subsystems. An image recognition process is generally separated into several steps or stages, both mathematically and implemented in hardware. A mathematical and engineering reason for this is to reduce an overwhelming complexity of the problem.

- Biological systems also are known to process visual information in stages, and many of our mathematical tools are patterned after biological vision systems. A typical breakdown includes detection of the region of interest, segmentation of the objects, enhancement, and recognition of the object class and pose.

- The following approaches have been discussed:

1. Professor D. Casasent discusses developing new powerful and efficient tools including new distortion-invariant filters, biologically-inspired Gabor wavelet filter techniques, hierarchical processing, new representation methods. Casasent also discusses mathematical methods of combining (fusing) these tools into a unified vision system. Practically important problems that still await their solutions include distortion-invariant detection and recognition; efficiently processing of time-sequential image frames; feature selection; and combining results from several algorithms, and efficient representation techniques.

2. Nonlinearity of human visual processing was recognized in the last century by scientists including Helmholtz. He found, for example, that a perceived color of an object depends on the average color of the visual field. But, nonlinear mathematical methods that are suitable for modeling vision and perception in general did not exist in the last century. These methods appeared only recently. Resurgence of neural network research in 1980s was due to emergence of powerful nonlinear neural paradigms. A neural network field can be viewed as a development of nonlinear mathematical tools suitable for modeling the mind. But, many of 
the currently popular neural network paradigms are limited in the basic type of a non-linear operation performed by a neuron to a nonlinear transformation applied to a weighted sum of neuronal input signals. Professor G. Ritter discusses a new morphological type of neural networks, which explores a different type of nonlinearity at the basic neuronal level. In morphological neurons, multiplication and addition are replaced with additions and $\max / \mathrm{min}$ operations.

3. The concept of morphological neural network has roots in image algebra and in an alternative hypothesis concerning properties of biological neurons. Hierarchical organization is another important aspect of intelligent systems. Specific mathematical techniques addressing hierarchical organization has received appreciable attention in the development of wavelet transforms. Professor H. Szu combines wavelet and neural network techniques to develop adaptive wavelet transforms. He discusses organization of human perceptual systems, the mathematical technique, hardware implementations and applications.

4. The roles of learning vs. a priori knowledge is one of the central and most controversial problems in the development of intelligent algorithms. While symbolic AI emphasizes apriority of knowledge acquisition, most pattern recognition and neural network techniques emphasize adaptivity of intelligent systems. In Prof. Minsky's words, "...theory of representation was developed independent from theory of learning." Model-based recognition paradigm has been developed to combine apriority of models with adaptivity of model parameters. Prof. E. Manolakos discusses model-based joint segmentation and recognition of objects. He develops this technique in the framework of parameter estimation of hierarchical mixture densities. The maximum a posteriori (MAP) estimate of the parameters is computed by the application of a modified version of the Expectation Maximization algorithm (EM with regularizing constraints applied to multiple level hierarchies). The approach is flexible. It allows for non-stationary pixel statistics, different noise models, translation and scale invariant and is well suited for recognition of partially occluded objects. An unsolved problem that should be addressed in the future is the development and use of complicated structural models.

5. Most recognition algorithms and neural networks can be viewed as seeking a minimum value of an appropriate objective function during recognition or learning phases. In which way is maximization of the goal function related to intelligence? In which way does it lead to an appropriate generalization from the past to future data? What type of intelligence could be attributed to different types of goal functions? Professor Golden addresses these questions by establishing a relationship of the goal function maximization to other mathematical concepts of intelligence. Specifically, he shows that the goal maximization is equivalent to an inference procedure on a relational system comprised of the data, event space, and relation operation. This theory is useful for practical applications, as well as for providing insights in formulating the sense in which a given recognition or neural network algorithm is making intelligent inferences. Future research should address establishing relationships between neural networks and the statistical pattern recognition framework, construction of statistical tests for testing hypotheses about the parameters of such networks, and statistical goodnessof-fit tests for deciding if a given neural network algorithm best fits a given statistical environment.

6. Genetic mechanisms have a fundamental similarity with the human Mind in producing adaptive systems based on a priori structures. This property of genetic mechanisms is used to develop improved recognition techniques in genetic algorithms (GA) and genetic programming (GP) techniques. Mr. W. Punch describes recognition techniques based on genetic mechanisms to find the properties of data that are important for data classification within large datasets. He combines GA with the K-nearest neighbor (knn) algorithm. The GA determines weights for each feature based on known examples so as to optimize knnclassification based on a linear combination of features. He also describes an extension of this work to GP. The combined GP-knn technique optimizes data classification by selecting linear combinations of features, as well as determines functional relationships among the features. He also compares the effectiveness of GA and GP for biological problems. 
What can be learned about intelligent recognition architectures from brain studies, and what type of brain organization is suggested by combining current psychological and neurophysiological data with mathematical models of neural networks? Prof. D. Levine discusses the mutual interaction between these fields and the neural models of the prefrontal function. The prefrontal cortex performs the "executive" function within the brain. It is roughly divided into three interacting parts: affective guidance of responses, linkage among working memories, and forming behavioral schemata. Neural models of each part is discussed within a theoretical framework.

\section{INTELLIGENCE OF LEARNING AND EVOLUTION}

- What is intelligent about our recognition and learning algorithms, neural networks, and intelligent system architectures? Which aspects of intelligence we understand and know how to use in our algorithms and neural networks, and which do we not? Does individual learning resemble evolution of species, and what are the mechanisms of learning and evolution? These were the questions posed to a group of distinguished scientists and leaders in the fields of recognition, intelligent systems, neural networks and learning who attended this workshop.

- The response was tremendous; the discussion of intelligence in our mathematical models of intellect seems to be timely. It is a matter of both hot debates and quickly emerging results. Both aspects of the problem are going to be discussed in the papers presented at the workshop: questions that are posed and questions that are being answered. Mathematical definitions and models of various aspects of intelligence are the most fascinating and exciting developments in the history of science.

- The question of intelligence of algorithms and neural networks is being answered today in at least five ways. First, mathematical models are developed to explain psychological experimental data on perceptual and behavioral phenomena. Second, mathematical tools are developed to simulate human perceptual functions, such as vision function. Third, mathematical models are developed for some of the functions of intellect. Functions of interest are associated with the internal working or mind, such as emergence of meaning and consciousness. Fourth, mathematical modeling of brain organization is used to search the understanding of mind. Fifth, mathematical and metaphysical analysis of intelligence is being undertaken. This establishes connections among various mathematical concepts, and their relationships to metaphysical concepts of intelligence.

- The following directions have been discussed at the workshop:

1. Prof. W. Freeman is concerned with the mechanisms of brains by which they construct meanings within themselves and representations of meanings by motor actions in order to communicate meanings to other brains: "A machine can only 'know' the inferences it constructs from the sensory consequences of its own actions into the world." This translates into the construction of machine intelligence by combining robotics and automata theory, in the context of operations that are modeled on brain dynamics and implemented by noisestabilized chaotic attractors for pattern generation and recognition. Simulations of animal and human performance in the laboratory are done by constructing sets of differential equations to represent cortical dynamics in pattern recognition and solving them with digital computers, with the emphasis on multisensory convergence in the combining of olfactory, visual, auditory, and tactile inputs. Future work is now being directed toward controlling chaotic dynamics by regulating the sensitivity to initial conditions, and also toward solving the equations with analog hardware, taking advantage of the speed and simplicity. Thereafter, the self-organizing machines will have increasing control of their own input through effectors by means of which they can control their sensors, and through internal 
feedback by which they can regulate the attractor landscapes in their sensory/perceptual processors for pattern recognition. Prof. W. Freeman calls the prototype of such devices modeled on animal brains a "meaning machine" that can make representations of its internal states to its trainer.

2. How do categories and symbolic structures develop? How is it possible to adapt and learn, while using a priori knowledge? What does constitute a priori knowledge? What are the differences and similarities between real-time adaptation and long-term learning of categories and complicated symbolic structures? Current research directions in this important area are represented in three talks in the workshop. Prof. R. Sun introduces a hybrid model for bottom-up skill learning in a situated way. The hybrid model combines connectionist, symbolic, and reinforcement learning within an integrated architecture. In this model, agents continuously learn from ongoing experience. Both procedural skills and highlevel knowledge are acquired through interaction with the world. The idea is that learning can be bottom-up: concepts and generic knowledge can be extracted on-line during interaction with the world, from low-level knowledge that is acquired through exploring the world. This idea is different from traditional top-learning learning theories in cognitive science, in which generic knowledge is given and then compiled into procedural skills through practice.

3. Prof. L. Goldfarb describes a fundamentally new mathematical model for inductive learning--the evolving transformation system. Although the model unifies the classical vector space and symbolic models; it suggests that the latter two classical paradigms are fundamentally inadequate to model inductive learning processes, which Goldfarb believes to be the central cognitive processes. The model also suggests, a simple formal mechanism responsible for the emergence of "fuzziness."

4. Dr. L. Perlovsky introduces modeling field theory, a model-based neural network whose learning is based on fuzzy a priori models. The modeling field theory combines a priori knowledge with adaptation and with structural learning. He also discusses interrelationships between various mathematical concepts of learning and their surprisingly close connections to metaphysical concepts of mind developed by philosophers since Plato and Aristotle. Future research directions include the development of multi-level hierarchical models of recognition in the context of recognition-behavior loop.

5. Prof. L. Levitin presents a dynamic model explaining Zipf's Law. Zipf's Law is a remarkable relationship observed in many complex systems of surprisingly different nature, including population growth and linguistics. For example, in linguistics the frequencies of the word usage are approximately inversely proportional to their ranks in decreasing frequency order. Levitin discusses an evolutionary model of emergent classification of objects into classes corresponding to concepts and denoted by words. The model leads to the emergence of a hierarchical two-tier structure with "superclasses" - groups of classes with almost equal populations. This model suggests that both, evolution and learning are based on a-priori concepts, while creating new concepts (new species and genera) is a relatively rare process. Future research directions include determination of the parameters of the model for various systems, for example of linguistic models of discourse in various areas and analysis of the relationships of model parameters and meanings of texts.

6. Does evolution of species resemble the process of individual learning? Is it random or directed? What is the role of a priori information coded in DNA in determining the direction of evolution and what mechanisms this process may employ? Prof. M. Lane discusses intergene variations of the minimal energy required for mutations and their roles in directing the evolution process.

7. What is intelligence? Is it possible to act intelligently without being intelligent? This question has been amplified in Searle's "Chinese Room" mental experiment. The nature of intelligence is addressed by Prof. L. Kanal. He discusses which type of intelligence can be now simulated by machines and what is truly "being intelligent?" 


\section{THEORETICAL ISSUES OF APPLIED SEMIOTICS}

- The main theme of the workshop on Theoretical Issues of Applied Semiotics was to further advance the existing theoretical premises as far applying semiotic methodology is concerned.

- Logical and philosophical component in the theory of semiotics should incorporate the relevant results obtained in the areas of mathematical logic since Peirce, as well as the views of the philosophers of our time.

- Application of semiotics is now focused upon a number of large complex ("open") systems.

- The following issues were discussed in detail:

1. In this paper, V. Stefanuk showed an example of, how ideas of semiotic sign expressed in the form of Frege triangular diagram, including the concept of similarity and multiresolutional approach, may be merged together to give a complete solution to the problem of discovery of good representation in a collection of problems, which are born by a famous AI puzzle.

2. Jan C.A. van der Lubbe showed the deduction, induction, and abduction were essentially the same logical procedures based on the Peircean notion of abduction. The difference emerges when based upon one (or a few) observations, we abductively construct a predicted value and the rule, which justifies the inference. In a pattern recognition task, he proposed to dynamically compare automatic abductive reasoning of the system constantly compare with the classification given by an export. This will greatly increase classification power.

3. Deborah Vakas Duong described a multi-agent simulation of a sample market of economics. The simulation showed an emergence of right decision on agent "investment" (trade or production). It also showed an emergence of a money-like property in this society. The author stressed that unlike general situation in AI, the social type models exhibit the emergence of new signs and their change in the process of social activity.

4. Leonid Perlovsky used a thoughtful survey of the greatest thinkers including Aristotle, Goedel, Turing, Penrose to show the genetics of complexity for a numbers of algorithms or predicates. Also, Perlovsky showed that the exponential combinatorial phenomena may be overcome by fuzzy logic and a multilevel artificial brain organization.

5. Mark F. Sulcoski, et. al., showed how various semiotic techniques should be combined together in a practical, military oriented problem. The authors stressed practical importance of Q-analyses, which allows representation of numerical relational data in a geometrical space.

6. Joseph Goguen described a mathematical framework which operates with signs in a programming domain. He showed that to describe the whole complexity of signs construction and transformation of one science system to another, one needs a model using the theory of categories. The presentation gave the ground for discussion at the end of the workshop. Another topic of an interesting discussion in which almost all people present in the workshop participated was understanding of the intrinsic complexity of signs in semiotics, their emergence, and their use in practical AI systems.

\section{SEMIOTIC ISSUES IN BIOLOGY, CONSTRUCTED SYSTEMS, AND SEMANTIC FOUNDATIONS OF MATHEMATICS}

The following issues were discussed at a group of workshops dedicated to biology, constructed systems and semantic foundations:

1. How do we develop systems engineering that can create and use 'specifications' for its human and artificial components? Alternatives were proposed: 
- Creating likable systems

- Next stage in notions of human-machine interfaces and human factors is required

2. The software analogy was useful for allowing cognitive science to get beyond strict American behaviorism. But can we invent better models for brain function that "manipulating representations" or "processing information?" We could then invent/learn new mechanisms/analogies for constructed systems.

3. How do we integrate, mesh different representations of the 'same' system?

- New ideas to understand the act of 'translation'

- For combining multiple sources of information/decisions

- Levels of representation

4. In many discussions, there was a strange tone of an incipient vitalism combined with an impoverished model of animal reasoning capabilities (the worse of both worlds). One way to examine these prejudices is to examine the concept of "reducibility". We started with the argument that intentionality is irreducible.

5. Intelligent living systems are emotional, social, and physically grounded. What role do each of these loosely described qualities play in what we call intelligence?

- Social, negotiated context, meanings, and understanding

- Emotional, caring, instructional, attentive systems

- Physically embodied, situated systems.

How do we represent/model?

6. Phantom limbs, "Neuralmatrix," skill acquisition, e.g. "knowing" how much you know, how well you know it, irreversible quality of knowing (can 'forget' but one doesn't return to the same ignorance) challenge present models of what it is to know and how it is represented.

7. We want to improve our models and mathematics with improved semantics. We want to create "shareable" semantics for combined human and automated systems. How do we need to alter current formal definitions of semantics?

In all topics above, mathematical issues were also addressed. This is the list of mathematical issues relevant to the themes of discussion:

- Integration Architecture in Constructed complex systems

- System Engineering (Processes and Artifacts)\& Validation

- Better Semantic Models of Systems, Behavior, and Time

- Computational Semiotics

- Treatment of Symbols by Computing Systems "Linguistic" Styles of Processing

- Cooperative Autonomous Systems (not control)

- Models of self and others in context

- Mathematical Methods for Self-Reference, Context and Situation

- Model Integration

- Mathematical Proof, Formality, Deductive Processes. 


\section{SYNERGISM/INTEGRATION}

1. The papers on attributes/classification for elements of fuzzy sets correlated very well with the paper by Y. Yufik who set forth a classification of neuron sets. Y. Yufik also noted some phenomena that resulted from or affected the human under stress.

2. P. Kugler's paper was seminal because he identified a major area of research in semiotics that has been obscured by the heavy attention to models, logic control and computation. This is because none of the above can be done without measuring the world and for open systems the major measurement component is the human.

3. S. Vahie/B. Ziegler's and N. Farhat/E. D. M. Hernandez'es papers were very important because they convey a promise that we may be able to create neural laboratories to establish new measurement systems.

4. Finally F. Brown's paper on logistics is promising; it demonstrates a computing language capable of handling massive amounts of information.

5. George Klir is on the path to integrating probabilistic, plausibilistic, and possibilistic into a reasoning integrated system.

6. D. Casasent's paper contained excellent results in the area of images fusion.

7. P. Werbos' paper on neural nets, semiotics and brain-like intelligence presented an issue of the existing duality: computational vs logic.

8. The triangular representation of triadicity was not used by C. Pierce. He used a prong

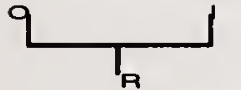

Burch showed that the prong was not reducible. The triangle $\Delta$ can be broken into a dyad $\langle\wedge\rangle$ and should be avoided to prevent a wrong conclusion.

8. L. Domatova's paper on combining models presented an application of information fusion.

\section{OPERATORS MODELING AS A SEMIOTIC PARADIGM}

The workshop has addressed the following issues:

1. Methods for designing on-line learning systems for acquiring diagnostic knowledge from manufacturing systems.

2. Methods for estimation of unmeasurable process variables by using fuzzy models of human operators.

3. Synthesis of supervised control strategies based on spatial aggregation theory.

4. Computational tools for diagnostic hypothesis generation. 
The conference ISAS'96 had a several plenary lectures and a tutorial.

The plenary lectures:

- J. Albus, A Roadmap to the Future of Intelligent Systems

- P. Antsaklis, Hybrid Control of Intelligent Systems

- T. A. Sebeok, Evolution of Semiosis and the Origin of Languages

- L. Zadeh, The Key Role of Information Granularity in Human Intelligence and the materials of tutorial:

- A. Meystel, Applied Semiotics: Theory, Methodology, Toolbox will be used for preparation of the White Paper. 
AUTHOR'S INDEX 



\section{AUTHOR'S INDEX}

A

Agre, Gennady 199

Albus, James $\mathbf{S}$. $3,75,151,531$

Alexander, Jr., John R...............................2239

Anlide, Cesar .................................................. 137

Anshakov, Oleg M .........................................87

$\boldsymbol{B}$

Baev, K.V. 499

Baggenstoss, Paul M...................................124

Balasubramanian, S....................................225

Barber, K. Suzanne ....................................263

Barnhart, Craig M.....................................175

Bellman, Kirstie L......................... 205,422,567

Brito, A.E.................................................157

Brooks, Bernard G. ...................................2275

Brown, Frank M.............................. 103, 252

Burke, Louis ................................................ 130

C

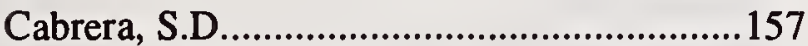

Casasent, David............................................57

Cassandras, Cristos G. .................................173

Chandler, Jerry L.R. .....................................211

Chen, Chun-Hung ................................. 185, 191

Chikrii, Arkadii A........................................443

Chikrii, Greta Ts.........................................450

Cotsaftis, Michel ..........................................517

D

Dai, Liyi

185,191

Davidson, Ian

DeClaris, $\mathbf{N}$.

Dmitriev, Victor ................................................. 333

Dolmatova, Ludmila M. .................................66

Donohue, Karen .............................................185

$\boldsymbol{E}$

Egbers, Emile

Ephremides, Anthony

$\boldsymbol{F}$

Ferregut, Carlos.........................................378

Fetzer, James H. .........................................493

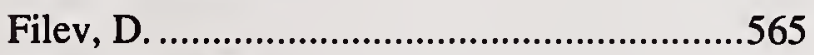

Finn, Victor K....................................8. 87, 92

Fogel, David...............................................2233

Fogel, Lawrence J......................................59

Frank, Rob ................................................395

Freeman, Walter J............................421, 487
G

George, Mary J. ........................................ 378

Goguen, J................................................. 567

Goldfarb, Lev................................................ 111

Golovina, E. Yu............................................ 50

Gomide, Fernando ...................................... 477

Gudwin, Ricardo........................................ 477

Gutierrez, Jose M....................................... 378

H

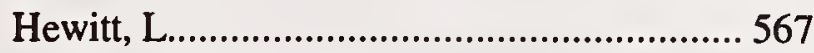

Ho, Yu-Chi ................................................. 12

Hofkirchner, Wolfgang .............................. 248

Hunt, Ken ..................................................... 391

Hutchison, William R............................... 287

$J$

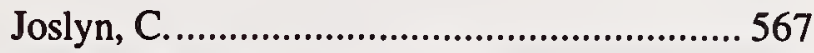

Juliano, Benjoe A.......................................... 313

$\boldsymbol{K}$

Kadtke, Kim ........................................... 349

Kamensky, Victor ...................................... 130

Kim, Eunjin............................................... 31

Klimontovich, Yu. L..............................14, 364

Kohout, Ladislav J.................................31, 567

Koshelev, M. .............................................. 383

Kosheleva, O. M. ........................................ 158

Kreinovich, Vladik ......................145, 378, 383

Kulikowski, Casimir .................................. 130

Küttner, Lars ............................................. 281

Kuznetsov, S.O..........................................96

$\boldsymbol{L}$

Lacaze, Alberto

$75,117,151,477,523$

Landauer, Christopher ............ 44, 205, 422, 567

Lau, T.W. Edward ........................................ 181

Lefebvre, Vladimir A.................................. 455

Lenhart, Suzanne ........................................ 349

Lewis, David................................................ 395

Linkins. Dennis ......................................... 395

$M$

Maturana, F............................................... 225

McKee, Gerard T......................................... 275

Merrell, Floyd................................................ 339

Meunier, Jean-Guy....................................... 325

Meystel, Alexander M. ............. 75,117,145,151,

477,505,523,567

Mikheyenkova, M.A. ................................... 6 
Modell, Mark

.130

Muchnik, llya

$N$

Nault, Georges...............................................325

Neves, Jose ....................................................... 137

Niloff, Jonathan........................................... 130

Nishina, Hiroyuki ........................................471

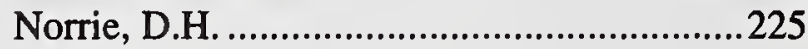

0

Osegueda, Roberto..........................................378

Oussedik, Sofiane

\section{$P$}

Panayiotou, Christos G.

Péntek, Áron 349

Perlovsky, Leonid I. $295,307,319,332,567$

Protopopescu, Vladimir .349

Prueitt, Paul S

\section{$\boldsymbol{R}$}

Rialle, Vincent 325

Rieger, Burghard B. $.331,541$

Ritter, Gerhard X.

\section{$S$}

Samad, Tariq .511

Schenker, Paul S 275

Schnelmer. Tim .252

Sebeok, Thomas A .354

Skvortsov, Dmitry P.

Smirnov, Evgueni. 137

Souza, Claisse Sieckenius de 461 Starks, S.A.
Stefanuk, V............................................ 567

Stilman, Boris .............................................. 552

Stoica, Adrian.............................................. 244

Stopp, Andreas ........................................... 281

Streit, Roy, L. ........................................... 301

Sussner, Peter .............................................. 60

$T$

Tadeusz, Szuba ........................................... 401

Tarassov, Valery ........................................ 162

Tasoluk, C. ............................................... 478

Tsoukalas, Lefteri H...................................... 434

U

Uhrig, Robert E. ....................................... 434

Ulieru, Mihaela

V

Vagin, V.N................................................... 50

van der Lubbe, Jan C.A. ............................... 37

$W$

Wang, Paul .................................................. 415

Weiss, Joe.................................................... 395

Werbos, Paul J. ...................................... 66, 82

Wieselthier, Jeffery E................................... 175

$Y$

Yucesan, Enver.............................................. 185

Yufuk, Y ................................................... 567

\section{$Z$}

Zabezhailo, M.I. ........................................... 100

Zadeh, Lotfi A............................................. 26 
LATE SUBMISSIONS 



\section{A Neural Iconizer for \\ Semiotic Processors}

\section{H. Jahn Caulfield}

Northeast Photosciences, Inc.

4626 Delina Road

Comersville, TY 37047

jcaulfield@vallnet.com

\section{Abstract}

A PCNN (Pulse Coupled Neural Network) is shown to moet all of the necessary needs of a semiotic processor for turning sensobr data into symbals or icons. At the same time, the single solution to the most long-lasting and vexing philosophical problems in cognitive science. These analyses lead us to spoculate that human symbalic processes which we designed computers to emulate (e.g. Boole's Laws of Thought) were alonost certainly based on an earlier human facility-semiotic processing.

\section{Introductian}

Semiotic processors deal with conncepts and symbols not numbers. Humans are inherently symbol makers, while modern, Turmg-equivalent, digital cnmputers are inherently symbol manipulators.

Dick's ago is twice Jano's. Dick's age (in years) plus Jane's age (in years) is 36 years. To solve this in a computer, we say

symbol $\{$ Let x symbolize Dick's ago, generation ( Let y symbolize Jane's age.

$$
\left.\begin{array}{l}
x=2 y \\
x+y=36
\end{array}\right)
$$

$$
\text { Thus } \begin{aligned}
& \text { symbal } \\
& x=24 \\
& y=12
\end{aligned}
$$

Symbol
Interpretation $\begin{gathered}\text { Thus Dlec is } 24 \text { yoars old. } \\ \text { Jane is } 12 \text { years old }\end{gathered}$ The total system (symbolization symbol manlpulation - resymbolization) is semiotic. The symbol manipulation is not.

Ta date most if not all semiatic processors have incorporated humans to symbolize resymbolize. Because that incorporation is universal, it passes unnoticed most of the time.

If we are to have fully-semiotic processors, we must replace
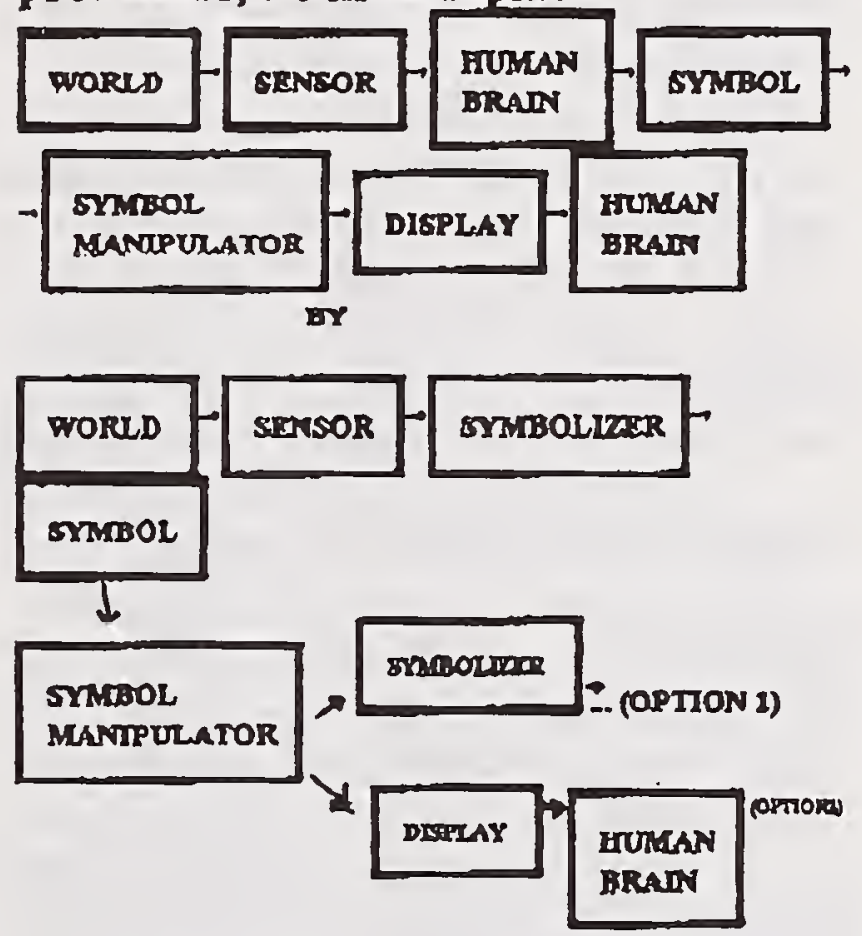

The full semiotic processor has no need of - human brain but allows human optiomal manitoring.

My goal here is to discuss symbolizers for semiotic processors. II. SYMDOLIZER REQUTREMENTS

The symbolizer converts sensed data about the natural and the technological wrorld and producos from those data symbals for further manipulation It is ungent to understand this powerful fumetionally of the human. brain before we try to build it.

Remack 1. The number of situatians wo may oncoumer is finito but effechivelyinfinite in the sense that look up tables are precluded in erincipie. Typical 2D spatial patterns csiptitin of the order of a million bytes per scene. Suppase re have a million pixels esch with 8 bits (128 shodos of gray) 
dynamic range. The number of possible scenes is then

$$
N=(128)^{1000000}
$$

This meets any derinition of effectivelyinfinithe.

Remark2. The goal of

symbolization is to convert any of the $N$ scenes to one of $M(<<N)$ symbols.

Symbolization is compressive. It involves a doliberate and irreversible loss of information to facilitate manipulation. For indtance, $M$ may be small enough to permit look up tables. As a human example, consider all of the scenes we might label with the word "dog." That set of scenes is itsolf elfectively infinite, yot very young, small humans do it routinely and inemntly.

Remark 3 There aro no God-given categaries in the world. Categories, up till now, have been human semiotic creations. The world and the images on our retinas simply are. Humans group them. We can make "self-orgeniaing" computers to group items according to human-selected criteria. But if we selected different criteris, the groups would be different. Canslder the figures below.

$$
\rightarrow \mathfrak{z}-5
$$

We may decide to put them in two groups (a. Muarn decision). Doos it make sense to ask how many groups are "really there"? Two obvious groupings are triangles/circles and big/small. The latter categories are valid but funzy. Is there a radius

$$
r \text {. such that circles with } r>r_{\text {, are }}
$$

big and circles with $p<p$. aro small?

Who chooses ? But thore are many

other valid groupings, for excample, figures with centroids above and below the centroid of the whale. All of these are "right" answers in the sense that they reduce $N=12$ figures to $M=2$ groups. None is more right than the other.

Remark 4. Utility is one way to select which symbolization ar categorization to choose. At least for the immediate future, artificial semiatic processors will be special purpose machbes. Gromphags reflecting our a prioci lmowledge of the dnta and problem damains will be preferred.

Remark5. Symbals must emphasize the relevant and ignore the irrelevant - cnce we decido what is and is not relevant If we want to classify the figures above as big or small, we want to tgnore their shapes.

Remack 6. Combining Remarks 4 and 5 , we seek "robust" symbolization in some problem-dependent sense. III. Philosophy of Symbolization

Semiotics can not avoid philosophy. As seen from Sec. I, semiotic computers invado a domain proviously forbidden to "mere" symbol-manipulating computers. Just analyzing the meaning and Implications of the previous sentence is a major undortaking.

Consider, for example, the strong version of Church's thesis (almost universally belleved and nelther proved nor disproved). Roughly, it says this: "Anyy physical process can be modeled to arbitrary accuracy with any digital computer having sufficient memory." But all digital camputers are just symbal manipulators. Given an input (data and instructions which aro human concopts not distingulshable by the computer), there is a unique output Every computer is equivalent to a giant look up table. Symbols (to tho computer) do not "mean" anything. That is the symbols do not refer to anything other than thomsolvos. 1 means 1. 0 means 0 . Nothing in a computer means anything or is a symbol 
for anything. Yet we humans mean things by our symbols and yet our brains are computers and subject to computer manipulation. Where does meaning enter the world?

In "cagnitive science," the question of symbolization is itself symbalized by Searle's story of "The Chinese Room." To most people outside that field, this story seems to add nothing to their understanding. The ultimate problem is this: "If computers mean nothing and if the human brain is a coxoputer, then the human brain means nothing. But humans do mean things. So how can these contradictions be accounted for?" Soarlo and othors seom to feel that a true semiotic computer (one that symbolizes things and associatos symbols in such a way that each "means" the other) cannot be based on current digital computers. We humans are somehow more than "just computers." My reply is computers can be far more than Searle envisions "mere computers" to be. Enter the semiotic computer.

N. A Spatial Pattem Symbolizer If the useful grouphing is spatial patterms (e.g. triangles and circles) or can be represented that way (e.g. a wavelet transform of a spoken word displayed as a 20 pattern), then "robustness" may be taken as the ability to ignore mild distartions, afine transformations, and the like.

If, in addition, we do not want to train the symbolizer, because we do not know what patterns it may encounter; then we want a symbalizer which is cantractivo, $(M<<N)$ for all possible input pattems.

We have experimented with PCNNs (Pulse Coupled Neural Networks) as symbolirers. We find that they are contractive for all inputs and extremely robust

Invented by Eckhom in Germany to omulate the observed behavior of the neurans in the visual cortex of cats, PCNTY offorts in America have been led by J.
Johnson at the U.S. Army Missile

Command A special issue of IEEE Transactions on Neural Networks edited by Dr. Johuson will be published in 1998. For now, however, the best way to leam more sad to use PCNNs is to access the web page of the author's previous employer (www.caos.anmu). The code is down lagdable or usable there.

The basic idea is that each input pirel drives its own neuron. The nearon is a classic intograto-fire-reset oscillator, so neurans driven by brighter pirels pulse more frequently. Then we perturb the situation by allowing each neuran to influence its neighbors. Regions of morear-less equal brightness begin to synchronize - beat together. What results is a complex $3 \mathrm{D}$ pattern (2D in spece, $1 D$ in timo). As we seek information compressian, this seems to carry us in the wrang direction

Suppose, however, we integrate that $3 D$ signal over $2 D$ space leaving only a $1 D$ time signal Experimentally, that time signal has a poriodic strange attractor - a pattern which more-or-less repeats indefinitely but nover exactly repeats. We call a single period of that time signal the uicon" of the imput pattern. Million bit patterns lead to very simple, e.g. 10position icons. You can see and create icons at the previously-noted URL.

Another experimental proporty of the icons is that they are quite robust against mild distortions and affine transformations nearly always. Yet there are sharp boundaries between attractor basin. Consider our running exrample of triangles and squaros. We can distort each into the other, e.g.

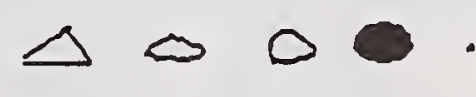

There is a very sharp dividing line between the attractor basin to which the "perfect" triangle bolongs and the aftractor basin to 
which the "perfect" circle belongs.

Note that the PCNN has attractors for triangles and circles even though it was not trained to recognize thom. If we want to racognize triangles and circles, all we have to do is recognizo (namo) the attractors.

In the Jewish/Christian/Mraslim story of Adam (man) and Eve (woman), God's first assignment to them was to name the animals. In PCNN terms, this may say wuch about the origin of language - a deep, unsolved problem which bears strongly on the uniqueness of humanos. Suppose our DNA directs the formation of sight and souvad PCNNs. We may come to recognize the sight of $2 \mathrm{dog}$ and the sound of the word "dog." If these two are linked by flebbian association, I am willing to assert that the sight comes to mean the sound and conversely.

It is true that a computer contemplating its own digits means nothine. But a special computer - a set of PCNNs - interacting with the world can came to mean things. The system - not just its CPU - and the warld come to "ground" meanings in reality. Thus yet another fundamental problem can be solved in principle. The PCNN may bo the rnissing link between the subsymbolic neural networks which we know are the basis for all brain functions and the symbolic processes you are using at this moment. The subsymbolic PCNN in interaction with the world produces a "starting set" of symbols on the basis of which symbalic processing arises.

\section{Conctussions}

I hope that I have convinced you that the following propositions are true,

(I) For ovents symbolizable in torms of spatial relationships, a PCNN is an iconizer which exhibits all of the prion desirable features of an iconizer.

(2) Thereione, the PCNN icanizer) for a semiotic processor.

(3) PCNNs offer a single, plausible answer to many deep, longstanding philosophical questions. 


\section{Information Needs And Its Impact On Medicine}

Thomas Hankins, CNM, C.P.

Douglas Frank, B.S., C.C.P.

Thomas Williams, M.D., Ph.D.

Until recently with the exception of the area of research, the only information that was pertinent to hospitals and practicioners was "How much do we bill and how much do we get paid". Now the medical community is quickly finding itself faced with a new threat of a double edged sword which many are not prepared to take on. That threat is Managed Care. Managed Care has and is turning the practice of medicine inside out. How does informational needs find itself smack in the middle of this battle? Every good general knows that the more you know about your enemy the better off you are and you never let your enemy know more about yourself than you know. That's where the informational needs fit in.

For years practicioners were always busy taking care of their patients the best way they could and usually having little more information on the patients than the patients medical record. While on the other hand the insurance carriers were busy keeping actuarial tables and different bits of information on large populations of patients. The insurance companies learned early on that "Those who control the information....control the game".

This paper will explore the tremendous impact information needs is having not only on the practice of medicine but its survival as well. 



\section{NIST-114 \\ (REV. 6-93) \\ ADMAN 4.09 \\ MANUSCRIPT REVIEW AND APPROVAL \\ U.S. DEPARTMENT OF COMMERCE}

Exix

INSTRUCTIONS: ATTACH ORIOIRN OF THIS FORM TO ONE (1) COPY OF MANUSCAIPT ANO SEND TO THE SECRETANY, APPROPHATE DOITORLL NIVIZW DOANO.

TTL AND SUDTIL (CTE IA PULU)

Proceedings of the 1997 International Conference on Intelligent Systems and Semiotics: A

Learning Perspective

\begin{tabular}{|l|l|l|l|l|}
\hline CONTAACT OR ORANT NUMDER & TYPE OF REPORT AND/OR PERIOD COVERED
\end{tabular}

AUTHOR(S) RAST MME, FIRST BHITLL, SECOHD INITLAL)

Meystel, A.M.

\begin{tabular}{|c|c|}
\hline EnEconTRDLUUDE & ONSIOH \\
\hline POLLCATIONASPOAT HUMDEA & catrophycoos \\
\hline
\end{tabular}

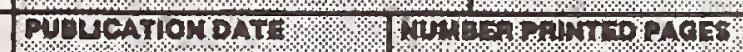

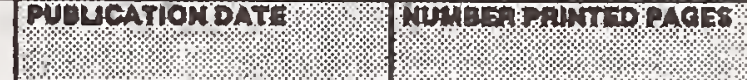

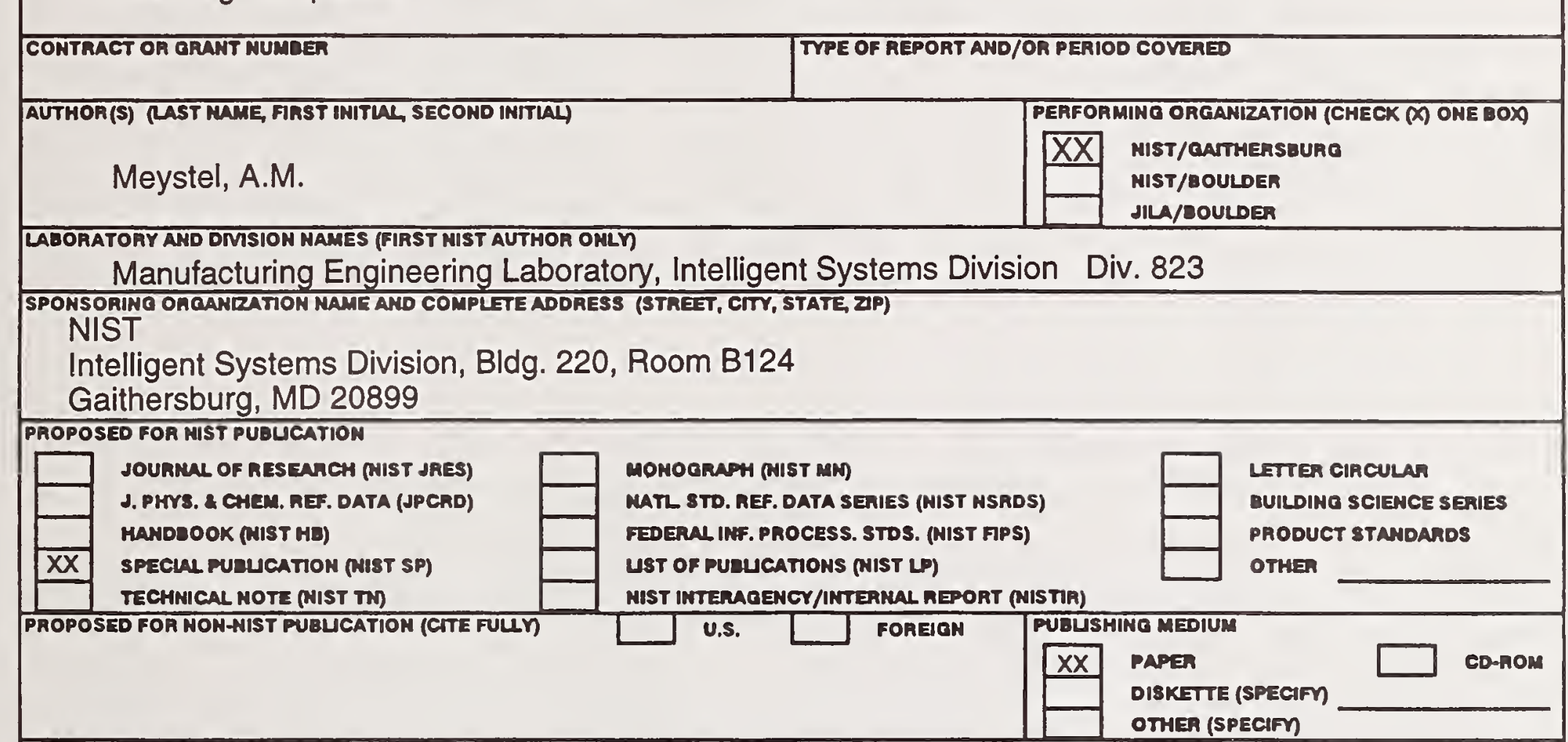

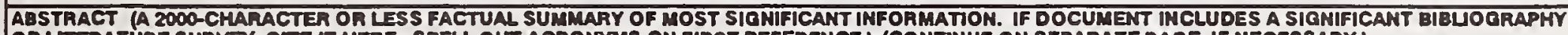
OR UTERATURE SURVEY, CTTE IT HERE. SPEU OUT ACRONVMS ON FIRST REFERENCE,) (CONTINUE ON SEPARATE PAOE, IF MECESSARY.)

This publication is the Proceedings of the International Conference ISAS'97, Intelligent Systems and Semiotics: A Learning Perspective. The Conference will be held at the National Institute of Standards and Technology, Gaithersburg, Md. from Sept. 22-25, 1997. The Proceedings include a list of participants, foreword, and the 83 papers written for presentation at the conference.

The papers are arranged within 20 themes: (I) Plenary Lectures (II)World Representations, (III) Neural Networks-Models, (IV)Logical Calculi for Semiosis, (V) Learning, (VI) Multiresolutional Semiosis, (VII) Ordinal Optimization, (VIII)Architectures, (IX) Evolution, (X)Autonomy, (XI)Likelihood, Mixture, and Symbols, (XII) Semiotics of Texts and Images, (XIII)Multidisciplinary Applications of Semiotics, (XIV)Semiotics of Open Systems, (XV) Soft Modeling, (XVI)Games and Reflexion,

(XVII)Computational Approaches in Semiotics, (XVIII) Brain, Mentality, and Meaning, (XIV)Semiotics in Intelligent Control, (XX) ISAS'96 Previously Unpublished papers

KEY WORDS (MWOMUM OF $9 ; 28$ CHARACTERS AND SPACES EACH; SEPAUTE WITH SEMICOLONS; ALPHABETIC ORDER; CAPITALIZE ONLY PROPER MUMES) brain; decision making; information; intelligent control; intelligent systems; knowledge representation; learning; manufacturing; open systems; optimization; planning; pragmatics; semantics; semiotics; symbol grounding; syntactics;

\section{AVALAOILTY}

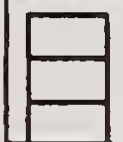

UNUMTTED $\square$ FON OFFCLA DISTRIDUTION - DO NOT RELEASE TO MTIS ORDER FROM SUPERINTENDENT OF DOCUMENTS, U.S. OPO, WASHINOTON, DC 20402 ORDER FROM NTIS, SPRINOFIELD, VA 22161

MOTE TO AUTHOR(S): IF YOU DO NOT WISH TMIS WWUS CRIPT ANMOUNCED BEFORE PUDUCATION, PLEASE CHECK hERE 








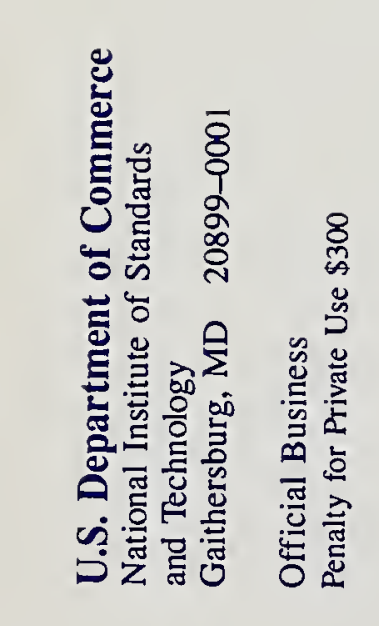

